\title{
DRUG-ACCEPTOR INTERACTIONS
}

Modeling theoretical tools to test and evaluate experimental equilibrium effects

\section{Niels Bindslev}

Department of Biomedical Sciences, Faculty of Health Sciences, University of Copenhagen 
First published 2008 by Co-Action Publishing

Published 2017 by Routledge

2 Park Square, Milton Park, Abingdon, Oxon OX14 4RN

711 Third Avenue, New York, NY 10017, USA

Routledge is an imprint of the Taylor \& Francis Group, an informa business

Copyright (C) 2008 by Niels Bindslev

The Open Access version of this book, available at www.tandfebooks.com, has been made available under a Creative Commons Attribution-Non Commercial-No Derivatives 3.0 license

Notice:

Product or corporate names may be trademarks or registered trademarks, and are used only for identification and explanation without intent to infringe.

Typeset by Datapage Ltd, Dublin, Ireland

Cover design: Julie Bindslev (with due permission from Scala Archives to reproduce Bernini's Rape of Proserpina)

Every possible effort has been made to ensure that the information contained in this book is accurate at the time of going to press. No responsibility for loss or damage occasioned to any person acting, or refraining from action, as a result of the material in this publication can be accepted by the author or the publisher.

ISBN: 978-91-977071-0-7 (pbk) 


\section{Contents}

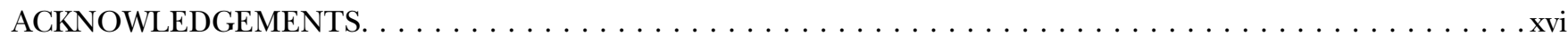

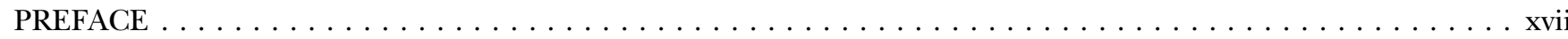

Part I ONE-STATE MODELS: SIMPLE AGONISM AND ANT-AGONISM $\ldots \ldots \ldots \ldots \ldots \ldots \ldots \ldots$

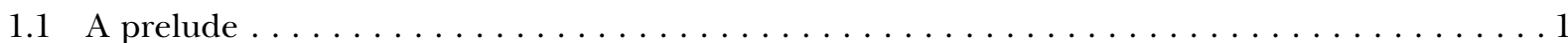

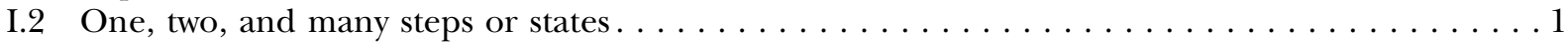

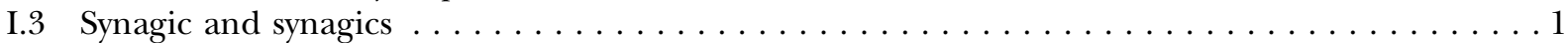

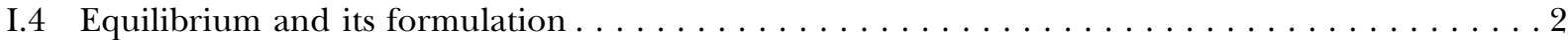

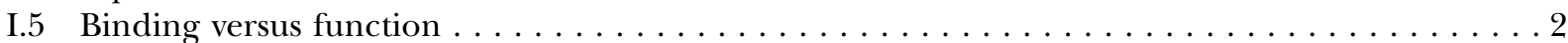

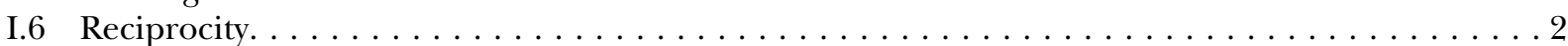

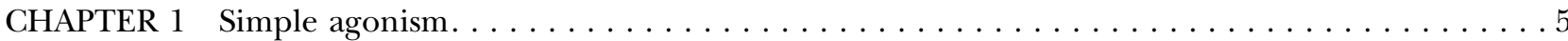

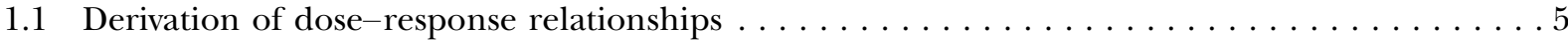

1.1.1 The binding proper of a ligand to a receptive unit $\ldots \ldots \ldots \ldots \ldots \ldots \ldots \ldots \ldots \ldots \ldots$

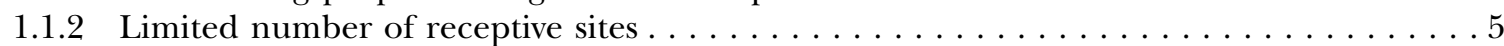

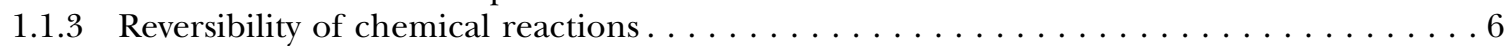

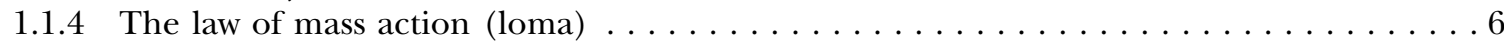

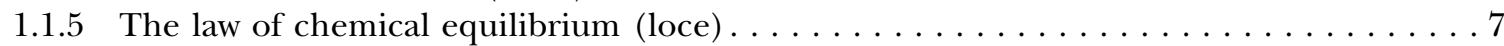

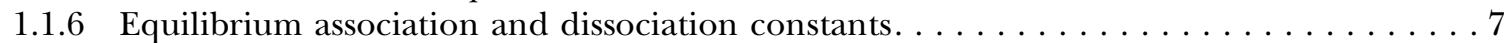

1.1 .7 Rules of regula detri $\ldots \ldots \ldots \ldots \ldots \ldots \ldots \ldots \ldots \ldots \ldots \ldots$

1.1.8 Langmuir's law of adsorption and desorption $(\mathrm{load}) \ldots \ldots \ldots \ldots \ldots \ldots \ldots \ldots$

1.1 .9 The Henri-Michaelis-Menten equation $(\mathrm{HMM}) \ldots \ldots \ldots \ldots \ldots \ldots \ldots \ldots$

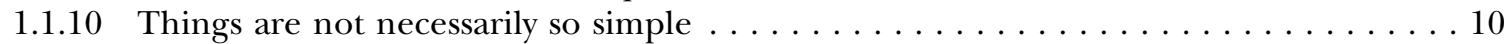

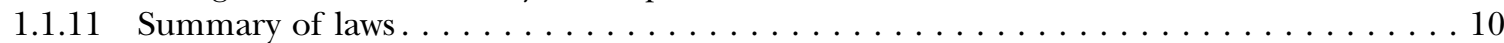

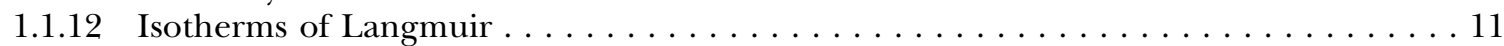

1.1.13 Plotting and analyzing simple synagic relationships. Parameters to be determined . . 11

1.1.14 Intermezzo on signal transduction by conformational induction or selection . . . . 12

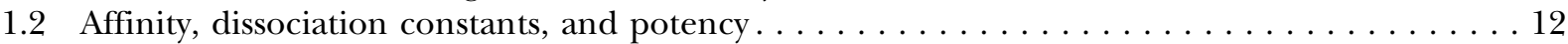

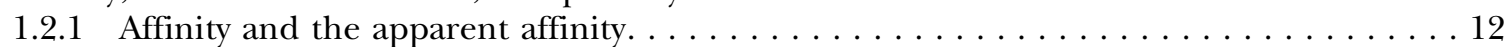

1.2.2 Dissociation constants and free energy change. . . . . . . . . . . . . . . . 12

1.2.3 Microscopic reversibility and detailed balance . . . . . . . . . . . . . . . . . . 13

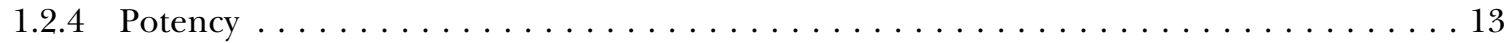

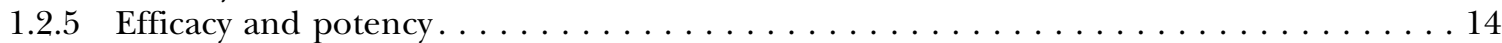

1.3 Partial agonists, efficacy, and spare receptors $\ldots \ldots \ldots \ldots \ldots \ldots \ldots \ldots \ldots \ldots \ldots$

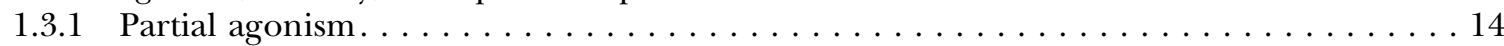

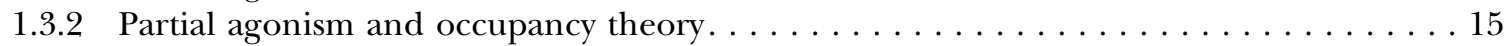

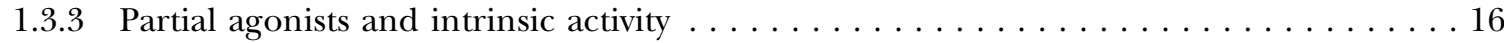

1.3.4 'Stimulus', 'efficacy' and partial agonism ad modum Stephenson . . . . . . . . . . . 17

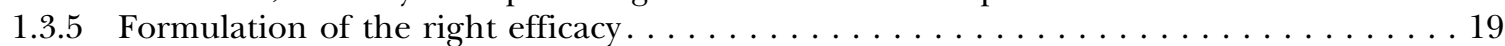

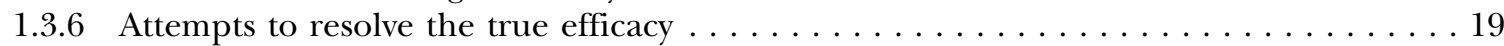

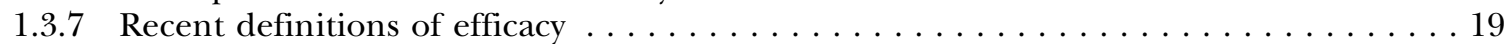

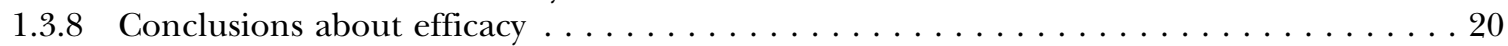

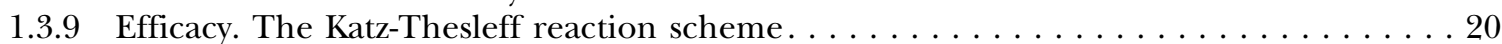

1.3 .10 Receptor reserve . . . . . . . . . . . . . . . . . . . . . . . . . . . 20 
1.4 The post-classic era. Allostery and two-state models. . . . . . . . . . . . . . . . . . . . . . . 22

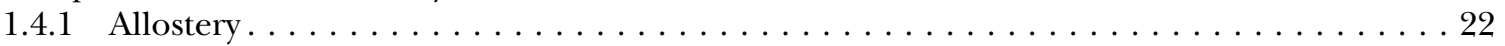

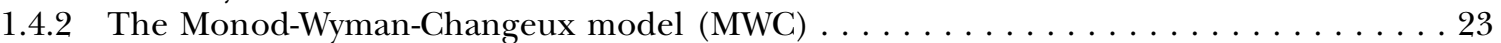

1.4 .3 The Koshland-Nemethy-Filmer (KNF) or sequential model . . . . . . . . . . . . . 23

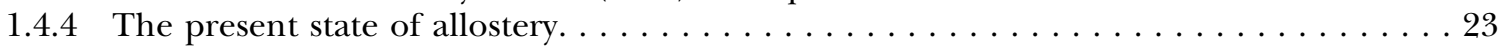

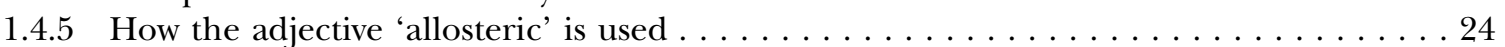

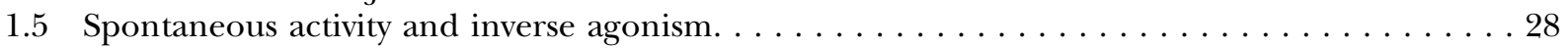

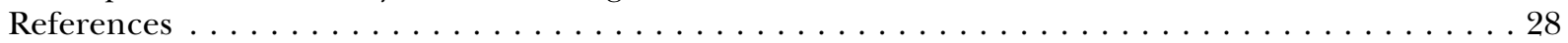

CHAPTER 2 Simple ant-agonism - simple intervention $\ldots \ldots \ldots \ldots \ldots$

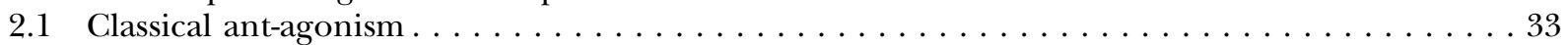

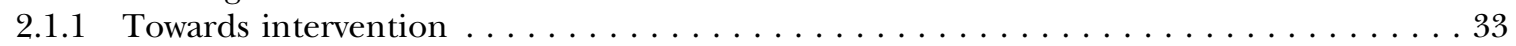

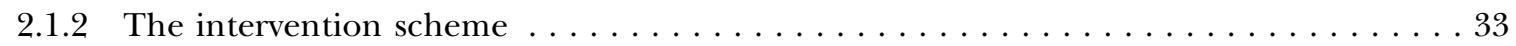

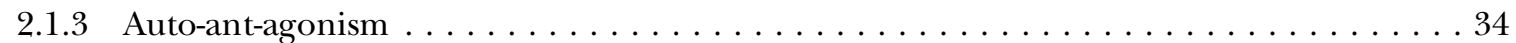

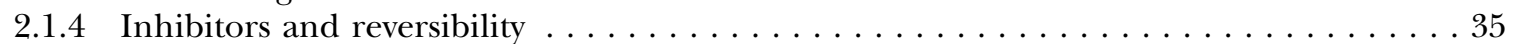

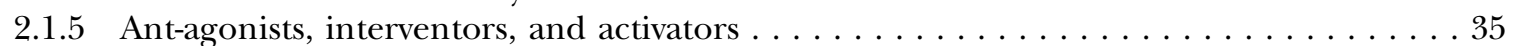

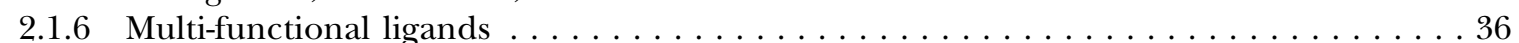

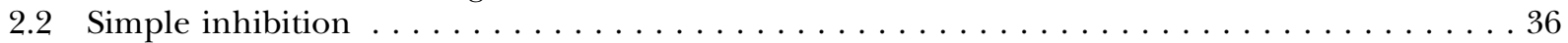

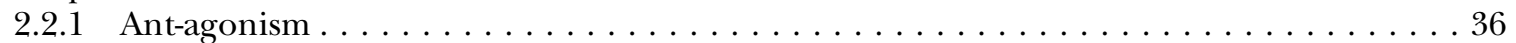

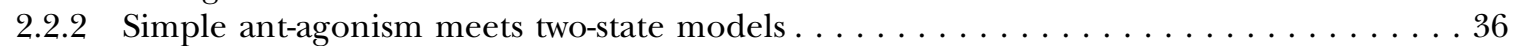

2.2.3 The 'full response' obtained at a fixed concentration of an agonist . . . . . . . . . . 39

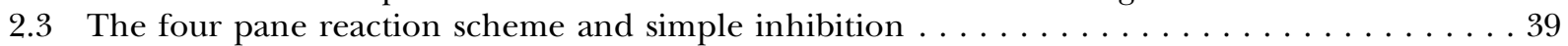

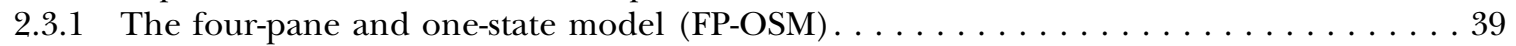

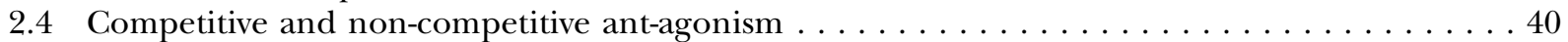

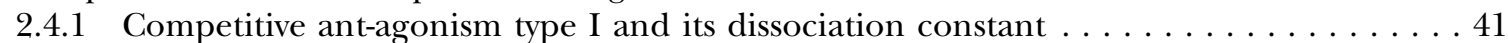

2.4.2 Competitive ant-agonism type II and its dissociation constant. . . . . . . . . . . 41

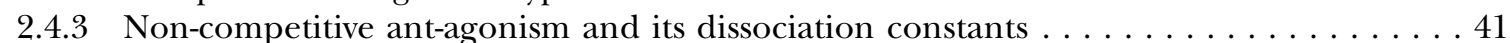

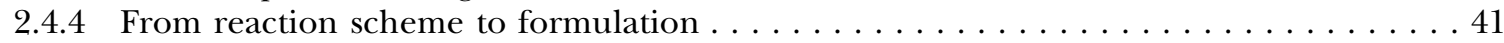

2.4.5 Formulation of functional non-competitive ant-agonism . . . . . . . . . . . . . . 42

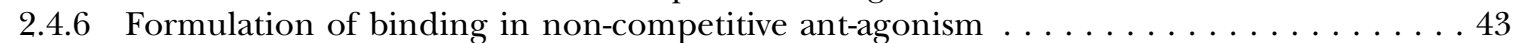

2.4.7 Actual remaining activity (ara) in non-competitive inhibition . . . . . . . . . . . 43

2.4.8 The actual inhibited response (air) in non-competitive inhibition . . . . . . . . . 43

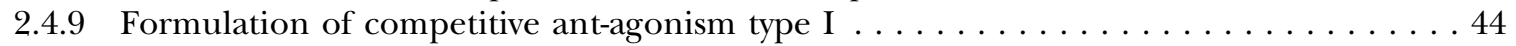

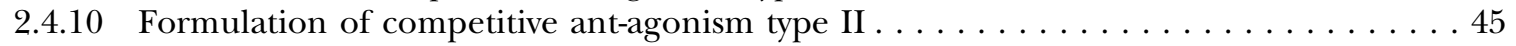

2.4.11 Actual remaining activity and actual inhibitory response

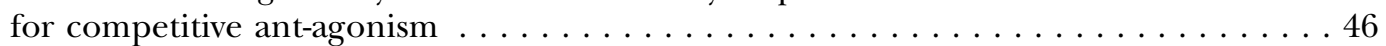

2.4.12 Competitive versus non-competitive studies. A summary . . . . . . . . . . . . 47

2.5 On simple ant-agonism and simple intervention $\ldots \ldots \ldots \ldots \ldots \ldots \ldots \ldots \ldots \ldots \ldots$

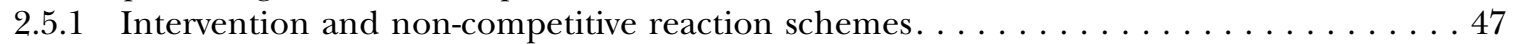

2.5.2 Switch from 'ant-agonist' to 'interventor' in binding and function . . . . . . . . . . . 48

2.5.3 Summary of parameter characteristics from simple intervention . . . . . . . . . . 5 50

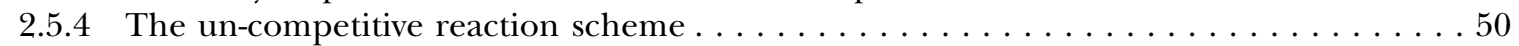

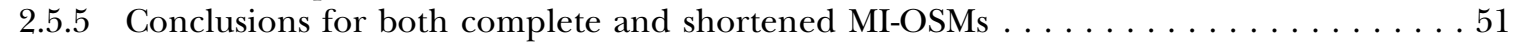

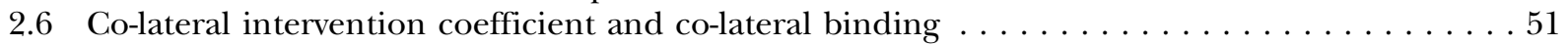

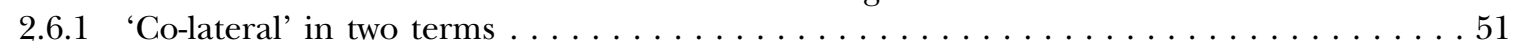

2.6.2 Co-lateral intervention constants and auto-intervention . . . . . . . . . . . . . 52

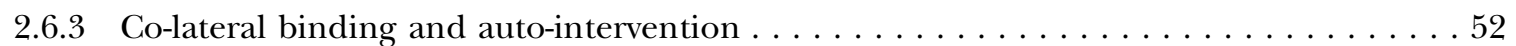

2.6.4 Co-lateral binding, co-lateral intervention and the HOTSM/ATSM . . . . . . . . . . . 53

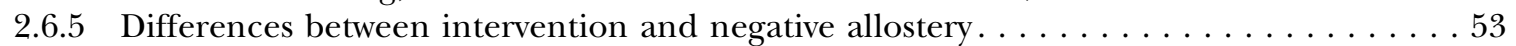

2.7 A history on the development of ternary-complex models $\ldots \ldots \ldots \ldots \ldots \ldots \ldots \ldots$

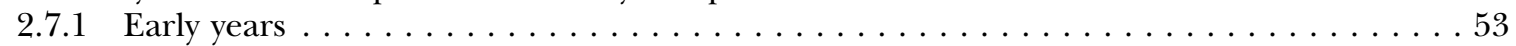

2.7.2 Terminology for the intervention scheme $\ldots \ldots \ldots \ldots \ldots \ldots \ldots \ldots \ldots \ldots \ldots \ldots \ldots \ldots \ldots$

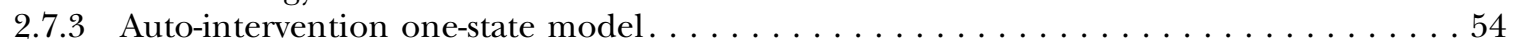

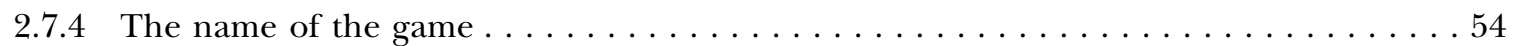

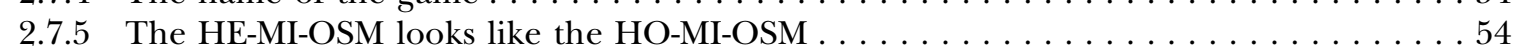




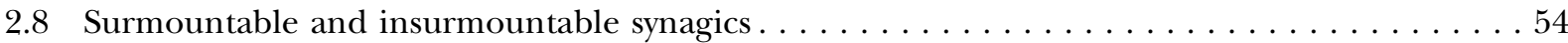

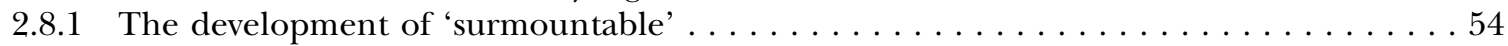

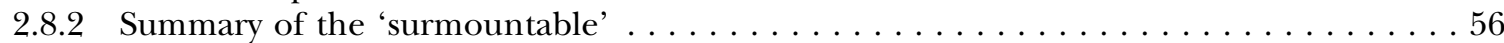

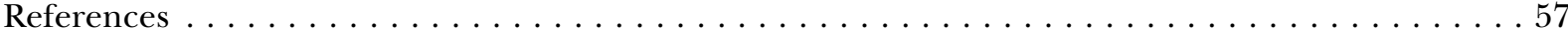

CHAPTER 3 Auto-inhibition and auto-intervention in one-state models $\ldots \ldots \ldots \ldots 1$

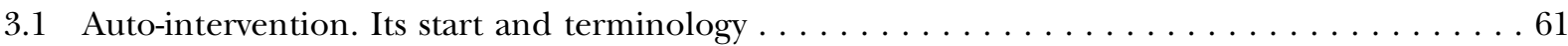

3.1.1 The start of formulating ligand-dependent auto-inhibition . . . . . . . . . . . . 61

3.1.2 A one-state model to analyze ligand-dependent auto-inhibition . . . . . . . . . . . . 62

3.1.3 Auto-inhibitory concentration-binding and dose-response relationships . . . . . . . 62

3.1.4 Auto-intervention may yield bell-shaped synagics and changes in apparent $K_{\mathrm{d}} \ldots \ldots 63$

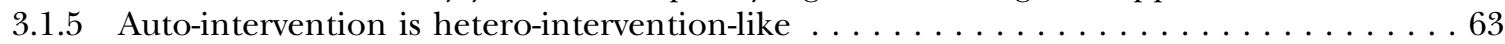

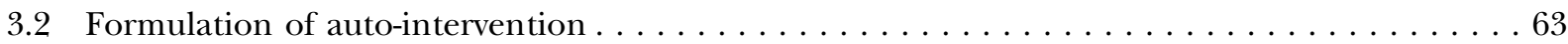

3.2.1 Equations of distribution for simple auto-intervention $\ldots \ldots \ldots \ldots \ldots \ldots \ldots \ldots \ldots \ldots$

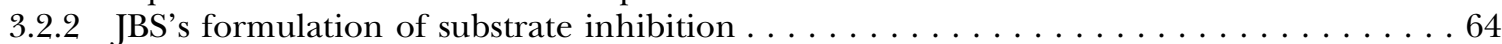

3.2.3 A more complete model for auto-intervention in functional assays . . . . . . . . . . . 64

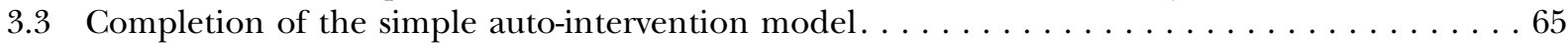

3.3.1 Auto-intervention for function with intervention constant $c^{\prime} \neq 1 \ldots \ldots \ldots \ldots$

3.3.2 Other model approaches to experimentation yielding Bell-shaped synagics. . . . . . . 66

3.3.3 Auto-intervention for binding with intervention constant $c^{\prime} \neq 1 \ldots \ldots \ldots \ldots$

3.3.4 Positive auto-intervention in functional studies $\ldots \ldots \ldots \ldots \ldots \ldots \ldots$

3.3.5 Conversion of hetero-intervention into a one-state substrate inhibition model . . . . 67

3.3.6 Switch of hetero-intervention in 3-D to auto-intervention in 2-D . . . . . . . . . 68

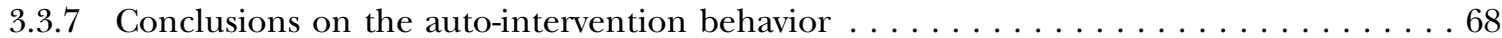

3.4 The 'low-dose hook effect' . . . . . . . . . . . . . . . . . . . . . . . . . . . . . . . . . . 69

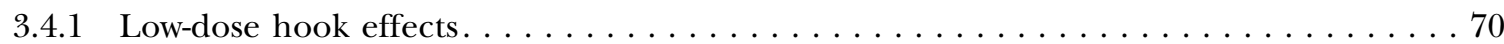

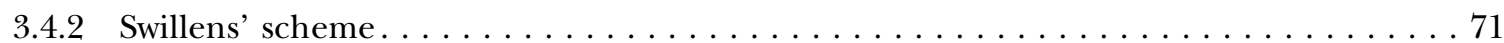

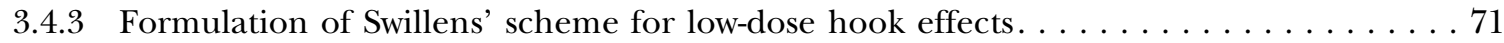

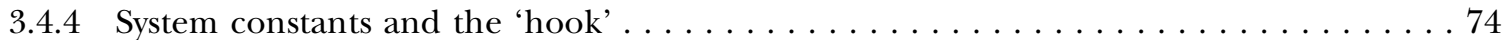

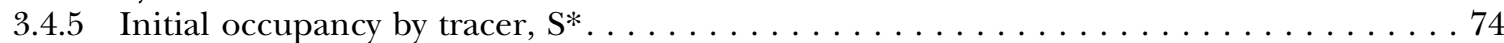

3.4.6 Virtual observations for $\mathrm{A}_{\mathrm{ss}}$ and $\mathrm{A}_{\mathrm{is}}$ and their related sites $\ldots \ldots \ldots \ldots \ldots \ldots$

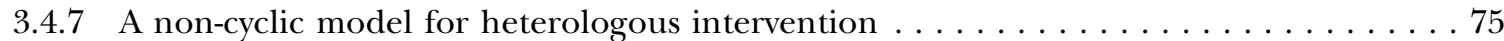

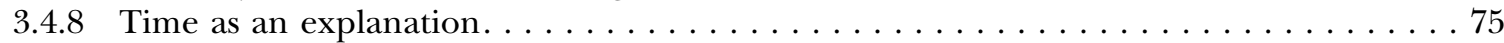

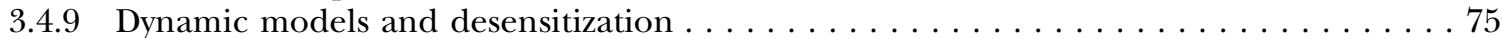

3.5 Prelude to biological auto-regulation $\ldots \ldots \ldots \ldots \ldots \ldots \ldots \ldots \ldots$

3.5 .1 Self-reference $\ldots \ldots \ldots \ldots \ldots \ldots \ldots \ldots \ldots \ldots \ldots \ldots \ldots$

3.5.2 Self-regulatory mechanisms, coupled tightly, loosely,

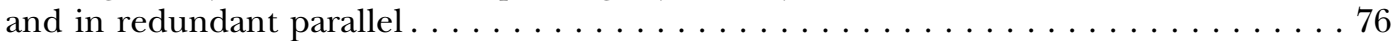

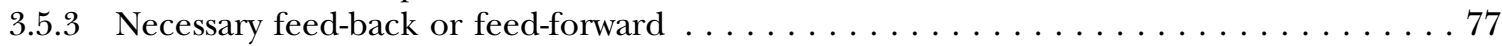

3.5 .4 Timing by turn-off $\ldots \ldots \ldots \ldots \ldots \ldots \ldots \ldots \ldots \ldots \ldots \ldots \ldots \ldots \ldots \ldots$

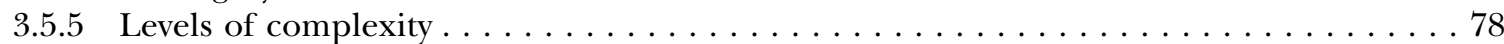

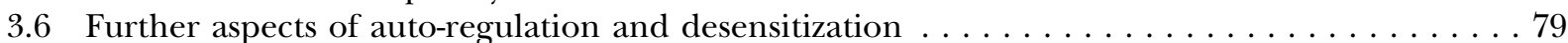

3.6.1 Dose-dependent and time-dependent regulation at a sub-/cellular level . . . . . . . . 79

3.6.2 The term auto-regulation and possible derivatives . . . . . . . . . . . . . . . . 79

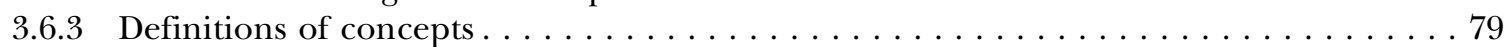

3.6.4 Homologous and heterologous desensitization for $\mathrm{G}$ protein-coupled

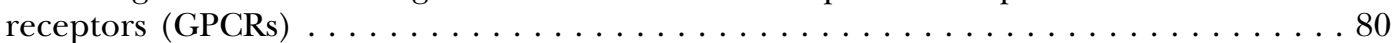

3.6.5 Intrinsic and extrinsic desensitization in receptive units including GPCRs. . . . . . . 8 80

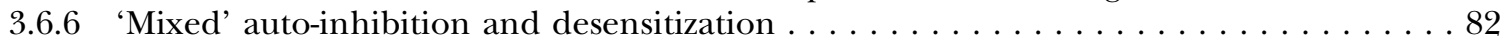

3.6.7 Two-state models (TSMs), two-site models (2SMs) and auto-inhibition . . . . . . . . . 83

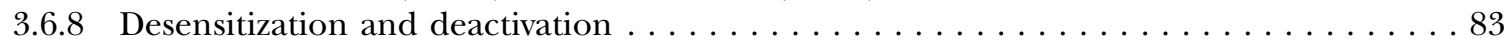

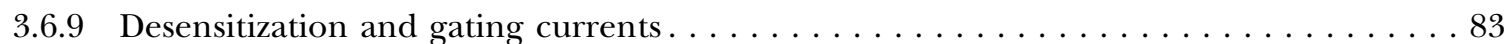

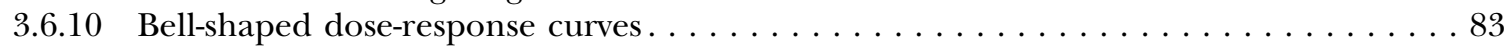

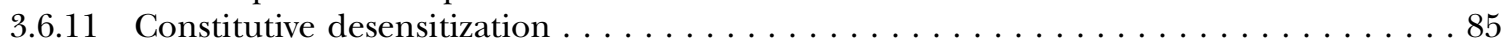

3.6.12 Desensitization-inactivation and the induction-versus-selection problem $\ldots \ldots \ldots$. . $\ldots 5$ 


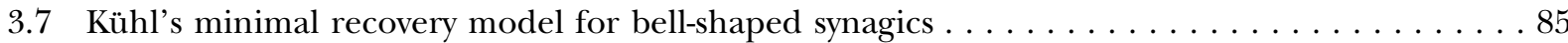

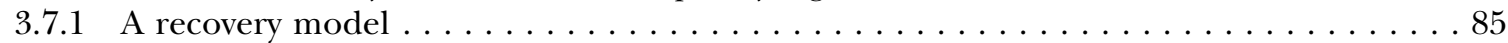

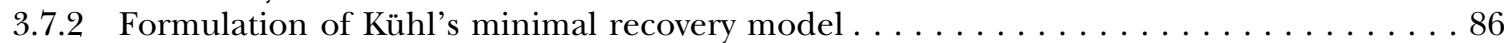

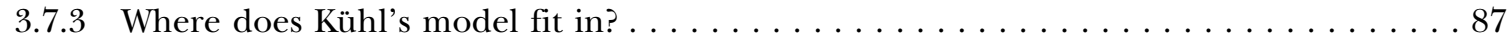

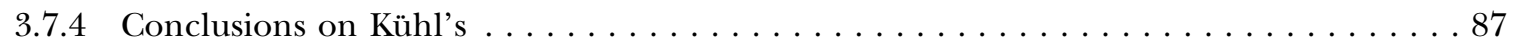

References .................................... 88

CHAPTER 4 Means of obtaining ant-agonist constants: Preliminary points of a personal view . . . . . 93

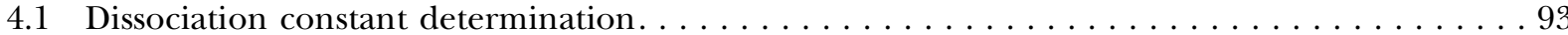

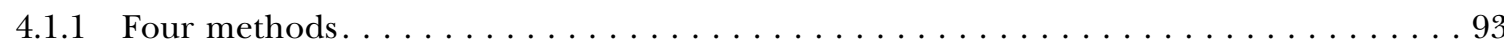

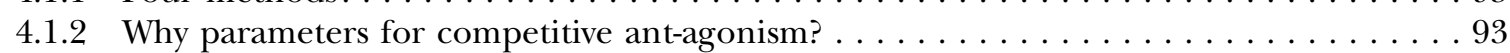

4.1.3 Constants for the hetero-intervention model . . . . . . . . . . . . . . . . . . . . . . . . . . 94

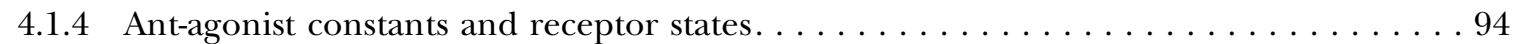

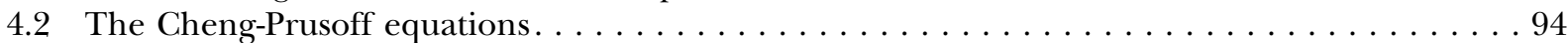

4.2.1 Cheng-Prusoff for $\mathrm{K}_{\mathrm{ii}}$ and $\mathrm{IC}_{50}$ in non-competitive ant-agonism $\ldots \ldots \ldots \ldots \ldots$

4.2.2 Explicit expressions for $K_{\mathrm{si}}, K_{\mathrm{ii}}$, and $\mathrm{IC}_{50}$ in competitive ant-agonism . . . . . . . 95

4.2.3 Direct derivation of the Cheng-Prusoff equation for competitive ant-agonism . . . . . . 95

4.2.4 Comparison of competitive and non-competitive inhibition curves . . . . . . . . . . 96

4.2.5 The functional equivalent of the Cheng-Prusoff equation (fe-C-P) $\ldots \ldots \ldots \ldots$

4.2.6 The validity of the Cheng-Prusoff equation for competitive ant-agonism . . . . . . . . . 98

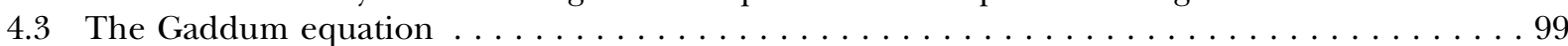

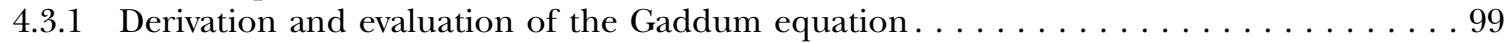

4.3.2 A comparison between the C-P and Gaddum equations . . . . . . . . . . . . . . . 99

4.4 Preliminary comparison of Schild-analysis with Cheng-Prusoff analysis . . . . . . . . . . . . . . .99

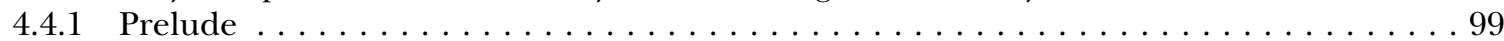

4.4 .2 The Schild regression. Initial considerations $\ldots \ldots \ldots \ldots \ldots \ldots \ldots \ldots$

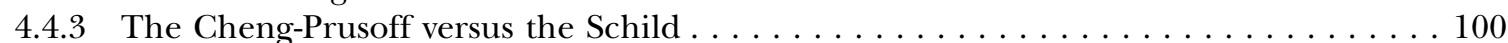

4.4.4 A provisional statement about Schild versus Cheng-Prusoff. . . . . . . . . . . . . . . 101

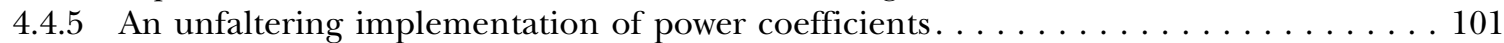

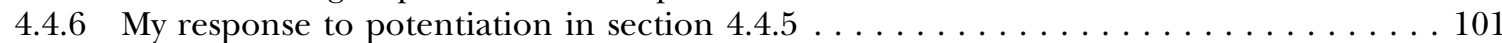

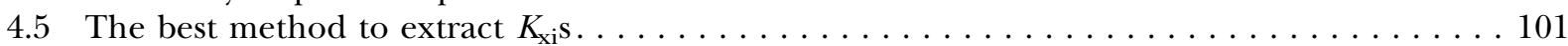

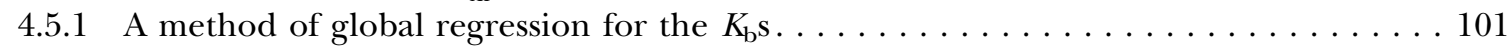

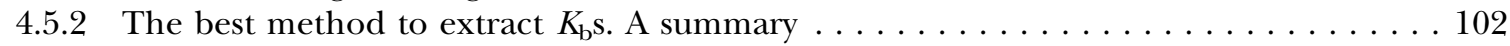

4.6 Multi-response ligands and ligand-dependent differential responses . . . . . . . . . . . . . 102

4.6.1 Functional selectivity and multi-response ligands . . . . . . . . . . . . . . . . . 102

4.6 .2 Multi-response ligands . . . . . . . . . . . . . . . . . . . . . . . . . . 102

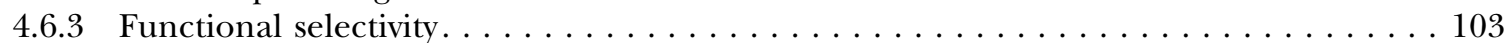

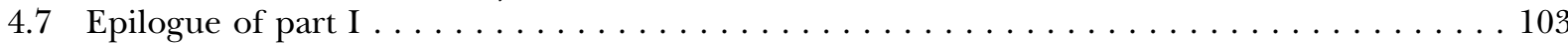

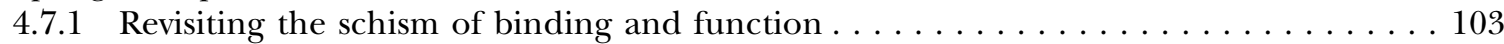

4.7.2 Revisiting the schism of competitive and non-competitive ant-agonism . . . . . . . . 104

References ....................................105

Part II TWO-STATE MODELS: COMPLEX AGONISM AND MODULATION $\ldots \ldots \ldots \ldots \ldots$

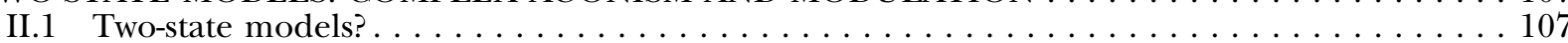

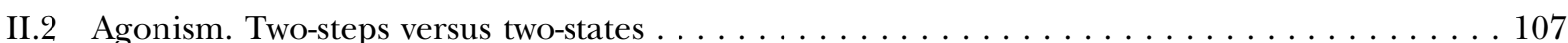

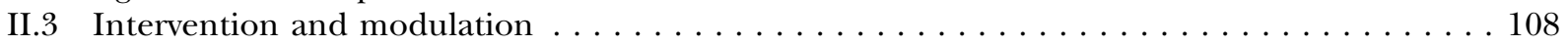

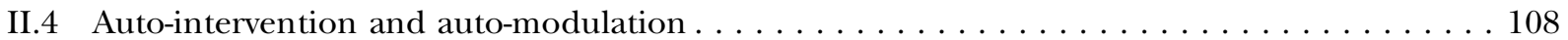

II.5 Dissociation versus association constants $\ldots \ldots \ldots \ldots \ldots \ldots \ldots \ldots$

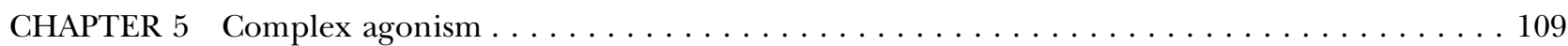

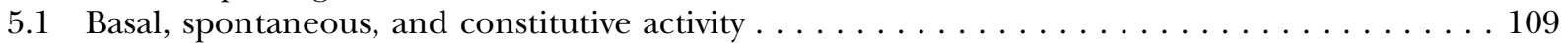

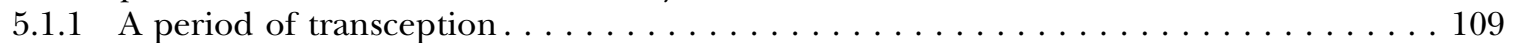

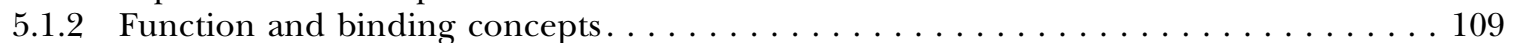

5.2 Inverse agonism and multi-functional drugs and receptive units $\ldots \ldots \ldots \ldots$

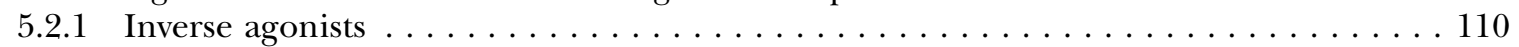




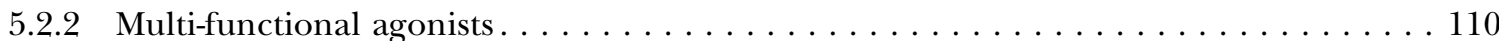

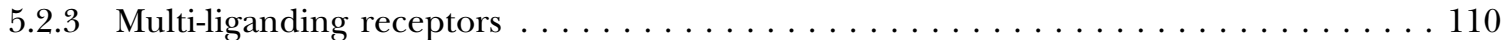

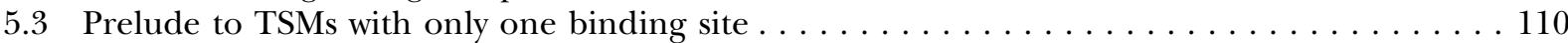

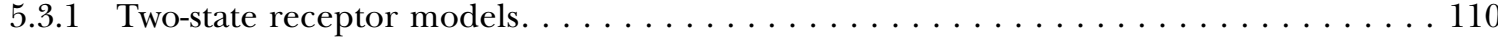

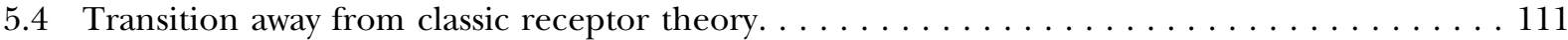

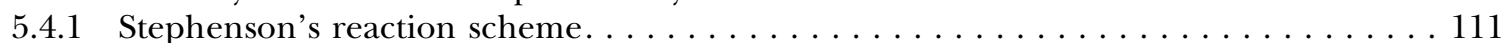

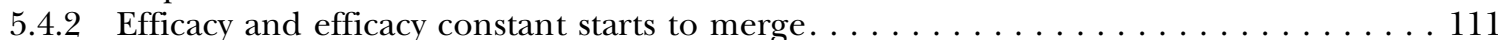

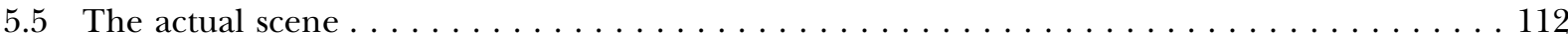

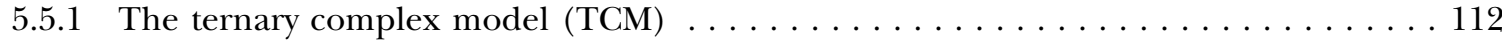

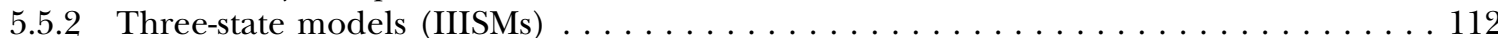

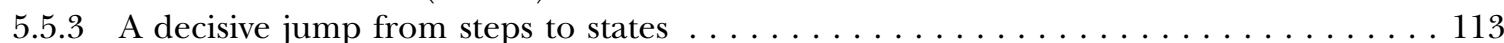

5.6 Two-steps intermediate complexes in non-active states . . . . . . . . . . . . . . . . . . . . 113

5.6 .1 The del Castillo \& Katz reaction scheme $(\mathrm{dC} \& \mathrm{~K}) \ldots \ldots \ldots \ldots \ldots \ldots \ldots \ldots$

5.6.2 The misconception induced by Stephenson's efficacy scheme . . . . . . . . . . . . . 114

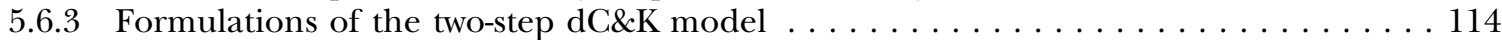

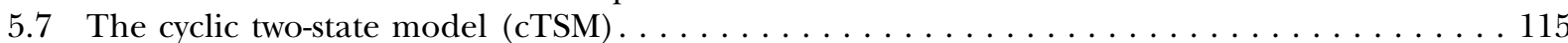

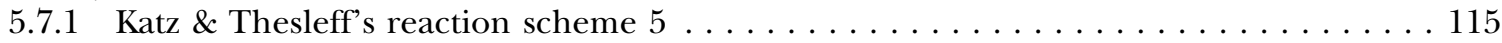

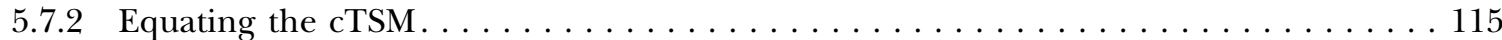

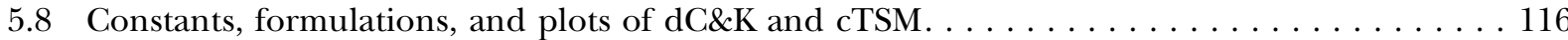

5.8.1 Constants and receptor conformations of the dC\&K and cTSM . . . . . . . . . 116

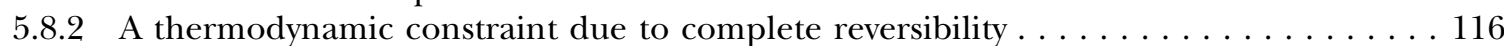

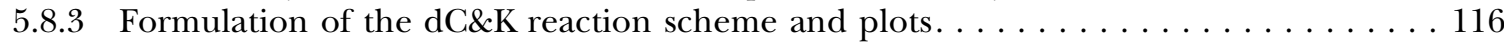

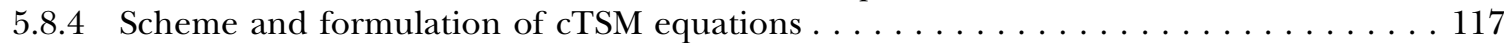

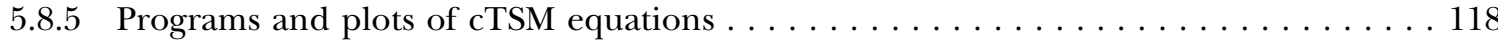

5.8 .6 Produce and analyze $d-r$ curves for function in cTSM. . . . . . . . . . . . . . . . . . . 120

5.8.7 Summary of the del Castillo \& Katz and Katz \& Thesleff reaction schemes . . . . . . . 120

5.8 .8 A comparison between cTSM and HI-OSM . . . . . . . . . . . . . . . . . . . . 120

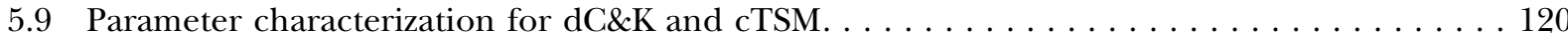

5.9.1 Parameter-dependent characterization of concentration-occupancy in dC\&K . . . . . 120

5.9.2 Parameter-dependent characterization of dose-response in dC\&K. . . . . . . . . . . 121

5.9.3 Parameter-dependent characterization of concentration-occupancy in cTSM. . . . . . . 122

5.9.4 Parameter-dependent characterization of dose-response in cTSM . . . . . . . . . . . . . 122

5.9 .5 Comparison between the cTSM and the dC\&K models . . . . . . . . . . . . . 123

5.9 .6 Extraction of parameter values from the functional cTSM . . . . . . . . . . . . . . . 124

5.9.7 Extraction of parameter values from the functional dC\&K . . . . . . . . . . . . . . . 124

5.9.8 What is a pure binding effect and a pure efficacy in terms of mechanisms? . . . . . . 124

5.9.9 Extraction of parameter values from binding studies. Hemoglobin $(\mathrm{Hb})$

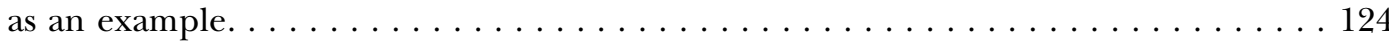

5.10 The general embedded load model. 'The operational model' . . . . . . . . . . . . . . 125

5.10 .1 Comparison between the dC\&K model and the B\&L model . . . . . . . . . . . . 125

5.10 .2 Formulations of del Castillo \& Katz and Black \& Leff models . . . . . . . . . . . . . . . 125

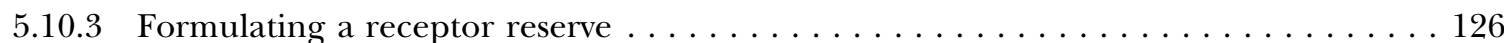

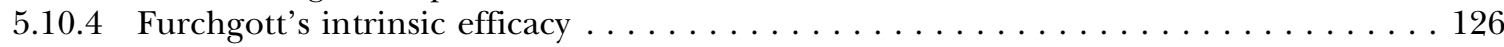

5.10 .5 Jargon or misuse of 'efficacy' for 'efficacy coefficient' . . . . . . . . . . . . . . . . 127

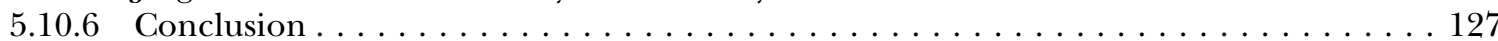

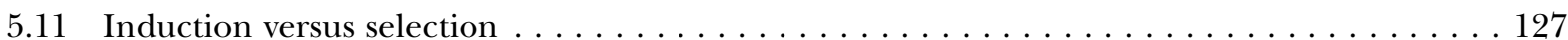

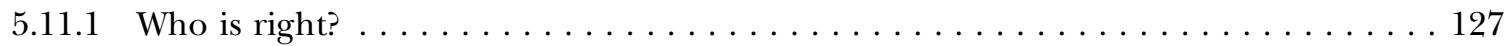

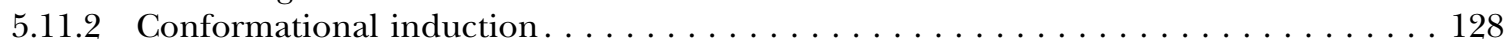

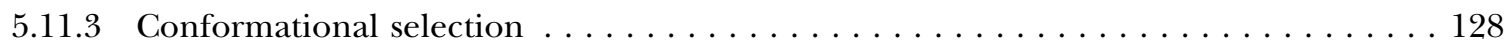

5.11 .4 The dilemma between selection and induction in enzymology . . . . . . . . . . . . 128

5.11 .5 The dilemma between selection and induction in pharmacology . . . . . . . . . 128

5.11 .6 Induction versus selection at equilibrium . . . . . . . . . . . . . . . . . . . . . . . 129

5.11.7 Solving the induction versus selection problem for enzymes with rate kinetics . . . . 129

5.11 .8 Induction versus selection at non-equilibrium in pharmacology . . . . . . . . . . . . 129

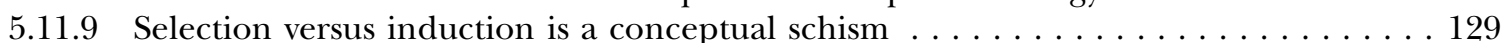


5.11.10 What is the impact of solving the induction-vs-selection problem (i-vs-s) $\ldots \ldots \ldots 130$

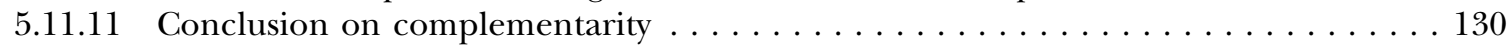

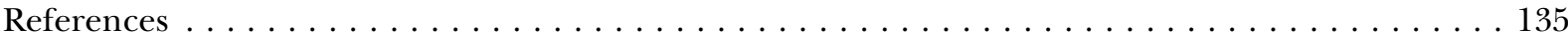

CHAPTER 6 Multi-step reaction schemes: extending the two-step mechanism of

del Castillo and Katz . . . . . . . . . . . . . . . . . . . . . . . . . . . . 139

6.1 Extending del Castillo-Katz or random and ordered models . . . . . . . . . . . . . . . . . . 139

6.1.1 Two-sites-and-more-than-one-step agonism. Extended reaction schemes . . . . . . . . 139

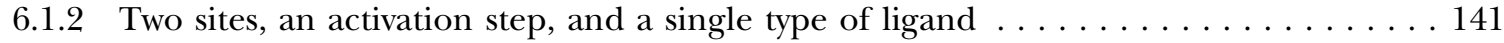

6.1.3 Random and ordered reaction schemes. . . . . . . . . . . . . . . . . . . . . 141

6.1 .4 When is 'sequential' sequential? . . . . . . . . . . . . . . . . . . . . . . . . . . . 142

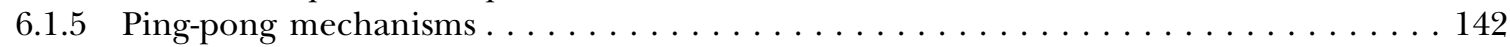

6.2 Random reaction schemes and extended with isomerization . . . . . . . . . . . . . . . . 142

6.2.1 The random two-site three-step reaction scheme . . . . . . . . . . . . . . . . . . . . . . 142

6.2 .2 Some examples of the random type reaction . . . . . . . . . . . . . . . . . 143

6.3 Ordered reaction schemes and now extended with isomerization . . . . . . . . . . . . 143

6.3.1 The ordered two-site three-step reaction scheme . . . . . . . . . . . . . . . . . . . . 143

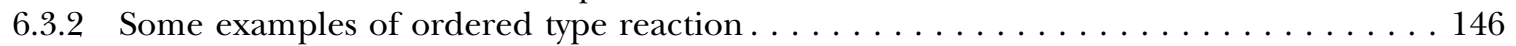

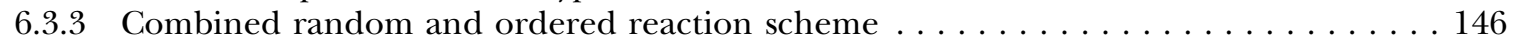

6.4 Analysis of parameters in the random and ordered reaction schemes . . . . . . . . . . . . . 149

6.4.1 Comparing simple random and ordered schemes by Hill fitting. . . . . . . . . . . . . . . 149

6.4.2 Comparing extended random and ordered schemes by Hill fitting. . . . . . . . . . . . 151

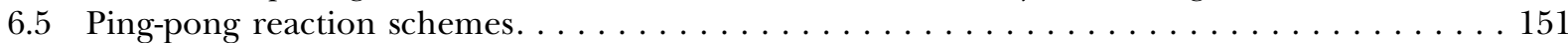

6.5.1 A circular two-site and more than two-step mechanism, the ping-pong model . . . . . 151

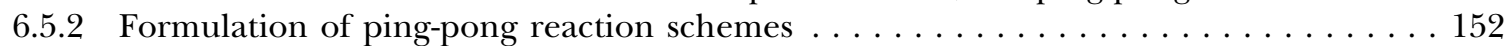

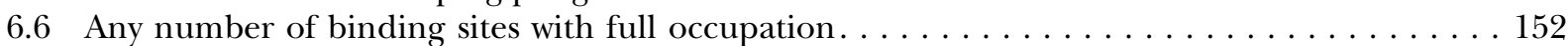

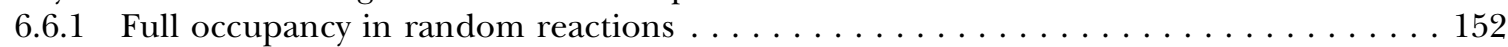

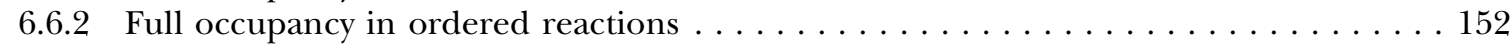

6.6.3 Pascal's triangle in random and ordered reaction schemes. . . . . . . . . . . . . . 152

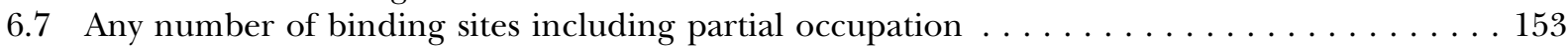

6.7.1 When functionality does not require full occupancy. . . . . . . . . . . . . . 153

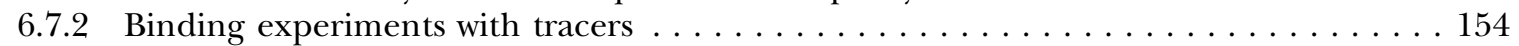

6.7.3 Functional experiments with efficacies varying between conformations . . . . . . . . 155

6.8 Random and ordered reactions combined with energized isomerization $\ldots \ldots \ldots \ldots$

6.8.1 Combination of ordered/random models with isomerization . . . . . . . . . . 155

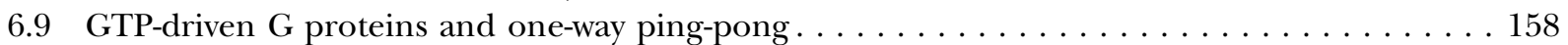

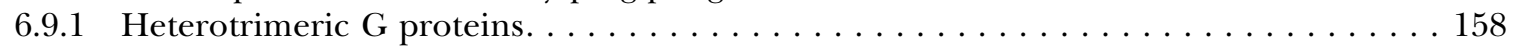

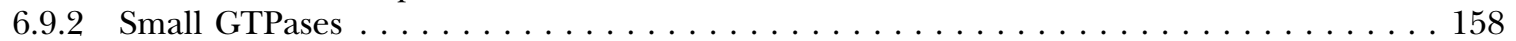

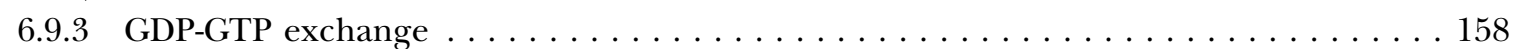

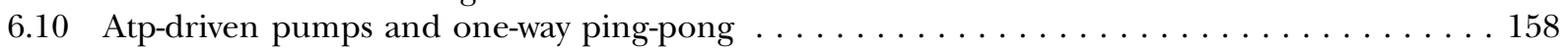

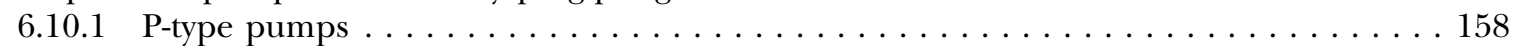

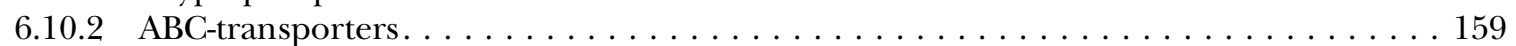

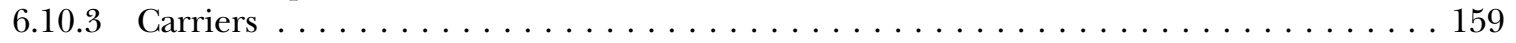

6.10.4 Development of models with two-states and ping-pong driven by ATP. . . . . . . . 159

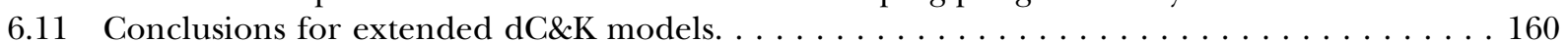

6.11 .1 Early models of non-fully occupied receptive units . . . . . . . . . . . . . . . 160

6.11 .2 Conclusions from extensions of the dC\&K scheme. . . . . . . . . . . . . . 160

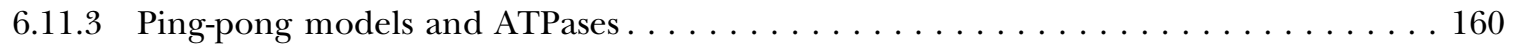

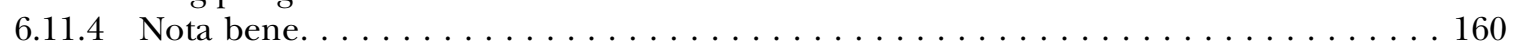

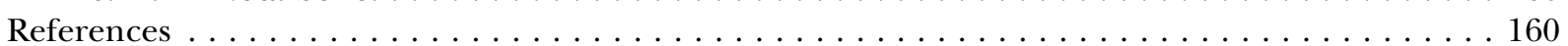

CHAPTER 7 Gubic reaction schemes. ATSM and HOTSM $\ldots \ldots \ldots \ldots \ldots$

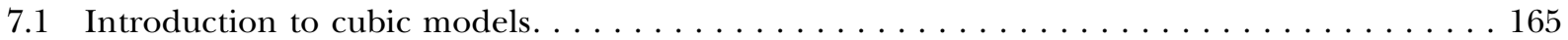

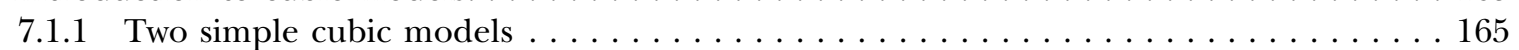

7.1.2 A promising model for the analysis of modulator action $\ldots \ldots \ldots \ldots \ldots$ 
7.1.3 Other cubic models and other models $\ldots \ldots \ldots \ldots \ldots \ldots \ldots \ldots$

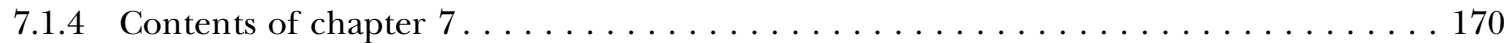

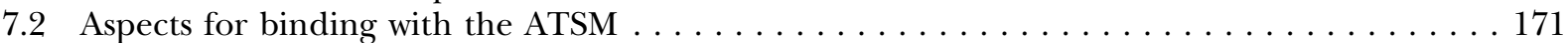

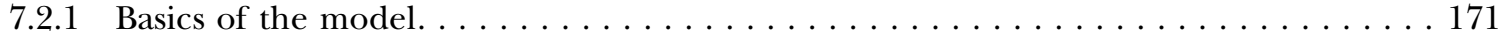

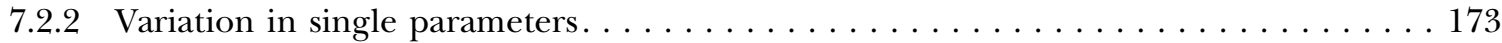

7.2.3 Conclusion on aspects of binding as simulated with the ATSM . . . . . . . . 173

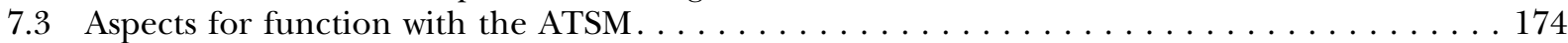

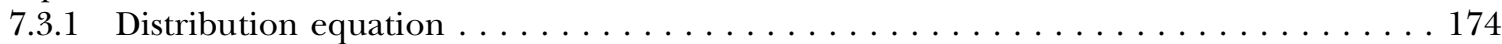

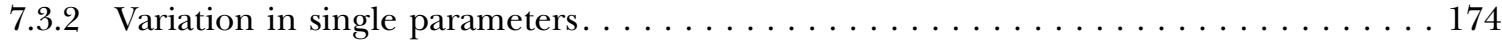

7.3.3 Conclusion on functional aspects as simulated with the ATSM . . . . . . . . . . . . 174

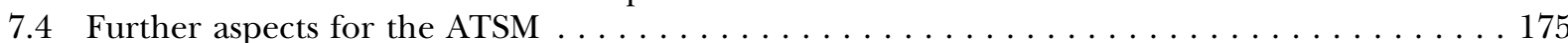

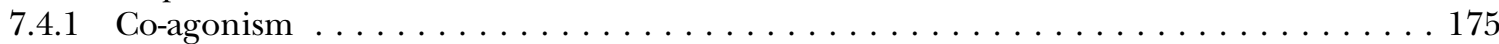

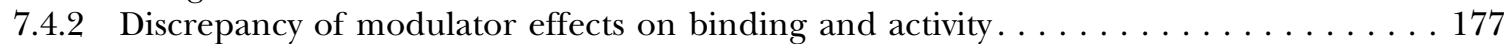

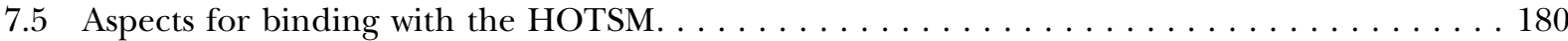

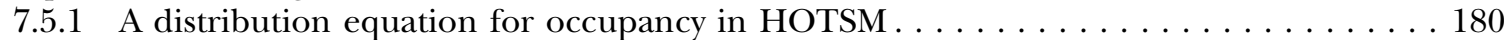

7.5.2 Effects of variation in single parameters of binding-HOTSM . . . . . . . . . . 182

7.5.3 Conclusion on aspects of binding as simulated

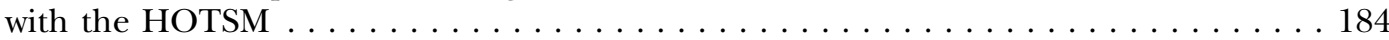

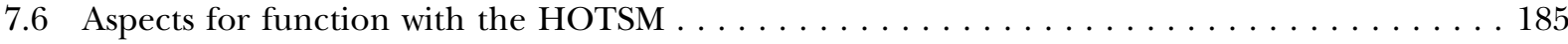

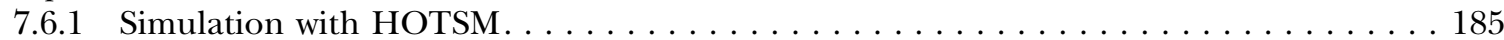

7.6.2 Examples of reverse bell-shaped dose-responses . . . . . . . . . . . . . . . . . . . . 186

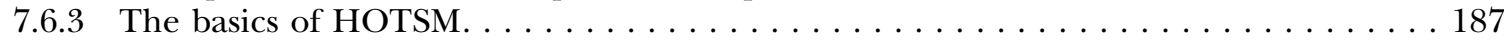

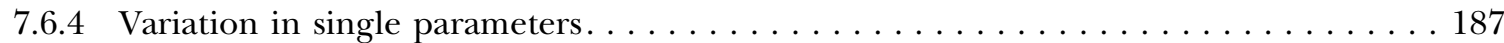

7.6.5 Conclusion on functional aspects as simulated with the HOTSM . . . . . . . . . 187

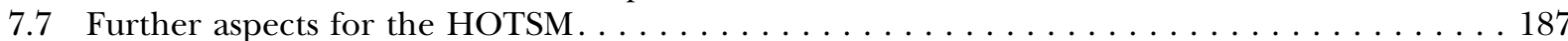

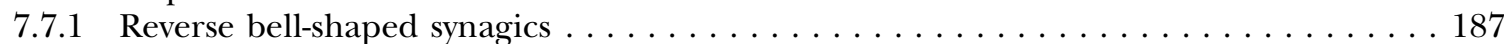

7.7 .2 The HOTSM against the Hill equation $\ldots \ldots \ldots \ldots \ldots \ldots \ldots \ldots \ldots \ldots \ldots$

7.7.3 Pseudo-homotropic two-state model (PHOTSM) . . . . . . . . . . . . . . . . . . . . 194

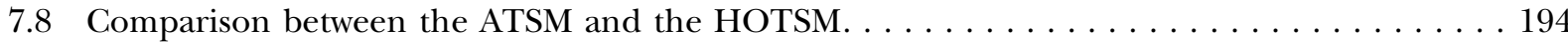

7.8.1 Functional studies. . . . . . . . . . . . . . . . . . . . . . . . . . . . . . . . . . . . 194

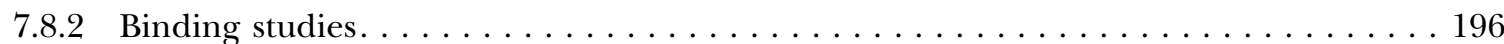

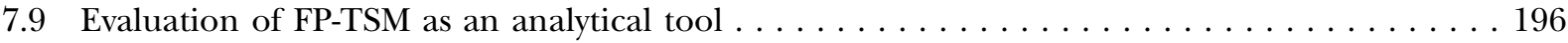

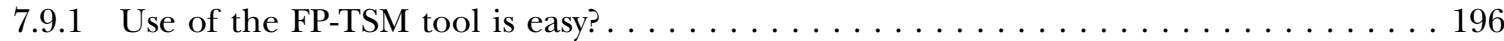

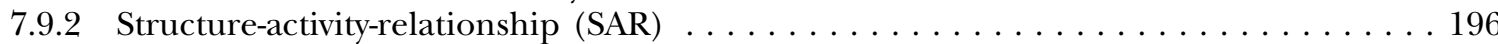

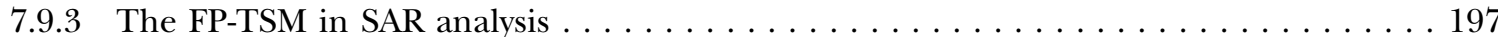

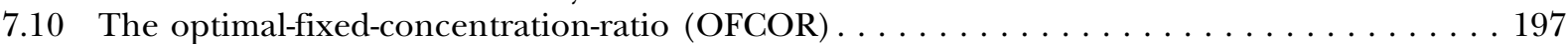

7.10 .1 Inhibition of negative auto-modification $\ldots \ldots \ldots \ldots \ldots \ldots \ldots \ldots \ldots \ldots$

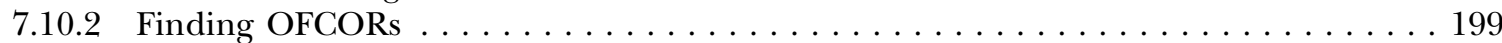

7.11 More on cubic models and GPCR-related models . . . . . . . . . . . . . . . . . . . . . . . 200

7.11.1 The extended ternary complex model $(\mathrm{ETCM}) \ldots \ldots \ldots \ldots \ldots$. . . . . . . . . . 200

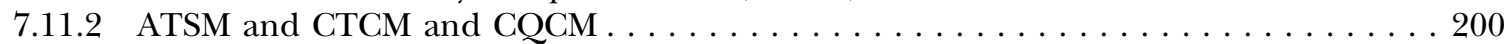

7.11.3 Models of 'ligand' numbers matching 'receptor' numbers . . . . . . . . . . . . . 200

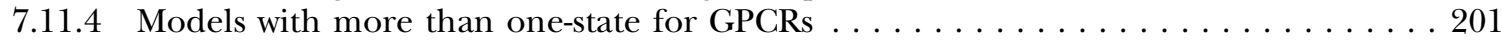

7.11 .5 Models for binding with dimerization as isomerization . . . . . . . . . . . 201

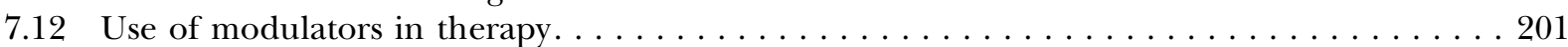

7.12.1 Benefits of modulators compared with orthosteric ligands . . . . . . . . . . . . 201

7.12 .2 Summary of modulator benefits. . . . . . . . . . . . . . . . . . . . . . . . . 202

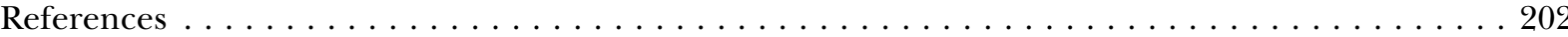

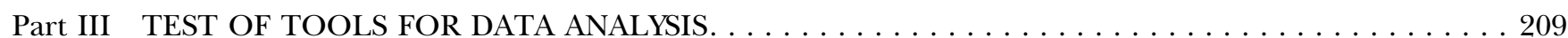

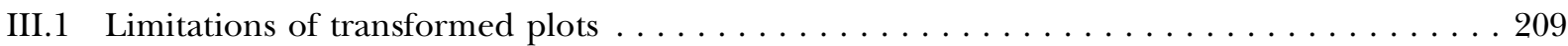

III.2 Reciprocal and non-reciprocal plots . . . . . . . . . . . . . . . . . . . . . . . . . 209

III.3 Stepping on the chips $\ldots \ldots \ldots \ldots \ldots \ldots \ldots \ldots \ldots \ldots \ldots \ldots \ldots \ldots \ldots \ldots \ldots \ldots \ldots \ldots \ldots$

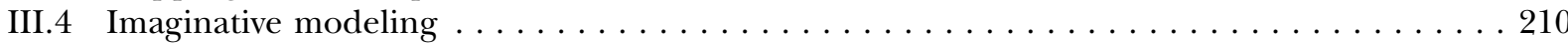

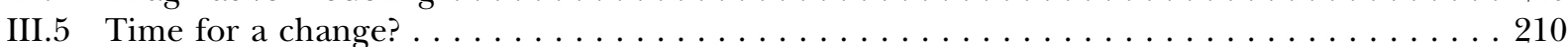




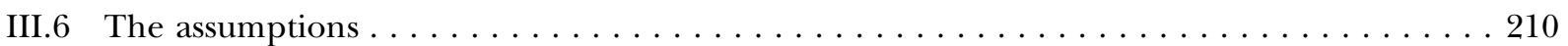

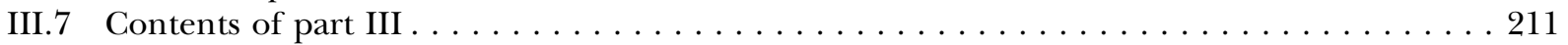

CHAPTER 8 Choosing and formulating relevant schemes $\ldots \ldots \ldots \ldots \ldots$

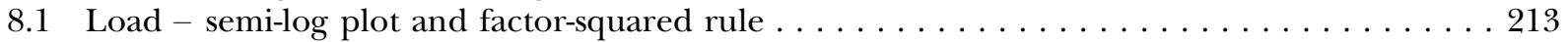

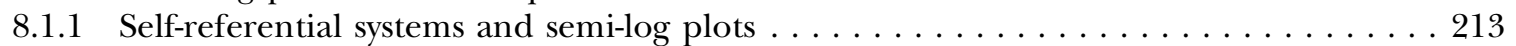

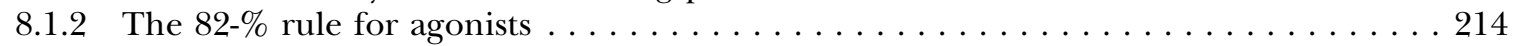

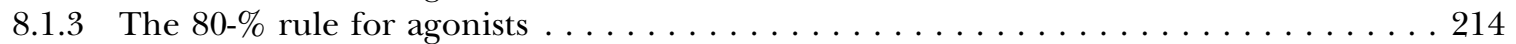

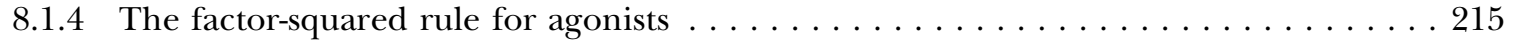

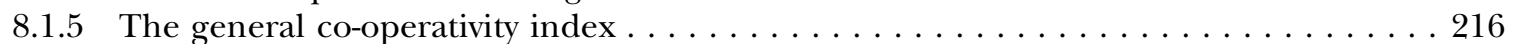

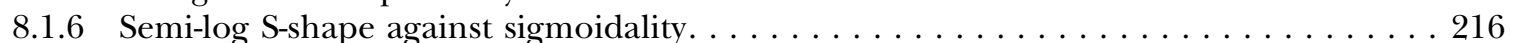

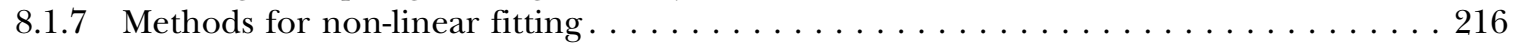

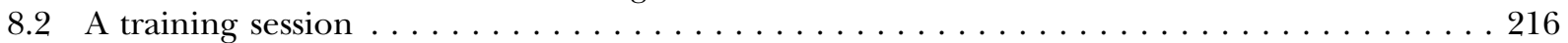

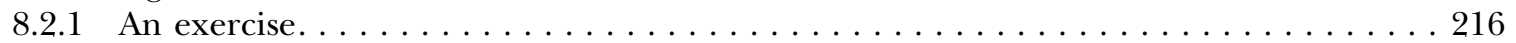

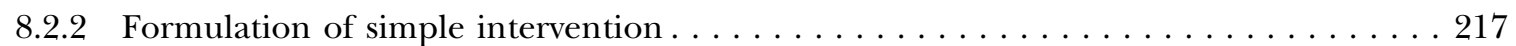

8.2 .3 The Hill and the reverse Hill . . . . . . . . . . . . . . . . . . . . . . . 217

8.2.4 Non-competitive versus competitive type II intervention or auto-intervention . . . . . 218

8.2.5 Actual plus remaining response and their logit formulation . . . . . . . . . . . . . . 219

8.2.6 Overlaid intervention involving Hill or reverse Hill equations. the Hill plot . . . . . . 220

8.2.7 When inhibition is mutually exclusive or that of allostery . . . . . . . . . . . . . 223

8.2 .8 Is it worth it? . . . . . . . . . . . . . . . . . . . . . . . . . . . . . . . . . . 223

8.3 Scatchard's plot and non-reciprocal tools with non-linear adjustments. . . . . . . . . . . . 225

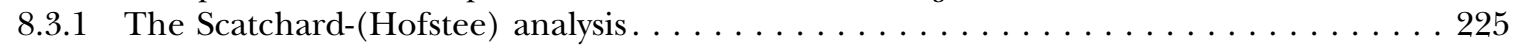

8.3.2 Historic development of reverse plots . . . . . . . . . . . . . . . . . . . . 225

8.3.3 Comparing semi-log plots with Hanes, L\&B and Eadie-Scatchard plots . . . . . . . . 225

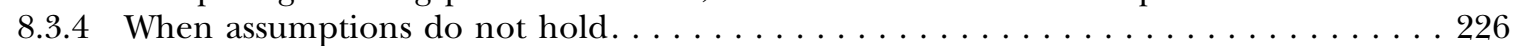

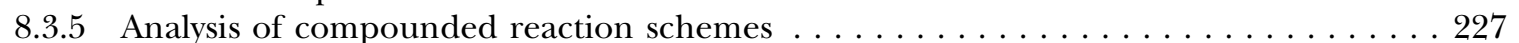

8.3.6 Eadie-Scatchard wins over non-reciprocal . . . . . . . . . . . . . . . . . . . . . . 227

8.3.7 A perspective on type I and type II competitive inhibition . . . . . . . . . . . . . . 228

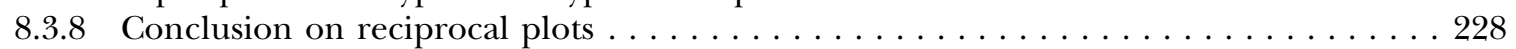

References . . . . . . . . . . . . . . . . . . . . . . . . . . . . . . . . . . . . . . . . . 229

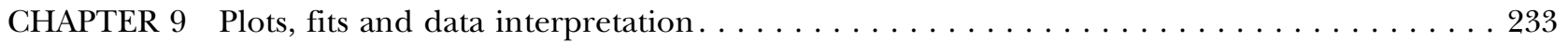

9.1 SigmaPlot software to plot, fit and generate curves $\ldots \ldots \ldots \ldots \ldots \ldots \ldots$

9.1.1 Using the SigmaPlot software to generate a plot . . . . . . . . . . . . . 233

9.1.2 Using the SigmaPlot software to generate a semi-log plot. . . . . . . . . . . . . 236

9.1.3 Using the SigmaPlot software to curve-fit to data . . . . . . . . . . . . . . . 236

9.1.4 Using the SigmaPlot software to generate the best-fit curve . . . . . . . . . . . . . . 240

9.1 .5 Non-linear fitting . . . . . . . . . . . . . . . . . . . . . . . . . . . . 240

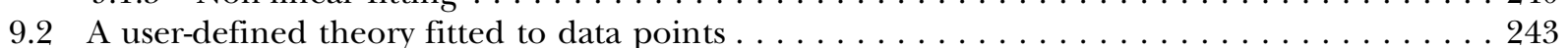

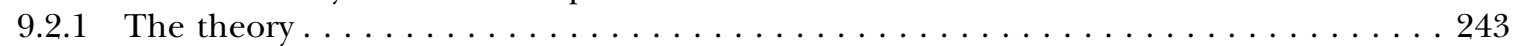

9.2 .2 Create a user-defined fit-routine . . . . . . . . . . . . . . . . . . . . . . . . . . . . . 243

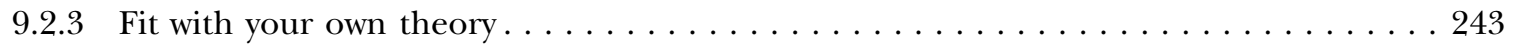

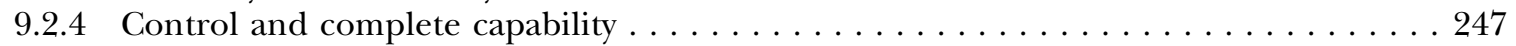

9.3 Test the realism of user-defined theories by their $2-\mathrm{D}$ and $3-\mathrm{D}$ plots $\ldots \ldots \ldots \ldots$. . . . . . 247

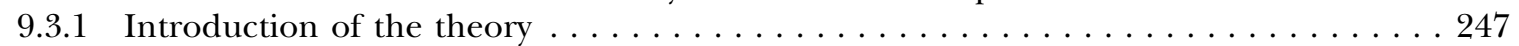

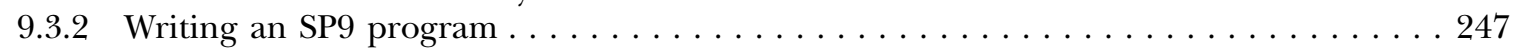

9.3.3 Subroutine for equidistant concentration values on a log-scale . . . . . . . . . . . . . 247

9.3.4 Subroutine for system constants, the so-called parameters . . . . . . . . . . . . . . . 248

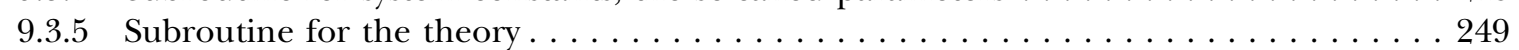

9.3.6 Simultaneous presentation of several plots for the user-defined theory . . . . . . . 251

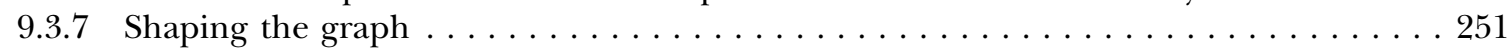

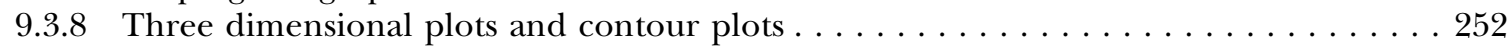

9.3.9 A user-defined 3-D subroutine . . . . . . . . . . . . . . . . . . . . . . . . . 252

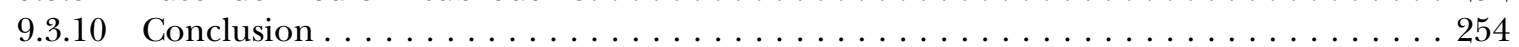




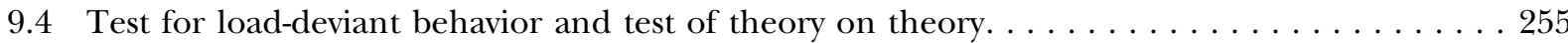

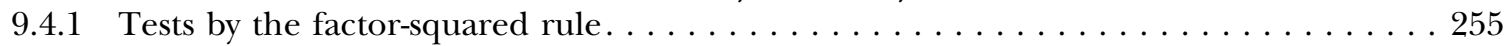

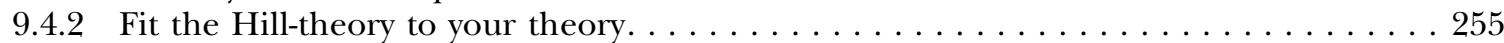

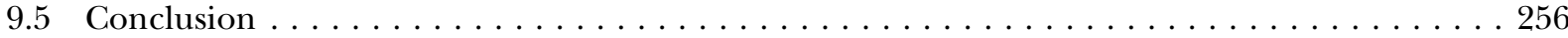

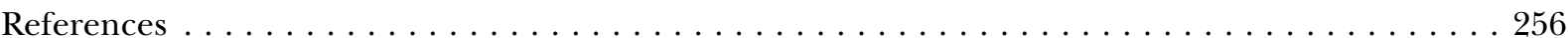

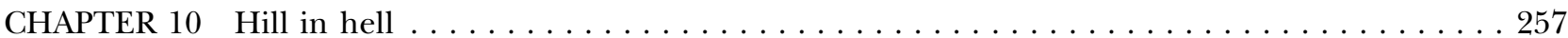

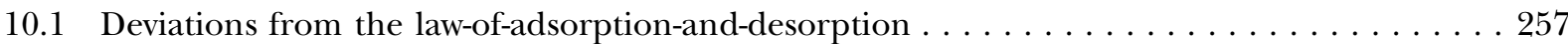

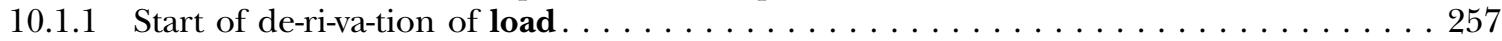

10.1.2 Start of de-vi-a-tions from load. Formulation of Hill's equation . . . . . . . . . . . . . 258

10.1.3 Hill's aggregation theory for hemoglobin complexation crumbles . . . . . . . . . 259

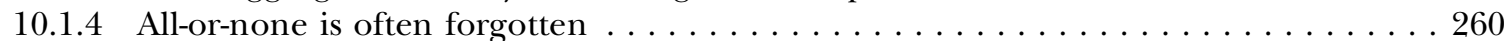

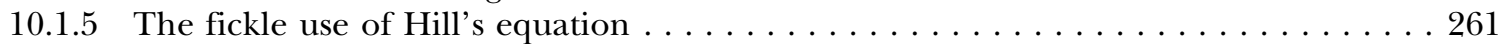

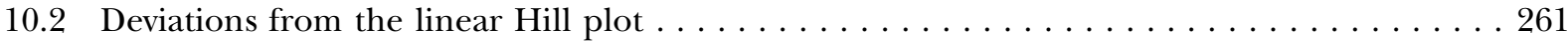

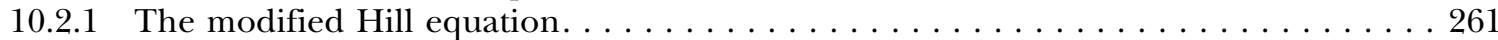

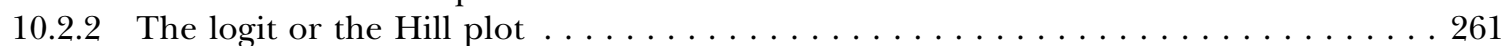

10.2 .3 Non-linear Hill plots . . . . . . . . . . . . . . . . . . . . . . . . . . . . . . . . . . . . . 262

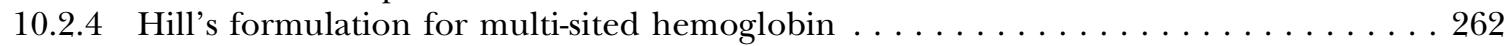

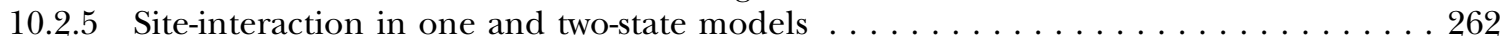

10.2.6 Adair's equation predicts non-linear Hill plots . . . . . . . . . . . . . . . . . . . . . 263

10.3 The original logistic equation and the original Hill equation $\ldots \ldots \ldots \ldots$

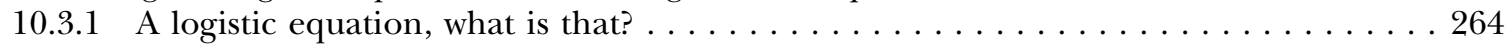

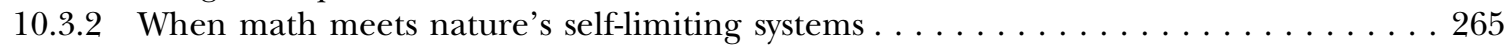

10.3.3 Transforming logistic equations to Hill's equations and vice versa . . . . . . . . . 268

10.3.4 Practical use of a 'logistic' Hill equation . . . . . . . . . . . . . . . . . . . . . . 269

10.3.5 'Logistic-Hill' is a technical term . . . . . . . . . . . . . . . . . . . . . . . . . . 269

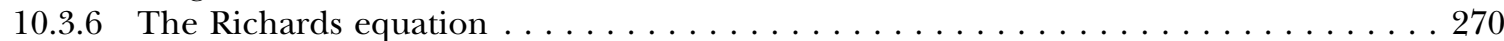

10.3.7 Use of double Hill or seriatim Hill equations. . . . . . . . . . . . . . . . . . . . . 272

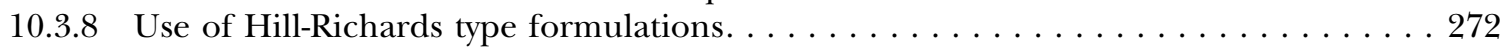

10.3.9 On the similarity between the Hill equation and the modified

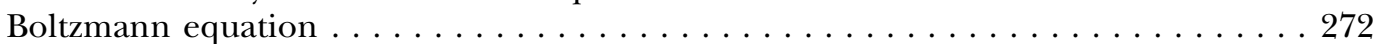

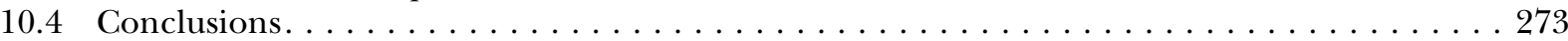

10.4.1 Details on the use of Hill's equation $\ldots \ldots \ldots \ldots \ldots \ldots \ldots \ldots \ldots \ldots \ldots \ldots \ldots \ldots$

10.4 .2 Is Hill in hell?. . . . . . . . . . . . . . . . . . . . . . . . . . . . . . . . . . . . . . . . . 274

10.A Appendix A: A short course on the term 'logistic' and its uses in statistics. . . . . . . . . 274

10.A.1. Statistical analysis. . . . . . . . . . . . . . . . . . . . . . . . . . . . . 274

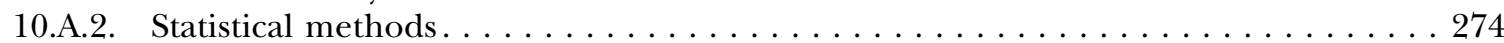

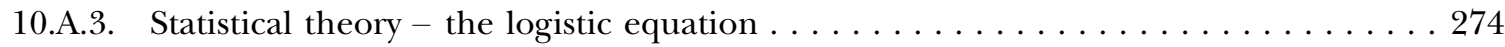

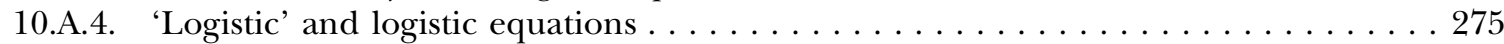

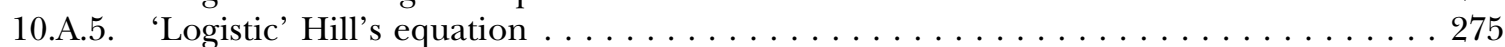

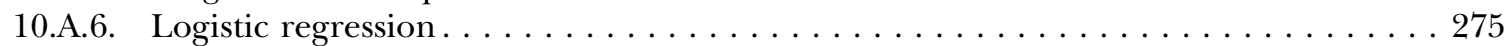

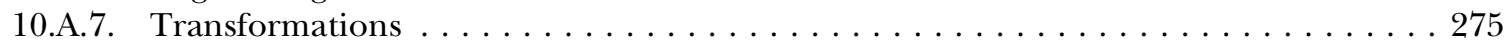

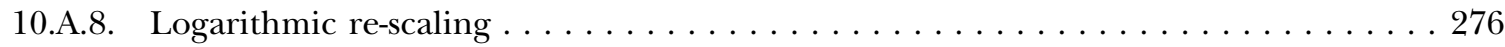

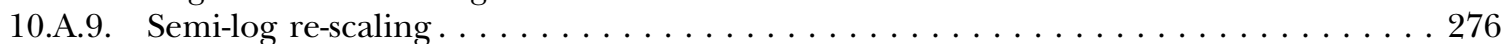

10.A.10. Log-linear re-scaling. . . . . . . . . . . . . . . . . . . . . . . . . . . . 276

10.A.11. Logit re-scaling . . . . . . . . . . . . . . . . . . . . . . . . . . . . . . . . 277

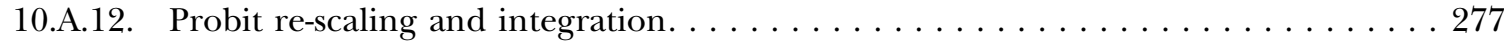

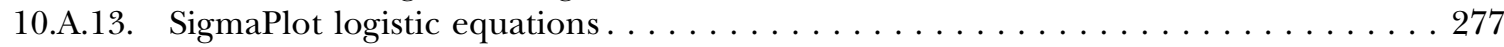

References . . . . . . . . . . . . . . . . . . . . . . . . . . . . . . . . . . . . 277

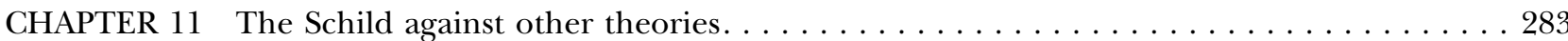

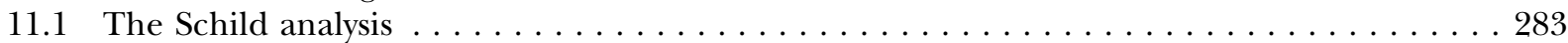

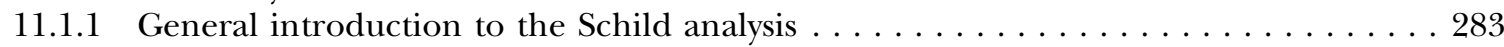

11.1.2 Null methods - comparison of equi-active responses in Schild analysis . . . . . . . . . 284

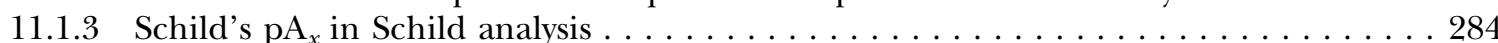




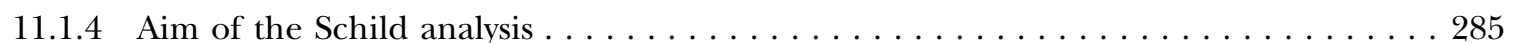

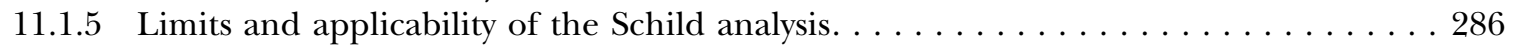

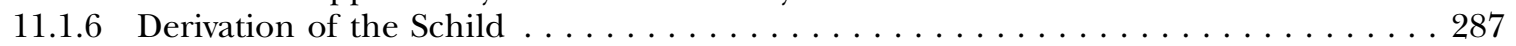

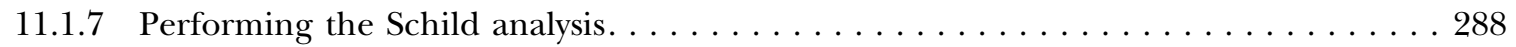

11.1 .8 A Schild analysis . . . . . . . . . . . . . . . . . . . . . . . . . . . . . . . 289

11.1 .9 Plots from the Schild analysis $\ldots \ldots \ldots \ldots \ldots \ldots \ldots \ldots \ldots \ldots \ldots \ldots$

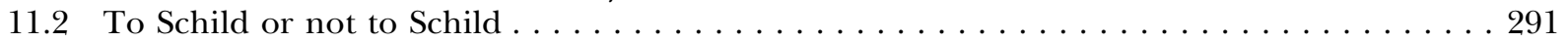

11.2.1 Inhibition curve experiments versus the Schild analysis . . . . . . . . . . . . . . 291

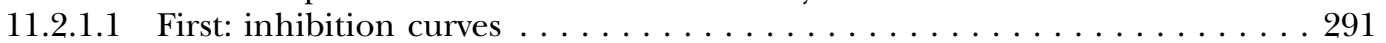

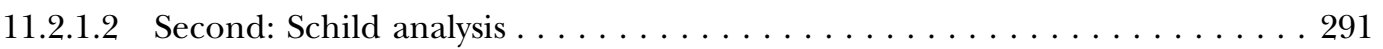

11.2.2 Other concerns about the Schild analysis. . . . . . . . . . . . . . . . . . . . . . 292

11.2.3 Inhibition curve strategy against Schild analysis . . . . . . . . . . . . . . . . . . . . . 292

11.2.4 An experimental evaluation of $\mathrm{pK}_{\mathrm{B}}$ by the Schild analysis . . . . . . . . . . . . 293

11.2.5 Deviation from slopes of unity and from linearity in the Schild analysis . . . . . . . 293

11.2 .6 To Schild or not to Schild? . . . . . . . . . . . . . . . . . . . . . . . . . . . 293

11.3 Time to abandon the Schild. . . . . . . . . . . . . . . . . . . . . . . . . . . . 294

11.3.1 How you can fool yourself with a Schild analysis . . . . . . . . . . . . . . . . . . . . 294

11.3.2 Other theoretical schemes and their Schild formulation. . . . . . . . . . . . . . . . . 294

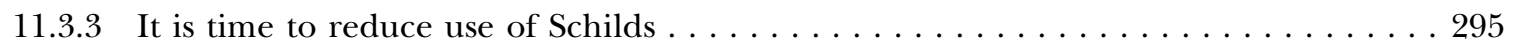

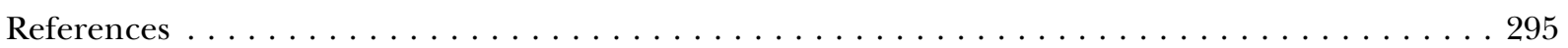

CHAPTER 12 Ties between synergy and two-state models . . . . . . . . . . . . . . . . . . 299

12.1 General synergy. A non-mechanistic approach . . . . . . . . . . . . . . . . . . . 299

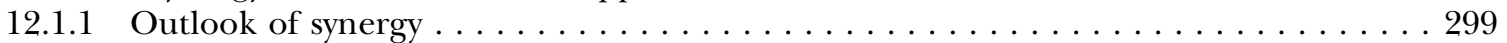

12.1.2 Three levels: Organ, cell, and receptor . . . . . . . . . . . . . . . . . . . . 299

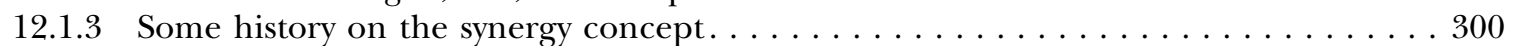

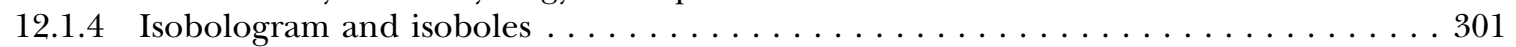

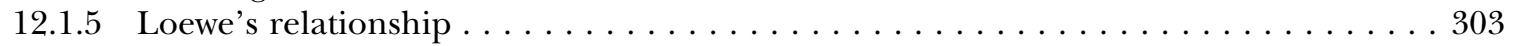

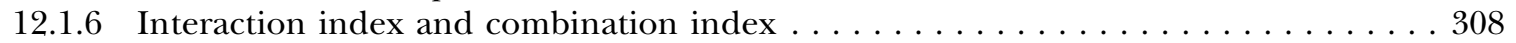

12.1.7 Random choice of models for integrated systems . . . . . . . . . . . . . . . . . 309

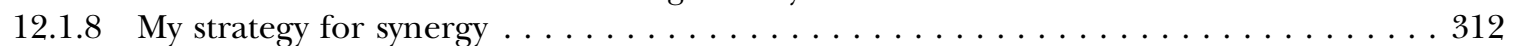

12.2 Mechanistic synergy at the receptor level. 1. Classic models. . . . . . . . . . . . . . 313

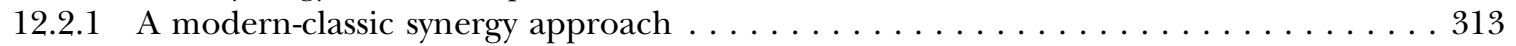

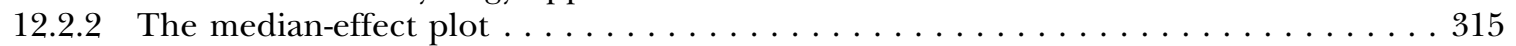

12.2.3 Bliss's formulation of independence a la Greco . . . . . . . . . . . . . . . . . 316

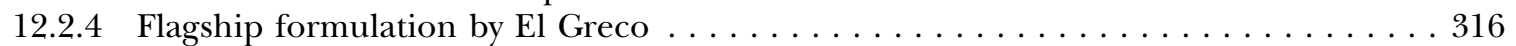

12.3 Mechanistic synergy at the receptor level. 2. The intervention model . . . . . . . . . 316

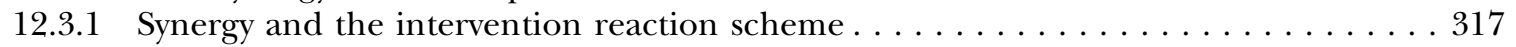

12.3.2 Modeling of two drugs with inclusive and co-lateral binding . . . . . . . . . . . . 317

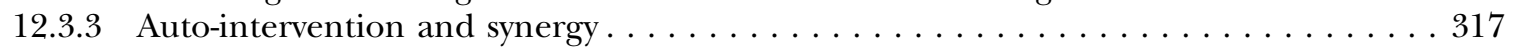

12.4 Mechanistic synergy at the receptor level. 3. The operational model . . . . . . . . . . . 318

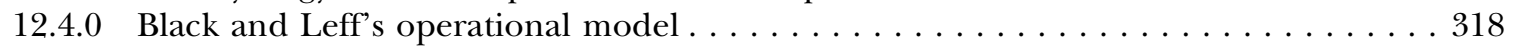

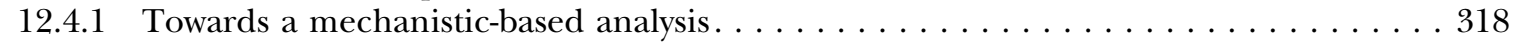

12.5 Mechanistic synergy at the receptor level. 4. The ATSM . . . . . . . . . . . . . . . . . 319

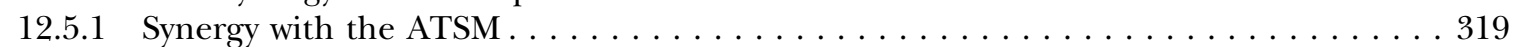

12.5.2 Contour plots of the ATSM without co-lateral binding . . . . . . . . . . . . . . . . 319

12.5.3 Contour plots of the ATSM with co-lateral binding . . . . . . . . . . . . . . . . . 319

12.5.4 Synergy, allosteric therapy, fixed-dose, and the OFCOR principle . . . . . . . . . . 320

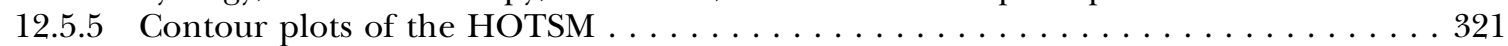

12.5.6 A discussion of linear-linear versus $\log -\log$ contour plots $\ldots \ldots \ldots \ldots \ldots \ldots \ldots \ldots$

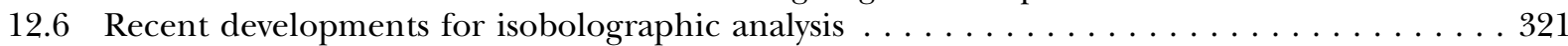

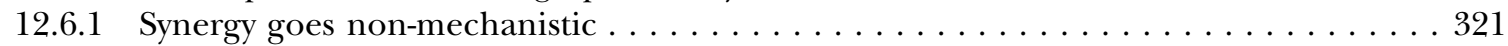

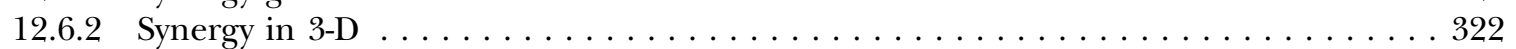

12.6.3 Actual software for synergy . . . . . . . . . . . . . . . . . . . . . . . . . . . . 322

12.6.4 Synergy in partial agonism with co-operativity $\ldots \ldots \ldots \ldots \ldots \ldots \ldots$ 
12.7 Conclusions on the analysis of synergy analysis . . . . . . . . . . . . . . . . . . . . . . . 324

12.7.1 Conclusions by Berenbaum . . . . . . . . . . . . . . . . . . . . . . . . . . . . . 324

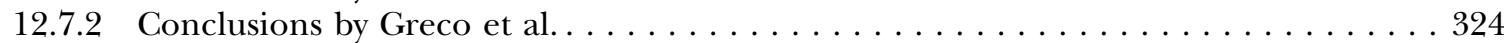

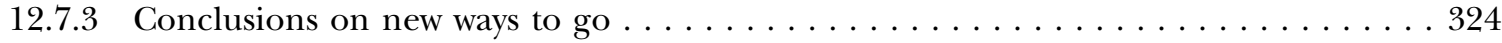

References . . . . . . . . . . . . . . . . . . . . . . . . . . . . . . . . . . . . . 324

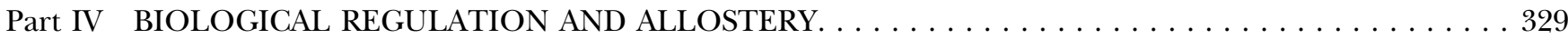

IV.1 Flicks and forces at a nano-scale . . . . . . . . . . . . . . . . . . . . . . . . . 329

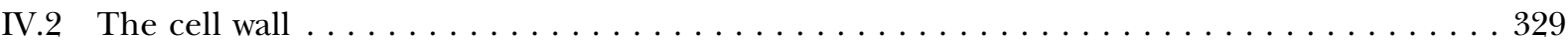

IV.3 Equilibrium is not 'static'. Synagics versus statics. . . . . . . . . . . . . . . . . . . . . . . 329

IV.4 Last three chapters . . . . . . . . . . . . . . . . . . . . . . . . . . . . . . . . . . . . . . . 329

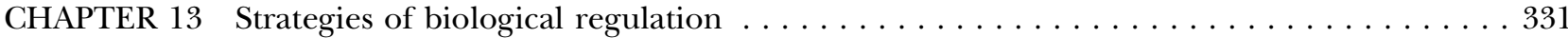

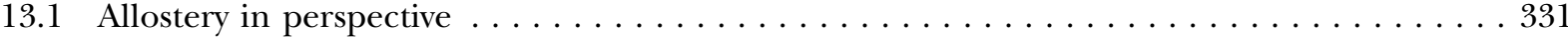

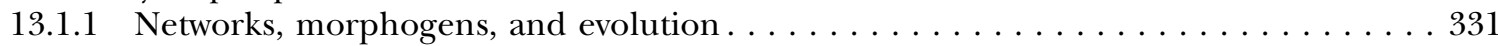

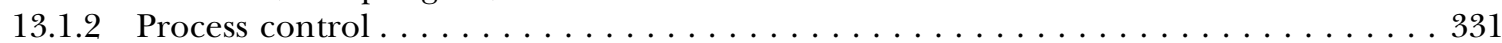

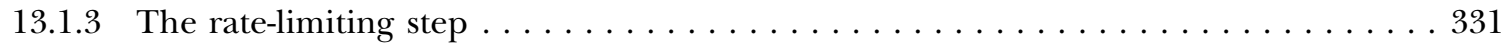

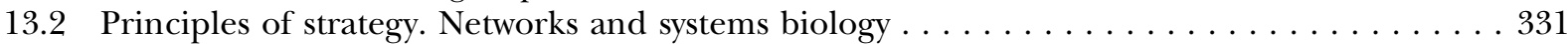

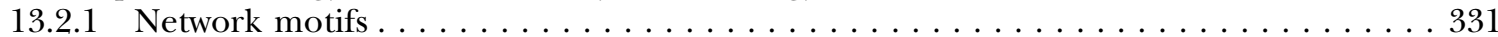

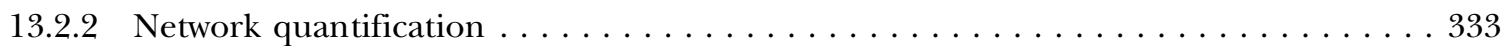

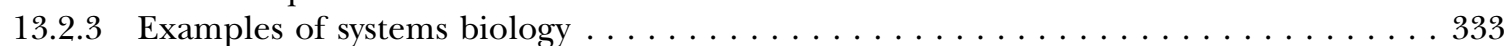

13.2.4 Brief statements on bioinformatics, networks, and systems biology . . . . . . . . 333

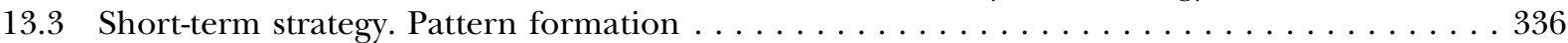

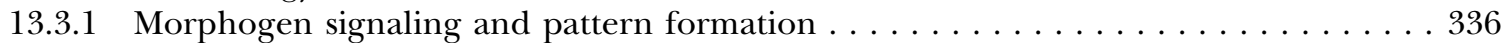

13.3.2 Production, release, and movement of morphogens . . . . . . . . . . . . . 336

13.3.3 Structural genes or homeo-box genes and their morphogens . . . . . . . . . . . . . 336

13.3.4 Regulated release of morphogens and extracellular binding or removal . . . . . . . 336

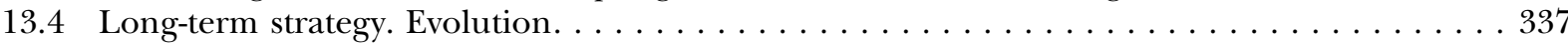

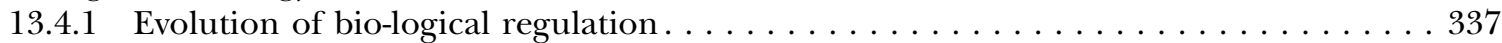

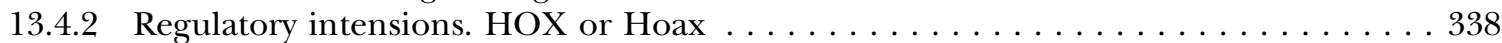

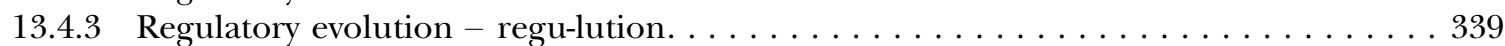

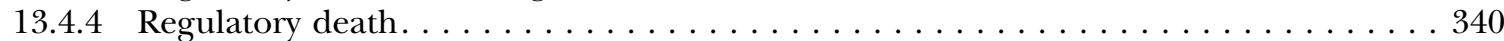

13.4.5 Mapping evolution. Human races?. . . . . . . . . . . . . . . . . . . . . . 340

13.4.6 Epigenetic inheritance systems $(\mathrm{EISs}) \ldots \ldots \ldots \ldots \ldots \ldots \ldots \ldots \ldots \ldots$

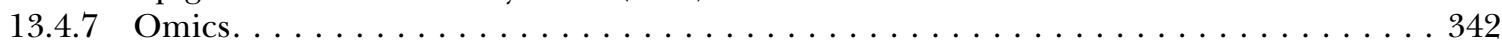

13.5 The present state of regulation by allostery and other affairs . . . . . . . . . . . . . 342

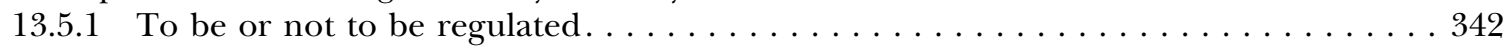

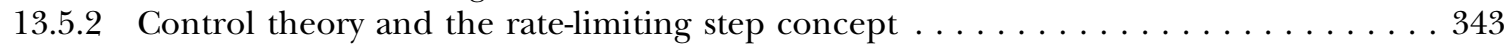

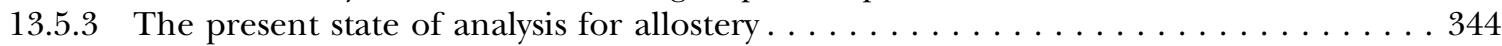

13.5.4 Will we be able to combine complexity, chaos, and criticality with allostery? . . . . 345

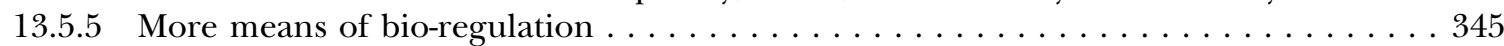

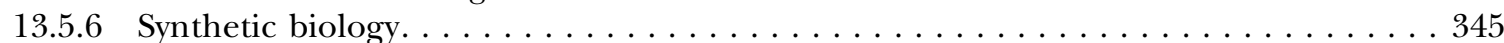

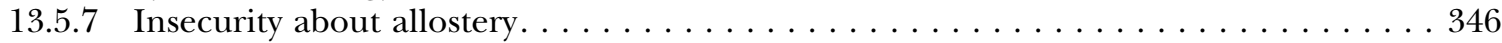

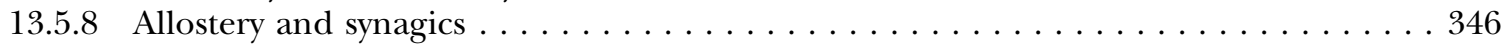

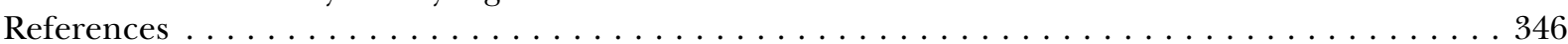

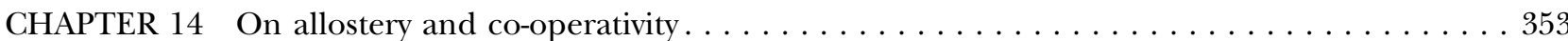

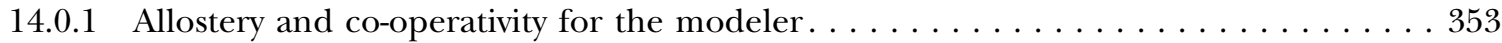

14.1 Historic accounts on the use of 'allosteric' and 'co-operative' . . . . . . . . . . . . . 353

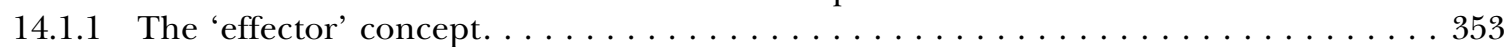

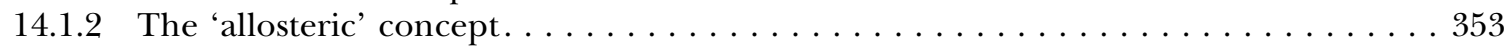

14.1.3 The adjective 'allosteric' and its noun 'allostery' or 'allosterism' . . . . . . . . . . . 354

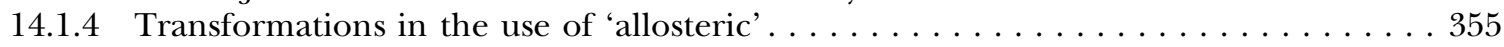

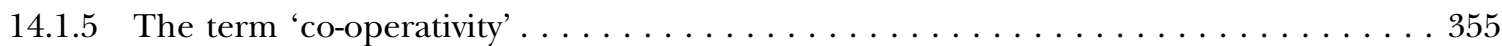

14.1.6 A comment on conformational selection versus conformational induction . . . . . 355 
14.1.7 The classical terms 'allostery' and 'co-operativity' . . . . . . . . . . . . . . . 356

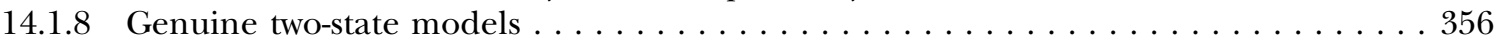

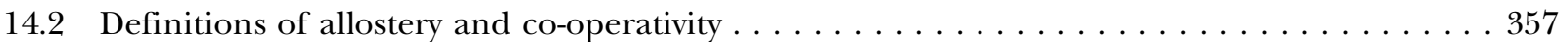

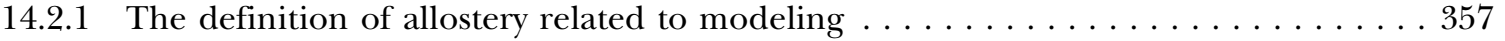

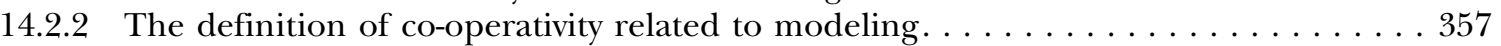

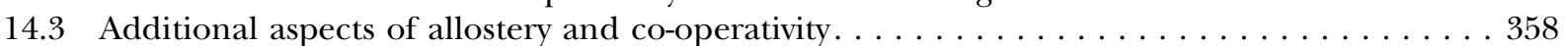

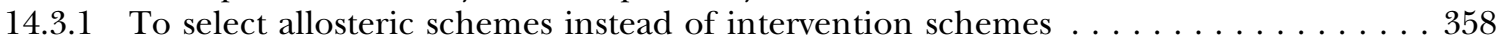

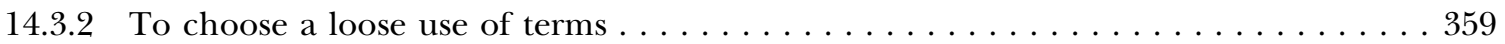

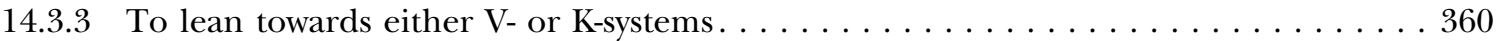

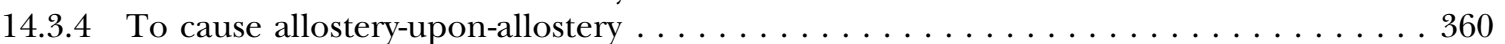

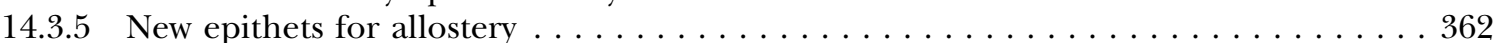

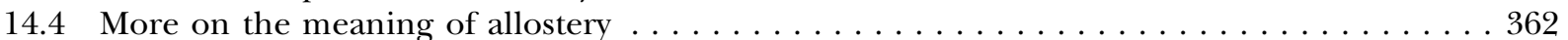

14.4.1 Allostery with multi-subunit complexes or multi-sited units . . . . . . . . . . . . . . . 362

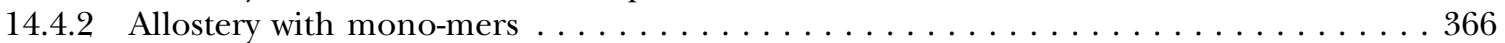

14.4.3 Allostery with two different primary ligands . . . . . . . . . . . . . . . . . . . 366

14.4.4 Allostery with reverse bell-shaped PTX-sensitive synagics . . . . . . . . . . . . . . . 366

14.4.5 Allostery with PTX-resistant reverse bell-shaped synagics . . . . . . . . . . . . . . . . . . . . 369

14.4.6 Allostery with covalent and intra-steric modifications . . . . . . . . . . . . . . . . 369

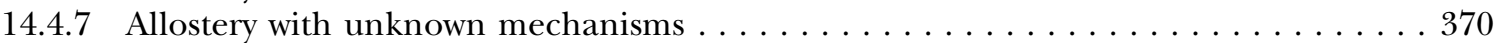

14.5 Summing up some views on allostery and co-operativity $\ldots \ldots \ldots \ldots \ldots \ldots$

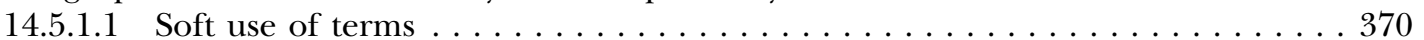

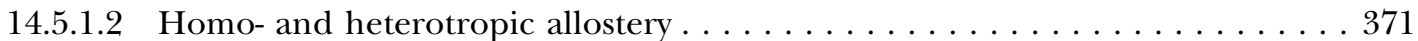

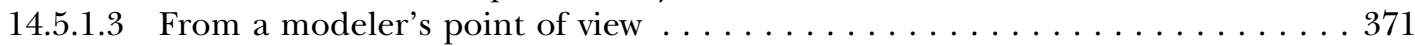

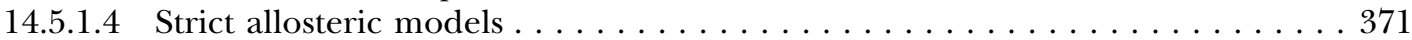

14.5.1.5 Allo- upon - allo- $\ldots \ldots \ldots \ldots \ldots \ldots \ldots \ldots \ldots \ldots \ldots$

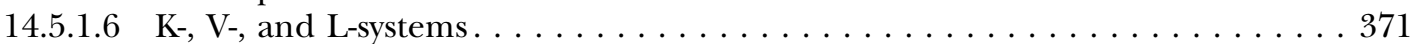

14.5.1.7 Reverse bell-shaped synagics and PTX poisoning . . . . . . . . . . . . . 371

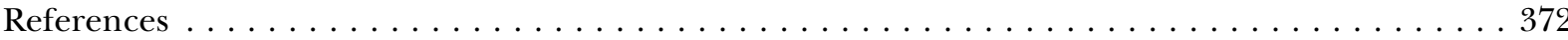

CHAPTER 15 Allostery and development of its models $\ldots \ldots \ldots \ldots \ldots \ldots \ldots \ldots$

15.1 Use, non-use, and misuse of the function of state $\mathrm{R} \ldots \ldots \ldots \ldots \ldots \ldots$

15.1.1 Hill-Adair-Pauling and Monod-Koshland models . . . . . . . . . . . . . . . . . . . 377

15.1.2 New allostery models FP-TSM and CQCM, and their hybrid model . . . . . . . . . . 378

15.2 The MWC model . . . . . . . . . . . . . . . . . . . . . . . . . . . . . . . 379

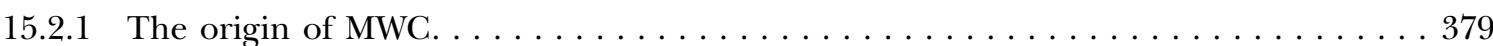

15.2.2 Details of Monod-Wyman-Changeux's model, the MWC model. . . . . . . . . . . . 379

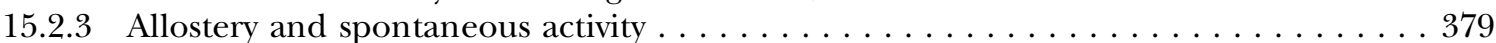

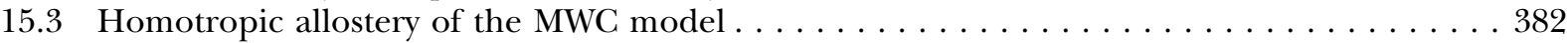

15.3.1 Consequences of the MWC model for homotropic allostery $=$ co-operativity $\ldots \ldots$. . 382

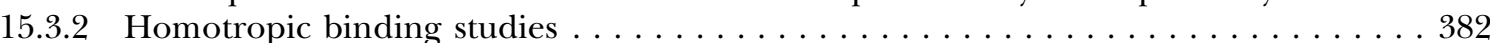

15.3.3 Validity of the homotropic Y-model . . . . . . . . . . . . . . . . . . . . . . . 384

15.3.4 Functional studies including some heterotropy . . . . . . . . . . . . . . . . . . . . . 384

15.3.5 Homotropic functional studies. A. Receptor studies . . . . . . . . . . . . . . . . . . 388

15.3.6 Homotropic functional studies. B. Enzyme and non-gated porter studies . . . . . . . 388

15.3.7 Functional bell-shaped synagics of the Cotr-enz formulation . . . . . . . . . . . . . . . 390

15.3.8 Other methods of suppressing spontaneous activity in the R-function. . . . . . . . . 390

15.4 Heterotropic allostery of the MWC model . . . . . . . . . . . . . . . . . . . . . . . . 393

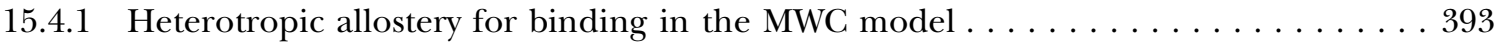

15.4.2 Heterotropic allostery for function in the MWC model . . . . . . . . . . . . . 393

15.4.3 Use of the R-function for enzymes displaying 'spontaneous activity' . . . . . . . . . . 396

15.4.4 Two-states and spontaneous activity in enzymes and non-ligand-gated transporters . . 396

15.5 Desensitization' as used by two different schools . . . . . . . . . . . . . . . . . . 396

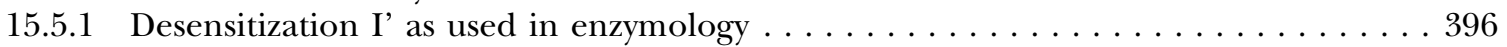

15.5.2 Desensitization II' as used in receptology and for some ligand gated channels . . . 396

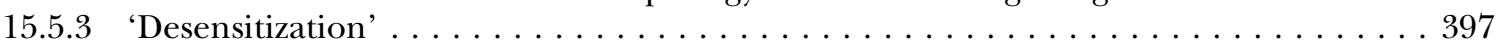


15.6 The KNF models . . . . . . . . . . . . . . . . . . . . . . . . . . 397

15.6.1 Homotropic binding studies in the KNF model . . . . . . . . . . . . . . . 397

15.6.2 Homotropic functional studies in the KNF model . . . . . . . . . . . . . . . . . 398

15.6.3 Heterotropic binding studies in the KNF model. . . . . . . . . . . . . . . . . 399

15.6.4 Heterotropic functional studies in the KNF model . . . . . . . . . . . . . . . . . 399

15.6.5 Disappointments with omitted details for the KNF model. . . . . . . . . . . . . . 399

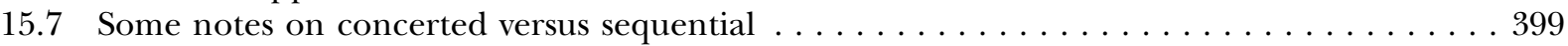

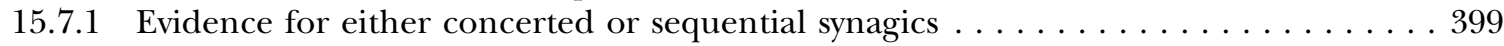

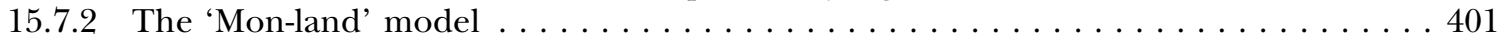

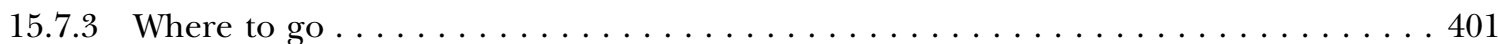

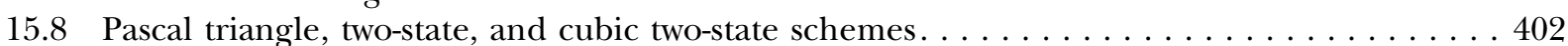

15.8.1 Combinatorial models and the Pascal-triangle extension . . . . . . . . . . . . . 402

15.8.2 Two-states - the MWC model versus the K\&T model . . . . . . . . . . . . . . . . . . 402

15.8.3 What differentiates the MWC model from other models? . . . . . . . . . . . . . . 403

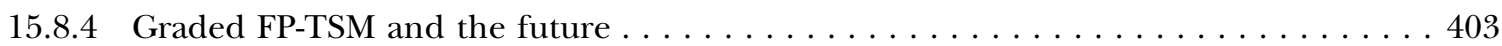

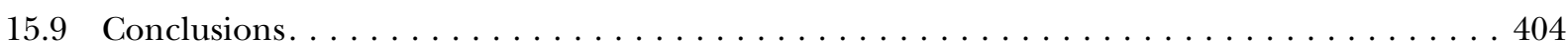

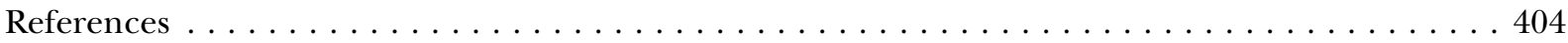




\section{Acknowledgements}

In finishing the book I think of:

Michael Lew, Melbourne, who willingly took on the tedious work of reviewing several parts of the book;

David Colquhoun, London, who also reviewed parts of the book;

Jean-Pierre Changeux, Paris, who took the time to discuss aspects of the MWC model with me;

Erik Skadhauge and Ernest M Wright, both of whom inspired me and taught me what Science is all about;

Mark Berner Hansen and Bent Winding, former graduate students in my laboratory, for fruitful collaboration;

The members of the New-Lacanian-School (NLSCopenhagen) - especially René Rasmussen - with whom I have analyzed the meaning of feelings and language;
Asger Bindslev, surgeon and theologian, my father, who constantly supplied me with texts on the existential questions of life during the writing process;

Lars Bindslev, MD (1944-1996), my twin-brother, to whom the book is dedicated;

The team at Co-Action Publishing, for their skillful and professional work;

Inge Hornung Pedersen, who helped with the timeconsuming work of obtaining permissions to republish figures and charts;

Thomas and Stine, my children, who will hopefully see more of me in the future; and

Helle, my wife, for her part in making the book unfold from dream to reality.

Niels Bindslev

Copenhagen, April 2008 


\section{Preface}

Our intuition tells us that in order to study the mechanisms of receptive molecules, their interactions with ligands, and their internal regulation, it is a sine qua non to perform and resolve rates of reaction as seen in non-steady-state experiments where time is the independent variable. However, a great deal of valuable information about these mechanisms may also be obtained from experiments at equilibrium, i.e., with ligand concentration as the sole independent variable.

This book is a survey of, and evaluates, analytical tools used to determine equilibrium effects of the interactions between ligands and receptive units as obtained from dose-response experimentation and from combinatorial drug therapy.

\section{Raîson d'être}

The book is intended as a help for students and researchers in the biomedical fields, enabling them to choose the best model for their dose-response data obtained from wet-lab experiments. The focus is on how to interpret and handle dose-response data, with a recommendation to down-play analytical methods developed before the era of personal computers, such as the Lineweaver-Burk and Scatchard data conversion, the null-methods by Gaddum and Schild, or the use of meaningless mathematical manipulations, as for instance the implementation of a Hill-exponentiation. Instead, when fishing for system constants, the readers learn how to get access to the free-way of analytical tools that offer forward formulated physical functions to be fixed with non-linear fitting procedures.

The approach I have taken is different from that of many other textbooks on the analysis of equilibrium dose-responses, which follow in the tradition of datalinearization developed more than 70 years ago. A book in point is Segel's 'Enzyme kinetics' (1975), reissued as a non-revised edition in 1993 and still considered a standard textbook on enzyme kinetics: it lacks almost completely in analysis of the so-called twostate models.

\section{Objectives}

The framework of this book is a so-called four-pane twostate model (FP-TSM), which is an equilibrium, twoligand and two-state model covering regulated ligandreceptor interactions at the level of receptive units. Meanwhile, as the model involves seventeen independent parameters it is a complex tool to use to determine parameters for dose-response relationships at equilibrium, even though it is probably one of the simplest tools for analysis of allosteric regulation and of combinatorial drug therapy by two ligands.

My goal has been to give a description of the FP-TSM that is as comprehensive as possible. All the implications of the FP-TSM are not yet solved, but in the process, the reader will learn about the usefulness of subsets of the four-pane framework for analysis of experimental data. Thus the road to the goal, is the goal.

\section{Regula detri}

There is heavy use of regula detri in the text. Regula de tribus is mediaeval Latin for equating elements by two equal fractions, e.g., $a / b=c / d$, such that one element, for instance $a$, is given by the knowledge of the three other elements $b, c$, and $d$. In the book the reader will learn how to tinker with regula detris.

As indicated above, both time-dependent and timeindependent experimentation can yield essential information about ligand-receptor interactions, and both types of experiments are necessary for the clarification of these mechanisms. Solutions to rate equation go through exponential terms, whereas equilibrium equations are solved by regula detri manipulations. Regula detris are much more robust for analysis than the exponential equations, and this is one major advantage of experiments at equilibrium. Meanwhile, the two types of approaches, equilibrium and non-steady-state, yield different and overlapping information about the mechanisms behind the dynamics of ligand-receptor interaction, and should of course both be performed. 
The excessive use of regula detris may be put in perspective by Lacan's four discourses for his model of the 'sujet' in psychoanalysis. ${ }^{1}$ Lacan's discourse contains extremely vague and willfully perplexing uses of regula detris, which are amusing pendants and counter-points to the physical regula detri tools employed in this book for dose-response analyses.

\section{Arrow diagrams}

Why tools of regula detri? Isn't molecular biology enough? Let me paraphrase from an editorial comment in Nature ${ }^{2}$ on pathway diagrams often employed to explain ideas imparted from molecular biology:

For cartoons of networks, pathways and complexes, indeed, superb papers have been written for the purpose of adding a single arrow to an existing scheme. But to really understand how the biological net works, one needs to have numbers attached to the arrows and equations (input functions) relating the system constants.

On a limited scale, this is what the book tries to do in the context of Systems Biology.

\footnotetext{
${ }^{1}$ The seminar of Jacques Lacan: Book XVII The Other Side of Psychoanalysis, translated by R. Grigg. New York: Norton, 2007.

2 Nature 397: 89, 1999.
} 

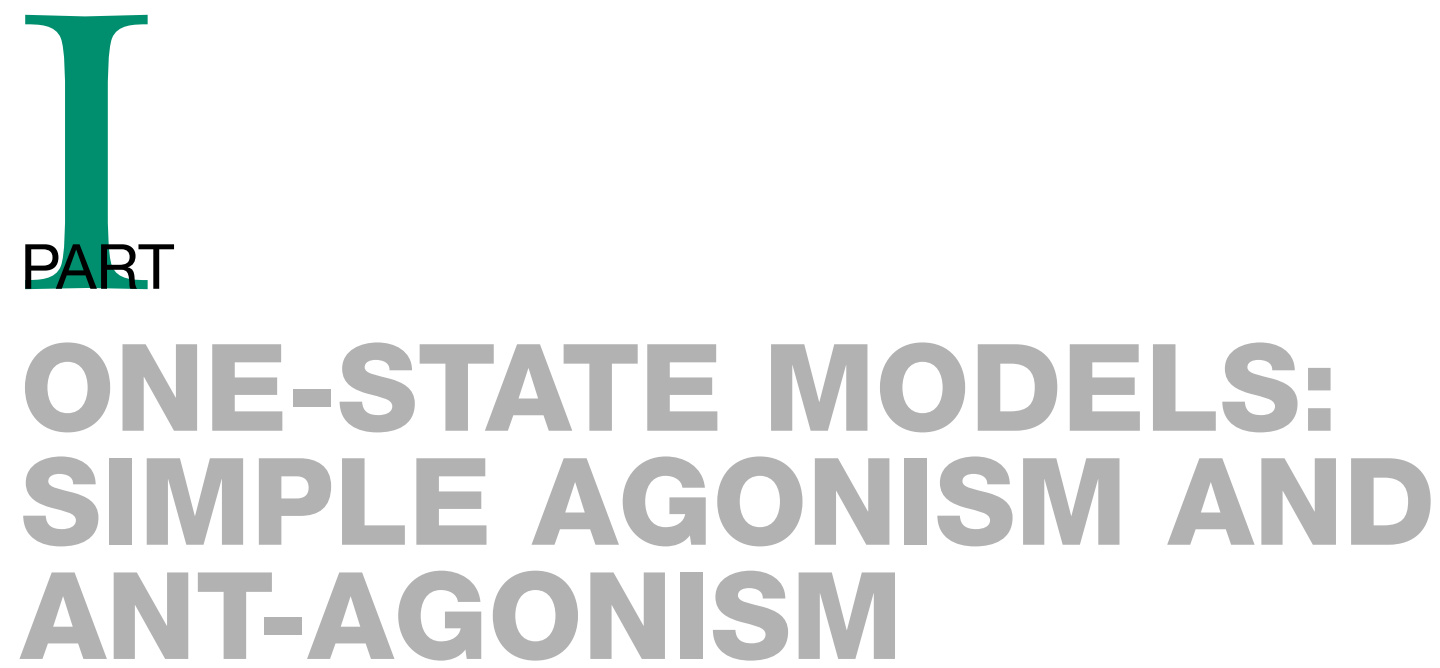

\section{I.1. A Prelude}

What happens when two drugs are given simultaneously? This book is about the analysis of data from ligandreceptor interactions obtained from dose-response experiments at equilibrium and about how theoretical schemes of mechanisms can be adopted to these data and with a main aim on how to simulate physically what happens when two drugs are given simultaneously. This for a comparison with reality and for a better implementation of combinational drug therapy. In essence, the entire book is an elaboration of the Michaelis-Menten equation.

The various concepts of ligand-receptor interaction were first developed during a so-called classic era ending circa 1945 , and then during a post-classic era, starting circa 1965, with what could be called a transition period between 1945 and 1965. Concepts from the classic era were mostly concerned with occupancy or function as a two-step mechanism while the post-classic era, besides binding and function, was and still is preoccupied with states of conformational changes for the un-liganded receptive unit, a two-state mechanism.

\section{I.2. One, Two, and Many Steps or States}

Concepts from the classic and post-classic eras are entwined. In Part I, concepts from the classic era are described in detail, delineating the step of binding per se. Concepts such as efficacy and partial agonism are also introduced. These latter concepts were developed in the transition period. Efficacy is related to conformational change in protein molecules that function as receptive units and effectors. Part II deals with the state of conformational change in un-liganded receptive units. A switch from one to two or more conformational states for an un-liganded receptive unit was introduced and expanded in the post-classic period.

Other terms for an initial classification of concepts from the classic era could be processes with single-state receptors or simple agonism and simple ant-agonism (cf. title of Part I), while for the post-classic era terms such as complex agonism and complex ant-agonism or two-state processes within a receptive unit are dealt with in Part II.

Terms are tentatively tabulated in a somewhat dogmatic manner in Table I.1 in an attempt to illuminate the distinction between the two eras, their concepts, and our terminology so far.

\section{I.3. Synagic and Synagics}

In this book, a dose-response relation will also be referred to as a 'synagic' relation. The term synagic is derived from the Greek substantive 'synagogé' meaning concentration, meeting, or gathering. We know this word from 'synagogue'. The verb to concentrate is 'synagein'. Instead of the somewhat cumbersome 'synagogic' as an adjective, we abbreviate it to synagic. The noun synagic signifies that concentration of a ligand is a driving force and the independent variable, rather than time as the independent variable. Note that synagic only refers to dose-response relations at equilibrium and steady-state (see Preface).

Time is a tacit independent variable in the adjectives 'kinetic' and 'dynamic'. Therefore, throughout most of the text, at equilibrium, synagic replaces kinetic and dynamic. Incidentally, remember that 'thermodynamics' 
Table I.1. Periods and concepts developed for interaction between ligands and receptive units

\begin{tabular}{lllll}
\hline Part in the book & Era & Agonism and ant-agonism & Process in focus & States for un-liganded receptive unit \\
\hline Part I & Classic & Simple & Occupancy & Single- \\
Part II & Post-classic & Complex & Conformation & Two- or multi- \\
\hline
\end{tabular}

When the subject is complex agonism, all the tabulated adjectives of Part II may come into play. Note, that simple ant-agonism may involve two sites on the receptive unit, as discussed in Part I Chapter 2, while mechanisms due to two states solely belong to the complex agonism and complex ant-agonism dealt with in Part II. Here, 'states' refer to the number of conformations for an un-liganded receptive unit.

most often refer to equilibrium situations, but not always (Katchalsky \& Curran 1965), and not necessarily to doseresponses.

So, synagic and synagics are specific terms that stand for concentration-occupancy and dose-responses at equilibrium.

\section{I.4. Equilibrium and its Formulation}

This tome is about the use of theories in the analysis of experimental dose-response data obtained at equilibrium. For the analysis, equilibrium theories are expressed as equations, synagic equations.

The formulations of concentration-dependent equilibria are based on a conservation principle, which means that the receptive units of interest, e.g., all activated receptors or actual response (ar), are expressed as a fraction of the total of all receptive units or total response $(T R)$. Thus, expressions such as ' $a r / T R=\ldots$ ' will commonly appear. Formulations with $\operatorname{ar} / T R=\ldots$ are also known as 'distribution equations'.

On equilibria, JBS Haldane wrote "The key to a knowledge of enzymes is the study of reaction velocities, not of equilibria" (Haldane 1930, p. 3), and, of course, one cannot assess the order of events in kinetic schemes by measurements at equilibrium (Fersht 1999, p. 125). Nevertheless, the present book tries to disprove Haldane's statement, arguing that theoretical analysis of concentration-dependent equilibria as well as timedependent velocities are key tools to our understanding of transducing molecules and their function. In addition, since the emphasis of this book is on equilibria, nearly all parts herein deal with dose-responses at equilibrium, that is, synagics.

\section{I.5. Binding versus Function}

In dose-response studies it is paramount to realize what type of experimental set-up is to be analyzed. When embarking on an analysis of agonism or ant-agonism at equilibrium, the first question to be answered is whether the experiments are performed as a binding response of a ligand against a change in concentration of the ligand, or as a functional response by a ligand against a change in concentration of the ligand. Recognition of the need to discern between binding and functional experiments is crucial (see, e.g., Sections 2.4.5, 2.4.6, and 2.4.12, and Table 5.1 in Chapter 5). Obviously, just because there is a conformational change when a ligand binds, this conformational change is not necessarily the one which activates the receptive unit for function. Meanwhile, since theories on binding and function have many overlapping and identical expressions (Sections 2.4.9 and 2.4.10), their analyses are easily confounded. For this reason, they must be sharply separated at all times. The very first question is, therefore, are your experiments based on binding or on functional observations?

\section{I.6. Reciprocity}

Lately, a clear realization that conformational changes also influence the binding-occupancy process has been established. Thus, if binding of a ligand induces a conformational change necessary for the functional activity, then that conformational change will affect the binding process. This mutual interaction is known

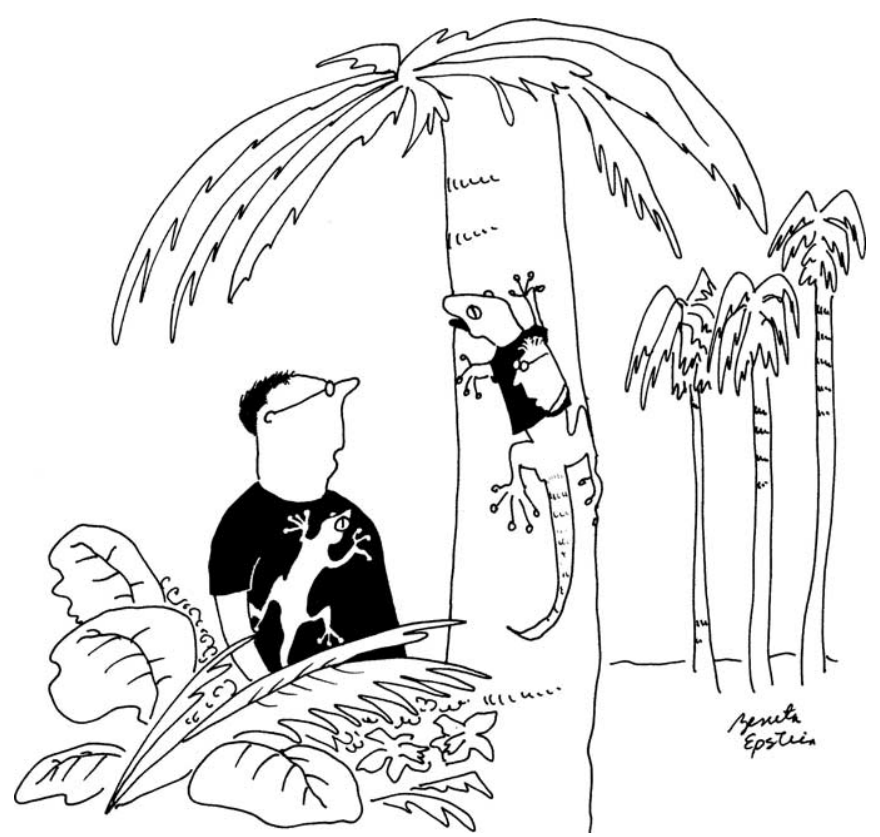

Figure I.1. Associative semiotics ... reciprocity? Geckoman (C) 1996. Benita Epstein, with permission. 
as reciprocity. Mechanistic reciprocity is a fundamental principle in all physico-chemical processes, including irreversible processes (Onsager 1931a,b). At equilibrium, and also in non-equilibrium situations, so-called cross-coefficients $L_{\mathrm{mn}}$ and $L_{\mathrm{nm}}$ are equal for $\mathrm{m} \neq \mathrm{n}$ :

$$
L_{\mathrm{mn}}=L_{\mathrm{nm}} \quad(\text { for } \mathrm{m} \neq \mathrm{n})
$$

when local entropy production is the sum of all effects. ${ }^{1}$

The consequences of reciprocity for the interaction between receptive molecules and ligand molecules have been discussed eloquently in a seminal paper (Colquhoun 1998). Colquhoun stated aptly that "If binding affects activation, then activation must affect binding". The reciprocity here is physical (Colquhoun 1987) and different from the associative reciprocity of Fig. I.1. Examples of a tight survey of reciprocity and its cross-coefficients for irreversible processes are given in Katchalsky and Curran (1965) and DeLaage (1975), and on "linked function"' in hemoglobin by Wyman (1964) and Wyman and Gill (1990).

\footnotetext{
1 Effect here is the product of flow and force. For more details on cross-coefficients, see for instance Katchalsky and Curran (1965).
} 
Part I: One-state Models: Simple Agonism and Ant-agonism 
Basic reaction schemes and their formulations are derived in Chapter 1. The derived equations are referred to as the 'law of ...' due to their axiomatic nature. Formulations are, of course, derived for both function and binding reaction schemes.

In this chapter, we will also look at partial agonism, receptor reserve, intrinsic activity, efficacy, and intrinsic efficacy from the transition period. Then, very briefly, the concept of 'allostery' will be touched upon, and the chapter ends with concepts of spontaneous activity and inverse agonism from late in the post-classic period. All these concepts will be discussed more thoroughly later on.

To start with the easiest, let us approach a derivation of equations for the interaction between a ligand and a one-state receptive unit: so called simple agonism.

\subsection{Derivation of Dose-response Relationships}

\subsubsection{The Binding Proper of a Ligand to a Receptive Unit}

The pure-process-proper of binding a ligand to its receptive unit is a chemical process theorized for equilibrium, and subsequently proven in experiments by Langmuir (1918) during World War I, from 1914 to 1918. The process is called 'adsorption' (Fig. 1.1). A subsequent release of the ligand back into solution is referred to as the desorption process.

What Langmuir showed was that single molecules moving freely and arriving at a solid material with a surface of receptive molecules would bind in a monomolecular layer at the exterior of the solid. Molecules in this surface layer had binding capabilities different from the underlying molecules of the solid. The extra forces of the surface molecules, as they had 'one side free', were the actual forces participating in the process of adsorption. As soon as the freely moving molecules, the ligands, passed the first monolayer of the solid, the process became an entirely different one, requiring conformational changes in the structure of the solid's material. This latter process was more of a physicochemical nature, and named 'absorption' (Fig. 1.2). Temporarily forgetting about absorption, the process of adsorption and desorption involves three entities, viz. free ligand molecules, e.g., designated S, non-occupied receptive units $(R)$, and receptive units with occupied sites bound by ligand (RS).

Since the adsorption process is a reversible one, there are two processes going on at any time, $\mathrm{R}$ and $\mathrm{S}$ combining to RS, and RS splitting into $\mathrm{S}$ and $\mathrm{R}$. We may write these two processes in a composite expression as:

$$
\mathrm{R}+\mathrm{S} \rightleftarrows \mathrm{RS}
$$

This is the simplest reaction scheme for an adsorption-desorption process.

\subsubsection{Limited Number of Receptive Sites}

In the above situation, the sum of all receptor conformations bound or unbound $\left(R_{\mathrm{tot}}\right)$ is equal to a fixed and 'limited' number. There will be a bombardment of a 'finite' number of receptive sites by ligand molecules with varying intensities as the ligand concentration varies.

When the symbols $\mathrm{R}$ and $\mathrm{RS}$ represent concentrations of the species R and RS, a 'finite' volume also means a 'limited' number of total Rs. Keep in mind as we go along that the description assumes a 'finite' number of receptive units, while the ligand source provisionally is inexhaustible.

In the following sections, the history shall be summarized from chemical mass action over equilibrium to the Langmuirian adsorption isotherms, describing doseresponse relations for a 'limited' number of receptive sites. The aim is to obtain expressions characterizing the studied system, and allowing, for example, an assessment of the 'finite' number of receptive sites, by determining the number of bound ligands at varying ligand concentrations. Purposely, 'limited' or 'finite' 


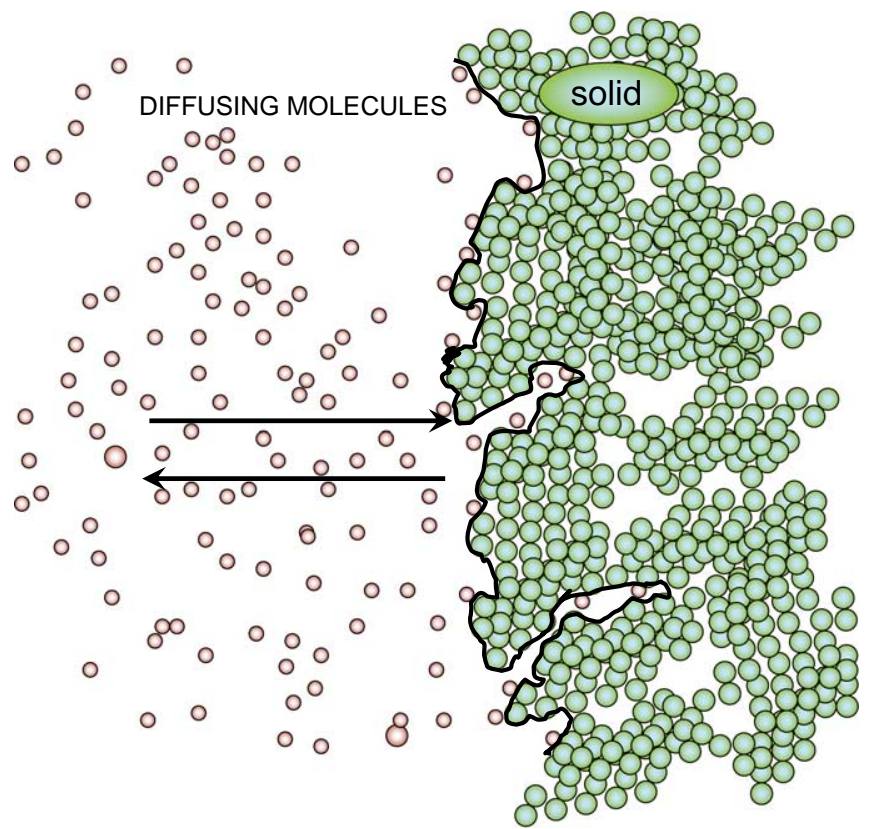

Figure 1.1. Adsorption. Diffusing molecules reach and bind to the outer layer of the fabric.

numbers of receptive sites have now appeared 10 times within about 25 lines of coherent text.

\subsubsection{Reversibility of Chemical Reactions}

Two hundred years ago, reversibility of chemical reactions and ideas about chemical equilibria were ventilated by Claude Louis Berthollet in his Essai de Statique Chimique (Berthollet 1803, pp. 1-67). In 1799, during a trip to Egypt, Berthollet noticed chemical reversibility

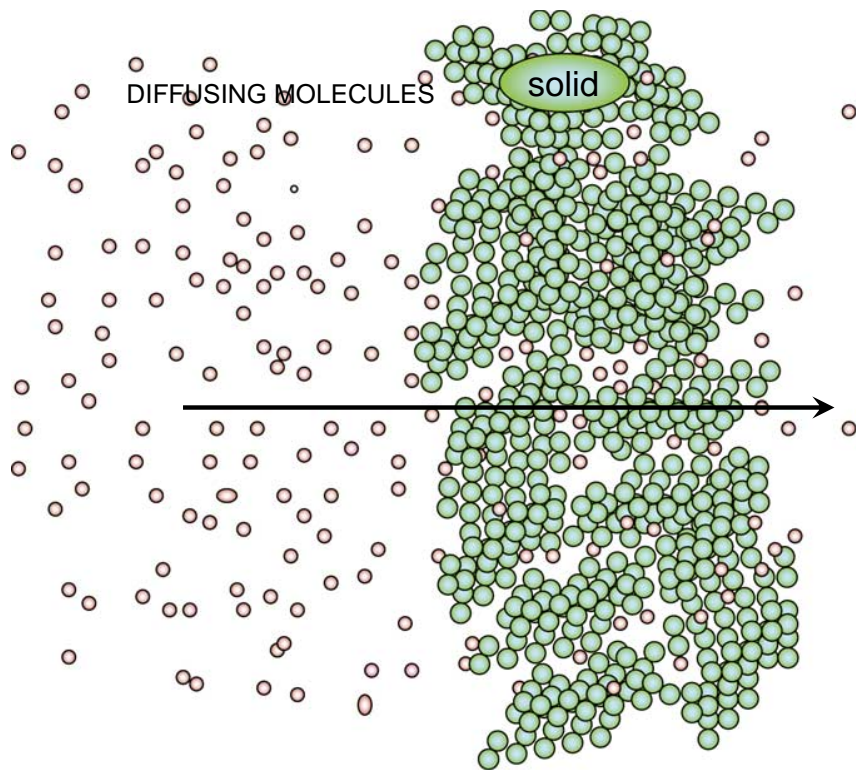

Figure 1.2. Absorption. Diffusing molecules penetrate the outer layer and interact with the fabric. while watching the effects of $\mathrm{NaCl}$ brine on deposits of limestone $\left(\mathrm{CaCO}_{3}\right)$ at the shores of salt lakes along the Nile. High salt concentrations could reverse the process of forming slaked lime. In passing, during his trip together with Bonaparte's campaign to Egypt, French soldiers recovered the Rosetta Stone (RS) (Hagar Rashid حسبر رشـيد at Rosetta (el Rashid)). This should remind us to read symbols and acronyms in their right context. ${ }^{1}$

\subsubsection{The Law of Mass Action (Loma)}

Around the middle of the nineteenth century, especially Wilhelmy (1850a, b), a German chemist known as the father of kinetics, and two French chemists, Berthelot and Péan de Saint-Gilles (1862a,b, 1863), helped establish the law of mass action, loma (see especially Berthelot 1862). Loma states that the chemical production rate is given by the product of concentrations of reactants $[\mathrm{S}]$ and $[\mathrm{R}]$ times a parameter, to describe the rate of that particular reaction, a so-called rate constant $(k)$. Thus, for the two processes, forward and backward in reaction scheme $\mathrm{S}+\mathrm{R} \rightleftarrows \mathrm{RS}$ given above, we have in terms of loma, first for the forward reaction:

$$
[\mathrm{R}] \cdot[\mathrm{S}] \cdot k_{1}=\text { production rate of }[\mathrm{RS}] \text {. }
$$

The expression to the left of the equal sign in Eq. 1.1 is the rate of production of occupied receptive sites (RS), given as the product of the concentrations of the reactants $\mathrm{R}$ and $\mathrm{S}$ plus a rate constant, $k_{1}$.

For the backward reaction we have:

$[\mathrm{RS}] \cdot k_{-1}=$ production rate of $[\mathrm{R}]+[\mathrm{S}]$.

This second equation gives the rate at which the complex RS is dissolved, as the $\mathrm{S}$ molecule leaves the RS receptor-ligand complex, and is expressed as the product of concentration of RS and a rate constant $\left(k_{-1}\right)$ for the rate of splitting. Equation 1.2 is another loma.

The squared parentheses [] indicate concentration. Recall that concentration is mass per (units of volume). In later formulations, squared parentheses will usually be omitted from expressions of concentration. As already mentioned, $k_{1}$ is a rate constant, characteristic for the adsorption process. The reaction rate constant $k_{1}$ for the formation of the complex RS is a parameter, ${ }^{2}$ and has the dimension per (unit time concentration). Rate constant $k_{-1}$ is another parameter characteristic of the desorption process of $\mathrm{S}$ leaving the RS complex. Parameter $k_{-1}$ is a coefficient for the rate of splitting complex RS into

\footnotetext{
${ }^{1}$ Recovery of the Rosetta Stone (RS) later gave the English an opportunity to bring the stone 'home' and 'forward' to the public, while many of the French soldiers and their progeny still remain in Egypt. Their descendants now wonder how to bring the RS 'backward'. On occasions RS is just RS.

${ }^{2}$ Recap that a 'system constant' is the same as a 'parameter'.
} 
its constituents. Constant $k_{-1}$ has the dimension per (unit time). Given a limited amount of receptive units, the time-dependent form of loma as an exponential-decay was also derived by Harcourt and Esson (1866).

\subsubsection{The Law of Chemical Equilibrium (Loce)}

At equilibrium, the two processes of fusion and fission are equally fast. From a rate of production point of view, at equilibrium, we can now write that the rate of production of RS, by combining $\mathrm{R}$ and $\mathrm{S}$, is just as fast as the elimination of RS over time, generating $\mathrm{R}$ and $\mathrm{S}$. For our reaction at equilibrium, we may write this statement as:

$$
[\mathrm{R}] \cdot[\mathrm{S}] \cdot k_{1}=[\mathrm{RS}] \cdot k_{-1} \text {. }
$$

Eq. 1.3 is a slightly circumscribed and simplified version of a $\mathrm{G}-\mathrm{W}$ equation (Guldberg \& Waage 1867). Equation 1.3 may be termed loce.

\subsubsection{Equilibrium Association and Dissociation Constants}

Omitting squared parentheses, Eq. 1.3 may be rewritten to:

$$
\frac{\mathrm{RS}}{\mathrm{R}}=\mathrm{S} \cdot \frac{k_{1}}{k_{-1}} \quad \text { or } \quad \frac{\mathrm{RS}}{\mathrm{R}}=\frac{\mathrm{S}}{k_{-1} / k_{1}} .
$$

This relationship for equilibrium was finally and firmly formulated by the two Norwegian chemists Guldberg and Waage (1867), equating $k_{-1} / k_{1}$ as a dissociation constant $K_{\mathrm{d}}$, with the dimension concentration. In the literature, $K_{\mathrm{d}}$ is often designated $K_{\text {eq }}$ (e.g., Fell 1997).

Note that time as a variable has disappeared in Eq. 1.4.

Eq. 1.4 states that the ratio between occupied receptive sites (RS) and non-occupied receptive sites (R) is equal to the ligand concentration $(\mathrm{S})$ times the ratio of association rate constant $\left(k_{1}\right)$ to dissociation rate constant $\left(k_{-1}\right)$, that is: $k_{1} / k_{-1}$.

By GW convention: $k_{1} / k_{-1}$ is the so-called equilibrium 'association' constant $K_{\mathrm{a}}$, and $k_{-1} / k_{1}$ is the equilibrium 'dissociation' constant $K_{\mathrm{d}}$ or $K_{\mathrm{eq}}$. Of course, in principle it does not matter if you use $K_{\mathrm{a}}$ or $K_{\mathrm{d}}$. The association constant is also called an affinity constant, ${ }^{3}$ and has the dimension of reciprocal concentration $(1 /$ concentration).

In the biological literature, more than fifty years ago it was en vogue to use $K_{\mathrm{a}}$, but today $K_{\mathrm{d}}$ is used most often. One reason to use $K_{\mathrm{d}}$ is the dimension of $K_{\mathrm{d}}$, which is

\footnotetext{
${ }^{3}$ On the history of 'affinity', earlier known as 'attraction', see Berthelot and Péan de Saint-Gilles (1862a,b, 1863), Guldberg and Waage (1867, 1899a,b), Ostwald (1902, pp 1-198), and Partington (1964, Vol 4, Chapter 18).
}

straightforward concentration. So, for instance, $K_{\mathrm{d}}$ is the parameter which appears right away on the concentration axis in dose-response graphs (see later). In chemical literature, the convention was (and still is) to have the forward rate constant in the numerator, as in the left half of Eq. 1.4, yielding $K_{\mathrm{a}}$ right away, that is:

$$
\frac{\mathrm{RS}}{\mathrm{R}}=\mathrm{S} \cdot \frac{k_{1}}{k_{-1}} \Rightarrow \frac{\mathrm{RS}}{\mathrm{R}}=\mathrm{S} \cdot K_{\mathrm{a}} \quad\left\{=\mathrm{S} / K_{\mathrm{d}}\right\} .
$$

As we shall see, this latter convention was also followed by Langmuir, and contaminated the biological literature to operate with $K_{\mathrm{a}}$ rather than $K_{\mathrm{d}}$. Reading older literature as well as advanced modern texts on receptor synagics, the above mentioned shift in convention from $K_{\mathrm{a}}$ to $K_{\mathrm{d}}$ is worth keeping in mind. Later in this book, as it becomes more complex, $K_{\mathrm{ss}}$ is used for $K_{\mathrm{d}}, A_{\mathrm{ss}}$ for $K_{\mathrm{a}}$, and $K_{\mathrm{ss}}$ and $A_{\mathrm{ss}}$ are used interchangeably as convenient. On the terminology for equilibrium constants as $K_{\mathrm{ss}}$ and $A_{\mathrm{ss}}$, see Box 2.1. in Chapter 2.

\subsubsection{Rules of Regula Detri}

Before proceeding with the development of doseresponse relationships, some simple arithmetic must be introduced. In this book, nearly all the equations are regula detri expressions (see Preface), the simplest form of math after addition and subtraction. Fractional terms or relative terms are expressed by regula detris (r.ds). Here is one:

$$
\frac{a}{b}=\frac{c}{d}
$$

Rules for this regula detri equation are presented in Box 1.1.

Employing regula detri rule (r-d-r) 1a from Box 1.1:

$$
\frac{a}{b+a}=\frac{c}{d+c}
$$

on the right-hand side of Eq. 1.4, and after inserting $K_{\mathrm{d}}$ for $k_{-1} / k_{1}$ as in Eq. 1.5, we get:

$$
\frac{\mathrm{RS}}{\mathrm{R}+\mathrm{RS}}=\frac{\mathrm{S}}{K_{\mathrm{d}}+\mathrm{S}} \text {. }
$$

Here $\mathrm{R}+\mathrm{RS}$ in the left-hand denominator is equal to the total number of 'limited' receptive units, $R_{\mathrm{tot}}$, (see next section).

\subsubsection{Langmuir's Law of Adsorption and Desorption (Load)}

So far, the adsorptive process is saturable because there is a 'limited' number of binding sites. The total sum of all receptive sites is equal to all free receptor sites $\mathrm{R}$ plus 


\section{Box 1.1. Regula detri rules (r-d-r)}

Rules for the regula detri in Eq. 1.6

Rule la $\frac{a}{b+a}=\frac{c}{d+c}$

Rule $1 \mathrm{~b} \quad \frac{a}{b-a}=\frac{c}{d-c}$

Rule $2 \mathrm{a} \quad \frac{a}{b}=\frac{c+a}{d+b}$

Rule $2 \mathrm{~b} \quad \frac{a}{b}=\frac{c-a}{d-b}$

Try these regula detri rules for example with:

$$
\frac{1}{3}=\frac{6}{18}
$$

The regular detri rules are also valid after a rotation of terms. An example: the above regula detri still holds after a counter-clock rotation to:

$$
\frac{6}{1}=\frac{18}{3}
$$

This is not the case for a similar rotation of signifiers between the Lacanian Master discourse and his Analytical discourse (see Preface).

all occupied receptor sites $\mathrm{RS}$, equal to $\mathrm{R}+\mathrm{RS}$, and we may write the following regula detri:

$$
\frac{\text { occupied receptive sites }}{\text { all receptive sites }}=\frac{\mathrm{RS}}{\mathrm{R}+\mathrm{RS}} \text {. }
$$

This equation states that the fraction of occupied binding sites to the total number of binding sites that can be occupied is equal to bound receptive sites (RS) divided by still free receptive sites $(\mathrm{R})$ plus the bound receptive sites (RS).

Combining Eqs. 1.8 and 1.9 yields:

$$
\frac{\text { occupied receptive sites }}{\text { total receptive sites }}=\frac{\mathrm{S}}{K_{\mathrm{d}}+\mathrm{S}} \text {. }
$$

Equation 1.10 states that the fraction of receptors in occupied form to all receptors is equal (directly proportional) to the ligand concentration $\mathrm{S}$, divided by the sum of equilibrium dissociation constant $K_{\mathrm{d}}$, plus ligand concentration S. Eq. 1.10 is a well-known simple and useful expression in order to determine $K_{\mathrm{d}}$. The maximal response one can expect is when all receptor sites are occupied. This will happen at high concentrations of ligand $\mathrm{S}$. Both sides of the above equation then become equal to 1 . When $\mathrm{S}=K_{\mathrm{d}}$, half of all the sites are occupied (cf. Eq. 1.10 and Fig. 1.3).
Replacing $K_{\mathrm{d}}$ with $1 / K_{\mathrm{a}}$ and total receptive sites $\mathrm{R}+\mathrm{RS}$ with $R_{\text {tot }}$, we can rewrite the last equation into:

$$
\text { occupied receptive sites }=\frac{R_{\mathrm{tot}} \cdot \mathrm{S} \cdot K_{\mathrm{a}}}{1+\mathrm{S} \cdot K_{\mathrm{a}}},
$$

which is Langmuir's equation for adsorption-desorption expressed in our terminology. It is the law of adsorptiondesorption = load. Langmuir confirmed this theory with experiments measuring adsorption of gas molecules to various solid surfaces by varying the gas pressure (Langmuir 1918).

Thus, the fraction of occupied binding sites in the load is not a simple proportional function of the gas or ligand concentration (see Eqs. 1.10 or 1.11 and Fig. 1.3), while the loma in Eqs. 1.1 and 1.2 is a simple proportional function of ligand concentration. In load, binding is not proportional to the ligand concentration due to the 'limited' number of total sites, $R_{\text {tot }}$. Moreover, loma is also a time-dependent expression, which load is not. Therefore, at any time, keep loma and load apart.

\subsubsection{The Henri-Michaelis-Menten Equation (HMM)}

A few years before Langmuir published his adsorption equation, Michaelis together with a colleague, Maud Menten, published equations for enzymatic reactions with an appearance analogous to Langmuir's adsorption equation, though $K_{\mathrm{a}}$ was presented with another constant (Michaelis \& Menten 1913). Furthermore, the Michaelis and Menten (M'nM) equations deviate in two fundamental aspects from that of the Langmuirian load.

(1) The M'nM equations are time-dependent expressions of initial rates, eventually at steady state, with a rate constant in the nominator, which we will ignore for the moment. ${ }^{4}$

(2) Further, the association constant $K_{\mathrm{a}}$ of the load equation is replaced in M'nM's equation with a dissociation constant that today is signified with $K_{\mathrm{m}}$. At equilibrium, ignoring the time-dependency, the $M^{\prime} n M$ concept may be formulated as:

$$
\frac{\text { occupied receptive sites }}{\text { total receptive sites }}=\frac{\mathrm{S}}{K_{\mathrm{m}}+\mathrm{S}} \text {. }
$$

Observe, that the $K_{\mathrm{d}}$ in Eq. 1.10 is replaced by a $K_{\mathrm{m}}$ in Eq. 1.12.

In M'nM terminology, the $K_{\mathrm{m}}$ was written as $K$. What is the difference between $K_{\mathrm{d}}, K_{\mathrm{m}}$, and $K$ ?

\footnotetext{
${ }^{4}$ In Chapter 10 in Part III a more accurate expression will be given for the M'nM equation, which was derived for steady-state situations and initial velocities, while here merely presented in its equilibrium form.
} 


\section{The hyperbolic function}

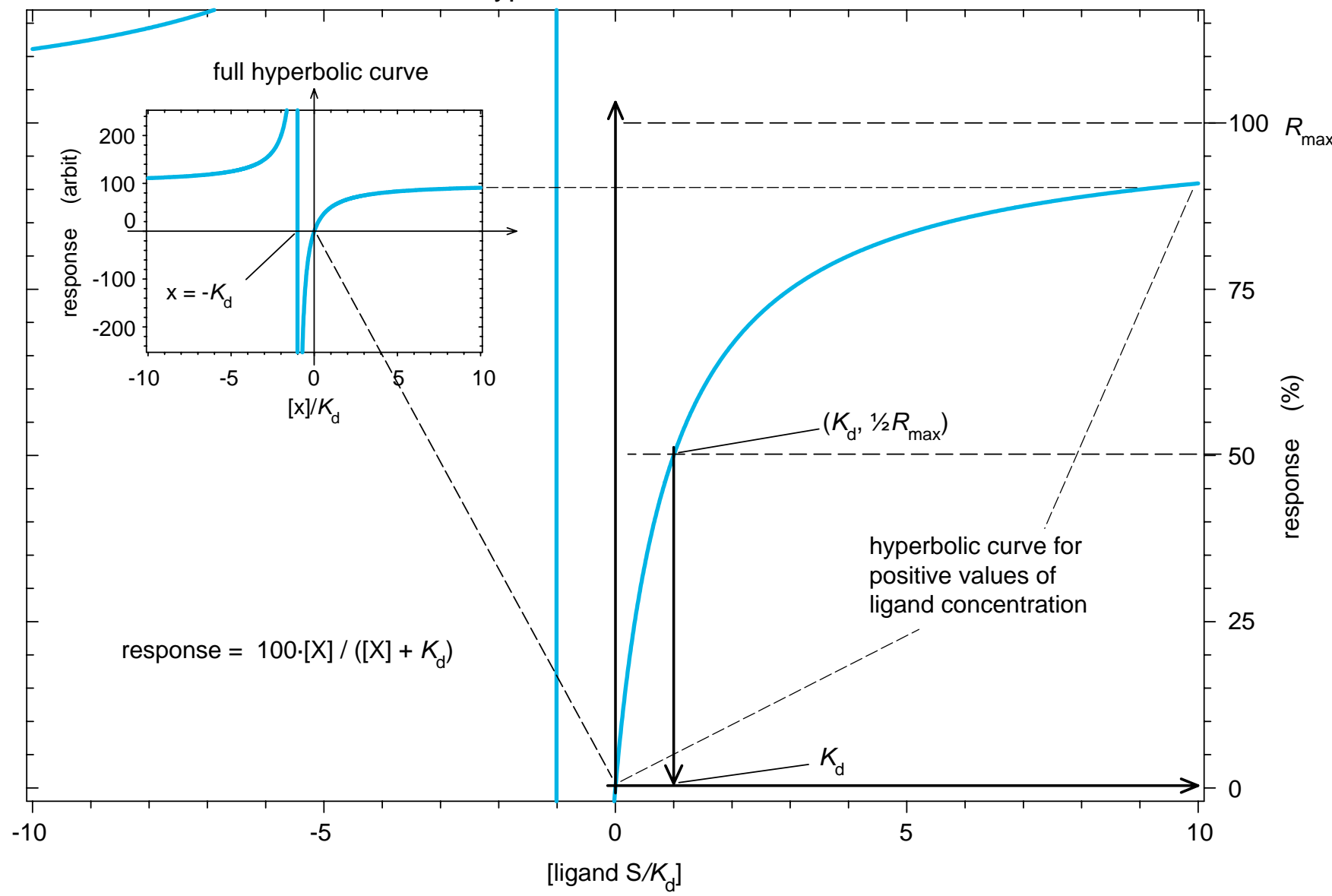

Figure 1.3. A full and a fractional rectangular hyperbolic relationship. The insert graph demonstrates the full hyperbolic function. The hyperbolic curve on the right-hand-side of the larger graph, which is a zoom-in of the insert graph, is defined for values of the independent variable $S \geq 0$. Parameter $K_{d}$ is 1 (in arbitrary units) in both graphs. Thus, the hyperbolic curve for positive values of $x$ or $\mathrm{S}$ is a fraction of the full hyperbolic function. Synagics as simple agonism is described by the fractional hyperbolic relationship.

Binding a substrate to an enzyme resembles the Langmurian adsorption process of gases binding to the surface texture of solids. But, M'nM also included a step for the conversion of the enzyme-substrate complex (ES) into free enzyme (E) and a product (P) being created with an additional rate constant $\left(k_{\mathrm{p}}\right)$ with the dimension of reciprocal time $(1 /$ time $)$ and assumed nearly irreversible (Fig. 1.4). Thus, focusing only on loma for production rate, i.e., production of $\mathrm{P}$ per time unit, we have:

$$
[\mathrm{ES}] \cdot k_{\mathrm{p}}=[\mathrm{E}] /(\text { time })+[\mathrm{P}] /(\text { time }) .
$$

We note that dissolving the complex ES into free $\mathrm{E}$ and $\mathrm{P}$ (Eq. 1.13), has the same form as loma for a simple desorption process. Meanwhile, instead of $[\mathrm{RS}] \cdot k_{-1}$, as assumed in the Langmurian load, M'nM have $[\mathrm{ES}] \cdot\left(k_{-1}+k_{\mathrm{p}}\right)$, indicating that ES could split into either $\mathrm{E}+\mathrm{S}$ with rate constant $k_{-1}$ or into $\mathrm{E}+\mathrm{P}$ with rate constant $k_{\mathrm{p}} . K_{\mathrm{d}}$ is equal to $k_{-1} / k_{1}$ as usual, while $K_{\mathrm{m}}$ (M'nM's $K$ ) is equal to $\left(k_{-1}+k_{\mathrm{p}}\right) / k_{1}$. However, when the desorption process is much faster than the product formation process, i.e., assuming $k_{-1} \gg k_{\mathrm{p}}$, then $K_{\mathrm{m}}$ simplifies to $K_{\mathrm{d}}$, as also formulated by Haldane (Briggs \& Haldane 1925). Detailed analyses of the fast equilibrium for enzymes, $k_{-1} \gg k_{\mathrm{p}}$, are main topics in Segel's book "Enzyme Kinetics" (1975/93) as well as herein for additional types of receptive units. Fast equilibrium is a tacit assumption throughout the text.

Now we can formulate a product- and time-independent form of the M'nM expression:

$$
\text { liganded enzymes }=\frac{E_{\mathrm{tot}} \cdot \mathrm{S}}{K_{\mathrm{d}}+\mathrm{S}} \text {. }
$$

In Eq. 1.14, $E_{\text {tot }}$ is equal to $\mathrm{ES}+\mathrm{E}$ and formally equal to $R_{\text {tot }}$ in Langmuir's Eq. 1.11.

At the turn of the previous century, A.J. Brown (1902) and H.T. Brown (Brown \& Glindinning 1902), two 


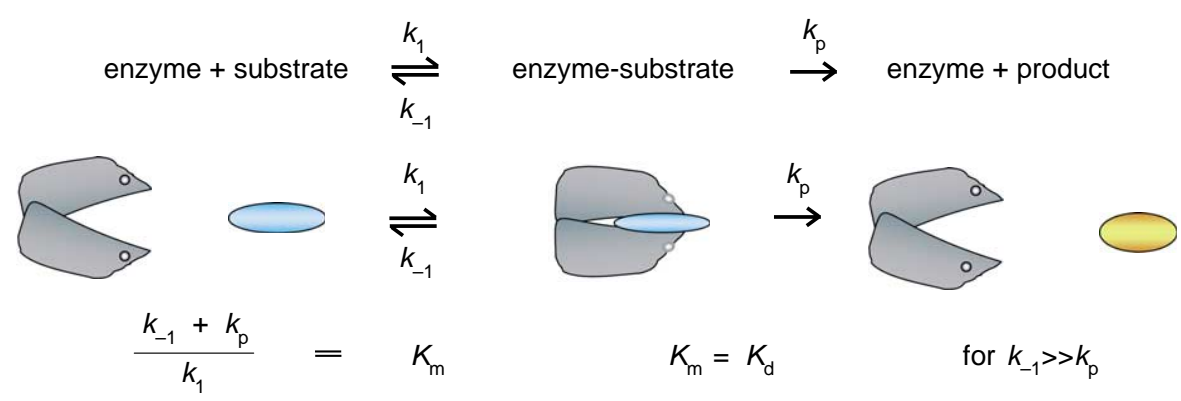

Figure 1.4. The Michaelis-'n-Menten reaction scheme for enzymes with catalytic product formation. The M'nM dissociation constant $K_{\mathrm{m}}$ is a parameter dependent on the sum of rate constant $k_{-1}$ for the dissociation reaction: $\mathrm{ES} \rightarrow \mathrm{E}+\mathrm{S}$, and the rate constant $k_{\mathrm{p}}$ for the forward product formation: $\mathrm{ES} \rightarrow \mathrm{E}+\mathrm{P}$. In case of a fast rate constant $k_{-1}$ compared with rate constant $k_{\mathrm{p}}$, the M'nM dissociation constant $K_{\mathrm{m}}$ reduces to the equilibrium dissociation constant $K_{\mathrm{d}}$ for the $\mathrm{E}+\mathrm{S} \rightleftharpoons \mathrm{ES}$ reaction.

English biochemists, and Henri (1903), a French biochemist, worked with the enzymatic splitting of saccharose (cane, caster, or table sugar) to D-glucose and D-fructose by the enzyme invertase (succhrase). Based on the work of the Browns and his own observations, Henri had already in 1902-1903 derived versions of the time-dependent M'nM expressions (Henri 1903, pp. 90-91). Yet, in spite of M'nM cited the works by Brown and Henri, in 1930 Haldane pin-pointed that Henri, Brown and others had already adumbrated the M'nM formulation ten years earlier than M'nM (Haldane 1930, p. 38; Segal 1959; Segel 1975, pp. 18-19). In accordance with history, we will appropriately refer to the expression in Eq. 1.14 as the HMM equation, although here it is not in its time-dependent form as it ought to be (see footnote 1 in Chapter 10).

\subsubsection{Things are Not Necessarily so Simple}

It may seem from the above that conformational changes in receptors are only pertinent to functional studies. In reality, however, binding of ligands to biological receptors will also involve actively controlled changes in the receptor affinity, equal to a liganddependent change in $K_{\mathrm{a}}$, i.e., a conformational change in the receptor complex with ligand binding, changing its own binding and controlled by the ligand-receptor complex. Part of the phenomenon was referred to as reciprocity in section I.6. This conformational change may not necessarily mean activation of a function. We shall come back to that later; for now, we will continue with the impression that only a naïve form of the adsorption process is relevant for binding, while functional measurements include both adsorption and conformational induction-to or selection-of an activated state of the receptive unit.

\subsubsection{Summary of Laws}

We can summarize the preceding sections by recapitulating

(1) the Wilhelmy-Berthelot \& Péan de Saint-Gilles lawof-mass-action (loma)

$$
\begin{aligned}
& {[\mathrm{R}] \cdot[\mathrm{S}] \cdot k_{1}=\text { production of }[\mathrm{RS}] \text { and }} \\
& {[\mathrm{RS}] \cdot k_{-1}=\text { production of }[\mathrm{R}]+[\mathrm{S}] \quad(1.1+2)}
\end{aligned}
$$

which are time-dependent expressions, ${ }^{5}$

(2) the Guldberg and Waage law-of-chemical-equilibrium (loce)

$[\mathrm{R}] \cdot[\mathrm{S}] \cdot k_{1}=[\mathrm{RS}] \cdot k_{1} \quad$ where $\quad \frac{k_{-1}}{k_{1}}=K_{\mathrm{d}}$.

For a system with a limited number of receptor sites, $R_{\mathrm{tot}}=\mathrm{R}+\mathrm{RS}$, we have

(3) the Langmuir law-of-adsorption-desorption (load)

$$
\mathrm{RS}=\frac{R_{\mathrm{tot}} \cdot \mathrm{S} \cdot K_{\mathrm{a}}}{1+\mathrm{S} \cdot K_{\mathrm{a}}}=\frac{R_{\mathrm{tot}} \cdot \mathrm{S}}{K_{\mathrm{d}}+\mathrm{S}}
$$

(4) and for enzymatic reactions with a potential product, we have so far the time-independent form of the Henri-Michaelis-Menten equation (HMM)

$$
\text { liganded enzymes }=\mathrm{ES}=\frac{E_{\mathrm{tot}} \cdot \mathrm{S}}{K_{\mathrm{m}}+\mathrm{S}},
$$

in which the sum of receptive units $\left(\mathrm{E}_{\mathrm{tot}}\right)$, is also a finite number (Section 1.1.2).

For a comparison of these four "laws-of ...", see footnote 5. Compare load in Eq. 1.11b with HMM in Eq. 1.14. This form of the HMM equation is equal to the Langmuirian load equation when $k_{-1} \gg k_{\mathrm{p}}$, equal to fast equilibrium.

\footnotetext{
${ }^{5}$ Note that loma is a time-related function. Under the right scope, loce and the original HMM equations are also time-related expressions, while the Langmurian load is independent of time.
} 
Table 1.1. Five Derived Laws

\begin{tabular}{lll}
\hline Law of & $\begin{array}{c}\text { Law acronym } \\
\text { (Eq. No.) }\end{array}$ & Reference \\
\hline $\begin{array}{l}\text { Mass action } \\
\text { Chemical equilibrium }\end{array}$ & $\begin{array}{l}\text { Loma (1.1, 1.2) } \\
\text { Loce }(1.3-5)\end{array}$ & $\begin{array}{c}\text { Wilhelmy (1850a, b) } \\
\text { Guldberg and Waage } \\
(1867)\end{array}$ \\
$\begin{array}{l}\text { Adsorption-desorption } \\
\text { Henri-Michaelis- } \\
\begin{array}{c}\text { Menten } \\
\text { Action-reaction }\end{array}\end{array}$ & Load (1.11a) $^{\text {LMM }(1.14 a)}$ & $\begin{array}{c}\text { Langmuir (1918) } \\
\text { Henri (1903), Michaelis } \\
\text { and Menten (1913) }\end{array}$ \\
& Loar (I.1) & $\begin{array}{c}\text { Onsager's reziprozi- } \\
\text { tätsbeziehung (1931) }\end{array}$
\end{tabular}

*See section 10.1.1. for a discussion of the equilibrium versus non-equilibrium version of the HMM.

$R_{\mathrm{tot}}$ or $E_{\mathrm{tot}}$ is the ceiling for every system with a limited number of receptive sites.

The Langmurian load equation is the simplest form at equilibrium for a dose-response relationship with a limited number of receptive units. It is the equation for receptive units occupied with ligands (RS) as a function of the varying concentration of free ligand $(\mathrm{S})$. So, we have derived the dose-occupation relationship, or more accurately, we have derived the simplest possible dose-response relationship when at equilibrium for a closed chemical or biological system, the simplest form of synagics.

(5) Finally, the law-of-reciprocity according to Onsager, as described in section I.6, we may refer to as the law-of-action-reaction (loar).

Loar states that phenomenological cross-coefficients of the general form $L_{\mathrm{ij}}$ are equal. Thus:

$$
L_{\mathrm{mn}}=L_{\mathrm{nm}} \quad(\text { for } \mathrm{m} \neq \mathrm{n}) \text {. }
$$

\subsubsection{Isotherms of Langmuir}

In summing up, the presentation of Langmuir's law-ofadsorption-desorption, it should be recognized that the derivation of the dose-response relationship is based on changing chemical concentrations at constant temperature and constant pressure in a so-called closed system. The original formulation by Langmuir, among others, was based on varying gas pressures, only keeping temperature constant - thus determining isotherms. Although pressure is most often a constant during biological experimentation, in spite of this, the doseresponse relationships in the form of load are often referred to as Langmuir's isotherms in biological literature, hence ignoring the isobaric conditions. Note also that even when at equilibrium, it is customary to refer to dose-responses as related to the law of mass action, loma. For a system at equilibrium, independent of time and with a limited number of receptive sites, the correct reference should rather be to the load (cf. last paragraph of section 1.1.8).

\subsubsection{Plotting and Analyzing Simple Synagic Relationships. Parameters to be Determined}

With a suitable number of experimentally determined values for pairs of $\mathrm{RS}$ and $\mathrm{S}$, using non-linear fitting routines, both $R_{\text {tot }}$ and $K_{\mathrm{a}}$ are easily determined (Chapter 9). The fitting requires a supplementation by the researcher of a mathematical formulation for the envisioned synagic scheme. At this stage, that formulation is the Langmuir law-of-adsorption, load. In the mathematical world, this type of expression is termed a rectangular hyperbolic function and is presented in Fig. 1.3, as well as in Part III on Data Analyses (Figs. 8.1 and 10.1).

The load relationship has the unknown parameters $R_{\text {tot }}$ and $K_{\mathrm{a}}$ (Eq. 1.11). $R_{\text {tot }}$ may be determined by measuring the number of bound ligands as the concentration of ligands increases. At saturating concentrations of ligands $\left(\mathrm{S} \gg K_{\mathrm{d}}\right)$, the occupied receptors are equal to the total number of receptors $\left(\mathrm{RS}=R_{\mathrm{tot}}\right)$. As $\mathrm{RS}$ approaches $R_{\text {tot }}$ in an asymptotic fashion, $R_{\text {tot }}$ and $K_{\mathrm{a}}$ may be obtained by non-linear fitting of the parameters in the load equation to match data (Part III).

Non-linear fitting of parameters, such as $R_{\text {tot }}$ and $K_{\mathrm{a}}$, has been made easy by modern computing, bringing theory as close as possible to experimentally determined data. Thus, unknown parameter values in the theory are adjusted until the theory matches the experimental data most closely. Before our supreme calculation capabilities on PCs, it was customary to present data in a linearized plot, such as that of Hanes, Lineweaver-Burk, or Scatchard-Hofstee, and read the parameter values off these plots. Due to power of lazy avant-garde, linearized plots are still in use. It is one of the aims of this book to advocate that synagic data, always, first be analyzed by non-linear fitting and then, eventually, for example by the Hofstee-Scatchard plot. The reasons are obvious. Non-linear fitting is the most accurate method, and it can always be used, no matter what the synagic scheme might be. Linearized methods are inaccurate, forcing researchers into short-cuts, and can often only be used under certain restrictive assumptions. For instance, Scatchard' and Schild' analyses are only for simple agonism and simple competitive ant-agonism. These assumptions are suffocating, tempting analysts to violate them (see Chapters 8 and 11). Besides, the fact that the non-linear fitting approach is more direct than linearized methods, and, thus, with better statistics, it allows for combining and integrating information obtained 
from experiment into the formulated equations. This makes non-linear fitting superior to other methods in analysis of synagic relationships. Non-linear fitting is a meaningful analytical tool as it allows for any synagic hypothesis to be evaluated intuitively.

I shall return to these postulates in Part III on Data Analyses.

\subsubsection{Intermezzo on Signal Transduction by Conformational Induction or Selection}

In functional studies, a signal such as a ligand, interacts with a receptive entity and induces a conformational change in the acceptor molecule. The conformational change carries the signal through the receptor. This is the concept of a classic transducing mechanism, where the signal gets transformed from one type of physical energy (the ligand concentration) to another type of physical energy (the number of receptive molecules in a certain activated state). Therefore, increasing the number of ligands may also increase the number of receptors in an activated state.

The conformation, which carries the signal in the receptive molecule, may be established spontaneously without a bound ligand, while the signaling ligand just stabilizes this conformation. Many researches in the field of ligand-receptology now believe that the stabilization of receptor conformation by ligands is the actual means by which information is transmitted through receptors, but it is debated (Kenakin 1996; Leff \& Scaramelli 1998; Gether 2000; Scaramelli \& Leff 2002; Hunyady et al. 2003; Giraldo 2004; Yao et al. 2006). As we shall see later, at equilibrium and steady-state, the question about change in conformation either by superiority of induction or of selection is irrelevant, as they are both equally relevant. Kenakin has developed the formulation of this problem over the last 20 years to a "continuum between the two alternatives, conformational induction and conformational selection" (Kenakin 1986, 1996, 2006). An ensemble of more or less active receptor states forming a complete continuum is an alternative introduction to a similar explanation for both conformational induction and selection (Kenakin 1996; Lutz \& Kenakin 1999, chapter 3; Kenakin \& Onaran 2002).

But, the theme of conformational selection and conformational induction of protein states is best discussed after a presentation of constitutive activity in mutant receptors and formulations of multi-states for receptive units. The subject of conformational induction and selection belongs to two-state modeling and, therefore, is only briefly touched upon here. Read more on conformational induction versus conformational selection in sub-chapter 5.11.
Instead, we will now continue with two basic concepts for pharmacology: 'potency' with relevance to affinity, and 'efficacy' with relevance to response.

\subsection{Affinity, Dissociation Constants, and Potency}

\subsubsection{Affinity and the Apparent Affinity}

Affinity is directly related to the binding process proper. As we have seen in the preceding sections, the equilibrium association constant $\left(K_{\mathrm{a}}\right)$ describes the condensation of a molecule with another receptive molecule, in a strict sense. 'Affinity' of an adsorption process is another term often used for the $K_{\mathrm{a}}$.

Nevertheless, when we speak about affinity, we may not mean $K_{\mathrm{a}}$. Affinity is taken a bit more relaxed. To indicate that we have not determined $K_{\mathrm{a}}$ in sensu strictu, as is often the case for composite systems, instead we tend to talk about apparent affinity or even apparent $K_{\mathrm{a}}$. If we use $K_{\mathrm{d}}$ instead of $K_{\mathrm{a}}$, likewise we will speak about apparent $K_{\mathrm{d}}$. The dissociation constant determined in binding or functional studies, where there is a feeling that knowledge about the true concentration in the systems is at hand, is also quoted as "effective concentration 50 " $=\mathrm{EC}_{50}$. In functional studies, where knowledge about the true concentration in the system is less secure, the apparent dissociation constant may be quoted as "effective dose $50 "=\mathrm{ED}_{50}$. In experiments with decaying saturation or response due to increase in opposing ligands, the comparable constants are "inhibitory concentration $50 "=\mathrm{IC}_{50}$ and "inhibitory dose $50 "=\mathrm{ID}_{50}($ Neubig et al. 2003).

\subsubsection{Dissociation Constants and Free Energy Change}

There is more than one type of Gibbs free energy for processes. Examples are the free energy change of a reaction $(\Delta G)$, and the free energy change of activation $\left(\Delta G^{*}\right)$ (Fig. 1.5).

The equilibrium dissociation constant $K_{\text {eq }}$ or $K_{\mathrm{d}}$ is related to the free energy change of reaction $(\Delta \mathrm{G})$ by a

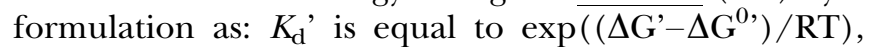
where $\Delta G^{0}$, is the standard free energy change of the reaction. ${ }^{6}$ At equilibrium, where $\Delta G$ ' equals zero, the dissociation constant is equal to the standard free energy. By convention, if $\Delta \mathrm{G}$ is positive, the process is said to be endergonic. It does not run spontaneously.

\footnotetext{
6 The apostrophe in the terms indicate that $\mathrm{pH}$ is sat at 7 . Note, that neither the $\Delta G$ nor the standard free energy $\Delta G^{0}$ are at equilibrium when formulated with the equilibrium dissociation constant. In RT, $\mathrm{R}$ is the gas constant (joule $\times \mathrm{mol}^{-1} \cdot$ kelvin $^{-1}$ ) and $\mathrm{T}$ is the temperature (kelvin).
} 


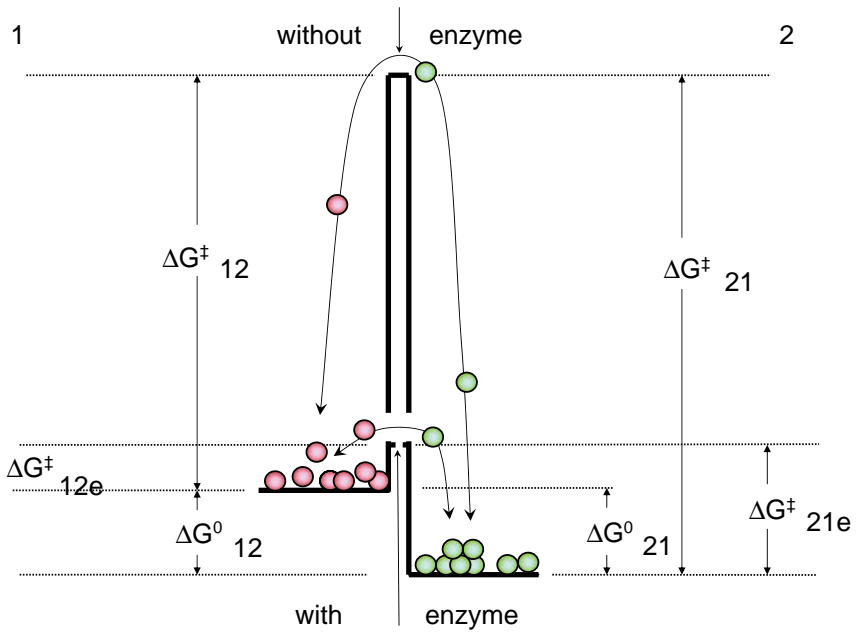

Figure 1.5. Two different Gibbs free energies. The difference in energy between state 1 and 2 is the standard free energy change of reaction $\left(\Delta \mathrm{G}_{12}^{0}\right)$. Between state 2 and 1 , the standard free energy change of reaction is $-\Delta G_{12}^{0}$ or $\Delta G_{21}^{0}$. The energy barrier for bringing reactants or ligands from condition 1 to 2 is equal to the free energy change of activation $\Delta \mathrm{G}_{12}$ without an enzyme, and $\Delta \mathrm{G}_{12 \mathrm{e}}$ with an enzyme present. In addition, $\Delta \mathrm{G}_{21}^{ \pm}$and $\Delta \mathrm{G}_{21 \mathrm{e}}^{*}$ are the activation energies for the reverse processes of bringing reactants from condition 2 to 1 . The activation energy may be lower than the standard free energy change of reaction, which is equal to $\mathrm{RT} \cdot \ln \left(K_{\mathrm{d}}\right)$. The reaction energy is usually not related to the activation energy (Berg et al. 2006, pp. 208-212). Enzymes and transporter molecules change the activation energy barrier - not the Gibbs standard free energy. This picture can be applied to receptors being activated by ligands.

Conversely, if $\Delta \mathrm{G}$ is negative, the process is exergonic, it will run spontaneously. $\Delta \mathrm{G}$ is determined by the standard free energy and the actual concentrations of reactants and products.

However, the equilibrium dissociation constant $K_{\mathrm{d}}$ is not related to the free energy change of 'activation' $\left(\Delta \mathrm{G}^{\ddagger}\right)$ for a reaction. $\Delta \mathrm{G}^{*}$ determines the rate of a reaction. Enzymes alter the barrier of Gibbs free energy of activation, i.e., the rate, but do not interfere with the free energy of reaction or the dissociation constant (Fig. 1.5).

For further details on the relation between free energy and equilibrium constants see, e.g., Nelson and Cox (2005, pp. 490-495) or, in particular, Berg et al. (2002, pp. 194 200; 2006, pp. 208-212). With the relation between free energy change in a reaction $\Delta \mathrm{G}$ and dissociation constant $K_{\mathrm{d}}$, it is possible to swiftly switch from equilibrium dissociation constants measured in moles/volume to a related free energy measured in joule/mol, representing an energy at non-equilibrium concentrations.

\subsubsection{Microscopic Reversibility and Detailed Balance}

The concepts of microscopic reversibility and detailed balance are descriptions of an equal probability or frequency in both directions for a two-state transition, and thus again directly related to the dissociation constant of reactions. As we shall see later for a twostate system, this also means that at equilibrium only three constants are necessary for a description instead of four (Chapter 5).

In relation to an equilibrium and non-equilibrium situation, the concept of "principles of microscopic reversibility" (Tolman 1924) was for a microscopic level and "detailed balance" was suggested for a macroscopic level; and also invoked for irreversible situations (Onsager 1931a). Other definitions separating the two terms have been proposed (Thomsen 1953). Walz and Caplan (1988) provide a good description of the historic development of the two concepts. Microscopic reversibility and detailed balance are distinguished by some authors, but can also be used interchangeably (Jackson et al. 1993; Fersht 1999; Colquhoun et al. 2004).

\subsubsection{Potency}

Potency is a term for especially comparing mutual $\mathrm{EC}_{50} \mathrm{~S}$ between drugs in binding and functional studies (Fig. $1.6 \mathrm{~A})$. Potency is related to the ligand-concentration axis, and is usually taken as a relative figure between $\mathrm{EC}_{50} \mathrm{~s}$ for ligands.

When the behavior of molecules are compared, we operate with the term 'potency' of a ligand in a particular process concerning either binding or elicited function, both with the possibility of being either stimulatory or inhibitory. Thus, in binding experiments, out of several ligands, some bind better and have a higher affinity, thus the potency to bind is higher than for the other ligands. In functional studies, the $\mathrm{EC}_{50}$ s are based on $50 \%$ of the maximal effects the ligands elicit (Fig. 1.6A).

When it comes to displacement and competition between ligands for binding, by a range of ligands, these ligands may have different potencies in their ability to displace each other. Now the scaling of their potency is based on the $\mathrm{IC}_{50} \mathrm{~s}$ of the ligands (Fig. 1.6B). In functional studies, it is the $\mathrm{IC}_{50}$ related to $50 \%$ of the maximal effect ligands elicit, and a potency range can be set up for a series of ligands. In both binding and functional agonism, one is interested in the ability of a drug from a series of ligands to either elicit a stimulatory (agonistic) or an inhibitory (ant-agonistic) response in a comparison with other drugs, and hence to insert the drug in a potency range. The maximal response or total binding may be the same, while the concentration needed for half maximal effect or saturation can vary between drugs (Fig. 1.6). Potency can also be related to the doses of ligands. When operating with doses, potency is based on the $\mathrm{ED}_{50} \mathrm{~s}$ or $\mathrm{ID}_{50} \mathrm{~s}$ between drugs in either binding or functional studies (Fig. 1.6). 
A

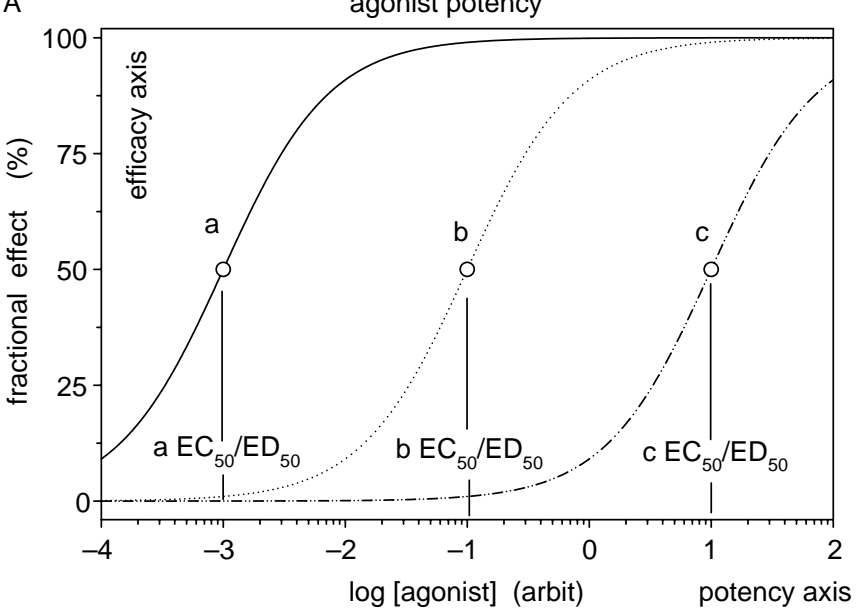

B

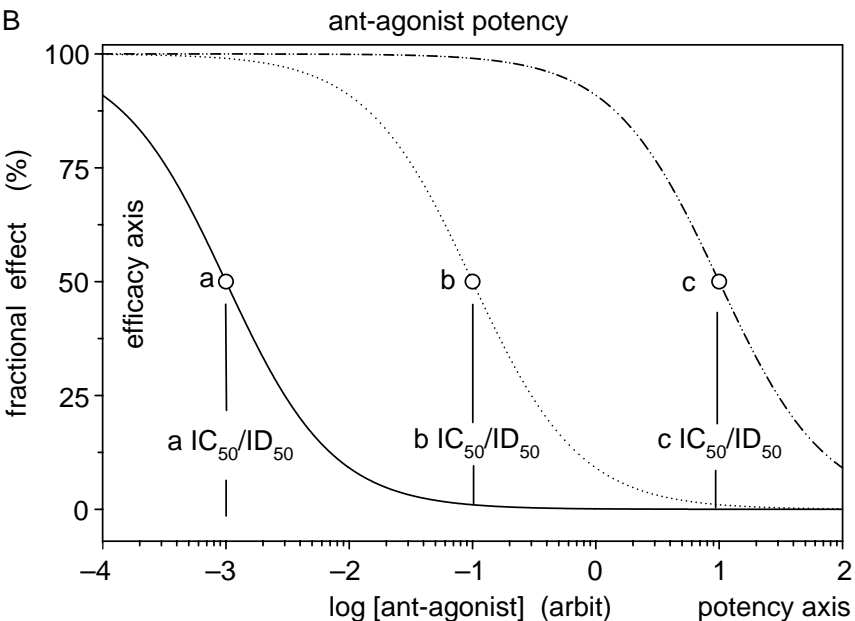

Figure 1.6. Potency of agonists and ant-agonists. (A) The potency based on $\mathrm{EC}_{50}$ of agonist $\mathbf{a}$ is higher than the potency of agonist $\mathbf{b}$ is higher than the potency of agonist $\mathbf{c}$ in both binding and functional studies. (B) The potency based on $I_{50}$ of ant-agonist $\mathbf{a}$ is higher than the potency of ant-agonist $\mathbf{b}$ is higher than the potency of ant-agonist $\mathbf{c}$ in both occupancy and functional studies.

\subsubsection{Efficacy and Potency}

Efficacy is defined in Sub-chapter 1.3. Efficacy is a relative rated ability of a ligand among other ligands to elicit a response in functional studies, and likewise in occupancy studies.

Like potency, efficacy is a normalizing concept comparing the maximal amplitude of effect between ligands. Drugs in a series of compounds with similar potencies, $\mathrm{EC}_{50} \mathrm{~s}$ or $\mathrm{IC}_{50} \mathrm{~s}$, may display different maximal effects (Fig. 1.7A, B).

Meanwhile, more often, both the efficacy and the potency represent a range of different values for a series of tested drugs (Fig. 1.8). A drug with high potency may thus not have a maximum response as high as for another drug with lower potency (Fig. 1.8; see also, e.g., Bourne \& von Zastrov 2004, Fig. 2.15).
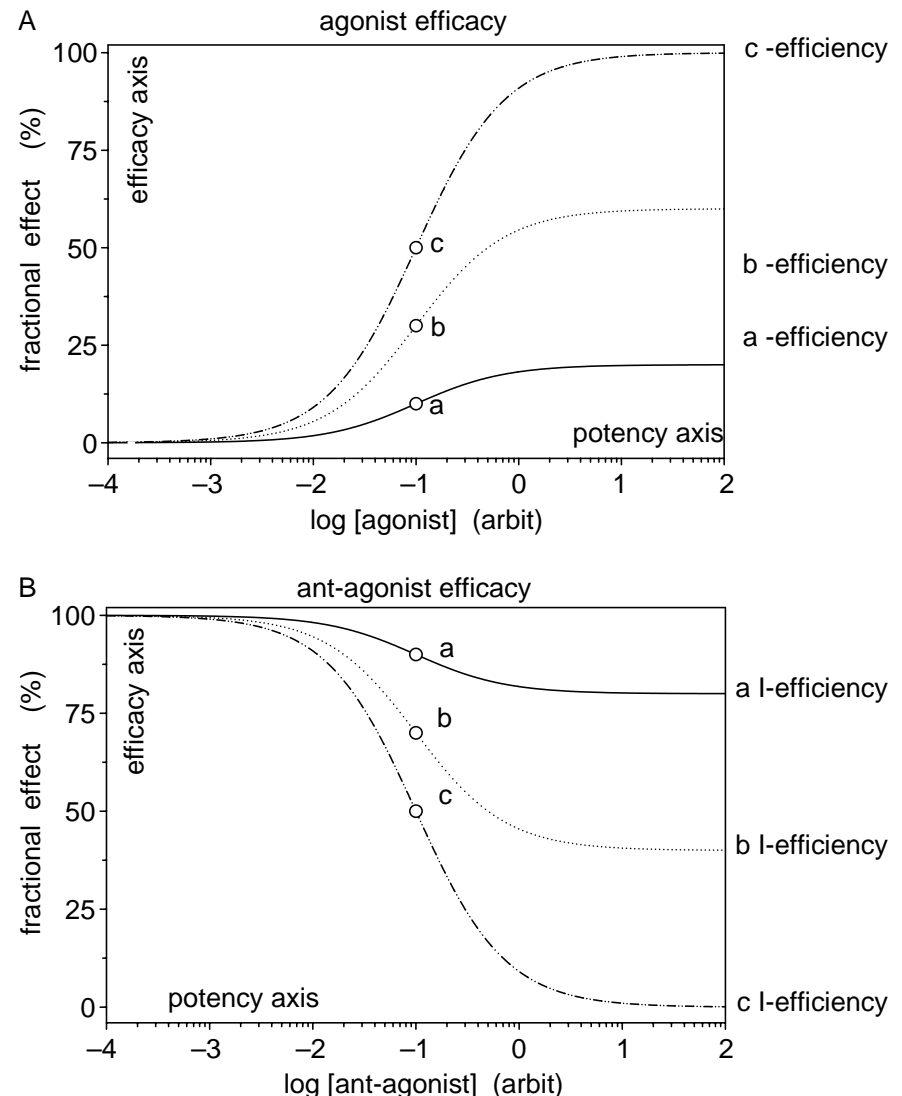

Figure 1.7. Efficacy of agonists and ant-agonists. (A) The efficiency (maximum stimulatory effect) of agonist $\mathbf{c}$ is higher than the efficiency of agonist $\mathbf{b}$ is higher than the efficiency of agonist a in both occupancy and functional studies. The maximal effect in a system is often defined by the efficiency of endogenous ligands. (B) The efficiency (maximum inhibitory effect) of ant-agonist (I-efficiency) $\mathbf{c}$ is higher than the Iefficiency of ant-agonist $\mathbf{b}$ is higher than the l-efficiency of antagonist $\mathbf{a}$ in both occupancy and functional studies.

When compounds elicit a maximal response less than the maximal response by the natural ligand for a receptive unit, it is a partial agonist (or partial inverse agonist). On the other hand, when a compound elicits a maximal response above the maximal response of the natural ligand, we are dealing with a super-agonist (Fig. 1.8).

\subsection{Partial Agonists, Efficacy, and Spare Receptors}

From section 1.3.4. onwards, the concepts related to efficacy, their meaning, and how concepts developed are somewhat tough to grasp. The reader may, however, skim through the text for later scrutiny and reference.

\subsubsection{Partial Agonism}

Drugs that do not elicit a maximal response, no matter how high their concentration is raised, are characterized 


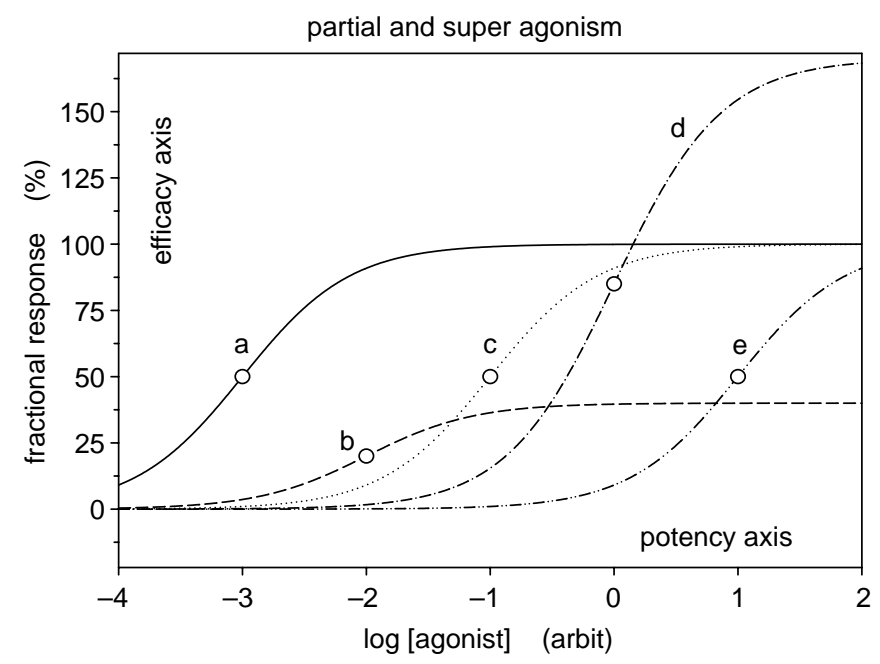

Figure 1.8. Illustration of potency and efficacy. Dose-response relations for five ligands $a, b, c, d$, and e activating the same receptive unit. The potency range is $a>b>c>d>e$, while the efficacy range is $d>a=c=e>b$. Compound $b$ is $a$ partial agonist and drug $d$ is a super-agonist.

as partial agonists (Fig. 1.9A). ${ }^{7}$ Thus, in a series of homologous drugs, there will often be ligands that on application give rise to a maximal obtainable response, while others will fall short of producing such a maximal response (Fig. 1.7).

Some ligands from a homologous series of compounds might bind with equal affinity to $100 \%$ occupancy, and still evoke a less than maximal response compared with other 'complete agonists' from the series (Fig. 1.9A). ${ }^{8}$ When combining effects of a complete agonist with that of a partial agonist, at high concentrations of the partial agonist, there can be a total displacement of the complete agonist resulting in a combined effect that matches that of the partial agonist (Fig. 1.9B).

The pharmacological concepts of partial agonists, spare receptors, and efficacy were developed in the midst of the transition period by Ariëns (1954), Furchgott (1955), Nickerson (1956), and Stephenson (1956). Examples of effects of partial agonists are shown in Fig. 1.10, and of receptor reserve in Fig. 1.11.

Actual insight into the mechanisms behind partial agonism can be found in a paper by Colquhoun and Sivilotti (2004). On partial agonism, see also Kenakin (2004b, pp. 23-26).

In modern day drug therapies, the principle of administering partial agonists has become an extremely important issue, since adverse effects of overdoses may be avoided (Lieberman 2004; Zablocki et al. 2004). Clinical

\footnotetext{
$\overline{7}$ A valid standard description of ideas about partial agonism and spare receptors can be found in Taylor and Insel (1990, pp 74-88).

8 The term "complete agonist" is used instead of the more familiar "full agonist", in order to save the predicate "full" for agonists in a different situation (see Section 2.2.3). Readers who prefer the convention can simply replace "complete" agonist with "full" agonist.
}
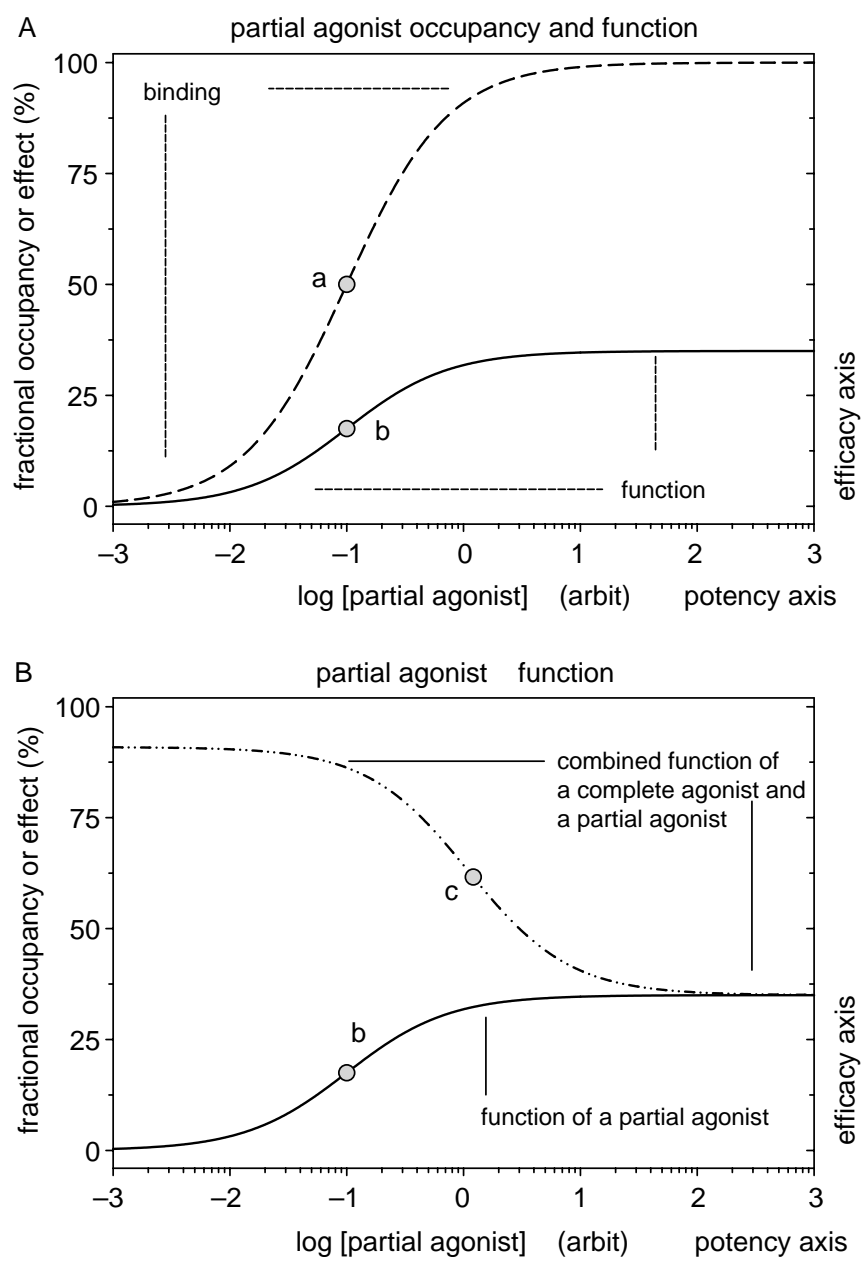

Figure 1.9. Theoretical dose-response relations of partial agonism in simple synagics. (A) An example of occupancy and function for a partial agonist as its concentration increases. Binding may reach $100 \%$ (curve a), while function only reaches $35 \%$. Although the partial agonist saturates receptive units $100 \%$, the efficiency of activation compared to that of a complete agonist is only partial (curve b). (B) Combining the function of a complete agonist at a fixed concentration yielding some $91 \%$ effect with a partial agonist at increasing concentration results in curve ' $c$ ', which large values of the partial agonist terminate at its maximal response (35\% efficiency), where occupancy for the partial agonist is almost $100 \%$ and nearly zero for the complete agonist (not shown). Curve $b$ is the same as in (A). Values for parameters were: $R_{\max }=100, K_{\mathrm{ss}}=1$, dissociation constant for partial agonist $K_{\mathrm{sp}}=0.1$, efficiency parameter for the partial agonist = 0.35 , and the agonist concentration $S^{\prime}$ fixed at 10 . Circles indicate the apparent half maximal response; in the combined function curve $\mathrm{c}$ for the partial and complete agonists, extracted by a quadratic equation.

benefits of partial agonists can be found in recent reviews by Kurtz (2006) and Tonini and Pace (2006).

\subsubsection{Partial Agonism and Occupancy Theory}

A.J. Clark, the father of classical 'occupation theory' for ligand-receptor interactions, together with Raventos 

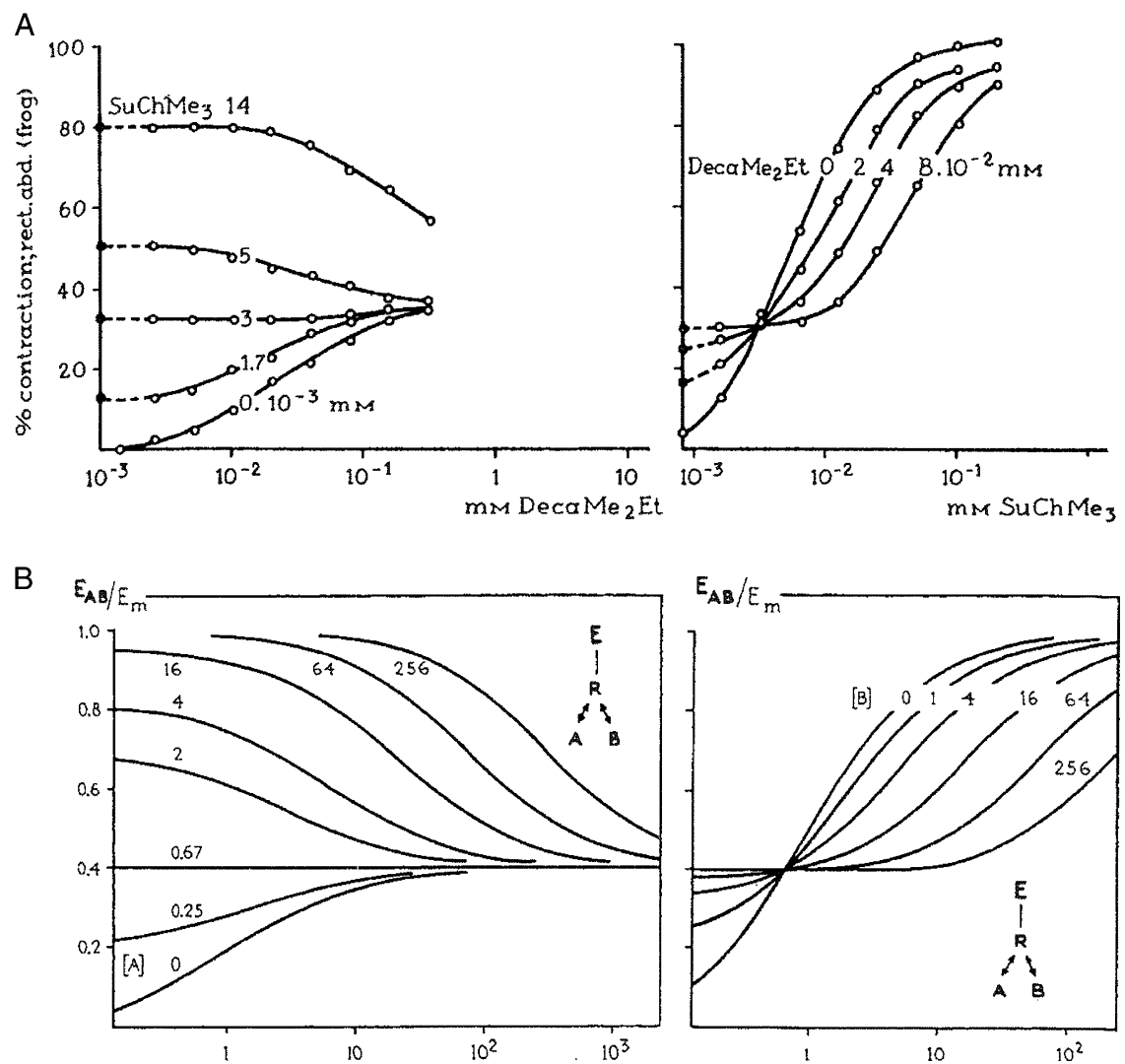

Figure 1.10. Some effects of partial agonism. (A) Examples of functional combination curves for a partial and a complete agonists when varying either the concentration of the partial agonist as independent variable, left panel, or the concentration of the complete agonist as independent variable, right panel. From Ariëns and de Groot (1954, Figs. 5 and 6; Ariëns et al. 1964, Figs. 25 and 26). (B) Theoretical curves describing the situation in (A). Taken from Ariëns et al. (1956, Figs. 7 and 8; Ariëns et al. 1964, Figs. 23 and 24). All figures reproduced with permission.

studied drugs dose-response behavior on the contraction of heart ventricle and auricle and of rectus abdominus muscle from frogs, including ant-agonists (Clark 1926a,b, 1933, 1937; Clark \& Raventos 1937). Although some drugs did not react in accordance with expectations from simple load theory, still, Clark maintained that the aberrant behavior could be resolved by a nonmechanistic formulation, raising the drug concentration to a power $(n)$ different from 1 , and thus implying that more than one binding site on receptive units was the cause of the aberrant behavior. Clark (1937) held on to the idea that dose-responses were somehow directly proportional (in the hyperbolic sense) to receptor occupancy:

$$
\text { response } \propto \text { load, }
$$

although he was well-aware of the limitations of the suggested formulations (Clark 1937, p. 216).

\subsubsection{Partial Agonists and Intrinsic Activity}

In an attempt to give a mechanistic explanation for loaddeviant dose-response behavior observed with series of drugs, Ariëns introduced the concept of 'intrinsic activity', described as $\alpha$ (Ariëns 1954; Ariëns \& de Groot 1954; Ariëns \& Simonis 1954; Ariëns et al. 1955; Ariëns 1966). Thus, dose-responses were expressed as a simple product of intrinsic activity $(\alpha)$ and occupancy:

$$
\operatorname{ar} / T R=\alpha \cdot \frac{\mathrm{S} / K_{\mathrm{s}}}{1+\mathrm{S} / K_{\mathrm{s}}}=\alpha \cdot \text { load }
$$

where $a r$ is the actual response, $T R$, the total response, $\mathrm{S}$, the agonist concentration, and $K_{\mathrm{s}}$, the equilibrium dissociation constant.

With this equation, Ariëns and co-workers could define partial agonists as compounds with an intrinsic activity $\alpha<1$ and classify drugs: full agonists $\alpha=1$, partial agonists $0<\alpha<1$, and ant-agonists $\alpha=0$ (Fig. 1.7). They termed the partial agonists 'dualists'. The type of Eq. 1.15 also gave room for a separation of occupancy and receptor activation, and thereby tempted experimenters to resolve and separate adsorption-affinity from activityconformational-change based on research data. 
A

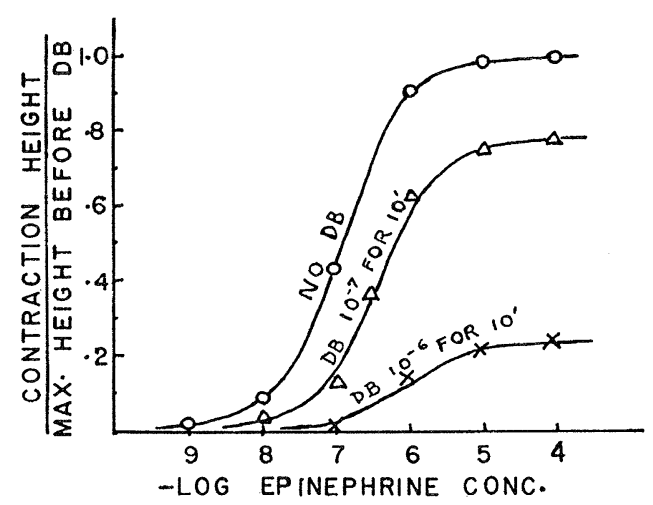

B

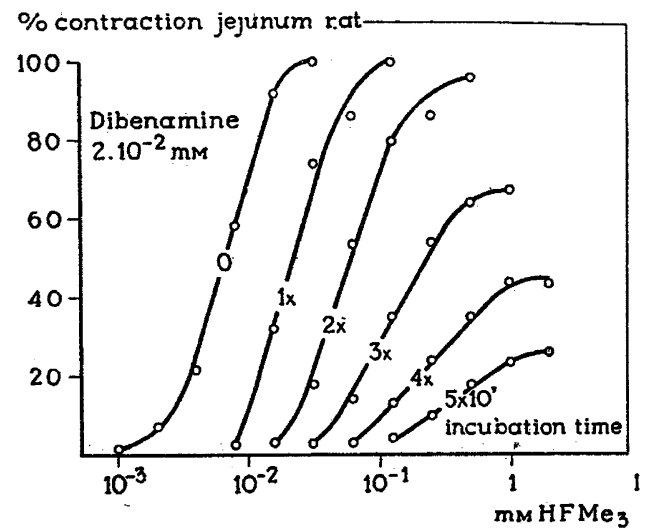

$\mathrm{C}$
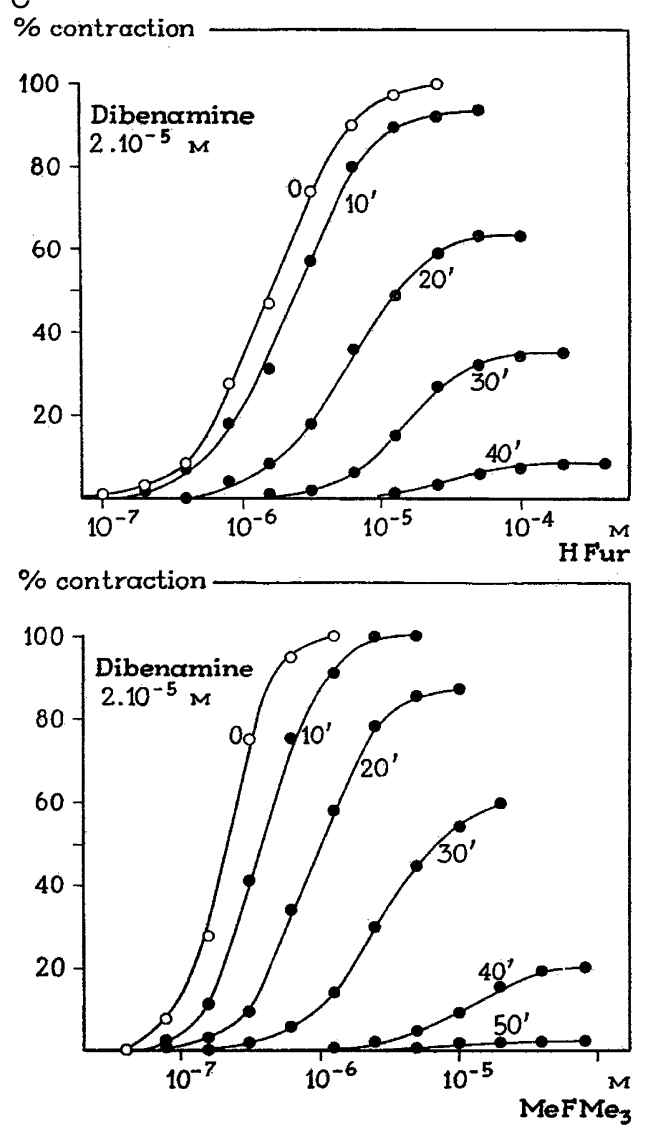

Figure 1.11. Examples of a receptor reserve and no receptor reserve. (A) Increasing concentration of Dibenamine (DB) eliminates more and more receptors and displaces dose-response curves for adrenergic effect to lower levels indicating no receptor reserve. From Furchgott (1955, Fig. 2). Contrary to the observations in panel A, with parasympathomimetic agonist HFMe3 in panel B (Ariëns et al. 1960, Fig. 6a) and parasympathomimetic agonist MeFMe3 in panel C (vanRossum and Ariëns 1962, Fig. 10c), Dibenamine, $40 \mu \mathrm{M}$, displaces the dose-response curves to the right by a factor up to 10. Especially doseresponse curves with HFMe3 in panel $B$ indicate a large receptor reserve of about $90 \%$. With Hfur as agonist in panel $C$ (from vanRossum \& Ariëns 1962, Fig. 10a), Dibenamine inhibition showed no receptor reserve. Dibenamine chemistry and irreversible elimination of reactive receptors was originally demonstrated by Nickerson and coworkers (Nickerson \& Goldman 1947; Harvey \& Nickerson 1954). Figures reproduced with permission.

\subsection{4. 'Stimulus', 'Efficacy' and Partial Agonism ad modum Stephenson}

For a modern understanding of the term efficacy, see Section 1.3.7 (Bourne \& Zastrov 2004, pp. 11-33; Kenakin 2004b, p. 11).

The aberrant behavior away from simple load theory of dose-response contraction in frog heart (Clark 1937), was re-examined by Stephenson (ST) (1956) on the contraction of guinea-pig ileum by a homologous series of alkyltrimethyl-ammonium salts with the purpose of better understanding partial agonism and receptor reserve (Fig. 1.12A) (Stephenson's Fig. 1, 1956), compare with Figs. 1.7A, 1.8, and Fig 11.
Based on his studies and with the purpose of providing his observations with a mechanistic interpretation, Stephenson broadened the occupancy theory by giving the following hypotheses (rephased by me):

(1) agonists may produce a maximal effect when only a small proportion of receptive units are occupied - meaning an inclusion of spare receptors.

(2) A response may not be linearly proportional to occupancy of receptive units, i.e., not proportional to load.

(3) Drugs have varying capacities to initiate a response, thus drugs occupy different 
A

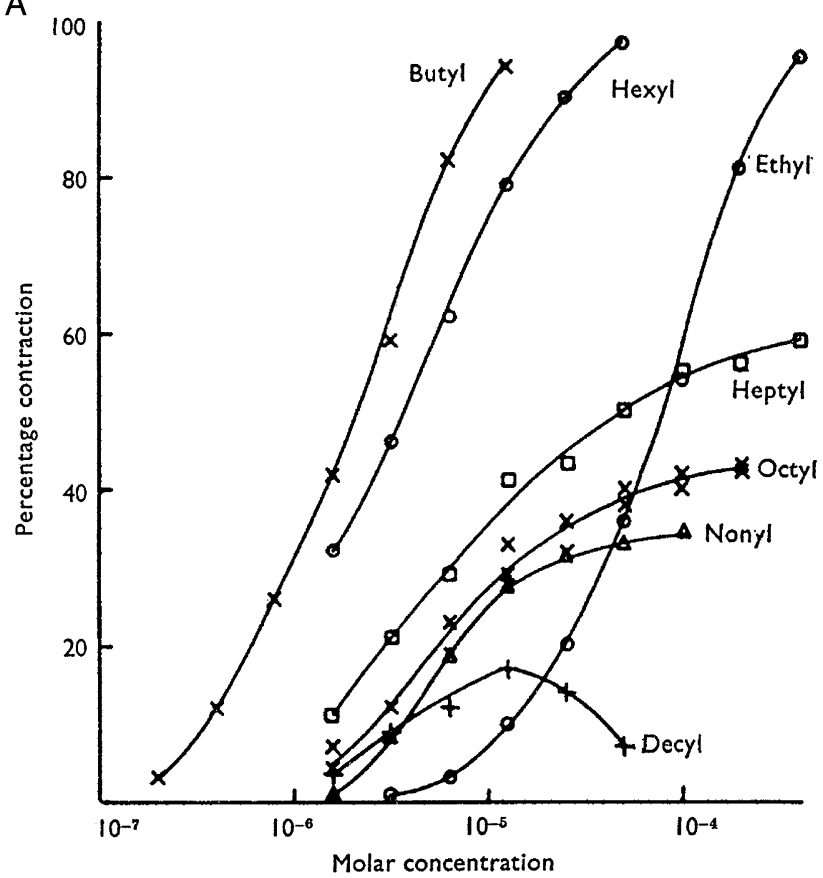

B

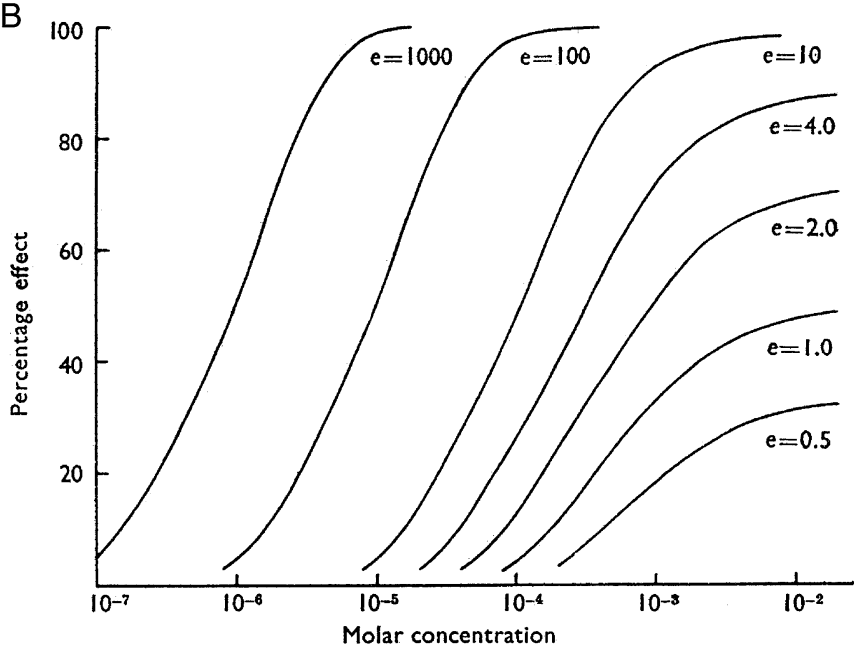

Figure 1.12. From Stephenson's dose-response experiments with a series of $\mathrm{C} 2$ through $\mathrm{C} 10$ alkyl-trimethylammonium ions on smooth muscle contraction in guinea pig ileum. (A) Experimental concentration-effect curves. (B) Theoretical concentration-effect curves with varying efficacy constant $e$ from 0.5 to 1000 . Observe, it is not the efficacy that varies between 0.5 and 1000, as indicated in the figure legend it is the efficacy coefficient $e$. The distinction is crucial. Reproduced from Stephenson (1956, Figs. 1 and 9) with permission.

proportions of receptive units when producing equal response. This relative property is the efficacy of the drug (ST's Fig. 1), shown in Fig. 1.12A.

In order to formulate his hypotheses, Stephenson presented two relationships. One for the actual response $(a r)$ as a function of a stimulus $(S t)$, a $S t$-ar relation:

$$
a r=f(S t),
$$

and another for the stimulus St equal to the product of an efficacy constant $e$ and of occupation given as load, an $e$ load-St relationship. Thus:

$$
S t=e \cdot \frac{\mathrm{S} / K_{\mathrm{S}}}{1+\mathrm{S} / K_{\mathrm{s}}} .
$$

Equation 1.16 explains Stephenson's hypothesis 2, in that the function of $f()$ is not necessarily a linear proportionality between response and stimulus. However, observe that Stephenson never presented a formula for his function $f(S t)$.

Meanwhile, interestingly, without an explicit formulation, Stephenson (1956) circumvents this lack of an equation by adopting, what he calls, a convention, viz. when stimulus $S t=1$, then the response is equal to $50 \%$. Based on this convention, Stephenson concludes that a drug, which at high concentration occupies all the receptors, load $=1$, and only reaches an actual response at $50 \%$ of maximum, must have an efficacy $=$ 1 - while Eq. 1.16 holds (Stephenson 1956). Thus, in reality, Stephenson's efficacy is both his efficacy constant $e$, and an efficacy equal to $f(e) .{ }^{9}$

Explicitly stated, Stephenson's $e$ is not efficacy; $e$ is an efficacy constant included in an equation of efficacy that was never formulated, except in a general form as efficacy $=f(e)$.

We may note that in the second equation by Stephenson (Eq. 1.17), there is an identity with Ariëns' formulation of intrinsic activity (Eq. 1.15). That is, $e$ in Eq. 1.17 seems to have the same significance as $\alpha$ in Eq. 1.15. But the point is that the $f(e)$ is not defined, so efficacy constant $e$ and intrinsic activity constant $\alpha$ are not necessarily the same. And they are not the same, as specified by Stephenson (1956) and later corroborated by van Rossum (1966). Intrinsic activity yields plots as in Fig. 1.7A, while ST knew that his dose-responses followed a pattern as in Fig. 1.12.

However, there is an understandable temptation to combine Eqs. 1.16 and 1.17, and forget about $f()$, which leads to the equation by Ariëns (Eq. 1.15). A temptation that has been purported since Stephenson did not resolve the $f(e)$ mystery. According to Colquhoun, this is 'Stephenson's short' (Colquhoun 1998). The tricky subject on efficacy is analyzed further in the following sections and in Sub-chapter 5.6.

\footnotetext{
${ }^{9}$ Stephenson did not differentiate clearly between the relative property (relative response) as "efficacy" and "efficacy" as an efficacy constant, $e$. Furthermore, to add to the confusion about Stephenson's "efficacy", Stephenson unsuitably formulated his stimulus as a product of the load function and the efficacy constant $e$. See Eq. 1.17. Rather, Stephenson's stimulus should be formulated as a function of $e$ and load, i.e., $S t=f(e$, load $)$, or explicitly as $S t=\left(e \cdot \mathrm{S} / K_{\mathrm{ss}}\right) /$ $\left(1+\mathrm{S} / K_{\mathrm{ss}}+\overline{e \cdot \mathrm{S}} / K_{\mathrm{ss}}\right)$, see Chapter 5.
} 


\subsubsection{Formulation of the Right Efficacy}

In the early part of the transition period (1945-1965), the literature especially on enzyme conditions was full of attempts to explicitly formulate the conformational transition for receptive units from a reactive state to an active state in the liganded condition (Tonomyra et al. 1952; Botts \& Morales 1953; Watanabe et al. 1953; Blum 1955; Botts 1958).

For the simplest possible reaction scheme including both binding and activation, we may take the reaction scheme suggested by delCastillo and Katz (dC\&K) (1957). Their reaction scheme states that:

$$
\mathrm{R}+\mathrm{S} \stackrel{K_{\mathrm{s}}}{\rightleftharpoons} \mathrm{RS} \stackrel{L^{\prime}}{\rightleftharpoons} \mathrm{RS},
$$

with a dissociation constant $K_{\mathrm{s}}$ in the left reaction and an isomerization constant $L^{\prime}$ in the right reaction. Using the conservation (distribution) principle for a formulation of the fractional response for this two-step reaction yields:

$$
\operatorname{ar} / T R=\frac{L^{\prime} \cdot \mathrm{S} / K_{\mathrm{s}}}{1+\mathrm{S} / K_{\mathrm{s}}+L^{\prime} \cdot \mathrm{S} / K_{\mathrm{s}}} .
$$

The formulation in Eq. 1.18, including an isomerization constant $L^{\prime}$, exactly covers the intentions behind the efficacy, as contemplated and tinkered with by Stephenson. Thus, Eq. 1.18 is the formulation Stephenson was seeking. Compare Fig. 9 in Stephenson (1956), Fig. 1.12B, with Fig. 5.6 in Chapter 5 for the delCastillo \& Katz regime (dC\&K 1957) and the efficacy constant $e$ and the isomerization constant $L^{\prime}$ sum out to be the same, and both are intrinsic or microscopic constants. They are inseparable from their pertinent affinity constants, and, therefore, they cannot be resolved experimentally (Colquhoun 1998; Colquhoun et al. 2004). As already mentioned, see Sub-chapter 5.6 for more details on this issue.

It is often forgotten that already a year before Stephenson's famous paper, Furchgott (1955) formulated an embedded load function for partial agonism, which presents the exact same kind of formulation as in Eq. 1.18 (see further in Section 1.3.6). ${ }^{10}$

\subsubsection{Attempts to Resolve the True Efficacy}

The efficacy concept has been defined and re-defined many times since its introduction in the transition era.

Equations have been set up to tackle the swap from stimulus St to response by using efficacy as a simple multiplier, $e$-occupancy in Eq. 1.17, to an efficacy with the characteristics as understood by Stephenson, such that at high ligand concentrations for $e=1$, the

\footnotetext{
${ }^{10}$ On embedded load functions, see the operational model of Black and Leff (1983) described here in Section 1.3.6., and in Chapter 5. On principles of embedded load, see Paton and Rothschild (1965); "a load in a load is a load".
}

response $=50 \%$ of maximum (Stephenson 1956), which is satisfied by Eq. 1.18. Inserting 1 for $L^{\prime}$ in Eq. 1.18 yields $50 \%$ response at high values of $[\mathrm{S}]$.

By embedding a hyperbolic expression for stimulus to effect-coupling within a hyperbolic expression for the concentration to occupancy-coupling, Black and Leff derive a so-called operational model (Black \& Leff 1983; Kenakin 2004). In this book, I have chosen to call their model an embedded model, since the operational model is based on embedding terms of load (see Sub-chapter 5.10). The Black and Leff embedded model (B\&L model) results in an expression near-identical to the formulation of the dC\&K model in Eq. 1.18. The dC\&K model has three system constants (see Chapter 5), while the B\&L models has four (Sections 5.10.1 and 5.10.2). Further, the reaction scheme for the $B \& L$ model has a different meaning to that of the dC\&K model. So, the derivation of the embedded (operational) model is the dC\&K mechanistic model created through a mathematical $\mathrm{b}$ (l)ack door. Thus, a so-called transducer ratio from Black and Leff's model, $\tau$, fulfills the function of an efficacy constant at face value, just like $L$ ' from the dC\&K model. However, the transducer ratio has no physical meaning, unless it is claimed to be identical to $L$ '. This was not done in 1983 by Black and Leff. On the contrary, Black and Leff (1983) unfortunately failed to see a difference between a dC\&K model and the Ariëns model for intrinsic activity (Ariëns 1954), as well as the similarity between a dC\&K model and their own simple operational model. As already stated, we know now, that the simple dC\&K model is exactly what Stephenson was unable to formulate mathematically, and that $L '$ from the dC\&K model is identical to Stephenson's efficacy constant.

We shall return to the similarity between $\mathrm{dC} \& \mathrm{~K}$ and B\&L in Chapter 5, where the prerequisites presented makes it easier to discuss the issue for a clearer understanding.

The literature on partial agonism and efficacy is somewhat unmanageable. In an attempt to bring some order into the many statements and texts on the subjects, a summary as in Table 1.2 with comments in Box 1.2, might be helpful.

\subsubsection{Recent Definitions of Efficacy}

The concept of efficacy is demonstrated in Figs. 1.7, 1.8, and 1.12 .

With the contemporary understanding, receptive units exist in a continuum of alternating conformations that may be represented as conformational topography maps in energy landscapes or represented by discontinuous receptor ensembles of distinct conformations (Onaran \& Costa 1997; Kenakin \& Onaran 2002). The understanding of 'efficacy' when related to conformational change or isomerization obviously have become somewhat more 
Table 1.2. Examples on partial agonism and parameterization of efficacy

\begin{tabular}{|c|c|c|c|c|}
\hline Reference on efficacy formulation and comment & $\#$ & Formulation type & Efficacy constant $L^{\prime}$ & Equation No. in reference \\
\hline $\begin{array}{l}\text { Furchgott (1955) } \\
\text { Stephenson (1956) } \\
\text { Furchgott (1966) } \\
\text { Black and Leff (1983) } \\
\text { Ehlert (1988) } \\
\text { Venter (1997) } \\
\text { Colquhoun (1998) } \\
\text { Christopoulos and El-Fakahany (1999) } \\
\text { Clark et al. (1999) } \\
\text { Present text }\end{array}$ & $\begin{array}{l}(1) \\
(2) \\
(3) \\
(4) \\
(5) \\
(6)\end{array}$ & $\begin{array}{l}\text { e-l } \\
f(e \cdot l o a d) \text { or just } f(e) \\
\text { e-l } \\
\text { e-l (dC\&K) } \\
\text { Null-method } \\
\text { Ariëns } \\
\text { dC\&K } \\
\text { e-I } \\
\text { e-I } \\
\text { dC\&K (1957) }\end{array}$ & $\begin{array}{l}\alpha\left(R_{\mathrm{T}}\right) / K_{\mathrm{R}} \\
e \\
f(e)^{£} \\
\tau=\mathrm{R}_{0} / K_{\mathrm{E}} \\
\varepsilon \text { and } \varepsilon^{\prime} \\
e^{\dagger} \\
E \\
\varepsilon_{\mathrm{A}} \cdot R_{\mathrm{T}} / \beta^{\$} \\
\varepsilon \cdot R_{\mathrm{T}} /\left(\varepsilon \cdot R_{\mathrm{T}}+\mathbf{a}\right)^{\S} \\
L^{\prime}\end{array}$ & $\begin{array}{l}10 \\
\text { No equation } \\
9 \\
5 \\
17 \\
1 \\
\text { Scheme } 1 \\
3 \\
1 \text { Box } 1 \\
1.18+5.10\end{array}$ \\
\hline
\end{tabular}

(1) Partial agonism formulated by embedded load (e-I, the simple operational model), (2) by null-method and partially ignoring 'function of', (3) by method of Ariëns, or (4) by conservation equation based on the delCastillo \& Katz scheme (dC\&K).

\#Comments 1 to 8 are listed in Box 1.2 .

'Here $e$ is not an efficacy constant, but an intrinsic activity constant similar to Ariëns' $\alpha$

${ }^{\mathrm{e}} \boldsymbol{e}$ is Furchgott's intrinsic efficacy relating efficacy to the single receptive unit.

$\$$ The $\beta$ coefficient inspired by Kenakin and Beck (1982) is a so-called fitting parameter.

§ The expression is termed intrinsic activity and is a transformation of the intrinsic efficacy $\boldsymbol{e}$ according to Furchgott (1966). In the expression, a is a so-called hyperbola-defining parameter. It can be shown that $\varepsilon \cdot \mathrm{R}_{T} / \mathbf{a}$ is equal to $L^{\prime}$. Meanwhile, the Clark et al. (1999) expression is not an explicit transformation from the Stephenson to the del Castillo and Katz expression (Subchapter 5.6). Additionally, the exact mechanistic meaning of a is not totally obvious, although it may be compared to the off-rate constant $k_{1}$ in the Cassell-Selinger scheme for G protein turn-over of GDP/GTP/GDP (Cassel \& Selinger 1976; Tolkovsky \& Levitzki 1981; Whaley et al. 1994; Clark et al. 1999).

of a concept than a measurable reality. It is informative to follow how Kenakin has developed the efficacy concept over the last 25 years (Kenakin 1982, 1984, 1985, 1988, 1990, 1994, 1995a,b, 1999, 2002a,b, 2004a,b, 2005a,c, 2006; Weiss et al. 1996c; Lutz \& Kenakin 1999, chapter 3; Kenakin \& Onaran 2002). The complementarity or uncertainty principle staged.

Although Stephenson never formulated efficacy mechanistically in an equation, his original understanding of the term efficacy is still valid: "drugs have varying capacities to initiate a response. This relative propensity is the efficacy of the drug'. ST's definition means that only when you compare between the amplitude of drugeffects on a receptive unit or the magnitude of drugbinding to a receptive unit can you speak about efficacy of a certain drug, much the same way as with the concept of potency. You can only speak about potency of a certain ligand when you compare between drugs, metabolites, or transportees, the amount of each ligand needed for identical response, i.e., concentration needed to obtain a certain level of binding, or more correctly, a certain level of response (Section 1.2.3). While potency is a relative measure on the concentration scale, efficacy is a relative measure on the response scale. Neither efficacy nor potency can be discussed as absolute values, and because efficacy is relative, the term "relative efficacy" is a pleonasm.

\subsubsection{Conclusions about Efficacy}

We can summarize that the easiest way to formulate efficacy, both conceptually and technically, is to employ the dC\&K reaction scheme and its functional formulation (see Eqs. 1.18 or 5.10). Thus, efficacy and isomerization are homologous concepts - in a stricter sense it is the relative ability to make a conformational change that is functional. Efficacy is a relative concept. Comparisons and conclusions on models of efficacy, including those of Furchgott, Stephenson, delCastillo and Katz, and Black and Leff, are summarized in Box 1.3.

\subsubsection{Efficacy. The Katz-Thesleff Reaction Scheme}

When we come to the so-called K\&T scheme in Chapter 5 (Katz \& Thesleff 1957), which is a cyclic and symmetrical reaction scheme, the ordered reaction of the dC\&K model disappears, and simultaneously $L$ ' and $K_{\mathrm{s}}$ become completely interdependent. They are microscopic constants which cannot be separated (see Sub-chapters 5.6-5.10) (Walz \& Caplan 1988; Fersht 1999, pp. 93-94). Congruent with load, which is for adsorption, the K\&T reaction scheme may be said to be a scheme for absorption involving a conformational change, although this conformational change may not necessarily lead to an activation. The idea of congruency is a reference to Langmuir' differentiation between adsorption and absorption in Section 1.1.1 (Langmuir 1918).

The real question is, are the formulated adsorption and absorption processes also processes in nature? An answer may materialize after reading to and through Chapter 15 .

\subsubsection{Receptor Reserve}

The notion of spare receptors was never recorded by the father of pharmacology, AJ Clark (1937). Subsequently, the existence of spare receptors has been demonstrated again and again. Examples of a receptor reserve and the 
Box 1.2. Comments 0 to 8 on symbols in Table 1.2.

(0) General comment. Efficacy is when the function $f()$ has operated on efficacy constant $e, f(e$, load). The symbol $f(e)$ is a brief notation for $f(e$, load $)=$ the real efficacy expression. Stephenson confused this by also writing $f(e \cdot$ load $)$. Now the parameter $e$ in itself is almost comparable to the Ariëns "intrinsic activity" $(\alpha)$. The mixing of $f(e \cdot$ load $)$ for $f(e$, load) has caused a lot of problems (see Section 1.3.6).

(1) $\alpha\left(R_{\mathrm{T}}\right) / K_{\mathrm{R}}$. The term $\alpha\left(R_{\mathrm{T}}\right)$ is the fraction of total receptors $\left(R_{\mathrm{T}}\right)$ that are activatable. $K_{\mathrm{R}}=k_{\mathrm{r}} / k_{\mathrm{c}}$, where $k_{\mathrm{r}}$ is a relaxation rate constant and $k_{\mathrm{c}}$ is a rate constant dependent on several factors, including energy immediately available for contraction. In fact, $K_{\mathrm{R}}$ is a reciprocal isomerization constant as well as a reciprocal efficacy constant.

(2) The real term for Stephenson' efficacy $f(e)$ is often mistaken by $e$. The two were confounded in Stephenson's definition of 'efficacy' and his use of 'efficacy' for the efficacy constant e. Furchgott (1966) makes explicit that $f(e)$ is related to response $=f(S t)=S t /(1+S t)$, as also demonstrated in Stephenson's Fig. 8 (1956).

(3) $f(\boldsymbol{e})$, Furchgott has accepted the Stephenson concept of 'efficacy'. With hindsight, this 'intrinsic efficacy' should rather be termed 'fractional intrinsic activity'.

(4) $\tau=\mathrm{R}_{0} / K_{\mathrm{E}} \cdot \tau$ is a so-called transducer ratio. Compare $K_{\mathrm{E}}$ with Furchgott's $K_{\mathrm{R}}$ (see comment 1 ).

(5) By taking the meaning of 'function of' as independent of an allosteric modulator and employing a nullmethod, Ehlert $(1988,2005)$ derives an expression for the relation between agonist concentration in the presence and absence of an 'allosteric modulator'. The exercises can yield the equilibrium dissociation constant for an interventor ligand and a so-called interventor constant in certain situations, as Ehlert's exercise actually belongs to binding and function for interventors treated in Chapter 2. Meanwhile, the procedures nicely illustrate the problems generated by Stephenson's approach and Schild's nullmethod.

(6) Venter's use of $e$ as efficacy is misleading. It is a mismatch of $e$ for $\alpha$. See general comment 0 and comment 2.

(7) $e_{\mathrm{A}} \cdot R_{\mathrm{T}} / \beta$. $e_{\mathrm{A}}$ has been termed 'intrinsic efficacy' with a dimension of reciprocal concentration. Here, the $e_{\mathrm{A}}$ ' $R_{\mathrm{T}}$ is also designated $e$. See the general comment 0 . Coefficient $e$ here is related to Ariëns' intrinsic activity, not real efficacy, while the parameter $\beta$ is related to efficacy and may be compared with Furchgott' $K_{\mathrm{R}}$. The authors call $\beta$ a fitting parameter inspired by Kenakin and Beck (1982). They further claim that 'spare receptors' ought to be replaced by 'non-linear stimulus response coupling'. My suggestion is that spare receptors ought to be treated as a separate reality. For instance, spare receptors are equal to total receptors $\left(R_{\mathrm{T}}\right)$ minus a fraction of total receptors, $\alpha \cdot R_{\mathrm{T}},=R_{\mathrm{T}} \cdot(1-\alpha)$.

(8) The efficacy constants in column 3 of Table 1.2 have different expressions and are sometimes referred to as intrinsic efficacy constants (see Furchgott 1966, Ehlert 1988, and comment 3 above). Some of the listed expressions for efficacy are correctly formulated based on a function of ' $f$ ' operating at the efficacy constant or intrinsic efficacy constant as well as the occupancy $y$. Thus, $f\left(1 / K_{\mathrm{R}}, y\right)=f(e, y)=f\left(1 / K_{\mathrm{E}}, y\right)=f(1 / \beta, y)=$ $f(\mathrm{E}, y)=f(L, y)$ are all expressions for efficacy.

lack of it are shown in Figs. 1.11 and 1.13. As mentioned in Section 1.3.1, several reports found a non-hyperbolic relationship between occupancy and response in functional studies of ligand-receptor interactions, indicating spare receptors. With spare receptors present, not all receptors have to be activated to obtain a maximal response (see especially Fig. 1.11B). One explanation for the rationale of a receptor reserve is that it increases ligand sensitivity (Ford \& Evavold 2004).

The amount of spare receptors is built into the efficacy concept, and it is hidden behind the parameter $e$ or $L '$. Compare a figure with changing receptor reserve (Fig. 1.11B), with a figure with changing $e$ (Fig. 1.12B), or a changing $L$ ' based on the dC\&K formulation (Fig. 5.2). Changing the efficacy constant $e$ or $L$, and therefore the efficacy, nicely follows a changing receptor reserve.
From these considerations and by reducing the spare receptors to a minimum, it seems that it would be possible to estimate the isomerization (efficacy) constant $L$ '. Meanwhile, the position of dose-response profiles on the concentration axis are also dependent on the system's dissociation constant $K_{\mathrm{s}}$. Of course, if only Ariëns' intrinsic activity constant $\alpha$ is considered in the theory, the amplitude of dose-response curves on the response axis is directly proportional with $\alpha$ (Fig. 1.7). Meanwhile, the concept of intrinsic activity does not satisfy the experimental observations (Fig. 1.12A). Therefore, the system constant $\alpha$ will not be able to elucidate the discrepancy. The amount of spare receptors is not built into the fractional intrinsic activity, i.e., parameter $\alpha$.

Spare receptors or varying efficacy between ligands in functional studies will result in a discrepancy between the theoretical dissociation constant for agonists, 
Box 1.3. Conclusions about Stephenson's efficacy expressed in the dC\&K model

(a) The $L^{\prime}$ constant of the delCastillo and Katz scheme (dC\&K, 1957) in Chapter 5 covers what Stephenson meant by his 'efficacy' as efficacy constant $e$. Thus, $f(e)=f(L$ ').

(b) Furchgott was the first to equate efficacy by employing an embedded load formulation (Furchgott 1955).

(c) Stephenson in the 1956 paper defined efficacy but never gave an explicit expression for this efficacy.

(d) Black and Leff (1983) failed to observed the parallelism between embedded load representing efficacy and the distribution equation for iso-merization in the $\mathrm{dC} \& \mathrm{~K}$ model, which they explicitly refer to.

(e) It is a clue to note that both embedded load and the dC\&K principle are based on an idea of ordered reaction - first binding then activation. Therefore, altering the efficacy constant or the isomerization constant affects the apparent dissociation constant, while altering the dissociation constant has no effect on apparent maximal efficacy, app $L^{\prime}=1 /\left(1+1 / L^{\prime}\right)$ (see Section 5.3.8) .

(f) That spare receptors exist and may as well be absent has been demonstrated by alkylating agents in many studies (Furchgott 1955 (Fig. 1.11A); Nickerson 1956; Stephenson 1956; van Rossum \& Ariëns 1960 (Fig. 1.11B); Eglen et al. 1994; Ford \& Evavold 2004; Winding (Fig. 1.13)).

(g) Compared with the embedded (operational) model (Black \& Leff 1983), the dC\&K model has one drawback, it does not discuss possible receptor reserves. The embedded model discusses such a receptor reserve.

$K_{\mathrm{ss}}$, and their experimentally determined effective concentration at $50 \%$ response $=\mathrm{EC}_{50}$ (Figs. 1.11 and 1.12). The phenomenon of spare receptors is also illustrated by the discussion of a functional equivalent of Cheng-Prusoff's equation (see Chapter 4).

\subsection{The Post-classic Era. Allostery and Two-state Models}

Ideas about conformational changes induced by ligand binding in receptive units of protein nature were discussed at length from early on in the 20th century, physically first demonstrated for hemoglobin binding oxygen by Harowitz (1938), and clearly formulated by Wyman and Allen (1951) for oxygen binding to hemoglobin (see also Edsall 1980; Perutz 1990, the Preface).

\subsubsection{Allostery}

The term 'allosteric' was introduced by Monod-Jacob in 1961 in connection with an explanation for the complex kinetic behavior of enzymes, as well as for the binding of oxygen to hemoglobin deviating from the HMM equation (Monod \& Jacob 1961; Monod et al. 1963, 1965). Allostery meant: (1) several independent homologous subunits (protomers) of protein molecules are present, (2) all subunits flip to a new conformation in a single step, that is, in a concerted fashion, and (3) binding affinity to each protomer is also changed simultaneously with the conformational change, while staying the same as ligands bind from protomer to protomer.

Due to this particular change in all dissociation constants at the same time, the model by Monod, Wyman, and Changeux is a 'concerted' model.

4-DAMP MUSTARD (nM)
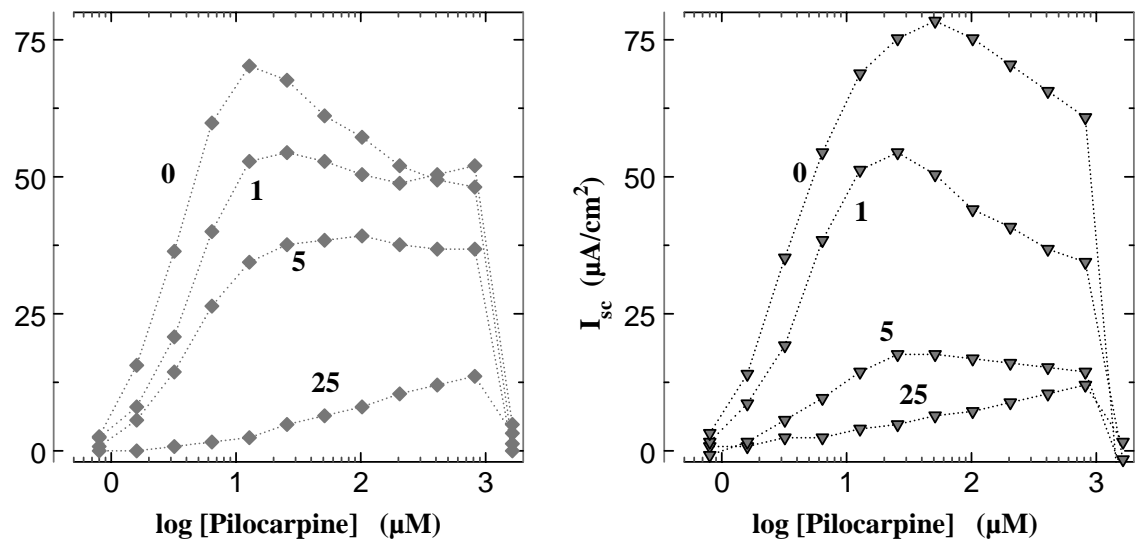

Figure 1.13. Two examples of no receptor reserve in tracheal epithelium. The alkylating reagent 4-DAMP mustard was used in increasing nano-molar concentration as indicated on the curves to eliminate functional muscarinic receptors stimulated by pilocarpine in hen tracheal epithelium. Pilocarpine maximal effect starts to drop already at the lowest dose of 4-DAMP. The function is measured as short circuit current, $I_{s c}$, in microamps per square $\mathrm{cm}$ (Courtesy of $\mathrm{dr}$. Winding, unpublished). 


\subsubsection{The Monod-Wyman-Changeux Model (MWC)}

The mechanistic reaction scheme by Monod et al. (1965) offered an explanation for the phenomenon called positive co-operativity - the term describing a behavior where the dose-occupancy relationship for a single type of ligand rises in a steeper fashion than that expected for the simple relationship of a synagic loadtype reaction. The binding is steeper than anticipated. Thus, allostery had a rather specific meaning and related directly to conformational changes in multimeric proteins. ${ }^{11}$

Meanwhile, the pivotal characteristics of the MWC model is its explicit formulation of an un-liganded receptive unit in two states (Table I.1). This means that a conformational change must take place 'before' a ligand is bound. This is contrary to the dC\&K scheme where binding of a ligand precedes a conformational change, which was also a possibility in a model by Koshland et al. (1966). Reaction schemes claimed to be MWC models are often presented, while violating the requirement of only permitting un-liganded flipping (Eigen 1968, Fig. 11; Laidler \& Bunting 1973, Fig. 11.8; Dixon \& Webb 1979, Fig VIII.31; Fell 1997, Fig. 3.11).

In modeling, allostery is a mechanistic model with two un-liganded states of the receptive unit (see Chapters 14 and 15 for details).

\subsubsection{The Koshland-Nemethy-Filmer (KNF) or Sequential Model}

Since the original allostery model did not explain negative co-operativity, which had also been observed experimentally as a more shallow dose-response relationship than expected from simple load, Koshland et al. (1966) introduced their sequential model for allostery with a differently imagined sequence for a conformational change. Here each subunit may undergo individual structural changes according to a certain order, induced by the consecutive binding of ligands. As mentioned for the KNF model, binding may 'precede' a conformational change (Fig. 1.14) (Eigen 1967). As noted by Eigen, in a sequential model for a four-subunit entity, the total number of possible conformations is 44 , all with related rate constants or dissociation constant. Thus, a pretty difficult scheme. Details on KNF are presented in Chapter 15.

\footnotetext{
11 Here, "multi-meric" just signifies that a protein consists of several subunits that are identical, or near identical in primary, secondary, and tertiary structure as well as when fixated in a quaternary structure together with other subunits.
}

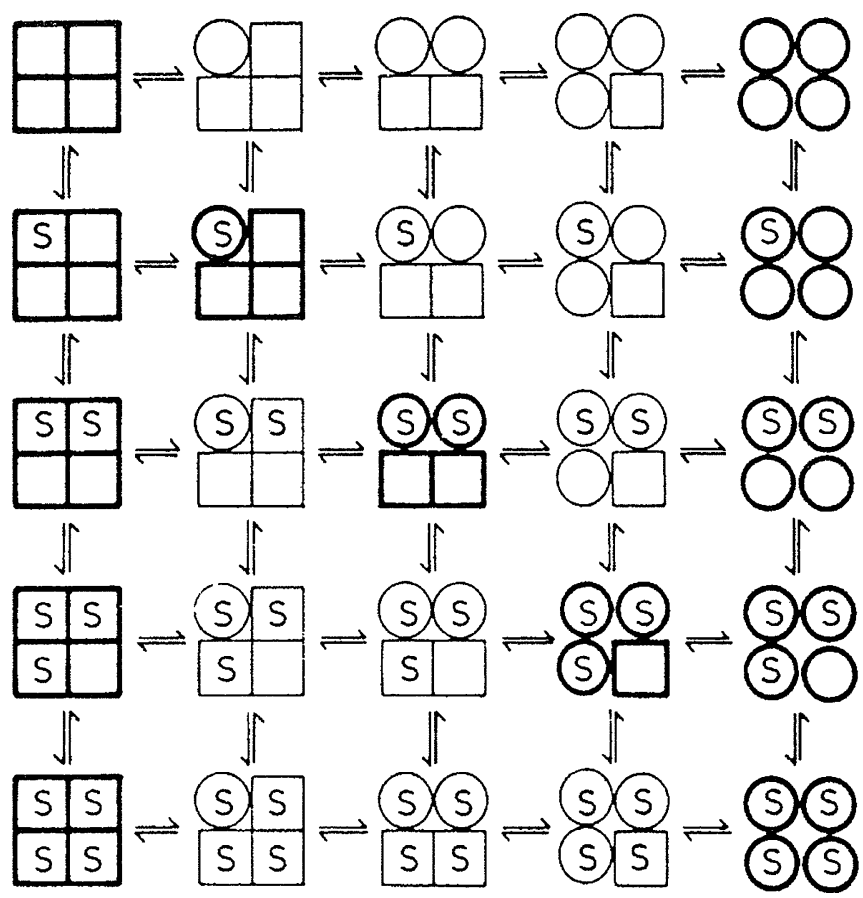

Figure 1.14. General allosteric reaction scheme for binding to a four subunit protein. All together there are 44 non-equivalent conformations of which only 25 are shown. Reproduced with permission from Eigen (1967).

\subsubsection{The Present State of Allostery}

So far, attempts to directly demonstrate the conformational changes in enzymes, in transport proteins or receptor molecules have come from X-ray diffraction (XRD), electron crystallography, and atomic force microscopy (AFM) (Stahlberg et al. 2002), and energy transfer measured by resonance between fluorescence probes (FRET), originally introduced for measurements of plasma membrane viscosity (Schnitsky \& Inbar 1974), and developed to measure conformational changes in, for instance, channels and co-transporters (Wright et al. 1981; Peerce \& Wright 1984), and later also in G proteincoupled receptors (GPCRs) (Gether 2000), as well as between GPCRs and G proteins (Oldham et al. 2006).

Although many receptive players are both dimeric as well as multimeric (Hermans 2003; Hogg et al. 2005), in enzymology, receptology (pharmacology) and transport physiology there is plenty of evidence that proteins binding a ligand or mediating a transport function are sometimes not multimeric (Kaiser 1980; Turk et al. 2000; Sintack et al. 2002; Trankle et al. 2005), although the status of muscarinic receptors is still debated (Huang \& Ellis 2007).

Finding the postulated structural changes in multimeric proteins that can cause allostery and co-operative phenomena is still a very active research field (Sivilotti \& Colquhoun 2004). In recent years, with X-ray crystallography, researchers in enzymology now find structural 
changes in support of the MWC model (Helmsteadt et al. 2001; Felter et al. 2007) and structural changes in favor of the KNF model (Helmsteadt 2001; Yao et al. 2006). That, to a certain extent, confirms the original postulates about allosteric changes in enzymes and hemoglobin. Allosteric concepts from the enzyme literature are also used for proven conformational changes in other types of heteromeric and monomeric proteins (Helmsteadt et al. 2001; Ikari \& Suketa 2002; Yao et al. 2006; Frauli et al. 2007). Examples of detailed conformational changes are shown for an enzyme in Fig. 1.15, for a GPCR in Fig. 1.16, for a transporter in Fig. 1.17, and a neurotransmitter co-transporter in Fig. 1.18. Thus, a detailed mosaic for sugar binding in the human sodium/D-glucose co-transporter can now be modeled (Hirayama et al. 2007).

\subsubsection{How the Adjective 'Allosteric' Is Used}

Lack of knowledge or failure to directly demonstrate conformational changes in vivo have resulted in the use of the term 'allosteric' in a more lax manner by researchers in the fields of enzymes, receptors, and molecular transporters. Thus, 'allostery' means something else when theoretical reaction schemes are not included in the data analysis. For example, allostery is invoked when the monomeric muscarinic receptor exhibits complex synagic behavior in the presence of a molecule, such as gallamine, that is, the non-agonist drug gallamine only influences the receptor function in the presence of a natural agonist (Stockton et al. 1983; Potter et al. 1989; Tucek \& Proska 1995; Ellis \& Seidenberg 2002; Huang \& Ellis 2007). This phenomenon has been designated as allosteric due to binding to secondary binding sites, and the non-agonist molecule as an allosteric modulator. More elaborate terms would be heterotropic positive/negative allostery and gallamine a negative heterotropic (allosteric) drug. Since cooperativity tacitly assumes that the deviation in synagics from a simple load is caused by the agonist molecule itself binding to a secondary site, the process may be specified as homotropic allostery (Chapter 14). Of course there are examples in the literature where allostery and allosteric are also invoked for theoretical models without a genuine two-state mechanism, i.e., without un-liganded receptors in two states (Chapter 2).

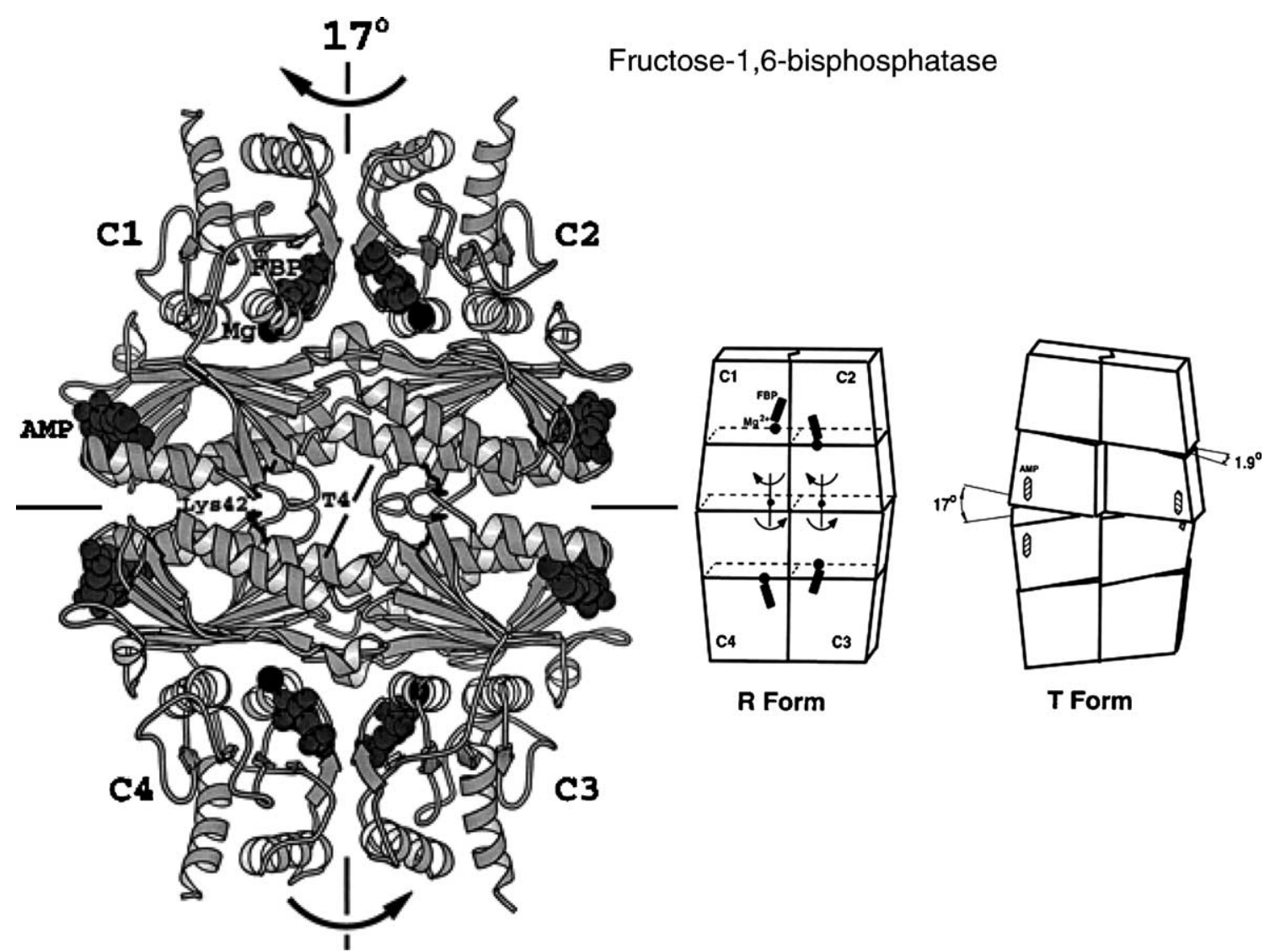

Figure 1.15. Structural changes in the porcine fructose-1,6-bisphosphatase enzyme. Shown to the right is the activating conformational twist in two planes between the relaxed $(R)$ and tense $(T)$ state. The quaternary tetrameric complex is shown to the left with indicated positions for four molecules of both fructose-1,6-bisphosphate (FBP), AMP, and Mg ${ }^{2+}$. Based on crystallographic resolution at 2.1-2.3 $\AA$. From Shyur et al. (1996, Fig 5 \& 6) with permission. See also the E. coli version of the enzyme with a comparable allosteric regulation (Hines et al. 2006). 

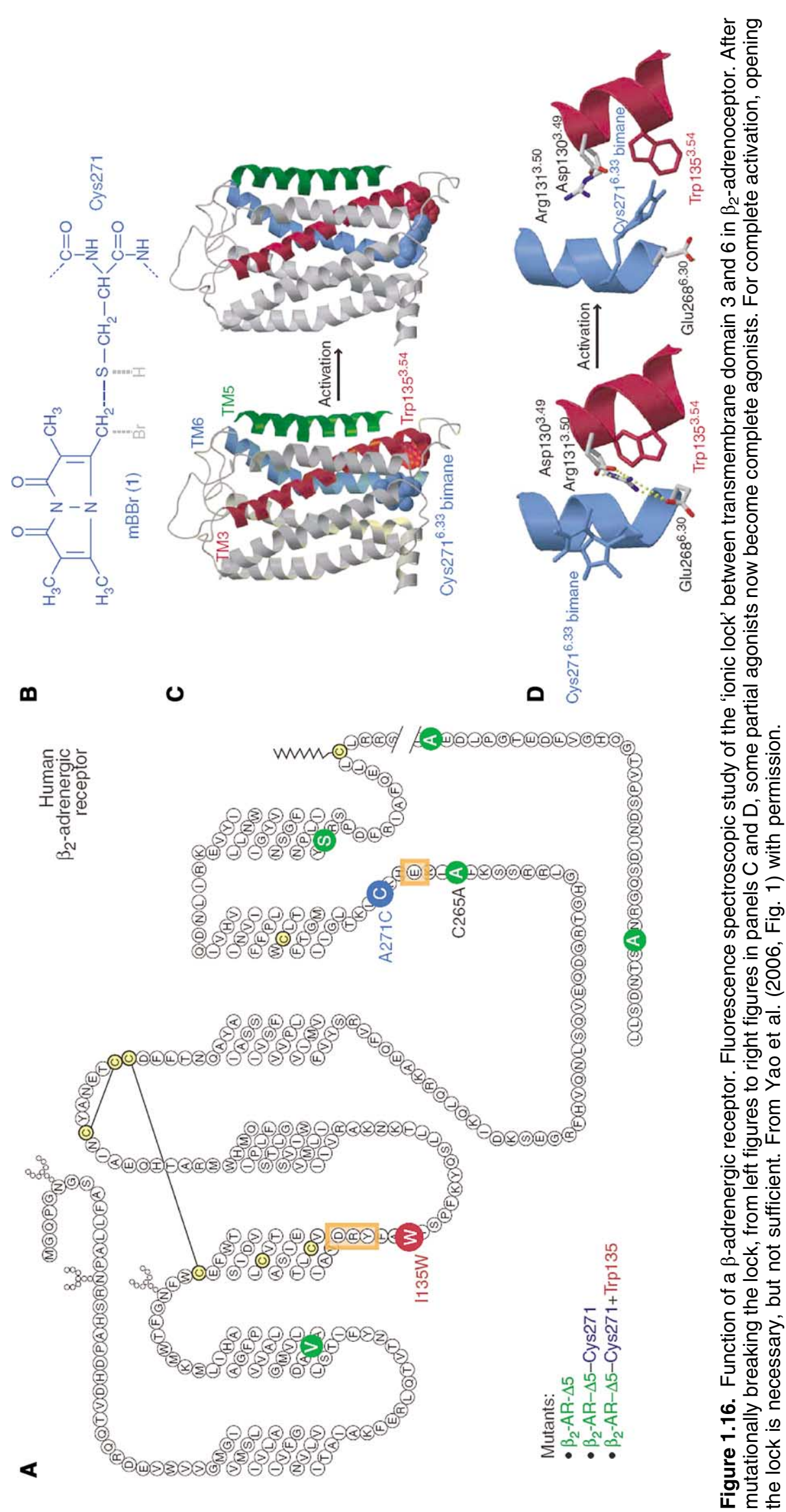

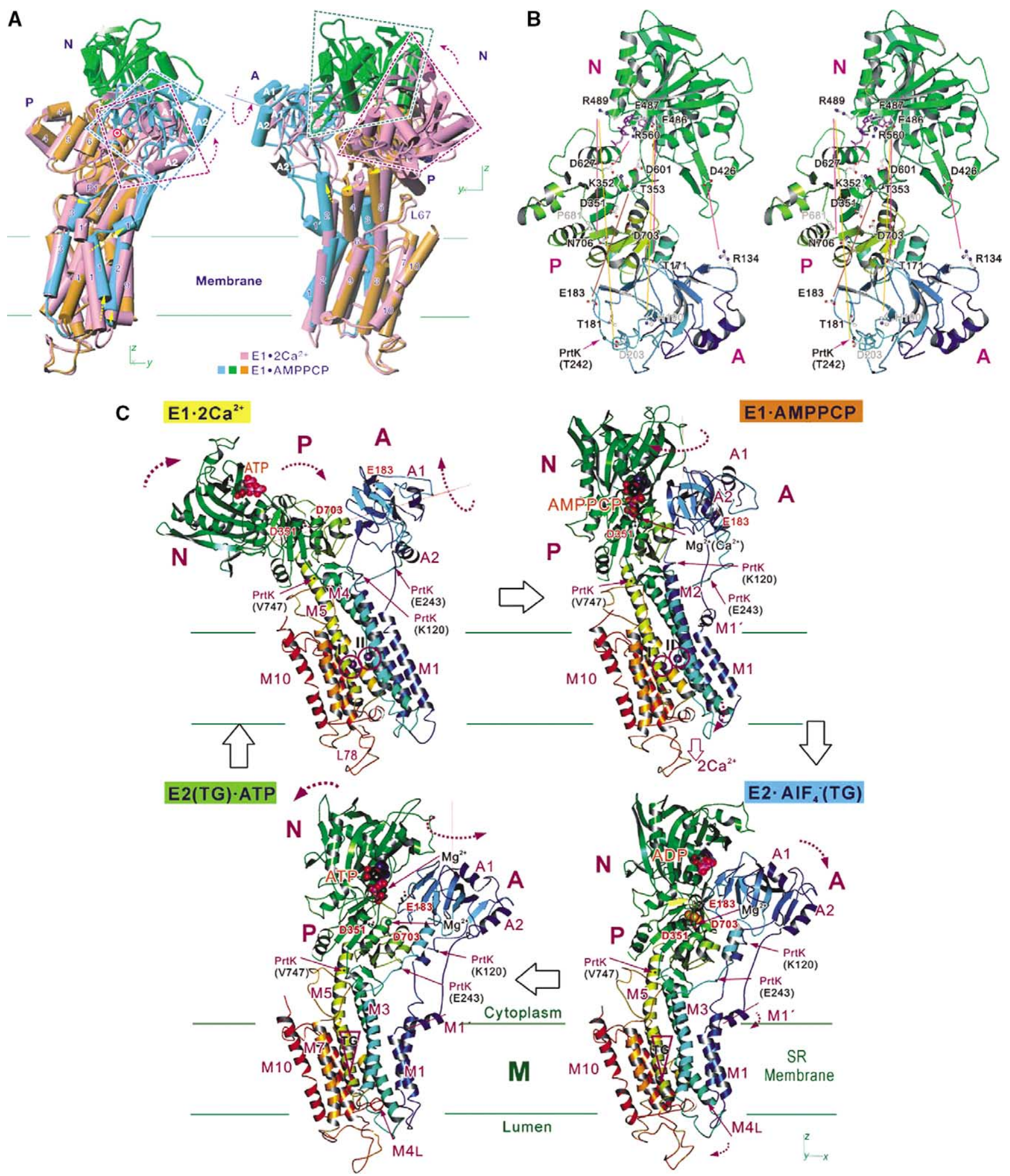

Figure 1.17. Structural changes in the sarcoplasmic calcium pump, SERCA. A switch of two calcium ions from crevices in the membrane part of SERCA to the lumen is obtained through reorientation of three cytoplasmic domains, N, $P$, and $A$ catalyzed by bound ATP. Based on crystallographic resolution at 2.6 $\AA$, (A) Side view of SERCA with the intracellular domain at the top. (B) Cross-section through the intracellular aspect of SERCA presenting domains $A, N$, and $P$. (C). Molecular reaction scheme of conformational changes in SERCA's one-way transport cycle. From (A) Toyoshima and Mizutani (2004, Fig. 2), (B) Ma et al. (2005, Fig. 1), and (C) Inesi et al. (2006, Fig. 1) with permissions. 


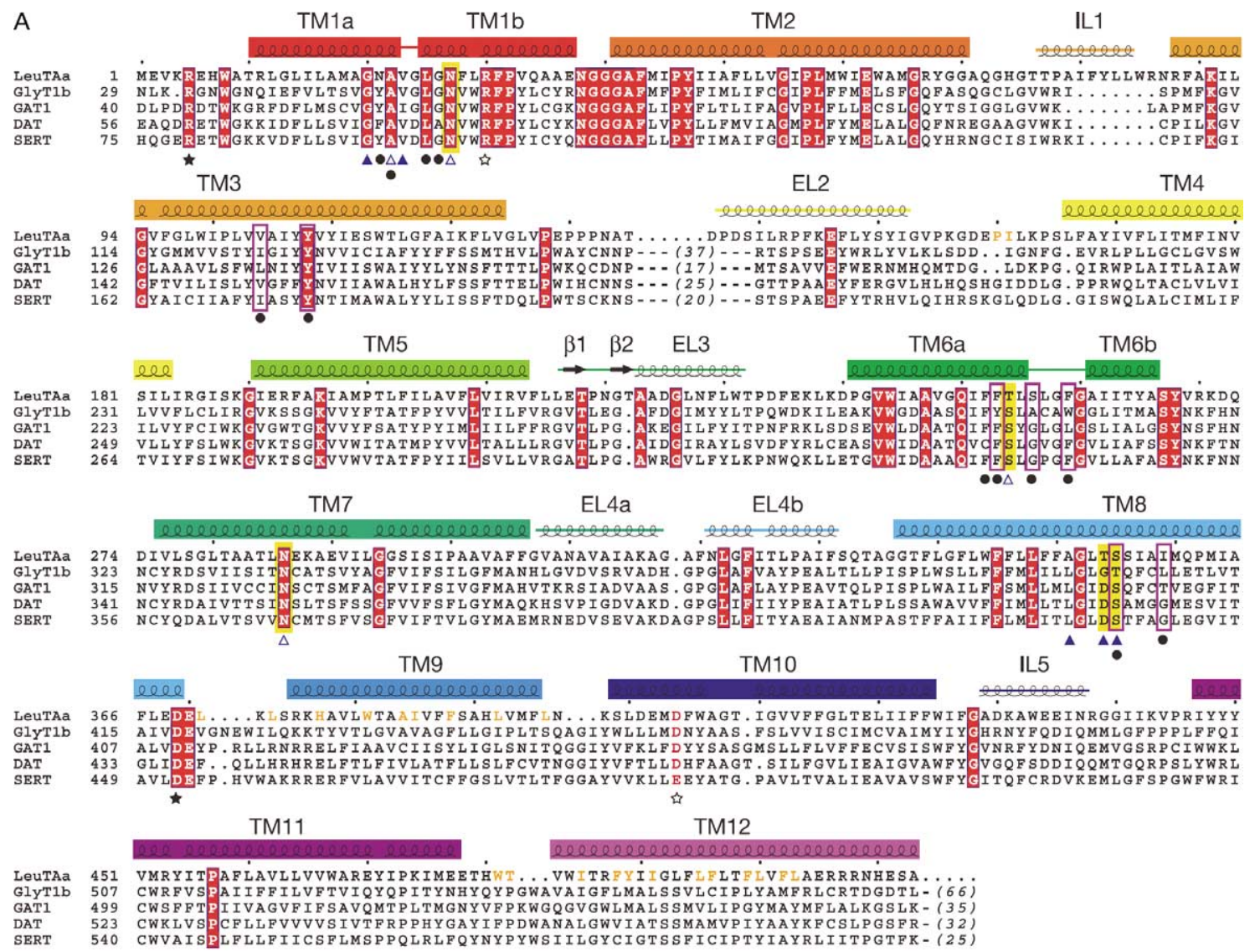

B

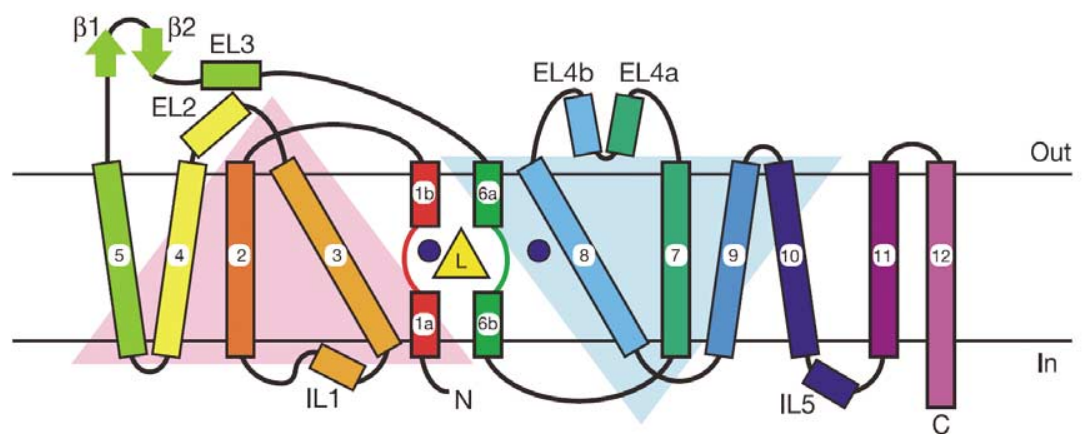

Figure 1.18. Structure of the bacterial Na/Cl dependent neurotransmitter co-transporter. From Yamashita et al. (2005, Fig 1) with permission.

In this text, the term 'allostery' will be used when talking about mechanisms and molecular models of doseresponses involving two states for un-liganded receptive units, and 'intervention' when talking about complex phenomena in one-state models, deviating from simple load. Using two different ligands in one-state models may elicit intervention (see Chapter 2). When two ligands interact in two-state models, modulation is the term to be used. One of two different ligands can modulate the effect of the other. Auto-modulation is the term we employ for two-state models to describe, for instance, bell-shaped or terraced dose-responses with a single ligand and detailed in Chapter 7 for the homotropic two-state model (HOTSM), while in one-state models to describe, for instance, bell-shaped or terraced dose-responses in the presence of a single ligand is termed 'auto-intervention', and is further treated in Chapter 3.

For now, let us just operate with 'an allosteric molecule' without specifying its mechanism. In two-state models, strict definitions for allostery, modulators, and co-operativity are postponed to Chapters 5, 7, and 15 . In one-state reaction schemes, the parallel terms are intervention, interventors, and auto-intervention (Chapters 2 and 3). 


\subsection{Spontaneous Activity and Inverse Agonism}

Late in the post-classic era (1990s), there was a significant surge in our understanding of the importance of explicitly formulating the spontaneous activity that can be observed for both receptors and ligandgated channels, seen in natural systems and often as well in gene-manipulated receptive systems (Costa \& Cotecchia 2005; Kenakin 2005b). Ligands reducing spontaneous or constitutive activity by binding to and thus directly interacting with primary binding sites are now designated as inverse agonists (Leff 1995).

Basal activity, constitutive activity, and inverse agonism are best handled by a so-called cyclic two-state model (cTSM), which is discussed in detail under complex agonism in Chapter 5. Moreover, the cTSM by its full thermodynamic nature covers the concept expressed in the loar (Sections I.1 and 1.1.12) giving perspective to the discussion of conformational induction versus conformational selection, as mentioned in Section 1.1.4 and detailed in sub-chapter 5.11.

\section{REFERENCES}

Ariëns EJ. Affinity and intrinsic activity in the theory of competitive inhibition. Part I. Problems and theory. Arch Int Pharmacodyn 99: 32-49, 1954.

Ariëns EJ. Receptor theory and structure-activity relationships. Adv Drug Res 3: 235-285, 1966.

Ariëns EJ \& de Groot WM. Affinity and intrinsic-activity in the theory of competitive inhibition. Part III. Homologous decamehoniumderivatives and succinyl-choline-esters. Arch Int Pharmacodyn 99: 193-205, 1954.

Ariëns EJ \& Simonis AM. Affinity and intrinsic-activity in the theory of competitive inhibition. Part II. Experiments with para-aminobenzoic acid derivatives. Arch Int Pharmacodyn 99: 175-187, 1954.

Ariëns EJ, Simonis AM \& de Groot WM. Affinity and intrinsic-activity in the theory of competitive- and non-competitive inhibition and an analysis of some forms of dualism in action. Arch Int Pharmacodyn 100: 298-322, 1955.

Ariëns EJ, Simonis AM \& van Rossum JM. Drug-receptor interaction: Interaction of one or more drugs with one receptor system. In Ariëns EJ (ed) Molecular Pharmacology. The mode of action of biologically active compounds. Vol I \& II. New York, Acad Press, 1964.

Ariëns EJ, van Rossum JM \& Koopman PC. Receptor reserve and threshold phenomena. I. Theory and experiments with autonomic drugs tested on isolated organs. Arch Int Pharmacodyn 127: 459-496, 1960.

Berg JM, Tymoczko JL \& Stryer L. Biochemistry. New York: WH Freeman and Company, 2006.

Berthelot M. Essai d'une théorie sur la formation des Éthers. Ann Chim Phys (3) 66: 110-128, 1862.

Berthelot M \& Péan de Saint-Gilles L. Recherches sur les affinités. De la formation et de la décomposition des éthers. Ann Chim Phys (3) 65: 385-422, 1862a.

Berthelot M \& Péan de Saint-Gilles L. Recherches sur les affinités. De la formation et de la décomposition des éthers. Ann Chim Phys (3) 66: 5-110, 1862b.
Berthelot M \& Péan de Saint-Gilles L. Recherches sur les affinités. De la formation et de la décomposition des éthers. Ann Chim Phys (3) 68: 225-359, 1863

Berthollet JC. Essai de Statique Chimique. Paris: DeMonville \& Soeurs, 1803.

Black JW \& Leff P. Operational models of pharmacological agonism. Proc $R$ Soc Lond B 220: 141-162, 1983.

Blum JJ. The enzymatic interaction between myosin and nucleotides. Arch Biochem Biophys 55: 486-511, 1955.

Botts J. Typical behavior of some simple models of enzyme action. Trans Faraday Soc 54: 593-604, 1958.

Botts J \& Morales M. Analytical description of the effects of modifiers and of enzyme multivalency upon the steady state catalyzed reaction rate. Trans Faraday Soc 49: 696-707, 1953.

Bourne HR \& von Zastrov M. Drug receptors and pharmacodynamics. In: Basic E Clinical Pharmacology, edited by Katzung BG. Stamford: McGraw Hill Co, 2004, pp. 11-33.

Briggs GE \& Haldane JBS. A note on the kinetics of enzyme action. Biochem J 19: 338-339, 1925.

Brown AJ. Enzyme action. J Chem Soc 81: 373-388, 1902.

Brown HT \& Glendinning TA. The velocity of hydrolysis of starch by diastase, with some remarks on enzyme action. J Chem Soc 81: 388400, 1902.

Burzomato V, Beato M, Groot-Kormelink PJ, Colquhoun D \& Sivilotti LG. Single-channel behavior of heteromeric alphalbeta glycine receptors: an attempt to detect a conformational change before the channel opens. J Neurosci 24: 10924-10940, 2004.

Cassel D \& Selinger Z. Catecholamine-stimulated GTPase activity in turkey erythrocyte membranes. Biochim Biophys Acta 452: 538-551, 1976.

Christopoulos A \& El-Fakahany EE. Qualitative and quantitative assessment of relative agonist efficacy. Biochem Pharmacol 58: 735-748, 1999

Clark AJ. The antagonism of acetyl choline by atropine. I Physiol 61: 547-556, 1926.

Clark AJ. The reaction between acetyl choline and muscle cells. J Physiol 61: 530-546, 1926

Clark AJ. The Mode of Action of Drugs on Cells. London: Arnold \& Co, 1933.

Clark AJ. General Pharmacology. Berlin: Springer, 1937.

Clark AJ \& Raventos J. The antagonism of acetyl choline and of quartenary ammonium salts. QJ Exp Physiol 26: 375-392, 1937.

Clark RB, Knoll BJ \& Barber R. Partial agonists and G protein-coupled receptor desensitization. Trends Pharmacol Sci 20: 279-286, 1999.

Colquhoun D. Affinity, efficacy and receptor classification: is the classical theory still useful? In: Perspectives on Hormone Receptor Classification, edited by Black JW, Jenkinson DH \& Gerskowitch VP. New York: Alan R. Liss Inc, 1987, pp. 103-114.

Colquhoun D. Binding, gating, affinity and efficacy: the interpretation of structure-activity relationships for agonists and of the effects of mutating receptors. Br J Pharmacol 125: 924-947, 1998.

Colquhoun D \& Sivilotti LG. Function and structure in glycine receptors and some of their relatives. Trends Neurosci 27: 337344, 2004.

Colquhoun D, Dowsland KA, Beato M \& Plested AJ. How to impose microscopic reversibility in complex reaction mechanisms. Biophys J 86: 3510-3518, 2004

Costa $\mathrm{T}$ \& Cotecchia S. Historical review: negative efficacy and constitutive activity of G-protein-coupled receptors. Trend Pharmacol Sci 26: 618-624, 2005.

del Castillo J \& Katz B. Interaction at end-plate receptors between different choline derivatives. J Physiol 146: 369-381, 1957.

Delaage MA. "Carrier"' theory and thermodynamics of irreversible processes. Biochim Biophys Acta 394: 493-503, 1975.

Dixon M \& Webb EC. Enzymes. London: Longman Group Ltd., 1979. 
Edsall JT. Hemoglobin and the origins of the concept of allosterism. Fed Proc 39: 226-235, 1980.

Eglen RM, Reddy H \& Watson N. Selective inactivation of muscarinic receptor subtypes. Int J Biochem 26: 1357-1368, 1994.

Ehlert FJ. Estimation of the affinities of allosteric ligands using radioligand binding and pharmacological null methods. Mol Pharmacol 33: 187-194, 1988.

Ehlert FJ. Analysis of allosterism in functional assays. J Pharmacol Exp Ther 315: 740-754, 2005.

Eigen M. Kinetics of reaction control and information transfer in enzymes and nucleic acids. In: Nobel symposium 5. Fast reactions and primary processes in chemical kinetics, edited by Claesson $\mathrm{S}$. Stockholm: Almqvist \& Wiksell, 1967, pp. 333-369.

Eigen M. New looks and outlooks on physical enzymology. Q Rev Biophys 1: 3-33, 1968.

Ellis J \& Seidenberg M. Interactions of alcuronium, TMB-8, and other allosteric ligands with muscarinic acetylcholine receptors: studies with chimeric receptors. Mol Pharmacol 58: 1451-1460, 2000.

Fell D. Understanding the Control of Metabolism. London: Portland Press, 1997.

Fersht AR. Structure and Mechanism in Protein Science. A Guide to Enzyme Catalysis and Protein Folding. New York: WH Freeman \& Co, 1999.

Fetler L, Kantrowitz ER \& Vachette P. Direct observation in solution of a preexisting structural equilibrium for a mutant of the allosteric aspartate transcarbamoylase. Proc Natl Acad Sci USA 104: 495-500, 2007.

Ford ML \& Evavold BD. Degenerate recognition of T cell epitopes: impact of $\mathrm{T}$ cell receptor reserve and stability of peptide:MHC complexes. Mol Immunol 40: 1019-1025, 2004.

Frauli M, Hubert N, Schann S, Triballeau N, Bertrand HO, Acher F, Neuville P, Pin JP \& Prezeau L. Amino-pyrrolidine tricarboxylic acids (APTCs) give new insight into group III metabotropic glutamate receptor activation mechanism. Mol Pharmacol 71: 704 712, 2007.

Furchgott RF. The pharmacology of vascular smooth muscle. Pharmacol Rev 7: 183-265, 1955.

Furchgott RF. The use of $\beta$-haloalkylamines in the differentiation of receptors and in the determination of dissociation constants of receptor-agonist complexes. Adv Drug Res 3: 21-55, 1966.

Gether U. Uncovering molecular mechanisms involved in activation of G protein-coupled receptors. Endocr Rev 21: 90-113, 2000.

Giraldo J. Agonist induction, conformational selection, and mutant receptors. FEBS Lett 256: 13-18, 2004.

Guldberg CM \& Waage P. Études sur les Affinités Chimiques. Programme de l'Université pour le 1er Semestre 1867. Christiania: Brøgger \& Christie, 1867.

Guldberg CM \& Waage P. Untersuchgen über die chemischen Affinitäten. Abhandlungen aus den Jahre 1864. In: Abegg R. Ostwald's Klassiker der exakten Wissenschaften 104, 3-9. Leipzig, Verlag von Wilhlem Engelmann, 1899.

Guldberg CM \& Waage P. Untersuchungen über die chemischen Affinitäten. Abhandlungen aus den Jahre 1879. In: Abegg R. 104, 126-171. Leipzig, Verlag von Wilhelm Engelmann, 1899.

Haldane JBS. Enzymes. London: Longmans, Green \& Co, 1930.

Harcourt AV \& Esson W. On the laws of connexion between the conditions of a chemical change and its amount. Philos Trans Roy Soc (London) 156: 193-221, 1866.

Harvey SC \& Nickerson M. Reactions of dibenamine and some congeners with substances of biological interest in relation to the mechanism of adrenergic blockade. J Pharmacol Exp Ther 112: 274-290, 1954.

Haurowitz F. Das Gleichgewicht zwischen Hämoglobin und Sauerstoff. Hoppe-Seyler Z Physiol Chem 254: 266-274, 1938.

Helmstaedt K, Krappmann S \& Braus GH. Allosteric regulation of catalytic activity: Escherichia coli aspartate transcarbamoylase versus yeast chorismate mutase. Microbiol Mol Biol Rev 65: 404-421, 2001.
Henri V. Lois Générales de l'Action des Diastases. Paris: A. Hermann, 1903.

Hermans E. Biochemical and pharmacological control of the multiplicity of coupling at G-protein-coupled receptors. Pharmacol Ther 99: 25-44, 2003.

Hines JK, Fromm HJ \& Honzatko RB. Novel allosteric activation site in Escherichia coli fructose-1,6-bisphosphatase. J Biol Chem 281: 18386-18393, 2006.

Hirayama BA, Loo DD, Diez-Sampedro A, Leung DW, Meinild A-K, LaiBing M, Turk E \& Wright EM. Sodium-dependent reorganization of the sugar-binding site of SGLT. Biochemistry 46:13391-13406, 2007.

Hogg RC, Buisson B \& Bertrand D. Allosteric modulation of ligandgated ion channels. Biochem Pharmacol 70: 1267-1276, 2005.

Huang XP \& Ellis J. Mutational disruption of a conserved disulfide bond in muscarinic acetylcholine receptors attenuates positive homotropic cooperativity between multiple allosteric sites and has subtype-dependent effects on the affinities of muscarinic allosteric ligands. Mol Pharmacol 71: 759-768, 2007.

Hunyady L, Vauquelin G \& Vanderheyden P. Agonist induction and conformational selection during activation of a G-proteincoupled receptor. Trends Pharmacol Sci 24: 81-86, 2003.

Ikari A \& Suketa Y. Expression of GFP-tagged low affinity $\mathrm{Na}^{+}$. dependent glucose transporter in Xenopus oocytes and $\mathrm{CHO}$ cells. Jpn J Physiol 52: 395-398, 2002.

Inesi G, Lewis D, Ma H, Prasad A \& Toyashima C. Concerted conformational effects of $\mathrm{Ca} 2+$ and ATP are requiired for activation of sequential reactions in the Ca2+ ATPase (SERCA) catalytic cycle. Biochemistry 45:13769-13778, 2006.

Jackson SE, el Masry N \& Fersht AR. Structure of the hydrophobic core in the transition state for folding of chymotrypsin inhibitor 2: a critical test of the protein engineering method of analysis. Biochemistry 32: 11270-11278, 1993.

Kaiser PM. Substrate inhibition as a problem of non-linear steady state kinetics with monomeric enzymes. J Mol Catal 8: 431-442, 1980.

Katchalsky A \& Curran PF. Non-equilibrium Thermodynamics in Biophysics. Cambridge, MA: Harvard University Press, 1965.

Katz B \& Thesleff S. A study of the 'desensitization' produced by acetylcholine at the motor end-plate. J Physiol 138: 63-80, 1957.

Kenakin T. Are receptors promiscuous? Intrinsic efficacy as a transduction phenomenon. Life Sci 43: 1095-1101, 1988.

Kenakin T. Drugs and receptors. An overview of the current state of knowledge. Drugs 40: 666-687, 1990.

Kenakin T. On the definition of efficacy. Trends Pharmacol Sci 15: 408409, 1994.

Kenakin T. Pharmacological proteus? Trend Pharmacol Sci 16: 256-258, 1995a.

Kenakin T. Agonist-receptor efficacy. I: Mechanisms of efficacy and receptor promiscuity. Trends Pharmacol Sci 16: 188-192, 1995b.

Kenakin T. Agonist-receptor efficacy. II. Agonist trafficking of receptor signals. Trends Pharmacol Sci 16: 232-238, 1995.

Kenakin T. Receptor conformational induction versus selection: all part of the same energy landscape. Trend Pharmacol Sci 17: 190$191,1996$.

Kenakin T. Pharmacological Analysis of Drug-Receptor Interaction. Philadelphia, PA: Lippincott-Raven Publishers, 1997.

Kenakin T. Efficacy in drug receptor theory: outdated concept or under-valued tool? Trends Pharmacol Sci 20: 400-405, 1999.

Kenakin T. Drug efficacy at G protein-coupled receptors. Annu Rev Pharmacol Toxicol 42: 349-379, 2002a.

Kenakin T. Efficacy at G-protein-coupled receptors. Nat Rev Drug Discov 1: 103-110, 2002b.

Kenakin T. Efficacy as a vector: the relative prevalence and paucity of inverse agonism. Mol Pharmacol 65: 2-11, 2004a.

Kenakin T. Principles: receptor theory in pharmacology. Trends Pharmacol Sci 25: 186-192, 2004b. 
Kenakin T. New concepts in drug discovery: collateral efficacy and permissive antagonism. Nat Rev Drug Discov 4: 919-927, $2005 a$.

Kenakin T. The physiological significance of constitutive receptor activity. Trend Pharmacol Sci 26: 603-605, 2005b.

Kenakin T. Data-driven analysis in drug discovery. $J$ Recept Signal Transduct Res 26: 299-327, 2006.

Kenakin T \& Onaran HO. The ligand paradox between affinity and efficacy: can you be there and not make a difference? Trends Pharmacol Sci 23: 275-280, 2002.

Kenakin TP. Theoretical and practical problems with the assessment of intrinsic efficacy of agonists: efficacy of reputed beta-1 selective adrenoceptor agonists for beta-2 adrenoceptors. J Pharmacol Exp Ther 223: 416-423, 1982.

Kenakin TP. The relative contribution of affinity and efficacy to agonist activity: organ selectivity of noradrenaline and oxymetazoline with reference to the classification of drug receptors. Br J Pharmacol 81: 131-141, 1984.

Kenakin TP. The quantification of relative efficacy of agonists. $J$ Pharmacol Methods 13: 281-308, 1985.

Kenakin TP. New eyes to see texture in ligand efficacy. Nat Methods 2: 163-164, 2005c.

Kenakin TP \& Beek D. In vitro studies on the cardiac activity of prenalterol with reference to use in congestive heart failure. $J$ Pharmacol Exp Ther 220: 77-85, 1982.

Koshland DE Jr, Nemethy G \& Filmer D. Comparison of experimental binding data and theoretical models in proteins containing subunits. Biochemistry 5: 365-385, 1966.

Kurtz TW. New treatment strategies for patients with hypertension and insulin resistance. Am J Med 119 (5 Suppl 1): S24-S30, 2006.

Laidler KJ and Bunting PS. The Chemical Kinetics of enzyme action. Oxford: Oxford University Press, 1973.

Langmuir I. The adsorption of gases on plane surfaces of glass, mica and platinum. J Am Chem Soc 40: 1361-1403, 1918.

Leff $\mathrm{P}$. The two state model of receptor activation. Trends Pharmacol Sci 16: 89-97, 1995.

Leff P \& Scaramellini C. Promiscuity, pre-coupling and instability. Trends Pharmacol Sci 19: 13, 1998.

Lieberman JA. Dopamine partial agonists: a new class of antipsychotic. CNS Drugs 18: 251-267, 2004.

Lutz M \& Kenakin T. Quantitative Molecular Pharmacology and Informatics in Drug Discovery. Chichester: Wiley \& Sons, 1999.

Ma H, Lewis D, Xu C, Inesi G \& Toyoshima C. Functional and structural roles of critical amino acids within the "N", "P", and "A" domains of the $\mathrm{Ca}^{2+}$ ATPase (SERCA) headpiece. Biochemistry 44: 8090-8100, 2005.

Michaelis L \& Menten ML. Die Kinetik der Invertinwirkung. Biochem Zeitsch 49: 333-369, 1913.

Monod J \& Jacob F. Teleonomic mechanisms in cellular metabolism, growth, and differentiation. Cold Spring Harb Symp Quant Biol 26: 389-401, 1961.

Monod J, Changeux JP \& Jacob F. Allosteric proteins and cellular control systems. J Mol Biol 6: 306-329, 1963.

Monod J, Wyman J \& Changeux J-P. On the nature of allosteric transitions: a plausible model. J Mol Biol 12: 88-118, 1965.

Nelson DL \& Cox MM. Lehninger Principles of Biochemistry. New York: WH Freeman, 2005.

Neubig RR, Spedding M, Kenakin T \& Christopoulos A. International Union of Pharmacology Committee on Receptor Nomenclature and Drug Classification. XXXVIII. Update on terms and symbols in quantitative pharmacology. Pharmacol Rev 55: 597-606, 2003.

Nickerson M. Receptor occupancy and tissue response. Nature 178: 697-698, 1956.

Nickerson M \& Goodman LS. Pharmacological properties of a new adrenergic blocking agent: N,N-dibenzyl-chloroethylamine (Dibenamine). J Pharmacol Exp Ther 89: 167-185, 1947.
Oldham WM, Van Eps N, Preininger AM, Hubbell WL \& Hamm HE. Mechanism of the receptor-catalyzed activation of heterotrimeric G proteins. Nat Struct Mol Biol 13: 772-777, 2006.

Onaran HO \& Costa T. Agonist efficacy and allosteric models of receptor action. Ann NY Acad Sci 812: 98-115, 1997.

Onsager L. Reciprocal relations in irreversible processes. I. Phys Rev 37 : 405-426, 1931.

Onsager L. Reciprocal relations in irreversible processes. II. Phys Rev 38: 2265-2279, 1931.

Ostwald W. Lehrbuch der Allgemeinen Chemie. Zweiten Bandes Zweiter Teil: Verwandtschaftslehere - Erster Teil. Leipzig: Verlag von W. Engelmann, 1902.

Partington JR. A History of Chemistry. London: MacMillan, 1964.

Paton WDM \& Rothschild AM. The effect of varying calcium concentration on the kinetic constants of hyoscine and mepyramine antagonism. Br J Pharmacol 24: 432-448, 1965.

Peerce BE \& Wright EM. Conformational changes in the intestinal brush border sodium-glucose cotransporter labeled with fluorescein isothiocyanate. Proc Natl Acad Sci USA 81: 2223-2226, 1984.

Perutz M. Structure of hemoglobin. Brookhaven Symp Biol 13: 165-183, 1960.

Perutz M. Mechanisms of Cooperativity and Allosteric Regulation in Proteins. Cambridge: Cambridge University Press, 1990.

Potter LT, Ferrendelli CA, Hanchett HE, Hollifield MA \& Lorenzi MV. Tetrahydroaminoacridine and other allosteric antagonists of hippocampal M1 muscarine receptors. Mol Pharmacol 35: 652$660,1989$.

Pratt WB \& Taylor P. Principles of Drug Action: The Basis of Pharmacology. Philadelphia, PA: Churchill Livingstone, 1990.

Scaramellini C \& Leff P. Theoretical implications of receptor coupling to multiple $\mathrm{G}$ proteins based on analysis of a three-state model. Methods Enzymol 343: 17-29, 2002.

Segal HL. The development of enzyme kinetics. In: The Enzymes, edited by Boyer PD, Lardy H \& Myrbäck K. New York: Academic Press, 1959, pp. 1-48.

Segel IH. Enzyme Kinetics. Behavior and Analysis of Rapid Equilibrium and Steady-state Enzyme Systems. New York: Wiley \& Sons (reissued in 1993), 1975.

Shinitzky M \& Inbar M. Difference in microviscosity induced by different cholesterol levels in the surface membrane lipid layer of normal lymphocytes and malignant lymphoma cells. J Mol Biol 85: 603-615, 1974

Shyur LF, Aleshin AE, Honzatko RB \& Fromm HJ. Biochemical properties of mutant and wild-type fructose-1,6-bisphosphatases are consistent with the coupling of intra- and intersubunit conformational changes in the T- and R-state transition. $J$ Biol Chem 271: 33301-33307, 1996.

Stahlberg H, Engel A \& Philippsen A. Assessing the structure of membrane proteins: combining different methods gives the full picture. Biochem Cell Biol 80: 563-568, 2002.

Stephenson RP. A modification of receptor theory. Br J Pharmacol 11: 379-393, 1956.

Stockton JM, Birdsall NJ, Burgen AS \& Hulme EC. Modification of the binding properties of muscarinic receptors by gallamine. Mol Pharmacol 23: 551-557, 1983.

Taylor P \& Insel PA. Principles of Drug Action, edited by Pratt WB and Taylor P. New York: Churchill Livingstone, 1990.

Thomsen JS. Logical relations among the principles of statistical mechanics and thermodynamics. Phys Rev 91: 1263-1266, 1953.

Tolkovsky AM \& Levitzki A. Mode of coupling between the betaadrenergic receptor and adenylate cyclase in turkey erythrocytes. Biochemistry 17: 3795-3810, 1978.

Tolkovsky AM \& Levitzki A. Theories and predictions of models describing sequential interactions between the receptor, the GTP regulatory unit, and the catalytic unit of hormone dependent adenylate cyclases. J Cyclic Nucleotide Res 7: 139-150, 1981. 
Tolman RC. Duration of molecules in upper quantum states. Phys Rev 23: 693-709, 1924

Tonini M \& Pace F. Drugs acting on serotonin receptors for the treatment of functional GI disorders. Dig Dis 24: 59-69, 2006.

Tonomura Y \& Watanabe S. Effect of adenosine triphosphate on the light-scattering of actinomysin solution. Nature 169: 112-113, 1952

Toyoshima C \& Mizutani T. Crystal structure of the calcium pump with a bound ATP analogue. Nature 430: 529-535, 2004.

Tucek S \& Proska J. Allosteric modulation of muscarinic acetylcholine receptors. Trends Pharmacol Sci 16: 205-212, 1995.

Turk E, Kim O, le Coutre J, Whitelegge JP, Eskandari S, Lam JT, Kreman M, Zampighi G, Faull KF \& Wright EM. Molecular characterization of Vibrio parahaemolyticus vSGLT: a model for sodium-coupled sugar cotransporters. J Biol Chem 275: 2571125716, 2000.

van Rossum JM. Limitations of molecular pharmacology. Some implications of the basic assumptions underlying calculations on drug-receptor interactions and the significance of biological drug parameters. Adv Drug Res 3: 189-234, 1966.

van Rossum JM \& Ariëns EJ. Pharmacodynamics of parasympathetic drugs; structure-action relationships in homologous series of quaternary ammonium salts. Arch Int Pharmacodyn Ther 118: 418-446, 1959

van Rossum JM \& Ariëns EJ. Receptor-reserve and threshold-phenomena. II. Theories on drug-action and a quantitative approach to spare receptors and threshold values. Arch Int Pharmacodyn 136: 385-413, 1962.

Venter DP. Efficacy. I: A new method for estimating relative efficacy of full agonists via a newly defined efficacy related parameter. Eur J Pharmacol 320: 223-231, 1997.

Walz D \& Caplan SR. Energy coupling and thermokinetic balancing in enzyme kinetics: microscopic reversibility and detailed balance revisited. Cell Biophys 12: 13-28, 1988.

Watanabe S, Tonomura Y \& Shiokawa H. Mechanism of muscular contraction. II. Kinetic studies on muscle ATP-ase. J Biochem 40: 387-402, 1953.
Weiss JM, Morgan PH, Lutz MW \& Kenakin TP. The cubic ternary complex receptor occupancy model. III. Resurrecting efficacy. $J$ Theor Biol 181: 381-397, 1996.

Whaley BS, Yuan N, Birnbaumer L, Clark RB \& Barber R. Differential expression of the beta-adrenergic receptor modifies agonist stimulation of adenylyl cyclase: a quantitative evaluation. Mol Pharmacol 45: 481-489, 1994.

Wilhelmy L. I. Ueber das Gesetz, nach welchem die Einwirkung der Säuren auf den Rohrzuker stattfindet. Ann Phys Chem 21: 413$428,1850 \mathrm{a}$.

Wilhelmy L. II. Ueber das Gesetz, nach welchen die Einwirkung der Säuern auf den Rohrzuker stattfindet. Ann Phys Chem 21: 499$526,1850 \mathrm{~b}$.

Wright SH, Krasne S, Kippen I \& Wright EM. $\mathrm{Na}^{+}$-dependent transport of tricarboxylic acid cycle intermediates by renal brush border membranes. Effects on fluorescence of a potentialsensitive cyanine dye. Biochim Biophys Acta 640: 767-778, 1981.

Wyman J. Linked functions and reciprocal effects in hemoglobin: A second look. Adv Protein Chem 19: 223-286, 1964.

Wyman J \& Allen DW. The problem of the heme interactions in hemoglobin and the basis of the Bohr effect. J Polym Sci 7: 499$518,1951$.

Wyman J \& Gill SJ. Binding and Linkage. Functional Chemistry of Biological Macromolecules. New York: Freeman, 1990.

Yamashita A, Singh SK, Kawate T \& Gouaux. Crystal structure of a bacterial homologue of $\mathrm{Na}^{+} / \mathrm{Cl}^{-}$dependent neurotransmitter transporters. Nature 437:215-223, 2005.

Yao X, Parnot C, Deupi X, Ratnala VR, Swaminath G, Farrens D \& Kobilka B. Coupling ligand structure to specific conformational switches in the beta2-adrenoceptor. Nat Chem Biol 2: 417-422, 2006.

Zablocki JA, Wu L, Shryock J \& Belardinelli L. Partial A(1) adenosine receptor agonists from a molecular perspective and their potential use as chronic ventricular rate control agents during atrial fibrillation (AF). Curr Top Med Chem 4: 839-854, 2004. 



\subsection{Classical Ant-agonism}

\subsubsection{Towards Intervention}

By introducing a secondary ligand as well as a secondary binding site, Chapter 2 examines inhibitors, also called ant-agonists. We shall deal with several aspects of a second ligand interfering with the binding of a primary ligand and/or with the function induced by a primary ligand. Inhibitory processes may be competitive or noncompetitive. Competitive ant-agonism only needs a single binding site, whereas non-competitive ant-agonism requires at least two binding sites. Un-competitive ant-agonism is a special case of the non-competitive reaction scheme. These concepts are dealt with in Subchapter 2.4. Meanwhile, the interaction between two ligands and a receptive unit may also involve a crosscorrelation, as exemplified in a so-called 'intervention' model described in the following section and detailed further in Sub-chapter 2.5.

\subsubsection{The Intervention Scheme}

Having introduced a secondary site and a secondary ligand, we must also operate with a possible interaction between the two binding sites as ligands bind. The interaction may be stimulatory or inhibitory for the subsequent processes of binding or function. Here, the interaction is expressed in a single system constant $c$ (Fig. 2.1A). Constant $c$ above unity is stimulatory, while below unity it is inhibitory. At present, in channel studies this type of interaction may be resolved into 18-system constants (Burzomato et al. 2004; Colquhoun 2006).

In order to cover both the stimulatory as well as the inhibitory aspect of interaction in a two-ligand/two-site reaction scheme (TLTS) as in Fig. 2.1A, and at the same time clearly differentiate it from the allosteric type of reaction scheme, which involves a TLTS with additional two-states and spontaneous activity (see Chapters 7 and 15), I have decided to designate the one-state TLTS reaction scheme in Fig. 2.1A - with binding site interaction but without spontaneous activity - as the intervention model. In this scheme, ligands participating in changing the binding or function of primary ligands will be referred to as interventors, reserving the term 'modulators' for secondary ligands in allosteric reaction schemes with two-sites and two-states (Chapter 14). The intervention model is depicted in Fig. 2.1A together with a simple two-state model with a single binding site shown in Fig. 2.1B, an allosteric model.

Derivation of formulas for the intervention model in enzymology started early with Michaelis (1914) and continued in the 1950s (Table 2.1) (Laidler \& Socquet 1950; Segal et al. 1952; Botts \& Morales 1953; Friedenwald \& Maengwyn-Davies 1954a,b, pp. 154-190; Ariëns et al. 1955; Laidler 1956, 1958, pp. 78-93; Webb 1963, pp. 54-60; Cleland 1963; Hijazi \& Laidler 1973; Laidler \& Bunting 1973; Cha 1975). ${ }^{1}$

In enzymology, intervention reaction schemes with negative interaction are mostly listed under the term 'mixed-inhibition' or just 'non-competitive' (Webb 1963; Cha 1975; Segel 1975, 1993, Chapter 4; CornishBowden 1995, 2004; Varon et al. 2002), while a stimulatory aspect in the intervention models is ill-fated as it is hardly observed, and therefore only sporadically dealt with as 'non-competitive synergism', 'partial noncompetitive', or 'mixed activation' (Ariëns et al. 1964; Mahler \& Cordes 1966, p. 252; Segel 1975, 1993, Chapter 5; Cornish-Bowden 1995, 2004). A more direct approach to the intervention model is the so-called

\footnotetext{
${ }^{1}$ Laidler $(1956,1958)$ operated with simple non-competitive reactions, which in this book are equal to non-competitive reactions, and noncompetitive reactions equal the present intervention scheme.
} 

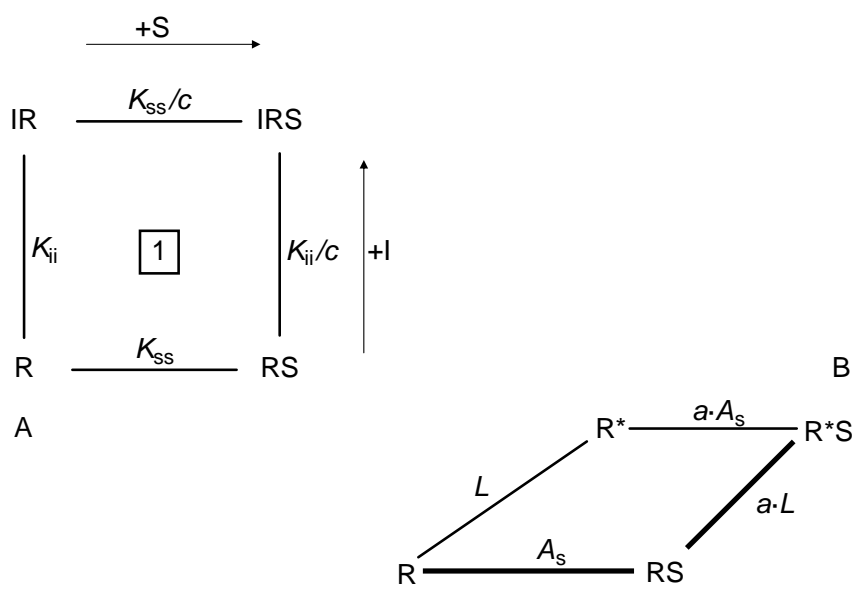

Figure 2.1. Two simple reaction schemes. (A) The Intervention model where an interaction constant $c$ may be (a) zero equal to 'competitive inhibition', (b) unity equal to 'noncompetitive inhibition', (c) above zero and below unity equal to 'negative intervention' and above unity equal to 'positive intervention'. Although the intervention model has two binding sites, it is not an allosteric model, it is a pro-allosteric model. Allostery further requires un-liganded activity, the same as spontaneous activity (see Chapter 15). (B) The simple twostate model with a single binding site. This model is also referred to as the cyclic two-state model with spontaneous activity (Chapter 5). This two-state model is not an allosteric model. Allostery also requires at least two binding sites (see Chapter 15 ). $S=$ primary ligand, I = interventor, $K_{\mathrm{ss}}=$ dissociation constant for $S$ at primary $s$-site, and $K_{\mathrm{ii}}=$ dissociation constant for interventor ligand at intervention i-site.

"general modifier mechanism of Botts and Morales" and its formulation (Botts \& Morales 1953; GarziaCanoves et al. 1987; Topham \& Brocklehurst 1992; Shou et al. 1999, Shou 2002; Varon et al. 2002).

In pharmacology, the intervention reaction schemes with site interaction were formulated by De Lean and coworkers (De Lean et al. 1980; Burgisser et al. 1982; Wreggett \& De Lean 1984) as the ternary complex-model (TCM), specifically related to G protein coupled receptors. More recently, the TCM has appeared as an 'allosteric' TCM (Ehlert 1988; Christopoulos 2002; Christopoulos \& Kenakin 2002; Avlani et al. 2004; Kenakin 2004a, Section 6.8.7, 2004b). Although terms including 'allosteric' have been sorted out (May \& Christopoulos 2003; May et al. 2004, 2007a), a term such as 'allosteric ternary complex-model' for plain TCM is still confusing (Scheer et al. 1996; Daeffler \& Landry 2000).

In modeling, including mixed-inhibition, mixed activation, TCM and 'allosteric' TCM, I recommend a new term for these kinds of reaction schemes - namely the intervention model. See Sub-chapter 2.7 for more details on the development of the intervention scheme.

\subsubsection{Auto-ant-agonism}

In this chapter, I have opted to examine ant-agonism or inhibition by compounds that are different from the
Table 2.1. On the roots to formulate the heterotropic mutually inclusive one-state model, HE-MI-OSM, and the homotropic mutually inclusive one-state model, HO-MI-OSM

\begin{tabular}{|c|c|c|}
\hline Reference & $\begin{array}{l}\text { Names or models for } \\
\text { intervention }\end{array}$ & Comment \\
\hline Michaelis (1914) & Substrate inhibition & Function \\
\hline Haldane (1930) & Substrate inhibition & Function \\
\hline Wyman $(1948,1964)$ & $\begin{array}{l}\text { Theory of linked } \\
\text { function }\end{array}$ & $\begin{array}{l}\text { Binding } \\
\text { HO-MI-OSM }\end{array}$ \\
\hline $\begin{array}{l}\text { Laidler and Socquet } \\
\text { (1950) }\end{array}$ & $\begin{array}{l}\text { Non-competitive } \\
\text { adsorption }\end{array}$ & Functional \\
\hline Segal et al. (1952) & An intervention scheme & Functional \\
\hline $\begin{array}{l}\text { Botts and Morales } \\
\text { (1953) }\end{array}$ & An intervention scheme & Functional \\
\hline $\begin{array}{l}\text { Friedenwald and } \\
\text { Maengwyn-Davies } \\
\text { (1954) }\end{array}$ & An intervention scheme & Functional \\
\hline $\begin{array}{l}\text { Ariëns et al. (1955, } \\
\text { 1964) }\end{array}$ & $\begin{array}{l}\text { Non-competitive } \\
\text { synergism }\end{array}$ & $\begin{array}{l}\text { Functional } \\
\text { HO-MI-OSM }\end{array}$ \\
\hline Laidler $(1956,1958)$ & An intervention scheme & Functional \\
\hline Webb (1963) & Mixed inhibition & Functional \\
\hline $\begin{array}{l}\text { Mahler and Cordes } \\
\text { (1966) }\end{array}$ & Partial non-competitive & Functional \\
\hline $\begin{array}{l}\text { Cheng and Prusoff } \\
\text { (1973) }\end{array}$ & Non-competitive & Functional \\
\hline $\begin{array}{c}\text { Cornish-Bowden (1974, } \\
\text { 1995, 2004, p 134) }\end{array}$ & $\begin{array}{l}\text { General modifier } \\
\text { mechanism }\end{array}$ & Functional \\
\hline Segel $(1975,1993)$ & $\begin{array}{l}\text { Mixed inhibition and } \\
\text { activation }\end{array}$ & Functional \\
\hline Cha (1975) & Non-competitive & Binding \\
\hline $\begin{array}{l}\text { Jacobs and } \\
\text { Cuatrecasas (1976) }\end{array}$ & Mobile receptor & Functional \\
\hline $\begin{array}{l}\text { Tolkovsky and Levitzki } \\
\text { (1978) }\end{array}$ & $\begin{array}{l}\text { Floating receptor } \\
\text { model }\end{array}$ & Functional \\
\hline Birdsall et al. (1978) & $\begin{array}{l}\text { Theory of linked } \\
\text { function }\end{array}$ & Binding \\
\hline $\begin{array}{l}\text { Boeynaems and } \\
\text { Dumont (1980, } \\
\text { pp. 143-147) }\end{array}$ & $\begin{array}{l}\text { Pseudo-competitive } \\
\text { ant-agonism/mixed } \\
\text { ant-agonism }\end{array}$ & Binding \\
\hline De Lean et al. (1980) & $\begin{array}{l}\text { Ternary complex } \\
\text { model }\end{array}$ & $\begin{array}{l}\text { Binding and } \\
\text { function }\end{array}$ \\
\hline Stockton et al. (1983) & Ternary complex & Binding \\
\hline $\begin{array}{l}\text { Wreggett and De Lean } \\
\quad(1984)\end{array}$ & Ternary complex & Binding \\
\hline Ehlert (1988) & 'Allosteric'* & Binding/functional \\
\hline MacKay (1990) & $\begin{array}{l}\text { Ternary complex, } \\
\text { G proteins }\end{array}$ & $\begin{array}{l}\text { Functional, } \\
\text { steady-state }\end{array}$ \\
\hline Topham (1990) & Mixed inhibition & Functional \\
\hline Wells (1992) & $\begin{array}{l}\text { Heterotropic } \\
\text { co-operativity }\end{array}$ & Binding \\
\hline Costa et al. (1992) & Negative ant-agonism & Functional \\
\hline Samama et al. (1993) & Extended TCM & Functional \\
\hline Trzeciakowski (1999) & $\begin{array}{l}\text { Standard TCM, } \\
\text { G proteins }\end{array}$ & Binding/function \\
\hline $\begin{array}{l}\text { Shou et al. (2001), } \\
\text { Shou (2002) }\end{array}$ & Partial inhibition** & $\begin{array}{l}\text { Functional } \\
\text { HO-MI-OSM }\end{array}$ \\
\hline $\begin{array}{l}\text { Present text on } \\
\text { one-state models }\end{array}$ & Intervention models & $\begin{array}{l}\text { Binding/ } \\
\text { function }\end{array}$ \\
\hline
\end{tabular}

*See Chapters 5 and 14 for use of the term 'allosteric' in general and in particular.

** 'Partial inhibition' frequently used in enzymology have different meanings, see, e.g., Webb (1963) and Segel $(1975,1993)$. Segel's Chapters 5 and 6 are on activation in HE-MI-OSM.

primary ligands, the agonists, while Chapter 3 will deal with self-inhibition. In self-inhibition, substrates or agonists at low concentration activate the function of relevant receptive units, enzymes, or transporters and so forth, while at increasing concentrations even in the absence of 
other ligands the agonist or substrate starts to ant-agonize its own action - this is also designated auto-ant-agonism, auto-inhibition, or negative auto-intervention. This selfinhibition is understood to take place from a secondary binding site in the receptive unit.

Means of obtaining parameters such as dissociation constants from experimental data are discussed in Chapter 4 for agonism, ant-agonism, and intervention as well as for auto-ant-agonism and auto-intervention in onestate models.

Inverse agonists also inhibit function, but they antagonize inherent activity by binding at the primary site. Since inverse agonists inhibit constitutive activity, they are therefore best treated in two-state models with spontaneous activity, which appears in Chapter 5 under complex agonism.

\subsubsection{Inhibitors and Reversibility}

In general, when a drug lowers one or more of the activities of a system, a cell, or a protein molecule, it is called an inhibitor. Inhibitors may be either reversible or irreversible. Often, the reversibility of an inhibitor is decided by the speed with which the interaction between the inhibitor and its target decays after washout of the ant-agonistic drug. The time of decay is held against the time of experimentation. That is to say, if the inhibitory effect does not reverse significantly during the observation period after the inhibitory compound is removed from the system, the compound is said to be an 'irreversible inhibitor'. For instance, regain of less than $10 \%$ of an initial response in the observation period following washout of a drug will probably be taken to mean that the drug has bound almost irreversibly or is distributed more permanently in an unstirred compartment, thereby preventing a reasonably short duration for washout and equilibration.

During washout, likely to be an exponential decay of binding or diffusion-limitation, the lapse of time to revert and regain $63 \%$ of a response equals one time constant, $\tau$. ${ }^{2}$ Thus, to name a compound 'reversible' typically requires an observation period in the range of a time constant or more for its washout process. The noncompetitive-like behavior of exponential decay or diffusion-limitation was explained by Rang (1966) in his description of dissociation-limited and biophase models.

In this context, a designation of irreversible inhibitor' may be employed about a high affinity ant-agonist not displaced by an agonist during its exposure that is too short to allow the displacement, also known

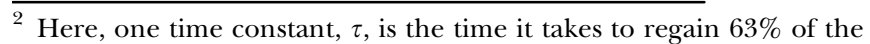
inhibited function. Percentage regain for an exponential function is: regain $(\%)=\left[1-\mathrm{e}^{-\tau}\right] \cdot 100$, where $\mathrm{e}$ is the base of natural logarithms $=2.718$. For an observation period equal $1 \tau$, the regain is $63 \%$.
}

as 'insurmountable ant-agonism' (see Sub-chapter 2.8) (Jenkinson 2003, p. 53).

\subsubsection{Ant-agonists, Interventors, and Activators}

Reversible inhibitors that affect agonist-induced activity are referred to as ant-agonists, and can be competitive, non-competitive, or un-competitive. Thus, for a compound to be an ant-agonist, it is required that it only works in the presence of an agonist, more or less preventing the agonist from activating or binding to its receptive unit. On the other hand, 'inhibitor' will, in general, refer to irreversible as well as reversible inhibitors of both agonist-induced and agonist-independent activity. The term 'inhibitor' is thus conveniently used for drugs that operate by irreversibly blocking spontaneous or agonist-induced activity, while 'ant-agonist' covers for reversible inhibitors dependent on agonistinduced activity (Table 2.2A), or eventually reversible inhibitors of a negative effect of inverse agonists.

Interventor molecules work at secondary sites in systems assumed not to possess spontaneous activity, i.e., in one-state models, similar to non-competitive antagonists. Meanwhile, interventors can be both inhibitory and stimulatory (Table 2.2A).

Drugs increasing the activity beyond constitutive or agonist-induced activities may be referred to as stimulators or activators. This is true in both binding and functional studies. Activators operate reversibly (Table 2.2A).

As demonstrated later, allosteric modulation at aberrant binding sites renders new possibilities in binding and function for the classification of drugs as activators, positive modulators, or inhibitors equal to negative modulators (Table 2.2A and Chapter 14).

Molecules that enhance binding or function in a constitutive manner will be referred to as enhancers. Auxiliary and accessory proteins, such as co-factors, beta-subunits, regulator-of-G protein-signaling (RGS), receptor-activity-modifying-proteins (RAMPs), scaffolding molecules, and chaperones (Sexton et al. 2001; Blumer \& Lanier 2003; Chidiak \& Roy 2003; Morfis et al. 2003; Rebois \& Hébert 2003; Fukoto et al. 2004; Udawela et al. 2004, 2006; Abramow-Newerly et al. 2006; Hill et al. 2008) that also have an activating function will fall under the category of enhancers. Several of these proteins are in fact part of the receptor complex (Hay et al. 2006).

Table $2.2 \mathrm{~A}$ is a preliminary and tentative summary of agonists, ant-agonist, inhibitors, interventors, inverse agonists, modulator molecules, activators, and enhancers. Ant-agonists and interventors are examined this chapter. Inverse agonists and neutral ant-agonists are first treated in Chapter 5, and modulator molecules in Chapters 7, 14 and 15. 
Table 2.2A. Activators, Inhibitors, Ant-agonists, Interventors, Modulators, Enhancers, and Repressors

\begin{tabular}{|c|c|c|c|c|c|}
\hline Type of interaction & Site of binding & Model & Augmentation by & Attenuation by & Auto-effects pos or neg by \\
\hline \multirow{4}{*}{ Reversible } & Primary & All & Agonist & Ant-agonist & - \\
\hline & Primary & All & $\begin{array}{l}\text { Agonist/ } \\
\text { neutral ant-agonist }\end{array}$ & $\begin{array}{l}\text { Neutral ant-agonist/ } \\
\text { inverse agonist }\end{array}$ & - \\
\hline & Secondary & OSM & Pos interventor ${ }^{\star \star}$ & Neg interventor ${ }^{\star \star}$ & Pos/neg auto-interventor \\
\hline & Secondary & TSM & Pos modulator ${ }^{\star \star \star}$ & Neg modulator $r^{\star \star *}$ & Pos/neg auto-modulator ${ }^{* \star * *}$ \\
\hline General & Not specified & - & Activator ${ }^{\star}$ & Inhibitor* & Auto-activator, auto-inhibitor \\
\hline Constitutive & Not specified & - & $\begin{array}{l}\text { Enhancer/ } \\
\text { Derepressor }\end{array}$ & $\begin{array}{r}\text { Desensitizor } \\
\text { Repressor }\end{array}$ & - \\
\hline
\end{tabular}

*The terms 'activator' and 'inhibitor' may also be used as general designators for augmenters and attenuators in all cases, including regulators of G protein-signaling (RGSs) and receptor-activity-modifying-proteins (RAMPs).

${ }_{\star \star *}^{*}$ If neither positive nor negative, we have a 'neutral interventor' equal to a non-competitive ant-agonist. Binding is unchanged.

***When neither positive nor negative, it is a 'neutral modulator', not the same as a 'neutral ant-agonist'.

****The process is designated positive or negative co-operativity.

\subsubsection{Multi-functional Ligands}

The complexity increases when ligands have more than one function. Activators may work synergistically with the possibility of positive co-operativity. Furthermore, ligands may modify the function or binding of other agonists while at the same time they are themselves substrates for enzyme reactions, transported species for mechanisms of pumping, transportees for co-, anti-, or uni-porters, if not ions funneled through a channel. At receptors, ligands may be either modulators or interventors - depending on the assumed reaction scheme and its modeling - while at the same time they are also agonists in their own right. Such drugs now rank as possible 'ago-modulators' (Schwartz \& Holm 2006) or 'allosteric agonists' (May et al. 2007b). Table 2.2B is a tentative proposal for the naming of multi-functional drugs (see Sub-chapter 4.6 for more details).

\subsection{Simple Inhibition}

\subsubsection{Ant-agonism}

In general, ant-agonism is generated by compounds inhibiting the effects of agonists - 'ant-agonists'. In classic literature on the subject, two possible forms of ant-agonism were often imagined, a competitive and a non-competitive ant-agonism.

With conceptions from the classic era, among others, Segel in his book 'Enzyme Kinetics' (1975, 1993, pp.

Table 2.2B. Possible Terms for Multi-functional Drugs

\begin{tabular}{ccc}
\hline $\begin{array}{l}\text { Ligands } \\
\text { Modelers choice }\end{array}$ & $\begin{array}{c}\text { Different } \\
\text { Heterotropic system }\end{array}$ & $\begin{array}{c}\text { Single } \\
\text { Homotropic system }\end{array}$ \\
\hline $\begin{array}{c}\text { One-state model } \\
\text { (-OSM) }\end{array}$ & Ago-interventor & $\begin{array}{c}\text { Ago-auto-interventor }= \\
\text { auto-interventor }\end{array}$ \\
$\begin{array}{c}\text { Two-state model } \\
\text { (-TSM) }\end{array}$ & $\begin{array}{c}\text { Ago-allosteric } \\
\text { modulators* }\end{array}$ & $\begin{array}{c}\text { Ago-co-operative }= \\
\text { co-operative drug }\end{array}$ \\
& allosteric agonists &
\end{tabular}

*See Schwartz and Holst (2006)

\$May et al. (2007b).
100-160) has presented ant-agonism as competitive, non-competitive, and un-competitive and explained them on a spatio-structural ground complete with derived formulations for each type (Fig. 2.2) (reproduced from Segel 1975, 1993, Figs. III-1.1-5, III-12, III-13, III-15, and III-21).

\subsubsection{Simple Ant-agonism Meets Two-state Models}

In the last few decades, ideas about how ligands can prevent agonist from activating receptors, enzymes from catalyzing substrates, or transporters from transporting transportees have been turned upside-down. Over the last fifteen years, theories have changed, especially in pharmacology, due to recognition of the importance of explicitly incorporating expressions for the spontaneous switching between active and non-active states of unliganded receptive units, 'two-state model' (Leff 1995). Thus, competitive ant-agonism is now revitalized and viewed in light of inverse agonists stabilizing the nonactive receptor state or new receptor states (Milligan et al. 1995; Vilardaga et al. 2005; Bond \& Ijzerman 2006; Dowling et al. 2006). A basic description of such doseresponses at equilibrium, or in another word synagics, is best done with a so-called cyclic-two-state-model (cTSM), which I shall deal with in detail in Part II on complex agonism and ant-agonism (Chapter 5).

Here we will derive the equations for simple antagonism, i.e., classic competitive, non-competitive and un-competitive ant-agonism (Sub-chapter 2.4). Furthermore, we shall develop a so-called mixed-inhibition/ mixed activation model into its complete potential in the form of the above-mentioned intervention model (Sub-chapter 2.5).

But first, a definition of what I call a 'full response' (see Section 2.2.3), and, in addition, a presentation of a complicated looking reaction scheme - a four-pane reaction scheme with receptive units in a single state, a so-called four-pane one-state model (FP-OSM) described in Sub-chapter 2.3. 
A

1.

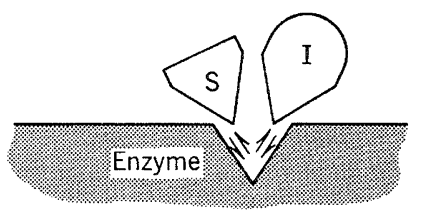

2.

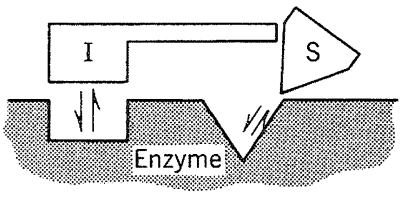

3.

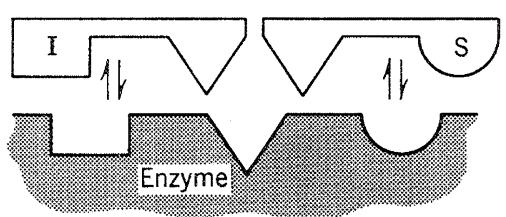

Models of competitive inhibition
4.

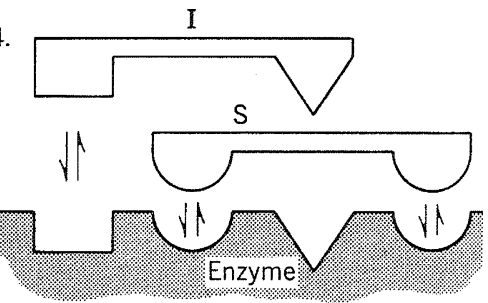

5.
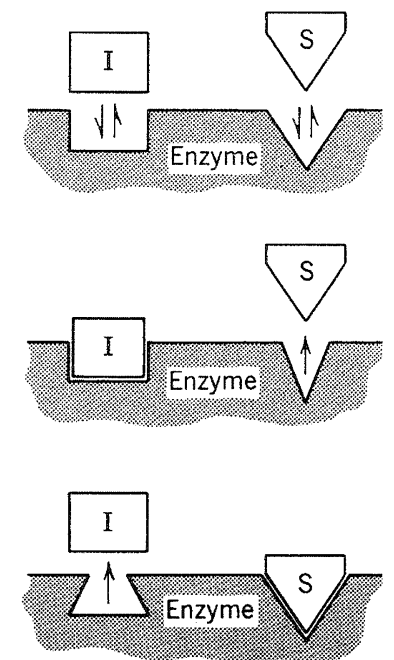

B

A model of non-competitive inhibition

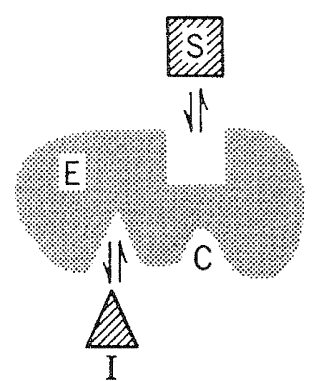

$\|$

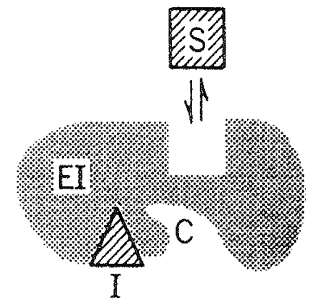

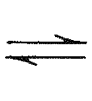

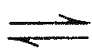

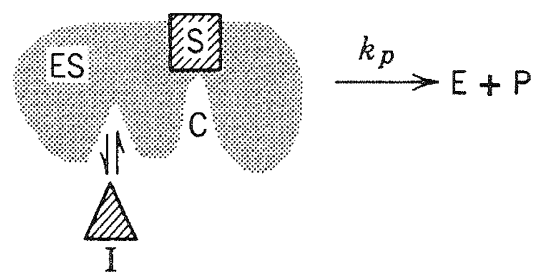

$\sqrt{1}$

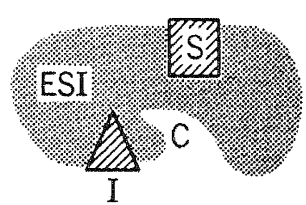

Figure 2.2. Physical models for competitive, non-competitive, and un-competitive inhibition. Panel A: models on competitive ant-agonism. Panels B and C: models for the non-competitive inhibition. Panel D: a model for the un-competitive inhibition (see text). Reproduced from Segel $(1975,1993)$ with permission. 
C

Figure 2.2 (Continued)

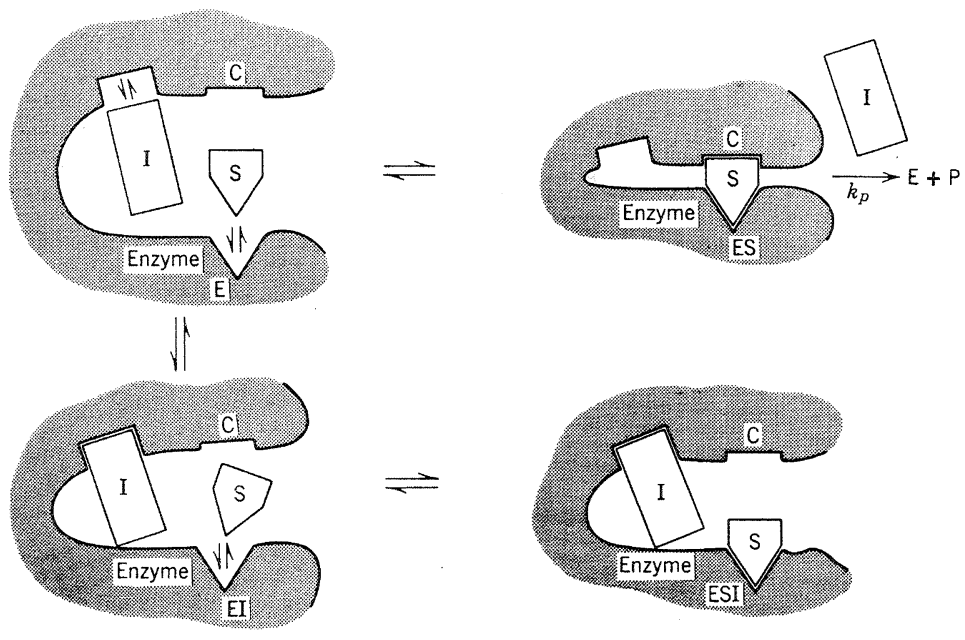

Two alternative models of non-competitive inhibition

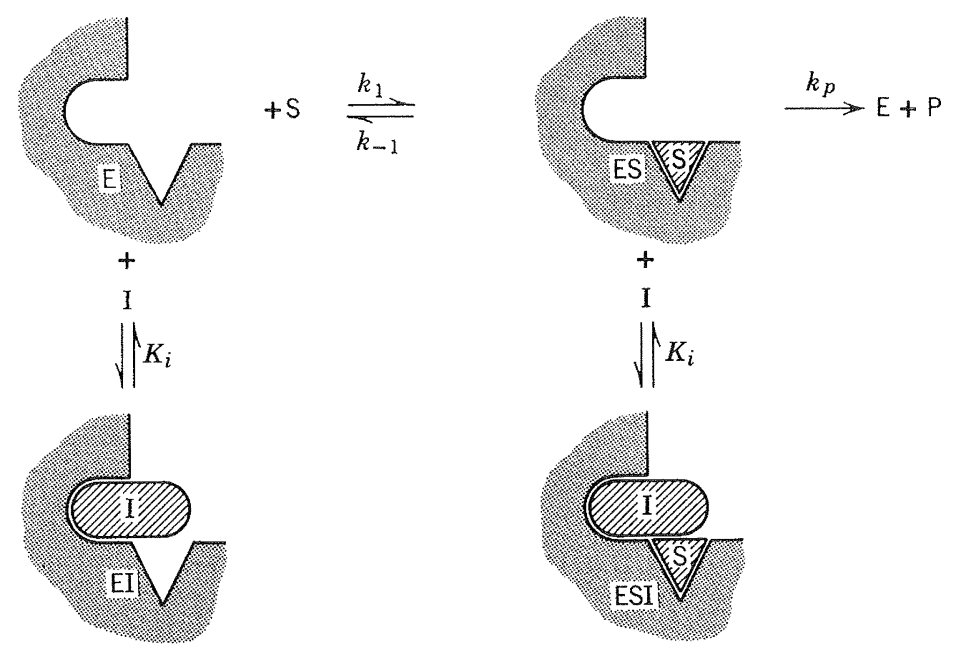

D
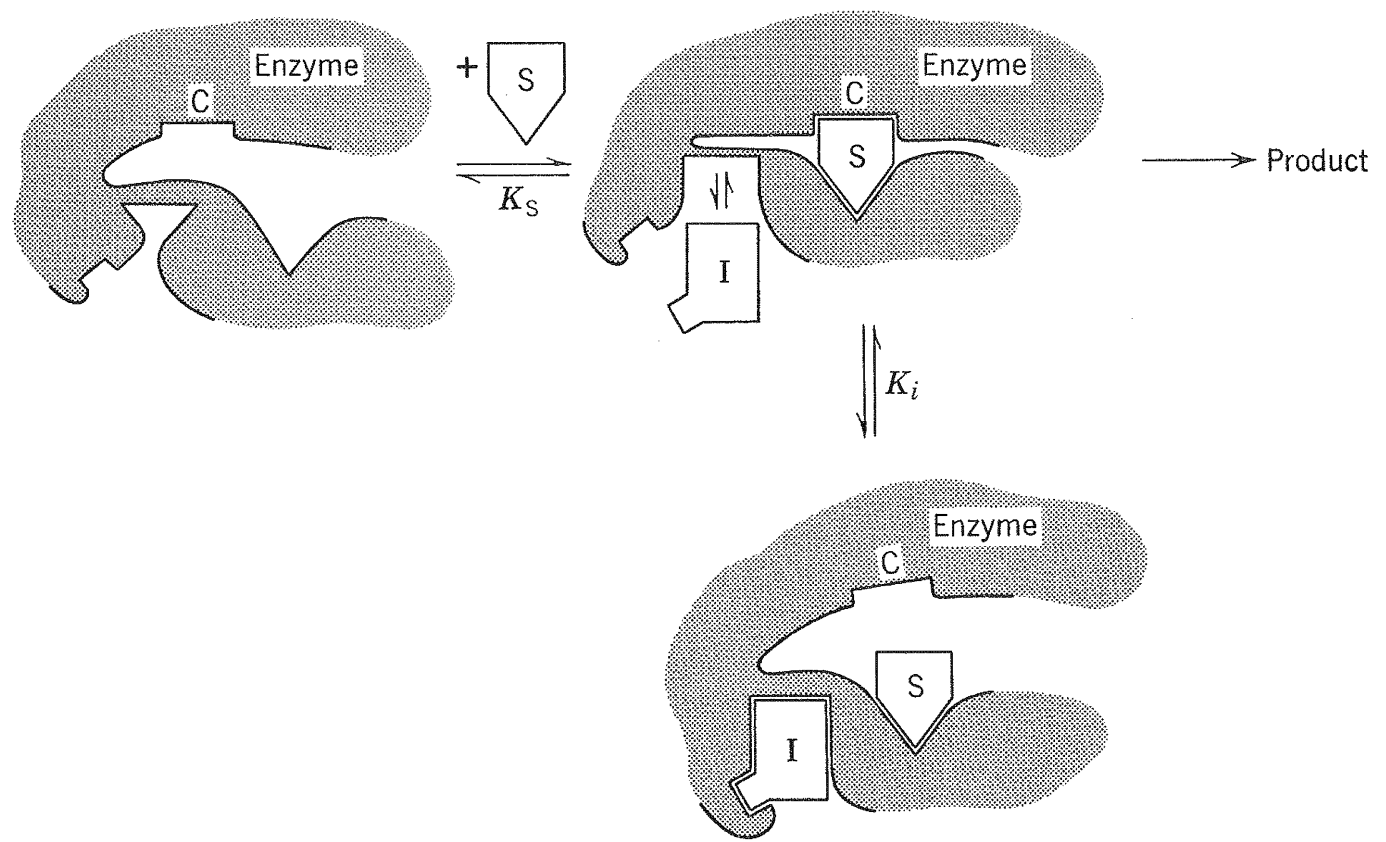

Model of un-competitive inhibition 


\subsubsection{The 'Full Response' Obtained at a Fixed Concentration of an Agonist}

An agonist-induced response at a fixed agonist concentration, $S^{\prime}$, is given by the load equation and represented in an [agonist]-vs-response plot, where the curve intersects with the ' $y$-axis for ant-agonists' before an antagonist $\mathrm{I}$ is introduced, i.e., for $[\mathrm{I}]=0$ (Fig. 2.3). This response by the fixed agonist concentration $\mathrm{S}^{\prime}$, we will call the full response. In a given situation, it is the full response that ant-agonists can work on. The full response is not necessarily the total response or maximal effect that can be obtained with higher concentrations of the agonist. That is, experiments of ant-agonism are carried out at a given agonist concentration, $\mathrm{S}^{\prime}$, which in the absence of ant-agonists yields a full response. The full response is lowered when an ant-agonist is introduced. The full response $(F R)$ may be equated as:

$$
\text { Full response }=F R=T R \cdot \frac{\mathrm{S}^{\prime}}{\mathrm{S}^{\prime}+K_{\mathrm{sS}}},
$$

where $T R$ is the total or maximal response, and $K_{\mathrm{ss}}$ is the equilibrium dissociation constant for ligand $S$. $S^{\prime}$ indi- cates a fixed concentration of S. Subscripts are explained in Box 2.1 (at the end of Chapter 2).

\subsection{The Four Pane Reaction Scheme and Simple Inhibition}

\subsubsection{The Four-pane and One-state Model (FP-OSM)}

To understand the following arguments about simple ant-agonism, it seems advantageous to introduce a reaction scheme as depicted in Fig. 2.4. Initially, this reaction scheme may look daunting with its ninereceptor conformations and eight independent system constants. But careful scrutiny reveals its basic simplicity. For the moment, we will ignore the lower half of the reaction scheme in Fig. 2.4, thus forgetting about all conformation where ligand $\mathrm{S}$ appears on the left side (site) of $\mathrm{R}$ (the receptive unit). This means that agonists are not allowed to bind to a secondary ant-agonistic (intervention) site at the left side of $\mathrm{R}$.
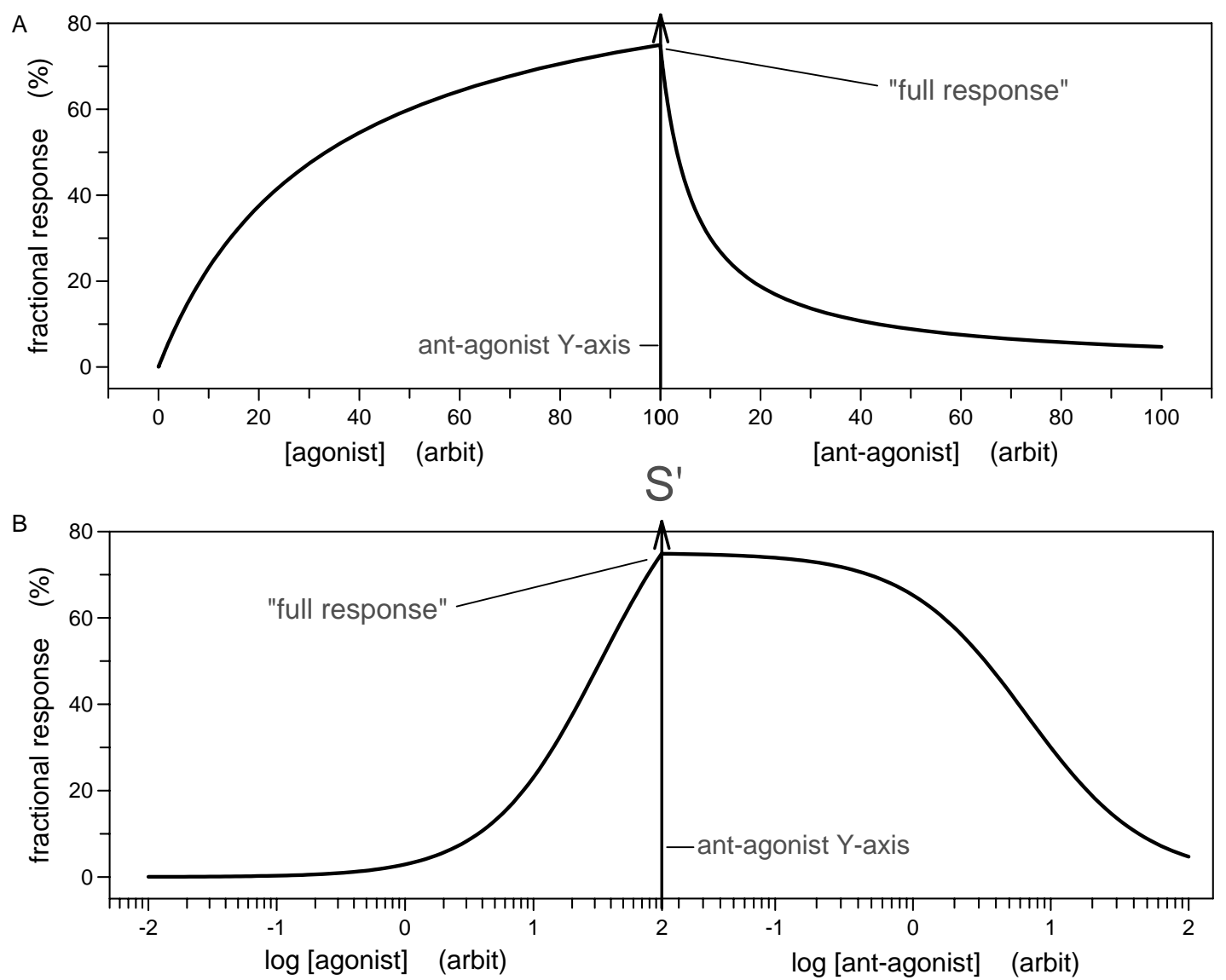

Figure 2.3. Illustration of the concept 'full response'. A fixed agonist concentration $S$ ' brings the system response to a given activity (or occupancy) level indicated at the ' $Y$-axis for ant-agonists' as full-response. At the right of the $Y$-axis for antagonists the concentration of ant-agonists starts to increase from zero and abolishes the full-response at high enough concentrations. Linear concentration scale in (A) and logarithmic concentration scale in (B). 


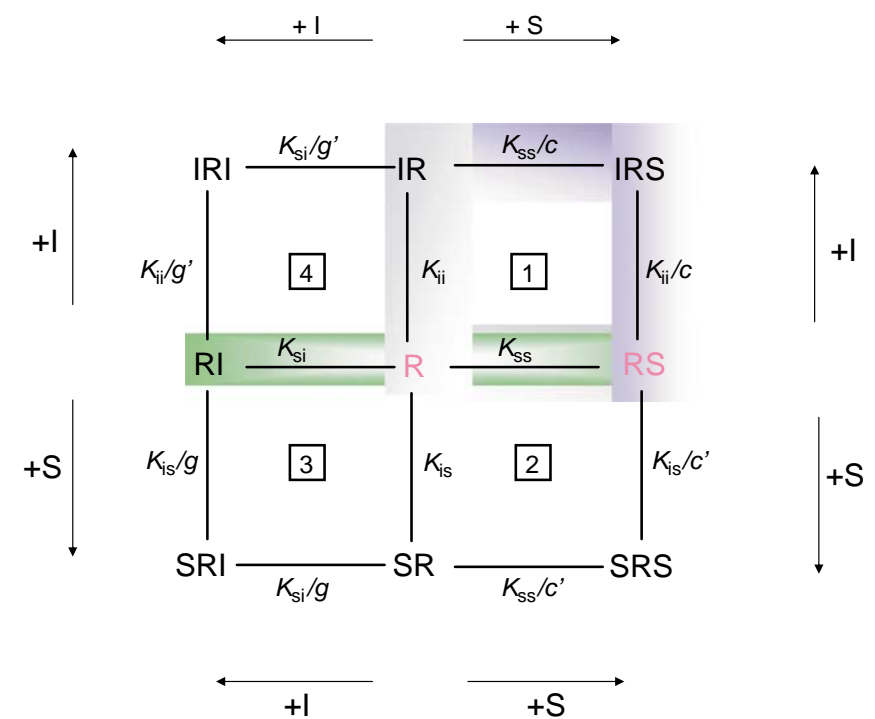

Figure 2.4. The four-pane one-state model (FP-OSM). This model consists of nine independent receptor conformations (not states) and has eight different and independent system constants, either as association constants $A_{\mathrm{xx}}$ or dissociation constants $K_{\mathrm{xx}}$ (see Box 2.1) and interaction constants $c, c^{\prime}, g$, and $g^{\prime}$.

In Fig. 2.4 the reaction scheme for a simple agonism with receptor symbols colored in maroon are easily identifiable:

$$
\mathrm{R}+\mathrm{S} \rightleftarrows \mathrm{RS}
$$

which was introduced in Chapter 1.

Next, identify the following three partial 'ant-agonistic' reaction schemes in the four-pane window scheme (Fig. 2.4):

1. The position at the right of the receptor symbol $\mathrm{R}$ is an agonist binding site, which may bind either the agonist $\mathrm{S}$ or an ant-agonist I. Binding the agonist can activate the receptor or stabilize the receptor in an active state. When an ant-agonist I binds to this site, it prevents activation by preventing the agonist from binding. Possible receptor conformations are R, RS or RI. We can write its reaction scheme and get:

$$
\mathrm{RI} \rightleftarrows \mathrm{I}+\mathrm{R}+\mathrm{S} \rightleftarrows \mathrm{RS},
$$

which is the reaction depicted in the middle, horizontal line of Fig. 2.4 (highlighted in green). Notice that all bound agents are to the right of receptor symbol R. S and I bind in a so-called mutually exclusive manner; they cannot bind simultaneously. Only the RS conformation is functionally active.

2. In Fig. 2.4 it may also be assumed that the receptor has two instead of only one binding site. At the position to the left of the receptor symbol $\mathrm{R}$ there is an additional binding site for ant-agonists/interventors. So far, we assume that the ant-agonist/ interventor site to the left of $\mathrm{R}$ cannot bind an agonist. Further, here we will assume that the antagonist/interventor I cannot bind at the agonist binding site. Binding at the agonist binding site is only for agonists and binding at the ant-agonist/interventor binding site is only for ant-agonists/interventors. This generates three possible receptor conformations R, IR, and RS, and for this we can also identify and extract a reaction scheme. The reaction scheme can be identified as the part highlighted in gray in the fourpane reaction scheme in Fig. 2.4, which is written as:

$$
\mathrm{IR} \rightleftarrows \mathrm{I}+\mathrm{R}+\mathrm{S} \rightleftarrows \mathrm{RS} .
$$

Again, the complex RS alone renders functional activity while the binding of agonists and ant-agonist/interventor are mutually exclusive.

3. Still assuming that agonists cannot bind to the ant-agonist/interventor site and ant-agonists/ interventors cannot bind to the agonist site, there is still another possibility, namely that when an agonist is bound at the agonist site, an ant-agonist/ interventor can simultaneously bind at the antagonist/interventor site. Binding of agonists and interventors is not mutually exclusive. This yields four possible receptor conformations, R, RS, IR, and IRS with a reaction scheme as shown in the upper right quadrant of Fig. 2.4 (quadrant labeled 1 and tinted in gray, green, and blue). This quadrant is equal to the reaction scheme in Fig. 2.1A; and again, RS is the only functionally active conformation.

With these three partial reaction schemes as possibilities, we are ready for the simple inhibition with competitive, non-competitive, and un-competitive antagonists.

\subsection{Competitive and Non-competitive Ant-agonism}

This Sub-chapter is relevant for functional studies to be analyzed by competitive and non-competitive theories. In an attempt to keep matters simple and separate, some of the parallel theories for binding studies are postponed to Sub-chapter 2.5, where the intervention model is also described.

As indicated in Sections 2.3.1.2 and 2.3.1.3, when a ligand binds to the secondary site it should more appropriately be termed an interventor instead of an ant-agonist. But, in the following I will stay with the term 
'ant-agonist' and follow the classical terminology for development of the competitive, non-competitive, and un-competitive ant-agonism. The term 'agonist' may be replaced with 'substrate' or 'transportee'.

\subsubsection{Competitive Ant-agonism Type I and Its Dissociation Constant}

In classic competitive ant-agonism, only the RS conformation is active, and both agonists and ant-agonists can bind to the agonist binding site of $\mathrm{R}$, that is to the right of symbol R. There is no secondary binding site. This is equal to reaction scheme $\mathrm{RI} \rightleftarrows \mathrm{I}+\mathrm{R}+\mathrm{S} \rightleftarrows \mathrm{RS}$ in Section 2.3.1.1, and is also depicted in Fig. 2.4 (see Segel 1975, 1993, Fig. III-1). Binding is mutually exclusive. There is no simultaneous binding of agonist and ant-agonist. The pertinent equilibrium dissociation constants are indicated with subscripts as follows: $K_{\mathrm{ss}}$ for the agonist $\mathrm{S}$ interaction and $K_{\mathrm{si}}$ for the ant-agonist I interaction at the agonist binding site. Note that with this choice of signification in the subscripts, the first position in the subscript signifies the binding site on the receptive unit, here s, while the second position in the subscript indicates the ligand bound to the site; $\mathrm{s}$ for the agonist and $\mathrm{i}$ for the ant-agonist. Box 2.1 specifies how these symbols are used and the conventions contained therein are used throughout the text.

\subsubsection{Competitive Ant-agonism Type II and Its Dissociation Constant}

As illustrated in Section 2.3.1.2, in case there are two separate binding sites on the receptive unit, one for agonists and another for ant-agonists, with the conditions that only agonists can bind at the agonist binding site and only ant-agonists can bind at the ant-agonistic binding site, then the reaction scheme $I R \rightleftarrows I+R+S \rightleftarrows R S$ is still without the possibility of simultaneous binding. Binding is mutually exclusive, and as indicated, only the RS conformation is functional. This reaction scheme is designated type II competitive ant-agonism. The scheme may be found in Figs. 2.1A and 2.4 (Segel 1975, 1993; Figs. III-1.2-5).

In the type II reaction scheme for competitive antagonism, binding of ant-agonists at the ant-agonistic binding site has its own dissociation constant $K_{\mathrm{ii}}$, which is different from $K_{\mathrm{si}}$ of the type I competitive antagonism. However, the type II competitive ant-agonism will be difficult to discern from the competitive type I ant-agonism described in Section 2.4.1.

Mutually exclusive binding is the virtue of type I and II competitive ant-agonism, and since both take place in a one-state model, their acronym is ME-OSM.

\subsubsection{Non-competitive Ant-agonism and Its Dissociation Constants}

In classic non-competitive ant-agonism there is also a secondary site on the receptive unit $R$, indicated by positioning ligand symbols to the left of $\mathrm{R}$, where only ant-agonists can bind. This renders an IR complex. At the agonist site only agonists can bind resulting in an RS complex. The difference between non-competitive antagonism and the just described competitive ant-agonism type II in Section 2.4.2 is the ability of the receptive unit in non-competitive ant-agonism to adapt binding of both agonists and ant-agonist at the same time. Binding is not mutually exclusive, binding is mutually inclusive (Levitski 1978). Meanwhile, activation of the receptive unit is prevented when an ant-agonist is bound, no matter what, thus only the RS conformation is active. Non-competitive ant-agonism also takes place in a onestate model and due to its mutual inclusiveness its acronym is MI-OSM.

This is the situation depicted and highlighted in Figs. 2.1A and 2.4-1 (Segel 1975, 1993; Figs. III-12, III-13, III15), with four receptor conformations, R, RS, IR, and IRS. The pertinent dissociation constants are indicated with subscripts as follows: $K_{\mathrm{ss}}$ for the agonist interaction with the agonist binding site and $K_{\mathrm{ii}}$ for the ant-agonist interaction with the ant-agonist binding site, in accordance with the above-mentioned conventions (Box 2.1). Compared with dissociation constants when no other ligand is bound, the equilibrium dissociation constant for binding of a ligand may be different when other ligands are already bound to the receptive unit. For the reaction scheme in Fig. 2.4-1, this condition is indicated by a factor $c$. The $c$ factor will be examined in more detail in Sections 2.5.1-3, but for now we will assume that $c$ is unity. Let an already bound ligand be indicated with a superscript, where the first position of the superscript indicates to which site the already-bound ligand is bound, and a second position in the superscript indicates which type of ligand is ligated, for instance agonists $=\mathrm{s}$ and ant-agonists $=\mathrm{i}$. Thus $K_{\mathrm{ii}}^{\mathrm{ss}}$ is the dissociation constant for an ant-agonistic ligand to bind at the ant-agonist binding site when an agonist is already bound to its agonist binding site (Box 2.1). When $c=1$, we have that $K_{\mathrm{ii}}=K_{\mathrm{ii}}^{\mathrm{ss}}$ and $K_{\mathrm{ss}}=K_{\mathrm{ss}}^{\mathrm{ii}}$. An explanation for $c \neq 1$ will be detailed in Sub-chapter 2.5 describing the intervention scheme.

\subsubsection{From Reaction Scheme to Formulation}

As soon as a specific reaction scheme has been defined, it is possible to derive its mathematical formulation. To formulate our reaction schemes, we need the system constants and the receptor conformations (see Figs. 2.1A, 2.4-1, and Box 2.1). 
The parameters are:

$\begin{array}{ll}R_{\max } \text { or } B_{\max } & \begin{array}{l}\text { maximal effect or maximal binding } \\ \text { equilibrium dissociation constant at } \\ K_{\mathrm{ss}}\end{array} \\ K_{\mathrm{si}} & \begin{array}{l}\text { the primary site for agonist } \mathrm{S} \\ \text { equilibrium dissociation constant at } \\ \text { the primary site for ant-agonist I }\end{array}\end{array}$

or $\quad$ equilibrium dissociation constant at the secondary site for ant-agonist I $c \quad$ intervention constant-first discussed in detail in Sub-chapter 2.5,

and the conformations of the receptive unit are:

$\mathrm{R}$

$\begin{array}{ll}\text { RS } & \mathrm{R} \cdot \mathrm{S} / K_{\mathrm{ss}} \\ \text { RI } & \mathrm{R} \cdot \mathrm{I} / K_{\mathrm{si}} \\ \text { or } & \mathrm{R} \cdot \mathrm{I} / K_{\mathrm{ii}}\end{array}$

and

IRS

$$
\mathrm{R} \cdot\left(\mathrm{S} / K_{\mathrm{ss}}\right) \cdot \mathrm{I} / K_{\mathrm{ii}}
$$

For the following formulations, in order to simply the equations, the letters $\mathrm{S}$ and I may also stand for the normalized ligand concentrations, $\mathrm{S} / K_{\mathrm{ss}}$ and $\mathrm{I} / K_{\mathrm{si}}$, as for instance in Eq. 2.2 $a$.

For reasons that will become obvious later, here, I first transcribe the reaction scheme for non-competitive antagonism into an equation before a transcription of the reaction schemes for competitive ant-agonism into its formulations.

\subsubsection{Formulation of Functional Non-competitive Ant-agonism}

A situation can be described as a non-competitive type of ant-agonism when a ligand solely binds to a secondary binding site and results in non-active receptive units even if an agonist is bound or not bound to its binding site on the receptive unit - the primary site (Section 2.4.3). For this, four receptor conformations are involved (see Figs. 2.1A or 2.4-1). To start, we will assume that the dissociation constants are identical for reactions $\mathrm{R}+\mathrm{S} \rightleftarrows \mathrm{RS}$ and $\mathrm{IR}+\mathrm{S} \rightleftarrows \mathrm{IRS}$, i.e., $c=1$, which as mentioned in Section 2.4.3 simplifies the analysis. Let us illustrate the issue by formulating a distribution equation involving all four receptor conformations of non-competitive ant-agonism and how they relate. With $T R$ as the total response and ar as the actual response, we have:

$$
\frac{a r}{T R}=\frac{\mathrm{RS}}{\mathrm{R}+\mathrm{RS}+\mathrm{IR}+\mathrm{IRS}}=\frac{\mathrm{S}}{1+\mathrm{S}+\mathrm{I}+\mathrm{I} \cdot \mathrm{S}},
$$

in which the $\mathrm{S}$ and I stand for the normalized ligand concentration. Eq. 2.2 $a$ can be rewritten to:

$$
\frac{a r}{T R}=\frac{\mathrm{S}}{(1+\mathrm{S}) \cdot(1+\mathrm{I})}=\frac{\mathrm{S}}{(1+\mathrm{S})} \cdot \frac{1}{(1+\mathrm{I})},
$$

or in explicitly normalized versions of Eqs. 2.2 $a$ and 2.2b, they become:

$$
\begin{aligned}
\frac{a r}{T R} & =\frac{\frac{\mathrm{S}}{K_{\mathrm{ss}}}}{1+\frac{\mathrm{S}}{K_{\mathrm{ss}}}+\frac{\mathrm{I}}{K_{\mathrm{ii}}}+\frac{\mathrm{S}}{K_{\mathrm{ss}}} \cdot \frac{\mathrm{I}}{K_{\mathrm{ii}}}} \\
& =\frac{\frac{\mathrm{S}}{K_{\mathrm{ss}}}}{\left(1+\frac{\mathrm{S}}{K_{\mathrm{ss}}}\right)} \cdot \frac{1}{\left(1+\frac{\mathrm{I}}{K_{\mathrm{ii}}}\right)} .
\end{aligned}
$$

We may rewrite Eq. $2.2 b$ as:

$$
a r=T R \cdot \frac{\mathrm{S}}{1+\mathrm{S}} \cdot \frac{1}{1+\mathrm{I}} .
$$

When there is no antagonist $[\mathrm{I}]=0$, from Eq. 2.4a we have $a r=T R \cdot \mathrm{S} /(1+\mathrm{S})$ or:

$$
a r_{\mathrm{I}=0}=T R \cdot \frac{\mathrm{S}}{1+\mathrm{S}}
$$

This again is a load expression. It yields responses obtained at a varying concentration of agonist $\mathrm{S}$.

When the agonist concentration is fixed at $\mathrm{S}^{\prime}$, the agonist-induced response is the intersection at the 'antagonist' $y$-axis in Fig. 2.3. In addition, when the agonist concentration is fixed at $\mathrm{S}^{\prime}$, Eq. $2.4 b$ can be written as a new version equal to Eq. 2.1, i.e.,

$$
a r_{\mathrm{I}=0}^{\prime}=T R \cdot \frac{\mathrm{S}^{\prime}}{1+\mathrm{S}^{\prime}} \vee F R=T R \cdot \frac{\mathrm{S}^{\prime}}{1+\mathrm{S}^{\prime}}
$$

Thus, the actual response $a r^{\prime}$ for $\mathrm{I}=0$ is now equal to the full response, i.e., $a r_{\mathrm{I}=0}^{\prime}=F R$.

In a given situation, this full response is the response that ant-agonists can work on. ${ }^{3}$

So, the analysis is now carried out at a given agonist concentration $\mathrm{S}^{\prime}$, while varying the ant-agonist concentration. For any ant-agonist concentration, the obtained responses can be referred to as 'actual remaining activity' (ara). A formula for the ara, as the ant-agonist concentration varies, is obtained by combining Eqs. $2.4 a$ and $2.4 c$ :

\footnotetext{
3 'Full response', given in Eq. 2.1, is not necessarily the maximal response that can be obtained with higher concentrations of the agonist (Section 2.2.3).
} 


$$
\operatorname{ara}=a r^{\prime} \cdot\left(\frac{1}{1+\mathrm{I}}\right)
$$

As explicitly demonstrated by Eq. 2.4d, not surprisingly, ara only varies with the term $1 /(1+\mathrm{I})$ when $\mathrm{S}$ is fixed, as depicted in Fig. 2.3. The level of fixed agonist concentration $\mathrm{S}^{\prime}$ determines from what level on the $y$ axis the response is reduced by ant-agonists, but note, without affecting the determination of $K_{\mathrm{ii}}$ for the antagonist on the $x$-axis. This conclusion works for the theory of functional studies with simple non-competitive ant-agonism.

For the moment, observe without insight that in case the above-mentioned $c$ coefficient is not equal to unity, as assumed, then the measured $K_{\mathrm{ii}}$ will be a function of $\mathrm{S}$, as demonstrated later for the intervention scheme in Sub-chapter 2.5.

\subsubsection{Formulation of Binding in Non-competitive Ant-agonism}

In the theory of non-competitive ant-agonism for binding experiment, it may be observed that it has a different formulation from the functional ant-agonism, since both conformations RS and IRS will be involved (Figs. 2.1A and 2.4-1). Both type I and II competitive ant-agonism are not hampered by this discrepancy between binding and functional studies in their formulations due to mutual exclusiveness in binding between agonist and ant-agonist (see Sections 2.4.9 and 2.4.10 and Subchapter 2.5).

For binding studies assuming a non-competitive reaction scheme we may derive the following equation:

$$
\frac{a r}{T R}=\frac{\mathrm{RS}+\mathrm{IRS}}{\mathrm{R}+\mathrm{RS}+\mathrm{IR}+\mathrm{IRS}}=\frac{\mathrm{S}}{\mathrm{S}+1} .
$$

This means, in contrast to functional studies, that binding is completely independent of possible noncompetitive ant-agonists (cf. Table 2.3).

\subsubsection{Actual Remaining Activity (ara) in Non-competitive Inhibition}

In functional studies, non-competitive activity drops as the ant-agonist concentration increases. The ara, which decreases as the ant-agonist concentration increases, was obtained in Eq. 2.4d by combining Eqs. 2.4 $a$ and $2.4 c$. Inserting $F R$ for $a r^{\prime}$ in Eq. 2.4d we may write:

$$
\operatorname{ara}=F R \cdot\left(\frac{1}{1+\mathrm{I}}\right)
$$

and in an explicitly normalized form:

$$
\operatorname{ara}=F R \cdot\left(\frac{1}{1+\frac{\mathrm{I}}{K_{\mathrm{ii}}}}\right)
$$

In a normalized and linear-linear co-ordinate system, the dose-response relation of the ara is viewed as a horizontally-flipped law of adsorption-desorption (load). That is, it is reciprocal to a dose-response following the load (Fig. 2.5A). In a normalized semilog co-ordinate system, a plot of simple dose-responses of actual remaining activity has the form as shown in Fig. 2.5B.

\subsubsection{The Actual Inhibited Response (air) in Non-competitive Inhibition}

Converse to the ara, the actual size of inhibited response (air) rises with increasing ant-agonist concentration. Given the scenario in Section 2.4.5 for functional studies, where the activity is induced by a fixed concentration of an agonist, air is the size of the eliminated response. In functional studies, air rises as a function of the actual ant-agonist concentration and follows a simple load, still with $F R$ as a 'maximal' obtainable response, now in the presence of an ant-agonist. We may equate the air as:

$$
\begin{aligned}
\text { air } & =F R-a r a=F R-F R \cdot\left(\frac{1}{1+\mathrm{I}}\right) \\
& =F R \cdot\left(\frac{\mathrm{I}}{1+\mathrm{I}}\right),
\end{aligned}
$$

and in an explicitly normalized form:

$$
\operatorname{air}=F R \cdot\left(\frac{\frac{\mathrm{I}}{K_{\mathrm{ii}}}}{1+\frac{\mathrm{I}}{K_{\mathrm{ii}}}}\right) \text {. }
$$

This equation is plotted in Fig. 2.5C and is a normal oriented Langmuirian dose-response relationship of the load type. The inhibitory response is rising with increasing concentration of the ant-agonist, and in a normalized semi-log co-ordinate system this simple ant-agonism has the form as shown in Fig. 2.5D.

We can summarize Sections 2.4.7 and 2.4.8 as follows:

$$
\operatorname{air}=F R \cdot\left(\mathrm{I} /\left(\mathrm{I}+K_{\mathrm{ii}}\right)\right),
$$

and

$\operatorname{ara}=F R-F R \cdot\left(\mathrm{I} /\left(\mathrm{I}+K_{\mathrm{ii}}\right)\right)=F R \cdot\left(K_{\mathrm{ii}} /\left(\mathrm{I}+K_{\mathrm{ii}}\right)\right), \quad(2.10 a)$

which are the equations for actual ant-agonistic or inhibitory response (air) and actual remaining activity ( $a r a)$, both as a function of the increasing ant-agonist 

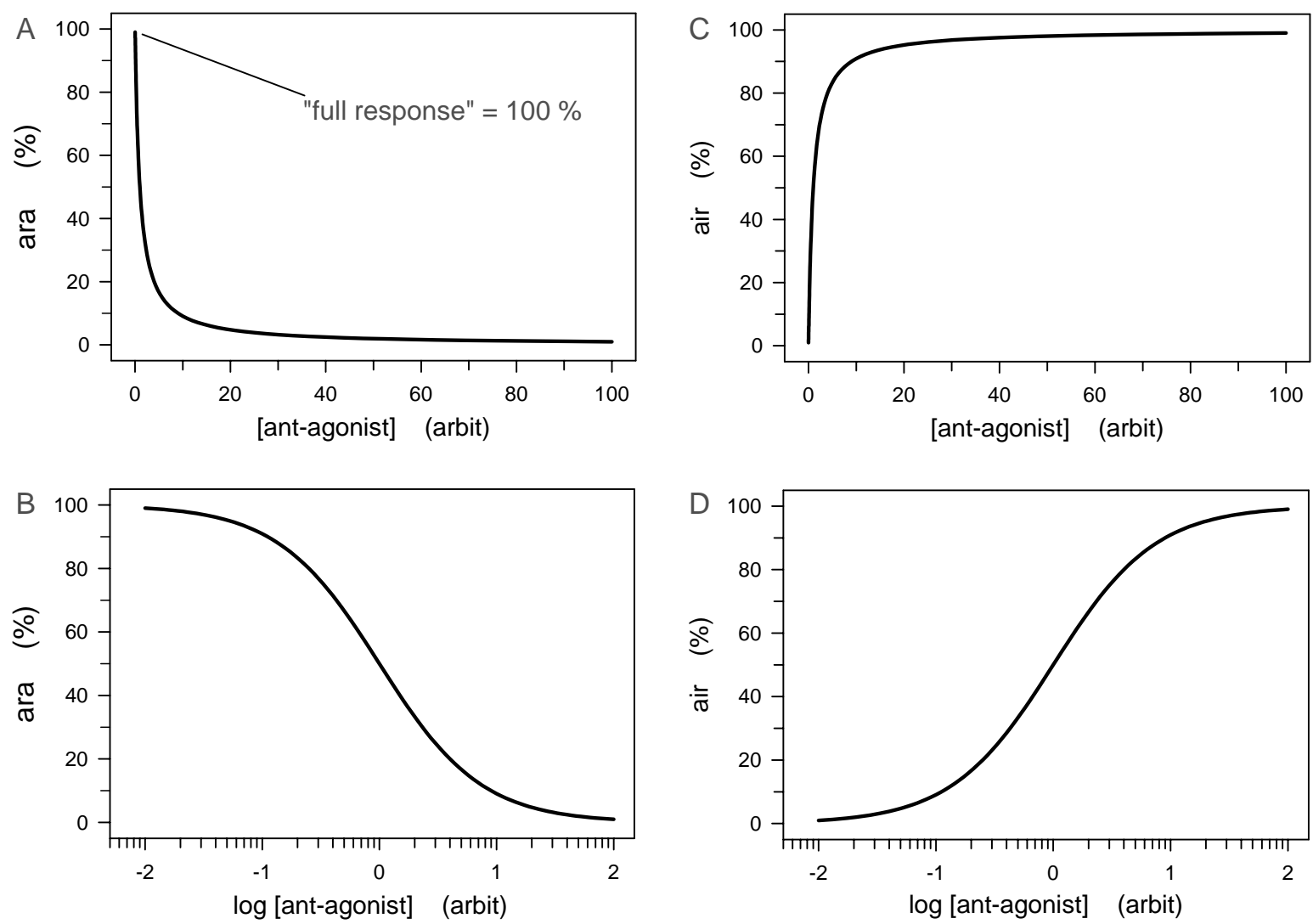

Figure 2.5. Non-competitive inhibition $(c=1)$ in a functional scheme. Panel $A$ is a linear-linear and Panel $B$ is a semi-log plot for the actual remaining activity (ara) in non-competitive ant-agonism as the concentration of the ant-agonist increases (Eq. 2.6). Panel $C$ is a linear-linear and panel $D$ is a semi-log plot of the actual inhibitory response (air) in non-competitive ant-agonism as the concentration of the ant-agonist increases (Eq. 2.8).

concentrations [I] in the functionally non-competitive reaction scheme.

The comparable formulations of theory for noncompetitive ant-agonism in binding studies, started in Section 2.4.6, are developed later in Section 2.5.1 and summarized in Table 2.3.

\subsubsection{Formulation of Competitive Ant-agonism Type I}

Now we turn to competitive ant-agonism. Although merely three receptor conformations are involved in the scheme for competitive ant-agonism (see reaction scheme in Section 2.3.1 and mid-horizontal line in Fig. 2.4), it is slightly more complicated than the non-competitive ant-agonism with four involved conformations. With a fixed agonist concentration $\mathrm{S}^{\prime}$, the antagonist competes with the agonist at the activating site in a mutually exclusive manner.

Below is the formulation for a fractional response of simple competitive ant-agonism as the ant-agonist concentration varies, while the agonist concentration is fixed at $\mathrm{S}^{\prime}$, and with the dissociation constants tacitly assumed:

$$
\frac{a r^{\prime}}{T R}=\frac{\mathrm{R} \cdot \mathrm{S}^{\prime}}{\mathrm{R}+\mathrm{R} \cdot \mathrm{S}^{\prime}+\mathrm{R} \cdot \mathrm{I}}=\frac{\mathrm{S}^{\prime}}{1+\mathrm{S}^{\prime}+\mathrm{I}},
$$

or in an explicitly normalized fashion by including dissociation constants:

$$
\frac{a r^{\prime}}{T R}=\frac{\frac{\mathrm{S}^{\prime}}{K_{\mathrm{ss}}}}{1+\frac{\mathrm{S}^{\prime}}{K_{\mathrm{ss}}}+\frac{\mathrm{I}}{K_{\mathrm{si}}}}=\frac{\mathrm{S}^{\prime}}{\mathrm{S}^{\prime}+K_{\mathrm{ss}} \cdot\left(1+\frac{\mathrm{I}}{K_{\mathrm{si}}}\right)} .
$$

We can identify that when $\mathrm{I}=0$, we have the full response $F R=a r^{\prime}=T R \cdot\left(\mathrm{S}^{\prime} /\left(\mathrm{S}^{\prime}+K_{\mathrm{ss}}\right)\right)$. With a fixed agonist concentration $[\mathrm{S}]^{\prime}$, it is only the so-called apparent dissociation appK $\mathrm{ss}_{\mathrm{ss}}$, equal to $K_{\mathrm{ss}} \cdot\left(1+\mathrm{I} / K_{\mathrm{si}}\right)$ in the denominator of Eq. 2.12 that varies as the [I] concentration varies, not the $F R$.

However, when $[\mathrm{S}]$ is not fixed, the right-hand term in Eq. 2.12 has the form of a load dose-response expression with an apparent dissociation constant for the ant-agonist, $\operatorname{appK}_{\mathrm{si}}=\mathrm{IC}_{50}$, which varies with the concentration of both the agonist and the ant-agonist; thus $\mathrm{IC}_{50}=K_{\mathrm{ss}} \cdot\left(1+\mathrm{I} / K_{\mathrm{si}}\right)$ is a function of both $\mathrm{I}$ and $\mathrm{S}$. The effect of $\mathrm{S}$ on the $\mathrm{IC}_{50}$ is most easily seen from a reformulation of Eq. 2.12: 
Table 2.3. Summary of the Relative Maximal Responses $\left(R_{\max }\right)$ and Maximal Occupancies $\left(B_{\max }\right)$ and the Apparent Dissociation Constant for Agonist S, appK $\mathrm{ss}_{\mathrm{s}}$, in the Simplest Mutually Exclusive and Inclusive Reaction Schemes

\begin{tabular}{|c|c|c|c|}
\hline $\begin{array}{l}\text { Summary of Sections } \\
2.4 .5-6 ; 2.4 .9 ; 2.4 .10 \\
\text { and } 2.5 .1\end{array}$ & $\begin{array}{l}\text { Competitive I+II ant-agonism } \\
c=0, \text { mutually exclusive } \\
\text { (Figs. 2.8A-2.9A) }\end{array}$ & $\begin{array}{l}\text { Non-competitive ant-agonism } \\
c=1, \text { mutually inclusive } \\
\text { (Figs. 2.8C-2.9C) }\end{array}$ & $\begin{array}{l}\text { Intervention model, intervention } \\
\quad>0 \text {, mutually inclusive } \\
\text { (Figs. 2.8-2.9) }\end{array}$ \\
\hline \multirow[t]{2}{*}{ Study type } & \multicolumn{3}{|c|}{ Parameters } \\
\hline & \multicolumn{2}{|c|}{ Fractional response $B_{\max } / R_{\max }$ for high [S] } & \\
\hline \multirow[t]{2}{*}{$\begin{array}{l}\text { Occupancy } \\
\text { Effect }\end{array}$} & $\begin{array}{l}1 \text { surmountable } \\
1 \text { surmountable }\end{array}$ & $\begin{array}{l}\text { 1, independent of [I] } \\
<1 ; 1 /\left(1+\mathrm{I} / K_{\mathrm{ii}}\right)\end{array}$ & $\begin{array}{l}1 \text { surmountable } \\
<1 ; 1 /\left(1+\left(1 / K_{\mathrm{ii}}\right) \cdot c\right)\end{array}$ \\
\hline & \multicolumn{3}{|c|}{ Apparent agonist dissociation constant appK $\mathrm{ss}_{\mathrm{ss}}=\mathrm{EC}_{50}$} \\
\hline Occupancy ${ }^{\S}$ & $K_{\mathrm{ss}} \cdot\left(1+\mathrm{l} / K_{\mathrm{l}}\right)^{\bullet}$ & $K_{\mathrm{ss}}$ & $\frac{K_{\mathrm{ss}} \cdot\left(1+\mathrm{I} / K_{\mathrm{ii}}\right)^{\#}}{\left(1+\left(\mathrm{I} / K_{\mathrm{ii}}\right) \cdot c\right)}$ \\
\hline Effect & $K_{\mathrm{ss}} \cdot\left(1+\mathrm{I} / K_{\mathrm{I}}\right)^{\bullet}$ & $K_{\mathrm{ss}}$ & $\frac{K_{\mathrm{ss}} \cdot\left(1+\mathrm{I} / K_{\mathrm{iij}}\right)^{\#}}{\left(1+\left(\mathrm{I} / K_{\mathrm{ii}}\right) \cdot c\right)}$ \\
\hline
\end{tabular}

Mutually inclusive inhibition =non-competitive ant-agonism, and mutually exclusive inhibition =competitive ant-agonism.

$K_{\mathrm{I}}=K_{\mathrm{si}}$ or $K_{\mathrm{ii}}$.

${ }^{\#}$ For $c>1$ appK $K_{\mathrm{ss}}<K_{\mathrm{ss}}$; for $c<1 \mathrm{appK}_{\mathrm{ss}}>K_{\mathrm{ss}}$. Compare these two equations with similar equations for 'allosteric' ligand binding and effect in Ehlert (1988). The co-lateral intervention constant $c$ here is equal to the inverse of constant $\alpha$ in Ehlert's paper.

Special: for competitive ant-agonism type I with $c>>1$ and $1 / c \approx \mathrm{I} / K_{\mathrm{ii}}$ all concentration-occupancy relationships lie on top of each other.

$$
\frac{a r}{T R}=\frac{1}{1+\frac{K_{\mathrm{ss}}}{\mathrm{S}} \cdot\left(1+\frac{\mathrm{I}}{K_{\mathrm{si}}}\right)} .
$$

Here $\left(K_{\mathrm{ss}} / \mathrm{S}\right) \cdot\left(1+\mathrm{I} / K_{\mathrm{si}}\right)$ in the denominator of Eq. 2.13 is a type of relative apparent dissociation constant dependent on the level of both $[\mathrm{S}]$ and $[\mathrm{I}]$.

Recap that we have assumed there is a steric hindrance for the agonist to bind at the agonist site and activate the receptive unit when an ant-agonist is bound to the agonist site of $\mathrm{R}$. This was for function.

In occupancy, the same equations as Eqs. 2.11 and 2.12 may be derived for comparable theories for binding studies. Therefore, conclusions about system constants for binding assays in competitive inhibition are the same as above (cf. Table 2.3).

\subsubsection{Formulation of Competitive Ant-agonism Type II}

As specified in Section 2.4.2, there is another reaction scheme that leads to competitive ant-agonism (Fig. 2.4-1, grey marking). We assume that there is a steric hindrance for the agonist to bind at the agonist site and activate the receptive unit when an ant-agonist is bound to an ant-agonist site on R. Development of the formulation for this type of reaction scheme is straightforward. It should result in:

$$
\frac{a r^{\prime}}{T R}=\frac{\mathrm{R} \cdot \mathrm{S}^{\prime}}{\mathrm{R}+\mathrm{R} \cdot \mathrm{S}^{\prime}+\mathrm{R} \cdot \mathrm{I}}=\frac{\mathrm{S}^{\prime}}{1+\mathrm{S}^{\prime}+\mathrm{I}},
$$

or in explicitly normalized form:

$$
\frac{a r^{\prime}}{T R}=\frac{\mathrm{S}^{\prime}}{\mathrm{S}^{\prime}+K_{\mathrm{ss}} \cdot\left(1+\frac{\mathrm{I}}{K_{\mathrm{ii}}}\right)} .
$$

There is an overlap between the above type I competitive ant-agonism in Section 2.4.9, and the formulations for the present regime of type II competitive ant-agonism in functional and binding studies. Compare Eq. 2.11 with Eq. 2.14 and Eq. 2.12 with Eq. 2.15; only $K_{\mathrm{ii}}$ is replaced with $K_{\mathrm{si}}$. This is contrary to the non-competitive ant-agonism where formulation from functional studies deviates from that of binding studies.

In occupancy, as mentioned, in either case where these two types of competitive ant-agonism operate, one with a single binding site and the other with two binding sites, the same equations as Eqs. 2.14 and 2.15 may be derived for comparable theories on competitive binding studies. Therefore, the conclusions above on system constants for functional studies are the same as for binding studies when competitive (cf. Table 2.3).

Due to complete thermodynamic reversibility in the competitive inhibition type II reaction scheme (Fig. 2.41 , in grey), the binding of an inhibitor to the secondary binding site can be surmounted by binding enough agonists to the primary binding site. Accordingly, also for occupancy studies, the two types of competitive antagonism will be hard to discern experimentally (for 'surmount-ability', see Section 2.8.2).

Competitive ant-agonism may also be developed for a system equal to the 'cross' in Fig. 2.4. In this type of 
reactions scheme, which is competitive with five receptor conformations, R, RS, IR, SR, and RI, there will be a discrepancy between function and binding, since only RS is functional, while both RS and SR count for binding. Producing formulations for this 'cross' reaction scheme and implementing its analysis are left to the reader.

\subsubsection{Actual Remaining Activity and Actual Inhibitory Response for Competitive Ant-agonism}

For the competitive reaction schemes type I and type II, the relative and actual remaining activity (ara) is determined at a fixed concentration of the agonist $S^{\prime}$, and an increasing concentration of an ant-agonist I, according to the equation for either type of competitive ant-agonism. Thus, for ara as a fraction in type I competitive ant-agonism, we have:
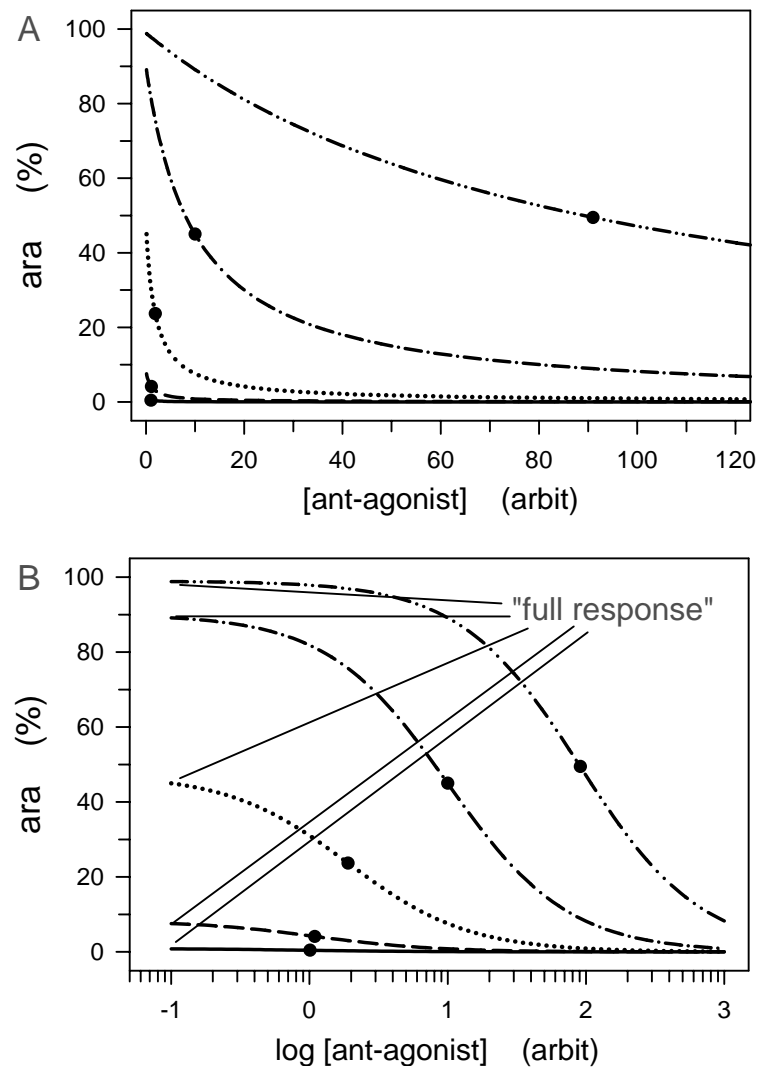

$$
\operatorname{ara}=\frac{\mathrm{S}^{\prime}}{\mathrm{S}^{\prime}+K_{\mathrm{ss}} \cdot\left(1+\mathrm{I} / K_{\mathrm{si}}\right)} .
$$

Eq. 2.10b is plotted in linear-linear and semi-log plots in Fig. 2.6A + B. Compare this equation with Eq. 2.10a for non-competitive ant-agonism.

If we equate the fractional level from which a competitive ant-agonist can operate, viz. $\mathrm{S}^{\prime} /\left(\mathrm{S}^{\prime}+K_{\mathrm{ss}}\right)=$ $F R$, then we can formulate the reverse function of ara, equal to the relative and actual inhibitory response (air) as a function of increasing ant-agonist concentration, i.e.,

$$
\text { air }=F R-\frac{\mathrm{S}^{\prime}}{\mathrm{S}^{\prime}+K_{\mathrm{ss}} \cdot\left(1+\mathrm{I} / K_{\mathrm{si}}\right)} .
$$

By incorporating the constant $F R$ into the fractional term, we get:

$$
\text { air }=\frac{F R \cdot\left[\mathrm{S}^{\prime}+K_{\mathrm{ss}} \cdot\left(1+\mathrm{I} / K_{\mathrm{si}}\right)\right]-\mathrm{S}^{\prime}}{\mathrm{S}^{\prime}+K_{\mathrm{ss}} \cdot\left(1+\mathrm{I} / K_{\mathrm{si}}\right)},
$$
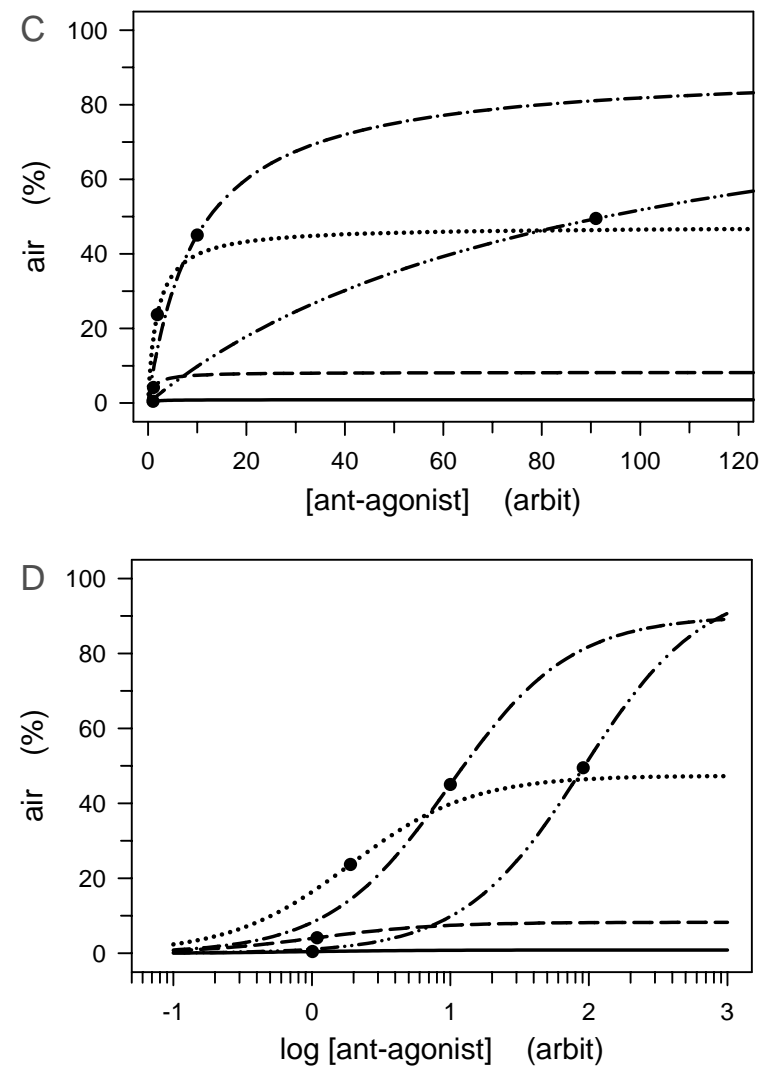

Figure 2.6. Competitive ant-agonism $(c=0)$ for both function and occupancy. Panel $A$ is linear-linear and panel $B$ is semi-log plots for the actual remaining activity (ara) in competitive ant-agonism as the concentration of the ant-agonist increases (Eq. $2.10 b$ ). Panel $C$ is linear-linear and panel $D$ is semi-log plots of the actual inhibitory response (air) in competitive ant-agonism as the concentration of the ant-agonist increases (Eq. 2.9d). The full response is varied in five steps by varying $S^{\prime}$ from $10^{-2}$ $(-)$ to $10^{2}(-\cdots \cdot)$ in steps of 10 yielding five different curves. Plots are for both competitive ant-agonism type I and type II, and the filled circles indicate the $K_{\mathrm{si}}$ or $K_{\mathrm{ii}}$ on the $x$-axes. 
and the nominator of Eq. $2.9 c$ can be reformulated to $F R \cdot\left(\mathrm{I} \cdot K_{\mathrm{ss}}\right) / K_{\mathrm{si}}$. Inserting the value for $F R$, the air is thus equal to:

$$
\operatorname{air}=\frac{\mathrm{S}^{\prime}}{\mathrm{S}^{\prime}+K_{\mathrm{ss}}} \cdot \frac{\mathrm{I} \cdot K_{\mathrm{ss}} / K_{\mathrm{si}}}{\mathrm{S}^{\prime}+K_{\mathrm{ss}} \cdot\left(1+\mathrm{I} / K_{\mathrm{si}}\right)} .
$$

Eq. $2.9 d$ is plotted in linear-linear and semi-log plots in Fig. 2.6C + D.

Notice that Eqs. $2.10 b$ and $2.9 d$ are both valid for any value of $S^{\prime} \geq 0$ and are mirror images of each other (compare Figs. 2.6A $+\mathrm{B}$ with 2.6C $+\mathrm{D}$ ).

The equations here in Section 2.4.11 are for both function and occupancy.

For competitive ant-agonism type II, we simply replace $K_{\mathrm{ss}}$ in Eqs. $2.10 b$ and $2.9 b-d$ with $K_{\mathrm{ii}}$.

\subsubsection{Competitive Versus Non-competitive Studies. A Summary}

It is traditional to conceive of the competitive and noncompetitive reaction schemes as opposites. This is based on the fact that in concentration-binding and dose-response studies of simple competitive ant-agonism (Section 2.4.9 and 2.4.10), it is only the apparent agonist-binding constant, $\operatorname{appK}_{\mathrm{ss}}=\mathrm{EC}_{50}$, which changes as the ant-agonist concentration increases, while the maximal agonist binding or maximal agonist-dependent response obtainable are unaffected at all ant-agonist concentrations. In simple competitive antagonism, the inhibitory effect of increasing an antagonist concentration on maximal binding or response of an agonist is always surmountable with high enough agonist concentrations as described in Sections 2.4.9 and 2.4.10.

Meanwhile, in the simple non-competitive ant-agonism of dose-response relations, only the maximal agonist response in the presence of a so-called non-competitive ant-agonist will be lower than the maximum of an agonist-dependent response in the absence of an antagonist, while the apparent agonist equilibrium dissociation constant, appK $\mathrm{K}_{\mathrm{ss}}$, is unaffected by the presence of a non-competitive ant-agonist and equal to $K_{\mathrm{ss}}$ (cf. Section 2.4.5, Fig. 2.5, and Table 2.3).

On the contrary, for non-competitive binding, neither the maximal binding nor the agonist dissociation constant is affected (Table 2.3). This is not the conventional understanding of 'non-competitive ant-agonism'.

\subsection{On Simple Ant-agonism and Simple Intervention}

In this sub-chapter, you will learn that when we develop a certain theory in the form of a reaction scheme to
Table 2.4. Effects in the Intervention Model on appK and $B_{\max }$ or $R_{\max }$ for Increasing Concentration of Interventor [l] (Compare with Table 2.6)

\begin{tabular}{llll}
\hline & $\operatorname{appK}_{\mathrm{s}}$ & $B_{\max }$ or $R_{\max }(\%)$ & Comment \\
\hline Binding $c=0$ & $\uparrow \uparrow$ & 100 & Competitive \\
$0<c<1$ & $\uparrow$ & 100 & \\
$c=1$ & $K_{\mathrm{s}}$ & 100 & Non-competitive \\
$c>1$ & $\downarrow \downarrow$ & 100 & \\
Function $c=0$ & $\uparrow \uparrow$ & 100 & Competitive \\
$0<c<1$ & $\uparrow$ & $\rightarrow 0$ & Non-competitive \\
$c=1$ & $K_{\mathrm{s}}$ & $\rightarrow 0$ & \\
$c>1$ & $\downarrow \downarrow$ & $\rightarrow 0$ & \\
\hline
\end{tabular}

Know your theoretical tools and what they can accomplish.

illustrate our concepts of competitive and non-competitive ant-agonism, as described in Sub-chapter 2.4, such a reaction scheme does not always yield the expected solutions. Further, the solutions may deviate between functional experiments and binding studies. This will be clear when we summarize the conclusions about simple ant-agonism and simple intervention in Tables 2.3 and 2.4. The theory developed and examined in this subchapter is a so-called intervention model. In this model the constant $c$, which was kept $=1$ in Sub-chapter 2.4, is now allowed to vary.

\subsubsection{Intervention and Non-competitive Reaction Schemes}

The non-competitive reaction scheme and variants thereof were presented in Sections 2.4.5, 2.4.6, 2.4.7, and 2.4.8. A condition for these schemes was the assumption that the constant $c$ equaled unity. Here, we will analyze the impact of constant $c$ varying freely above as well as below unity in the full cyclic scheme of Figs. 2.1A or 2.4-1.

When the co-lateral intervention constant is equal to unity, $c=1$, it is the same as saying that binding of an interventor to a secondary site does not change the binding of an agonist to its orthosteric binding site and vice versa when an agonist binds to the orthosteric binding site it does not change the equilibrium binding constant for an interventor to its binding site, the secondary binding site. Observe in this connection, that a non-competitive ant-agonist/inhibitor is not the same as a neutral ant-agonist. ${ }^{4}$

The non-competitive ant-agonist/inhibitor should in a model-related sense be a 'neutral interventor' (see Table 2.2).

\footnotetext{
4 A neutral ant-agonist (see Milligan et al. 1995; Milligan \& Bond 1997; Richard et al. 2001; Foucaud et al. 2006) is something other than a non-competitive ant-agonist, ə: non-competitive ant-agonists/ inhibitors are defined by $c=1$, while neutral ant-agonists are defined by system constant $b=1$. For constants $b$ and $c$, see for instance the allosteric two-state model (ATSM) in Chapter 7.
} 
However, that $c$ is equal to unity is unlikely to be the general case, since one would expect an ant-agonist/ inhibitor, on binding to the secondary binding site, to cause a conformational change in the receptive unit, and thereby a change in agonist-dependent binding and activity. A quantification of this expected change is taken care of by the co-lateral intervention constant $c$, as shown in Figs. 2.1A and 2.4-1, equal to a full intervention reaction scheme.

Function. Therefore, in functional studies, when $c$ is different from unity, both the apparent agonist dissociation constant, $\operatorname{appK}_{\mathrm{ss}}$, and the maximal agonist-dependent response, $R_{\max }$, are affected. As already stated, in classic theory this is referred to as mixed-inhibition (antagonism). Thus, for $c \neq 1$ (Fig. 2.4-1) which is the most likely condition for ant-agonism/inhibition of mutually inclusive ant-agonists/inhibitors, the relationship of doseresponse by agonist in the presence of an ant-agonist/ interventor will most likely demonstrate mixed behavior, i.e., both $R_{\max }$ and $K_{\mathrm{ss}}$ are altered concomitantly.
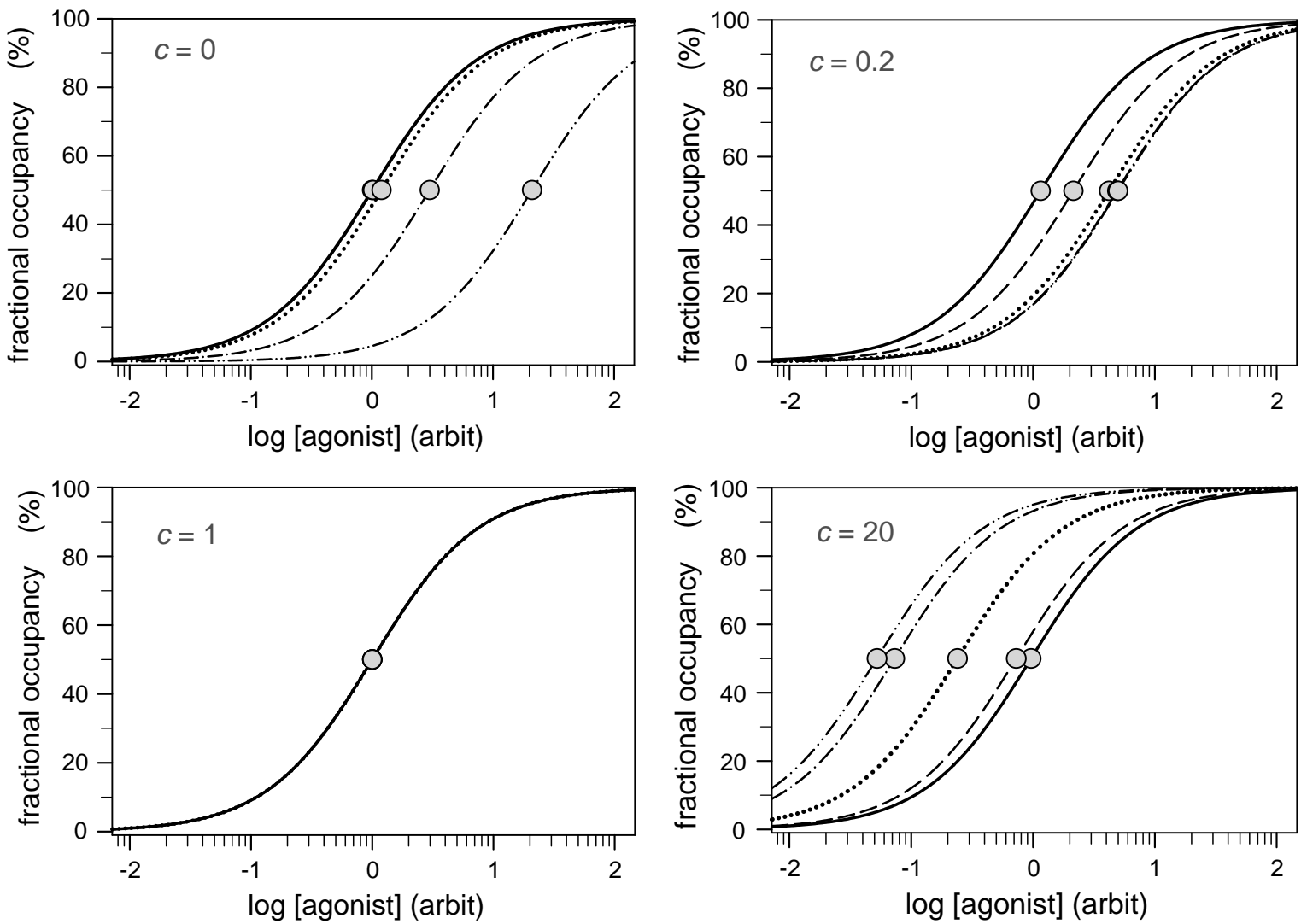

Figure 2.7. Plots of the intervention model for binding. In the first three panels the interventor concentration [I] is varied by a factor 10 in five steps between $2 \cdot 10^{-1}(-)$ and $2 \cdot 10^{3}(\cdots \cdots)$. In the last panel $(c=20)$, the interventor concentration [I] is varied by a factor 10 in five steps between $2 \cdot 10^{-3}(-)$ and $2 \cdot 10(\cdots \ldots)$ yielding five plots in each graph. In $c=0$, the plots are for the competitive situation. In $c=0.2$, the plots are for a negative intervention situation. In $c=1$, the plots are for a non-competitive situation. In $c=20$, the plots are for a positive intervention situation. Tinted circles indicate the $\mathrm{ED}_{50}$ at $50 \%$ of the effective binding $\left(\mathrm{EB}_{50}\right) . K_{\mathrm{ss}}=$ dissociation constant for $\mathrm{S}$ at primary $\mathrm{s}$-site and $K_{\mathrm{ii}}=$ dissociation constant for interventor ligand at intervention i-site are both $=1$.

\subsubsection{Switch from 'Ant-agonist' to 'Interventor' in Binding and Function}

For intervention schemes the fractional binding of an agonist as the interventor concentration varies is given by:

$$
\frac{\text { occupancy }}{\text { total }}=\frac{\mathrm{S}}{\mathrm{S}+K_{\mathrm{ss}} \cdot\left(\frac{1+\mathrm{I} / K_{\mathrm{ii}}}{1+\left(\mathrm{I} / K_{\mathrm{ii}}\right) \cdot c}\right)}
$$

and, as the concentration of an interventor changes, the fractional activity, which is dependent on an agonist, is:

$$
\frac{\text { response }}{\text { total }}=\frac{\mathrm{S} /\left(1+\left(\mathrm{I} / K_{\mathrm{ii}}\right) \cdot c\right)}{\mathrm{S}+K_{\mathrm{ss}} \cdot\left(\frac{1+\mathrm{I} / K_{\mathrm{ii}}}{1+\left(\mathrm{I} / K_{\mathrm{ii}}\right) \cdot c}\right)} .
$$

Now I is not only symbol for an ant-agonist/inhibitor but also for an interventor ligand.

Examples of semi-log plots of Eq. 2.16 for bindingagainst-agonist concentration are shown in Fig. 2.7, and 
response-against-agonist concentration (Eq. 2.17), is shown in Fig. 2.8 when the interventor concentration [I] is varied in five steps and with four different values for constant $c$ as well.

From Eq. 2.16 for concentration-occupancy it is fairly straightforward to deduce that the apparent dissociation constant $\operatorname{appK}_{\mathrm{ss}}$ for intervention is equal to $K_{\mathrm{ss}} \cdot(1+\mathrm{I} /$ $\left.K_{\mathrm{ii}}\right) /\left(1+\left(\mathrm{I} / K_{\mathrm{ii}}\right) \cdot c\right)$, and thus may be above or below $K_{\mathrm{ss}}$ and that the maximal occupancy is reached at $100 \%$ as the agonist concentration $\mathrm{S}$ increases (Fig. 2.7).

For intervention in the dose-effect relationship (Eq. 2.17, Fig. 2.8), we can show that the apparent dissociation constant $\operatorname{appK}_{\mathrm{ss}}=K_{\mathrm{ss}} \cdot\left(1+\mathrm{I} / K_{\mathrm{ii}}\right) /\left(1+\left(\mathrm{I} / K_{\mathrm{ii}}\right) \cdot c\right)$ may be above or below $K_{\mathrm{ss}}$ and the fractional effect at high agonist concentration is always $<1$ in the presence of an interventor for $c>0: \quad R_{\max }=1 /\left(1+\left(\mathrm{I} / K_{\mathrm{ii}}\right) \cdot c\right)$ (Fig. 2.8). Thus, the interventor appears as a non-sur mountable inhibitor.

For both the binding and functional schemes, it is worth noting that the non-competitive ant-agonism/ inhibition appears for $c=1$ (Figs. 2.7C and 2.8C), and competitive ant-agonism/inhibition type II for $c=0$ (Figs. 2.7A and 2.8A). Thus, non-competitive and competitive ant-agonism are merely special cases of the more general intervention theory.

In non-competitive ant-agonism/inhibition only the $R_{\text {max }}$ in functional studies is affected (Fig. 2.8C), while neither the maximal binding $B_{\max }$ nor its agonist equilibrium dissociation constant $K_{\mathrm{ss}}$ are affected (Fig. 2.7C). The fractional effect at high $[\mathrm{S}]$ in functional studies will be $R_{\max }=1 /\left(1+\left(\mathrm{I} / K_{\mathrm{ii}}\right)\right.$, and in binding studies the occupancy reaches maximum and is always completely independent of [I] (Tables 2.3 and 2.4). Obviously when $c=1-$ the non-competitive condition - then appK $\mathrm{ss}_{\mathrm{ss}}$ is equal to $K_{\mathrm{ss}}$ for both occupancy and response studies (Table 2.4). System constants for all these derived conditions, based on theories, each from a portion of the four-pane reaction scheme with one state as in Fig. 2.4-1, are summed up in the Section 2.5.3.
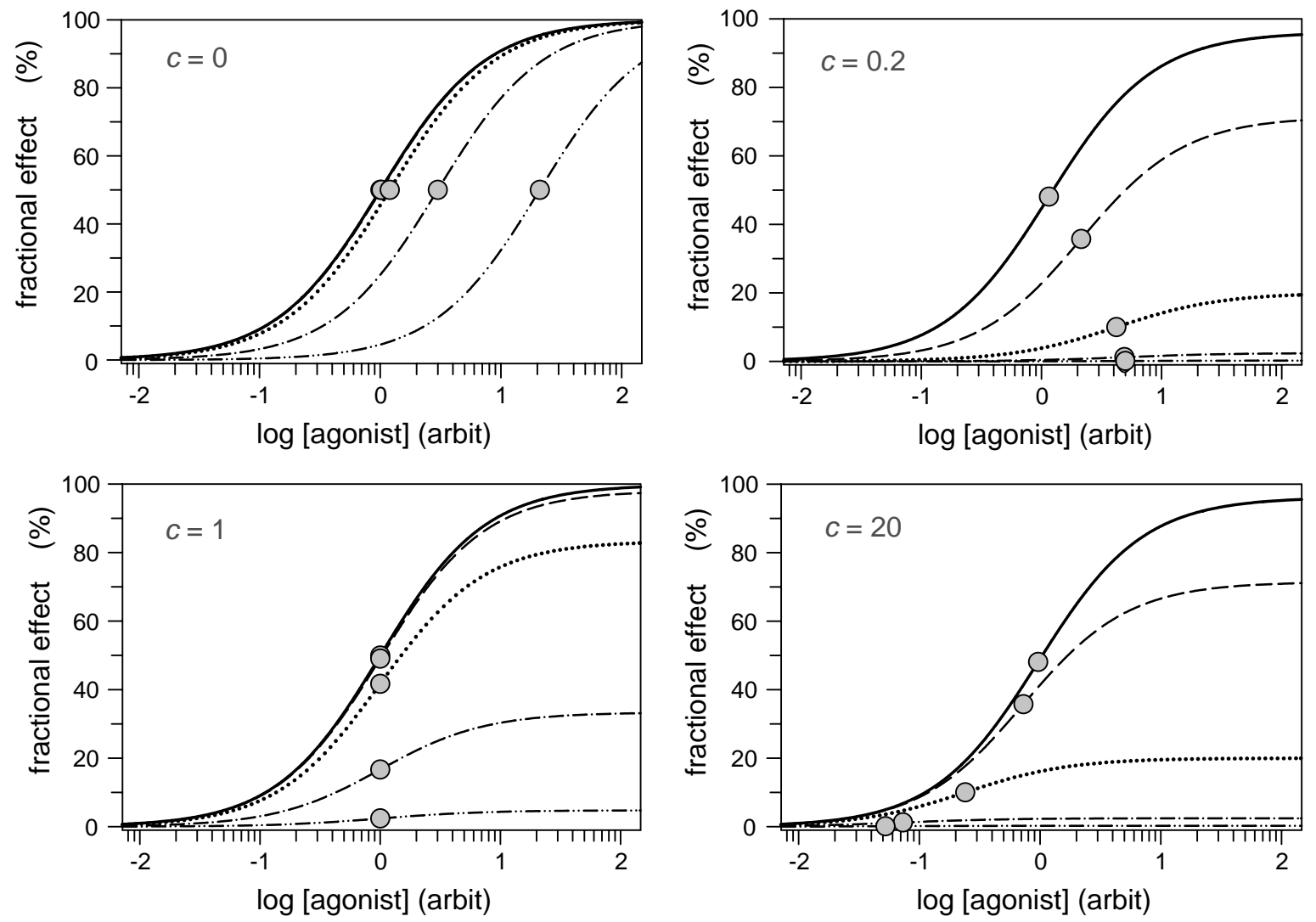

Figure 2.8. Plots of the intervention model for function. In the first three panels the interventor concentration [I] is varied by a factor 10 in five steps between $2 \cdot 10^{-1}(\longrightarrow)$ and $2 \cdot 10^{3}(\cdots \cdots)$. In the last panel $(c=20)$, the interventor concentration [I] is varied by a factor 10 in five steps between $2 \cdot 10^{-3}(-)$ and $2 \cdot 10(-\cdots)$ yielding five plots in each graph. In $c=0$, the plots are for a competitive situation. In $c=0.2$, the plots are for a negative intervention situation. $\ln c=1$, the plots are for a non-competitive situation. In $c=20$, the plots are for a positive intervention situation. Tinted circles indicate the $\mathrm{ED}_{50}$ at $50 \%$ of the effective response $\left(\mathrm{ER}_{50}\right) . K_{\mathrm{ss}}=$ dissociation constant for $\mathrm{S}$ at primary s-site and $K_{\mathrm{ii}}=$ dissociation constant for interventor ligand at intervention i-site are both $=1$. 


\subsubsection{Summary of Parameter Characteristics from Simple Intervention}

Based on both mutually exclusive and inclusive one-state models, ME-OSM and MI-OSM, with an interventor different from the agonist, we reach a conclusion on the various effects an interventor may have on agonistinduced maximal binding or maximal response and on agonist affinity, $1 / K_{\mathrm{ss}}$, with different types of intervention, as analyzed in Sections 2.4.5-6 and 2.4.9-10, and for intervention in Sections 2.5.1 and 2.5.2. Examples of plots of the intervention model are shown in Figs. 2.7 and 2.8. Conclusions from these sections are summarized in Tables 2.3, 2.4 and 2.5. These results may be compared with similar solutions and conclusions reached by other authors on competitive and noncompetitive inhibition as well as mixed-inhibition and activation (Ariëns et al. 1964, pp. 287-321; Cheng \& Prusoff 1973; Laidler \& Bunting 1973; Segel 1975, 1993 - mixed-inhibition, pp. 136-143; Levitzki 1978, pp. 1127; Dixon \& Webb 1979, pp. 332-353; Varon et al. 2002; Cornish-Bowden 2004, Chapter 5; Shou 2005). Examples in enzymology are of a more complex nature as they include product formation and additional pathways.

\subsubsection{The Un-competitive Reaction Scheme}

The suggested reaction scheme for un-competitive ${ }^{5}$ antagonism/inhibition (see Segel 1975, 1993, Fig. III-21; Cornish-Bowden 1995, 2004) is a special case, a shortened version of the MI-OSM described in Section 2.4.5 (Fig. 2.4-1). In the shortened version of the MI-OSM, the receptive unit conformation IR is excluded. Now, the un-competitive reaction scheme has the parameter $c=1$. In the 'un-competitive' reaction of the shortened version of MI-OSM, the interventor cannot bind to the unliganded receptive unit. This means that the receptive unit must first bind an agonist before a second interventor ligand can bind. That is, an ordered reaction. Parameter $c$ is allowed to vary above and below unity in the shortened version of MI-OSM, but in reality, in the classical un-competitive scheme $c=1$. It is subsumed into the dissociation constant $K_{\mathrm{ii}}$ and we can put $c$ as 1 . The reaction scheme for the shortened version of the mutually inclusive one-state model is:

$$
\mathrm{R}+\mathrm{S}+\mathrm{I} \rightleftarrows \mathrm{R}^{\prime} \mathrm{S}+\mathrm{I} \rightleftarrows \mathrm{IR}^{\prime \prime} \mathrm{S}
$$

where only the $\mathrm{R}^{\prime} \mathrm{S}$ conformation is active or activatable. $\mathrm{R}^{\prime}$ and $\mathrm{R}^{\prime \prime}$ in this reaction scheme signify conformations different from $\mathrm{R}$ and from each other.

We can derive the fractional concentration-binding and dose-response equations for this shortened mutually-

\footnotetext{
5 The term 'un-competitive' was introduced by Ebersole et al. (1944).
}

Table 2.5. Summary of the Relative Maximal Responses $\left(\mathrm{R}_{\max }\right)$ and Maximal Occupancies $\left(B_{\max }\right)$ and the Apparent Dissociation Constant for Agonist $S$, appK $\mathrm{ss}_{\mathrm{s}}$, in Two Simple Mutually Inclusive Reaction Schemes, the Shortened Heterotropic Mutually Inclusive One-state Model (amp-HE-MI-OSM) and the Full Heterotropic Mutually Inclusive One-state Model (HE-MI-OSM)

\begin{tabular}{|c|c|c|}
\hline $\begin{array}{l}\text { Type of study } \\
\text { with } c \neq 1\end{array}$ & amp-HE-MI-OSM & HE-MI-OSM \\
\hline & \multicolumn{2}{|c|}{ Parameters } \\
\hline & \multicolumn{2}{|c|}{$\begin{array}{c}\text { Fractional response } B_{\max } / R_{\max }(\text { in } \%) \\
\text { for high }[\mathrm{S}]\end{array}$} \\
\hline $\begin{array}{l}\text { Concentration- } \\
\text { occupancy }\end{array}$ & 100; surmountable & 100; surmountable \\
\hline Dose-response & $100 /\left(1+\left(\mathrm{I} / K_{\mathrm{ii}}\right) \cdot c\right)$ & $100 /\left(1+\left(\mathrm{I} / K_{\mathrm{ii}}\right) \cdot c\right)$ \\
\hline & \multicolumn{2}{|c|}{$\begin{array}{l}\text { Apparent dissociation constant } \\
\qquad \operatorname{appK}_{\mathrm{ss}}=\mathrm{EC}_{50}\end{array}$} \\
\hline $\begin{array}{l}\text { Concentration- } \\
\text { occupancy }\end{array}$ & $K_{\mathrm{ss}} /\left(1+\left(\mathrm{I} / K_{\mathrm{ii}}\right) \cdot c\right)$ & $\frac{K_{\mathrm{ss}} \cdot\left(1+\mathrm{I} / K_{\mathrm{ii}}\right)^{\#}}{\left(1+\left(\mathrm{I} / K_{\mathrm{ii}}\right) \cdot c\right)}$ \\
\hline Dose-response & $K_{\mathrm{ss}} /\left(1+\left(\mathrm{I} / K_{\mathrm{ii}}\right) \cdot c\right)$ & $\frac{K_{\mathrm{ss}} \cdot\left(1+\mathrm{I} / K_{\mathrm{ii}}\right)^{\#}}{\left(1+\left(\mathrm{I} / K_{\mathrm{ii}}\right) \cdot c\right)}$ \\
\hline
\end{tabular}

For the 'un-competitive' situation, amp-HE-MI-OSM, parameter $K_{\mathrm{ii}}$ varies with $c$ as there are only two independent system constants (see Figs. 2.9 and 2.10). ${ }^{\#}$ For $c>1 \mathrm{appK}_{\mathrm{ss}}<K_{\mathrm{ss}}$; for $c<1 \mathrm{appK}_{\mathrm{ss}}>K_{\mathrm{ss}}$.

inclusive and one-state reaction scheme. For binding one gets:

$$
\frac{\text { occupancy }}{\text { total }}=\frac{\mathrm{S}}{\mathrm{S}+K_{\mathrm{ss}} /\left(1+\left(\mathrm{I} / K_{\mathrm{ii}}\right) \cdot c\right)} \text {, }
$$

and for the functional shortened MI-OSM it is:

$$
\frac{\text { response }}{\text { total }}=\frac{\mathrm{S} /\left(1+\left(\mathrm{I} / K_{\mathrm{ii}}\right) \cdot c\right)}{\mathrm{S}+K_{\mathrm{ss}} /\left(1+\left(\mathrm{I} / K_{\mathrm{ii}}\right) \cdot c\right)} .
$$

The system parameters for the fractional binding and response of the shortened MI-OSM are summarized in Table 2.5, its behavior in Table 2.6, and examples of plots are presented in Fig. 2.9 for occupancy and Fig. 2.10 for function. All panels in Figs. 2.9 and 2.10 are for the 'un-competitive' situation because when $c$ varies it is the same as $K_{\mathrm{ii}}$ varies.

Table 2.6. Effects of the Shortened Intervention Model on $\mathrm{appK}_{\mathrm{s}}$ and $B_{\max }$ or $R_{\max }$ for Increasing Concentration of Interventor [I] (Compare with Table 2.4)

\begin{tabular}{llll}
\hline & $\operatorname{appK}_{\mathrm{s}}$ & $B_{\max }$ or $R_{\max }(\%)$ & Comment \\
\hline Binding $c=0$ & $K_{\mathrm{s}}$ & 100 & Competitive \\
$0<c<1$ & $\downarrow$ & 100 & \\
$c=1$ & $\downarrow \downarrow$ & 100 & Un-competitive \\
$c>1$ & $\downarrow \downarrow \downarrow$ & 100 & \\
Function $c=0$ & $K_{\mathrm{s}}$ & 100 & Competitive \\
$0<c<1$ & $\downarrow$ & $\rightarrow 0$ & Un-competitive \\
$c=1$ & $\downarrow \downarrow$ & $\rightarrow 0$ & \\
$c>1$ & $\downarrow \downarrow \downarrow$ & $\rightarrow 0$ & \\
\hline
\end{tabular}



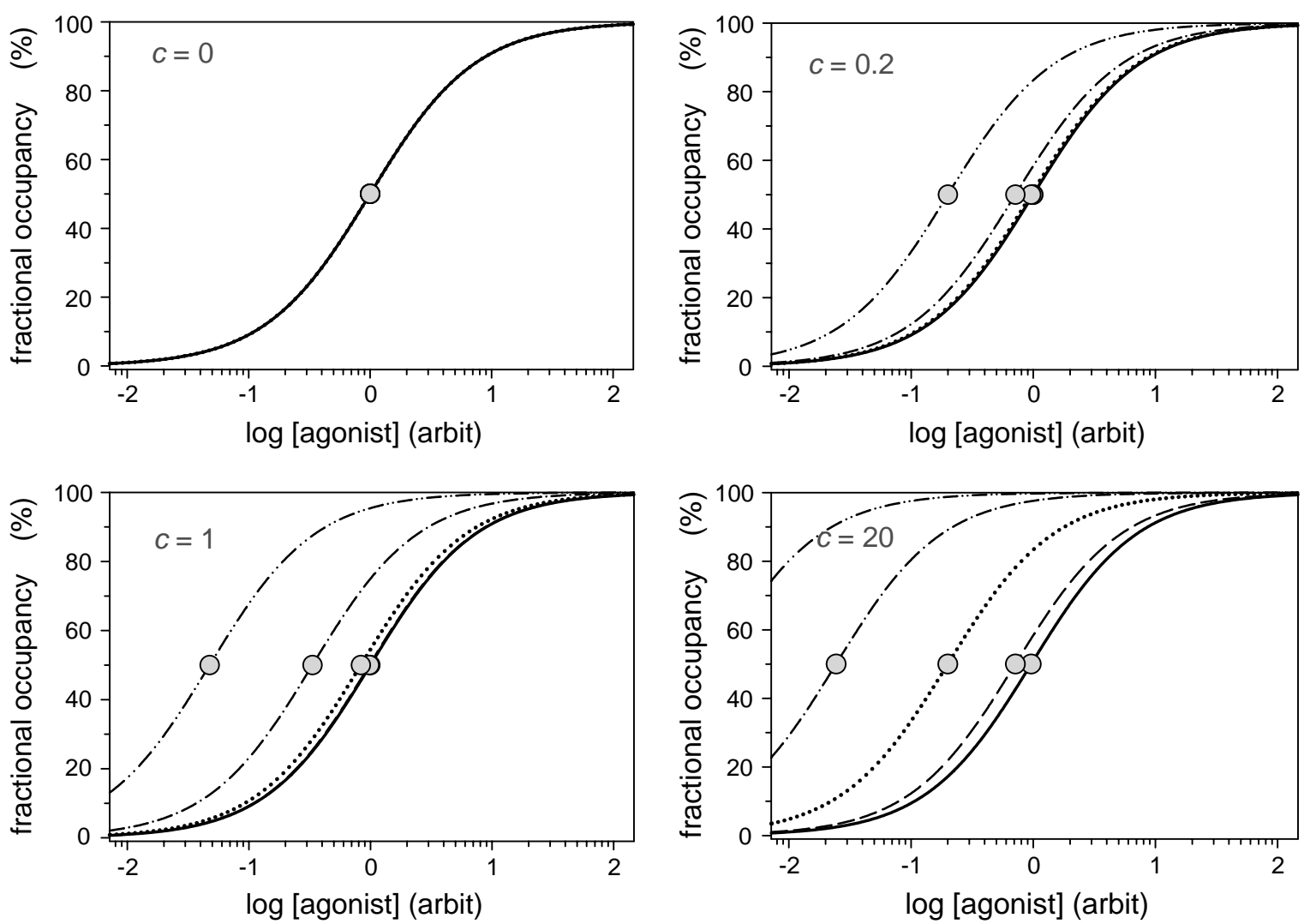

Figure 2.9. Plots of the shortened intervention model for binding. In all four panels the interventor concentration [I] is varied by a factor 10 in five steps between $2 \cdot 10^{-1}\left({ }_{-}\right.$and $2 \cdot 10^{3}(\ldots .$.$) yielding five plots in each graph. In c=0$, the plots are for an 'ordered' competitive situation. In $c=0.2$, the plots are for the negative shortened intervention situation. In $c=1$, the plots are for an 'ordered' non-competitive, un-competitive, situation. In $c=20$, the plots are for the positive shortened intervention situation. Tinted circles indicate the $\mathrm{ED}_{50}$ at $50 \%$ of the effective binding $\left(\mathrm{EB}_{50}\right) . K_{\mathrm{ss}}=$ dissociation constant for $\mathrm{S}$ at primary s-site and $K_{\mathrm{ii}}=$ dissociation constant for interventor ligand at intervention $\mathrm{i}$-site are both $=1$.

\subsubsection{Conclusions for Both Complete and Shortened MI-OSMs}

For comparison, Table 2.6 lists the parameter characteristics based on the complete heterotropic MI-OSM together with the equivalent terms for the shortened heterotropic MI-OSM.

With a definition of 'un-competitive' as a concomitant lowering of both the maximal response and the affinity for agonists in the presence of an interventor, if we base our modeling of such a behavior on either the shortened or the complete MI-OSM, the conclusion is that only in the functional versions of these models is the 'un-competitive' definition fulfilled. In the binding versions of these models, we simply have 'competitive' ant-agonism (Tables 2.3, 2.4, 2.5 and 2.6).

A theory for non-competitive behavior in functional studies is described by the heterotropic MI-OSM when the co-lateral intervention constant is equal to unity, $c=$ 1 , while non-competitive behavior in binding studies does not have a correlate in MI-OSM (Table 2.3).

Competitive ant-agonism/inhibition both in binding and functional studies may be analyzed by the ME-OSM.
Strangely enough, competitive behavior in binding studies can also be analyzed by the shortened MI-OSM as well as the complete MI-OSM for all values of $c$ (Tables 2.3 and 2.4).

\subsection{Co-lateral Intervention Coefficient and Co-lateral Binding}

\subsection{1. 'Co-lateral' ${ }^{\mathbf{6}}$ in Two Terms}

Caveat: The terms 'co-lateral intervention coefficient' and 'co-lateral binding' are two totally different concepts.

Co-lateral intervention coefficient $c$ appears in the intervention reaction scheme (Fig. 2.4-1) with mutually inclusive binding, meaning that both an agonist and an interventor can bind simultaneously to their respective binding sites and affect binding or function of the other ligand. Meanwhile, they cannot bind co-laterally in this scheme.

\footnotetext{
${ }^{6}$ Co-lateral or collateral? 'Co-lateral' is chosen in favor of 'collateral'.
} 

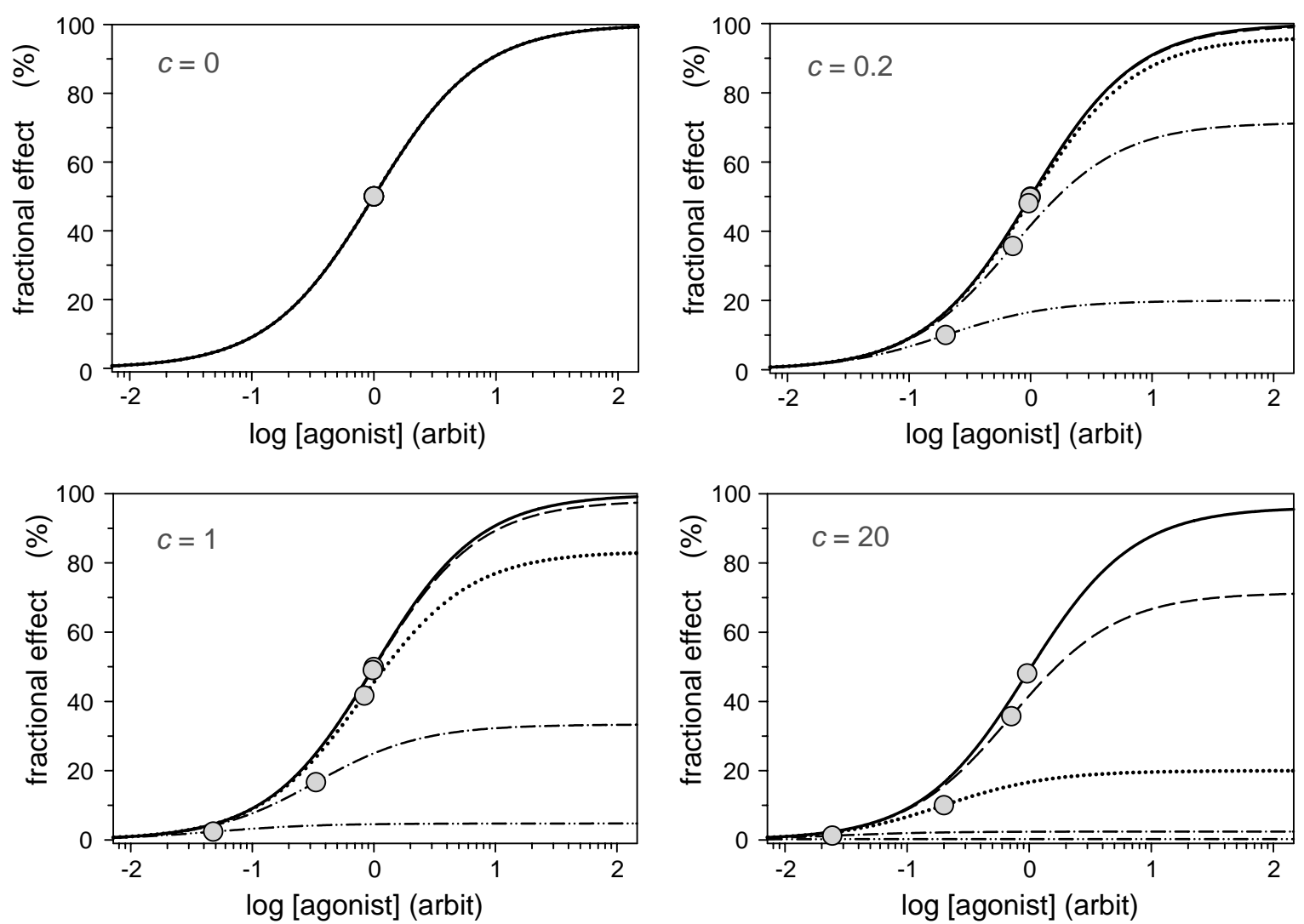

Figure 2.10. Plots of the shortened intervention model for function. In all four panels the interventor concentration [I] is varied by a factor 10 in five steps between $2 \cdot 10^{-1}\left({ }_{-}\right.$and $2 \cdot 10^{3}(-\cdots .$.$) yielding five plots in each graph. All plots are for variations in$ agonist concentration. In $c=0$, the plots are for an 'ordered' competitive situation. In $c=0.2$, the plots are for the negative shortened intervention situation. In $c=1$, the plots are for an 'ordered' non-competitive, un-competitive, situation. In $c=20$, the plots are for the positive shortened intervention situation. Tinted circles indicate the $E D_{50}$ at $50 \%$ of the effective response $\left(\mathrm{ER}_{50}\right) . K_{\mathrm{ss}}=$ dissociation constant for $\mathrm{S}$ at primary s-site and $K_{\mathrm{ii}}=$ dissociation constant for interventor ligand at intervention i-site are both $=1$.

Co-lateral binding signifies that the substrate or agonist is allowed to bind to both the primary binding site as well as the secondary binding site (Figs. 2.4-2 and 2.4-3, and Section 2.6.3) and that the interventor can also bind to both binding sites, including the orthosteric binding site (Figs. 2.4-3 and 2.4-4). The two ligands can switch sites, and this ability of ligands to switch sites is defined as co-lateral binding. Thus, co-lateral binding goes beyond 'mutually inclusive' binding, which only covers simultaneity in binding.

\subsubsection{Co-lateral Intervention Constants and Auto-intervention}

In this chapter, ant-agonism and intervention by ligands have, thus far, solely been induced by molecules other than the agonists - i.e., hetero-intervention. We shall deal further with intervention that is elicited by the agonist itself. This type of intervention is also known as 'auto-intervention' (Fig. 2.4-2), and will be examined in Chapter 3. In this reaction scheme there is an obligatory co-lateral binding, RS or SR and SRS.
The co-lateral intervention constant for hetero-intervention is described by $c$, while the co-lateral intervention constant for auto-intervention is designated as $c^{\prime}$, in order to differentiate between the two co-lateral intervention constants (Table 2.7 ). See Figs. 2.4-1 and 2.4-2 of the four-pane scheme for reasons to operate with both $c$ and $c^{\prime}$.

\subsubsection{Co-lateral Binding and Auto-intervention}

A reaction scheme with co-lateral binding as well as mutually inclusive binding-simultaneous binding for both agonists and interventors - is illustrated in Fig. 2.4. It is the complete four-pane one-state-model (FP-OSM). The FP-OSM was used to derive the reaction schemes for competitive ant-agonism/inhibition types I and II (MEOSMs) and for non-competitive and un-competitive antagonism/inhibition as well, and, furthermore, expanding into intervention models where both $c$ and $c^{\prime}$ are above 0 (MI-OSMs). In Chapter 3, I describe an auto-intervention model where only one type of ligand (substrate, agonist) can bind to both an orthosteric and a secondary site 
Table 2.7. Models With and Without Co-lateral Intervention and With and Without Co-lateral Binding

\begin{tabular}{lll}
\hline \multirow{2}{*}{ Type of model } & \multicolumn{2}{c}{ Co-lateral } \\
\cline { 2 - 3 } & Intervention constant & Binding \\
\hline ME-OSM (Chapter 2) & None $(c=0)$ & No \\
HE-MI-OSM (Chapter 2) & $c$ & No \\
HO-MI-OSM (Chapter 3) & $c^{\prime}$ & Yes \\
FP-OSM (Chapter 2) & Both $c$ and $c^{\prime}$ & Yes \\
ATSM (Chapter 7) & $c$ & No \\
HOTSM (Chapter 7) & $c^{\prime}$ & Yes \\
\hline
\end{tabular}

simultaneously. It is an auto-intervention one-state model (HO-MI-OSM), with co-lateral binding for the ligand (Fig. 2.4-2). Obviously, for interventors that can bind to orthosteric as well as secondary binding sites, there is also co-lateral binding, equal to the upper left quadrant of the FP-OSM (Fig. 2.4-4).

\subsubsection{Co-lateral Binding, Co-lateral Intervention and the HOTSM/ATSM}

After the FP-OSM has been transformed to its two-state variant, a four-pane two-state model (FP-TSM; see Figs. 5.9 and 7.2), it will be employed to derive the homotropic two-state model (HOTSM, Fig 7.1B) in Part II, Chapter 7 (Bindslev 2004), which is a part of the FP-TSM with co-lateral binding. However, note that an allosteric two-state model (ATSM) (Hall 2000), which is also part of the FP-TSM, and likewise described in Chapter 7, does not allow co-lateral binding for the interventor, modulator or agonist (Fig. 7.1A and Table 2.7). This, as opposed to the lower right quadrant in the FP-OSM for a primary ligand (Fig. 2.4-2), and the upper left quadrant of the FP-OSM for a secondary ligand, an interventor (Fig. 2.4-4). In these two quadrants co-lateral binding is allowed and comparable to co-lateral binding in the FPTSM for an agonist and a modulator (Fig. 7.2).

\subsubsection{Differences Between Intervention and Negative Allostery}

Negative allostery is first defined in Chapter 15 and demonstrated in Fig. 15.10. Briefly, in functional studies of negative allostery, a modulator molecule can lower the maximal response similar to an interventor in the intervention scheme (Fig. 2.8 and Table 2.3).

The difference between negative intervention and negative allosteric modulation is that allosteric modulation conceptually is based on two-state models, while the intervention including un-competitive and non-competitive ant-agonism/inhibition is in the realm of one-state models. Two-state against one-state models is the difference. For the sake of organizing models in a meaningful and manageable lattice that is also crystal clear, the crucial concept of the difference between two-state and one-state is further discussed in Chapters 5 and 15. If it is not the key issue of the book, it is one of them.

\subsection{A History on the Development of Ternary-complex Models}

\subsubsection{Early Years}

From 1914 models were suggested for the action (function) of enzymes altered through two different substrates or ligands that interfere with the action of each other (Michaelis 1914; Burk 1944 cited by Ebersole et al 1944; and by Friedenwald \& Maengwyn-Davis 1954a, pp. 163). Burk termed the aberrant behavior "anti-competitive coupling activation'. Formalism for ternary complexes with two substrates was developed in the following years (Laidler \& Socquet 1950; Friendenwald \& MaengwynDavis 1954a,b, pp. 154-190; Webb 1963; Mahler \& Cordes 1966; Cheng \& Prusoff 1973; Segel 1975, 1993; Birdsall et al. 1978; Tolkovski \& Levitzki 1978; Dixon \& Webb 1979, pp. 79-82). In pharmacological literature, similar and not always complete derivations starting with Ariëns et al. (1955) appeared in the 1970s and 1980s (Jacobs \& Cuatrecasas 1976; Boeynaemes \& Dumont 1980; Burgisser et al. 1982; Stockton et al. 1983).

\subsubsection{Terminology for the Intervention Scheme}

The fully reversible reaction scheme for two ligands binding at a single receptive unit is presented in Figs. 2.1A and 2.4-1, and appears in different settings. I call it the intervention model. In principle, it is a heterotropic, onestate ternary-complex model that is also 'inclusive', which indicates that it allows binding of two ligands simultaneously. All these features are also part of the noncompetitive reaction scheme presented in Sub-chapter 2.4. The difference between a non-competitive reaction scheme and the intervention scheme is that constant $c$ may deviate from unity in the intervention scheme.

The intervention scheme in Fig. 2.4-1 is described by numerous authors, and is given different names depending on the text it appears in (Laidler 1956, 1958; Mahler \& Cordes 1966; Segel 1975, 1993; Birdsall et al. 1978; Dixon \& Webb 1979; De Lean et al. 1980; Stockton et al. 1983; Wreggett \& De Lean 1984; Ehlert 1988; Costa et al. 1992; Cornish-Bowden 1995, 2004; Varon et al. 2002; Shou 2005; see additional references in Table 2.1). For instance, it is now standard in pharmacology to refer to the scheme as 'The ternary-complex model', TCM, when invoked for ligand and $G$ protein interaction with a $G$ protein-coupled receptor (De Lean et al. 1980), and as the 'allosteric ternary-complex model' when explicitly 
dealing with parameter $c$ (Ehlert 1988). Meanwhile in enzymology, when focusing on both its functional as well as ant-agonistic possibilities, it is referred to as the 'general modifier mechanism' in order to avoid the term 'competitive' (Topham 1990; Topham \& Brocklehurst 1992), while when merely paying attention to its ant-agonistic aspects it is referred to as the 'mixedcompetitive reaction scheme' (e.g., Webb 1963; Laidler \& Bunting 1973; Segel 1975, 1993, Chapters 5 and 6; Dixon \& Webb 1979; Cornish-Bowden 2004, Chapter 5) or the 'general non-competitive inhibition mechanism' (Cha 1975). In enzymology in general, there are added reaction paths of one-way product formation in relation to the intervention scheme in Fig. 2.1A, and often the term ternary-complex model in enzymology refers to other types of reaction schemes (Cornish-Bowden 1995, 2004, Chapter 7).

Initially, and in particular with Ehlert's derivations in 1988 for ligand interaction in pharmacology, we got the full picture of formalism for the intervention model with two different ligands at receptors, a primary ligand and a secondary ligand, a G protein (Ehlert 1988; see also Costa et al. 1992).

Ehlert (1988) has treated the ternary-complex model in much detail and derived its formulation both for binding and functional experimentation. Ehlert's derivations were performed with the specific purpose of extracting affinity constants of occupancy and activity for GPCRs on binding ligands, including $\mathrm{G}$ proteins. Meanwhile, with regard to the terminology in this book, Ehlert's use of the word 'allosteric' is misleading. Observed from a modeler's view, Ehlert's 'allostery' is unfortunately purported by others (Kenakin 2004a,b, 2007; Christopoulos et al. 2004, Vauquelin \& von Mentzer 2007, Fig. 213).

I want to use a novel expression to designate the reaction scheme in Fig. 2.4-1. This in order to avoid imprecise designation and confounding connotations, such as (1) 'inhibition' and 'competition' used in enzymology, and (2) 'ternary-complex' and 'allosteric ternary-complex' as used in receptology. Therefore, I suggest to call the reaction scheme in Fig. 2.4-1 'the intervention scheme', hopefully leaving room for possible interactive measures as well as associations that include both positive and negative effects in the epithet 'intervention'. A designation that fully covers the reaction scheme in Fig. 2.4-1 is a "heterotropic mutually inclusive one-state ternary-complex model with interaction parameter $c \geq 0$ - possibly different from unity - and with no 'co-lateral binding' of ligands'. This is too cumbersome, justifying a short designator as 'intervention' (see Subchapter 2.6 for more on co-lateral binding).

Note that the intervention scheme in Fig. 2.1A is an integral part of the FP-OSM (Fig. 2.4-1), a reaction scheme in which co-lateral binding is allowed in some quadrants.
Can you find the quadrants in Fig. 2.4 where the colateral binding is allowed? The answers appear in Section 2.6.1.

\subsubsection{Auto-intervention One-state Model}

For the homotropic mutually inclusive and intervention one-state model (HO-MI-OSM) in binding and action with identical ligands in a ternary complex, derivations started early on with the equation by Hill (1910), the formulation by Adair (1925), and Haldane's 'substrate inhibition' (Haldane 1930), and was then followed by Pauling and Wyman, culminating with a derivation given by Laidler (Laidler \& Hoare 1949; Laidler 1956, 1958, pp. 77-80) invoking an intervention constant equal to $c^{\prime}$ as described in more detail in Chapter 3.

\subsubsection{The Name of the Game}

In this section, in an attempt to clarify which model is treated, I shall use the designation HE-MI-OSM for the simple TCM with heterologous ligands and HO-MI-OSM for the simple TCM with homologous ligands. Thus, the simplest TCM with full reciprocity where two different ligands interact simultaneously (mutually inclusive) (Figs. 2.1A and 2.4-1) but not co-laterally (see Section 2.6.2) is HE-MI-OSM. When two identical ligands interact, the model is the HO-MI-OSM (Fig. 2.4-2). The HE-MI-OSM is described in Sub-chapter 2.5, while the HO-MI-OSM is treated in Chapter 3. The behavior of parameters in the two models, HE-MI-OSM and HO-MIOSM, for both binding and function are listed in Tables 2.3, 2.4, 2.5, and 3.1.

\subsubsection{The HE-MI-OSM Looks Like the HO-MI-OSM}

It is worth noting that plots of a HE-MI-OSM may become equal to plots for the HO-MI-OSM. In case the two different ligand, agonist and interventor, in the HEMI-OSM are combined in a mixture and this mixture is used for changing the ligand concentration, then [S] and [I] are changed simultaneously and the plots become identical to plots of the HO-MI-OSM (Fig. 2.11) (see Chapter 3 for more details).

\subsection{Surmountable and Insurmountable Synagics}

\subsubsection{The Development of 'Surmountable'}

The two terms 'surmountable' and 'insurmountable' were originally introduced for functional assays by Gaddum et al. (1955) to characterize the behaviour of 
agonist and interventor in fixed concentration ratio

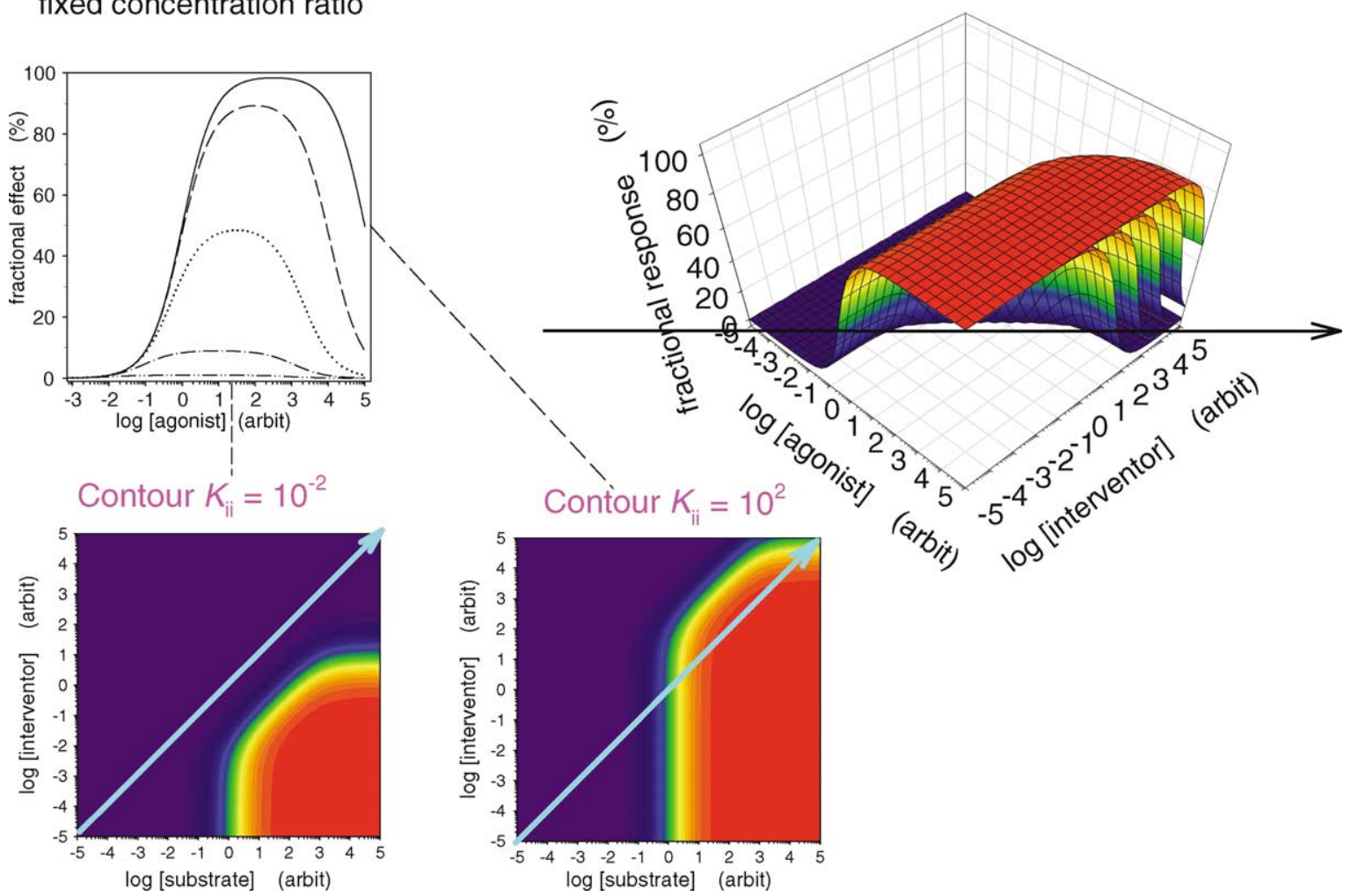

Figure 2.11. Plots of the intervention model in three-dimensions. Upper right panel shows dose-response plots for the intervention model in 3-D, where the substrate (agonist) and the interventor concentrations are the independent variables and also vary independently of each other. The interventor concentration is varied by a factor 10 in five steps from $10^{-2}$ to $10^{2}$ yielding five 3-D surface plots. In this model (Fig. 2.4-1) there is no co-lateral binding possible. The two lower panels are contour plots of the model for $\mathrm{I} / K_{\mathrm{ii}}=10^{-2}$ and $\mathrm{I} / K_{\mathrm{ii}}=10^{2}$. With a fixed concentration ratio between substrate (agonist) and interventor a 2-D graph as in the upper left panel may appear with an interventor concentration varied by a factor 10 in five steps from $10^{-2}$ (-) to $10^{2}(-\cdots \cdots)$. A graph similar to the 2-D graph will also be the consequence in case a single ligand (agonist) is capable of co-lateral binding that may lead to 'auto-ant-agonism' (substrate inhibition) (see Figs. 3.2 and 3.3 and auto-intervention functions in Chapter 3). Maximal response $=100 \%$ and co-lateral intervention coefficient $c=10^{-3} . K_{\mathrm{ss}}=$ dissociation constant for $S$ at primary $\mathrm{s}$-site and $K_{\mathrm{ii}}=$ dissociation constant for interventor ligand at intervention i-site are both $=1$.

dose-responses equal to that expected for competitive (surmountable) and non-competitive (insurmountable) ant-agonism/inhibition. Surmountable synagics were characterized by a rightward shift in dose-responses always reaching the maximum level by a high enough agonist concentration (Fig. 2.7), while insurmountable synagics had decreasing maximum responses as the antagonist concentration increased, independent of increasing agonist concentrations (Fig. 2.8).

The physical realities behind observed surmountable and insurmountable functional dose-responses are much more diverse than merely being explained on the grounds of competitive and non-competitive synagics. Vauquelin et al. (2002) provide a good review on the subject, however, their use of the concept 'allosteric', as based on a so-called Kaumann model (Kaumann \& Frenken 1988), is not in line with its use in this text. The Kaumann model does not include un-liganded activity, which is a hallmark of allosteric models. Reasons for insurmountable dose-responses such as dissociation/ limitation or diffusion/limitation (biophase) were detailed by Rang (1966) and, recently, a detailed description for assessing system constants in insurmountable analysis has reappeared (Kenakin et al. 2006).

The concepts of surmountability/insurmountability remain (Christopoulos 2001; Takezako et al. 2004; Mathiesen et al. 2006). Today, it is often assumed to relate to non-equilibrium or hemi-equilibrium explanations and therefore more often suggests an analysis of time-dependence in the models to analyse this kind of non-competitive-like behaviour (Paton \& Rang 1965; Lew et al 2000; Avlani et al. 2004; Mathiesen et al. 2006, Kenakin et al. 2006; Vauquelin \& Szczuka 2007), while others lean towards other explanations of a conformational change in the receptor per se, one being so-called 'network-leaning' (Takezako et al. 2004). 


\subsubsection{Summary of the 'Surmountable'}

The two types of simple ant-agonism/inhibition, competitive and non-competitive, may be summarized in a 'narrow' manner by statements of 'surmount-ability':

In non-competitive ant-agonism/inhibition, the relative effect of ant-agonists/inhibitors is always independent of varying concentrations of the agonist evoking a response. No matter what the value of a fixed concentration of an agonist, $S^{\prime}$, the full response is always reduced by the fraction $1 /(1+\mathrm{I})$ in the presence of non-competitive ant-agonists/inhibitors. The ant-agonism/inhibition is said to be insurmountable (Figs. 2.7C and 2.8C).

In competitive antagonism/inhibition, the relative effect of ant-agonists/inhibitors is always dependent on the concentrations of the agonist evoking a response, with an apparent dissociation constant for the ant-agonist/ inhibitor $K_{\mathrm{xi}}$ equal to $\left(K_{\mathrm{ss}} / \mathrm{S}\right) \cdot\left(1+\mathrm{I} / K_{\mathrm{xi}}\right)$, where $K_{\mathrm{xi}}$ is either $K_{\mathrm{si}}$ or $K_{\mathrm{ii}}$. The ant-agonism/inhibition is said to be surmountable, since with an increasing concentration of $\mathrm{S}$, the effect of competitive ant-agonism/inhibition may

\section{Box 2.1. Some papers on HAP- and Mon-land models for allostery and co-operativity}

Parameters. The letter $K$ is most often used to indicate an equilibrium dissociation constant and the letter $A$ an equilibrium association constant. The present text varies between association and dissociation constants as most convenient. At $K$ and $A$, subscripts are nearly always included as signifiers, while superscripts are often omitted. For subscripts and superscripts, see below.
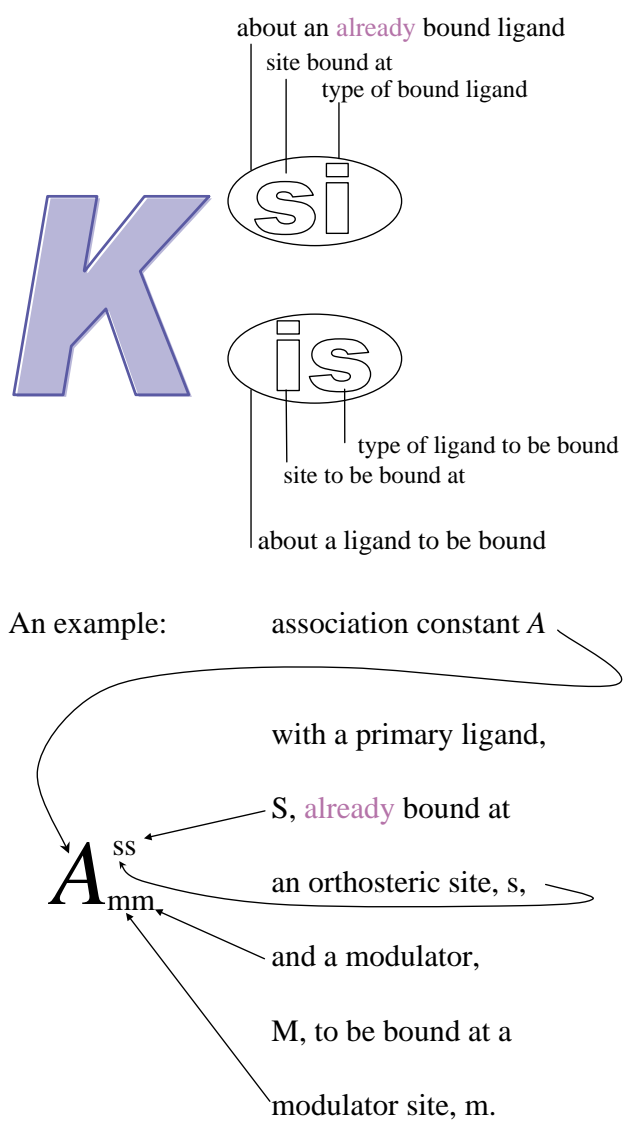

Ligands. A typical symbol for ligands such as substrates, agonists or transportees (transported molecules) is $\mathrm{S}$, while inhibitors, ant-agonists or interventors are designated with I, and modulators equal to allosteric ligands with M.

Receptive units. The symbol for a receptive unit as an enzyme, a receptor, or a transporter is usually $\mathrm{R}$. Receptive units often have more than one binding site. For a receptive unit with two binding sites, see, e.g., Fig. 2.1A. In reaction schemes, symbols to the right of the $\mathrm{R}$ term mean that they stand for ligands bound to a primary site on the receptive unit, for instance RS. When ligand symbols are to the left of the symbol $\mathrm{R}$ they indicate binding to a secondary site on the receptive unit, for instance IR. A primary binding site is also termed an 'orthosteric' 


\section{Box 2.1 (Continued)}

site, and a secondary binding site may be termed an 'intervention', 'modulation', or 'allosteric' site. For a receptive unit with two states in un-liganded conformations, see, e.g., Fig. 2.1B. These are represented by, for instance, the following two symbols: $\mathrm{R}$ and $\mathrm{R}^{*}$, where $\mathrm{R}$ is the reactive (not yet active) conformation and $\mathrm{R}^{*}$ is an active conformation.

Subscripts and superscripts. Symbols and conventions are described for subscripts and superscripts at $K$ and $A$. See Box 2.1 figure with $K$ as an example.

Subscripts may contain one or two signifiers. In case only one position is filled it refers to the ligand to be bound, for instance $K_{\mathrm{s}}$ is a dissociation constant for ligand $\mathrm{S}$ to be bound. If both positions in the subscript are presented, the first signifier indicates the site to be occupied, and the second signifier indicates the ligand to be bound.

The primary binding site is signified by $\mathrm{s}$, and the secondary binding site is signified by $\mathrm{i}$ or $\mathrm{m}$. Signifier $\mathrm{i}$ for the secondary site is related to intervention models (see text), while signifier $\mathrm{m}$ is related to the secondary site in allosteric models.

Three examples: (1) $A_{\mathrm{ss}}$ designates an association constant with the primary binding site to be occupied by ligand $\mathrm{S}$; (2) $K_{\mathrm{is}}$ designates a dissociation constant for binding to the secondary binding site by a ligand $\mathrm{S}$; and (3) $A_{\mathrm{ii}}$ indicates an association constant for binding to the secondary site of ligand I.

Superscripts. When a superscript is included, the same rules as those for subscripts apply except it is now for ligands already bound to the receptive unit, see Box 2.1 figure. The $K$ in Box-figure signifies a dissociation constant for ligand $S$ ready to bind to a secondary site in a receptive unit to which another ligand $I$ is already bound at the primary binding site.

For practice, first write (A) the equilibrium dissociation constant for an agonist $\mathrm{S}$ binding to a secondary binding site, assuming intervention models, and include the reaction scheme for such a process. Next write (B) the dissociation constant for a modulator, i.e., assuming allosteric models, binding at a primary site with an agonist already bound to the secondary site. The solutions are at the bottom of the Box.

Additional examples of parameter signifiers. The conformational constant for a switch between a reactive (not yet active) receptor state $\mathrm{R}$ and an active receptor state $\mathrm{R}^{*}$ is usually designated with an $L$. Thus, $\mathrm{R}^{*} / \mathrm{R}=L$. In case an agonist is already bound to an orthosteric site $\mathrm{m}$ the receptive unit before a conformational change, this is indicated in superscript. Thus, for instance, $L^{\mathrm{s}}, L^{\mathrm{ss}}$, or $L^{\prime}$ are for $\mathrm{RS} \rightleftharpoons \mathrm{R}^{*} \mathrm{~S}$ as in the dC\&K model (Chapter 5 ), and $L^{\text {is }}$ is for $\mathrm{SR} \rightleftharpoons \mathrm{SR}^{*}$ as in the homotropic two-state model (Chapter 7).

always be overcome (Figs. 2.7A and 2.8A). Since its introduction (Gaddum et al. 1955), the concepts of being surmountable/insurmountable have developed and, currently, seem to be employed in a more loose sense than defined above (Vauquelin et al. 2002 and references therein; Vauquelin \& Szczuka 2007).

\section{REFERENCES}

Abramow-Newerly M, Roy AA, Nunn C \& Chidiac P. RGS proteins have a signalling complex: interactions between RGS proteins and GPCRs, effectors, and auxiliary proteins. Cell Signal 18: 579-591, 2006.

Ariëns EJ. Molecular Pharmacology. The Mode of Action of Biologically Active Compounds. New York: Academic Press, 1964.

Ariëns EJ, Simonis AM \& de Groot WM. Affinity and intrinsic-activity in the theory of competitive- and non-competitive inhibition and an analysis of some forms of dualism in action. Arch Int Pharmacodyn 100: 298-322, 1955.
Avlani V, May LT, Sexton PM \& Christopoulos A. Application of a kinetic model to the apparently complex behavior of negative and positive allosteric modulators of muscarinic acetylcholine receptors. J Pharmacol Exp Ther 308: 1062-1072, 2004.

Bindslev N. A homotropic two-state model and auto-antagonism. BMC Pharmacol 4: 11(pp 1-22), 2004.

Birdsall B, Burgen AS, Rodrigues de Miranda J \& Roberts GC. Cooperativity in ligand binding to dihydrofolate reductase. Biochemistry 17: 2102-2110, 1978.

Blumer JB \& Lanier SM. Accessory proteins for G protein-signaling systems: activators of $\mathrm{G}$ protein signaling and other nonreceptor proteins influencing the activation state of $\mathrm{G}$ proteins. Receptors Channels 9: 195-204, 2003.

Boeynaems JM \& Dumont JE. Outlines of Receptor Theory. Amsterdam: Elsevier, 1980.

Bond RA \& Ijzerman AP. Recent developments in constitutive receptor activity and inverse agonism, and their potential for GPCR drug discovery. Trends Pharmacol Sci 27: 92-96, 2006.

Botts J \& Morales M. Analytical description of the effects of modifiers and of enzyme multivalency upon the steady state catalyzed reaction rate. Trans Faraday Soc 49: 696-707, 1953. 
Burgisser E, De Lean A \& Lefkowitz RJ. Reciprocal modulation of agonist and antagonist binding to muscarinic cholinergic receptor by guanine nucleotide. Proc Nat Acad Sci USA 79: 1732-1736, 1982.

Burzomato V, Beato M, Groot-Kormelink PJ, Colquhoun D \& Sivilotti LG. Single-channel behavior of heteromeric alphalbeta glycine receptors: an attempt to detect a conformational change before the channel opens. J Neurosci 24: 10924-10940, 2004.

Cha S. Tight-binding inhibitors-I. Kinetic behavior. Biochem Pharmacol 24: 2177-2185, 1975.

Cheng Y-C \& Prusoff WH. Relationship between the inhibition constant $(K I)$ and the concentration of inhibitor which causes 50 per cent inhibition (I50) of enzymatic reaction. Biochem Pharmacol 22: 3099-3108, 1973.

Chidiac P \& Roy AA. Activity, regulation, and intracellular localization of RGS proteins. Receptors Channels 9: 135-147, 2003.

Christopoulos A. From 'captive' agonism to insurmountable antagonism: demonstrating the power of analytical pharmacology. Clin Exp Pharmacol Physiol 28: 223-229, 2001.

Christopoulos A. Allosteric binding sites on cell-surface receptors: novel targets for drug discovery. Nat Rev Drug Discov 1: 198-210, 2002.

Christopoulos A \& Kenakin T. G protein coupled receptor allosterism and complexing. Pharmacol Rev 54: 323-374, 2002

Christopoulos A, May LT, Avlani VA \& Sexton PM. G-protein-coupled receptor allosterism: the promise and the problem(s). Biochem Soc Trans 32: 873-877, 2004.

Cleland WW. The kinetics of enzyme-catalyzed reactions with two or more substrates or products. Biochim Biophys Acta 67: 104-137, 1963.

Colquhoun D. Agonist-activated ion channels. BrJ Pharmacol 147: S17S26, 2006.

Cornish-Bowden A. A simple graphical method for determining the inhibition constants of mixed, uncompetitive and non-competitive inhibitors. Biochem J 137: 143-144, 1974.

Cornish-Bowden A. Fundamentals of Enzyme Kinetics. London: Portland Press, 1995.

Cornish-Bowden A. Fundamentals of Enzyme Kinetics. Colchester: Portland Press Ltd., 2004.

Costa T, Ogino Y, Munson PJ, Onaran HO \& Rodbard D. Drug efficacy at guanine nucleotide-binding regulatory protein-linked receptors: thermodynamic interpretation of negative antagonism and of receptor activity in the absence of ligand. Mol Pharmacol 41: 549-560, 1992.

Daeffler L \& Landry Y. Inverse agonism at heptahelical receptors: concept, experimental approach and therapeutic potential. Fundam Clin Pharmacol 14: 73-87, 2000.

De Lean A, Stadel JM \& Lefkowitz RJ. A ternary complex model explains the agonist specific binding properties of the adenylate cyclase coupled beta-adrenergic receptor. J Biol Chem 255: 7108$7117,1980$.

Dixon M \& Webb EC. Enzymes. London: Longman Group Ltd., 1979.

Dowling MR, Willets JM, Budd DC, Charlton SJ, Nahorski SR \& Challiss RA. A single point mutation (N514Y) in the human M3 muscarinic acetylcholine receptor reveals differences in the properties of antagonists: evidence for differential inverse agonism. J Pharmacol Exp Ther 317: 1134-1142, 2006.

Ebersole ER, Guttentag C \& Wilson PW. Nature of carbon monoxide inhibition of biological nitrogen fixation. Arch Biochem 3: 399418, 1944.

Ehlert FJ. Estimation of the affinities of allosteric ligands using radioligand binding and pharmacological null methods. Mol Pharmacol 33: 187-194, 1988.
Foucaud M, Tikhonova IG, Langer I, Escrieut C, Dufresne M, Seva C, Maigret B \& Fourmy D. Partial agonism, neutral antagonism, and inverse agonism at the human wild-type and constitutively active cholecystokinin-2 receptors. Mol Pharmacol 69: 680-690, 2006.

Friedenwald JS \& Maengwyn-Davies GD. Elementary kinetic theory of enzymatic activity. First order theory. In: The Mechanism of Enzyme Action, edited by McElroy WD \& Glass B. Baltimore: Johns Hopkins Press, 1954a, pp. 154-179.

Friedenwald JS \& Maengwyn-Davies GD. Elementary kinetic theory of enzymatic activity. Second order theory. In: The Mechanism of Enzyme Action, edited by McElroy WD \& Glass B. Baltimore: Johns Hopkins Press, 1954b, pp. 180-190.

Fukuto HS, Ferkey DM, Apicella AJ, Lans H, Sharmeen T, Chen W, Lefkowitz RJ, Jansen G, Schafer WR \& Hart AC. G Proteincoupled receptor kinase function is essential for chemosensation in C. elegans. Neuron 42: 581-593, 2004.

Gaddum JH, Hameed KA, Hathway DE \& Stephens FF. Quantitative studies of antagonists for 5-hydroxytryptamine. QJ Exp Physiol 40: 49-74, 1955.

Garcia Canovas F, Tudela J, Martinez Madrid C, Varon R, Garcia Carmona F \& Lozano JA. Kinetic study on the suicide inactivation of tyrosinase induced by catechol. Biochim Biophys Acta 912: 417423, 1987.

Haldane JBS. Enzymes. London: Longmans, Green \& Co., 1930.

Hall DA. Modeling the functional effects of allosteric modulators at pharmacological receptors: an extension of the two-state model of receptor activation. Mol Pharmacol 58: 1412-1423, 2000.

Hay DL, Christopoulos G, Christopoulos A \& Sexton PM. Determinants of BIBN4096BS affinity for CGRP and amylin receptors; the role of RAMP1. Mol Pharmacol 70: 1984-1991, 2006.

Hijazi NH \& Laidler KJ. Transient-phase and steady-state kinetics for enzyme systems involving two substrates. Can J Biochem 51: 832$840,1973$.

Hill C, Brownlie Z, Davey J, Milligan G \& Ladds G. Isolation and characterization of novel human RGS mutant displaying gain-offunction activity. Cell Signal 20: 323-336, 2008.

Jacobs S \& Cuatrecasas P. The mobile receptor hypothesis and 'cooperativity' of hormone binding. Application to insulin. Biochim Biophys Acta 433: 482-495, 1976.

Jenkinson DH. Classical approaches to the study of drug-receptor interactions. In: Textbook of Receptor Pharmacology, edited by Foreman JC \& Johansen T. Boca Raton: CRC Press, 2003, pp. 3-62.

Kaumann AJ \& Frenken M. ICI 169,369 is both a competitive antagonist and an allosteric activator of the arterial 5-hydroxytryptamine2 receptor system. J Pharmacol Exp Ther 245: 1010-1015, 1988.

Kenakin T. A Pharmacology Primer: Theory, Application, and Methods. Amsterdam: Elsevier Academic Press, 2004a.

Kenakin T. Allosteric modulators: the new generation of receptor antagonist. Mol Interv 4: 222-229, 2004b.

Kenakin T. Allosteric agonist modulators. J Recept Signal Transduct Res 27: 247-259, 2007.

Kenakin TP, Jenkinson S \& Watson C. Determining the potency and molecular mechanism of action of insurmountable antagonists. $J$ Pharmacol Exp Ther 319: 710-723, 2006.

Laidler KJ. General steady-state equations in enzyme and other catalyzed reactions. Trans Faraday Soc 52: 1374-1382, 1956.

Laidler KJ. The Chemical Kinetics of Enzyme Action. London: Oxford University Press, 1958.

Laidler KJ \& Socquet IM. Kinetic laws in catalysed systems. II. Reactions involving two substrates or a substrate and a coenzyme. $J$ Phys Colloid Chem 54: 530-536, 1950.

Laidler KJ \& Bunting PS. The chemical kinetics of enzyme action. Oxford: Oxford University Press, 1973. 
Leff P. The two state model of receptor activation. Trends Pharmacol Sci 16: 89-97, 1995

Levitzki A. Quantitative Aspects of Allosteric Mechanisms. Berlin: SpringerVerlag, 1978.

Lew MJ. Ziogas J \& Christopoulos A. Dynamic mechanisms of nonclassical antagonism by competitive AT(1) receptor antagonists. Trends Pharmacol Sci 21:376-381, 2000.

MacKay D. Interpretation of relative potencies, relative efficacies and apparent affinity constants of agonist drugs estimated from concentration-response curves. J Theor Biol 142: 415-427, 1990.

Mahler HR \& Cordes EH. Biological Chemistry. London: Harper \& Row, Ltd., 1966.

Mathiesen JM, Christopoulos A, Ulven T, Royer J, Campillo M, Heinemann A, Pardo L \& Kostenis E. On the mechanism of interaction of potent, surmountable and insurmountable antagonists with the prostaglandin D2 receptor CRTH2. Mol Pharmacol 69: 1441-1453, 2006.

May LT \& Christopoulos A. Allosteric modulators of G-protein-coupled receptors. Curr Opin Pharmacol 3: 551-556, 2003.

May LT, Avlani VA, Sexton PM \& Christopoulos A. Allosteric modulation of $\mathrm{G}$ protein-coupled receptors. Curr Pharm Des 10: 2003-2013, 2004.

May LT, Leach K, Sexton PM \& Christopoulos A. Allosteric modulation of G protein-coupled receptors. Annu Rev Pharmacol Toxicol 47: 151, 2007a.

May LT, Avlani VA, Langmead CJ, Herdon HJ, Wood MJ, Sexton PM \& Christopoulos A. Structure-function studies of allosteric agonism at M2 muscarinic acetylcholine receptors. Mol Pharmacol 72: 463$476,2007 \mathrm{~b}$

Michaelis L. Zur theorie der elektrolytischen dissociation der fermenten. Biochem Zeitschrift 60: 91-96, 1914.

Milligan G \& Bond RA. Inverse agonism and the regulation of receptor number. Trends Pharmacol Sci 18: 468-474, 1997.

Milligan G, Bond RA \& Lee M. Inverse agonism: pharmacological curiosity or potential therapeutic strategy? Trends Pharmacol Sci 16: $10-13,1995$

Morfis M, Christopoulos A \& Sexton PM. RAMPs: 5 years on, where to now? Trends Pharmacol Sci 24: 596-601, 2003.

Paton WD \& Rang HP. The uptake of atropine and related drugs by intestinal smooth muscle of the guinea-pig in relation to acetylcholine receptors. Proc R Soc Lond B Biol Sci 163: 1-44, 1965.

Rang HP. The kinetics of action of acetylcholine antagonists in smooth muscle. Proc R Soc Lond B Biol Sci 164: 488-510, 1966.

Rebois RV \& Hebert TE. Protein complexes involved in heptahelical receptor-mediated signal transduction. Receptors Channels 9: 169194, 2003.

Richard F, Barroso S, Martinez J, Labbe-Jullie C \& Kitabgi P. Agonism, inverse agonism, and neutral antagonism at the constitutively active human neurotensin receptor 2. Mol Pharmacol 60: 13921398, 2001.

Samama P, Cotecchia S, Costa T \& Lefkowitz RJ. A mutation induced activated state of the beta 2 adrenergic receptor. Extending the ternary complex model. J Biol Chem 268: 4625-4636, 1993.

Scheer A, Fanelli F, Costa T, De Benedetti PG \& Cotecchia S. Constitutively active mutants of the alpha $1 \mathrm{~B}$-adrenergic receptor: role of highly conserved polar amino acids in receptor activation. EMBO J 15: 3566-3578, 1996.

Schwartz TW \& Holst B. Ago-allosteric modulation and other types of allostery in dimeric 7TM receptors. J Recept Signal Transduct Res 26: 107-128, 2006.

Segal HL, Kachmar JF \& Boyer PD. Kinetic analysis of enzyme reactions. I. Further considerations of enzyme inhibition and analysis of enzyme activation. Enzymologia 15: 187-198, 1952.
Segel IH. Enzyme Kinetics. Behavior and Analysis of Rapid Equilibrium and Steady-state Enzyme Systems. New York: Wiley \& Sons (reissued 1993), 1975.

Sexton PM, Albiston A, Morfis M \& Tilakaratne N. Receptor activity modifying proteins. Cell Signal 13: 73-83, 2001.

Shou M. Kinetic analysis for multiple substrate interaction at the active site of cytochrome P450. Methods Enzymol 357: 261-276, 2002.

Shou M. Prediction of pharmacokinetics and drug-drug interactions from in vitro metabolism data. Curr Opin Drug Discov Dev 8: 66-77, 2005.

Shou M, Mei Q, Ettore MW Jr, Dai R, Baillie TA \& Rushmore TH. Sigmoidal kinetic model for two co-operative substrate-binding sites in a cytochrome P450 3A4 active site: an example of the metabolism of diazepam and its derivatives. Biochem J 340: 845853, 1999.

Shou M, Lin Y, Lu P, Tang C, Mei Q, Cui D, Tang W, Ngui JS, Lin CC, Singh R, Wong BK, Yergey JA, Lin JH, Pearson PG, Baillie TA, Rodrigues AD \& Rushmore TH. Enzyme kinetics of cytochrome P450-mediated reactions. Curr Drug Metab 2: 17-36, 2001.

Stockton JM, Birdsall NJ, Burgen AS \& Hulme EC. Modification of the binding properties of muscarinic receptors by gallamine. Mol Pharmacol 23: 551-557, 1983.

Takezako T, Gogonea C, Saad Y, Noda K \& Karnik SS. 'Network leaning' as a mechanism of insurmountable antagonism of the angiotensin II type 1 receptor by non-peptide antagonists. J Biol Chem 279: 15248-15257, 2004

Tolkovsky AM \& Levitzki A. Mode of coupling between the betaadrenergic receptor and adenylate cyclase in turkey erythrocytes. Biochemistry 17: 3795-3810, 1978 .

Topham CM. A generalized theoretical treatment of the kinetics of an enzyme-catalysed reaction in the presence of an unstable irreversible modifier. J Theor Biol 145: 547-572, 1990.

Topham CM \& Brocklehurst K. In defence of the general validity of the Cha method of deriving rate equations. The importance of explicit recognition of the thermodynamic box in enzyme kinetics. Biochem J 282: 261-265, 1992.

Trzeciakowski JP. Stimulus amplification, efficacy, and the operational model. Part II-ternary complex occupancy mechanisms. J Theor Biol 198: 347-374, 1999.

Udawela M, Hay DL \& Sexton PM. The receptor activity modifying protein family of $\mathrm{G}$ protein coupled receptor accessory proteins. Semin Cell Dev Biol 15: 299-308, 2004.

Udawela M, Christopoulos G, Tilakaratne N, Christopoulos A, Albiston A \& Sexton PM. Distinct receptor activity modifying protein (RAMP) domains differentially modulate interaction with calcitonin receptors. Mol Pharmacol 70: 1750-1760, 2006.

Varon R, Garcia-Canovas F, Garcia-Moreno M, Valero E, MolinaAlarcon M, Garcia-Meseguers MJ, Vidal de Labra JA \& Garridodel Sol C. Kinetic analysis of the general modifier mechanism of Botts and Morales involving a suicide substrate. J Theor Biol 218: 355-374, 2002

Vauquelin G \& Szczuka A. Kinetic versus allosteric mechanisms to explain insurmountable antagonism and delayed ligand dissociation. Neurochem Int 51: 254-260, 2007.

Vauquelin G, Van Liefde I \& Vanderheyden P. Models and methods for studying insurmountable antagonism. Trends Pharmacol Sci 23: 514-518, 2002

Vauquelin G \& von Mentzer B. G protein-coupled receptors. Molecular pharmacology from academic concept to pharmaceutical research. Chichester, Wiley \& Sons, 2007.

Vilardaga JP, Steinmeyer R, Harms GS \& Lohse MJ. Molecular basis of inverse agonism in a G protein-coupled receptor. Nat Chem Biol 1: 25-28, 2005. 
Walz D \& Caplan SR. Energy coupling and thermokinetic balancing in enzyme kinetics: microscopic reversibility and detailed balance revisited. Cell Biophys 12: 13-28, 1988.

Webb JL. Enzyme and Metabolic Inhibitors. Vol 1. General Principles of Inhibition. New York: Academic Press, 1963.

Weber G. Ligand binding and internal equilibria in proteins. Biochemistry 11: 878, 1972.

Weber G. Energetics of ligand binding to proteins. Adv Protein Chem 29: $1-83,1975$.

Wells JW. Analysis and interpretation of binding at equilibrium. In: Receptor-ligand Interactions. A Practical Approach, edited by Hulme
EC. Oxford: IRL Press at Oxford University Press, 1992, pp. 289 395.

Wreggett KA \& De Lean A. The ternary complex model. Its properties and application to ligand interactions with the D2-dopamine receptor of the anterior pituitary gland. Mol Pharmacol 26: 214 227, 1984.

Wyman J. Heme proteins. Adv Protein Chem 4: 407-531, 1948.

Wyman J. Linked functions and reciprocal effects in hemoglobin: A second look. Adv Protein Chem 19: 223-286, 1964.

Wyman J. Allosteric linkage. J Am Chem Soc 89: 2202-2218, 1967. 


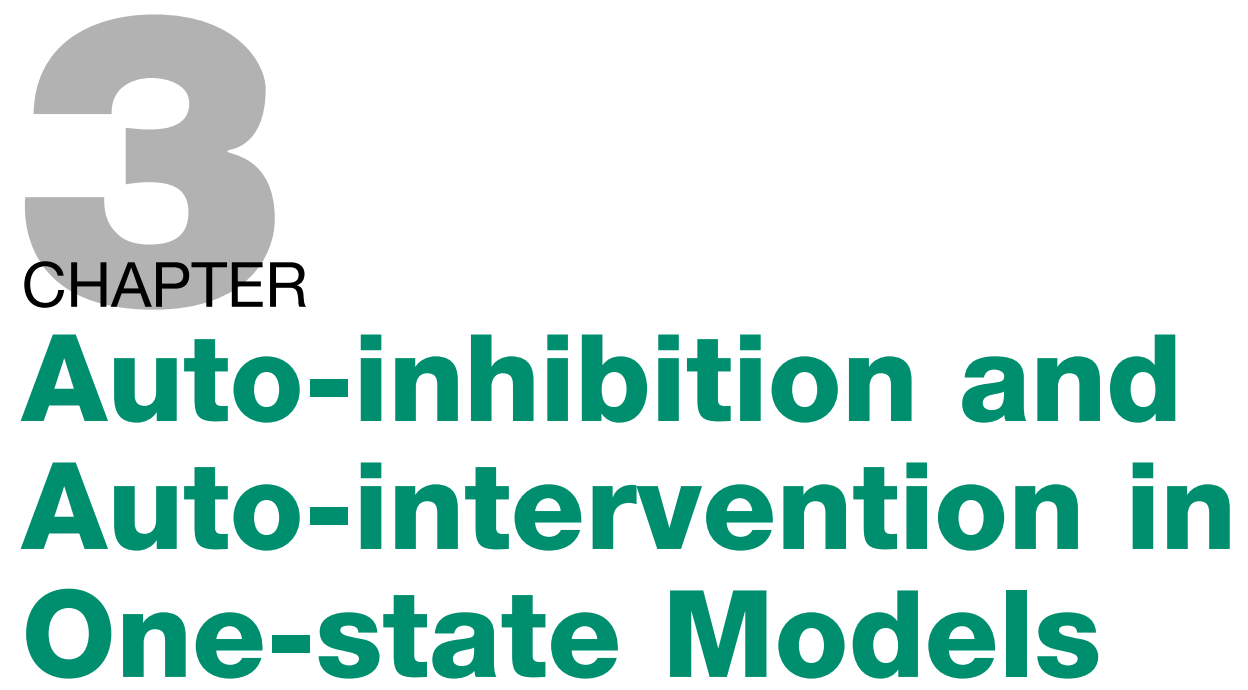

Several separate issues relate to biological self-regulation. In the field of ligand-receptor interactions, auto-regulation may relate to either ligand-induced alterations of a receptive unit or to control induced within receptive units per $s e$; before further down-stream regulations through feedback.

The themes of this chapter are divided into four divisions. The first division comprises of formulations for the modeling of ligand-dependent auto-inhibition (sub-chapters 3.1-3.3), as well as for a so-called 'lowdose hook-effect' (sub-chapter 3.4); all related to simple auto-intervention in one-state models.

The second division is a general introduction to biological auto-regulation and control (sub-chapter 3.5) which includes reflections on the meaning of 'self'.

The third division deals with concentration-dependent auto-inhibition and time-dependent phenomena, such as ligand-induced desensitization, both types eliciting autoregulatory inhibition in receptive systems with only one type of ligand present (sub-chapter 3.6). Sub-chapter 3.6 also treats various aspects of desensitization including homologous and heterologous desensitization with actual examples of intrinsic (receptor-dependent) and extrinsic (phosphorylation-dependent) desensitization.

The fourth division includes additional examples of models related to ligand-dependent auto-inhibition and desensitization (sub-chapter 3.7).

\subsection{Auto-intervention. Its Start and Terminology}

The formulation of auto-intervention has its own history, it requires certain conditions, and uses a special termi- nology. Therefore, before formulation of auto-intervention in sub-chapter 3.2, I will recount some conditions and give examples of terms related to auto-intervention.

\subsubsection{The Start of Formulating Ligand-dependent Auto-inhibition}

Enzymatic substrate-inhibition was originally formulated in 1930 by Sir John Burdon Sanderson Haldane in his treatise 'Enzymes' (Haldane 1930) (see Section 3.2.2).

Long before scientists from other fields, including physiology, enzymologists were formulating a selfinduced inhibition by activating ligands, i.e., substrates. Evidently, inhibition can take place for enzymes at high substrate - 'agonist' - concentrations, even when feedback inhibition due to product generation is ruled out (Kaiser 1980; Kuhl 1994). Thus, no regulatory mechanism can explain the concentration-dependent attenuation of enzymatic activity at high substrate concentration 'substrate inhibition' - other than through the substrate itself at a likely regulatory site. The result is bell-shaped dose-response curves. Similarly, ligand-induced inhibition at high concentration, which might yield bell-shaped synagics, is seen in many other fields including receptor studies (Trist \& Leff 1985; Winding \& Bindslev 1993; Bronnikov et al. 1999; Accomazzo et al. 2002; Hornigold et al. 2003; Schlee et al. 2006) and transport experiments with channels (Jow \& Numann 1999; Murayama et al. 1999; Zwart \& Vijverberg 2000; Hapfelmeier et al. 2003; Hong \& Wang 2005), with pumps (Andersen et al. 2001; Bucher et al. 2005; Einholm et al. 2007), with cotransporters (Berthelot et al. 2005), with uniporters (Alpers 2005; Vieira et al. 2005), and in motor string formation (Hayashi et al. 2007) 


\subsubsection{A One-state Model to Analyze Ligand- dependent Auto-inhibition}

Chapter 2 focused on simple inhibition by ligands different from the stimulatory agonist. In competitive type II and non-/ un-competitive intervention, binding of an interventor ligand at a secondary site prevents either the binding of agonists at the primary site or the possible conformational change needed for activation of the receptive unit. This is the hetero-intervention model without co-lateral binding presented in sub-chapter 2.5. On the contrary, as described here, in auto-intervention just one ligand is present and this ligand can now bind at the same time to an orthosteric site as well as to a secondary binding site. That is, co-lateral binding is possible and efficacy and affinity interactions between sites may be invoked concomitantly with a single type of ligand (depicted in Section 2.6.3 and shown in Fig. 2.4-2). Again, I'll use the terms ligand, agonist, and substrate interchangeably.

\subsubsection{Auto-inhibitory Concentration-binding and Dose-response Relationships}

Three simple reaction schemes of auto-inhibition are shown in Fig. 3.1A, B, and C. The scheme in Fig. 3.1C is equal to the lower right quadrant of the four-pane onestate model (FP-OSM), shown as quadrant number 2 of Fig. 2.4. This scheme has simply three parameters due to microscopic reversibility (e.g., Colquhoun et al. 2004).

C

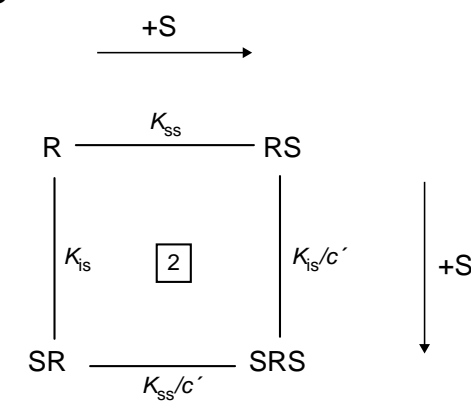

B

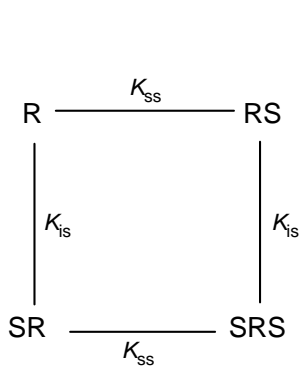

$\mathrm{D}$
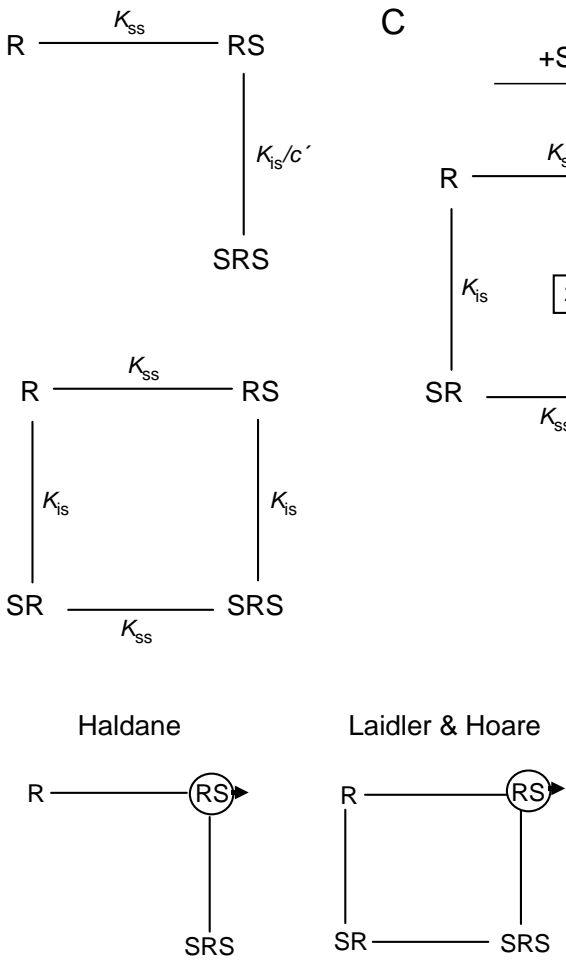

Complete

Negative intervention
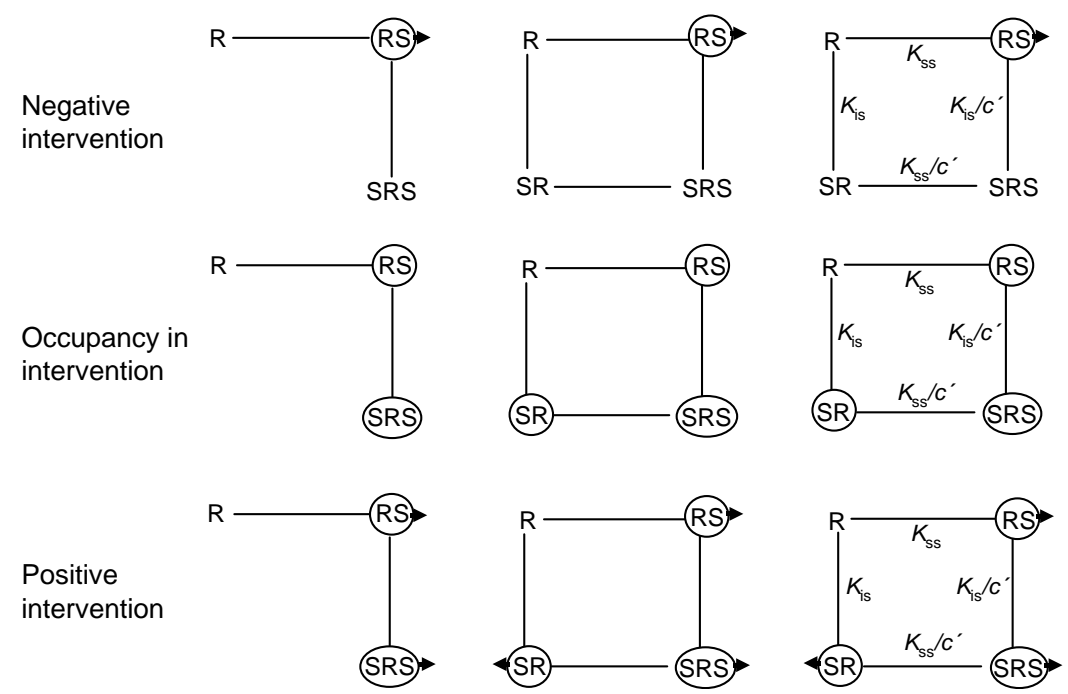

Figure 3.1. Forms of the auto-intervention one-state reaction scheme (Al-OSM). The models have two binding sites for the same ligand in a receptive unit R. (A) Haldane's model for substrate inhibition with the conformation SR missing (Haldane 1930). (B) Laidler-Hoare's model for substrate inhibition (Laidler \& Hoare 1949). (C) The complete auto-intervention model. Panel D details the liganded species in negative and positive functional intervention and in intervention occupancy by circling the species. Function, activity, is indicated by arrowheads. Parameter $c^{\prime}$ is a co-lateral interaction coefficient in a single-ligand system. Parameter $c^{\prime}$ is $\geq 0$ in the complete model. 
The three reaction schemes in Fig. 3.1 have a receptive unit with a primary and a secondary binding site, where a ligand $\mathrm{S}$ can bind to both the primary site and to the secondary site simultaneously. As mentioned, this means that the binding of a ligand $\mathrm{S}$ is both co-lateral and mutually inclusive, as defined in sub-chapter 2.6. Binding of a ligand to the secondary site is assumed to prevent activation of the receptive unit, i.e., the receptor conformations SR and SRS are possible but not activatable $($ Fig. $3.1 B+C)$. In such reaction schemes, only the RS conformation is ready to undergo the change to an active conformation, while SR and SRS are dead, functionally speaking. Such a behavior is designated 'auto-inhibition' or as 'negative co-operativity' by some, and in enzyme literature it is known as 'substrate-inhibition'.

In our present model of functional studies, since both conformations SR and SRS are non-active, the activity will always drop to zero at high enough agonist concentration, yielding a bell-shaped dose-response curve (Sections 3.2.2 and 3.2.3) (Haldane 1930; Laidler \& Hoare 1949; Laidler 1956, 1958; Harper 1971).

\subsubsection{Auto-intervention May Yield Bell-shaped Synagics and Changes in Apparent $K_{\mathbf{d}}$}

In auto-inhibition schemes as in Fig. 3.1, the agonist $S$ behaves both as an agonist when binding to the primary site and as a negative interventor when it binds to the secondary binding site of a receptor and prevents function. Therefore, in functional studies, it turns out that the negative intervention always results in bellshaped dose-responses, while in binding assays only the apparent dissociation constant differs from the real $K_{\mathrm{d}}$. In models representing binding studies, the maximum occupancy is unaffected by auto-inhibition.

In receptor literature without a specific model, autoinhibition is known as 'negative co-operativity', while in literature on carriers such as hemoglobin, 'positive cooperativity' is the characterizing term for augmented binding of $\mathrm{O}_{2}$ at increasing $\mathrm{O}_{2}$ concentration. Notice though, for carriers such as hemoglobin, as the concentration of the ligand increases, only the binding assay come into play where the result in comparison with simple load is an accelerated binding. Therefore, in the present context with one-state models, I suggest the term auto-intervention, which covers both changes in apparent $K_{\mathrm{d}}$, shallow or accelerated binding in concentration-occupancy relations, and full-blown bell-shaped dose-effects of self-inhibition. Accordingly, throughout this text, where one-state models are assumed, I will use the term 'auto-intervention' and reserve similar terms as 'co-operative' or 'auto-modulation' for allosteric twostate models (ATSMs) (Chapters 7 and 15). Herewith, we can discriminate between one- and two-state models and indicate which type of model is employed in our analysis. Of note, terms such as 'auto-intervention', 'co-operativity', and 'auto-modulation' do not preclude positive effects. Thus, with a one-state model for $\mathrm{O}_{2^{-}}$ binding to hemoglobin there is "positive auto-intervention' revealed as steepened dose-response curves.

Usually the term 'negative co-operativity' does not cover for bell-shaped dose-responses, but rather for shallow synagics below the load-hyperbolic doseresponse profile. Therefore, when analyzed by one-state models, a bell-shaped dose-response is also better designated with 'negative auto-intervention' or 'auto-inhibition'. Auto-intervention is either positive or negative.

The diagram in Fig. 3.1A is a scheme for 'auto-uncompetitive inhibition' and the diagram in Fig. 3.1B is a scheme for 'auto-non-competitive inhibition' (autoant-agonism). However, since both these reaction schemes are merely special cases of Fig. 3.1C, that is $c^{\prime} \neq 1$ versus $c^{\prime}=1$, we can include Fig. $3.1 \mathrm{~A}+\mathrm{B}$ reaction schemes under the term 'auto-intervention'.

For auto-intervention as presented in Fig. 3.1, observe that the terms 'positive' and 'negative' are related directly to which conformations are active or non-active and not to parameter $c^{\prime}$, cf. for instance with top and bottom row in Fig. 3.1D.

\subsubsection{Auto-intervention is Hetero-intervention-like}

The reaction scheme of auto-intervention in Fig. 3.1C is also depicted in a quadrant of the FP-OSM reaction scheme shown earlier (Fig. 2.4-2). Furthermore, the system to be analyzed as shown in Fig. 3.1C, is a look-alike of the hetero-intervention type reaction scheme shown in Fig. 2.4-1 and discussed in sub-chapter 2.5. Auto-intervention in one-state models is hetero-intervention-like because binding at the two sites are mutually inclusive and the co-lateral intervention constant $c$ is not necessarily equal to unity, which is also the case for the co-lateral intervention constant $c$ of the hetero-interventory reaction scheme (cf Fig. 2.4-1). The novelty in the present auto-intervention one-state model, as mentioned above, is the possibility of the same ligand interacting with both a primary site and a secondary site, equal to colateral binding. Co-lateral binding was not possible in the one-state hetero-intervention reaction scheme (subchapter 2.5 and Fig. 2.4-1).

\subsection{Formulation of Auto-intervention}

\subsubsection{Equations of Distribution for Simple Auto-intervention}

For the formulation of a one-state reaction scheme with one-ligand and a two-site receptive unit with co-lateral binding as shown in Fig. 3.1C, I first list the possible receptor conformations and system constants: 
$\mathrm{R}$ A receptive unit with two-binding-sites, an agonist $S$ can bind both to a primary site (RS) as well as to a secondary site (SR).

As before, symbols for receptor species in reaction schemes may also stand for the concentration of receptors and receptor-complexes, thus, $\mathrm{R}$ is also [R].

$\mathrm{RS}=\mathrm{R} \cdot \mathrm{S} / K_{\mathrm{ss}}$

The agonist, $\mathrm{S}$, is bound at a primary site in the receptive unit $\mathrm{R}$. Tacitly, $\mathrm{RS}$ is also symbol for the active form $\mathrm{R} * \mathrm{~S}$, which gives functional life to the receptor through a conformational isomerization. $K_{\mathrm{ss}}$ is the dissociation constant for $S$ at the primary site.

$\mathrm{SR}=\mathrm{R} \cdot \mathrm{S} / K_{\mathrm{is}} \quad \mathrm{SR}$ is a complex of the receptive unit $\mathrm{R}$ with an agonist $\mathrm{S}$ bound at a secondary or intervention site. $K_{\text {is }}$ is the dissociation constant for $\mathrm{S}$ at a secondary site. SR is assumed nonactive.

$\mathrm{SRS}=\mathrm{R} \cdot\left(\mathrm{S} / K_{\mathrm{ss}}\right) \cdot \quad \mathrm{SRS}$ is the complex of a receptive $\left(\mathrm{S} \cdot c^{\prime} / K_{\mathrm{is}}\right) \quad$ unit with an agonist bound simultaneously to its primary and secondary sites. SRS is assumed non-active.

$c^{\prime}$

A co-lateral intervention constant for ligand binding at one site when the other site is already liganded with the same type of ligand. Compare parameters $c$ and $c^{\prime}$ in Figs. 2.4-1 and 2.4-2.

As we shall soon experience, the assumption that both conformations SR and SRS are non-active is what leads to negative auto-intervention in the model.

To simplify matters, it is tacitly assumed that the reactive $\mathrm{RS}$ complex is in fast equilibrium with its activated state $\mathrm{R} * \mathrm{~S}$, although this conformation of the receptive unit will not be included in our formulation. Since we operate with a one-state model for autointervention, explicit formulations of $\mathrm{R}^{*}$ in general will not appear here; only later in TSMs (Chapters 5 and 7).

\subsubsection{JBS's Formulation of Substrate Inhibition}

Haldane $^{1}$ (1930, pp. 84-85) formulated the following equation in order to describe inhibition of enzymes at high substrate concentrations:

\footnotetext{
1 JBS Haldane held a professorship in physiology and was a reader in biochemistry when his book 'Enzymes' was published. He is one of my favorite milestone-idols in natural sciences, including population genetics. Among many other qualities, he was physically strong, smart, stubborn, sturdy, Stalinist, super-kind humanist, and bloodyminded. Quite an impossible man with a ferocious memory and intellect. JBS is decently depicted in a biography by Roland W. Clark (1968).
}

$$
\frac{p}{e}=\frac{\mathrm{x}}{K_{1}+\mathrm{x}+\mathrm{x}^{2} / K_{2}}
$$

which may by transcribed to the terminology used in this book for the receptor reaction scheme as highlighted in Fig. 3.1A:

$$
\begin{aligned}
\frac{a r}{T R} & =\frac{\mathrm{S} / K_{\mathrm{ss}}}{1+\mathrm{S} / K_{\mathrm{ss}}+\left(\mathrm{S} / K_{\mathrm{ss}} \cdot \mathrm{S} / K_{\mathrm{is}}^{\prime}\right)} \\
& =\frac{\mathrm{S}}{K_{\mathrm{ss}}+\mathrm{S}+\mathrm{S}^{2} / K_{\mathrm{is}}^{\prime}},
\end{aligned}
$$

where $p / e$ equals the fractional activity of actual response over total receptive units $=a r / T R, \mathrm{x}=\mathrm{S}=$ substrate or agonist concentration, $K_{1}=K_{\mathrm{ss}}$, and $K_{2}=$ $K_{\text {is }}^{\prime}$. The parameter $K_{\text {is }}^{\prime}$ is the equilibrium dissociation constant for $\mathrm{S}$ at an intervention site when $\mathrm{S}$ is already bound to the orthosteric site. $K_{\mathrm{is}}^{\prime}$ is also equal to $K_{\mathrm{is}} \cdot 1 / c^{\prime}$ (cf Fig. 3.1A+C). The value of $c^{\prime}$ may thus be included in the new parameter $K_{\text {is }}^{\prime}$ (see Section 3.2.3).

The functional dose-response relation according to Eq. 3.2 is shown in Figs. 3.2A $+\mathrm{B}$ and $3.3 \mathrm{~A}+\mathrm{B}$.

In his reaction scheme, Haldane disregarded a fourth conformation of the receptor, viz. SR, by only including the R, RS, and SRS forms of the enzyme, and therefore only included two equilibrium constants. Haldane's model resembles un-competitive ant-agonism, where binding to the secondary site is only possible when the primary site is liganded (Section 2.5.4) (Segel 1975, 1993, pp. 136-143).

Haldane's scheme is an ordered reaction scheme with a single type of ligand (see Chapter 6).

Lineweaver and Burk expanded the Haldane scheme slightly by allowing an exponentiation to a higher order than 2 (Lineweaver \& Burk 1934; Nayyar \& Glick 1956).

\subsubsection{A More Complete Model for Auto- intervention in Functional Assays}

Although assuming that $c^{\prime}=1$, a more complete distribution equation in functional assays of auto-intervention than that of Haldane can be formulated by including the SR conformation of the reaction scheme as shown in Fig. 3.1B. Its dose-response equation reads:

$$
\begin{aligned}
\frac{a r}{T R} & =\frac{\mathrm{S} / K_{\mathrm{ss}}}{1+\mathrm{S} / K_{\mathrm{ss}}+\mathrm{S} / K_{\mathrm{is}}+\left(\mathrm{S} / K_{\mathrm{ss}} \cdot \mathrm{S} / K_{\mathrm{is}}\right)} \\
& =\frac{\mathrm{S}}{K_{\mathrm{ss}}+\mathrm{S}+\mathrm{S} \cdot K_{\mathrm{ss}} / K_{\mathrm{is}}+\mathrm{S}^{2} / K_{\mathrm{is}}}
\end{aligned}
$$

with a denominator representing all four receptor conformations. In 1949 (Laidler \& Hoare), a similar equation had already been derived tacitly assuming parameter $c^{\prime}$ to be 1 . 
Plots of Laidler and Hoare's dose-response curves for function are shown in Figs. 3.2C $+\mathrm{D}$ and 3.3C $+\mathrm{D}$. The Haldane and Laidler-Hoare dose-response curves in functional studies are always bell-shaped. In both schemes when $K_{\mathrm{is}} / K_{\mathrm{ss}}>1000$, the maximal response approaches $100 \%$ and there is a broadening of the maximum plateau as the ratio increases (Fig. 3.3 panels $\mathrm{A}+\mathrm{B}$ and $\mathrm{C}+\mathrm{D})$. When the ratio $K_{\mathrm{is}} / K_{\mathrm{ss}}$ falls below 1000 , the two legs of the bell merge and the maximum response falls towards zero (Fig. 3.2 panels A+B and C+ D). Furthermore, as $K_{\mathrm{ss}}$ increases separately, the left leg of the bell is right-shifted (Figs. 3.2C and 3.3C), whereas when $K_{\text {is }}$ increases separately, the right leg of the bell moves to the right (Figs. 3.2D and 3.3D). When both $K_{\mathrm{ss}}$ and $K_{\text {is }}$ increase (move to the right) in a fixed ratio, the bell moves unchanged to the right as well (not shown).

Compared with the Laidler-Hoare scheme, Haldane's omission of SR does not dramatically alter the shape of the negative auto-intervention curve as long as $K_{\text {is }} / K_{\mathrm{ss}}>100$ (Figs. $3.2 \mathrm{~A}+\mathrm{B}$ versus $\mathrm{C}+\mathrm{D}$ and $3.3 \mathrm{~A}+\mathrm{B}$ versus $\mathrm{C}+\mathrm{D})$.

Notice, conformations involving the term $\mathrm{S} / K_{\text {is }}$ do not appear in the nominator of functional expressions (Eqs. 3.2 and 3.3), as they are considered non-functional conformations, while in binding studies, these terms
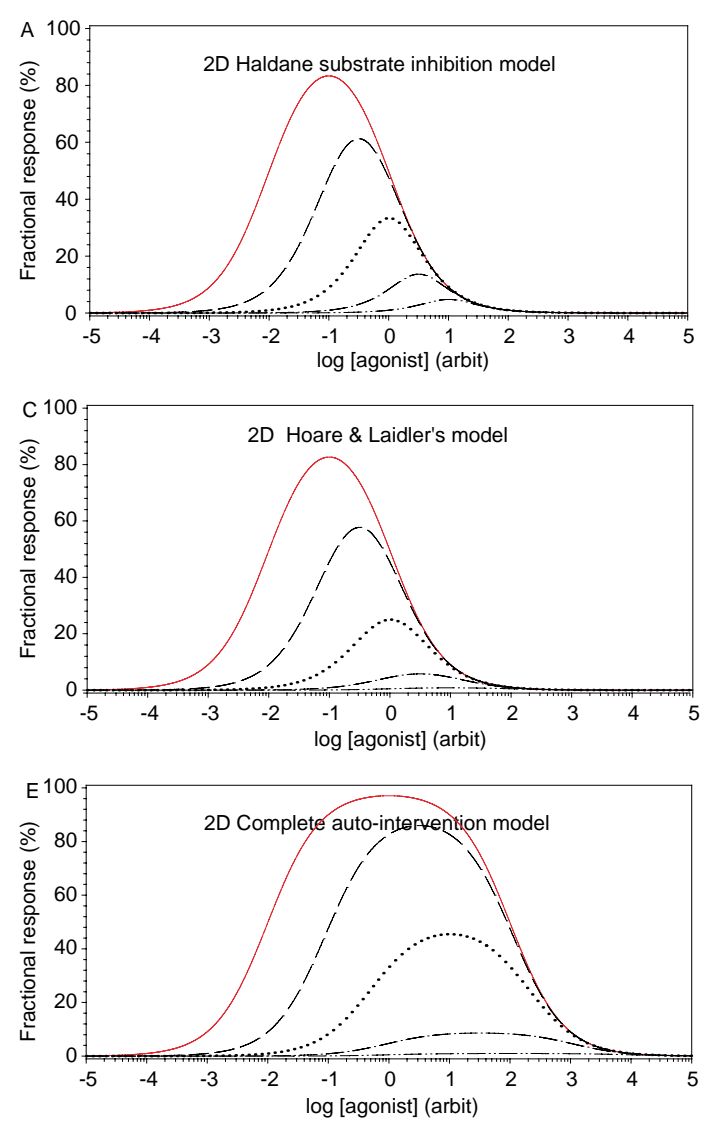

must appear in the nominator! Therefore, the above equations are valid for functional studies, but not for binding studies.

So far, we have assumed that $c^{\prime}$ is equal to 1 , meaning that $K_{\text {is }}^{\prime}=K_{\text {is }}$ or $K_{\text {is }}^{\text {ss }}=K_{\text {is }}$. See Box 2.1 on parameter sub- and superscripts.

\subsection{Completion of the Simple Auto-intervention Model}

\subsubsection{Auto-intervention for Function with Intervention Constant $C^{\prime} \neq 1$}

It is time to derive a formulation of the complete distribution function for the auto-inhibition one-state reaction scheme by including a co-lateral intervention parameter that deviates from unity, $c^{\prime} \neq 1$, as presented for functional studies in Fig. 3.1C. I re-emphasize that the co-lateral intervention constant $c$ in the heterointervention reaction schemes is now replaced with $c$ apostrophe $\left(c^{\prime}\right)$, just to indicate that they are two different parameters in two different reaction schemes. The apostrophed intervention constant $c^{\prime}$ is for two
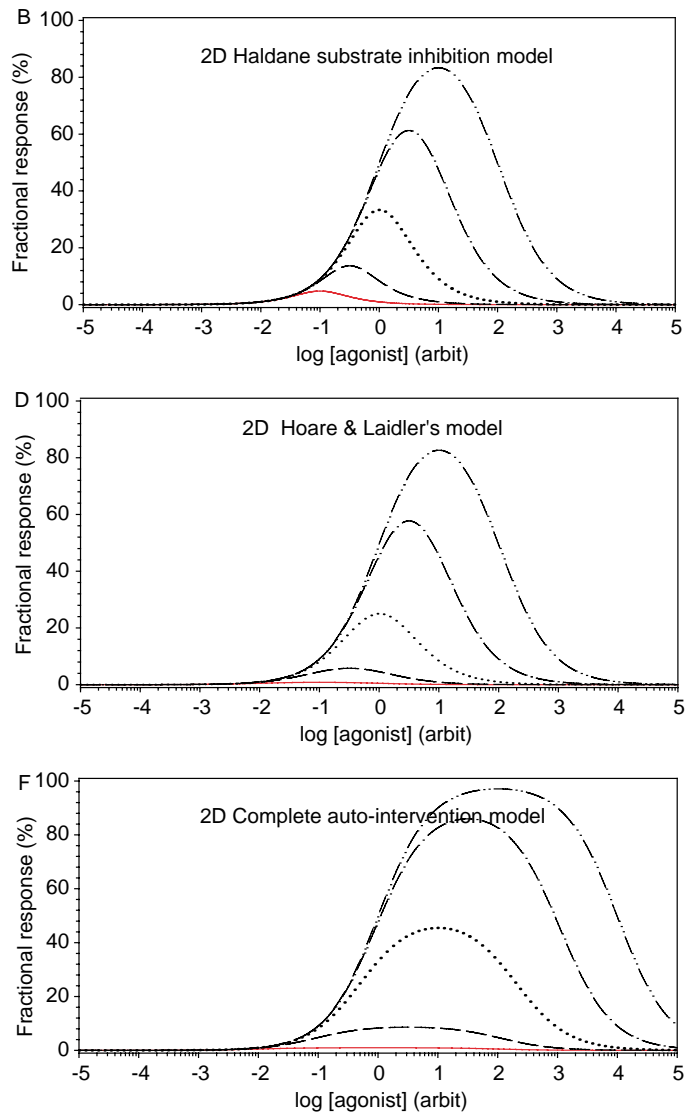

Figure 3.2. Functional dose-response curves of the three reaction schemes in Fig. 3.1. In A, C, and $\mathrm{E}, K_{\mathrm{ss}}$ vary between $10^{-2}$ $(-)$ and $10^{2}(-\cdots-)$ in five steps by a factor 10 , while $K_{\text {is }}=1$. In B, D and F, $K_{\text {is }}$ vary between $10^{2}(-)$ and $10^{-2}(-\cdots-)$ in five steps by a factor 10 , while $K_{\mathrm{ss}}=1$. Co-lateral coefficient $c^{\prime}$ is 1 in $\mathrm{A}, \mathrm{B}, \mathrm{C}$, and $\mathrm{D}$, and $c^{\prime}$ is 0.01 in $\mathrm{E}$ and $\mathrm{F}$. 

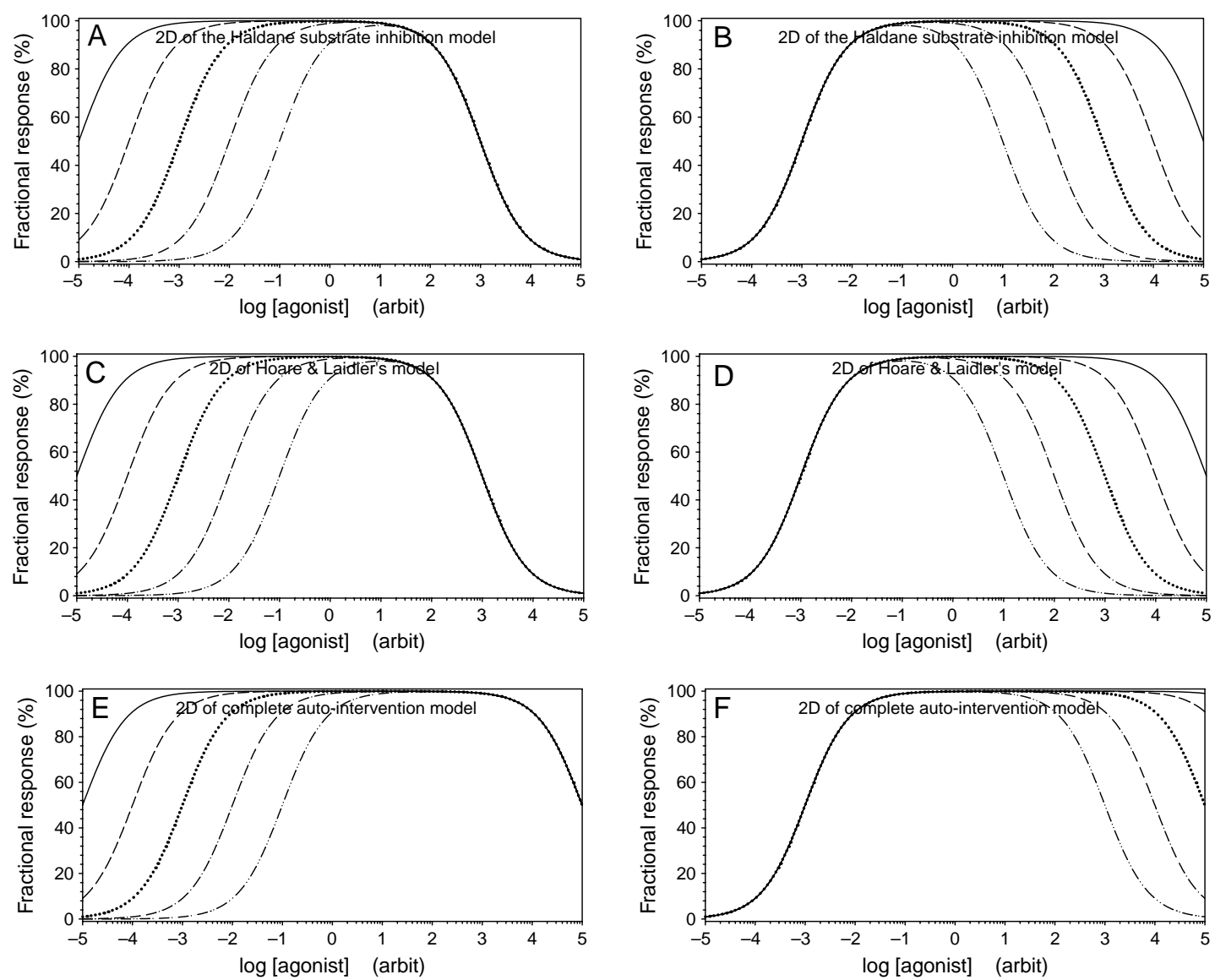

Figure 3.3. Functional dose-response curves of the three negative intervention schemes in Fig. $3.1 \mathrm{D}$. In A, C, and E, $K_{\mathrm{ss}}$ vary between $10^{-5}(-)$ and $10^{-1}(-\cdots-)$ in five steps by a factor 10 , while $K_{\text {is }}=1000$. In B, D, and F, $K_{\text {is }}$ vary between $10^{5}(-)$ and $10^{1}(-\cdots-)$ in five steps by a factor 10 , while $K_{\mathrm{ss}}=0.001$. Co-lateral coefficient $c^{\prime}$ is 1 in $\mathrm{A}, \mathrm{B}, \mathrm{C}$, and $\mathrm{D}$, and $c^{\prime}$ is 0.01 in $\mathrm{E}$ and $\mathrm{F}$.

identical ligands (Figs. 2.4-2 and 3.1C), whereas the intervention constant $c$, without an apostrophe, is for two different ligands (Fig 2.4-1).

Furthermore, I continue formulations by still employing dissociation constants instead of association constants in order to compare with formulations by Haldane and Laidler-Hoare in Sections 3.2.2 and 3.2.3.

For an equation of dose-response for the complete functional auto-intervention scheme (Fig. 3.1C), with the terminology given in Section 3.2.1, you should get:

$$
\frac{a r}{T R}=\frac{\mathrm{S} / K_{\mathrm{ss}}}{1+\mathrm{S} / K_{\mathrm{ss}}+\mathrm{S} / K_{\mathrm{is}}+\left(\mathrm{S} / K_{\mathrm{ss}}\right) \cdot\left(\mathrm{S} \cdot c^{\prime} / K_{\mathrm{is}}\right)},
$$

which may be rewritten to:

$$
=\frac{\mathrm{S}}{\mathrm{S}+\mathrm{S} \cdot K_{\mathrm{ss}} / K_{\mathrm{is}}+\mathrm{S}^{2} \cdot c^{\prime} / K_{\mathrm{is}}+K_{\mathrm{ss}}} .
$$

The dose-response curves of this equation for the complete reaction scheme of negative auto-intervention is depicted in Figs. $3.2 \mathrm{E}+\mathrm{F}$ and $3.3 \mathrm{E}+\mathrm{F}$. When an agonist is also a negative interventor in its own transduction pathway, it can be both a stimulator at low concentrations and an auto-inhibitor at higher concen- trations. Again, the negative intervention is due to nonactive conformations with $\mathrm{S}$ bound at the i-site.

The manner in which the three parameters $K_{\mathrm{ss}}, K_{\mathrm{is}}$, and $c^{\prime}$ affect the bell-shaped dose-responses is written up in Table 3.1A.

The distribution function in Eq. 3.5 of a negative complete auto-intervention model for activity was derived in the 1950s (Segal et al. 1952; Botts \& Morales 1953; Laidler 1956, 1958, pp. 77-79), and later even more elaborately analyzed by Harper (1971). Modern versions may be found in Shou (2005).

\subsubsection{Other Model Approaches to Experimentation Yielding Bell-shaped Synagics}

Mathematical modeling and parameter determination for bell-shaped synagics is possible with either a doubleload or a double-Hill formulation (see, e.g., Szabadi 1974; Jarv et al. 1993; Rovarti \& Nicosia 1994; Tucek et al. 2002; Griffen et al. 2003; Hornigold et al. 2003). However, a mechanistic interpretation of obtained parameter values is meaningless with these approaches. Therefore, let us return to aspects of the auto-intervention model. 
Table 3.1A. Function in three negative intervention models. The related reaction schemes are shown in the upper row of Fig. 3.1D. All dose-responses of the negative intervention models are bell-shaped (see Fig. 3.3A-C). As listed in the table, the bell height and the broadness of the bell-plateau are altered by varying one of three parameters, $K_{\mathrm{ss}}, K_{\text {is }}$, and $c^{\prime}$ indicated in parentheses, while keeping the others at unity. Haldane's model is described in Eq. 3.2, Laidler-Hoare's model (L\&H) is described in Eq. 3.3, and the complete intervention model is described in Eqs. 3.4 and 3.5.

\begin{tabular}{|c|c|c|c|c|c|c|c|}
\hline Model & Parameter value & $R_{\max }\left(K_{\mathrm{ss}}\right)(\%)$ & Bell move & $R_{\max }\left(K_{\mathrm{is}}\right)(\%)$ & Bell move & $R_{\max }\left(c^{\prime}\right)(\%)$ & Bell move \\
\hline \multirow[t]{3}{*}{ Haldane } & $<<1$ & 100 & Down to the Right* & 0 & Up to the Right* & 35 & No \\
\hline & $=1$ & 35 & & 35 & & & \\
\hline & $>>1$ & 0 & & 100 & & & \\
\hline \multirow[t]{3}{*}{ L\&H } & $<<1$ & 100 & Down to the Right* & 0 & Up to the Right* & 25 & No \\
\hline & $=1$ & 25 & & 25 & & & \\
\hline & $>>1$ & 0 & & 100 & & & \\
\hline \multirow[t]{3}{*}{ Complete } & $<<1$ & 100 & Down to the Right* & 0 & Up to the Right* & 50 & Down Left* \\
\hline & $=1$ & 25 & & 25 & & 25 & \\
\hline & $>>1$ & 0 & & 100 & & 0 & \\
\hline
\end{tabular}

*Lower values of $K_{\mathrm{ss}}$ and $c^{\prime}$ and increasing values of $K_{\mathrm{is}}$ result in a broadening of the bell-plateau (see also Fig. 3.3).

\subsubsection{Auto-intervention for Binding with Intervention Constant $\mathrm{c}^{\prime} \neq 1$}

Next, let us look at the reaction scheme for autointervention in a concentration-binding regime, where receptive units with two bound ligands count twice (Fig. 3.1C). The fractional binding in this reaction scheme has the following equation:

$\frac{\text { occupancy }}{\text { total }}=\frac{\mathrm{S} / K_{\mathrm{ss}}+\mathrm{S} / K_{\mathrm{is}}+2 \cdot\left(\mathrm{S} / K_{\mathrm{ss}}\right) \cdot\left(\mathrm{S} \cdot c^{\prime} / K_{\mathrm{is}}\right)}{1+\mathrm{S} / K_{\mathrm{ss}}+\mathrm{S} / K_{\mathrm{is}}+2 \cdot\left(\mathrm{S} / K_{\mathrm{ss}}\right) \cdot\left(\mathrm{S} \cdot c^{\prime} / K_{\mathrm{is}}\right)}$,

which also reads:

$$
\frac{\text { occupancy }}{\text { total }}=\frac{\mathrm{S}}{\mathrm{S}+\frac{K_{\mathrm{ss}}}{1+K_{\mathrm{ss}} / K_{\mathrm{is}}+2 \cdot \mathrm{S} \cdot c^{\prime} / K_{\mathrm{is}}}} .
$$

The terms multiplied by a factor 2 take care of the double-liganded receptive units.

Similar equations for binding in the Laidler-Hoare and Haldane's reaction schemes are as follows:

$$
\frac{\text { occupancy }}{\text { total }}=\frac{\mathrm{S}}{\mathrm{S}+\frac{K_{\mathrm{ss}}}{1+K_{\mathrm{ss}} / K_{\mathrm{is}}+2 \cdot \mathrm{S} / K_{\mathrm{is}}}} .
$$

for the Laidler-Hoare scheme shown in Fig. 3.1B, and:

$$
\frac{\text { occupancy }}{\text { total }}=\frac{\mathrm{S}}{\mathrm{S}+\frac{K_{\mathrm{ss}}}{1+2 \cdot \mathrm{S} / K_{\mathrm{is}}^{\prime}}} \text {. }
$$

for the Haldane scheme shown in Fig. 3.1A.

Examples of plots for these concentration-binding relationships are shown in Figs. $3.4 \mathrm{~A}+\mathrm{B}$ and $3.5 \mathrm{~A}+\mathrm{B}$ for Haldane, Figs. 3.4C+D and 3.5C $+\mathrm{D}$ for LaidlerHoare, and Figs. 3.4E $+\mathrm{F}$ and $3.5 \mathrm{E}+\mathrm{F}$ for the complete auto-intervention scheme. Table $3.1 \mathrm{~B}$ gives a summary of the effects on binding steepness by varying one of the three parameters $K_{\mathrm{ss}}, K_{\mathrm{is}}$, and $c^{\prime}$.
When occupancy is by a radioactive isotope and displacement is by an identical cold isotope, Eq. $3.7 a$ is expanded and can demonstrate the so-called 'low-dose hook-effect' (see sub-chapter 3.4).

\subsubsection{Positive Auto-intervention in Functional Studies}

Positive auto-intervention of function is described by a set of equations similar to Eqs. 3.7 $a-c$ for binding with the sole modification that the factor ' 2 ' is omitted. Therefore, plots of positive functional auto-intervention are almost identical to plots of the concentration-binding relations shown in Figs. 3.4 and Fig. 3.5. The steepness of the doseresponse relations are determined by the value of parameters $K_{\mathrm{ss}}, K_{\mathrm{is}}$, and $c^{\prime}$. The effects of varying one of the three parameters on this dose-response steepness are quantified by the Hill coefficient $n_{\mathrm{H}}$ in Table 3.1C.

\subsubsection{Conversion of Hetero-intervention into a One-state Substrate Inhibition Model}

You should realize that in an auto-intervention model for substrate inhibition, the dose-response formula for a ligand $\mathrm{S}$ that is both an agonist at an s-site and a negative interventor at an i-site, is in principle similar to doseresponse equations for hetero-intervention in a mutually exclusive one-state model (ME-OSM). Here an agonist S can only bind to an s-site and a different ligand, I, can only bind to an i-site on the receptive unit (see subchapter 2.6.3 and Section 3.1.5). Thus, the formula for negative functional hetero-intervention (Eq. 2.17 in subchapter 2.5), may easily be re-formulated to functional auto-intervention by simply substituting 'I' with 'S', keeping ' $i$ ' in the subscripts first position, and in the second subscript position replacing ' $i$ ' with 's' at relevant positions in the pertinent equations. This, for instance, results in $K_{\mathrm{ii}}$ transformed to $K_{\mathrm{is}}$. Further, $c$ is now $c^{\prime}$. With these changes, Eq. 2.17 is equal to Eq. 3.4. 

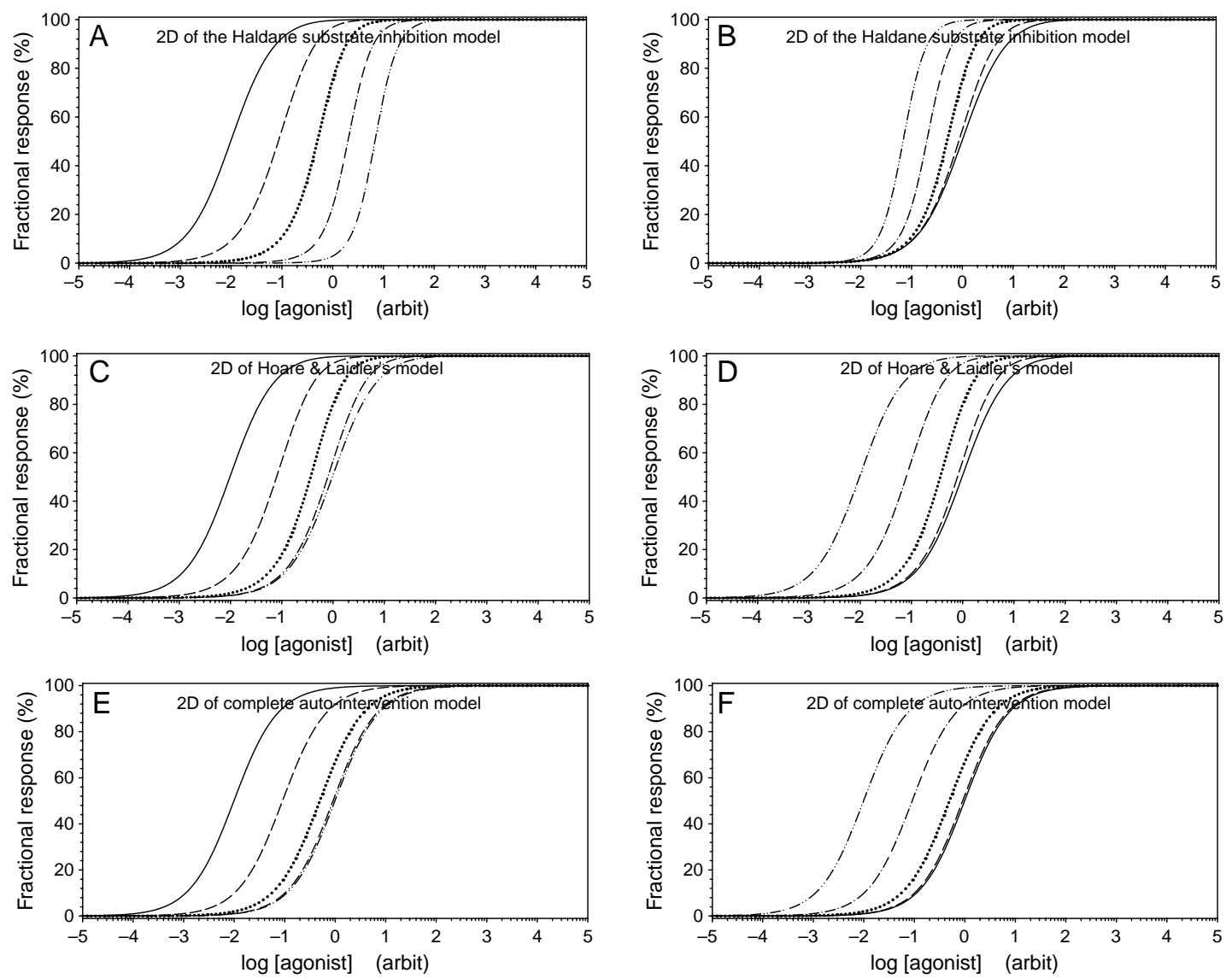

Figure 3.4. Binding concentration-occupancy curves of the three intervention schemes in Fig. 3.1D. In A, C, and E, $K_{\mathrm{ss}}$ vary between $10^{-2}(-)$ and $10^{2}(-\cdots-)$ in five steps by a factor 10 , while $K_{\text {is }}=1$. In B, D, and F, $K_{\text {is }}$ vary between $10^{2}(-)$ and $10^{-2}$ $(-\cdots-)$ in five steps by a factor 10 , while $K_{\mathrm{ss}}=1$. Co-lateral coefficient $c^{\prime}$ is 1 in $\mathrm{A}, \mathrm{B}, \mathrm{C}$, and $\mathrm{D}$, and $c^{\prime}$ is 0.01 in $\mathrm{E}$ and $\mathrm{F}$.

\subsubsection{Switch of Hetero-intervention in 3-D to Auto-intervention in 2-D}

Three-dimensional surface-plots for the functional version of the hetero-intervention model are shown in Fig. 2.11. In experiments where a mixture of substrate and interventor in a fixed ratio of concentrations is used to change ligand concentration, the surface-plots change into 2-D plots, as shown by the arrows in Fig. 2.11. Hence, hetero-intervention in 3-D in Fig. 2.11 is converted to auto-intervention in 2-D as in Figs. 3.2 and 3.3. The conclusion from Figs. 2.11 and 3.2-3.3 is that auto-intervention is a special case of the more general hetero-intervention scheme.

\subsubsection{Conclusions on the Auto-intervention Behavior}

The behavior of the auto-intervention reaction scheme for concentration-binding and of the auto-intervention reaction scheme for the dose-response relation is summarized in Table 3.1A, B, and $\mathrm{C}$ while examples of the impact of varying single parameter values of $K_{\mathrm{ss}}, K_{\mathrm{is}}$, or $c^{\prime}$ in the functional scheme are shown in Figs. 3.2 and 3.3 and for the binding scheme in Figs. 3.4 and 3.5.
For functional dose-response curves, all the fractional response curves display bell-shaped behavior. The drop in activity at high ligand concentrations for the doseresponse curves is simply due to the fact that neither the SR nor the SRS conformations of the receptive units contribute to the activity (Figs. 3.2 and 3.3).

As the value of $c^{\prime}$ decreases below unity, the co-lateral intervention parameter $c^{\prime}$ has a broadening effect on the bell-shaped dose-responses (compare panel E with panels $\mathrm{A}$ and $\mathrm{C}$ and panel $\mathrm{F}$ with panels $\mathrm{B}$ and $\mathrm{D}$ in Figs. 3.2 and 3.3). Parameter $c^{\prime}$ also determines the steepness of concentration-occupancy and positive autointervention dose-responses in functional relations. Compare these effects of $c^{\prime}$ with the effects of the same parameter in the functional homotropic two-state model (HOTSM) (Chapter 7). The effects of constant $c^{\prime}$ may also be compared with the effects of the similar parameter $c$ in the functional hetero-intervention model (sub-chapter 2.5), and in dose-responses of the ATSM described in Chapter 7.

All the fractional concentration-binding curves increase continuously towards unity as the ligand concentration increases and the apparent dissociation constant is dependent on the relation between $K_{\mathrm{ss}}, K_{\mathrm{is}}$, and $c^{\prime}$, as 
Table 3.1B. Occupancy in three intervention models. The related reaction schemes are shown in the middle row of Fig. 3.1D. All dose-binding curves of the occupancy intervention models reach maximal binding with increasing ligand concentration (see Figs. 3.4A-C). As listed in the table, the steepness of curves and the position on the concentration axis are altered by varying one of three parameters, $K_{\mathrm{ss}}, K_{\mathrm{is}}$, and $c^{\prime}$ indicated in parentheses, while keeping the others at unity. Haldane's model is described in Eq. 3.7c, Laidler-Hoare's model (L\&H) is described in Eq. 3.7b, and the complete intervention model is described in Eq $3.7 a$.

\begin{tabular}{llllllll}
\hline Model & Parameter & $n_{\mathrm{H}}\left(K_{\mathrm{ss}}\right)$ & Curve move & $n_{\mathrm{H}}\left(K_{\mathrm{is}}\right)$ & Curve move & $n_{\mathrm{H}}\left(c^{\prime}\right)$ & Curve move \\
\hline Haldane & $<<1$ & 1.0 & Right & 2.0 & Right & $1.468\left(c^{\prime}=0\right)$ & No \\
& $=1$ & 1.468 & & 1.468 & & & \\
& $>>1$ & 2.0 & & 1.0 & & & No \\
\hline L\&H & $<<1$ & 1.0 & Right & 1.0 & Right & $1.267\left(c^{\prime}=1\right)$ & \\
& $=1$ & 1.267 & & 1.267 & & & \\
& $>>1$ & 1.0 & & 1.0 & & & Left \\
\hline Complete & $<<1$ & 1.0 & Right & 1.0 & Right & 1.0 & \\
& $=1$ & 1.267 & & 1.267 & & 1.267 & \\
& $>>1$ & 1.0 & & 1.0 & & 2.0 & \\
\hline
\end{tabular}

indicated in Table 3.1 and shown by examples in Figs. 3.4 and 3.5.

When the hetero-intervention reaction scheme is conducted with a mixture of agonist and interventor in a fixed ratio (Fig. 2.11), the result is equal to the autointervention dose-response relationship as shown in Figs. 3.2 and 3.3 (see Sections 3.3.4 and 3.3.5).
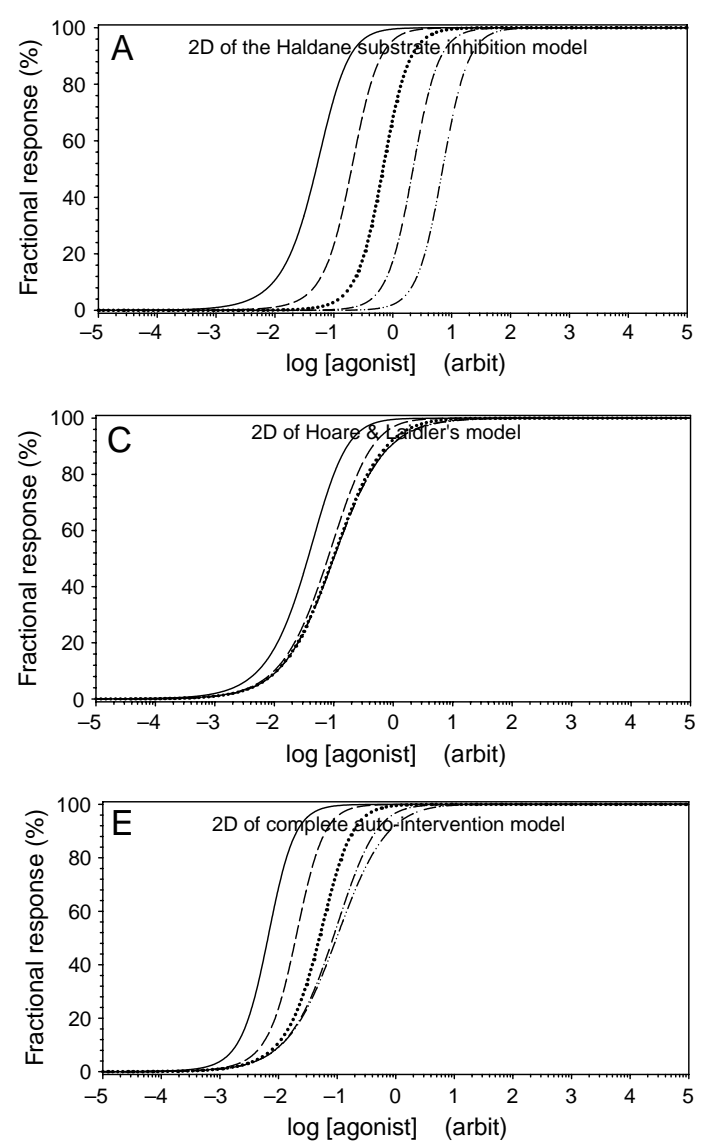

\subsection{The 'Low-dose Hook Effect'}

In concentration-occupancy studies, there are two hook effects-the 'high' and the 'low' hook effect.

In binding studies, observed convex bell-shaped relations are described in the literature as a 'high-dose hook effect' when using high doses of antigen in so-called 2-site
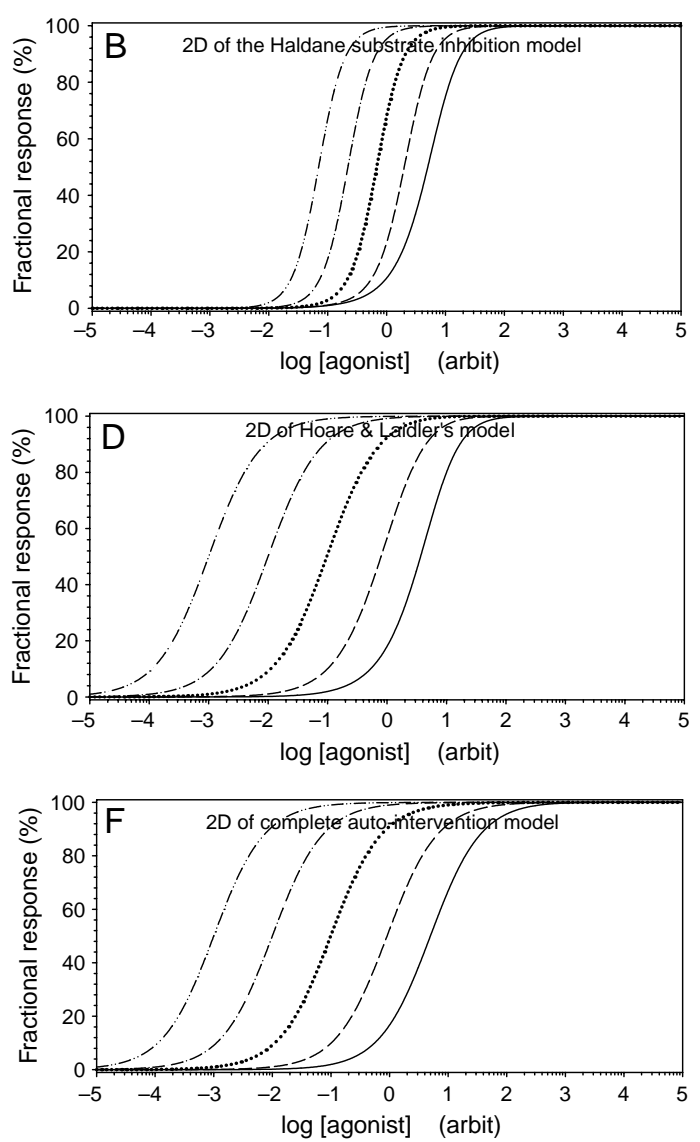

Figure 3.5. Functional dose-response curves of the three positive intervention schemes in Fig. 3.1D. In A, C, and E, $K_{\mathrm{ss}}$ vary between $10^{-1}(-)$ and $10^{3}(-\cdots)$ in five steps by a factor 10 , while $K_{\text {is }}=0.1$. In B, D, and F, $K_{\text {is }}$ vary between $10^{1}(-)$ and $10^{-3}$ $(-\cdots-)$ in five steps by a factor 10 , while $K_{\mathrm{ss}}=10$. Co-lateral coefficient $c^{\prime}$ is 1 in $\mathrm{A}, \mathrm{B}, \mathrm{C}$, and $\mathrm{D}$, and $c^{\prime}$ is 0.01 in $\mathrm{E}$ and $\mathrm{F}$. 
Table 3.1C. Function in three positive intervention models. The related reaction schemes are shown in the lower row of Fig. 3.1D. All dose-responses of the functional form of positive intervention models reach maximal activation with increasing ligand concentration (see Fig. 3.5A-C). As listed in the table, the steepness of curves and the position on the concentration axis are altered by varying one of three parameters, $K_{\mathrm{ss}}, K_{\mathrm{is}}$, and $c^{\prime}$ indicated in parentheses, while keeping the others at unity. By removing a factor 2 from the following three equations: Haldane's model is described in Eq. 3.7c, Laidler-Hoare's model (L\&H) is described in Eq. 3.7b, and the complete intervention model is described in Eq. 3.7a.

\begin{tabular}{llllllll}
\hline Model & Parameter & $n_{\mathrm{H}}\left(K_{\mathrm{ss}}\right)$ & Curve move & $n_{\mathrm{H}}\left(K_{\mathrm{is}}\right)$ & Curve move & $n_{\mathrm{H}}\left(c^{\prime}\right)$ & Curve move \\
\hline Haldane & $<<1$ & 1.0 & Right & 2.0 & Right & $1.364\left(c^{\prime}=0\right)$ & No \\
& $=1$ & 1.364 & & 1.364 & & & \\
& $>>1$ & 2.0 & & 1.0 & & & \\
\hline L\&H & $<<1$ & 1.0 & Right & 1.0 & Right & $1.184\left(c^{\prime}=1\right)$ & No \\
& $=1$ & 1.184 & & 1.184 & & & \\
& $>>1$ & 1.0 & & 1.0 & & & \\
\hline Complete & $<<1$ & 1.0 & Right & 1.0 & Right & 1.0 & Left \\
& $=1$ & 1.184 & & 1.184 & & 2.0 & \\
\hline
\end{tabular}

immuno-radio-metric assays (2-site IRMA) curves (Fig. 3.6) (Miles et al. 1974; Rodbard 1988; Leboeuf et al. 2006, and references therein). Miles and coworkers first suggested an explanation for the 'high-dose hook effect' seen in 2-site IRMAs. At high antigen concentration, a non-specific lowaffinity binding of the antigen to the solid-phase antibody in a first reaction may interfere with available labeled antibody in a second step, thus reducing the number of labeled antibodies present for binding to insolubilized antigen (Miles et al. 1974; Miles 1975).

Another 'hook' relation is seen in homologous displacement studies in radio-immuno assays (RIAs). This other type of bell-shaped displacement curve is

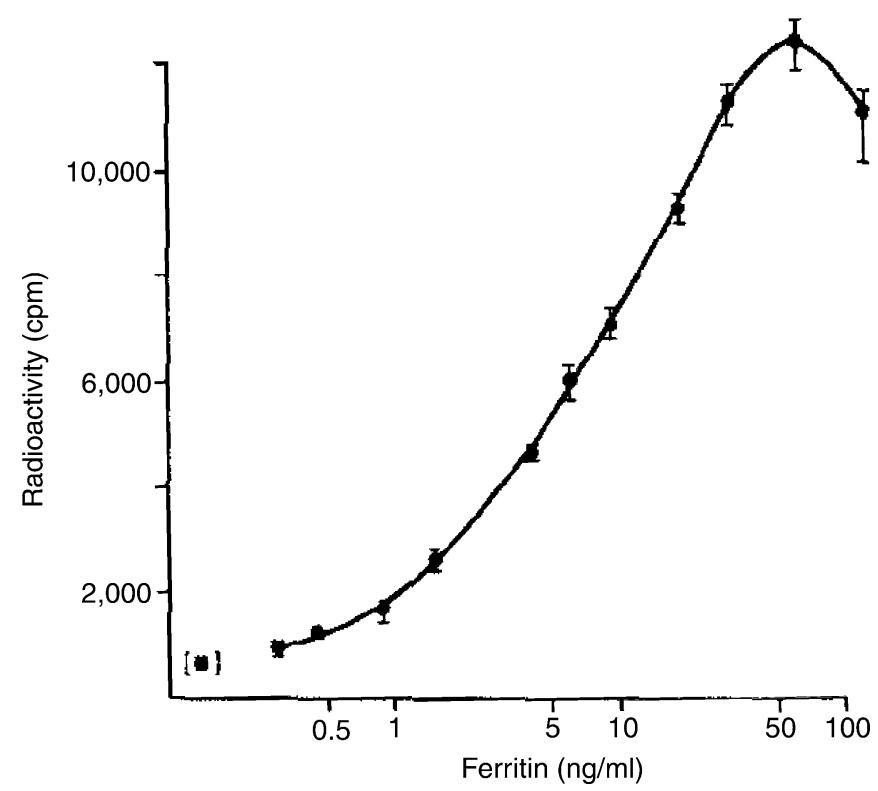

Figure 3.6. High-dose hook effect. This dose-response relation is the first described example of a 'high-dose hook effect' in a 2-site immuno-radiometric assay (2-site IRMA; Miles et al. 1974, Fig. 6A). See text for an explanation. Reproduced with permission. sometimes observed at low concentrations of cold ligand used to displace a homologous radio-ligand (Fig. 3.7) (Matsukura et al. 1971). I call this response the 'low-dose hook effect'.

An explanation for the 'low-dose hook effect' was given by Swillens et al. (1995) as due to positive interaction between two binding sites resulting in bellshaped displacement curves (Fig. 3.8). The Swillens scheme is developed further in Sections 3.4.2-3.4.4.

Non-equilibrium condition is another explanation for low-dose hook effects, as formulated by Lazareno and coworkers (Lazareno \& Birdsall 1995; Lazareno et al. 2000) and may be an explanation for the observed hooks in Fig. 3.7. Non-equilibrium conditions were likewise suggested as an explanation for an observed low-dose hook effect in a heterologous setup with KT5720 as interventor in the binding of muscarinic toxin MT-7 (Onali et al. 2005) to the M1 muscarinic subtype receptor (Fig. 3.9) (Fruchart-Gaillard et al. 2006, Fig. 8A). Meanwhile, a two-site hypothesis for the 'low-dose hook effect', to be discussed and analyzed in more details in the following sections, was not tested by these authors.

Note, the heterotropic association-dissociation bellshaped relations, described by Monod and coworkers (Monod et al. 1963; Fig. 3), are a different story.

\subsubsection{Low-dose Hook Effects}

In RIA-displacement studies, an increase instead of a decrease in radio-ligand binding is sometimes observed at the start of increasing concentrations of cold isotope displacer. Thus, at concentrations in proximity to the common dissociation constant for a homologous and non-radioactive ligand used as a displacer, an increase is sometimes observed in bound radio-activity before a drop in bound radio-activity as concentrations of the cold isotope are increased. Swillens and co-workers have presented a simple reaction scheme involving two 

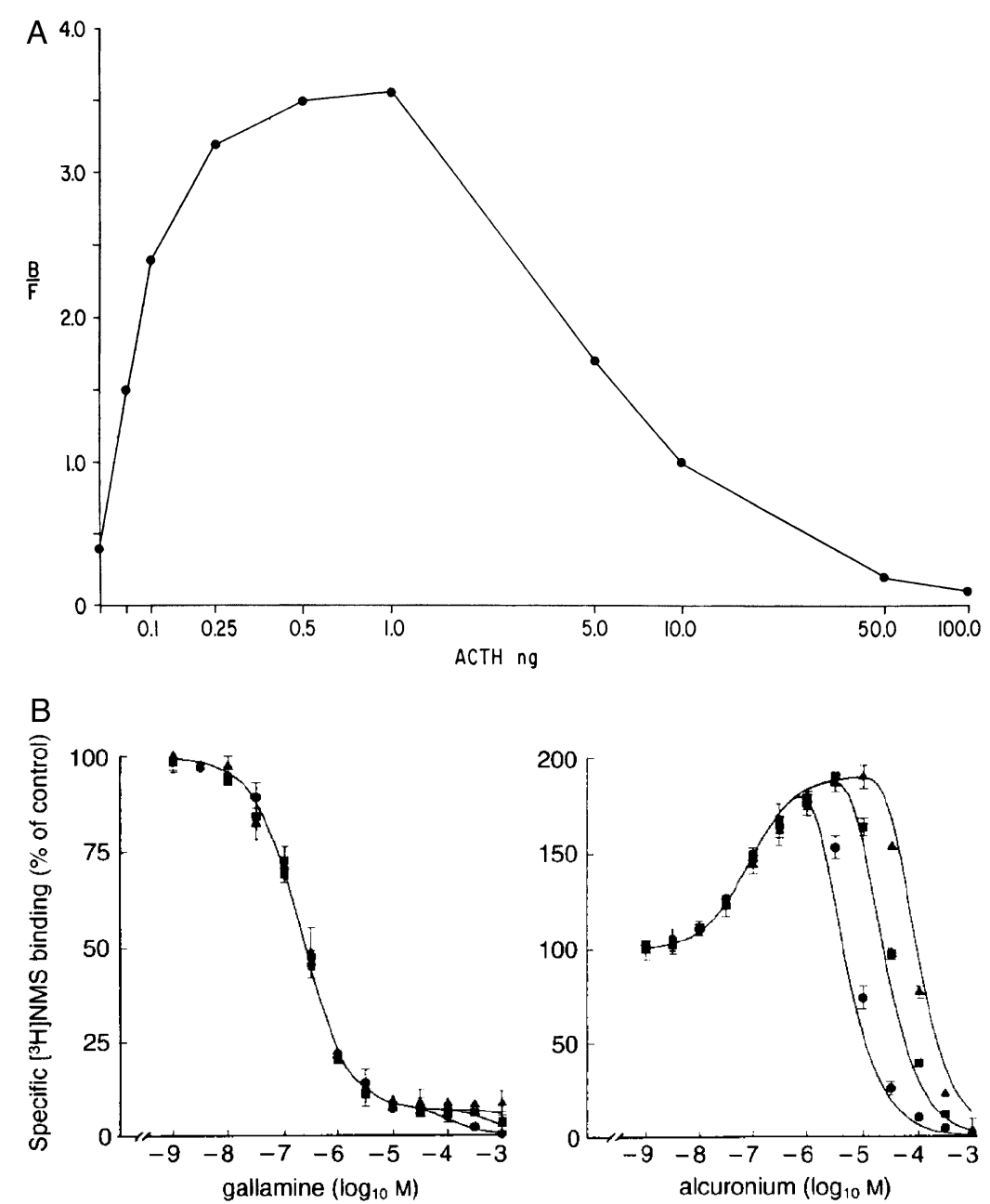

Figure 3.7. Low-dose hook effects. (A) Matsukura et al. (1971, Fig 2) as some of the first observed what looks like a low-dose hook effect in radioactive displacement studies. (B) Bell-shaped displacement of tracer NMS by cold gallamine or alcuronium. Tucek and Proska (1995, Fig. 2) with permission.

interacting binding sites, which for certain parameter values behave as described above (Swillens et al. 1995).

\subsubsection{Swillens' Scheme}

According to Swillens et al. (1995), the reaction scheme for a radio-active ligand symbolized as $S^{*}$ and binding to a two-sited receptive unit $\mathrm{R}$ where the sites interact has the following formulation when transcribed to our terminology:

$$
\mathrm{R}+2 \mathrm{~S}^{*} \stackrel{A_{\mathrm{s}}}{\rightleftharpoons} \mathrm{RS}^{*}+\mathrm{S}^{*}\left(\text { or } \mathrm{S}^{*} \mathrm{R}+\mathrm{S}^{*}\right) \stackrel{A_{\mathrm{s}} \cdot c^{\prime}}{\rightleftharpoons} \mathrm{S}^{*} \mathrm{RS}^{*}
$$

where $A_{\mathrm{s}}$ is the association constant for binding either the cold isotope $\mathrm{S}$ or the tracer $\mathrm{S}^{*}$ to the receptive unit $\mathrm{R}$, and $A_{\mathrm{s}} \cdot c^{\prime}$ is a combined association constant and an autointervention constant. Constant $c^{\prime}$ is an interaction coefficient for enhancement or attenuation in binding affinity for a second ligand following binding of a first ligand. The constant $A_{\mathrm{s}} \cdot c^{\prime}$ is again the same for both tracer and cold displacer molecule. For training purposes, I have replaced dissociation constants and switched to association constants. The above reaction scheme is the same as a compact version of the auto-intervention schemes presented in sub-chapter 3.3 (Fig. 3.1), but now with association constants. In the above version of the autointervention scheme, it is undecided whether binding is ordered or random. If binding at a primary site takes place before binding at the secondary site, it is an ordered reaction (Fig. 3.1A). See Chapter 6 for more on ordered and random reaction schemes.

Naturally, there are other examples of models with 'hook-effects' for a single ligand. One example with hook effects for a single ligand is Kühl's minimal recovery model (sub-chapter 3.7), where rate constants are invoked instead of, as here, Swillens et al.'s multi-sited interaction.

\subsubsection{Formulation of Swillens' Scheme for Low-dose Hook Effects}

The complete reaction scheme for the displacement of radio-active ligands with cold isotope at a two-sited 


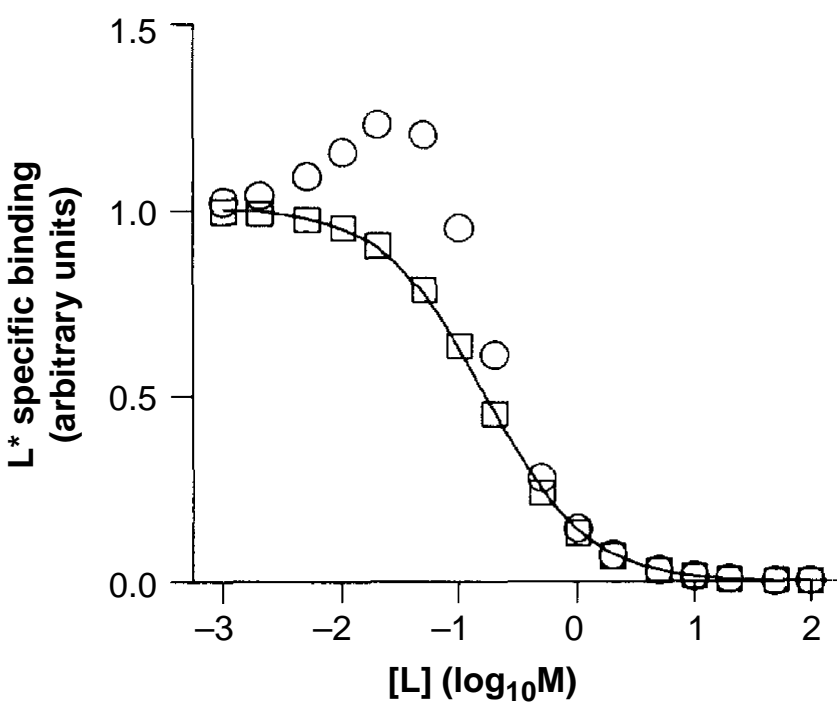

Figure 3.8. Theoretical low-dose hook effect. Assuming a two-sited receptive unit with 'positive co-operativity' between sites, radioactive binding displacement experiment may display a 'low-dose hook effect' at low to moderate concentrations of the radioactive ligand as demonstrated by Swillens et al. (1995; Fig. 3b). See subchapter 3.4 for further details. Reproduced with permission.

receptor system is shown in Fig. 3.10, and includes the possibility of site interaction, $c^{\prime} \neq 1$. Compare Fig. 3.10 with a similar heterologous four-pane one-state reaction scheme in Fig. 2.4.

As mentioned above, we assume that the association constants for both tracer and cold isotope are the same on either site, i.e., $A_{\mathrm{ss}}=A_{\mathrm{ss}}$ and $A_{\mathrm{is} *}=A_{\mathrm{is}}$, and binding is random. In case the binding is ordered, conformations $\mathrm{SR}$ and $\mathrm{S} * \mathrm{R}$ will disappear from the scheme in Fig. 3.10 , and the reaction will resemble an un-competitive reaction, but in principle with two identical ligands, tracer and cold isotope.

Now we will further assume that $A_{\mathrm{ss}}$ is also equal to $A_{\mathrm{is}}$ and derive the related equation.

The equation for displacement of a radioactive isotope, $\mathrm{S}^{*}$, by cold isotope, $\mathrm{S}$, in a random four-pane one-state scheme (Fig. 3.10), only contains the liganded conformations with cold isotope in its denominator. Thus, for $A_{\mathrm{ss}}=A_{\mathrm{is}}$, the sum:

$$
\begin{aligned}
& \text { of unlabeled species }=\mathrm{UL}_{\mathrm{s}=\mathrm{i}}=1+2 \cdot \mathrm{S} \cdot A_{\mathrm{ss}}+\left(\mathrm{S} \cdot A_{\mathrm{ss}}\right)^{2} \cdot c^{\prime} \text {, } \\
& \text { in the nominator }=\mathrm{N}_{\mathrm{s}=\mathrm{i}}, \quad=2 \cdot \mathrm{S}^{*} \cdot A_{\mathrm{ss}} \cdot\left(1+\mathrm{S}^{*} \cdot A_{\mathrm{ss}} \cdot c^{\prime}\right. \\
& \left.+\mathrm{S} \cdot A_{\mathrm{ss}} \cdot c^{\prime}\right) \text {, and }
\end{aligned}
$$

and the fractional bound tracer is:

$$
\frac{\text { bound tracer }}{\text { total ligand }}=\frac{\mathrm{N}_{\mathrm{s}=\mathrm{i}}}{\mathrm{UL}_{\mathrm{s}=\mathrm{i}}+\mathrm{N}_{\mathrm{s}=\mathrm{i}}} \text {. }
$$

$\mathrm{S}^{*}$ will be fixed for the single experiment, while $\mathrm{S}$ varies. The displacement of tracer for $A_{\mathrm{ss}}=A_{\mathrm{is}}$, is shown in Fig. 3.11. As shown in panel 3.11A, for certain values of association constant $A_{\mathrm{ss}}$ and of coupling coefficient $c^{\prime}$ and the level of initial tracer occupancy, there is a 'hook'

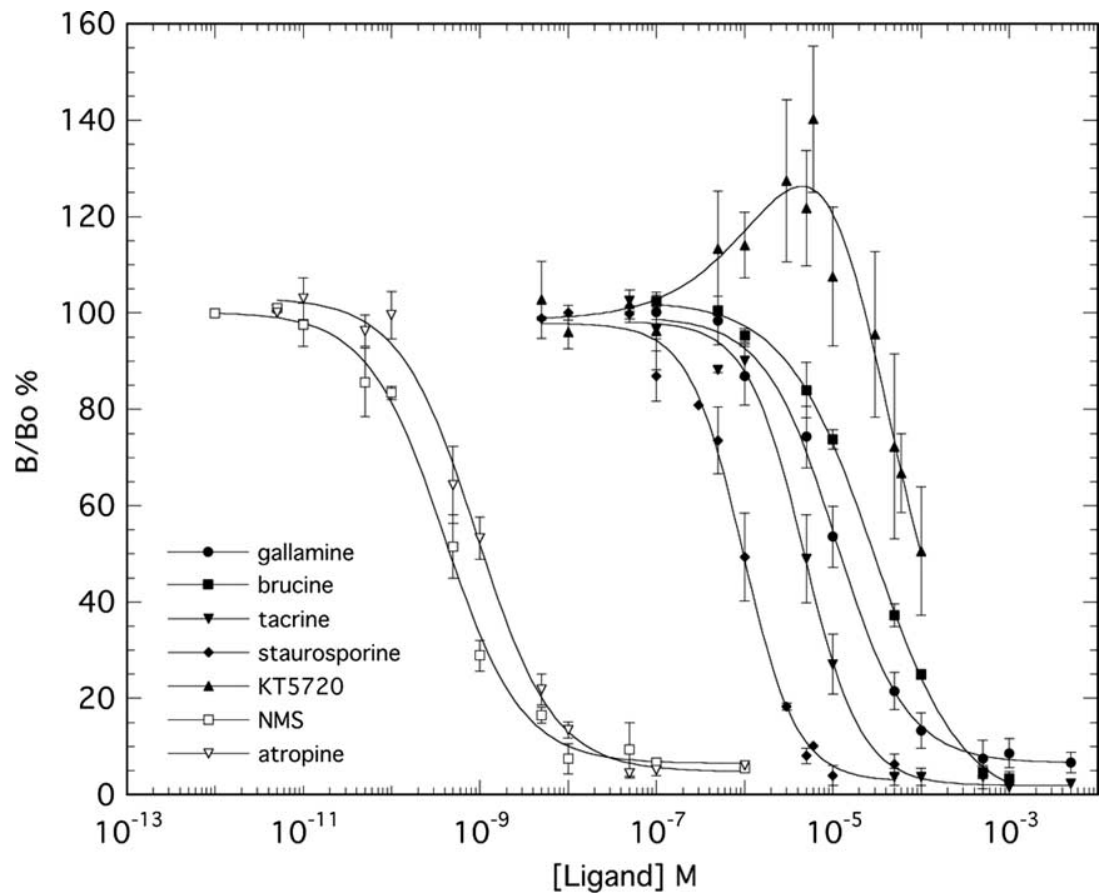

Figure 3.9. Non-equilibrium may explain 'low-dose hook effects'? The observed low-dose bell-shaped relation could possibly be explained by a heterologous displacement in a one-state intervention model (see text). However, the authors interpreted the observed hook effect as a non-equilibrium phenomenon. From Fruchart-Gaillard et al. (2006, Fig. 8A) with permission. 


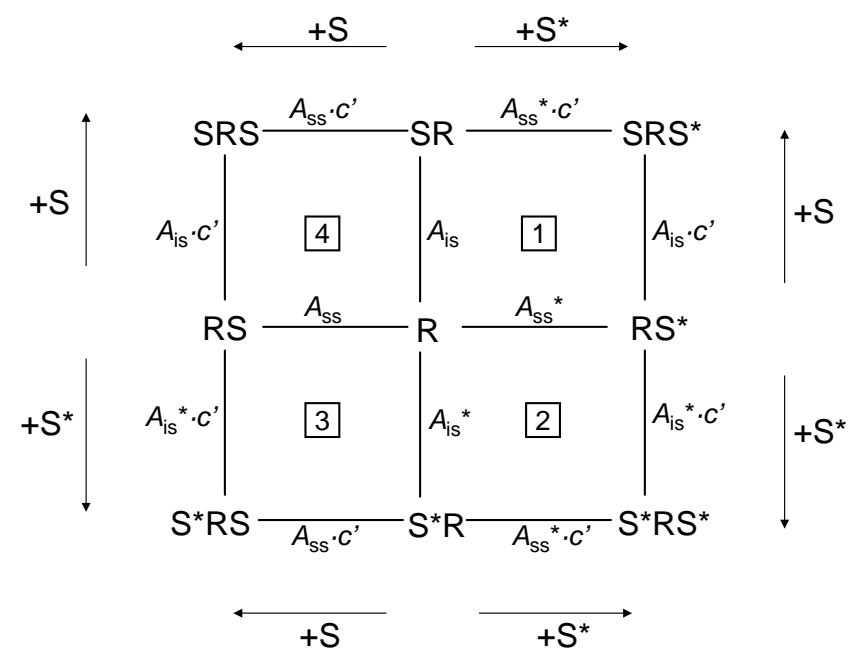

Figure 3.10. Reaction scheme for ligand displacement of tracer by cold isotope. The reaction scheme is a two-sited one-state model in an isotopic four-pane model (I-FP-OSM). The scheme may explain 'low-dose hook effects' seen in RIA assays.

on the binding displacement-curve as the concentration of cold isotope is raised.

The 3-D surface plot in Fig. 3.11B is only realistic in theory, where the concentration of cold isotope may be assumed to change separately either at the primary site, s-site, following the $x$-axis or at the intervention site, i-site, following the $y$-axis. In real life, the relationship will follow a cut through the surface topology along the indicated arrow in the $\mathrm{x}-\mathrm{y}$ plane, yielding curves as shown in the 2-D graph in Fig. 3.11A.

\section{A TRACER DISPLACEMENT in BINDING}

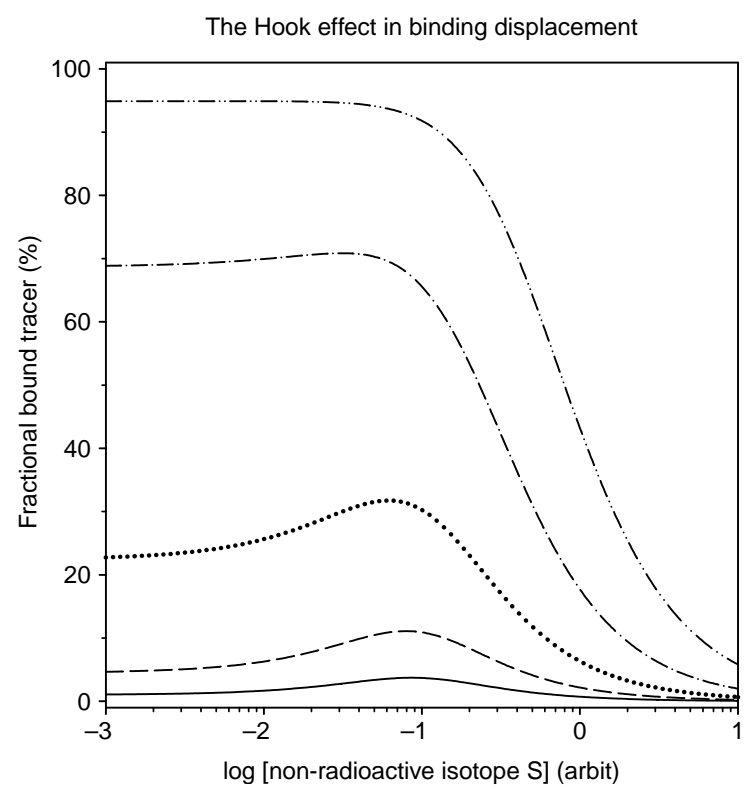

Next, in the isotopic FP-OSM (I-FP-OSM; Fig. 3.10), we assume that $A_{\mathrm{ss}} \neq A_{\mathrm{is}}$, therefore its fractional occupancy equation will be slightly different from Eq. 3.8. The complete I-FP-OSM for binding in Fig. 3.10 now has the following sum:

of unlabeled species $=\mathrm{UL}_{\mathrm{s} \neq \mathrm{I}}$

in the nominator

$$
\begin{aligned}
= & 1+\mathrm{S} \cdot A_{\mathrm{ss}} \cdot\left(1+\mathrm{S} \cdot A_{\mathrm{is}} \cdot c^{\prime}\right)+\mathrm{S} \cdot A_{\mathrm{is}}, \\
= & \mathrm{N}_{\mathrm{s} \neq \mathrm{I}} \\
= & \mathrm{S}^{*} \cdot A_{\mathrm{ss}} \cdot\left(1+2 \cdot \mathrm{S}^{*} \cdot A_{\mathrm{is}} \cdot c^{\prime}\right. \\
& \left.+2 \cdot \mathrm{S} \cdot A_{\mathrm{is}} \cdot c^{\prime}\right)+\mathrm{S}^{*} \cdot A_{\mathrm{is}},
\end{aligned}
$$

in the denominator $=\mathrm{D}_{\mathrm{s} \neq \mathrm{I}}=\mathrm{UL}_{\mathrm{s} \neq \mathrm{i}}+\mathrm{N}_{\mathrm{s} \neq \mathrm{i}}$,

and the fractional bound tracer is:

$$
\frac{\text { bound tracer }}{\text { total ligand }}=\frac{\mathrm{N}_{\mathrm{s} \neq \mathrm{i}}}{\mathrm{UL}_{\mathrm{s} \neq \mathrm{i}}+\mathrm{N}_{\mathrm{s} \neq \mathrm{i}}} \text {. }
$$

The tracer binding or its displacement with cold isotope will be almost the same based on either Eq. 3.9 or Eq. 3.8.

Plots of the relation in Eq. 3.9 are shown in Fig. 3.12A. Again, for certain values of the initial level of tracer occupancy and of the association constant $A_{\text {ss }}$, of the coupling coefficient $c^{\prime}$ and for the association constant $A_{\text {is }}$, there is a 'hook' on the binding displacement-curve as the concentration of cold isotope is raised.

For both schemes in Eqs. 3.8 and 3.9 the hook appears around a value of $[\mathrm{S}]$ equal $A_{\mathrm{s}} \cdot\left[\mathrm{S}^{*}\right]$, however it disappears at higher doses of $\mathrm{S}$.

\section{B The low-dose Hook effect in 3D}

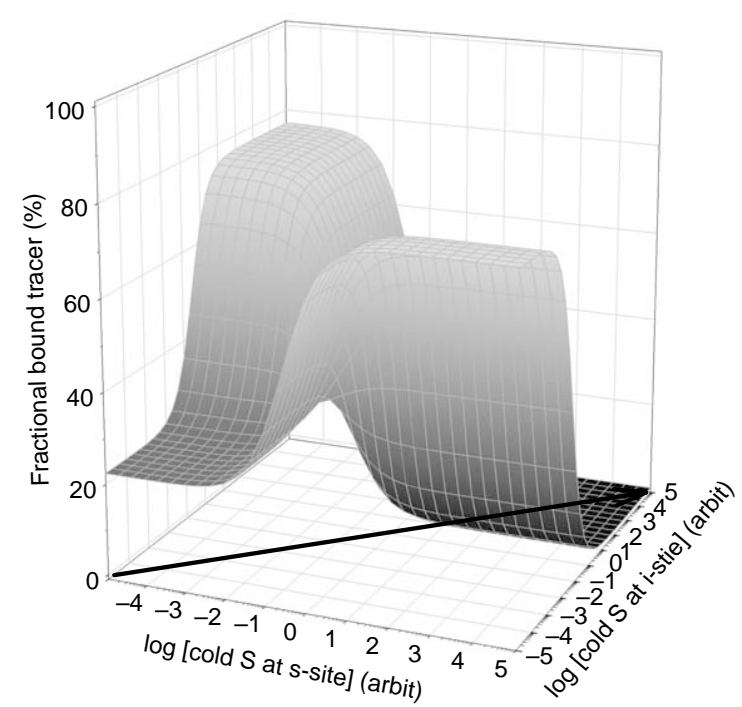

Figure 3.11. Theoretical demonstration of the hook-effect for the two-site one-state model in Fig. 3.10. Association constants $A_{\mathrm{ss}}$ and $A_{\text {is }}$ are assumed identical. In the 3-D plot tracer concentrations follow the $x$-axis while cold isotope follow the $y$-axis. Parameter $C^{\prime}$ in 2-D plots varied in 5 steps from $10^{-2}(-)$ to $10^{2}(-\cdots-)$ by a factor 10. 
The plot in 3-D of the low-dose hook effect for $A_{\mathrm{ss}} \neq$ $A_{\text {is }}$ (Fig. 3.12B) has the same explanation as for the 3-D plot in Fig. 3.11B.

\subsubsection{System Constants and the 'Hook'}

According to the present 'low-dose hook effect' model, for $A_{\mathrm{ss}}=A_{\mathrm{is}}$ the hook will appear with increasing concentrations of cold isotope $\mathrm{S}$ only when the coupling coefficient starts to increase above a value of circa 10 , $c^{\prime}>10$, and the product $A_{\mathrm{ss}} \cdot \mathrm{S}^{*}$ is less than $1 / 5$, though often clearly visible when the product is $1 / 10$ and below. Absolute requirements for observing the hook are $c^{\prime}>1$ and $A_{\mathrm{ss}} \cdot \mathrm{S}^{*}<1$.

For the 'low-dose hook model' with association constants not identical at the two binding sites, i.e., $A_{\text {ss }} \neq A_{\text {is }}$, the above statements are almost true once the two products $A_{\mathrm{ss}} \cdot \mathrm{S}^{*}$ and $A_{\mathrm{is}} \cdot \mathrm{S}^{*}<0.2$.

\subsubsection{Initial Occupancy by Tracer, $S^{\star}$}

For the two-sited low-dose hook model above, the level of bound radio-activity at low concentration of the displacing ligand $\mathrm{S}$ or in its absence is given by inserting zero for [S] in Eq. 3.8, which gives us:

$$
\begin{aligned}
& \frac{\text { bound radio-actitvity }}{\text { total radio-actitvity }} \\
& \quad=\frac{2 \cdot A_{\mathrm{ss}} \cdot \mathrm{S}^{*} \cdot\left(1+A_{\mathrm{ss}} \cdot c^{\prime} \cdot \mathrm{S}^{*}\right)}{1+2 \cdot A_{\mathrm{ss}} \cdot \mathrm{S}^{*} \cdot\left(1+A_{\mathrm{ss}} \cdot c^{\prime} \cdot \mathrm{S}^{*}\right)},
\end{aligned}
$$

\section{A TRACER DISPLACEMENT in BINDING}

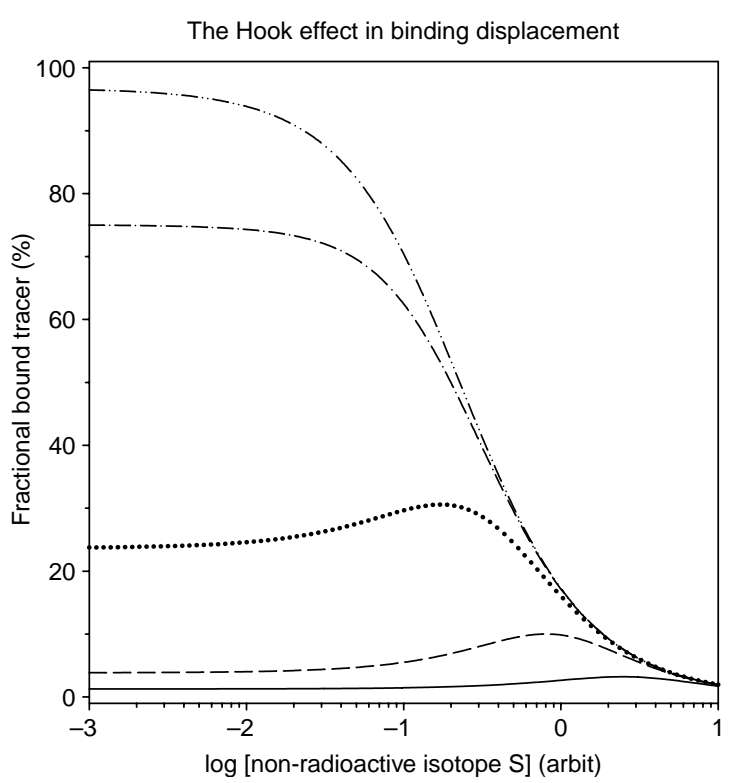

for the initial level of occupancy of the tracer when $A_{\mathrm{ss}}=A_{\text {is. }}$.

When $A_{\mathrm{ss}} \neq A_{\mathrm{is}}$, the initial occupancy by radioactive isotope is given by inserting zero for $[\mathrm{S}]$ in Eq. 3.9:

$$
\begin{aligned}
& \frac{\text { bound radio-actitvity }}{\text { total radio-actitvity }} \\
& \quad=\frac{A_{\mathrm{ss}} \cdot \mathrm{S}^{*} \cdot\left(1+2 \cdot A_{\mathrm{ss}} \cdot c^{\prime} \cdot \mathrm{S}^{*}\right)}{1+A_{\mathrm{ss}} \cdot \mathrm{S}^{*} \cdot\left(1+2 \cdot A_{\mathrm{ss}} \cdot c^{\prime} \cdot \mathrm{S}^{*}\right)} .
\end{aligned}
$$

Therefore, in a two-sited receptive system with a large co-lateral intervention coefficient $c^{\prime}$ equal to strong site interaction, the initial level of bound radio-activity is given by these expressions. The fractional level of bound radio-activity is of interest in RIA assays for two-sited receptive units, as it may be used to choose optimal working conditions (see, e.g., protocols at http://www. gehealthcare.com).

\subsubsection{Virtual Observations for $A_{\text {ss }}$ and $A_{\text {is }}$ and Their Related Sites}

As a consequence of the two-sited 'low-dose hook model', there is an astonishing relationship between the two association constants $A_{\mathrm{ss}}$ and $A_{\mathrm{is}}$ and their pertinent orthosteric and intervention sites, the 's-site' and the 'i-site'. Under the theoretical assumption that the concentration of the cold isotope can change separately at either the s-site or the i-site, i.e., the concentration follows either the $x$-axis or the $y$-axis as indicated in Section 3.4.3 (Figs. 3.11B and 3.12B), there

B The low-dose Hook effect in 3D

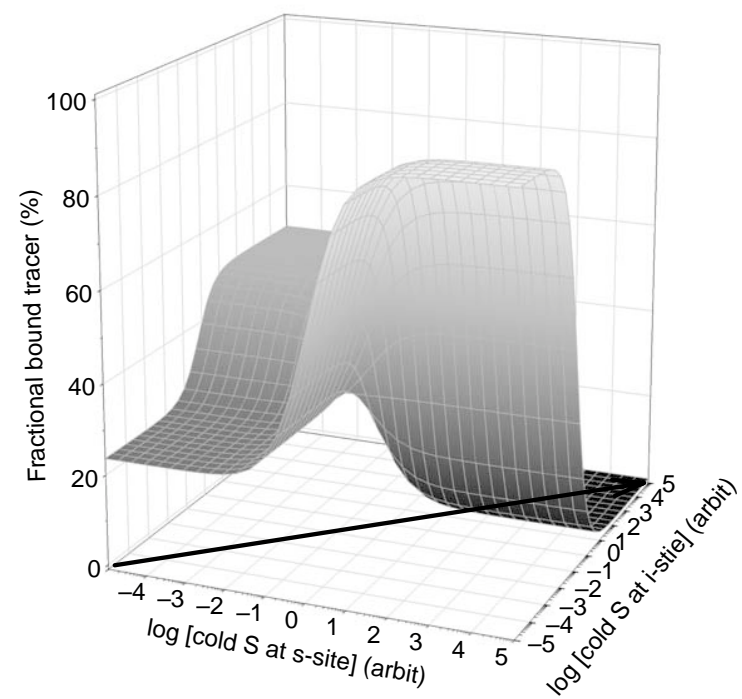

Figure 3.12. Theoretical demonstration of the hook-effect for the two-site one-state model in Fig. 3.10. Association constants $A_{\mathrm{ss}}$ and $A_{\text {is }}$ are assumed different. In the 3-D plot tracer concentrations follow the $x$-axis while cold isotope follow the $y$-axis. Parameter $c^{\prime}$ in 2-D plots varied in 5 steps from $10^{-2}(-)$ to $10^{2}(-\cdots-)$ by a factor 10. 
is an unforeseeable consequence. Thus, merely changing $\mathrm{S}$ concentration at the s-site (following the $x$-axis), the binding-occupancy profile is solely dependent on the association constant of the other site, $A_{\text {is }}$, and not its own association constant, $A_{\mathrm{ss}}$ ! This can be seen in Fig. 3.12B. And, vice versa. Only changing $S$ concentration at the isite (following the $y$-axis), the binding-occupancy curve is solely dependent on the association constant of the opposite site, $A_{\mathrm{ss}}$, and not its own association constant, $A_{\text {is }}$ ! This is also demonstrated in Fig. 3.12B.

\subsubsection{A Non-cyclic Model for Heterologous Intervention}

Another model of the intervention scheme is a model in which one of its legs is amputated, as shown in Fig. 3.13. Compare this model with the un-competitive reaction scheme in Chapter 2 (Fig. 2.2D). For convenience, I designate this model the "non-cyclic model for intervention' (NC-MI). The model is nearly identical to a model by Segel, reproduced in Fig. 2.2C. Segel discussed his model in relation to function. However, we shall look at it as a model for occupancy. Although the NC-MI belong to models with two different ligands in a ternarycomplex, and therefore should appear in Chapter 2, I have decided to discuss it here in relation to low-dose hook effects, since the model has been implemented in attempts to explain bell-shaped displacement of bound tracer compounds (Tucek et al. 1990; Proska \& Tucek 1994, 1995; Tucek \& Proska 1995).

At equilibrium the NC-MI closely resembles the ordinary intervention model. In order to differentiate between the two models, we may write up equations for receptor conformations in both directions of the cyclic scheme and realize that the double set of conformations cancel out and we obtain the equations presented in Chapter 2 (Eqs. 2.16 and 2.17). Conversely, in the NC-MI reaction

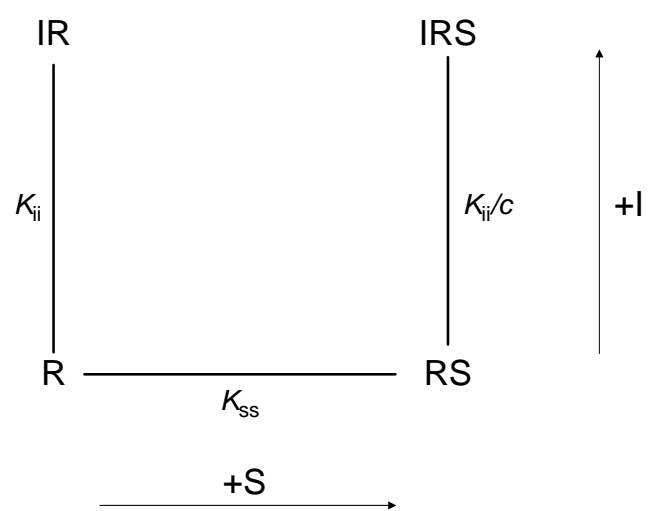

Figure 3.13. A 'non-cyclic model for intervention' (NC-MI). Cutting off the upper leg of the intervention model in Chapter 2 (Fig. 2.1A) results in the NC-MI reaction scheme. Formulation of a distribution equation for this NC-MI reaction scheme is in Eq. 3.12. Compare the NC-MI with a Segel model reproduced in Fig. 2.2C. scheme at equilibrium, the derivations for occupancy are slightly different as the terms for the reaction IR $\rightleftarrows$ IRS are eliminated from the double listing, which result in the following equation for occupancy:

$$
\frac{\text { occupancy }}{\text { total }}=\frac{\mathrm{S} / K_{\mathrm{ss}}}{\mathrm{S} K_{\mathrm{ss}}+\frac{2+\mathrm{I} / K_{\mathrm{ii}}}{2+c \cdot \mathrm{I} / K_{\mathrm{ii}}}} .
$$

Compare Eq. 3.12 with Eq. 2.16. The effects on occupancy in the NC-MI due to variation in the parameter $c$ are illustrated in Fig. 3.14 together with the occupancy variation for the ordinary intervention model; both as a function of the concentration of an interventor.

The difference between the two models is minor. More importantly, the NC-MI at equilibrium cannot explain bell-shaped hook effect behavior by interventor-displacement of occupancy as claimed by Proska and Tucek (1994). The interventor can merely increase or decrease binding of a substrate or an agonist, as demonstrated by Proska and Tucek (1995). Thus, it is unclear to me, which model Proska and Tucek used in their 1994 publication to explain low-dose hook effects in heterologous displacement by alcuronium of $\left[{ }^{3} \mathrm{H}\right]-\mathrm{NMS}$ occupancy at equilibrium (Proska \& Tucek 1994, Figs. 5, 6b and 10; Tucek \& Proska 1995; Figs. Box 2b and 2).

The NC-MI was also introduced for reverse bell-shaped occupancy found with gallamine on the $\left[{ }^{3} \mathrm{H}\right]-\mathrm{NMS}$ binding in membrane fraction of rat heart atria, although not implemented (Proska \& Tucek 1995, Fig. 7).

\subsubsection{Time as an Explanation}

More complex models and models involving non-equilibrium schemes have been suggested for the aberrant behavior of heterologous displacement studies demonstrating convex and/or concave bell-shaped concentration-binding relationships (for more details see, e.g., Waelbroeck et al. 1988; Tomlinson \& Hnatowich 1988; Marvizon \& Baudry 1994; Wreggett \& Wells 1995; Lazareno \& Birdsall 1995; Ellis 1997; Lazareno et al. 2000; Armstrong \& Strange 2001; Neuman-Tancredi et al. 2002; Avlani et al. 2004; Durroux 2005; Franco et al. 2006; Albizu et al. 2006; Fruchart-Gaillard et al. 2006; May et al. 2007).

A play on rate constants as an explanation for the functional substrate inhibition is discussed in more detail for a model in sub-chapter 3.7.

\subsubsection{Dynamic Models and Desensitization}

To explain non-classical dose-response effects, such as partial agonism and insurmountability, various simple models assuming non-equilibrium conditions have been introduced. In many of these models, desensitization is a through theme. No doubt, on suspicion of non-equilibrium conditions, kinetic (dynamic) models are preferable to all 

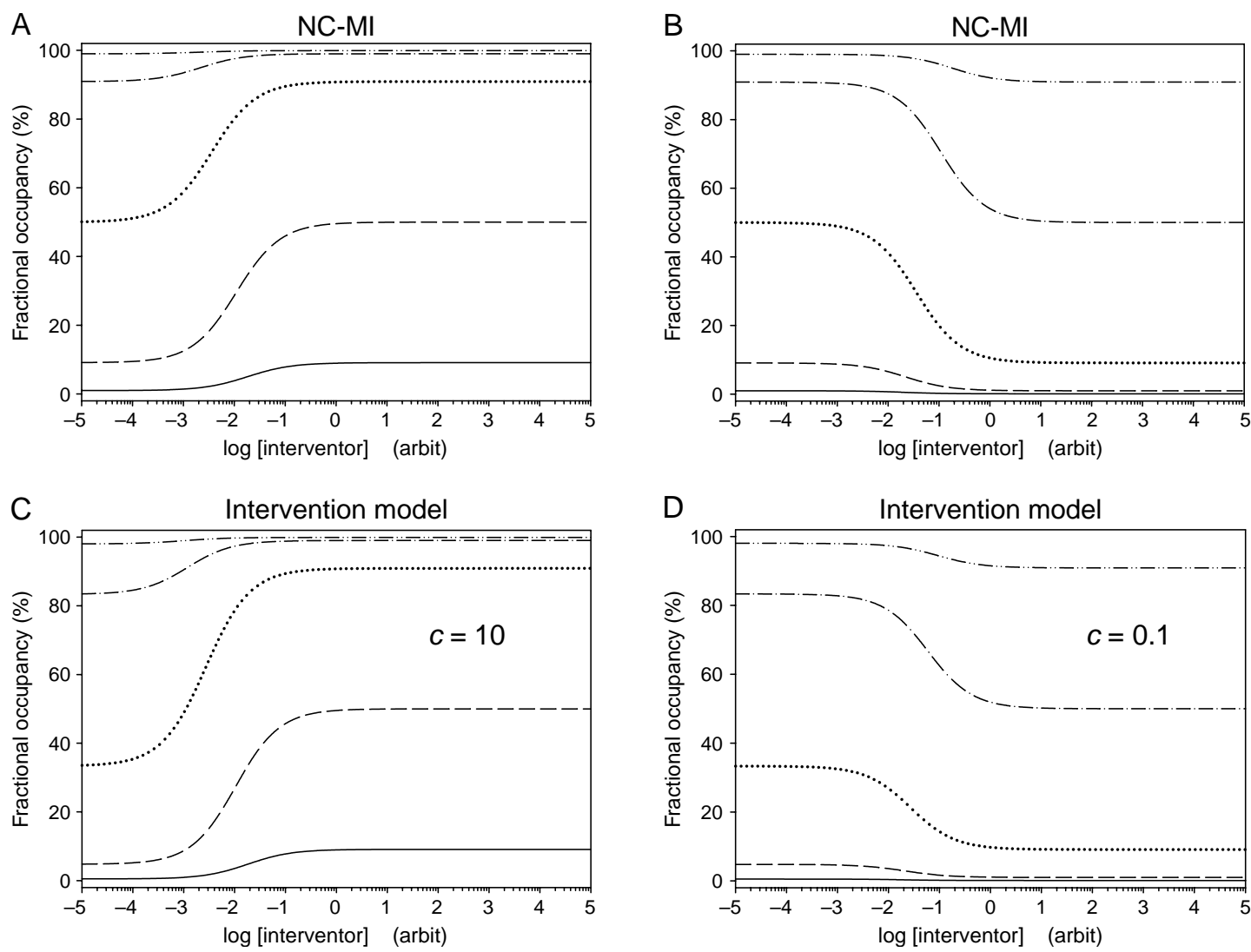

Figure 3.14. Concentration-occupancy relation for the non-cyclic model for intervention (NC-MI; Fig. 3.11), and the intervention model (IM-OSM; Fig. 2.1A). Plots of NC-MI are in panels $A$ and $B$ and plots of IM-OSM are in panels $C$ and D. The heterologous interventor is the independent variable in all plots, while the interaction parameter $c$ is either $>1$ (panels $A$ and $C$ ), or $<1$ (panels $B$ and $D$ ). The fixed substrate or agonist concentration vary between $10^{-1}(-)$ and $10^{3}(-\cdots-)$ in five steps by a factor 10 with parameters $K_{\mathrm{ss}}=10$ and $K_{\mathrm{ii}}=100$. In both models, the interventor can increase or decrease occupancy of substrate or agonist in a mono-phasic fashion. Bell-shaped or hooked concentration-binding is not possible with these models (see text).

the equilibrium models discussed in this book. However, it is not my intention to analyze dynamic models, therefore, such models should be sought for scrutiny elsewhere in the literature. Examples of dynamic models are Paton (1961), Paton and Rang (1966), Rang (1966), Rang and Ritter (1969), Lüllmann and Ziegler (1973), Gosselin (1977, pp. 325-356), Robertson et al. (1994), Kukkonen et al. (1998), Lew et al. (2000), Vauquelin and Szczuka (2007), and May et al. (2007). A follow-up discussion of the key-paper by Lew et al. (2000) can be found in Trends Pharmacol Sci 22; February and July 2001.

\subsection{Prelude to Biological Auto-regulation}

\subsubsection{Self-reference}

Self-reference in a general sense is an integral necessity and a prerequisite for regulated and recursive functions of our life and our soul.

Therefore, before I deal with actual examples on hardwired coupling between concentration-dependent auto-regulation and time-dependent auto-inhibition as desensitization, let me briefly introduce some associative aspects related to self-adjustment and homeostatic control in the biosphere together with some substantial examples. Readers who dislike my bona fide digressions, can without breaking the continuity skip to the next subchapter.

\subsubsection{Self-regulatory Mechanisms, Coupled Tightly, Loosely, and in Redundant Parallel}

In nature auto-regulatory mechanisms in the biosphere have developed through evolution to a high degree of sophistication. Following less complicated rules, autoregulatory principles are also found in the non-living nature as 'self-organizing criticality' (e.g., Bak 1997; Jensen 1998; Camazine et al. 2001; Feltz et al. 2006).

Bacteria originated about 1 billion years after the formation of Earth. Notwithstanding, if the intergalactic theory about panspermia (Wickramasinghe 2003, 2005; Hoyle 2005) is correct, creation of auto-poietic (Maturana \& Varela 1980) organic life may have taken several billion years to develop instead of just 1 billion years. After 
Table 3.2. The development of multicellularity

\begin{tabular}{lcl}
\hline Epoch of & Ca. years ago $\times 10^{9}$ & References/comments \\
\hline Earth formation & 4.6 & Schopf (2002) \\
Unicellular life as & & \\
Prokaryotes & 3.8 & \\
Eukaryotes & 3.5 & \\
Multicellular life as & & \\
Homocytics & 3.5 & \\
Heterocytics & $3.0-2.0$ & Schopf (2002) \\
Early animals & 1 & \\
\hline
\end{tabular}

Mainly based on Kirk (1998, Chapter 1)

bacteria, just over 2 billion years elapsed to get through unicellular eukaryotes and primitive forms of homo- and hetero-cytics to multicellular organisms (Table 3.2 and Fig. 13.8) (Kirk 1998, Chapter 1; Stephenson et al. 2000; Kessin 2001; Schopf 2002; King 2004; Embley \& Martin 2006, Hoenisberg et al. 2008; Abedin \& King 2008).

What is organic life? Life is a self-replicating mode based on nucleic acids and on their code; thus including viruses - and may be even smaller entities (Ma \& Yu 2006).

Self-reference in auto-poietic or self-replicating organisms is part of organic life, and self-reference expressed in biological activity may be formulated and analyzed in biosemiosis (Hoffmeyer 2008), in memetics, or by functional magnetic resonance imaging (fMRI) (Johnson et al. 2005).

In humanities, in contrast, self-reference and its dominance in intentionality and in the personality appear in signifier-chains of symbolic language without self-reference (Lacan 2007, seminar Feb 18, 1970), in Peirce-related representation (Wenz 2003), also in memetics, and is even afforded by The Providence emerging from 'The mind of God' (Davies 1993).

From a logical point of view, self-reference often ends up in paradoxes (Hofstadter 2007). In the Arts and Letters, covering psycho-analysis, the most elegant paradox in relation to self-reference is the statement 'I lie'. Descartes among others divided the subject of the subject into a duality of body-'n-soul. Of course, this view has been disputed repeatedly. The modern understanding of body-'n-soul is a unification of the two, while operating with a divided subject (see, e.g., Lacan 1969; Nordtug 2004; Cavell 2006). Another formulation of the body-"n-soul unification is: "and the Word (the Father) became Flesh (the Son) ", 2

Satiety is an example of a mechanism where the description of the coupling between sense, motoraction, and behavior narrows in on the psycho-physical players (Morton et al. 2006). However, I expect that a deeper understanding of the coupling between percep-

\footnotetext{
$\overline{2}$ 'Homoousion to patri' ('of one substance with the father'). Council of Nicaea AD 325.
}

tion, sense, apperception, ${ }^{3}$ affect, intention, and behavior will be debated by our descendants for as long as there are humans around.

\subsubsection{Necessary Feed-back or Feed-forward}

Initiating a response by an action does not necessarily render an efficient re-action, therefore new recurrent and parallel adjustments are constantly selected to obtain optimal or favorable leverage in living organisms. Designlike, although serendipitous, evolutionary forms of species that have survived depend on smart strategies in all aspects of reproduction. A delicate balance of homeostasis in evo-devo is maintained by regulatory means, such as differential feedback, auto-regulation, and epigenetics (Allis et al. 2007). In addition, coherent control (Shapiro \& Brumer 2003; Alon 2006; Palsson 2006) and ordered chaos (Peitgen et al. 1992; Kingsland 1995; Camarzine et al. 2000; Ford 2000, Chapter 12; Turchin 2005, Chapter 5) may begin.

Guiding a single message is often done by parallel, interacting, and self-adjustable pathways in elaborate networks at a molecular scale. Regulated through mechanical or chemical signals and electrical impulses, biological processes have these characteristics. The signal may be a single sub-atomic particle, such as the photon in regulated coherent control of retinal chemistry (Prokhorenko et al. 2006), or atomic scale movements of stereocilia on hair cells in our inner ear (Narayan et al. 1998; Hudspeth 2005). Signaling is sometimes even elicited by quantal release of few molecules (Mallet et al. 2007), although the exact interpretation has been questioned (Ninio 2007). To be sure, quantum mechanics is a base for our understanding of the fabric of the Cosmos (Green 2005) and in the description of coherent control in retinal isomerization. However, quantum mechanics behind our souls has still not reached a trustworthy argumentation for an explanation of our personality. Zohar and Marshall (1990) have argued that human 'self' emerges with Bose-Einstein condensates in our brains. For the single reason that these condensates only exist at extremely low temperatures, the Zohar-Marshall postulates seem to be sheer nonsense.

I believe that the complexity of the human neuronal network at an atomic scale is sufficient for conscious 'self', a so-called apperception (see Section 3.5.2 and footnote 3.3).

\subsubsection{Timing by Turn-off}

In pathways of biochemical and bio-electrical signaltransduction there are always stringent and severe

\footnotetext{
3 Apperception ə: human neuronal net's perception of itself as a conscious agent (paraphrased by author).
} 


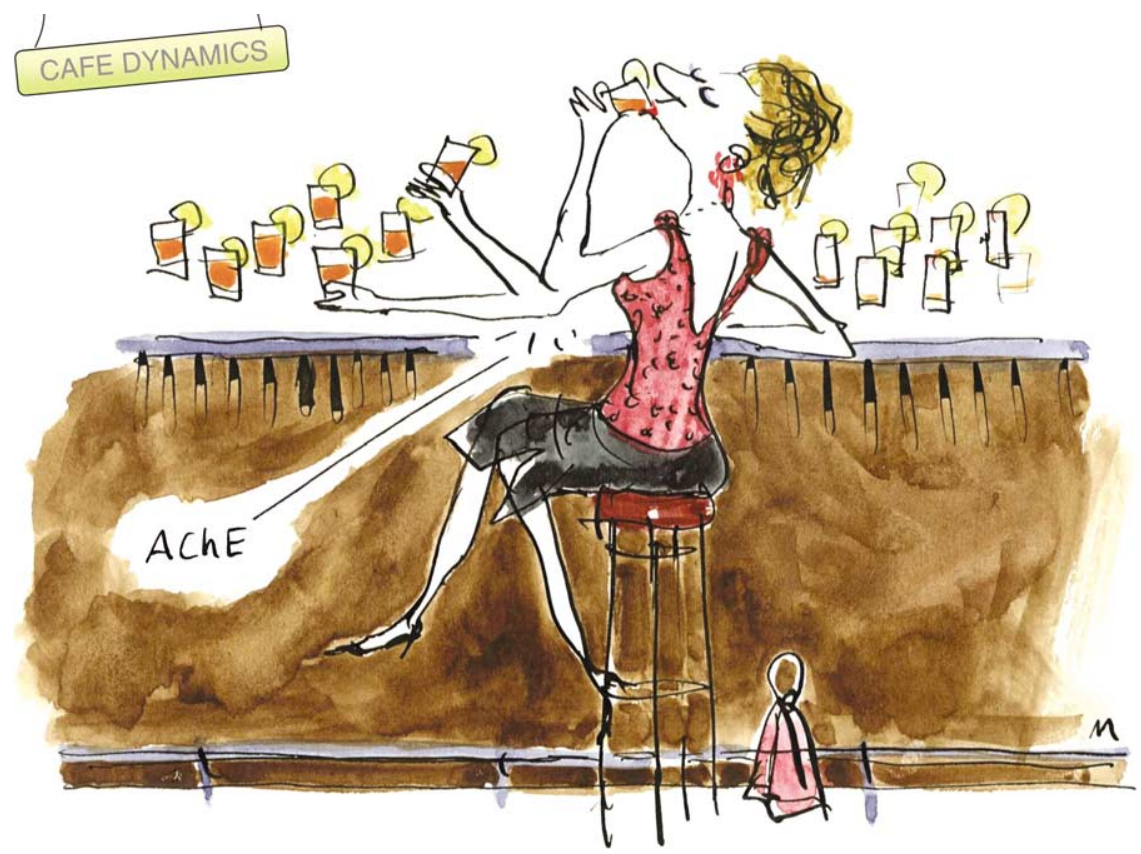

Figure 3.15. Does the elbow Ache? No, no, no, it displays DYNAMICS. Arm does not hurt - uses acetylcholine-esterase (AChE) to eliminate ACh and perform dynamic and repetitive movements. Drawing by Mette Dreyer (2006). Rights to the figure reside with the author.

turn-off mechanisms, since turn-off is a sine qua non for dynamic and repetitive responses. Examples are degradation of acetylcholine by choline esterases at the neuromuscular junction (Fig. 3.15), removal of choline by synaptic re-uptake in a $\mathrm{Na}$ and $\mathrm{Cl}$ coupled co-transport and of other monoamines (Ribiero et al. 2006; Vialou et al. 2007), decyclization of cyclic nucleotides by phospho-diesterases (Francis et al. 2001), and dephosphorylation of phosphorylated effectors by phosphatases (Forrest et al. 2006).

For the purposeful adaptation of functions, such as the beat of hearts, coordinated visual inputs and outputs, or sudden flight-or-fight reflexes, it has been a prerequisite by haphazard mutation schemes to construct and select feedback and feed-forward regimes with very different dissociation and time constants for turn on-'n-off in most dissimilar environments, in order to create the creatures that have prevailed. Self-control exist widespread in living organisms as feed-back and feed-forward circuits with a span of on-'n-off time constants from pico-seconds, to minutes, to days, to years, and even longer and with dissociation constants over many orders of magnitude. Photo-isomerization of a retinal chromophore, closure of an ion channel in nerve conduction, beat of hearts, division of a cell, hunger-satiety circles, circadian rhythms, menstrual cycle, hibernation, début of puberty, function of memory and long-term reinforcement and reward mechanisms are examples at either end of our time scale as well as from the middle of it (Table 3.3).
A poignant example of the importance of timing is the function of ion channels orchestrating the heart rhythm in interplay with ion exchangers, pumps, and auxiliary proteins as phopholamban and second messengers. A simple mutation in either a $\mathrm{Na}^{+}$- or $\mathrm{a} \mathrm{K}^{+}$-channel eliciting an ostensible innocent delay in transport shutting may lead to an early death (Sanguinetti \& TristaniFirouzi 2006; Thomas et al. 2006; Gupta et al. 2007).

\subsubsection{Levels of Complexity}

On one scale of complexity, we have the classic examples of enzyme feedback control, limiting overgrowth via product and waste material, and enzyme feed-forward substrate inhibition - controlling against metabolic overstimulation (Cornish-Bowden 2004, e.g., Chapter 8),

Table 3.3. Span of time constants by examples from biological functions

\begin{tabular}{lll}
\hline Physiological system & Time constant =1/rate constant & $(\mathrm{sec})$ \\
\hline Retinal isomerization & Pico- to nanosecond & $10^{-9}$ \\
Action potential & Millisecond & $10^{-3}$ \\
Heart beat & Second & $10^{0}$ \\
Cell cycle & Minute & $6 \times 10^{1}$ \\
Hunger recursion & Hours & $7 \times 10^{3}$ \\
Circadian rhythm & Day and night & $3 \times 10^{4}$ \\
Menstruation & Month & $2.6 \times 10^{6}$ \\
Hibernation/migration & Months & $10^{7}$ \\
Lag of puberty & Years & $4 \times 10^{8}$ \\
Memory & Life time & $2 \times 10^{9}$ \\
\hline
\end{tabular}

The span of time constants from short to long is a factor of about $10^{20}$. 
while on other scales of complexity, there is control of seasonal alterations in a single individual of a colony, exemplified by the regulated life cycle of a social slime mold (Dictyostelium discoideum) (Stephenson et al. 2000; Bonner 2001; Kessin 2001), control of time for menopause or for parts regulated in Gaia's body (Volk 1997; Lovelock 2000; 2006). ${ }^{4}$

Presentations herein are chiefly drawn from the cellular and sub-cellular level, and will deal mainly with examples of modest complexity, though complex. See, for instance, the description by Agnati et al. (2007) of such complexities by Boolean logic.

In essence, in regulatory systems, the value of TIME CONSTANTS and DISSOCIATION CONSTANTS are keys to optimal function. Features of the time and 'concentration-space' in biology are discussed in subchapter 3.6 and presented by examples of time constants in Table 3.3.

\subsection{Further Aspects of Auto-regulation and Desensitization}

\subsubsection{Dose-dependent and Time-dependent Regulation at a Sub-/cellular Level}

Responses elicited by agonists in biological systems are often either attenuated or augmented (accelerated) with time, in spite of a maintained and constant stimulatory signal. Langley (1905) described such timedependent descending responses. The slope of attenuation/acceleration with time may also be dependent on the dose of the agonist. It turns out that lowering of an agonist or metabolite-mediated response, with both time and dose, may involve regulatory knots at a multitude of levels in the signal-transduction chain of events, and these regulatory units can operate by different mechanisms at each level. Behind the nets of knots, the interconnecting regulatory threads have time constants varying from picoseconds to a life span; about 20 orders of magnitude (Table 3.3).

Furthermore, time-dependent fade may require the permanent presence of the eliciting drug, while in other systems, when the stimulatory process is first started, attenuation of a response is by an intrinsic automaticity in the activated molecules. Or, attenuation of the response is incorporated in the function of molecules associated with the signaling pathway, such as auxiliary and scaffolding proteins that can turn the transducing passage on-'n-off (Bhattacharyya et al. 2006; Kolch

\footnotetext{
${ }^{4}$ Natural self-control in the reproduction of Man is sabotaged by Man. Laws announced in letters of declaration in politics or in the name of God lead indirectly to a malignant overpopulation. Religious and research-based decrees are players in this deregulation - or are they just part of Nature's plan?
}

2006). Thus, self-regulatory processes are often not dependent on a maintained concentration of an exogenous primary stimulant, not to speak of the stimulants very presence, which might not be needed at all. An example is the spontaneous oscillation in self-sustained $\mathrm{Ca}^{2+}$ sensitization underlying Starling's law of the heart. The process includes synchrony of $\mathrm{Ca}^{2+}$ channels, $\mathrm{Ca}^{2+}$ pumps, $\mathrm{Ca}^{2+}$ exchangers, $\mathrm{Ca}^{2+}$ buffers, and $\mathrm{Ca}^{2+}$ receptors (Armoundas et al. 2007).

\subsubsection{The Term Auto-regulation and Possible Derivatives}

As mentioned in the Introduction to Chapter 3, 'autoregulation' in relation to ligand-receptor interactions may cover both ligand-induced alterations in the receptor as well as intrinsic self-control within the receptive unit per se. Furthermore, auto-regulation may be CONCENTRATION-dependent and/or TIME-dependent, as will be discussed in the following sections.

In our context of ligand-induced auto-regulation in one-state models, the term is 'auto-intervention' and models for this were presented in sub-chapters 3.1-3.4. In multi-state allosteric auto-regulation, I will use the term concentration-dependent 'auto-modulation' (see Chapters 14 and 15). When there is negative feedback in these situations because of the addition of ligands, the term is 'auto-inhibition' both for CONCENTRATIONdependent auto-regulation and for TIME-dependent desensitization or inactivation. For an overview of the employed terminology, see Table 3.4.

\subsubsection{Definitions of Concepts}

The above is a rather general introduction to regulation in time and with dose and needs a couple of examples to illustrate the subject at a cellular or subcellular level. However, to obtain a good overview on the subject of auto-modification for ligand-receptor interactions, it is wise to first realize a few additional concepts and to make these concepts operative through examples.

Thus, I will introduce a simple definition of (a) autoinhibition, (b) desensitization, and in addition some definitions of (c) related terms (Table 3.4).

Pure auto-ant-agonism ${ }^{5}$ appears in steady-state and equilibrium experiments of response versus CONCENTRATION of a ligand as a lower response than the loadrelation, while pure desensitization shows its many faces as a spontaneous decay of a response with TIME, following immediately after the addition of the drug that elicits the response. After wash-out, a mix of the

\footnotetext{
5 Ant-agonism is defined in Chapters 2 and 5, while auto-ant-agonism is concentration-dependent auto-inhibition or concentrationdependent negative auto-modification (Table 3.4).
} 
Table 3.4. Tentative derivatives for the term 'auto-regulation'

\begin{tabular}{|c|c|c|}
\hline & Auto-regulation & \\
\hline \multirow[t]{2}{*}{ Ligand-, voltage- or state-dependent } & TIME-dependent T-dep & CONCENTRATION-dependent C-dep \\
\hline & Model-independent & \\
\hline \multirow[t]{2}{*}{ Ligand-dependent (LGCs, GPCRs, TKRs) } & $\begin{array}{l}\text { Sensitization } \\
\text { desensitization }= \\
\text { T-dep auto-inhibition } \\
\text { Sensitization } \\
\text { Inactivation }\end{array}$ & $\begin{array}{l}\text { positive auto-modification } \\
\text { negative auto-modification }= \\
\text { C-dep auto-inhibition also equal to 'auto-ant-agonism' } \\
\text { auto-activation } \\
\text { auto-inhibition }\end{array}$ \\
\hline & Model-dependent & \\
\hline Two-state or multi-state models & $\begin{array}{l}\text { intrinsic activation/desensitization/ } \\
\text { inactivation } \\
\text { Extrinsic desensitization/inactivation } \\
\text { intrinsic desensitization/inactivation } \\
\text { Extrinsic desensitization/inactivation }\end{array}$ & $\begin{array}{l}\text { positive or negative } \\
\text { auto-intervention } \\
\text { positive or negative } \\
\text { auto-modulation } \\
\text { in a restricted form it is positive or negative } \\
\text { co-operativity }\end{array}$ \\
\hline
\end{tabular}

'Auto-modification' covers auto-intervention and auto-modulation and may also cover bell-shaped synagics, whereas 'co-operativity' is related solely to steeper (positive) or shallower (negative) slopes of the load relationship.

The terms 'intrinsic' and 'extrinsic' seem to refer to 'ligand-independent' and 'ligand-dependent'.

In the table there is no discrimination between models of binding and function.

two, auto-ant-agonism and desensitization, is eventually seen as a reduced response to a second application of the same or another drug.

Desensitization is defined and detailed below and illustrated in Figs. 3.16 and 15.13B, while auto-ant-agonism is shown in Figs. 3.2 and 3.3 and depicted in Fig. 3.16A.

\subsubsection{Homologous and Heterologous Desensitization for $G$ protein-coupled receptors (GPCRs)}

Here, it suffices to briefly mention the time-dependent desensitization for GPCRs either by the agonist itself, homologous desensitization, or alternatively as a reduced response to a given agonist engendered by exposure to agonists of distinct receptor systems, heterologous desensitization. In other words, desensitization may be detected as a lowering of the response to a second application of a drug shortly after wash-out of the drug from a first application. In case the drug used is the same in two test additions, lowering of the response in the second application to the drug is named homologous desensitization (Fig. 3.16C).

On the other hand, when it is two different agonists used in two test applications, with removal of the first drug in between, showing a lowering in response to the application of a second drug when compared to an earlier control stimulus by this second drug, then the attenuation is said to be due to a 'heterologous desensitization' (Fig. 3.16C+D) (Lefkowitz et al. 1986; Sibley et al. 1986; Freedman \& Lefkowitz 1996; Chuang et al. 1996; Pitcher et al. 1998; Ferguson \& Caron 1998; Bunemann
\& Hosey 1999; Bunemann et al. 2001; Ehlert 2003; Fortin \& De Lean 2006; Lefkowitz 2007).

Heterologous desensitization is especially induced by shut-down of the receptor function due to phosphorylation by second-messenger protein kinases, such as PKA and PKC, while the homologous desensitization is also effectuated through phosphorylation by members of the family of $G$ protein-coupled receptor kinases (GRKs), a family consisting of seven subfamilies (Premont et al. 1995; Hisatomi et al. 1998; Weiss et al. 1998; Bunemann \& Hosey 1999).

So far, in both writing and in reality, the most decimated mechanisms for homologous and heterologous desensitization seem to be those of serine-/threonine- or tyrosine-phosphorylation/dephosphorylation. The phosphorylation of serine/threonine $(\mathrm{S} / \mathrm{T})$ and tyrosine $(\mathrm{Y})$ residues goes through activation of GRKs and second messenger activated kinases, SMPK $=$ PKA and PKC, while signal turn-off is dephosphorylation by S/ T- and Y-phosphatases, PP1-2 and PTPs (Shi et al. 1998). In signal-transduction, phospho-relay systems of phosphorylation and dephosphorylation involving basic and acidic amino acid residues as histidine and aspartic acid are more often seen in two-component signaling in prokaryotes (Hoch 2000; Maeda et al. 2006; Szurmant et al. 2007), although also described for eukaryotes (Curien et al. 2007), and sporadically for mammalian systems (Klumpp \& Kringlstein 2005).

\subsubsection{Intrinsic and Extrinsic Desensitization in Receptive Units Including GPCRs}

Rearrangement of intra-molecular residues with intrinsic inactivation mechanisms for voltage-operated channels 

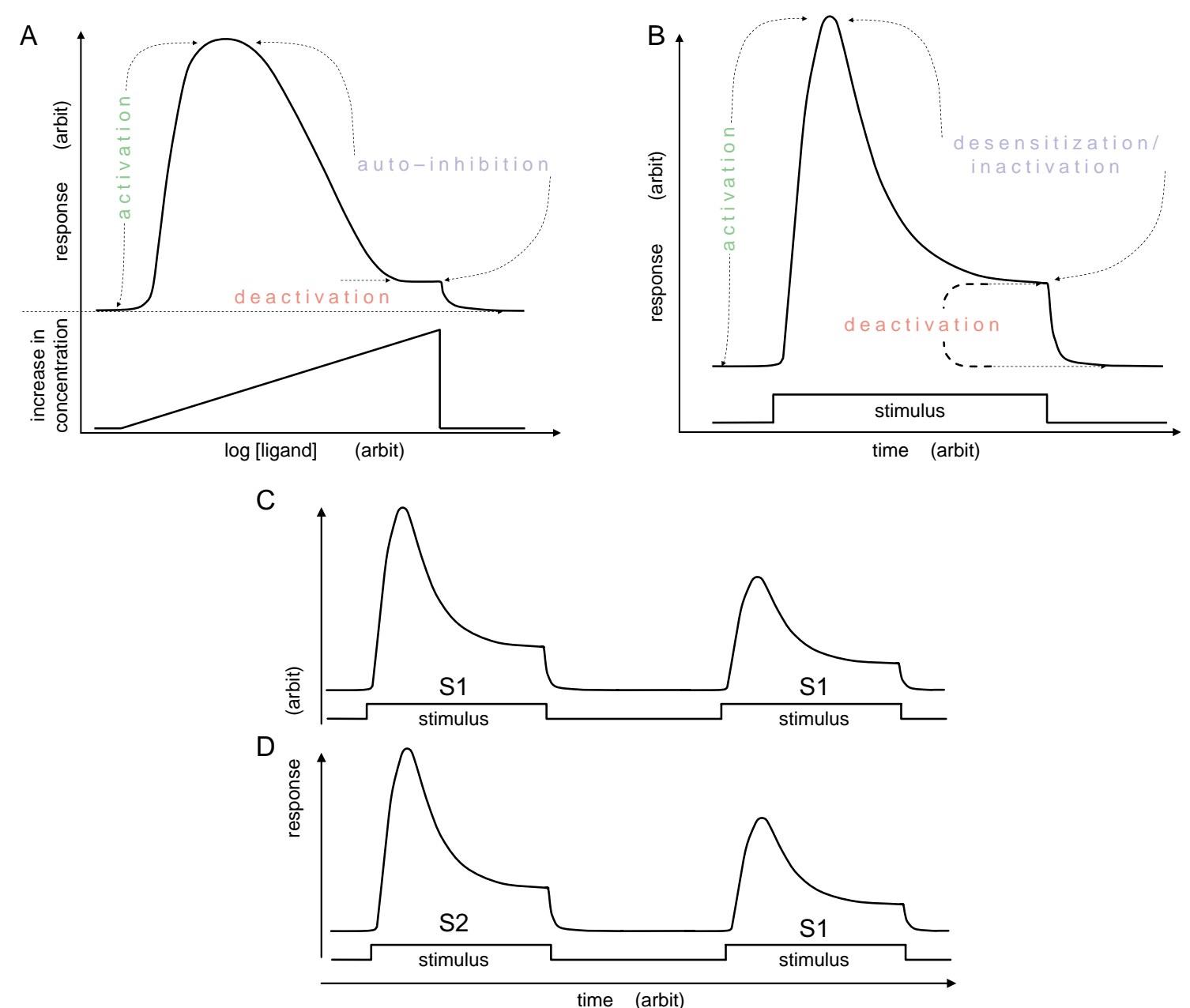

Figure 3.16. Principal diagram of auto-inhibition. Panel A: auto-inhibition due to rising ligand CONCENTRATION. Assuming a one-state reaction scheme it is 'negative auto-intervention', whilst based on a two-state model it is a 'negative auto-modulation'. Panel B: Auto-inhibition as a function of TIME is either a 'desensitization' or an 'inactivation' of the induced activation, some times during a constant stimulus. Panel C: a control response by a ligand S1 is reduced in a second application of the same ligand. This phenomenon is termed 'tachphylaxia' or 'desensitization'. Panel D: a ligand S2 that reduces a control response of a different ligand S1 is characterized as a 'heterologous desensitization'; opposite to the desensitization in panel C that is a 'homologous desensitization'. In general, when a stimulus is removed, there is a 'deactivation' of the response.

(VOCs) (Zagotta et al. 1990) is also seen for intrinsic desensitization in ligand-operated channels (LOCs) (Revah et al. 1991; Devillers-Thiery et al. 1993; Galzi et al. 1996; Mourot et al. 2006a,b). Meanwhile, molecular rearrangements within GPCRs and other desensitizing receptive molecules, such as tyrosine kinase receptors (TKR), are mainly regulated by external modulation with a longer half-time. Altered structural arrangements globally or of specific amino acid segments in $\mathrm{G}$ protein-coupled receptor molecules upon activation may induce lowered activity of the receptive unit. Lowered activity by phosphorylation and internalization of the receptive unit is based on $\mathrm{G}$ protein-coupled receptor kinases, second messenger kinases, arrestins, SNAREs, and dynamin (DeWire et al. 2007; Lefkowitz 2007; Rizo \& Dai 2007; Violin \& Lefkowitz 2007). Other means of auto-attenuation or auto-abrogation of the GPCRsignal are by control of the GTPase activity in the related $\mathrm{G}$ proteins regulated by 'regulators-of-G protein-signaling' proteins (RGSs) (Dhami \& Ferguson 2006). An intrinsic desensitization similar to VOC inactivation or intrinsic desensitization of LOCs has still not been described for the GPCRs or TKRs. The intrinsic desensitization is usually a fast process, within milliseconds to seconds (Giniatullin et al. 2005), while the extrinsic desensitization by phosphorylation mechanisms in the GPCRs takes place within minutes, although both sub-minute and hour-long half-times have been reported. As an example, homologous desensitization is often fast with a $\mathrm{t}_{1 / 2}<20 \mathrm{~s}$, whereas the heterologous desensitization is a slower process with a $\mathrm{t}_{1 / 2}>2 \mathrm{~min}$ (Roth et al. 1991). 
For many enzymes, and especially for members within the superfamily of kinases, there is self-inhibition due to intra-molecular sequences that are substrate-like (Francis et al. 2002). This mechanism may rather be classified under enzymes with intrinsic auto-ant-agonism for activation.

\subsection{6. 'Mixed' Auto-inhibition and Desensitization}

Desensitization is also a concentration-dependent phenomenon, i.e., the greater the stimulus, the larger the ensuing decay. The higher the concentration of agonists and the more prolonged the stimulus employed in the initial exposure, the more dramatic the reduction in response to a second test solution of an agonist.

Cumulative dose application may yield a dose-response relationship as shown in Fig. 3.17A, where each of the steady-state levels of response may be plotted against the actual agonist concentration (Fig. 3.17B). For this type of cumulative dose-response curve, we could use the term 'concentration-dependent desensitization', but since it would likely confuse some the term is not recommended.

In a narrow sense, the mixed auto-inhibition and desensitization is equal to substrate inhibition, while in a more general sense it may also involve, for example, product inhibition, feedback control through pre-synaptic auto-receptors, removal of the stimulus through degradation or re-uptake of signaling molecules, and phosphorylation by $\mathrm{G}$ protein coupled receptor kinases that leads to self-inhibition, through altered GTPase activity induced by RGSs, by guanine nucleotide exchange factors (GEFs), and by GTPase activating proteins (GAPs) or even downregulation of receptors by internalization (Koenig \& Edwardson 1997; Zhang et al. 1999) via endocytosis (Shimada et al. 2007)

You have or will experience that although autoinhibition has CONCENTRATION of an agonist as its independent variable and desensitization has TIME as its independent variable for a triggered response induced for instance by a fixed agonist concentration, the recording and analysis of these two phenomena are often mixed. Thus, in the practical and daily use of the two terms, they refer in general to an attenuation of a response elicited by a permanently present ligand - a substrate, an agonist, or a transportee. That is what you will be acquainted with. Here, I shall try to keep a clear distinction between concentration-dependent auto-inhibition and time-dependent desensitization, as illustrated in Figs. 3.16 and 3.17 and listed in Table 3.4, in an attempt to analyze the mechanisms underlying selfinhibition by synagic models.
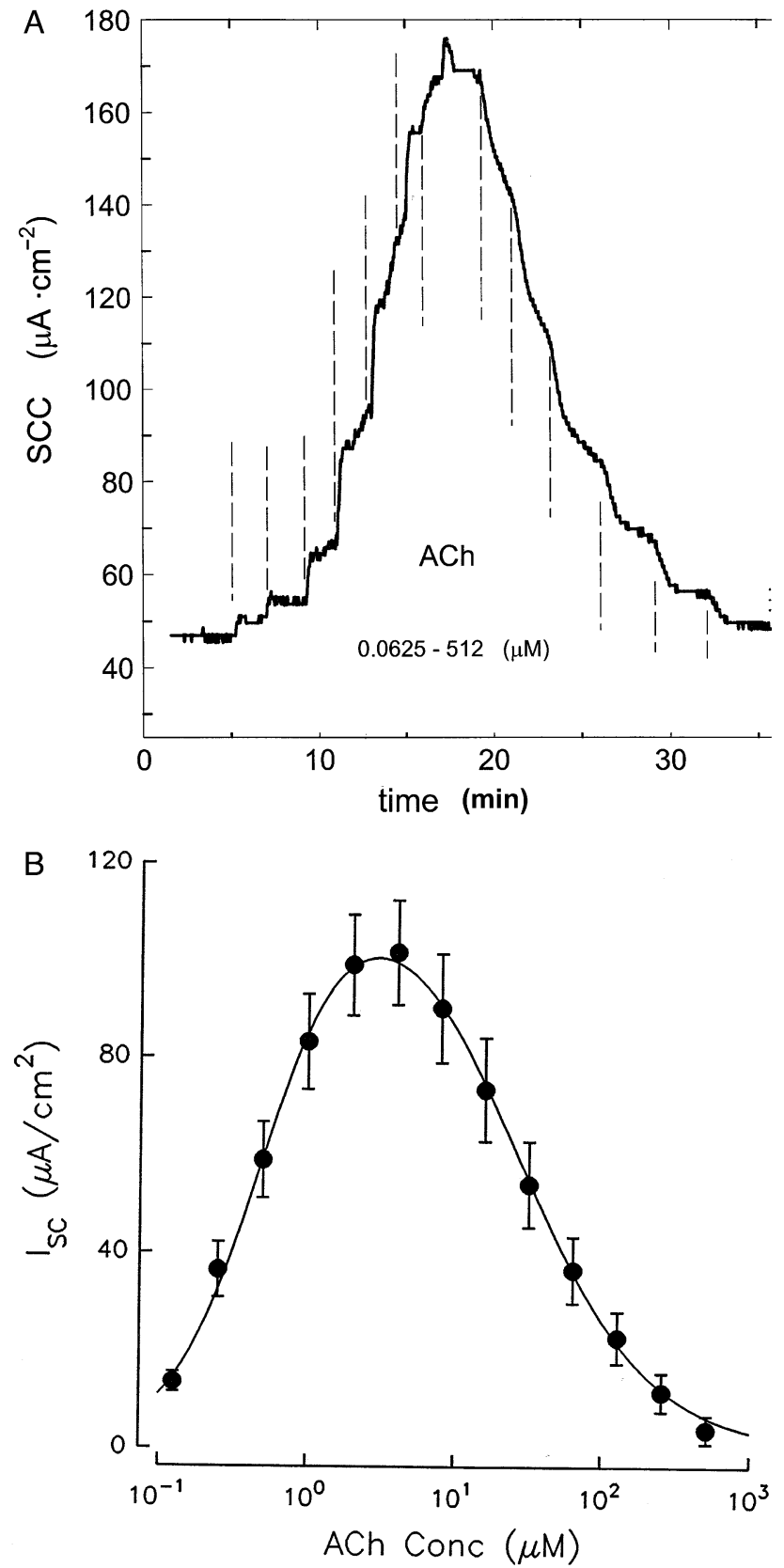

Figure 3.17. A mixture of desensitization and negative auto-modulation. Short circuit current (SCC) induced by acetylcholine in stripped tracheal epithelium from hens. The dose-response curve in panel $B$ is based on data from pseudo-steady-state or equilibrium conditions similar to the induced short circuit current with increasing concentrations of acetylcholine (Ach) in panel $A$. The time component in $A$ is eliminated in $\mathrm{B}$. Extracting the pure concentration-dependent attenuating effect, auto-inhibition, as shown in Fig. 3.17B, is not an illustration of TIME-dependent desensitization in sensu strictu. The 'pure' concentration-dependent attenuating effect in Fig. 3.17B should, therefore, be referred to as concentration-dependent auto-inhibition or auto-ant-agonism. (Courtesy of Dr. B. Winding, unpublished). 
Before I continue with examples of desensitization and auto-ant-agonism, it is worth reminding ourselves about definitions of a few other related reactions as illustrated in Fig. 3.16 and presented in Section 3.6.7.

\subsubsection{Two-state Models (TSMs), Two-site Models (2SMs) and Auto-inhibition}

Two-state models with only one-site (shown in Table 3.5 and described in Chapter 5), belong to the field of intrinsic and TIME-dependent desensitization, while two- (or more) site models, including the intervention model described in Chapter 2, more naturally go along with the ideas of CONCENTRATION-dependent autoinhibition. Thus, strictly speaking, auto-inhibition requires models with at least two binding sites.

A reversible and cyclic model that combines both two states and two sites is described in Chapter 7. This reversible, cyclic TSM may belong to a spontaneous, intrinsic type TIME-desensitization mechanism as well as to an intervention CONCENTRATION-dependent autoinhibitory mechanism, where the resultant conformational change gives a lower activity at higher concentrations of an interventor or a modulator. Auto-inhibition, contrary to desensitization and heterotropic competitive inhibition type I, still requires an additional binding site, i.e., a receptive unit with a minimum of two sites. Thus, the two-state reaction scheme in its basic form (Fig. 2.1B), with only one binding site, is only relevant for intrinsic desensitization or competitive inhibition type I.

\subsubsection{Desensitization and Deactivation}

'Deactivation' is the disappearance of a response with time upon removal of a triggering stimulus such as a substrate, an agonist, or a transportee. Thus, deactivation is the reverse mechanism of the activating conformational change, while mechanisms of desensitization for ligand gated channels or mechanisms of inactivation for VOCs result in 'tail' currents that have their base in mechanisms that are separate from the tail currents of deactivating conformational changes.

Desensitization and deactivation are separate phenomena, although they may be interconnected (Barberis et al. 2007). The desensitizing response in channels may be resolved from the process of deactivation, by experimentally determining their individual time constants, i.e. $\tau_{\text {off }}$ for deactivation from $\tau_{\text {on }}$ for desensitization. This can be a challenging experiment, when time constants for both activation and desensitization are in the millisecond range. Deactivation and desensitization have been studied in detail in for instance GABA-A receptor-channels (Jones \& Westbrook 1995; Barberis et al. 2007). As an example from one such study, the deactivation time constant was determined to be $0.6 \mathrm{~ms}$ and the desensitizing time constant resolved was $4 \mathrm{~ms}$ for a ligand gated GABA-A channel (Silver et al. 1996).

\subsubsection{Desensitization and Gating Currents}

Even two humps in I-V diagrams may be due to charge movements in the electrical field across the cell membrane as the gate of VOCs are activated for the channel opening. These charges sit in the fourth TM domain, S4, of cation channels such as the Shaker $\mathrm{K}^{+}$channel, and the movement of these charges in the electrical field is referred to as gating current. Gating currents for VOC channels have been studied in great detail by Bezanilla, Armstrong, and co-workers as well as by many others (Blunck et al. 2005; Armstrong 2006; Bezanilla 2006; Campos et al. 2007; Savalli et al. 2007). Also 'gating' currents that are found, for instance, in relation to co-transfer of ions and neutral nutrients, may be used for the detailed analysis of transport mechanisms in co-transporters (Eskandria et al. 2005; Loo et al. 2006). However, a prototype cotransporter, such as the sodium/D-glucose transporter (SGLT) (Wright et al. 2007) and the sodium-calcium exchanger (NCX) (Pott et al. 2007) normally do not 'desensitize'.

Finally, the deactivating tail current, as indicated in Fig. 3.16, and overshoot 'tail' current are due to channel and capacitative charge (ion) movements following the opening and closing of channels (Clay 1989; Hong \& Wang 2005; Zhang et al. 2005). These two transient currents must be kept separate from accelerated gating and inactivating/desensitizing current changes.

\subsubsection{Bell-shaped Dose-response Curves}

Auto-inhibition as the concentration of a ligand is raised may display bell-shaped dose-responses as depicted in Fig. 3.16. This regulatory behavior at high agonist or substrate concentration may have different mechanisms.

It may be due to an allosteric site at the receptor itself with a lower affinity for the agonist. Thus, with a negative influence on receptor activity, we may observe either negative co-operativity or a full-blown bell-shaped response. This mechanism is described by the complete intervention model in sub-chapter 3.3 (Eq. 3.5) and relevant for substrate inhibition of enzymes.

Another possibility is a withheld dissociation of GPCRs from the cogent $\mathrm{G}$ proteins when agonist-activated. The maintained dissociation is due to a phosphorylation and an association of beta-arrestins at the cytoplasmic loops and tails of GPCRs preventing their re-association to $\mathrm{G}$ proteins. The higher the agonist concentration, 
Table 3.5. Examples of receptors and effectors as enzymes and transporters in six categories. (For acronyms search in PubMed.)

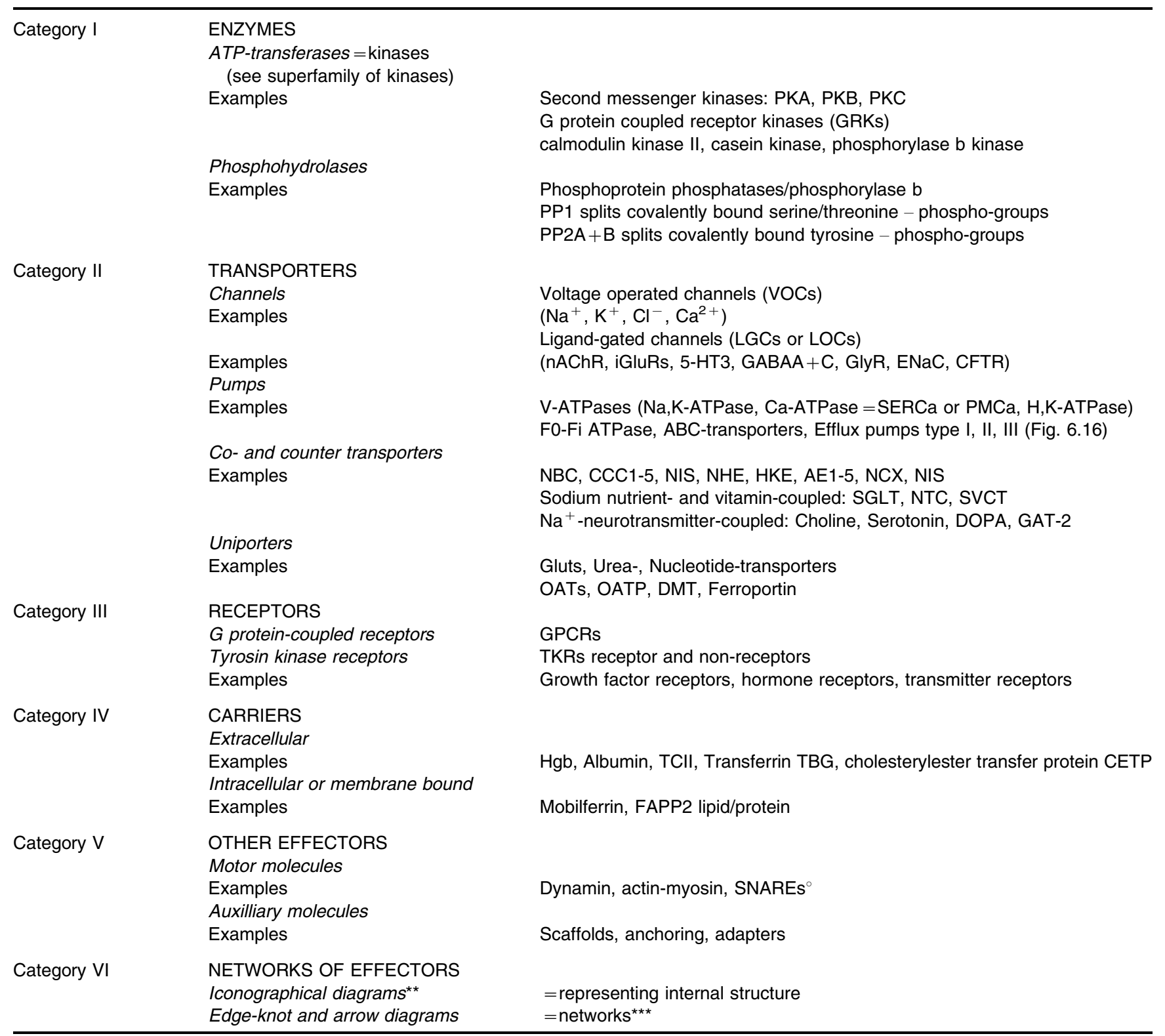

On SNAREs as motors see Rizo and Dai (2007).

${ }^{* *}$ On logical diagrams see, e.g., Vidler (2006, pp. 120-129) and Stjernfelt (2007). ${ }^{* * *}$ On networks see chapter 13 .

the higher the number of phosphorylated and betaarrestin-associated GPCRs; locked as non-functional.

A third possibility is a reduction in the GPCRs at the surface of cells due to internalization of activated receptors (Koenig \& Edwardson 1997; Zhang et al. 1999; Newman-Tancredi et al. 2002; Shimada et al. 2007). This process is referred to as 'down-regulation' and the removal of the receptors may even include a degradation of the internalized receptors in lysosomes instead of recycling to the cell membrane (Urbe 2005; Zhou et al. 2007). This results in a more permanent auto-inhibition.
As a fourth possibility of bell-shaped dose-response curves, a popular theory is invoked for growth factors and other peptide agonists as well as for antibodies. The model is based on the factual required dimerization for activation of antigen and growth factor receptors in a mono-ligand-dimer-receptor complex. As the concentration of either antibody or peptide ligand rises, chances increase of forming inactive mono-ligand-mono-receptor complexes (Fig. 3.18). This mono-mono complexation at increasing ligand concentrations can explain the decaying leg of observed bell-shaped dose-response curves 


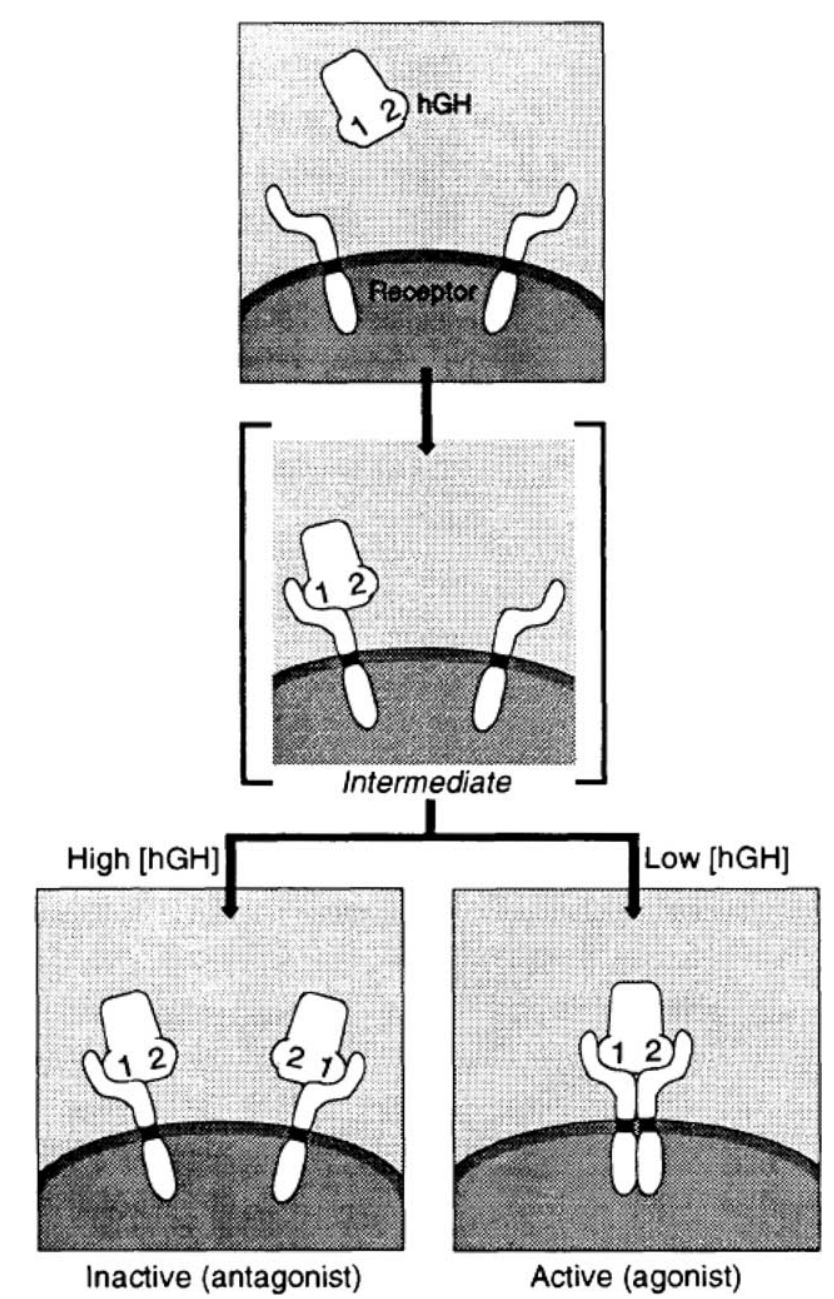

Figure 3.18. A model for bell-shaped synagics in functional dimerization. High dose of human growth factor (hGH) or antibody favors $\mathrm{hGH} / \mathrm{hGH}$-receptor or antigen-antibody monomeric complexes and prevents the formation of active dimeric complexes. From Fuh et al. (1992; Fig. 1) with permission.

(Fig. 3.19) (Maeyama et al. 1988; Fuh et al. 1992; Ilondo et al. 1994; de Boer et al. 1996; Urso et al. 2003).

A fifth possibility for upward bell-shaped dose-response curves is negative regulation through auxiliary receptor proteins as the RGSs (Cladman \& Chidiac 2002; Dhami \& Ferguson 2006).

Finally, downward or reverse bell-shaped dose-responses with an accelerating activity at increasing concentrations of a single ligand are also frequently seen for GPCRs (Accamzzo et al. 2002; Hornigold et al. 2003), and a suggestion for the underlying mechanism was offered by Bindslev (2004).

\subsubsection{Constitutive Desensitization}

Artificially mutated or 'naturally' mutated G protein coupled receptors may display constitutively desensitized receptor activity, and thus be the cause of illnesses
(Pei et al. 1994; Barak et al. 2001, 2003; Rankin et al. 2006). Spontaneously desensitized receptor units are also found for LOCs, as the nicotinic acetylcholine receptor (Changeux \& Edelstein 2005, p. 97).

\subsubsection{Desensitization-inactivation and the Induction-versus-selection Problem}

The conformational induction versus conformational selection problem described in sub-chapter 5.11 is the same for desensitization-inactivation as for activation, except that desensitization-inactivation requires the activation step preceding the inactivation-desensitization step (see Fig. 5.2). Therefore, in essence, inactivationdesensitization is a three-state model. The three states are (1) a reactive state to which agonist can bind, ${ }^{6}$ (2) spontaneous or induced conformational flip-flop between a reactive and an active state, which leads to (3) a spontaneous or induced inactivated-desensitized state, by an intrinsic or an extrinsic mechanism (Fig. 5.2).

\subsection{Kühl's Minimal Recovery Model for Bell-shaped Synagics}

\subsubsection{A Recovery Model}

Another model for bell-shaped dose-responses in functional studies was formulated by Kühl (1994).

In this book substrate inhibition or auto-inhibition at high ligand concentrations is termed auto-intervention or auto-modulation; conditioned on the selected reaction scheme. Kühl introduced the subject of substrate inhibition with the following statement: "although recognized early on as an almost universal phenomenon, it has nevertheless met an almost universal disinterest" and "Probably the main reason for this neglect is that the majority of enzymologists and many authorities in the field regard substrate inhibition as being almost always a non-physiological phenomenon".

Besides auto-inhibition, other terms for high substrate inhibition are (1) high-dose inhibition, (2) auto-antagonism, (3) auto-desensitization, (4) self-blockade, (5) excess ligand inhibition, (6) bell-shaped dose-response relationship, and (7) the Arndt-Schulz law. The ArndtSchulz law is, however, on the sideline. It stems from the homeopathic field - stating that small doses may have a beneficiary effect, while large doses may be self-inhibitory (Clark 1937).

\footnotetext{
6 The reactive receptor in itself actually has two conformations or states, an unbound and a bound state, thus adding one more state to the above counting (see Fig 5.2). In reality there are even more states. Observe though, that for modeling of allostery, 'states' are only for un-liganded receptor conformations as defined and described in Chapters 14 and 15 .
} 


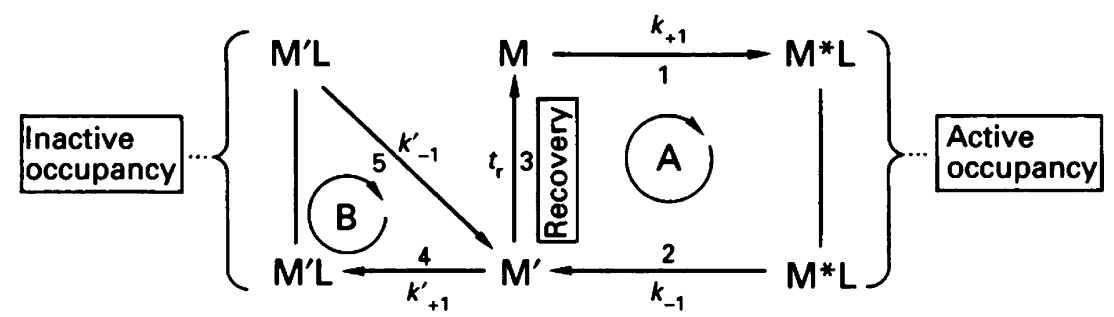

Figure 3.19. Kühl's minimal recovery model. Reproduced from Kühl (1994; Fig. 3) with permission.

Kühl suggests a reaction scheme for auto-inhibition, which he calls 'the recovery model'. This model also suggests new ways to explain such diverse phenomenon as partial agonism, pulse generation, desensitization, memory effects, and ultra-sensitivity.

Certain liganded states are shown twice in Kühl's general reaction scheme in order to emphasize steps in his recovery model. For instance $\mathrm{R} * \mathrm{~S}$ equals both ligand bound and activated, and R'S equals both ligand bound and non-activatable (desensitized) states. Thus, Kühl models deviate on several points from an ordinary reversible kinetic scheme (Kuhl 1994; Kuhl \& Jobmann 2006).

\subsubsection{Formulation of Kühl's Minimal Recovery Model}

Kühl's minimal recovery model is shown in Fig. 3.19.

Direct return from an activated state $\mathrm{R} * \mathrm{~S}$ to a reactive (reactivatable) state $\mathrm{R}$ is not possible in Kühl's general model. Thus, $\mathrm{R}^{*} \rightarrow \mathrm{R}$, termed deactivation, ${ }^{7}$ is not allowed, while recovery in a step from a non-activatable (refractory) state $R^{\prime}$ to an activatable (reactive) state $R$, i.e., $\mathrm{R}^{\prime} \rightarrow \mathrm{R}$, is allowed. Further, $\mathrm{R}^{*}$ and $\mathrm{R}^{*} \mathrm{~S}$ are lumped together as well as $\mathrm{R}$ and RS. Thus, by release from the conventional symbolization, certain elements and pathways are eliminated, and with lumped conformations we reach a rather simple graphical representation, a socalled minimal recovery model (Fig. 3.19).

Notice, in Kühl's minimal recovery model for autoinhibition even the RS complex is lumped together with the free conformation of the receptor in the resting state, $\mathrm{R}$, and both referred to as $\mathrm{R}$.

Essential features of the minimal recovery model by Kühl are the association of agonist $\mathrm{S}$ with $\mathrm{R}$ and with $\mathrm{R}^{\prime}$ having rates $k_{+1} \cdot \mathrm{S} \cdot \mathrm{R}$ and $k_{+1}^{\prime} \cdot \mathrm{S} \cdot \mathrm{R}^{\prime}$, and the dissociation of $\mathrm{RS}$ and $\mathrm{R}^{\prime} \mathrm{S}$ with the rate constants $k_{-1}$ and $k_{-1}^{\prime}$. Only the complex $\mathrm{R}^{*} \mathrm{~S}$ is active. The activity is proportional to the concentration of $\mathrm{R} * \mathrm{~S}$ and/or the time spent in this configuration. $\mathrm{R}$ is locked in its refractory or nonactivatable form $\mathrm{R}^{\prime}$, when an agonist, $\mathrm{S}$, binds to this isomeric conformation.

\footnotetext{
7 Kühl use the term 'deactivation' at variance with its use in this book (see, e.g., Fig. 3.16).
}

The essence of the minimal recovery model is the balance between the time constant, $t_{\mathrm{r}}$, for recovery from $\mathrm{R}$ ' to $\mathrm{R}$ and the association constant $k_{+1}^{\prime} \mathrm{S} / k_{-1}^{\prime}$ for the association with, and dissociation of the agonist from the refractory state of the receptor, $R^{\prime}$.

The probability that reaction $R^{\prime} \rightarrow R$ happens instead of the reaction $R^{\prime} \rightarrow R^{\prime} S$ is therefore given by the stochastic frequency that reaction $R^{\prime} \rightarrow R^{\prime} S$ does not occur within the fixed recovery time of $t_{\mathrm{r}}$. The recovery is a waiting process described by a Poissonian distribution

$$
P=\exp \left(-k_{+}^{\prime} \cdot \mathrm{S} \cdot t_{\mathrm{r}}\right)
$$

and the $\mathrm{R}^{\prime} \rightarrow \mathrm{R}$ isomerization has a fixed duration called $t_{\mathrm{r}}$. The recovery time $t_{\mathrm{r}}$ may be put equal to $1 / k_{\mathrm{r}}$, where $k_{\mathrm{r}}$ is the frequency of the recovery, although not a genuine rate constant.

In the formulation of the minimal recovery model, $\mathrm{S}$ designates concentration of substrate/agonist $S$, $[S]$, or concentration of substrate/agonist $\mathrm{S}$ normalized by its equilibrium dissociation constant $K_{\mathrm{s}}$. To simplify the analysis, we assume that $K_{\mathrm{s}}=1$; thus $\mathrm{S}$ is $[\mathrm{S}]$ or $[\mathrm{S}] / K_{\mathrm{s}}$.

Parameters in Kühl's minimal recovery model are:

$\lambda=\mathrm{S} / K_{\mathrm{s}}$, and $\mathrm{S} / K_{\mathrm{s}}=\mathrm{S}$, since as indicated above $K_{\mathrm{s}}$ is assumed $=1$.

$$
\begin{aligned}
& F=k_{+1} \cdot t_{\mathrm{r}} \\
& P=\exp (-F \cdot \mathrm{S}) \\
& A=k_{-1} / k_{+1} \cdot k_{+1}^{\prime} / k_{-1}^{\prime}=K_{\mathrm{d}} / K_{\mathrm{d}}^{\prime} \\
& B=k_{-1}^{\prime} / k_{-1} \\
& T=k_{-1} \cdot t_{\mathrm{r}}
\end{aligned}
$$

The product of $A$ and $B$ is $A \cdot B=k_{+1}^{\prime} / k_{+1}$ which we may express as a new constant $=C$.

Kühl's equation A9 for the response of his model then becomes:

$R=$

$$
\frac{P \cdot \mathrm{S}}{P \cdot(1+\mathrm{S}+\mathrm{S} \cdot T)+\frac{1-P}{A \cdot B} \cdot[1+A \cdot \mathrm{S}-(1+A B T \mathrm{~S}) \cdot P]},
$$

which can be slightly contracted and thus reformulated to: 
response $=\frac{\mathrm{S}}{\mathrm{S} \cdot\left(1+P \cdot T+\frac{(1-P) \cdot A}{P \cdot C}\right)+1+\frac{(1-P)^{2}}{P \cdot C}}$.

Here $1-P$ is the probability of not recovering. Although the Kühl model is hampered by non-reversibility, examples of dose-response relations for the model are shown in Fig. 3.20 with a resumé.

On particular features in his model, Kühl states that the presence of an exponential term introduces an asymmetry into the dose-response curves and can elicit an inhibitory leg of the dose-response curve, which can be very steep; steeper than derived for ordinary reaction schemes with conventional rate constants. This is true as demonstrated in Fig 3.20.

\subsubsection{Where Does Kühl's Model Fit In?}

Kühl's minimal recovery model only requires that high agonist concentration favors an inactive state of a less active isomer of the receptor or effector molecule in order to simulate bell-shaped synagics. Meanwhile,
Kühl's model cannot describe activation of a substrateinduced auto-inhibition state by an interventor of the studied system; as found in several systems and discussed in sub-chapter 7.9.

\subsubsection{Conclusions on Kühl's}

Kühl's model puts forward a simple model for autoant-agonism and desensitization by assuming slow reaction kinetics for certain association and dissociation steps. The model omits a selection of generally accepted molecular conformations. Furthermore, pathways between receptor conformations are banned and most steps are irreversible. Kühl derives a hypothetical probability expression based on the relation between rate constants, rates, and recovery time. The latter appears in the exponential function $\mathrm{P}$ - or even as a Poissonian expression, see above.

It is commendable to include rates and rate constants, which for simplicity have been heavily suppressed in this tome. However, in the case of Kühl's model, including rates does not necessarily tangle us out of the snares that hamper insight into synagics and kinetics.
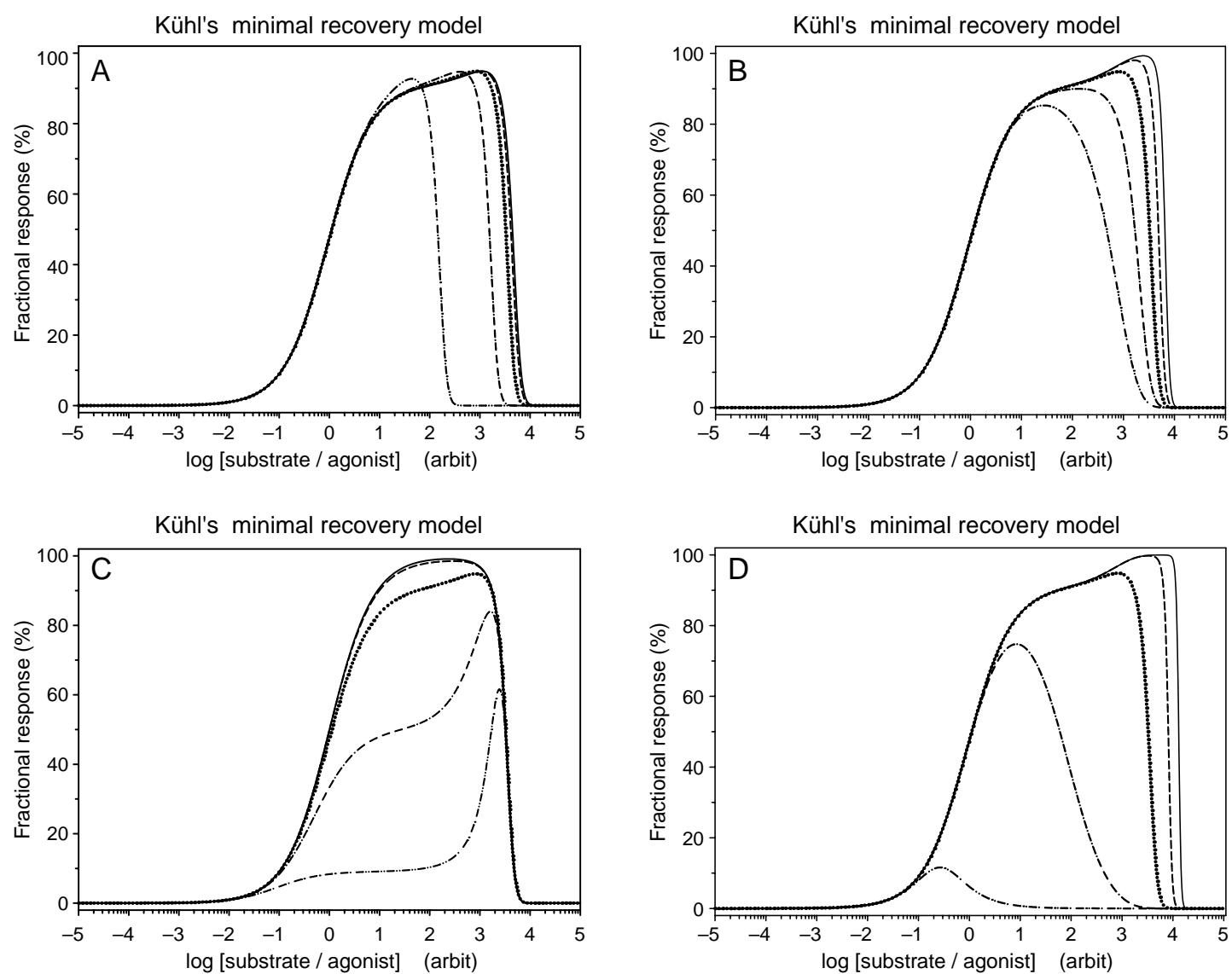

Figure 3.20. Dose-response plots of the Kühl model. Parameters in all plot, when not varied in five steps, were: $F=1.414, A=1$, $T=0.1$, and $C=0.01$. (A) Parameter $F$ is varied in five steps from $1.035 \cdot 10^{-3}(-)$ to $3.20 \cdot 10^{-2}(-\cdots-)$ by a factor square root of 3. (B) Parameter $A$ is varied in five steps from $10^{-2}(-)$ to $10^{2}(-\cdots-)$ by a factor 10 . (C) Parameter $T$ is varied in five steps from $10^{-3}(-)$ to $10^{1}(-\cdots-)$ by a factor 10 . (D) Parameter $C$ is varied in five steps from $10^{-8}(-)$ to $10^{4}(-\cdots-)$ by a factor $10^{3}$. 
Rather, you should start by choosing a fully reversible reaction scheme, as also strongly advocated by Colquhoun (1998). Select the simplest, e.g., the K\&T model, and then, as Kühl, add an extra loop with binding of the substrate that somehow inhibits the activated state of the receptor. It may be by binding and conformational change at a secondary site, or as with Kühl, binding of the agonist to a refractory state, and stabilizing that conformation by preventing recovery back to the reactivatable state - the resting state. Study this type of model, even though they do not include time, before engaging in non-reversible models. Of course, TIME is a parameter indispensable for the full analysis of kinetics, but its introduction may be postponed until the full implications of synagic analysis are grasped.

Once again, the purpose of this book is to demonstrate that in order to engage in understanding molecular kinetics dependent on time, it is beneficial to start at equilibrium with fully reversible models, before omitting natural pathways and involving more complicated reaction schemes including irreversibility.

\section{REFERENCES}

Abedin M \& King N. The premetazoan ancestry of cadherins. Science 319: 946-948, 2008.

Accomazzo MR, Cattaneo S, Nicosia S \& Rovati GE. Bell-shaped curves for prostaglandin-induced modulation of adenylate cyclase: two mutually opposing effects. Eur J Pharmacol 454: 107-114, 2002.

Agnati LF, Guidolin D, Leo G \& Fuxe K. A Boolean network modelling of receptor mosaics relevance of topology and cooperativity. J Neural Transm 114: 77-92, 2007.

Albizu L, Balestre MN, Breton C, Pin JP, Manning M, Mouillac B, et al. Probing the existence of $\mathrm{G}$ protein-coupled receptor dimers by positive and negative ligand-dependent cooperative binding. $\mathrm{Mol}$ Pharmacol 70: 1783-1791, 2006.

Allis CD, Jenuwein T \& Reinberg D. Epigenetics, 1st ed. Cold Spring Harbor, NY: Cold Spring Harbor Laboratory Press, 2007.

Alon U. An Introduction to Systems Biology. Design Principles of Biological Circuits. Boca Raton, Chapman \& Hall/CRC, 2006.

Alpers DH. What is new in vitamin B(12)? Curr Opin Gastroenterol 21: 183-186, 2005.

Andersen JP, Sorensen TL, Povlsen K \& Vilsen B. Importance of transmembrane segment M3 of the sarcoplasmic reticulum $\mathrm{Ca}^{2+}$. ATPase for control of the gateway to the $\mathrm{Ca}^{2+}$ sites. J Biol Chem 276: 23312-23321, 2001.

Armoundas AA, Rose J, Aggarwal R, Stuyvers BD, O'rourke B, Kass DA, Marban E, Shorofsky SR, Tomaselli GF and William Balke C. Cellular and molecular determinants of altered $\mathrm{Ca}^{2+}$ handling in the failing rabbit heart: primary defects in $\mathrm{SR} \mathrm{Ca}^{2+}$ uptake and release mechanisms. Am J Physiol Heart Circ Physiol 292: H1607H1618, 2007.

Armstrong CM. Na channel inactivation from open and closed states. Proc Natl Acad Sci USA 103: 17991-17996, 2006.

Armstrong D \& Strange PG. Dopamine D2 receptor dimer formation: evidence from ligand binding. J Biol Chem 276: 22621-22629, 2000.

Avlani V, May LT, Sexton PM \& Christopoulos A. Application of a kinetic model to the apparently complex behavior of negative and positive allosteric modulators of muscarinic acetylcholine receptors. J Pharmacol Exp Ther 308: 1062-1072, 2004.
Bak P. How Nature Works. The Science of Self-organized Criticality, 1st ed. Oxford: Oxford University Press, 1997.

Barak LS, Oakley RH, Laporte SA \& Caron MG. Constitutive arrestinmediated desensitization of a human vasopressin receptor mutant associated with nephrogenic diabetes insipidus. Proc Natl Acad Sci USA 98: 93-98, 2001.

Barak LS, Wilbanks AM \& Caron MG. Constitutive desensitization: a new paradigm for $\mathrm{G}$ protein-coupled receptor regulation. Assay Drug Dev Technol 1: 339-346, 2003.

Barberis A, Mozrzymas JW, Ortinski PI \& Vicini S. Desensitization and binding properties determine distinct alphalbeta2gamma2 and alpha3beta2gamma2 GABA(A) receptor-channel kinetic behavior. Eur J Neurosci 25: 2726-2740, 2007.

Berteloot A. Kinetic mechanism of $\mathrm{Na}+$-glucose cotransport through the rabbit intestinal SGLT1 protein. J Membr Biol 192: 89-100, 2003.

Bezanilla F. The action potential: from voltage-gated conductances to molecular structures. Biol Res 39: 425-435, 2006.

Bhattacharyya RP, Remenyi A, Good MC, Bashor CJ, Falick AM \& Lim WA. The Ste5 scaffold allosterically modulates signaling output of the yeast mating pathway. Science 311: 822-826, 2006.

Bindslev N. A homotropic two-state model and auto-antagonism. BMC Pharmacol 4: 11, 2004.

Blunck R, Chanda B \& Bezanilla F. Nano to micro-fluorescence measurements of electric fields in molecules and genetically specified neurons. J Membr Biol 208: 91-102, 2005.

Bonner JT. First Signals: the Evolution of Multicellularity. Princeton, NJ: Princeton University Press, 2001.

Botts J \& Morales M. Analytical description of the effects of modifiers and of enzyme multivalency upon the steady state catalyzed reaction rate. Trans Faraday Soc 49: 696-707, 1953.

Bronnikov GE, Zhang SJ, Cannon B \& Nedergaard J. A dual component analysis explains the distinctive kinetics of cAMP accumulation in brown adipocytes. J Biol Chem 274: 37770-37780, 1999.

Bucher K, Besse CA, Kamau SW, Wunderli-Allenspach H \& Kramer SD. Isolated rafts from adriamycin-resistant P388 cells contain functional ATPases and provide an easy test system for P-glycoproteinrelated activities. Pharm Res 22: 449-457, 2005.

Bunemann M \& Hosey MM. G-protein coupled receptor kinases as modulators of G-protein signalling. J Physiol 517: 5-23, 1999.

Bunemann M \& Hosey MM. Novel signalling events mediated by muscarinic receptor subtypes. Life Sci 68: 2525-2533, 2001.

Camazine S, Deneubourg J-L, Franks NR, Sneyd J, Theraulaz G \& Bonabeau E. Self-organization in Biological Systems, 1st ed. Princeton, NJ: Princeton University Press, 2001.

Campos FV, Chanda B, Roux B \& Bezanilla F. Two atomic constraints unambiguously position the S4 segment relative to S1 and S2 segments in the closed state of Shaker K channel. Proc Natl Acad Sci USA 130: 257-268, 2007.

Cavell S. The Claim of Reason: Wittgenstein, Skepticism, Morality, and Tragedy, New ed. Oxford: Oxford University Press, 1999.

Changeux J-P \& Edelstein SJ. Nicotinic Acetylcholine Receptors: from Molecular Biology to Cognition, English ed. Baltimore: Johns Hopkins University Press, 2005.

Chuang TT, Iacovelli L, Sallese M \& De Blasi A. G protein-coupled receptors: heterologous regulation of homologous desensitization and its implications. Trends Pharmacol Sci 17: 416-421, 1996.

Cladman W \& Chidiac P. Characterization and comparison of RGS2 and RGS4 as GTPase-activating proteins for $\mathrm{m} 2$ muscarinic receptor-stimulated G(i). Mol Pharmacol 62: 654-659, 2002.

Clark AJ. General Pharmacology, Vol. 4, 1st ed. Berlin: Springer, 1937.

Clark RW. J.B.S. The Life and Work of J.B.S. Haldane. London: Hodder \& Stoughton, 1968.

Clay JR. Slow inactivation and reactivation of the $\mathrm{K}+$ channel in squid axons. A tail current analysis. Biophys J 55: 407-414, 1989. 
Colquhoun D. Binding, gating, affinity and efficacy: the interpretation of structure-activity relationships for agonists and of the effects of mutating receptors. Br J Pharmacol 125: 924-947, 1998.

Colquhoun D, Dowsland KA, Beato M and Plested AJ. How to impose microscopic reversibility in complex reaction mechanisms. BiophysJ 86: 3510-3518, 2004.

Cornish-Bowden A. The Pursuit of Perfection. Aspects of Biochemical Evolution, 1st ed. Oxford: Oxford University Press, 2004.

Curien G, Laurencin M, Robert-Genthon M \& Dumas R. Allosteric monofunctional aspartate kinases from Arabidopsis. FEBS J 274: 164-176, 2007.

Davies P. The Mind of God. Science and the Search for Ultimate Meaning, 1st ed. London: Penguin Books, 1993.

De Boer RJ, Boerlijst MC, Sulzer B \& Perelson AS. A new bell-shaped function for idiotypic interactions based on cross-linking. Bull Math Biol 58: 285-312, 1996.

Devillers-Thiery A, Galzi JL, Eisele JL, Bertrand S, Bertrand D \& Changeux JP. Functional architecture of the nicotinic acetylcholine receptor: a prototype of ligand-gated ion channels. J Membr Biol 136: 97-112, 1993.

DeWire SM, Ahn S, Lefkowitz RJ \& Shenoy SK. Beta-arrestins and cell signaling. Annu Rev Physiol 69: 483-510, 2007.

Dhami GK \& Ferguson SS. Regulation of metabotropic glutamate receptor signaling, desensitization and endocytosis. Pharmacol Ther 111: 260-267, 2006.

Durroux T. Principles: a model for the allosteric interactions between ligand binding sites within a dimeric GPCR. Trends Pharmacol Sci 26: $376-384,2005$.

Ehlert FJ. Contractile role of M2 and M3 muscarinic receptors in gastrointestinal, airway and urinary bladder smooth muscle. Life Sci 74: 355-366, 2003.

Einholm AP, Andersen JP \& Vilsen B. Importance of Leu99 in transmembrane segment $\mathrm{M} 1$ of the $\mathrm{Na}+, \mathrm{K}+$-ATPase in the binding and occlusion of $\mathrm{K}+. J$ Biol Chem 282: 23854-23866, 2007.

Ellis J. Allosteric binding sites on muscarinic receptors. Drug Dev Res 40: 193-204, 1997.

Embley TM \& Martin W. Eukaryotic evolution, changes and challenges. Nature 440: 623-630, 2006.

Eskandari S, Wright EM \& Loo DD. Kinetics of the reverse mode of the $\mathrm{Na}+$ /glucose cotransporter. J Membr Biol 204: 23-32, 2005.

Feltz B, Crommelinck M \& Goujon P. Self-organization and Emergence in Life Sciences, 1st ed. Dordrecht: Springer, 2006.

Ferguson SS \& Caron MG. G protein-coupled receptor adaptation mechanisms. Semin Cell Dev Biol 9: 119-127, 1998.

Ford ED. Scientific Method for Ecological Research. Cambridge: Cambridge University Press, 2000.

Forrest AR, Taylor DF, Fink JL, Gongora MM, Flegg C, Teasdale RD, Suzuki H, Kanamori M, Kai C, Hayashizaki Y \& Grimmond SM. PhosphoregDB: the tissue and sub-cellular distribution of mammalian protein kinases and phosphatases. BMC Bioinformatics 7: 82, 2006

Fortin Y \& De Lean A. Role of cyclic GMP and calcineurin in homologous and heterologous desensitization of natriuretic peptide receptor-A. Can J Physiol Pharmacol 84: 539-546, 2006.

Francis SH, Turko IV \& Corbin JD. Cyclic nucleotide phosphodiesterases: relating structure and function. Prog Nucleic Acid Res Mol Biol 65: 1-52, 2001.

Francis SH, Poteet-Smith C, Busch JL, Richie-Jannetta R \& Corbin JD. Mechanisms of autoinhibition in cyclic nucleotide-dependent protein kinases. Front Biosci 7: 580-592, 2002.

Franco R, Casado V, Mallol J, Ferrada C, Ferre S, Fuxe K, Cortes A, Ciruela F, Lluis C \& Canela EI. The two-state dimer receptor model: a general model for receptor dimers. Mol Pharmacol 69: 1905-1912, 2006.
Freedman NJ \& Lefkowitz RJ. Desensitization of G protein-coupled receptors. Recent Prog Horm Res 51: 319-351, 1996.

Fruchart-Gaillard C, Mourier G, Marquer C \& Menez A. Identification of various allosteric interaction sites on $\mathrm{M}_{1}$ muscarinic receptor using ${ }^{125}$ I-met35-oxidized muscarinic toxin 7. Mol Pharmacol 69: 1641-1651, 2006.

Fuh G, Cunningham BC, Fukunaga R, Nagata S, Goeddel DV \& Wells JA. Rational design of potent antagonists to the human growth hormone receptor. Science 256: 1677-1680, 1992.

Galzi JL, Edelstein SJ \& Changeux J. The multiple phenotypes of allosteric receptor mutants. Proc Natl Acad Sci USA 93: 1853-1858, 1996.

Giniatullin R, Nistri A \& Yakel JL. Desensitization of nicotinic ACh receptors: shaping cholinergic signaling. Trends Neurosci 28: 371378, 2005.

Gosselin RE. Drug-receptor interaction: a new kinetic model. In: Kinetics of Drug Action, 1st ed, edited by van Rossum JM. Berlin: Springer Verlag, 1977.

Green B. The Fabric of the Cosmos. Space, Time, and the Texture of Reality, First Vintage Books edition. New York: Vintage Books, 2005.

Griffin MT, Hsu JC, Shehnaz D \& Ehlert FJ. Comparison of the pharmacological antagonism of M2 and M3 muscarinic receptors expressed in isolation and in combination. Biochem Pharmacol 65: 1227-1241, 2003.

Gupta A, Lawrence AT, Krishnan K, Kavinsky CJ \& Trohman RG. Current concepts in the mechanisms and management of druginduced QT prolongation and torsade de pointes. Am Heart $J 153$ : 891-899, 2007.

Haldane JBS. Enzymes, 1st ed. London: Longmans, Green \& Co, 1930. Hapfelmeier G, Tredt C, Haseneder R, Zieglgansberger W, Eisensamer B, Rupprecht R \& Rammes G. Co-expression of the 5-HT3B serotonin receptor subunit alters the biophysics of the 5-HT3 receptor. Biophys J 84: 1720-1733, 2003.

Harper ET. Kinetics of the two-sited enzyme. I. Activation and inhibition by substrate. J Theor Biol 32: 405-414, 1971.

Hayashi I, Plevin MJ \& Ikura M. CLIP170 autoinhibition mimics intermolecular interactions with p150(Glued) or EB1. Nat Struct Mol Biol 14: 980-981, 2007.

Hisatomi O, Matsuda S, Satoh T, Kotaka S, Imanishi Y \& Tokunaga F. A novel subtype of G-protein-coupled receptor kinase, GRK7, in teleost cone photoreceptors. FEBS Lett 424:159-164, 1998.

Hoch JA. Two-component and phosphorelay signal transduction. Curr Opin Microbiol 3: 165-170, 2000.

Hoenisberg HF, Tijaro MH \& Sanabria C. From unicellularity to multicellularity - molecular speculations about early animal evolution. Genet Mol Res 7: 50-59, 2008.

Hofstadter D. I Am a Strange Loop, 1st ed. New York: Basic Books, 2007.

Hong Z, Wang DS. Potentiation, activation and blockade of GABAA receptors by etomidate in the rat sacral dorsal commissural neurons. Neuroscience 132: 1045-1053, 2005.

Hornigold DC, Mistry R, Raymond PD, Blank JL \& Challiss RA. Evidence for cross-talk between M2 and M3 muscarinic acetylcholine receptors in the regulation of second messenger and extracellular signal-regulated kinase signalling pathways in Chinese hamster ovary cells. Br J Pharmacol 138: 1340-1350, 2003.

Hoyle F. Of Men and Galaxies. Amhent, Prometheus Books, 2005.

Hudspeth AJ. How the ear's works work: mechanoelectrical transduction and amplification by hair cells. C R Biol 328: 155-162, 2005.

Ilondo MM, Damholt AB, Cunningham BA, Wells JA, De Meyts P \& Shymko RM. Receptor dimerization determines the effects of growth hormone in primary rat adipocytes and cultured human IM-9 lymphocytes. Endocrinology 134: 2397-2403, 1994.

Jarv J, Toomela T \& Karelson E. Dual effect of carbachol on the muscarinic receptor. Biochem Mol Biol Int 30: 649-654, 1993. 
Jensen HJ. Self-Organized Criticality: Emergent Complex Behavior in Physical and Biological Systems, 1st ed. Cambridge, Cambridge University Press 1998.

Johnson SC, Schmitz TW, Kawahara-Baccus TN, Rowley HA, Alexander AL, Lee J \& Davidson RJ. The cerebral response during subjective choice with and without self-reference. J Cogn Neurosci 17: 18971906, 2005.

Jones MV \& Westbrook GL. Desensitized states prolong GABAA channel responses to brief agonist pulses. Neuron 15: 181-191, 1995.

Jow B \& Numann R. The effects of ZD6169 on the ATP-dependent $\mathrm{K}(+)$ current $(\mathrm{I}(\mathrm{K})(\mathrm{ATP}))$ in isolated cat ventricular myocytes. Eur J Pharmacol 383: 197-202, 1999.

Kaiser PM. Substrate inhibition as a problem of non-linear steady state kinetics with monomeric enzymes. J Mol Catal 8: 431-442, 1980.

Kessin RH. Dictyostelium: Evolution, Cell Biology, and the Development of Multicellularity, 1st ed. Cambridge: Cambridge University Press, 2001.

King N. The unicellular ancestry of animal development. Dev Cell 7: 313-325, 2004.

Kingsland SE. Modeling Nature. Episodes in the History of Population Ecology, 2nd ed. Chicago, IL: The University of Chicago Press, 1995.

Kirk D. Volvox. Molecular-genetic Origins of Multicalluarity and Cellular Differentiation, 1st ed. Cambridge: Cambridge University Press, 1998.

Klumpp S \& Krieglstein J. Reversible phosphorylation of histidine residues in vertebrate proteins. Biochim Biophys Acta 1754: 291$295,2005$.

Koenig JA \& Edwardson JM. Endocytosis and recycling of G proteincoupled receptors. Trends Pharmacol Sci 18: 276-287, 1997.

Kolch W. Coordinating ERK/MAPK signalling through scaffolds and inhibitors. Nat Rev Mol Cell Biol 6: 827-837, 2006.

Kukkonen JP, Näsman J, Rinken A, Dementjev A \& Akerman KE. Pseudo-noncompetitive antagonism of M1, M3, and M5 muscarinic receptor-mediated $\mathrm{Ca} 2+$ mobilization by muscarinic antagonists. Biochem Biophys Res Commun 243: 41-46, 1998.

Kühl PW. Excess-substrate inhibition in enzymology and high-dose inhibition in pharmacology: a reinterpretation. Biochem J 298: 171-180, 1994.

Kühl PW \& Jobmann M. Receptor-agonist interactions in service theoretic perspective, effects of molecular timing on the shape of dose-response curves. I Recept Signal Transduct Res 26: 1-34, 2006.

Lacan J. The Seminar of Jacques Lacan. Book XVII. The Reverse Side of Psychoanalysis (1969-1970), English translation by R. Grigg. New York: WW Norton, 2007.

Laidler KJ. General steady-state equations in enzyme and other catalyzed reactions. Trans Faraday Soc 52: 1374-1382, 1956.

Laidler KJ. The Chemical Kinetics of Enzyme Action, 1st ed. London: Oxford University Press, 1958.

Laidler KJ \& Hoare JP. The molecular kinetics of the urea-urease system. I. The kinetic laws. J Am Chem Soc 71: 2699-2702, 1949.

Langley NJ. On the reaction of cells and of nerve-endings to certain poisons, chiefly as regards the reaction of striated muscle to nicotine and to curari. J Physiol 33: 374-413, 1905.

Lazareno S \& Birdsall NJ. Detection, quantitation, and verification of allosteric interactions of agents with labeled and unlabeled ligands at $\mathrm{G}$ protein-coupled receptors: interactions of strychnine and acetylcholine at muscarinic receptors. Mol Pharmacol 48: 362378, 1995.

Lazareno S, Popham A \& Birdsall NJ. Allosteric interactions of staurosporine and other indolocarbazoles with $\mathrm{N}$-[methyl- $\left.{ }^{3} \mathrm{H}\right]$ scopolamine and acetylcholine at muscarinic receptor subtypes: identification of a second allosteric site. Mol Pharmacol 58: 194 207, 2000 .
Leboeuf R, Langlois MF, Martin M, Ahnadi CE \& Fink GD. 'Hook effect' in calcitonin immunoradiometric assay in patients with metastatic medullary thyroid carcinoma: case report and review of the literature. J Clin Endocrinol Metab 91: 361-364, 2006.

Lefkowitz RJ. Seven transmembrane receptors: something old, something new. Acta Physiol (Oxf) 190: 9-19, 2007.

Lefkowitz RJ, Benovic JL, Kobilka B \& Caron MG. Beta-adrenegic receptors and rhodopsin: shedding new light on an old subject. Trends Pharmacol Sci 7: 444-448, 1986.

Lew MJ, Ziogas J \& Christopoulos A. Dynamic mechanisms of nonclassical antagonism by competitive AT(1) receptor antagonists. Trends Pharmacol Sci 21: 376-381, 2000.

Lineweaver H \& Burk D. The determination of enzyme dissociation constants. J Am Chem Soc 56: 658-666, 1934.

Loo DD, Hirayama BA, Karakossian MH, Meinild AK \& Wright EM. Conformational dynamics of hSGLT1 during Na+/glucose cotransport. J Gen Physiol 128: 701-720, 2006.

Lovelock J. Gaia: a New Look at Life on Earth. Oxford: Oxford Paperbacks, 2000.

Lovelock J. The Revenge of Gaia: Why the Earth Is Fighting Back - and How We Can Still Save Humanity, 1st ed. London: Allen Lane, 2006.

Lüllmann H \& Ziegler A. A transient state concept of drug receptor interaction. Naunyn Schmiedebergs Arch Pharmacol 280: 1-21, 1973.

Ma W \& Yu C. Intramolecular RNA replicase: possibly the first selfreplicating molecule in the RNA world. Orig Life Evol Biosph 36: 413-420, 2006

Maeda S, Sugita C, Sugita M \& Omata T. A new class of signal transducer in His-Asp phosphorelay systems. J Biol Chem 281: 37868-37876, 2006.

Maeyama K, Hohman RJ, Ali H, Cunha-Melo JR \& Beaven MA. Assessment of IgE-receptor function through measurement of hydrolysis of membrane inositol phospholipids. New insights on the phenomena of biphasic antigen concentration-response curves and desensitization. J Immunol 140: 3919-3927, 1988.

Mallet A, Faber DS \& Korn H. Statistical analysis of visual fits: answer to J. Ninio. J Neurophysiol 98: 1836-1840, 2007.

Marvizon JC \& Baudry M. Allosteric interactions and modulator requirement for NMDA receptor function. Eur J Pharmacol 269: 165-175, 1994.

Matsukura S, West CD, Ichikawa Y, Jubiz W, Harada G \& Tyler FH. A new phenomenon of usefulness in the radioimmunoassay of plasma adrenocorticotropic hormone. J Lab Clin Med 77: 490$500,1971$.

Maturana HR \& Varela FJ. Autopoiesis and Cognition: the Realization of the Living, 1st ed. Dordrecht: Reidel Publishing Co., 1980.

May LT, Avlani VA, Langmead CJ, Herdon HJ, Wood MJ, Sexton PM \& Christopoulos A. Structure-function studies of allosteric agonism at M2 muscarinic acetylcholine receptors. Mol Pharmacol 72: 463 476, 2007.

Miles LE. Properties, variants, and applications of the immunoradiometric assay method. Ric Clin Lab 5: 59-72, 1975.

Miles LE, Lipschitz DA, Bieber CP \& Cook JD. Measurement of serum ferritin by a 2-site immunoradiometric assay. Anal Biochem 61: 209-224, 1974

Monod J, Changeux JP \& Jacob F. Allosteric proteins and cellular control systems. J Mol Biol 6: 306-329, 1963.

Morton GJ, Cummings DE, Baskin DG, Barsh GS \& Schwartz MW. Central nervous system control of food intake and body weight. Nature 443: 289-295, 2006a.

Mourot A, Rodrigo J, Kotzyba-Hibert F, Bertrand S, Bertrand D \& Goeldner M. Probing the reorganization of the nicotinic acetylcholine receptor during desensitization by time-resolved covalent labeling using [3H]AC5, a photoactivatable agonist. Mol Pharmacol 69: 452-461, 2006b.

Mourot A, Rodrigo J, Bertrand S, Bertrand D, Goeldner M \& KotzybaHibert F. Reorganization of the nicotinic acetylcholine receptor 
during desensitization probed with a photoactivatable agonist. J Mol Neurosci 30: 13-14, 2006.

Murayama T, Oba T, Katayama E, Oyamada H, Oguchi K, Kobayashi M, Otsuka K \& Ogawa Y. Further characterization of the type 3 ryanodine receptor (RyR3) purified from rabbit diaphragm. J Biol Chem 274: 17297-17308, 1999.

Narayan SS, Temchin AN, Recio A \& Ruggero MA. Frequency tuning of basilar membrane and auditory nerve fibers in the same cochleae. Science 282: 1882-1884, 1998.

Nayyar SN \& Glick D. Effect of alcohols on beta-glucuronidase activity. J Biol Chem 222: 73-83, 1956.

Newman-Tancredi A, Cussac D, Marini L \& Millan MJ. Antibody capture assay reveals bell-shaped concentration-response isotherms for h5-HT(1A) receptor-mediated Galpha(i3) activation: conformational selection by high-efficacy agonists, and relationship to trafficking of receptor signaling. Mol Pharmacol 62: 590601, 2002.

Ninio J. Doubts about quantal analysis. J Neurophysiol 98: 1827-1835, 2007.

Nordtug B. Subjectivity as an unlimited semiosis: Lacan and Peirce. Studies Phil Edu 23: 87-102, 2004.

Onali P, Adem A, Karlsson E \& Olianas MC. The pharmacological action of MT-7. Life Sci 76: 1547-1552, 2005.

Palsson BØ. Systems Biology. Properties of Reconstructed Networks, 1st ed. Cambridge: Cambridge University Press, 2006.

Paton WD \& Rang HP. The uptake of atropine and related drugs by intestinal smooth muscle of the guinea-pig in relation to acetylcholine receptors. Proc R Soc Lond B Biol Sci 163: 1-44, 1965.

Paton WDM. A theory of drug action based on the rate of drug/ receptor combination. Proc R Soc Lond B 154: 21-69, 1961.

Pei G, Samama P, Lohse M, Wang M, Codina J \& Lefkowitz RJ. A constitutively active mutant beta 2-adrenergic receptor is constitutively desensitized and phosphorylated. Proc Natl Acad Sci USA 91: 2699-2702, 1994.

Peitgen H-O, Jürgens H \& Saupe D. Classical Fractals and Self-Similarity, Chaos and Fractals: New Frontiers of Science, 1st ed. Berlin: SpringerVerlag, 1992.

Pitcher JA, Freedman NJ \& Lefkowitz RJ. G protein-coupled receptor kinases. Anпu Rev Biochem 67: 653-692, 1998.

Pott C, Goldhaber JI \& Philipson KD. Homozygous overexpression of the $\mathrm{Na}+-\mathrm{Ca} 2+$ exchanger in mice: evidence for increased transsarcolemmal Ca2+ fluxes. Ann N Y Acad Sci 1099: 310-314, 2007.

Premont RT, Inglese J \& Lefkowitz RJ. Protein kinases that phosphorylate activated G protein-coupled receptors. FASEB J 9: 175-182, 1995.

Prokhorenko VI, Nagy AM, Waschuk SA, Brown LS, Birge RR \& Miller RJ. Coherent control of retinal isomerization in bacteriorhodopsin. Science 313: 1257-1261, 2006

Proska J \& Tucek S. Mechanisms of steric and cooperative actions of alcuronium on cardiac muscarinic acetylcholine receptors. Mol Pharmacol 45: 709-717, 1994.

Proska J \& Tucek S. Competition between positive and negative allosteric effectors on muscarinic receptors. Mol Pharmacol 48: 696-702, 1995.

Rang HP. The kinetics of action of acetylcholine antagonists in smooth muscle. Proc R Soc Lond B Biol Sci 164: 488-510, 1966.

Rang HP \& Ritter JM. On the mechanism of desensitization at cholinergic receptors. Mol Pharmacol 6: 357-382, 1970.

Rankin ML, Marinec PS, Cabrera DM, Wang Z, Jose PA \& Sibley DR. The D1 dopamine receptor is constitutively phosphorylated by G protein-coupled receptor kinase 4. Mol Pharmacol 69: 759-769, 2006.

Revah F, Bertrand D, Galzi JL, Devillers-Thiery A, Mulle C, Hussy N, Bertrand S, Ballivet M \& Changeux JP. Mutations in the channel domain alter desensitization of a neuronal nicotinic receptor. Nature 353: 846-849, 1991

Ribeiro FM, Black SA, Prado VF, Rylett RJ, Ferguson SS \& Prado MA. The 'ins' and 'outs' of the high-affinity choline transporter CHT1. J Neurochem 97: 1-12, 2006.

Rizo J \& Dai H. How much can SNAREs flex their muscles? Nat Struct Mol Biol 14: 880-882, 2007.

Robertson MJ, Wragg A \& Clark KL. Modulation of tachyphylaxis to angiotensin II in rabbit isolated aorta by the angiotensin AT1 receptor antagonist, losartan. Regul Pept 50: 137-145, 1994.

Rodbard D. Radioimmunoassays and 2-site immunoradiometric 'sandwich' assays: basic principles. Radioisotopes 37: 590-594, 1988.

Roth NS, Campbell PT, Caron MG, Lefkowitz RJ \& Lohse MJ. Comparative rates of desensitization of beta-adrenergic receptors by the beta-adrenergic receptor kinase and the cyclic AMPdependent protein kinase. Proc Natl Acad Sci USA 88: 6201$6204,1991$.

Rovati GE \& Nicosia S. Lower efficacy: interaction with an inhibitory receptor or partial agonism? Trends Pharmacol Sci 15: 140-144, 1994.

Sanguinetti MC \& Tristani-Firouzi M. hERG potassium channels and cardiac arrhythmia. Nature 440: 463-469, 2006.

Savalli N, Kondratiev A, de Quintana SB, Toro L \& Olcese R. Modes of operation of the BKCa channel beta2 subunit. J Gen Physiol 130: 117-131, 2007.

Schlee S, Carmillo P \& Whitty A. Quantitative analysis of the activation mechanism of the multicomponent growth-factor receptor Ret. Nat Chem Biol 2: 636-644, 2006.

Schopf JW. Life's Origin: the Beginnings of Biological Evolution, 1st ed. Berkeley, CA: University of California Press, 2002.

Segel IH. Enzyme Kinetics. Behavior and Analysis of Rapid Equilibrium and Steady-State Enzyme Systems. New York: Wiley \& Sons (reissued 1993), 1975.

Segal HL, Kachmar JF \& Boyer PD. Kinetic analysis of enzyme reactions. I. Further considerations of enzyme inhibition and analysis of enzyme activation. Enzymologia 15: 187-198, 1952.

Shapiro M \& Brumer P. Principles of the Quantum Control of Molecular Processes. Hoboken, NJ: Wiley, 2003.

Shi L, Potts M \& Kennelly PJ. The serine, threonine, and/or tyrosinespecific protein kinases and protein phosphatases of prokaryotic organisms: a family portrait. FEMS Microbiol Rev 22: 229-253, 1998.

Shimada A, Niwa H, Tsujita K, Suetsugu S, Nitta K, Hanawa-Suetsugu K, Akasaka R, Nishino Y, Toyama M, Chen L, Liu ZJ, Wang B, Yamamoto M, Terada T, Miyazawa A, Tanaka A, Sugano S, Shirouzu M, Nagayama K, Takenawa T \& Yokoyama S. Curved $\mathrm{EFC} / \mathrm{F}-\mathrm{BAR}$-domain dimers are joined end to end into a filament for membrane invagination in endocytosis. Cell 129: 761-772, 2007.

Shou M. Prediction of pharmacokinetics and drug-drug interactions from in vitro metabolism data. Curr Opin Drug Discov Dev 8: 66-77, 2005 .

Sibley DR, Strasser RH, Benovic JL, Daniel K \& Lefkowitz RJ. Phosphorylation/dephosphorylation of the beta-adrenergic receptor regulates its functional coupling to adenylate cyclase and subcellular distribution. Proc Natl Acad Sci USA 83: 9408-9412, 1986.

Silver RA, Colquhoun D, Cull-Candy SG \& Edmonds B. Deactivation and desensitization of non-NMDA receptors in patches and the time course of EPSCs in rat cerebellar granule cells. J Physiol 493, 1996. Erratum in: J Physiol 496: 891: 167-173, 1996.

Stephenson SL, Stempen H \& Stephen H. Myxomycetes: Handbook of Slime Moulds. Portland: Timber Press, 2000.

Stjernfelt F. Diagrammatology: an Investigation on the Borderlines of Phenomenology, Ontology, and Semiotics, 1st ed. Dordrecht: Springer Verlag, 2007. 
Swillens S, Waelbroeck M \& Champeil P. Does a radiolabelled ligand bind to a homogenous population of non-interacting receptor sites? Trend Pharmacol Sci 16: 151-155, 1995.

Szabadi E. A model of two functionally antagonistic receptor populations activated by the same agonist. J Theor Biol 69: 101-112, 1977.

Szurmant H, Mohan MA, Imus PM \& Hoch JA. YycH and YycI interact to regulate the essential YycFG two-component system in Bacillus subtilis. J Bacteriol 189: 3280-3289, 2007.

Thomas D, Karle CA \& Kiehn J. The cardiac hERG/IKr potassium channel as pharmacological target: structure, function, regulation, and clinical applications. Curr Pharm Des 12: 2271-2283, 2006.

Tomlinson G \& Hnatowich MR. Apparent competitive inhibition of radioligand binding to receptors: experimental and theoretical considerations in the analysis of equilibrium binding data. J Recept Res 8: 809-830, 1988.

Trist DG \& Leff P. Quantification of H2-agonism by clonidine and dimaprit in an adenylate cyclase assay. Agents Actions 16: 222-226, 1985.

Tucek S \& Proska J. Allosteric modulation of muscarinic acetylcholine receptors. Trends Pharmacol Sci 16: 205-212, 1995.

Tucek S, Michal P \& Vlachova V. Modelling the consequences of receptor-G-protein promiscuity. Trends Pharmacol Sci 23:171-176, 2002.

Tucek S, Musilkova J, Nedoma J, Proska J, Shelkovnikov S \& Vorlicek J. Positive cooperativity in the binding of alcuronium and $\mathrm{N}$ methylscopolamine to muscarinic acetylcholine receptors. Mol Pharmacol 38: 674-680, 1990.

Turchin P. Complex population dynamics: A theoretical/empirical synthesis. Princeton: Princeton University Press, 2003.

Urbe S. Ubiquitin and endocytic protein sorting. Essays Biochem 41: 8198, 2005.

Urso B, Ilondo MM, Holst PA, Christoffersen CT, Ouwens M, Giorgetti S, Van Obberghen E, Naor D, Tornqvist H \& De Meyts P. IRS-4 mediated mitogenic signalling by insulin and growth hormone in LB cells, a murine T-cell lymphoma devoid of IGF-I receptors. Cell Signal 15: 385-394, 2003.

Vauquelin G \& Szczuka A. Kinetic versus allosteric mechanisms to explain insurmountable antagonism and delayed ligand dissociation. Neurochem Int 51: 254-260, 2007.

Vialou V, Balasse L, Dumas S, Giros B \& Gautron S. Neurochemical characterization of pathways expressing plasma membrane monoamine transporter in the rat brain. Neuroscience 144: 616-622, 2007.

Vidler A. What is a diagram anyway? In: Peter Eisenman: Feints, 1st ed, edited by Cassara S. Milan: Skira, 2006.

Vieira OV, Verkade P, Manninen A, Simons K. FAPP2 is involved in the transport of apical cargo in polarized MDCK cells. J Cell Biol 170: 521-526, 2005.
Violin JD \& Lefkowitz RJ. Beta-arrestin-biased ligands at seventransmembrane receptors. Trends Pharmacol Sci 28: 416-422, 2007.

Volk T. Gaia's Body. Toward a Physiology of Earth, 1st ed. New York: Copernicus/Springer Verlag, 1997.

Waelbroeck M, Robberecht P, De Neef P \& Christophe J. Effects of D-tubocurarine on rat cardiac muscarinic receptors: a comparison with gallamine. J Recept Res 8: 787-808, 1988.

Weiss ER, Raman D, Shirakawa S, Ducceschi MH, Bertram PT, Wong F, Kraft TW \& Osawa S. The cloning of GRK7, a candidate cone opsin kinase, from cone- and rod-dominant mammalian retinas. Mol Vis 4: 27, 1998.

Wenz K. Representation and self-reference: Peirce's sign and its application to the computer. Semiotica 143: 199-209, 2003.

Wickramasinghe C. A Journey with Fred Hoyle: the Search for Cosmic Life. Singapore: World Scientific Publishing Co. Pte. Ltd., 2005.

Wickramasinghe NC. Fred Hoyle's Universe. Proceedings of a Conference celebrating Fred Hoyle's Extraordinary Contribution to Science, 25-26 June 2002. Cardiff: Cardiff University, 2003.

Winding B \& Bindslev N. Desensitization and reactivation of AChregulated exocrine secretion in hen tracheal epithelium. Am J Physiol 264: C342-C351, 1993.

Wood D. Time After Time, 1st ed. Bloomington, IN: Indiana University Press, 2007.

Wreggett KA \& Wells JW. Cooperativity manifest in the binding properties of purified cardiac muscarinic receptors. J Biol Chem 270: 22488-22499, 1995.

Wright EM, Hirayama BA \& Loo DF. Active sugar transport in health and disease. J Intern Med 261: 32-43, 2007.

Zagotta WN, Hoshi T \& Aldrich RW. Restoration of inactivation in mutants of Shaker potassium channels by a peptide derived from ShB. Science 250: 568-571, 1990.

Zhang J, Barak LS, Anborgh PH, Laporte SA, Caron MG \& Ferguson SS. Cellular trafficking of $\mathrm{G}$ protein-coupled receptor/betaarrestin endocytic complexes. J Biol Chem 274: 10999-21006, 1999.

Zhang M, Houamed K, Kupershmidt S, Roden D \& Satin LS. Pharmacological properties and functional role of Kslow current in mouse pancreatic beta-cells: SK channels contribute to Kslow tail current and modulate insulin secretion. I Gen Physiol 126: 353-363, 2005.

Zhou J, Livak MF, Bernier M, Muller DC, Carlson OD, Elahi D, Maudsley S \& Egan JM. Ubiquitination is involved in glucosemediated downregulation of GIP receptors in islets. Am J Physiol Endocrinol Metab 293: E538-E547, 2007.

Zohar D, Marshall IN. The Quantum Self, 1st ed. London: Bloomsbury Publishing Plc, 1990.

Zwart R, Vijverberg HP. Potentiation and inhibition of neuronal alpha4beta4 nicotinic acetylcholine receptors by choline. Eur J Pharmacol 393: 209-214, 2000. 


\section{1}

CHAPTER

Means of Obtaining Ant-agonist Constants PRELIMINARY POINTS OF A PERSONAL VIEW

This chapter deals with methods to determine dissociation constants for ant-agonists and interventors. The focus is on the analyses of present theoretical tools based on one-state models used for synagic evaluation of the mentioned constants, rather than an analysis of data from synagic experimentation as such.

Sub-chapter 4.6 introduces novel concepts from the experimental fields that point to a future with implementation of two-state models presented in Part II.

\subsection{Dissociation Constant Determination}

\subsubsection{Four Methods}

Out of several proposals for obtaining the ant-agonist dissociation constants in functional studies with proven or assumed simple competitive inhibition, four methods in particular have been suggested.

(1) The Cheng \& Prusoff (1973) equation (C-P).

(2) Its functional equivalent (fe-C-P) that corrects for potential receptor reserve (Craig 1993).

(3) The Gaddum (1937) equation (Gaddum et al. 1955).

(4) The Schild $(1947,1949)$ analysis (Arunlakshana \& Schild 1959).

As we shall see, Cheng \& Prusoff (1973) also derived methods for the analysis of non-competitive and classical un-competitive ant-agonism ${ }^{1}$ in functional studies (Section 4.2.1).

\footnotetext{
${ }^{1}$ Classical un-competitive ant-agonism (Segel 1975, pp. 136-143) is equal to the amputated form of the hetero-intervention model with co-lateral coefficient $c=1$ (see sub-chapter 2.4 and Table 2.6).
}

In this text, equilibrium dissociation constants for competitive ant-agonism are designated $K_{\mathrm{si}}, K_{\mathrm{ii}}$ or $K_{\mathrm{xi}}$, while in the literature $K_{\mathrm{b}}$ or $K_{\mathrm{B}}$ is often used.

In the non-competitive scheme, constants related to the ant-agonist or interventor will appear as $K_{\mathrm{si}}, K_{\mathrm{ii}}, K_{\mathrm{si}}^{\text {is }}$ or $K_{\mathrm{ii}}^{\mathrm{ss}}$, and a so-called 'co-lateral intervention' constant $c$ comes into play.

More details on the methods above, in particular on the Schild analysis, can be found in Chapter 11. To appreciate and judge 'powered' versions of the methods, discussions on the Hill equation in Chapter 10 should be consulted.

\subsubsection{Why Parameters for Competitive Ant-agonism?}

In the course of developing methods and analytical tools to determine the dissociation constants for ant-agonists in functional studies, there has been a peculiar preference for systems with competitive inhibition, and accordingly a host of methods to determine equilibrium dissociation constants based on the competitive reaction scheme. Incidentally, the mystery of the preference may disappear once it is realized that competitive antagonism is a popular if not compulsive prerequisite for derived theories in binding studies. In binding studies, non-competitive ant-agonists do not alter agonist concentration-occupancy relationships (cf. Table 2.2), therefore, perhaps the variants of Cheng-Prusoff and the Gaddum/Schild analyses, all with assumed competitive reaction schemes, are also the preferred kind of analyses when data from functional studies of ant-agonism are to be analyzed. Although Gaddum's and Schild's equations are based on null methods (see Section 4.3.2), in 
principle, they are to be applied exclusively to studies of competitive ant-agonism (Kenakin 1997, Chapter 10).

Of the four means of obtaining the ant-agonist dissociations constants in functional studies, the Cheng-Prusoff was once fashionable due to its simplicity. In later years, the method of Schild has become increasingly popular, and is currently the accepted method of assessing $K_{\mathrm{xi}} \mathrm{s}\left(K_{\mathrm{b}} \mathrm{s}\right)$, while respect for the C-P has decayed concomitantly, if not dramatically (Lazareno \& Birdsall 1993b; Wyllie \& Chen 2007).

To extract the system constants, Lazareno \& Birdsall (1993b) wisely suggested using an entire data set from a Schild performed experiment and its analysis based on Waud's formulations (Waud 1976, 1978) using nonlinear regression.

Nevertheless, all the methods are based on assumptions, and all the methods are fine as long as their assumptions are full-filled and respected.

\subsubsection{Constants for the Hetero-intervention Model}

Ehlert (1988) has listed methods for extracting equilibrium dissociation constants for the complete ternarycomplex model (TCM). ${ }^{2}$

Thus, for:

(1) agonists. $K_{\mathrm{ss}}$ is extracted in binding and in functional studies with reference to Furchgott's method (1966; Furchgott \& Bursztyn 1967) after elimination of a possible receptor reserve.

(2) partial agonists. $K_{\mathrm{ss}}$ is extracted in binding and in functional studies with reference to methods by Barlow et al. (1967) and Waud (1969).

(3) ant-agonists. $K_{\mathrm{si}}$ or $K_{\mathrm{ii}}$ is extracted in competitive reaction schemes of binding and functional studies with reference to the null methods by Arunlakshana \& Schild (1959).

(4) interventors (sub-chapter 2.5). $K_{\mathrm{ii}}$, of the complete ternary-complex with co-lateral intervention constant $c \neq 1$, is extracted in binding, radio-ligand binding, and functional studies according to Ehlert's scheme (see formulations in Table 2.3).

The fourth category of methods was developed by Ehlert (1988) for extracting affinity constants as well as the intervention constant $c$ based on the complete TCM identical to the intervention model derived in Chapter 2. The present co-lateral intervention constant $c$ is equal to parameter $1 / \alpha$ in Ehlert's paper.

${ }^{2}$ The complete ternary-complex model (TCM) with three independent system constants and a co-lateral coefficient $c \neq 1$, cf. Ehlert (1988), is still a one-state hetero-intervention model (sub-chapter 2.5).
Ehlert's four categories of methods are based on classical occupancy theory with one-state reaction schemes. In functional studies, response is therefore equated as a simple product of occupancy and efficacy, where affinity constants are imagined as separable from efficacy constants. This view is repeated for instance in Griffin et al. (2003). The idea is in accord with onestate models as presented here in Part I of the book, but at variance with Stephenson's understanding of efficacy (Stephenson 1956) and with the two-state allosteric models presented in Parts II and IV (see Sections 1.3.4, 1.3.5, 1.3.8, Box 1.3, Chapter 5 and Chapter 14).

\subsubsection{Ant-agonist Constants and Receptor States}

Observe, therefore, that the simple paradigm of a universal affinity constant for a particular ant-agonist ligand and receptor interaction (Ehlert 1988; Kenakin et al. 1995; Kenakin 2004; Hill 2006) has been expanded due to receptive units in more than one state and is eagerly debated (Baker 2005a,b; Baker \& Hill 2007a,b; Giraldo et al. 2007).

Meanwhile, in Chapter 4, I will only discuss antagonism at a basal or 'classical' level with receptors in a single state, thereby ignoring, thus far, the complications of a receptive unit in two or more states, initiated particularly in Chapter 5.

\subsection{The Cheng-Prusoff Equations}

At the outset, we will go through the Cheng \& Prusoff (1973) derivation of formulae for extracting ant-agonist dissociation constants from experimentation assuming competitive and non-competitive ant-agonism, supplemented by recent suggestions for a functional equivalent of the Cheng-Prusoff equation (fe-C-P) (Craig 1993). The derivations by Cheng \& Prusoff are not completely new (see, e.g., Kirschner \& Stone 1951; Webb 1963, p. 106). From the formulae and a determination of the apparent ant-agonist dissociation constant $\mathrm{IC}_{50}$, parameters such as $K_{\mathrm{ii}}$ and $K_{\mathrm{si}}$ may be extracted. In subchapter 4.3, derivation and discussion of the Gaddum equation are detailed for extracting ant-agonist dissociation constant $K_{\mathrm{si}}$, followed by the Schild analysis introduced in sub-chapter 4.4. However, a meticulous presentation of the Schild method is postponed. Use of Schild's null method for extracting relevant parameters and the method's raison d'être are discussed in Part III Chapter 11.

Extracting parameter values by the C-P formulation of non-competitive ant-agonism (intervention) is presented first. 


\subsubsection{Cheng-Prusoff for $K_{\mathrm{ii}}$ and $\mathrm{IC}_{50}$ in Non-competitive Ant-agonism}

In functional studies, non-competitive ant-agonism (intervention) developed in Section 2.4.5 yielded Eq. 2.3, which is repeated here:

$$
\frac{a r}{T R}=\frac{\frac{\mathrm{S}}{K_{\mathrm{ss}}}}{\left(1+\frac{\mathrm{S}}{K_{\mathrm{ss}}}\right)} \cdot \frac{1}{\left(1+\frac{\mathrm{I}}{K_{\mathrm{ii}}}\right)}
$$

Here $a r$ is the actual response and $T R$ the maximal response.

The first fraction on the right hand side of Eq. 2.3 is the activity in the absence of ant-agonists, equal to a simple load-expression at equilibrium. It is multiplied by a second fraction, $1 /\left(1+\mathrm{I} / K_{\mathrm{ii}}\right)$, which is a correction term in the presence of a non-competitive ant-agonist (interventor). When the ant-agonist concentration, I, lowers the stimulated response by half, then for any value of $\mathrm{S}$, I is equal to $\mathrm{IC}_{50}$ by definition. Half of the response occurs when the correction term equals $1 / 2$, which exclusively happens for $\mathrm{IC}_{50}=K_{\mathrm{ii}}$.

Thus, in functional, non-competitive intervention, $\mathrm{IC}_{50}$ is always equal to $K_{\mathrm{ii}}$, and independent of $[\mathrm{S}]$ :

$$
\mathrm{IC}_{50}=K_{\mathrm{ii}} \text { or }=K_{\mathrm{ii}}^{\mathrm{ss}},
$$

(Kirschner \& Stone 1951; Cheng \& Prusoff 1973).

Note that the assumption here is that an initial binding of a ligand ' $S$ ' to the receptive unit does not change the dissociation constant for the ensuing binding of an ant-agonist 'I'. Thus $K_{\mathrm{ii}}=K_{\mathrm{ii}}^{\mathrm{ss}}$ is equal to the assumption that the co-lateral intervention constant $c=1$ (cf. sub-chapter 2.4).

\subsubsection{Explicit Expressions for $K_{\mathrm{si}}, K_{\mathrm{ii}}$, and $\mathrm{IC}_{50}$ in Competitive Ant-agonism}

For competitive ant-agonism (formulated in Eqs. 2.12 and 2.13 in Section 2.4.9), ${ }^{3}$ a similar type of analysis as for non-competitive intervention yields that half a response, when no ant-agonist is present, can be written as $(1 / 2) \cdot\left(\mathrm{S} / K_{\mathrm{ss}}\right) /\left(1+\left(\mathrm{S} / K_{\mathrm{ss}}\right)\right)$ and must equal the response when ant-agonist concentration $\mathrm{I}=\mathrm{IC}_{50}$ (Fig. 2.6). That is, inserting $\mathrm{IC}_{50}$ for ' $\mathrm{I}$ ' in Eq. 2.12 gives us: $\left(\mathrm{S} / K_{\mathrm{ss}}\right) /\left(1+\left(\mathrm{S} / K_{\mathrm{ss}}\right)+\left(\mathrm{IC}_{50} / K_{\mathrm{si}}\right)\right)$ at a given concentration of S. Therefore, as these two $50 \%$ or half maximal responses must be equal, we have:

\footnotetext{
${ }^{3}$ In this context, to keep things on a reasonable level, only $K_{\mathrm{si}}$ for the competitive ant-agonism type $\mathrm{I}$, as surveyed in Section 2.4.9, is analyzed. This leaves an analysis of the competitive ant-agonism type II, as in Section 2.4.10, with its constant $K_{\mathrm{ii}}$ to be evaluated by the reader. Note, here $K_{\mathrm{ii}}$ is not $K_{\mathrm{ii}}^{\mathrm{ss}}$ as in Eq. 4.1.
}

$$
\begin{aligned}
& (1 / 2) \cdot\left(\mathrm{S} / K_{\mathrm{ss}}\right) /\left(1+\left(\mathrm{S} / K_{\mathrm{ss}}\right)\right) \\
& \quad=\left(\mathrm{S} / K_{\mathrm{ss}}\right) /\left(1+\left(\mathrm{S} / K_{\mathrm{ss}}\right)+\left(\mathrm{IC}_{50} / K_{\mathrm{si}}\right)\right)
\end{aligned}
$$

or

$$
1 /\left(1+\left(\mathrm{S} / K_{\mathrm{ss}}\right)\right)=2 /\left(1+\left(\mathrm{S} / K_{\mathrm{ss}}\right)+\left(\mathrm{IC}_{50} / K_{\mathrm{si}}\right)\right) \text {. }
$$

Subtracting the left nominator from the right nominator and the left denominator from the right denominator in Eq. 4.3, i.e., implementing regula detri rule b2 in Box 1.1, gives us:

$$
1 /\left(1+\left(\mathrm{S} / K_{\mathrm{ss}}\right)\right)=1 /\left(\mathrm{IC}_{50} / K_{\mathrm{si}}\right),
$$

and for all values of $\mathrm{S}$ by rearrangement:

$$
\mathrm{IC}_{50}=K_{\mathrm{si}} \cdot\left(1+\left(\mathrm{S} / K_{\mathrm{ss}}\right)\right) \text { or } K_{\mathrm{si}}=\mathrm{IC}_{50} /\left(1+\mathrm{S} / K_{\mathrm{ss}}\right) \text {. }
$$

$\mathrm{IC}_{50}$ in this case is appK $\mathrm{K}_{\mathrm{s}}$, equal the apparent dissociation constant for a type 1 competitive ant-agonist. For a type II competitive ant-agonist, $K_{\mathrm{ii}}$ replaces $K_{\mathrm{si}}$ in Eq. 4.5 (see footnote 4.3).

Eq. 4.5 is the renowned Cheng-Prusoff equation for competitive ant-agonism in mono-substrate enzymatic reactions (Cheng \& Prusoff 1973). ${ }^{4}$ As long as the assumptions for using this C-P formulation are met, the relationship in Eq. 4.5 holds for both competitive ant-agonism in functional studies and for competitive displacement in binding studies.

From Eq. 4.5 we have for competitive ant-agonism

when $\mathrm{S}>>K_{\mathrm{ss}} \quad$ then $K_{\mathrm{si}} \simeq \mathrm{IC}_{50} \cdot K_{\mathrm{ss}} / \mathrm{S}$,

when $\mathrm{S}=K_{\mathrm{ss}} \quad$ then $K_{\mathrm{si}}=\mathrm{IC}_{50} / 2$, and

when $\mathrm{S}<<K_{\mathrm{ss}} \quad$ then $K_{\mathrm{si}}=\mathrm{IC}_{50}$.

All the relations for competitive type I ant-agonism hold for competitive type II ant-agonism by replacing $K_{\mathrm{si}}$ with $K_{\mathrm{ii}}$.

\subsubsection{Direct Derivation of the Cheng-Prusoff Equation for Competitive Ant-agonism}

Above is the classical way of obtaining the C-P equation for competitive ant-agonism. However, it may be extracted in a more direct manner, as demonstrated here.

For a fixed concentration of an agonist $S^{\prime}$, the actual inhibitory response (air) in competitive ant-agonism was derived earlier in Eq. $2.9 d$. That equation is valid for both studies of function and binding. I repeat the equation in a slightly modified form:

$$
\text { air }=F R \cdot \frac{\mathrm{I} / K_{\mathrm{si}}}{1+\mathrm{S}^{\prime} / K_{\mathrm{ss}}+\mathrm{I} / K_{\mathrm{si}}},
$$

where the full response $(F R)$ is equal to $\mathrm{S}^{\prime} /\left(\mathrm{S}^{\prime}+K_{\mathrm{ss}}\right)$. Compare Eq. 4.6 with Eq. $2.9 d$.

\footnotetext{
${ }^{4}$ As already indicated, a slightly different form of this C-P equation for competitive ant-agonism was derived by Kirschner \& Stone (1951).
} 

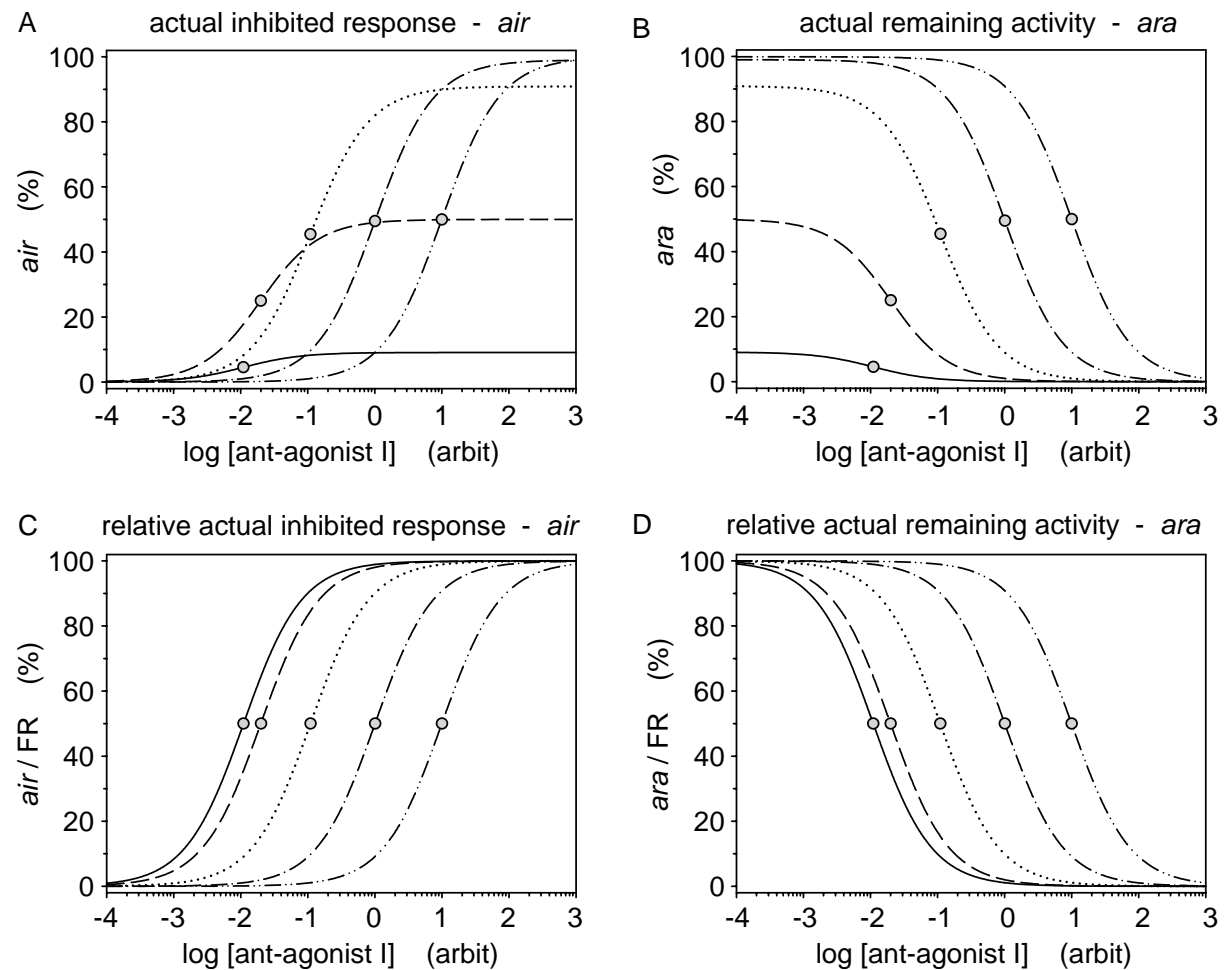

Figure 4.1. Examples of dose-responses for competitive ant-agonism of actual-inhibitory-response (air) and actual-remainingactivity (ara) as a function of the ant-agonist concentration [I]. (A) The air d-r relation is given in Eq. 4.6. (B) The ara d-r is formulated in Eq. 4.8b. (C) Expression for the relative air/FR as in Eq. 4.7. (D) The relative ara/FR is obtained by dividing both sides of Eq. $4.8 b$ with the full response: $F R=S /\left(S+K_{\mathrm{ss}}\right)$. In all four panels, the agonist concentration [S] is varied in five steps from $10^{-2}(-)$ to $10^{2}(-\cdots-)$ by a factor 10 . Parameters in all four panels are: $K_{\mathrm{ss}}=0.1$ and $K_{\mathrm{si}}=0.01$. Grey dots indicate half maximal effects.

Thus, depending on one's focus, from Eq. 4.6 with a fixed concentration of agonist, $\mathrm{S}^{\prime}$, you also have:

$$
\frac{a i r}{F R}=\frac{\mathrm{I}}{\mathrm{I}+K_{\mathrm{si}} \cdot\left(1+\mathrm{S}^{\prime} / K_{\mathrm{sS}}\right)},
$$

and in parallel with this, for the actual remaining activity (ara), from Eq. $2.10 \mathrm{~b}$ in studies of competitive antagonism we can formulate:

$$
\operatorname{ara}=\frac{\mathrm{S}^{\prime}}{\mathrm{S}^{\prime}+K_{\mathrm{ss}} \cdot\left(1+\mathrm{I} / K_{\mathrm{si}}\right)} .
$$

These two functions (Eqs. 4.7 and 4.8) are mirror images, horizontally-flipped, as shown in Fig. 4.1A+B, and in a relative form in panels $\mathrm{C}+\mathrm{D}$. All graphs in Fig. 4.1 are for both functional and binding assays.

The second term in the denominator of Eq. $4.8, K_{\mathrm{ss}}$. $\left(1+\mathrm{I} / K_{\mathrm{si}}\right)$, is equal to the appearant disociation constant for a substrate or an agonist $K_{\mathrm{ss}}$, $\operatorname{appK}_{\mathrm{ss}}=\mathrm{EC}_{50}$.

The second term in the denominator of the right hand expression in Eq. $4.7, K_{\mathrm{si}} \cdot\left(1+\mathrm{S}^{\prime} / K_{\mathrm{ss}}\right)$, is equal to the apparent equilibrium dissociation constant for a competitive ant-agonist, $\operatorname{appK}_{\mathrm{si}}=\mathrm{IC}_{50}$, at a fixed agonist concentration $\mathrm{S}^{\prime}$. We obtain directly:

$$
\operatorname{appK}_{\mathrm{si}}=\mathrm{IC}_{50}=K_{\mathrm{si}} \cdot\left(1+\mathrm{S}^{\prime} / K_{\mathrm{ss}}\right)
$$

and rearranged equal to:

$$
K_{\mathrm{si}}=\mathrm{IC}_{50} /\left(1+\mathrm{S}^{\prime} / K_{\mathrm{ss}}\right) .
$$

Eq. 4.10 is the Cheng-Prusoff equation for $K_{\mathrm{si}}$ in competitive ant-agonism with $\mathrm{IC}_{50}$ determined at a single fixed concentration of the agonist $\mathrm{S}^{\prime}$. Since Eq. 4.10 is valid for any value of $S \geq 0$, it is identical to Eq. 4.5 , which is the general form of the Cheng-Prusoff equation for $K_{\mathrm{si}}$ in competitive ant-agonism both in functional and in binding studies.

A generalized form of the equation for ara (Eq. 4.8) can be obtained by replacing the fixed concentration of agonist, $\mathrm{S}$ ', with a non-fixed agonist concentration ' $\mathrm{S}$ ' (see Eq. $4.8 b$ ).

\subsubsection{Comparison of Competitive and Non-competitive Inhibition Curves}

A parallel can be made between a generalized form of the C-P equation for competitive ant-agonism as formulated here: 


$$
a r a=\frac{\mathrm{S}}{\mathrm{S}+K_{\mathrm{ss}} \cdot\left(1+\mathrm{I} / K_{\mathrm{si}}\right)},
$$

equal to:

$$
\operatorname{ara}=\frac{\mathrm{S}}{\mathrm{S}+\mathrm{EC}_{50}}
$$

and the non-competitive ant-agonism, Eq. 2.3 or Eq. 4.0.

If $K_{\mathrm{si}}$ in the equation for competitive ant-agonism (Eq. $4.8 b$ ) is substituted by the C-P expression in Eq. 4.5, the general formulation for actual remaining activity of competitive ant-agonism in Eq. $4.8 b$ can be rewritten to $\mathrm{S} /\left(\left(\mathrm{S}+K_{\mathrm{ss}}\right) \cdot\left(1+\mathrm{I} / \mathrm{IC}_{50}\right)\right)$. Try to derive this expression.

Thus, two parallel expressions, one for competitive ant-agonism and one for non-competitive intervention, become:

$$
\text { competitive: } \operatorname{ara}=\frac{\mathrm{S}}{\left(\mathrm{S}+K_{\mathrm{ss}}\right)} \cdot \frac{1}{\left(1+\frac{\mathrm{I}}{\mathrm{IC}_{50}}\right)}
$$

and by rewriting Eq. 4.0 from Section 4.2.1:

$$
\text { non-competitive: } \frac{a r}{T R}=\frac{\mathrm{S}}{\left(\mathrm{S}+K_{\mathrm{ss}}\right)} \cdot \frac{1}{\left(1+\frac{\mathrm{I}}{K_{\mathrm{ii}}}\right)} \text {. }
$$

In Eqs. 4.11 and 4.12, both [S] and [I] may vary.

The two expressions for the competitive ara and noncompetitive $a r / T R$ are identical, except for $\mathrm{IC}_{50}$ versus $K_{\mathrm{ii}}$. In the competitive expression, $\mathrm{IC}_{50}$ is a function of the agonist concentration $\mathrm{S}$ and the agonist dissociation constant $K_{\mathrm{ss}}$, since $\mathrm{IC}_{50}=K_{\mathrm{is}} \cdot\left(1+\mathrm{S} / K_{\mathrm{ss}}\right)$, while $K_{\mathrm{ii}}$ for non-competitive ant-agonism is not a function of either the agonist concentration $\mathrm{S}$ or its dissociation constant $K_{\mathrm{ss}}$. Non-competitive $K_{\mathrm{ii}}$ is a constant and equal to $K_{\mathrm{ii}}^{\mathrm{ss}}$.

Thus, in order to determine $K_{\mathrm{ii}}$ in the non-competitive situation, knowledge of $\mathrm{S}$ and $K_{\mathrm{ss}}$ values is not necessary since a functional inhibition curve may be obtained for any value of agonist stimulation induced by $\mathrm{a}$ fixed $\mathrm{S}$ concentration. Further, the initial fullresponse in the absence of an interventor-concentration [I] equals zero - is given by $F R=R_{\text {tot }} \cdot \mathrm{S}^{\prime} /\left(\mathrm{S}^{\prime}+K_{\mathrm{ss}}\right)$, where $F R$ is the so-called full response. Therefore, increasing the concentration I of an ant-agonist reduces the $F R$ by $1 /\left(1+\mathrm{I} / K_{\mathrm{ii}}\right)$ in a non-competitive reaction. The $\mathrm{IC}_{50}$ of this 'inhibition curve' is equal to $K_{\mathrm{ii}}$.

In order for systems to be analyzed by the competitive scheme, the ratio $\mathrm{S} / K_{\mathrm{ss}}$ must be known before one can evaluate $K_{\mathrm{si}}$. The ratio $\mathrm{S} / K_{\mathrm{ss}}$ is usually obtained from initial studies of agonist dose-response characteristics, ensuring a competitive relationship for the studied system. Following this analysis, a dose of the agonist may be added to induce its so-called full-response. In functional studies, at a given agonist concentration $\left[\mathrm{S}^{\prime}\right]$, the $\mathrm{IC}_{50}$ for the ant-agonist can be determined by increasing the concentration of the ant-agonist I, and with knowledge of $K_{\mathrm{ss}}$, the dose-response relation for competitive synagics may be analyzed in the same manner as for non-competitive dose-responses (Eqs. 4.11 and 4.12).

In functional studies with a receptor reserve the measured dissociation constant, $\mathrm{EC}_{50}$, may be a distortion of the $K_{\mathrm{ss}}$. Therefore, in functional studies with competitive ant-agonism, $\mathrm{EC}_{50}$ should be used instead of $K_{\mathrm{ss}}$. This will correct for a potential receptor reserve (see Section 4.2.5).

\subsubsection{The Functional Equivalent of the Cheng- Prusoff Equation (fe-C-P)}

In the C-P regime of competitive antagonism, it is necessary to obtain the equilibrium dissociation constant for the employed agonist in order to determine a dissociation constant for an ant-agonist (Eq. 4.5). Meanwhile, as pointed out by Craig (1993), in case there is a receptor reserve, it is necessary to replace the agonist dissociation constant, $K_{\mathrm{ss}}$, with its equivalent $\mathrm{EC}_{50}$ obtained in functional studies.

The equivalent of the C-P equation for functional competitive ant-agonism as a formulation of increasing ant-agonist concentration with unknown receptor reserve, is obtained by inserting $\mathrm{EC}_{50}$ for $K_{\mathrm{ss}}$ in the denominator of Eq. 4.7, which now writes:

$$
\frac{\text { air }}{F R}=\frac{\mathrm{I}}{\mathrm{I}+K_{\mathrm{si}} \cdot\left(1+\mathrm{S}^{\prime} / \mathrm{EC}_{50}\right)} \text {. }
$$

Compare Eq. 4.8 b with Eq. 4.13.

The term $K_{\mathrm{si}} \cdot\left(1+\mathrm{S}^{\prime} / \mathrm{EC}_{50}\right)$ in the denominator of Eq. 4.13 is equal to $\mathrm{IC}_{50}$ at a given agonist concentration $\left[\mathrm{S}^{\prime}\right]$, and we can deduce that the ant-agonist dissociation constant is given by:

$$
K_{\mathrm{si}}=\frac{\mathrm{IC}_{50}}{\left(1+\mathrm{S}^{\prime} / \mathrm{EC}_{50}\right)} .
$$

This equation is identical to the functional equivalent of Cheng-Prusoff's equation derived by McKinney et al. (1991) and Craig (1993). Accordingly, Eq. 4.14 is designated the 'functional equivalent of Cheng-Prusoff' (fe-C-P).

Similarly, compare Eq. 4.14 with Eq. 4.10. To obtain the ant-agonist dissociation constant in competitive reaction schemes, $K_{\mathrm{si}}$ or $K_{\mathrm{ii}}$, it is now sufficient to perform a 'functional inhibitor curve' experiment based on a single fixed agonist concentration, $\mathrm{S}^{\prime}$, and increasing the ant-agonist concentration from zero to $>90 \%$ inhibition (Fig. 4.2).

Eq. 4.14 is valid, but only when its assumptions are fulfilled.

Observe that knowledge of $\mathrm{EC}_{50}$ for an agonist under study is necessary for any kind of analyses for ant-agonist 


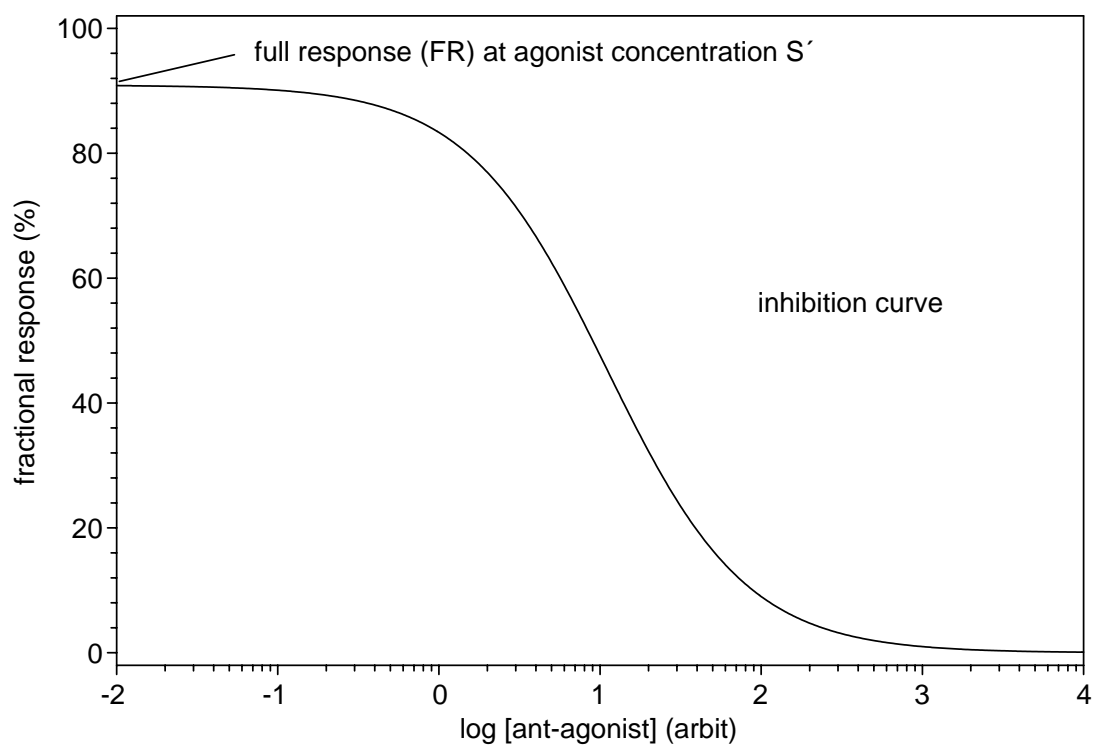

Figure 4.2. An inhibition curve based on simple competitive ant-agonism. Half of the full response $(F R=90.9 \%)$ is at $45.45 \%$ corresponding to an ant-agonist concentration of $K_{\mathrm{si}}$ (here equal 10) in arbitrary units.

parameters, although it may be argued that knowledge about receptor reserve is not needed for the two null methods of Gaddum and Schild (sub-chapter 4.2 and Chapter 11).

\subsubsection{The Validity of the Cheng-Prusoff Equation for Competitive Ant-agonism}

The C-P (Eqs. 4.5 or 4.10) and its functional equivalent Eq. 4.14, are based on the assumption of competitive ant-agonism and covers for both binding and functional studies.

Logically, in case the Cheng-Prusoff equations-C-P and fe-C-P - are supplied with a theory such as Hill's reaction scheme (Chapter 10) as suggested by some authors (Leff \& Dougall 1993; Lazareno \& Birdsall 1993a,b), they are, mechanistically speaking, bound to result in spurious results. We should remember that Hill's reaction scheme is only valid as a quantitative tool, not as a theoretical reaction scheme (see Chapter 10). However, this is not an argument for rejecting Eqs. 4.10 and 4.14 without Hillian exponentiation (Lazareno \& Birdsall 1993a,b).

In comparing the C-P approach with the null methods of Gaddum and Schild (Lazareno \& Birdsall 1993a,b), it is true that the complete Schild analysis will reveal deviations from simple competitive reaction schemes, while an analysis of dose-response relations will miss that, if only inducing, for instance $<50 \%$ of maximal response, and continuing with an inhibition curve'. Therefore, the correct C-P analysis requires a thorough determination of dose-responses for agonists and ant-agonist, after which it may be just as valid as a complete Schild analysis. Meanwhile, observe that a Schild method too requires application of both agonist and ant-agonist over extended ranges. Thus, on equal basis the C-P has its advantages over a Schild analysis as it requires fewer data points than the Schild procedure (see Section 4.4.2 and Chapter 11).

The thorough Schild analysis requires a good amount of data point, which is often limited by a requirement for impracticable high agonist as well as ant-agonist concentrations. A Schild slope with values deviant from unity is often the outcome of a functional Schild analysis. The deviant slope may be formulated as a Hill coefficient. This has been used as an argument for the validity of Schild's analysis, and the rejection of other analyses based on for instance the C-P/'inhibition curve experiments' (Leff \& Dougall 1993; Lazareno \& Birdsall 1993a,b).

For me, this argumentation does not hold. When Hill's reaction scheme is evoked as potentiation either of a C-P or a Schild-type derivation (Leff \& Dougall 1993; Lazareno \& Birdsall 1993a; Cheng 2004) and used not in a process of semi-quantitation, but as a tool for analysis, then valid dissociation constant for antagonists, $K_{\mathrm{B}} \mathrm{s}$, are not obtained from either a Schild or a C-P analysis, only their estimates. Admittedly, when a system cannot be treated for its receptor reserve, a Schild analysis, if possible, might be performed instead of a C-P analysis. On the other hand, in case unreasonably high concentrations of either agonists or ant-agonists are needed for the Schild analysis, which is often the case, then a much simpler C-P 
analysis may be valid with the right precautions, such as ruling out a receptor reserve by use of the fe-C-P equation.

On a different issue, concerning tracer bindingdisplacement studies, Munson \& Rodbard (1988, erratum: J Receptor Res 9: 511, 1989-1990) have argued for a correction to the Cheng-Prusoff correlation between $\mathrm{IC}_{50}$ and $K_{\mathrm{si}}$, Eq. 4.5, in case total and free concentrations of either receptor or labeled ligand are not the same (also see http://www.orc.ru/ yur77/refine.htm).

\subsection{The Gaddum Equation}

\subsubsection{Derivation and Evaluation of the Gaddum Equation}

Based on experiments performed and published in 1926, Gaddum was the first to derive an equation in 1937 (Gaddum 1926, 1937, 1943), which when reformulated and expressed in symbols used presently, yields:

$$
\frac{\operatorname{ara}}{F R}=\frac{\mathrm{S}^{\prime}}{\mathrm{S}^{\prime}+K_{\mathrm{ss}}+K_{\mathrm{ss}} \cdot \mathrm{I} / K_{\mathrm{si}}} .
$$

Compare Eq. 4.15 with Eq. 4.8b. They are identical and the derivation of both is parallel. Thus, Gaddum's equation is derived based on arguments already given in Sections 2.4.11, 4.2.3 and 4.2.4.

\subsubsection{A Comparison Between the $C-P$ and Gaddum Equations}

The Gaddum analysis (Gaddum et al. 1955; Gaddum 1957) together with the Schild equations (Schild 1947, 1949; Arunlakshana \& Schild 1959) are so-called null methods. The hallmark of null methods is that they eliminate complexing factors from the analysis of dissociation constants in binding studies, although the Gaddum and Schild formulations are particularly developed for functional studies. The complexing factors may be efficacy-related conformational changes and other conditions pertinent to functional studies (Chapter 5). However, it is still paramount to check for all other kinds of deviations from simple load before embarking upon a particular analysis. With this in mind, it seems that the C-P analysis and its functional equivalent, fe-CP, are satisfactory (McKinney et al. 1991; Craig 1993).

\footnotetext{
${ }^{5}$ Colquhoun (2006) has pointed out that the Gaddum equation was presented much earlier than 1937 by Michaelis and coworkers in 1914 and quoted in Haldane's book from 1930. Colquhoun further claims that Gaddum's equation was merely derived for binding studies. I don't read Gaddum's 1937 paper to that effect. Anyhow, observe that a Gaddum equation for fractional binding is identical to one for fractional response when based on a competitive reaction scheme.
}

\subsection{Preliminary Comparison of Schild-analysis with Cheng-Prusoff Analysis}

\subsubsection{Prelude}

Here we will discuss a comparison of the four methods suggested for determining and extracting $K_{\mathrm{b}}$ values including the Schild analysis (see Section 4.1.1). For this, one needs a provisional understanding of how the Schild regression operates. Therefore, such an insight is presented in Section 4.4.2. Chapter 11 provides a more detailed description of the Schild method, including newer transformed Schild formulations, such as that devised by Waud (1975, 1976) and reinstated by Lazareno \& Birdsall (1993b).

\subsubsection{The Schild Regression. Initial Considerations}

The standard procedure for extracting $K_{\mathrm{b}} \mathrm{s}$ with the Schild dose-ratio (DR) method is as follows. Preferably in the same assay or on the same tissue, perform a cumulative agonist dose-response control curve and continue with a single dose of an ant-agonist included in consecutive agonist dose-response relations, i.e., an ant-agonist concentration which varies for each of the following agonist dose-response curves (Fig. 4.3A). Calculate a DR based on equi-active agonist responses, as for instance $60 \%$ in Fig. 4.3A, from the ratio of concentration $\mathrm{S}^{\prime}$ of agonist in the presence of an antagonist over the equi-active concentration of agonist, $\mathrm{S}_{0}$, selected in the absence of an ant-agonist. The doseratio is $\mathrm{DR}=\mathrm{S}^{\prime} / \mathrm{S}_{0}$. Collect $\mathrm{DRs}$ from each agonist dose-response curve with a single ant-agonist concentration present, which varies from curve to curve. From these DRs at each of the agonist $\mathrm{S}^{\prime}$ concentrations rendering equi-active responses, make a plot of (DR-1) against the actual ant-agonist concentration (Fig. 4.3B). The curve of the Schild regression should be a straight line with a slope of unity. If this is the case, then extend the Schild curve to the $y$-axis (i.e., $\mathrm{DR}-1=1$ ) and read an estimate of $K_{\mathrm{b}}$ for the ant-agonist as the value of intercept with the $x$-axis. In Fig. $4.3 \mathrm{~B}$, which is a linear-linear plot, this happens at the point $(1,1)$; meaning that $K_{\mathrm{b}}=1$. A log-log plot of (DR-1) versus [I] in Fig. 4.3B is shown in Fig. 4.4 as a Schild regression. A Schild regression, which is a straight line with slope one, is a good indication that the assumptions for the Schild analysis are fulfilled, i.e., simple competitive interaction, type I or type II, between agonist and ant-agonist. Conversely so when a Schild regression is not a straight line or does not have a slope of unity or both. In such situations, 


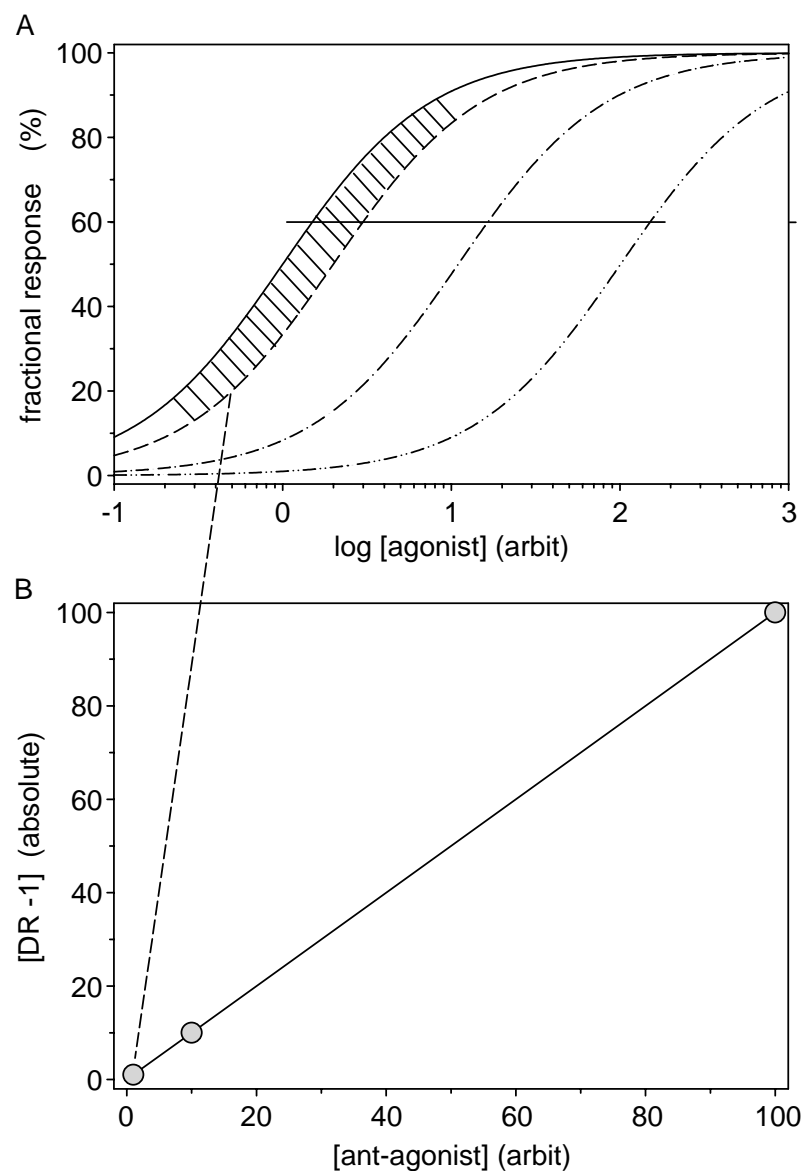

Figure 4.3. An illustration of the Schild analysis. (A) A control dose-response curve for an agonist $S$ is established with the ant-agonist concentration $[I]=0$. At $60 \%$ response, arbitrarily chosen and indicated by a horizontal line, the agonist concentration is 1.5 in arbitrary units. With an ant-agonist present at either of three maintained concentrations, three additional agonist dose-response curves are provided with fixed ant-agonist concentration at for instance 1,10, or 100 in arbitrary units. For these three latter curves, [S] is 3.0, 16.5 and 151.5 when the response is at $60 \%$ for all three curves and comparable to the same effect for the control curve, equiactive. The dose ratios (DRs), which are independent of the selected response level for equi-activity, are $3.0 / 1.5=2,16.5 /$ $1.5=11$, and $151.5 / 1.5=101$ for the three experimental curves. A plot of the DRs against their pertinent selected ant-agonist concentrations is in panel B. For DR-1 equal 1 on the ordinate, the abscissa value is equal to $K_{\mathrm{si}}$, which was chosen here as 1. Note, the plot is a linear-linear graph, while a Schild regression is a log-log plot of the same curve as shown in Fig. 4.4. The hatched area in panel $A$ is for doseresponses with ant-agonist concentrations below or equal 1 (see Chapter 11 for more details).

information does not allow for an estimation of the ant-agonist equilibrium dissociation constant $K_{\mathrm{b}}$. Nevertheless, the type of deformation in the Schild regression, away from linearity, may give hints about the underlying reaction scheme. Kenakin has meticulously listed and illustrated many of the possible deviations in the Schild regime (Kenakin 1997, Chapter 10).

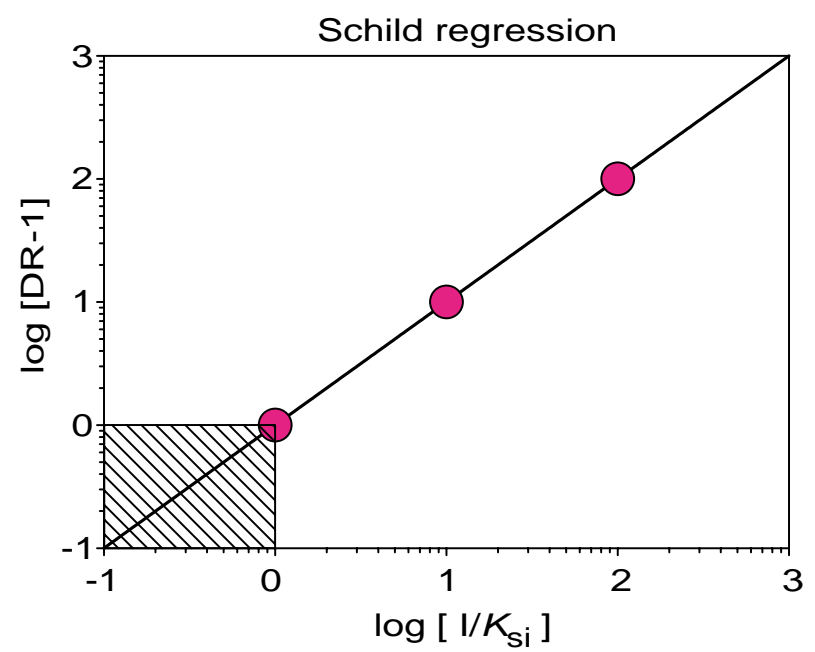

Figure 4.4. A plot of 'Schild regression' based on the plot in Fig. 4.3. Here both axes are logarithmic. Since $K_{\mathrm{si}}$ was chosen as 1 , we can read the log (affinity) for the ant-agonist compound as zero directly of the $x$-axis equal - log $\left(K_{\mathrm{si}}\right)$ or $\mathrm{pA}_{2}$ at a response level equal to log $(\mathrm{DR}-1)=0$. The hatched area is for dose-responses with normalized ant-agonist concentrations below or equal 1 (see Chapter 11 for more details).

\subsubsection{The Cheng-Prusoff Versus the Schild}

At face value, the Schild method has two advantages over the C-P scheme. (1) The Schild regression or Schild plot ${ }^{6}$ steps over problems of receptor reserve. For the C-P scheme, receptor reserve may be solved by employing the fe-C-P instead of C-P (McKinney et al. 1991; Craig 1993). (2) The Schild regression inherently reveals deviations from the assumptions expected of the studied system, which is that it must follow simple, one-state competitive ant-agonism. Incidentally, it can be argued that if the C-P analysis is performed as it ought to be, correctly with a complete agonist dose-response curve, this will also reveal deviations of the studied system from the assumptions, namely that it must follow simple, one-state competitive ant-agonism.

On the other hand, Schild analyses have at least the following three maladies, which do not hamper the C-P 'functional-inhibition curve' analysis. (1) To displace the apparent dissociation constant of a competitive doseresponse curve, $\operatorname{appK}_{\mathrm{ss}}$, by a mere factor of 2 requires an ant-agonist concentration equal to $K_{\mathrm{b}}$, meaning that an analysis of DRs at ant-agonist concentrations below $K_{\mathrm{b}}$ is not very accurate (see hatched area in Fig. 4.3 and 4.4). (2) For a proper Schild analysis, frequently it is impossible to reach high enough agonist does-responses with ant-agonists present, while the Cheng-Prusoff functionalinhibition curve analysis may be easily performed. (3) The many data points needed for a proper Schild analysis may also cause problems due to a prolonged duration of data collection and several separate preps may be

\footnotetext{
${ }^{6}$ On the difference between a Schild regression and a Schild plot, see Fig. 11.7 and Pratt \& Taylor (1990, p. 63).
} 
needed. Pros and cons for the two methods will depend on the system under exploration (see Chapter 11).

\subsubsection{A Provisional Statement about Schild Versus Cheng-Prusoff}

As already alluded to in Section 4.2.6, Leff \& Dougall (1993) and Lazareno \& Birdsall (1993a,b) argued for the Schild analysis in favor of the Cheng-Prusoff analysis. Their argumentation was partially based on implementing the 'logistic' function onto the Schild, as already suggested by several others (Arunlakshana \& Schild 1959; Ariëns et al. 1964, p. 146), thus providing a correspondence between the Hill factor and the Schild slope. However, to me, this transformation is not an advantage for the null method of Schild. Why? It is due to the convoluted acceptance for implementing such a transformation of the Schild theory as an explanation for a non-load behavior. Moreover, the 'logistic' transformation could just as well be implemented for the Cheng-Prusoff regime.

The Hill factor is generally not a parameter for a realistic system constant, but rather can be a practical measure of 'co-operative' activity, that is, load-deviant behavior. Accepting Hill's analysis may prevent one from searching methods that are more useful. I shall return to this claim in Chapters 10 and 11.

\subsubsection{An Unfaltering Implementation of Power Coefficients}

Similar to Leff \& Dougall (1993) and Lazareno \& Birdall (1993a), HC Cheng has argued for the use of a powered version of the Cheng-Prusoff formulation along with the functional-equivalent of Cheng-Prusoff (Cheng 2002). Furthermore, Cheng has argued for a potentiation of the Scatchard formulation and a complete potentiation of the Schild equation in order to extract $K_{\mathrm{B}}$ s from synagics that deviate from the simple competitive dose-response relations (Cheng 2004). Cheng has listed different potentiations of the Schild DR equations in the literature (Cheng 2004, Table 6), and his potentiation of Schild is similar to a suggestion by Ariëns and co-workers (1964, p. 146). As pointed out by Giraldo et al. (2007), a proper warning against an unreflected acceptance of 'powered' regimes should specify their mere empirical status.

Credibility for the implementation of power coefficients by Cheng to extract $K_{\mathrm{B}}$ 's from non-conform synagics is based on a 'proportionality approach' (Cheng 2002; Cheng \& Lai 2003). This proportionality approach is the same as using expressions of regula detri for actual response ar compared to remaining response $r r$ which is equal to the ratio between ligand concentration and it dissociation constant as developed later in Chapter 8, e.g., Eqs. 8.8 and 8.16, and repeated here:

$$
\frac{a r}{T R-a r}=\frac{\mathrm{S}}{\mathrm{S}+K_{\mathrm{ss}}-\mathrm{S}} \rightarrow \frac{a r}{r r}=\frac{\mathrm{S}}{K_{\mathrm{ss}}} .
$$

This type of double fractional formulation as in the right side regula detri of Eq. 4.16 may be raised to a power $n_{\mathrm{s}}$ for the agonist [S] and a power $n_{\mathrm{i}}$ for the ant-agonist [I] Thus, for instance:

$$
\frac{a r}{T R-a r}=\frac{\mathrm{S}^{n_{\mathrm{s}}}}{\mathrm{S}^{n_{\mathrm{s}}}+K_{\mathrm{ss}}^{n_{\mathrm{s}}}-\mathrm{S}^{n_{\mathrm{s}}}} \rightarrow \frac{a r}{r r}=\left(\frac{\mathrm{S}}{K_{\mathrm{ss}}}\right)^{n_{\mathrm{s}}} .
$$

This type of "proportionality with potentiation approach' was also suggested by Ariëns and co-workers (1964, p. 429) and has been extensively and successfully used by Chou \& Talalay (1977, 1981; Chou \& Hayball 1996; Chou et al. 2005; Chou 2006) for analysis of synergy (Chapter 12, Section 12.2.1). However, as also pointed out in Chapter 12, implementing this type of Hill approach will yield quantitative estimates of virtual system constants, but not a solution.

\subsubsection{My Response to Potentiation in Section 4.4 .5}

We use math to simulate Nature. Sometimes Nature deviates from our math. To solve such a discrepancy, a 'nice trick' is to raise the function variables and/or the function constants to powers that now become new parameters of the system. In pharmacology, the classical example of this approach is the Hill equation, which I will comment on in greater detail in other chapters of this book (see, e.g., sub-chapter 8.2 and Chapter 10, Sections 10.1.1-10.1.4). Also mentioned several times, potentiation (raising to a power) of variables and parameters is a legitimate approach when assumptions are taken into account, i.e., questioning whether the selected arithmetics cover the physics of the studied system. Meanwhile, this cautious standpoint seems to be ignored, when Cheng (2004) writes 'Although the Hill equation is originally derived for allosteric enzymes with multiple subunits, the Hill coefficient is now believed to signify a co-operativity of the drug-receptor interaction'. Compare this with Hill's statements about his own coefficient (quoted in Section 10.1.2). Hill's coefficient is merely a qualitative estimate of co-operativity and an empirical assessment (Giraldo et al. 2007).

\subsection{The Best Method to Extract $K_{\mathrm{xi}} \mathrm{s}$}

\subsubsection{A Method of Global Regression for the $K_{b} s$}

The best use of experimental data in an analysis for system parameters seems to be the method suggested by Lew \& Angus (1995, 1997); see also Lazareno \& Birdsall 1993b). These authors suggest a global non-linear 
analysis of the composite data obtained from a C-P functional inhibition curve assays together with a Schildtype dose-response data collection, and an equal weighting of all data points. This is a correct view, but again I have my doubts about their suggestion to also implement a logistic (Hill-type) theory for the reaction scheme to be the basis for an extraction of the ant-agonist $K_{\mathrm{b}}$ s. I hope to convince you that my uncalled-for objection can be substantiated (cf. sub-chapter 8.2 and Chapters 10 and 11). Thus, the Lew-Angus method is recommended, but the applied theory in such an analysis should be as realistic as possible, not a Hill transformation. Especially, after the emergence of two-state reaction schemes, such as the Hall (2000) allosteric two-state model (ATSM), it seems more valid to implement two-state theories for ligand-receptor interactions when observing deviations from simple load (Chapter 7). This is also true for inverse agonists as simple competitive ant-agonists in the cyclictwo-state model (Chapter 5) (Giraldo et al. 2007).

\subsubsection{The Best Method to Extract $K_{\mathrm{b}} s$. A Summary}

At this point, a conclusion on the discussion in preceding sections is that data obtained both from dose-response curves with increasing ant-agonist concentrations (Schild-type data) and from functional-inhibition curve experiment (C-P-type data) should be analyzed by a global non-linear regression of the total data and based on the most realistic theory available for the studied system. Often this can be just the simple one-state competitive ant-agonist reaction scheme and its formulation, as presented in Sections 2.4.9 and 2.4.10. In functional studies, hetero-interventory interaction tools, as formulated in Eq. 2.17, may also be evoked in the analysis.

Extra: The C-P analysis in its simple form may, at least in principle, be analyzed by a single data point determination (van Rossum 1963). However, in real life it is necessary to supply the C-P analysis. Thus, a determination of a full dose-response relation is required in order to supply a value for the dissociation constant for the agonist, $K_{\mathrm{ss}}, K_{\mathrm{m}}$, or $K_{\mathrm{d}}$. Accomplishing this task will reveal if the interaction with the agonist follows the assumed simple load, just as the analysis of the inhibitory curve will also reveal deviation from the simple load for competitive ant-agonism (Fig. 4.1B + D). To judge deviations, one can employ the factor-squared rule described in Section 8.1.4 from experimental doseresponse relations, as idealized in Fig. 4.1. Various forms of non-competitive and hetero-intervention models are aberrant possibilities that will show up in the simple analysis. This still leaves other alternatives undetected, such as receptor reserve, non-equilibrium conditions, functional competition, or heterogeneous receptor populations. One should check for these possibilities. In an ordinary Schild analysis, also based on simple competitive ant-agonism, many of these deviations from the assumed scheme will reveal themselves (Chapter 11).

\subsection{Multi-response Ligands and Ligand-dependent Differential Responses}

To impart perspective, here in Chapter 4 dealing with classic one-state ligand-receptor interactions, their parameters, and their determination, it may be appropriate to briefly consider new trends and conceptions in the synagic field, pointing to a need for parameter analyses in two-state models as described in detail in Part II.

\subsubsection{Functional Selectivity and Multi-response Ligands}

Following the confusion about inverse agonists, which was settled in the mid-1990s (sub-chapter 5.1), the phenomena of multi-response ligands and functional selectivity related to 7TM receptors have emerged and are being debated eagerly (see the 2007 August issue of Trends Pharmacol Sci). In particular, it is a question of how to designate these two conceptions in order to nail their substance.

\subsubsection{Multi-response Ligands}

Techniques for determining ligand responses and expanded drug design-development-and-discovery have spurred the recognition of multi-response ligands.

Table 4.1. Multi-response ligands - also designated 'allosteric agonists' or 'ago-modulators'

\begin{tabular}{llll}
\hline Multi-response ligands & No specific model & One-state model & Two-state model \\
\hline Positive/negative & Modifier agonist & Interventor agonist & Modulator agonist \\
Positive/negative & Modifier inverse agonist & Interventor inverse agonist & Modulator inverse agonist \\
\hline
\end{tabular}

The table suggests nomenclature for ligands that possess both modifier and agonist/inverse agonist activity elicited from an allosteric binding site and/or by overlap with a primary site. 
Table 4.2. Variety of functional ways. Terms for assay systems that due to specific ligands elicit or dampen some of the responses associated with the functional repertoire of the system either independent of the presence and absence of other ligands or dependent on the presence of other ligands

\begin{tabular}{|c|c|c|c|}
\hline Other ligands & On binding and functionality & Definition & Reference \\
\hline \multirow[t]{9}{*}{ Independence of other ligands } & Functional selectivity & Broad functionality & Urban et al. (2006) \\
\hline & Collateral efficacy ${ }^{7}$ & Phenomenological & Kenakin (2005) \\
\hline & Stimulus trafficking & Mechanistic & Kenakin (1995) \\
\hline & Ligand-directed trafficking & Focus on the ligand & Galandrin et al. (2007) \\
\hline & Biased agonism & General & Alpin et al. (2007) \\
\hline & & & Schulte \& Levy (2007) \\
\hline & Differential efficacy & Applied science & Goldin et al. (2007) \\
\hline & Differential response* & Broad & This book \\
\hline & $\begin{array}{l}\text { Protean agonism } \\
\text { at the same receptor }\end{array}$ & $\begin{array}{l}\text { Positive and inverse } \\
\text { Agonism }\end{array}$ & Kenakin (2001) \\
\hline \multirow[t]{2}{*}{ Dependence on the presence of other ligands } & Permissive agonism & & Leach et al. (2007) \\
\hline & Permissive ant-agonism & & Kenakin (2005) \\
\hline
\end{tabular}

*In order to cover 'broad', my suggestion for the 'functional selectivity' would probably be (pos/neg), (primary, allosteric, co-lateral) 'ligand-dependent differential response'. The term 'response' covers both functionality and binding. If adopted, the colloquial version of this designator is likely to be 'differential response'.

Although the subject of multi-response ligands is not completely new (Jakubik et al. 1993), it has been invoked recently by Schwartz \& Holst (2006, 2007), who termed these ligands 'ago-allosteric modulators' (Schwartz et al. 2006; Teng et al. 2007), followed by Christopoulos and co-workers who talk about 'allosteric agonists' (Langmead \& Christopoulos 2006; May et al. $2007 a, b)$. In principle, dual effects of modifier molecules at 7TM receptors cover both positive and negative action on binding and function of present primary ligands, while the modifiers at the same time, in the absence of other ligands, can appear as either agonist or inverse agonist in their own right in binding and functional assays with all responses elicited from a secondary site - the allosteric site - equal to 'allosteric agonists' (see http://www.IUPHAR.org).

Notice in this connection, in the understanding and description of this type of dual response ligands there is also possible co-lateral binding, i.e., simultaneous binding at primary and secondary sites (sub-chapter 2.6). Hence, classical agonist and inverse agonists bind to a primary orthosteric site, while the new 'multi-response ligands' may bind to a secondary allosteric site and a primary site, and the overlap at a primary site may even be a dynamic one (Schwartz \& Holst 2007). Actually, such a possibility can explicitly be formulated by the fourpane one-state model (FP-OSM) depicted in Fig. 2.4.

For multi-response ligands in relation to my strict separation of intervention models, with interventors in one-state schemes, from allosteric models, with modulators in two-state schemes, as presented in Chapter 14, I suggest a scheme of designators that follows the

\footnotetext{
7 'Collateral' indicates parallel processes and should not be mixed with 'co-lateral' in 'co-lateral binding' (sub-chapter 2.6). Hence, collateral is related to differential responses, while co-lateral is related to 'binding of multi-response' ligands (listed in Table 4.1).
}

segregation of ligands into 'interventors' and 'modulators', and furthermore with the two combined under the term 'modifiers' (Table 4.1).

\subsubsection{Functional Selectivity}

'Functional selectivity' is a broad designator for the conception that ligands in general may activate some elements of the repertoire of functions that a receptive system can engage with endogenous ligands (Urban et al. 2006). This selective functionality by ligands is not new (Jones et al. 1991; Meller et al. 1992; Spengler 1993; Migeon \& Nathanson 1994; Kenakin 1995), but due to new combinatorial assay systems and high throughput screening programs, it is revealed more often and in growing complexity (Kenakin 2007; Leach et al. 2007). In this connection, several descriptive terms have appeared, such as 'collateral efficacy' and 'permissive ant-agonism' (Kenakin 2005), which I list in Table 4.2 and supply with a new term that might specifically characterize the paradigm of 'functional selectivity'. I thus follow a suggestion by Urban et al. (2006) to try to find the most appropriate term. The new term is 'liganddependent differential response' (Table 4.2).

Note that enzymes that are multi-sited may also respond in different ways upon activation by pertinent ligands (Fig. 4.5) (Krejewski et al. 2005, 2008).

\subsection{Epilogue of Part I \\ 4.7.1. Revisiting the Schism of Binding and Function}

As stated earlier, it was provisionally assumed that binding is only a matter of adsorption, while function is established through adsorption and absorption due to an additional conformational change (Figs. 1.2 and 1.3). 

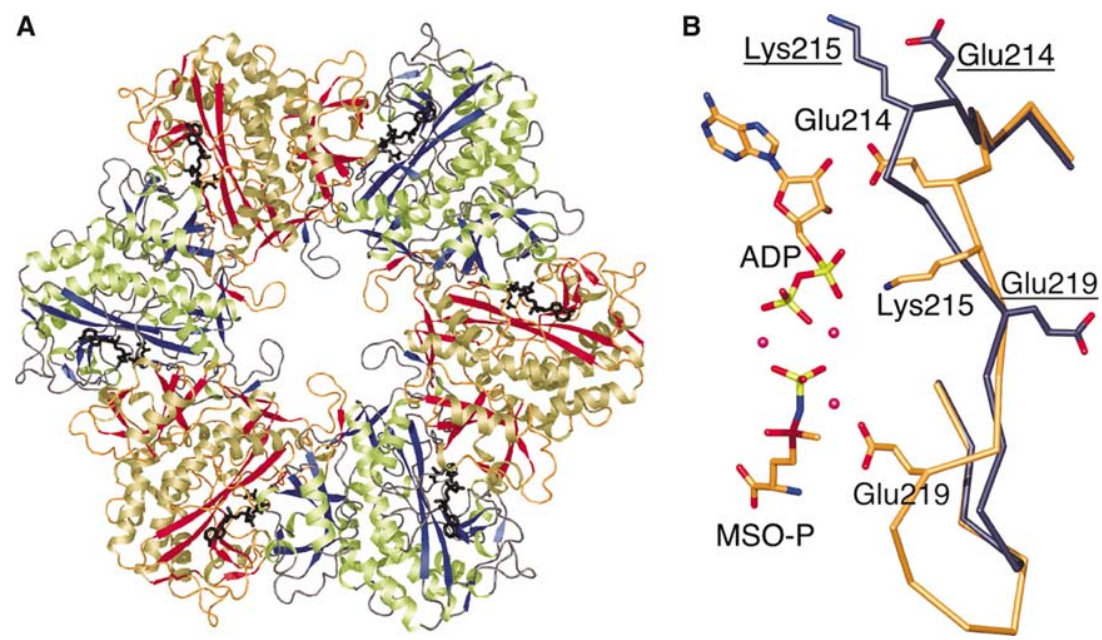

Figure 4.5. 'Multi-response receptive units'. Overall structure of multi-sited glutamine synthetase (GS). For more details on GS, see Chapter 13. (A) The subunits of the MtGS hexamer are colored either gold/red or green/blue. Ball-and-stick representations of MSO-P and ADP (black) show the location of the active sites at subunit-subunit interfaces. (B) The $\beta$-strand register-shift that distinguishes the current taut structure of MtGS (gold carbons) from the previous relaxed one (dark gray carbons) is shown. Residues of the relaxed structure are underlined. From Krajewski et al. (2005, Fig. 1) with permission.

This is also somewhat implied by the function of the colateral intervention coefficient $c-$ a conformational parameter related to efficacy.

The best way to illustrate the difference between a binding experiment and an assay for a functional response is to contrast the simplest forms of doseresponse relationship for the two types of experiment. This is presented in reaction schemes, in formulas, and in plots (Figs 1.8 and 1.9A). Several of the features are quite obviously different between binding and function from the presented figures.

Allow these statements to stand for a short moment as they are valid for the synagic models of Part I.

In a seminal paper, Colquhoun discussed the schism between binding and function, or affinity and efficacy, and how to visualize, prove, and measure the related molecular mechanistic changes (1998, Figs. 1A+B and $2 \mathrm{~A}+\mathrm{B})$. These changes are equal to conformational changes of receptors, separation into subunits of heterotrimeric $\mathrm{G}$ proteins, and gating in channels. Colquhoun concluded that we have almost completely failed in demonstrating the structural changes that lie behind the observed deviations measured for all kinds of proteins.

Colquhoun's 1998 paper is a milestone paper in receptology. Colquhoun made it clear, that we are not in a position to separate affinity and efficacy, as it was imagined in the classic period and still purported in many one-state models. This paper is essential reading for all those interested in the analysis of synagic and kinetic data. Colquhoun's insight has taught me a lot. Keep in mind, binding like function is dependent on conformational changes at the gating site and not necessarily solely at the binding site in effector proteins.
Insecurity about our ability to separate affinity and efficacy or binding-gating is still actual (Colquhoun 2007). This knowledge together with an implementation of genuine two-state models will be exploited in Part II.

\subsubsection{Revisiting the schism of competitive and non-competitive ant-agonism}

Recently, for GPCRs, Baker and coworkers have meticulously reconfirmed that not all agonist-dependent inhibition by ant-agonists is by classical competitive ant-agonism (Baker 2005a, b, 2008; Baker \& Hill 2007a, b). These and other observations have spurred terms such as "orthosteric ant-agonist" and "allosteric ant-agonist" (Jakubik \& Dolezal 2006; Hemstapat et al 2007; May et al. 2007b; Kenakin 2008). These terms imbue our minds with the conceptions that ant-agonist in the presence of agonists may work at a primary or a secondary binding site. In modeling, as I have demonstrated in chapter 2, classical competitive ant-agonism may be induced from a primary (orthosteric) binding site, type I competitive inhibition, or from a secondary ("allosteric") binding site, type II competitive antagonism. Thus both intervention, non-competitive inhibition and type II competitive ant-agonism may fall under the term "allosteric ant-agonism". When we model for analysis of synagics, it is worth (recommended) to operate with a strict terminology. Thus, in modeling, naming of interventors (chapter 2) and modulators (chapter 14 and 15) operating from a secondary site should be considered carefully. Who knows, maybe "orthosteric ant-agonist" and "allosteric ant-agonist" is for the future. 


\section{REFERENCES}

Abramow-Newerly M, Roy AA, Nunn C \& Chidiac P. RGS proteins have a signalling complex: interactions between RGS proteins and GPCRs, effectors, and auxiliary proteins. Cell Signal 18: 579-591, 2006.

Aplin M, Christensen GL, Schneider M, Heydorn A, Gammeltoft S, Kjolbye AL, Sheikh SP \& Hansen JL. The angiotensin type 1 receptor activates extracellular signal-regulated kinases 1 and 2 by $\mathrm{G}$ protein-dependent and -independent pathways in cardiac myocytes and Langendorff-perfused hearts. Basic Clin Pharmacol Toxicol 100: 289-295, 2007.

Ariëns EJ. Molecular Pharmacology. The Mode of Action of Biologically Active Compounds, Vol. I+ II, 1st ed. New York: Academic Press, 1964.

Arunlakshana $\mathrm{O}$ \& Schild HO. Some quantitative uses of drug antagonism. Br J Pharmacol 14: 48-58, 1959.

Baker JG. Site of action of beta-ligands at the human betal-adrenoceptor. J Pharmacol Exp Ther 313: 1163-1171, 2005a.

Baker JG. Evidence for a secondary state of the human beta3adrenoceptor. Mol Pharmacol 68: 1645-1655, 2005b.

Baker JG. A study of antagonist affinities for the human histamine H(2) receptor. Br J Pharmacol 153: 1011-1021, 2008.

Baker JG \& Hill SJ. A comparison of the antagonist affinities for the Giand Gs-coupled states of the human adenosine A1-receptor. $J$ Pharmacol Exp Ther 320: 218-228, 2007a.

Baker JG \& Hill SJ. Multiple GPCR conformations and signalling pathways: implications for antagonist affinity estimates. Trends Pharmacol Sci 28: 374-381, 2007b.

Barlow RB, Scott NC \& Stephenson RP. The affinity and efficacy of onium salts on the frog rectus abdominis. Br J Pharmacol Chemother 31: 188-196, 1967.

Cheng HC. The power issue: determination of $\mathrm{K}_{\mathrm{B}}$ or $\mathrm{K}_{\mathrm{i}}$ from $\mathrm{IC}_{50}$. A closer look at the Cheng-Prusoff equation, the Schild plot and related power equations. J Pharmacol Toxicol Methods 46: 61-71, 2002.

Cheng HC. The influence of cooperativity on the determination of dissociation constants: examination of the Cheng-Prusoff equation, the Scatchard analysis, the Schild analysis and related power equations. Pharmacol Res 50: 21-40, 2004.

Cheng HC \& Lai RW. Use of the proportionality equations for analyses of dose-response curves. Pharmacol Res 47: 163-173, 2003.

Cheng Y-C \& Prusoff WH. Relationship between the inhibition constant $\left(K_{\mathrm{I}}\right)$ and the concentration of inhibitor which causes 50 per cent inhibition $\left(I_{50}\right)$ of enzymatic reaction. Biochem Pharmacol 22: 3099-3108, 1973.

Chou TC. Theoretical basis, experimental design, and computerized simulation of synergism and antagonism in drug combination studies. Pharmacol Rev 58: 621-681, 2006.

Chou TC \& Talalay P. A simple generalized equation for the analysis of multiple inhibitions of Michaelis-Menten kinetic systems. J Biol Chem 252: 6438-6442, 1977.

Chou TC \& Talalay P. Generalized equations for the analysis of inhibitions of Michaelis-Menten and higher-order kinetic systems with two or more mutually exclusive and nonexclusive inhibitors. Eur J Biochem 115: 207-216, 1981.

Chou TC \& Hayball MK. Calcusyn for Windows, Multiple-drug-effect Analyzer and Manual, 1st ed. Cambridge: Biosoft Cambridge Place, 1996.

Chou TC, Guan Y, Soenen DR, Danishefsky SJ \& Boger DL. Potent reversal of multidrug resistance by ningalins and its use in drug combinations against human colon carcinoma xenograft in nude mice. Cancer Chemother Pharmacol 56: 379-390, 2005.

Colquhoun D. Binding, gating, affinity and efficacy: the interpretation of structure-activity relationships for agonists and of the effects of mutating receptors. Br J Pharmacol 125: 924-947, 1998.
Colquhoun D. What have we learned from single ion channels? $J$ Physiol 581: 425-427, 2007

Colquhoun D. The quantitative analysis of drug-receptor interactions: a short history. Trends Pharmacol Sci 27: 149-157, 2006.

Craig DA. The Cheng-Prusoff relationship: something lost in the translation. Trends Pharmacol Sci 14: 89-91, 1993.

Ehlert FJ. Estimation of the affinities of allosteric ligands using radioligand binding and pharmacological null methods. Mol Pharmacol 33: 187-194, 1988.

Figler H, Olsson RA \& Linden J. Allosteric enhancers of A1 adenosine receptors increase receptor-G protein coupling and counteract guanine nucleotide effects on agonist binding. Mol Pharmacol 64: $1557-1564,2003$.

Furchgott RF. The use of $\beta$-haloalkylamines in the differentiation of receptors and in the determination of dissociation constants of receptor-agonist complexes. Adv Drug Res 3: 21-55, 1966.

Furchgott RF and Bursztyn P. Comparison of dissociation constants and of relative efficacies of selected agonists acting on parasympathetic receptors. Ann N Y Acad Sci 144: 882-899, 1967.

Gaddum JH. The action of adrenalin and ergotamine on the uterus of the rabbit. J Physiol 61: 141-150, 1926.

Gaddum JH. The quantitative effects of antagonistic drugs. J Physiol 89: 7P-9P, 1937.

Gaddum JH. Introductory address. Part I. Biological aspects: the antagonism of drugs. Trans Faraday Soc 39: 332, 1943.

Gaddum JH. Theories of drug antagonism. Pharmacol Rev 9: 211-218, 1957.

Gaddum JH, Hameed KA, Hathway DE \& Stephens FF. Quantitative studies of antagonists for 5/hydroxytryptamine. QJExp Physiol 40: $49-74,1955$.

Galandrin S, Oligny-Longpre G \& Bouvier M. The evasive nature of drug efficacy: implications for drug discovery. Trends Pharmacol $\mathrm{Sci}$ 28: 423-430, 2007.

Giraldo J, Serra J, Roche D \& Rovira X. Assessing receptor affinity for inverse agonists: Schild and Cheng-Prusoff methods revisited. Curr Drug Targets 8: 197-202, 2007.

Goldin PR, McRae K, Ramel W \& Gross JJ. The neural bases of emotion regulation: reappraisal and suppression of negative emotion. Biol Psychiatry 63: 577-586, 2008.

Griffin MT, Hsu JC, Shehnaz D \& Ehlert FJ. Comparison of the pharmacological antagonism of M2 and M3 muscarinic receptors expressed in isolation and in combination. Biochem Pharmacol 65: 1227-1241, 2003

Hall DA. Modeling the functional effects of allosteric modulators at pharmacological receptors: an extension of the two-state model of receptor activation. Mol Pharmacol 58: 1412-1423, 2000.

Hemstapat K, Da Costa H, Nong Y, Brady AE, Luo Q, Niswender CM, Tamagnan GD \& Conn PJ. A Novel Family of Potent Negative Allosteric Modulators of Group II Metabotropic Glutamate Receptors. J Pharmacol Exp Ther 322: 254-264, 2007.

Hill SJ. G-protein-coupled receptors: past, present and future. $\mathrm{Br} J$ Pharmacol 147: S27-S37, 2006.

Jakubik J, Bacakova L, Lisa V, El-Fakahany EE \& Tucek S. Activation of muscarinic acetylcholine receptors via their allosteric binding sites. Proc Natl Acad Sci USA 93: 8705-8709, 1996.

Jakubík J \& Dolezal V. Determinants of positive cooperativity between strychnine-like allosteric modulators and N-methylscopolamine at muscarinic receptors. J Mol Neurosci 30: 111-112, 2006.

Jones SV, Heilman CJ \& Brann MR. Functional responses of cloned muscarinic receptors expressed in CHO-K1 cells. Mol Pharmacol 40: 242-247, 1991.

Kenakin T. Pharmacological Analysis of Drug-receptor Interaction, 3rd ed. New York: Raven Press, 1997.

Kenakin T. Inverse, protean, and ligand-selective agonism: matters of receptor conformation. FASEB J 15: 589-611, 2001. 
Kenakin T. New concepts in drug discovery: collateral efficacy and permissive antagonism. Nat Rev Drug Discov 4: 919-927, 2005.

Kenakin T. Collateral efficacy in drug discovery: taking advantage of the good (allosteric) nature of 7TM receptors. Trends Pharmacol Sci 28: 407-415, 2007.

Kenakin T. What systems can and can't do. Br J Pharmacol 153: 841843, 2008.

Kenakin T, Morgan P \& Lutz M. On the importance of the 'antagonist assumption' to how receptors express themselves. Biochem Pharmacol 50: 17-26, 1995.

Kirschner LB \& Stone WE. Action of inhibitors at the myoneural junction. J Physiol 34: 821-834, 1951.

Krajewski WW, Jones TA \& Mowbray SL. Structure of Mycobacterium tuberculosis glutamine synthetase in complex with a transitionstate mimic provides functional insights. Proc Natl Acad Sci USA 102: 10499-10504, 2005.

Krajewski WW, Collins R, Holmberg-Schiavone L, Jones TA, Karlberg T \& Mowbray SL. Crystal structures of mammalian glutamine synthetases illustrate substrate-induced conformational changes and provide opportunities for drug and herbicide design. $J \mathrm{Mol}$ Biol 375: 217-228, 2008.

Langmead CJ \& Christopoulos A. Allosteric agonists of 7TM receptors: expanding the pharmacological toolbox. Trends Pharmacol Sci 27: 475-581, 2006.

Lazareno S \& Birdsall NJ. Estimation of competitive antagonist affinity from functional inhibition curves using the Gaddum, Schild and Cheng-Prusoff equations. Br J Pharmacol 109: 1110-1119, 1993a.

Lazareno S \& Birdsall NJ. Estimation of antagonist Kb from inhibition curves in functional experiments: alternatives to the ChengPrusoff equation. Trends Pharmacol Sci 14: 237-239, 1993b.

Leach K, Sexton PM \& Christopoulos A. Allosteric GPCR modulators: taking advantage of permissive receptor pharmacology. Trends Pharmacol Sci 28: 382-389, 2007.

Leff P \& Dougall IG. Further concerns over Cheng-Prusoff analysis. Trends Pharmacol Sci 14: 110-112, 1993.

Lew MJ \& Angus JA. Analysis of competitive agonist-antagonist interactions by non-linear regression. Trends Pharmacol Sci 16: 328-337, 1995.

Lew MJ \& Angus JA. An improved method for analysis of competitive agonist/antagonist interactions by non-linear regression. Ann N Y Acad Sci 812: 179-181, 1997.

May LT, Leach K, Sexton PM \& Christopoulos A. Allosteric modulation of G protein-coupled receptors. Annu Rev Pharmacol Toxicol 47: $1-51,2007 \mathrm{a}$

May LT, Avlani VA, Langmead CJ, Herdon HJ, Wood MJ, Sexton PM \& Christopoulos A. Structure-function studies of allosteric agonism at M2 muscarinic acetylcholine receptors. Mol Pharmacol 72: 463$476,2007 \mathrm{~b}$.

McKinney M, Anderson DJ, Vella-Rountree L, Connolly T \& Miller JH. Pharmacological profiles for rat cortical M1 and M2 muscarinic receptors using selective antagonists: comparison with N1E-115 muscarinic receptors. J Pharmacol Exp Ther 257: 1121-1129, 1991.

Meller E, Puza T, Diamond J, Lieu HD \& Bohmaker K. Comparative effects of receptor inactivation, 17 beta-estradiol and pertussis toxin on dopaminergic inhibition of prolactin secretion in vitro. J Pharmacol Exp Ther 263: 462-469, 1992.

Migeon JC \& Nathanson NM. Differential regulation of cAMPmediated gene transcription by $\mathrm{ml}$ and $\mathrm{m} 4$ muscarinic acetylcholine receptors. Preferential coupling of $\mathrm{m} 4$ receptors to $\mathrm{Gi}$ alpha-2. J Biol Chem 269: 9767-9773, 1994.
Munson PJ \& Rodbard D. An exact correction to the 'Cheng-Prusoff' correction. J Recept Res 8: 533-546, 1988.

Schild HO. pA, a new scale for the measurement of drug antagonism. Br J Pharmacol 2: 189-206, 1947.

Schild HO. pAx and competitive drug antagonism. Br J Pharmacol 4: 277-280, 1949

Schild HO. Drug antagonism and $\mathrm{pA}_{\mathrm{x}}$. Pharmacol Rev 9: 242-246, 1957.

Schulte G \& Levy FO. Novel aspects of G-protein-coupled receptor signalling - different ways to achieve specificity. Acta Physiol (Oxf) 190: 33-38, 2007.

Schwartz TW \& Holst B. Ago-allosteric modulation and other types of allostery in dimeric 7TM receptors. J Recept Signal Transduct Res 26: 107-128, 2006.

Schwartz TW \& Holst B. Allosteric enhancers, allosteric agonists and ago-allosteric modulators: where do they bind and how do they act? Trends Pharmacol Sci 28: 366-373, 2007.

Schwartz TW, Frimurer TM, Holst B, Rosenkilde MM \& Elling CE. Molecular mechanism of 7TM receptor activation - a global toggle switch model. Annu Rev Pharmacol Toxicol 46: 481-519, 2006.

Segel IH. Enzyme Kinetics. Behavior and Analysis of Rapid Equilibrium and Steady-state Enzyme Systems. New York: Wiley \& Sons (reissued 1993), 1975.

Spengler D, Waeber C, Pantaloni C, Holsboer F, Bockaert J, Seeburg $\mathrm{PH}$, et al. Differential signal transduction by five splice variants of the PACAP receptor. Nature 365: 170-175, 1993.

Stephenson RP. A modification of receptor theory. Br J Pharmacol 11: 379-393, 1956.

Teng M, Johnson MD, Thomas C, Kiel D, Lakis JN, Kercher T, Aytes S, Kostrowicki J, Bhumralkar D, Truesdale L, May J, Sidelman U, Kodra JT, Jørgensen AS, Olesen PH, de Jong JC, Madsen P, Behrens C, Pettersson I, Knudsen LB, Holst JJ and Lau J. Small molecule ago-allosteric modulators of the human glucagon-like peptide-1 (hGLP-1) receptor. Bioorg Med Chem Lett 17: 5472-5478, 2007.

Urban JD, Clarke WP, von Zastrow M, Nichols DE, Kobilka B, Weinstein H, Javitch JA, Roth BL, Christopoulos A, Sexton PM, Miller KJ and Mailman RB. Functional selectivity and classical concepts of quantitative pharmacology. J Pharmacol Exp Ther 320: 1-13, 2007.

van Rossum JM. Cumulative dose-response curves: II. Techniques for making of dose-response curves in isolated organs and the evaluation of drug parameters. Arch Int Pharmacodyn Ther 143: 299-330, 1963

Waud DR. On the measurement of the affinity of partial agonists for receptors. J Pharmacol Exp Ther 170: 117-122, 1969.

Waud DR. Analysis of dose-response curves. In: Methods in Pharmacology: Smooth Muscle, Vol. 3, 1st ed, edited by Daniel EE \& Paton DM. New York: Plenum Press, 1975.

Waud DR. Analysis of dose-response relationships. In: Advances in General and Cellular Pharmacology, 1st ed, edited by Narahashi T \& Bianchi CP. New York: Plenum Press, 1976.

Waud DR, Son SL \& Waud BE. Kinetic and empirical analysis of doseresponse curves illustrated with a cardiac example. Life Sci 22: 1275-1285, 1978.

Webb JL. Enzyme and metabolic inhibitors. In: General Principles of Inhibition, Vol. 1, 1st ed. New York: Academic Press, 1963.

Wyllie DJ \& Chen PE. Taking the time to study competitive antagonism. Br J Pharmacol 150: 541-545, 2007. 

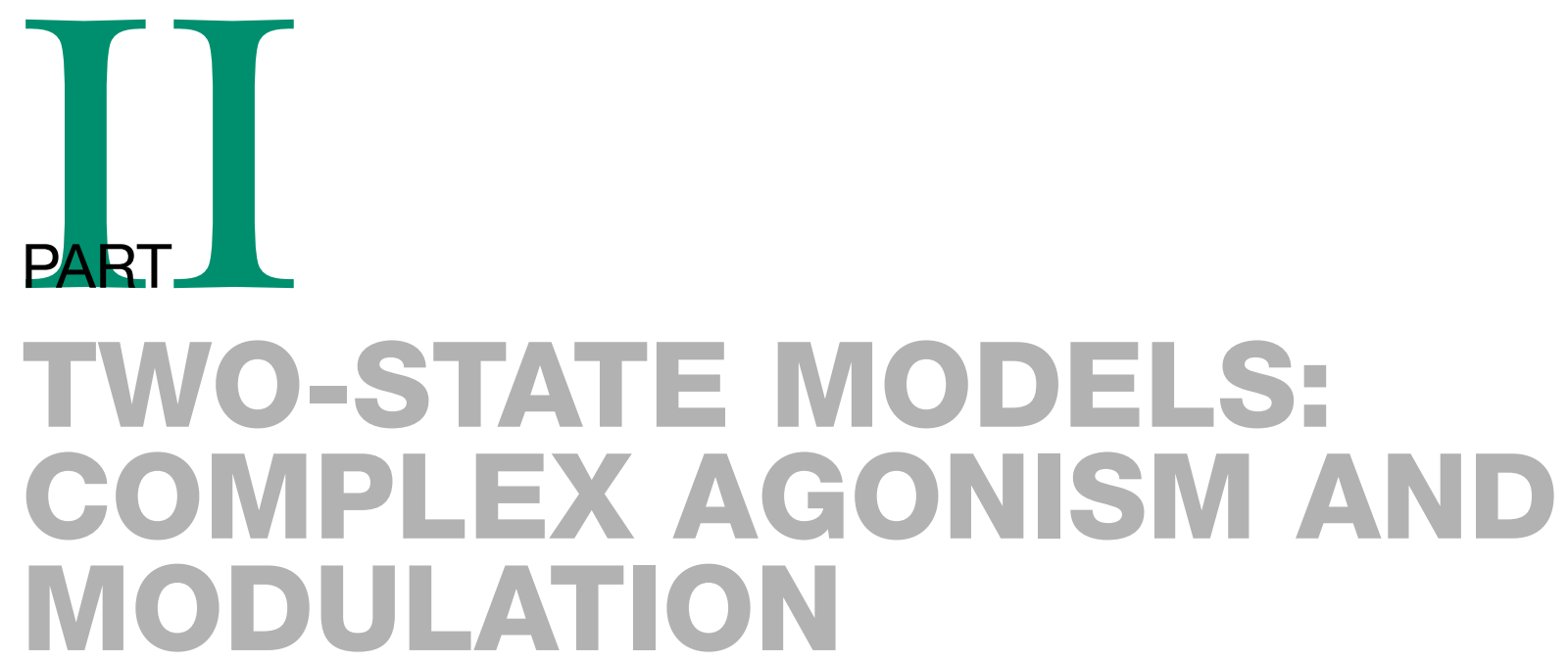

In Part II we are still at the receptor level. In Part IV on super-complex agonism and regulation, we will move beyond the confines of the ligand-receptor level.

The word 'complex' in the title to Part II is a reference to reaction schemes that involve two-state receptor models. Thus, in reality, the adjective 'complex' covers the concept of 'two-state' reaction schemes.

The two-state model, developed for ion-channels and enzymes during the 1950 s, has recently undergone a dramatic re-emergence in and impact on pharmacology (Rang 2006). Paul Leff writes (1995) 'Until recently pharmacological receptor theory developed along nonmechanistic lines and largely overlooked the two-state concept'.

\section{II.1. Two-state Models?}

What do we mean by 'two-state' reaction schemes? The intervention reaction scheme in Chapter 2 consisted of four symbols for a receptive unit, R, RS, IR, and IRS (Fig. 2.1). These symbols signify that the receptive unit exists in four different states. The same is true for the four receptor symbols of the ternery-complex model described for $\mathrm{G}$ protein-coupled receptors (GPCRs) (see Section 2.7.2). However, to make it very clear, the designation 'two-state' in the term 'two-state model' (TSM) is a reference to the number of states for the unliganded receptive unit. Therefore, as we shall see, a model by Katz and Thesleff from 1957 is a TSM, since it explicitly operates with the un-liganded receptor in two conformations, R and R* (Figs. 5.1A and 5.4). Similarly, when we come to the allosteric and the homotropic TSMs, as well as the extended-ternary-complex and the cubic-ternary-complex models in Chapter 7, these additional four models are also true 'two-state' reaction schemes even though they each cover a total of six to eight different conformations for the receptive unit. Their sole un-liganded receptive units are $\mathrm{R}$ and $\mathrm{R}^{*}$, i.e., they are genuine TSMs (cf. Chapters 7 and 14). Note, that the intervention reaction scheme analyzed in Chapter 2 (Fig. 2.1), and the ternary-complex model (TCM) described for GPCRs (front quadrant of Fig. 7.4A) are not genuine TSMs. In these two models, there is only one form of the un-liganded receptive unit.

Thus, the reader should realize that 'complex' and 'TSMs' need a context to be perceived correctly, and certainly within the frame of this book.

\section{II.2. Agonism. Two-steps Versus Two-states}

A simple two-step model, the del Castillo \& Katz (dC\&K) model (Figs. 5.1B, 5.2A and 5.3B), and a simple two-state model, such as the original by Katz \& Thesleff (Figs. 5.1A and 5.2C), are developed in sub-chapters 5.6-5.9. 'Twostep' covers reaction schemes in which intermediate conformations of liganded but non-active receptive units are involved together with liganded conformations that are active (Fig. 5.3B). Meanwhile, a receptive unit in both an un-liganded and an active state, $\mathrm{R}^{*}$, is not necessarily part of the two-step reaction, as it has to be in TSMs (Fig. 5.4). The simple one-state reaction scheme, as formulated by del Castillo \& Katz (1957) (Fig. 5.1B), supports the classic view of first-binding-then-activation (see sub-chapter 5.6). Three additional two-step models, described in Chapter 6, follow the first-binding-then- 
activation scheme and are the so-called ordered, random, and ping-pong reaction schemes.

\section{II.3. Intervention and Modulation}

Chapter 2 in Part I outlined ant-agonism and inhibition by intervention. To recapitulate, 'intervention' means mutually inclusive, i.e., two different ligands can bind simultaneously to their respective binding sites, while the two ligands do not bind at either site. There is no colateral binding possible (Section 2.6.3 and Fig. 2.4). On the other hand, when such a two-sited receptive unit for both interventor and agonist is also capable of a "twostate'-switch, $\mathrm{R} \rightleftharpoons \mathrm{R}^{*}$, its full thermodynamic reaction scheme involves eight receptor conformations, and ligand interaction at the secondary binding site is now denoted 'modulation' rather than 'intervention'. This type of reaction scheme is covered in Chapter 7 by the allosteric two-state model (ATSM).

\section{II.4. Auto-intervention and Auto-modulation}

Chapter 3 in Part I covered a simple auto-intervention reaction scheme, in which a single ligand could bind to either of two separate sites. Therefore, for such an autointervention reaction scheme, it was possible to have both mutually inclusive as well as co-lateral binding. A ligand, which can bind to either site and simultaneously to both sites, will affect the binding characteristics for the very same type of ligand at the opposite binding site. If we assume that this receptive unit with auto-intervention can also switch between 'two-states', then ligand interaction at the secondary binding site is now denoted 'automodulation' rather than 'auto-intervention'. The full thermodynamic reaction scheme for such an automodulatory receptive unit may be equated by the homotropic two-state model (HOTSM), surveyed in Chapter 7. In Chapter 5 on complex agonism, we shall first deal with a receptive unit in two-states, but only with a single binding site.

\section{II.5. Dissociation Versus Association Constants}

In developing the two-state concept in Part II, we will also switch freely between equilibrium dissociation and association constants. At equilibrium the parameters can be dissociation constants, such as $K_{\mathrm{s}}, K_{\mathrm{d}}, K_{\mathrm{ss}}, K_{\mathrm{si}}$, $K_{\mathrm{is}}$, and $K_{\mathrm{ii}}$, plus $K_{\mathrm{sm}}, K_{\mathrm{ms}}$, and $K_{\mathrm{mm}}$, and association constants, such as $A_{\mathrm{s}}, A_{\mathrm{m}}, A_{\mathrm{ss}}, A_{\mathrm{is}}, A_{\mathrm{si}}$, and $A_{\mathrm{ii}}$, plus $A_{\mathrm{ms}}$, $A_{\mathrm{sm}}$, and $A_{\mathrm{mm}}$; thus far used for normalizing ligand concentrations. In these subscripts ' $\mathrm{i}$ ' signifies an inhibitor/interventor or its binding site and ' $\mathrm{m}$ ' stands for a modulator or its binding site (Fig. II.1). For rules on subscript nomenclature see Box 2.1 in Chapter 2.

The choice of either dissociation or association is selected $a d$ hoc as it simplifies formulation and understanding in the theoretical analyses of models. Thus, in theoretical analysis of slightly complicated reaction schemes with 'two-state' isomerization, it is sometimes convenient and more straightforward to use 'forward' association constants, although 'backward' dissociation constants have the useful dimension of concentration. In principle, they are equally valid. Van Rossum (1966) has given arguments for selecting either association or dissociation constants.

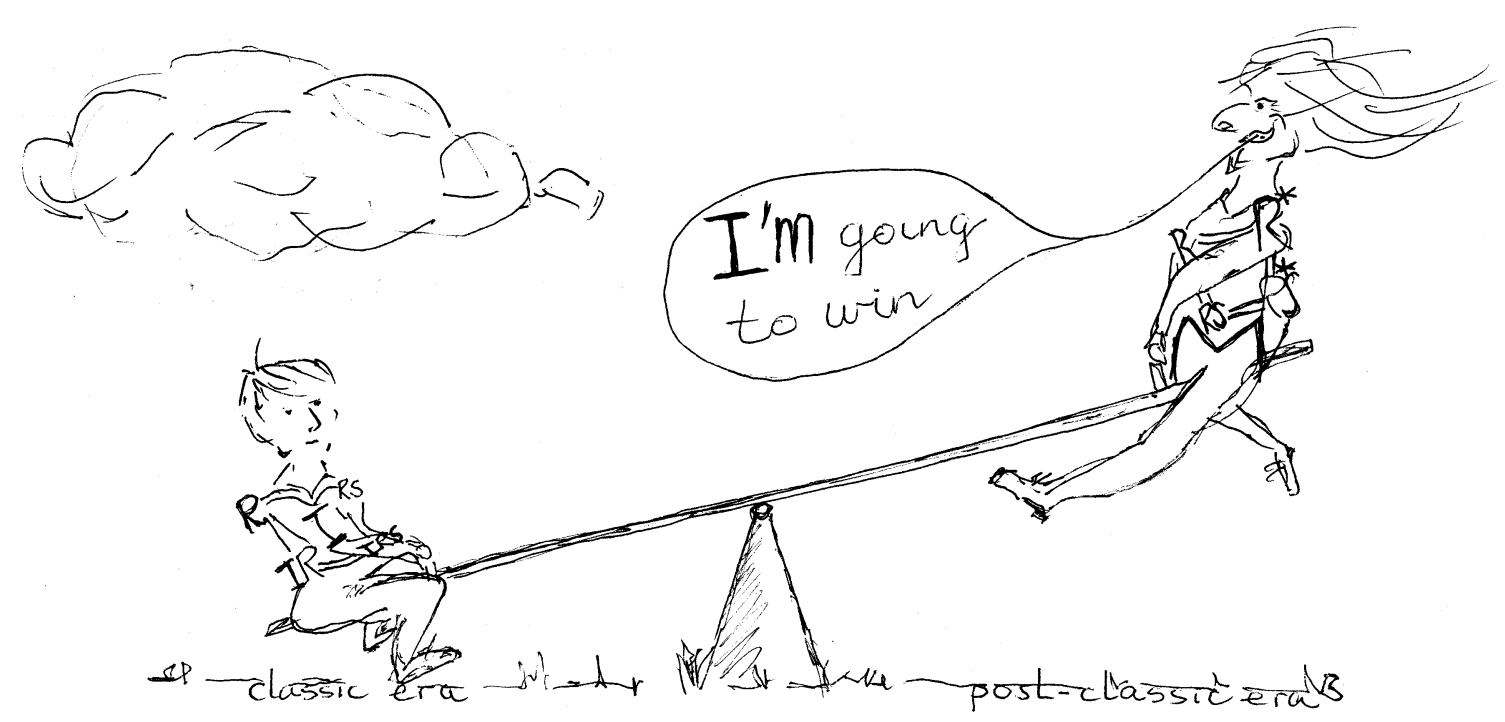

Figure II.1. Modulator (M) against interventor (I). 


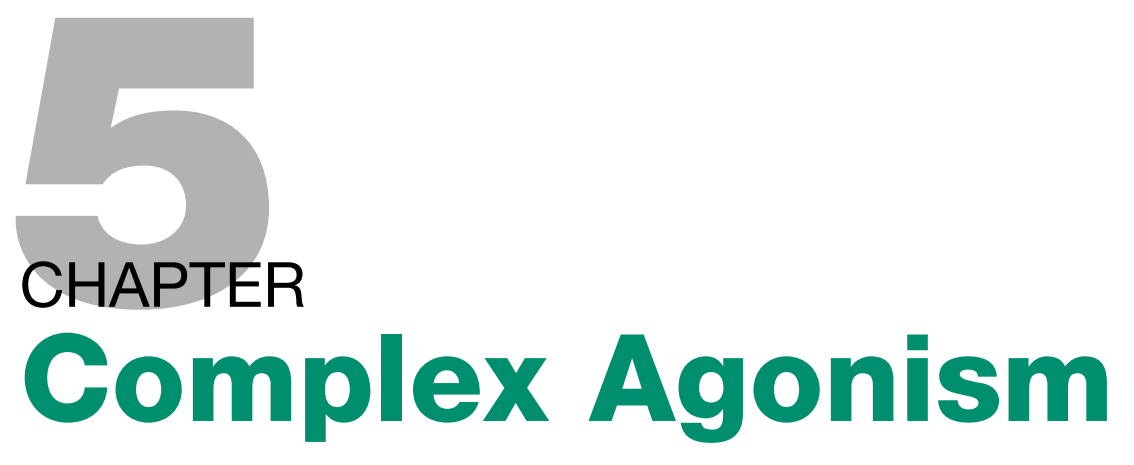

\subsection{Basal, Spontaneous, and Constitutive Activity}

Many activities run through effector molecules in the natural state of resting cells. Channels tunnel molecules, pumps pump ions, and cotransporters shovel nutrients to the enzyme catalyzed furnace. Let us think of these activities at rest as basal activity by the various effector molecules.

Viewing the regulated function of effector proteins from the perspective of receptive units, the receptor molecules are mostly thought of as quiescent until the moment they are stimulated by an agonist, which now affect and alter the effector activity - a view which due to its simplicity more or less dominated pharmacology and its textbooks until about a decade ago. In a way, such a view is even more obvious in enzymology and transport physiology, since substrates or transported molecules in many types of experiments must be present in order to allow measurement of function. However, the above dogmatism about receptors seems to have vanished with the recent realization that even in the absence of agonists, many receptor systems including systems of over-expressed receptors (Costa \& Herz 1989) display spontaneous activity, while mutated receptors might be or become constitutively active (Kenakin et al. 1995; Milligan \& Bond 1997), and explain the behavior of inverse agonists (Bond et al. 1995; Kenakin 2004).

\subsubsection{A Period of Transception}

From 1989 to 1995, pharmacology experienced a renaissance in its understanding of molecules that stimulate or inhibit receptor function due to the recognition that many receptors, and therefore also their effector proteins, are already basically, spontaneously, or constitutively active.
Understandably, during this period of transception, ${ }^{1}$ the discoveries and recognition of spontaneous receptor activity briefly confused the definition of what agonists and ant-agonists should be, how to designate them, and furthermore, how to design experiments, including which drugs to choose for ones own experiments (Kenakin 1987; Jenkinson 1991; Hoyer \& Boddeke 1993; Kenakin et al. 1995). In those years, new notions and formulations were introduced, e.g., concepts appeared such as 'inverse agonism', 'negative efficacy', and 'negative intrinsic activity' as well as 'negative antagonism' (Costa et al. 1992; Samama et al. 1993; Kenakin 1994; Milligan et al. 1995; Bond et al. 1995; Kenakin 1995a,b; Kenakin et al. 1995; Kenakin 2004). Those lacunae in our understanding almost evaporated again with the re-recognition of the cyclic two-state model (cTSM) (Robertson et al. 1994; Leff 1995), and later developed reaction schemes, such as the ATSM (Hall 2000), the HOTSM (Bindslev 2004), and the cubic ternary-complex model (CTCM) (Weiss et al. 1996 a,b,c) (Chapter 7).

\subsubsection{Function and Binding Concepts}

When we focus on receptor controlled protein function, as mentioned, activity in the absence of agonists can be either basal or spontaneous/constitutive activity. The constitutive activity in mutated receptor/effector systems is most easily seen when receptors are overexpressed. Thus, the acronym CAM, standing for 'constitutively active mutants', has emerged. However, the three concepts 'basal', 'spontaneous', and 'constitutive' are often used interchangeably. For the modeler of reaction schemes, activity observed in functional studies can be either (1) basal/spontaneous/constitutive, or (2) agonist-induced activity.

\footnotetext{
1 Transception is synonymous with a jump in conception and sometimes even with a paradigm-shift.
} 
In binding studies, the term 'activity' is meaningless. Therefore, for those experimental situations where a binding assay is used, 'spontaneous activity' may be replaced by a term such as 'high-or-low-affinity-state'. Thus, spontaneous high-or-low-affinity-states in occupancy studies has a direct parallel to the spontaneous activity in functional studies and is delineated here for TSMs in Section 5.8.4.

\subsection{Inverse Agonism and Multi-functional Drugs and Receptive Units}

\subsubsection{Inverse Agonists}

Increasing numbers of compounds, considered agonists, are observed to inhibit spontaneous or constitutive activity instead of stimulating the activity. In addition, ant-agonists have been observed to prevent the inhibition induced by thought-to-be agonist and rather to stimulate receptor activity in the presence of these inhibitory agonists.

The thought-to-be agonists with a reducing effect on spontaneous activity are now known as 'inverse agonists', whereas compounds that ant-agonize the inhibitory effect of inverse agonists, but without an effect on their own in the absence of inverse agonists, are still signified as ant-agonists or even better as neutral ant-agonists (Milligan et al. 1995). Moreover, many competitive antagonists have been found to be inverse agonists (Chidiac 2002; Strange 2002; Kenakin 2004).

Since the reappearance of spontaneous receptor activity and its cognate inverse agonism, the cTSM has experienced a revitalization (Leff 1995). As we shall see, the effects of inverse agonists are immanent in the cTSM and its formulation. Thus, the TSM has taken center stage, while concepts from Stephenson's efficacy scheme as partial agonism and receptor reserve (sub-chapter 1.3) still live on in parallel.

\subsubsection{Multi-functional Agonists}

New models are required as novel ligand-receptor interactions are discovered. Ligands that bind to primary sites as agonists and to secondary sites as modulators may behave as auto-modulators, described for the HOTSM in Chapter 7. Acetylcholine at muscarinic receptors is one example (Winding \& Bindslev 1993). Other drugs are developed that both stimulate as agonists and concurrently affect the response to an endogenous agonist, so-called 'ago-modulators' (Table 2.2B and 4.1) (Schwartz \& Holm 2006). To date, the behavior of these types of drugs has not been well described and therefore demands new modeling.
Several papers on this theme appeared in the August 2007 issue of Trends in Pharmacological Sciences.

\subsubsection{Multi-liganding Receptors}

Just as new concepts on multi-functional ligands appear, novel possibilities for multi-functional binding and catalysis in receptive units emerge. Thus, binding of ligands in the binding sites of a receptive unit is often more than the simple view of a 'key-in-the-lock'. As an example of this complexity, the enzyme Cyt P450 3A4, which is induced by glucocorticoids and degrades about half of all ingested drugs in humans, seems to adapt its binding site to more than one drug, at the same time exhibiting both homotropic and heterotropic allostery (Isin \& Guengerich 2005; Ekroos \& Sjogren 2006). Again, innovative modeling may be required for this catalytic system (Roberts \& Atkins 2007).

\subsection{Prelude to TSMs with Only One Binding Site}

\subsubsection{Two-state Receptor Models}

As we start to examine dose-response relationships that do not follow simple load theory, some of the problems and challenges we face have already been presented in preceding sections on partial agonism, on possible spare receptors, on allostery, on spontaneous activity, and on inverse agonism (Chapter 1).

Before 1950, ideas about complex receptor function advanced slowly. Although A.J. Clark, the farther of pharmacology, hinted at receptor reserve as an explanation for the linear drug concentration-response for narcotics and had his doubts about the applicability of a simple load theory in the mechanistic Langmuirian sense (Clark 1937, p. 64 and pp. 215-217), he tenaciously purported formulations along the lines of a power equation, i.e., response $=$ occupancy raised to a power of $n$ (Clark 1937).

From the 1950s on, things started to move. Based on earlier observations and with sharp insight, Wyman imagined a concerted conformational change in hemoglobin subunits as oxygen would bind, affecting the binding of the next oxygen to bind (Wyman \& Allen 1951; Edsall 1980). Likewise, at this time in the field of muscle enzymes, a wakening insight began to crystallize among biochemists. They drew reaction schemes involving intermediate states for activity of the enzyme (Tonomura \& Watanabe 1952; Watanabe et al. 1953; Botts \& Morales 1953; Blum 1955; Botts 1958). This culminated in the late 1950 s, when a cTSM for enzymes was finally formulated. Botts and Drain (1958, reaction scheme 14) first described a cTSM for enzymes with the 
A $S+R \rightleftharpoons S R \rightleftharpoons S R^{\prime}$

в $A c h+R \stackrel{\text { (1) }}{\rightleftharpoons} A c h R \stackrel{\text { (ii) }}{\rightleftharpoons} A c h R^{\prime} \stackrel{\text { (iii) }}{\longrightarrow} A c h+R^{\prime}$
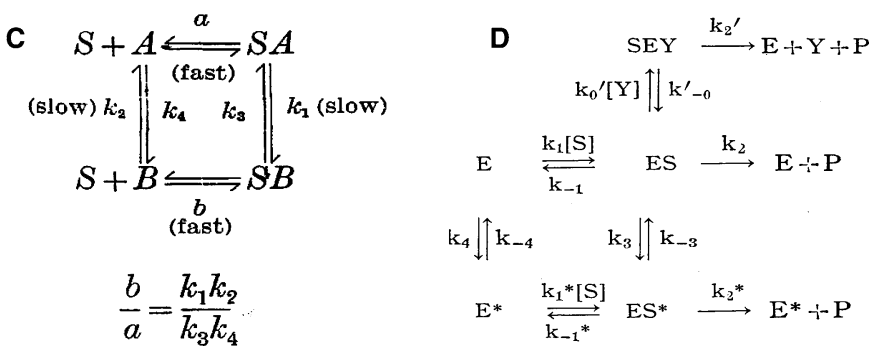

Figure 5.1. Four original reaction schemes. $(A+B)$ DelCastillo and Katz (1957, p. 369 and p. 380) reaction schemes 2 and 3 $(\mathrm{dC} \& \mathrm{~K}) . \mathrm{Ach}=\mathrm{S}, \mathrm{R}=$ initial reactive receptor, $\mathrm{SR}=$ intermediate reactive ('inactive') conformation, and $\mathrm{SR}^{\prime}=$ an active form (open), that may desensitize. In panel $B$ a nearly irreversible step $\rightarrow S+R^{\prime}$ is added. $R^{\prime}$ is the receptive unit in its un-liganded form, a non-reactive conformation, i.e., desensitized. (C) Katz and Thesleff (1957) reaction scheme 5 (K\&T5). Note the balanced equilibrium condition in panel C: $b / a=k_{1} k_{2} / k_{3} k_{4}$. (D) Botts and Drain (1958) reaction scheme 14 (B\&D14). The authors comment 'Free energy considerations also require that, for generality, the deformation steps in the cycle be reversible'.

explicit involvement of a conformational change for unliganded receptive units between a reactive and an active state in a fully reversible reaction scheme (Fig. 5.1D).

\subsection{Transition Away from Classic Receptor Theory}

\subsubsection{Stephenson's Reaction Scheme}

Recognition of a need to explicitly express the existence of a conformational change for the un-liganded receptor ran in parallel with observations of partial agonism and receptor reserve (Furchgott 1955; Nickerson 1956; Stephenson 1956). Ariëns (1954) introduced the parameter 'intrinsic activity' $\alpha$, a system constant between 0 and 1, that could account for partial agonism. However, to incorporate both partial agonism and receptor reserve, Stephenson (1956) gave a simple approach for the formulation of response as a function of a stimulus. Stephenson wrote the stimulus expressed as adsorption in the form of a Langmurian occupancy, the load, times activation in the form of an efficacy constant, $e$ (Eqs. 5.1 and 1.17), i.e. not the property 'efficacy' as used in relation to response (see also the following discussion and sub-chapter 1.3). Furthermore, the actual response or activity was now an unknown function of this stimulus (see Eq. 5.2).

Stephenson's stimulus, consisting of a binding process and an activation process, was equated as:

$$
\text { stimulus = occupancy } \cdot \text { efficacy constant, }
$$

and a response, related to the concept termed 'efficacy' as a property, was then imagined by Stephenson as a yet unknown function of the stimulus:

$$
\begin{aligned}
\text { response }= & \text { unknown function of } \\
& (\text { occupancy.efficacy constant }) .
\end{aligned}
$$

Note in Eq. 5.2 that 'response' or its related property efficacy is not the efficacy constant but an unknown function of the efficacy constant.

Thus for various agonists, dialing on a nob-of-efficacy, that is, varying the efficacy constant in Eq. 5.2, Stephenson could explain the observation of response for partial agonism including a possible receptor reserve (Fig. 1.12A+B) (Stephenson 1956, Figs. 1 and 9). Similar plots are generated later from the functional form of the dC\&K model (Fig. 5.6).

\subsubsection{Efficacy and Efficacy Constant Starts to Merge}

Although, the two terms 'efficacy' as a property and 'efficacy' as a constant were intended as separate concepts, a slip of the mind due to wording later brought the two together as equal. Thus, the relative response due to an agonist 'property', equal to 'efficacy' (Stephenson 1956, p. 380), was unfortunately not clearly differentiated from 'efficacy' as a constant used throughout most of the paper by Stephenson (cf. for instance the legend to Stephenson's Fig. 9 (1956), reproduced in Fig. 1.12B, and his Table V). See also Sections 1.3.4 1.3.8 and more details on this subject in Section 5.6.2.

In the intervening years, Stephenson's model for efficacy has been refined by several authors in an attempt to experimentally isolate and determine an entity such as the efficacy (Furchgott 1966; MacKay 1966, 1977; Black \& Leff 1983; Clarke \& Bond 1998; Clark et al. 1999). Why the effort? Well, if one can obtain experimental data for the activation process per se, i.e., the efficacy in absolute 
terms as an efficacy constant, that would give us an opportunity to assay and understand the conformational change that is elicited in a protein molecule when it activates. Others have questioned Stephenson's model (Colquhoun 1987, Colquhoun \& Farrant 1993). In addition, as pointed out by several authors, all efforts to dis-entangle dose-response relations and isolate an efficacy constant will be in vain (Kenakin 1994, 2002; Colquhoun 1998). Notwith-standing, with a different perspective, playing on the affinity and efficacy of drugs as separate and on a model for their combined effects, Black and co-researchers came up with cimetidine, the money-making drug for ulcer treatment (Kenakin 2004, pp. 6-7).

\subsection{The Actual Scene}

Before moving on to the development of models equipped with two conformations for the un-liganded receptive unit and cartooned as the so-called cTSM, let us pause for a moment and look at the actual scene for TSMs and other similar reaction schemes.

Two main trends are presently being pursued, the TCM and three-state models (IIISMs).

\subsubsection{The Ternary Complex Model (TCM)}

One trend is that of the TCM, detailed as the INTERVENTION model in Chapter 2. The TCM originally took care of the realization that in order to elicit function, receptors had to couple to other protein molecules, with $\mathrm{G}$ proteins as the prime example (Abramowitz et al. 1980; Birnbaumer et al. 1980; DeLean et al. 1980), and further detailed by Ehlert (1988). The TCM for G proteins was preceded by a so-called 'two-step receptor model' (Boyneames \& Dumount 1975, 1980), a 'mobilereceptor model' (Jacobs \& Cuatrecasas 1976), and 'precoupled and collision-coupled models' (Tolkovsky \& Levitzki 1978, 1981). Shortly after, the TCM expanded to the extended ternary complex model (ETCM) (Iyengar et al. 1980; Lefkovitz et al. 1993; Samama et al. 1993; Kenakin et al. 1995; Weiss et al. 1996a), to a cubic ternary-complex model, CTCM (Frieden 1970; Laidler \& Bunting 1973, p 318; DeHaën 1976) later derived especially for $\mathrm{G}$ protein as the second ligand (Kenakin 1995a; Fong 1996; Weiss et al. 1996a,b,c; Onaran \& Costa 1997), and further to a dynamic variation on the CTCM theme in the form of a cubic ternary-complex activation model (CTCAM) (Shea et al. 2000). Both the ETCM and the CTCM take into account the idea of two states for the receptor in a fully reversible scheme, the cTSM. Thus, TCM people on their part are bending towards the paradigm of cTSMs.
In the field of $\mathrm{G}$ protein coupled receptors, the discussion about which model to use is ongoing (Kenakin et al. 2000, Chapter 5, p. 156), although the new ways of thinking about dose-response relations with ETCM and CTCM have reached maturity and are ready to be exploited in an unfolding of the cubic TCM and its quaternary sibling, the cubic quarternary-complex model (CQCM), Fig 7.5, (Christopoulos et al. 1998; Christopoulos \& Kenakin 2002). This goes together with other variations on the theme (Rusch et al. 2004; Rusch \& Forman 2005; Downing et al. 2005; Durroux 2005), and is also combined with receptor ensemble hypotheses (Onaran \& Costa 1997; Kenakin \& Onaran 2002). Some details on these models are described in Chapter 7 (Table 7.3).

\subsubsection{Three-state Models (IIISMs)}

The other trendsetting development is multi-state models, where receptor molecules are complexed with $\mathrm{G}$ proteins in a precoupled fashion, i.e., receptor and $\mathrm{G}$ protein complexation precedes the binding of ligands. In these theories, two different active states are postulated for the receptor and each binds to a different $G$ protein allowing for the experimentally observed bifurcation of signals for GPCRs. With an additional non-active receptor state, the total comes to three un-liganded receptor states, the IIISMs (Leff et al. 1997; Scaramellini \& Leff 1998, 2002; Strange 1998; Surya et al. 1998; Kameda 2003). Thus, two-state modelers engaged in GPCR transduction mechanisms are bending towards the context of a TCM, a model including a complexation step with a third component, the $\mathrm{G}$ protein, for both the receptor as such and the ligand-receptor complex before the receptor performs its action (Scaramellini \& Leff 2002). Giraldo (2004) analyzed a IIISM in more detail and concludes that the model can be useful for assessing quantitatively the changes in activity following receptor mutation. The IIISM keeps the stick-shift of efficacy within the receptive unit, so to speak, contrary to other models that leave the efficacy transmission to a step beyond the receptor as in classic receptor theory, for instance in the TCM, where the efficacy coupling is down-stream of the receptor in the chain of events for signal transduction.

For more about models on signal bifurcation, see the quarternary-complex model (QCM) by Kukkonen et al. (2001) and the models by Tucek et al. (2002) in Chapter 11. These latter models for promiscuity are all one-state models. Thus, the QCM mentioned here is not the same as the CQCM mentioned in Section 5.5.1, which is a genuine TSM, look at Fig 7.5. See Table 7.3 for a survey and segregation of these models. 


\subsubsection{A Decisive Jump from Steps to States}

Before moving on to these types of exciting new models, we need an insight into their base, which are the cTSM and its modulatory versions in the form of the ATSM and HOTSM. Accordingly, we now continue with the development of the cTSM starting with the dC\&.K model, a two-step reaction scheme, which transforms into the cyclic-two-state mechanism (cTSM).

\subsection{Two-steps Intermediate Complexes in Non-active States}

\subsubsection{The del Castillo \& Katz Reaction Scheme (dC\&K)}

At the same time that Stephenson introduced his efficacy term, in order to accommodate theory to dose-response of partial agonists, another approach was invoked to handle the expected conformational changes during activation of receptor channels. Katz together with del Castillo suggested a hypothesis in which they envisioned that binding of a ligand to a receptor formed an intermediate complex, RS, which could switch to a secondary active state, R*S, Fig. 5.1A. Their model was also proposed in an attempt to explain the competitive ant-agonism between choline derivatives at nicotinic acetylcholine receptors in the neuro-muscular junction (del Castillo \& Katz 1957, p. 369). The two authors further extended their model for the desensitization phenomenon of the nicotinic receptor channel (Fig. 5.1B) (del Castillo \& Katz 1957, p. 380), as described earlier by several researchers (Fatt 1950; Thesleff 1955). At the time, desensitized receptor channels were termed 'non-reactive' receptors. The complete del Castillo \& Katz reaction scheme, which explicitly included a transition from a liganded reactive state - non-active intermediate state - to a liganded and active state, was described as:

$$
\mathrm{R}+\mathrm{S} \leftrightharpoons \mathrm{RS} \leftrightharpoons \mathrm{R} * \mathrm{~S},
$$

in which $\mathrm{R} * \mathrm{~S}$ is an active conformation of the receptive entity including a possible desensitized state.

This dC\&K reaction scheme is the simplest two-step model with a ligand involved in an activating isomerization. However, it is not a TSM. $^{2}$ Without a ligand involved as for voltage-operated channels, the reaction scheme is even simpler (Fig. 5.2B).

For enzymatic activity, two-step models with their intermediate state or conformation were already proposed in the early 1940s (Glasstone et al. 1941) and

\footnotetext{
$\overline{2}$ Definition: two-state models operate with the un-liganded receptor in two conformations.
}

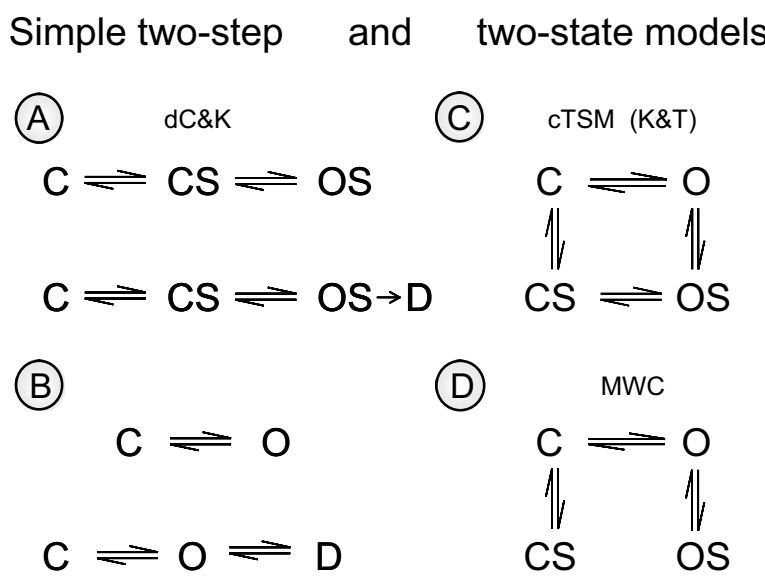

Figure 5.2. Simple reaction schemes including transcribed originals from Fig. 5.1. Models in A are one-state models (OSMs). Models in B-D are genuine two-state models (TSMs). $\mathrm{S}$ is a substrate or agonist. (A) Transcription of the two dC\&K schemes from Fig. 5.1A+B. (B) Simple reaction scheme between closed, $\mathrm{C}$, and open, $\mathrm{O}$, conformations that may also desensitize, D (inactivate). (C) Transcription of the K\&T5 scheme from Fig. 5.1C. (D) Simplified Monod-Wyman-Changeux (MWC)-scheme. Note that this scheme is not fully reversible as for instance the $\mathrm{K} \& \mathrm{~T} 5$ and $\mathrm{B} \& \mathrm{D} 14$ reaction schemes in Fig. 5.1.

ideas about two-step mechanisms appeared increasingly compelling. Impetus for this came from the development of techniques to measure fast reaction kinetics by Eigen in Germany, Tonomura in Japan, Chance in the USA, and Roughton in England. Eyring's transitionstate theory was also instrumental (Eyring 1935, Glasstone et al. 1941) as was Wyman's reflections on the response in hemoglobin upon oxygen-binding (Wyman \& Allen 1951). The treated and tested systems included enzymes, hemoglobin, receptor channels and transporters, including ion channels. Inspired by the Perutz (1942) studies on hemoglobin, now receptive units were described as existing in 'aramagnetic' to a 'diamagnetic' configuration (Wyman 1948; Allen et al. 1950), in non-deformed to deformed states or as in non-active intermediate states (Tonomura \& Watanabe 1952; Hodgkin \& Huxley 1952; Watanabe et al. 1953; Shaw 1954; Blum 1955; Glynn 1955; Botts 1958). Later these terms became 'tense' equal to nondeformed or reactive, $\mathrm{T}$, and 'relaxed' equal to deformed or active, $\mathrm{R}$, states of the receptive units (Fig. 5.2D) (Monod et al. 1965) (also see Section 5.9.9 and Chapter 15).

The state of affairs gave birth to two thermodynamically complete two-state receptor models, one developed for desensitization at the nicotinic acetylcholine receptor (Fig. 5.1C) (Katz \& Thesleff 1957) (see sub-chapter 5.7), and another for enzyme catalytic activity (Fig. 5.1D) (Botts \& Drain 1958). 


\subsubsection{The Misconception Induced by Stephenson's Efficacy Scheme}

At this point in our development of models, it would be beneficial to compare the scheme by Stephenson, described in Chapter 1 and Eqs. 5.1 and 5.2, with the scheme by del Castillo \& Katz.

Let efficacy parameter for conformational change be given by the symbol ' $e$ ' and occupancy by the symbol ' $y$ '. Then we can abbreviate Stephenson's formula (Eq. 5.2) into:

$$
r=f(y \cdot e),
$$

while the del Castillo \& Katz hypothesis may be written by a near-identical expression, in an abstract form, as:

$$
r=f(y, e) \text {. }
$$

In these two equations, $r$ is the response and $f($ ) means 'function of'.

The difference between Stephenson and dC\&K is a product operator in Stephenson's expression, '.' (Eq. 5.3) versus a separation or listing operator, ', (Eq. 5.4), for the del Castillo \& Katz interpretation of receptor states (Fig. 5.3). The conceptual difference between a 'Stephenson' and a 'Katz-et-al.' formulation is subtle, but the influence on the outcome of the formulated equations is more than dramatic. It is mind-blowing.

For the dC\&K model, the derivation of formulas takes in its origin including explicitly a 'new' conformation of the receptive unit. In the Stephenson scheme, the effect of a conformational change is simply multiplied as an efficacy constant $e$ onto the occupancy term $y$ (Eq. 5.3), and the product is often taken as the response to cover efficacy, not just the stimulus. That is, Eqs. 5.1 and 5.2 in sub-chapter 5.4 are combined, thus omitting the 'function of'. The misuse is exerted due to impatience with

A

$$
\begin{aligned}
& \text { Stephenson's } \\
& \text { occupancy }=y=\mathrm{S} /\left(\mathrm{S}+K_{\mathrm{s}}\right) \\
& \text { stimulus }=e \cdot y \\
& \text { relative response }=f\left\{e \cdot\left[\mathrm{S} /\left(\mathrm{S}+K_{\mathrm{S}}\right)\right]\right\}
\end{aligned}
$$

B

del Castillo \& Katz's

relative response $=f\left\{L^{\prime},\left[S /\left(S+K_{S}\right)\right]\right\}$

$$
\mathrm{R}+\mathrm{S} \stackrel{A_{\mathrm{S}}}{\rightleftharpoons} \mathrm{RS} \stackrel{L^{\prime}}{\rightleftharpoons} \mathrm{R}^{*} \mathrm{~S} \quad \begin{aligned}
& A_{\mathrm{s}}=1 / K_{\mathrm{S}} \\
& L^{\prime}=e
\end{aligned}
$$

Figure 5.3. Formulation of the Stephenson and dC\&K reaction schemes. (A) Occupancy, stimulus, and relative response efficacy as formulated by Stephenson. (B) The dC\&K schemes were published without indication of rate constants, dissociation constant or isomerization constant. S is ligand concentration. $K_{\mathrm{s}}$ is a dissociation constant, and $e$ an efficacy constant. $A_{\mathrm{S}}$ is an association constant for $\mathrm{S}$, and $L^{\prime}$ ' an isomerization constant, equal to Stephenson's efficacy constant $e$. Note that constant $L^{\prime}$ is homologous to parameter $a \cdot L$ in the cyclic twostate model (cTSM) in Fig. 5.4. the lack of an explicit formulation for Stephenson's 'function of'.

There is a world of difference between this misconception of Stephenson's efficacy scheme due to its ambiguity, and the scheme by del Castillo \& Katz. This difference will be made even clearer by the end of subchapter 5.10 on 'operational models'.

As mentioned, the temptation to ignore the 'function of' operator in Eqs. 5.2 and 5.3 often results in equations where binding and conformational changes appear as a simple product (see Section 1.3.4). Users of the Stephenson scheme are misled by Eq. 5.3, and as mentioned in Chapter 1, Colquhoun (1998) has referred to the temptation to misinterpret Stephenson's scheme as 'Stephenson's error'. With Stephenson's error, the efficacy coefficient easily becomes identical to Ariëns 'intrinsic activity' coefficient $\alpha$ (Ariëns 1954) which it is not supposed to be (Stephenson 1956; van Rossum 1966).

From conceptually being separable in the misconceived Stephenson scheme (Furchgott 1966; Venter 1997), the system entities $y$ and $e$, insensibly, become inseparable in the del Castillo \& Katz scheme (shown later in sub-chapter 5.8 and discussed further in subchapter 5.10) (Fig. 5.3B).

\subsubsection{Formulations of the Two-step $d C \& K$ model}

The expression in Eq. 5.4 is open to many interpretations, one of which is the formulation of del CastilloKatz's reaction scheme with an intermediate receptor conformation, RS, and an additional conformation of the bound receptor in an active form, $\mathrm{R} * \mathrm{~S}$.

Although not originally formulated by dC\&K, their two-step reaction scheme may be equipped with an association constant, $A_{\mathrm{s}}$, for the first step, and governed by an equilibrium isomerization constant, $L^{\prime}$, for the second step. Thus:

$$
\mathrm{R}+\mathrm{S} \stackrel{A_{S}}{\rightleftharpoons} \mathrm{RS} \stackrel{L^{\prime}}{\rightleftharpoons} \mathrm{R} * \mathrm{~S},
$$

(Fig. 5.3B). At a glance, this dC\&K scheme may suddenly look as if we have separated binding and efficacy. Meanwhile, when equating this reaction scheme, the system constants $A_{\mathrm{s}}$ and $L^{\prime}$ become microscopic constants, i.e., they become inseparable (see Sections 5.8.1-5.8.3). Inseparable system constants are the reverse of 'Stephenson's error'.

In terms of a distribution formulation in the Langmurian sense, we can write the fraction of receptors in an active form for a dC\&K response as:

$$
\frac{\text { response }}{\text { total }}=\frac{\mathrm{R} * \mathrm{~S}}{\mathrm{R}+\mathrm{RS}+\mathrm{R} * \mathrm{~S}},
$$

and this represents the actual functional level. 
Eq. 5.5 is the expression for the simplest two-step receptor model in functional studies with ligand binding and isomerization.

In case the experiment on our lab-bench is a binding assay, involving a receptive unit that is activated due to the binding, then, assuming that the underlying receptor kinetics follow the two-step dC\&K model at equilibrium, we have:

$$
\frac{\text { bound }}{\text { total }}=\frac{\mathrm{RS}+\mathrm{R} * \mathrm{~S}}{\mathrm{R}+\mathrm{RS}+\mathrm{R} * \mathrm{~S}}
$$

for the fraction of receptors in a bound form.

Compare Eqs. 5.5. and 5.6 and observe the difference between response in functional experiments and the amount of bound receptors in binding experiments.

We shall scrutinize the consequences of these two equations, but before that, let me introduce the distribution formulation for reaction scheme 5 by Katz and Thesleff (K\&T5), shown in Fig. 5.1C, transcribed in Fig. 5.2C, and reconfigured to the cTSM in Fig. 5.4

\subsection{The Cyclic Two-state Model (cTSM)}

\subsubsection{Katz \& Thesleff's Reaction Scheme 5}

In 1957, a few months after del Castillo and Katz published their simple two-step model, Bernhard Katz and a young visiting Swede, Stephen Thesleff, put out a model for the desensitization process at nicotinic receptor sites in the neuro-muscular junction of skeletal muscle, their reaction scheme 5 (K\&T5) (Figs. 5.1C, 5.2C, or Fig. 5.4) (Katz \& Thesleff 1957). In this model, Katz and Thesleff extended the del Castillo and Katz model to include the thermodynamically, fully reversible, reaction scheme of a two-state receptor model.

$\mathrm{K} \& \mathrm{~T} 5$ is a milestone model in receptor theory. Tacitly, this scheme expresses reciprocity, i.e., if binding affects activation, then activation affects binding. Furthermore, a consequence of a reaction scheme with complete reversibility is that one of the receptor conformations must be an unbound active form, $\mathrm{R}^{*}$. This is the same as spontaneous activity! A similar model for enzymes was published a year later (Fig. 5.1D) (Botts \& Drain 1958).

Since the model by Katz and Thesleff involve desensitization, I shall refer to similar models describing activation rather than the time-dependent desensitization as cyclic two-state models (cTSM) (Fig. 5.4). There was no direct equal base for the birth of the two cTSMs above. One was for activation and desensitization of ion channels and the other was related to product formation by enzymes. However, one might argue that the time was

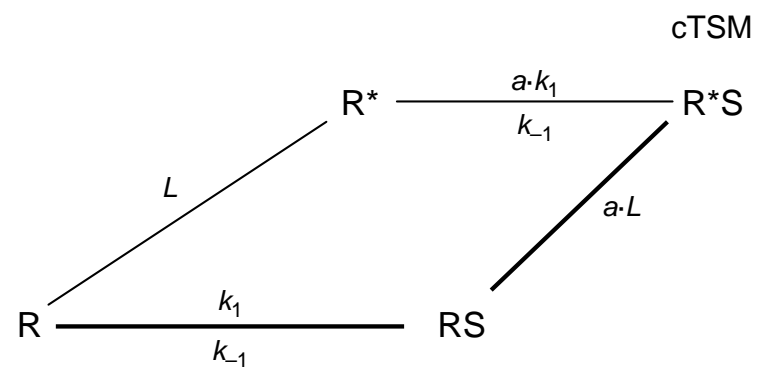

$A_{\mathrm{s}}=k_{1} / k_{-1}=1 / K_{\mathrm{s}} \quad A_{\mathrm{s}}{ }^{\prime}=a \cdot A_{\mathrm{s}}, \quad L^{\prime}=a \cdot L$

Figure 5.4. Reaction scheme for the cyclic two-state model (cTSM). The reaction paths are indicated with rate constants, association and dissociation constants, and isomerization constants.

ripe for their simultaneous emergence (see Section 5.6.1).

Compare the cTSM in Fig. 5.2C with a simplified version of the Monod-Wyman-Changeux (MWC) model in Fig. 5.2D. This latter model does not comprise complete cyclic reversibility. The MWC model is described in detail in Chapters 14 and 15.

\subsubsection{Equating the cTSM}

That an active state of the un-liganded receptors certainly exists has, to put it mildly, burgeoned in recent years in pharmacology (see literature cited in subchapters 5.1, 5.2, 5.4, and 5.5). In the cTSM there are four receptor conformations, and the distribution equations based on the conservation principle for this reaction scheme is therefore given for functional studies as:

$$
\frac{\text { response }}{\text { total }}=\frac{\mathrm{R}^{*}+\mathrm{R} * \mathrm{~S}}{\mathrm{R}+\mathrm{RS}+\mathrm{R}^{*}+\mathrm{R} * \mathrm{~S}},
$$

and for binding studies as:

$$
\frac{\text { bound }}{\text { total }}=\frac{\mathrm{RS}+\mathrm{R} * \mathrm{~S}}{\mathrm{R}+\mathrm{R} *+\mathrm{RS}+\mathrm{R} * \mathrm{~S}} .
$$

Again, notice the difference between Eqs. 5.7 and 5.8 for the two types of experiment, functional versus occupancy.

We will now analyze the consequences of $\mathrm{dC} \& \mathrm{~K}$ and cTSM and plot them in graphs (Sections 5.8.3-5.8.5). But, first, I must define some of the constants in the reaction schemes in Figs. 5.3B and 5.4. 


\subsection{Constants, Formulations, and Plots of dC\&K and cTSM}

\subsubsection{Constants and Receptor Conformations of the $d C \& K$ and $C T S M$}

There are three independent system constants in the cTSM (Fig. 5.4). In the following derivation of equations for the dC\&T and the cTSM, we will operate with both dissociation and association constants as illustrated in Figs. 5.3 and 5.4. The reason for this redundancy is given in the introduction to Part II, II.5.

In the terminology of this text, the three system constants of the CTSM are:

$A_{\mathrm{s}}$ an equilibrium association constant for ligand binding to non-active receptive unit. $A_{\mathrm{s}}$ is an equilibrium 'forward' constant for a bi-molecular process.

$L$ an isomerization constant representing the ratio between the activated unbound state of the receptive unit, $\mathrm{R}^{*}$, and the non-active and unbound state of the receptive unit; $L=\mathrm{R} * \mathrm{R}$. $L$ is an equilibrium 'forward' constant for a mono-molecular process. ${ }^{3}$

$a$ an intrinsic efficacy constant at equilibrium as a ratio of bound conformations times the ratio of unbound conformations, $\left\{\mathrm{R}^{*} \mathrm{~S} / \mathrm{RS}\right\} \cdot\left\{\mathrm{R} / \mathrm{R}^{*}\right\}$ or an intrinsic association constant as a ratio of activated conformations times the ratio of reactive conformations of the receptive unit at equilibrium, $\{\mathrm{R} * \mathrm{~S} / \mathrm{R} *\} \cdot\{\mathrm{R} / \mathrm{RS}\}$ (see Fig. 5.4).

In the literature on the cTSM, the three constants above may be expressed in different ways:

$K_{\mathrm{s}}$ the dissociation constant for the binding of an agonist and equal to the reciprocal association constant, $1 / A_{\mathrm{s}} . K_{\mathrm{s}}$ is an 'equilibrium backward' constant for a bi-mono-molecular process.

$A_{\mathrm{s}}{ }^{\prime}$ an equilibrium association constant when the receptive unit is already activated. $A_{\mathrm{s}}^{\prime}$ is an equilibrium 'forward' constant for a bi-molecular process when receptive units are in an activated state. Thus $A_{\mathrm{s}}^{\prime}$ is equal to $a \cdot A_{\mathrm{s}}$

$K_{\mathrm{s}}^{\prime}$ the dissociation constant for the binding of an agonist when the receptive unit is in an activated state and equal to the reciprocal association constant, $1 / A_{\mathrm{s}}^{\prime}$. Thus $K_{\mathrm{s}}^{\prime}=K_{\mathrm{s}} / a$. The $K_{\mathrm{s}}^{\prime}$ is an equilibrium 'backward' constant for a bi-monomolecular process.

\footnotetext{
$L=\mathrm{R} * / \mathrm{R}$ may be compared with Monod-Wyman-Changeux's allosteric constant, $L$ (Fig. 15.3). Both can be seen as identical, although in MWC's terminology, $L$ is a reverse isomerization constant of the $L$ used here. The $L$ of MWC which is also for un-liganded receptive units is equal to $\mathrm{R} / \mathrm{R} *$ or in their terminology $\mathrm{T}_{0} / \mathrm{R}_{0}$ (Monod et al. 1965). To distinguish between the $L$ symbols, the MWC $L$ is designated $L^{\mathrm{MWC}}$ (see Chapters 14 and 15).
}

$L^{\prime}$ an isomerization constant representing the ratio between the activated bound state and the reactive, not yet active, but bound state of the receptive unit, $L^{\prime}=\mathrm{R} * \mathrm{~S} / \mathrm{RS}$. Also $L^{\prime}=a \cdot L$. The $L^{\prime}$ is an equilibrium 'forward constant' for a mono-molecular process. This constant is directly related to the isomerization constant of the del Castillo \& Katz reaction scheme (Fig. 5.3B), as well as to the efficacy constant in Stephenson's scheme (Fig. 5.3A). ${ }^{4}$

Consequently, the parameter $a$ is also the ratio between the two dissociation constants, $K_{\mathrm{s}} / K_{\mathrm{s}}^{\prime}$, between the two isomerization constants, $L^{\prime} / L$, and the ratio between the two association constants $A_{\mathrm{s}}^{\prime} / A_{\mathrm{s}}$ (Fig. 5.4).

For the four receptor conformation at equilibrium in cTSM we have:

$\mathrm{R}$

$\mathrm{RS}=\mathrm{R} \cdot A_{\mathrm{s}} \cdot \mathrm{S}$

$\mathrm{R}^{*}=\mathrm{R} \cdot L$

$\mathrm{R}^{*} \mathrm{~S}=\mathrm{R} \cdot L \cdot a \cdot A_{\mathrm{s}} \cdot \mathrm{S}$

(This receptor conformation is missing in the dC\&K)

\subsubsection{A Thermodynamic Constraint Due to Complete Reversibility}

It follows that due to thermodynamic equilibrium in the cTSM, there is a constraint on the parameters. Thus, the ratio of equilibrium constants for association must be equal to the ratio of isomerization constants, i.e., $A_{\mathrm{s}}^{\prime} /$ $A_{\mathrm{s}}=L^{\prime} / L=a$. A more detailed description of this constraint is given by Fersth (1999, pp. 126-131).

In the cTSM, apparent affinity constants are altered by a conformational change at binding, as the apparent activation constants are modulated by the binding process. The true affinity and conformational constants are microscopic constants, i.e. they cannot be separated experimentally.

For convenience we will now switch from equilibrium dissociation constants to mainly using forward association equilibrium constants, such that all system constants become forward parameters. Thus $A_{\mathrm{s}}$, or $A_{\mathrm{ss}}$, is the agonist association constant $=$ affinity constant or colloquially just 'affinity'. Subscript 'ss' indicates that the constant is for the binding at an s-site by an agonist $\mathrm{S}$ (Box 2.1). Unless needed otherwise, we will just use $A_{\mathrm{s}}$ for the agonist affinity constant at the primary binding site.

\subsubsection{Formulation of the $d C \& K$ Reaction Scheme and Plots}

To start, we reformulate the two equations for the dC\&K reaction scheme, one for binding (Eq. 5.6), and another for function (Eq. 5.5), by taking the agonist

\footnotetext{
${ }^{4}$ See Sections 1.3.4-1.3.8. In channel literature $L$ ' is equal to $\beta / \alpha$ (Gibb 1996, Hille 2001) (cf. Section 6.1.2). The constant $\beta$ is the opening rate constant while $\alpha$ is the shutting rate constant (Cymes et al. 2002).
} 
concentration in relative terms, $A_{\mathrm{s}} \cdot \mathrm{S}$, and replacing the bound and activated state $\mathrm{R}^{*} \mathrm{~S}$ by $\mathrm{R} \cdot\left(A_{\mathrm{s}} \cdot \mathrm{S}\right) \cdot L^{\prime}$. Further, we arrange the equations such that the apparent dissociation constant $=\mathrm{appK}_{\mathrm{s}}$, or $\mathrm{EC}_{50}$, can easily be extracted for both binding experiments and for functional experiments.

In accordance with these conditions, formulating the $\mathrm{dC \& K}$ reaction scheme for binding experiment (Eq. 5.6) gives us:

$$
\begin{aligned}
\frac{\text { bound }}{\text { total }} & =\frac{\mathrm{R} \cdot A_{\mathrm{s}} \cdot \mathrm{S}+\mathrm{R} \cdot A_{\mathrm{s}} \cdot L^{\prime} \cdot \mathrm{S}}{\mathrm{R}+\mathrm{R} \cdot A_{s} \cdot \mathrm{S}+\mathrm{R} \cdot A_{s} \cdot L^{\prime} \cdot \mathrm{S}} \\
& =\frac{\mathrm{S}}{\mathrm{S}+\frac{1}{A_{\mathrm{s}} \cdot\left(1+L^{\prime}\right)}}
\end{aligned}
$$

where $\mathrm{EC}_{50}$ for fractional binding is the last term in the denominator of the right hand side fraction, $\operatorname{appK}_{\mathrm{s}}=1$ / $\left(A_{\mathrm{s}} \cdot\left(1+L^{\prime}\right)\right)$ or $K_{\mathrm{s}} /\left(1+L^{\prime}\right)$. The maximal fraction of liganded receptors, $B_{\max }$, is equal to unity, as the total number of receptors becomes liganded at high $[\mathrm{S}]$.

In functional experiments for a dC\&K reaction scheme (Eq. 5.5), we get:

$$
\begin{aligned}
\frac{\text { response }}{\text { total }} & =\frac{\mathrm{R} \cdot A_{\mathrm{s}} \cdot L^{\prime} \cdot \mathrm{S}}{\mathrm{R}+\mathrm{R} \cdot A_{\mathrm{S}} \cdot \mathrm{S}+\mathrm{R} \cdot A_{\mathrm{s}} \cdot L^{\prime} \cdot \mathrm{S}} \\
& =\frac{\mathrm{S} /\left(1+1 / L^{\prime}\right)}{\mathrm{S}+\frac{1}{A_{\mathrm{s}} \cdot\left(1+L^{\prime}\right)}} .
\end{aligned}
$$

Here $\mathrm{EC}_{50}$ for fractional response in functional studies is the last term in the denominator of the right hand side fraction, equal to $1 /\left(A_{\mathrm{s}} \cdot\left(1+L^{\prime}\right)\right)$ or $K_{\mathrm{s}} /\left(1+L^{\prime}\right)$, while the maximal response $R_{\max }$ is equal to $1 /\left(1+1 / L^{\prime}\right)$.

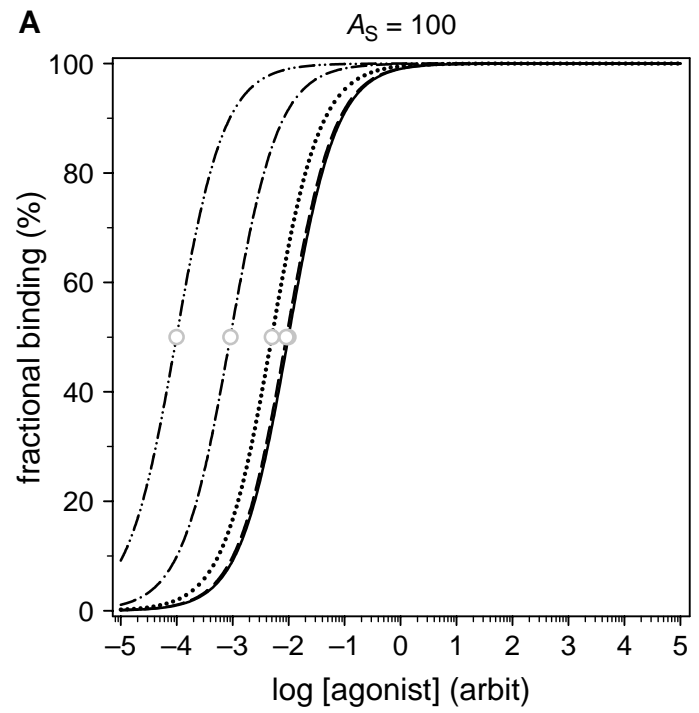

Figs. 5.5 and 5.6 are presentations of Eqs. 5.9 and 5.10, respectively. Both graphs show examples of varying constants $A_{\mathrm{s}}$ and $L^{\prime}$. Increasing the association constant $A_{\mathrm{s}}$ will move the concentration-occupancy (Fig. 5.5) and dose-effect (Fig. 5.6) curves to the left. In binding for dC\&K's model, increasing $L^{\prime}$ displaces the concentrationoccupancy curve to the left (Fig. 5.5), while in functional types for $\mathrm{dC \& K}$, increasing $L^{\prime}$ increases the relative maximal response from zero to 1 (Fig. 5.6). Compare Fig. 5.6 with Stephenson's Fig. 9 shown in Fig. 1.11B.

Recipes for drawing the figures are given in subchapter 9.1.

For certain parameter values, the binding and functional dose-responses of the dC\&K may appear identical, with the possibility of leading to erroneous conclusions about mutations in the binding site instead of the effector site in the receptive unit (Colquhoun 1998).

\subsubsection{Scheme and Formulation of cTSM Equations}

First, the reaction scheme for cTSM is presented in a drawing (Fig. 5.4). Next, by equating the reaction scheme started in Eqs. 5.7 and 5.8 and elaborated here by inserting the system constants into Eq. 5.8, we get for occupancy in cTSM:

$$
\begin{aligned}
\frac{\text { bound }}{\text { total }} & =\frac{\mathrm{R} \cdot A_{\mathrm{s}} \cdot \mathrm{S}+\mathrm{R} \cdot A_{\mathrm{s}} \cdot a \cdot L \cdot \mathrm{S}}{\mathrm{R}+\mathrm{R} \cdot A_{\mathrm{s}} \cdot \mathrm{S}+\mathrm{R} \cdot L+\mathrm{R} \cdot A_{\mathrm{s}} \cdot a \cdot L \cdot \mathrm{S}} \\
& =\frac{\mathrm{S}}{\mathrm{S}+\frac{1+L}{A_{\mathrm{s}} \cdot(1+a \cdot L)}}
\end{aligned}
$$

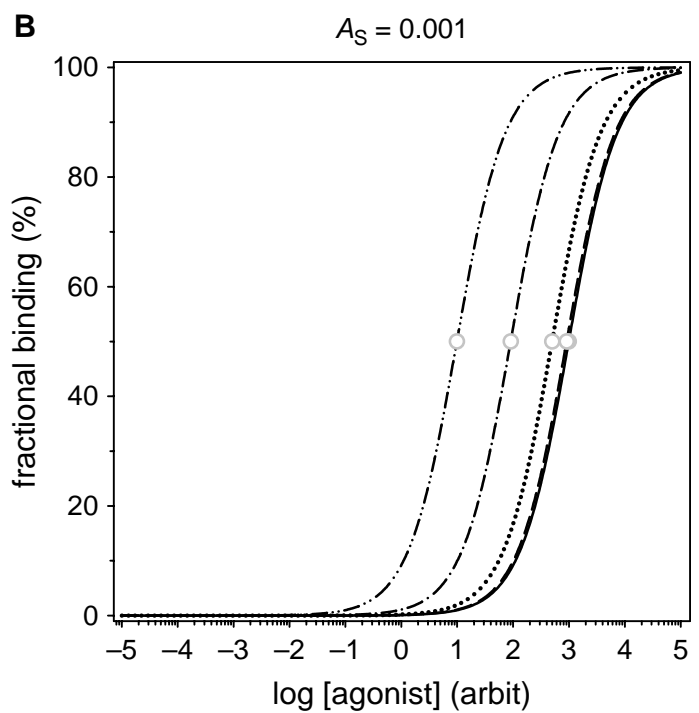

Figure 5.5. Examples of plots of binding in the dC\&K reaction scheme. The association constant $A_{\mathrm{S}}$ for binding of ligand $\mathrm{S}$ to the receptive unit is 100 in panel $A$ and 0.001 in panel $B$. The isomerization parameter $L$ 'varies in five steps from $10^{-2}(-)$ to $10^{2}(-\cdot-)$ by a factor 10 between steps. Circles indicate the $\mathrm{EC}_{50}$. 

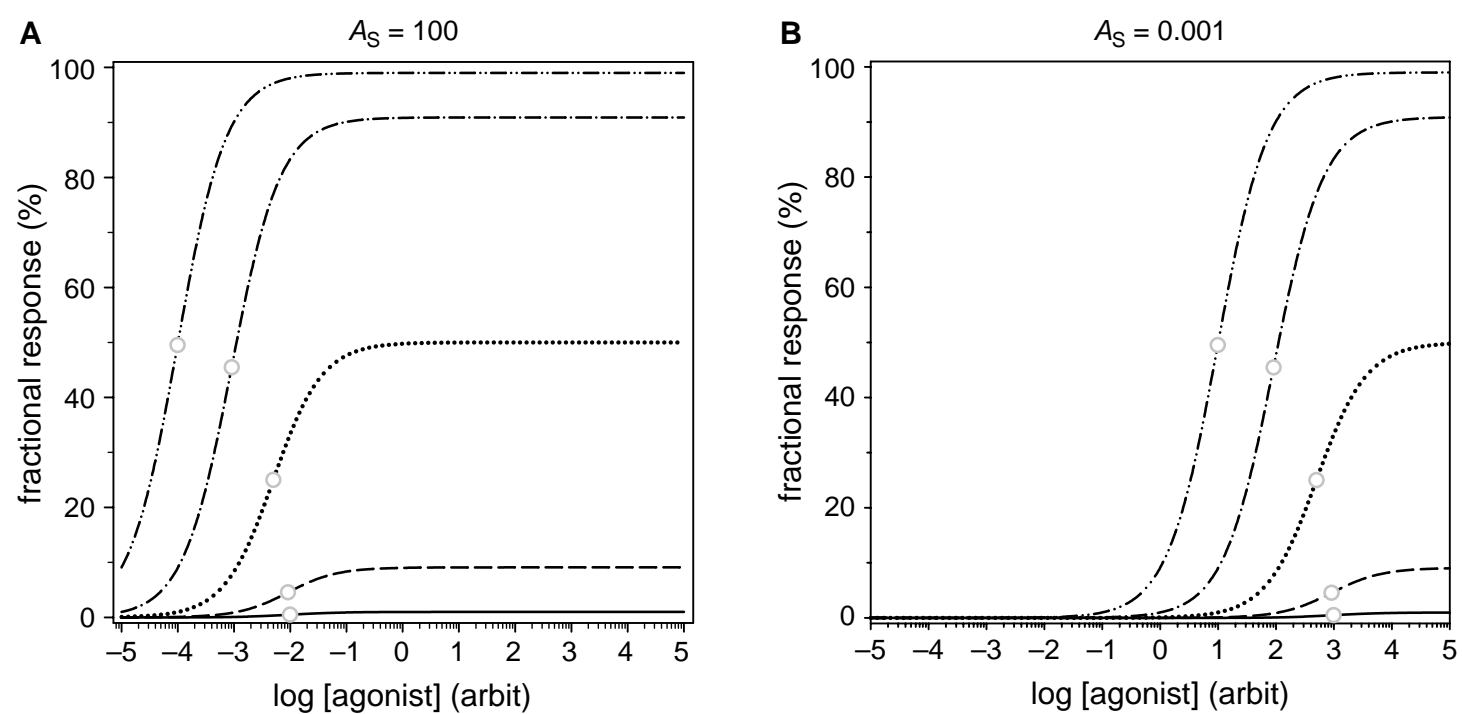

Figure 5.6. Examples of plots for function in the dC\&K reaction scheme. The association constant $A_{\mathrm{s}}$ for binding of ligand $\mathrm{S}$ to the receptive unit is 100 in panel $A$ and 0.001 in panel $B$. The isomerization parameter $L^{\prime}$ 'varies in five steps from $10^{-2}(-)$ to $10^{2}(-\cdots-)$ by a factor 10 between steps. Circles indicate the $\mathrm{EC}_{50}$. Compare this figure with Stephenson's Fig. 9 reproduced in Fig. 1.11B. Also, compare with panel A in Fig. 5.8.

and by inserting system constants into Eq. 5.7 for function in cTSM we get:

$$
\begin{aligned}
\frac{\text { response }}{\text { total }} & =\frac{\mathrm{R} \cdot L+\mathrm{R} \cdot A_{\mathrm{s}} \cdot a \cdot L \cdot \mathrm{S}}{\mathrm{R}+\mathrm{R} \cdot A_{\mathrm{S}} \cdot \mathrm{S}+\mathrm{R} \cdot L+\mathrm{R} \cdot A_{\mathrm{s}} \cdot a \cdot L \cdot \mathrm{S}} \\
& =\frac{L \cdot\left(1+a \cdot A_{\mathrm{s}} \cdot \mathrm{S}\right)}{1+A_{\mathrm{s}} \cdot \mathrm{S}+L \cdot\left(1+a \cdot A_{\mathrm{s}} \cdot \mathrm{S}\right)},
\end{aligned}
$$

or in a different form as:

$$
\frac{\text { response }}{\text { total }}=\frac{L}{L+\frac{1+A_{\mathrm{s}} \cdot \mathrm{S}}{\left(1+a \cdot A_{\mathrm{s}} \cdot \mathrm{S}\right)}}
$$

The $\operatorname{appK}_{\mathrm{s}}$ in the binding expression is the last term in the denominator of Eq. 5.11, $\operatorname{appK}_{\mathrm{s}}=(1+L) /\left(A_{\mathrm{s}} \cdot(1+\right.$ $a \cdot L))$. Formulating the last term product in the denominator in the right hand side of Eq. 5.11 with equilibrium dissociation constant $K_{\mathrm{s}}$ instead gives the appK $\mathrm{s}_{\mathrm{s}}=(1+$ $\left.\mathrm{S} / K_{\mathrm{s}}\right) /\left(\left(1+\mathrm{S} / K_{\mathrm{s}}^{\prime}\right) \cdot L\right)$

The $\operatorname{appK}_{\mathrm{s}}$ in the functional expression (Eq. 5.12), is more difficult to extract. It turns out to be the same as for the binding equation: $\operatorname{appK}_{\mathrm{s}}=(1+L) /\left(A_{\mathrm{s}} \cdot(1+\right.$ $a \cdot L)$ ), derived in Box 5.1.

After transcription of the fractional response equation for function in cTSM (Eq. 5.12) into Katz \& Thesleff's nomenclature, it becomes identical with the K\&Tequation derived for their 5 th reaction scheme for desensitization (Katz \& Thesleff 1957). Thus, by replacing $\mathrm{R}$ and $\mathrm{R}^{*}$ with $\mathrm{A}$ and $\mathrm{B}$ as in the K\&Ts nomenclature, and setting $A_{\mathrm{s}}=$ their a, $a \cdot A_{\mathrm{s}}=\mathrm{b}, L=\mathrm{k}_{4} / \mathrm{k}_{2}$, and $a \cdot L=\mathrm{k}_{1} / \mathrm{k}_{3}$, the Katz \& Thesleff equation for desensitization is obtained.
As an exercise, try to derive this $\mathrm{K} \& \mathrm{~T}$ equation on your own and obtain an in depth sensation for the thermodynamic constraints described in Section 5.8.2.

\subsubsection{Programs and Plots of cTSM Equations}

Third is programming of concentration-response relationships for cTSM in binding and function. Transcribing reaction schemes to programs for computer software is described in sub-chapter 9.3.

Fourth is plotting of the concentration-response curves for cTSM, as shown in Figs 5.7 and 5.8. Recipes for this are given in sub-chapter 9.1 .

\section{Box 5.1. Further analysis of the functional cTSM}

This box is for readers with an extended interest in details about the functional cTSM.

It has already been demonstrated that in its functional form the cTSM, as built-in, has spontaneous activity, partial agonism, and inverse agonism. In the following, we shall see how an expression may be derived for the apparent dissociation constant, $\operatorname{appK}_{\mathrm{s}}$, and the related $\mathrm{EC}_{50}$ of the functional cTSM. The influence of varying the parameters $L$ and $a$ on the appK $\mathrm{s}_{\mathrm{s}}$ and $\mathrm{EC}_{50}$ in the functional cTSM is detailed in Box 5.1.4. Relevance of ant-agonism in cTSM is briefly touched upon in Box 5.1.6, and bifurcation of signaling through an extended cTSM is mentioned in Box 5.1.7, in the form of a cyclic three-state model (Scaramellini \& Leff 2002).

The Box continues on page 133 . 

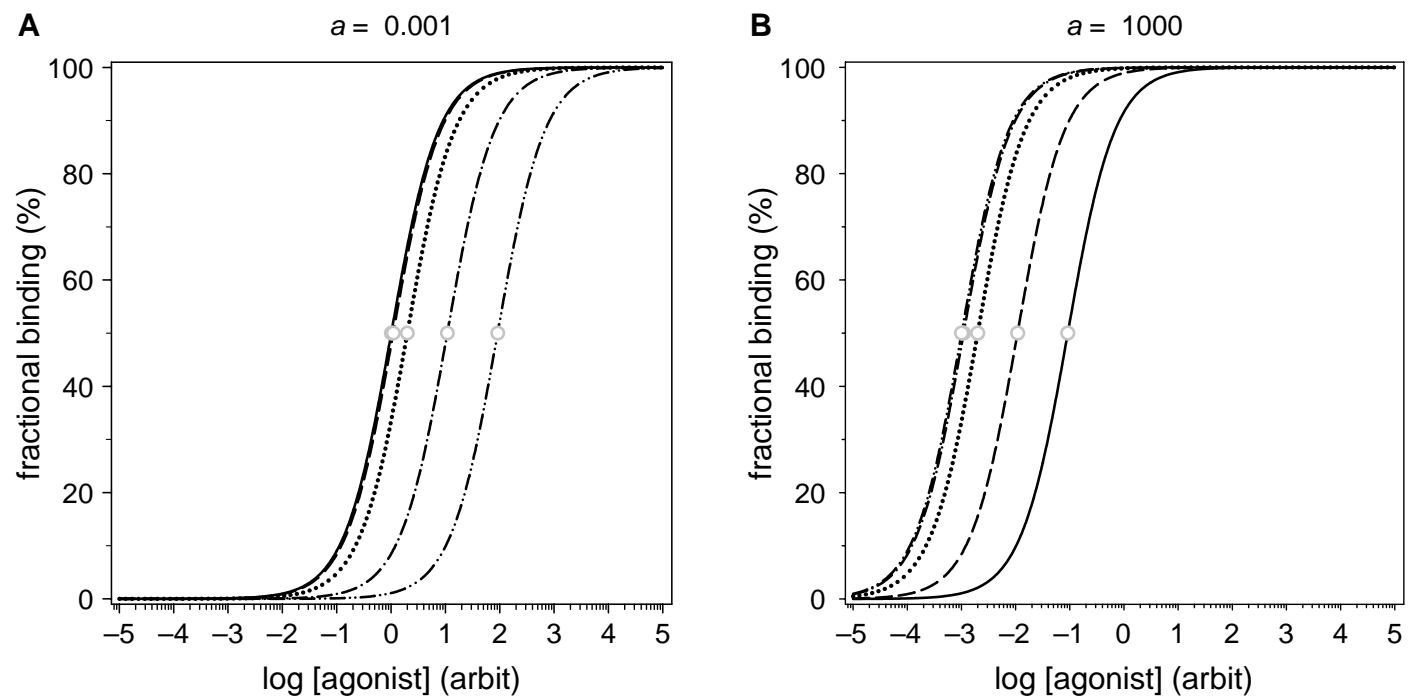

Figure 5.7. Examples of plots for the binding in the cTSM. The association constant $A_{\mathrm{S}}$ for binding of ligand $\mathrm{S}$ to the receptive unit is 1 in panels $A$ and $B$, while parameter $a$ (see Fig. 5.4), is 0.001 in panel $A$ and 1000 in panel $B$. The isomerization parameter $L$ varies in five steps from $10^{-2}(-)$ to $10^{2}(-\cdot-)$ by a factor 10 between steps. Circles indicate the $\mathrm{EC}_{50}$. Changes in $A_{\mathrm{s}}$ move the assembly of plots in a proportional manner along the concentration axis (not shown).

In Fig. 5.7 of the cTSM for binding, parameter $A_{\mathrm{s}}$ is kept constant at 1 . Changing this parameter moves the concentration-occupancy (c-o) relations in a proportional fashion along the concentration axis to the left. For parameter $a<1$, increasing $L$ displaces the c-o curves to the right (Fig. 5.7A), while for $a>1$, increasing $L$ pushes the c-o relations to the left (Fig. 5.7B).

In the functional aspect of the cTSM (Fig. 5.8), the basal response is solely dependent on parameter $L$. In Fig. $5.8 \mathrm{~A}, L$ is $1 / 1000$ and the dose-effect curve starts at near zero. For $L=1$ the initial level starts at $50 \%$ of the maximal response (Fig. 5.8B). For higher values of $L$, the initial response approximates the initial $R_{\max }$ (see Fig. 5.12).

As before, $A_{\mathrm{s}}$ moves the dose-effect curve in a proportional fashion along the concentration axis (Fig. 5.8B). With $L$ fixed, it is parameter $a$ that determines the maximal response of the functional cTSM (Fig. 5.8). The $\operatorname{appK}_{\mathrm{s}} \mathrm{s}$ described in Box 5.1 are indicated by circles at each curve.

A detail discussion of the implications of changing cTSM parameters is presented in Section 5.9.4.
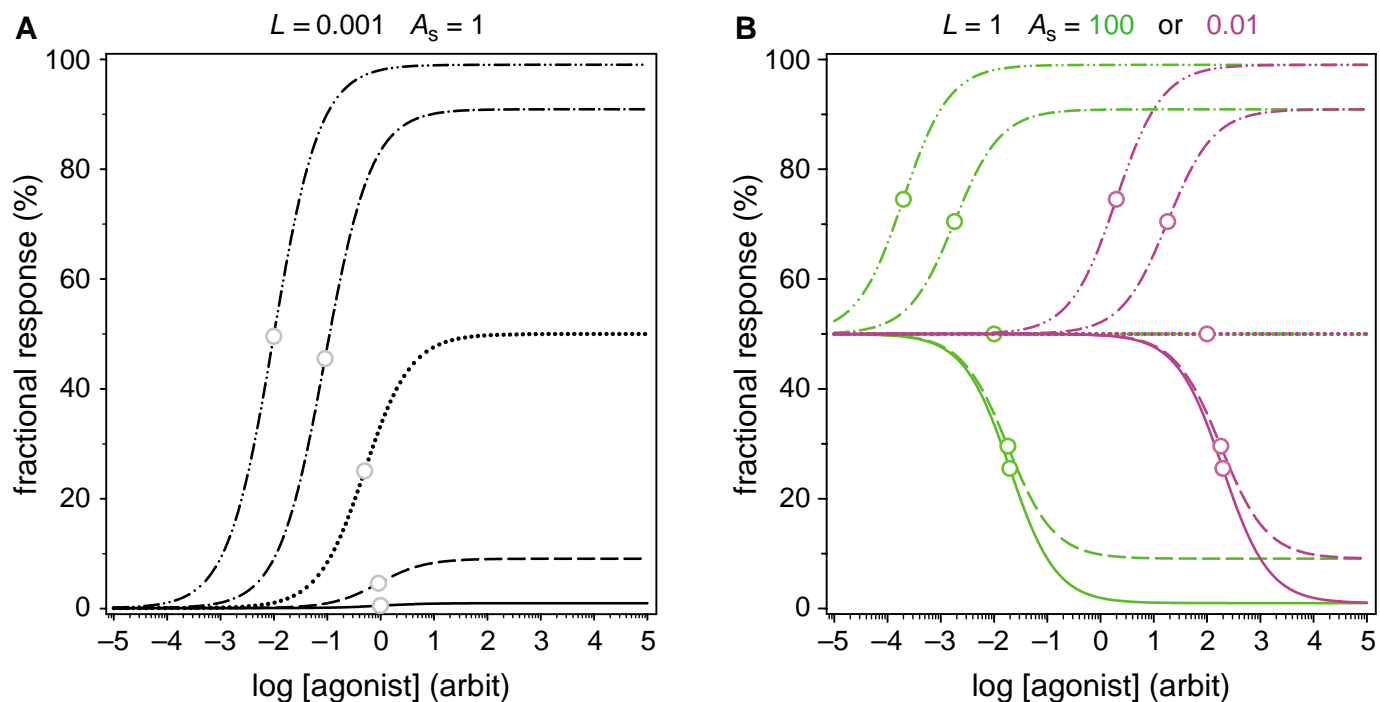

Figure 5.8. Examples of plots for function in the cTSM. Parameter $L$ is 0.001 in panel $\mathrm{A}$ and 1 in panel $\mathrm{B}$. Parameter $A_{\mathrm{s}}$ is 1 in panel $A$ and is either 100 or 0.01 in panel $B$ as indicated by color code. The five plots in both panels vary with values of parameter $a$, which changes in five steps from $10^{-2}(-)$ to $10^{2}(-\cdots-)$ by a factor 10 between steps. Circles indicate the $\mathrm{EC}_{50}$. Compare panel A with Fig. 5.6. 
Table 5.1. Occupational and functional parameters for two models, the dC\&K and the cTSM

\begin{tabular}{|c|c|c|c|c|}
\hline \multirow[b]{2}{*}{ Binding (Figs. 5.5, 5.7 and 5.11) } & \multicolumn{2}{|c|}{ dC\&K } & \multicolumn{2}{|c|}{ cTSM } \\
\hline & Fractional $B_{\max }$ for high $\mathrm{S}$ & $\mathrm{appK}_{\mathrm{s}} \dagger$ & Fractional $B_{\max }$ for high $\mathrm{S}$ & $\mathrm{appK}_{\mathrm{s}} \dagger$ \\
\hline & 1 & $1 /\left(A_{\mathrm{s}} \cdot\left(1+L^{\prime}\right)\right)$ & 1 & $\frac{1+L}{A_{\mathrm{s}} \cdot(1+a \cdot L)}$ \\
\hline \multirow{4}{*}{ Functional (Figs. 5.6, 5.8 and 5.12) } & $=0$ for $\mathrm{S} \rightarrow 0$ & - & $=0$ for $S \rightarrow 0$ & - \\
\hline & Fractional $R_{\max }$ for high $\mathrm{S}$ & $\mathrm{appK}_{\mathrm{s}} \dagger$ & Fractional $R_{\max }$ for high $\mathrm{S}$ & $\mathrm{appK}_{\mathrm{s}}^{\dagger} \dagger$ \\
\hline & $1 /\left(1+1 / L^{\prime}\right)$ & $1 /\left(A_{\mathrm{s}} \cdot\left(1+L^{\prime}\right)\right)$ & $1 /(1+1 /(a \cdot L))$ & $\frac{1+L}{A_{\mathrm{s}} \cdot(1+a \cdot L)}$ \\
\hline & $=0$ for $\mathrm{S} \rightarrow 0$ & - & $1 /(1+1 / L)$ for $S \rightarrow 0$ & - \\
\hline
\end{tabular}

$L^{\prime}$ is equal to $a \cdot L$.

tappK is the agonist concentration yielding a response midway between the level of basal activity and the maximal response. For dC\&K this midway response is (1/ $2) \cdot\left(1 /\left(1+1 / L^{\prime}\right)\right)$, while for cTSM the response is midway between $1 /(1+1 / L)$ and $1 /(1+1 /(a \cdot L))$. Expression for the appK $K_{s}$ in the functional cTSM is derived in Section 5.10 .2 , and dose-response points for $[S]=a{ }^{2} \mathrm{ap}_{\mathrm{s}}$ appears in figures where it is relevant (Figs. 5.8 and 5.12 ).

A note on the terminology: in this text we have $A_{\mathrm{s}}, L$, and $a \cdot A_{\mathrm{s}}$, while in the paper by Leff (1995) the same parameters are $1 / K_{\mathrm{A}}, 1 / L$, and $1 / K_{\mathrm{A}}{ }^{*}$. See also, e.g., Chang \& Weiss (1999), Chang et al. (2002) and Buck et al. (2004).

\subsubsection{Produce and Analyze d-r Curves for Function in CTSM}

For novices who have the time, I highly advice going through the details of the four-step exercise for the cTSM as stipulated in Sections 5.8.4 and 5.8.5. The four steps are:

1. suggesting and manipulating reaction schemes,

2. formulating their fractional response into equations,

3. writing programs for their dose-response relationships, and

4. plotting and analyzing their curves for evaluation, relevance, and possible new strategies.

To obtain a good understanding of what agonism is in the realm of TSMs, the listed exercise is paramount. Just one example of its benefits: inverse agonism at the receptor level will be very clear after handling these four steps of the functional cTSM reaction scheme. I strongly recommend it to all beginners.

Detailed recipes for involved procedures are described in Chapter 9.

\subsubsection{Summary of the del Castillo \& Katz and Katz \& Thesleff Reaction Schemes}

The system constants of maximum binding and maximum response and the $\operatorname{appK}_{\mathrm{s}} \mathrm{s}$ in studies of binding and response for both the $\mathrm{dC \& K}$ and the cTSM reaction schemes are listed in Table 5.1

Parts of the results and conclusions in Table 5.1 have also been reported by Gero (1983) ${ }^{5}$ and Leff (1995).

\footnotetext{
${ }^{5}$ Gero (1983) focusing on the desensitization mechanism missed the spontaneous activity inherent in K\&T5 by only equating the complex $\mathrm{R} * \mathrm{~S}(\mathrm{RD})$ as active, not the $\mathrm{R} *(\mathrm{R})$.
}

\subsubsection{A Comparison Between CTSM and HI-OSM}

The hetero-intervention and mutually-inclusive onestate model (HI-OSM) was presented as part of the four-pane reaction scheme (FP-OSM) (Fig. 2.4). The FP-OSM may be expanded with an additional dimension, viz. with a dimension of activated conformations for the receptive unit. The result of such an expansion is a four-pane two-state model (FP-TSM) (Fig. 5.9). To understand how the cTSM relates to the HI-OSM, it is helpful to compare the FP-OSM in Fig. 2.4 with the figure for the FP-TSM, of which the HI-OSM, the FPOSM, and the cTSM are parts (Fig. 5.10). The FP-TSM in Fig. 5.9 also covers the so-called ATSM and the HOTSM (Fig. 5.10), both detailed further in Chapter 7.

\subsection{Parameter Characterization for dC\&K and CTSM}

\subsubsection{Parameter-dependent Characterization of Concentration-occupancy in $d C \& K$}

The maximum occupancy for the dC\&K model in binding studies always reaches $100 \%$ at high ligand concentration, independent of system parameters $A_{\mathrm{s}}$ and $L^{\prime}$.

Affinity constant $A_{\mathrm{s}}$ shifts the concentration-occupancy relations in a proportional fashion, that is, for increasing $A_{\mathrm{s}}$-values there is a proportional left shift of the concentration-occupancy curves and a similar right shift as $A_{\mathrm{s}}$ decreases (Fig. 5.5). The isomerization constant, $L^{\prime}$, only moves the concentration-occupancy relations to the left as it increases (Fig. 5.5). Thus, the apparent dissociation constant is a function of both $A_{\mathrm{s}}$ and $L^{\prime}$ (Table 5.1). When the $A_{\mathrm{s}}$ is fixed at unity, appK $\mathrm{s}_{\mathrm{s}}$ is given by $1 /\left(1+L^{\prime}\right)$. Clearly as $L^{\prime}$ increases, the value of appK $\mathrm{K}_{\mathrm{s}}$ decreases identical with an increase in apparent affinity. For small values of $L^{\prime}, \operatorname{appK}_{\mathrm{s}}$ is equal to $1 / A_{\mathrm{s}}$ (Fig. 5.5). 


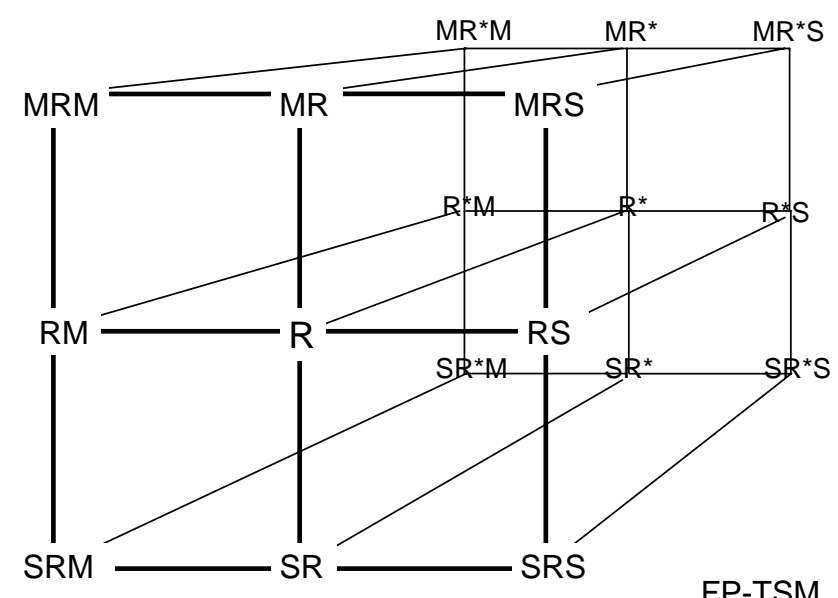

Figure 5.9. Reaction scheme for the four-pane two-state model (FP-TSM). Symbol 'M' stands for a bound modulator ligand. The four-pane scheme in front is a one-state model (FPOSM), similar to the FP-OSM in Fig. 2.4 in which the modulator symbol ' $M$ ' is replaced with the interventor symbol ' $l$ '.

\subsubsection{Parameter-dependent Characterization of Dose-response in dC\&K}

In the functional scheme of $\mathrm{dC} \& \mathrm{~K}$, the maximum response is only dependent on parameter $L^{\prime}$, while the apparent affinity constant $\operatorname{appK}_{\mathrm{s}}$ is dependent on both system constants $L^{\prime}$ and $A_{\mathrm{s}}$ (Table 5.1). As for the binding studies, affinity constant $A_{\mathrm{s}}$ shifts the concentration-activity relations in a proportional fashion (Fig. 5.6) and again as $L^{\prime}$ increases the $\operatorname{appK}_{\mathrm{s}}$ decreases, thus left-shifting the dose-response curves and increasing the apparent affinity (Fig. 5.6A+B, compare Section 5.9.1).

The initial activity level with no ligand present is zero. The level of maximum response is given by $1 /$ $\left(1+\left(1 / L^{\prime}\right)\right)$, obtained as the concentration of agonists reaches high values. This second level increases with increasing $L^{\prime}$, yielding $50 \%$ of the possible $100 \%$ maximum when $L^{\prime}=1$ (dotted curves in Fig. 5.6A+ B). Since both the apparent affinity and the fractional maximum increase with increasing values of $L^{\prime}$, there is a spree of apparent affinities from a value equal to $A_{\mathrm{s}}$ at near-zero activity for $L<<1$ to constantly increasing values of the apparent affinity at $50 \%$ of maximum activity as $L^{\prime}$ increases (see left-most curves in Fig. 5.6A+B). As mentioned, the apparent affinity is dependent on both $L^{\prime}$ and $A_{\mathrm{s}}$. For $A_{\mathrm{s}}$ fixed at unity, it is easy to see that increasing $L^{\prime}$ from zero to unity lifts the apparent affinity from $A_{\mathrm{s}}$ by a factor of 2, while the apparent affinity increases unlimited as $L^{\prime}$ increases above unity (Fig. 5.6A+B).
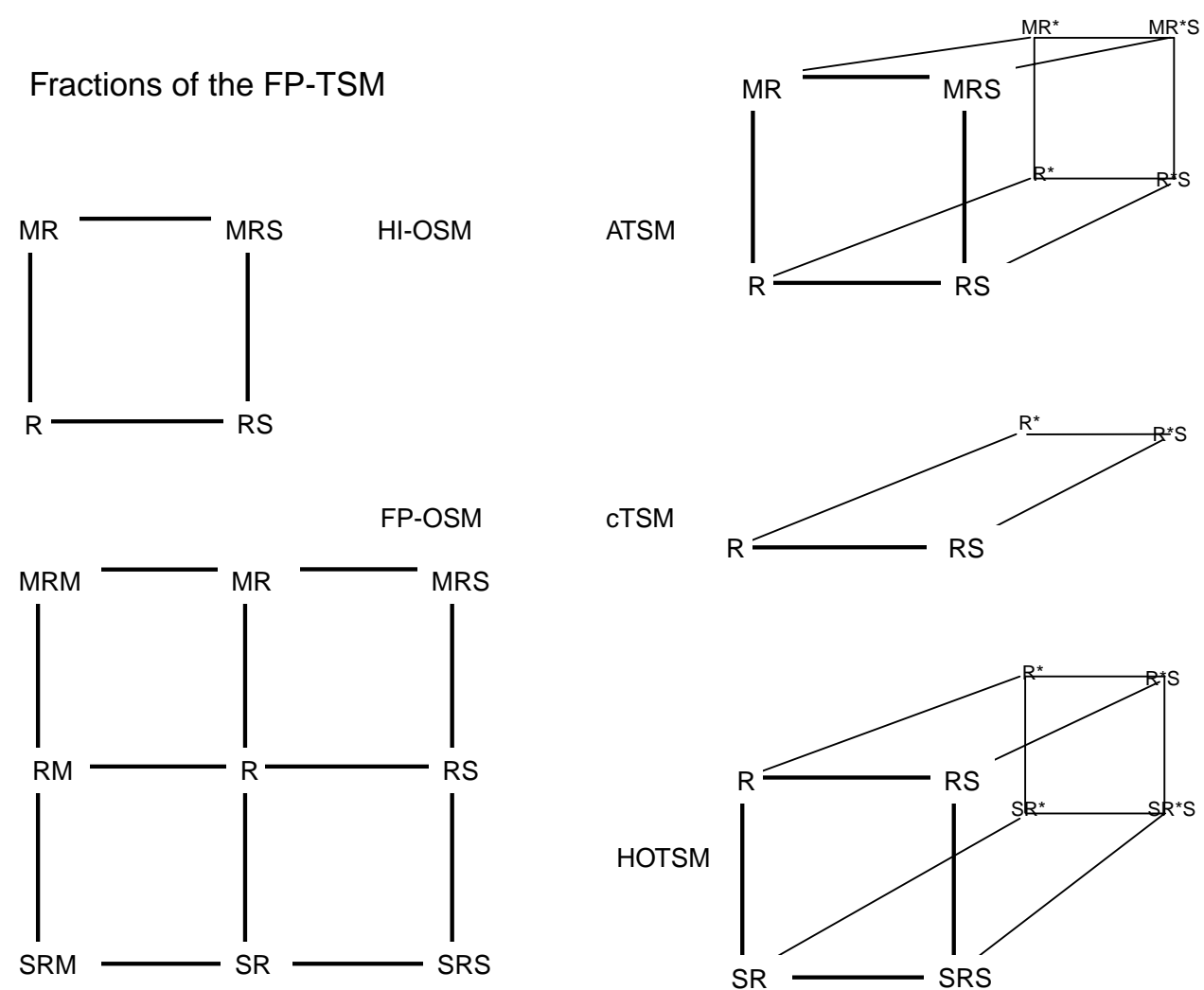

Figure 5.10. Parts of the FP-TSM in Fig. 5.9. The partial models are: the ternary-complex model (TCM), the four-pane one-state model (FP-OSM), the cyclic two-state model (CTSM), the allosteric two-state model (ATSM), and the homotropic two-state model (HOTSM). The 'M' symbol in TCM and FP-OSM may be replaced with interventor symbol 'l'. 
Furthermore, notice the similarities between the dependence on $L^{\prime}$ of dose-responses for this $\mathrm{dC \& K}$ model (Figs. 5.3B and 5.6) with the dependence of doseresponses on the efficacy constant $e$ in the hypothetical efficacy model by Stephenson (see Section 1.3.5, Figs. $5.3 \mathrm{~A}$ and 1.11B). In fact, Stephenson efficacy constant $e$ may be compared directly with constant $L^{\prime}$ of the functional dC\&K (Figs. 5.3A + B).

Compare also the effects here of varying $L^{\prime}$ on the apparent affinity with the effect of varying $L^{\prime}(=a \cdot L)$ on the apparent affinity in the cTSM in Section 5.9.4 (Fig. 5.8). For the cTSM it is only the system constant $a$ that has the same function as $L^{\prime}$ for the dC\&K regime. This is discussed further in Section 5.9.5.

\subsubsection{Parameter-dependent Characterization of Concentration-occupancy in CTSM}

Similar to the dC\&K scheme as indicated in Section 5.9.1 and Table 5.1, the maximum occupancy in the cTSM at high ligand concentration always reaches $100 \%$, independent of values for the three system constants, $A_{\mathrm{s}}$, $L$ and $a$ (Fig. 5.7).

Conversely, the apparent dissociation constant is dependent on all three parameters and given by $\operatorname{appK}_{\mathrm{s}}=(1+L) /\left(A_{\mathrm{s}} \cdot(1+a \cdot L)\right) \quad($ Table 5.1$)$. This is demonstrated in Figs. 5.7 and 5.11 for changes in parameters $a$ and $L$. The concentration-occupancy curves are shifted proportionally to the left or right with changes in parameter $A_{\mathrm{s}}$. This is not observed when $A_{\mathrm{s}}$ is kept at 1 in Figs. 5.7 and 5.11.

Now all three constants are intrinsic or microscopic constants. Since $A_{\mathrm{s}}, L$ or $a$ cannot in principle be

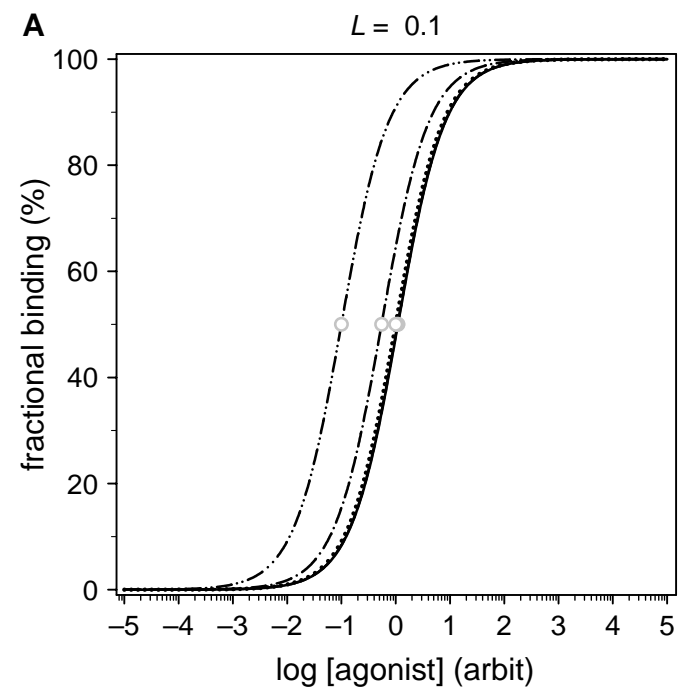

measured separately (sub-chapter 5.11), they are 'microscopic' constants.

For parameter $a<1$, increasing the value of $L$ gives a non-proportional right-shift in the concentrationoccupancy (c-o) curves with appK moving from $1 / A_{\mathrm{s}}$ towards $1 /(a \cdot A \mathrm{~s})$ (Fig. 5.7A). For $a>1$, increasing $L$ results in a left-shift of the c-o curves with appK moving from $1 / A_{\mathrm{s}}$ towards $1 /(a \cdot A \mathrm{~s})$ (Fig. 5.7B). On the other hand, for all values of $L$, increasing $a$ only drives the c-o curves to the left (Fig. 5.11A+B), in accordance with $\operatorname{appK}_{\mathrm{s}}=1 /\left(a \cdot A_{\mathrm{s}}\right)$. Therefore, when $L$ is fixed, the apparent affinity increases with increasing values of $a$; confer with the expression for binding appK $\mathrm{s}_{\mathrm{s}}$ in 5.1.

\subsubsection{Parameter-dependent Characterization of Dose-response in cTSM}

Dose-response curves for the cTSM in its functional version have two varying levels of activity.

The initial or first level of activity (FL) with no ligand present is solely dependent on the value of parameter $L$. Thus, response at the first level is given by $1 /(1+(1 / L))$ (Table 5.1 and Figs. 5.8 and 5.12). This initial level represents the spontaneous activity of the non-stimulated system. For an isomerization constant equal to unity, $L=1$, the spontaneous activity is equal to $50 \%$ of the potentially possible $100 \%$ activity (Figs. 5.8B and 5.12).

The second level of activity (SL) at high concentration of a ligand is the maximal response level for that particular ligand and given by $1 /(1+(1 /(a \cdot L)))$ (Table 5.1 and Fig. 5.12). Note, $L$ and $a$ are both dependent on the receptor and the ligand. The intrinsic association constant $A_{\mathrm{s}}$ merely shifts the dose-responses proportionally to the left or right along the concentration axis

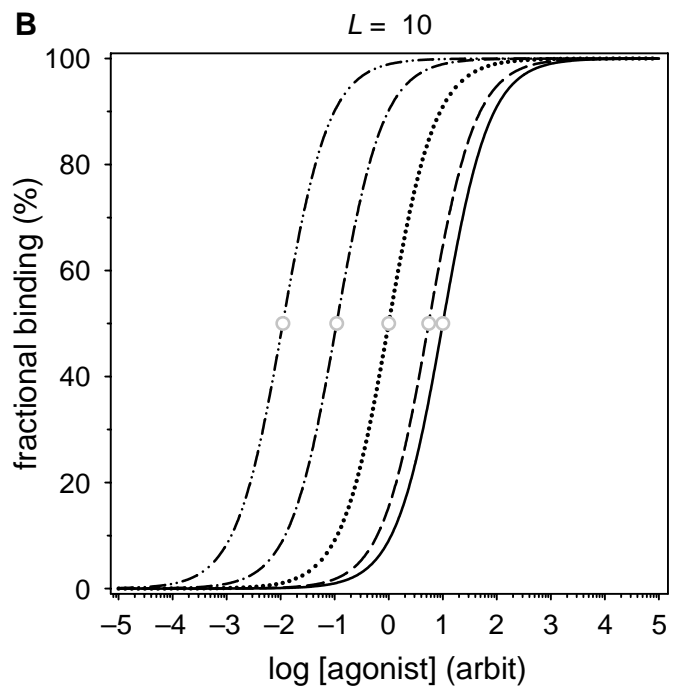

Figure 5.11. Binding aspects of CTSM. Effects of varying parameter $a$ in 5 steps from $10^{-2}(-)$ to $10^{2}(-\cdot-)$ by a factor 10 between steps. $A_{\mathrm{s}}=1$ while parameters $L$ as indicated $=0.1$ in panel $\mathrm{A}$ and 10 in panel $\mathrm{B}$. Circles indicate the $\mathrm{EC}_{50}$. Compare with Figs. 5.5 and 5.7 . 

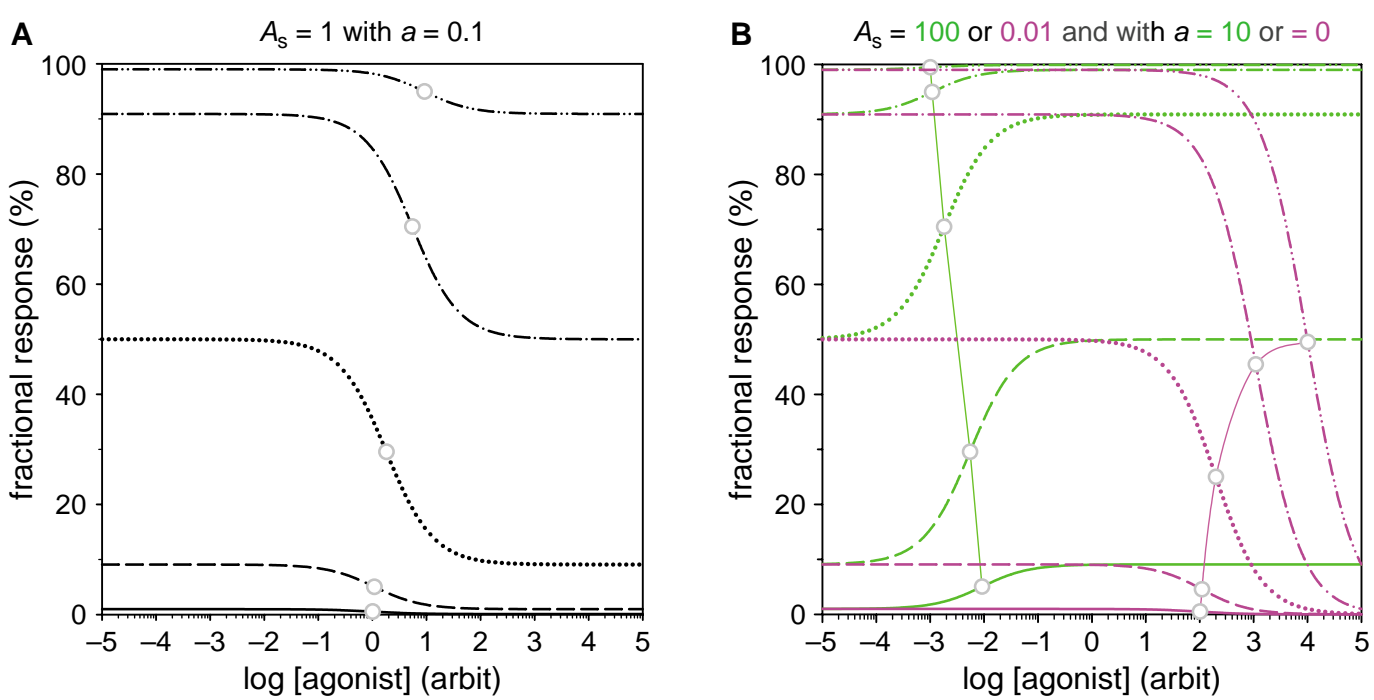

Figure 5.12. Functional aspect of the cTSM. Panels $A$ and $B$ show the effects of varying parameter $L$ in five steps from $3 \times 10^{-1}$ $(-)$ to $3 \times 10^{3}(-\cdots-)$ by a factor 10 between steps. (A) Parameter $A_{\mathrm{s}}$ is 1 and $a$ is 0.1 . (B) Two situations are overlaid. Parameters $A_{\mathrm{s}}$ is either 100 or 0.01 and $a$ is either 0.01 or zero as indicated by color code. For a equal zero only the $\mathrm{R}^{\star}$ can bind ligand $\mathrm{S}$ (see Fig. 5.4). Circles indicate the $\mathrm{EC}_{50}$. Compare with Figs. 5.6 and 5.8.

(Fig. 5.8B). SL is not dependent on the intrinsic affinity constant $A_{\mathrm{s}}$. Higher values of $A_{\mathrm{s}}$ means higher affinities, thus moving dose-response curves unchanged to the left (Fig. 5.8B). Similar to $L$ and $a, A_{\mathrm{s}}$ is also dependent on both the receptor and the ligand.

Comparing the minimum response at the first level with the maximum response at the second level in the functional version of the cTSM (Table 5.1) demonstrates that the difference between the two levels is determined by parameters $a$ and $L$ (Fig. 5.12). Thus, $L$ may change both FL and SL (Fig. 5.12). However, this also means that the second 'maximum' response level (SL) due to a high ligand concentration is above, at, or below the initial 'spontaneous' response level (FL) exclusively determined by $a$, as this parameter varies from above to below unity (Figs. 5.8 and 5.12). Therefore, for $a>1$, the second level is above the initial level, for $a=1$ : SL $=\mathrm{FL}$, and for $a<1$ the 'maximum' level SL is below the spontaneous level FL (Figs. 5.8 and 5.12).

This functional behavior of the cTSM due to variations in system constant $a$ is exactly related to the behavior of agonists, ant-agonists, and inverse agonists. Thus, if a ligand increases the second level to above the spontaneous level of activity, $a>1$, we are dealing with an agonist (by definition). If after the addition of a ligand, the two levels are identical with no change in response, $a=1$, but the compound changes the response induced by agonists, then it is an antagonist (neutral ant-agonist by definition). Third, when the second level elicited by a ligand is below the spontaneous activity, $a<1$, the ligand is an inverse agonist (per definition).

\subsubsection{Comparison Between the cTSM and the dC\&K Models}

By inspecting the formulations for maximum response and apparent affinity in the functional models of dC\&K and cTSM (Table 5.1) and by inspecting their reaction schemes (Figs. 5.3B and 5.4 and Section 5.6.3), realize that parameter $L^{\prime}$ in the $\mathrm{dC} \& \mathrm{~K}$ model essentially corresponds to the $a \cdot L$ parameter of the cTSM. The major difference between the two models is the initial level of response. When the agonist is absent, the initial level in dC\&K's model is zero, while in cTSM it is dependent on $L$ and recognizable for values of $L$ above $1 / 100$. Therefore, functional dose-response relations for the two models may resemble each other when parameter $L$ in the cTSM has a low value, thus suppressing the first level of response for the cTSM towards zero. Additionally, in case values of $L^{\prime}$ and $a \cdot L$ are kept identical with $L<<1$, we will observe that dose-response curves for the two models are congruent (Figs. 5.6 and $5.8 \mathrm{~A})$. Furthermore, for small values of $L$, the conclusions about the apparent dissociation constant, appK both for the binding and functional response of the dC\&K model also holds for the cTCM as long as the product $a \cdot L$ of cTSM is close to $L^{\prime}$ of the dC\&K reaction scheme (see Table 5.1). For the functional versions of the two models, there will be a spree of appK $\mathrm{K}_{\mathrm{s}}$ constants as the system constant $a$ for cTSM and $L^{\prime}$ for the dC\&K increase. Parameter $a$ for the cTSM has taken over the effects of the $L^{\prime}$ constant on $\operatorname{appK}_{\mathrm{s}}$ of the dC\&K reaction scheme (compare Figs. 5.6 and 5.8A). The differences between the two schemes enter when $L$ is 
not significantly less than unity. For $L$ above $1 / 100$, the response level begins to differ between the two reaction schemes for values of concentration for the ligand at appK $_{\mathrm{s}}$. Thus, although the two maximum levels are comparable, that is $1 /\left(1+\left(1 / L^{\prime}\right)\right)$ is comparable to $1 /$ $(1+(1 /(a \cdot L)))$, by keeping $a \cdot L$ nearly equal to $L^{\prime}$, the initial levels are now different, zero for the dC\&K versus $1 /(1+(1 / L))$ for the cTSM (Figs. 5.6 and 5.12). For $L<<1$, i.e., $\mathrm{R}^{*}$ near zero, then the cTSM actually becomes a dC\&K model (confer with reaction schemes in Figs. 5.3B and 5.4). Therefore, for $L<<1$ the cTSM also describes the Stephenson model in Section 1.3.5 (compare Figs. 5.8A and 1.11B).

\subsubsection{Extraction of Parameter Values from the Functional CTSM}

In functional systems where it is possible to record the maximal level of response in absolute values and simultaneously measure a spontaneous activity that is significantly different from zero, it will be possible to determine the $L$ and $a$ system constants. Therefore, with $L$ and $a$ obtained, the system is left with one independent variable to be assessed, parameter $A_{\mathrm{s}}$. Since $A_{\mathrm{s}}$ only vary the apparent affinity, these parameters may be extracted for added ligands by mutual comparison of their dose-response relationships. Thus, all three parameters may be assessed in such systems when analyzing experimental data. This sounds captivating, but the requirement is knowledge of both $L$ and $R_{\max }$ in absolute terms, which is not possible to obtain for most physiological systems. What is more, these studies may also require tests for spare receptors to be excluded by alkylating agents before an analysis for $A_{\mathrm{s}}$ and $a$ can be undertaken.

In order to separate the microscopic constants of isomerization and affinity, $L$ and $A_{\mathrm{s}}, L$ should be $<1$. If values of the isomerization constant $L$ are $>1$, it will be difficult to separate the microscopic constants. Binding of ACh to nAChRs inducing conformational changes is such a process. This process has $L$ values well above 30 , preventing the separation of $A_{\mathrm{s}}$ and $L$ (Leff 1995; Colquhoun 1998).

\subsubsection{Extraction of Parameter Values from the Functional $d C \& K$}

It will be possible to extract relative parameter values for $A_{\mathrm{s}}$ and $L^{\prime}$ based on dose-response profiles of full and partial agonists from the functional form of the dC\&K model, where there is no spontaneous activity level, but for which a maximum level $R_{\max }$ can be established as an absolute value.

\subsubsection{What is a Pure Binding Effect and a Pure Efficacy in Terms of Mechanisms?}

One may question if it is possible to vary a single parameter in the functional or binding regime of either the dC\&K model or the cTSM, that will only affect either affinity, i.e., potency, or efficacy (Colquhoun 1998). In the binding regime of the two models, both $A_{\mathrm{s}}$ and $L^{\prime}$, or $L \cdot a$, affect the apparent affinity (potency), without changes in relative efficacy, the $B_{\max }$. All parameters are thus involved in the 'potency' of binding, while the 'efficacy' of binding is independent of variations in either parameter (Table 5.1).

From Table 5.1 we can also draw conclusions about the functional regimes. Thus, in the functional theory of both models, $A_{\mathrm{s}}$ is a pure parameter for apparent affinity variations, but not the only parameter affecting affinity (Table 5.1). Variations in both $L^{\prime}$ and $L \cdot a$ also affect the apparent affinity. Both $L^{\prime}$ and $L \cdot a$ are concurrently involved in the occupancy and effect level. Therefore, pure binding effects may be seen by varying $A_{\mathrm{s}}$ solely, while, due to parameter variations without affinity effects, pure efficacy effects are impossible.

\subsubsection{Extraction of Parameter Values from Binding Studies. Hemoglobin $(\mathrm{Hb})$ as an Example}

Hemoglobin has two states, a deoxy-state equal to the tense (T-) state, which is comparable to the reactive state of other receptors, and an oxy-state equal to the relaxed (R-) state which may be characterized as an 'active' state. These two states are $T_{0}$ and $\mathrm{R}_{0}$ when $\mathrm{Hb}$ is un-liganded. Comparable terms for $\mathrm{Hb}$ and other receptive units are listed in Table 5.2.

Below is a survey of the four steps of $\mathrm{O}_{2}$ binding to hemoglobin interpreted in a two-step model, the dC\&K. In pure binding experiments, increased 'efficacy' is part of an isomerization to a higher 'affinity' state.

The constants have been measured (Roughton et al. 1955; Edelstein 1975) and represent apparent affinities or efficacies (Colquhoun 1998). From binding of the first $\mathrm{O}_{2}$ molecule, each binding step increases by a factor of about 70-fold (Colquhoun 1998). The total change in apparent binding affinity is thus $70^{3}$, some $3.5 \times 10^{5}$-fold. In reality, it is merely 300 -fold (Table 5.3) (Edelstein 1975).

Table 5.2. Symbols for the un-liganded conformations of twostate models

\begin{tabular}{lll}
\hline $\begin{array}{l}\text { Two-state terminology } \\
\text { 'to have identity, } \\
\text { is to be alienated' }\end{array}$ & \multicolumn{1}{c}{ 'Reactive' } & 'Active' \\
\hline $\begin{array}{l}\text { Receptors } \\
\text { un-liganded }\end{array}$ & $\mathrm{R}$ (non-deformed) & $\mathrm{R}^{*}$ (deformed) \\
$\begin{array}{l}\text { Hemoglobin } \\
\text { un-liganded enzymes }\end{array}$ & $\mathrm{T}_{0}$ (tense) & $\mathrm{R}_{0}$ (relaxed) \\
\hline
\end{tabular}


Table 5.3. The relative affinity constants for $\mathrm{O}_{2}$ binding to hemoglobin

\begin{tabular}{lcc}
\hline No. of bound $\mathrm{O}_{2}$ molecules & Constants & $\begin{array}{c}\text { Enhancement factor } \\
\text { (relative affinity) }\end{array}$ \\
\hline $\begin{array}{l}\text { First oxygen molecule binds } \\
\text { Second oxygen molecule binds }\end{array}$ & $A_{\mathrm{so}}$ & 1 \\
Third oxygen molecule binds & $A_{\mathrm{so}}^{\text {mo }}$ & 4.7 \\
Fourth oxygen molecule binds & $A_{\mathrm{s}}^{\text {mooo }}$ & 7.9 \\
\hline The association constant $A_{\mathrm{so}}=1 / K_{\mathrm{s}}$ for binding of the first $\mathrm{O}_{2}$ molecule. For the \\
other association constants, see Box 2.1. \\
\\
$\begin{array}{l}\text { 5.10. The General Embedded Load Model. } \\
\text { 'The Operational Model' }\end{array}$ \\
\hline
\end{tabular}

Here I compare the del Castillo and Katz (1957) model with the so-called operational model by Black and Leff (1983).

\subsubsection{Comparison Between the dC\&K Model and the B\&L Model}

In order to understand the arguments in a comparison of models developed for spare receptors by Furchgott (1955, 1964, 1966) and Black \& Leff (1983) with the model posited by del Castillo \& Katz (1957) it is helpful to have the model-related formulations listed next to each other. Hence, symbols for the dC\&K model are listed here for the receptor conformations, the ligand, and the system constants, while corresponding terminology for symbols and responses from the B\&L model appear in parenthesis when relevant. The formulations of the $\mathrm{dC} \& \mathrm{~K}$ and $\mathrm{B} \& \mathrm{~L}$ regimes are compared in Section 5.10.2.

Symbols for the dC\&K regime:

\begin{tabular}{|c|c|c|}
\hline $\mathrm{R}$ & free receptive unit $(\mathrm{R})$, and $S$ an agonist & $(\mathrm{A})$ \\
\hline RS & $\begin{array}{l}\text { receptive unit bound with an agonist in } \\
\text { a reactive state }\end{array}$ & $(\mathrm{AR})$ \\
\hline $\mathrm{R} * \mathrm{~S}$ & $\begin{array}{l}\text { receptive unit bound with an agonist in } \\
\text { an active state }\end{array}$ & $\begin{array}{l}\text { see a } \\
\text { below }\end{array}$ \\
\hline $\mathrm{R}_{\mathrm{tot}}$ & $\begin{array}{l}\text { sum of all possible receptor conforma- } \\
\text { tions in the system }\end{array}$ & $\left(R_{0}\right)^{6}$ \\
\hline $\mathrm{R}_{\mathrm{tot}}^{*}$ & $\begin{array}{l}\text { sum of active receptors needed for } \\
\text { maximal response }\end{array}$ & \\
\hline ar & actual response & $(\mathrm{E})$ \\
\hline TR & total or maximal response, $R_{\max }$ & $\left(E_{\mathrm{m}}\right)$ \\
\hline$L^{\prime}$ & $\begin{array}{l}\text { isomerization constant for liganded } \\
\text { receptive unit }\end{array}$ & $(\tau)$ \\
\hline
\end{tabular}

${ }^{6} R_{0}$ here is similar to $R_{\max }$ and not the same as $R_{0}$ described in Section 5.9 .9 which is for an un-liganded receptor conformation in a relaxed state.
$K_{\mathrm{E}}$ the value of $\mathrm{RS}$ (AR) that elicits half-maximal effect. This parameter is without physical implications.

$R_{0} /$ defined as the 'transducer ratio' $=\tau$. It turns out

$K_{\mathrm{E}}$ that $\tau$ in the B\&L formulation is equal to $L^{\prime}$ in the dC\&K formulation (see Section 5.10.2). $\mathrm{R} * \mathrm{~S}$ is equal to Stephenson's stimulus ' $\mathrm{St}$ ' and since the fractional response $a r / T R$ is a function of this stimulus, we have that $\operatorname{ar} / T R=f\left(\mathrm{R}^{*} \mathrm{~S}\right)$.

\subsubsection{Formulations of del Castillo \& Katz and Black \& Leff Models}

For the dC\&K reaction scheme we have already derived its formulation (see Eq. 5.10), repeated here in a slightly different form:

$$
\frac{\text { response }}{\text { total response }}=\frac{a r}{T R}=\frac{\mathrm{S} \cdot L^{\prime}}{S \cdot\left(1+L^{\prime}\right)+K_{\mathrm{s}}},
$$

while a lightly reformulated B\&L expression based on their Eq. 5 is given as:

$\frac{\text { response }}{\text { total response }}=\frac{\mathrm{E}}{E_{\mathrm{m}}}=\frac{\mathrm{S} \cdot\left(R_{\mathrm{tot}} / K_{\mathrm{E}}\right)}{\mathrm{S} \cdot\left(1+\left(R_{\mathrm{tot}} / K_{\mathrm{E}}\right)\right)+K_{\mathrm{s}}}$

Black \& Leff (1983). Examples of the B\&L model are shown in Fig. 5.13.

Transcribed from B\&L83, Eq. 5.14 is obtained by embedding two rectangular hyperbolic expressions. The $\mathrm{B} \& \mathrm{~L}$ model is derived based on an argument of logical deduction, namely that the fractional effect $\mathrm{E} / E_{\mathrm{m}}$ is related to the agonist concentration [A] by a hyperbolic relation as well as the fractional occupancy $\mathrm{AR} / R_{0}$, which is also a rectangular hyperbolic function of the agonist concentration. Meanwhile, the formulation is not based on mechanistic grounds, such as the dC\&K formulation in Eq 5.13. Clearly, Black and Leff in their 1983 paper on embedded load models employed a mathematical solution rather than a mechanistic one with the intention to incorporate information about receptor reserve. ${ }^{7}$ The mathematical approach in B\&L is exemplified moreover by use in general of the describing term 'operational' for their models, including a subsequent, hasty introduction of Hill's exponentiation.

With the purpose of discarding the dC\&K model in favor of their own B\&L model, Black and Leff argued that the 'the Castillo \& Katz model reduces to the form of Ariëns' model (1954)'. However, this is incorrect, as we have already seen in Section 5.6.2.

The dC\&K model is sound and should be implemented instead of the B\&L general hyperbolic model, the socalled 'operational model'. Let us see why.

\footnotetext{
7 See Paton and Rothschild (1965) on embedded loads resulting in a single load relation.
} 


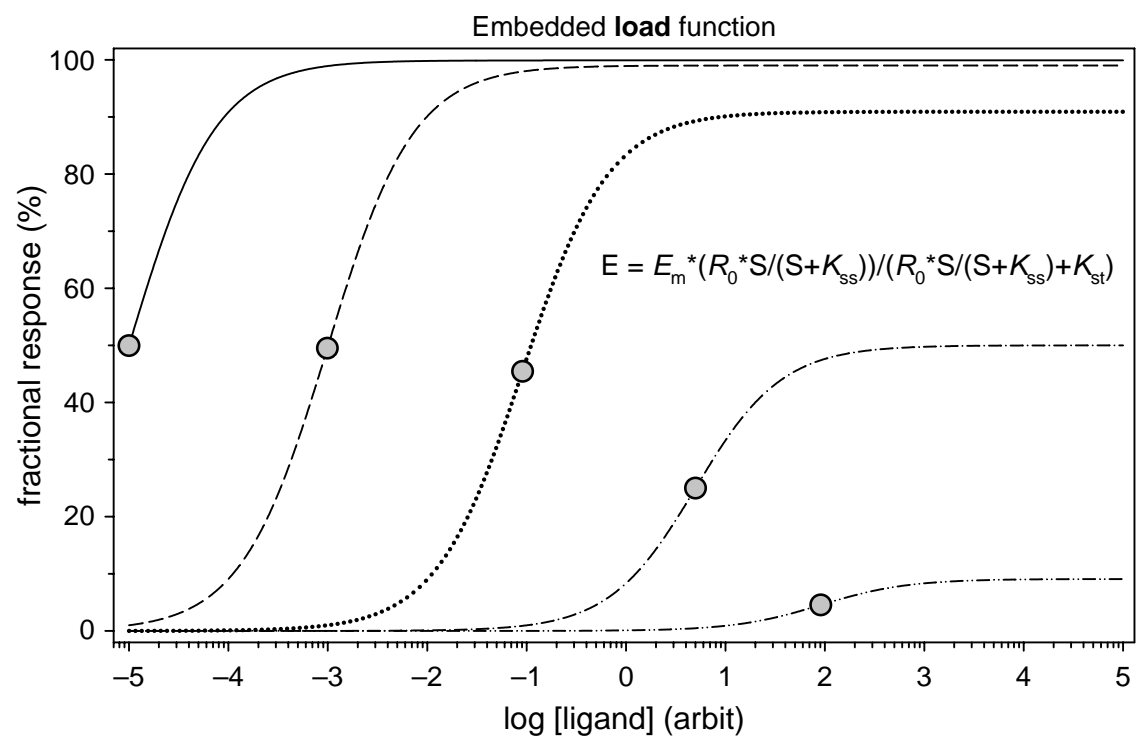

Figure 5.13. The embedded load function equal to the operational model, B\&L. The embedded load formulation is indicated on the graph. The parameters $\mathrm{E}, E_{\mathrm{m}}, R_{0}, K_{\mathrm{E}}=K_{\mathrm{st}}$, and $K_{\mathrm{A}}=K_{\mathrm{ss}}$ are as defined in the original paper including the transducer ratio $\tau=R_{0} / K_{E}$ (Black \& Leff 1983). The occupied receptors, here designated 'St', is a fraction of the total receptors $R_{0}$ and formulated as: $S t=R_{0}{ }^{*} S /\left(S+K_{\mathrm{sS}}\right)$ in which $S$ is the ligand concentration. The total receptor concentration $R_{0}$ is fixed at 1 , the maximum effect $E_{\mathrm{m}}$ at 100 , while the maximum fractional response is $E_{\mathrm{m}}{ }^{*} S t /\left(S t+K_{\mathrm{st}}\right)$ for $\mathrm{S} \rightarrow \infty$. Dissociation constant $K_{\mathrm{ss}}$ is varied in 5 steps from $10^{-2}(-)$ to $10^{2}(-\cdot-)$ by a factor 10 between steps, and dissociation constant $K_{\mathrm{st}}$ is varied at the same time in 5 steps from $10^{-3}(-)$ to $10^{1}(-\cdot-)$ by a factor 10 between steps. Circles indicate the $\mathrm{EC}_{50}$. The embedded load function is itself a load function (Paton \& Rothschild 1965). See text on a comparison between embedded load and dC\&K's regime.

\subsubsection{Formulating a Receptor Reserve}

The models by Furchgott (1966) and Black and Leff (1983) were derived in an attempt to address the question of a receptor reserve. On face value, receptor reserve seems axiomatic in the B\&L formulation, where by diminution of $R_{\text {tot }}\left(R_{0}\right)$ in the transducer ratio $\tau$ (cf. Section 5.10.1 and Eq. 5.14), it is possible to reduce the maximal efficacy the same way as for 4-DAMP mustard, $\beta$-haloalkylamines, and other irreversible inhibitors. Hence, manipulating the size of $R_{0}$ appears as a direct tuning of the receptor reserve. Meanwhile, it is possible to compensate for a reduction of $R_{0}$ by decreasing the value of coefficient $K_{\mathrm{E}}$. Thus, even with a receptor reserve less than sufficient for regular maximal response, according to the formulation, we can decrease $K_{\mathrm{E}}$, keep the parameter $\tau$ constant, and regain a former maximal response. However, that is not the manner in which experimentation operates or how the coefficient $K_{\mathrm{E}}$ should be conceived. How should $K_{\mathrm{E}}$ be conceived?

In this context and to answer that question, parameter $L^{\prime}$ can be regarded as a product of an absolute parameter $R_{0}$ and a relative constant $1 / K_{\mathrm{E}}$, where $K_{\mathrm{E}}$ is a virtual factor while $L^{\prime}$ has a physical meaning. $K_{\mathrm{E}}$ emerges from a mere algebraic manipulation and therefore is not very useful. Efficacy coefficients $e$ or $L^{\prime}$ are not mixed up with numbers of receptors, $R_{\mathrm{tot}}$ or $R_{0}$, as in the transducer ratio $R_{0} / K_{\mathrm{E}}$, which is also an efficacy coefficient.
Conversely, the concept efficacy is a mixture of both the total number of receptors needed for maximal effect, $R_{\text {tot }}^{*}$, and the efficacy coefficient $e$. However, the pool of receptors that can be activated and the efficacy coefficient are kept separate in the expression for efficacy.

What does our derived dC\&K formulation say about a receptor reserve? The present $\mathrm{dC} \& \mathrm{~K}$ formulation simply states that if $R_{\mathrm{tot}}$ is larger than $R_{\mathrm{tot}}^{*}$ then there is a proportionally larger receptor reserve (RR). First, when $R_{\text {tot }}$ is reduced to equal $R_{\text {tot }}^{*}$, then there is no receptor reserve. Should $R_{\text {tot }}$ be reduced further below the actual $R_{\text {tot }}^{*}$, then $R_{\text {tot }}^{*}$ is also reduced in parallel to a new $R_{\text {tot }}^{*}$. Observing these conditions, we can formulate a relation between all receptive units and units needed for maximal response as: $R_{\text {tot }}^{*}=R_{\text {tot }}-\mathrm{RR}$. The B\&L transducer ratio $\tau$ is equal to $L^{\prime}$ or Stephenson's $e$ and determines the maximum fractional response by $\mathrm{E} / E_{\mathrm{m}}=\operatorname{ar} / T R=1 /(1+1 / \tau)=1 /\left(1+1 / L^{\prime}\right)($ Fig. 5.13)

\subsubsection{Furchgott's Intrinsic Efficacy}

Furchgott derived his formulation for spare receptors based on embedded load equations as later copied in the operational model by Black and Leff (Furchgott 1955, 1964; Black \& Leff 1983). However, Furchgott (1966) paid more attention to tissue-dependent and ligand-dependent parameters by introducing his 'intrinsic efficacy' concept, wherein Stephenson's efficacy 
constant $e$ was normalized by the total number of receptors, $R_{\text {tot }}$. Thus 'intrinsic efficacy' $\varepsilon$ is equal to $e /$ $R_{\text {tot }}$, to $L^{\prime} / R_{\text {tot }}$, or if you will, to $1 / K_{\mathrm{E}}$. Just as $1 / K_{\mathrm{E}}$ is without physical meaning, so is $L^{\prime} / R_{\mathrm{tot}}$.

On experimental extraction of values for $\varepsilon$ see, for instance, MacKay (1977). The understanding of $K_{\mathrm{E}}$ and $\varepsilon$ is not made clearer by introducing a 'fitting constant' $\beta$ from Kenakin and Beck (1982), since now $K_{\mathrm{E}}$ is suddenly equal to $\beta / \varepsilon$ (Black \& Leff 1983).

Operational modeling may be elaborated further, involving Furchgott's 'efficacy', encompassing his intrinsic efficacy coefficient $\varepsilon$, and B\&L's 'operational efficacy' subsuming their transducer ratio coefficient $\tau$ (Trzechiakowski 1999a,b; Frigyesi \& Hossjer 2005 Kenakin 2007). However, my understanding of the issue moves me in an opposite direction, which is to keep the formulation of a receptor reserve as simple as possible, i.e., $R_{\mathrm{tot}}^{*}=R_{\mathrm{tot}}-\mathrm{RR}$.
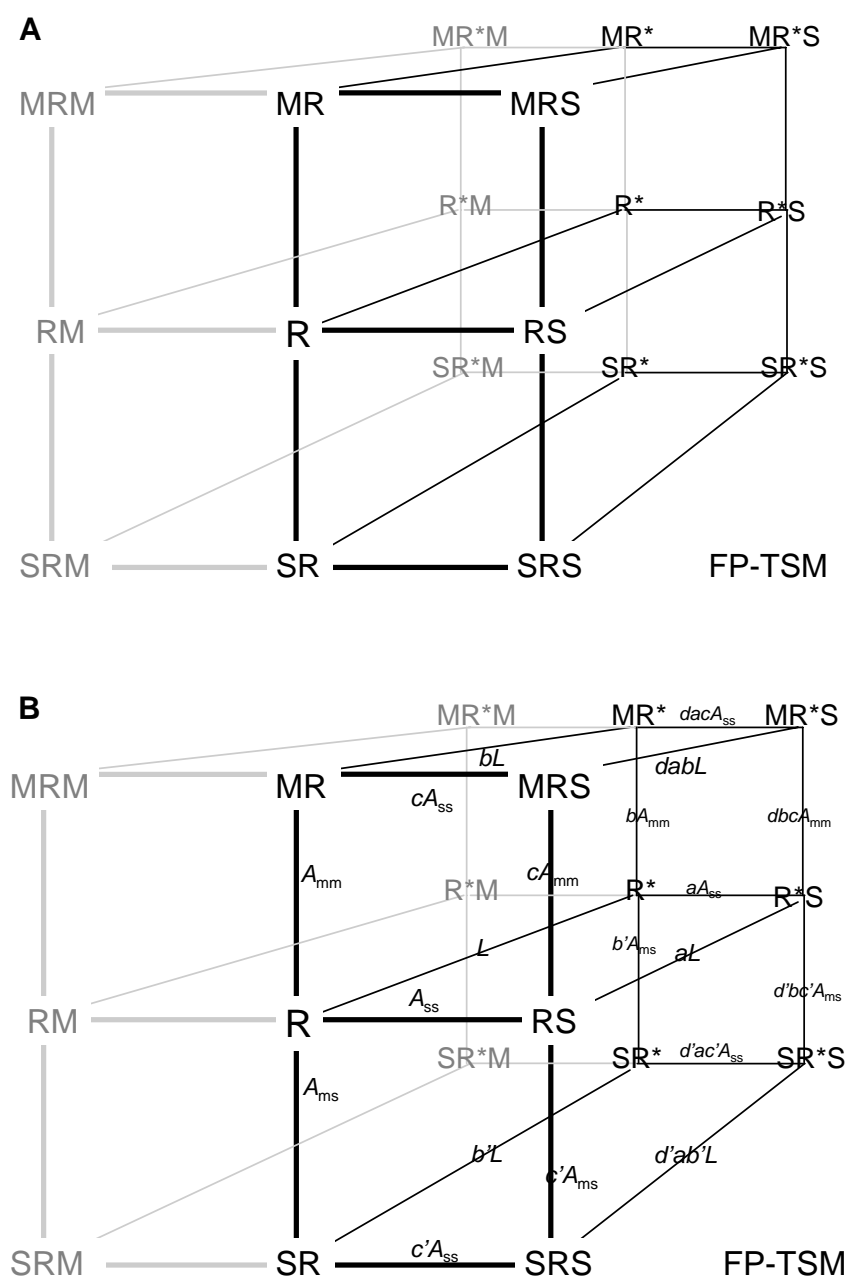

Figure 5.14. The combined allosteric two-state and homotropic two-state model. Part of the four-pane two-state model (FP-TSM) consists of the ATSM and HOTSM. Panel A without system constants, and panel B with system constants.

\subsubsection{Jargon or Misuse of 'Efficacy' for 'Efficacy Coefficient'}

In the writings of Furchgott (1966) and Black and Leff (1983), it can be observed that there is no clean differentiation, as there should be, between Stephenson's 'efficacy' as a property and 'efficacy' as a coefficient, $e$. For example, 'intrinsic efficacy' is equal to the constant $\varepsilon$ according to Furchgott (1966) instead of the more precise 'intrinsic efficacy constant', and ' $\tau$ is equivalent to efficacy' (Black \& Leff 1983) instead of the more precise ' $\tau$ is equivalent to an efficacy constant' or 'efficacy is a function of $\tau$ '. Also, in the general operational model, according to Black and Leff the transducer ratio links the number of receptors to the 'intrinsic efficacy' of the agonist. Is this latter 'intrinsic efficacy' an 'efficacy' or is it an 'efficacy coefficient'? What is more, their use of a term such as 'relative efficacy' is redundant since 'efficacy' is relative (cf. Sections 1.3.4 and 5.4.2). In spite of this, ' $K_{\mathrm{E}}$ is generally used to quantify relative agonist efficacy' (Trist \& Corsi 2000).

\subsubsection{Conclusion}

In my opinion, the solution to Stephenson's search is simply the dC\&K model, and to make Stephenson's 'efficacy' operative I suggest a clear differentiation between the property 'efficacy' (Stephenson 1956, p. 380) and 'efficacy' as an efficacy constant, used mostly throughout Stephenson’s paper (1956).

\subsection{Induction Versus Selection}

\subsubsection{Who is Right?}

The title to this sub-chapter, 'induction versus selection', covers an age-old problem that has reappeared many times. It was again on the agenda for enzymologists in the 1950s, as measurements with fast reaction kinetics became available (Eigen 1968), and further debated in enzymology in the 1960s with the introduction of the Monod-concerted selection model (Monod et al. 1965) and Koshland-sequential induction model (Koshland 1966; Harber \& Koshland 1967) for protein activation. Views by keyoriginators of induction and selection theories do not appear to have changed (Changeux 2004; Koshland 2004; Changeux \& Edelstein 2005). Changeux has argued once again for the selection model by observing that channels can switch between closed and open states in the absence of a ligand, i.e., demonstrate spontaneous activity, "which is readily explained in terms of a selection mechanism' (Changeux 2004, p. 232). Meanwhile, his argument is irrelevant for the question of what happens when 
a ligand is introduced. Does it stabilize the open conformation and thereby increase the likelihood of being open or does it induce the becoming of new openings? That is the question.

\subsubsection{Conformational Induction}

When a receptive unit is activated by induction, it is a process where the ligand first binds to the primary binding site, induces a conformational change, transforming the receptor into an active state. The scheme for conformational induction may be written as:

$$
\text { (1) } \mathrm{R}+\mathrm{S} \rightleftharpoons \mathrm{R}+\mathrm{S} \rightleftharpoons \mathrm{R} * \mathrm{~S} \text {. }
$$

We have already met this scheme in the dC\&K model (sub-chapter 5.6).

\subsubsection{Conformational Selection}

Conversely, when a receptive unit is activated in selection, it is a process where the receptive unit spontaneously flips between a resting (reactive) state and an active state. The active state has a higher affinity for the agonist than the reactive state. Thus, the ligand selects and stabilizes the active state, i.e., increases its concentration. This increases the activity of the receptive unit by conformational selection. The scheme for conformational selection may be written as:

$$
\text { (2) } \mathrm{R} \rightleftharpoons \mathrm{R} *+\mathrm{S} \rightleftharpoons \mathrm{R} * \mathrm{~S} \text {. }
$$

Combining the reaction schemes of conformational induction and selection results in the cTSM (Fig. 5.3). The two schemes tells us that we can go from $\mathrm{R}$ to $\mathrm{R} * \mathrm{~S}$ via two pathways. Analyses of the cTSM have shown us that it is impossible to separate and distinguish between the two paths. They are interlocked conceptually as well as in reality (sub-chapter 5.8).

\subsubsection{The Dilemma Between Selection and Induction in Enzymology}

In the famous models for concerted action (Monod et al. 1965) and sequential action (Koshland et al. 1966), the choice between induction and selection is tacitly assumed. In Monod's model, the choice is a conformational selection, whereas in Koshland's model the choice is for the conformational induction. The question concerning the concerted-vs-sequential behavior of proteins is different from, but related to, the dilemma of induction-vs-selection, and further discussed in Part IV Chapter 15 . When a number of ligands one by one each induces a conformational change on binding to a receptive system, it is a sequential model. From the start, Koshland favored the conformational induction also inherent in his early models with an induced fit
(Koshland 1958). This was also assumed in the dC\&K model from 1957. On the other hand, in the 1940s Monod had already advocated for a conformational selection as the right way of thinking (Changeux 1993), and incorporated it in the concerted model, where conformational change in all binding sites flip simultaneously in one step before binding. The spontaneous activity in the absence of a ligand, as mentioned above by Changeux, was never excluded from the modeling by Koshland and coworkers (Harber \& Koshland 1967), although 'induced fit' became synonymous with 'Koshland' (Bennett \& Steitz 1978). Bennett and Steitz discussed the concept 'occluded' in relation to the i-vss dichotomy, but their 'selection' must be understood in a different manner than an immediate intuitive fashion (see Bennett \& Steitz 1978, their Fig. 3).

\subsubsection{The Dilemma Between Selection and Induction in Pharmacology}

The induction-selection schism has been a conceptual dilemma in pharmacology as well. Working with ligandreceptor interaction, one essential question is "what is the real action of an agonist'? Does the agonist ligand first bind to the non-active receptor, thereby inducing an activation of the receptive unit, or does the receptive unit already exist in both reactive and active conformations of which the agonist prefers the active conformation, which it binds to and stabilizes? Spontaneous activity was excluded from the understanding of the conformational induction in receptors by Kenakin, who writes about the mechanism for conformational induction 'the different conformation of the receptor is not present in the cell membrane until the molecule (ligand) is present' (Kenakin 1995b).

Burgen (1981) revived the discussion about the mechanism of how agonists increase the number of active receptors - was it by induction or was it by selection? The question arose again in the 1990s as it was realized that receptors also had to be considered as spontaneously active in an agonist-independent manner (Costa \& Herz 1989). As a result of this, the induction-vs-selection discussion re-appeared together with a re-definition of an agonist, caused by the impact of mounting realization of the existence of spontaneous activity with high expression of receptors or constitutive activity due to mutated receptors, as mentioned earlier. Kenakin summarized the issue by a review on the subject, concluding that both conformational induction and conformational selection are possible mechanisms (Kenakin 1995b).

Based on experimental observations with allosteric enhancers, Bruns (1996) argued in favor of an induction mechanism, and challenged Terry Kenakin to a comment. Instead of a direct response to Bruns, Kenakin introduced the multi-state receptor and concluded that 
there were no dichotomy - rather induction and selection 'are extremes of the same mechanism' (Kenakin 1996) or 'of a continuum' (Lutz \& Kenakin 1999, Chapter 3.2, pp. 64-68) of receptor conformations. Multiple conformations of the receptive unit now existed in 'energy landscapes' (Kenakin 1996; Kumar et al. 2000; Kenakin \& Onaran 2002).

The multi-state continuum for receptor states is certainly a concept to be considered in connection with how receptors are activated and how ligands and receptors interact, but hardly necessary as an argument not to favor either induction or selection.

\subsubsection{Induction Versus Selection at Equilibrium}

In case we accept the cTSM, an answer to the dilemma is very easy and may be recognized by considering the two legs of the cTSM reaction scheme. When the two reaction schemes in Section 5.11.1 are combined, they constitute the cTSM reaction scheme (Haber \& Koshland 1967), but not the original MWC model (Fig $5.2 \mathrm{C}+\mathrm{D})$. The cTSM reaction scheme is thermodynamically the simplest cyclic or fully reversible two-statemodel. At equilibrium the clockwise reaction, equal to induction (1), is equally as fast as the anticlockwise reaction, equal to selection (2). Thus, the quest for a choice between supremacy of either induction or selection at equilibrium is a futile endeavor.

\subsubsection{Solving the Induction Versus Selection Problem for Enzymes with Rate Kinetics}

When a system is perturbed away from equilibrium, one might expect that the two mechanisms, induction and selection, can be studied separately, thus yielding a solution to the induction-versus-selection (i-vs-s) problem.

Using temperature and concentration jumps, Halford (1972) tried to distinguish between conformational induction and conformational selection. Halford studied the activation of the alkaline phosphatase enzyme for which a substrate-enzyme complex is formed by a conformational change and/or by selection of the active conformational form of the enzyme. The question is which step precedes the other or which one is the most rapid? The question was further studied by Hull and colleagues (1976), but remained unresolved. The schism about induction before selection or vice versa is still debated by enzymologists (Gutfreund 1995; Changeux \& Edelstein 2005; Wang et al. 2005, 2007) and by protein structuralists (Kumar et al. 2000). The conclusions are that relaxation kinetics does not seem to resolve the dichotomy of selection before induction or vice versa (Gutfreund 1995, pp. 217-219; Kumar et al. 2000).

\subsubsection{Induction Versus Selection at Non-equilibrium in Pharmacology}

Bruns (1996) rendered a scenario where allosteric enhancers, in conformational induction, should increase agonist affinity solely by slowing the dissociation rate constant $k_{-1}$ in Fig. 5.4. Further, according to Burns, for allosteric enhancers in conformational selection, one could specifically predict an acceleration of agonist association rate constant $k_{1}$ in Fig. 5.4, without an effect on the dissociation rate constant. Therefore, since it is generally observed that allosteric enhancers reduce the agonist off-rate of the ligand-receptor interaction, the induction scheme is the most likely.

Let us look at Bruns' argument. First, in case binding of $\mathrm{S}$ is a matter of stabilizing the $\mathrm{R} * \mathrm{~S}$ complex as shown in Fig. 5.4, such binding will drive the whole process to the right. This shift in process rate may be obtained by either an increase in $k_{1}$ or a decrease in $k_{-1}$ in the reaction scheme, and for that matter by an increase in the isomerization constants $L^{\prime}$ and $L$. Therefore, when Bruns writes about the conformational selection scheme that the enhancers cannot influence dissociation of agonists, since the proportion of agonist-occupied receptors is already fully in the activated conformation and cannot be further shifted' and when he continues 'Conformational selection thus predicts an acceleration of agonist-receptor association without any effect on dissociation', this is not justified. It is nonsense, possibly due to a mix-up of rates and equilibrium conditions.

The debate about conformational selection versus conformational induction appears still to be actual in pharmacology (Fong 1996; Hunyady et al. 2003, Le et al. 2003; del Carmine et al. 2004). No doubt, both the cTSM and the double TSM, equal to IIISMs, due to their thermodynamic nature cannot differentiate between selection and induction (Giraldo 2004). The belief remains that dynamic studies will be able to differentiate between the two concepts (Gether \& Kobilka 1998; Gether 2000; Kobilka \& Gether 2002; Kobilka 2002; Urban et al. 2007), now with a wording in favor of induction (Swaminath et al. 2004, 2005; Giraldo 2004; Feng et al. 2005; Lu et al. 2005; Sosa-Peinado \& Gonzalez-Andrade 2005; Ellis et al. 2006; Yao et al. 2006; Granier et al. 2007; Urban et al. 2007), although it is realized that the schism is difficult to get rid off (Peleg et al. 2001). Some groups convincingly present substrate binding as induced fit or secondary induced fit, but based on static X-ray images (Wang et al. 2005, 2007).

\subsubsection{Selection Versus Induction is a Conceptual Schism}

Definitions of conformational selection and induction at equilibrium, with inherent complementarity, dissolve 
when systems are perturbed away from equilibrium. In return, dynamic complementarities emerge, and the complementarity problem, which is virtual, remains.

Therefore, since the induction-vs-selection (i-vs-s) dilemma is a conceptual enigma, measurements of fast reaction kinetics and conformational changes cannot solve the dichotomy.

The answer to the question about i-vs-s does not boil down to resolving which process is the fastest, the preequilibration between the two states or the binding process proper. As Colquhoun has pointed out, due to reciprocity, the two processes are inseparable and should be considered two phenomena of the same process (1998); an understanding that is upheld today (Colquhoun 2007). This is also my understanding. For another balanced view see Giraldo et al (2006).

\subsubsection{What is the Impact of Solving the Induction-vs-selection Problem (i-vs-s)}

From an intellectual point of view, the above i-vs-s question is an intriguing one, while we may question if it is also an important distinction from a practical standpoint. In studying biomedical and industrial drugs as well as pharmaceuticals during the drug discovery and development process, what is of interest and useful for in vivo application is the quantitation of on- and off-rates in chiralty, of racemic shifts, and of ligand-receptor interactions including observations in vitro from kinetic and dose-response experiments and their analysis (Giraldo 2004; Baranczewski et al. 2006), and not the i-vs-s schism.

In the theoretical field, the i-vs-s quandary has left our modeling with a complexity we refer to as 'microscopic', i.e., non-separable system constants.

\subsubsection{Conclusion on Complementarity}

In our cTSM, induction and selection are but two aspects of the same phenomenon. If this conclusion is still unsatisfactory and counter-intuitive to you, I recommend that you work further at non-equilibrium with the inherent complementarity of i-vs-s in the cTSM.

\section{Box 5.1.1. Apparent dissociation constant and its related efficacy}

In deriving explicit expressions for the $\operatorname{appK}_{\mathrm{s}}$ in the functional form of the cyclic two-state model, the parameters used are as before:

$\mathrm{S}=\quad$ ligand concentration

$\mathrm{R} *, \mathrm{R}, \mathrm{RS}, \mathrm{R} * \mathrm{~S}$ receptor conformations or their mass/volume = concentration

$A_{\mathrm{s}}=\quad$ ligand equilibrium association constant. A forward constant

$L=\quad$ isomerization constant for unliganded receptors. A forward constant

$a=\quad$ an intrinsic efficacy as well as an intrinsic affinity constant

$\operatorname{appK}_{\mathrm{s}}=\quad$ the parameter we want to find. It is the ligand concentration for which the response is midway up between the maximum level and the spontaneous level of activity (Box 5.1 figure). This middle level of response we may call pssE $\mathrm{E}_{50}$

$\operatorname{pssE}_{50}=\quad$ is a new constant. As already defined, pssE $_{50}$ is the mean response level between initial activity level without ligand, equal to $L /(L+1)$, and maximal activity level at high ligand concentration equal to $L /(L+1 / a)$ or $1 /(1+1 /(a \cdot L))$. Convince yourself that this maximum level is identical with the maximum level for functional cTSM listed in Table 5.1. Thus: $\operatorname{pss}_{50}=1 / 2 \cdot[(L /(L+1 / a))+(L /(1+L))]$ 


\section{Box 5.1.2. Deriving the apparent dissociation constant, appK $\mathrm{s}_{\mathrm{s}}$}

The efficacy midway between the initial level and the maximum level, pssE $\mathrm{E}_{50}$, is, as mentioned, also the response at which the ligand concentration $\mathrm{S}$ is equal to $\operatorname{appK}_{\mathrm{s}}$. The half-way response pssE $\mathrm{E}_{50}$ on the actual d-r curve is shown in Box 5.1 figure. Inserting appK $\mathrm{K}_{\mathrm{s}}$ for $\mathrm{S}$ in the expression for fractional response of the cTSM (Eq. 5.12b) yields an equation for the midway response pssEC $5_{50}$ equal to $L /(L+Q)$; in which $\mathrm{Q}=\left(1+A_{\mathrm{s}} \cdot \operatorname{appK}_{\mathrm{s}}\right) /\left(1+a \cdot A_{\mathrm{s}} \cdot \operatorname{appK}_{\mathrm{s}}\right)$. Thus, two expressions for the possible mean efficacy, pssE 50 , may be set equal:

$$
\frac{1}{2} \cdot\left[\frac{L}{L+1 / a}+\frac{L}{L+1}\right]=\frac{L}{L+\mathrm{Q}}
$$

Isolating for $\operatorname{appK}_{\mathrm{s}}$ tacit in the $\mathrm{Q}$ term in Eq. Box 5.1-1, we get:

$$
\operatorname{appK}_{\mathrm{s}}=\frac{(1+L)}{A_{\mathrm{s}} \cdot(1+a \cdot L)} .
$$

Compare with the appK $\mathrm{s}_{\mathrm{s}}$ listed in Table 5.1. The appK $\mathrm{s}_{\mathrm{s}}$ is a function of $A_{\mathrm{s}}, a$, and $L$ as depicted in Box 5.1 figure. For an elegant derivation of this $\operatorname{appK}_{\mathrm{s}}$ see Giraldo (2004).

\section{Box 5.1.3. What is the limiting value of $\mathrm{pss}_{50}$ and $a p p K_{s}$ ?}

Limiting values for the efficacy measure $\mathrm{pssE}_{50}$ and the potency term $\operatorname{appK}_{\mathrm{s}}$ may be obtained by inserting values for parameter $a$, approaching either zero or infinity, into the functional response expressed in the left hand-side of Eq. Box 5.1-1, which is equal to $\operatorname{pssE}_{50}$, for all $L$, i.e., find lim pssE $_{50}$ for $a \rightarrow \infty$ or $a \rightarrow 0$. Values for lim pssEC $_{50}$ are listed in column 2 of Box 5.1 (Table 0). The limiting values for $\operatorname{appK}_{\mathrm{s}}$ may be obtained from Eq. Box 5.1-2. They are listed in column 3 of Box 5.1 table for all values of $L$.

Since the level of active receptors before adding any ligand vary with $L$, and the level of active receptors after adding a ligand vary with the values of both $L$ and $a$, the limiting $\operatorname{pss}_{50}$ is a function of these two parameters, as also indicated in Box 5.1 table for general and specific cases. For parameter $a$ approaching the value 1, both from below and from above, the value of limiting appK $\mathrm{s}_{\mathrm{s}}$ is equal to $1 / A_{\mathrm{s}}$, as also indicated in Box 5.1 table for $a=1$ and shown in Box 5.1 figure, although, at $a$ exactly equal unity, the limiting $\operatorname{appK}_{\mathrm{s}}$ is not defined.

From Box 5.1 table it is obvious that $A_{\mathrm{s}}$ is always involved in the position of the limiting appK $\mathrm{s}_{\mathrm{s}}$, that $L$ by itself determines the starting level on the response axis (initial efficacy), while $a$ is the parameter which sovereignly determines if the ligand is an agonist, an inverse agonist, or a competitive ant-agonist (neutral ant-agonist). Due to natural reciprocity, or thermodynamic equilibrium, in the equilibrated cTSM, $a$ and $L$ are bound together in determining the efficacy level in the presence of an agonist, see columns 1 and 2 in Box 5.1 table. 


\section{Box 5.1.4. The special case of $L<<1$ and $a>1$ yields partial agonism}

Let us look at some consequences of the cTSM for function. In Nature, where receptor-guided regulatory mechanisms have evolved, the most abundant type of tabulated cases is the one in Box 5.1 (Table 1 ) for $L<<1$ and $a>1$. This case is illustrated in Fig. 5.8. It also demonstrates what partial agonism means in the realm of the cTSM. Further analysis will show that partial agonists operate in the range of $0.1<a \cdot L>10$. For partial agonism to occur in a classical display, based on functional cTSM, $L$ must be much smaller than $1, L<<1$, while $a$ must be larger than 1, $a>1$ (Fig. 5.8). Therefore, since $L$ is probably $<0.01$ in many natural systems without spontaneous activity, we can conclude that $a$ must be larger than 10 when partial agonism is observed (Fig. 5.8). Further for agonism to show up with a reasonable efficacy, $a$ should be $\geq 10 \cdot L$. Typically, thus, for the full agonist $a$ is $>100$ and efficacy may be said to be dependent on the product of $a \cdot L$, see $\mathrm{R}_{\text {max }}$ for functional cTSM response in Table 5.1. As $a \cdot L$ increases above 0.1 , the dose-response relationships rise in a fashion comparable to the dose-response of a series of partial agonists with increasing apparent affinities from values of $A_{\mathrm{s}}$ towards values approaching $a \cdot L \cdot A_{\mathrm{s}}$ (Fig. 5.8). When $a \cdot L>>10$ complete agonism is obtained. Partial agonists with $<10 \%$ efficacy of a complete agonist have appA close to the genuine $A_{\mathrm{s}}$. Stated in a different manner, for a series of homologous drugs to display partial agonism (Nickerson 1956) in cTSMs, the requirements are that $a$ through the series of less and less efficacious ligands decreases towards a value such that $a \cdot L$ become $<0.1$. See the sweep of theoretical curves for partial agonist as depicted in Fig. 5.8.

For the case of $L<<1$ and $a>1$, we can further state that the affinity for an agonist is highest in the active receptor, equal to most systems occurring in Nature. Additionally, the activating conformational change is the most likely event for the liganded conformation compared with the non-liganded form of the receptor. Consulting Fig. 5.3, you will see that the affinity of the ligand is much larger for the active non-liganded receptor than for the reactive (non-active) non-liganded receptor state, while the tendency for the receptor to go into the active state spontaneously in the non-liganded configuration, $\mathrm{R} \rightarrow \mathrm{R}^{*}$, is a less likely event compared to the liganded receptor flip-flopping into the active state, $\mathrm{RS} \rightarrow \mathrm{R} * \mathrm{~S}$.

When we trust the cTSM to be the underlying reaction scheme for our experimental observations at equilibrium, it is justified to claim that the agonist stabilizes the active conformation, as it is to state that the binding of an agonist to the non-active receptor conformation is that, which induces the conformational change to an active state of the liganded receptor (confer sub-chapter 5.11).

\section{Box 5.1.5. Partial agonism in Nature and the cTSM}

Partial agonism is widespread in Nature and in test tubes. This ought to trigger your curiosity and pose the question: is the cTSM really a relevant rôle-model for what goes on in the real world? Meanwhile, I would formulate the question in a different way. Using functional cTSM, what are the constraints on, or in, evolution that have predisposed ligand-receptor interaction such that $a \cdot L$ is between $1 / 10$ and 10 , allowing us to detect partial agonism? From an analytical point of view, should the two parameters $a$ and $L$ not be totally independent? Is the constraint, $1 / 10<a \cdot L<10$, linked to survival of the fittest?

The answer is: Yes, of course. Receptor conformations and natural ligands that do not follow the $0.1<a$. $L>10$ constraint have been eliminated during evolution, since $1 / 10<a \cdot L<10$ is a hallmark of mechanisms involved in regulatory actions in biology. Likewise the condition, that $L<<1$, is also fixed by evolution in most natural systems - in particular when we believe in the cTSM.

In conclusion, the simple K\&T5 reaction scheme is a matrix for the functional cTSM, which can explain spontaneous/constitutive activity, partial agonism, and inverse agonism. In case there are spare receptors, the $\operatorname{appA}_{\mathrm{s}}$ and the true $A_{\mathrm{s}}$ deviate. 


\section{Box 5.1.6. Ant-agonists and the cTSM}

Paul Leff published a key-paper on the cTSM, summarizing several aspects on the consequences of applying a cTSM for the analysis of doseresponse data from functional studies (Leff 1995). His paper is certainly worth consulting. Meanwhile, his discussion of ant-agonists interfering with effects of the functional cTSM should be treated in the realm of modulation in the allosteric two-state model by Hall (the ATSM in Chapter 7), rather than by Schild's method as done by Leff.

\section{Box 5.1.7. The three-state model}

Leff and Scaramellini et al. have developed the cTSM into a genuine three-state model (IIISM) (Leff et al. 1997; Scaramellini \& Leff 1998, 2002). This model belongs to the category ternary-complex models for GPCRs, and should therefore be discussed in relation to bifurcation of $G$ protein coupled receptors. See Giraldo (2004) for another approach to three-state models.

Box 5.1. Table. Limiting values of $\mathrm{pssEC}_{50}$ and $\mathrm{appK}_{\mathrm{s}}$ in the cyclic two-state model

Table 0. General cases

\begin{tabular}{lll}
\hline Parameters in cTSM for all values of $L$ & \multicolumn{1}{c}{$\operatorname{lim~pssE_{50}}$} & \multicolumn{1}{c}{$\lim \operatorname{appK}_{\mathrm{s}}$} \\
\hline$a \rightarrow 0$ & $L /[2 \cdot(L+1)]$ & $(L+1) / A_{\mathrm{s}}$ \\
$a \rightarrow \infty$ & $(2 L+1) /[2 \cdot(L+1)]$ & $(L+1) /\left(A_{\mathrm{s}} \cdot a \cdot L\right)$ \\
$a=1$ & $L /(L+1)$ & Not defined \\
\hline
\end{tabular}

Table 1. Special cases

\begin{tabular}{lcc}
\hline For $L<<1$ & $\lim \operatorname{pssE}_{50}$ & $\lim \operatorname{appK}_{\mathrm{s}}$ \\
\hline$a \rightarrow 0$ & $L / 2$ & $1 / A_{\mathrm{s}}$ \\
$a \rightarrow \infty \dagger$ & $1 / 2$ & $1 /\left(A_{\mathrm{s}} \cdot a \cdot L\right)$ \\
$a=1$ & $L$ & $1 / A_{\mathrm{s}}$ \\
\hline
\end{tabular}

$\dagger$ The natural situation.

Table 2. Special cases

\begin{tabular}{lcc}
\hline For $L>>1$ & $\lim \mathrm{pssE}_{50}$ & $\lim \mathrm{appK}_{\mathrm{s}}$ \\
\hline$a \rightarrow 0$ & $1 / 2$ & $L / A_{\mathrm{s}}$ \\
$a \rightarrow \infty$ & 1 & $1 /\left(A_{\mathrm{s}} \cdot a\right)$ \\
$a=1$ & 1 & $' 1 / A_{\mathrm{s}}$ ' \\
\hline
\end{tabular}

Table 3. Special cases

\begin{tabular}{lcc}
\hline For $L=1$ & $\lim \operatorname{pssE}_{50}$ & $\lim \operatorname{appK}_{\mathrm{s}}$ \\
\hline$a \rightarrow 0$ & $1 / 4$ & $2 / A_{\mathrm{s}}$ \\
$a \rightarrow \infty$ & $3 / 4$ & $2 /\left(A_{\mathrm{s}} \cdot a\right)$ \\
$a=1$ & 1 & $1 / A_{\mathrm{s}}$ ' \\
\hline
\end{tabular}


Box 5.1. Figure. Principal plot of the functional cTSM. As indicated in the figure there is a basic spontaneous activity level determined by $L /(1+L)=1 /(1+1 / L)$ and a maximum activity level equal to $a L /(1+a L)=1 /$ $(1+1 / a L)$. The $\mathrm{EC}_{50}$ is given by a level mid-way between the basic and the maximum levels, pssE $\mathrm{E}_{50}=1 / 2 \cdot[1 /$ $(1+1 / L)+1 /(1+1 / a L)]$. Circles indicate the actual $\mathrm{EC}_{50}$. The apparent dissociation constant appK $\mathrm{s}_{\mathrm{s}}$ is equal to $\left(K_{\mathrm{s}}\right) \cdot[(1+L) /(1+a L)]$. (A) Parameter values are $L=0.2, A_{\mathrm{s}}=0.1$ and $a$ vary from $10^{-2}$ (bottom curve) to $10^{2}$ (top curve) in 5 steps by a factor 10 between steps. (B) Parameter values are $a=30, A_{\mathrm{s}}=0.1$ and $L$ vary from $10^{-2}$ (bottom curve) to $10^{2}$ (top curve) in 5 steps by a factor 10 between steps.
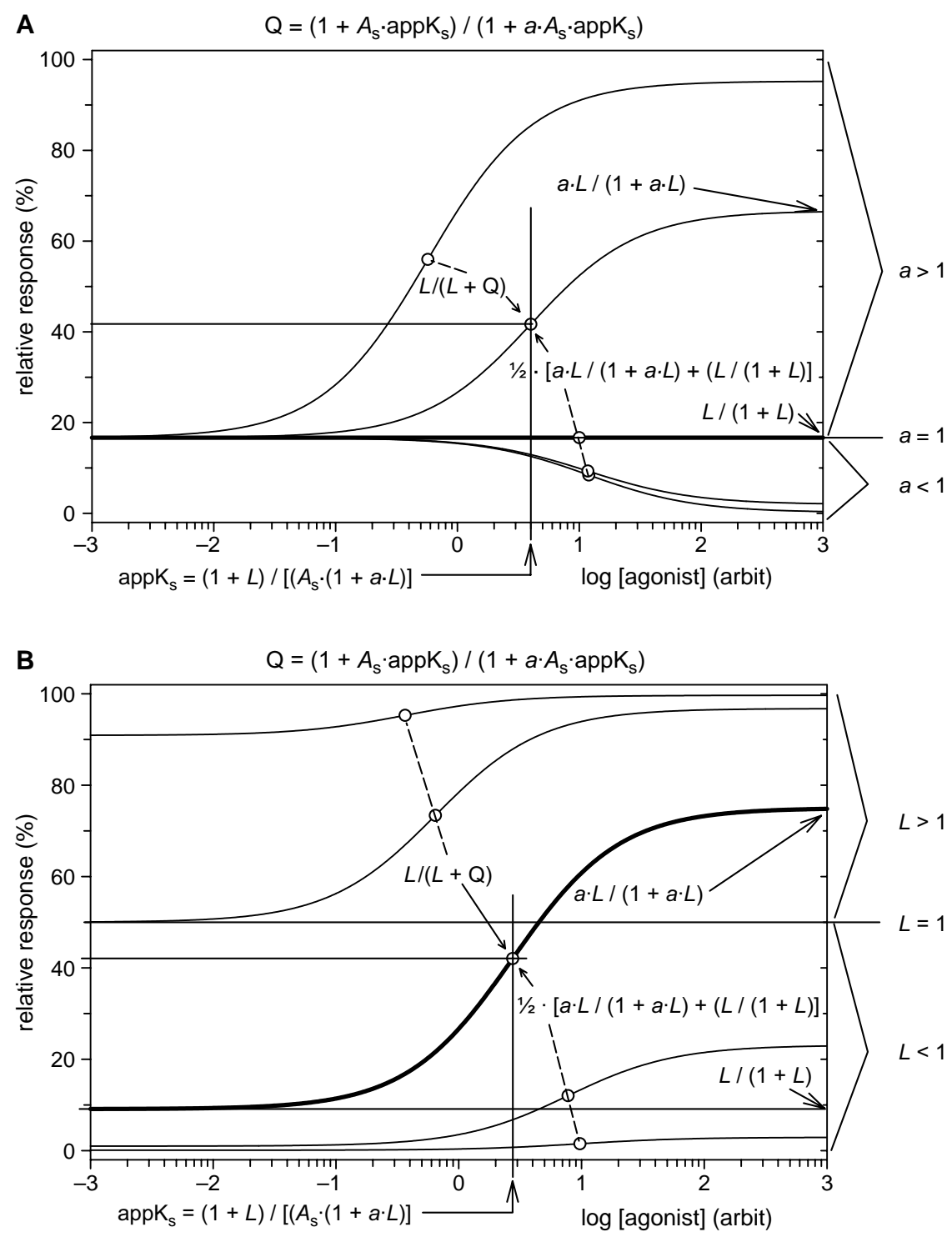


\section{REFERENCES}

Abramowitz J, Iyengar R \& Birnbaumer L. On the mode of action of catecholamines on the turkey erythrocyte adenylyl cyclase. Evaluation of basic activity states after removal of endogenous GDP and interpretation of nucleotide regulation and hormone activation in terms of a two-state model. J Biol Chem 255: 8259$8265,1980$.

Allen DW, Guthe KF \& Wyman J Jr. Further studies on the oxygen equilibrium of hemoglobin. J Biol Chem 187: 393-410, 1950.

Ariëns EJ. Affinity and intrinsic activity in the theory of competitive inhibition. Part I. Problems and theory. Arch Int Pharmacodyn 99: 32-49, 1954.

Baranczewski P, Stanczak A, Sundberg K, Svensson R, Wallin A, Jansson $\mathrm{J}$, et al. Introduction to in vitro estimation of metabolic stability and drug interactions of new chemical entities in drug discovery and development. Pharmacol Rep 58: 453-472, 2006.

Bennett WS Jr \& Steitz TA. Glucose-induced conformational change in yeast hexokinase. Proc Natl Acad Sci USA 75: 4848-4852, 1978.

Bindslev N. A homotropic two-state model and auto-antagonism. BMC Pharmacol 4: 11, 2004

Birnbaumer L, Swartz TL, Abramowitz J, Mintz PW \& Iyengar R. Transient and steady state kinetics of the interaction of guanyl nucleotides with the adenylyl cyclase system from rat liver plasma membranes. Interpretation in terms of a simple two-state model. J Biol Chem 255: 3542-3551, 1980.

Black JW \& Leff P. Operational models of pharmacological agonism. Proc R Soc Lond B 220: 141-162, 1983.

Blum JJ. The enzymatic interaction between myosin and nucleotides. Arch Biochem Biophys 55: 486-511, 1955.

Boeynaems JM \& Dumont JE. Quantitative analysis of the binding of ligands to their receptors. J Cyclic Nucleotide Res 1: 123-142, 1975.

Boeynaems JM \& Dumont JE. Outlines of Receptor Theory. Amsterdam: Elsevier, 1980.

Bond RA, Leff P, Johnson TD, Milano CA, Rockman HA, McMinn TR, et al. Physiological effects of inverse agonists in transgenic mice with myocardial overexpression of the beta 2-adrenoceptor. Nature 374: 272-276, 1995.

Botts J. Typical behavior of some simple models of enzyme action. Trans Faraday Soc 54: 593-604, 1958.

Botts J \& Morales M. Analytical description of the effects of modifiers and of enzyme multivalency upon the steady state catalyzed reaction rate. Trans Faraday Soc 49: 696-707, 1953.

Botts J \& Drain GF. An illustration of a kinetic analysis: the myosin B-ATP-EDTA system. The committee of muscle chemistry of Japan. Conference on the chemistry of muscular contraction (1957), Tokyo, Igaku Shoin. 1958.

Bruns RF. Conformational induction versus conformational selection: evidence from allosteric enhancers. Trend Pharmacol Sci 17: 189, 1996.

Buck M, Xu W \& Rosen MK. A two-state allosteric model for autoinhibition rationalizes WASP signal integration and targeting. J Mol Biol 338: 271-285, 2004.

Burgen AS. Conformational changes and drug action. Fed Proc 40: 2723-2728, 1981.

Chang Y \& Weiss DS. Allosteric activation mechanism of the alphalbeta2gamma2 gamma-aminobutyric acid type A receptor revealed by mutation of the conserved M2 leucine. Biophys J 77: 2542-2551, 1999.

Chang Y, Ghansah E, Chen Y, Ye J \& Weiss DS. Desensitization mechanism of GABA receptors revealed by single oocyte binding and receptor function. J Neurosci 22: 7982-7990, 2002.

Changeux J-P. The Physiology of Truth: Neuroscience and Human Knowledge (English ed.). Cambridge: Belkamp Press of Harvard University Press, 2004.
Changeux JP. Allosteric proteins: from regulatory enzymes to receptorspersonal recollections. Bioessays 15: 625-634, 1993.

Changeux JP \& Edelstein SJ. Allosteric mechanisms of signal transduction. Science 308: 1424-1428, 2005.

Chidiac P. Considerations in the evaluation of inverse agonism and protean agonism at $\mathrm{G}$ protein-coupled receptors. Methods Enzymol 343: 3-16, 2002.

Christopoulos A \& Kenakin T. G protein coupled receptor allosterism and complexing. Pharmacol Rev 54: 323-374, 2002.

Christopoulos A, Lanzafame A \& Mitchelson F. Allosteric interactions at muscarinic cholinoceptors. Clin Exp Pharmacol Physiol 25: 185194, 1998.

Clark AJ. General Pharmacology, Vol. 4, 1st ed. Berlin: Springer, 1937.

Clark RB, Knoll BJ \& Barber R. Partial agonists and G protein-coupled receptor desensitization. Trends Pharmacol Sci 20: 279-286, 1999.

Clarke WP \& Bond RA. The elusive nature of intrinsic efficacy. Trend Pharmacol Sci 19: 270-276, 1998.

Colquhoun D. Affinity, efficacy and receptor classification: is the classical theory still useful? In: Perspectives on Hormone Receptor Classification, edited by Black JW, Jenkinson DH \& Gerskowitch VP. New York: Alan R. Liss Inc., 1987.

Colquhoun D. Binding, gating, affinity and efficacy: the interpretation of structure-activity relationships for agonists and of the effects of mutating receptors. Br J Pharmacol 125: 924-947, 1998.

Colquhoun D. What have we learned from single ion channels? J Physiol 581: 425-427, 2007.

Colquhoun D \& Farrant M. Molecular pharmacology. The binding issue. Nature 366: 510-511, 1993.

Costa T \& Herz A. Antagonists with negative intrinsic activity at delta opioid receptors coupled to GTP binding proteins. Proc Natl Acad Sci USA 86: 7321-7325, 1989.

Cymes GD, Grosman C \& Auerbach A. Structure of the transition state of gating in the acetylcholine receptor channel pore: a phi-value analysis. Biochemistry 41: 5548-5555, 2002.

De Haën C. The nonstoichiometric floating receptor model for hormone sensitive adenylyl cyclase. J Theor Biol 58: 383-400, 1976.

De Lean A, Stadel JM \& Lefkowitz RJ. A ternary complex model explains the agonist specific binding properties of the adenylate cyclase coupled beta-adrenergic receptor. J Biol Chem 255: 71087117,1980

del Castillo J \& Katz B. Interaction at end-plate receptors between different choline derivatives. J Physiol 146: 369-381, 1957.

Del Carmine R, Molinari P, Sbraccia M, Ambrosio C \& Costa T. 'Induced-fit' mechanism for catecholamine binding to the beta2adrenergic receptor. Mol Pharmacol 66: 356-363, 2004.

Downing SS, Lee YT, Farb DH \& Gibbs TT. Benzodiazepine modulation of partial agonist efficacy and spontaneously active GABA(A) receptors supports an allosteric model of modulation. $\mathrm{Br} J$ Pharmacol 145: 894-906, 2005.

Durroux T. Principles: a model for the allosteric interactions between ligand binding sites within a dimeric GPCR. Trends Pharmacol Sci 26: 376-384, 2005.

Edelstein SJ. Cooperative interactions of hemoglobin. Annu Rev Biochem 44: 209-232, 1975.

Eigen M. Kinetics of reaction control and information transfer in enzymes and nucleic acids, In: Fast Reactions and Primary Processes in Chemical Kinetics, edited by Claesson S. Nobel Symposium 5, 1st ed. Stockholm: Almquist \& Wiksell, 1967.

Ellis J, Warburton P, Donnelly D \& Balmforth AJ. Conformational induction is the key process for activation of the AT1 receptor. Biochem Pharmacol 71: 464-471, 2006.

Eyring H. The activated complex in chemical reactions. J Chem Phys 3: 107-115, 1935.

Fatt P. The electromotive action of acetylcholine at the motor endplate. J Physiol (Lond.) 111: 408-422, 1950. 
Feng YH, Zhou L, Qiu R \& Zeng R. Single mutations at Asn295 and Leu305 in the cytoplasmic half of transmembrane alpha-helix domain 7 of the AT1 receptor induce promiscuous agonist specificity for angiotensin II fragments: a pseudo-constitutive activity. Mol Pharmacol 68: 347-355, 2005.

Fersht A. Structure and Mechanism in Protein Science. A Guide to Enzyme Catalysis and Protein Folding. New York: WH Freeman \& Co., 1999.

Fetler L, Kantrowitz ER \& Vachette P. Direct observation in solution of a preexisting structural equilibrium for a mutant of the allosteric aspartate transcarbamoylase. Proc Natl Acad Sci USA 104: 495-500, 2007.

Fong TM. Mechanistic hypotheses for the activation of G-proteincoupled receptors. Cell Signal 8: 217-224, 1996.

Frieden C. Kinetic aspects of regulation of metabolic processes. The hysteretic enzyme concept. J Biol Chem 245: 5788-5799, 1970.

Furchgott RF. The pharmacology of vascular smooth muscle. Pharmacol Rev 7: 183-265, 1955.

Furchgott RF. The use of $\beta$-haloalkylamines in the differentiation of receptors and in the determination of dissociation constants of receptor-agonist complexes. Adv Drug Res 3: 21-55, 1966.

Gero A. Desensitization, two-state receptors and pharmacological parameters. J Theor Biol 103: 137-161, 1983.

Gether U. Uncovering molecular mechanisms involved in activation of G protein-coupled receptors. Endocr Rev 21: 90-113, 2000.

Gether U \& Kobilka BK. G protein-coupled receptors. II. Mechanism of agonist activation. J Biol Chem 273: 17979-17982, 1998.

Gibb AJ. Receptors linked to ion channels: activation and block. In: Textbook of Receptor Pharmacology, edited by Foreman JC \& Johansen T. Boca Raton: CRC Press, 1996.

Giraldo J. Agonist induction, conformational selection, and mutant receptors. FEBS Lett 256: 13-18, 2004.

Giraldo J, Roche D, Rovira X and Serra J. The catalytic power of enzymes: conformational selection or transition state stabilization? FEBS Lett 580: 2170-2177, 2006.

Glasstone S, Laidler KJ \& Eyring H. Theory of Rate Processes, 1st ed. New York: McGraw-Hill Book Co., 1941.

Glynn IM. The ionic permeability of the red cell membrane. Prog Biophys 8: 241-307, 1955.

Gonzalez-Lebrero RM, Kaufman SB, Montes MR, Norby JG, Garrahan PJ \& Rossi RC. The Occlusion of $\mathrm{Rb}(+)$ in the $\mathrm{Na}(+) / \mathrm{K}(+)-$ ATPase. I. The identity of occluded states formed by the physiological or the direct routes: occlusion/deocclusion kinetics through the direct route. J Biol Chem 277: 5910-5921, 2002.

Granier S, Kim S, Shafer AM, Ratnala VR, Fung JJ, Zare RN, et al. Structure and conformational changes in the C-terminal domain of the beta2-adrenoceptor: insights from fluorescence resonance energy transfer studies. J Biol Chem 282: 13895-13905, 2007.

Gutfreund H. Kinetics for the Life Sciences. Receptors, Transmitters and Catalysts, 1st ed. Cambridge: Cambridge University Press, 1995.

Haber JE \& Koshland DE Jr. Relation of protein subunit interactions to the molecular species observed during cooperative binding of ligands. Proc Natl Acad Sci USA 58: 2087-2093, 1967.

Halford SE. Escherichia coli alkaline phosphatase. Relaxation spectra of ligand binding. Biochem J 126: 727-738, 1972.

Hall DA. Modeling the functional effects of allosteric modulators at pharmacological receptors: an extension of the two-state model of receptor activation. Mol Pharmacol 58: 1412-1423, 2000.

Hille B. Ionic Channels of Excitable Membranes, 3rd ed. Sunderland: Sinauer Associates, 2001

Hodgkin AL \& Huxley AF. A quantitative description of membrane current and its application to conduction and excitation in nerve. J Physiol (Lond.) 117: 500-544, 1952.

Hoyer D \& Boddeke HW. Partial agonists, full agonists, antagonists: dilemmas of definition. Trends Pharmacol Sci 14: 270-275, 1993.
Hull WE, Halford SE, Gutfreund H \& Sykes BD. ${ }^{31} \mathrm{P}$ nuclear magnetic resonance study of alkaline phosphatase: the role of inorganic phosphate in limiting the enzyme turnover rate at alkaline $\mathrm{pH}$. Biochemistry 15: 1547-1561, 1976.

Hunyady L, Vauquelin G \& Vanderheyden P. Agonist induction and conformational selection during activation of a G-proteincoupled receptor. Trends Pharmacol Sci 24: 81-86, 2003.

Iyengar R, Abramowitz J, Bordelon-Riser M \& Birnbaumer L. Hormone receptor-mediated stimulation of adenylyl cyclase systems. Nucleotide effects and analysis in terms of a simple two-state model for the basic receptor-affected enzyme. J Biol Chem 255: 3558-3564, 1980.

Jacobs S \& Cuatrecasas P. The mobile receptor hypothesis and 'cooperativity' of hormone binding. Application to insulin. Biochim Biophys Acta 433: 482-495, 1976.

Jenkinson DH. How we describe competitive antagonists: three questions of usage. Trends Pharmacol Sci 12: 53-54, 1991.

Kameda T. Importance of sequence specificity for predicting protein folding pathways: perturbed Gaussian chain model. Proteins 53: 616-628, 2003.

Katz B \& Thesleff S. A study of the desensitization produced by acetylcholine at the motor end-plate. J Physiol 138: 63-80, 1957.

Kenakin T. Agonists, partial agonists, inverse agonists and agonist/ antagonists? Trend Pharmacol Sci 8: 423-426, 1987.

Kenakin T. On the definition of efficacy. Trends Pharmacol Sci 15: 408409, 1994.

Kenakin T. Pharmacological Proteus? Trend Pharmacol Sci 16: 256-258, 1995a.

Kenakin T. Agonist-receptor efficacy. I: Mechanisms of efficacy and receptor promiscuity. Trends Pharmacol Sci 16: 188-192, 1995b.

Kenakin T. Receptor conformational induction versus selection: all part of the same energy landscape. Trend Pharmacol Sci 17: 190191, 1996.

Kenakin T. Pharmacological Analysis of Drug-receptor Interaction, 3rd ed. NY: Raven Press, 1997.

Kenakin T. Drug efficacy at G protein-coupled receptors. Annu Rev Pharmacol Toxicol 42: 349-379, 2002.

Kenakin T. Efficacy as a vector: the relative prevalence and paucity of inverse agonism. Mol Pharmacol 65: 2-11, 2004.

Kenakin T. Functional Selectivity through Protean and Biased Agonism: Who Steers the Ship? Mol Pharmacol 72: 1393-1401, 2007.

Kenakin T \& Onaran HO. The ligand paradox between affinity and efficacy: can you be there and not make a difference? Trends Pharmacol Sci 23: 275-280, 2002.

Kenakin T, Morgan P \& Lutz M. On the importance of the 'antagonist assumption' to how receptors express themselves. Biochem Pharmacol 50: 17-26, 1995

Kenakin T, Morgan P, Lutz M \& Weiss J. The evolution of drugreceptor models: the cubic ternary complex model for $\mathrm{G}$ protein-coupled receptors. In: Kenakin T \& Angus JA, editors. The Pharmacology of Functional, Biochemical, and Recombinant Receptor Systems, 1st ed. Berlin: Springer Verlag, 2000.

Kobilka BK. Agonist-induced conformational changes in the beta2 adrenergic receptor. J Pept Res 317-321, 2002.

Kobilka BK \& Gether U. Use of fluorescence spectroscopy to study conformational changes in the beta 2-adrenoceptor. Methods Enzymol 343: 170-182, 2002.

Koshland DE Jr. Application of a theory of enzyme specificity to protein synthesis. Proc Natl Acad Sci USA 44: 98-104, 1958.

Koshland DE Jr. Crazy, but correct. Nature 432: 447, 2004.

Koshland DE Jr, Nemethy G \& Filmer D. Comparison of experimental binding data and theoretical models in proteins containing subunits. Biochemistry 5: 365-385, 1966

Kukkonen JP, Nasman J \& Akerman KE. Modelling of promiscuous receptor-Gi/Gs-protein coupling and effector response. Trends Pharmacol Sci 22: 616-622, 2001 
Kumar S, Ma B, Tsai C-J, Sinha N \& Nussinov R. Folding and binding cascades: dynamic landscapes and population shifts. Protein Sci 9: 10-19, 2000.

Laidler KJ and Bunting PS. The chemical kinetics of enzyme action. Oxford: Oxford University Press, 1973.

Le MT, Vanderheyden PM, Szaszak M, Hunyady L, Kersemans V \& Vauquelin G. Peptide and nonpeptide antagonist interaction with constitutively active human AT1 receptors. Biochem Pharmacol 65: 1329-1338, 2003.

Leff P. The two state model of receptor activation. Trends Pharmacol Sci 16: 89-97, 1995.

Leff P \& Scaramellini C. Promiscuity, pre-coupling and instability. Trends Pharmacol Sci 19: 13, 1998.

Leff P, Scaramellini C, Law C \& McKechnie K. A three-state receptor model of agonist action. Trends Pharmacol Sci 18: 355-362, 1997.

Lefkowitz RJ, Cotecchia S, Samama P \& Costa T. Constitutive activity of receptors coupled to guanine nucleotide regulatory proteins. Trends Pharmacol Sci 14: 303-307, 1993.

Lu ZL, Gallagher R, Sellar R, Coetsee M \& Millar RP. Mutations remote from the human gonadotropin-releasing hormone (GnRH) receptor-binding sites specifically increase binding affinity for GnRH II but not GnRH I: evidence for ligand-selective, receptoractive conformations. J Biol Chem 280: 29796-29803, 2005.

Lutz M \& Kenakin T. Quantitative Molecular Pharmacology and Informatics in Drug Discovery, 1st ed. Chichester: Wiley \& Sons, 1999.

MacKay D. A critical survey of receptor theories of drug action. In: Kinetics of Drug Action, edited by van Rossum JM. Berlin: SpringerVerlag, 1977.

Milligan G \& Bond RA. Inverse agonism and the regulation of receptor number. Trends Pharmacol Sci 18: 468-474, 1997.

Milligan G, Bond RA \& Lee M. Inverse agonism: pharmacological curiosity or potential therapeutic strategy? Trends Pharmacol Sci 16: 10-13, 1995.

Monod J, Wyman J \& Changeux J-P. On the nature of allosteric transitions: a plausible model. J Mol Biol 12: 88-118, 1965.

Nickerson M. Receptor occupancy and tissue response. Nature 178: 697-698, 1956.

Onaran HO \& Costa T. Agonist efficacy and allosteric models of receptor action. Ann N Y Acad Sci 812: 98-115, 1997.

Paton WDM \& Rothschild AM. The effect of varying calcium concentration on the kinetic constants of hyoscine and mepyramine antagonism. Br J Pharmacol 24: 432-448, 1965.

Peleg G, Ghanouni P, Kobilka BK \& Zare RN. Single-molecule spectroscopy of the beta(2) adrenergic receptor: observation of conformational substates in a membrane protein. Proc Natl Acad Sci USA 98: 8469-8474, 2001.

Perutz M. X-ray analysis of haemoglobin. Nature 149: 491-494, 1942.

Rang HP. The receptor concept: pharmacology's big idea. $\mathrm{Br} J$ Pharmacol 147: S9-S16, 2006.

Roberts AG \& Atkins WM. Energetics of heterotropic cooperativity between alpha-naphthoflavone and testosterone binding to CYP3A4. Arch Biochem Biophys 463: 89-101, 2007.

Robertson MJ, Dougall IG, Harper D, McKechnie KC \& Leff P. Agonistantagonist interactions at angiotensin receptors: application of a two-state receptor model. Trends Pharmacol Sci 15: 364-369, 1994.

Roughton FJW, Otis AB \& Lyster RLJ. The determination of the individual equilibrium constants of the four intermediate reactions between oxygen and sheep haemoglobin. Proc Roy Soc B 144: 29-54, 1955.

Rusch D \& Forman SA. Classic benzodiazepines modulate the openclose equilibrium in alphalbeta2gamma2L gamma-aminobutyric acid type A receptors. Anesthesiology 102: 783-792, 2005.

Rusch D, Zhong H \& Forman SA. Gating allosterism at a single class of etomidate sites on alphalbeta2gamma2L GABA A receptors accounts for both direct activation and agonist modulation. J Biol Chem 279: 20982-20992, 2004.
Samama P, Cotecchia S, Costa T \& Lefkowitz RJ. A mutation induced activated state of the beta 2adrenergic receptor. Extending the ternary complex model. J Biol Chem 268: 4625-4636, 1993.

Scaramellini C \& Leff P. A three-state receptor model: predictions of multiple agonist pharmacology for the same receptor type. Ann $N$ Y Acad Sci 861: 97-103, 1998.

Scaramellini C \& Leff P. Theoretical implications of receptor coupling to multiple $\mathrm{G}$ proteins based on analysis of a three-state model. Methods Enzymol 343: 17-29, 2002.

Schwartz TW \& Holst B. Ago-allosteric modulation and other types of allostery in dimeric 7TM receptors. J Recept Signal Transduct Res 26: 107-128, 2006.

Segel IH. Enzyme kinetics. Behavior and Analysis of Rapid Equilibrium and Steady-state Enzyme Systems. New York: Wiley \& Sons (reissued 1993), 1975.

Shaw. Na-pump (cited in Glynn 1955 op. cit.). Dissertation, Cambridge, Cambridge University, 1954.

Shea LD, Neubig RR \& Linderman JJ. Timing is everything the role of kinetics in G protein activation. Life Sci 68: 647-658, 2000.

Sosa-Peinado A \& Gonzalez-Andrade M.Site-directed fluorescence labeling reveals differences on the R-conformer of glucosamine 6-phosphate deaminase of Escherichia coli induced by active or allosteric site ligands at steady state. Biochemistry 44: 15083-15092, 2005.

Stephenson RP. A modification of receptor theory. BrJ Pharmacol 11: 379-393, 1956.

Strange PG. Three-state and two-state models. Trends Pharmacol Sci 19: $85-86,1998$.

Strange PG. Mechanisms of inverse agonism at G-protein-coupled receptors. Trends Pharmacol Sci 23: 89-95, 2002.

Surya A, Stadel JM \& Knox BE. Evidence for multiple, biochemically distinguishable states in the $\mathrm{G}$ protein-coupled receptor, rhodopsin. Trends Pharmacol Sci 19: 243-247, 1998.

Swaminath G, Xiang Y, Lee TW, Steenhuis J, Parnot C \& Kobilka BK. Sequential binding of agonists to the beta2 adrenoceptor. Kinetic evidence for intermediate conformational states. J Biol Chem 279: 686-691, 2004.

Swaminath G, Deupi X, Lee TW, Zhu W, Thian FS, Kobilka TS, et al. Probing the beta2 adrenoceptor binding site with catechol reveals differences in binding and activation by agonists and partial agonists. J Biol Chem 280: 22165-22171, 2005.

Thesleff S. The effects of acetylcholine, decamethonium and succinylcholine on neuromuscular transmission in the rat. Acta Physiol Scand 34: 386-392, 1955

Tolkovsky AM \& Levitzki A. Theories and predictions of models describing sequential interactions between the receptor, the GTP regulatory unit, and the catalytic unit of hormone dependent adenylate cyclases. J Cyclic Nucleotide Res 7: 139-150, 1981.

Tonomura Y \& Watanabe S. Effect of adenosine triphosphate on the light-scattering of actinomysin solution. Nature 169: 112-113, 1952.

Tucek S, Michal P \& Vlachova V. Modelling the consequences of receptor-G-protein promiscuity. Trends Pharmacol Sci 23: 171-176, 2002.

Urban JD, Clarke WP, von Zastrow M, Nichols DE, Kobilka B, Weinstein H, Javitch JA, Roth BL, Christopoulos A, Sexton PM, Miller KJ and Mailman RB. Functional selectivity and classical concepts of quantitative pharmacology. J Pharmacol Exp Ther 320: $1-13,2007$.

van Rossum JM. Limitations of molecular pharmacology. Some implications of the basic assumptions underlying calculations on drug-receptor interactions and the significance of biological drug parameters. Adv Drug Res 3: 189-234, 1966.

Venter DP. Efficacy. I: A new method for estimating relative efficacy of full agonists via a newly defined efficacy related parameter. Eur J Pharmacol 320: 223-231, 1997. 
Wang J, Stieglitz KA, Cardia JP \& Kantrowitz ER. Structural basis for ordered substrate binding and cooperativity in aspartate transcarbamoylase. Proc Natl Acad Sci USA 102: 8881-8886, 2005.

Wang Y, McMahon AP \& Allen BL. Shifting paradigms in Hedgehog signaling. Curr Opin Cell Biol 19: 159-165, 2007.

Watanabe S, Tonomura Y \& Shiokawa H. Mechanism of muscular contraction. II. Kinetic studies on muscle ATP-ase. J Biochem 40: 387-402, 1953.

Weiss JN. The Hill equation revisited: uses and misuses. FASEB J 11: 835-841, 1997.

Weiss JM, Morgan PH, Lutz MW \& Kenakin TP. The Cubic Ternary Complex Receptor-Occupancy Model I. Model description. J Theor Biol 178: 151-167, 1996.

Weiss JM, Morgan PH, Lutz MW \& Kenakin TP. The Cubic Ternary Complex Receptor-Occupancy Model II. Understanding apparent affinity. J Theor Biol 178: 169-182, 1996.
Weiss JM, Morgan PH, Lutz MW \& Kenakin TP. The Cubic Ternary Complex Receptor-Occupancy Model III. Resurrecting efficacy. J Theor Biol 181: 381-397, 1996.

Whaley BS, Yuan N, Birnbaumer L, Clark RB \& Barber R. Differential expression of the beta-adrenergic receptor modifies agonist stimulation of adenylyl cyclase: a quantitative evaluation. Mol Pharmacol 45: 481-489, 1994.

Wyman J Jr. Heme proteins. Adv Protein Chem 4: 430-438, 1948.

Wyman J \& Allen DW. The problem of the heme interactions in hemoglobin and the basis of the Bohr effect. J Polym Sci 7: 499 518, 1951.

Yao X, Parnot C, Deupi X, Ratnala VR, Swaminath G, Farrens D and Kobilka B. Coupling ligand structure to specific conformational switches in the beta2-adrenoceptor. Nat Chem Biol 2: 417-422, 2006. 


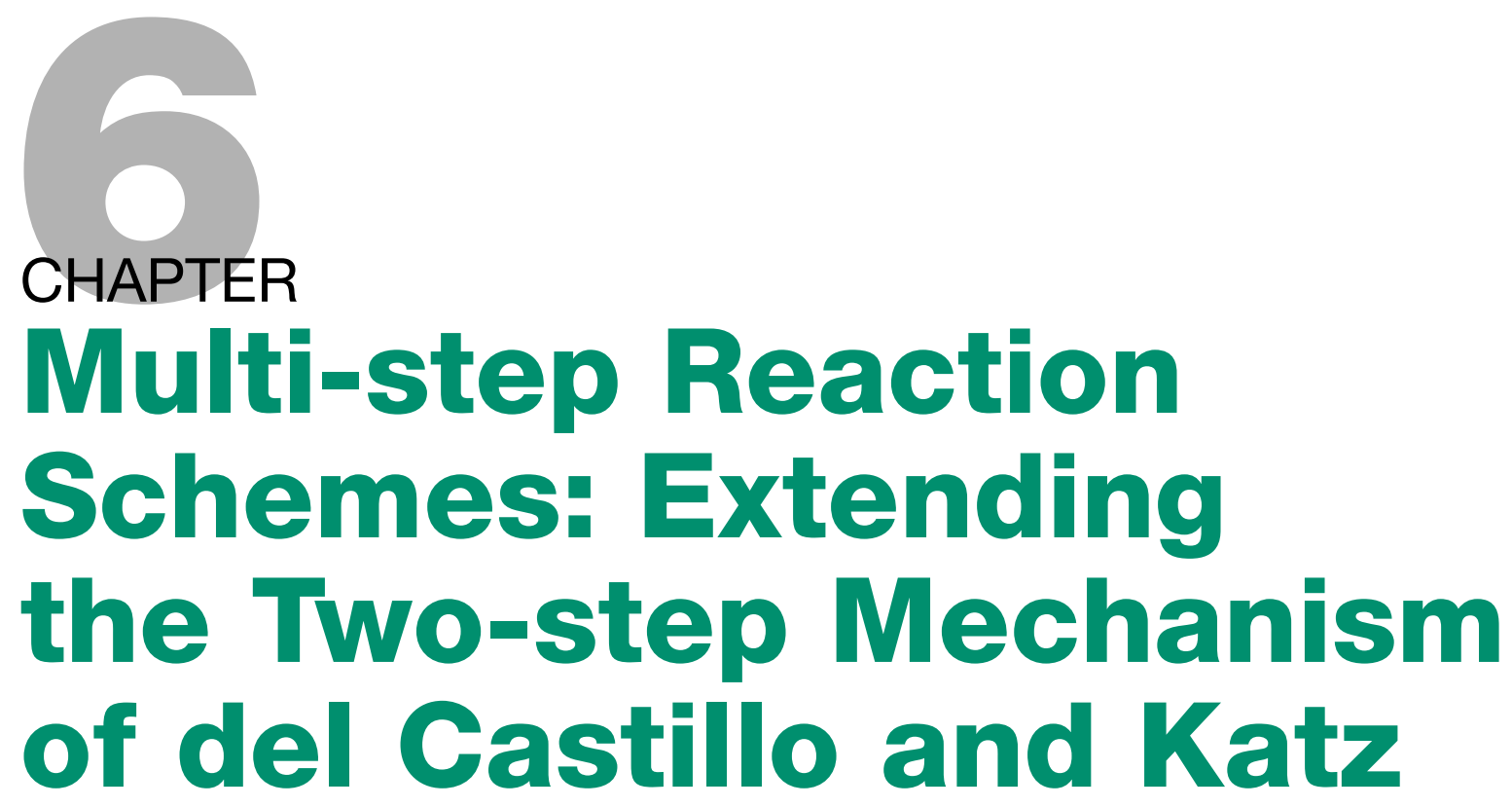

Logically we should continue with complex models, that is, the models with at least two-states for the un-liganded receptive unit. However, in this chapter, I will look at classical reaction schemes as the random, ordered and ping-pong mechanisms (Cornish-Bowden 2004).

\subsection{Extending del Castillo-Katz or Random and Ordered Models}

\subsubsection{Two-sites-and-more-than-one-step Agonism. Extended Reaction Schemes}

The del Castillo and Katz (dC\&K) model (described in Chapter 5) for data analysis and evaluation is employed in the field of transporters, receptors, enzymes, and other acceptor molecules.

Mechanistic investigation of ligand-gated ion channels is popular, since their response seems to be directly proportional to receptor activation by a 'supposedly twostep mechanism', only consisting of a binding and an activation step, a pure dC\&K reaction scheme. Therefore, the dC\&K model in Figs. 5.1A+B, 5.2 and 5.3B has become a prototype model for substrates at enzymes and drug interactions at ligand-gated ion channels (Weiss 1997; Hille 2001; Jenkinson 2003; Colquhoun 2006a,b).

However, there are variations on the theme. Some channels need two identical ligands to bind before they will open. Examples of this are the hetero-multimeric

cation transporters $\mathrm{nAChR}, 5-\mathrm{HT}_{3}-\mathrm{R}$, and ionotropic glutamateR channels, as well as the $\mathrm{GABA}_{\mathrm{A}}-\mathrm{R}$ channel, which transports anions (Changeux \& Edelstein 2005). Other channels need the presence of two different ligands to open, a mechanism called co-agonism (Johnson \& Ascher 1987; Corsi et al. 1996; Jenkinson 2003, pp. 63-64). Thus, for full function in certain systems, ternary complexation is necessary with simultaneous binding of two ligands, either identical or different, to one channel molecule, and this is covered by extended versions of the dC\&K model.

In a similar fashion, signal-transduction also requires at least ternary complexation to function through heteromultimeric complexed receptors (GPCRs) coupled to hetero-trimeric $\mathrm{G}$ protein (Pin et al. 2007), through the small G protein signaling systems (Bustelo et al. 2007), and through two-component signaling in bacteria (Hoch 1995; Bekker et al. 2006; Mitrophanov et al. 2006). But these processes differ from that of the channels. In the $G$ protein signaling pathway, activation involves at least a three-component complex: a ligand, a receptor, and a $\mathrm{G}$ protein containing a GDP molecule, with a minimum of three steps: (1) G protein binding to receptor, (2) ligand binding to receptor-G protein complex, forming a ternary complex of ligand-receptor-G protein, and (3) an activation step of the receptor to a new state, where the words 'activation step' cover a mechanism of a conformational change allowing an exchange of GDP for GTP. After receptor activation, the $G$ protein is released and 

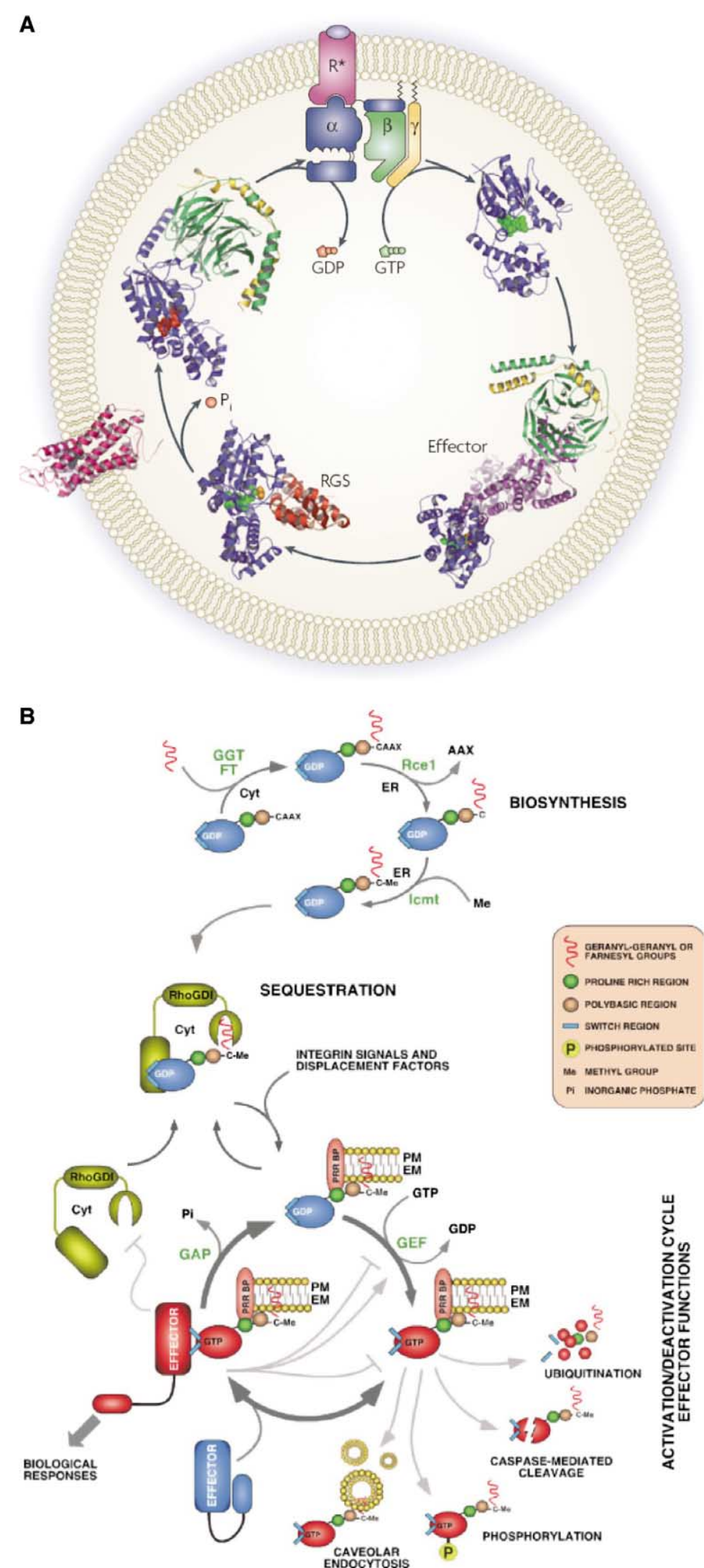

Figure 6.1. Cycles of $G$ protein from reactive to active and back again. Panel $A$ of a hetero-trimeric $G$ protein is from Oldham and Hamm (2008, Fig. 1). Panel B of a small G protein is from Bustelo et al. (2007, Fig. 2). Both with permission. 
with its GTPase activity switches to containing a GDP molecule, which turns the $G$ protein activation off. The above step (1) in this scheme, the re-association of the GDP-form of a $G$ protein $\alpha$-subunit with the receptor is sometimes the rate-limiting step although it varies from system to system (Cassel \& Seelig 1976; Sagoo \& Lagnado 1997; Tolkowsky \& Levitzki 1981; Whaley et al. 1994; Oldham \& Hamm 2006; Sprang et al. 2007; Lohse et al. 2008). The one-way irreversible activation and turn off transduction cycle for $\mathrm{G}$ protein signaling is cartooned for GPCRs and small G proteins in Fig. 6.1. Nonreversibility in the cycles is obtained by an energy input from the GTP to GDP conversion. Thus, the G protein cycle also requires an extended form of the dC\&K model.

Furthermore, there are additional variations on the theme. Hetero-multimeric GPCRs possesing modulator sites (Pin et al. 2007) must be modeled by higher than ternary complexation (Parmentier et al. 2002; Pin et al. 2005).

In the enzyme field, the lactate dehydrogenase and the chymotrypsin enzymes are classical examples of enzymes that catalytically have intermediate ternary complexes (Cleland 1963; Cleland et al. 1998). Catalysis by lactate dehydrogenase follows a so-called ordered reaction scheme, while for instance that of creatine kinase follows a random reaction scheme with ternary complexation. Both catalytic processes may be described by slightly extended forms of the dC\&k scheme (see Section 6.1.3).

Let us look at some simple examples of mechanisms extending the dC\&K reaction scheme with two binding sites and involving more than two receptor conformations, equal to at least two steps in the reaction. Observe, however, that compared with the cyclic two-state model in Chapter 5, these reaction schemes, including the simple dC\&K model, are not genuine two-state models if they do not include two different conformations of an un-liganded receptive unit.

\subsubsection{Two Sites, an Activation Step, and a Single Type of Ligand}

Some receptor channels have two binding sites for full activation. Both sites must be occupied simultaneously by two identical ligands for the channels to open. The current of ions through nAChR-, glutamateR- (iGluR), and $5-\mathrm{HT}_{3}-\mathrm{R}$ channels is carried by ions including $\mathrm{Na}^{+}$, $\mathrm{K}^{+}$, and $\mathrm{Ca}^{2+} \cdot \mathrm{Na}^{+}$and $\mathrm{Ca}^{2+}$ ions flow from the outside to the inside. This movement of either $\mathrm{Na}^{+}$or $\mathrm{Ca}^{2+}$ ions is generally excitatory and leads to depolarization of cells. The $\mathrm{K}^{+}$ion flows from the inside to the outside, hyperpolarizes and stabilizes the electrical resting potential of the plasma membrane. $\mathrm{Cl}^{-}$ions, somewhat conduced with $\mathrm{HCO}_{3}^{-}$ions, are the major carriers of charge through the $\mathrm{GABA}_{\mathrm{A}}-\mathrm{R}$ and glycine receptor channels. Frequently, this $\mathrm{Cl}^{-}$current results in a clamping at, or return to, the resting membrane potential in cells, e.g., in the form of inhibitory postsynaptic potentials (IPSPs).

In the proteins with ligand controlled opening and closing of the channel pore, channel gating, the reaction scheme may be formulated as:

$$
\mathrm{R}+2 \mathrm{~S} \underset{k_{-1}}{\stackrel{k_{+1}}{\rightleftharpoons}} \mathrm{R}^{\prime} \mathrm{S}+\mathrm{S} \underset{k_{-2}}{\stackrel{k_{+2}}{\rightleftharpoons}} \mathrm{R}^{\prime \prime} \mathrm{S}_{2} \underset{\alpha}{\stackrel{\beta}{\rightleftharpoons}} \mathrm{R}^{*} \mathrm{~S}_{2}
$$

where the $k$ symbols stand for rate constants for association and dissociation, parameter $\beta$ is the rate constant for channel opening, and parameter $\alpha$ is a rate constant for its closing (Gibb 1996; Hille 2001, pp. 191192; Cymes et al. 2002; Jenkinson 2003). The ratio $\beta / \alpha$ ( $=L^{2 \mathrm{~S}}$, see Sections 6.2.1 and 6.3.1) is a conformational constant when two agonists have bound to the receptor. It is a forward isomerizaton constant. In this reaction scheme the receptive unit appears in four different conformations: $\mathrm{R}, \mathrm{R}^{\prime} \mathrm{S}, \mathrm{R}^{\prime \prime} \mathrm{S}_{2}$, and $\mathrm{R}^{*} \mathrm{~S}_{2}$.

The above reaction scheme may be considered either as an extension of the one-site dC\&K model with an extra binding site or as a two-sited random/ordered process extended with an isomerization step. Random and ordered processes are described in Section 6.1.3.

In the following derivation of equations for this and related reaction schemes, I return to dissociation constants instead of association constants.

\subsubsection{Random and Ordered Reaction Schemes}

In certain contexts, the term 'sequential' is used for processes where at least two ligands must be bound simultaneously for a reaction to occur. The sequential process may further be divided into two types of mechanisms - random and ordered. In the random type sequential process, two ligands can bind in either order before the second ligand binds (Fig. 6.2A), while in the ordered type sequential process one ligand always binds to a certain site before a second ligand binds to the other site (Fig. 6.2B).

Random concentration-binding models are equal to reaction schemes described by Tuk and van Oostenbrugge (1996) as well as one of the models by Weiss (1997, so-called 'independent'), where ligands can bind with equal frequency to either of the empty sites and dissociate with equal probability from occupied sites (Fig. 6.2A). An ordered reaction scheme, equal to a so-called 'dependent' concentration-binding model with non-random binding, is another model detailed by Weiss (1997), so-called 'sequential' in his terminology (Fig. 6.2B).

Thus, for channel gating, the ligand binding may be in random order at two sites, designated an independent reaction scheme, or for channel-passage of ions as seen in single-file systems, the binding at several sites may be 
Sequential models

A Random

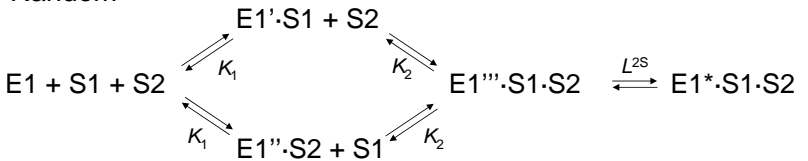

B Ordered

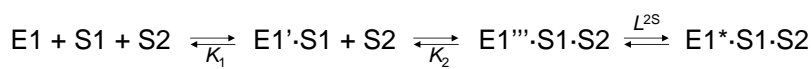

Figure 6.2. Simple forms of the random and ordered reaction schemes. Both reaction schemes have varying dissociation constants for sequential binding steps, $K_{1}$ may be different from $K_{2}$, and the schemes are therefore 'sequential'.

in an ordered fashion, also designated a dependent reaction scheme (Weiss 1997). Both the random and ordered reaction schemes may be extended with an isomerization step as depicted in the reaction schemes in Fig. 6.2. These are two-site three-step mechanisms.

In the random scheme in Fig. 6.2A, a factor 2 will appear at the rate constants $k_{+1}$ and $k_{-2}$ as their rates result from the assumption that the two sites, prior to agonist binding, are equally prepared for reception of the ligand (see Section 6.2.1). Thus, the probability for binding in the random reaction scheme is double that for binding in the ordered reaction scheme (Fig 6.2B). In addition, this is true for functional studies and binding studies.

\subsubsection{When is 'Sequential' Sequential?}

The term 'sequential' is used about reaction schemes where dissociation constants are not necessarily identical from binding step to binding step. 'Sequential' is used as a term with this meaning for a classical distinction between multi-step binding models, as for instance differentiating between the $\mathrm{KNF}$ and MWC models (see Chapter 15). Thus, Adair's scheme is a sequential process (Adair 1925), and both the ordered and random schemes mentioned above and detailed below, which are simple variations on the Adair model, are also sequential in that their first dissociation constant, $K_{1}$, may differ from the next constant, $K_{2}$. As an example, Kobilka's lab now assumes this kind of sequential process for GPCR-signaling (Swaminath et al. 2004).

If we accept the KNF term 'sequential' in allostery as a term differentiating Koshland synagic reaction schemes from models with identical dissociation constants between binding steps, assumed in the MWC model (see Chapter 15), bear in mind that this may be confused with the term 'sequential' in relation to other processes mentioned above (Weiss 1997; Weiner 2006). In addition, 'sequential' for multi-step mechanisms in Weiss' schemes, meaning ordered reaction (Weiss 1997), does not cover the same ideas as for the multi-step

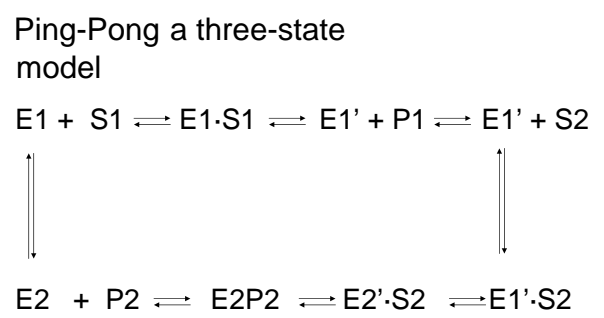

Figure 6.3. One form of the ping-pong reaction scheme. In principle, ping-pong schemes include a minimum of a twostate model. Here with three unliganded receptor conformations, $\mathrm{E} 1, \mathrm{E} 1^{\prime}$, and $\mathrm{E} 2$, it is a three-state model.

models by Koshland et al. (1966), namely, that dissociation constants may differ from step to step. Here the term 'sequential' will be used in the classical sense.

\subsubsection{Ping-pong Mechanisms}

The random and ordered processes may be contrasted with a reaction scheme denoted 'ping-pong' where again two ligands are involved in a coupled reaction, but where first one ligand is bound to and released from an effector, followed by binding of another ligand and its release before the effector returns to its initial conditions (Fig. 6.3). The ping-pong scheme is considered by some as 'non-sequential' (Segel 1975, 1993). The transaminase-catalyzed reaction is a typical ping-pong mechanism (Coomes 2006; Weiner 2006). Observe that ping-pong mechanisms are often also two-state or multistate mechanisms (Fig. 6.3).

Cornish-Bowden (1995, Chapter 6; 2004, Chapter 7) has an excellent discussion of the validity of these models.

The mathematical formulation of the ordered and the random reaction schemes are detailed in the following Sub-chapters.

\subsection{Random Reaction Schemes and Extended with Isomerization}

\subsubsection{The Random Two-site Three-step Reaction Scheme}

As described for the reaction scheme above with channel gating, a two-site and three-step random mechanism seems to be operating. For this random reaction scheme the following receptor types exist:

$\mathrm{R} \quad$ empty reactive receptor

$\mathrm{R}^{\prime} \mathrm{S}=2 \cdot \mathrm{R} \cdot \mathrm{S} / K_{1} \quad$ one agonist bound to reactive $\mathrm{R}$

$\mathrm{R}^{\prime \prime} \mathrm{S}_{2}=\left[2 \cdot \mathrm{R} \cdot \mathrm{S} / K_{1}\right]$ two identical agonists bound to $\cdot\left[\mathrm{S} / 2 \cdot K_{2}\right] \quad$ reactive $\mathrm{R}$

$\mathrm{R} * \mathrm{~S}_{2}=L^{2 \mathrm{~S}} \cdot \mathrm{R} \quad$ two identical agonists bound to $\cdot \mathrm{S}^{2} /\left(K_{1} \cdot K_{2}\right) \quad$ active $\mathrm{R}^{*}$ 
Receptor symbol 'R' (or ' $E$ ' for enzyme) may also indicate the mass of a particular receptor (or enzyme) conformation. Actually the random reaction scheme in Fig. 6.2A is part of the homotropic two-state model (HOTSM) described in Chapter 7 and shown in Figs. 5.10 and 7.1B. System constants listed here have their pendant in the HOTSM. Thus, $K_{1}=K_{\mathrm{ss}}=K_{\mathrm{ms}}=1 / A_{\mathrm{ss}}$, $K_{2}=K_{\mathrm{ss}} / c^{\prime}=1 /\left(A_{\mathrm{ss}} \cdot c^{\prime}\right)$, and $L^{2 \mathrm{~S}}=a \cdot b^{\prime} \cdot d^{\prime} \cdot L$. Note, that $K_{\mathrm{ss}}$ is equal to $K_{\mathrm{ms}}$, while $K_{1}$ is not necessarily equal to $K_{2}$ in the random reaction scheme. The random reaction scheme is also part of the FP-TSM reaction scheme (Fig. 5.9).

Several authors have argued that due to random access to two sites on the receptive unit, i.e., $\mathrm{R}^{\prime} \mathrm{S}+\mathrm{SR}^{\prime}$, the probability of the $\mathrm{R}^{\prime}$ conformation increases by a factor of 2 (Segel 1975, 1993; Cornish-Bowden 1995, 2004; Weiss 1997). Realize that $2 \cdot \mathrm{R}^{\prime} \mathrm{S}$ is the same as combining receptor conformations $\mathrm{R}^{\prime} \mathrm{S}$ and $\mathrm{SR}^{\prime}$, as the two steps to reach them are identical in the random reaction scheme. $\mathrm{SR}^{\prime}$ is the symbol for the receptive unit with a ligand bound at the modulator site, equal to SR, while the apostrophe indicates a change in $\mathrm{R}$ upon ligand binding. Compare this with the derivation of the HOTSM in Chapter 7, where two ligands can bind randomly and simultaneously as well. However, in this model there is no factor 2 .

Equating the random two-site three-step reaction scheme for functional studies gives us:

$$
\frac{\text { response }}{\text { total }}=\frac{\mathrm{S}^{2} \cdot L^{2 \mathrm{~S}} /\left(K_{1} \cdot K_{2}\right)}{1+2 \cdot \mathrm{S} / K_{1}+\mathrm{S}^{2} /\left(K_{1} \cdot K_{2}\right)+\mathrm{S}^{2} \cdot L^{2 \mathrm{~S}} /\left(K_{1} \cdot K_{2}\right)},
$$

when abbreviated comes to:

$$
\frac{\text { response }}{\text { total }}=\frac{\mathrm{S}}{\mathrm{S}+\frac{1}{L^{2 \mathrm{~S}}}\left[\mathrm{~S}+K_{2} \cdot\left(\frac{K_{1}}{\mathrm{~S}}+2\right)\right]},
$$

and for binding studies of the random two-site three-step reaction scheme, we obtain:

$$
\frac{\text { bound }}{\text { total }}=\frac{2 \cdot \mathrm{S} / K_{1}+2 \cdot \mathrm{S}^{2} /\left(K_{1} \cdot K_{2}\right)+2 \cdot \mathrm{S}^{2} \cdot L^{2 \mathrm{~S}} /\left(K_{1} \cdot K_{2}\right)}{1+2 \cdot \mathrm{S} / K_{1}+2 \cdot \mathrm{S}^{2} /\left(K_{1} \cdot K_{2}\right)+2 \cdot \mathrm{S}^{2} \cdot L^{2 \mathrm{~S}} /\left(K_{1} \cdot K_{2}\right)},
$$

when abbreviated gives us:

$$
\frac{\text { bound }}{\text { total }}=\frac{\mathrm{S}}{\mathrm{S}+\frac{K_{1} K_{2}}{2\left[K_{2}+\mathrm{S}+\mathrm{S} \cdot L^{2 \mathrm{~S}}\right]}} .
$$

Semi-log plots of Eqs. 6.2 and 6.4 for function and binding are shown in Figs. 6.4 and 6.5 and are commented on in Section 6.4.1.

\subsubsection{Some Examples of the Random Type Reaction}

The random type of binding is typical for multi-meric proteins, which besides hemoglobin (Perutz 1990) and enzymes (Hindson \& Shaw 2003) include ligand-operated

channels, $\mathrm{Rad} / \mathrm{Gem} / \mathrm{Kir}-\mathrm{G}$ protein-gated channels (Kelly 2005; Beguin et al. 2007; Kobayashi et al. 2007), tyrosine-kinase-receptors (Cowan-Jacob 2006), and others such as antibodies (Gronski et al. 2007).

Models of random reactions apply to ligand-gated ion channels - where for instance ACh can open for channel activity via two binding sites distributed among the subunits (Unwin 2003, 2005) (Fig. 6.6 A+B). Another example of the random reaction scheme is the proteins of the ClC-protein family with double-barreled ClCvoltage gated $\mathrm{Cl}^{-}$channels and $\mathrm{H} / \mathrm{Cl}$ exchangers, where two pores work in concert (Miller 1982; Lin \& Chen 2000, Jentsch et al. 2005; Chen 2005; Miller 2006). This assumption was recently confirmed by a crystal structure of the ClC channel (Dutzler et al. 2002, 2003; Estevez et al. 2004; Miloshevsky \& Jordan 2004) (Fig. 6.6C), although the exact mechanism is still debated (Lin \& Chen 2000; Chen et al. 2003; Chen 2005; Bykova et al. 2006). Two chloride ions can bind in an ordered fashion to each subunit of the $\mathrm{ClC}$ channel before a transfer, but the order of binding to either subunit is probably random. On the other hand, exchange of ions in the ClC-exchanger seems to be through two separate channels. However, the exact mechanism is still unknown (Accardi et al. 2006; Nguitragool \& Miller 2006).

Since many GPCRs are dimerized (e.g., Zeng \& Wess 2000; Rios et al. 2001; Ciarkowski et al. 2005; Fotiadis et al. 2006) and, therefore have two binding sites, ligands may bind in a random manner according to a so-called 'two-state interacting receptor model' (Onaran \& Gurdel 1999).

\subsection{Ordered Reaction Schemes and Now Extended with Isomerization}

\subsubsection{The Ordered Two-site Three-step Reaction Scheme}

Transport of two or more ions through a channel at a time by a single file mechanism resembles the ordered two-site three-step mechanism as a possibility. Other examples of ordered mechanisms in effectors such as channels, catalysts and pumps (Forge et al. 1995; Canet et al. 1996; MacKinnon 2004; Allen et al. 2004; Grottesi et al. 2005) are mentioned in Sections 6.3.2 and 6.5.2.

Receptor conformations of the ordered binding mechanism are:

$\mathrm{R}$

$\mathrm{R}^{\prime} \cdot \mathrm{S}=\mathrm{R} \cdot \mathrm{S} / K_{1}$

$\mathrm{R}^{\prime \prime} \mathrm{S}_{2}=\left[\mathrm{R} \cdot \mathrm{S} / K_{1}\right]$

$\cdot\left[\mathrm{S} / K_{2}\right]$

$\mathrm{R} * \mathrm{~S}_{2}=L^{2 \mathrm{~S}} \cdot \mathrm{R}$

$\cdot \mathrm{S}^{2} /\left(K_{1} \cdot K_{2}\right)$ empty reactive receptor

one agonist bound to reactive $\mathrm{R}$

two identical agonists bound to reactive $R$

two identical agonists bound to an active $\mathrm{R}^{*}$ 
Functional response in random reaction scheme

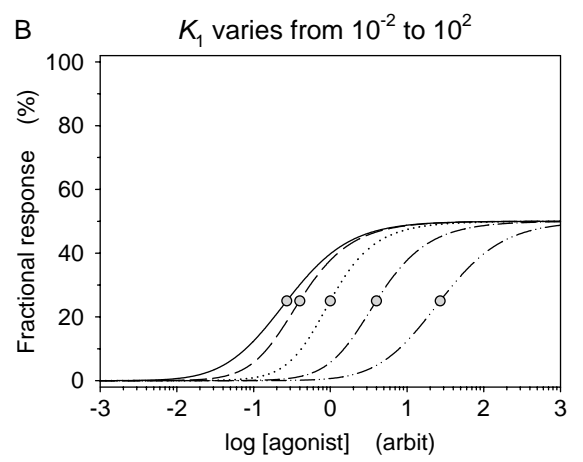

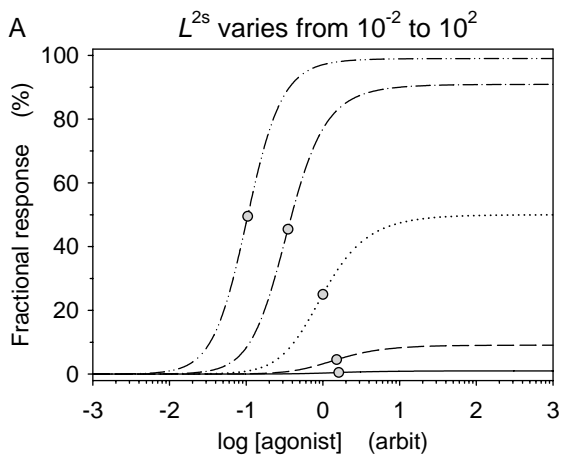

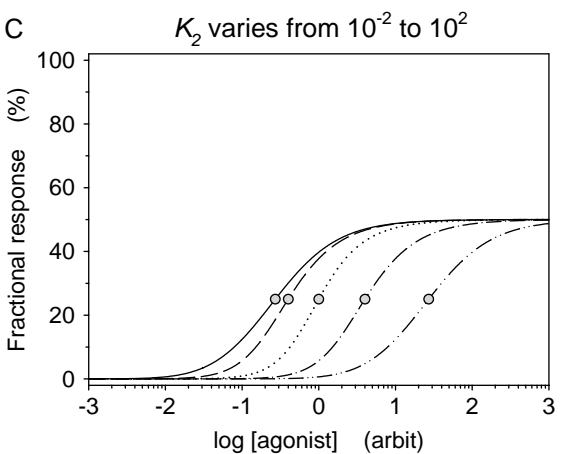

Figure 6.4. Examples of varying parameters $L^{2 S}, K_{1}$, or $K_{2}$ in the functional version of an extended random reaction scheme. Parameters varied in five steps from $10^{-2}(-)$ to $10^{2}(-\cdot-)$ by a factor 10 between steps, as indicated in $(A)$ for $L^{2 S}$, (B) for $K_{1}$, and $(C)$ for $K_{2}$. The other parameters were kept at unity. The appK $s$ are indicated by open circles. Calculation of the appK co-ordinates require solution to a quadratic equation with one unknown: $a \cdot x^{2}+b \cdot x+c=0$, where $x_{(1,2)}=\left[-b \pm \sqrt{\left.b^{2}-4 a \cdot c\right]} / 2 a\right.$. For the discriminant $\left(b^{2}-4 a \cdot c\right)<0$ there is no rational solution.

Binding response in random reaction scheme
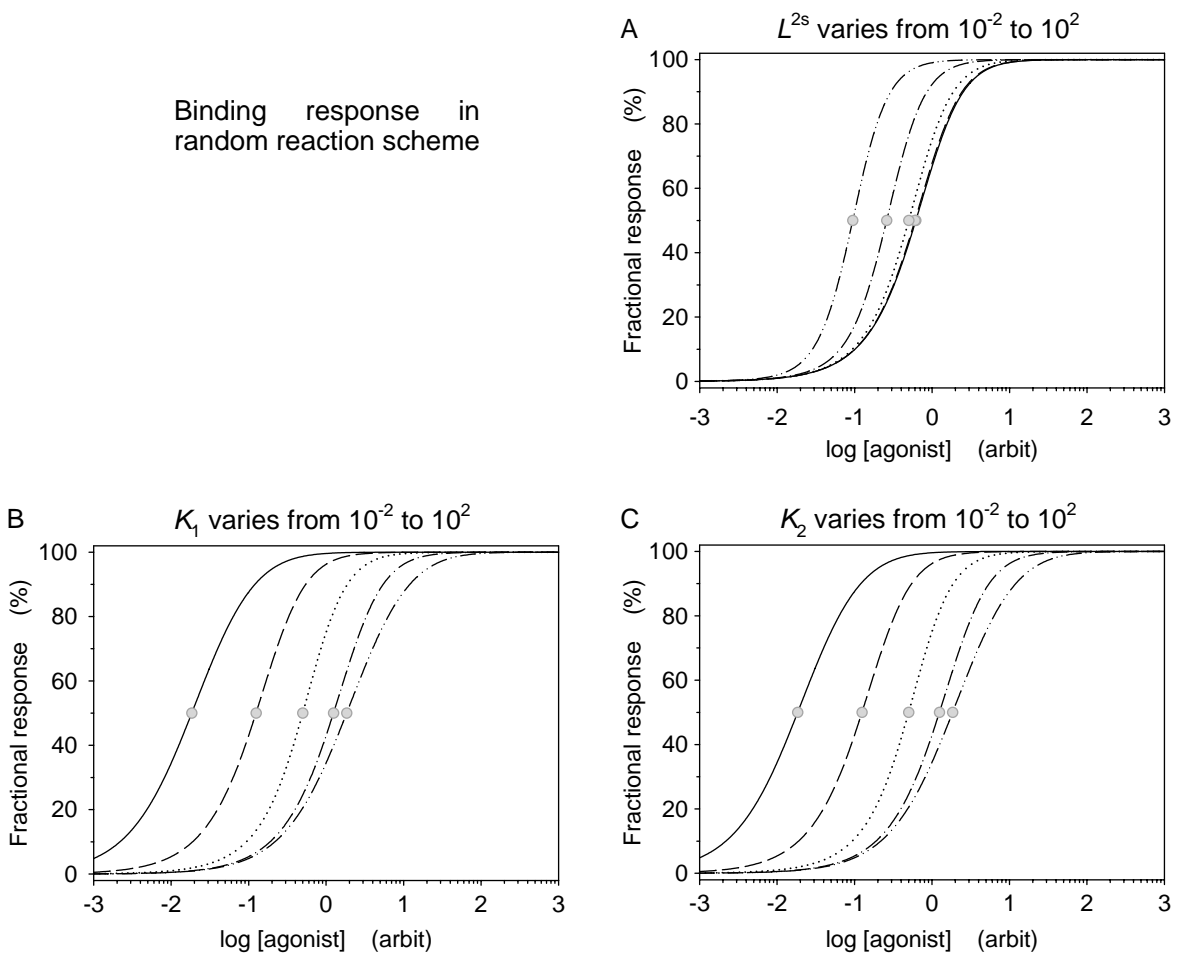

Figure 6.5. Examples of varying parameters $L^{2 S}, K_{1}$, or $K_{2}$ in the binding version of an extended random reaction scheme. Parameters varied in five steps from $10^{-2}(-)$ to $10^{2}(-\cdots-)$ by a factor 10 between steps as indicated in $(A)$ for $L^{2 S}$, (B) for $K_{1}$, and $(C)$ for $K_{2}$. The other parameters were kept at unity. The appK $K_{s}$ is indicated by open circles; see Fig. 6.4 to derive its co-ordinates. 
As before, receptor symbol ' $\mathrm{R}$ ' (or ' $\mathrm{E}$ ' for enzymes) may indicate the mass of the particular receptor conformation. Compared with the HOTSM described in Chapter 7 (Fig. 7.3A), the constants are: $K_{1}=K_{\mathrm{ss}}=1$ / $A_{\mathrm{ss}}, K_{2}=K_{\mathrm{ss}} / c^{\prime}=1 /\left(c^{\prime} \cdot A_{\mathrm{ss}}\right)$, and $L^{2 \mathrm{~S}}=a \cdot b^{\prime} \cdot d^{\prime} \cdot L$. Similar to the random reaction scheme, for the ordered reaction scheme $K_{1}$ can differ from $K_{2}$, equal to $c^{\prime} \cdot A_{\mathrm{ss}} \neq c^{\prime} \cdot A_{\mathrm{ms}}$. The ordered reaction scheme, as shown in Fig. 6.2B, is also a part of the FP-TSM reaction scheme (Fig. 5.9).
Equating the ordered two-site three-step reaction scheme for functional studies, we get:

$$
\frac{\text { response }}{\text { total }}=\frac{\mathrm{S}}{\mathrm{S}+\frac{1}{L^{2 \mathrm{~S}}}\left[\mathrm{~S}+K_{2} \cdot\left(\frac{K_{1}}{\mathrm{~S}}+1\right)\right]}
$$

and for binding studies of the ordered two-site three-step reaction scheme, the equation is:
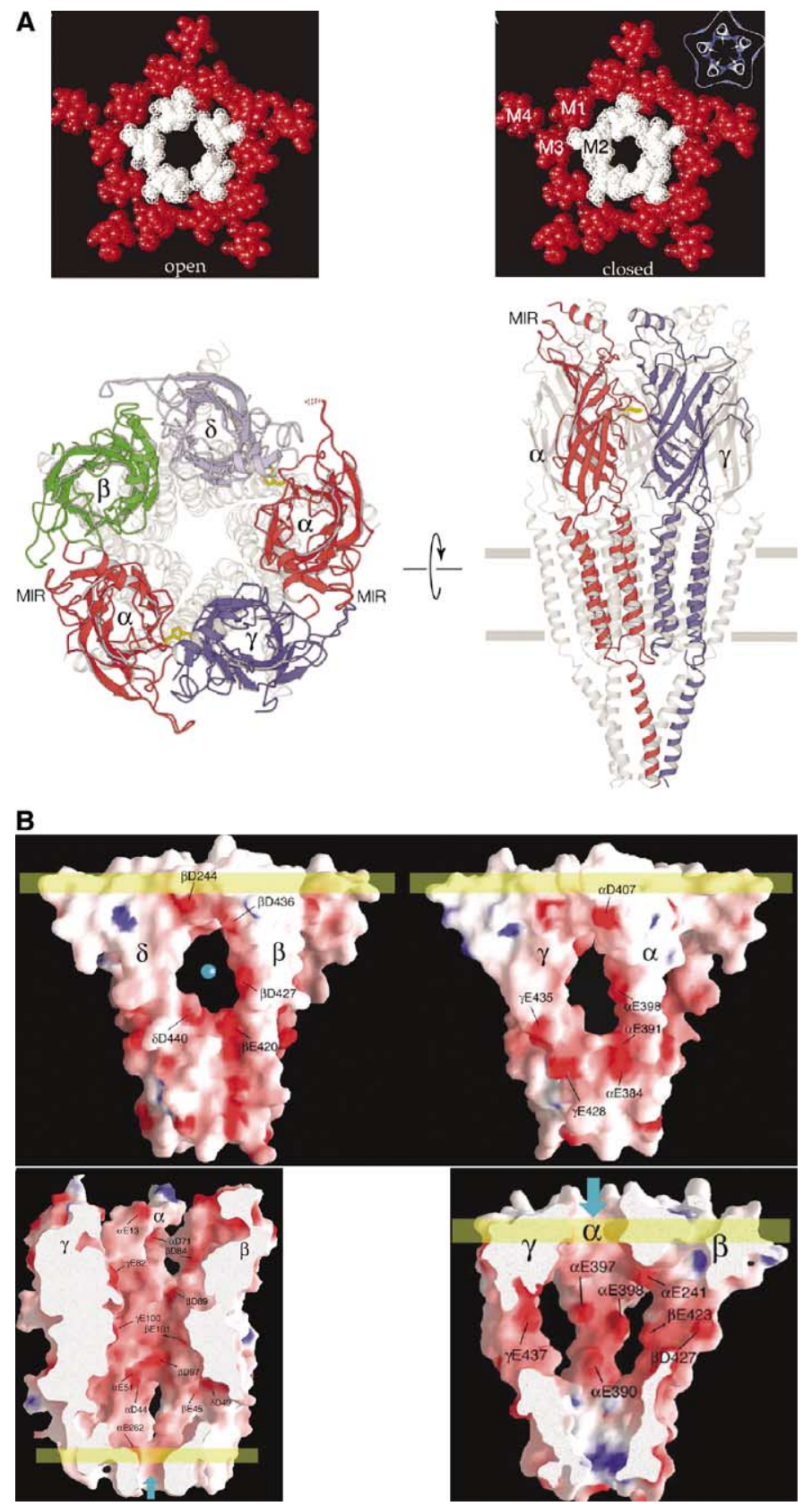

Figure 6.6. Two different channels. Figures in panels $A$ and $B$ are different aspects of a ligand-gated channel with random binding of two ligands for activation. It is the nicotinic acetylcholine receptor (nAChR) at a $4 \AA$ resolution for parts of the structure. Two binding sites for the agonist ACh are at the $\alpha$ subunits. From Unwin (2003, Fig. 2, 2005, Figs. 3, 7, and 8) with permission. Figures in panel C are various aspects of a double-barreled chloride channel CIC with 'single-file'. From Dutzler (2006, Fig. 2) with permission. 
Figure 6.6 (Continued)

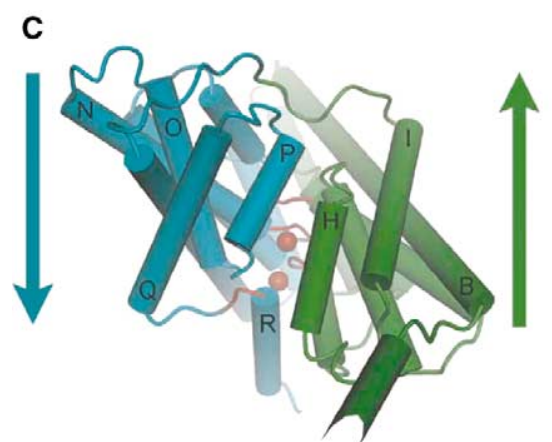

$\frac{\text { bound }}{\text { total }}=\frac{\mathrm{S}}{\mathrm{S}+\frac{K_{1} K_{2}}{K_{2}+2 \cdot\left[\mathrm{S}+\mathrm{S} \cdot L^{2 \mathrm{~S}}\right]}}$.

Plots of Eqs. 6.5 and 6.6 are in Figs. 6.7 and 6.8.

Note that the only difference between the random and the ordered reaction schemes is a factor of two in the expressions for the receptor conformation of the random reaction scheme when the first ligand binds. However, the $\operatorname{app} K_{\mathrm{s}}$ and the slope of the two regimes differ as $K_{1}$ and $K_{2}$ vary (compare Figs. 6.4 and 6.7, and Figs. 6.5 and 6.8). The dose-response relations in Eqs. 6.5 and 6.6 are further described in Sub-chapter 6.4 .

\subsubsection{Some Examples of Ordered Type Reaction}

Enzyme examples of the ordered reaction is the citrate synthetase catalyzed reaction and the "catalysis by approximation' in adenylate cyclase (Berg et al. 2002, pp. 472$473,255)$. Another example is the lactate dehydrogenase (Cleland et al. 1998). In the aspartate transcarbamoylase enzyme (ATCase), there is an ordered association of first carbomoyl phosphate inducing a fit for the second substrate aspartate (Wang et al. 2005, 2007).

In the category of transporters, such as voltageoperated $\mathrm{K}$ channels, muscarinic $\mathrm{G}$ protein-activated $\mathrm{K}$ channels, Ca-activated $\mathrm{K}$ channels, ATP-sensitive $\mathrm{K}$ channels (Packer et al. 2000), and H,K-pumps, the single-file type transport in channels and pumps, can be viewed as an ordered reaction scheme in Fig. 6.9A+ B (McKinnon 2003, 2004; Nishida et al. 2007) and Fig. 6.9C (Munson et al. 2007).

Some transporters of the cotransport type also have ordered binding and release of transportees (Parent et al. 1992; Falk et al. 1998). Loo et al. (2005) elegantly combined detection of conformational changes in the human sodium-glucose cotransporter (hSGLT1) by simultaneously recording fluorescence from a tagged probe and transient currents by stepped jumps in membrane voltage. The hSGLT1 undergoes a major

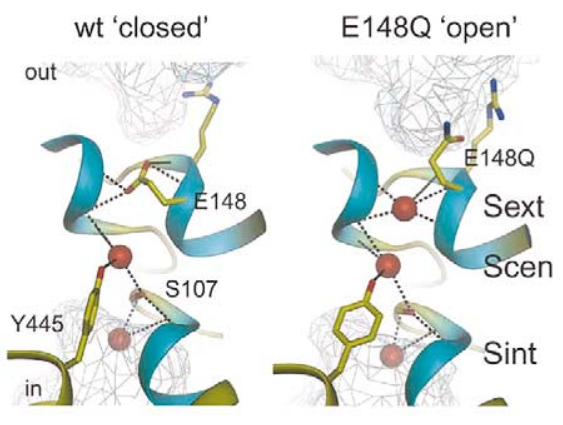

conformational change between two states of the un-liganded cotransporter when opening for ligand reception to either side of the cell membrane (Fig. $6.9 \mathrm{D}+\mathrm{E})$. Two sodium ions bind before a D-glucose molecule, and are released in reverse order, D-glucose first (Fig. 6.9D+E). Transport in the hSGLT1 is so far by an ordered two-state mechanism. An unresolved question is the type of binding for the two Na ions in SGLT1. Cotransport may additionally be a genuine two-state mechanism with unliganded SGLT in two conformations (Wright et al. 2007).

Several other nutrient cotransporters and ion-cotransporters are likely to follow a similar ordered binding scheme (Baekgaard \& Bindslev 1998; Abuladze et al. 2005; Bernardo et al. 2006; Reig et al. 2007; Piermarini et al. 2007).

The anion exchanger (AE) the sodium-proton exchanger (NHE) and the Na,Ca-exchanger (NCX) also without ATP-energy input are other examples of probable ordered association-dissociation, but with countertransport instead of co-transport (Lemieux et al. 2002; Abuladze et al. 2005; Hunte et al. 2005; Nicoll et al. 2006; Olkhova et al. 2006; Screpanti et al. 2006).

Finally, as discussed by Weiss (1997), the last step in filling the n-sites of a pump or an enzyme and activating it may be passive or driven by input of energy from ATP (Fig. 6.10), which may change the ordered reaction into a ping-pong reaction as for the Na,K-ATPase (Fig. 6.3) (Jorgensen \& Pedersen 2001; Jorgensen et al. 2003). This and other examples of the ping-pong mechanism are commented on in Sub-chapters 6.5, 6.9 and 6.10 .

\subsubsection{Combined Random and Ordered Reaction Scheme}

There are examples for enzymes that have a random association of two ligands but an ordered release of the products (Hanes et al. 1972). See also the discussion by Cornish-Bowden of the random and ordered mechanisms (Cornish-Bowden 1995, Section 6.2.3 or 2004, Section 7.2.3). 
Functional response in ordered reaction scheme

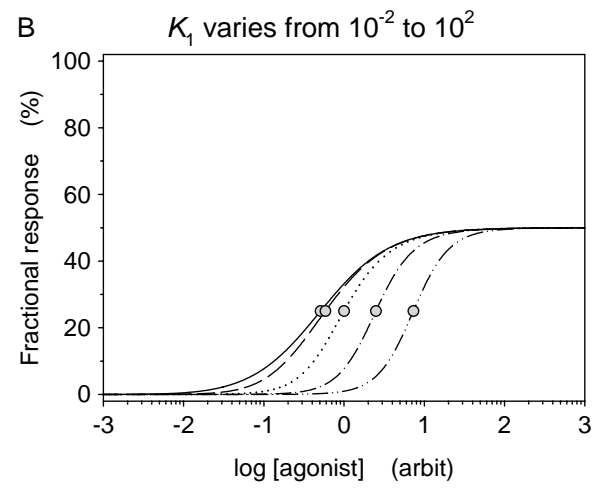

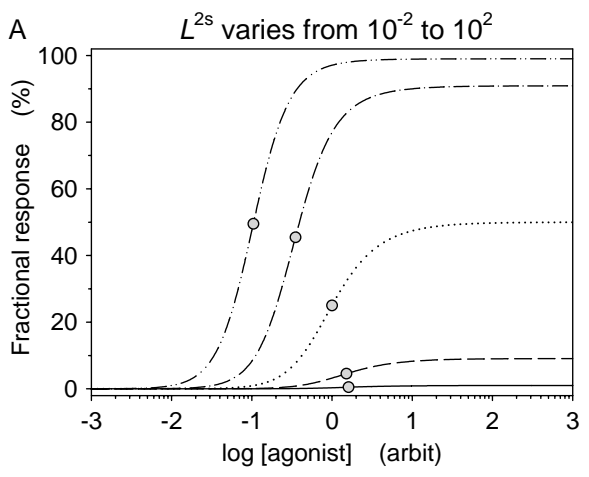

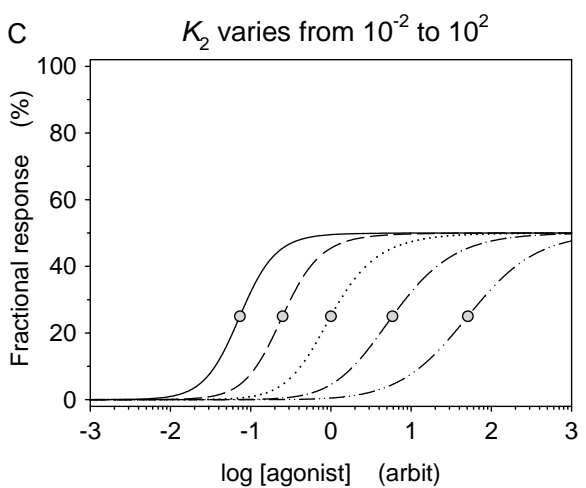

Figure 6.7. Examples of varying parameters $L^{2 S}, K_{1}$, or $K_{2}$ in the functional version of an extended ordered reaction scheme. Parameters varied in five steps from $10^{-2}(-)$ to $10^{2}(-\cdot-)$ by a factor 10 between steps as indicated in $(A)$ for $L^{2 S}$, (B) for $K_{1}$, and $(C)$ for $K_{2}$. The other parameters were kept at unity. The appK $K_{s}$ is indicated by open circles; see Fig. 6.4 to derive its co-ordinates.

Binding response in
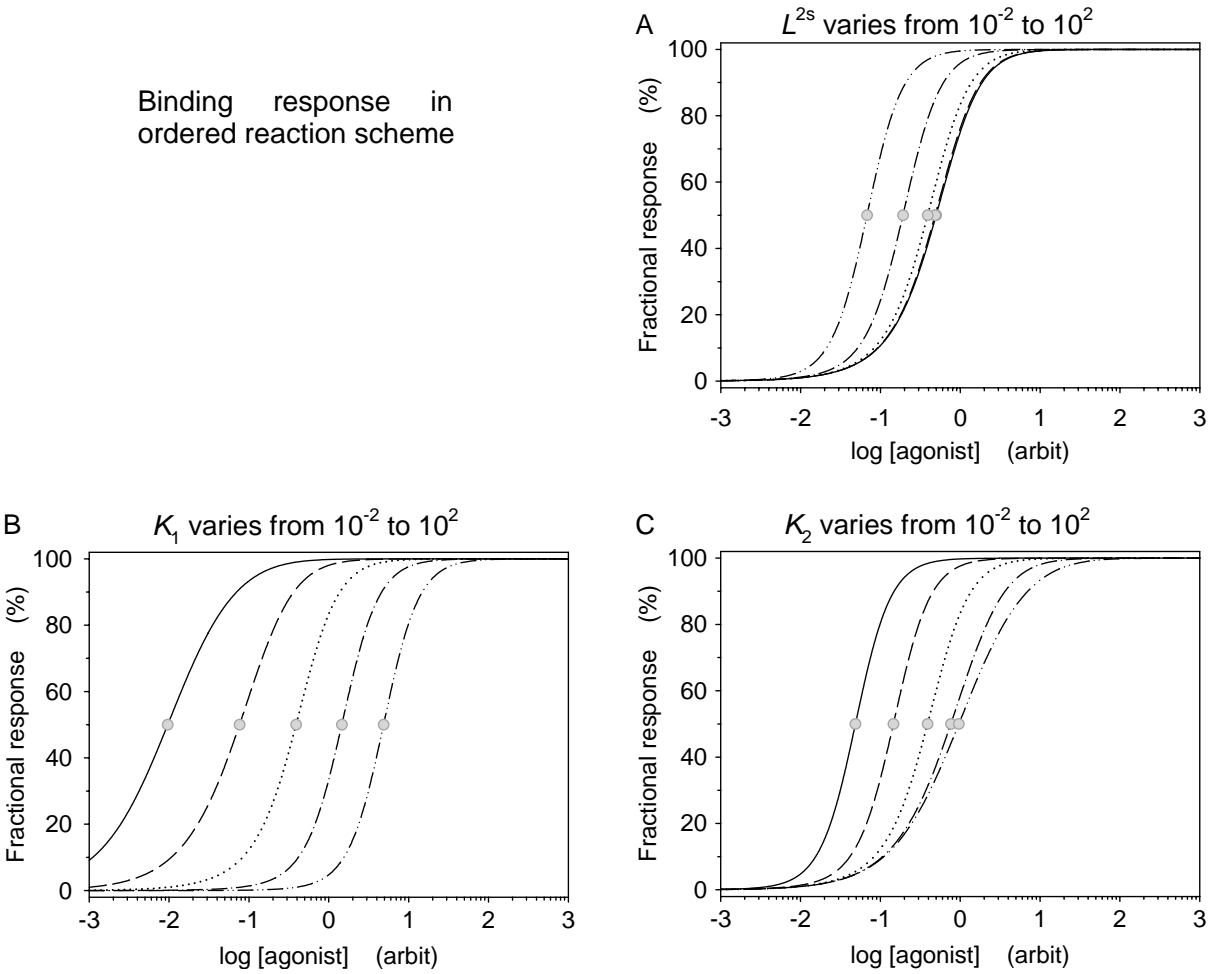

Figure 6.8. Examples of varying parameters $L^{2 S}, K_{1}$, or $K_{2}$ in the binding version of an ordered reaction scheme. Parameters varied in five steps from $10^{-2}(-)$ to $10^{2}(-\cdots)$ by a factor 10 between steps as indicated in $(\mathrm{A})$ for $L^{2 S}$, (B) for $K_{1}$, and $(\mathrm{C})$ for $K_{2}$. The other parameters were kept at unity. The $\mathrm{appK}_{\mathrm{s}}$ is indicated by open circles; see Fig. 6.4 to derive its co-ordinates. 
A
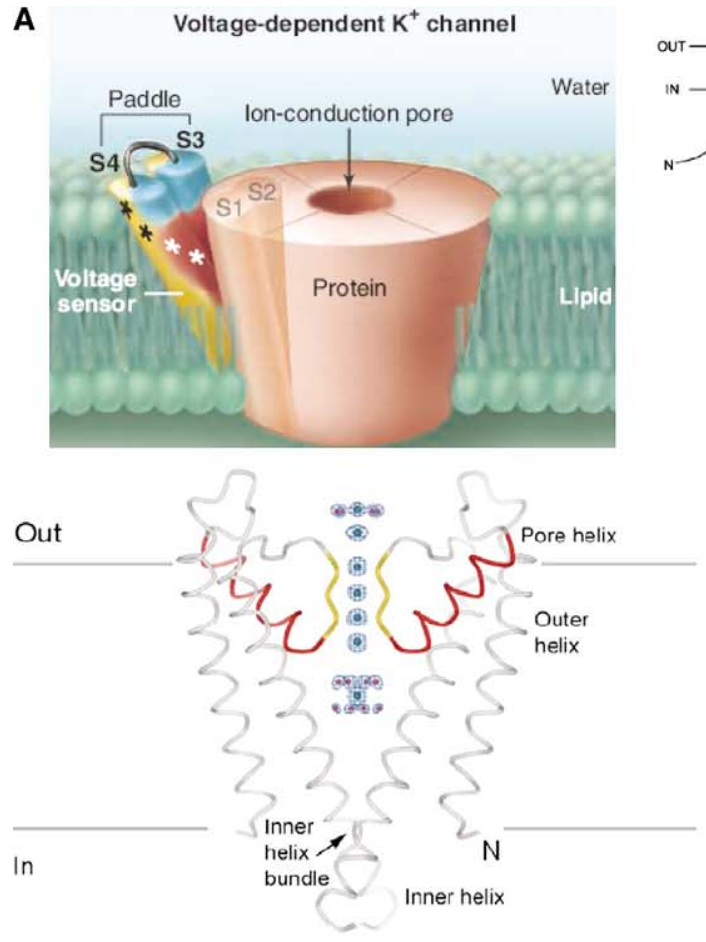

B
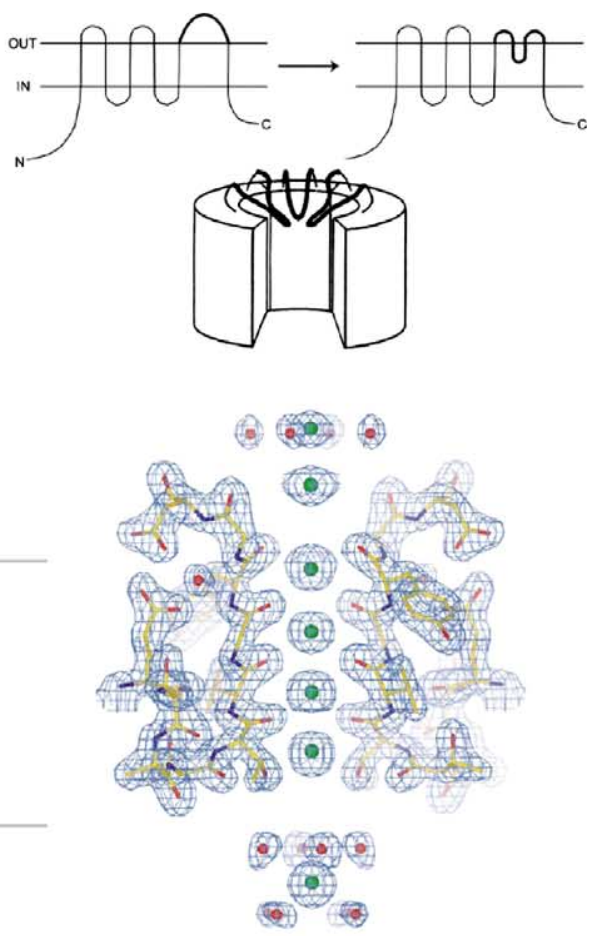

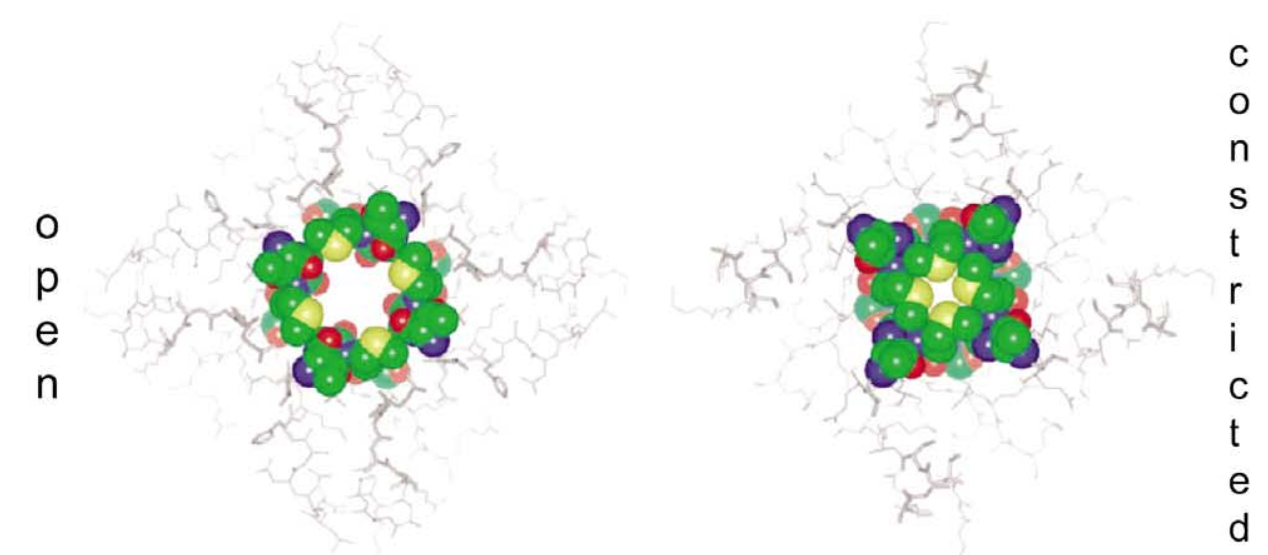

Figure 6.9. Examples of receptive units following ordered binding. Panel A shows ordered single file ion transport in a potassium channel (McKinnon 2004a, Fig., 2004b, Figs. 2, 4, and 5b with permission). Panel B shows a narrow view of a change from an open to a closed conformation of the pore in a chimeric $\mathrm{K}$ channel (Nishida et al. 2007, Fig. 5, with permission). Panel C shows a model of possible $\mathrm{K}^{+}$ion exit to cytoplasmic side of the $\mathrm{H}^{+}, \mathrm{K}^{+}$-ATPase in single-file (Munson et al. 2007, Fig. 7C, with permission). The construction is based on a homology model of the $\mathrm{E}_{1} 2 \mathrm{Ca}^{2+}$ conformation of the SERCA ATPase (Toyoshima et al. 2000), shown in Fig. 1.17. Panel $D$ is a reaction scheme, and panel $E$ is a cartoon of coupled ion and nutrient transport in a sodium/D-glucose cotransporter (SGLT). The association and dissociation of ions and nutrients are ordered and fully reversible. Panel D from Loo et al. (2005, Fig. 15A). Panel E from Wright et al. (2007, Fig. 3). Both with permission. 
Figure 6.9 (Continued)

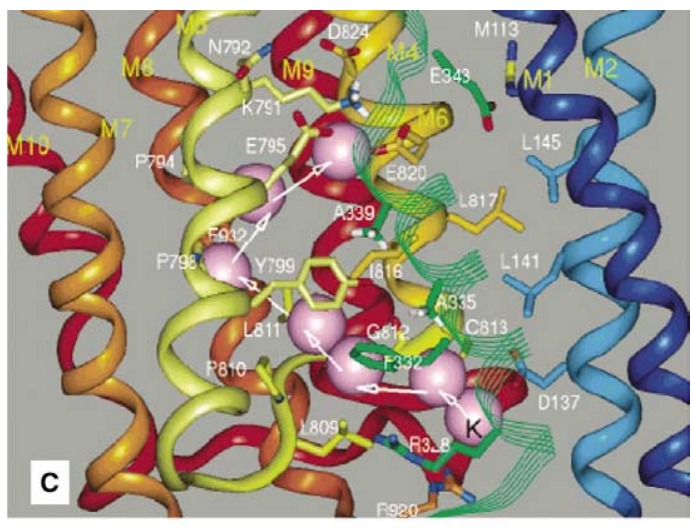

D
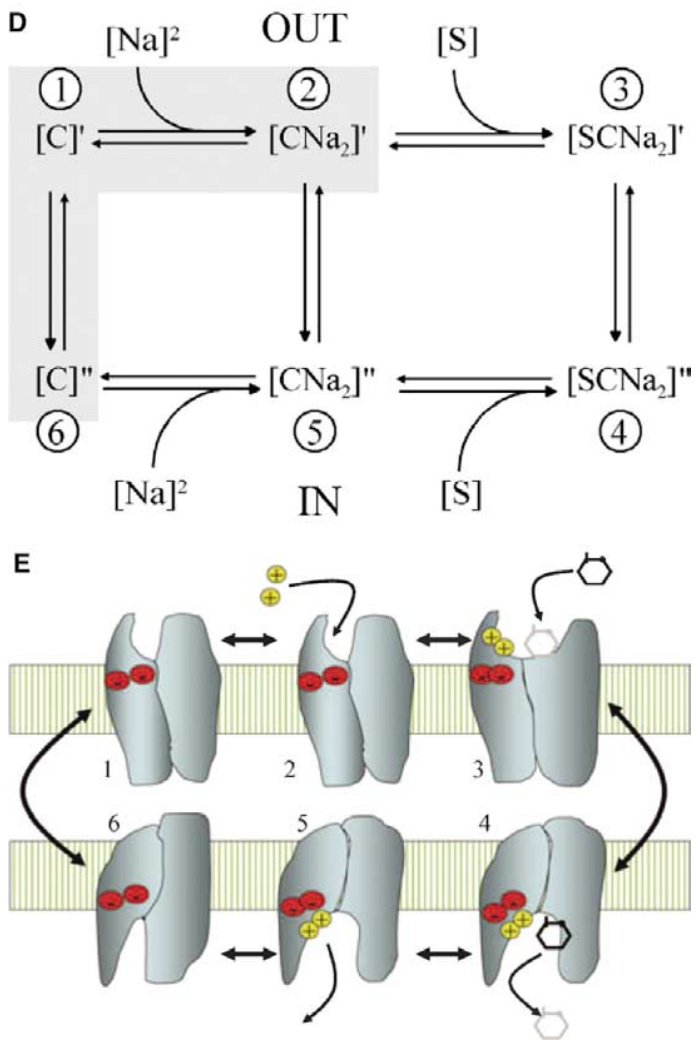

Weiss reaction scheme

$\mathrm{R}(\mathrm{S})_{\mathrm{n}} \stackrel{L^{\mathrm{nS}}}{\rightleftharpoons} \mathrm{R}^{*}(\mathrm{~S})_{\mathrm{n}}$

$$
\begin{aligned}
\mathrm{R}^{*} \mathrm{~S}_{\mathrm{n}-1} & \rightleftharpoons \mathrm{R}^{*} \mathrm{~S}_{\mathrm{n}} \\
\mathbb{1} \quad & \mathbb{1} \quad \mathrm{L}^{\mathrm{nS}} \\
\mathrm{R} \rightleftharpoons \mathrm{RS} \rightleftharpoons \mathrm{RS}_{2} \ldots & \mathrm{RS}_{\mathrm{n}-1} \rightleftharpoons \mathrm{RS}_{\mathrm{n}}
\end{aligned}
$$

Figure 6.10. A sequential reaction scheme with a final isomerization step in form of an $\mathrm{n}-1$ to $\mathrm{n}$-liganded receptive unit in two conformations. Modified from Weiss (1997).

\subsection{Analysis of Parameters in the Random and Ordered Reaction Schemes}

\subsubsection{Comparing Simple Random and Ordered Schemes by Hill Fitting}

Fig. 6.11 is an illustration of plots according to the fullbinding models of either the random, the ordered, or the Hill model with a single or up to four sites per functional unit, but, for simplicity, with identical dissociation constants $=$ no co-operativity. All three models are 'simple' as they do not include an isomerization step.

Notice that in contrast to Hill's formulation, the curves of random and ordered models never cross each other, no matter how high the number of sites in the functional unit; also illustrated for the random scheme by Tuk and van Oostenbruggen (1996, Fig. 1).

It is often stated that the Hill reaction scheme with implied simultaneity is physically unlikely and only relevant when intermediate liganded states never accumulate (Chapter 10). Put in a different way, Hill's scheme as a mechanism is only for extreme positive co-operativity, that is, $K_{1}>>K_{2}$, in random and ordered models with two sites, or in functional studies where function is only released when all sites are occupied. After reflecting on the initiation of activity, maybe this Hillian type of function is not as unlikely. Think for example of the regulated activation of protein kinase A by four molecules of cyclic AMP.

Unless a co-operativity of decreasing dissociation constants (increasing affinity) of about an order of magnitude between each step in binding is implicated, the number of sites, for instance 4 in a quaternary binding unit, is always underestimated by the fitted Hill coefficient (Weiss 1997). Strong co-operativity will yield a Hill coefficient close to the number of binding site, since this is a situation of simultaneity described by the Hill equation. The Hill coefficient cannot exceed the number of physical sites on the receptor (Monod et al. 1965).

In the absence of co-operativity or even with negative co-operativity, $K_{1}<K_{2}$, a Hill coefficient still above unity may be derived for multi-sited binding regimes of the random reaction scheme (Tuk \& van Oostenbruggen 1996). Indeed, Tuk and van Oostenbruggen showed that when the $K_{2}$ dissociation constant is 10 fold larger than the $K_{1}$, i.e., the affinity for the second ligand decreases as the first ligand binds, the fitted Hill coefficient is still above unity (Table 6.1) (Tuk \& van Oostenbruggen 1996, Table 2). A similar conclusion was obtained by Weiss for both random and ordered reactions schemes, and, in fact, the Hill coefficient in these two schemes never drops below unity (Weiss 1997, Fig. 2C). 

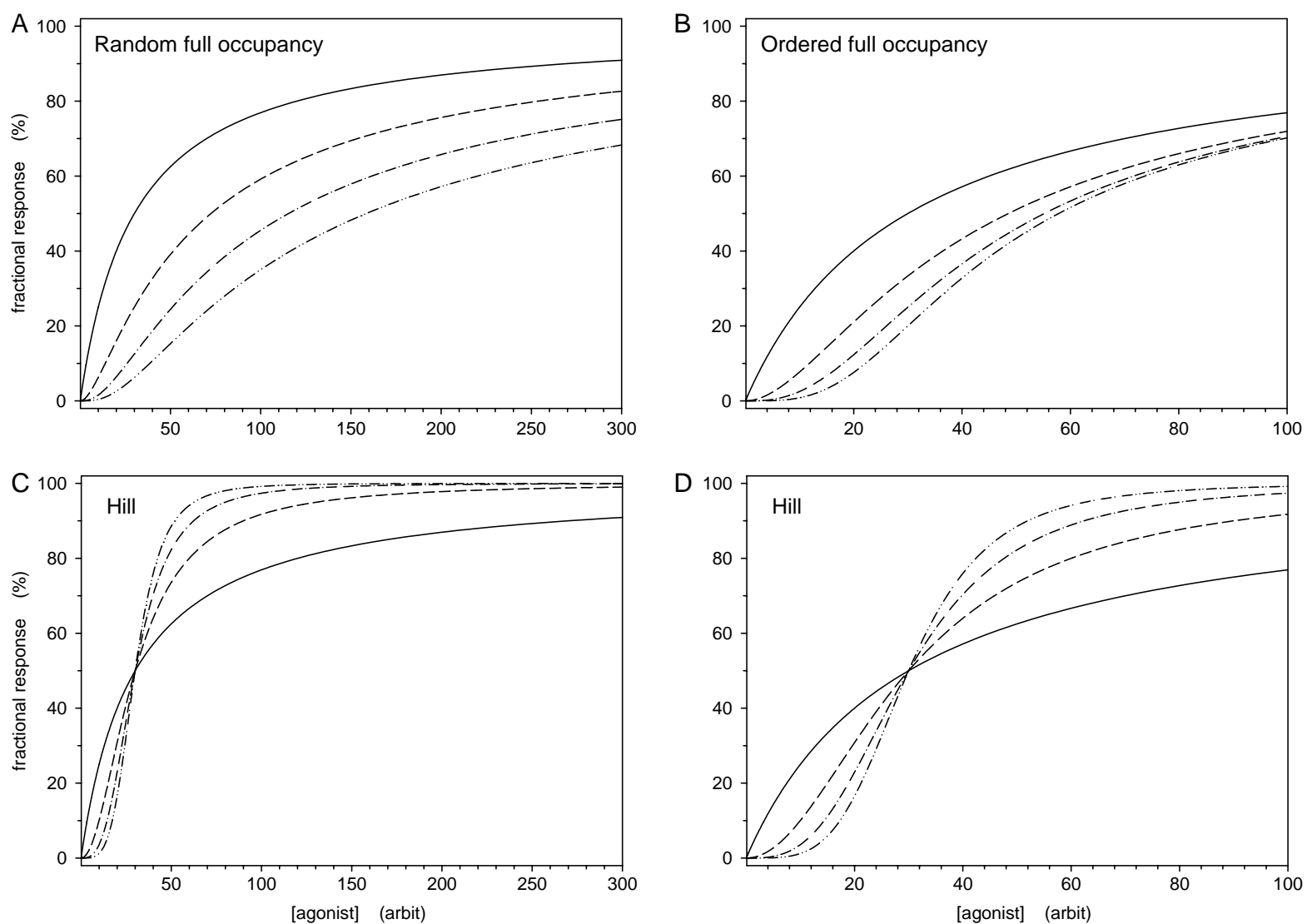

Figure 6.11. Comparison of dose-responses in three different models. Models are the simple random and simple ordered reaction schemes with no co-operativity and compared with the Hill scheme as indicated in the graphs. The number of sites varied from $1(-)$ to $4(-\cdots-)$ in all three models. For the ordered and random models, the curves never cross each other and their Hill coefficients are always above unity. Plots in panels $C$ and $D$ are identical with differently scaled $x$ axes.

As an example, the fitted Hill coefficient for the random reaction scheme with four binding sites and all sites with the same dissociation constant, i.e., $K_{1}=K_{2}=$ $K_{3}=K_{4}$ and equal to non-co-operativity, is between 1.32 and 1.35. With the same conditions for the ordered reaction scheme, the fitted Hill coefficient is between 1.77 and 1.81 , as the size of the dissociation constants vary (Fig. 6.11).

Table 6.1. Hill-parameters Fitted to the Extended Random and Ordered Reaction schemes

\begin{tabular}{|c|c|c|c|c|c|c|}
\hline & \multicolumn{6}{|c|}{ Increasing a parameter in } \\
\hline & \multicolumn{3}{|c|}{ Functional dose-response curves } & \multicolumn{3}{|c|}{ Binding concentration-occupancy curves } \\
\hline & $R_{\max }$ & Move appK & & $B_{\max }$ & & \\
\hline Random reaction scheme & & & Fig $6.4 n_{\mathrm{H}}$ & & & Fig $6.5 n_{\mathrm{H}}$ \\
\hline$K_{1}$ & for $L^{2 S}=10.5$ & Right & $1 \rightarrow 1.493 \rightarrow 1$ & 1 & Right & $1.088 \rightarrow 1.468 \rightarrow 1.085$ \\
\hline$K_{2}$ & for $L^{2 S}=10.5$ & Right & $1 \rightarrow 1.493 \rightarrow 1$ & 1 & Right & $1.088 \rightarrow 1.468 \rightarrow 1.085$ \\
\hline$L^{2 S}$ & $0 \rightarrow 1$ & Left & $1.390 \rightarrow 2$ & 1 & Left & $1.364 \rightarrow 2$ \\
\hline Ordered reaction scheme & & & Fig $6.7 n_{\mathrm{H}}$ & & & Fig $6.8 n_{\mathrm{H}}$ \\
\hline$K_{1}$ & for $L^{2 S}=10.5$ & Right & $1 \rightarrow 2$ & 1 & Right & $1 \rightarrow 2$ \\
\hline$K_{2}$ & for $L^{2 S}=10.5$ & Right & $2 \rightarrow 1$ & 1 & Right & $2 \rightarrow 1$ \\
\hline$L^{2 S}$ & $0 \rightarrow 1$ & Left & $1.390 \rightarrow 2^{\star *}$ & 1 & Left & $1.468 \rightarrow 2^{\star \star}$ \\
\hline
\end{tabular}


Weiss (1997) reached the same conclusion. He cites that a fit of the Hill equation to dose-response curves for the random reaction scheme with 4 binding sites per functional unit without co-operativity, yields a Hill coefficient of 1.31, and similarly, the fitting of a Hill equation to an ordered reaction scheme yields a Hill coefficient of 1.74 .

Thus, even when there is no co-operativity, the Hill coefficient for both the random and the ordered reaction schemes deviate from unity.

To summarize, in the random and the ordered schemes, negative co-operativity, that is $K_{1}<<K_{\mathrm{n}}$, reduces the fitted Hill coefficient towards unity, while positive co-operativity, $K_{1}>>K_{\mathrm{n}}$, brings the fitted Hill coefficient towards the number of binding sites.

\subsubsection{Comparing Extended Random and Ordered Schemes by Hill Fitting}

Parameters for the fitted Hill equation, including the slope factor $n_{\mathrm{H}}$, are extracted and listed in Table 6.1 for the isomerization-extended random and ordered reaction schemes, both with $\mathrm{n}=2$ (Fig. 6.2). Examples are for increasing $K_{1}, K_{2}$, or $L^{2 \mathrm{~S}}$ while keeping the two other constants at unity.

The effects on the maximal effect and maximal binding as well as the impact on the apparent dissociations constant and the slope of curves are summarized in Table 6.1 when increasing parameters in the extended random and ordered reactions schemes with two binding sites and analyzed by fitting to the Hill equation.

The apparent equilibrium dissociation constant, $\mathrm{appK}_{\mathrm{s}}$, increases in functional as well as in binding studies for both the random and the ordered reactions schemes when parameters $K_{1}$ or $K_{2}$ increase, while the apparent dissociation constant appK $\mathrm{s}_{\mathrm{s}}$ decreases as $L^{2 \mathrm{~S}}$ increases in all four situations. Examples of this are shown in panel A of Figs. 6.4, 6.5, 6.7, and 6.8.

The maximal fractional binding is always $100 \%$ for high ligand concentrations independent of the values of $K_{1}, K_{2}$, and $L^{2 \mathrm{~S}}$ (Figs. 6.5 and 6.8).

In the functional dose-responses, the fractional maximum effect is determined by the value of parameter $L^{2 \mathrm{~S}}$ in both random and ordered reactions schemes. Varying values of $L^{2 \mathrm{~S}}$ in functional studies from high values towards 1 will reduce the maximal effect from a maximal fraction equal to $100 \%$ to $50 \%$ for $L^{2 \mathrm{~S}}=1$, and further towards zero as $L^{2 \mathrm{~S}}$ goes towards zero both in random and ordered reactions scheme (panel A in Figs. 6.4 and 6.7).

The slope of curves for the random and ordered relations are obtained as Hill coefficients by fitting Hill's theory formulated in Eq. 10.2 to plots of Eqs. 6.2, 6.4 6.5, and 6.6. Principal results are listed in Table 6.1.

In general, for the functional form of the random scheme, when $L^{2 \mathrm{~S}}$ is 1 , the Hill slope moves from 1 towards nearly 1.5 and back again to 1 as either $K_{1}$ or $K_{2}$ increases. For increasing $L^{2 \mathrm{~S}}$ with $K_{1}$ and $K_{2}$ equal 1 , the slope starts at 1.39 and increases towards 2 .

For binding in the random scheme with $L^{2 \mathrm{~S}}=1$, the slope of the random relation moves up from close to 1.1 towards 1.47 and back to circa 1.1 as either $K_{1}$ or $K_{2}$ increases. For increasing $L^{2 \mathrm{~S}}$, with $K_{1}$ and $K_{2}$ equal 1 , the Hill slope of the curves move from about 1.36 towards 2 .

For the ordered reaction scheme there are differences in the behavior of Hill slopes compared with the random scheme. Thus, in both the functional and binding form of the ordered relation, for $L^{2 \mathrm{~S}}=1$, increasing $K_{1}$ brings the slope from 1 up towards 2, while increasing $K_{2}$ brings the Hill slope from 2 down towards 1 .

In the functional form of the ordered scheme, when increasing $L^{2 \mathrm{~S}}$ as $K_{1}$ and $K_{2}$ is 1 , the Hill slope starts at 1.39 and increases towards 2, while the slope in the binding form of the ordered reaction scheme starts at 1.47 and move towards 2 as $L^{2 \mathrm{~S}}$ increases (see Table 6.1). Note that for $K_{1}>>1$ or $K_{2}<<1$ as $L^{2 \mathrm{~S}}$ increases in both types of the ordered reaction scheme, the slope moves towards 2, while when $K_{1}<<1$ or $K_{2}>>1$, the slope in both types of the ordered scheme moves towards 1 as $L^{2 \mathrm{~S}}$ increases (Table 6.1).

\subsection{Ping-pong Reaction Schemes}

\subsubsection{A Circular Two-site and More than Two-step Mechanism, the Ping-pong Model}

Fig. 6.3 is a classical display of an ordinary ping-pong mechanism, without energy input and, therefore, in principle a completely reversible reaction scheme. Pingpong is frequently considered for irreversible reaction schemes where one step is driven by a GTP- or an ATPenergy input. Thus, a mechanism such as the ping-pong variant of the two-site and three-step mechanism seems a plausible model in transport of a few single molecules through co- and counter-transporters across membranes as well as in enzyme-catalyzed reactions without energy input, while with energy input for $G$ protein cycles as shown in Fig. 6.1 and for pumps in Fig. 6.16. Genuine ping-pong for enzyme reactions are described as 'double-displacement reaction' (Berg et al. 2002, p. 208), and part of the membrane transfer by a ping-pong mechanism is also known as an 'iso-uni-uni system' (Segel 1975, 1993, pp. 534-543). 


\subsubsection{Formulation of Ping-pong Reaction Schemes}

The ping-pong mechanism presented in Fig. 6.3 is actually a three-state model by definition, since it includes three un-liganded receptor forms, E1, E2, and E1'.

There is a wealth of various schemes for the so-called ping-pong mechanism. The purpose of this book is to give the reader a feel for the complexity even in simple reaction schemes. However, for the scope of the present text, I have judged that the derivation of equations for ping-pong mechanisms will divert our focus too much. Therefore, on the subject of ping-pong formulation, the interested reader is referred to Chapter 9 in Segel's book (1975, 1993, pp. 606-845), where he presents and formulates several ping-pong mechanisms. King and Altman (1956) and Cleland (1963) provide methods to write an efficient shorthand of more complex reaction schemes. This is exploited as well by Segel for his pingpong reactions.

Segel's book $(1975,1993)$ contains examples of the ping-pong, such as a 'uni uni uni uni ping pong' system (pp. 606-612), an 'iso bi bi' system (pp. 634-639), a 'hybrid ping pong bi bi' system (pp. 626-634), a 'hybrid Theorell-Chance ping pong' system (pp. 639-643), a 'bi uni uni uni ping pong ter bi' system (pp. 684-699), a 'bi bi uni uni ping pong ter ter' system (pp. 719-727), and even a 'hexa uni ping pong' system (pp. 727-736), with added plotting methods. Some of the schemes also include substrate inhibition. (On dead-end inhibition, also covering ping-pong, see pp. 767-845 in Segel's book.)

In this connection it is wise to remember some prudent words by Cornish-Bowden (1995, p. 134; 2004, p. 162): "The Method of King and Altman (1956) ${ }^{1}$ can be applied to unreasonable as easily as to reasonable mechanisms, and if one regards kinetics as a branch of algebra, largely unrelated to chemistry, one risks having to deal with a bewildering array of possibilities. For this reason one should always regard algebra as the servant of enzyme kinetics and not its master'.

\subsection{Any Number of Binding Sites with Full Occupation}

\subsubsection{Full Occupancy in Random Reactions}

Tuk and van Oostenbruggen (1996) and Weiss (1997), among others, have listed the principles for deriving formulas for random binding including up to any number of binding sites (see Table 6.2). Tuk and van

\footnotetext{
1 Op. cit.
}

Ostenbruggen (1996) termed their model the reformulated occupation model. These two authors derived functional equations under the assumption that receptor function requires all of its sites to be occupied, similar to the Hill scheme, although not in a process of one single step as required for the Hill reaction, but in an independent manner, and allowing for variety in the values of individual equilibrium dissociation constants among the different sites of a receptive unit, a random reaction.

\subsubsection{Full Occupancy in Ordered Reactions}

Furthermore, Weiss derived the general expression for any number of binding sites in each receptive unit, when only fully occupied receptors relative to the total number of receptors are present in both the random model (as mentioned in Section 6.6.1) and in a strictly ordered model (Weiss 1997).

\subsubsection{Pascal's Triangle in Random and Ordered Reaction Schemes}

In the following, I will discuss the general distribution equations for models with either random or ordered binding and activation only at full occupancy, and in Sub-chapter 6.7, I discuss the random and ordered binding and function when alternatively all intermediate stages of binding counts, including those of full occupation. Both the denominator and the nominator of such distributed functions are expressions of the Pascal triangle. This type of expression was first suggested by Pauling (1935) for a description of the random binding of $\mathrm{O}_{2}$ to hemoglobin. Two examples of Pascal's triangle are shown in Fig. 6.12.

Weiss (1997) has the general expression for the fractional response $(f r)$ of a fully occupied system that is random (Table 6.2):

$$
f r=\frac{\frac{[\mathrm{S}]^{\mathrm{n}}}{\Pi_{\mathrm{i}}^{\mathrm{n}} K_{\mathrm{i}}}}{1+\sum_{\mathrm{i}}^{\mathrm{n}} \frac{\frac{\mathrm{n} ![\mathrm{S}]^{\mathrm{i}}}{(\mathrm{n}-\mathrm{i}) ! \mathrm{i} !}}{\Pi_{\mathrm{j}}^{\mathrm{i}} K_{\mathrm{j}}}},
$$

as well as the $f r$ for a fully occupied and ordered system (Table 6.2):

$$
f r=\frac{\frac{[\mathrm{S}]^{\mathrm{n}}}{\Pi_{\mathrm{i}}^{\mathrm{n}} K_{\mathrm{i}}}}{1+\sum_{\mathrm{i}}^{\mathrm{n}} \frac{[\mathrm{S}]^{\mathrm{i}}}{\Pi_{\mathrm{j}}^{\mathrm{i}} K_{\mathrm{j}}}} .
$$

Plots of these two fractional responses are shown in Fig. $6.13 \mathrm{~A}+\mathrm{B}$ for $\mathrm{n}=4$. 
Table 6.2. Formulae for Fraction of Either Fully Occupied or Just liganded Conformations with Numbers of Ligands for Any Number of Binding Sites in the Random and Ordered Reaction Schemes

One-state models

\begin{tabular}{|c|c|c|}
\hline \multicolumn{3}{|c|}{ One-state models } \\
\hline & Fraction of full occupancy & Fraction of bound ligands \\
\hline Random/independent ${ }^{\star}$ & $f r=\frac{\frac{[\mathrm{S}]^{\mathrm{n}}}{\prod_{i}^{\mathrm{n}} K_{\mathrm{i}}}}{1+\sum_{\mathrm{i}}^{\mathrm{n}} \frac{\frac{\mathrm{n} ![\mathrm{S}]^{\mathrm{i}}}{(\mathrm{n}) ! \mathrm{i} !}}{\prod_{\mathrm{j}}^{\mathrm{n}} K_{\mathrm{j}}}}($ Eq. 6.8, Fig. 6.13A) & $f b=\frac{\frac{\sum_{\mathrm{i}}^{\mathrm{n}} \frac{(\mathrm{n}-1) !}{(\mathrm{n}-\mathrm{i}) !(\mathrm{i}-1) !}[\mathrm{S}]^{\mathrm{i}}}{\prod_{\mathrm{j}}^{\mathrm{i}} K_{\mathrm{j}}}}{1+\frac{\sum_{\mathrm{i}}^{\mathrm{n}} \frac{(\mathrm{n}-1) !}{(\mathrm{n}-\mathrm{i}) !(\mathrm{i}-1) !}[\mathrm{S}]^{\mathrm{i}}}{\prod_{\mathrm{j}}^{\mathrm{i}} K_{\mathrm{j}}}}$ (Eq. 6.10b, Fig. 6.14) \\
\hline Ordered/dependent ${ }^{\star \star}$ & $f r=\frac{\frac{[\mathrm{S}]^{\mathrm{n}}}{\prod_{\mathrm{i}}^{\mathrm{n}} K_{\mathrm{i}}}}{1+\sum_{\mathrm{i}}^{\mathrm{n}} \frac{[\mathrm{S}]^{\mathrm{i}}}{\prod_{\mathrm{j}}^{\mathrm{n}} K_{\mathrm{j}}}}($ Eq. 6.9, Fig. 6.13B) & $f b=\frac{\sum_{\mathrm{i}}^{\mathrm{n}} \frac{(\mathrm{i} / \mathrm{n}) \cdot[\mathrm{S}]^{\mathrm{i}}}{\prod_{\mathrm{j}}^{\mathrm{i}} K_{\mathrm{j}}}}{1+\sum_{\mathrm{i}}^{\mathrm{n}} \frac{(\mathrm{i} / \mathrm{n}) \cdot[\mathrm{S}]^{\mathrm{i}}}{\prod_{\mathrm{j}}^{\mathrm{i}} K_{\mathrm{j}}}}($ Eq. 6.11b, Fig. 6.15) \\
\hline Ping-pong (often multi-states) & You can derive it. & Can you derive it? \\
\hline $\begin{array}{l}\text { Two-state models: all } K_{\mathrm{R}} \mathrm{s} \text { are equal } \\
\text { and all } K_{\mathrm{T}} \mathrm{s} \text { are equal }\end{array}$ & $\begin{array}{l}\text { You can derive it. If not, see Monod et al. } \\
\text { (1965) or Berg et al. (2006, pp. 201-202) }\end{array}$ & Can you derive it? \\
\hline
\end{tabular}

*Tuk and van Oostenbruggen (1996) called this model the reformulated occupancy model.

**Weiss (1997) designated this model the 'sequential' model.

The table expressions are generalized forms of those in Tuk and van Oostenbruggen (1996) Eq. 6.8, and in Weiss (1997) Eqs. 6.8 and 6.9.

If fractional response not only requires full occupancy, but a count of every bound ligand, as for instance for tracer binding, expression in the third column of the table may be relevant for this type of experimental set up.

Eq. $6.10 \mathrm{~b}$ in column 3 is a generalized formulation of a Tuk-vanOostenbruggen scheme, now with terms for summation in both nominator and denominator factored by the actual number of bound ligands $=\mathrm{i}$, and normalized by the number of binding sites $\mathrm{n}$, i.e., $\mathrm{i} / \mathrm{n}$, also relevant for the equation derived for the ordered reaction in Eq. 6.11b. Compare with Michel (2007).

\subsection{Any Number of Binding Sites Including Partial Occupation}

\subsubsection{When Functionality Does Not Require Full Occupancy}

In case all liganded conformations of the receptive unit must be included and not just the fully occupied receptive units as described in Sub-chapter 6.6, Tuk and van Oostenbrugge demonstrate how the nominator and denominator for the random dose-response distribution function must be formulated (Eq. 6.10a) (Tuk \& van Oostenbruggen 1996, Box 2). For the corresponding ordered binding studies, I derive the parallel expression in Eq. 6.11a.

When the requirement described in Sub-chapter 6.6 with fully occupied receptors is not in effect for functionality, then in studies where function is activated as ligands bind, and in some binding experiments, formulation of the dose-response always requires a summation of all of the liganded forms.

As pointed out by Tuk and van Oostenbruggen for the random reaction scheme, a general distribution equations for fractional binding $(\mathrm{fb})$ is readily derived (Tuk \& van Oostenbruggen 1996, Box 2):

$$
f b=\frac{\sum_{\mathrm{i}}^{\mathrm{n}} \frac{\frac{(\mathrm{n}-1) !}{(\mathrm{n}-\mathrm{i}) !(\mathrm{i}-1) !}[\mathrm{S}]^{\mathrm{i}}}{\Pi_{\mathrm{j}}^{\mathrm{i}} K_{\mathrm{j}}}}{1+\sum_{\mathrm{i}}^{\mathrm{n}} \frac{\frac{\mathrm{n} ![\mathrm{S}]^{\mathrm{i}}}{(\mathrm{n}-\mathrm{i}) ! \mathrm{i} !}}{\Pi_{\mathrm{j}}^{\mathrm{i}} K_{\mathrm{j}}}} .
$$

Compared to Eq. 6.8, the summation in the nominator of Eq. 10a is obtained by correcting the denominator summation of Eq. 6.8 with a normalized number of bound ligand equal to a running number $\mathrm{i}$ divided by the total number $\mathrm{n} ;=\mathrm{i} / \mathrm{n}$.

Examples of graphs for varying the dissociation constants $K_{1}, K_{2}$ and so forth with up to four binding sites in Eq. 6.10a are shown in the upper panels of Fig. 6.14.

The parallel equation for fractional binding in the ordered reaction schemes is given by:

$$
f b=\frac{\sum_{\mathrm{i}}^{\mathrm{n}} \frac{(\mathrm{i} / \mathrm{n}) \cdot[\mathrm{S}]^{\mathrm{i}}}{\Pi_{\mathrm{j}}^{\mathrm{i}} K_{\mathrm{j}}}}{1+\sum_{\mathrm{i}}^{\mathrm{n}} \frac{[\mathrm{S}]^{\mathrm{i}}}{\prod_{\mathrm{j}}^{\mathrm{i}} K_{\mathrm{j}}}} .
$$




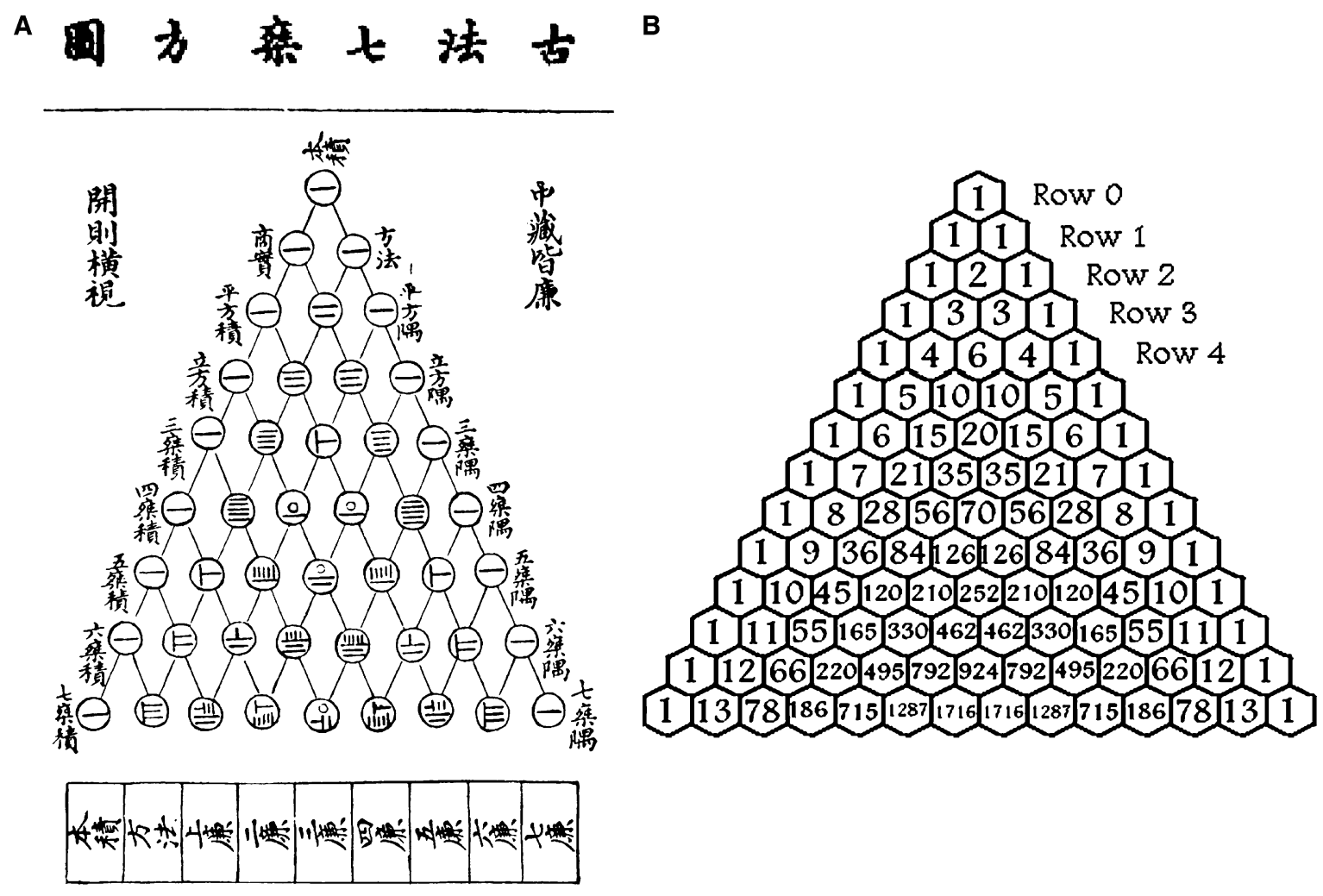

Figure 6.12. Pascal's triangle. ${ }^{2}$ According to Halayudha (c. 975 AD), who was an Indian mathematician, Pingala (c. 200 BC) in his analysis of prosody (chandas-sutra) in Sanscrit Vedanga is supposed, as the first, to have introduced the principle of Pascal's triangle, called mera-prastaara. Later the triangle appeared in Persian writings (Omar Khayyám c. 1080 AD) and in Chinese writings (Yang Hui c. 1250 AD and Zhu Shijie 1303 AD). Panel A from the front cover of Zhu Shijie's (Chu Shih-Chieh's) Ssu Yuan Yii Chien (Precious Mirror of the Four Elements or True Reflections of the Four Unknowns) published in China (1303 AD). Taken from Petigen et al. (1992, p. 84) with permission. Shijie also explains the rule of three in Introduction to Mathematical Studies (1299 AD). Panel B is from http://briton.disted.camosun.bc.ca/pascal/pascal.html.

Examples of graphs for varying the dissociation constants $K_{1}, K_{2}$ and so forth with up to four binding sites in Eq. 6.11a are shown in the upper panels of Fig. 6.15

\subsubsection{Binding Experiments with Tracers}

For binding expressions in both ordered and random reactions where the number of bound ligands counts, there is a need for summation of numbers of ligand in case more than one ligand is bound per receptor. However, this requires multiplication by a value of every summation term with the single term's number of bound ligands in both nominator and denominator of the distribution equations This will be relevant for nominators and denominators in formulation for binding studies with, for instance, tracers.

In order to account for every single liganded tracer molecule, an additional term is necessary in the

\footnotetext{
${ }^{2}$ Blaise Pascal (Traité du Triangle Arithmétique 1655).
}

denominators of Eqs. 6.10a and 6.11a. In the two equations, expressions under the summation signs in both the nominator and denominator must be multiplied by the normalized running number $i$, as already formulated by Adair (1925), Pauling (1935), and Rougton et al. (1955). Thus, for the fraction of any liganded species in random binding, and taking into account that each bound tracer molecule counts in both the nominator and the denominator, we get:

$$
f b=\frac{\sum_{\mathrm{i}}^{\mathrm{n}} \frac{\frac{(\mathrm{n}-1) !}{(\mathrm{n}-\mathrm{i}) !(\mathrm{i}-1) !}[\mathrm{S}]^{\mathrm{i}}}{\Pi_{\mathrm{j}}^{\mathrm{i}} K_{\mathrm{j}}}}{1+\sum_{\mathrm{i}}^{\mathrm{n}} \frac{(\mathrm{n}-1) ![\mathrm{S}]^{\mathrm{i}}}{(\mathrm{n}-\mathrm{i}) !(\mathrm{i}-1) !}} .
$$

This is a combination of the Pascal triangle and Adair's idea. A plot of this theoretical model for actual binding to receptive units with up to four binding sites is shown in the lower panels of Fig. 6.14. The difference between a Tuk and van Oostenbruggen model (Eq. 6.10a), and the 
A

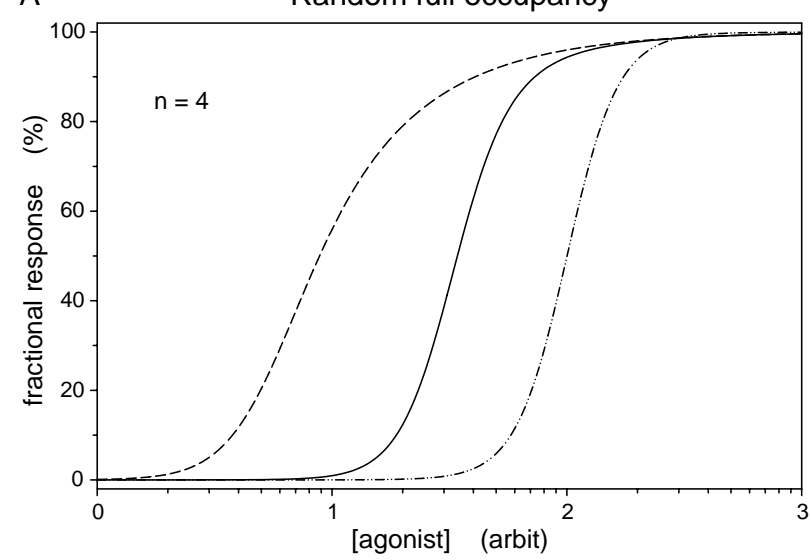

B

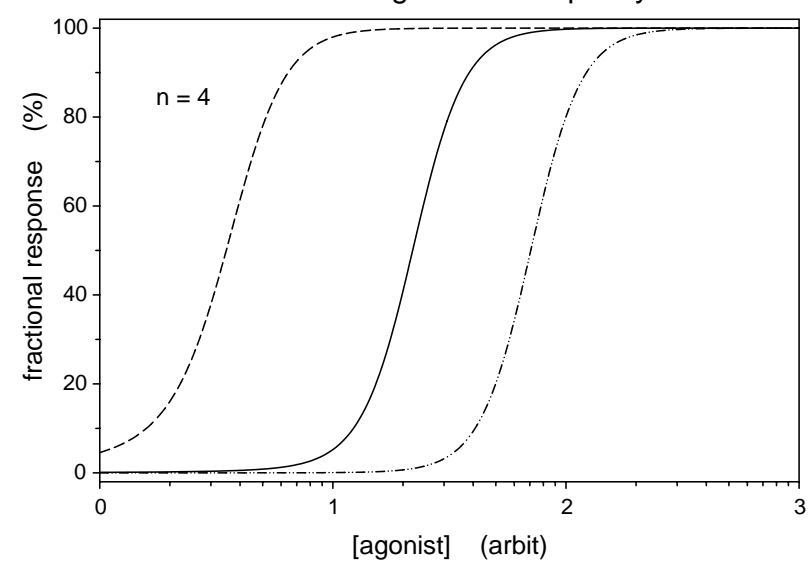

Figure 6.13. Fractional reaction for random and ordered schemes with four binding sites $(n=4)$ and full occupancy. The three plots in each graph are generated according to Eqs. 6.8-6.9 in Table 6.2. Panel A is for the random and panel $B$ for the ordered reaction scheme. The responses are shown for varying values of the four independent dissociation constants $K_{1}, K_{2}, K_{3}$, and $K_{4}$. Values for the four dissociation constants in plots were $30,10,3$, and $1(--), 1000,100,10,1(-)$, and $100000,1000,10$, and $0.1(-\cdot-)$. Using Hill's equation theory for non-linear fitting to each single plot for parameters $R_{\max }$ $(\%), n_{\mathrm{H}}$, and $\mathrm{appK}_{\mathrm{s}}$ (arbit) yields: $98.9 \pm 0.1,1.99 \pm 0.01$, $9.49 \pm 0.04 ; 99.9 \pm 0.1,3.33 \pm 0.01,34.7 \pm 0.04$; and $100 \pm 0$, $3.98 \pm 0.00,100.2 \pm 0.0$ for the random, and $99.4 \pm 0.0,3.10 \pm$ $0.02,6.29 \pm 0.01 ; 99.8 \pm 0.01,3.82 \pm 0.00,32.3 \pm 0.01$; and $100 \pm 0, \quad 3.99 \pm 0.00, \quad 100.1 \pm 0.0$ for the ordered scheme. "arbit" = normalized concentration.

model presented in Eq. 6.10b is not dramatic, as long as the last binding step has an affinity, $1 / K_{4}$, much higher than the preceding affinities (compare upper and lower graphs in panel 6.14C). However, the theoretical approach in Eqs. 6.10a and 6.10b deviate dramatically when the last binding step has an affinity, $1 / K_{4}$, equal to or larger than the preceding steps (see upper and lower graphs in panels B and A in Fig. 6.14).

The equation comparable to Eq. $6.10 \mathrm{~b}$ for ordered binding is:

$$
f b=\frac{\sum_{\mathrm{i}}^{\mathrm{n}} \frac{(\mathrm{i} / \mathrm{n}) \cdot[\mathrm{S}]^{\mathrm{i}}}{\Pi_{\mathrm{j}}^{\mathrm{i}} K_{\mathrm{j}}}}{1+\sum_{\mathrm{i}}^{\mathrm{n}} \frac{(\mathrm{i} / \mathrm{n}) \cdot[\mathrm{S}]^{\mathrm{i}}}{\Pi_{\mathrm{j}}^{\mathrm{i}} K_{\mathrm{j}}}} .
$$

A plot of the relation in Eq. 6.11b with up to four binding sites is shown in the lower panels of Fig. 6.15 with varying dissociation constants.

Eqs. 6.8, 6.9, 6.10b and $6.11 \mathrm{~b}$ are summarized in Table 6.2. Compare Eqs. 6.9 and $6.11 \mathrm{~b}$ with a recent and similar derivation by Michel (2007).

\subsubsection{Functional Experiments with Efficacies Varying Between Conformations}

In order to acknowledge potential differences in efficacy between the liganded conformations expressed as a fraction in column 3 of Table 6.2, it may be necessary to multiply each term under the summation signs with an efficacy coefficient, e.g., $e_{\mathrm{i}}$. Therefore, in Eqs. $6.10 \mathrm{~b}$ and $6.11 \mathrm{~b}$ the newly introduced multiplier of a running number i may be replaced by an efficacy term $e_{\mathrm{i}}$. Such an implementation equals Ariëns' introduction of 'intrinsic activity' (Ariëns 1954), and is more an algebraic than an actual physical modeling that involve new multi-site interactions.

\subsection{Random and Ordered Reactions Combined with Energized Isomerization}

\subsubsection{Combination of Ordered/random Models with Isomerization}

Weiss (1997) also treats an extended random/ordered model with a consideration of an activation step for the receptor, that is, an isomerization step for a fully occupied receptive unit as in the $\mathrm{K} \& \mathrm{~T}$ scheme in Chapter 5, but with more than one ligand bound (see model in Fig. 6.10).

The model in Fig. 6.10 is a compulsory reaction scheme with binding of ligands to $n$ numbers of binding sites coupled to an isomerization step, a conformational change between a reactive state and an active state, where the Hill coefficient can approach the number of binding sites. The requirement is that the allosteric coefficient $L^{\mathrm{ns}}$ is dramatically favoring the active state, $L^{\mathrm{ns}}>>10^{4} \cdot L^{1}$ to $L^{\mathrm{ns}-1} \cdot L^{\mathrm{ns}}$ is the equilibrium constant between two inter-convertible conformations, a reactive, $\mathrm{RS}_{\mathrm{n}}$, and an active, $\mathrm{R} * \mathrm{~S}_{\mathrm{n}}$, state with $\mathrm{n}$ bound ligands. Thus, $L^{\mathrm{ns}}=\left[\mathrm{R}^{*} \mathrm{~S}_{\mathrm{n}}\right] /\left[\mathrm{RS}_{\mathrm{n}}\right]$ (Fig. 6.10).

To illustrate, the Hill coefficient may come close to the theoretical maximal value of 4 in a tetrameric 

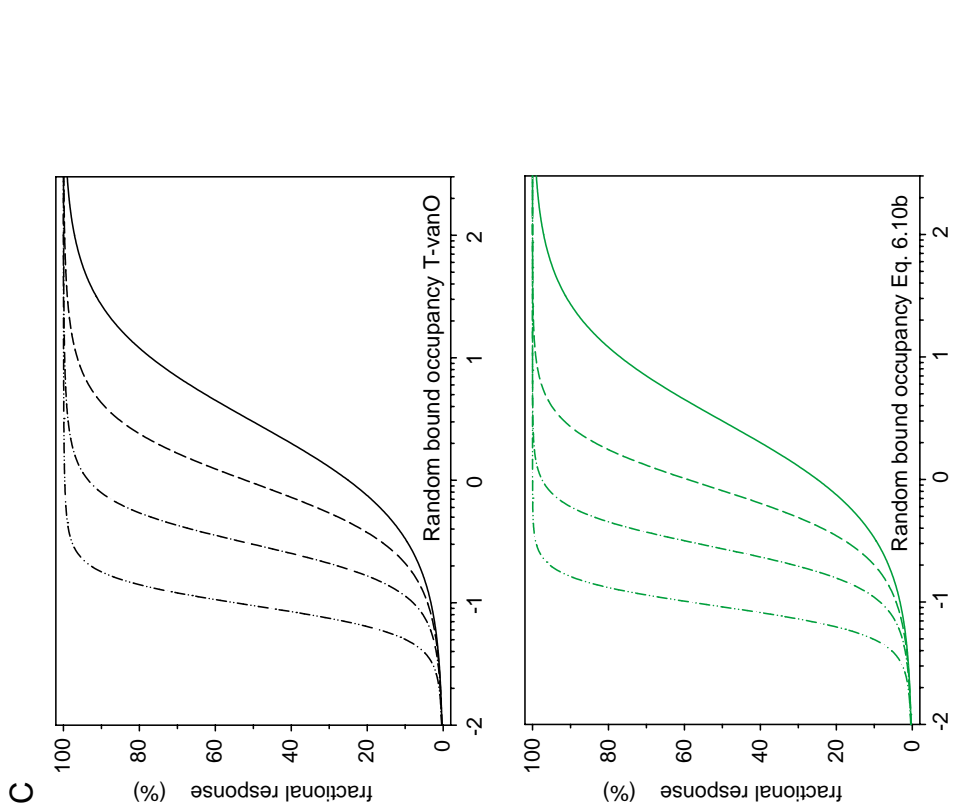

$\frac{0}{\omega}+\frac{\omega}{0} \geqq$

舟需

की

ब

은

융유워

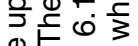

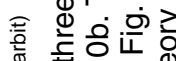

ष

家

空

용 웡 क

흥휴 당

के

¿.

苋

$=\pi$ m

(\%) әsuodsəג ןeuo!̣юеג!

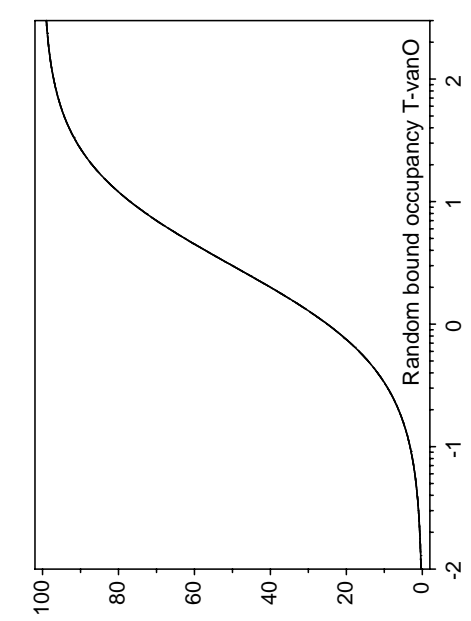

m

(\%) әsuodsəג ןеuo!̣əeג!

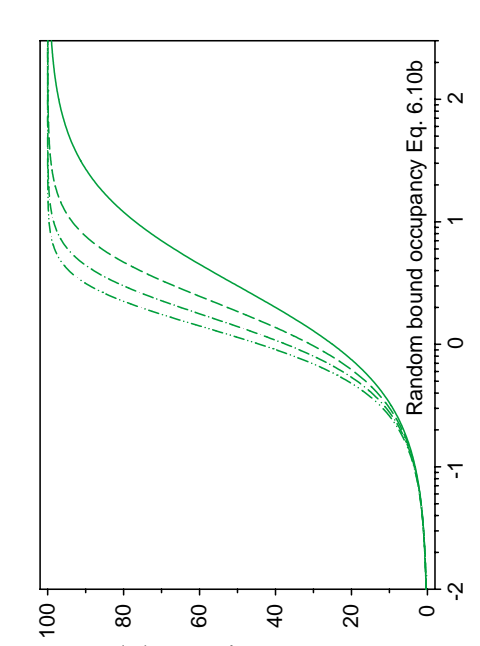

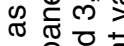

응 은 훙

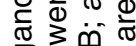

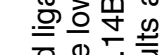

원

음 훈

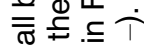

₹.

홇

ठㅁ. लि

एक

落 $\frac{0}{3} \mathrm{~m}^{-1} \frac{\hat{0}}{0}$

잉

용

耐

등 윙 응

(\%) əsuodsəג ןeuo!̣’eג
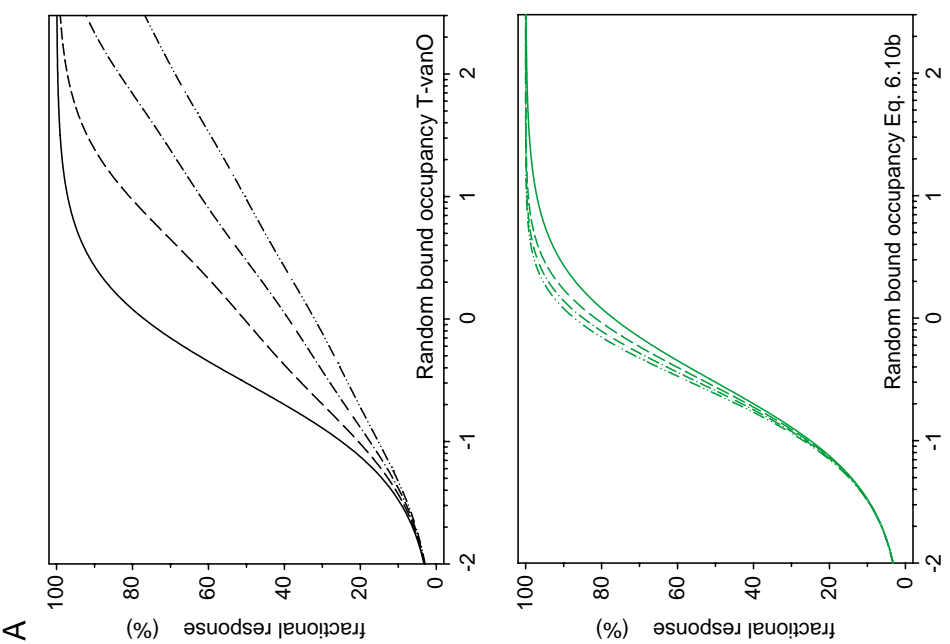

\&

응 융

๘

ช $>$ 융 ฮ

文亭可

胥比

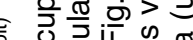

这

7 वृ क्ष

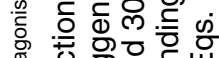

요 $\stackrel{\pi}{=}$ 공

齐

के

ช

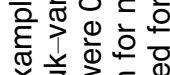

凶थ $3 \leq$

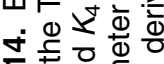

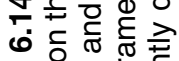

웡 

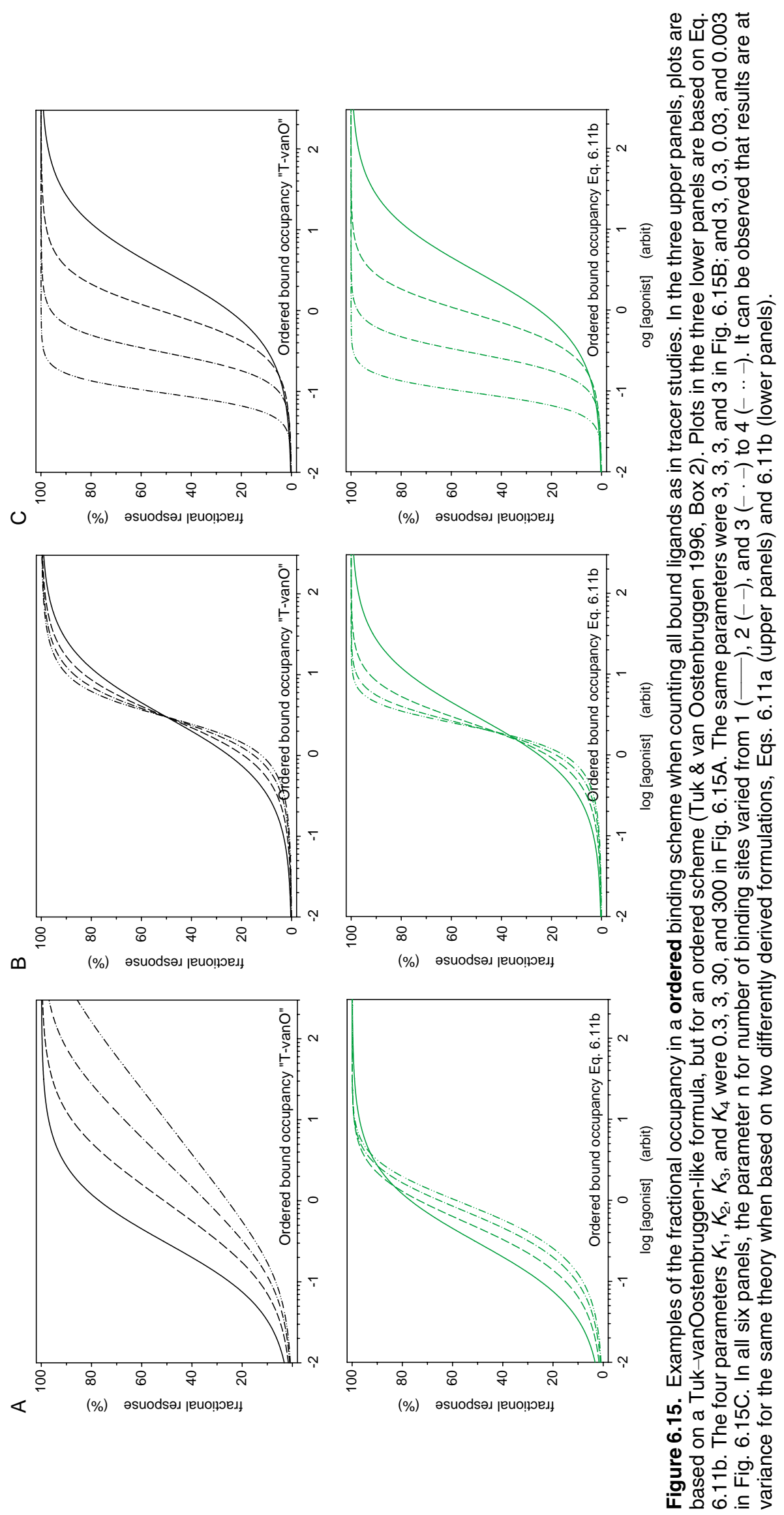
binding site system for an ordered system equated in Eq. 6.11 and for a random reaction scheme, as equated in Eq. 6.10, with the added final activation, even without a genuine co-operativity (i.e., with $K_{1}=K_{2}=\cdots=K_{4}$ ), in case the isomerization constant, $L^{\mathrm{ns}}$, is in favor of an activated state, $\mathrm{RS}_{\mathrm{n}} \rightleftharpoons \rightarrow \rightarrow \mathrm{R} * \mathrm{~S}_{\mathrm{n}}$. Another possibility is a deactivation by a high rate for the return step in the reaction scheme in Fig. $6.10, \mathrm{R}^{*} \mathrm{~S}_{\mathrm{n}-1} \rightleftharpoons \mathrm{RS}_{\mathrm{n}-1}$. This will function as a fast final step for activation and asymptoting the value of the Hill coefficient towards the number of binding sites.

When the cyclic reaction, depicted in the reaction scheme in Fig. 6.16, is driven by input of ATP-energy more complex behaviors can arise, the dose-response curve can for example take on biphasic shapes (Weiss 1997).

In the next two Sub-chapters I exemplify random and ordered reaction schemes combined with isomerization and energized by GTPase or ATPase activity leading to irreversible one-way ping-pong mechanisms.

\subsection{GTP-driven G Proteins and One-way Ping-pong}

Most $G$ proteins are found in two superfamilies, the heterotrimeric $\mathrm{G}$ protein superfamily and the monomeric G protein or small GTPase superfamily (Fig. 6.1).

\subsubsection{Heterotrimeric G Proteins}

The superfamily of heterotrimeric $\mathrm{G}$ proteins consists of $\mathrm{G}_{\alpha}^{\prime} \mathrm{s}$ in five families or subtypes consisting of $\mathrm{G}_{t}, \mathrm{G}_{s}, \mathrm{G}_{o / i}$, $\mathrm{G}_{q / 11}$ and $\mathrm{G}_{12}$ (Kristiansen 2004). When bound to various beta-gamma subunits, $\mathrm{G}_{\beta \gamma}$, they are in an inactive state in the form of a loosely associated heterotrimeric alfa-beta-gamma $\mathrm{G}$ protein with bound GDP. GPCRs are allosteric modulators of $\mathrm{G}$ protein activity, and the association of $\mathrm{G}_{\alpha}$ with $\mathrm{G}_{\beta \gamma}$ stabilizes the $\mathrm{G}_{\alpha}-\mathrm{GPCR}$ interface (Fig. 6.1A).

The one-way ping-pong mechanism for heterotrimeric $\mathrm{G}$ protein is as follows. The inactive state of the $\mathrm{G}$ proteins is activated by stimulating GPCRs, which, in turn, may be activated by extracellular first messengers such as neurotransmitters, hormones, paracrine and autocrine signaling molecules as well as odorants, light, and mechanical stimuli. During activation, there is an exchange of GDP for GTP at the $\mathrm{G}_{\alpha}^{\prime}$ s.

The activation also triggers a dissociation of $\mathrm{G}_{\alpha}{ }^{\prime} \mathrm{s}$ from the beta-gamma subunits and, through the activated $\mathrm{G}_{\alpha^{-}}$ GTP and the released $\mathrm{G}_{\beta \gamma}$, initiates an effector activation such as increased enzyme catalysis or ion flow through transporters as pumps and channels with an appearance or production of second messengers, like $\mathrm{Ca}^{2+}$ and
cyclicAMP. This includes changes in the electrical potential of the cell membrane. The activated $\mathrm{G}_{\alpha}-\mathrm{GTP}$ moiety is inactivated by catalysis of GTP to GDP by an intrinsic GTPase activity of the $\mathrm{G}_{\alpha}$ subunit. This catalysis delivers the energy for an irreversible one-way reaction (Kristiansen 2004; Oldham \& Hamm 2006, 2008).

\subsubsection{Small GTPases}

The monomeric G protein superfamily, or Ras superfamily, consists of five families of small GTPase: Ras, Rho/Rac, Arf, Rab, and the Ran families, and within each there are several subfamilies (Mitin et al. 2005; D'Souza-Schorey \& Chavrier 2006; ten Klooster \& Hordijk 2007; Bustelo et al. 2007). Many of the small GTPases are involved in cell cycle, development, proliferation, movement, and apoptosis (Fig. 6.1B).

\subsubsection{GDP-GTP Exchange}

The exchange of GDP-GTP both at $\mathrm{G}_{\alpha} \mathrm{s}$ in the heterotrimeric as well as at all the $\mathrm{G}$ proteins of the monomeric superfamily, where GDP jumps off before GTP docks (Fig. 6.1A+B), may therefore be described as a double displacement process - ping-pong (Heck \& Hofmann 2001). The exchange is regulated by cytoplasmic proteins such as the guanine nucleotide exchange factor (GEF). The rate of turn-off of activated $\mathrm{G}$ proteins, which is the energy producing process of the intrinsic GTPase activity of $G$ proteins, is also regulated by other types of cytoplasmic proteins for example the GTPase activating protein (GAP), the family or superfamily of regulators of $G$ protein signaling (RGS) (Nunn et al. 2006), or the receptor activity modifying proteins (RAMPs) (Parameswaran \& Spielman 2006).

Other regulatory proteins are the GDP dissociation inhibitors (GDIs), which inhibit the dissociation of GDP from the $\mathrm{G}$ protein. These molecules also prevent the trafficking and association of the Rho/Rac and Rab G proteins to the plasma membrane as well as interaction between GEF, GAP or pertinent effector molecules and the $G$ proteins (DerMardirossian \& Bokoch 2005; DerMardirossian et al. 2006; Oldham \& Hamm 2006, 2008; Bustelo et al. 2007).

\subsection{ATP-driven Pumps and One-way Ping-pong}

\subsubsection{P-type Pumps}

Following crystallization and structural resolution at a $2.6 \AA$ scale of a calcium pump from sarcoplamatic reticulum, SERCA (Fig. 1.17) (Toyoshima et al. 2000; Jensen et al. 2006; Toyoshima 2007), this ATP-driven 
enzyme has become the prototype for modeling ion transfer in P-type pumps as the sodium pump, Na,KATPase (Ogawa \& Toyoshima 2002; Jorgensen 2003; Artigas \& Gadsby 2003a,b; Reynes \& Gadsby 2006), the proton-potassium pump, H,K-ATPase (Fig. 6.9C) (Munson et al. 2007), and others including plant $\mathrm{H}^{+}$-ATPases (Buch-Pedersen \& Palmgren 2003; Gaxiola et al. 2007).

Both the binding to and release of two calcium ions from the Ca-ATPase pump seem to be in an ordered fashion (Forge et al. 1995), although the two ions are randomized during phosphorylated isomerization of the pump (Canet et al. 1996).

A closing rear door mechanism involved in the transfer of ions through the pumping systems is part of the one-way ping-pong movement of ions in pumpATPases.

Thus, the earlier back door occlusion of ions described by Glynn and Karlish (1990) for the Na,KATPase, now has its molecular correlate (Fig. 1.17) (Toyoshima 2007).

\subsection{2. $A B C$-Transporters}

The growing superfamily of ATP-binding cassette $(\mathrm{ABC})$ transporters also possesses a one-way movement of a whole host of different molecules due to the input of ATP energy (Al-Shawi \& Omote 2005). Although many details have been collected about the transfer of drugs and molecules through the $\mathrm{ABC}$ transporters (Saito et al. 2006; Higgens 2007), the molecular understanding of the passage is still on the drawing board. The efflux pump in Gram negative bacteria including an $\mathrm{ABC}$ transporter is further complex and exists in at least four general types, Types I-IV (Gerlach \& Hensel 2007) (Fig. 6.16).

\subsubsection{Carriers}

Of course, reaction schemes such as the ping-pong transfer mechanism may also describe conditions for other enzymes, signaling systems, and transporters including carriers. A recent example of a bi-way pingpong mechanism by a carrier is the transfer of cholesteryl esters (CEs), and triglycerides (TGs), between VLDL- and HDL-particles via a cholesteryl ester transfer protein (CETP), where two molecules of CE or two molecules of TG may be shoveled through a $60 \AA$ A-tunnel in the CETP carrier (Qiu et al. 2007).

\subsubsection{Development of Models with Two-states and Ping-pong Driven by ATP}

For pumps, the first ping-pong scheme, actually with a two-state model, was suggested by Shaw in 1954, and referred to and presented by Glynn (1955, 2002). Glynn did not derive any formulation for a two-state ping-pong reaction scheme by Shaw. Two-state formulation first appeared with the K\&T model two years later (Katz \& Thesleff 1957).

A cyclic reaction scheme with the receptive unit in two different conformations, but going in only one direction, seems to be a characteristic of some enzyme catalyzed processes (Segel 1975, 1993) and for most of the ATPase transporters, including pumps such as the sodium pump, the Na,K-ATPases (Jorgensen \& Pedersen 2001; Jorgensen et al. 2003), the calcium pump,

Type I secretion in Gram-negative bacterium

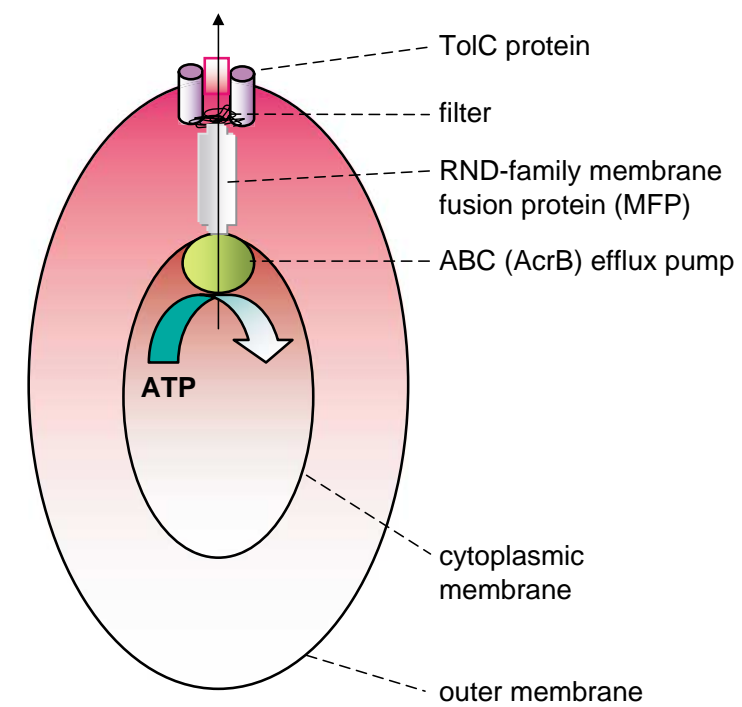

Figure 6.16. Schematic type I efflux pump of Eschericia coli. TolC refers to a protein encoded from tolC locus in the E. Coli K-12 genome. RND = resistance-nodulatin-cell division. Detailed descriptions in Murakami et al. (2006) and Seeger et al. (2006). 
Table 6.3. Survey of Models Discussed in Chapter 6

\begin{tabular}{|c|c|c|c|}
\hline \multirow[b]{2}{*}{ Reaction scheme } & \multicolumn{3}{|c|}{ Fractional number of } \\
\hline & Fully occupied receptive units & Intermediate + fully occupied & All bound ligands \\
\hline Simple random & Eq. 6.8 Weiss/T-vanO** & Eq. 6.10a T-vanO & Eq. $6.10 \mathrm{~b}$ Table 6.2 \\
\hline Simple ordered & Eq. 6.9 Weiss & Eq. $6.11 \mathrm{a}$ & Eq. $6.11 \mathrm{~b}$ Table 6.2 \\
\hline Extended $\mathbf{r}$./o.* & Sub-chapters $6.2-6.4$ & & \\
\hline Ping-pong & $\begin{array}{l}\text { Sub-chapter } 6.5 \text {, see Segel } \\
\quad(1975,1993, \text { Chapter } 9)\end{array}$ & & \\
\hline
\end{tabular}

*Extending simple two-site random and ordered reaction schemes with a step of isomerization is the same as an extension of a one-site dC\&K model with an extra binding site; now with two binding sites.

**Weiss (1997) and T-vanO (Tuk \& van Oostenbruggen 1996).

Ca-ATPases (Andersen et al. 2001; Antonisen et al. 2006; Dode et al. 2006) as well as the mammalian and bacterial multidrug resistance transporters (Borst \& Elferink 2002; Litman et al. 2003; Al-Shawi \& Omote 2005; Dawson \& Locher 2006; Murakami et al. 2006; Seeger et al. 2006; Lobedanz et al. 2007; Higgins 2007).

For enzymes and pumps, this type of reaction scheme evolved in the 1950s (Watanabe et al. 1953; Glynn 1955; Blum 1955; Albers et al. 1962; Post et al. 1965) and preceded the fully reciprocal two-state model (cTSM), developed for enzymes by Botts and Drain (1958).

The full scheme for the Na,K-ATPase is much more complex than presented here, as the regulation of the enzyme activity is dependent on $\left[\mathrm{Na}^{+}\right]$at the inside to the third power and on $\left[\mathrm{K}^{+}\right]$at the outside to the second power as well as the inside concentration of Mg-ATP. In addition, the membrane potential influences the energy barriers for the transport function of the Na-pump. It seems to require a dynamic time-dependent formulation and thus lies outside the content and intent of this text.

\subsection{Conclusions for Extended dC\&K Models}

\subsubsection{Early Models of Non-fully Occupied Receptive Units}

The scenario with a combination of sequential multisteps, including conformational changes as for reaction schemes in Fig. 6.10, yields many possibilities. Early examples of such combined model treated with independent binding and without the requirement for fully occupied receptor units for functionality are the two historical key papers by Monod-Wyman-Changeux (Monod et al. 1965) and Koshland-Nemthy-Filmer (Koshland et al. 1966) (see Chapters 14 and 15).

\subsubsection{Conclusions from Extensions of the $d C \& K$ Scheme}

A survey of the discussed models in the chapter is listed in Table 6.3.
A comparison between the simple random and ordered models with an ordinary Hill model is demonstrated in Fig. 6.11 (see Tuc \& van Oostenbruggen 1996 and Weiss 1997). The Hill coefficient never drops below unity in the simple random and ordered reaction schemes with full occupancy.

The two-site and three-step models in the form of the extended $\mathrm{dC \& K}$ or the simple random/ordered reaction schemes extended with an isomerization step were analyzed with the Hill model (Chapter 10), see Fig. 6.13. This analysis is certainly informative. A similar exercise was carried out by Weiss (1997).

\subsubsection{Ping-pong Models and ATPases}

Many enzymes, cotransporters and pumps seem to operate according to so-called ping-pong models. There are still debates about the realism of such models for certain enzymes. One such recent example, the Nacetyltransferase in E. Coli may rather be described by a random model (Hindson \& Shaw 2003) supported by additional observations (Delgado et al. 2003).

Since the understanding of ion movement in ATPasedriven systems has recently developed at a high rate to an exquisitely sophisticated level, its literature should be consulted directly (Glynn \& Karlish 1990; Apell et al. 1996; Gropp et al. 1998; Andersen et al. 2001; GonzalezLebrero et al. 2002; Jorgensen et al. 2003; Antonisen et al. 2006; Dode 2006; Picard et al. 2006; Reyes \& Gadsby 2007; Munson et al. 2007; Toyoshima 2007).

\subsubsection{Nota Bene}

As a memento to what has happened in Chapter 6, it is recommended to introduce new models with care.

\section{REFERENCES}

Abuladze N, Azimov R, Newman D, Sassani P, Liu W, Tatishchev S, Pushkin A and Kurtz I. Critical amino acid residues involved in the electrogenic sodium-bicarbonate cotransporter kNBC1mediated transport. J Physiol 565: 717-730, 2005. 
Accardi A, Walden M, Nguitragool W, Jayaram H, Williams C \& Miller C. Separate ion pathways in a $\mathrm{Cl}-/ \mathrm{H}+$ exchanger. $J$ Gen Physiol 126: 563-570, 2005.

Accardi A, Lobet S, Williams C, Miller C \& Dutzler R. Synergism between halide binding and proton transport in a CLC-type exchanger. J Mol Biol 362: 691-699, 2006.

Adair GS. The hemoglobin system. VI. The oxygen dissociation curve of hemoglobin. J Biol Chem 63: 529-545, 1925.

Al-Shawi MK \& Omote $H$. The remarkable transport mechanism of P-glycoprotein: a multidrug transporter. J Bioenerg Biomembr 37: 489-496, 2005.

Albers RW \& Koval GJ. Properties of the sodium-dependent ATPase of electrophorus electricus. Life Sci 5: 219-222, 1962.

Allen TW, Andersen OS \& Roux B. Energetics of ion conduction through the gramicidin channel. Proc Natl Acad Sci USA 101: 117122, 2004.

Andersen JP, Sorensen TL, Povlsen K \& Vilsen B. Importance of transmembrane segment M3 of the sarcoplasmic reticulum $\mathrm{Ca} 2+$-ATPase for control of the gateway to the $\mathrm{Ca} 2+$ sites. J Biol Chem 276: 23312-23321, 2001.

Anthonisen AN, Clausen JD \& Andersen JP. Mutational analysis of the conserved TGES loop of sarcoplasmic reticulum Ca2+-ATPase. J Biol Chem 281: 31572-31582, 2006.

Apell HJ, Roudna M, Corrie JE \& Trentham DR. Kinetics of the phosphorylation of Na,K-ATPase by inorganic phosphate detected by a fluorescence method. Biochemistry 35: 10922-10930, 1996.

Ariëns EJ. Affinity and intrinsic activity in the theory of competitive inhibition. Part I. Problems and theory. Arch Int Pharmacodyn 99: 32-49, 1954

Artigas P \& Gadsby DC. Ion occlusion/deocclusion partial reactions in individual palytoxin-modified Na/K pumps. Ann N Y Acad Sci 986 : 116-126, 2003a.

Artigas P \& Gadsby DC. Ion channel-like properties of the $\mathrm{Na}+/ \mathrm{K}+$ Pump. Ann N Y Acad Sci 976: 31-40, 2003b.

Baekgaard A \& Bindslev N. Novel protein kinase C, nPKC, inhibition of murine bumetanide-sensitive $\mathrm{Na}+, \mathrm{K}+, 2 \mathrm{Cl}$-cotransporter $\mathrm{BSCl}$ in Xenopus oocyte. Pflugers Arch 436: 189-198, 1998.

Beguin P, Ng YJ, Krause C, Mahalakshmi RN, Ng MY \& Hunziker W. RGK small GTP-binding proteins interact with the nucleotide kinase domain of $\mathrm{Ca} 2+$-channel beta-subunits via an uncommon effector binding domain. J Biol Chem 282: 11509-11520, 2007.

Bekker M, Teixeira de Mattos MJ \& Hellingwerf KJ. The role of twocomponent regulation systems in the physiology of the bacterial cell. Sci Prog 89: 213-242, 2006.

Berg JM, Tymoczko JL \& Stryer L. Biochemistry, 5th ed. New York: WH Freeman, 2002.

Berg JM, Tymoczko JL \& Stryer L. Biochemistry, 6th ed. New York: WH Freeman, 2006.

Bernardo AA, Bernardo CM, Espiritu DJ \& Arruda JA. The sodium bicarbonate cotransporter: structure, function, and regulation. Semin Nephrol 26: 352-360, 2006.

Blum JJ. The enzymatic interaction between myosin and nucleotides. Arch Biochem Biophys 55: 486-511, 1955.

Borst P \& Elferink RO. Mammalian ABC transporters in health and disease. Annu Rev Biochem 71: 537-592, 2002.

Botts J \& Drain GF. An illustration of a kinetic analysis: the myosin B-ATP-EDTA system. The Committee of Muscle Chemistry of Japan, 1958, pp. 33-41. Tokyo, Igaku Shoin: Conference on the Chemistry of Muscular Contraction, 1957.

Buch-Pedersen MJ \& Palmgren MG. Mechanism of proton transport by plant plasma membrane proton ATPases. J Plant Res 116: 507515, 2003.

Bustelo XR, Sauzeau V \& Berenjeno IM. GTP-binding proteins of the Rho/Rac family: regulation, effectors and functions in vivo. Bioessays 29: 356-370, 2007.
Bykova EA, Zhang XD, Chen TY \& Zheng J. Large movement in the C terminus of CLC-0 chloride channel during slow gating. Nat Struct Mol Biol 13: 1115-1119, 2006.

Canet D, Forge V, Guillain F \& Mintz E. Ca2 + translocation across sarcoplasmic reticulum ATPase randomizes the two transported ions. J Biol Chem 271: 20566-20572, 1996.

Cassel D \& Selinger Z. Catecholamine-stimulated GTPase activity in turkey erythrocyte membranes. Biochim Biophys Acta 452: 538-551, 1976.

Changeux JP \& Edelstein SJ. Allosteric mechanisms of signal transduction. Science 308: 1424-1428, 2005

Chen TY. Structure and function of clc channels. Annu Rev Physiol 67: 809-839, 2005.

Chen TY, Chen MF \& Lin CW. Electrostatic control and chloride regulation of the fast gating of ClC-0 chloride channels. J Gen Physiol 122: 641-651, 2003.

Ciarkowski J, Witt M \& Slusarz R. A hypothesis for GPCR activation. J Mol Model 11: 407-415, 2005.

Cleland WW. The kinetics of enzyme-catalyzed reactions with two or more substrates or products. Biochim Biophys Acta 67: 104-137, 1963.

Cleland WW, Frey PA \& Gerlt JA. The low barrier hydrogen bond in enzymatic catalysis. J Biol Chem 273: 25529-25532, 1998.

Colquhoun D. Agonist-activated ion channels. Br J Pharmacol 147: S17S26, 2006a.

Colquhoun D. The quantitative analysis of drug-receptor interactions: a short history. Trends Pharmacol Sci 27: 149-157, $2006 \mathrm{~b}$.

Coomes MW. Amino acid metabolism. In: Textbook of Biochemistry with Clinical Correlations, 6th ed., edited by Devlin TM. New York: Wiley-Liss, 2006.

Cornish-Bowden A. Fundamentals of Enzyme Kinetics, 2nd ed. London: Portland Press Ltd., 1995.

Cornish-Bowden A. Fundamentals of Enzyme Kinetics, 3rd ed. Colchester: Portland Press Ltd., 2004.

Corsi M, Fina P \& Trist DG. Co-agonism in drug-receptor interaction: illustrated by the NMDA receptors. Trends Pharmacol Sci 17: 220 222, 1996

Cowan-Jacob SW. Structural biology of protein tyrosine kinases. Cell Mol Life Sci 63: 2608-2625, 2006.

Cymes GD, Grosman C \& Auerbach A. Structure of the transition state of gating in the acetylcholine receptor channel pore: a phi-value analysis. Biochemistry 41: 5548-5555, 2002.

D'Souza-Schorey C \& Chavrier P. ARF proteins: roles in membrane traffic and beyond. Nat Rev Mol Cell Biol 7: 347-358, 2006.

Dawson RJP \& Locher KP. Structure of a bacterial multidrug ABC transporter. Nature 443: 180-185, 2006.

Delgoda R, Lian LY, Sandy J \& Sim E. NMR investigation of the catalytic mechanism of arylamine N-acetyltransferase from Salmonella typhimurium. Biochim Biophys Acta 1620: 8-14, 2003.

DerMardirossian C \& Bokoch GM. GDIs: central regulatory molecules in Rho GTPase activation. Trends Cell Biol 15: 356363, 2005.

DerMardirossian C, Rocklin G, Seo JY \& Bokoch GM. Phosphorylation of RhoGDI by Src regulates Rho GTPase binding and cytosolmembrane cycling. Mol Biol Cell 17: 4760-4768, 2006.

Dode L, Andersen JP, Vanoevelen J, Raeymaekers L, Missiaen L, Vilsen B \& Wuytack F. Dissection of the functional differences between human secretory pathway $\mathrm{Ca} 2+/ \mathrm{Mn} 2+$-ATPase (SPCA) 1 and 2 isoenzymes by steady-state and transient kinetic analyses. J Biol Chem 281: 3182-3189, 2006

Dutzler R. The ClC family of chloride channels and transporters. Curr Opin Struct Biol 16: 439-446, 2006.

Dutzler R, Campbell EB \& MacKinnon R. Gating the selectivity filter in ClC chloride channels. Science 300: 108-112, 2003 
Estevez R, Pusch M, Ferrer-Costa C, Orozco M \& Jentsch TJ. Functional and structural conservation of CBS domains from CLC channels. J Physiol 557: 363-378, 2004.

Falk S, Guay A, Chenu C, Patil SD \& Berteloot A. Reduction of an eight-state mechanism of cotransport to a six-state model using a new computer program. Biophys J 74: 816-830, 1998.

Forge V, Mintz E, Canet D \& Guillain F. Lumenal Ca2+ dissociation from the phosphorylated $\mathrm{Ca}(2+)$-ATPase of the sarcoplasmic reticulum is sequential. J Biol Chem 270: 18271-18276, 1995.

Fotiadis D, Jastrzebska B, Philippsen A, Muller DJ, Palczewski K \& Engel A. Structure of the rhodopsin dimer: a working model for G-protein-coupled receptors. Curr Opin Struct Biol 16: 252-259, 2006.

Gaxiola RA, Palmgren MG \& Schumacher K. Plant proton pumps. FEBS Lett 581: 2204-2214, 2007.

Gerlach RG \& Hensel M. Protein secretion systems and adhesins: the molecular armory of Gram-negative pathogens. Int J Med Microbiol 297: 401-415, 2007.

Gibb AJ. Receptors linked to ion channels: activation and block. In: Textbook of Receptor Pharmacology, edited by Foreman JC \& Johansen T. Boca Raton, FL: CRC Press, 1996, pp. 159-185.

Glynn IM. The ionic permeability of the red cell membrane. Prog Biophys 8: 241-307, 1955.

Glynn IM. A hundred years of sodium pumping. Annu Rev Physiol 64: 1-18, 2002.

Glynn IM \& Karlish SJ. Occluded cations in active transport. Annu Rev Biochem 59: 171-205, 1990.

Gonzalez-Lebrero RM, Kaufman SB, Montes MR, Norby JG, Garrahan PJ \& Rossi RC. The occlusion of $\mathrm{Rb}(+)$ in the $\mathrm{Na}(+) / \mathrm{K}(+)$ ATPase. I. The identity of occluded states formed by the physiological or the direct routes: occlusion/deocclusion kinetics through the direct route. J Biol Chem 277: 5910-5921, 2002.

Gronski P, Schridde C \& Kanzy EJ. Off-rate and concentration diversity in multidonor-derived dimers of immunoglobulin G. Mol Immunol 44: 2528-2540, 2007.

Gropp T, Cornelius F \& Fendler K. Abstract K+-dependence of electrogenic transport by the NaK-ATPase. Biochim Biophys Acta 1368: 184-200, 1998.

Grottesi A, Domene C, Haider S \& Sansom MS. Molecular dynamics simulation approaches to K channels: conformational flexibility and physiological function. IEEE Trans Nanobiosci 4: 112-120, 2005.

Hanes CS, Bronskill PM, Gurr PA \& Wong JT. Kinetic mechanism for the major isoenzyme of horse liver alcohol dehydrogenase. Can J Biochem 50: 1385-1413, 1972.

Heck M \& Hofmann KP. Maximal rate and nucleotide dependence of rhodopsin-catalyzed transducin activation: initial rate analysis based on a double displacement mechanism. J Biol Chem 276: 10000-10009, 2001

Higgins CF. Multiple molecular mechanisms for multidrug resistance transporters. Nature 446: 749-757, 2007.

Hille B. Ionic Channels of Excitable Membranes, 3rd ed. Sunderland: Sinauer Associates, 2001.

Hindson VJ \& Shaw WV. Random-order ternary complex reaction mechanism of serine acetyltransferase from Escherichia coli. Biochemistry 42: 3113-3119, 2003

Hoch JA. Control of cellular development in sporulating bacteria by the phosphorelay two-component signal transduction system. In: Two-component Signal Transduction, edited by Hoch JA \& Silhavy TJ. Washington, DC: ASM Press, 1995, pp. 129-144.

Hunte C, Screpanti E, Venturi M, Rimon A, Padan E \& Michel H. Structure of a Na $+/ \mathrm{H}+$ antiporter and insights into mechanism of action and regulation by pH. Nature 435: 1197-1202, 2005.

Jenkinson DH. Classical approaches to the study of drug-receptor interactions. In: Textbook of Receptor Pharmacology, 2nd ed., edited by Foreman JC \& Johansen T. Boca Raton, FL: CRC Press, 2003, pp. 3-62.

Jensen AM, Sorensen TL, Olesen C, Moller JV \& Nissen P. Modulatory and catalytic modes of ATP binding by the calcium pump. EMBOJ 25: 2305-2314, 2006

Jentsch TJ, Poet M, Fuhrmann JC \& Zdebik AA. Physiological functions of CLC Cl- channels gleaned from human genetic disease and mouse models. Annu Rev Physiol 67: 779-807, 2005.

Johnson JW \& Ascher P. Glycine potentiates the NMDA response in cultured mouse brain neurons. Nature 325: 529-531, 1987.

Jorgensen PL. Transmission of E1-E2 structural changes in response to $\mathrm{Na}+$ or K+ binding in Na,K-ATPase. Ann N Y Acad Sci 986: 2230, 2003.

Jorgensen PL \& Pedersen PA. Structure-function relationships of $\mathrm{Na}(+), \mathrm{K}(+)$, ATP, or $\mathrm{Mg}(2+)$ binding and energy transduction in Na,K-ATPase. Biochim Biophys Acta 1505: 57-74, 2001.

Jorgensen PL, Hakansson KO \& Karlish SJ. Structure and mechanism of Na,K-ATPase: functional sites and their interactions. Annu Rev Physiol 65: 817-849, 2003.

Katz B \& Thesleff S. A study of the desensitization produced by acetylcholine at the motor end-plate. J Physiol 138: 63-80, 1957.

Kelly K. The RGK family: a regulatory tail of small GTP-binding proteins. Trends Cell Biol 15: 640-643, 2005.

Kim SK \& Jacobson KA. Computational prediction of homodimerization of the A3 adenosine receptor. J Mol Graph Model 25: 549-561, 2006.

Kim SK, Gao ZG, Jeong LS \& Jacobson KA. Docking studies of agonists and antagonists suggest an activation pathway of the A3 adenosine receptor. J Mol Graph Model 25: 562-577, 2006.

King EL \& Altman C. A schematic method of deriving the rate laws for enzyme-catalyzed reactions. J Am Chem Soc 60: 1377-1381, 1956.

Kobayashi T, Yamada Y, Fukao M, Tsutsuura M \& Tohse N. Regulation of $\mathrm{ca}(\mathrm{v}) 1.2$ current: interaction with intracellular molecules. J Pharmacol Sci 103: 347-353, 2007.

Koshland DE Jr, Nemethy G \& Filmer D. Comparison of experimental binding data and theoretical models in proteins containing subunits. Biochemistry 5: 365-385, 1966.

Kristiansen K. Molecular mechanisms of ligand binding, signaling, and regulation within the superfamily of G-protein-coupled receptors: molecular modeling and mutagenesis approaches to receptor structure and function. Pharmacol Ther 103: 21-80, 2004

Lemieux MJ, Reithmeier RA \& Wang DN. Importance of detergent and phospholipid in the crystallization of the human erythrocyte anion-exchanger membrane domain. J Struct Biol 137: 322-332, 2002.

Lin CW \& Chen TY. Cysteine modification of a putative pore residue in ClC-0: implication for the pore stoichiometry of ClC chloride channels. J Gen Physiol 116: 535-546, 2000.

Litman T, Skovsgaard $\mathrm{T}$ \& Stein WD. Pumping of drugs by P-glycoprotein: a two-step process? J Pharmacol Exp Ther 307: 846-853, 2003.

Lobedanz S, Bokma E, Symmons MF, Koronakis E, Hughes C \& Koronakis V. A periplasmic coiled-coil interface underlying TolC recruitment and the assembly of bacterial drug efflux pumps. Proc Natl Acad Sci USA 104: 4612-4617, 2007.

Lohse MJ, Nikolaev VO, Hein P, Hoffmann C, Vilardaga JP \& Bünemann M. Optical techniques to analyze real-time activation and signaling of G-protein-coupled receptors. Trends Pharmacol Sci 29: $159-165,2008$

Loo DDF, Hirayama BA, Cha A, Bezanilla F \& Wright EM. Pertubation analysis of the voltage-sensitive conformational changes of the $\mathrm{Na}^{+}$/glucose cotransporter. J Gen Physiol 125: 13-36, 2005.

MacKinnon R. Potassium channels. FEBS Lett 555: 62-65, 2003.

Mackinnon R. Structural biology. Voltage sensor meets lipid membrane. Science 306: 1304-1305, 2004a. 
MacKinnon R. Nobel Lecture. Potassium channels and the atomic basis of selective ion conduction. Biosci Rep 24: 75-100, 2004b.

Michel D. Cooperative equilibrium curves generated by ordered ligand binding to multi-site molecules. Biophys Chem 129: 284-288, 2007.

Miller C. Open-state substructure of single chloride channels from Torpedo electroplax. Phil Trans R Soc Lond B 299: 401-411, 1982.

Miller C. ClC chloride channels viewed through a transporter lens. Nature 440: 484-489, 2006.

Miloshevsky GV \& Jordan PC. Anion pathway and potential energy profiles along curvilinear bacterial $\mathrm{ClC} \mathrm{Cl}(-)$ pores: electrostatic effects of charged residues. Biophys J 86: 825-835, 2004.

Mitin N, Rossman KL \& Der CJ. Signaling interplay in Ras superfamily function. Curr Biol 15: R563-R574, 2005.

Mitrophanov AY, Churchward G \& Borodovsky M. Control of Streptococcus pyogenes virulence: modeling of the CovR/S signal transduction system. J Theor Biol 246: 113-128, 2007.

Monod J, Wyman J \& Changeux J-P. On the nature of allosteric transitions: a plausible model. J Mol Biol 12: 88-118, 1965.

Munson K, Law RJ \& Sachs G. Analysis of the gastric H,K ATPase for ion pathways and inhibitor binding sites. Biochemistry 46: 53985417, 2007.

Murakami S, Nakashima R, Yamashita E, Matsumoto T \& Yamaguchi A. Crystal structures of a multidrug transporter reveal a functionally rotating mechanism. Nature 443: 173-179, 2006.

Nguitragool W \& Miller C. Uncoupling of a CLC $\mathrm{Cl}(-) / \mathrm{H}(+)$ exchange transporter by polyatomic anions. J Mol Biol 362: 682690, 2006.

Nicoll DA, Sawaya MR, Kwon S, Cascio D, Philipson KD \& Abramson J. The crystal structure of the primary $\mathrm{Ca} 2+$ sensor of the $\mathrm{Na}+/$ $\mathrm{Ca} 2+$ exchanger reveals a novel $\mathrm{Ca} 2+$ binding motif. J Biol Chem 281: 21577-21581, 2006.

Nishida M, Cadene M, Chait BT \& MacKinnon R. Crystal structure of a Kir3.1-prokaryotic Kir channel chimera. EMBO J 26: 4005-4415, 2007.

Nunn C, Mao H, Chidiac P \& Albert PR. RGS17/RGSZ2 and the RZ/A family of regulators of G-protein signaling. Semin Cell Dev Biol 17: 390-399, 2006.

Ogawa H \& Toyoshima C. Homology modeling of the cation binding sites of $\mathrm{Na}+\mathrm{K}+$-ATPase. Proc Natl Acad Sci USA 99: 15977-15982, 2002.

Oldham WM \& Hamm HE. Structural basis of function in heterotrimeric G proteins. Q Rev Biophys 39: 117-166, 2006.

Oldham WM \& Hamm HE. Heterotrimeric G protein activation by Gprotein-coupled receptors. Nat Rev Mol Cell Biol 9: 60-71, 2008.

Olkhova E, Hunte C, Screpanti E, Padan E \& Michel H. Multiconformation continuum electrostatics analysis of the NhaA $\mathrm{Na}+/ \mathrm{H}+$ antiporter of Escherichia coli with functional implications. Proc Natl Acad Sci USA 103: 2629-2634, 2006.

Onaran HO \& Gurdal H. Ligand efficacy and affinity in an interacting 7TM receptor model. Trends Pharmacol Sci 20: 274-278, 1999

Packer J, Conley E, Castle N, Wray D, January C \& Patmore L. Diversity of potassium channels. Trends Pharmacol Sci 21: 2000, Poster.

Parameswaran N \& Spielman WS. RAMPs: the past, present and future. Trends Biochem Sci 31: 631-638, 2006.

Parent L, Supplisson S, Loo DDF \& Wright EM. Electrogenic properties of the cloned $\mathrm{Na}+/$ glucose cotransporter. II. A transport model under nonrapid equilibrium conditions. J Membr Biol 125: 63-79, 1992

Parmentier ML, Prezeau L, Bockaert J \& Pin JP. A model for the functioning of family 3 GPCRs. Trends Pharmacol Sci 23: 268-274, 2002.

Pauling L. The oxygen equilibrium of hemoglobin and its structural interpretation. Proc Nat Acad Sci USA 21: 186-191, 1935.

Peitgen H-O, Jürgens H \& Saupe D. Classical Fractals and Selfsimilarity, Chaos and Fractals: New Frontiers of Science, 1st ed. Berlin: Springer-Verlag, 1992.
Perutz M. Stereochemistry of cooperative effects in haemoglobin. Nature 228: 726-739, 1970

Picard M, Toyoshima C \& Champeil P. Effects of inhibitors on luminal opening of Ca2 + binding sites in an E2P-like complex of sarcoplasmic reticulum Ca22+-ATPase with Be22+-fluoride. J Biol Chem 281: 3360-3369, 2006.

Piermarini PM, Choi I \& Boron WF. Cloning and characterization of an electrogenic $\mathrm{Na} / \mathrm{HCO} 3$ cotransporter from the squid giant fiber lobe. Am J Physiol Cell Physiol 292: C2032-C2045, 2007.

Pin JP, Kniazeff J, Liu J, Binet V, Goudet C, Rondard P \& Prezeau L. Allosteric functioning of dimeric class C G-protein-coupled receptors. FEBS J 272: 2947-2955, 2005.

Pin JP, Neubig R, Bouvier M, Devi L, Filizola M, Javitch JA, Lohse MJ, Milligan G, Palczewski K, Parmentier M \& Spedding M. International Union of Basic and Clinical Pharmacology. LXVII. Recommendations for the recognition and nomenclature of G protein-coupled receptor heteromultimers. Pharmacol Rev 59: $1-13,2007$.

Post RL, Sen AK \& Rosenthal AS. A phosphorylated intermediate in adenosine triphosphate-dependent sodium and potassium transport across kidney membranes. J Biol Chem 240: 1437-1445, 1965

Qiu X, Mistry A, Ammirati MJ, Chrunyk BA, Clark RW, Cong Y, Culp JS, Danley DE, Freeman TB, Geoghegan KF, Griffor MC, Hawrylik SJ, Hayward CM, Hensley P, Hoth LR, Karam GA, Lira ME, Lloyd DB, McGrath KM, Stutzman-Engwall KJ, Subashi AK, Subashi TA, Thompson JF, Wang IK, Zhao H \& Seddon AP. Crystal structure of cholesteryl ester transfer protein reveals a long tunnel and four bound lipid molecules. Nat Struct Mol Biol 14: $106-113,2007$

Reig N, Del Rio C, Casagrande F, Ratera M, Gelpi JL, Torrents D, Henderson PJ, Xie H, Baldwin SA, Zorzano A, Fotiadis D \& Palacin M. Functional and structural characterization of the first prokaryotic member of the L-amino acid transporter (LAT) family: A model for APC transporters. J Biol Chem 282: 1327013281, 2007.

Reyes N \& Gadsby DC. Ion permeation through the Na+ ,K+-ATPase. Nature 443: 470-474, 2006.

Rios CD, Jordan BA, Gomes I \& Devi LA. G-protein-coupled receptor dimerization: modulation of receptor function. Pharmacol Ther 92: 71-87, 2001.

Roughton FJW, Otis $\mathrm{AB}$ \& Lyster RLJ. The determination of the individual equilibrium constants of the four intermediate reactions between oxygen and sheep haemoglobin. Proc $R$ Soc B 144: 29-54, 1955 .

Sagoo MS \& Lagnado L. G-protein deactivation is rate-limiting for shutoff of the phototransduction cascade. Nature 389: 392-395, 1997.

Saito H, Hirano H, Nakagawa H, Fukami T, Oosumi K, Murakami K, Kimura H, Kouchi T, Konomi M, Tao E, Tsujikawa N, Tarui S, Nagakura M, Osumi M \& Ishikawa T. A new strategy of high-speed screening and quantitative structure-activity relationship analysis to evaluate human ATP-binding cassette transporter ABCG2-drug interactions. J Pharmacol Exp Ther 317: 1114-1124, 2006.

Screpanti E, Padan E, Rimon A, Michel H \& Hunte C. Crucial steps in the structure determination of the $\mathrm{Na}+/ \mathrm{H}+$ antiporter NhaA in its native conformation. J Mol Biol 362: 192-202, 2006.

Seeger MA, Schiefner A, Eicher T, Verrey F, Diederichs K \& Pos KM. Structural asymmetry of AcrB trimer suggests a peristaltic pump mechanism. Science 313: 1295-1298, 2006

Segel IH. Enzyme Kinetics. Behavior and Analysis of Rapid Equilibrium and Steady-state Enzyme Systems. New York: Wiley \& Sons (reissued 1993), 1975.

Shijie Z. Precious Mirror of the Four Elements. 1303. (published in China 1303 no publisher)

Sprang SR, Chen Z \& Du X. Structural basis of effector regulation and signal termination in heterotrimeric Galpha proteins. Adv Protein Chem 74: 1-65, 2007. 
Swaminath G, Xiang Y, Lee TW, Steenhuis J, Parnot C \& Kobilka BK. Sequential binding of agonists to the beta2 adrenoceptor. Kinetic evidence for intermediate conformational states. J Biol Chem 279: 686-691, 2004.

ten Klooster JP \& Hordijk PL. Targeting and localized signalling by small GTPases. Biol Cell 99: 1-12, 2007.

Tolkovsky AM \& Levitzki A. Theories and predictions of models describing sequential interactions between the receptor, the GTP regulatory unit, and the catalytic unit of hormone dependent adenylate cyclases. J Cyclic Nucleotide Res 7: 139-150, 1981.

Toyoshima C. Ion pumping by calcium ATPase of sarcoplasmic reticulum. Adv Exp Med Biol 592: 295-303, 2007.

Toyoshima C, Nakasako M, Nomura H \& Ogawa H. Crystal structure of the calcium pump of sarcoplasmic reticulum at $2.6 \mathrm{~A}$ resolution. Nature 405: 647-655, 2000.

Tuk B \& van Oostenbruggen MF. Solving inconsistencies in the analysis of receptor-ligand interactions. Trend Pharmacol Sci 17: 403-409, 1996.

Unwin N. Structure and action of the nicotinic acetylcholine receptor explored by electron microscopy. FEBS Lett 555: 91-95, 2003.
Unwin N. Refined structure of the nicotinic acetylcholine receptor at 4 A resolution. I Mol Biol 346: 967-989, 2005.

Wang J, Stieglitz KA, Cardia JP \& Kantrowitz ER. Structural basis for ordered substrate binding and cooperativity in aspartate transcarbamoylase. Proc Natl Acad Sci USA 102: 8881-8886, 2005.

Watanabe S, Tonomura Y \& Shiokawa H. Mechanism of muscular contraction. II. Kinetic studies on muscle ATP-ase. J Biochem 40: 387-402, 1953.

Weiner H. Enzymes: classification, kinetics, and control. In: Textbook of Biochemistry, edited by Devlin TM. Hoboken, NJ: Wiley-Liss, 2006.

Weiss JN. The Hill equation revisited: uses and misuses. FASEB J 11: 835-841, 1997.

Whaley BS, Yuan N, Birnbaumer L, Clark RB \& Barber R. Differential expression of the beta-adrenergic receptor modifies agonist stimulation of adenylyl cyclase: a quantitative evaluation. $\mathrm{Mol}$ Pharmacol 45: 481-489, 1994.

Wright EM, Hirayama BA \& Loo DF. Active sugar transport in health and disease. J Intern Med 261: 32-43, 2007.

Zeng F \& Wess J. Molecular aspects of muscarinic receptor dimerization. Neuropsychopharmacology 34: S19-S31, 2000. 


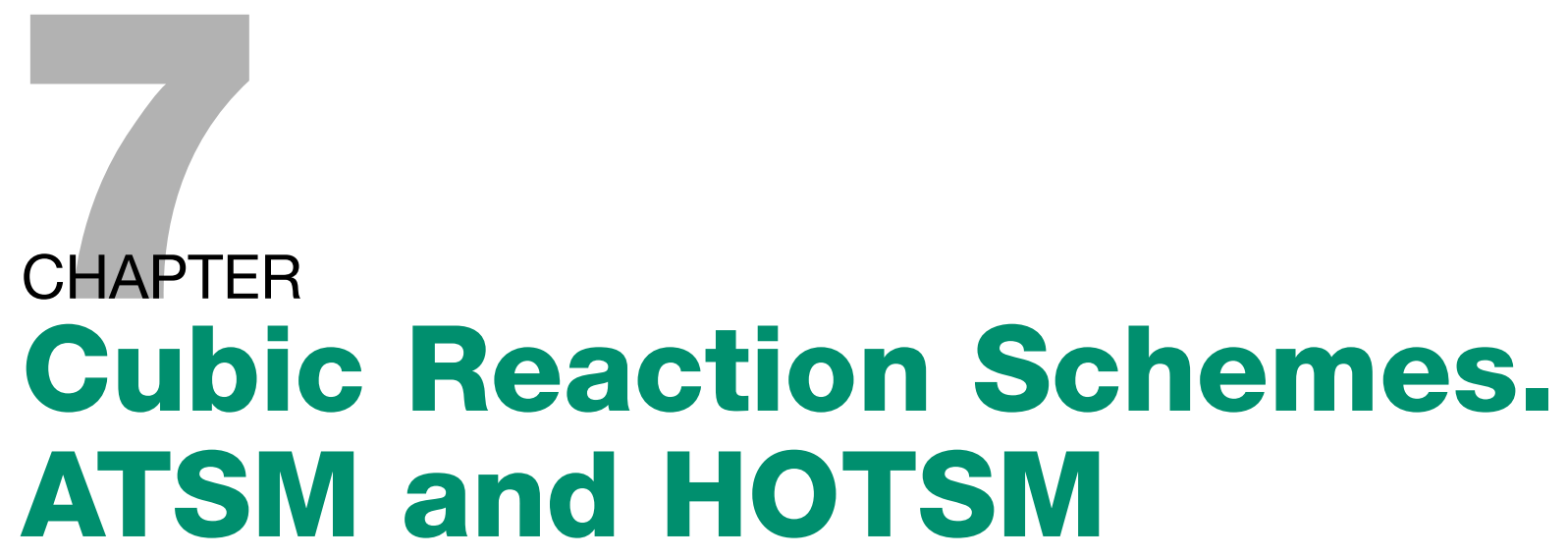

Some general considerations about cubic models are described in sub-chapter 7.1, followed by the derivation and application of some cubic models, especially the allosteric two-state model (ATSM) and the homotropic two-state model (HOTSM).

\subsection{Introduction to Cubic Models}

\subsubsection{Two Simple Cubic Models}

We have now advanced to two two-state models that are essential for our conception of mechanisms in modulatory regulation at the receptor level. In both models, two ligands can interact via a receptive unit and the receptor may exist unliganded in two isomeric forms, a reactive or an active conformation. The two models are the allosteric two-state model (ATSM) and the homotropic two-state model (HOTSM) (Fig. 7.1). Two different (heterotropic) ligands act in the ATSM, while two identical (homotropic) ligands are active in the

HOTSM. One could argue that a better name for the allosteric two-state model would be the 'heterotropic two-state model' (HETSM), but currently I will adhere to the name settled in the literature - the allosteric twostate model (ATSM).

The ATSM was first analyzed by Hall (2000) varying either the agonist or the modulator concentration separately. Although the concentration of the heterotropic ligands in ATSM may be varied simultaneously in a fixed ratio (see later), this possibility was not explored by Hall (2000).

The HOTSM was developed by Bindslev (2004). In this model, the agonist and the modulator concentration by necessity vary simultaneously, since agonists and modulators will be identical molecules.

As we shall see, when a mixture of agonist and modulator molecules with a fixed concentration ratio are employed for dose-responses in the functional form of ATSM, this model behaves as a HOTSM.

Both models are explored in more detail in the two papers mentioned above, and should be consulted for
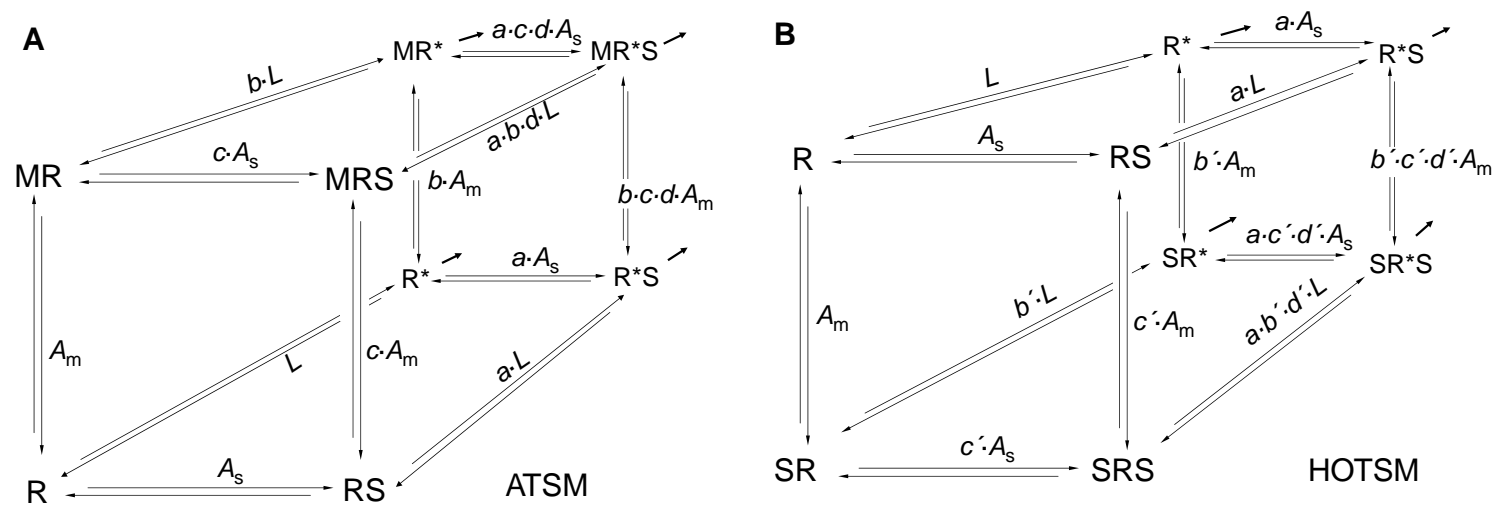

Figure 7.1. (A) The allosteric two-state reaction scheme (ATSM). (B) The homotropic two-state reaction scheme (HOTSM). Arrows indicate the flow of information or path of activation. Receptor symbols and parameters are listed and explained in Table 7.1 and Box 7.1.

(C) $2008 \mathrm{~N}$ Bindslev. This book and all matter and items published therein are distributed under the terms of the Creative Commons Attribution-Noncommercial 3.0 Unported License (http://creativecommons.org/licenses/by-nc/3.0/), permiting all non-commercial use, distribution, and reproduction in any medium, provided the original work is properly cited. 

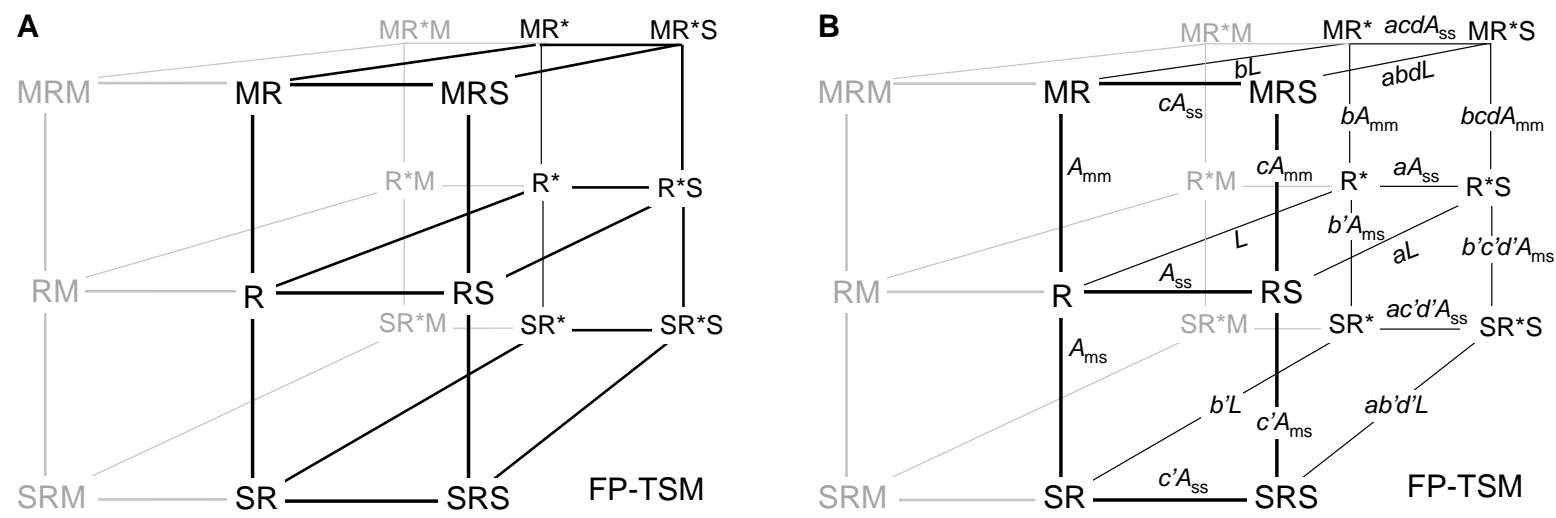

Figure 7.2. ATSM and HOTSM as parts of the four-pane two-state model (FP-TSM). (A) Without system constants. (B) With system constants.

an in-depth understanding of the model characteristics and capabilities as tools for synagic analysis. Here I shall present some highlights and additional possibilities for the two models.

Table 7.1. Ligands, receptor conformations and parameters in ATSM and HOTSM

\begin{tabular}{|c|c|c|c|c|}
\hline \multicolumn{2}{|c|}{ Receptor complexes } & \multicolumn{3}{|c|}{ Ligands and system constants* } \\
\hline ATSM & HOTSM & ATSM & Hall's design & HOTSM \\
\hline & & S & $A$ & $\mathrm{~S}$ \\
\hline & & M & $\mathrm{B}$ & $\mathrm{S}$ \\
\hline $\mathrm{R}$ & $\mathrm{R}$ & & & \\
\hline $\mathrm{RS}$ & RS & $A_{\mathrm{ss}}$ & $K$ & $A_{\mathrm{ss}}$ \\
\hline $\mathrm{MR}$ & SR & $A_{\mathrm{mm}}$ & $M$ & $A_{\mathrm{ms}}$ \\
\hline MRS & SRS & $c$ & $\gamma$ & $c^{\prime}$ \\
\hline $\mathrm{R}^{*}$ & $\mathrm{R}^{*}$ & $L$ & $L$ & $L$ \\
\hline$R^{\star} S$ & $R^{*} S$ & $a$ & $\alpha$ & $a$ \\
\hline $\mathrm{MR}^{*}$ & $\mathrm{SR}^{*}$ & $b$ & $\beta$ & $b^{\prime}$ \\
\hline$M R^{\star} S$ & $S R^{\star} S$ & $d$ & $\delta$ & $d^{d}$ \\
\hline
\end{tabular}

*The system constants are all forward constants. Compare Table 7.1 with Box 7.1.
There are three principal conditions in both the ATSM and the HOTSM: (1) binding of one ligand, (2) binding of two ligands simultaneously, and (3) a conformational isomerization for activation. Reaction schemes to describe the possible combinations of these three conditions result in cubic networks with seven independent system constants, as shown in Fig. 1A+B.

The ATSM is a cubic combination of the intervention model (Chapter 2) and the cyclic two-state model (cTSM) (Chapter 5). The HOTSM is a cubic combination of the auto-intervention model (Chapter 3) and again the cTSM described in Chapter 5 .

The ATSM and HOTSM are both parts of and thus included in the four-pane two-state model (FP-TSM) in Fig. 7.2. The designation of system constants in modelpair ATSM and HOTSM in Fig. 7.1 is transferred from the FP-TSM in Fig. 7.2 in order to maintain a consistency of terms for the ATSM and HOTSM with those of the mother-model, FP-TSM. Terms for the receptor complexes and the parameters for ATSM and HOTSM are listed in Table 7.1 and Box 7.1. Apostrophes and

Box 7.1. Receptor conformations and parameters in ATSM and HOTSM

ATSM

Acronyms are as listed here for the eight receptor conformations together with symbols for the seven independent parameters of ATSM in Fig. 7.1A:

\section{Receptor conformations in ATSM}

$\mathrm{R} \quad$ unbound reactive receptor

RS agonist-bound reactive receptor

$\mathrm{R}^{*} \quad$ unbound active receptor

$\mathrm{R} * \mathrm{~S}$ agonist-bound active receptor

MR modulator-bound reactive receptor

MR* modulator-bound isomerized receptor with activity

MRS agonist- and modulator-bound reactive receptor

$\mathrm{MR} * \mathrm{~S}$ agonist- and modulator-bound receptor with activity 


\section{Parameters in ATSM}

$L \quad$ isomerization constant-efficacy constant

$A_{\mathrm{ss}} \quad$ equilibrium association constant at the primary site for agonist $\mathrm{S}$

$A_{\mathrm{mm}}$ equilibrium association constant at the secondary site for modulator $\mathrm{M}$

$a \quad$ intrinsic efficacy constant with an agonist already bound

$b \quad$ intrinsic efficacy constant with a modulator already bound

$c \quad$ co-operativity coefficient for binding of a second ligand when a first ligand is already bound

$d \quad$ co-operativity coefficient for binding of a second ligand when a first ligand is already bound to an active HOTSM receptor or an intrinsic efficacy constant when two ligands are bound

Acronyms are as listed here for the eight receptor conformations together with symbols for the seven independent parameters of HOTSM in Fig. 7.1B:

\section{Receptor conformations in HOTSM \\ $\mathrm{R} \quad$ unbound reactive receptor \\ RS substrate-bound reactive receptor \\ $\mathrm{R}^{*} \quad$ unbound active receptor \\ $\mathrm{R} * \mathrm{~S} \quad$ substrate-bound active receptor \\ SR modulator-bound reactive receptor without activity \\ SR* modulator-bound isomerized receptor with or without activity \\ SRS substrate- and modulator-bound reactive receptor \\ $\mathrm{SR} * \mathrm{~S} \quad$ substrate- and modulator-bound receptor with or without activity}

\section{Parameters in HOTSM}

$L \quad$ isomerization constant-efficacy constant

$A_{\text {ss }}$ equilibrium association constant at the primary site for substrate $\mathrm{S}$

$A_{\mathrm{ms}}$ equilibrium association constant at the secondary site for substrate $\mathrm{S}$

$a$ intrinsic efficacy constant with a substrate already bound at ' $\mathrm{O}$ '

$b$ ' intrinsic efficacy constant with a substrate already bound at 'M'

c' co-operativity coefficient for binding of a second ligand when a first ligand is already bound

$d$ ' co-operativity coefficient for binding of a second ligand when a first ligand is already bound to either site of an active receptor or an intrinsic efficacy constant when two ligands are bound

' $\mathrm{O}$ ' stands for the orthosteric or primary binding site, and ' $\mathrm{M}$ ' designates the modulatory or secondary binding site.

All parameters are forward constants for both ATSM and HOTSM.

subscripts at certain parameter symbols secure the difference between the two models (compare panels A and B in Fig. 7.1 and Fig. 7.2).

The ATSM and the HOTSM are close siblings. In fact, it may be said that the HOTSM is a special model variant of the ATSM, since from a functional aspect, as already indicated, for ATSM with mixes of the two different ligands, when their ratio of concentration is kept constant during application, the ATSM becomes a copy of the HOTSM. This aspect of the ATSM/HOTSM pair was analyzed by Bindslev (2004), and discussed further in Section 7.8.1. However, formulation of occupancy in the two models results in completely different expressions (see Section 7.8.2).

\subsubsection{A Promising Model for the Analysis of Modulator Action}

Hall's ATSM is a clear choice for analyzing modulator effects and will probably be exploited in the coming years. ATSM is a modification of the ternary complex model of 'allosteric' interaction by Ehlert (1988). In the words of Hall (2000), the ATSM 'provides a framework in which each of the receptor species in the ternary complex model can cause downstream functional effects with different efficacies'. In a fixed concentration ratio mode, both ATSM and HOTSM have three plateaus of effect, and the models predict that an allosteric ligand which affect the affinity but not the efficacy of a primary ligand will only influence a second plateau of the 
model's dose-response curves, and neither its third plateau at high ligand concentrations nor its initial first plateau before ligand application (see later in Fig. 7.27). It is only modulator ligands with an efficacy constants $L$ different from unity that can affect the third level of function induced by a primary ligand.

Thus far, only sporadic implementation of the ATSM has been reported (see, e.g., May et al. 2004; Jensen \& Spalding 2004; Franco et al. 2006; Langmead \& Christopoulos 2006; Ehlert \& Griffin 2008; and partially by Hoare et al. 2008). However, with the accelerated development of allosteric drugs and their clinical application (Table 7.2) (Gao \& Jacobson 2006, sub-chapter 7.12), the relevance of ATSM as an analytical tool justifies a more extended examination of this model. Therefore, in Chapter 7, I will scrutinize the ATSM and its sibling the HOTSM for some general principles and some details.

\subsubsection{Other Cubic Models and Other Models}

Other reaction schemes have been presented which also result in cubic models when based on the three principal conditions mentioned above. For instance, for $G$ protein coupled receptors (GPCRs), a cubic ternary-complex model (CTCM), was scrutinized by Weiss and co-workers (Weiss et al. 1996a,b,c). On its development see Fig. 7.3. In the CTCM it is merely the activated receptor complexes when coupled to $G$ protein $\left(\mathrm{GR}^{*}\right)$ that are considered functional in the transduction pathway (arrows in Fig. 7.4A). Nevertheless, even in the absence of agonists but with functionality linked to $\mathrm{G}$ proteins coupled to the receptor, the CTCM includes the possibilities for simulation of both spontaneous activity and inverse agonism, as inherent in ATSM and HOTSM.

Table 7.2. Examples of receptors for which there is development of allosteric drugs for better therapy (see also Raddatz et al. 2007)

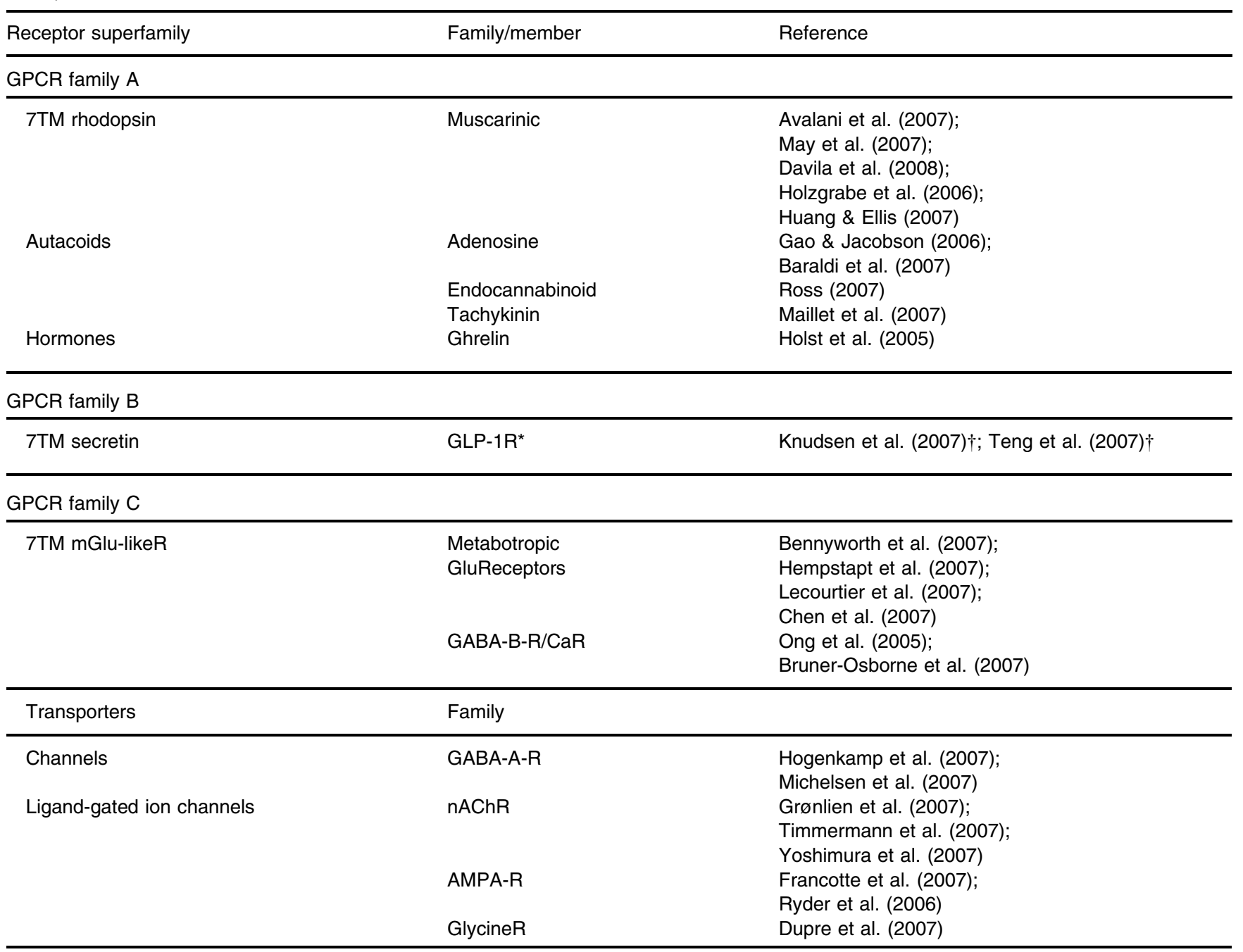

The table does not include allosteric compounds for enzymes or growth factor receptors

*See also Baggio \& Drucker (2007), especially pp. 2146-2149.

${ }^{\dagger}$ Small peptide ago-allosteric modulator for a GPCR family B member. 
(a) Classical Model

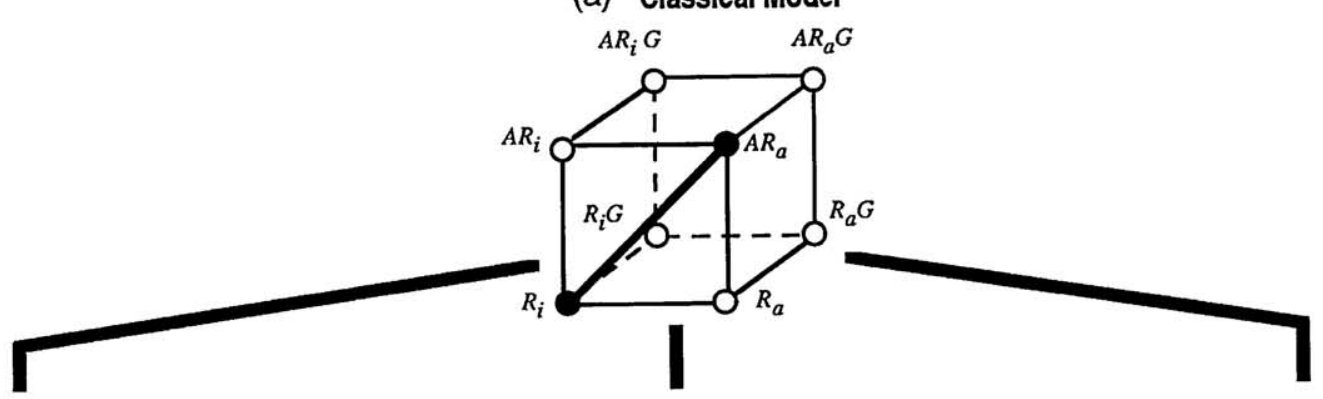

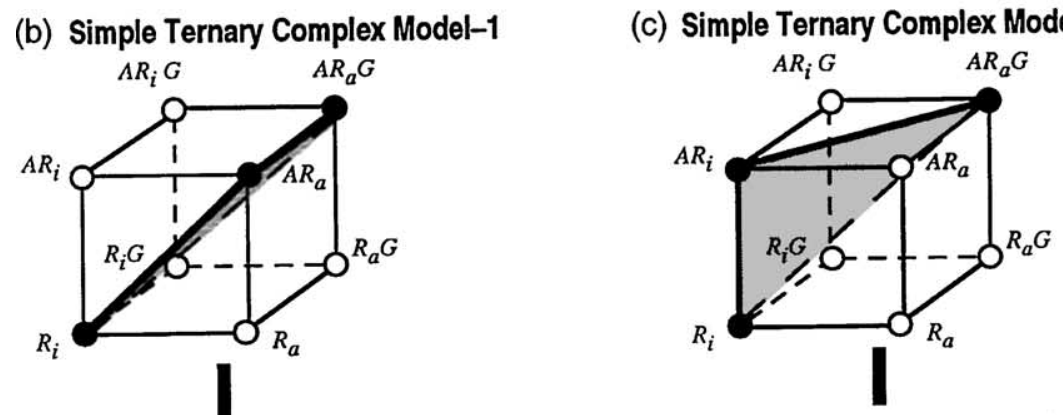

(e) Ternary Complex Model

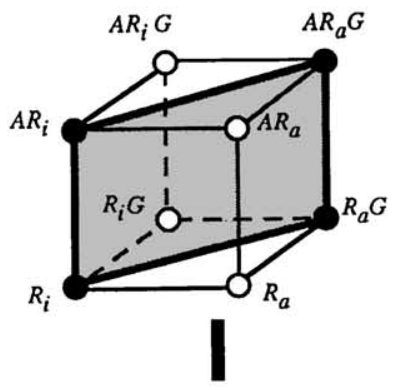

(d) Simple Two-State Model

$A R_{i} G \quad A R_{a} G$

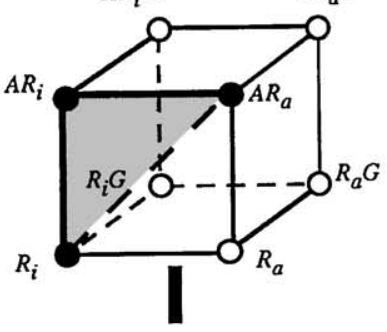

(f) Full Two-State Model

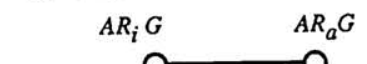

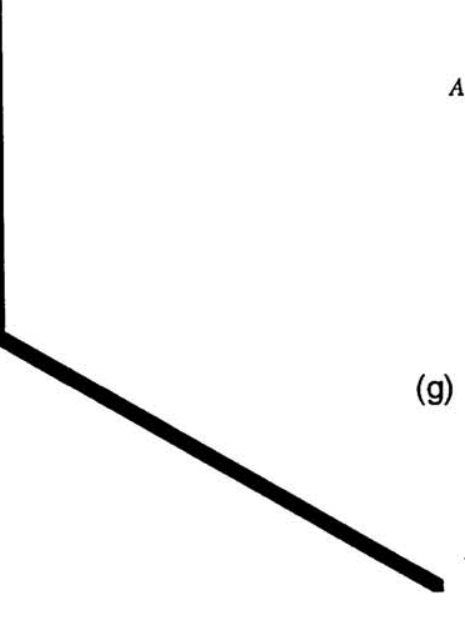

(g) Extended Ternary Complex Model

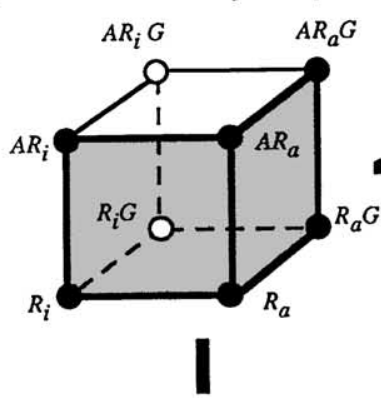

(h) Cubic Ternary Complex Model

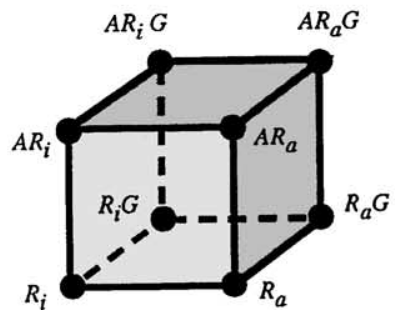

Figure 7.3. Historic development of the cubic ternary-complex model (CTCM). The ternary complex model (TCM) (de Lean et al. 1980), and the extended ternary-complex model (ETCM) (Samama et al. 1993) appear on the road towards the CTCM. Taken from Weiss et al. (1996, p. 160, Fig. 12) with permission. 

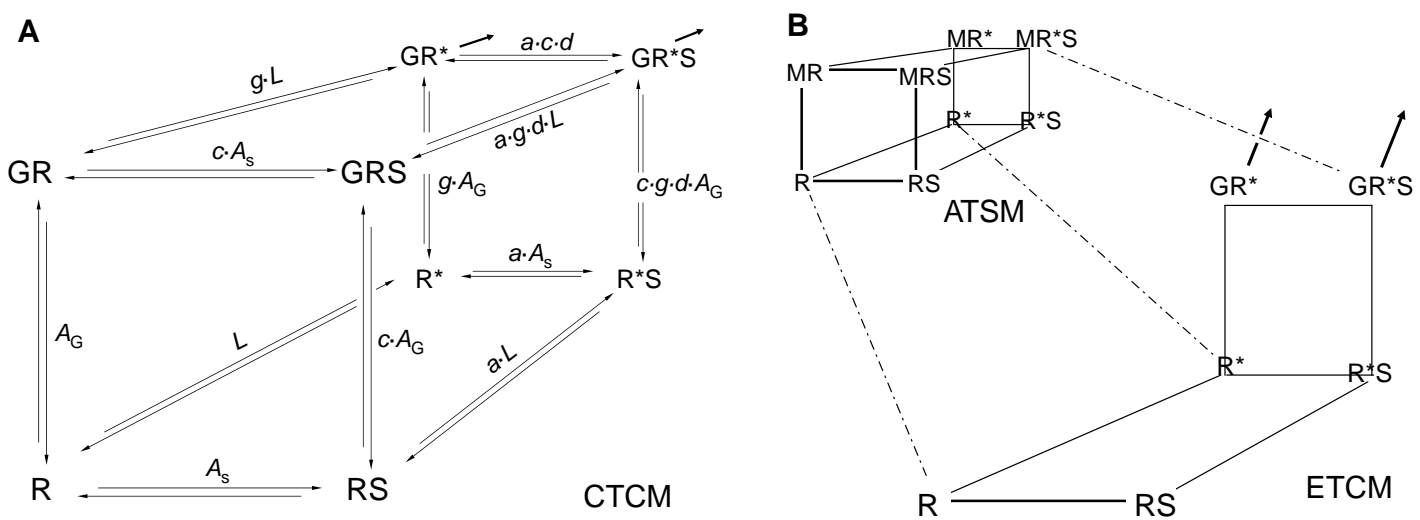

Figure 7.4. The cubic ternary-complex model (CTCM). (A) Arrows indicate the flow of information or path of activation. The symbol R stands for G protein coupled receptor (GPCR), G for G protein, and parameter $g$ replaces parameter $b$ of the ATSM. (B) The lower panel shows the extended ternary complex model (ETCM), which is part of the CTCM in (A). The allosteric two state model is included to demonstrate that ECTM can be derived from ATSM as well.

To date, the CTCM has been implemented by few research groups (e.g., Bruheim et al. 2003; Monczor et al. 2003; Fitzsimmons et al. 2004; O'Brien et al. 2004; Pineyro et al. 2005).

Adding another ligand to the CTCM scheme, that is, operating with three different ligands, but baring simultaneity in binding, i.e., excluding tri-ligand binding, was also considered by Weiss and co-workers (1996a) and visualized in their Fig. 5. Other variations without explicit conformational isomerization have been suggested on the theme of cubic complex modeling, but with three ligands interacting by simultaneous binding (Marvizon \& Baudry 1993; Kukkonen et al. 2001).

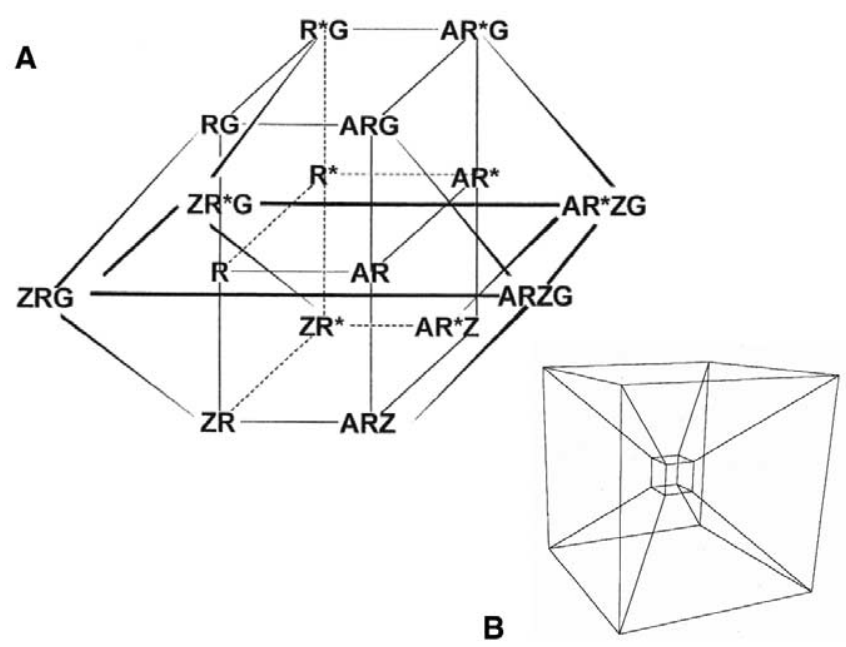

Figure 7.5. The cubic quaternary-complex model (CQCM). (A) From Christopoulos et al. (1998, Fig. 1B) with permission. (B) In a double cubic version, cube-in-a-cube (courtesy of CHETAN $^{\circledR}$ 2003). Both versions of the model assume the possibility of three ligands bound simultaneously, excluding co-lateral binding, and a conformational switch between a reactive and an active state. The model has 16 receptor conformations and 15 independent parameters.
A model more complete than the CTCM for GPCRs was suggested by Christopoulos et al. (1998), and again by Christopoulos \& Kenakin (2002), as before, requesting $G$ protein coupling for activity and including spontaneous activity, inverse agonism, but now with triligand binding, the same as simultaneous binding of three different ligands including (1) an agonist, (2) the $\mathrm{G}$ protein, and (3) a modulator molecule. It is a socalled cubic quaternary-complex model (CQCM) (Fig. 7.5A), with four principal conditions and still, as mentioned, implicating both inverse agonism and spontaneous activity in the presence of bound G protein. The CQCM may alternatively be represented as a cube-in-a-cube (Fig 7.5B). The cube-in-a-cube was discussed in a different setting as a 'hyper-cube' model with four ligands, that is, replacing the possibility of conformational isomerization by one extra ligand binding, leading to a pentanary-complex model (van Rijn \& Willems-van Bree 2004).

The CTCM and its offspring, the CQCM, are further characterized in sub-chapter 7.11.

\subsubsection{Contents of Chapter 7}

As usual, both the ATSM and the HOTSM are analyzed for their characteristics related to binding studies (subchapters 7.2 and 7.5), as well as functional experimentation (sub-chapters 7.3 and 7.6). Further aspects on HOTSM are in sub-chapter 7.7. Overlap between ATSM and HOTSM is considered and visualized in sub-chapter 7.8.

A combination and extension of ATSM and HOTSM into the FP-TSM (Fig. 7.2), and this model's possibilities for simulating synagics are considered in sub-chapter 7.9. A subject related to the FP-TSM, the so-called OFCOR principle, is looked into in sub-chapter 7.10. Differences and similarities between ATSM/HOTSM and other 
Table 7.3. Some examples of one-, two- (dimer), or multi-sited models in one-, two-, or three states involving one-, two-, or more ligands - and some in cubic representations. See also for example Casadó et al. (2007).

\begin{tabular}{|c|c|c|}
\hline \# of sites, states, ligands & References & Comments \\
\hline One-state model Two-sites & Haldane (1930); Laidler \& Hoare (1949) & Auto-intervention (termed \\
\hline OTCM - one ligand & See chapter 3 & "desensitization" by some) \\
\hline One-state TCM & de Lean et al. (1980, Fig 6); Waelbroeck & Non-competitive, \\
\hline Two-sites or three sites & (1994); Proska \& Tucek (1995); & Intervention \\
\hline$c \neq 1$ & $\begin{array}{l}\text { Ehlert (1988, 2001, 2005); Costa et al. } \\
\text { (1992) }\end{array}$ & Two or three ligands \\
\hline One-state three-sited & $\begin{array}{l}\text { Ehlert \& Rathbun (1990); } \\
\text { Lanzafame et al. (2006); } \\
\text { Onaran et al. (1993) }\end{array}$ & $\begin{array}{l}\text { Three ligands } \\
\text { with ligand complexation }\end{array}$ \\
\hline One-state 3-bladed propeller & Christopoulos et al. $(1999,2000)$ & Four ligands \\
\hline Cubic quaternary model QCM & $\begin{array}{l}\text { Kukkonen et al. (2001); } \\
\text { Kukkonen (2004a); Ehlert (2008) }\end{array}$ & Three different ligands \\
\hline One-state site interaction & Tucek et al. $(2001,2002)$ & Adair-type product \\
\hline One-state Multi-ligand & Giraldo et al. (2006) & A ligand in 3 conformations \\
\hline Two-states One-sited & Katz \& Thesleff (1957); & Spontaneous activity and \\
\hline $\begin{array}{l}\text { Cyclic-TSM - one ligand } \\
\text { plus G protein-coupling }\end{array}$ & $\begin{array}{l}\text { Botts \& Drain (1958); } \\
\text { Haber \& Koshland (1967); Leff (1995); } \\
\text { Buck et al. (2004, auto-inhibition); } \\
\text { Scheer et al. (1996) }\end{array}$ & possible inverse agonism \\
\hline $\begin{array}{l}\text { Two-state Non-cyclic-TSM } \\
\text { one ligand multi-sited }\end{array}$ & Monod et al. (1965) & $\begin{array}{l}\text { Co-operativity } \\
\text { possible inverse agonism }\end{array}$ \\
\hline HOTSM two-states one ligand & Bindslev (2004) & Four active conformations \\
\hline Dimer interaction model & Durroux (2005) & Interaction between subunits \\
\hline Two-state Two-site ETCM & Samama et al. (1993); Chang \& Weiss & Modulation, auto-modulation, \\
\hline Dimer models & $\begin{array}{l}\text { (1999); Franco et al. }(2005,2007 a, b) \text {; } \\
\text { Ehlert (2008) }\end{array}$ & One or two ligands \\
\hline $\begin{array}{l}\text { Two-state Three-site } \\
\text { double-cubic }\end{array}$ & $\begin{array}{l}\text { Rusch \& Forman (2005); Scampo-Soria } \\
\text { et al. (2006) }\end{array}$ & Two ligand types, three ligands \\
\hline $\begin{array}{l}\text { Three-state model III-SM + } \\
\text { Four-state model IV-SM }\end{array}$ & $\begin{array}{l}\text { Leff et al. (1997); Scaramellini \& Leff } \\
\text { (1999, 2002); Mak et al. 2003; } \\
\text { Ehlert \& Griffin (2008) }\end{array}$ & One or two ligands \\
\hline Two or three parallel TSM & $\begin{array}{l}\text { Giraldo (2004); Giraldo et al. (2007); } \\
\text { Rovira et al. (2008). }\end{array}$ & One or two ligands \\
\hline Two parallel CTCMs & de Hain (1976) & Receptor/ ligand in two-states \\
\hline The allosteric two-state model & Himoe et al. (1967); Frieden (1970); & Two ligands \\
\hline (ATSM) & Hall (2000) & Four active conformations \\
\hline СТCM (G protein) & Weiss et al. (1996a, b, c); Fong (1996) & Two active conformations \\
\hline ATSM plus ETCM-dimer & Franco et al. (2006), Casadó et al. (2007) & \\
\hline ETCM-dimer, site-interaction & Durroux (2005) & "FP-TSM" + "HOTSM" * \\
\hline Composite TSM & Wreggett \& Wells & Four ligands \\
\hline $\begin{array}{l}\text { Composite TSM } \\
\text { Multi-sited three-state }\end{array}$ & $\begin{array}{l}\text { Burzamato et al. (2004), Colquhoun et al. } \\
\text { (2006), Plested et al. (2007) Solt et al. (2007) }\end{array}$ & One ligand \\
\hline $\begin{array}{l}\text { Cubic quaternary-complex } \\
\text { model (CQCM) }\end{array}$ & $\begin{array}{l}\text { Christopoulos et al. (1998, Fig. 1b) } \\
\text { Christopoulos \& Kenakin (2002) }\end{array}$ & Fig $7.5 \mathrm{~A}$ and $\mathrm{B}$ \\
\hline $\begin{array}{l}\text { Four parameter logistic Eq. } \\
\text { FP-OSM shown in Fig. } 2.4\end{array}$ & $\begin{array}{l}\text { Hay et al. (2005), Langmead et al. (2006) } \\
\text { Wells (1992), Winding \& Bindslev } \\
\text { (1993), Rusch et al. (2004) } \\
\text { Half-FP-TSM (Downing et al. 2005) }\end{array}$ & Non-mechanistic approach \\
\hline FP-TSM & shown in Figs. 5.9, 7.2 and 15.2 & FP-TSM not yet developed! \\
\hline
\end{tabular}

${ }^{*}$ cross-talk between binding sites.

cubic models are debated in sub-chapter 7.11. Finally, sub-chapter 7.12 exposes how a 10-year old market for allosteric drugs in therapy is longing for the implementation of a two-state model à la Hall's ATSM. For an overview, together with other models, some cubic models are listed in Table 7.3.

\subsection{Aspects for Binding with the ATSM}

\subsubsection{Basics of the Model}

The ATSM by Hall (2000) is shown in Fig. 7.1A. Receptor conformations and parameters for the ATSM are given in 

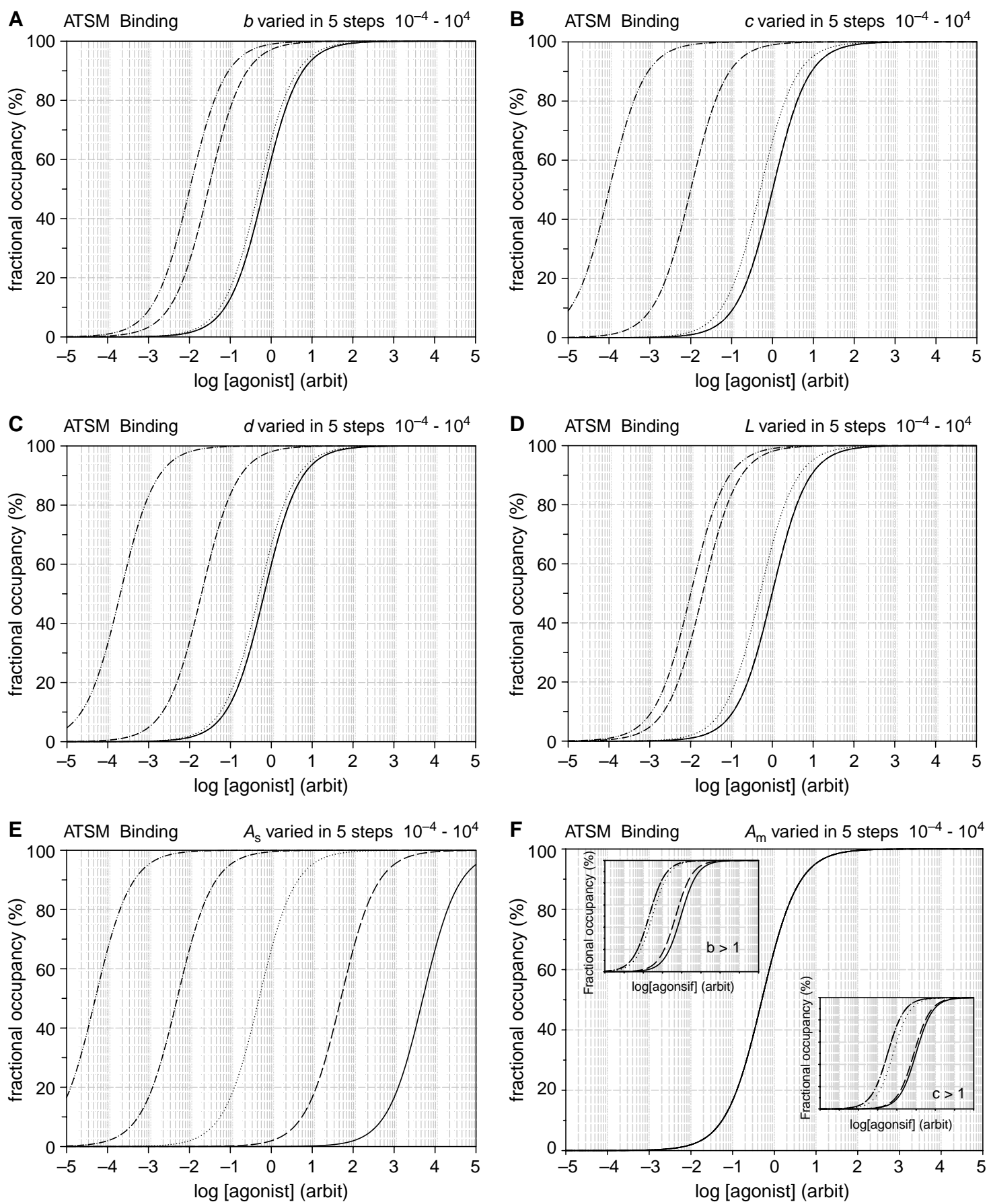

Figure 7.6. Examples of parameter changes in ATSM for binding with the agonist [S] as independent variable. (A) In panels A-F, parameters $b, c, d, L, A_{\mathrm{ss}}$, and $A_{\mathrm{mm}}$, as indicated, vary in five steps between $10^{-2}(-)$ and $10^{2}(-\cdot \cdot-)$, except for $L$ between $10^{-6}$ and $10^{2}$. The other parameters are kept constant at unity except for $a=100$ and $L=1 / 100$. Varying parameter $A_{\mathrm{m}}$ in panel $\mathrm{F}$ is the same as changing the modulator concentration [M]. As can be observed, changing [M] with the above fixed parameter values has no effect on the concentration-occupancy curve. Inserts in panel $\mathrm{F}, b>1$ and $c>1$. $A_{\mathrm{m}}$ or $[\mathrm{M}]$ is varied as before, but now with $b=100$ and $a=1000$ in the left insert. Compare with Fig. $3 b$ in Hall (2000). In the right insert $c=30$ with $a=1$. Compare with Hall's Fig. $4 b$ (2000). 

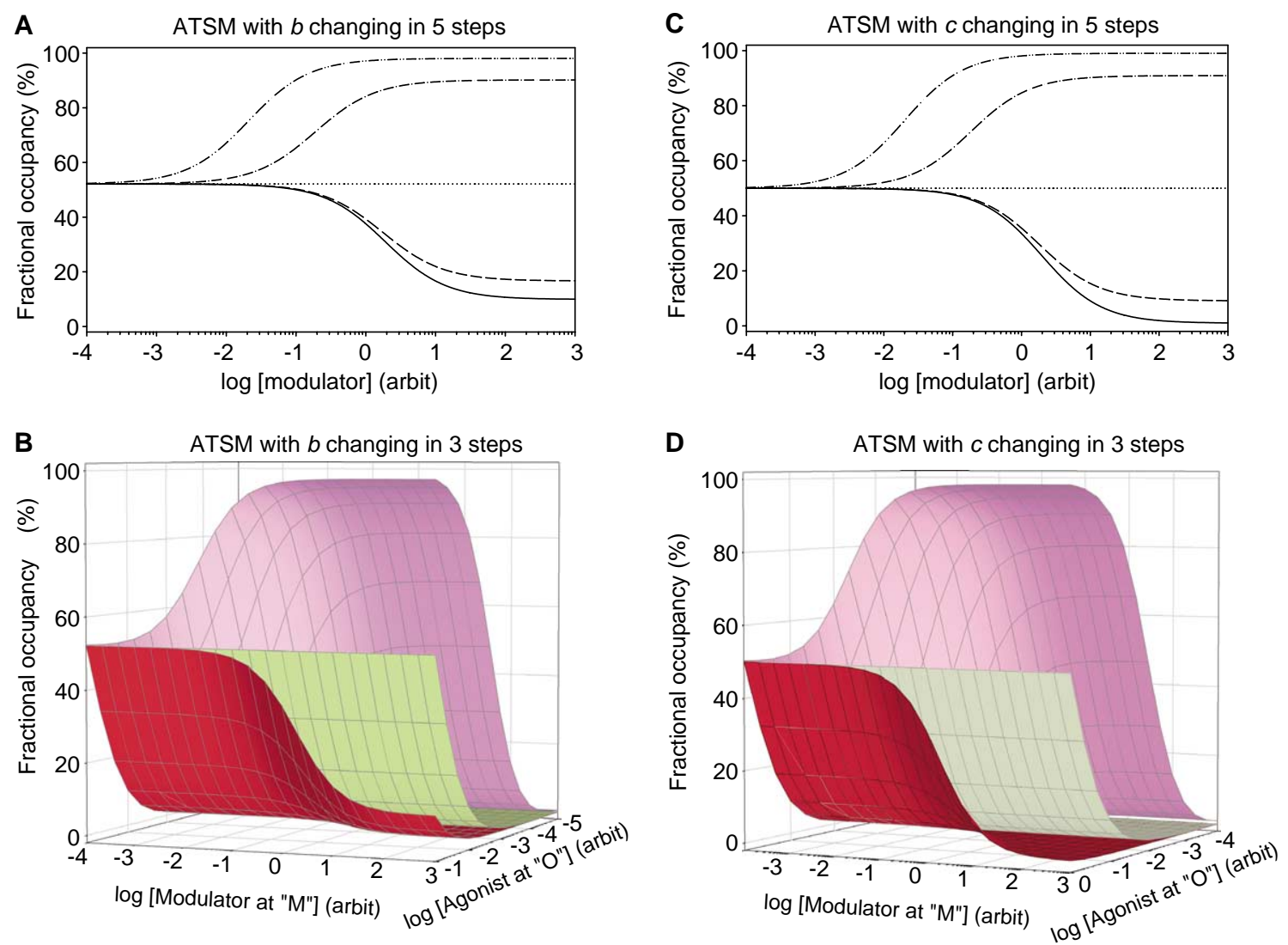

Figure 7.7. Variation of parameters $b$ and $c$ in ATSM for binding with the modulator $[\mathrm{M}]$ as independent variable. (A) System constant $b$ is varied in five steps between $10^{-2}(-)$ and $10^{2}(-\cdot-)$ and the other parameters were $a=1000, c=1, d=1, L=$ $0.01, A_{\mathrm{ss}}=1$, and $A_{\mathrm{mm}}=1$ with $\mathrm{S}$ fixed at $=0.1$. Compare with Hall's Fig. 3a (2000). (B) A 3-D presentations of ATSM with the same parameter values as in panel $A$ for $b$ varying in 3 steps: $10^{-2}, 10^{\circ}$, and $10^{2}$. The $3-D$ presentations are cut off at $S$ ([Agonist at ' $\mathrm{O}$ ']) above 0.1. (C) System constant $c$ is varied in five steps between $10^{-2}(-)$ and $10^{2}(-\cdot \cdot-)$ and the other parameters were, $a=1, b=1, d=1, L=0.01, A_{\mathrm{ss}}=1$, and $A_{\mathrm{mm}}=1$ with [S] fixed at $=1$. As indicated by Hall (2000), $a=1$ may be taken as a neutral ant-agonist and $c$ is independent of $a$. Compare with Hall's (2000) Fig. 4a. (D) A 3-D presentation of ATSM with the same parameter values as in panel $C$ for $c$ varying in 3 steps: $10^{-2}, 10^{\circ}$, and $10^{2}$. The $3-D$ presentation is cut off at $S$ ([agonist at ' $O$ ']) above 1.

Table 7.1 and Box 7.1. A distribution equation for the binding aspect of ATSM with $a o$ as actual occupancy is:

$$
\begin{aligned}
& A_{\mathrm{ss}} \cdot \mathrm{S}+c \cdot A_{\mathrm{ss}} \cdot \mathrm{S} \cdot A_{\mathrm{mm}} \cdot \mathrm{M}+L \cdot\left(a \cdot A_{\mathrm{ss}} \cdot \mathrm{S}\right. \\
& \frac{a o}{T R}=\frac{\left.+a \cdot b \cdot c \cdot d \cdot A_{\mathrm{ss}} \cdot \mathrm{S} \cdot A_{\mathrm{mm}} \cdot \mathrm{M}\right)}{1+A_{\mathrm{ss}} \cdot \mathrm{S}+A_{\mathrm{mm}} \cdot \mathrm{M}+c \cdot A_{\mathrm{ss}} \cdot \mathrm{S} \cdot A_{\mathrm{mm}} \cdot \mathrm{M}} . \\
& +L \cdot\left(1+a \cdot A_{\mathrm{ss}} \cdot \mathrm{S}+b \cdot A_{\mathrm{mm}} \cdot \mathrm{M}\right. \\
& \left.+a \cdot b \cdot c \cdot d \cdot A_{\mathrm{ss}} \cdot \mathrm{S} \cdot A_{\mathrm{mm}} \cdot \mathrm{M}\right)
\end{aligned}
$$

Compare Eq. 7.1 with the corrected Hall Eq. 3 (Hall 2000, Erratum in: Mol Pharmacol 59: 161, 2001). In Hall's analysis it is the concentration of either an agonist or a modulator that is varied as the independent variable.

\subsubsection{Variation in Single Parameters}

Variations in single model parameters for the binding aspect of ATSM are shown in Fig. 7.6 for five different values of each parameter while increasing the agonist concentration.

\subsubsection{Conclusion on Aspects of Binding as Simulated with the ATSM}

The ATSM can model the effects of a modulator molecule on reduced as well as enhanced binding of an orthosteric ligand by changes in apparent affinity. The ATSM is well suited for a simulation of a modulator molecule that can either increase or decrease (a) binding of a radio-ligand, and it can also imitate (b) positive or negative effect on binding affinity of a neutral ant-agonist, and (c) positive or negative effects on binding affinity of an inverse agonist. All these effects can be observed in laboratory experiments with agonist, ant-agonist, and inverse agonist both as radio-ligands and non-labeled compounds. For binding, compare Fig. 7.7A with Hall's Fig. 3a, Fig. 7.7C with Hall's Fig. 4a, and Fig. 7.8A with Hall's Fig. 5a. 
A

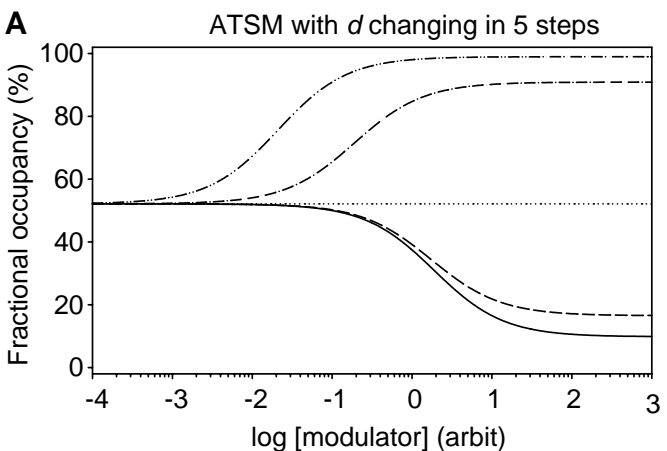

B

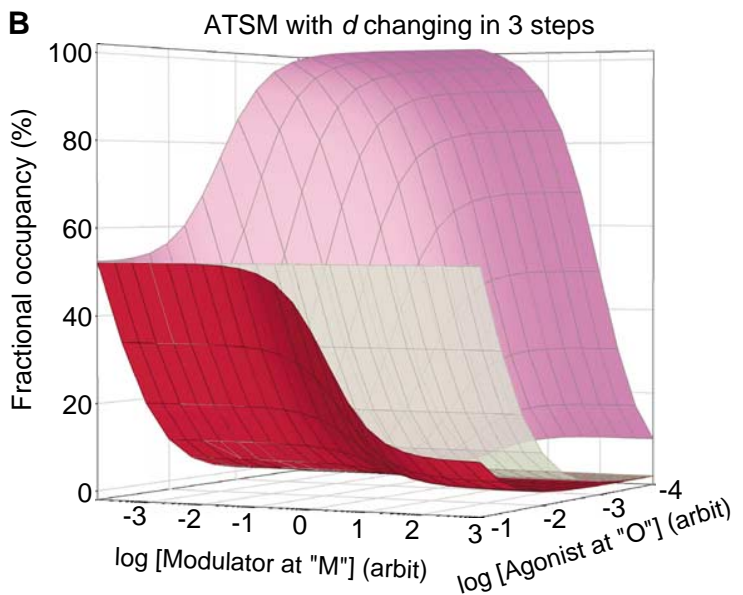

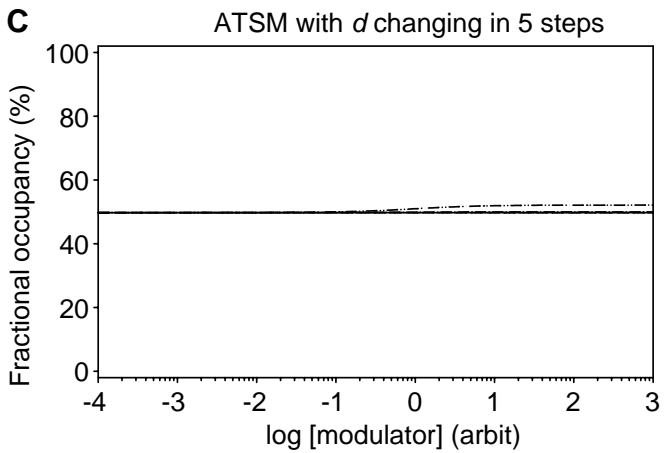

D

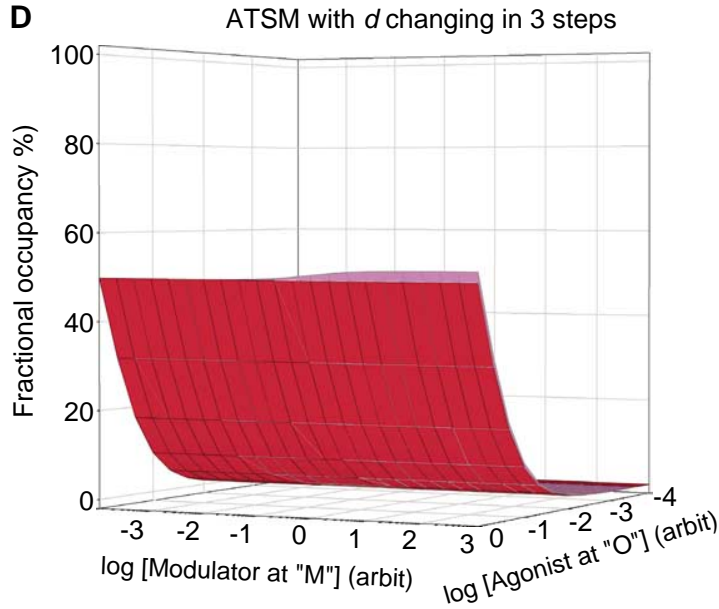

Figure 7.8. Variation of parameter $d$ in ATSM for binding with the modulator as independent variable. (A) System constant $d$ is varied in five steps between $10^{-2}(-)$ and $10^{2}(-\cdot-)$ and the other parameters were $a=10,000, b=1, c=1, L=0.01, A_{\mathrm{ss}}=1$, and $A_{\mathrm{mm}}=1$ with [S] fixed at $=0.01$. Compare with Hall's (2000) Fig. 5a. (B) A 3-D presentation of ATSM with the same parameter values as in panel $A$ for $d$ varying in 3 steps: $10^{-2}, 10^{\circ}$, and $10^{2}$. The 3-D presentation is cut of at $S$ ([Agonist at 'O']) above 0.01. (C) The same as in panel A but with $[S]=1$ instead of 0.01 and $a=0.1$ instead of 10,000. Compare with Hall's (2000) Fig. 5d. (D) A 3-D presentation of ATSM with the same parameter values as in panel C for $d$ varying in 3 steps: $10^{-2}, 10^{\circ}$, and $10^{2}$. The 3-D presentation is cut off at $S$ ([Agonist at ' $O$ ']) above 1.

\subsection{Aspects for Function with the ATSM}

\subsubsection{Distribution Equation}

The conservation principle for function in the ATSM (Fig. 7.1A) may be formulated in a distribution equation given here as:

$$
\begin{gathered}
L \cdot\left(1+a \cdot A_{\mathrm{ss}} \cdot \mathrm{S}+b \cdot A_{\mathrm{mm}} \cdot \mathrm{M}\right. \\
\frac{+a r}{T R}=\frac{\left.a \cdot b \cdot c \cdot d \cdot A_{\mathrm{ss}} \cdot \mathrm{S} \cdot A_{\mathrm{mm}} \cdot \mathrm{M}\right)}{1+A_{\mathrm{ss}} \cdot \mathrm{S}+A_{\mathrm{mm}} \cdot \mathrm{M}+c \cdot A_{\mathrm{ss}} \cdot \mathrm{S} \cdot A_{\mathrm{mm}} \cdot \mathrm{M}} \\
+L \cdot\left(1+a \cdot A_{\mathrm{ss}} \cdot \mathrm{S}+b \cdot A_{\mathrm{mm}} \cdot \mathrm{M}\right. \\
\left.+a \cdot b \cdot c \cdot d \cdot A_{\mathrm{ss}} \cdot \mathrm{S} \cdot A_{\mathrm{mm}} \cdot \mathrm{M}\right)
\end{gathered}
$$

Symbols used are listed in Box 7.1 and Table 7.1. Compare Eq. 7.2 with Hall's equivalent expression (Hall 2000, Eq. 10). As for binding, in Hall's analysis it is the concentration of either an agonist or a modulator that is varied as independent variable, not concomitantly.

\subsubsection{Variation in Single Parameters}

Examples of variation in single model parameters for the functional ATSM (listed in Box 7.1) are shown in Fig. 7.9 for increasing concentrations of the agonist. See also the legend to Fig. 7.9 for further details.

\subsubsection{Conclusion on Functional Aspects as Simulated with the ATSM}

Observing the effects of parameter variation in the ATSM demonstrates how ATSM can simultaneously explain both positive and negative heterotropic modulation of function; by variation in $b, c$ and $d$. For function, compare Fig. 7.10A + C with Hall's Fig. 6a,b, Fig. 7.11A + C with Hall's Fig. 7a,b, and Fig. 7.12A + C with Hall's Fig. 8a,c. In Fig. 7.13, there are plots of the functional ATSM for parameter $L$ below and above $1 / 10$ with modulator concentration $[\mathrm{M}]$ varying in five steps. Notice the spontaneous activity in plots of Fig. 7.13C $+\mathrm{D}$ for $L$ at 0.2. 

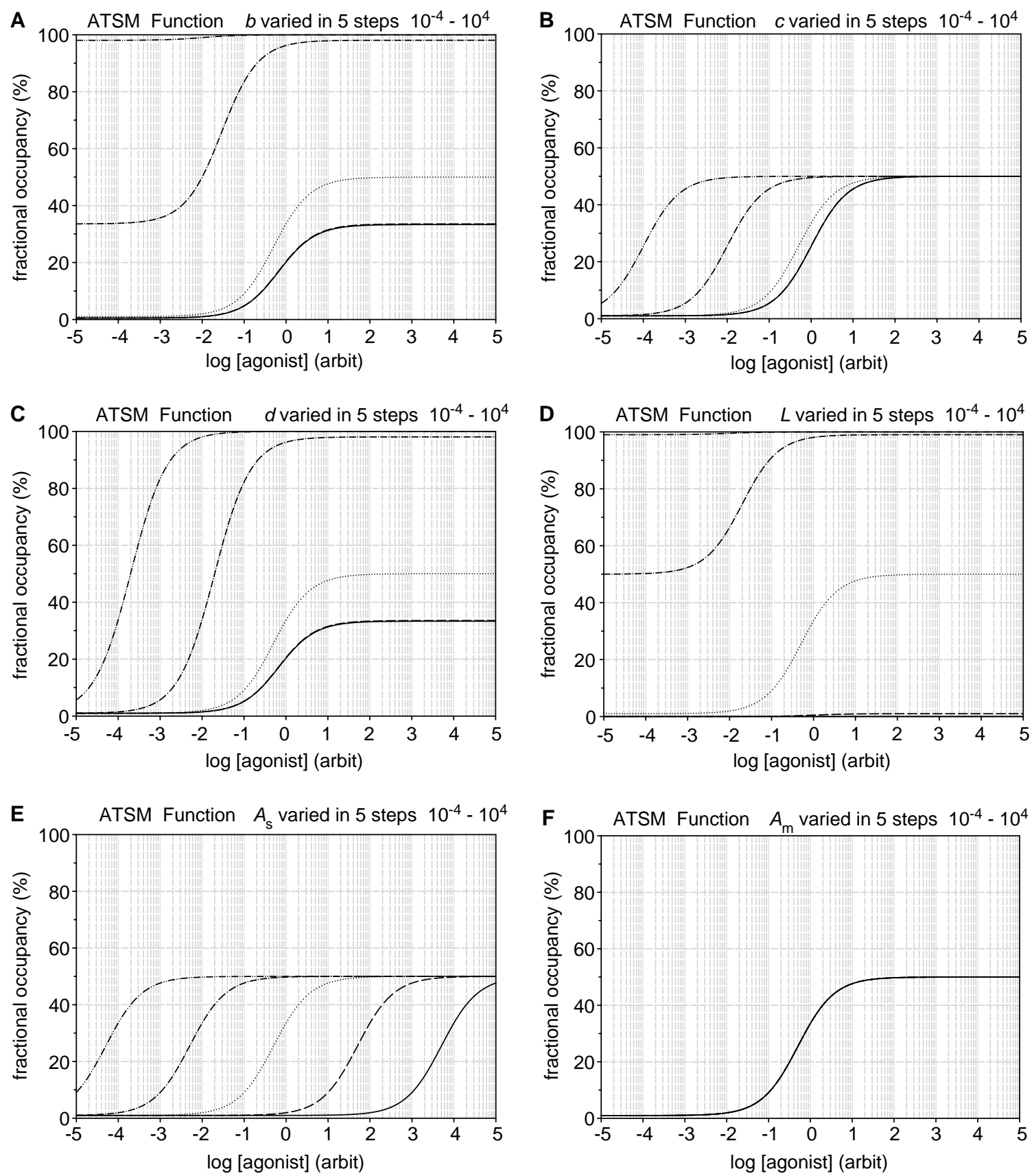

Figure 7.9. Examples of parameter changes in ATSM for function with the agonist [S] as independent variable. (A) In panels A-F, parameters $b, c, d, L, A_{\mathrm{ss}}$, and $A_{\mathrm{mm}}$, as indicated, vary in five steps between $10^{-4}\left(\stackrel{-}{-}\right.$ and $10^{4}(-\cdot \cdot)$, for $L$ between $10^{-6}$ and $10^{2}$. The other parameters are kept constant at unity except for $a=100$ and $L=1 / 100$. Varying parameter $A_{\mathrm{m}}$ in panel $\mathrm{F}$ is the same as changing the modulator concentration [M]. As can be observed, changing [M] with the above fixed parameter values has no effect on the dose-response curve. Changing [M] with either $b, c, d$, or $L$ different from unity is demonstrated in Figs. 7.10-13.

\subsection{Further Aspects for the ATSM}

Hall (2000) has analyzed the behavior of the ATSM for binding and function when either the agonist concentration or the modulator concentration varies as the independent variable. Some aspects on his analyses are presented and debated below.

\subsubsection{Co-agonism}

Co-agonism is seen for ligand-gated receptor channels (LGC) where a simultaneous presence of two different ligands is necessary to open the channel (Johnson \& Ascher 1987; Dingledine et al. 1990; Marvizon \& Baudry 1993; Corsi et al. 1996, Hall 2000). Other systems have been suggested with co-agonism, for instance, GABA-A 
A

$$
\text { B }
$$

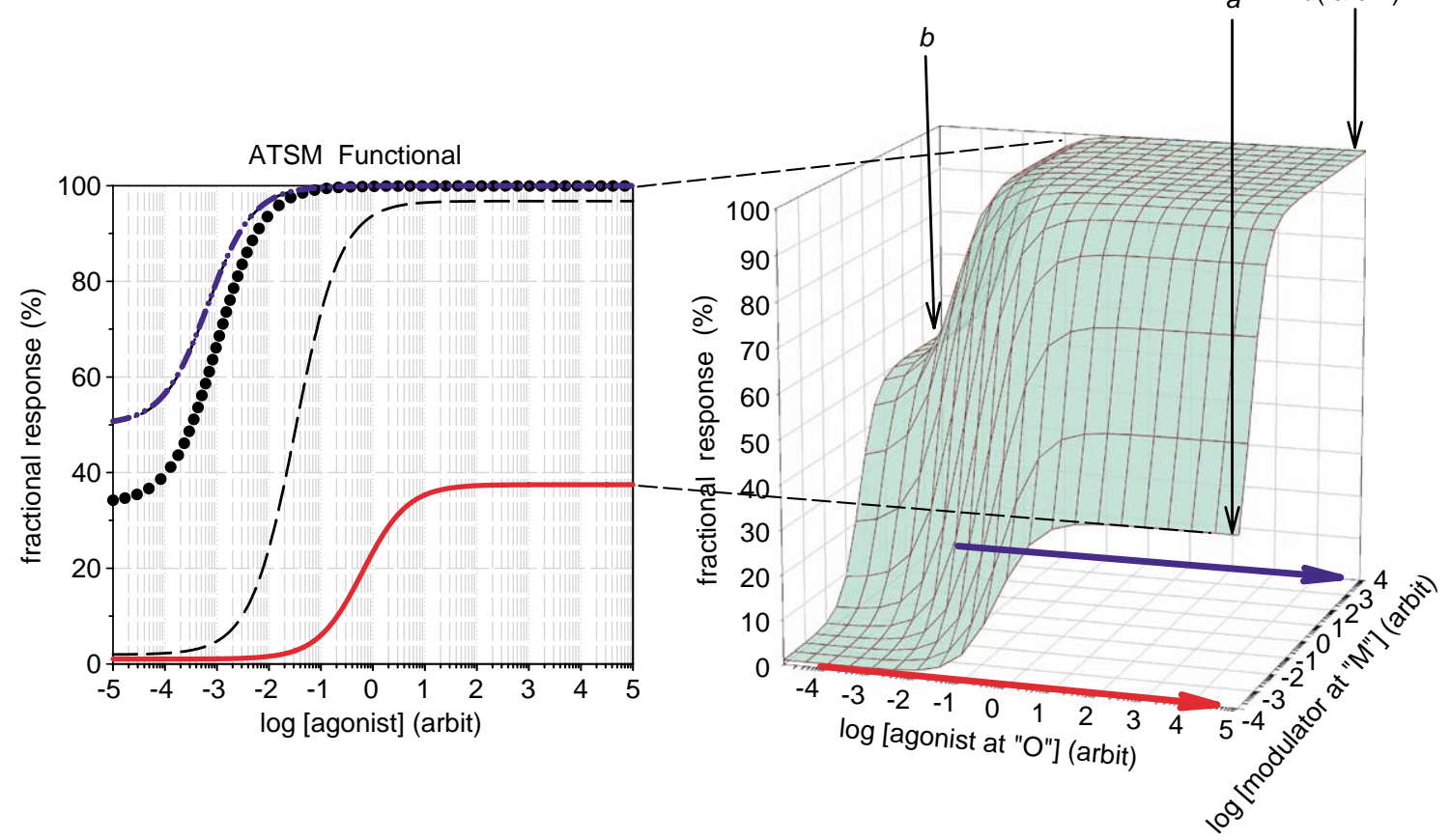

C

D
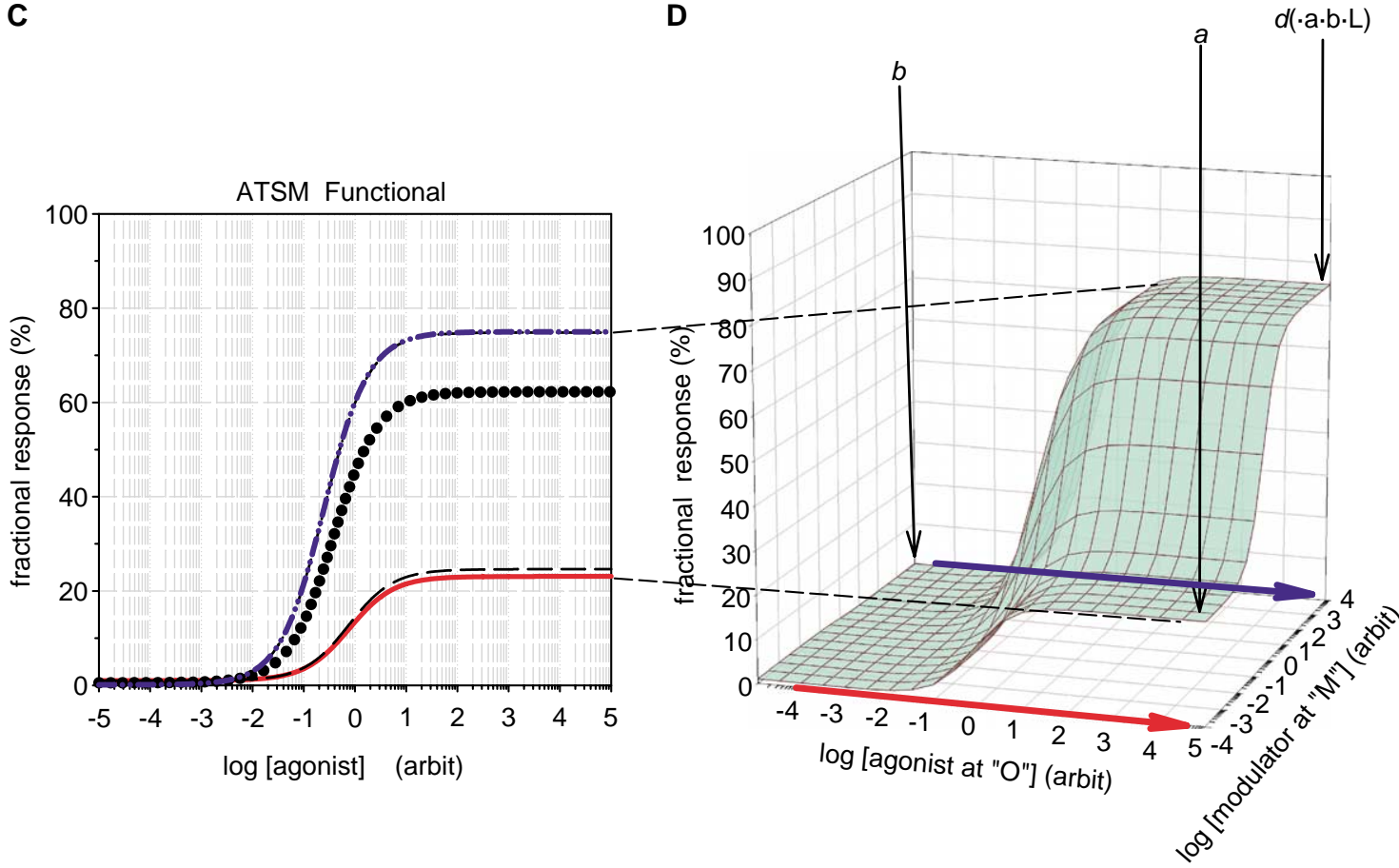

Figure 7.10. Variation of modulator concentration [M] in ATSM for function with the primary ligand $S$ as independent variable. In $\mathrm{A}$ and $\mathrm{B}: b>1$, in $\mathrm{C}$ and $\mathrm{D}: b<1$. System constants were as follows: $a=30, b=100$ or $0.1, c=1, d=100, L=0.01, A_{\mathrm{ss}}=1$, and $A_{\mathrm{mm}}=1$. [M] was varied in five steps between $10^{-4}(-)$ and $10^{4}(-\cdot-)$ in panels $\mathrm{A}$ and $\mathrm{C}$ by a factor $10^{2}$ and in the $3-\mathrm{D}$ plots arrows indicate $[\mathrm{M}]$ at $10^{-4}$ and $10^{4}$. The arrows in the 3-D concentration plane illustrate the [M] used for the two extreme plots in panels $A$ and $C$, thus, the cut off in topography in 3-D by a plane raised at the arrows will produce the indicated plots in 2-D. Compare panels A and C with Hall's (2000) Fig. 6a,b. 
A
B

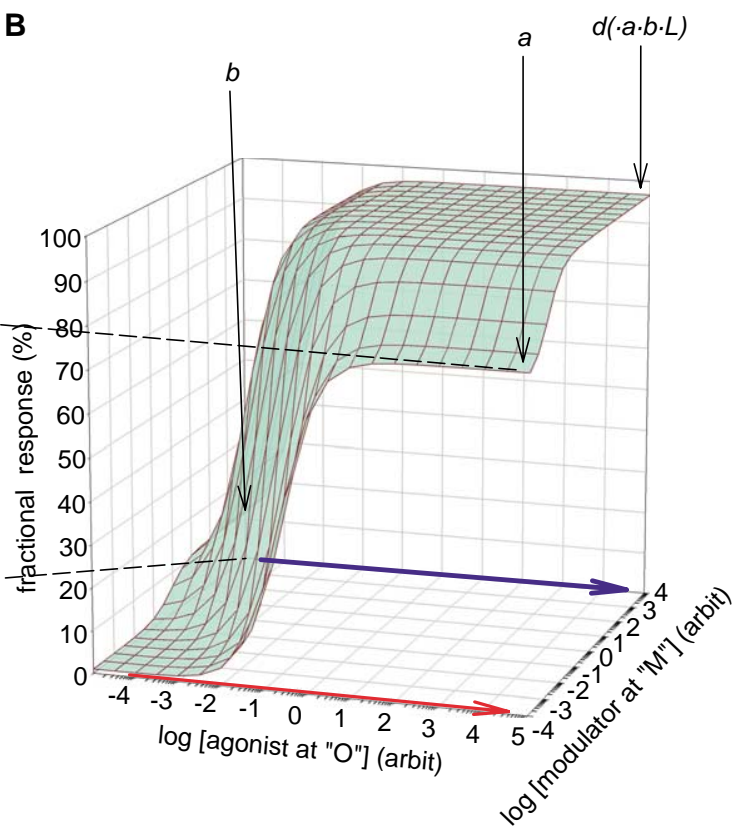

C

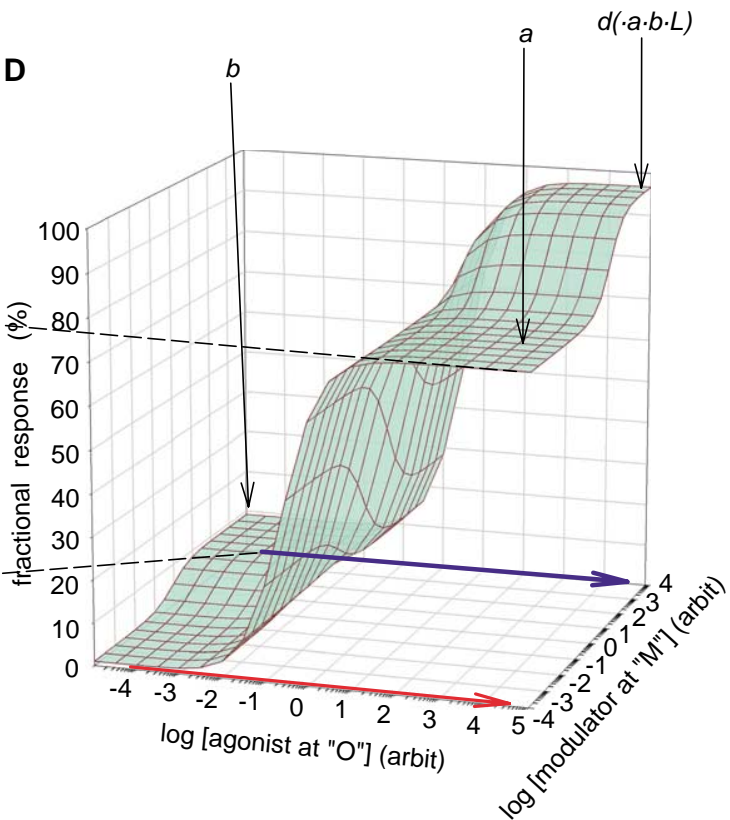

Figure 7.11. Variation of modulator concentration $[\mathrm{M}]$ in ATSM for function with the primary ligand $\mathrm{S}$ as independent variable. In $\mathrm{A}$ and $\mathrm{B}: c>1$, in $\mathrm{C}$ and $\mathrm{D}: c<1$. System constants were as follows: $a=300, b=10, c=100$ or $0.01, d=1, L=0.01, A_{\mathrm{ss}}=1$, and $A_{\mathrm{mm}}=1$. [M] was varied in five steps between $10^{-4}(-)$ and $10^{4}(-. .-)$ in panels $\mathrm{A}$ and $\mathrm{C}$ by a factor $10^{2}$ and in the $3-\mathrm{D}$ plots arrows indicate $[\mathrm{M}]$ at $10^{-4}$ and $10^{4}$. The arrows in the 3-D concentration plane illustrate the [M] used for the two extreme plots in panels $A$ and $C$, thus, the cut off in topography in 3-D by a plane raised at the arrows will produce the indicated plots in 2-D. Compare panels A and C with Hall's (2000) Fig. 7a,b.

channel receptors (Chang \& Weiss 1999; Rusch et al. 2004; Rusch \& Forman 2005). ${ }^{1}$

\footnotetext{
$\overline{1}$ Co-agonism is not always defined as a strict simultaneous presence of both a neutral modulator molecule and an agonist required for activation of an effector according to Johnson \& Ascher (1987); see, e.g., Finch et al. (1991) and the GABA-A literature mentioned above.
}

\subsubsection{Discrepancy of Modulator Effects on Binding and Activity}

In the ATSM with the right selection of parameters, as shown by Hall (2000), the occupancy with primary ligand $\mathrm{S}$ in a double-sited receptive unit can be almost independent of the concentration of an allosteric ligand, 
A

B
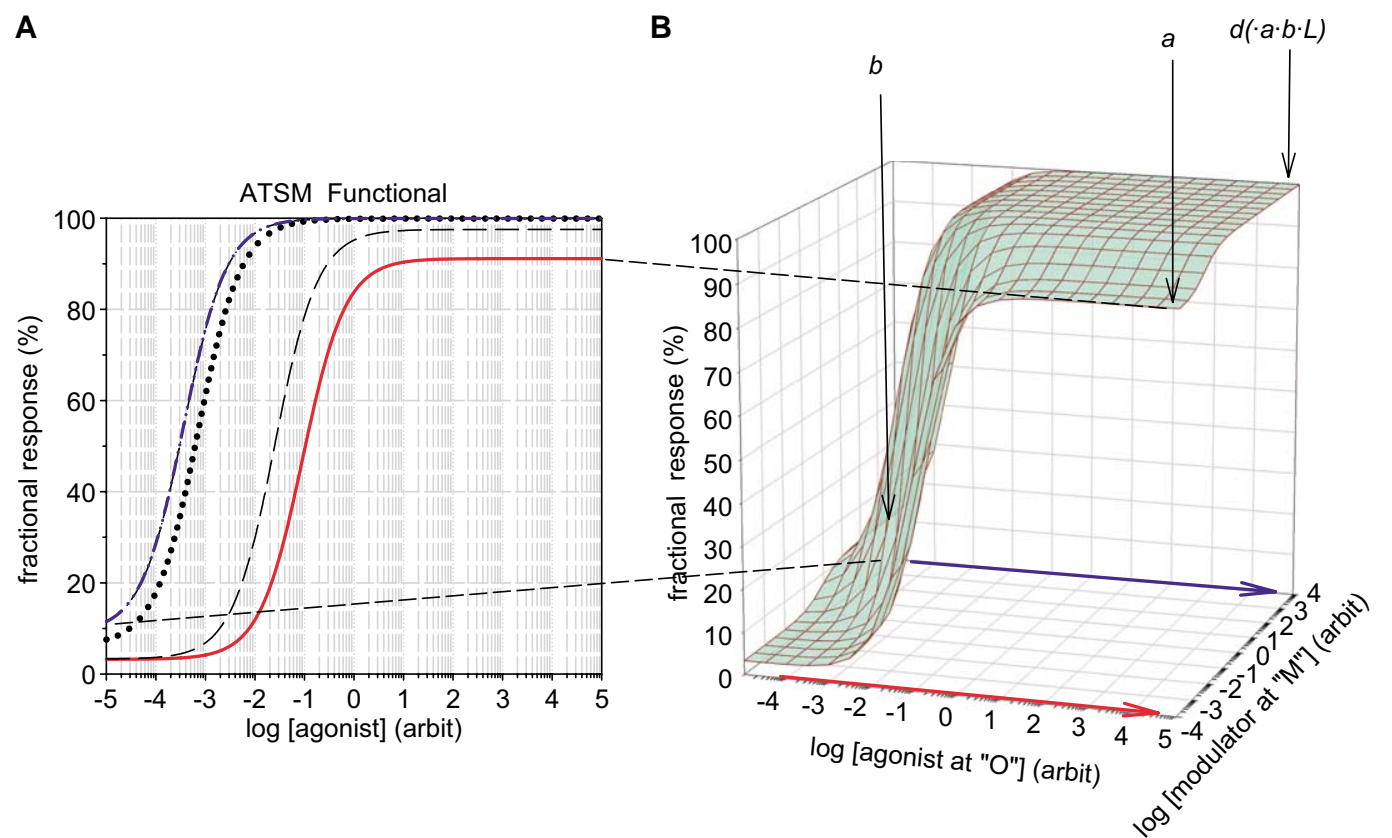

C

D

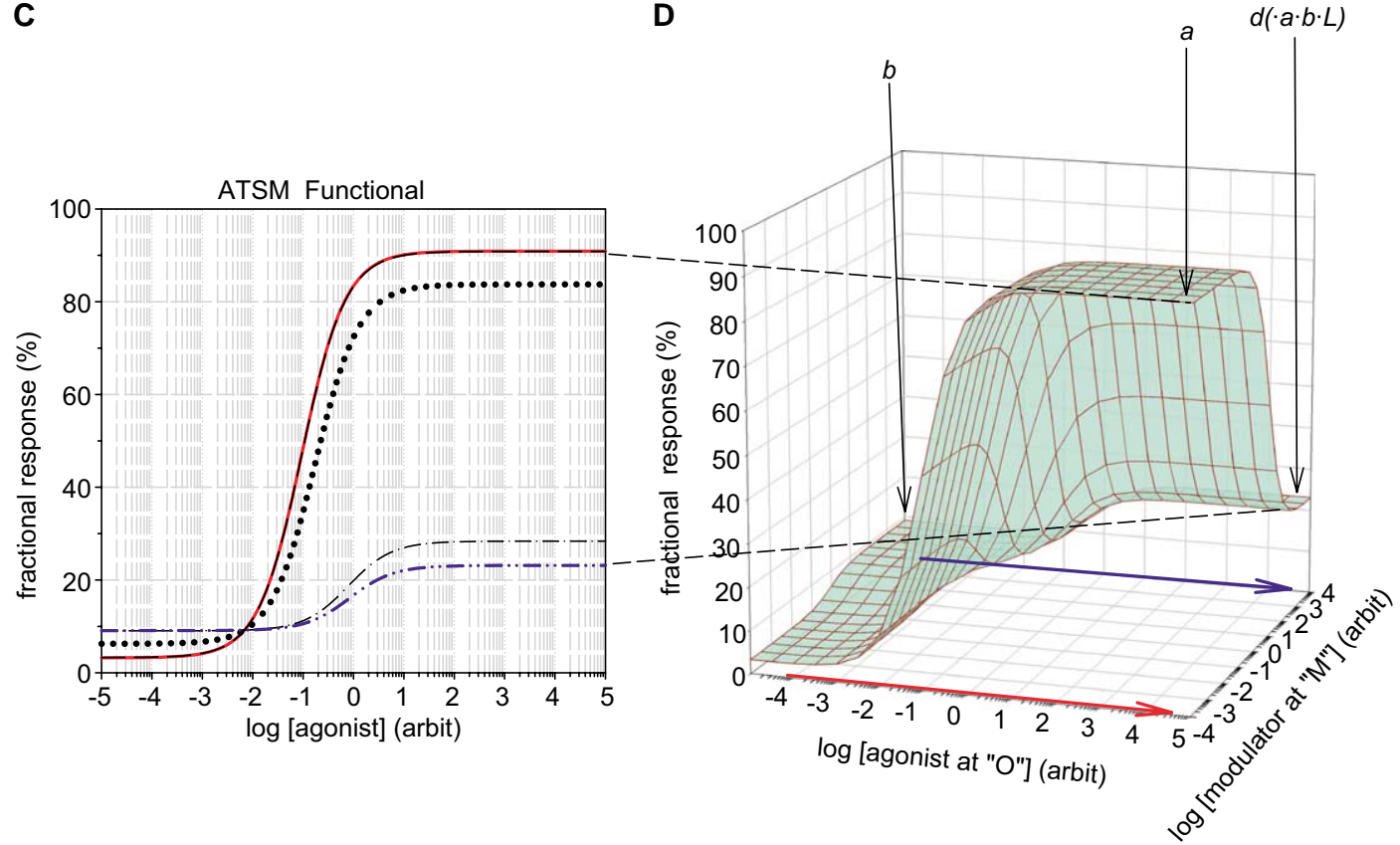

Figure 7.12. Variation of modulator concentration $[\mathrm{M}]$ in ATSM for function with the primary ligand $\mathrm{S}$ as independent variable. In $\mathrm{A}$ and $\mathrm{B}: d>1$, in $\mathrm{C}$ and $\mathrm{D}: d<1$. System constants were as follows: $a=300, b=3, c=1, d=100$ or $0.01, L=0.033, A_{\mathrm{ss}}=1$, and $A_{\mathrm{mm}}=1$. [M] was varied in five steps between $10^{-4}(-)$ and $10^{4}(-\cdot-)$ in panels $\mathrm{A}$ and $\mathrm{C}$ by a factor $10^{2}$ and in the $3-\mathrm{D}$ plots arrows indicate $[\mathrm{M}]$ at $10^{-4}$ and $10^{4}$. The arrows in the 3-D concentration plane illustrate the [M] used for the two extreme plots in panels $A$ and $C$, thus, the cut off in topography in 3-D by a plane raised at the arrows will produce the indicated plots in 2-D. Compare panels A and C with Hall's (2000) Fig. 8a,b.

ranging over several orders of magnitude. Thus, the activity of the receptive unit may vary with changing concentrations of the allosteric ligand M, [M] (Fig. 7.13A and Hall's Fig. 10a) with parameters $a=10,000, b=1$, $c=8.5, d=0.03$ and $L=0.001$, while the binding of $\mathrm{S}$ is almost constant for certain parameter values over an extended span of concentrations for [M] (Fig. 7.14A and Hall's Fig. 10b). This is an illustration of the situation for allosteric compound CPCCOEt at the human mGluR1b receptor, where there is no detectable change in glutamate binding for changing concentrations of CPCCOEt, while the activity changes with the change in [CPCCOEt] (Litschig et al. 1999; Hall 2000).

However, for this to occur with the ATSM, demands are high on selected parameters for the ATSM. Parameter $L$ must be $\leq 1 / 1000$, $a$ must be $\geq 10,000$, and 

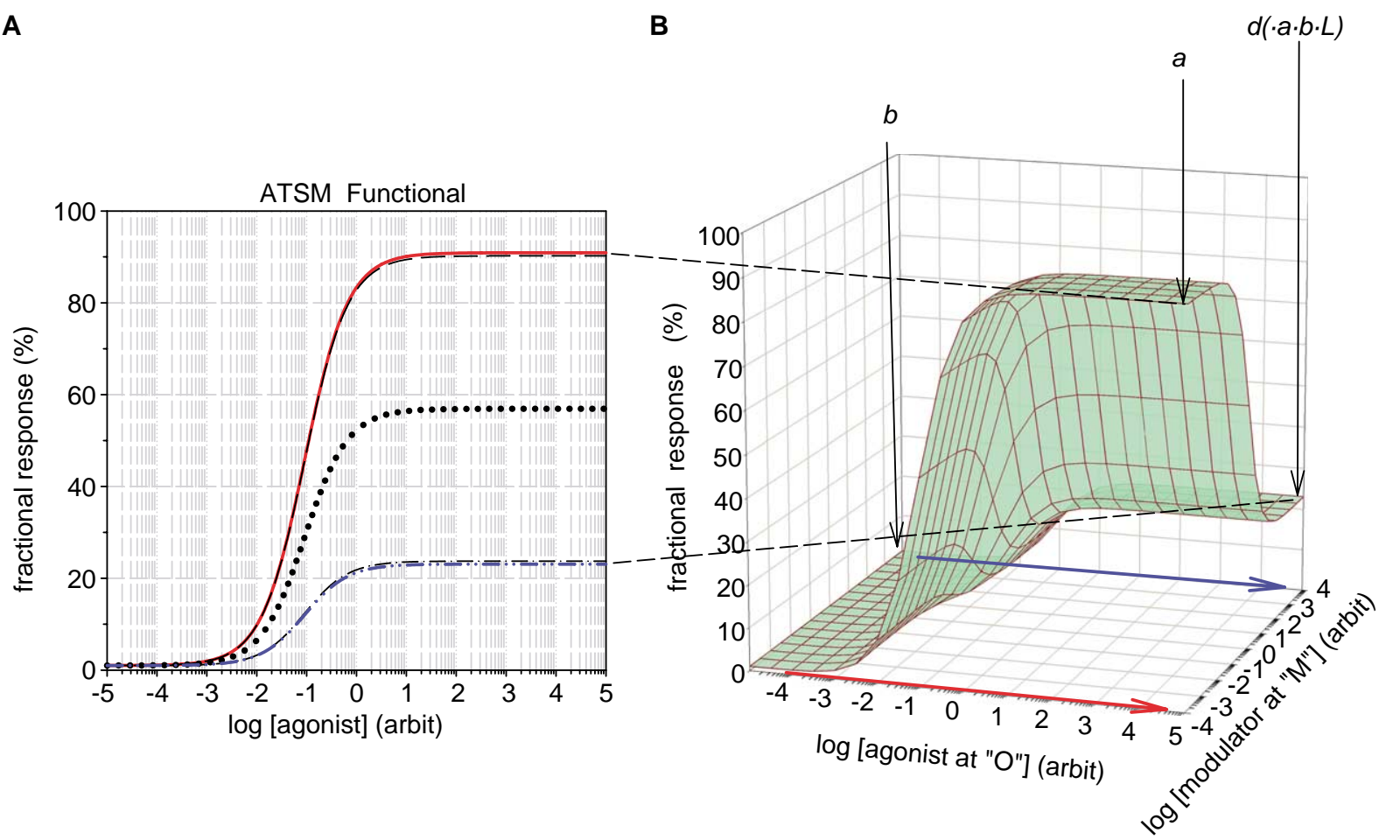

$\mathbf{C}$

D

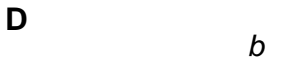

ATSM Functional<smiles>CCC</smiles>

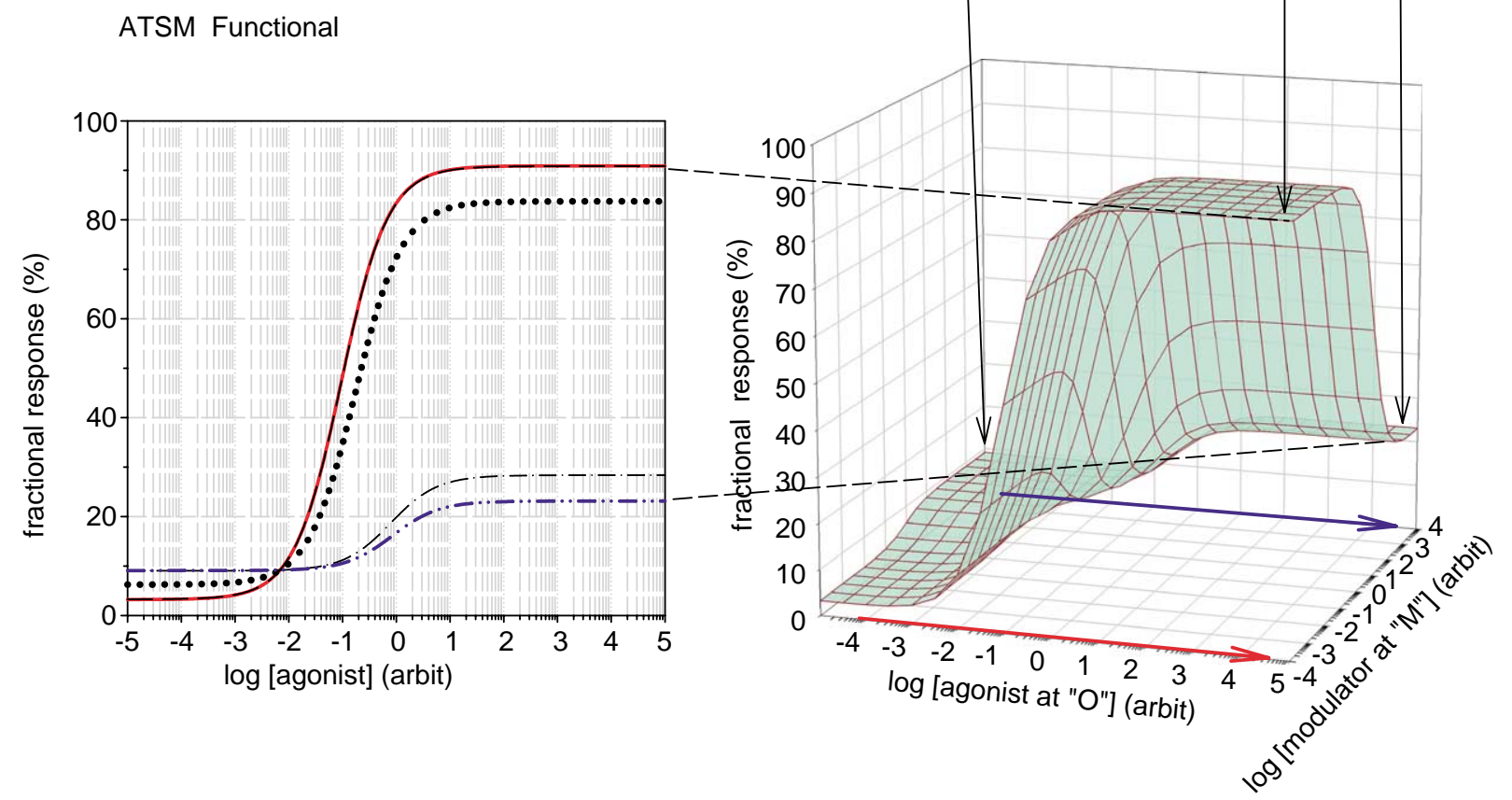

Figure 7.13. Variation of modulator concentration $[\mathrm{M}]$ in ATSM for function with the primary ligand $\mathrm{S}$ as independent variable. In $\mathrm{A}$ and $\mathrm{B}$ system constants were as follows, $L=0.01, a=1000, b=1, c=8.5, d=0.03, A_{\mathrm{ss}}=1$, and $A_{\mathrm{mm}}=1$. In $\mathrm{C}$ and $\mathrm{D}, L=1 / 3$ and the other system constants were as follows: $a=50, b=0.5, c=1, d=0.01, A_{\mathrm{ss}}=1$, and $A_{\mathrm{mm}}=1$. [M] was varied in five steps between $10^{-4}(\longrightarrow)$ and $10^{4}(-\cdot \cdot)$ in panels $A$ and $C$ by a factor $10^{2}$ and in the $3-D$ plots arrows indicate $\left[\mathrm{M}\right.$ ] at $10^{-4}$ and $10^{4}$. The arrows in the 3-D concentration plane illustrate the $[\mathrm{M}]$ used for the two extreme plots in panels $A$ and $C$, thus, the cut off in topography in 3-D by a plane raised at the arrows will produce the indicated plots in 2-D. Compare panel A with Hall's (2000) Fig. 10a. 

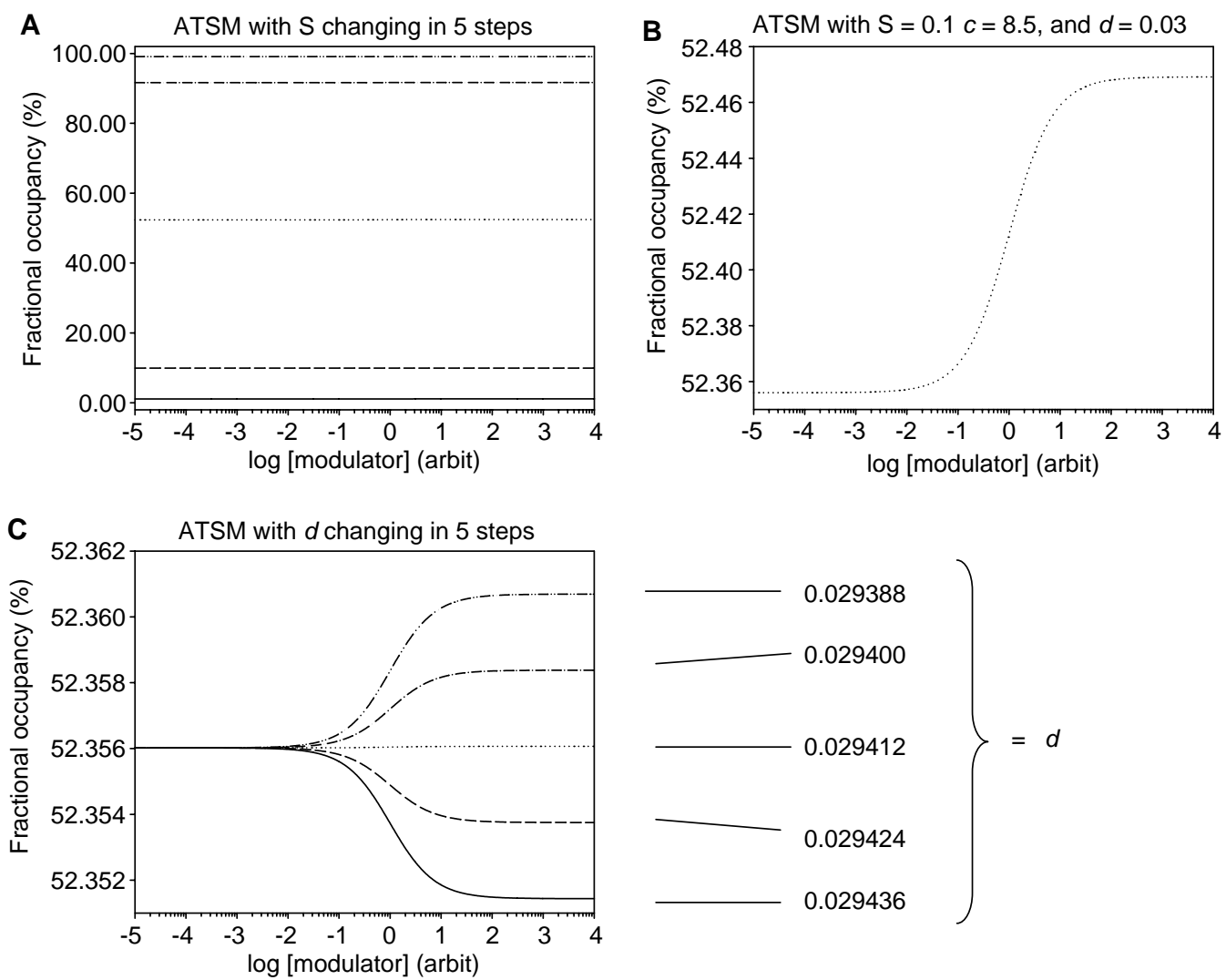

Figure 7.14. The fractional binding in ATSM as a function of modulator concentration [M]. (A) Parameter values are $a=10,000$ $b=1, c=8.5, d=0.03, L=0.001, A_{\mathrm{ss}}=1$, and $A_{\mathrm{mm}}=1$ with $S$ varying between $10^{-5}$ and $10^{3}$. Thus, for certain values of parameters, the occupancy appears independent of modulator concentration over a large range of agonist concentrations. Compare with Hall's Fig. 10b. Conversely, as demonstrated in Fig. 7.13A, the activity changes with variation in [M] as a function of [S]. See Hall's Fig. 10a. This discrepancy between occupancy and function of the ATSM explains observed effects with modulator CPCCOEt (Litschig et al. 1999). (B) However, observe that with [S] for instance fixed at $=0.1$, the concentrationoccupancy relation in panel $A$ is not absolutely straight as demonstrated by expanding the ordinate just above $50 \%$ in panel $B$. (C) To keep the concentration-occupancy constant for variations in [M] is a delicate balance of parameter selection as in panel A. It is a balance which easily breaks down with minor changes, here for instance in parameter $d$, with [S] still at 0.1 and the other parameters as in panel A. The ATSM is a 'cliff-hanger' model for effects of modulators as CPCCOEt.

concomitantly only narrow value ranges for $d$ and $c$ will qualify. The span for the right selection of parameter values is minute, as already indicated in Fig. 7.14B. Additionally, observe for instance the effects of minute variations of parameter $d$ in Fig. 7.14C. The ATSM parameter values have to be within extremely narrow ranges to follow the action of CPCCOEt, therefore the above explanation for the behavior of CPCCOEt does not appear the most likely one, although it is a possibility.

\subsection{Aspects for Binding with the HOTSM}

\subsubsection{A Distribution Equation for Occupancy in HOTSM}

The HOTSM is shown in Fig. 7.1B, and its parameters and receptor conformations are listed in Table 7.1 and Box 7.1. A distribution equation for receptor occupancy in HOTSM based on the conservation principle must include factorization of receptor conformations where two identical ligands are bound at the same time.

We may list two slightly different occupancy models for the homotropic two-state reaction scheme. One in which we double the value of conformations in the reaction scheme with due respect to the fact that in conformations with two ligands bound, SRS and SR*S, they count twice. This first model merely includes the possible conformations. Therefore, the binding version of HOTSM is formulated with $a o$ as actual occupancy:

$$
\begin{aligned}
& A_{\mathrm{ss}} \cdot \mathrm{S}+A_{\mathrm{ms}} \cdot \mathrm{S}+2 \cdot c^{\prime} \cdot A_{\mathrm{ss}} \cdot \mathrm{S} \cdot A_{\mathrm{ms}} \cdot \mathrm{S}+L \cdot\left(a \cdot A_{\mathrm{ss}} \cdot \mathrm{S}\right. \\
& \frac{a o}{T R}=\frac{\left.+b^{\prime} \cdot A_{\mathrm{ms}} \cdot \mathrm{S}+2 \cdot a \cdot b^{\prime} \cdot c^{\prime} \cdot d^{\prime} \cdot A_{\mathrm{ss}} \cdot \mathrm{S} \cdot A_{\mathrm{ms}} \cdot \mathrm{S}\right)}{1+A_{\mathrm{ss}} \cdot \mathrm{S}+A_{\mathrm{ms}} \cdot \mathrm{S}+2 \cdot c^{\prime} \cdot A_{\mathrm{ss}} \cdot \mathrm{S} \cdot A_{\mathrm{ms}} \cdot \mathrm{S}} \\
& +L \cdot\left(1+a \cdot A_{\mathrm{ss}} \cdot \mathrm{S}+b^{\prime} \cdot A_{\mathrm{ms}} \cdot \mathrm{S}\right. \\
& \left.+2 \cdot a \cdot b^{\prime} \cdot c^{\prime} \cdot d^{\prime} \cdot A_{\mathrm{ss}} \cdot \mathrm{S} \cdot A_{\mathrm{ms}} \cdot \mathrm{S}\right)
\end{aligned}
$$

The second formulation of binding in HOTSM further takes into account that all conformations have 

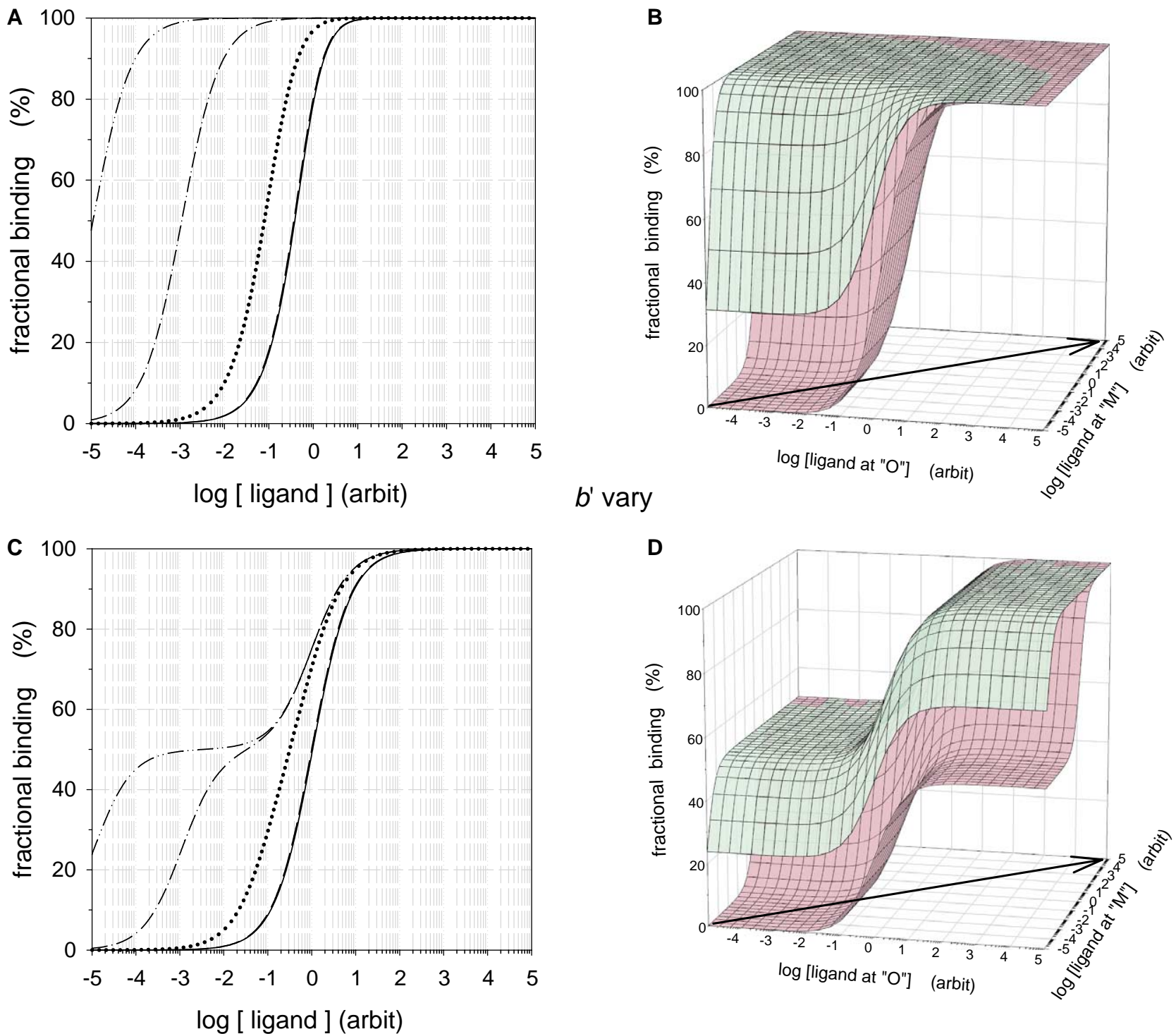

Figure 7.15. Variation of parameter $b$ ' in HOTSM for binding with the agonist/modulator as independent variable. A and B are based on the conformation model for HOTSM, C and D on the probability model for binding (e.g., Cornish-Bowden 2004, Eq. 11.30). Parameter $b^{\prime}$ ' was varied in five steps between $10^{-2}(\longrightarrow)$ and $10^{6}(-\cdot-)$ in panels $A$ and $C$, while the other system constants were as follows: $a=1, c^{\prime}=1, d^{\prime}=1, L=0.1, A_{\mathrm{ss}}=1$, and $A_{\mathrm{ms}}=1$. Compare with Bindslev's Fig. 10 (2004). In panel $\mathrm{B}$ and $\mathrm{D}$ the two surface plots are for $b^{\prime}=10^{-2}$ and $10^{6}$. The arrows in the 3-D concentration plane illustrate the agonist/modulator concentration used for the two extreme plots in panels $A$ and $C$, thus, the cut off in topography in 3-D by a plane raised at the arrows will produce the indicated plots in 2-D.

a double possibility of binding ligands, therefore binding is related to a double set of conformations by simply doubling all conformations in the denominator of the distribution equation equal to all possible binding situations (see, e.g., Cornish-Bowden 2004, Eq. 11.30 or Cornish-Bowden 1995, Eq. 9.27). ${ }^{2}$ This distribution equation is thus given by:

\footnotetext{
${ }^{2}$ This is in line with the MWC model discussed in Chapter 15. A difference is the allowed variation in association constants on binding for the HOTSM, which is banned for dissociation constants in the MWC.
}

$$
\begin{gathered}
A_{\mathrm{ss}} \cdot \mathrm{S}+A_{\mathrm{ms}} \cdot \mathrm{S}+2 \cdot c^{\prime} \cdot A_{\mathrm{ss}} \cdot \mathrm{S} \cdot A_{\mathrm{ms}} \cdot \mathrm{S} \\
+L \cdot\left(a \cdot A_{\mathrm{ss}} \cdot \mathrm{S}+b^{\prime} \cdot A_{\mathrm{ms}} \cdot \mathrm{S}\right. \\
\frac{a o}{T R}=\frac{\left.2 \cdot a \cdot b^{\prime} \cdot c^{\prime} \cdot d^{\prime} \cdot A_{\mathrm{ss}} \cdot \mathrm{S} \cdot A_{\mathrm{ms}} \cdot \mathrm{S}\right)}{2 \cdot\left[1+\cdot A_{\mathrm{ss}} \cdot \mathrm{S}+A_{\mathrm{ms}} \cdot \mathrm{S}+c^{\prime} \cdot A_{\mathrm{ss}} \cdot \mathrm{S} \cdot A_{\mathrm{ms}} \cdot \mathrm{S}\right.} \\
+L \cdot\left(1+a \cdot A_{\mathrm{ss}} \cdot \mathrm{S}+b^{\prime} \cdot A_{\mathrm{ms}} \cdot \mathrm{S}\right. \\
\left.\left.+a \cdot b^{\prime} \cdot c^{\prime} \cdot d^{\prime} \cdot A_{\mathrm{ss}} \cdot \mathrm{S} \cdot A_{\mathrm{ms}} \cdot \mathrm{S}\right)\right]
\end{gathered}
$$

Note that in Eqs. 7.3 and 7.4, some system constants now appear with an apostrophe in order to differentiate them from similar parameters in the ATSM. Further, here for HOTSM, the association constant $A_{\mathrm{ms}}$ replaces $A_{\mathrm{mm}}$ of the ATSM. 

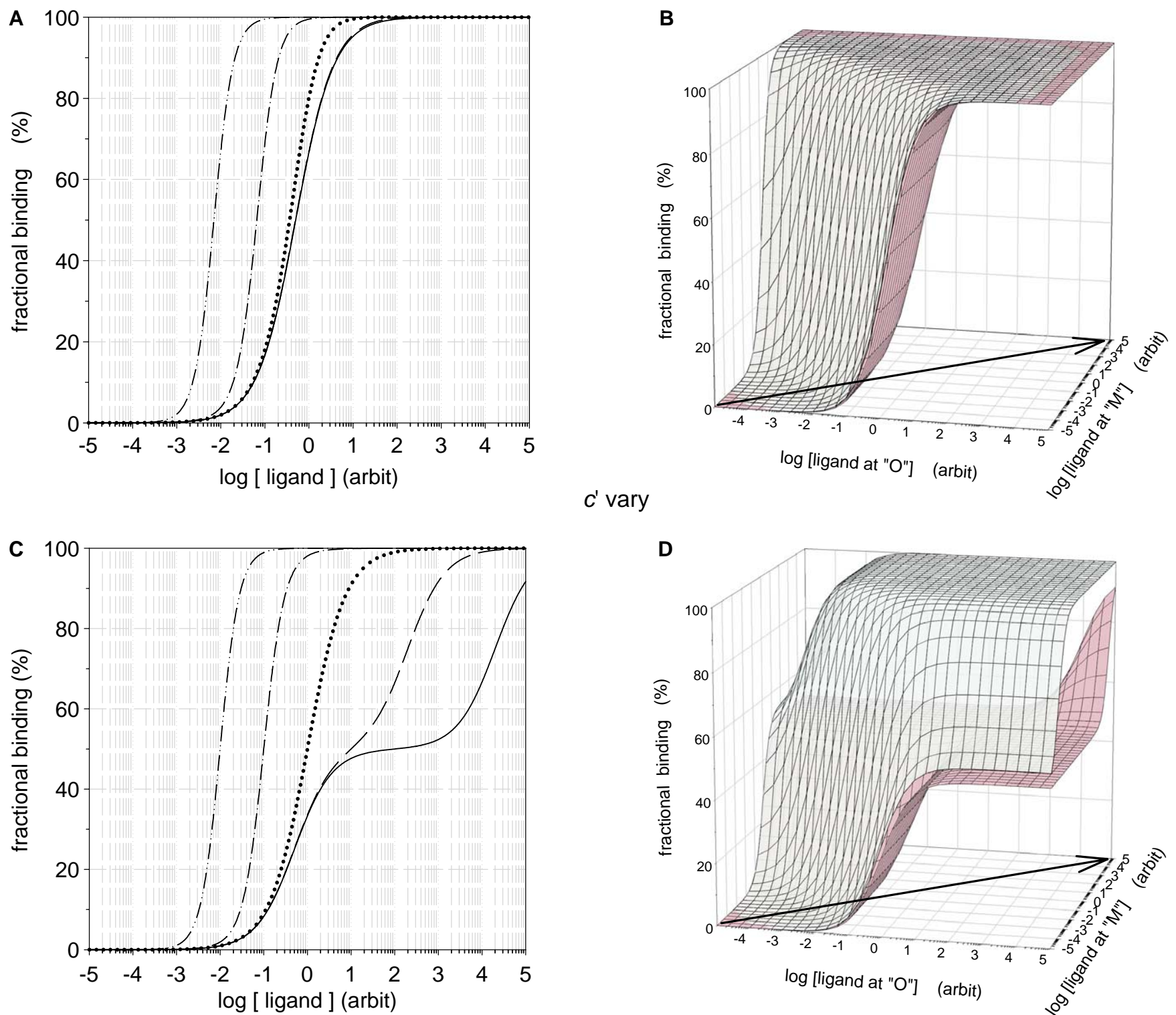

Figure 7.16. Variation of parameter $c^{\prime}$ in HOTSM for binding with the agonist/modulator as independent variable. A and B are based on the conformation model for HOTSM, C and D on the probability model for binding (e.g., Cornish-Bowden 2004, Eq. 11.30). Parameter $c^{\prime}$ was varied in five steps between $10^{-4}(-)$ and $10^{4}(-\cdot \cdot)$ in panels $A$ and $C$, while the other system constants were as follows: $a=1, b^{\prime}=1, d^{\prime}=1, L=0.1, A_{\mathrm{ss}}=1$, and $A_{\mathrm{ms}}=1$. Compare with Bindslev's Fig. 10 (2004). In panel B and $D$, the two surface plots are for $c^{\prime}=10^{-4}$ and $10^{4}$. The arrows in the 3-D concentration plane illustrate the agonist/modulator concentration used for the two extreme plots in panels $A$ and $C$, thus, the cut off in topography in 3-D by a plane raised at the arrows will produce the indicated plots in 2-D.

A general form of Eq. 7.4 is given by Cornish-Bowden (2004, Eq. 11.32, 1995, Eq. 9.29) and a variant form is derived by Kurganow (1982, Eq. 3.41). Compare this with the equations for the random reaction scheme for binding in Chapter 6 - Eq. 6.4 for two ligands and Eq. 6.8 for $n$ ligands.

\subsubsection{Effects of Variation in Single Parameters of Binding-HOTSM}

A preliminary analysis of varying single parameters in the occupancy version of the HOTSM has been carried out (Bindslev 2004). Meanwhile in binding, this analysis did 

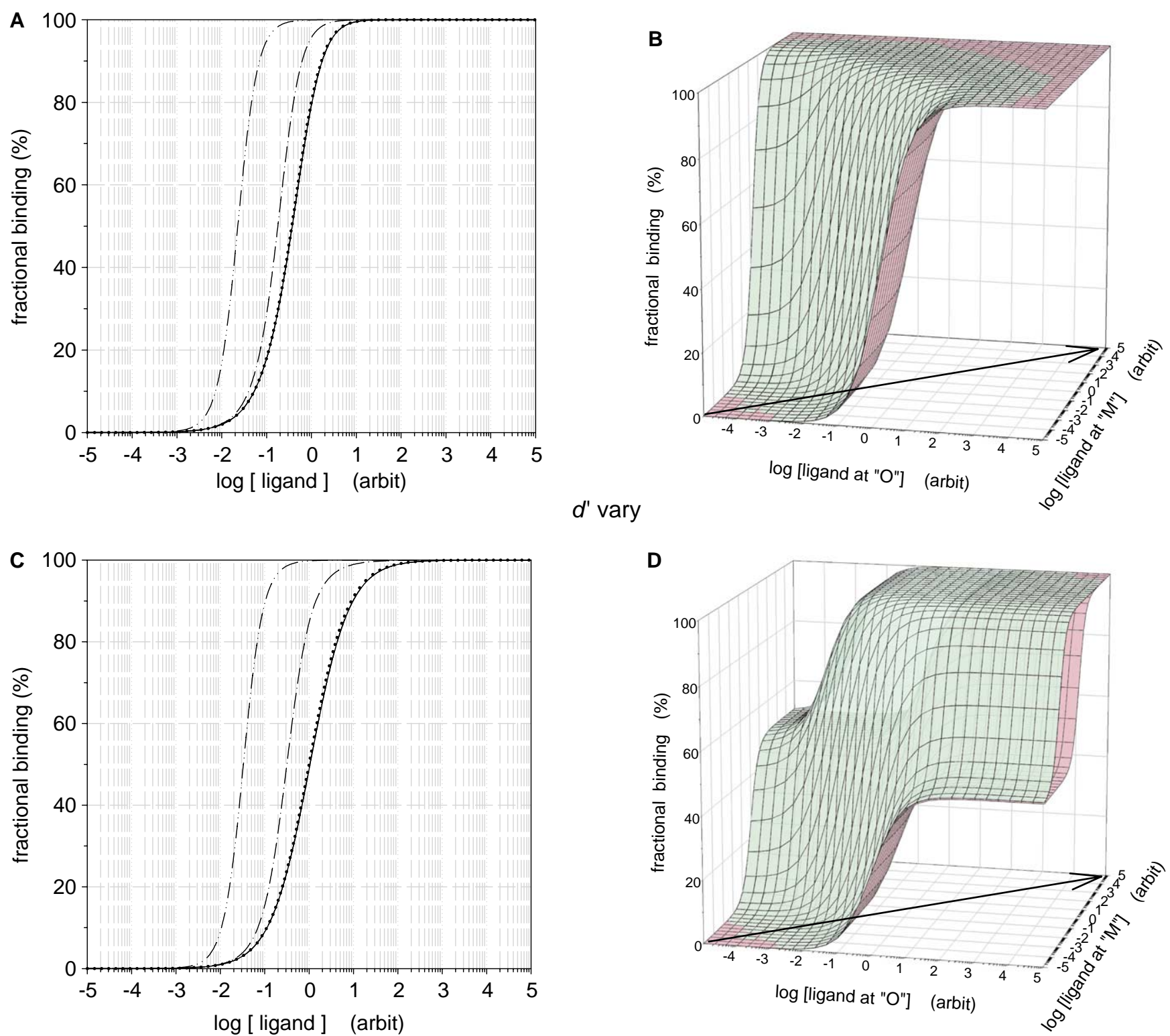

Figure 7.17. Variation of parameter $d^{\prime}$ in HOTSM for binding with the agonist/modulator as independent variable. A and B are based on the conformation model for HOTSM, C and D on the probability model for binding (e.g., Cornish-Bowden 2004 , Eq. 11.30). Parameter $d^{d}$ was varied in five steps between $10^{-4}(-)$ and $10^{4}(-\cdot-)$ in panels $A$ and $C$, while the other system constants were as follows: $a=1, b^{\prime}=1, c^{\prime}=1, L=0.1, A_{\mathrm{ss}}=1$, and $A_{\mathrm{ms}}=1$. Compare with Bindslev's Fig. 10 (2004). In panel B and $D$, the two surface plots are for $d^{\prime}=10^{-4}$ and $10^{4}$. The arrows in the 3-D concentration plane illustrate the agonist/modulator concentration used for the two extreme plots in panels $A$ and $C$, thus, the cut off in topography in 3-D by a plane raised at the arrows will produce the indicated plots in 2-D.

not take into account that some of the receptor conformations have to count twice when two agonist molecules are bound simultaneously, and as a further possibility that all bound conformations should be related relative to all possible conformation multiplied by a factor 2, as in Eq. 7.4 (see the arguments for Eq. 7.4 above).

An analysis of these aspects of the HOTSM in binding is presented here. A demonstration of the effects of varying single parameters in occupancy-HOTSM by the two principles in Eqs. 7.3 and 7.4 are shown for parameters $b^{\prime}, c^{\prime}, d^{\prime}, L, A_{\mathrm{ss}}$, and $A_{\mathrm{ms}}$ in Figs. 7.15-7.20 and detailed comments may be found in the figure legends.

With the extension in Eq. 7.4 compared to Eq. 7.3, the theory predicts a plateau level at $50 \%$ occupancy for certain parameter values, shown for $b$ ' in Fig. 7.15, for $c$ ' in Fig. 7.16, for $A_{\mathrm{ss}}$ in Fig. 7.19, and for $A_{\mathrm{ms}}$ in Fig. 7.20. However, in spite of the modification of Eq. 7.3 to Eq. 7.4 , the introduced 'flexibility' of a plateau at $50 \%$ is not worth much in binding displacement experiments, 

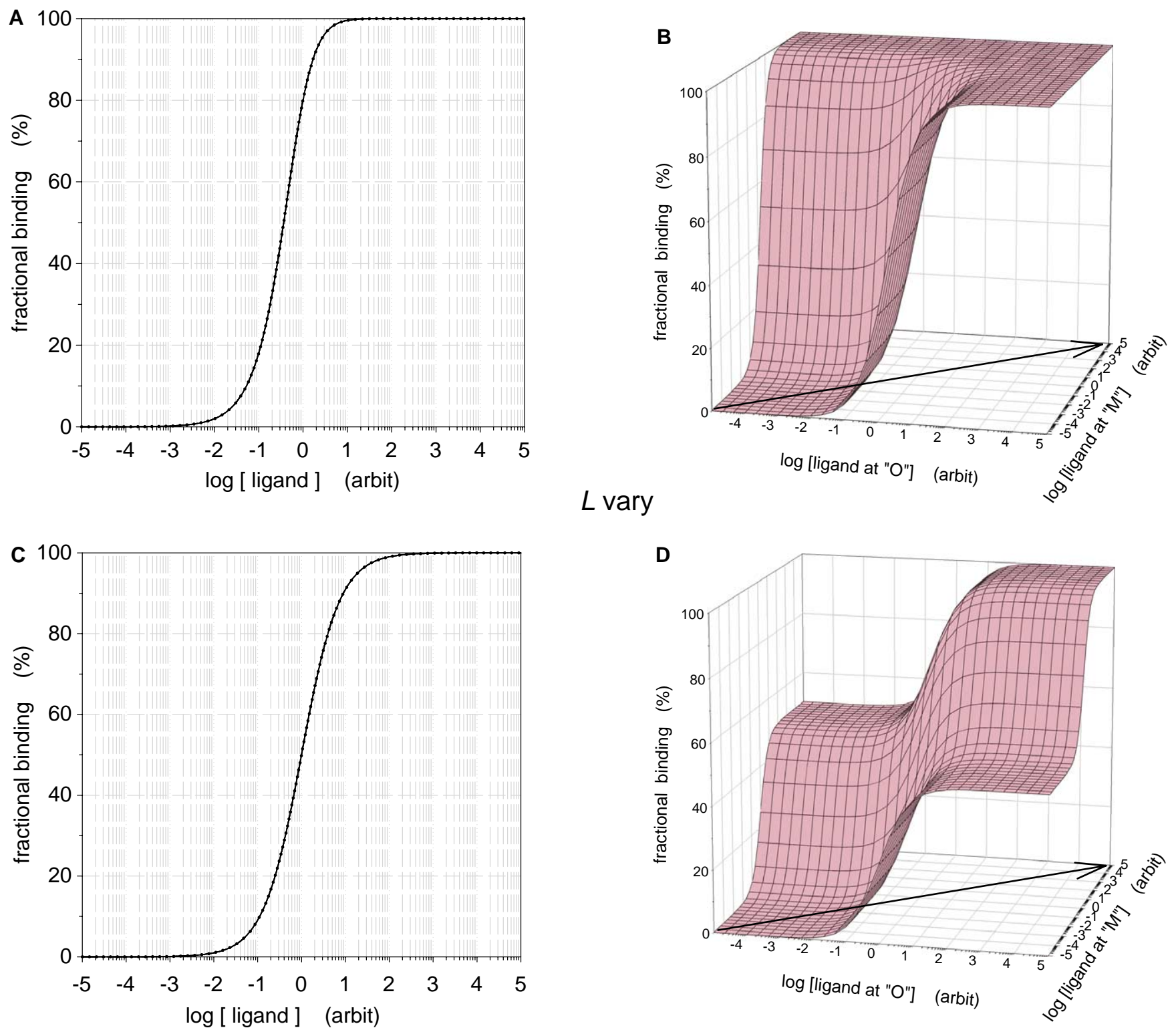

Figure 7.18. Variation of parameter $L$ in HOTSM for binding with the agonist/modulator as independent variable. A and B are based on the conformation model for HOTSM, C and D on the probability model for binding (e.g., Cornish-Bowden 2004, Eq. 11.30). Parameter $L$ was varied in five steps between $10^{-4}(-)$ and $10^{4}(-\cdot-)$ in panels $A$ and $C$, while the other system constants were as follows: $a=1, b^{\prime}=1, c^{\prime}=1, d^{\prime}=1, A_{\mathrm{ss}}=1$, and $A_{\mathrm{ms}}=1$. Compare with Bindslev's Fig. 10 (2004). In panel B and $D$, two overlaid surface plots are for $L=10^{-4}$ and $10^{4}$. The arrows in the 3-D concentration plane illustrate the agonist/ modulator concentration used for the two extreme plots in panels $A$ and $C$, thus, the cut off in topography in 3-D by a plane raised at the arrows will produce the indicated plots in 2-D.

which do not seem restrained to a plateau phase at exactly $50 \%$ occupancy; though, it may reasonably be argued to include this correction for the distribution equation of the binding-HOTSM.

\subsubsection{Conclusion on Aspects of Binding as Simulated with the HOTSM}

Positive co-operativity in occupancy can be simulated with HOTSM. A variant form of the HOTSM can describe terraced concentration binding curves, but merely terraced for binding around 50\% occupancy. Also, compare these $50 \%$ plateaus with similar published plateaus for the square geometry in the KNF model by Koshland et al. (1966).

How is binding in HOTSM different from binding in ATSM? Binding in the HOTSM includes double occupancy with co-lateral binding, whereas in the ATSM colateral binding is excluded (compare plots in Figs. 7.157.20 with plots in Fig. 7.6)

The positive co-operativity of the HOTSM is insufficient for positive co-operativity in, for instance, hemoglobin 

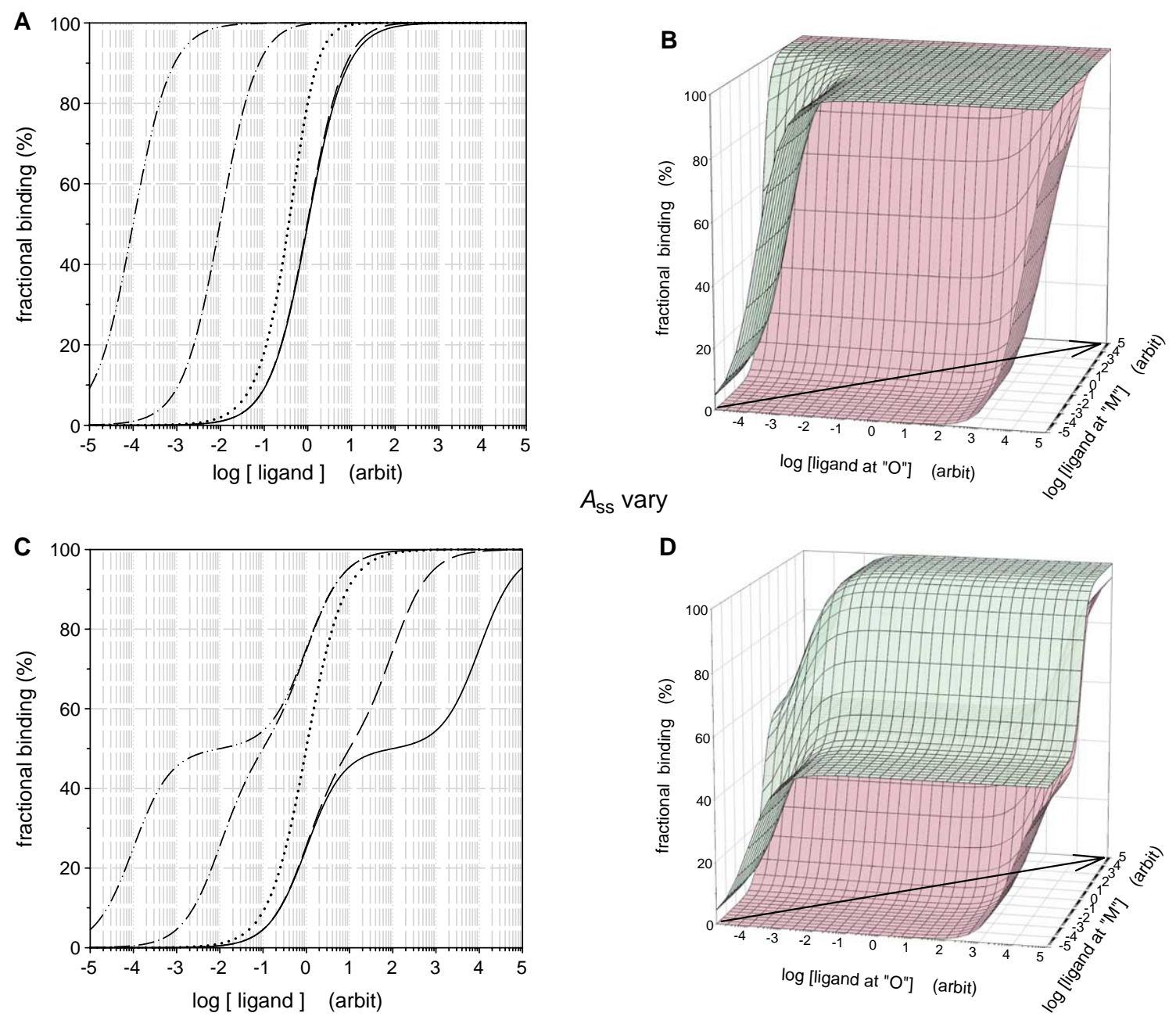

Figure 7.19. Variation of parameter $A_{\mathrm{SS}}$ in HOTSM for binding with the agonist/modulator as independent variable. A and B are based on the conformation model for HOTSM, C and D on the probability model for binding (e.g., Cornish-Bowden 2004, Eq.

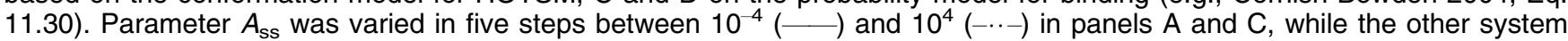
constants were as follows: $a=1, b^{\prime}=1, c^{\prime}=1, d^{\prime}=1, L=0.1$, and $A_{\mathrm{ms}}=1$. Compare with Bindslev's Fig. 10 (2004). In panel B and $\mathrm{D}$ the two surface plots are for $A_{\mathrm{ss}}=10^{-4}$ and $10^{4}$. The arrows in the 3-D concentration plane illustrate the agonist/modulator concentration used for the two extreme plots in panels $A$ and $C$, thus, the cut off in topography in 3-D by a plane raised at the arrows will produce the indicated plots in 2-D.

that operates with four binding sites. Here, the HOTSM needs to be developed further.

\subsection{Aspects for Function with the HOTSM}

\subsubsection{Simulation with HOTSM}

The functional form of the homotropic two-state reaction model has several and surprising possible simulations available (Bindslev 2004). To illustrate, a research example, that is discussed in more detail in sub-chapter 7.7, is brought up here in the introduction (section 7.6.2), for which the HOTSM seems relevant in simulation of experimental self-inhibited and self-enhanced effects as reverse bell-shaped dose-responses.
Intrinsic self-enhancement of oxygen binding to hemoglobin following a deviation from the ordinarily hyperbolic load relation is not relevant for the functionalform of HOTSM, while self-inhibition in the form of substrate inhibition, as described in Chapter 3, is relevant. The former is designated as positive co-operative, while the latter may be denoted as negative co-operative. However, since substrate inhibition can result in bell-shaped doseresponses (see Figs. 3.2 and 3.3), a designation such as 'auto-ant-agonism', 'auto-inhibition', or 'negative autointervention' for the decaying leg of bell-shaped doseresponses are more valid than 'negative co-operativity' because negative co-operativity is usually a reference to merely shallow dose-responses (Chapter 15) (Bindslev 2004). A general term when assuming genuine allostery which covers all the observed deviations from simple load 


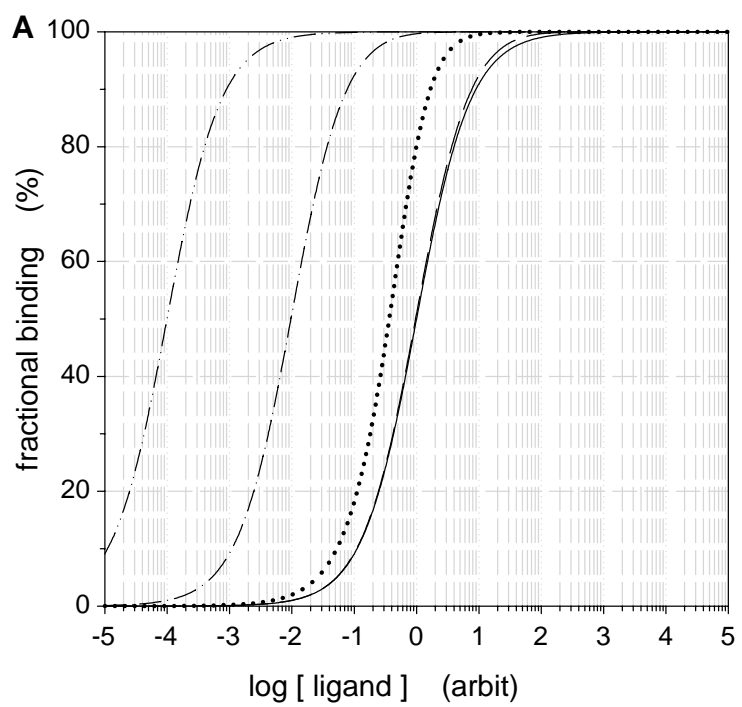

B

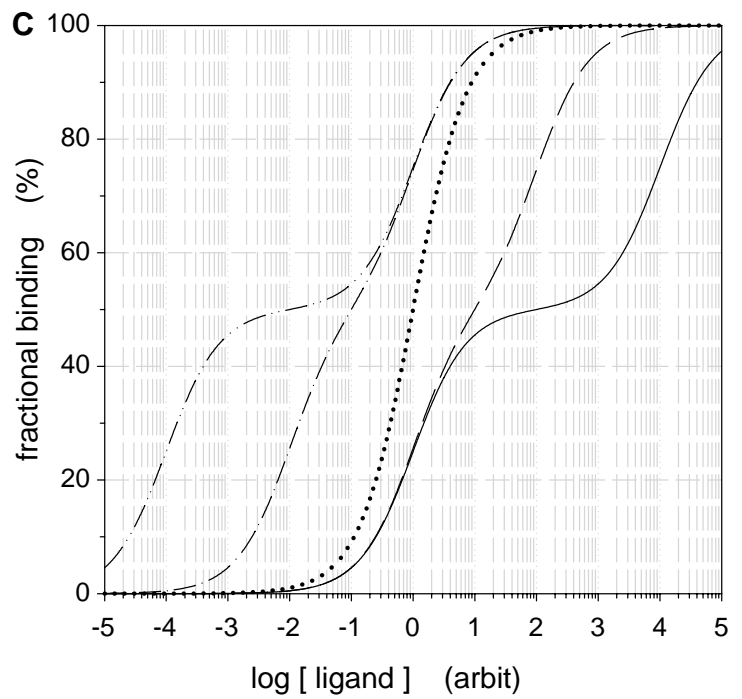

$A_{\mathrm{ms}}$ vary
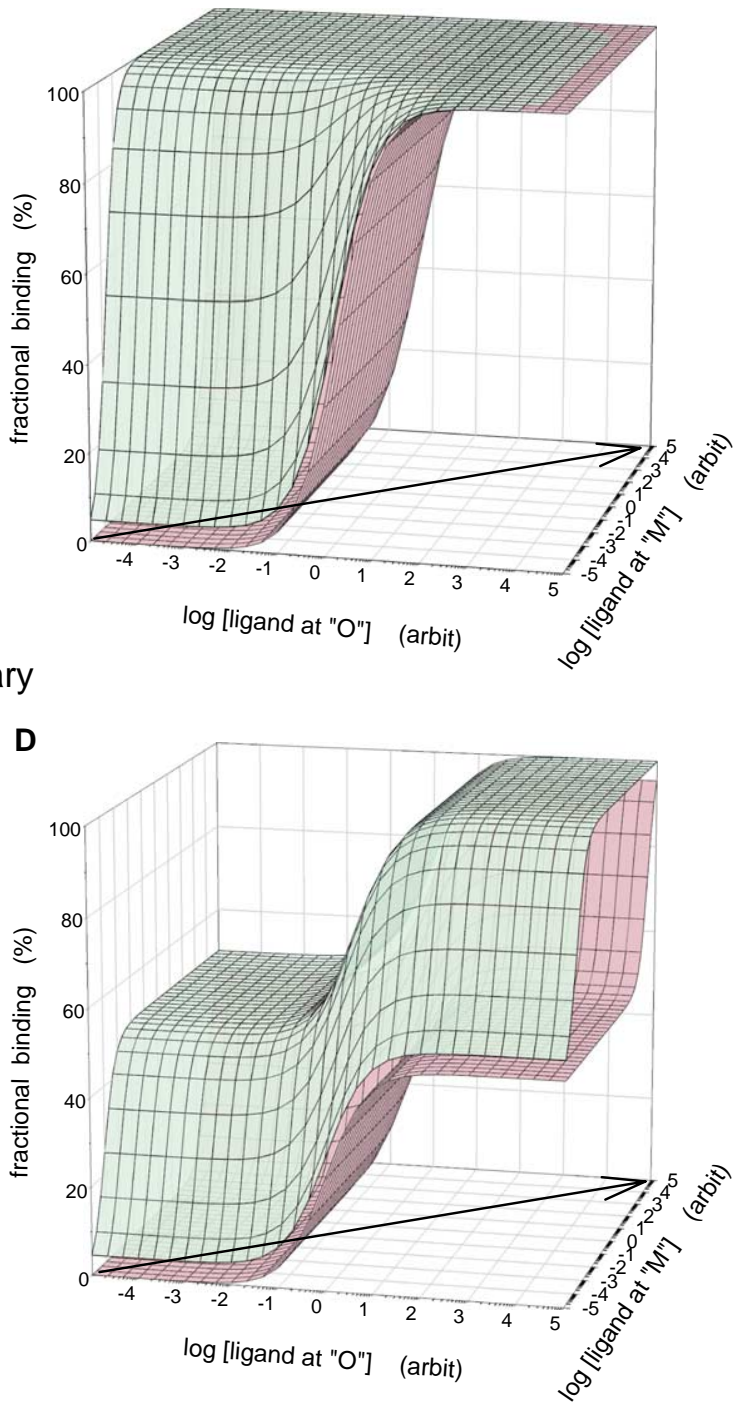

Figure 7.20. Variation of parameter $A_{\mathrm{ms}}$ in HOTSM for binding with the agonist/modulator as independent variable. A and B are based on the conformation model for HOTSM, C and D on the probability model for binding (e.g., Cornish-Bowden 2004, Eq. 11.30). Parameter $A_{\mathrm{ms}}$ was varied in five steps between $10^{-4}(-)$ and $10^{4}(-\cdot-)$ in panels $A$ and $C$, while the other system constants were as follows: $a=1, b^{\prime}=1, c^{\prime}=1, d^{\prime}=1, L=0.1$, and $A_{\mathrm{ss}}=1$. Compare with Bindslev's Fig. 10 (2004). In panel B and $\mathrm{D}$, the two surface plots are for $A_{\mathrm{ms}}=10^{4}$ and $10^{4}$. The arrows in the 3-D concentration plane illustrate the agonist/modulator concentration used for the two extreme plots in panels $A$ and $C$, thus, the cut off in topography in 3-D by a plane raised at the arrows will produce the plots indicated in 2-D.

relations, even reversed bell-shaped dose-responses, is auto-modulation. We may operate with positive and negative auto-modulation. HOTSM is well suited to simulate both positive and negative functional automodulation, including, as we shall see, reverse bellshaped and reverse terraced dose-response relationships.

\subsubsection{Examples of Reverse Bell-shaped Dose-responses}

Due to significant spontaneous activity, typically in model systems of transfected cell, it is possible to observe reverse bell-shaped dose responses (Migeon \& Nathanson 1994; Michal et al. 2001; Christopoulos et al. 2001; Accomazzo et al. 2002; Nasman et al. 2002; Hornigold et al. 2003; Holmqvist et al. 2005). These reverse bellshaped dose-responses are not necessarily explained, as usual, by a $G$ protein signal-bifurcation between for instance $G_{s}$ and $G_{i}$ complexes, since the bell-shaped dose-response may be present even after eliminating the function of $\mathrm{G}_{\mathrm{i}}$ proteins by treatment with PTX of the receptor-transfected cells (Hornigold et al. 2003). Meanwhile, the observed reverse bell-shaped behavior is easily modeled by the HOTSM, suggesting an intrinsic 
receptor modulation as explanation. Thus, the HOTSM may also be used as an analytical tool for reverse bell-shaped dose-responses. This aspect of the HOTSM together with its inherent reversed terraced doseresponses is further discussed in sub-chapter 7.7. Models are discussed in Section 7.11.3 for the reverse bellshaped dose-response of GPCRs where only a single type of $\mathrm{G}$ protein is present.

\subsubsection{The Basics of HOTSM}

The HOTSM is shown in Fig. 7.1B. It is a combination of the auto-intervention model described in Chapter 3 and the cTSM presented in Chapter 5. The receptor conformations and parameters for HOTSM are listed in Table 7.1 and Box 7.1, and its distribution equation for funcion can be formulated as:

$$
\begin{gathered}
L \cdot\left(1+a \cdot A_{\mathrm{ss}} \cdot \mathrm{S}+b^{\prime} \cdot A_{\mathrm{ms}} \cdot \mathrm{S}\right. \\
\frac{a r}{T R}=\frac{\left.+a \cdot b^{\prime} \cdot c^{\prime} \cdot d^{\prime} \cdot A_{\mathrm{ss}} \cdot \mathrm{S} \cdot A_{\mathrm{ms}} \cdot \mathrm{S}\right)}{1+A_{\mathrm{ss}} \cdot \mathrm{S}+A_{\mathrm{sm}} \cdot \mathrm{S}+c^{\prime} \cdot A_{\mathrm{ss}} \cdot \mathrm{S}} \\
\cdot A_{\mathrm{ms}} \cdot \mathrm{S}+L \cdot\left(1+a \cdot A_{\mathrm{ss}} \cdot \mathrm{S}+b^{\prime}\right. \\
\left.\cdot A_{\mathrm{ms}} \cdot \mathrm{S}+a \cdot b^{\prime} \cdot c^{\prime} \cdot d^{\prime} \cdot A_{\mathrm{ss}} \cdot \mathrm{S} \cdot A_{\mathrm{ms}} \cdot \mathrm{S}\right)
\end{gathered}
$$

see Bindslev (2004, Eq. 2). Again, note the parameters with apostrophe in order to differentiate them from similar parameters in the ATSM. The association constant $A_{\mathrm{ms}}$ replaces the $A_{\mathrm{mm}}$ of ATSM.

\subsubsection{Variation in Single Parameters}

In contrast to the ATSM, both concentrations of ligands at either binding site are varied simultaneously as they change concomitantly. This is compulsory for the HOTSM. I shall return to how this can be mimicked in the ATSM in sub-chapter 7.8.

For the functional form of the HOTSM, variations in parameters $b^{\prime}, c^{\prime}, d^{\prime}, L, A_{\mathrm{ss}}$, and $A_{\mathrm{ms}}$ are shown in Figs. 7.21-7.26. Details of the parameter variations are commented on in the figure legends.

\subsubsection{Conclusion on Functional Aspects as Simulated with the HOTSM}

The functional HOTSM is a versatile tool for simulation of single-ligand dose-responses rendering load-deviant curves. Experimental curve forms that may be simulated with HOTSM are summarized in Fig. 7.27. In the past, such load-deviant curve forms of experimental synagics triggered use of the Hill and sums of the Hill equation as analytical tools (e.g., Bronnikov et al. 1999; Accomazzo et al. 2002; Hornigold et al. 2003). The HOTSM offers a mechanistic approach to the analysis, while the Hillian approach is nearly always a pure mathematical description lacking any mechanistic relevance (Chapter
10). Hence, future modeling ought to consider variations on the themes of the HOTSM, replacing versions of the Hill equation.

\subsection{Further Aspects for the HOTSM}

With the right combination of parameter values, the functional HOTSM possesses both bell-shaped and terraced as well as reverse bell-shaped and reverse terraced relationships (Fig. 7.27). A prerequisite for observing inverse agonism, reverse terraced curves, and reverse bell-shaped dose-response relations is spontaneous activity which is detectable for $L>1 / 100$. Parameter $L$ is the sole system constant to determine the initial level of activity in both HOTSM (Figs. 7.23 and 7.24) and ATSM (Fig. 7.13C + D).

\subsubsection{Reverse Bell-shaped Synagics}

Hornigold et al. (2003) described a system only expressing a single receptor subtype, m3, coupled to producing cAMP as spontaneous activity and affected in a reverse bell-shaped dose-response relation by methacholine (Fig. 7.29A). Even after treatment with PTX in order to eliminate possible coupling to inhibitory $G$ proteins, this system still displays reverse bell-shaped synagics (Fig. 7.29B). This excludes bifurcation via $G_{s}$ and $G_{i}$ as an explanation for the observed reverse bellshaped dose-response relationship.

The concave bell-shaped dose-response in Fig. 7.29A can be simulated by the HOTSM (see curve in the figure) and may thus be interpreted as a receptor intrinsic modulation in line with the evidence of more than one binding site for ligands in functional muscarinic receptors (Huang \& Ellis 2007).

Therefore, for a possible alternative explanation of the observed reverse bell-shaped dose-response curve, as mentioned above, the HOTSM is a simple, elegant, and sufficient analytical tool, as also pointed out by Bindslev (2004, Figs. 2 and 9).

\subsubsection{The HOTSM Against the Hill Equation}

The change between three plateau levels of the HOTSM in function (Fig. 7.27), and also the co-operative rise of the concentration-occupancy relation for the model in binding (Figs. 7.15-7.20), are not as steep as doseresponses described by a single or a combination of two or more Hill equations. This is not surprising, as the whole analysis of the HOTSM is based on a system with only two binding sites. For a fair comparison, the HOTSM should be allowed to have as many sites as the Hill analyses in such situations (Bindslev 2004). 
A

$$
b^{\prime} \text { varied in } 5 \text { steps, } 10^{0}-10^{4}
$$

B

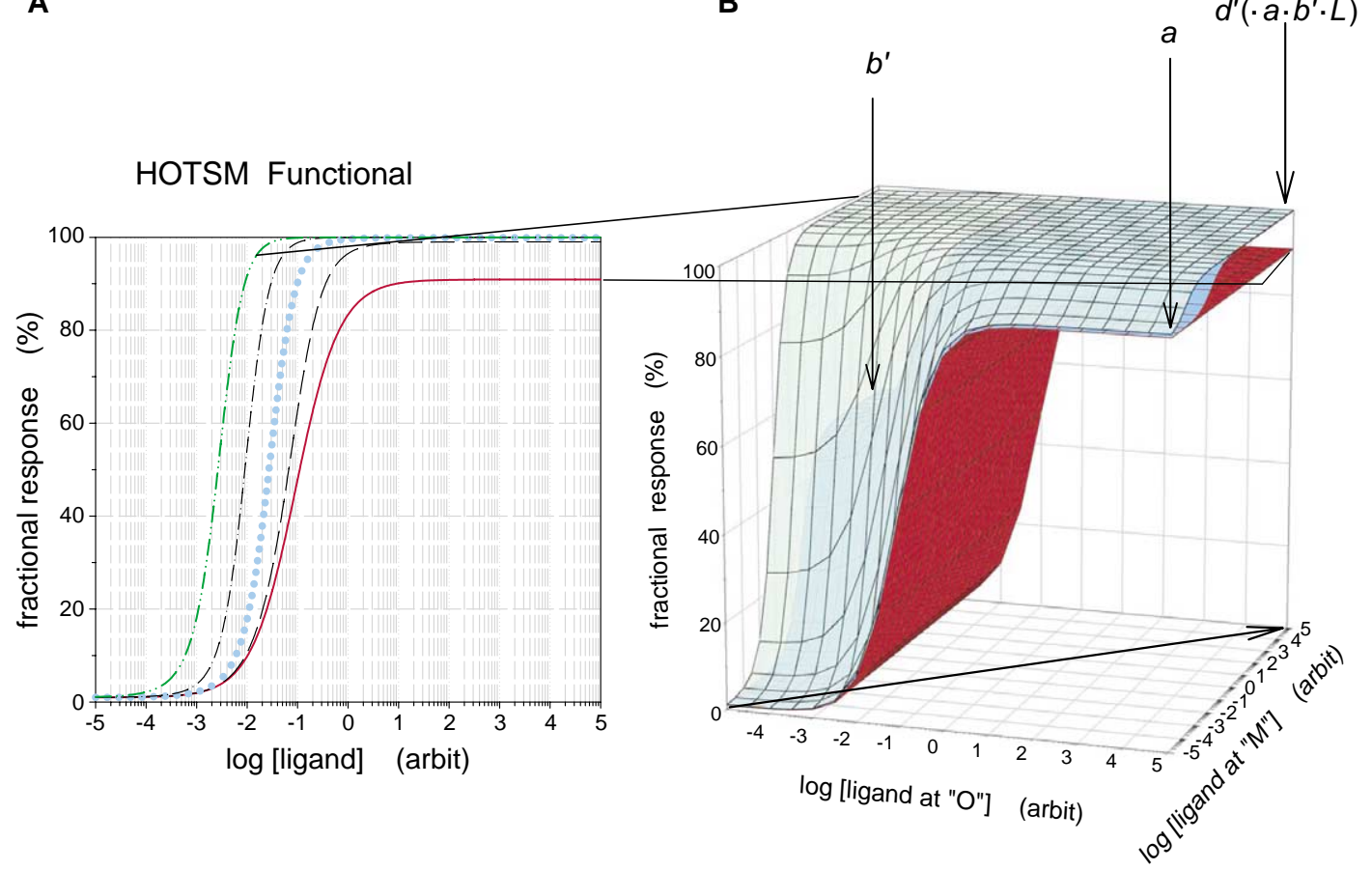

C $b^{\prime}$ varied in 5 steps, $10^{-4}-10^{0}$

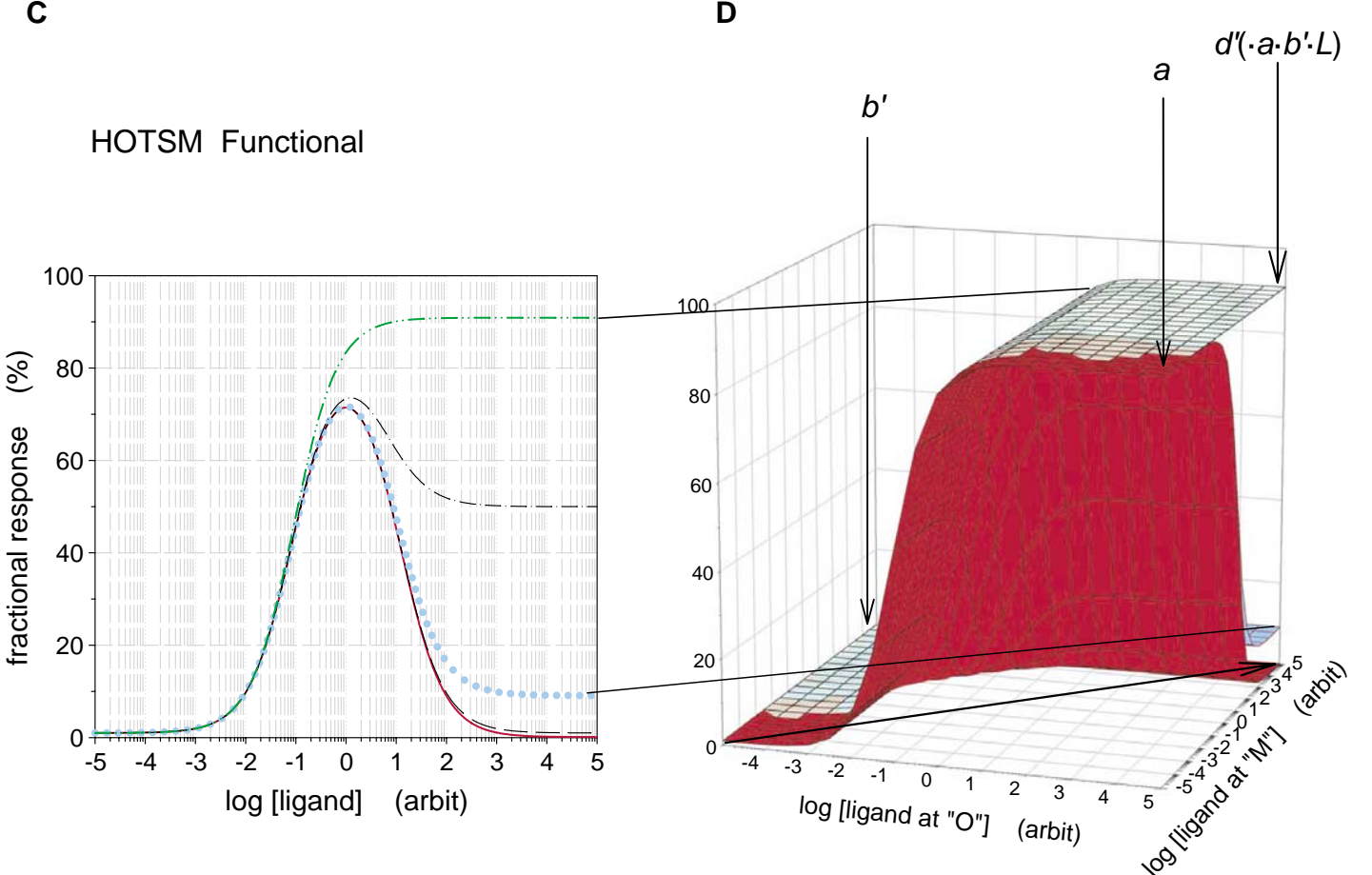

Figure 7.21. Variation of parameter $b^{\prime}$ in HOTSM for function with increasing concentration of agonist/modulator as independent variable. For $b^{\prime}>1$ in $A$ and $B$, and for $b^{\prime}<1$ in $C$ and $D$. Parameter $b^{\prime}$ was varied in five steps between $10^{\circ}(-)$ and $10^{4}(-\cdot \cdot-)$ in panel $A$, and between $10^{-4}(\longrightarrow)$ and $10^{\circ}(-\cdot-)$ in panel $C$, while the other system constants were as follows: $a=1000, c^{\prime}=1$, $d^{\prime}=1, L=0.01, A_{\mathrm{ss}}=1$, and $A_{\mathrm{ms}}=1$. Compare with Bindslev's Fig. 3 (2004). The three surface plots in panel $\mathrm{B}$ are for $b^{\prime}=10^{\circ}$, $10^{2}$, and $10^{4}$ and in panel $D$ for $b^{\prime}=10^{-4}, 10^{-2}$, and $10^{\circ}$. The arrows in the $3-D$ concentration plane in panels $B$ and $D$ illustrate the agonist/modulator concentration used for the plots in panels $A$ and $C$, thus, the cut off in topography in 3-D by a plane raised at the arrows will produce the indicated plots in 2-D. 
A

$C^{\prime}$ varied in 5 steps, $10^{0}-10^{4}$

B

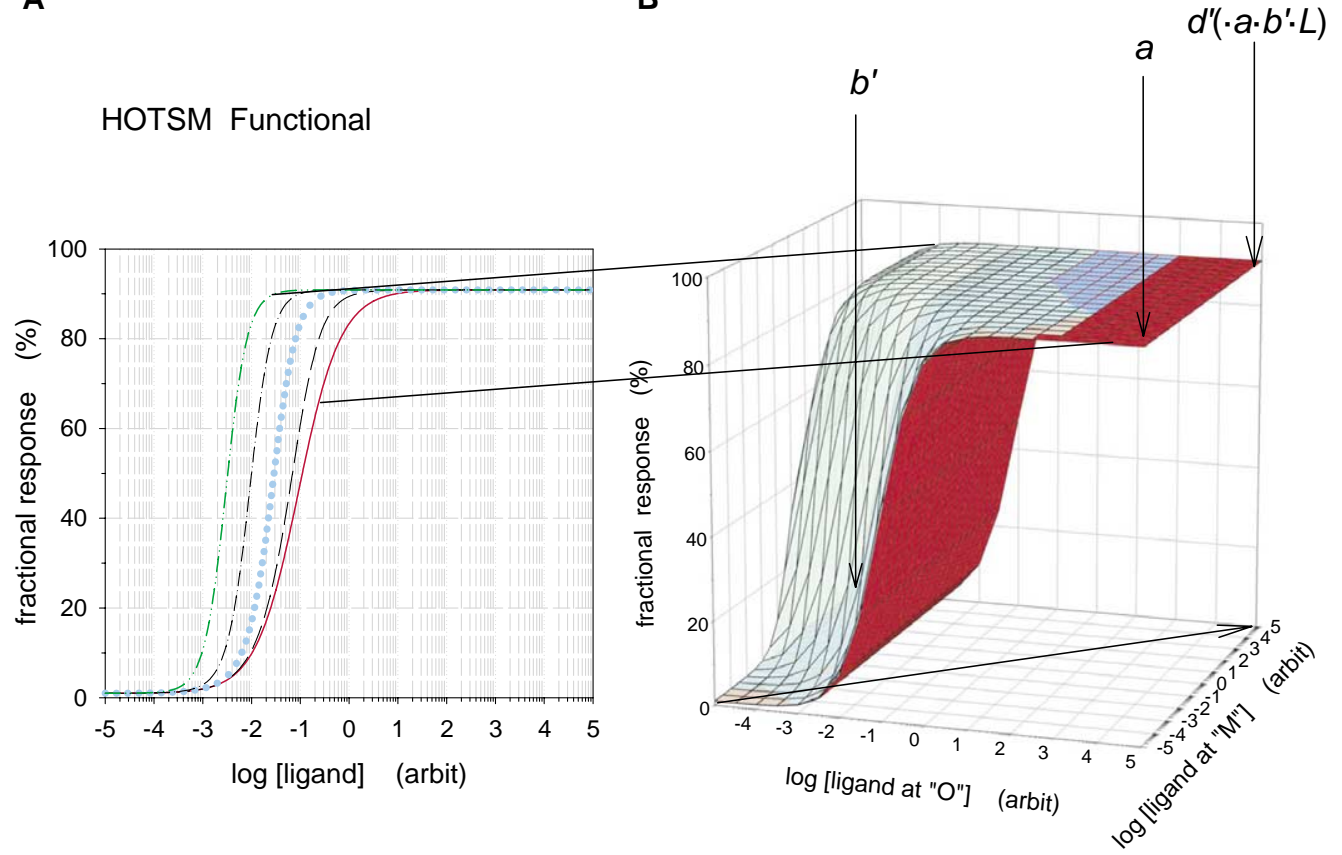

C

$C^{\prime}$ varied in 5 steps, $10^{-4}-10^{0}$

D

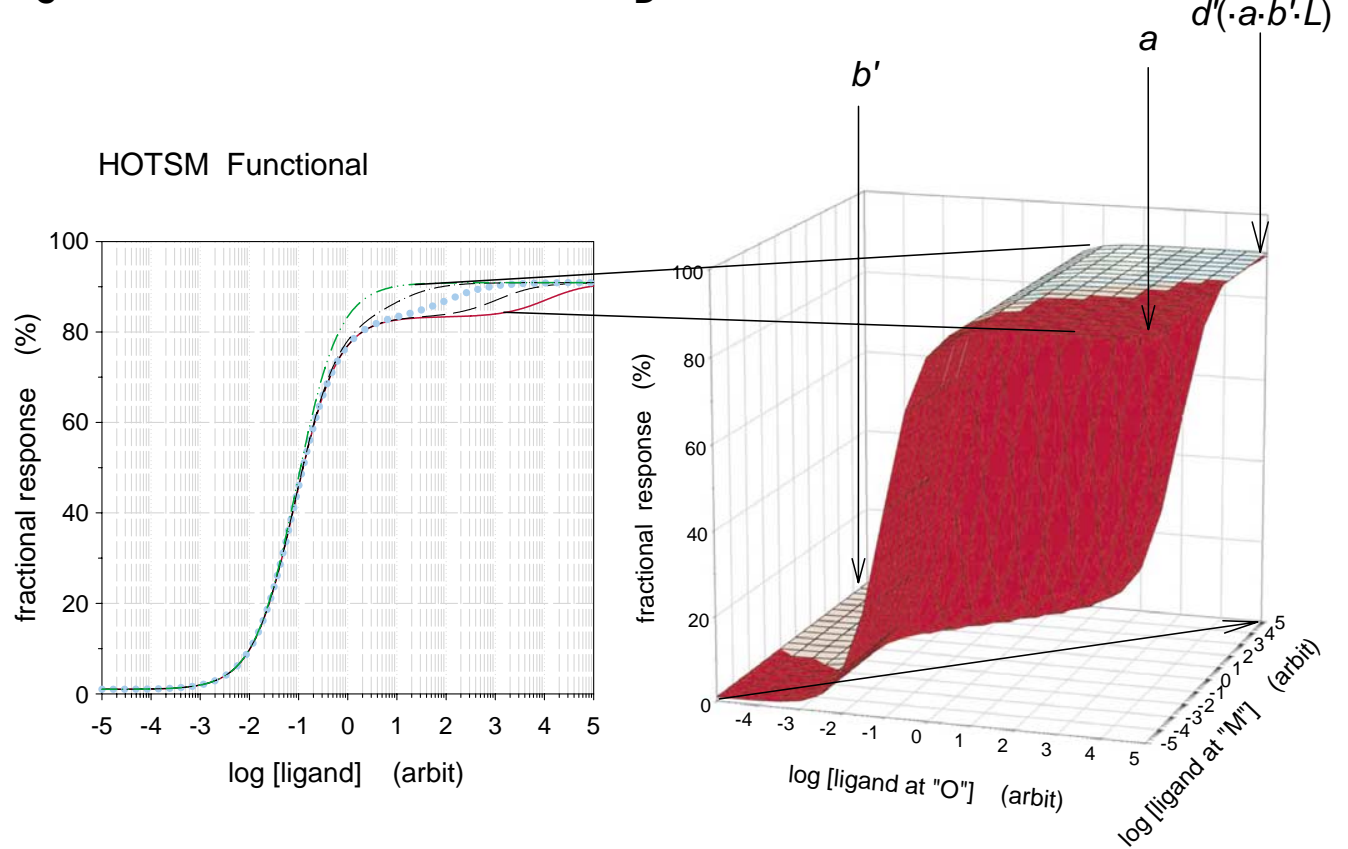

Figure 7.22. Variation of parameter $c^{\prime}$ in HOTSM for function with increasing concentration of agonist/modulator as independent variable. For $c^{\prime}>1$ in $A$ and $C$, and for $c^{\prime}<1$ in $C$ and $D$. Parameter $c^{\prime}$ was varied in five steps between $10^{\circ}(-)$ and $10^{4}(-\cdot \cdot-)$ in panel $\mathrm{A}$, and between $10^{-4}(-)$ and $10^{\circ}(-\cdot-)$ in panel $\mathrm{C}$, while the other system constants were as follows: $a=1000, b^{\prime}=1$, $d^{\prime}=1, L=0.01, A_{\mathrm{ss}}=1$, and $A_{\mathrm{ms}}=1$. Compare with Bindslev's Fig. 7 (2004). The three surface plots in panel $\mathrm{B}$ are for $c^{\prime}=10^{\circ}$, $10^{2}$, and $10^{4}$, and in panel $D$ for $c^{\prime}=10^{-4}, 10^{-2}$, and $10^{\circ}$. The arrows in the 3-D concentration plane in panels $B$ and $D$ illustrate the agonist/modulator concentration used for the plots in panels $A$ and $C$, thus, the cut off in topography in 3-D by a plane raised at the arrows will produce the indicated plots in 2-D. 
A

$$
d^{\prime} \text { varied in } 5 \text { steps, } 10^{0}-10^{4}
$$

B

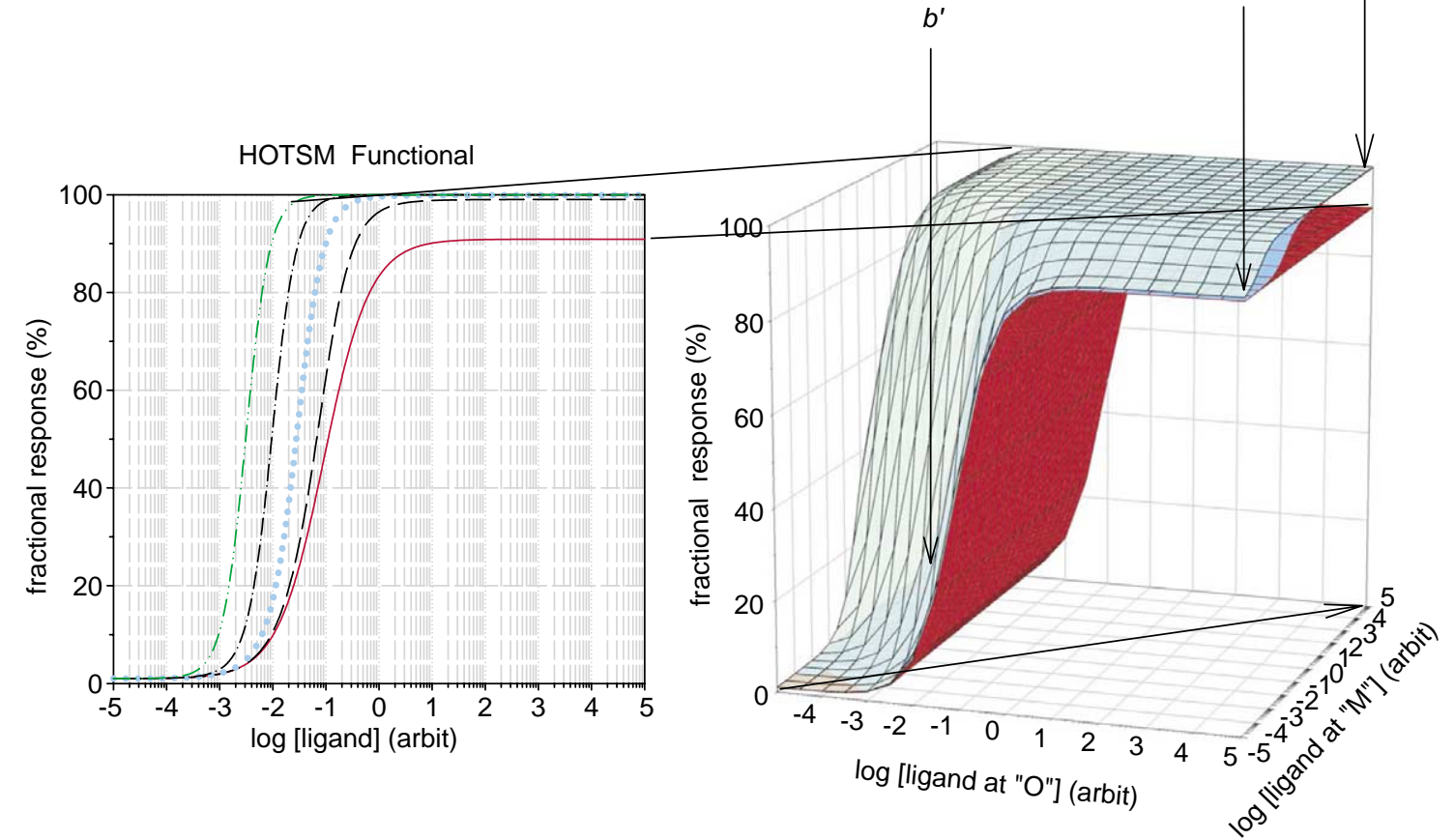

$d^{\prime}$ varied in 5 steps, $10^{-4}-10^{0}$

C

D

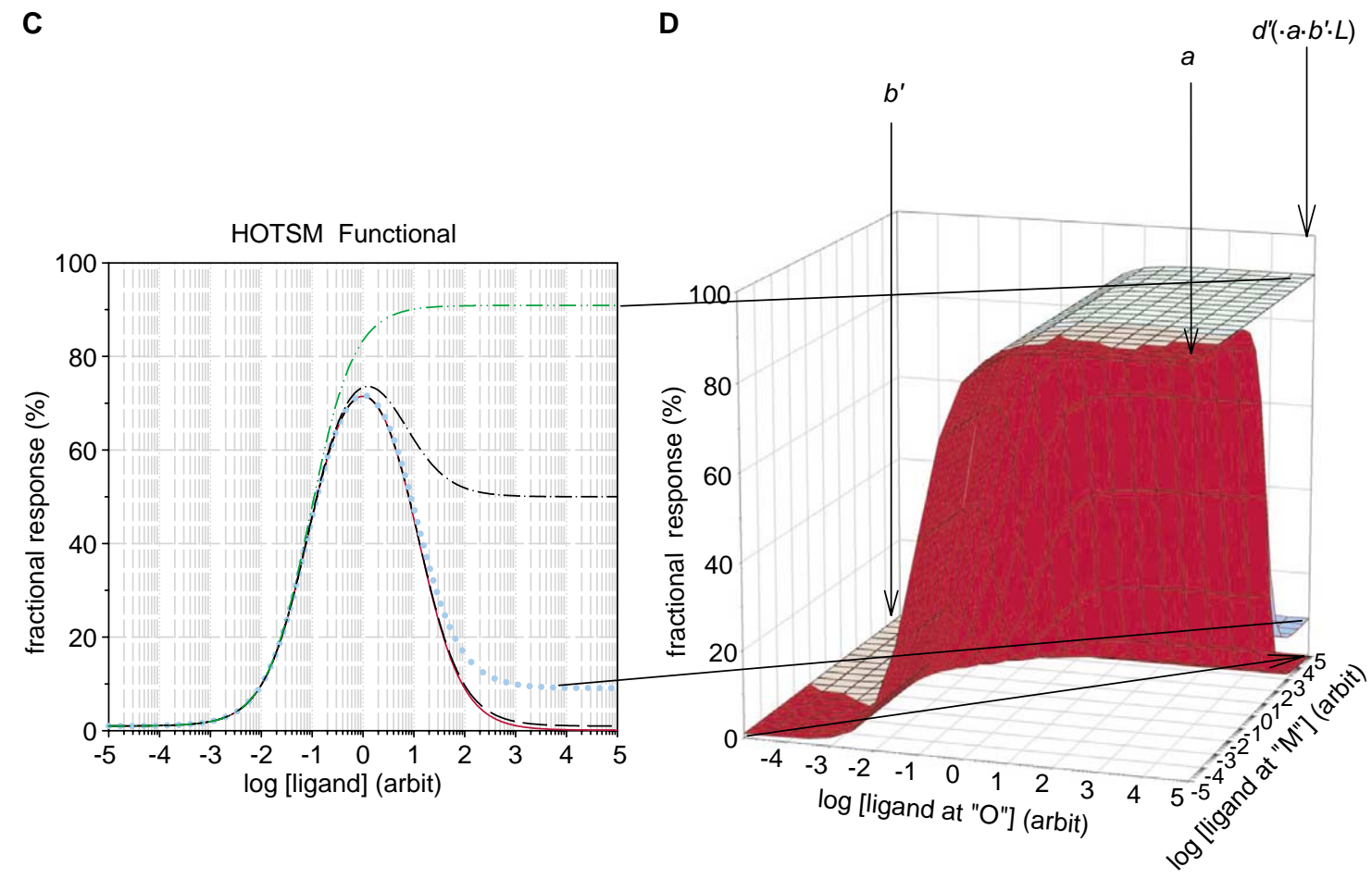

Figure 7.23. Variation of parameter $d$ ' in HOTSM for function with increasing concentration of agonist/modulator as independent variable. For $d^{\prime}>1$ in $A$ and $B$, and for $d^{\prime}<1$ in $C$ and $D$. Parameter $d$ was varied in five steps between $10^{\circ}$ $(-)$ and $10^{4}(-\cdot \cdot)$ in panel $A$, and between $10^{-4}(-)$ and $10^{\circ}(-\cdot \cdot-)$ in panel $\mathrm{C}$, while the other system constants were as follows: $a=30, b^{\prime}=3, c^{\prime}=0.1, L=1 / 9, A_{\mathrm{ss}}=1$, and $A_{\mathrm{ms}}=100$. Compare with Bindslev's Fig. 4 (2004). The three surface plots in panel $B$ are for $d^{\prime}=10^{\circ}, 10^{2}$, and $10^{4}$, and in panel $D$ for $d^{\prime}=10^{-4}, 10^{-2}$, and $10^{\circ}$. The arrows in the 3-D concentration plane in panels $B$ and $D$ illustrate the agonist/modulator concentration used for the plots in panels $A$ and $C$, thus, the cut off in topography in $3-D$ by a plane raised at the arrows will produce the indicated plots in 2-D. 
A

$L$ varied in 5 steps, $0.2 \times 10^{-2}-0.2 \times 10^{2}$

B

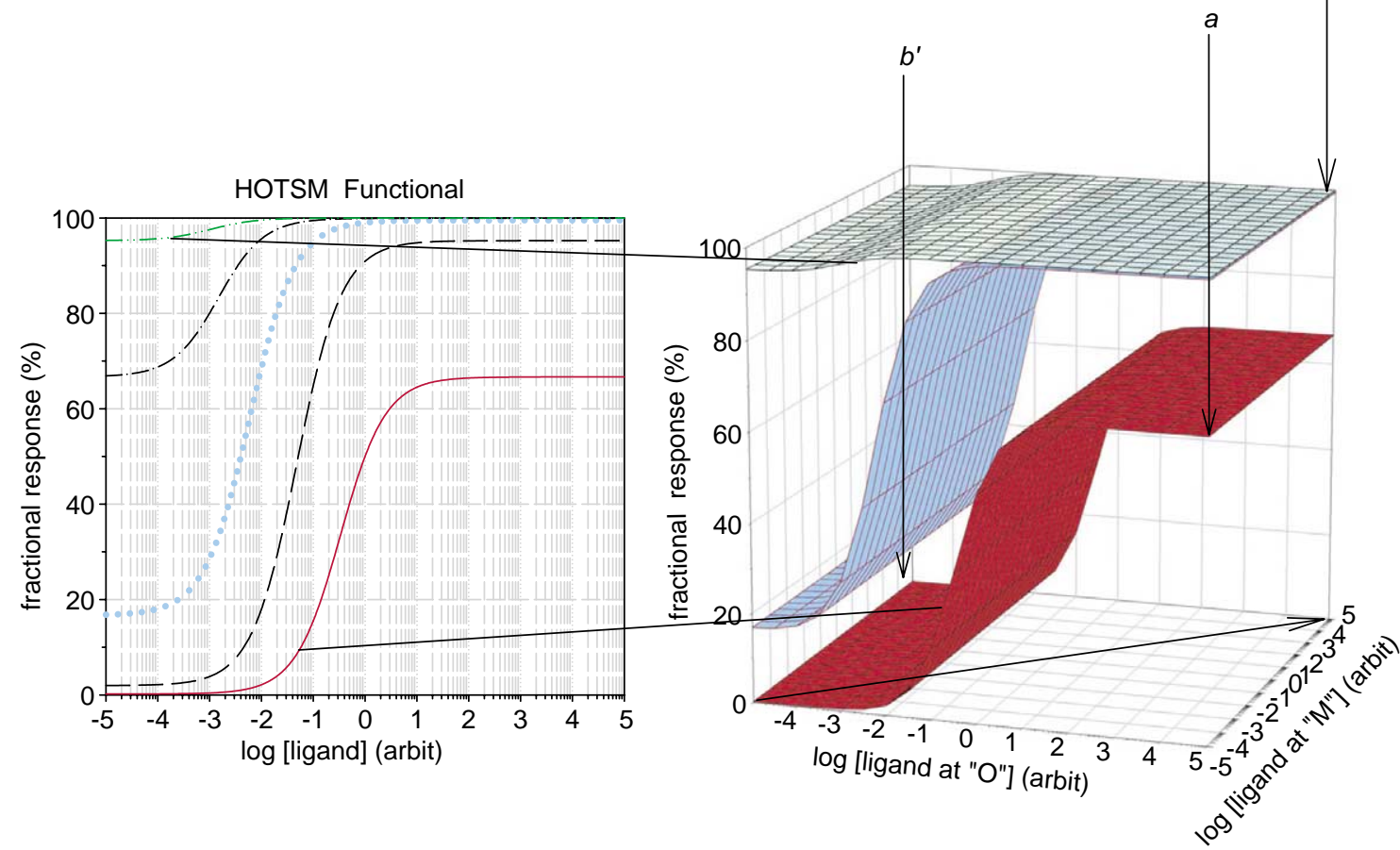

C

$L$ varied in 5 steps, $0.2 \times 10^{-2}-0.2 \times 10^{2}$

D

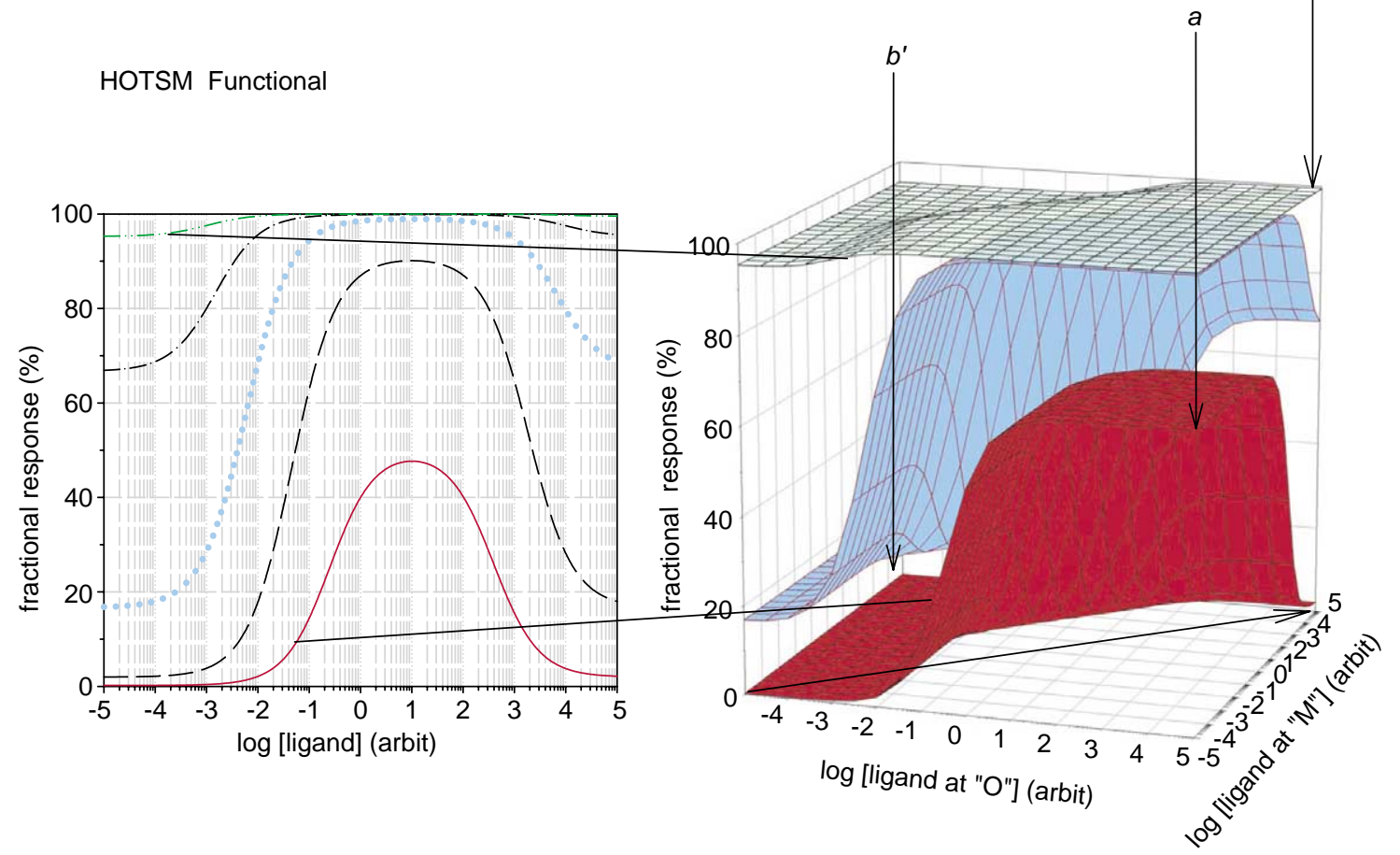

Figure 7.24. Variation of parameter $L$ in HOTSM for function with increasing concentration of agonist/modulator as independent variable. Parameter $L$ varied in five steps from $0.2 \times 10^{-2}(-)$ to $0.2 \times 10^{2}(-\cdot-)$ by a factor 10 in panels $A$ and $C$ and in three steps from $0.2 \times 10^{-2}$ to $0.2 \times 10^{2}$ in panels $B$ and $D$ by a factor $10^{2}$. Other parameter values were as follows in $A$ and $B$ : $a=$ $1000, b^{\prime}=1, c^{\prime}=1, d^{\prime}=1, A_{\mathrm{ss}}=1$, and $A_{\mathrm{ms}}=1$. In panels $\mathrm{C}$ and $\mathrm{D}$ for $a=1000, b^{\prime}=1, c^{\prime}=0.01, d^{\prime}=0.01, A_{\mathrm{ss}}=1$, and $A_{\mathrm{ms}}=1$. See for instance Fig. 2 in Bindslev (2004). The arrows in the 3-D concentration plane in panels B and D illustrate the agonist/ modulator concentration used for the plots in panels $A$ and $C$, thus, the cut off in topography in 3-D by a plane raised at the arrows will produce the indicated plots in 2-D. 
$A_{\mathrm{ss}}$ varied in 5 steps, $10^{0}-10^{4}$

A

B

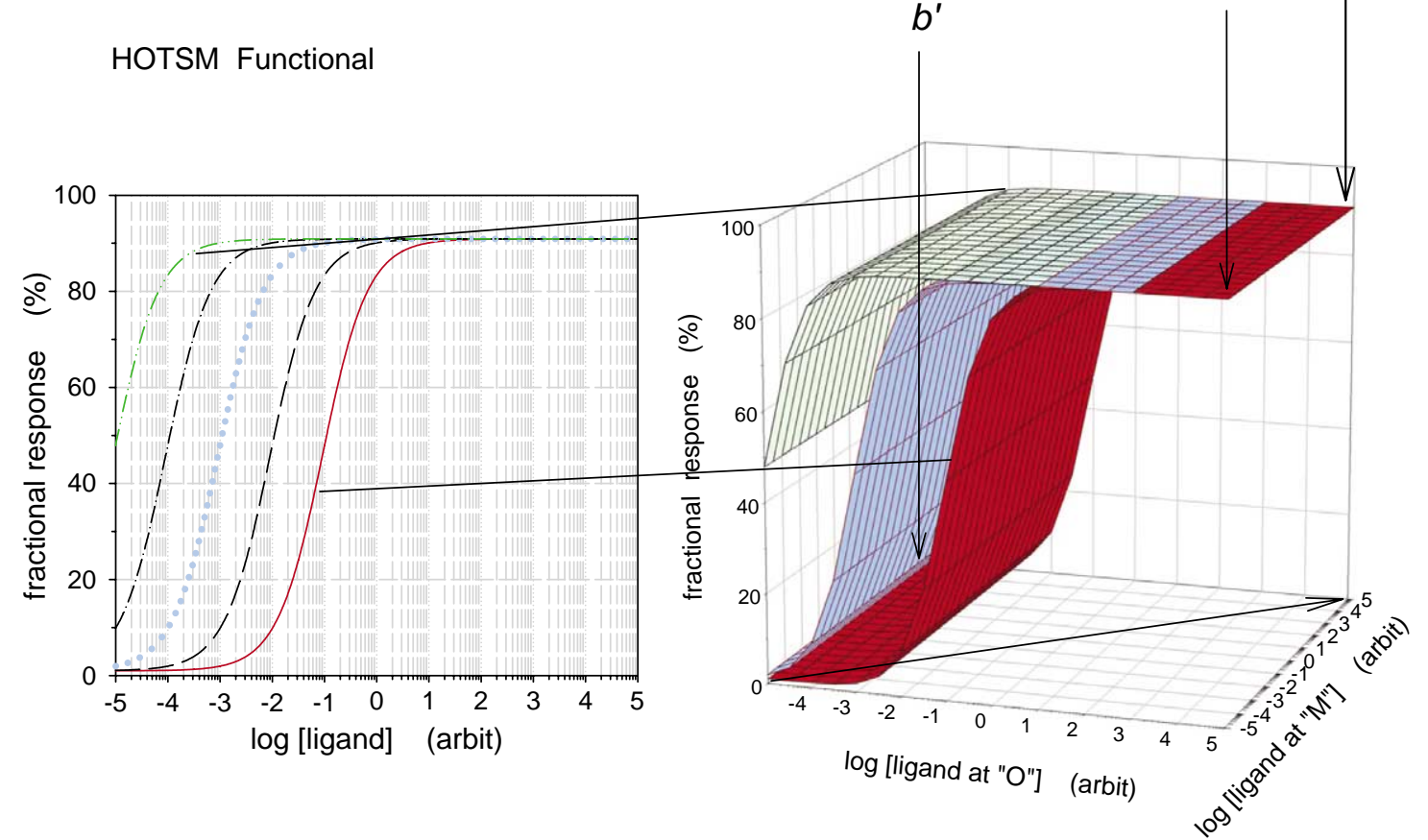

$A_{\mathrm{ss}}$ varied in 5 steps, $10^{-4}-10^{0}$

C

D

$b^{\prime}$

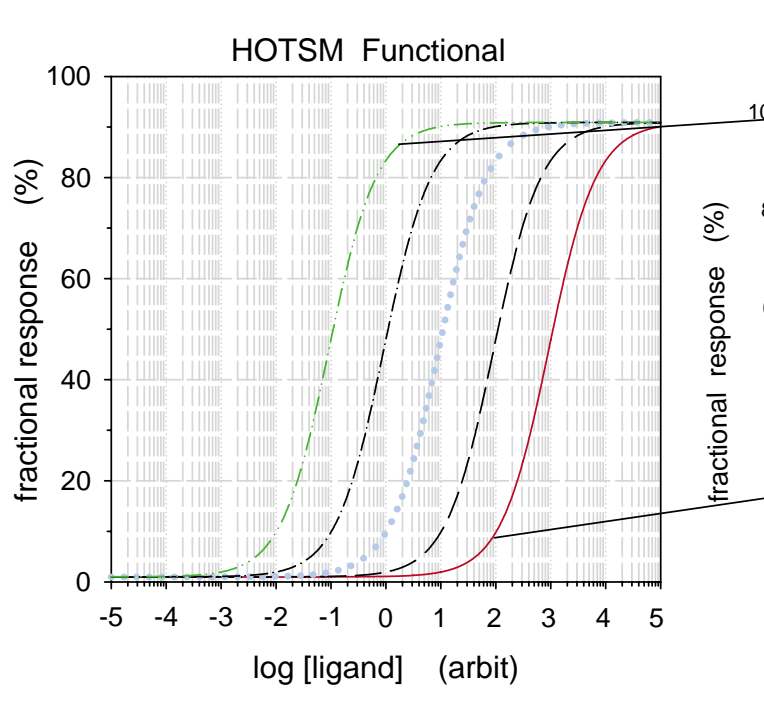

Figure 7.25. Variation of parameter $A_{\mathrm{ss}}$ in HOTSM for function with increasing concentration of agonist/modulator as independent variable. $A_{\mathrm{ss}}$ was varied in five steps between $10^{\circ}(-)$ and $10^{4}(-\cdot \cdot)$ in panel $\mathrm{A}$, and between $10^{-4}(-)$ and $10^{0}$ $(-\cdot-)$ in panel $C$, while the other system constants were as follows: $a=1000, b^{\prime}=1, c^{\prime}=1, d=1, L=0.01$, and $A_{\mathrm{ms}}=1$. Compare with Bindslev's Fig. 5 (2004). The three surface plots in panel B are for $A_{\mathrm{ss}}=10^{0}, 10^{2}$, and $10^{4}$ and in panel $\mathrm{D}$ for $A_{\mathrm{ss}}=10^{-4}, 10^{-2}$, and $10^{\circ}$. The arrows in the $3-\mathrm{D}$ concentration plane in panels $\mathrm{B}$ and $\mathrm{D}$ illustrate the agonist/modulator concentration used for the plots in panels $A$ and $C$, thus, the cut off in topography in 3-D by a plane raised at the arrows will produce the indicated plots in 2-D. 
A

$A_{\mathrm{ms}}$ varied in 5 steps, $10^{0}-10^{4}$

B

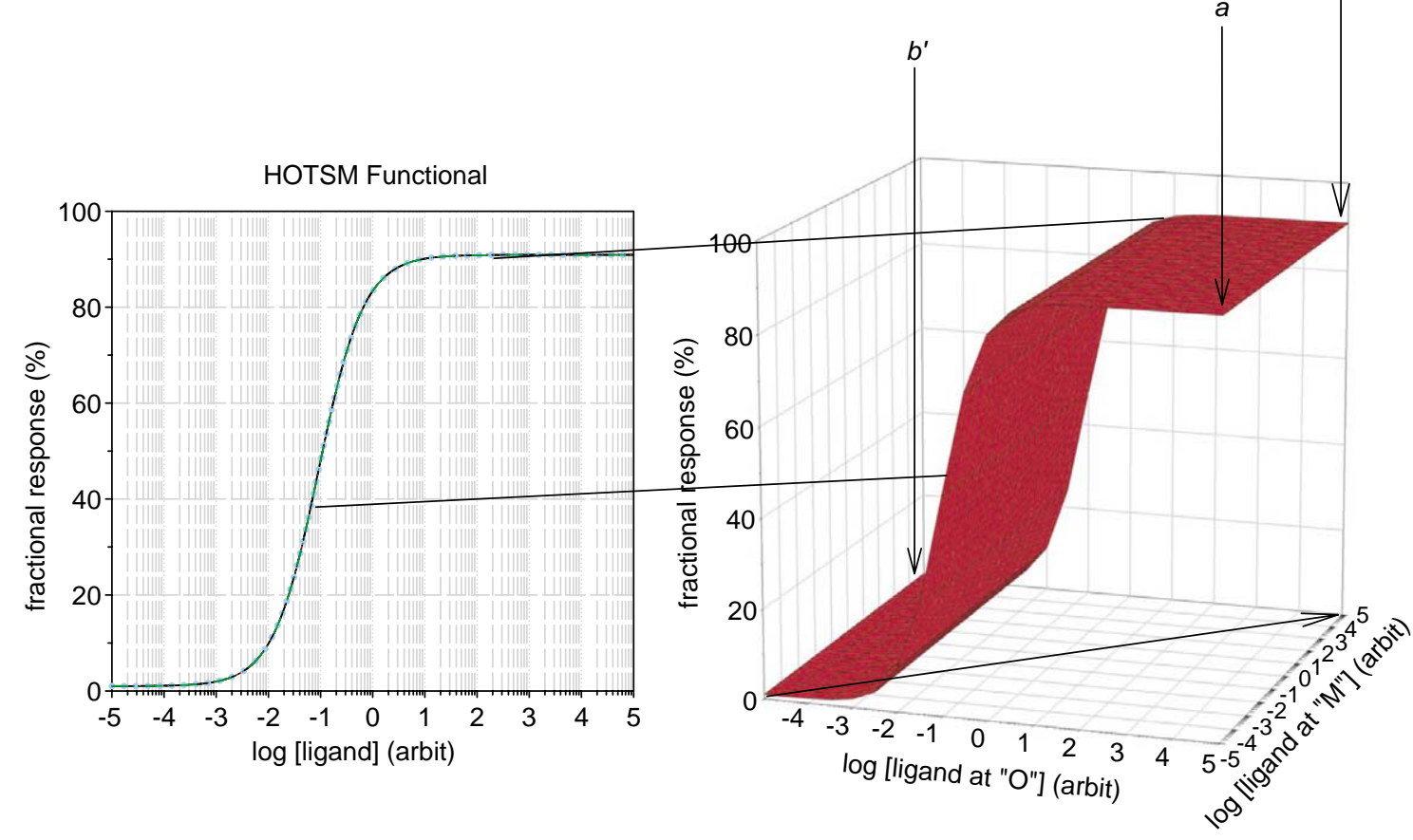

$A_{\mathrm{ms}}$ varied in 5 steps, $10^{-4}-10^{0}$

C

D

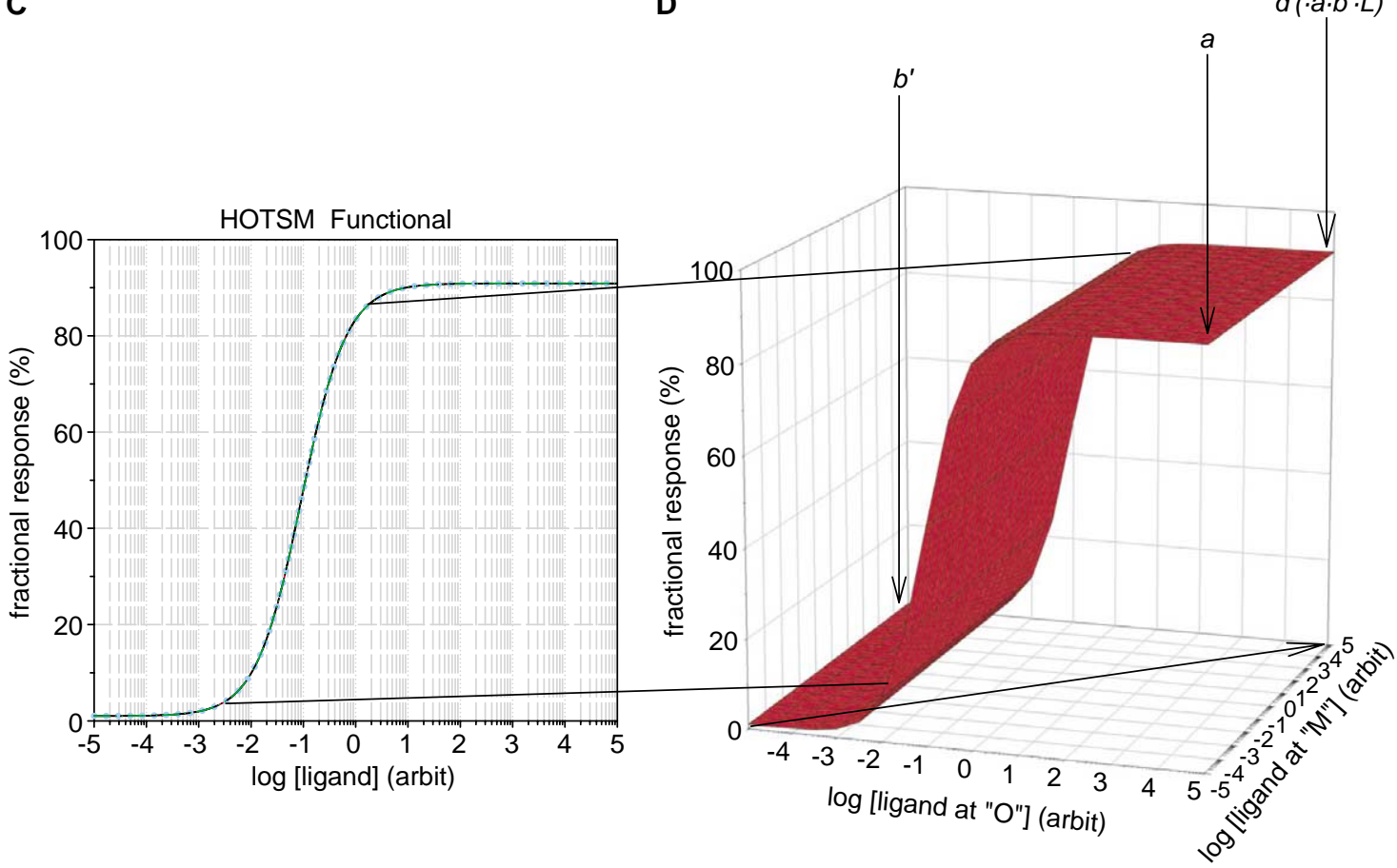

Figure 7.26. Variation of parameter $A_{\mathrm{ms}}$ in HOTSM for function with increasing concentration of agonist/modulator as independent variable. $A_{\mathrm{ms}}$ was varied in five steps between $10^{\circ}(-)$ and $10^{4}(-\cdot-)$ in panel $\mathrm{A}$, and between $10^{-4}(-)$ and $10^{\circ}$ $(-\cdot-)$ in panel $\mathrm{C}$, while the other system constants were as follows: $a=1000, b^{\prime}=1, c^{\prime}=1, d^{\prime}=1, L=0.01$, and $A_{\mathrm{ss}}=1$. Compare with Bindslev's Fig. 6 (2004). The three surface plots in panel B are for $A_{\mathrm{ms}}=10^{\circ}, 10^{2}$, and $10^{4}$, and in panel $\mathrm{D}$ for $A_{\mathrm{ms}}=10^{-4}$, $10^{-2}$, and $10^{\circ}$. The arrows in the 3-D concentration plane in panels $B$ and $D$ illustrate the agonist/modulator concentration used for the plots in panels $A$ and $C$, thus, the cut off in topography in 3-D by a plane raised at the arrows will produce the indicated plots in 2-D. 
A

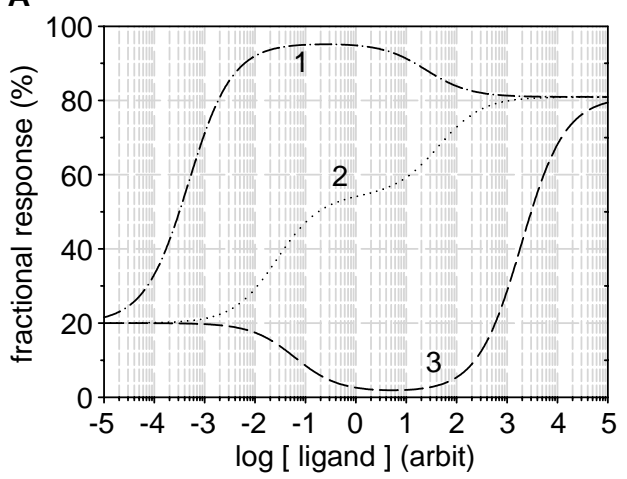

C

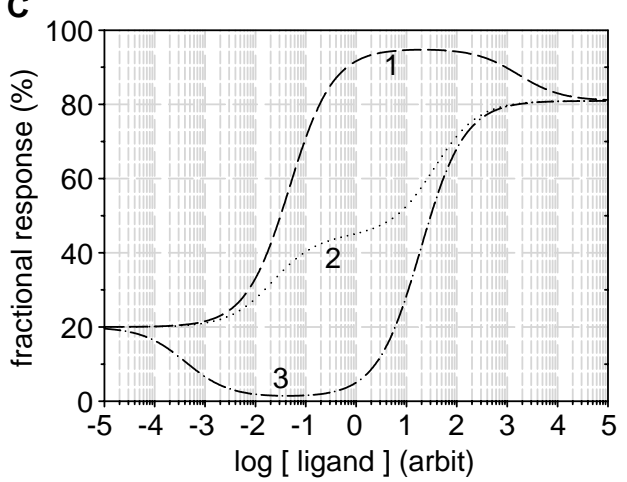

B

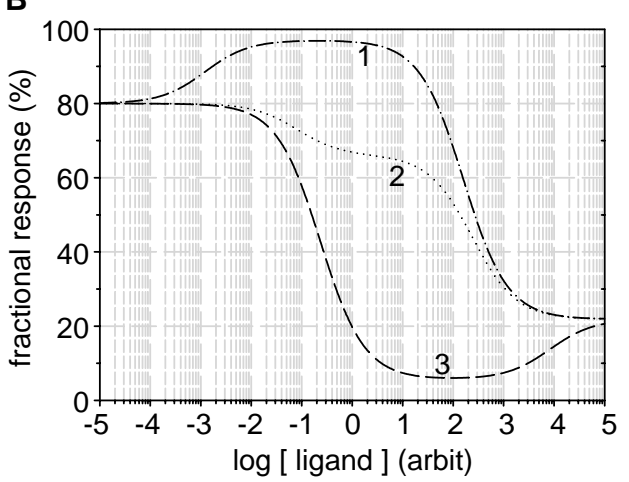

D

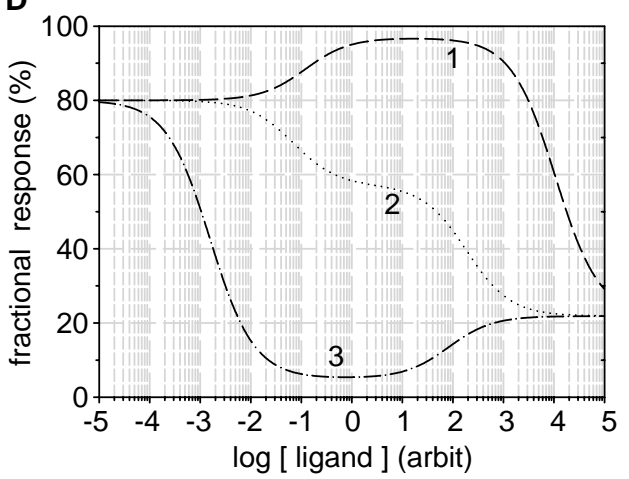

Figure 7.27. The response level of the functional-HOTSM has three plateaus. A first response-plateau in the absence of ligands is equal to $1 /[1+1 /(L)]$. A third response-plateau at high agonist concentrations is given by $1 /\left[1+1 /\left(L \cdot a \cdot b^{\prime} \cdot d^{\prime}\right)\right]$. Finally, a secondresponse plateau at intermediate ligand concentrations is dependent on all seven system constants. The three plateaus may be above, at, or below each other, panels A-D, rendering dose-response curves that are positive or negative co-operative, bellshaped or terraced, as well as reverse bell-shaped or reverse terraced. Parameter values in panels $A-D$ were arbitrarily chosen in order to demonstrate several aspects of the model. $A_{\mathrm{ss}}$ varied in three steps from $10^{-2}(---)$ to $10^{2}(-\ldots .$.$) by a factor 100$ in panels $\mathrm{A}$ and $\mathrm{B}$, while $A_{\mathrm{ms}}$ varied in three steps from 0.3 (---) to $3000(-\ldots .$.$) by a factor 100$ in panels $\mathrm{C}$ and $\mathrm{D}$. The remaining parameters were fixed. Thus in panel $\mathrm{A}: L^{\prime}=1 / 4, A_{\mathrm{ms}}=20, a=100, b^{\prime}=0.01, c^{\prime}=0.01$, and $d^{\prime}=17$. In panel $\mathrm{B}: L^{\prime}=4, A_{\mathrm{ms}}=20$, $a=10, b^{\prime}=0.01, c^{\prime}=0.01$, and $d^{\prime}=0.7$. In panel C: $L=1 / 4, A_{\mathrm{ss}}=1, a=100, b^{\prime}=0.01, c^{\prime}=0.01$, and $d^{\prime}=17$. In panel $\mathrm{D}: L^{\prime}=4$, $A_{\mathrm{ss}}=1, a=10, b^{\prime}=0.01, c^{\prime}=0.01$, and $d^{\prime}=0.7$. (After Bindslev (2004), Fig. 2).

\subsubsection{Pseudo-homotropic Two-state Model (PHOTSM)}

A model similar to the extended two-state model for heterotropic interaction (ETCM) in Section 7.10.1, but now for homotropic behavior, co-operativity, has been put forward recently as a two-state two-site model or a 'two-state-dimer-model' (Franco et al. 2005; Franco et al. 2007a,b; Ehlert 2008). The former model, which is a fraction of the HOTSM, is capable of simulating reverse bell-shaped dose-response relations without an overshoot (compare Fig. 3 in Franco et al. (2005) and Fig. 7.27). Obviously, the 'two-state-dimer-model' and the two-state two-site model are covered by the HOTSM. See Table 7.3 for more models.

\subsection{Comparison between the ATSM and the HOTSM}

From a functional viewpoint, the ATSM overlap with the HOTSM. From the aspect of occupancy, the two models cover separate issues and are very different.

\subsubsection{Functional Studies}

The HOTSM can be said to be a special version of the ATSM. The ATSM is congruent with the HOTSM when doses are applied from a mixture made of an orthosteric ligand and a modulatory ligand (Figs. 7.21-7.26). As the concentration of ligands in the mixture is increased, a 
A

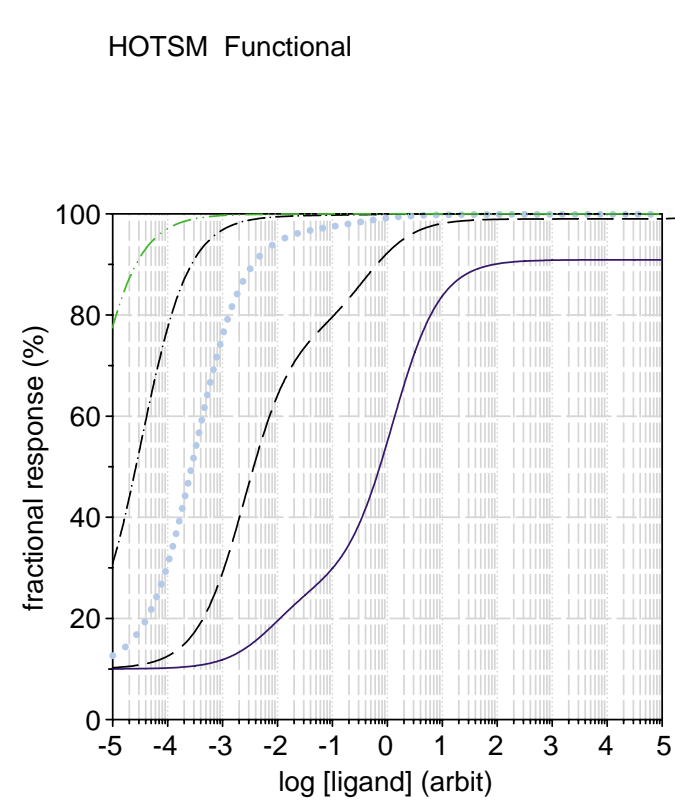

B

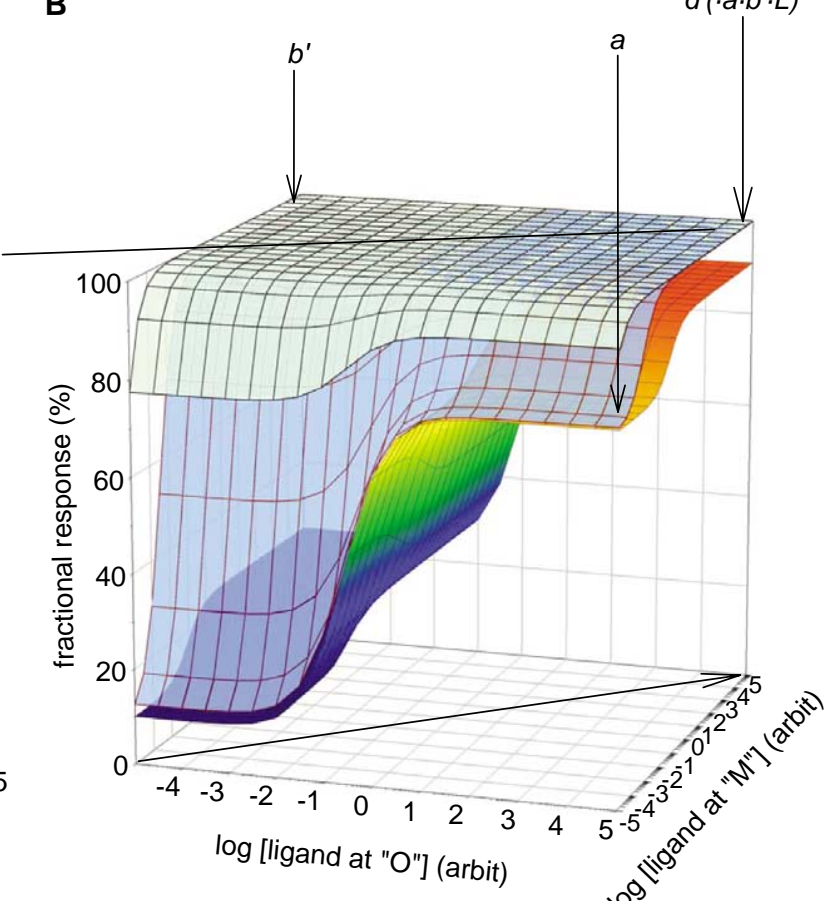

$b^{\prime}$ varied in 5 steps, $10^{-4}-10^{0}$

C

D
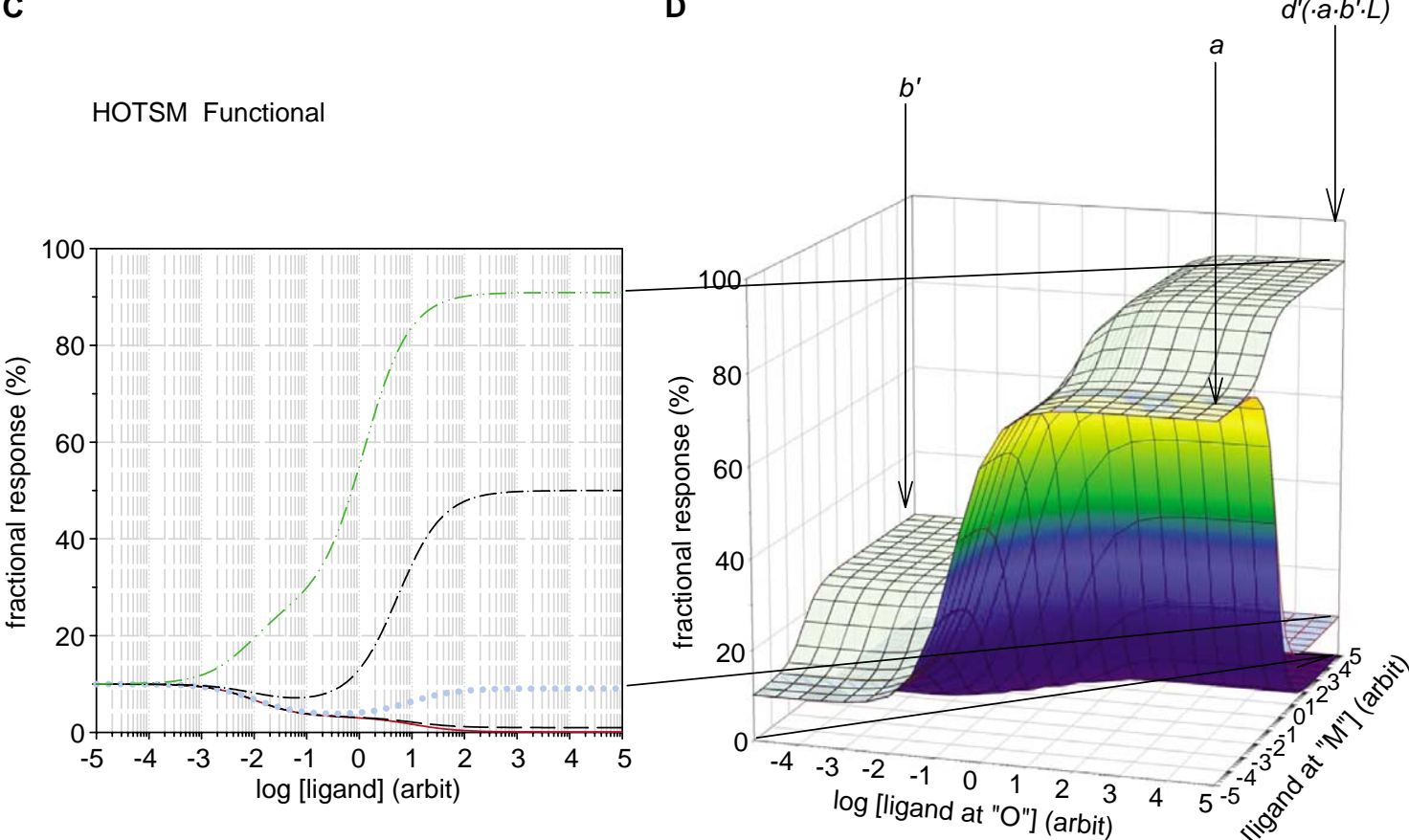

م)

Figure 7.28. An additional example of HOTSM in function, where $b^{\prime}$ vary. Parameters were $a=30, c^{\prime}=0.1, d^{\prime}=1, A_{\mathrm{ss}}=1$, $A_{\mathrm{ms}}=100$, and $L=1 / 9$. Parameters $b^{\prime}$ was varied as indicated in the panels. Unbroken line-curves represent the lowest $b^{\prime}$ value.

fixed proportion is kept between doses of the two ligands in the ATSM, just as for the HOTSM regime. Such an experiment with a primary ligand and a modulator in a fixed ratio will, in theory, follow the
HOTSM when corrections are made for possible differences between affinity constants $A_{\mathrm{mm}}$ and $A_{\mathrm{ms}}$, which in the model may simply be taken into account by multiplying either by a factor. Now the fixed ratio experiment 
has become a single-ligand study, and its analysis by the ATSM is identical to an analysis by the HOTSM. Aspects of the behavior of ATSM/HOTSM as system tools were presented by Bindslev (2004).

\subsubsection{Binding Studies}

The ATSM is a prime example for the simulation and analysis of dose-responses of occupancy in radio-ligand binding studies as affected by cold allosteric ligands, demonstrated by Hall (2000). In the HOTSM, application of a ligand that can bind to two sites simultaneously is different from the drug application described by Hall (compare Figs. 7.6-7.8 with Figs. 7.15-7.20).

To recapitulate, there is no feasible comparison between the ATSM and the HOTSM reaction schemes used in simulations for binding. The reaction scheme of ATSM can only bind ligands of interest for occupancy studies in a single site. Co-lateral binding is excluded. In the HOTSM, two ligands of interest can bind simultaneously in a co-lateral fashion by just adding one type of ligand. This clearly separates the two models from each other in binding studies.

\subsection{Evaluation of FP-TSM as an Analytical Tool}

\subsubsection{Use of the FP-TSM Tool is Easy?}

The FP-TSMs in Figs. 7.2 and 5.9 has 18 conformations and 17 independent system constants, and besides its genuine two-state isomerization, it involves two different ligands, an agonist or a substrate and a modulator, with mutual co-lateral binding. The possibility of co-lateral binding in FP-TSM differentiates it from the CQCM in Fig. 7.5.

Armed with some reasonable assumptions, the 17 constants can be reduced to around 12 .

Still, to analyze the FP-TSM with 12 independent system constants sounds horrendous, but I believe it is only a matter of time before this model with unliganded spontaneous activity will be employed in the analysis of ligand-receptor interactions. How can I trust such a development? Well, consider all the efforts being put into drug design and drug development based and performed on the modeled dynamics of steric coordinates in ligand binding sites, and hold this against the immense degrees of freedom in possible alignment for receptive systems and their cognate ligands at an Angstrom scale. The possibilities are legio and will keep scientists and support floating for years - not necessarily with any foretold causal solutions.
A

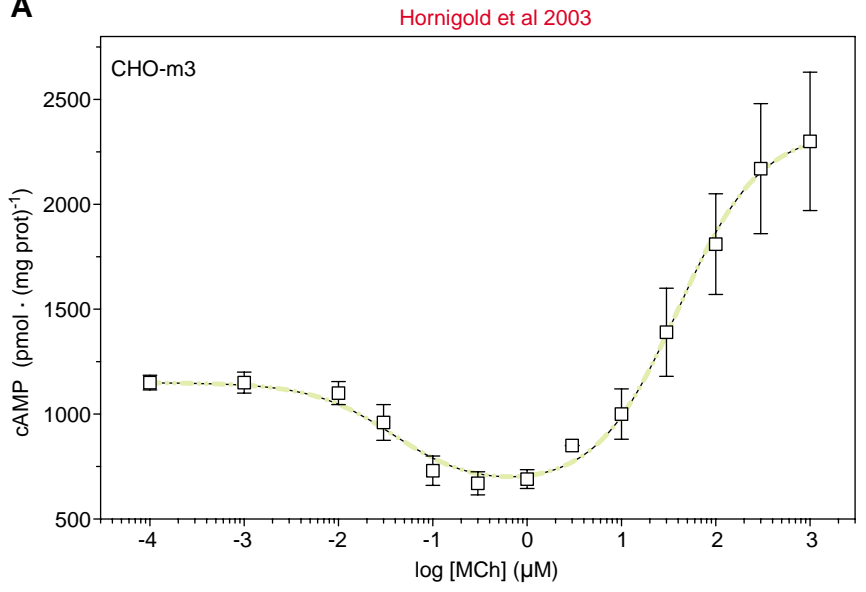

B

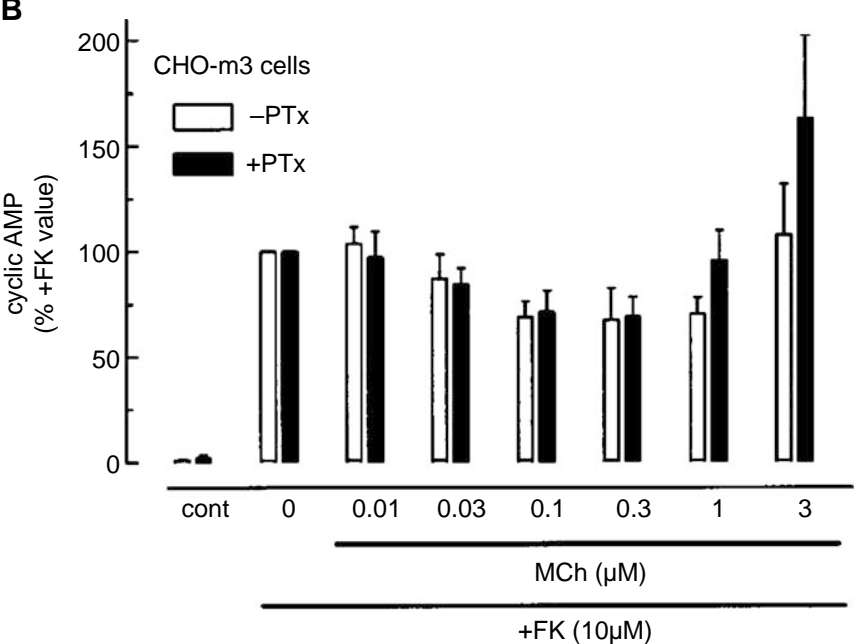

Figure 7.29. A fit of HOTSM to experimental data. (A) Doseresponse data of cAMP production in Chinese hamster ovary $(\mathrm{CHO})$ cells expressing muscarinic $\mathrm{m} 3$ receptor subtypes and a HOTSM curve fitted by adjusting parameters. Fitted parameters were $a=0.401, b^{\prime}=0.534, c^{\prime}=0.145, d^{\prime}=11.1, A_{\mathrm{ss}}=$ 0.142 , and $A_{\mathrm{ms}}=27.3$ with $L$ sat at $1 / 3$. Data taken from Hornigold et al. (2003). (B) Control and petussis toxin (PTX), effects on cAMP production in $\mathrm{CHO}$ cells expressing only $\mathrm{m} 3$ receptor subtypes. If any effect, PTX augments the concave bell-shaped dose-response relation. Copy of Fig. 5a in Hornigold et al. (2003) with permission.

\subsubsection{Structure-activity-relationship (SAR)}

The whole G protein-coupled receptor community eagerly awaits more details of the structural resolution of GPCRs, both for ligand binding sites and for the coupling between GPCRs and G proteins (Oldham et al. 2006; Kobilka \& Scherkler 2008), as well as the interaction between GPCRs - G proteins and other proteins (Ferguson 2007), and looks enviously to the advancements of nearly 40 years in structural resolution of enzyme function. As the structural secrets are being revealed for transporters as well (Toyoshima 2007), porter-people are alert and expectations are high. 
Meanwhile, even with recent promising breakthroughs in the structural resolution for receptors (Palechewski et al. 2000; Okada et al. 2002, 2004; Palechewski 2006; Cherezov et al. 2007) and for several different transporters (Gouaux \& MacKinnon 2005) as channels (Dutzler et al. 2002, 2003; MacKinnon et al. 2003, 2004; Unwin 2005; Jentsch et al. 2005a,b; Miller 2006; Ramjeesingh et al. 2006; Jasti et al. 2007), pumps (Toyoshima et al. 2000; Obara et al. 2005; Jensen et al. 2006), exchangers (Dutzler et al. 2002, 2003; Hunte et al. 2005; Jentsch et al. 2005a,b; Accardi et al. 2006; Miller 2006; Nicoll et al. 2006; Nguitragool et al. 2006), ABC-transporters (Yu et al. 2003; Dawson \& Locher 2006), and co-transporters (Yernool et al. 2004; Yamashita 2005; Indarte et al. 2007) leading the way to synthesis of new lead-compounds, dynamic possibilities in the binding pockets are still immense and overwhelming when it comes to the dynamic stereochemistry at a subatomic scale needed for the ultimate useful structure-activity-relationship (SAR).

\subsubsection{The FP-TSM in SAR Analysis}

In comparison with the efforts described in the preceding sections, to me it seems a modest task to develop the FP-TSM, which among other issues mechanistically may describe the reversal of auto-ant-agonism (reversal of negative auto-modulation or negative auto-intervention) by a classical competitive ant-agonist. An example of such reversal by a competitive ant-agonist has been observed for atropine at muscarinic receptors (Fig. 7.30A) (Winding \& Bindslev 1993), where atropine can reverse auto-inhibition induced by acetylcholine within one to two minutes, thus reactivating an autoinhibited acetylcholine response of a cell membrane displayed muscarinic receptor pool (Fig. 7.30B).

In developing a scheme for this kind of 'ant-agonism', the terminology of designators in FP-TSM such as 'competitive' inhibitor, 'non-competitive' inhibitor, 'interventor', and 'modulator' may start to mingle. Atropine is a competitive ant-agonist in the classical sense (Brimblecombe 1974, Chapter 1; Carrijo et al. 1977; Hulme et al. 1978), but atropine will also appear as a non-competitive inhibitor or an interventor in intervention models, or a modulatory ligand in the ATSM and the FP-TSM. Although atropine is a 'neutral' ant-agonist, as it does not in itself evoke or prevent any activity in the absence of agonists or inverse agonists, it may have the characteristics of all four types of ligands mentioned above. However, atropine does not qualify as an agomodulator, since it does not act on its own.

In the realm of $\mathrm{G}$ protein function, a start has been made with the implementation of a pre-FP-TSM (Downing et al. 2006). Furthermore, an alternative FP-TSM reaction scheme has been invoked for GABA-A channels
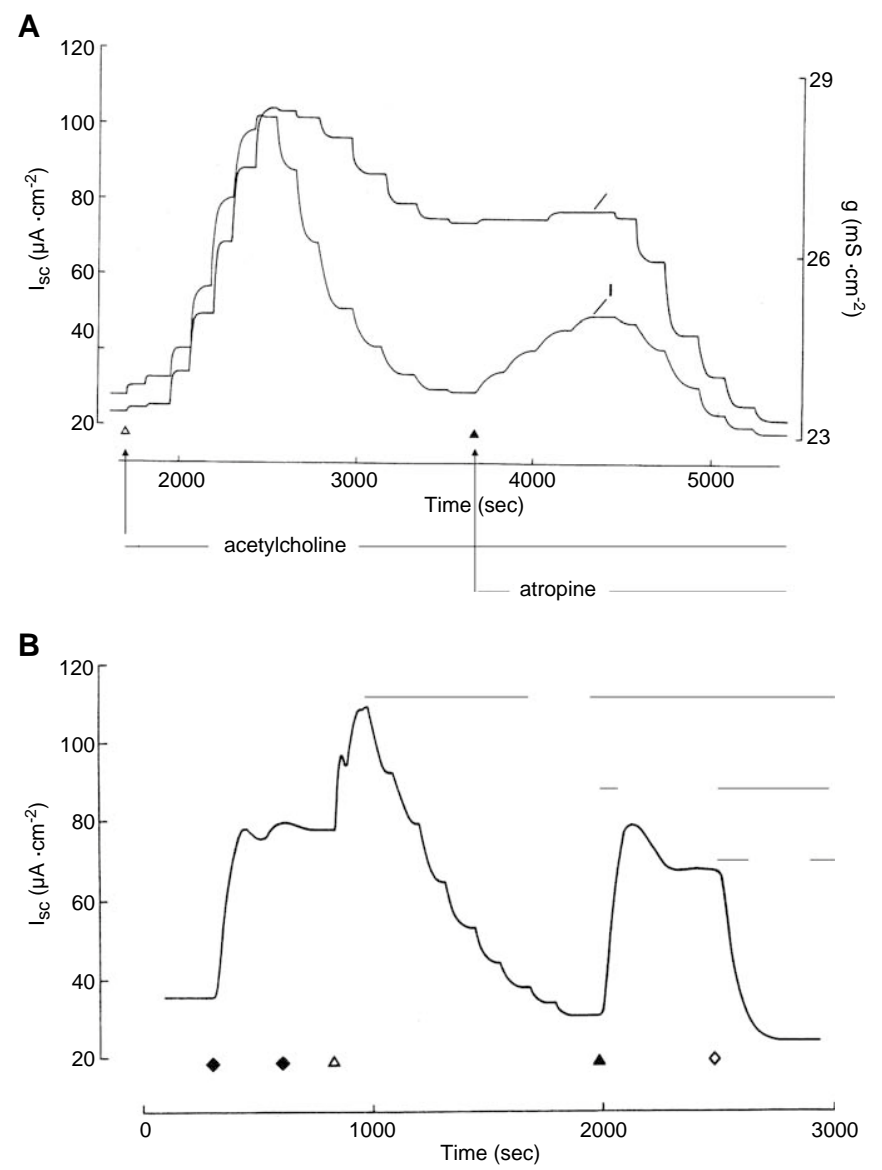

Figure 7.30. Auto-inhibition by acetylcholine of tracheal secretion reactivated by atropine. (A) Low dose activation and high dose auto-inhibition of secretion (Isc) in hen tracheal epithelium by acetylcholine $(A C h) \triangle$, followed by a reactivation-inhibition by atropine $\mathbf{A}$. (B) Inhibition of secretion (Isc) by acetylcholine $\Delta$ at high doses, total $512 \mu \mathrm{M}$, and fast reactivation, $\mathrm{t}^{1} \frac{2}{2} \approx 60 \mathrm{~s}$, by a single dose of atropine $\boldsymbol{\Delta}, 100 \mu \mathrm{M} . \diamond=$ bumetanide at $10 \mu \mathrm{M}$. Modified from Winding \& Bindslev (1993, Figs. 1 and 3) with permission.

(Rusch et al. 2004). The future looks bright for the FP-TSM as an analytical tool.

\subsection{The Optimal-fixed-concentration- ratio (OFCOR)}

\subsubsection{Inhibition of Negative Auto-modification}

In systems with negative auto-intervention or negative auto-modulation in the form of bell-shaped doseresponses at high agonist concentrations, this inhibition by the agonist itself may be counteracted by introducing a modulator or even a competitive ant-agonist, both with access to more than one agonist binding site.

As described above, a reactivation of such a negative auto-modulatory response was found for the ligand-pair 

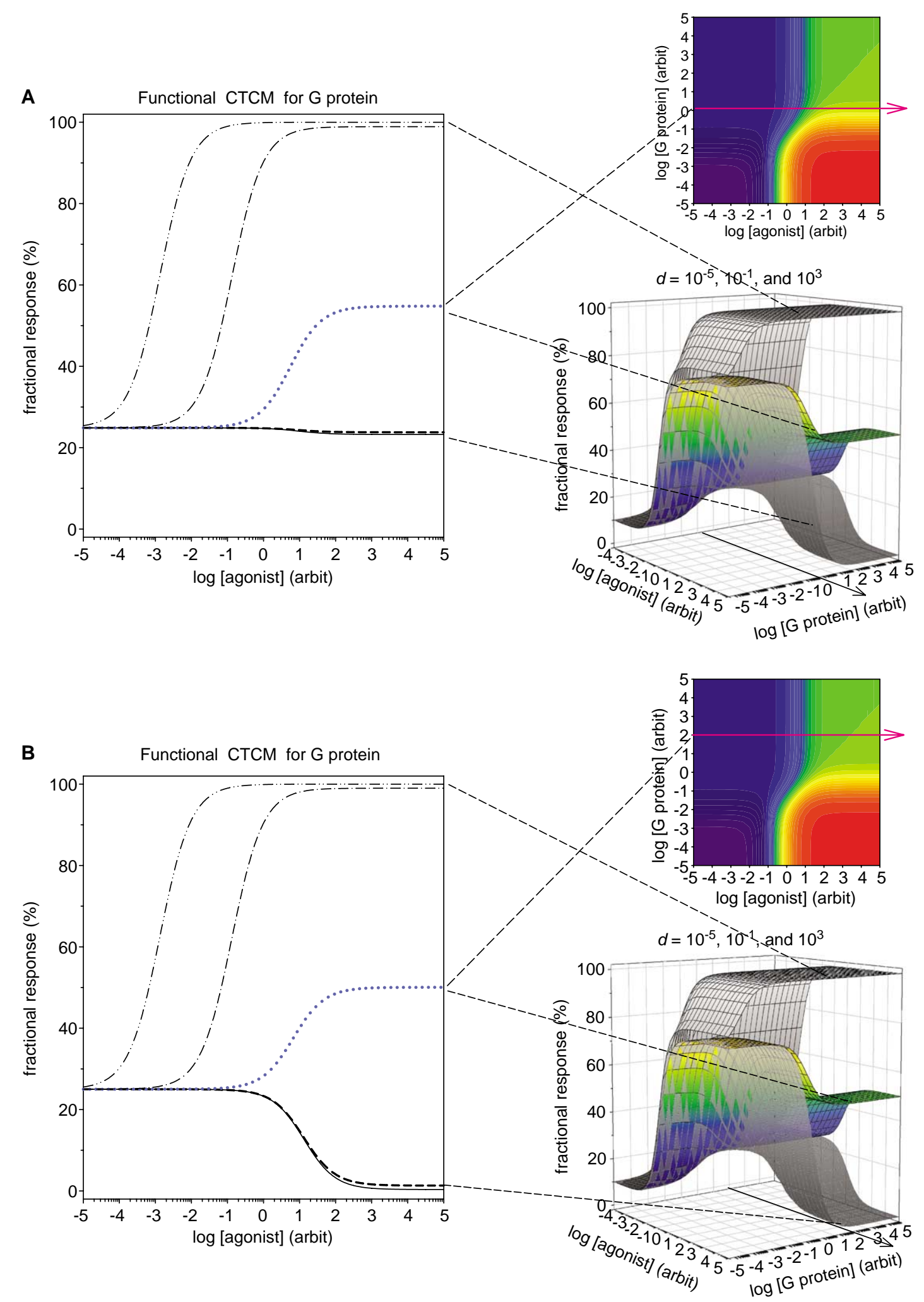

Figure 7.31. Examples of dose-response relation in the cubic ternary-complex model for G proteins (CTCM). Parameter $d$ was varied in five steps. Values for parameters were as follows: $a=30, b=3, c=0.1$, Parameter $d$ was varied in five steps between $10^{-5}$ $(-)$ and $10^{3}(-\cdot-)$ in both panels $\mathrm{A}$ and $\mathrm{B}$, while the other system constants were as follows: $a=30, b=3, c^{\prime}=0.1, L=1 / 9$, $A_{\mathrm{ss}}=1$, and $A_{\mathrm{ms}}=100$. The concentration of the $\mathrm{G}$ protein as modulator, [G protein] at different expression levels, was fixed at 1 (arbitrary units) in A and at 100 (arbitrary units) in B as indicated by an arrow in the 3-D panels. The related contour plots are for $d=10^{-1}$ in both panels with arrows indicating the G protein concentration. Compare the 3-D plots here with a 3-D plot in Fig. 7.23. 


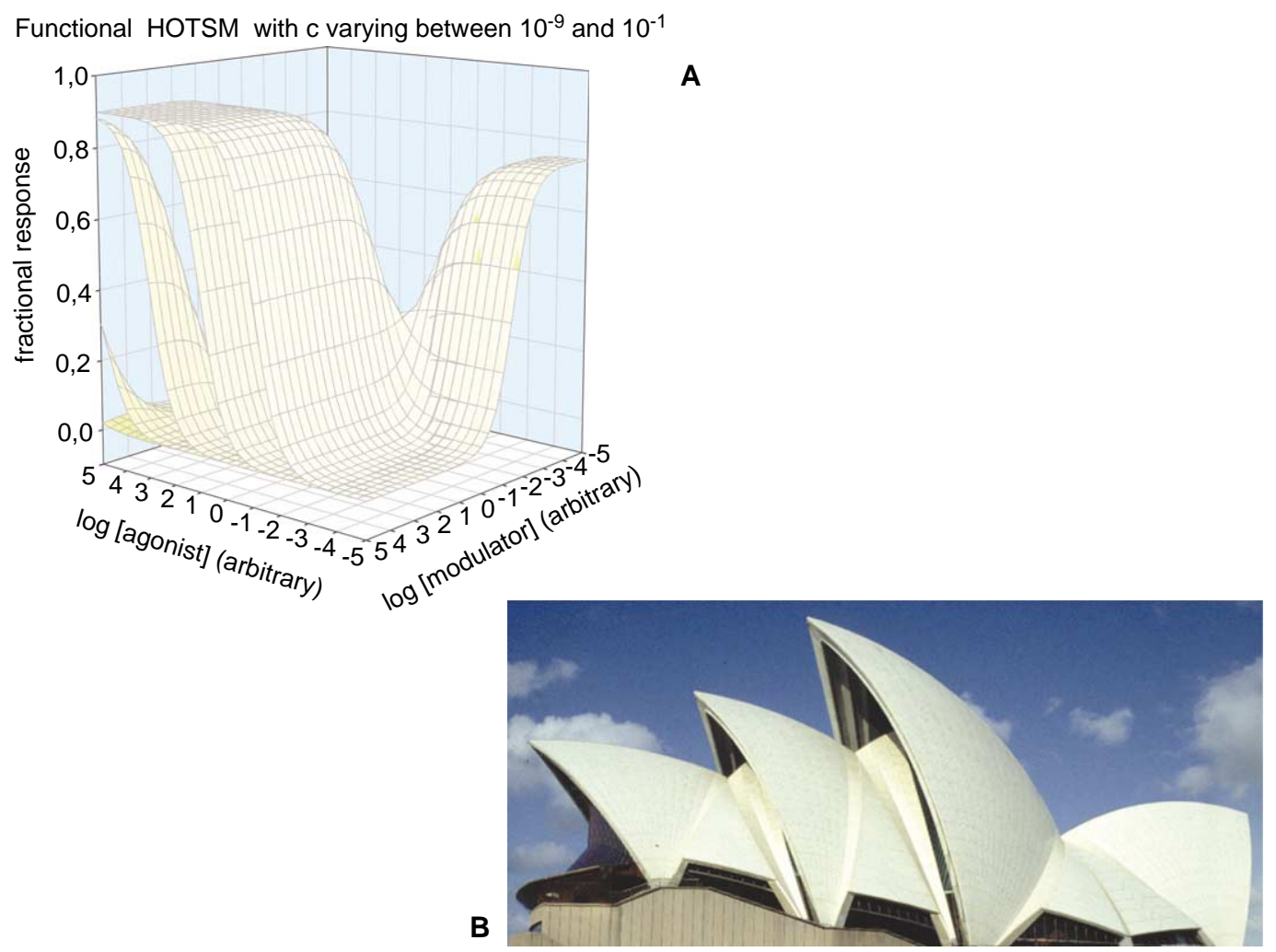

Figure 7.32. All kinds of models and mock-ups are possible, but not necessarily correct. (A) Parameter values for the ATSM $a=30, b=3, c^{\prime}=$ vary in five steps by a factor 100 between $10^{-9}$ and $10^{-1}, L=1 / 9, A_{\mathrm{ss}}=1$, and $A_{\mathrm{ms}}=100$. (B) Can the allosteric two-state model describe Sidney's Opera house with the right architect?

acetylcholine/atropine in tracheal secretion (Fig. 7.30) (Winding \& Bindslev 1993). There are several possible molecular explanations for this reactivation mechanism by atropine, one being competition at a secondary binding site with access for both inhibitory primary ligand (auto-inhibitory agonist acetylcholine) and a modulator (atropine). The FP-TSM is suited for such an analysis (see below).

Internalization of receptors as an explanation for the auto-inhibition in this system seems to be ruled out by the speedy reactivation within a minute or two by atropine (Fig. 7.30). Desensitization, converted to an attenuating dose-response, is another obvious possibility for the auto-inhibition by acetylcholine. Meanwhile, in this scenario, we have to explain how atropine behaves as a 'competitive ant-agonist' and how it resensitizes the auto-inhibition by acetylcholine in a time dependent manner.

As mentioned, the reactivation behavior can be addressed by a FP-OSM, which includes co-lateral binding at either site (Winding \& Bindslev 1993). Ligand-relief of negative auto-modulation may of course also be simulated with the FP-TSM, which will be useful in case systems moreover comprise spontaneous activity.

\subsubsection{Finding OFCORs}

In case agonist concentrations in clinical treatment elicit auto-inhibition, thereby tempting a higher dose application with possible adverse effects, such auto-inhibition may be prevented by interventors, modulators, or 'competitive ant-agonists' with a 'positive' effect elicited from a secondary site. It will be possible to avoid adverse effects due to high concentrations of primary ligands that also elicit auto-inhibition by use of a single mixture of 'agonist' and 'auto-modulator' with an optimal concentration ratio of the two drugs. Of course, using such mixtures in dose-response experiments with optimum is the same as using OFCORs. The challenge is, of course, to find the right OFCORs for a given individual; his of hers drug-mixture.

Determining an optimal concentration ratio for experiments and treatments may be obtained by assaying the dose-response relations for and between the agonist and the modulator in several combinations of the two drugs. As an example, the OFCOR for a mixture was determined and verified in the study by Winding and Bindslev (1993) using a version of the FP-OSM. To date, a similar analysis using the FP-TSM is not in the literature. 


\subsection{More on Cubic Models and GPCR-related Models}

\subsubsection{The Extended Ternary Complex Model (ETCM)}

In order to describe the dose-response relations obtained for GPCRs with constitutive activity and varied G protein levels, Samama and co-workers developed a model combining the original ternary-complex model equal to an intervention model (Chapter 2) for $G$ proteins, with the CTSM (Chapter 5), in the simplest possible scheme (Samama et al. 1993; Lefkowitz et al. 1993). The ETCM for GPCRs is shown in Fig. 7.4B. Development of the ETCM was a stage on the road to the thermodynamically full model with modulators, including $\mathrm{G}$ proteins, and two-states combined in the CTCM (Fig. 7.3, Table 7.3) (Weiss et al. 1996a).

\subsubsection{ATSM and CTCM and CQCM}

When occupancy is considered, the ATSM (Hall 2000) for allosteric modulators is in principle identical to the CTCM for 'modulation' of GPCRs by G proteins (Fig. 7.1A and 7.4A) (Weiss et al. 1996a,b). Examples of occupancy that cover both models are shown in Fig. 7.6.

The parallel occupancy between ATSM and CTCM is true also in simulation of experiments where the number of total receptors differs between radio-ligand agonist and radio-ligand ant-agonist data due to $G$ protein-induced affinity changes (Kenakin 1997; Baker \& Hill 2007; Ferguson 2007). Here, adjustments of parameter $A_{\mathrm{mm}}$ come in to play.

On the other hand, in functional studies the two tools, ATSM and CTCM, behave somewhat differently (Weiss et al. 1996a,c; Hall 2000). An illustration of this can be acknowledged by comparing the surface plot in 3-D panels of Figs. 7.23 and 7.31, where both concentrations of an agonist and the $\mathrm{G}$ protein as modulators are varied, as independent variables yielding the 3-D surface plots. Following the arrows in the 3-D concentration planes in Fig. $7.31 \mathrm{~A}+\mathrm{B}$ at an arbitrary concentration (expression level) of $\mathrm{G}$ protein exemplified with ' 1 ' in Fig. 7.31A or at ' 100 ' in Fig. 7.31B results in 2-D plots in $A$ and $B$ of Fig. 7.31, and should not be compared with the 2-D plots in Fig. 7.23 which are for the HOTSM. However, with simple modifications, the ATSM may as well be a model for GPCRs.

Experiments with varying expressions (concentrations) of $\mathrm{G}$ proteins are less common but have been performed, modeled, and discussed (Chidiac et al. 1996; Kenakin 1997; Tucek et al. 2001, 2002; Kukkonen et al. 2001; Kukkonen 2004b).

With the CTCM it is possible to study GPCR doseresponse relations with varying concentrations of the agonist as the independent variable and $\mathrm{G}$ proteins at different levels. The CTCM for G protein coupled to activated receptors, even in the absence of agonists GR*, obviously has possibilities of simulating spontaneous activity and inverse agonism, but in relation to non-G protein regulators, the CTCM is clearly unsatisfactory. Therefore, models such as the CTCM with an added third ligand are in demand and have been suggested as the CQCM (Christopoulos et al. 1998; Christopoulos \& Kenakin 2002; May et al. 2004), but, to date, are not fully developed.

An amputated version of the CQCM with two G proteins, a GPCR, and an agonist yielding quaternary complexes $^{3}$ but without an isomerization between two states, was implemented by Kukkonen et al. (2001) and Kukkonen (2004a), introducing a factor $F$ controlling the level of receptors chelated by $\mathrm{G}$ proteins. Other simpler models, also without isomerization, including variations in $\mathrm{G}$ protein expression and a factor controlling maximal inhibition (MI) and maximal stimulation (MS) have been suggested (Tucek et al. 2002).

Needless to say, the CQCM illustrated in Fig. 7.5 should be developed sooner rather than later.

\subsubsection{Models of 'Ligand' Numbers Matching 'Receptor' Numbers}

In the latest development of models addressing the reverse bell-shaped dose-response relations for GPCRs, 'dual effects' (Jones et al. 1991; Kashihara et al. 1992; Migeon \& Nathanson 1994; Vogel et al. 1995; Jakubik et al. 1996), two parallel approaches have been advocated for a limited $\mathrm{G}$ protein activation (Tucek et al. 2001, 2002; Nasman et al. 2001; Kukkonen et al. 2001; Kukkonen 2004a). Tucek et al. (2002) and especially Kukkonen (2004a) give elegant descriptions of these models' development and simulation capabilities; notwithstanding, arithmetical treatment in these two sources mutually overlook each others similarities in analysis.

Following Kukkonen, three principal models are invoked. The shuttling model, earlier described as a mobile receptor model (Jacobs \& Cuatrecasas 1976) or collision coupling model (Tolkovsky \& Levitzki 1978, 1981), the complexing model (Chidiac 1998), and the precoupled model (Neubig 1994; Kukkonen 2004a). The latter comes in two versions; a precoupled-dependent and a precoupled-independent model. Somewhat different models on precoupling were also presented by Jacobs and Cuatrecassas (1976) and by Tolkovsky and Levitzki (1978).

\footnotetext{
${ }^{3}$ Quaternary complexation results in cubic reaction schemes. Meanwhile, to keep model terminology consistent, an acronym for this quaternary complex model is QCM not CQCM.
} 
In the recent models, the variation in a limited number of single type $\mathrm{G}$ protein that can be activated, is accomplished by a coefficient resembling the intrinsic activity factor of Ariens (1954) both in the Czech and the Swedish approach (Tucek et al. 2002; Kukkonen 2004a,b). Thus, the limited number of active $G$ proteins and the ratio between $G_{s}$ and $G_{i}$ proteins result in simulations that can match the observed reverse bell-shaped dose-responses. The simulated 'spontaneous' activity at $100 \%$ was obtained experimentally by forskolin stimulation.

\subsubsection{Models with More than One-state for GPCRs}

Model approaches to cover multi-states in GPCRs were recently presented, Table 7.3. For instance, one by Leff and co-workers (Leff et al. 1997; Scaramellini \& Leff 1998, 2002), another by Pin and co-workers (Parmentier et al. 2002) covering both two-states of a binding domain and two-state of an effector domain (see also Rovira et al. 2008), and a third by Giraldo (2004). The models by Leff et al. and Giraldo both play on the theme of combining two cTSM for the sake of simulating GPCR functional behavior. These models operate with three or more states for the unliganded receptive unit, and may be elaborated further into multi-state models - a project left for the reader with some help from a recently published four-state model (Ehlert \& Griffin 2008). A double-cubic model encompassing two states for both the receptor and its effector was already suggested in the 1970s (de Haen 1976).

\subsubsection{Models for Binding with Dimerization as Isomerization}

Dimerization of receptive units may lead to an allosteric interaction between binding sites in the dimer complex, thus resembling the switch between a reactive and an active state for a double-sited receptor. Recently, Durroux (2005) has suggested different and attractive models for such dimerization with 'allosteric' interaction, including cubic reaction schemes and four-pane two-state-like models. It will be interesting to follow development of Durroux' models as they appear most relevant for a description and possible simulation of activity in dimerized GPCRs.

\subsection{Use of Modulators in Therapy}

\subsubsection{Benefits of Modulators Compared with Orthosteric Ligands}

An early example of modulators used in therapy with great success and few side-effects is the treatment of anxiety, depression, epilepsy, and sleeplessness with benzodiazepines, which started more than forty years ago. Nevertheless, the clinical aspect of the use of modulators or allosteric compounds is a relatively new issue, and has been advocated since the mid 1990s (Proska \& Tucek 1994; Tucek \& Proska 1995, Birdsall et al. 1995; Lazareno \& Birdsall 1995). Some of the advantages of using modulator compounds instead of the natural endogenous agonist or artificial agonists with receptor subtype specificity can be listed as follows (Birdsall \& Lazareno 2005):

(a) As the function of a modulator to a great extent is dependent on the presence and action of the physiological signal molecule, it is likely to mimic normal biological effects. The modulator is playing on the activity of an endogenous primary ligand.

(b) The enhancement of natural agonist activation or inhibition by modulators is less likely to reach adverse effects as for agonist drugs, even at high concentrations of the modulator compound, and therefore prolonged stimulation with high modulator concentrations may be induced without adverse effects as often seen with high concentrations of developed agonist drugs. There is 'ceiling' and 'tune down'.

Besides reuptake and decay inhibitors, some of the expected therapeutic advantages with use of modulatory drugs may also be obtained with partial and partial inverse agonists (Fisas et al. 2006).

In clinical work with modulators, it is the functional aspect that is in focus. The picture of modulatory drug therapy has developed rapidly within the last few years for several receptor subfamilies, and many developed drugs are in various phases of evaluation for introduction to the clinics (Table 7.2) (Gao \& Jacobson 2006). Examples of biotech firms that develop such drugs are the NPS Pharmaceutical Company with a modulator for Ca sensors, and Xytis with a glycine site enhancer for NMDA channels under development (see homepages of NPS Pharmaceuticals Inc. and Xytis Inc.).

Of course, examples may appear where modulators are less beneficial than, for instance, weak partial agonists (Metha \& Ticku 1999).

(c) Highly selective modulators for specific receptor subtypes are more likely to be found than subtype specific agonists, primary ligands, due to the evolutionary conservation of the primary binding sites for endogenous ligands (Birdsall et al. 1999; Spalding et al. 2002; Lanzafame 
et al. 2006; Langmead et al. 2006; Langmead \& Christopoulos 2006). This is especially true for the muscarinic bindings sites. Actually, development of allosteric modulator drugs is an exploding field involving several effector molecules as indicated by recent publications listed in Table 7.2.

The receptor subtype selectivity by modulators, when judged to be based mainly on the conformational changes by induced fits or stabilization rather than on (high) affinity, is a kind of conformational change dubbed 'absolute subtype selectivity' (Lazareno et al. 2004).

During evolution, modulation may have been adapted for several secondary ligands with a more loose structure of their secondary binding sites. It has been difficult to find selectivity much above a factor 10 between primary ligands developed for muscarinic receptor subtypes (Alexander et al. 2006), whereas it seems likely that much higher selectivity may be found for modulators at the various muscarinic receptor subtypes (Birdsall \& Lazareno 2005; Langmead et al. 2006). Other examples of allosteric modulators are for adenosine (Jacobson \& Gao 2006; Childers et al. 2006), metabotropic glutamate (Kew 2004; Ritzen 2005; Shipe et al. 2005; Vauquelin \& Liefde 2005; Vieira et al. 2005; Pin et al. 2005; Foster \& Kemp 2006), and dopamine (Schetz 2005) receptor subtypes. Examples for ligand-gated ion channels (LGICs) are reviewed by Hogg et al. (2005) with benzodiazepines as the earliest prototype of modulators with allosteric responses at the GABA-A receptor (Olsen et al. 2004). For transporters, see, e.g., Maki \& Dey (2006), Ghosh et al. (2006) and Borst et al. (2006), and other examples mentioned in sub-chapter 7.9 and Table 7.2.

Protocols and screens for new allosteric modulators for GPCRs are evolving (Langmead 2007; Raddatz et al. 2007; May et al. 2007; Avlani et al. 2007). Moreover, analytical tools are created to solve complex behavior in GPCR signaling (Fig. 7.31; Ehlert \& Griffin 2008).

\subsubsection{Summary of Modulator Benefits}

A summary of the advantages of developed modulators compared with drug agonists is: (1) they mimic the function of endogenous primary ligands, (2) they are less likely to elicit adverse effects, and (3) they have possible greater receptor subtype selectivity than agonists and other molecules operating at a primary binding sites with its evolutionary conserved geometry.

Due to this rapidly expanding and potentially huge market for allosteric drugs, in the future, on theoretical grounds, it will be necessary to firmly implicate the ATSM and HOTSM for analysis of (allosteric) modulators in basic as well as applied approaches.

\section{REFERENCES}

Accardi A, Walden M, Nguitragool W, Jayaram H, Williams C \& Miller C. Separate ion pathways in a $\mathrm{Cl}-/ \mathrm{H}+$ exchanger. J Gen Physiol 126: 563-570, 2005.

Accardi A, Lobet S, Williams C, Miller C \& Dutzler R. Synergism between halide binding and proton transport in a CLC-type exchanger. J Mol Biol 362: 691-699, 2006.

Accomazzo MR, Cattaneo S, Nicosia S \& Rovati GE. Bell-shaped curves for prostaglandin-induced modulation of adenylate cyclase: two mutually opposing effects. Eur J Pharmacol 454: 107-114, 2002.

Alexander SPH, Mathie A \& Peters JA. Guide to receptors and channels. Br J Pharmacol 147: S8, 2006.

Ariëns EJ \& de Groot WM. Affinity and intrinsic-activity in the theory of competitive inhibition. Part III. Homologous decamehoniumderivatives and succinyl-choline-esters. Arch Int Pharmacodyn 99: 193-205, 1954.

Avlani VA, Gregory KJ, Morton CJ, Parker MW, Sexton PM \& Christopoulos A. Critical role for the second extracellular loop in the binding of both orthosteric and allosteric G proteincoupled receptor ligands. I Biol Chem 282: 25677-25686, 2007.

Baggio LL \& Drucker DJ. Biology of incretins: GLP-1 and GIP. Gastroenterology 132: 2131-2157, 2007.

Baker JG \& Hill SJ. A comparison of the antagonist affinities for the Gi- and Gs-coupled states of the human adenosine A1-receptor. J Pharmacol Exp Ther 320: 218-228, 2007.

Baraldi PG, Iaconinoto MA, Moorman AR, Carrion MD, Cara CL, Preti D, Lopez OC, Fruttarolo F, Tabrizi MA \& Romagnoli R. Allosteric enhancers for A1 adenosine receptor. Mini Rev Med Chem 7: 559-569, 2007.

Benneyworth MA, Xiang Z, Smith RL, Garcia EE, Conn PJ \& SandersBush E. A selective positive allosteric modulator of metabotropic glutamate receptor subtype 2 blocks a hallucinogenic drug model of psychosis. Mol Pharmacol 72: 477-484, 2007.

Bindslev N. A homotropic two-state model and auto-antagonism. BMC Pharmacol 4: 11, 2004.

Birdsall NJ \& Lazareno S. Allosterism at muscarinic receptors: ligands and mechanisms. Mini Rev Med Chem 5: 523-543, 2005.

Birdsall NJ, Cohen F, Lazareno S \& Matsui H. Allosteric regulation of G-protein-linked receptors. Biochem Soc Trans 23: 108-111, 1995.

Birdsall NJ, Farries T, Gharagozloo P, Kobayashi S, Lazareno S \& Sugimoto M. Subtype-selective positive cooperative interactions between brucine analogs and acetylcholine at muscarinic receptors: functional studies. Mol Pharmacol 55: 778-786, 1999.

Borst P, Zelcer N \& van de Wetering K. MRP2 and 3 in health and disease. Cancer Lett 234: 51-61, 2006.

Botts J \& Drain GF. An illustration of a kinetic analysis: the myosin BATP-EDTA system. The committee of muscle chemistry of Japan. Conference on the chemistry of muscular contraction (1957), Tokyo, Igaku Shoin. 1958.

Brauner-Osborne H, Wellendorph P \& Jensen AA. Structure, pharmacology and therapeutic prospects of family C G-protein coupled receptors. Curr Drug Targets 8: 169-184, 2007.

Brimblecombe RW. Drug Action on Cholinergic Systems. London: The Macmillan Press Ltd., 1974.

Bronnikov GE, Zhang SJ, Cannon B \& Nedergaard J. A dual component analysis explains the distinctive kinetics of cAMP accumulation in brown adipocytes. J Biol Chem 274: 37770-37780, 1999.

Bruheim S, Krobert KA, Andressen KW \& Levy FO. Unaltered agonist potency upon inducible 5-HT7(a) but not 5-HT4(b) receptor expression indicates agonist-independent association of 5-HT7 (a) receptor and Gs. Receptors Channels 9: 107-116, 2003.

Buck M, Xu W \& Rosen MK. A two-state allosteric model for autoinhibition rationalizes WASP signal integration and targeting. J Mol Biol 338: 271-285, 2004. 
Burzomato V, Beato M, Groot-Kormelink PJ, Colquhoun D \& Sivilotti LG. Single-channel behavior of heteromeric alphalbeta glycine receptors: an attempt to detect a conformational change before the channel opens. J Neurosci 24: 10924-10940, 2004.

Campo-Soria C, Chang Y \& Weiss DS. Mechanism of action of benzodiazepines on GABAA receptors. Br J Pharmacol 148: 984 990, 2006.

Carrijo JB, Antonio A \& Rocha e Silva M. On the nature of the antagonism atropine-acetylcholine on the guinea pig heart. Acta Physiol Lat Am 27: 207-214, 1977.

Casadó V, Cortés A, Ciruela F, Mallol J, Ferré S, Lluis C, Canela EI \& Franco R. Old and new ways to calculate the affinity of agonists and antagonists interacting with G-protein-coupled monomeric and dimeric receptors: the receptor-dimer cooperativity index. Pharmacol Ther 116: 343-354, 2007.

Chang Y \& Weiss DS. Allosteric activation mechanism of the alphalbeta2gamma2 gamma-aminobutyric acid type A receptor revealed by mutation of the conserved M2 leucine. Biophys $J 77$ : 2542-2551, 1999.

Chen Y, Nong Y, Goudet C, Hemstapat K, de Paulis T, Pin JP, \& Conn PJ. Interaction of novel positive allosteric modulators of metabotropic glutamate receptor 5 with the negative allosteric antagonist site is required for potentiation of receptor responses. $\mathrm{Mol}$ Pharmacol 71: 1389-1398, 2007.

Cherezov V, Rosenbaum DM, Hanson MA, Rasmussen SG, Thian FS, Kobilka TS, Choi HJ, Kuhn P, Weis WI, Kobilka BK \& Stevens RC. High-resolution crystal structure of an engineered human beta2adrenergic G protein-coupled receptor. Science 318: 1258-1265, 2007.

Chidiac P. Rethinking receptor-G protein-effector interactions. Biochem Pharmacol 55: 549-556, 1998.

Chidiac P, Nouet S \& Bouvier M. Agonist-induced modulation of inverse agonist efficacy at the beta 2-adrenergic receptor. $\mathrm{Mol}$ Pharmacol 50: 662-669, 1996.

Childers SR, Li X, Xiao R \& Eisenach JC. Allosteric modulation of adenosine Al receptor coupling to G-proteins in brain. $J$ Neurochem 93: 715-723, 2006.

Christopoulos A \& El-Fakahany EE. Qualitative and quantitative assessment of relative agonist efficacy. Biochem Pharmacol 58: 735-748, 1999.

Christopoulos A \& Kenakin T. G protein coupled receptor allosterism and complexing. Pharmacol Rev 54: 323-374, 2002.

Christopoulos A, Lanzafame A \& Mitchelson F. Allosteric interactions at muscarinic cholinoceptors. Clin Exp Pharmacol Physiol 25: 185194, 1998.

Christopoulos A, Grant MK \& El-Fakahany EE. Transducer abstraction: a novel approach to the detection of partial agonist efficacy in radioligand binding studies. J Pharmacol Toxicol Methods 43: 55-67, 2000.

Christopoulos A, Grant MK, Ayoubzadeh N, Kim ON, Sauerberg P, Jeppesen L \& El-Fakahany EE. Synthesis and pharmacological evaluation of dimeric muscarinic acetylcholine receptor agonists. J Pharmacol Exp Ther 298: 1260-1268, 2001.

Colquhoun D. Agonist-activated ion channels. Br J Pharmacol 147: S17S26, 2006.

Cornish-Bowden A. Fundamentals of Enzyme Kinetics, 2nd ed. London: Portland Press, 1995.

Cornish-Bowden A. Fundamentals of Enzyme Kinetics, 3rd ed. Colchester: Portland Press Ltd., 2004.

Corsi M, Fina P \& Trist DG. Co-agonism in drug-receptor interaction: illustrated by the NMDA receptors. Trends Pharmacol Sci 17: 220222, 1996.

Costa T, Ogino Y, Munson PJ, Onaran HO \& Rodbard D. Drug efficacy at guanine nucleotide-binding regulatory protein-linked receptors: thermodynamic interpretation of negative antagonism and of receptor activity in the absence of ligand. Mol Pharmacol 41: 549-560, 1992.

Davila DF, Donis JH, Davila LA, Odreman WA, de Bellabarba GA \& Villarroel V. Anti-muscarinic autoantibodies and vagal modulation in Chagas disease: positive allosteric modulators vs desensitization and downregulation of M2 cardiac acetylcholine receptors. Int J Cardiol 123: 328-329, 2008.

Dawson RJP \& Locher KP. Structure of a bacterial multidrug ABC transporter. Nature 443: 180-185, 2006.

De Haën C. The nonstoichiometric floating receptor model for hormone sensitive adenylyl cyclase. J Theor Biol 58: 383-400, 1976.

De Lean A, Stadel JM \& Lefkowitz RJ. A ternary complex model explains the agonist specific binding properties of the adenylate cyclase coupled beta-adrenergic receptor. J Biol Chem 255: 71087117,1980 .

Dingledine R, Kleckner NW \& McBain CJ. The glycine coagonist site of the NMDA receptor. Adv Exp Med Biol 268: 17-22, 1990

Downing SS, Lee YT, Farb DH \& Gibbs TT. Benzodiazepine modulation of partial agonist efficacy and spontaneously active GABA(A) receptors supports an allosteric model of modulation. $\mathrm{Br} J$ Pharmacol 145: 894-906, 2005.

Dupre ML, Broyles JM \& Mihic SJ. Effects of a mutation in the TM2TM3 linker region of the glycine receptor alphal subunit on gating and allosteric modulation. Brain Res 1152C: 1-9, 2007.

Durroux T. Principles: a model for the allosteric interactions between ligand binding sites within a dimeric GPCR. Trends Pharmacol Sci 26: 376-384, 2005.

Dutzler R, Campbell EB, Chait BT \& MacKinnon R. X-ray structure of a $\mathrm{ClC}$ chloride channel at 3.0 A reveals the molecular basis of anion selectivity. Nature 415: 287-294, 2002.

Dutzler R, Campbell EB \& MacKinnon R. Gating the selectivity filter in ClC chloride channels. Science 300: 108-112, 2003.

Ehlert FJ. Estimation of the affinities of allosteric ligands using radioligand binding and pharmacological null methods. Mol Pharmacol 33: 187-194, 1988.

Ehlert FJ. The ternary complex model. In: Biomedical Application of Computer Modeling, 1st ed., edited by Christopoulos A. Boca Raton: CRC Press, 2001.

Ehlert FJ. Analysis of allosterism in functional assays. J Pharmacol Exp Ther 315: 740-754, 2005 .

Ehlert FJ. On the analysis of ligand-directed signaling at G proteincoupled receptors. Naunyn Schmiedebergs Arch Pharmacol Feb 6; [Epub ahead of print]: 2008.

Ehlert FJ \& Rathbun BE. Signaling through the muscarinic receptoradenylate cyclase system of the heart is buffered against GTP over a range of concentrations. Mol Pharmacol 38: 148-158, 1990.

Ehlert FJ \& Griffin MT. Two-state Models and the Analysis of the Allosteric Effect of Gallamine at the M2 Muscarinic Receptor. J Pharmacol Exp Ther Feb 27; [Epub ahead of print]: 2008.

Ferguson SS. Phosphorylation-independent attenuation of GPCR signalling. Trends Pharmacol Sci 28: 173-179, 2007.

Finch EA, Turner TJ \& Goldin SM. Calcium as a coagonist of inositol 1,4,5-trisphosphate-induced calcium release. Science 252: 443-446, 1991.

Fisas A, Codony X, Romero G, Dordal A, Giraldo J, Merce R, Holenz J, Heal D, Buschmann H \& Pauwels PJ. Chronic 5-HT(6) receptor modulation by E- 6837 induces hypophagia and sustained weight loss in diet-induced obese rats. Br J Pharmacol 148: 973-983, 2006.

Fitzsimons CP, Monczor F, Fernandez N, Shayo C \& Davio C. Mepyramine, a histamine $\mathrm{H} 1$ receptor inverse agonist, binds preferentially to a $\mathrm{G}$ protein-coupled form of the receptor and sequesters G protein. J Biol Chem 279: 34431-34439, 2004.

Fong TM. Mechanistic hypotheses for the activation of G-proteincoupled receptors. Cell Signal 8: 217-224, 1996.

Foster AC \& Kemp JA. Glutamate- and GABA-based CNS therapeutics. Curr Opin Pharmacol 6: 7-17, 2006. 
Franco R, Casado V, Mallol J, Ferre S, Fuxe K, Cortes A, Ciruela F, Lluis C \& Canela EI. Dimer-based model for heptaspanning membrane receptors. Trends Biochem Sci 30: 360-366, 2005.

Franco R, Casado V, Mallol J, Ferrada C, Ferre S, Fuxe K, Cortes A, Ciruela F, Lluis C \& Canela EI. The two-state dimer receptor model: a general model for receptor dimers. Mol Pharmacol 69: 1905-1912, 2006.

Franco R, Casadó V, Cortés A, Mallol J, Ciruela F, Ferré S, Lluis C \& Canela EI. G-protein-coupled receptor heteromers: function and ligand pharmacology. Br J Pharmacol 153: S90-S98, 2007a.

Franco R, Casadó V, Cortés A, Ferrada C, Mallol J, Woods A, Lluis C, Canela EI \& Ferré S. Basic concepts in G-protein-coupled receptor homo- and heterodimerization. ScientificWorldJournal 7: 48-57, 2007b.

Francotte P, Tullio P, Goffin E, Dintilhac G, Graindorge E, Fraikin P, Lestage P, Danober L, Thomas JY, Caignard DH \& Pirotte B. Design, synthesis, and pharmacology of novel 7-substituted 3,4dihydro-2H-1,2,4-benzothiadiazine 1,1-dioxides as positive allosteric modulators of AMPA receptors. J Med Chem 50: 31533157, 2007.

Frieden C. Kinetic aspects of regulation of metabolic processes. The hysteretic enzyme concept. J Biol Chem 245: 5788-5799, 1970.

Gao ZG \& Jacobson KA. Keynote review: allosterism in membrane receptors. Drug Discov Today 11: 191-202, 2006.

Ghosh P, Moitra K, Maki N \& Dey S. Allosteric modulation of the human P-glycoprotein involves conformational changes mimicking catalytic transition intermediates. Arch Biochem Biophys 450: 100-112, 2006.

Giraldo J. Agonist induction, conformational selection, and mutant receptors. FEBS Lett 256: 13-18, 2004.

Giraldo J, Roche D, Rovira X \& Serra J. The catalytic power of enzymes: conformational selection or transition state stabilization? FEBS Lett 580: 2170-2177, 2006.

Giraldo J, Serra J, Roche D \& Rovira X. Assessing receptor affinity for inverse agonists: Schild and Cheng-Prusoff methods revisited. Curr Drug Targets 8: 197-202, 2007.

Gouaux E \& MacKinnon R. Principles of selective ion transport in channels and pumps. Science 310: 1461-1465, 2005.

Gronlien JH, Haakerud M, Ween H, Thorin-Hagene K, Briggs CA, Gopalakrishnan M, et al. Distinct profiles of \{alpha\}7 nAChR positive allosteric modulation revealed by structurally diverse chemotypes. Mol Pharmacol 72: 715-724, 2007.

Haber JE \& Koshland DE Jr. Relation of protein subunit interactions to the molecular species observed during cooperative binding of ligands. Proc Natl Acad Sci USA 58: 2087-2093, 1967.

Hall DA. Modeling the functional effects of allosteric modulators at pharmacological receptors: an extension of the two-state model of receptor activation. Mol Pharmacol 58: 1412-1423, 2000.

Hay DL, Christopoulos G, Christopoulos A, Poyner DR \& Sexton PM. Pharmacological discrimination of calcitonin receptor: receptor activity-modifying protein complexes. Mol Pharmacol 67: 1655$1665,2005$.

Hemstapat K, Da Costa H, Nong Y, Brady AE, Luo Q, Niswender CM, et al. A novel family of potent negative allosteric modulators of group II metabotropic glutamate receptors. J Pharmacol Exp Ther 322: 254-264, 2007.

Himoe A, Parks PC \& Hess GP. Investigations of the chymotrypsincatalyzed hydrolysis of specific substrates. I. The $\mathrm{pH}$ dependence of the catalytic hydrolysis of N-acetyl-L-tryptophanamide by three forms of the enzyme at alkaline pH. I Biol Chem 242: 919-929, 1967.

Hoare SR, Fleck BA, Gross RS, Crowe PD, Williams JP \& Grigoriadis DE. Allosteric ligands for the corticotropin releasing factor type 1 receptor modulate conformational states involved in receptor activation. Mol Pharmacol Jan 31; [Epub ahead of print]: 2008.
Hogenkamp DJ, Johnstone TB, Huang JC, Li WY, Tran M, Whittemore ER, Bagnera RE \& Gee KW. Enaminone amides as novel orally active GABA(A) receptor modulators. J Med Chem 50: 3369-3379, 2007.

Hogg RC, Buisson B \& Bertrand D. Allosteric modulation of ligandgated ion channels. Biochem Pharmacol 70: 1267-1276, 2005.

Holst B, Brandt E, Bach A, Heding A \& Schwartz TW. Nonpeptide and peptide growth hormone secretagogues act both as ghrelin receptor agonist and as positive or negative allosteric modulators of ghrelin signaling. Mol Endocrinol 19: 2400-2411, 2005.

Holzgrabe U, De Amici M \& Mohr K. Allosteric modulators and selective agonists of muscarinic receptors. J Mol Neurosci 30: 165168, 2006.

Hornigold DC, Mistry R, Raymond PD, Blank JL \& Challiss RA. Evidence for cross-talk between M2 and M3 muscarinic acetylcholine receptors in the regulation of second messenger and extracellular signal-regulated kinase signalling pathways in Chinese hamster ovary cells. Br J Pharmacol 138: 1340-1350, 2003.

Huang XP \& Ellis J. Mutational disruption of a conserved disulfide bond in muscarinic acetylcholine receptors attenuates positive homotropic cooperativity between multiple allosteric sites and has subtype-dependent effects on the affinities of muscarinic allosteric ligands. Mol Pharmacol 71: 759-768, 2007.

Hulme EC, Birdsall NJ, Burgen AS \& Mehta P. The binding of antagonists to brain muscarinic receptors. Mol Pharmacol 14: 737$750,1978$.

Hunte C, Screpanti E, Venturi M, Rimon A, Padan E \& Michel H. Structure of a $\mathrm{Na}+/ \mathrm{H}+$ antiporter and insights into mechanism of action and regulation by pH. Nature 435: 1197-1202, 2005.

Indarte M, Madura JD \& Surratt CK. Dopamine transporter comparative molecular modeling and binding site prediction using the LeuT(Aa) leucine transporter as a template. Proteins 70: 10331046, 2007.

Jacobs S \& Cuatrecasas P. The mobile receptor hypothesis and 'cooperativity' of hormone binding. Application to insulin. Biochim Biophys Acta 433: 482-495, 1976.

Jacobson KA \& Gao ZG. Adenosine receptors as therapeutic targets. Nat Rev Drug Discov 5: 247-264, 2006.

Jakubik J, Bacakova L, Lisa V, El-Fakahany EE \& Tucek S. Activation of muscarinic acetylcholine receptors via their allosteric binding sites. Proc Natl Acad Sci USA 93: 8705-8709, 1996.

Jasti J, Furukawa H, Gonzales EB \& Gouaux E. Structure of acid-sensing ion channel 1 at 1.9 A resolution and low pH. Nature 449: 316 323, 2007.

Jensen AA \& Spalding TA. Allosteric modulation of G-protein coupled receptors. Eur J Pharm Sci 21: 407-420, 2004.

Jensen AM, Sorensen TL, Olesen C, Moller JV \& Nissen P. Modulatory and catalytic modes of ATP binding by the calcium pump. EMBOJ 25: 2305-2314, 2006.

Jentsch TJ, Neagoe I \& Scheel O. CLC chloride channels and transporters. Curr Opin Neurobiol 15: 319-325, $2005 \mathrm{a}$.

Jentsch TJ, Poet M, Fuhrmann JC \& Zdebik AA. Physiological functions of CLC Cl-channels gleaned from human genetic disease and mouse models. Annu Rev Physiol 67: 779-807, 2005b.

Johnson JW \& Ascher P. Glycine potentiates the NMDA response in cultured mouse brain neurons. Nature 325: 529-531, 1987.

Jones SV, Heilman CJ \& Brann MR. Functional responses of cloned muscarinic receptors expressed in CHO-K1 cells. Mol Pharmacol 40: 242-247, 1991.

Kashihara K, Varga EV, Waite SL, Roeske WR \& Yamamura HI. Cloning of the rat M3, M4 and M5 muscarinic acetylcholine receptor genes by the polymerase chain reaction (PCR) and the pharmacological characterization of the expressed genes. Life Sci 51: 955971, 1992. 
Katz B \& Thesleff S. A study of the desensitization produced by acetylcholine at the motor end-plate. J Physiol 138: 63-80, 1957.

Kenakin T. Differences between natural and recombinant $G$ proteincoupled receptor systems with varying receptor/G protein stoichiometry. Trends Pharmacol Sci 18: 456-464, 1997.

Kew JN. Positive and negative allosteric modulation of metabotropic glutamate receptors: emerging therapeutic potential. Pharmacol Ther 104: 233-244, 2004.

Knudsen LB, Kiel D, Teng M, Behrens C, Bhumralkar D, Kodra JT, Holst JJ, Jeppesen CB, Johnson MD, de Jong JC, Jorgensen AS, Kercher T, Kostrowicki J, Madsen P, Olesen PH, Petersen JS, Poulsen F, Sidelmann UG, Sturis J, Truesdale L, May J \& Lau J. Small-molecule agonists for the glucagon-like peptide 1 receptor. Proc Natl Acad Sci USA 104: 937-942, 2007.

Koshland DE Jr, Nemethy G \& Filmer D. Comparison of experimental binding data and theoretical models in proteins containing subunits. Biochemistry 5: 365-385, 1966.

Kukkonen JP. Explicit formulation of different receptor-G-protein interactions and effector regulation. Bioinformatics 20: 2411-2420, 2004a.

Kukkonen JP. Regulation of receptor-coupling to (multiple) G proteins. A challenge for basic research and drug discovery. Receptors Channels 10: 167-183, 2004b.

Kukkonen JP, Nasman J \& Akerman KE. Modelling of promiscuous receptor-Gi/Gs-protein coupling and effector response. Trends Pharmacol Sci 22: 616-622, 2001.

Kurganov BI. Allosteric enzymes. Kinetic Behaviour (1st English edition). Chichester: Wiley \& Sons, 1982.

Langmead CJ. Screening for positive allosteric modulators: assessment of modulator concentration-response curves as a screening paradigm. J Biomol Screen 12: 668-676, 2007.

Langmead CJ \& Christopoulos A. Allosteric agonists of 7TM receptors: expanding the pharmacological toolbox. Trends Pharmacol Sci 27: 475-581, 2006.

Langmead CJ, Fry VA, Forbes IT, Branch CL, Christopoulos A, Wood MD \& Herdon HJ. Probing the molecular mechanism of interaction between 4-n-butyl-1-[4-(2-methylphenyl)-4-oxo-1-butyl]piperidine (AC-42) and the muscarinic $\mathrm{M}(1)$ receptor: direct pharmacological evidence that AC-42 is an allosteric agonist. $\mathrm{Mol}$ Pharmacol 69: 236-246, 2006.

Lanzafame AA, Sexton PM \& Christopoulos A. Interaction studies of multiple binding sites on M4 muscarinic acetylcholine receptors. Mol Pharmacol 70: 736-746, 2006.

Lazareno S \& Birdsall NJ. Detection, quantitation, and verification of allosteric interactions of agents with labeled and unlabeled ligands at $\mathrm{G}$ protein-coupled receptors: interactions of strychnine and acetylcholine at muscarinic receptors. Mol Pharmacol 48: 362378, 1995.

Lazareno S, Dolezal V, Popham A \& Birdsall NJ. Thiochrome enhances acetylcholine affinity at muscarinic M4 receptors: receptor subtype selectivity via cooperativity rather than affinity. Mol Pharmacol 65: 257-266, 2004.

Lecourtier L, Homayoun H, Tamagnan G \& Moghaddam B. Positive allosteric modulation of metabotropic glutamate 5 (mGlu5) receptors reverses $\mathrm{N}$-methyl-D-aspartate antagonist-induced alteration of neuronal firing in prefrontal cortex. Biol Psychiatry 62: 739-746, 2007.

Leff P. The two state model of receptor activation. Trends Pharmacol Sci 16: 89-97, 1995.

Leff P, Scaramellini C, Law C \& McKechnie K. A three-state receptor model of agonist action. Trends Pharmacol Sci 18: 355-362, 1997.

Lefkowitz RJ, Cotecchia S, Samama P \& Costa T. Constitutive activity of receptors coupled to guanine nucleotide regulatory proteins. Trends Pharmacol Sci 14: 303-307, 1993.

Litschig S, Gasparini F, Rueegg D, Stoehr N, Flor PJ, Vranesic I, Prezeau L, Pin JP, Thomsen C \& Kuhn R. CPCCOEt, a noncompetitive metabotropic glutamate receptor 1 antagonist, inhibits receptor signaling without affecting glutamate binding. Mol Pharmacol 55: 453-461, 1999.

MacKinnon R. Potassium channels. FEBS Lett 555: 62-65, 2003.

MacKinnon R. Nobel Lecture. Potassium channels and the atomic basis of selective ion conduction. Biosci Rep 24: 75-100, 2004.

Maillet EL, Pellegrini N, Valant C, Bucher B, Hibert M, Bourguignon JJ \& Galzi JL. A novel, conformation-specific allosteric inhibitor of the tachykinin NK2 receptor (NK2R) with functionally selective properties. FASEB J 21: 2124-2134, 2007.

Mak DO, McBride SM \& Foskett JK. Spontaneous channel activity of the inositol 1,4,5-trisphosphate (InsP3) receptor (InsP3R). Application of allosteric modeling to calcium and InsP3 regulation of InsP3R single-channel gating. J Gen Physiol 122: 583-603, 2003.

Maki N, Moitra K, Ghosh P \& Dey S. Allosteric modulation bypasses the requirement for ATP hydrolysis in regenerating low affinity transition state conformation of human P-glycoprotein. I Biol Chem 281: 10769-10777, 2006.

Marvizon JC \& Baudry M. Receptor activation by two agonists: analysis by nonlinear regression and application to N-methyl-D-aspartate receptors. Anal Biochem 213: 3-11, 1993.

May LT, Avlani VA, Sexton PM \& Christopoulos A. Allosteric modulation of G protein-coupled receptors. Curr Pharm Des 10 2003-2013, 2004

May LT, Leach K, Sexton PM \& Christopoulos A. Allosteric modulation of G protein-coupled receptors. Annu Rev Pharmacol Toxicol 47: 151, 2007.

Mehta AK \& Ticku MK. An update on GABAA receptors. Brain Res Brain Res Rev 29: 196-217, 1999.

Michal P, Lysikova M \& Tucek S. Dual effects of muscarinic M(2) acetylcholine receptors on the synthesis of cyclic AMP in CHO cells: dependence on time, receptor density and receptor agonists. Br J Pharmacol 132: 1217-1228, 2001.

Michelsen S, Sanchez C \& Ebert B. Lack of generalisation between the GABA(A) receptor agonist, gaboxadol, and allosteric modulators of the benzodiazepine binding site in the rat drug discrimination procedure. Psychopharmacology (Berl) 193: 151-157, 2007.

Migeon JC \& Nathanson NM. Differential regulation of cAMPmediated gene transcription by $\mathrm{ml}$ and $\mathrm{m} 4$ muscarinic acetylcholine receptors. Preferential coupling of $\mathrm{m} 4$ receptors to $\mathrm{Gi}$ alpha2. J Biol Chem 269: 9767-9773, 1994

Miller C. ClC chloride channels viewed through a transporter lens. Nature 440: 484-489, 2006

Monczor F, Fernandez N, Legnazzi BL, Riveiro ME, Baldi A, Shayo C \& Davio C. Tiotidine, a histamine $\mathrm{H} 2$ receptor inverse agonist that binds with high affinity to an inactive G-protein-coupled form of the receptor. Experimental support for the cubic ternary complex model. Mol Pharmacol 64: 512-520, 2003.

Monod J, Wyman J \& Changeux J-P. On the nature of allosteric transitions: a plausible model. J Mol Biol 12: 88-118, 1965.

Murakami S, Nakashima R, Yamashita E, Matsumoto T \& Yamaguchi A. Crystal structures of a multidrug transporter reveal a functionally rotating mechanism. Nature 443: 173-179, 2006.

Nasman J, Kukkonen JP, Ammoun S \& Akerman KE. Role of G-protein availability in differential signaling by alpha 2-adrenoceptors. Biochem Pharmacol 62: 913-922, 2001.

Neubig RR. Membrane organization in G-protein mechanisms. FASEB 8: 939-946, 1994

Nguitragool W \& Miller C. Uncoupling of a CLC $\mathrm{Cl}(-) / \mathrm{H}(+)$ exchange transporter by polyatomic anions. J Mol Biol 362: 682690, 2006.

Nicoll DA, Sawaya MR, Kwon S, Cascio D, Philipson KD \& Abramson J. The crystal structure of the primary $\mathrm{Ca} 2+$ sensor of the $\mathrm{Na}+/$ $\mathrm{Ca} 2+$ exchanger reveals a novel Ca2 + binding motif. J Biol Chem 281: 21577-21581, 2006. 
O'Brien JA, Lemaire W, Wittmann M, Jacobson MA, Ha SN, Wisnoski DD, Lindsley CW, Schaffhauser HJ, Rowe B, Sur C, Duggan ME, Pettibone DJ, Conn PJ \& Williams DL Jr. A novel selective allosteric modulator potentiates the activity of native metabotropic glutamate receptor subtype 5 in rat forebrain. J Pharmacol Exp Ther 309: 568-577, 2004.

Obara K, Miyashita N, Xu C, Toyoshima I, Sugita Y, Inesi G \& Toyoshima C. Structural role of countertransport revealed in $\mathrm{Ca}(2+)$ pump crystal structure in the absence of $\mathrm{Ca}(2+)$. Proc Natl Acad Sci USA 102: 14489-14496, 2005.

Okada T, Fujiyoshi Y, Silow M, Navarro J, Landau EM \& Shichida Y. Functional role of internal water molecules in rhodopsin revealed by X-ray crystallography. Proc Natl Acad Sci USA 99: 5982-5987, 2002.

Okada T, Sugihara M, Bondar AN, Elstner M, Entel P \& Buss V. The retinal conformation and its environment in rhodopsin in light of a new 2.2 A crystal structure. J Mol Biol 342: 571-583, 2004.

Oldham WM, Van Eps N, Preininger AM, Hubbell WL \& Hamm HE. Mechanism of the receptor-catalyzed activation of heterotrimeric G proteins. Nat Struct Mol Biol 13: 772-777, 2006.

Olsen RW, Chang CS, Li G, Hanchar HJ \& Wallner M. Fishing for allosteric sites on GABA(A) receptors. Biochem Pharmacol 68: 1675-1684, 2004.

Onaran HO, Costa T \& Rodbard D. Beta gamma subunits of guanine nucleotide-binding proteins and regulation of spontaneous receptor activity: thermodynamic model for the interaction between receptors and guanine nucleotide-binding protein subunits. Mol Pharmacol 43: 245-256, 1993.

Ong J \& Kerr DI. Clinical potential of GABAB receptor modulators. CNS Drug Rev 11: 317-334, 2005.

Palczewski K. G protein-coupled receptor rhodopsin. Annu Rev Biochem 75: 743-767, 2006.

Palczewski K, Kumasaka T, Hori T, Behnke CA, Motoshima H, Fox BA, Le Trong I, Teller DC, Okada T, Stenkamp RE, Yamamoto M \& Miyano M. Crystal structure of rhodopsin: A G protein-coupled receptor. Science 289: 739-745, 2000.

Parmentier ML, Prezeau L, Bockaert J \& Pin JP. A model for the functioning of family 3 GPCRs. Trends Pharmacol Sci 23: 268-274, 2002.

Pin JP, Kniazeff J, Liu J, Binet V, Goudet C, Rondard P \& Prezeau L. Allosteric functioning of dimeric class C G-protein-coupled receptors. FEBS J 272: 2947-2955, 2005.

Pineyro G, Azzi M, deLean A, Schiller PW \& Bouvier M. Reciprocal regulation of agonist and inverse agonist signaling efficacy upon short-term treatment of the human delta-opioid receptor with an inverse agonist. Mol Pharmacol 67: 336-348, 2005.

Plested AJ, Groot-Kormelink PJ, Colquhoun D \& Sivilotti LG. Singlechannel study of the spasmodic mutation alpha1A52S in recombinant rat glycine receptors. J Physiol 581: 51-73, 2007.

Proska J \& Tucek S. Mechanisms of steric and cooperative actions of alcuronium on cardiac muscarinic acetylcholine receptors. $\mathrm{Mol}$ Pharmacol 45: 709-717, 1994.

Proska J \& Tucek S. Competition between positive and negative allosteric effectors on muscarinic receptors. Mol Pharmacol 48: 696-702, 1995.

Raddatz R, Schaffhauser H \& Marino MJ. Allosteric approaches to the targeting of G-protein-coupled receptors for novel drug discovery: a critical assessment. Biochem Pharmacol 74: 383-391, 2007.

Ramjeesingh M, Li C, She YM \& Bear CE. Evaluation of the membranespanning domain of ClC-2. Biochem J 396: 449-460, 2006.

Ritzen A, Mathiesen JM \& Thomsen C. Molecular pharmacology and therapeutic prospects of metabotropic glutamate receptor allosteric modulators. Basic Clin Pharmacol Toxicol 97: 202-213, 2005.

Ross RA. Tuning the endocannabinoid system: allosteric modulators of the CB(1) receptor. Br J Pharmacol 152: 565-566, 2007.
Ross RA. Allosterism and cannabinoid CB(1) receptors: the shape of things to come. Trends Pharmacol Sci 28: 567-572, 2007.

Rovira X, Roche D, Serra J, Kniazeff J, Pin JP \& Giraldo J. Modeling the Binding and Function of Metabotropic Glutamate Receptors. $J$ Pharmacol Exp Ther Feb 20; [Epub ahead of print]: 2008.

Rusch D \& Forman SA. Classic benzodiazepines modulate the openclose equilibrium in alphalbeta2gamma2L gamma-aminobutyric acid type A receptors. Anesthesiology 102: 783-792, 2005.

Rusch D, Zhong H \& Forman SA. Gating allosterism at a single class of etomidate sites on alphalbeta2gamma2L GABA A receptors accounts for both direct activation and agonist modulation. $J$ Biol Chem 279: 20982-20992, 2004.

Ryder JW, Falcone JF, Manro JR, Svensson KA \& Merchant KM. Pharmacological characterization of cGMP regulation by the biarylpropylsulfonamide class of positive, allosteric modulators of alpha-amino-3-hydroxy-5-methyl-4-isoxazolepropionic acid receptors. J Pharmacol Exp Ther 319: 293-298, 2006.

Samama P, Cotecchia S, Costa T \& Lefkowitz RJ. A mutation induced activated state of the beta 2adrenergic receptor. Extending the ternary complex model. J Biol Chem 268: 4625-4636, 1993.

Scaramellini C \& Leff P. A three-state receptor model: predictions of multiple agonist pharmacology for the same receptor type. Ann $N$ Y Acad Sci 861: 97-103, 1998.

Scaramellini C \& Leff P. Theoretical implications of receptor coupling to multiple $\mathrm{G}$ proteins based on analysis of a three-state model. Methods Enzymol 343: 17-29, 2002.

Scheer A, Fanelli F, Costa T, De Benedetti PG \& Cotecchia S. Constitutively active mutants of the alpha $1 \mathrm{~B}$-adrenergic receptor: role of highly conserved polar amino acids in receptor activation. ЕМВO J 15: 3566-3578, 1996.

Schetz JA. Allosteric modulation of dopamine receptors. Mini Rev Med Chem 5: 555-561, 2005.

Shipe WD, Wolkenberg SE, Williams DL Jr \& Lindsley CW. Recent advances in positive allosteric modulators of metabotropic glutamate receptors. Curr Opin Drug Discov Dev 8: 449-457, 2005.

Solt K, Ruesch D, Forman SA, Davies PA \& Raines DE. Differential effects of serotonin and dopamine on human 5-HT3A receptor kinetics: interpretation within an allosteric kinetic model. $J$ Neurosci 27: 13151-13160, 2007.

Spalding TA, Trotter C, Skjaerbaek N, Messier TL, Currier EA, Burstein ES, Li D, Hacksell U \& Brann MR. Discovery of an ectopic activation site on the $\mathrm{M}(1)$ muscarinic receptor. Mol Pharmacol 61: 1297-1302, 2002.

Teng M, Johnson MD, Thomas C, Kiel D, Lakis JN, Kercher T, Aytes S, Kostrowicki J, Bhumralkar D, Truesdale L, May J, Sidelman U, Kodra JT, Jørgensen AS, Olesen PH, de Jong JC, Madsen P, Behrens C, Pettersson I, Knudsen LB, Holst IJ \& Lau J. Small molecule ago-allosteric modulators of the human glucagon-like peptide-1 (hGLP-1) receptor. Bioorg Med Chem Lett 17: 5472-5478, 2007.

Timmermann DB, Grønlien JH, Kohlhaas KL, Nielsen EØ, Dam E, Jørgensen TD, Ahring PK, Peters D, Holst D, Chrsitensen JK, Malysz J, Briggs CA, Gopalakrishnan M \& Olsen GM. An allosteric modulator of the alpha7 nicotinic acetylcholine receptor possessing cognition-enhancing properties in vivo. J Pharmacol Exp Ther 323: 294-307, 2007.

Tolkovsky AM \& Levitzki A. Mode of coupling between the betaadrenergic receptor and adenylate cyclase in turkey erythrocytes. Biochemistry 17: 3795-3810, 1978.

Tolkovsky AM \& Levitzki A. Theories and predictions of models describing sequential interactions between the receptor, the GTP regulatory unit, and the catalytic unit of hormone dependent adenylate cyclases. J Cyclic Nucleotide Res 7: 139-150, 1981.

Toyoshima C. Ion pumping by calcium ATPase of sarcoplasmic reticulum. In Regulatory mechanisms of striated muscle contraction 
vol 592, Adv Exp Med Biol Editor Ebashi S, Tokyo, Springer Verlag, 2007, pp295-303.

Toyoshima C, Nakasako M, Nomura H \& Ogawa H. Crystal structure of the calcium pump of sarcoplasmic reticulum at 2.6 A resolution. Nature 405: 647-655, 2000.

Tucek S \& Proska J. Allosteric modulation of muscarinic acetylcholine receptors. Trends Pharmacol Sci 16: 205-212, 1995.

Tucek S, Michal P \& Vlachova V. Dual effects of muscarinic M2 receptors on the synthesis of cyclic AMP in CHO cells: background and model. Life Sci 68: 2501-2510, 2001.

Tucek S, Michal P \& Vlachova V. Modelling the consequences of receptor-G-protein promiscuity. Trends Pharmacol Sci 23: 171-176, 2002.

Unwin N. Refined structure of the nicotinic acetylcholine receptor at 4A resolution. J Mol Biol 346: 967-989, 2005.

van Rijn CM \& Willems-van Bree E. A four-ligand hypercube model to quantify allosteric interactions within the GABAA receptor complex. Eur J Pharmacol 485: 43-51, 2004.

Vauquelin G \& Van Liefde I. G protein-coupled receptors: a count of 1001 conformations. Fundam Clin Pharmacol 19: 45-56, 2005.

Vieira E, Huwyler J, Jolidon S, Knoflach F, Mutel V \& Wichmann J. 9HXanthene-9-carboxylic acid [1,2,4] oxadiazol-3-yl- and (2H-tetrazol-5-yl)-amides as potent, orally available mGlul receptor enhancers. Bioorg Med Chem Lett 15: 4628-4631, 2005.

Vogel WK, Mosser VA, Bulseco DA \& Schimerlik MI. Porcine m2 muscarinic acetylcholine receptor-effector coupling in Chinese hamster ovary cells. J Biol Chem 270: 15485-15493, 1995.

Waelbroeck M. Identification of drugs competing with D-tubocurarine for an allosteric site on cardiac muscarinic receptors. Mol Pharmacol 46: 685-692, 1994.

Weiss JM, Morgan PH, Lutz MW \& Kenakin TP. The cubic ternary complex receptor occupancy model. III. Resurrecting efficacy. I Theor Biol 181: 381-397, 1996a.
Weiss JM, Morgan PH, Lutz MW \& Kenakin TP. The cubic ternary complex receptor-occupancy model II. Understanding apparent affinity. J Theor Biol 178: 169-182, 1996b.

Weiss JM, Morgan PH, Lutz MW \& Kenakin TP. The cubic ternary complex receptor-occupancy model I. Model description. J Theor Biol 178: 151-167, 1996c.

Wells JW. Analysis and interpretation of binding at equilibrium. In: Receptor-ligand Interactions. A Practical Approach, 1st ed., edited by Hulme EC. Oxford: IRL Press at Oxford University Press, 1992.

Winding B \& Bindslev N. Desensitization and reactivation of AChregulated exocrine secretion in hen tracheal epithelium. Am J Physiol 264: C342-C351, 1993.

Wreggett KA \& Wells JW. Cooperativity manifest in the binding properties of purified cardiac muscarinic receptors. J Biol Chem 270: 22488-22499, 1995 .

Yamashita A, Singh SK, Kawate T, Jin Y \& Gouaux E. Crystal structure of a bacterial homologue of $\mathrm{Na}+/ \mathrm{Cl}-$-dependent neurotransmitter transporters. Nature 437: 215-223, 2005.

Yernool D, Boudker O, Jin Y \& Gouaux E. Structure of a glutamate transporter homologue from Pyrococcus horikoshii. Nature 431: 811-818, 2004.

Yoshimura RF, Hogenkamp DJ, Li WY, Tran MB, Belluzzi JD, Whittemore ER, Leslie FM \& Gee KW. Negative allosteric modulation of nicotinic acetylcholine receptors blocks nicotine self-administration in rats. J Pharmacol Exp Ther 323: 907-915, 2007

Yu EW, Aires JR \& Nikaido H. AcrB multidrug efflux pump of Escherichia coli: composite substrate-binding cavity of exceptional flexibility generates its extremely wide substrate specificity. $J$ Bacteriol 185: 5657-5664, 2003. 
Part II: Two-State Models: Complex Agonism and Modulation 


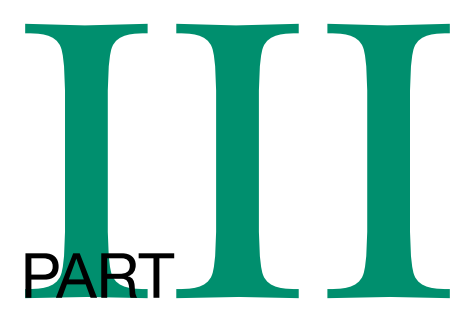

TEST OF TOOLS FOR DATA
ANALYSIS

\section{III.1. Limitations of Transformed Plots}

What happens when you ask students who have just finished a course in biochemistry how to analyze dose-response data assumed to follow the simple law-of-adsorption-desorption (load)? In the year 2008, the most frequent answer, if any, is that one should employ the Lineweaver-Burk plot.

Some 40 years ago, it was recognized that the Lineweaver-Burk (L\&B) plot with double reciprocal scales for analysis of simple ligand-receptor interactions is the least reliable of all linearizing techniques (Dowds \& Riggs 1965; Eisenthal \& Cornish-Bowden 1974; CornishBowden 1974). With present computing capabilities, we must teach our young peers to use non-linear fitting routines on plots without reciprocal scales. Such instructions ought to be our common objective. Only as an aside should we provide information about when, for example, to use the reciprocal Scatchard-Hofstee plot or the nullmethod of a Schild plot. The Lineweaver-Burk (reciprocal) plot should only be mentioned as a historic curiosity.

The Scatchard-(Hofstee or Eadie-Scatchard) plot for agonist action is restricted to analysis of simple load (Chapter 1), while the Schild plot is restricted to analysis of simple competitive ant-agonist action (Chapters 2, 4, and 11). In spite of these restrictions, the two plots are nonetheless in general the most abundant methods used in certain fields of life sciences for analysis of the interaction between ligands and receptive units.

\section{III.2. Reciprocal and Non-reciprocal Plots}

The load function is changed from a hyperbolic plot to a linear plot by reciprocal transformation of the independent variable ( $x$-axis in the Eadie-Hofstee plot), the dependent variable ( $y$-axis in the Hanes plot), or both the independent and dependent variable ( $x$ and $y$ axes in the Lineweaver-Burk plot) (see Subchapter 8 and Table 8.3). These plots are reciprocal plots. The reciprocal plot methods were developed with the purpose of making the analyses for maximum effect and drug affinity more manageable (Table 8.3), and were somewhat justified before the advent of easy computing.

Non-reciprocal transformation of the load function to achieve more evenly distributed data points than the hyperbolic curve, and thereby a better analysis, may be attained by so-called 'semi-log' or 'logit' plotting (see Subchapters 8.1 and 8.2). These are non-reciprocal plots. Table III.1 summarizes the plot types for load and Hill relations.

To implement a versatile tool, this tome is also written with the purpose of convincing the reader and future scientists to use non-reciprocal formulations and plots with non-linear routines for parameter fitting of the most appropriate hypothesis, while skipping the 'reciprocal' methods developed for load $^{1}$ as well as the 'nullmethod' of Schild. The latter is strictly related to simple competitive ant-agonism. See Marangoni (2003, pp. 3336) for a balanced opinion on the use of non-linear as opposed to linearized methods.

Algebraic analysis of synagic data beyond the HenriMichaelis-Menten, the Hill, and the Schild is appalling to most laboratory scientists, as, in general, mathematical modeling is suspect to many researchers (Keller 2002, preface).$^{2}$ Meanwhile, as pointed out by Keller (2002) and May (2004), theoretical work is experiencing a new dawn.

\footnotetext{
${ }^{1}$ I am aware that leading kineticists may not agree with me (see Cornish-Bowden in his review of Marangoni's 'Enzyme Kinetics: A Modern Approach' 7 January 2005, Amazon books, Amazon.com).

${ }^{2}$ In her book, Keller presents a sovereign and enlightening discussion of why d'Arzy Thomson's view on form in nature is obsolete.
} 
Table III.1. Plots of the law of adsorption-desorption, Load, and Hill Function

\begin{tabular}{lll}
\hline Type of transformation & Relation & Name of plot \\
\hline None or & Load & $\begin{array}{l}\text { Ordinary linear-linear } \\
\text { hyperbolic } \\
\text { Semi-log/S-shaped }\end{array}$ \\
logarithmic $x$-axis & Hill & $\begin{array}{l}\text { Sigmoidal } \\
\text { Semilog or 'Logistic' plot } \\
\text { Logit plot or Hill plot }\end{array}$ \\
\hline $\begin{array}{l}\text { None or } \\
\text { logarithmic } x \text {-axis or } \\
\text { relative }\end{array}$ & & $\begin{array}{l}\text { Eadie Hofstee or Scatchard } \\
\text { Hanes } \\
\text { Lineweaver-Burk }\end{array}$ \\
\hline Reciprocal & Load & \\
\hline
\end{tabular}

Reciprocal indicates that at least one of the system variables is reversed, either the independent variable, i.e., $1 /$ [ligand], or the dependent variables, i.e. $1 / B$ (ound) and $1 / R$ (esponse), or even both independent and dependent variables are reciprocal at the same time.

This is exemplified very well by the recent hype around and boom in 'Systems Biology' (see Sub-chapter 13.1).

\section{III.3. Stepping on the Chips}

The aforementioned convenience and justification for earlier employing linearizing strategies such as Hanes' plot, Lineweaver-Burk's plot, Eadie-Scatchard's plot, or null-methods such as Schild's plot, when analyzing equilibrium data, have evidently evaporated with the emergence of chip-based computers and their software (Marangoni 2003). In addition, this development has parallels in biology, ecology, economy, evolutionism, embryology, sociology, and several other sciences (May 1976; Keller 2002, especially Part 3; Cramer 2003; May 2004). In all these areas, analysis of data is completely integrated with chip-based interactive software and is unthinkable without stepping on the chips as a prerequisite for future advancement of data analysis. For a more elaborate example, look for instance into efforts on interoperability of software for simulation of biochemical networks (Hucka et al. 2001). The field of bioinformatics likewise has exploded in the last ten years (see Chapter 13 and Table 13.3) due to computational capabilities. For example, browse at random in new journals as BMC Systems Biology, Systems and Synthetic Biology, Biosystems, the Intl Journal of Bioinformatics, and Bioinformatics (available at http://bioinformatics. oxfordjournals.org).

\section{III.4. Imaginative Modeling}

On a banal level, suitable computer programs allow nonlinear adjustment of the parameters in theoretical reaction schemes to experimental dose-response data based on various minimum-likelihood or least squares methods. These chip-based programs can determine system constants just as easily as the earlier linear regression analyses. Therefore, in synagics, non-reciprocal plotting and non-linear parameter fitting should initially be the method of choice, solely for the reason that non-linear fitting yield more accurate estimates of parameter values compared with the linearized methods of reciprocally transformed data. However, most importantly, the implementation of non-reciprocal graphing and non-linear parameter fitting at the same time allow a direct application and appreciation of much more versatile reaction schemes and their intuitive algorithms. In this way, imaginative modeling is permissible and penetrable with non-reciprocal/non-linear analysis, whereas the linearized methods mostly assume simple models or offer rather indirect solutions (Segel 1975, 1993).

\section{III.5. Time for a Change?}

Highly skilled scribes in Pharaonic dynasties used syllabic writing with hieroglyphs of the outmost sophistication and subtlety. Hieroglyphs died out due to the supremacy of a pure alphabet that evolved from around 1700 to 1200 BC (Healey 1990). Likewise, I predict that the use of reciprocal transforms in the analysis of dose-response data at equilibrium will succumb to meaningful modeling inspired by probing techniques combined with powerful computation based on the chip's non-linear procedures applied to non-reciprocal plots.

However, complete elimination of the hieroglyphic writing lasted more than a 1000 years after the invention of a 'digitized' ABC. ${ }^{3}$ Similarly, in spite of the present surge in chip-based cities and digitalization of almost everything, we may ask - will the continued use of Hanes, Lineweaver-Burk, Eadie-Scatchard or Schild plots also last that long?

\section{III.6. The Assumptions}

The lesson that should be drawn from Chapter 8 is that it is the assumptions involved in setting up hypothetical schemes at equilibrium that are crucial for the whole analysis of dose-responses and not so much the method of analysis; although it is worth advocating for non-linear fitting. For every synagic or kinetic hypothesis, experiments with built-in falsification procedures should

\footnotetext{
3 The Chinese syllabic/pictographic 'ABC' is still in use. Reading it creates a completely different activity in the brain compared to the brain pattern during Western reading (Siok et al. 2004).
} 
be implemented and tested before one settles for a particular reaction scheme. In this, non-reciprocal/ non-linear parameter fitting is the instrument to probe for possible hypotheses and test whether an assumption holds - for instance, when analyzing a particular form of modulatory allostery. Non-linear parameter fitting is altogether more simple to use and by far the most superior evaluation tool. Although repeatedly recommended (Marangoni 2003; Neubig et al. 2003; Motulsky \& Christopoulos 2004), non-linear fitting is often not implemented.

\section{III.7. Contents of Part III}

For an analysis of dose-response data, Chapter 8 demonstrates how to construct your own theory based on a hypothesis. When we move from the intervention model in Chapter 2 to the allosteric models in Chapter 15, there is a shift in nomenclature; that is 'site interaction' described as 'intervention' or 'auto-intervention' in the former may become 'modulation' or 'co-operativity' in the latter (Table III.2 and 8.1). While reading Chapter 8, it is advantageous to have these and related terms present when models involve interactions between sites. Subchapter 8.1 is valuable for an appreciation of the contents of Chapter 9, while Sub-chapters 8.2 and 8.3 may be postponed for later studies. Sub-chapter 8.2 contains a training session with a detailed description of how to ana-

Table III.2. Comparison of Nomenclature for Intervention and Allostery Schemes (An Abbreviated Form of this Table is in Table 8.1)

\begin{tabular}{|c|c|c|}
\hline $\begin{array}{l}\text { Type of reaction } \\
\text { schemes }\end{array}$ & Intervention schemes & Allosteric schemes \\
\hline Two-site models & $\begin{array}{l}\text { One-state models } \\
\text { (OSM) (see Chapters } \\
2 \text { and } 3 \text { ) }\end{array}$ & $\begin{array}{l}\text { Two-state models } \\
\text { (TSM) (see Chapters } \\
7 \text { and 15) }\end{array}$ \\
\hline $\begin{array}{l}\text { Binding and } \\
\text { function with } \\
\text { different ligands }\end{array}$ & $\begin{array}{l}\text { Mixed competitive } \\
\text { inhibition } \\
\text { (intervention) } \\
\text { Mixed competitive } \\
\text { acceleration } \\
\text { (intervention) }\end{array}$ & $\begin{array}{l}\text { Negative heterotropic } \\
\text { allostery } \\
\text { (modulation) } \\
\text { Positive heterotropic } \\
\text { allostery } \\
\text { (modulation) }\end{array}$ \\
\hline $\begin{array}{l}\text { Binding and } \\
\text { function with a } \\
\text { single ligand }\end{array}$ & $\begin{array}{l}\text { Substrate inhibition; } \\
\text { negative auto- } \\
\text { intervention } \\
\text { Substrate acceleration; } \\
\text { positive auto-inter- } \\
\text { vention }\end{array}$ & $\begin{array}{l}\text { Negative co-operativity } \\
\text { or homo-tropic } \\
\text { modulation } \\
\text { Positive co-operativity } \\
\text { or homotropic } \\
\text { modulation }\end{array}$ \\
\hline \multicolumn{3}{|l|}{$\begin{array}{l}\text { General } \\
\text { designators for: }\end{array}$} \\
\hline (1) Ligand & $\begin{array}{l}\text { Positive or negative } \\
\text { interventor }\end{array}$ & $\begin{array}{l}\text { Positive or negative } \\
\text { modulator }\end{array}$ \\
\hline (2) Process & $\begin{array}{l}\text { Positive or negative } \\
\text { intervention }\end{array}$ & $\begin{array}{l}\text { Positive or negative } \\
\text { modulation }\end{array}$ \\
\hline
\end{tabular}

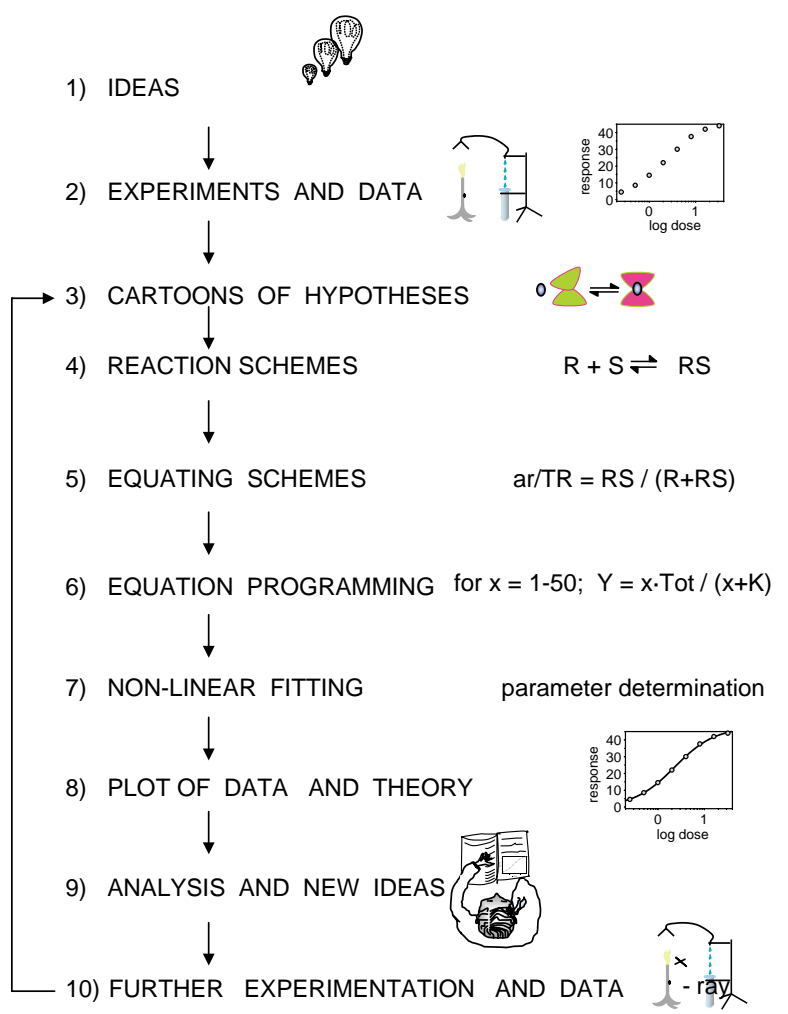

Figure III.1. A simple flowchart on the scientific progress of ideas. For a more detailed layout also defining theory, postulate and hypothesis, see Ford (2000, especially Chapter 3 ).

lyze an example of a theory for dose-response data. Subchapter 8.3 is a summary of some of the still current but out-dated reciprocal methods and their equations describing different set-ups, as well as a brief look at various transformations of these equations and their plots.

The procedures in Sub-chapters 8.2 and Chapter 9 follow the flowchart depicted in Fig. III.1.

The essence of Part III is in Chapter 9 on 'Plots, Fits, and Data Interpretation'. Chapter 9 is a step-by-step instruction on how to obtain estimates for system constants, such as the maximal obtainable effect $\left(R_{\max }\right)$ or maximal binding $\left(B_{\max }\right)$, and the apparent affinity of a drug $\left(\operatorname{appK}_{\mathrm{s}}\right)$, when based on one's selection of the most likely hypothesis for an underlying reaction scheme. Thus, Chapter 9 is a hands-on of how to present data, fit parameters of theory to data, and interpret the results.

Chapter 10 is a detailed description of Hill's equation and variations on the theme of Hill's equation. Chapter 11 takes care of Gaddum's and Schild's null methods (Gaddum 1937; Schild 1947, 1949). Finally, Chapter 12 in Part III treats the 'synergy' concept. Here, 'synergy' covers the effects of applying two or more drugs simultaneously in, for instance combinatorial drug therapy, and the analysis of data form such procedures.

Motulsky and Christopoulos (2004) have issued an extensive and useful guide to analyze synagic data, and this guide should be consulted. 



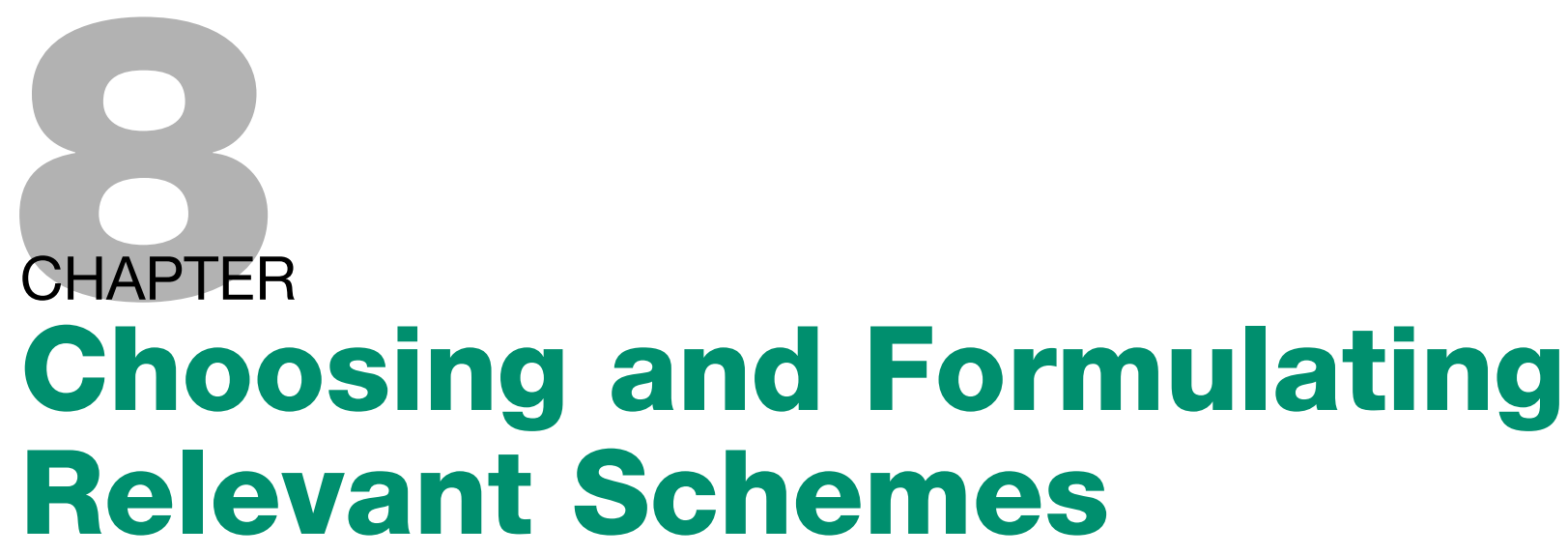

One main conclusion in this chapter is that there is a general relationship between responses and their concentrations presented as the factor-squared rule, which goes beyond a so-called $80-\%$ rule (Sub-chapter 8.1 ). Other conclusions are that Hill plots are also logit plots and a plot of data employing a 'logistic' Hill equation is simply the same as a semi-log plot of data (see also Chapter 10). Furthermore, the chapter will take you through a training session on how to implement an analysis of your own synagic data by an example (Subchapter 8.2 ). Sub-chapter 8.3 recounts out-dated plots.

\subsection{Load - Semi-log Plot and Factor-squared Rule}

\subsubsection{Self-referential Systems and Semi-log Plots}

Many biological phenomena as well as chemical and physical responses are based on self-referring systems that may exhaust themselves or have limiting maxima. Such systems can often be described by logarithmic build-up or decay (Jacquez 1972, 1985), deterministic chaos (Peitgen et al. 1992), or self-organized criticality (Bak 1997). This also means that the outcome of a change in an independent variable in these systems is dependent on the immediate preceding events due to an earlier change of the very same independent variable. Several such systems have dependent variables, i.e., responses, which saturate asymptotically to a plateau with increasing concentration, with running time, or with other evolving independent variables. The dose-response relation of load is also a sort of self-referential system due to its limited number of receptive units (see Sections 1.1.2 and 1.1.8).

Exponential functions as well as non-linear chaosmath are suitable for formulating certain self-referential systems (Riggs 1970; Peitgen et al. 1992). A good example is population growth described with the logistic equation involving a self-exhausting exponential growth as a function of time, where time is the independent variable on a linear scale and the dependent variable growth is on a relative or logarithmic scale (see Subchapter 10.3, Fig. 10.6). An obvious choice for presenting such data is therefore the semi-log plot, which is also relevant for dose-response data. However, for doseresponses it is the independent variable concentration that is made 'logistic'. Thus, the semi-log plot of concentration-binding data or dose-function data is a plot of the amount of ligand bound $(B)$ or enzyme catalytic rate at steady-state $(v)$ on a linear $y$-axis against free concentration (S) or against free dose (D) of the ligand on a logarithmic transformed $x$-axis. In other words, plots of symbols as:

$$
B \text { versus } \log [\mathrm{S}] \text { or } v \text { versus } \log [\mathrm{D}] \text {. }
$$

Concentration-binding/response data that follow the simple load relationship may be plotted in a semi-log plot out of many other plots (Boeyneams \& Dumont 1975). The semi-log plot of the simple hyperbolic Langmuirian load isotherm (Fig. 8.1A) yields a symmetrical S-shaped curve (Fig. 8.1B). Features of the semilog plot are described further in Sections 8.1.2-8.1.6.

Plotting data in a semi-log co-ordinate system was already introduced with Sørensen's pH concept (1909, pp. 28-29) and for the pH-effect on oxygen binding to hemoglobin (Adair 1925). For dose-responses in general, early examples are by Michaelis and Davidsohn (1911) and Kuhn (1923a, b). Regarding concentration versus complex-formation data in chemistry, the semi-log plot was initially suggested by Bjerrum in the early 1930s (1941, p. 21 'formation curve'), and in growth analysis by Berkson (Reed \& Berkson 1929; Berkson 1944).

Among several researchers in biochemistry, Klotz (1982) has strongly advocated the use of semi-log 


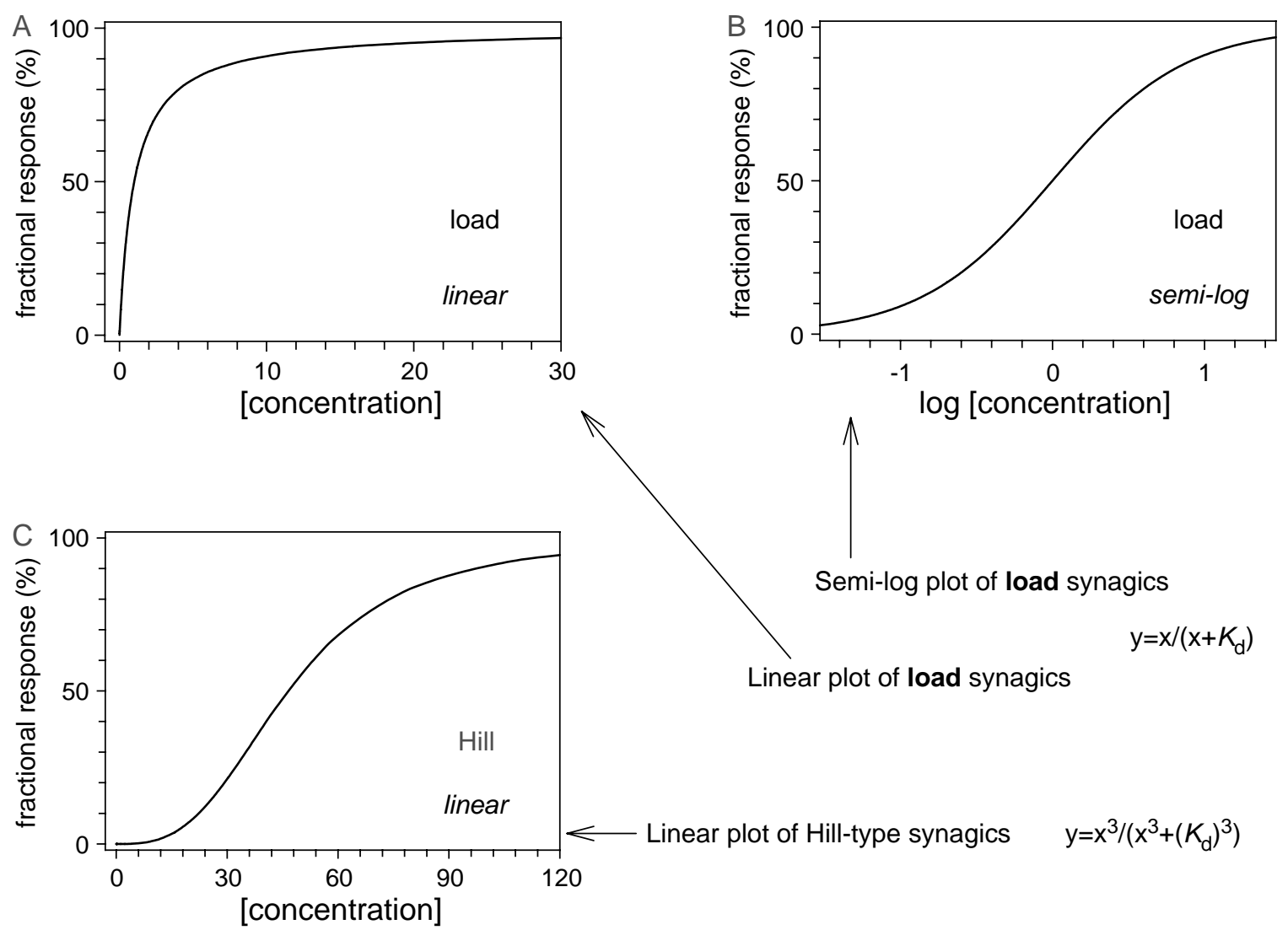

Figure 8.1. A semi-log plot and a plot of sigmoid synagics. Panel A is a linear-linear plot of the simple load function. Panel $B$ is a semi-log plot of the simple load function resulting in an $S$-shaped curve. Panel $C$ is a linear-linear plot of an allosteric function. This plot is generated based on a Hill-type equation with a Hill coefficient of 3 . Other formulations of allostery, for instance by varying dissociation constants, will also result in sigmoid shaped curves in linear-linear plots (see Chapter 15). The $S$-shaped form of a simple load function in a semi-log plot (panel B) should be differentiated from the sigmoid-shaped form of a genuine co-operative reaction in a linear-linear plot (panel C).

presentation of dose-response data, particularly in relation to analysis of ligand-receptor or substrateenzyme binding experiments intended at determining the absolute numbers of binding sites in a sample. The alert reader will have noticed that the present author also favors semi-log plots for the presentation of concentration-binding/dose-response data. Of course, from a principal point of view, the choice of plot method does not matter as long as it is handled correctly. For statistical evaluation, both semi-log and logit transformations may be advantageous (see Sections 10.5.4, 10.A.9, and 10.A.11).

\subsubsection{The $82-\%$ Rule for Agonists}

Data from a reaction scheme following the simple Langmuirian load is most simply presented in a semi$\log$ plot (Fig. 8.2A). The agonist concentration corresponding to the inflection point of the curve and read of the $x$-axis is identical to the equilibrium dissociation constant $\left(K_{\mathrm{d}}\right)$. Moving an order of magnitude down in concentration from this midpoint, which is an agonist concentration equal to $1 / 10$ th of the equilibrium dissociation constant $K_{\mathrm{d}}$, reduces the response to $1 / 11=$ $0.09090909 \ldots$ (aso), which is at about $9.1 \%$ of the full effect. Moving an order of magnitude up in concentration above the same midpoint, equal to 10 times the $K_{\mathrm{d}}$, yields a response of $10 / 11=0.90909090 \ldots$ (aso), equal at about $91 \%$ of the maximum response. Ergo, over two orders of magnitude in concentration and with a midpoint concentration of $K_{\mathrm{d}}$, the response for Langmuirian load will change in absolute values from $0.90909 \ldots$ aso to $0.09090 \ldots$ aso; a difference equal to 0.81818 . . aso; close to $82 \%$ of the total response. This is the 82-\% rule for an absolute change in response (Fig. 8.2A). As a relative change in response, 0.9090/0.0909, it is a paltry factor of 10 for a 100 -fold relative change in concentration.

\subsubsection{The $80-\%$ Rule for Agonists}

Another rule is the $\mathbf{8 0 - \%}$ rule, where for a change in response or binding going from exactly 10 to $90 \%$ of the response in absolute terms, equal to $80 \%$ of the total 
A

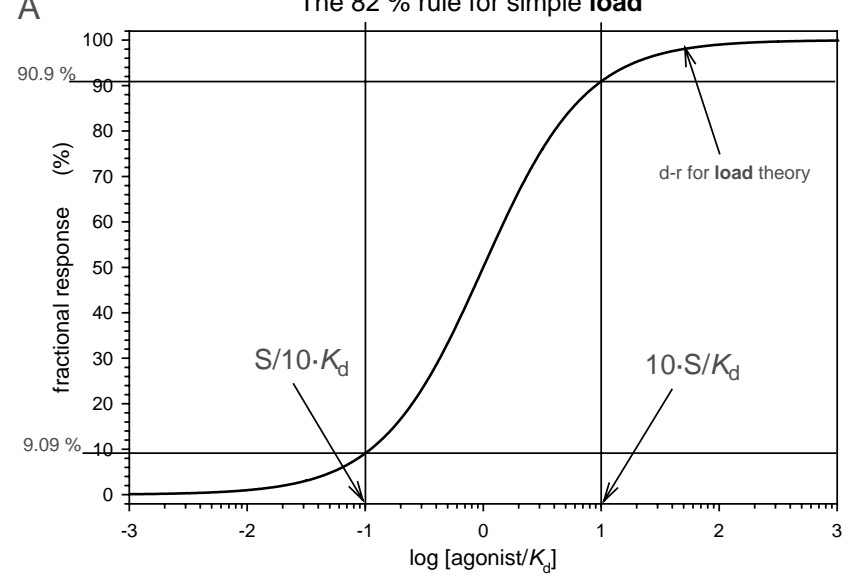

B

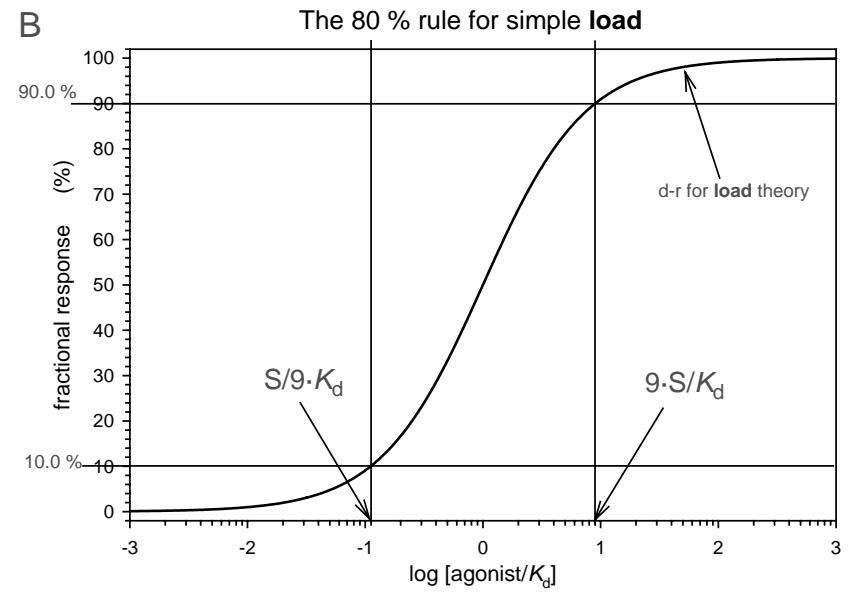

Figure 8.2. Illustrations of the $82-\%$ and $80-\%$ rule for simple load. (A) A 100 -fold change in relative concentration around $K_{d}$ results in a nearly $82 \%$ absolute change or 10 -fold relative change in response, from about $9 \%$ to about $91 \%$. (B) An 81 fold change in relative concentration around $K_{d}$ results in exactly $80 \%$ absolute change or in a 9 -fold relative change in response, from $10 \%$ to $90 \%$.

effect, this change corresponds to moving from $K_{\mathrm{d}} / 9$ to $9 \cdot K_{\mathrm{d}}$ in concentration, which is exactly equal to an 81 fold relative change in concentration (Fig. 8.2B) (Segel 1975,1993 , p. 362). Thus, a relative change in response from 10 to $90 \%$ is a mere factor 9 for an 81 -fold relative increase in concentration.

In case the data are understood as following a Hillian relationship with a Hill coefficient $\left(n_{\mathrm{H}}\right)$, in relation to the $80-\%$ rule, then the factor 9 change in relative effect, i.e., going from 10 to $90 \%$, corresponds to the $n_{\mathrm{H}^{-}}$th root of the concentration ratio between $[\mathrm{S}]_{0.9}$ and $[\mathrm{S}]_{0.1}$ for a load scheme (Fig. 8.2B).

The $n_{\mathrm{H}^{-}}$th root of the load-concentration ratio $[\mathrm{S}]_{0.9} /$ $[\mathrm{S}]_{0.1}$ may be written as:

$$
\sqrt[n_{\mathrm{H}}]{81} \vee 81^{1 / n_{\mathrm{H}}}
$$

(Taketa \& Pogell 1965; Kurganov 1982, pp. 43-45; Segel $1975,1993$, p. 362$)$. The actual ratio $[\mathrm{S}]_{0.9}^{\text {Hill }} /[\mathrm{S}]_{0.1}^{\text {Hill }}$ in a
Hill scheme is the so-called co-operativity index $R_{\mathrm{a}}$ for a 9-fold relative change in response (Cornish-Bowden 1995 , p. 209). Thus, $[\mathrm{S}]_{0.9}^{\mathrm{Hill}} /[\mathrm{S}]_{0.1}^{\mathrm{Hill}}$ equal to $R_{\mathrm{a}}$ is the same as $\sqrt[n_{\mathrm{H}}]{[S]_{0.9} /[S]_{0.1}}$.

For instance, for $n_{\mathrm{H}}=2$ a 9-fold relative change in concentration is enough to yield a 9-fold relative change in response. This is the $80-\%$ rule for a 9-fold response change describing its related relative change in concentration when taking Hillian synagics into account.

\subsubsection{The Factor-squared Rule for Agonists}

A third and general rule for load is the squared relation between a change in response ratio $\left(R_{2} / R_{1}\right)$, and its pertinent relative change in concentration ratio $\left(\mathrm{S}_{2} / \mathrm{S}_{1}\right)$, around the inflection point. $S_{2}$ and $S_{1}$ are above and below $K_{\mathrm{d}}$ with the same factor. Thus, for a given ratio $(x)$ between responses symmetrically above and below the midpoint of a load relation, the ratio of matching concentrations is the $x$-ratio squared, that is $x^{2}$. In other words, for a relative change in load-response of a factor $x$, the required relative change in concentration is the same factor squared, $x^{2}$. For load dose-responses, this is the factor-squared rule (Fig. 8.3). In Sections 8.1.2 and 8.1 .3 , we observed that a relative 10 - or 9 -fold change in response is equal to a relative 100- or 81-fold change in concentration, complying with the factor-squared rule.

A general expression for the load reaction scheme is therefore $\left(R_{2} / R_{1}\right)^{2}=\mathrm{S}_{2} / \mathrm{S}_{1}$, where $\mathrm{S}_{2}$ and $\mathrm{S}_{1}$ are distributed with an equal factor at either side of $\mathrm{S} / K_{\mathrm{d}}$. Fig. 8.3 is an example of this with the ratio of response $x$ or $R_{2} / R_{2}=$ square-root of 10 for $\mathrm{S}_{2} / \mathrm{S}_{1}=10$.

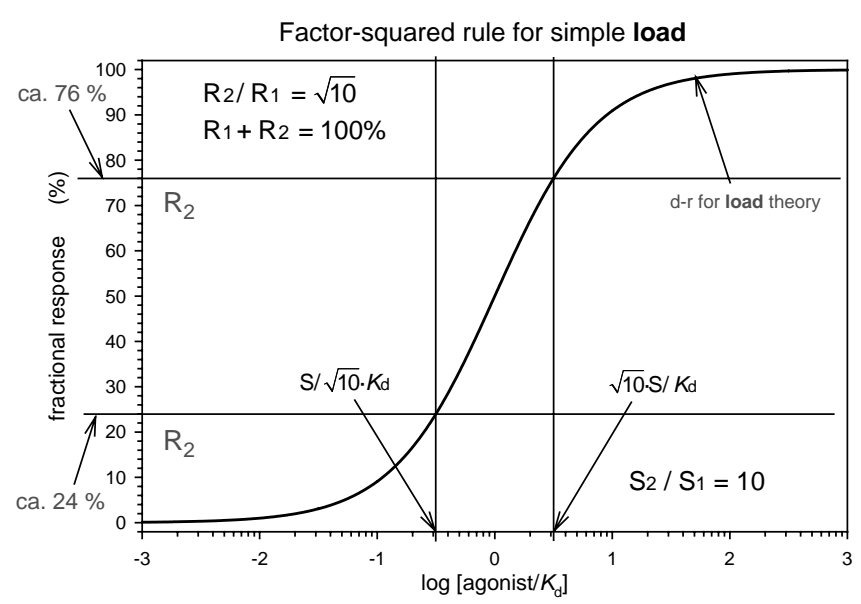

Figure 8.3. Illustration of the factor-squared rule for simple load reaction schemes. For any set of values for the ligand concentration symmetrically distributed around $[S] / K_{d}$ in a load reaction scheme, i.e., with an equal factor $x$ above and below $[\mathrm{S}] / K_{\mathrm{d}}$, such that the ratio $\left[\mathrm{S}_{2}\right] /\left[\mathrm{S}_{1}\right]$ is equal $x^{2}$, the ratio of pertinent responses, $R_{2} / R_{1}$, is the square root of $S_{2} / S_{1}$, equal $x$. The relationship between response ratio and concentration ratio is thus: $\left(R_{2} / R_{1}\right)^{2}=\mathrm{S}_{2} / \mathrm{S}_{1}$. Compare $\mathrm{S}_{2} / \mathrm{S}_{1}$ with the cooperativity index, $R_{\mathrm{a}}$, for the $80-\%$ rule in Section 8.1.3. 
If semi-log plots or ordinary linear plots of doseresponse data deviate significantly from the factorsquared rule, including the $82-$ and $80-\%$ rules, then the underlying mechanism for the observed doseresponse is not a simple load reaction scheme, a plain Langmuirian adsorption interaction or, for that matter, neither a simple ant-agonistic displacement (Figs. 2.5 and 2.6) nor a simple hyperbolic desaturation process.

Using plot programs or training the eye, it is easy to decide on such deviations. However, compared with the 82- and 80-\% rules both describing absolute levels of response change the factor-squared rule when it comes to visual judgment is less obvious as it is based on relative changes in the response (see Figs. 8.2 and 8.3).

\subsubsection{The General Co-operativity Index}

For a genuine Hill scheme, we can now formulate a general relation for the ratio of two responses $\left(R_{2} / R_{1}\right)$ related to two concentrations $\left(\mathrm{S}_{2} / \mathrm{S}_{1}\right)$ symmetric around $K_{\mathrm{d}}$. Thus, a given concentration ratio $\mathrm{S}_{2} / \mathrm{S}_{1}$, identical to the co-operativity index $R_{\mathrm{a}}$ is equal to:

$$
\sqrt[n_{\mathrm{H}}]{\left(R_{2} / R_{1}\right)^{2}} \vee R_{2} / R_{1}^{\left(2 / n_{\mathrm{H}}\right)},
$$

but now $R_{\mathrm{a}}$ is a general co-operativity index. Accordingly, for a Hill-type reaction with a Hill coefficient equal 2, a mere ratio of $x$ for $\mathrm{S}_{2} / \mathrm{S}_{1}\left(=R_{\mathrm{a}}\right)$ is necessary for an $x$-fold change in response (Fig. 8.4).

Observe that the sum of absolute responses $R_{1}$ and $R_{2}$ is always $100 \%$ (Figs. 8.3 and 8.4 ).

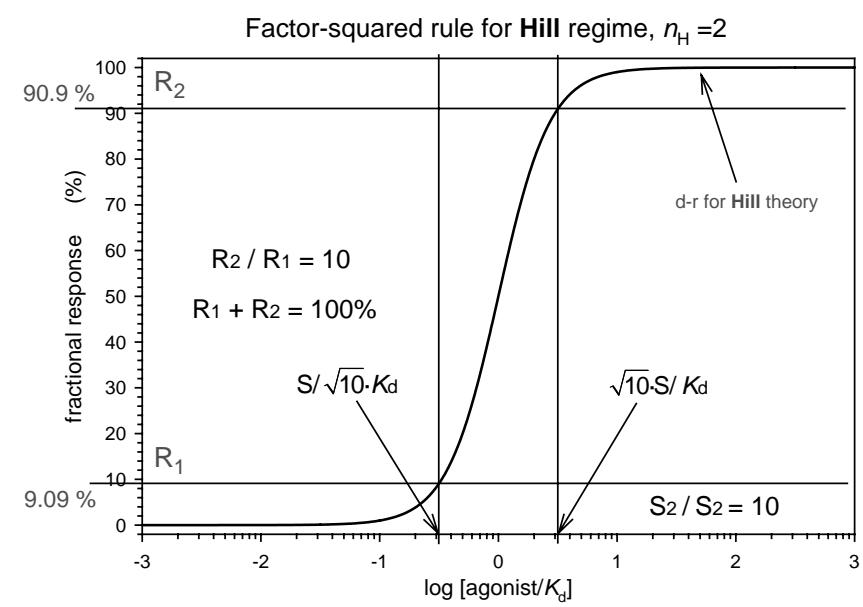

Figure 8.4. Illustration of the factor-squared rule for Hill reaction schemes. In Hill reaction schemes with a Hill coefficient equal $n_{\mathrm{H}}$, for any set of values for the ligand concentration symmetrically distributed around $[S] / K_{\mathrm{d}}$, i.e., with an equal factor above and below $[S] / K_{d}$, such that the ratio $\left[S_{2}\right] /\left[S_{1}\right]$ is equal to $x$ and the ratio of pertinent responses, $R_{2} / R_{1}$, is the square root of $\left(\mathrm{S}_{2} / \mathrm{S}_{1},\right)^{n_{\mathrm{H}}}$. Thus: $\left(R_{2} / R_{1}\right)^{2 / n_{\mathrm{H}}}=\mathrm{S}_{2} /$ $S_{1}$. Here $S_{2} / S_{1}$ is a general co-operativity index for any value of $x$. Compare with $R_{\mathrm{a}}$ for $\mathrm{S}_{2} / \mathrm{S}_{1}$ in Fig. 8.3. In the present example, $x=10, n_{\mathrm{H}}=2$ and therefore $R_{2} / R_{1}=10$.
Further, realize that the above relation between $\mathrm{S}_{2} / \mathrm{S}_{1}$ $\left(=R_{\mathrm{a}}\right)$ and $R_{2} / R_{1}$ also works for inhibitor ligands in reverse Hill schemes (see, for instance, Eq. 8.4 and Section 8.2.4).

\subsubsection{Semi-log S-shape Against Sigmoidality}

My zealous readers should realize that a semi-log plot of ones own data from a hyperbolic reaction scheme as the load would result in a symmetric $S$-shaped curve, which may look just like the sigmoidality of an allosteric doseresponse relation in a linear-linear plot (Fig. 8.1B+C). Recognize that the S-shaped curve of hyperbolic load synagics is due to a simple logarithmic scale-manipulation and is quite different from the real sigmoid relationship of allosteric synagics, except for the appearance of their plotted curves (Fig. 8.1B+C).

\subsubsection{Methods for Non-linear Fitting}

Guidelines on how to apply non-linear methods for parameter fitting and statistical evaluation of synagic data - that is data from dose-responses at equilibrium, are not detailed here. For these guidelines consult a couple of the following texts: Waud (1975), Johnson and Frasier (1985), Ratkowsky (1990), Wells (1992), Bevington and Robinson (1992), Lawson and Hanson (1995), Winzor and Sawyer (1995), Lew and Angus (1997), Severini (2000), Grafen and Hails (2002), Samuels and Witmer (2002), Marangoni (2003, pp. 33-36), Kenakin (2004), Motulsky and Christopoulos (2004), Witte and Witte (2004) and Fox (2008). Also, see Eisenthal and Cornish-Bowden (1974) and CornishBowden and Eisenthal (1974).

\subsection{A Training Session}

\subsubsection{An Exercise}

As a training session, it is my intention in this Subchapter to demonstrate how you may select appropriate tools to analyze data from experiments.

As mentioned in the introduction to Part III, we will differentiate between the terms 'intervention' and 'modulation' in order to keep one- and two-state models apart, OSM versus TSM (Table 8.1).

I have chosen an experimental situation where an antagonist is suspected of inhibiting a process already activated by an agonist in an interventory or modulatory manner. The goal of this examination is to train you to pick out a suitable formulation for the analysis of your own experimental data, to test it, and to eventually reject the employed hypothesis. Thus, the aim is to allow you later on, on your own, to select or from the very start set 
Table 8.1. Comparable Terms from OSM and TSM Modeling

\begin{tabular}{|c|c|c|}
\hline $\begin{array}{l}\text { One-state models } \\
\text { (mixed inhibition/ } \\
\text { acceleration) }\end{array}$ & $\begin{array}{l}\text { Two-state } \\
\text { models } \\
\text { (allostery) }\end{array}$ & Comments \\
\hline Intervention & Modulation & $\begin{array}{l}\text { Heterotropic site interaction } \\
\text { parameter } c \neq 1\end{array}$ \\
\hline Auto-intervention & Co-operativity & $\begin{array}{l}\text { Homotropic site interaction } \\
\text { parameter } c^{\prime} \neq 1\end{array}$ \\
\hline
\end{tabular}

Parameters $c$ and $c^{\prime}$ are defined in Chapters 2, 3 and 7 .

up a theory formulated as an equation that will describe the observed data points of an actual dose-response relationship and, if matching the experimental data, based on its formulation enable you to estimate the value of parameters in the selected theory; mediating additional insight into the regulatory processes of your system.

\subsubsection{Formulation of Simple Intervention}

For our exercise, I have selected intervention with suspected site interaction as an example. From earlier experience, we know that intervention described in Chapter 2 (or modulation, see Chapter 15) may be dealt with by simply raising the symbols for concentration in our load formulations to a power. The power exponent is a parameter in our equation. This type of exponentiation was first implemented by Hill in an attempt to deal with the load-deviant behavior of oxygen-binding to hemoglobin; not for the purpose of describing the mechanism behind the deviant behavior, but merely as a practical handle (Hill 1910). (More on the Hill equation can be found in Chapter 10.) Although such a procedure may not have the right physical meaning, we will use it as an instructive instrument. What we are seeking to extract are estimates for the parameters of a theory, our tool, such that the equation of the theory, with these estimates inserted, best fits the experimental data.

In the realm of allosteric schemes, there are numerous models possible, with or without immanent cooperativity and/or heterotropic modulation. But, with a choice of inhibition due to simple negative intervention and not to allosteric modulation, it turns out to be a selection between three special theories related to socalled 'mixed-inhibition' (Tables III.2 and 8.1). Within the general intervention scheme (Fig. 2.1), it is a model of non-competitive inhibition, of competitive inhibition type II, or of full and negative interventory interactions when $c>0$ (Chapter 2).

For our exercise, to start simple, the equations required are: (1) the Langmuirian load, (2) the reverse-Langmuir or hyperbolic decay equation, (3) the
Hill equation, and (4) the reverse Hill equation. What I mean by all this can be explained by Eqs. 8.1-8.4.

The exercise will equip you with a better feel for formulating a reaction scheme that matches your own particular experimental system.

\subsubsection{The Hill and the Reverse Hill}

The Langmuir Eq. 1.11 for the load for agonists may be written as:

$$
y=\frac{y_{\max }}{1+\left(K_{\mathrm{ss}} / \mathrm{S}\right)},
$$

(Fig. 8.5A), while the hyperbolic decay equation for inhibitors $^{1}$ may be written as:

$$
y=\frac{y_{\max }}{1+\left([\mathrm{I}] / K_{\mathrm{ii}}\right)},
$$

(Fig. 8.5B), a companion Hill equation to Eq. 8.1 can be equated as:

$$
y=\frac{y_{\max }}{1+\left(K_{\mathrm{ss}} / \mathrm{S}\right)^{n_{\mathrm{s}}}},
$$

(Fig. 8.5C), and a reversed Hill equation as a pendant to Eq. 8.2 is equal to:

$$
y=\frac{y_{\max }}{1+\left([\mathrm{I}] / K_{\mathrm{ii}}\right)^{n_{\mathrm{i}}}},
$$

(Fig. 8.5D). Eq. 8.4 is the so-called reverse Hill equation and is simply a formulation of an inhibitor displacing an agonist binding or blocking an agonist response by involving more than one binding site for the inhibitor and formulated for a negative intervention or a modulatory reaction scheme.

In the Hill Eq. 8.3, $n_{\mathrm{s}}$ is Hill's coefficient for a number of agonist binding sites, and in the reverse Hill Eq. 8.4, $n_{\mathrm{i}}$ is the Hill coefficient for the number of interventor or modulator binding sites. Both the Hill equation and the reverse Hill equation have the underlying assumption of an all-or-none reaction equal to simultaneity (Chapter 10: Hill-in-Hell). 'Hill' describes a multi-site phenomenon and not the site-interaction effects of intervention or modulation. Still, the Hill equations can fit to doseresponses with underlying site interaction. The best fit value of the Hill coefficients $n_{\mathrm{s}}$ and $n_{\mathrm{i}}$ is a semiquantitative estimate of the underlying processes of site interaction. An example of the discrepancy between 'Hill' and site-interaction is illustrated in Fig. 6.11.

In case the agonist itself renders a display of 'site interaction' in its dose-response curve, we analyze socalled auto-intervention or co-operativity with Eq. 8.3.

\footnotetext{
${ }_{1}$ Linearized forms of the hyperbolic decay equation are the CornishBowden and Dixon plots (Table 8.3).
} 

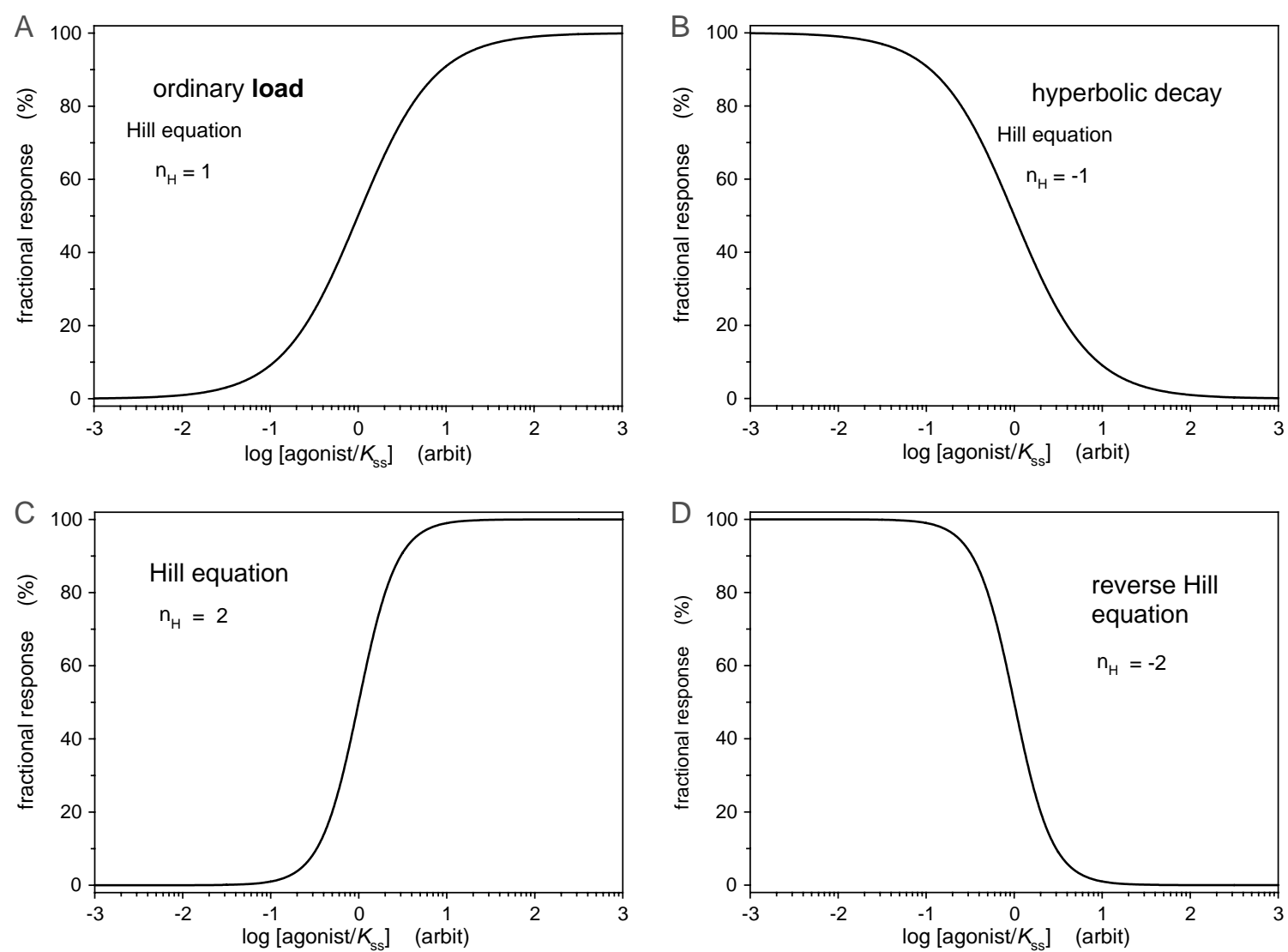

Figure 8.5. Semi-log plots of agonist load (A), inhibitor load (B), Hill (C), and reverse Hill (D). The inhibitor or ant-agonist load $(B)$ is known as 'hyperbolic decay', while the semi-log plot of a Hill equation (C) by some is called a 'logistic equation'. Consequently, in fact all four semi-log presentations (A-D) are 'logistic equations' or 'logistic plots' because the independent variable concentration is transformed to a logarithmic scale in all four and not just for the Hill equation.

Eq. 8.4 is for the analysis of dose-response curves with indication of intervention or modulation, similar to Eq. 8.3, but now for an inhibitory action with a molecule different from the agonist and binding to it own sites with dissociation constant $K_{\mathrm{ii}}$.

Therefore, in practice, the Hill equation is used conveniently for evaluating agonistic site interactions, for example, when there is auto-intervention or cooperativity, just as the reverse Hill equation may be employed conveniently for the study of inhibitory relations, when dose-responses for alien agents display possible negative intervention or modulation in the presence of an agonist.

\subsubsection{Non-competitive Versus Competitive Type II Intervention or Auto-intervention}

In our exercise, an issue that must be addressed initially is which type of inhibition is at play in the hyperbolic decay and its deviation described in Eqs. 8.2 and 8.4. Which intervention or modulation do the hyperbolic decay equations describe? Alternatively, how are we to understand the dissociation constant $K_{\mathrm{ii}}$ in Eq. 8.2 as well as in the reverse Hill Eq. 8.4, likely measured as an experimental $\mathrm{IC}_{50}$ ? In Chapter 2, we saw that $K_{\mathrm{ii}}$ could be the dissociation constant for competitive inhibition type II, for non-competitive inhibition reactions, or for full intervention schemes where $c \geq 0 . K_{\mathrm{ii}}$ may thus have different meanings. Of course, only the experience of our experimentation can guide us in selecting the best formulation and the most appropriate understanding of the $\mathrm{IC}_{50}$ and its underlying $K_{\mathrm{ii}}$.

As an example, in competitive type II inhibition, the $\mathrm{IC}_{50}$ is dependent on the concentration used for the agonist, while the $\mathrm{IC}_{50}$ is independent of agonist concentration used for non-competitive synagics. In this latter case, the apparent $K_{\mathrm{ii}}$ remains the same as the real $K_{\mathrm{ii}}$ no matter what concentration of agonist is used (confer with equations in Sections 2.4.5 and 2.4.10). Thus, for an inhibitor, the hyperbolic decay Eq. 8.2 and the reverse Hill Eq. 8.4 are for noncompetitive reactions (cf. with inhibition parameters in Table 8.3), whereas, the dose-response curve for a competitive inhibitor is breaking the factor-squared rule (Section 8.1.4).

Since the present reverse Hill Eq. 8.4 for noncompetitive synagics is not a reverse Hill equation for 
Table 8.2. Characteristics of Theoretical Dose-response Curves According to Eq. 8.5b

\begin{tabular}{llll}
\hline $\begin{array}{l}\text { Altering fixed ratio } A_{\mathrm{s}} / A_{\mathrm{i}} \\
\text { by increasing parameter }\end{array}$ & Top-point of $d-r$ curve move & Broadness of bell & Fitted Hill coefficient $\left(n_{\mathrm{H}}\right)$ \\
\hline & For $n_{\mathrm{s}}<n_{\mathrm{i}}$ & & \\
\cline { 2 - 4 }$A_{\mathrm{s}}$ & No & Increase & - \\
$A_{\mathrm{i}}$ & Left & Decrease & \\
& For $n_{\mathrm{s}}>n_{\mathrm{i}}$ & - & Increase towards $n_{\mathrm{s}}$ \\
\cline { 2 - 4 }$A_{\mathrm{s}}$ & Left & - & Decrease towards $n_{\mathrm{i}}$ (zero) \\
\hline
\end{tabular}

Principal effects on curve appearance due to changes in values of parameter $A_{\mathrm{s}}$ or $A_{\mathrm{i}}$ for either $n_{\mathrm{s}}<n_{\mathrm{i}}$ or $n_{\mathrm{s}}>n_{\mathrm{i}}$. The observed shifts in curve appearance are for the top-point and broadness of bell-shaped dose-responses, i.e., for increasing [S] when $n_{\mathrm{s}}<n_{\mathrm{i}}$. For continuously increasing dose-responses from 0 to $100 \%$ response , shifts are in positioning and change of fitted Hill coefficients for the curves, i.e., for increasing [S] when $n_{\mathrm{s}}>n_{\mathrm{i}}$. Examples of the appearance of theoretical doseresponse curves with altered parameters for Eq. $8.5 \mathrm{~b}$ are shown in Fig. 8.9 (panels E-H).

competitive inhibition type II, which equation will describe competitive inhibition type II with possible 'site interaction'? Furthermore, what about a doseresponse with possible auto-intervention for an agonist also deviating from the factor-squared rule?

With its base in competitive synagics, the apparent $K_{\mathrm{ii}}$ is a function of the agonist concentration, where the apparent $K_{\mathrm{ii}}$ equals $\mathrm{IC}_{50}=K_{\mathrm{ii}} \cdot\left(1+\mathrm{S} / K_{\mathrm{ss}}\right)$ (see Section 4.2.2 and 4.2.4), even when it is assumed there is no agonist-induced intervention. When intervention is involved as well, the apparent $K_{\mathrm{ii}}$ may be approximated with $\mathrm{IC}_{50}=\left(K_{\mathrm{ii}}\right)^{n_{\mathrm{i}}} *\left(1+\left(\mathrm{S} / K_{\mathrm{ss}}\right)^{n_{\mathrm{s}}}\right)$. The question now is whether this means that our reverse Hill equation for competitive inhibition type II with different multi-site effects for agonist and ant-agonist molecules should be:

$$
y=\frac{y_{\max }}{1+\left(\mathrm{I}^{n_{\mathrm{i}}} / \mathrm{IC}_{50}\right)}=\frac{y_{\max }}{1+\frac{[\mathrm{I}]^{n_{\mathrm{i}}}}{K_{\mathrm{ii}}^{n_{\mathrm{i}}} \cdot\left(1+\left(\mathrm{S} / K_{\mathrm{ss}}\right)^{n_{\mathrm{s}}}\right)}}
$$

In the right-hand side of Eq. $8.5 a$, the $\mathrm{IC}_{50}$ is replaced with $\left(K_{\mathrm{ii}}\right)^{n_{\mathrm{i}}} \cdot\left(1+\left(\mathrm{S} / K_{\mathrm{ss}}\right)^{n_{\mathrm{s}}}\right)$.

A similar description with an alternative derivation was carried out and used for experimental data analysis by Lazarenao and Birdsall (1993a,b). Their derivation was built on Waud's inclusion of the Schild approach (Waud 1975, 1976; Waud et al. 1978) and was completed with Hill's multi-site 'interaction'.

Another possible derivation is to take the distribution function of competitive inhibition type II (Eq. 2.15), and simply raise the concentration terms to a power. Thus, Eq. 2.15 becomes:

$$
y=\frac{y_{\max }}{1+\frac{1+\left([\mathrm{I}] / K_{\mathrm{ii}}\right)^{n_{\mathrm{i}}}}{\left([\mathrm{S}] / K_{\mathrm{ss}}\right)^{n_{\mathrm{s}}}}} .
$$

Eqs. $8.5 a$ and $8.5 b$ we may baptize the 'vent-auto-ventII' equation, since it is supposed to apply when a synagic scheme is a competitive inhibition type II displaying intervention and/or negative auto-intervention or rather multi-site effects for both agonist and ant-agonist. However, is it the right formulation? We will examine this in Section 8.2.7.

\subsubsection{Actual Plus Remaining Response and Their Logit Formulation}

In Chapter 2 on simple competitive, non-competitive, and interventory inhibition, we saw how it is possible to switch between the actual response and the not-yet induced effect - the remaining response - for possible responses that operate between 0 and $100 \%$, where $100 \%$ is equal to the maximal response for a given system. Reaction schemes for the remaining activity or occupancy are in Sections 2.4.7 and 2.4.11, and a plot of this is shown in Fig. 2.5.

I have named the induced effect due to a substrate or an agonist at a given concentration $\left[S^{\prime}\right]$, the actual response $(a r)$, and the remaining not-yet induced effect we can call the remaining response $(r r)$. If the total possible response is $T R$, then we have $T R=a r+r r$ (Fig. 8.6A).

At a given interventor concentration equal [ $\left.\mathrm{I}^{\prime}\right]$ eliciting an inhibitory response in an already induced response due to an agonist, the actual inhibitory response for an interventor is called air, and the remaining not-yet induced inhibitory response we may call the remaining inhibitory response ( rir). The total inhibitory response is $T I R$, therefore we have $T I R=$ air + rir (Fig. 8.6B).

We can now write a general and simple expression for the responses to agonists and inhibitors. Later we will deal with both intervention/modulation and auto-intervention/co-operativity involving reversible but different types of interaction between separate sites for binding and induced by either agonists, interventors or modulators, where constants $c$ and $c^{\prime}$ differ from unity (Chapters 2, 7, and 15). Here, in order to simplify matters, we will initially assume no competitive reversible interaction between agonist and inhibitor, and no 

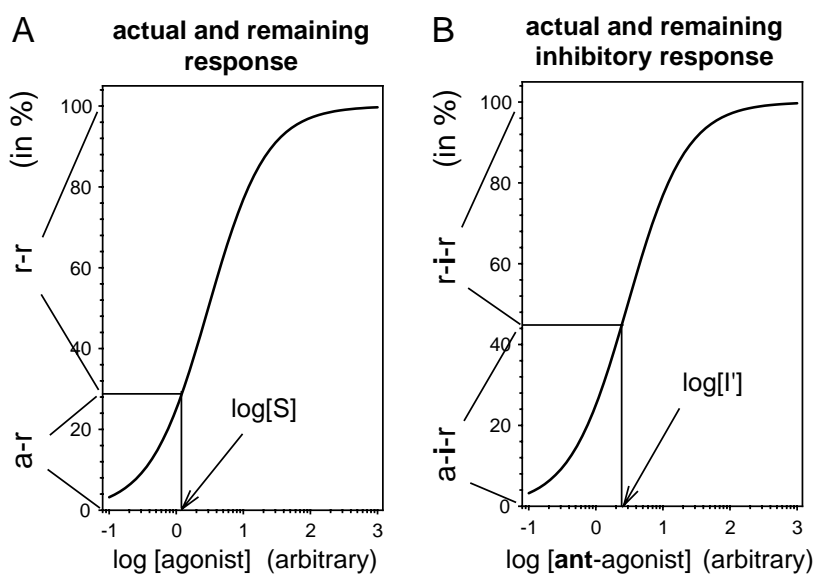

Figure 8.6. Semi-log plots of actual and remaining responses. (A) The dose-response ' $a-r$ ' for an agonist concentration [S'] and the remaining response ' $r-r$ ' for agonist concentrations higher than [S']. The total response $T R=a-r+r-r$. (B) The dose-response ' $a-i-r$ ' for ant-agonist concentration $\left[I^{\prime}\right]$ and the remaining inhibitory response ' $r-i-r$ ' for ant-agonist concentrations higher than $\left[\mathrm{I}^{\prime}\right]$. The total inhibitory response $T I R=a-i-r+r-i-r$.

auto-intervention or co-operativity for either interventor or agonist, i.e., both constants $c$ and $c^{\prime}$ are at unity in the intervention reaction Schemes 2.1 and 3.1, and $n_{\mathrm{s}}$ and $n_{\mathrm{i}}$ are also assumed at unity. This leaves us with a noncompetitive interaction (Fig. 2.5).

For the agonist we have:

(i) the ar given by a Langmurian equation:

$$
\frac{a r}{T R}=\frac{\mathrm{S}}{\mathrm{S}+K_{\mathrm{ss}}},
$$

(ii) and its $r r$, which is given by a reversal of the hyperbolic expression and obtained by for instance subtracting from 1 the expressions on either side of the equal sign:

$$
1-\frac{a r}{T R}=1-\frac{\mathrm{S}}{\mathrm{S}+K_{\mathrm{ss}}} \rightarrow \frac{r r}{T R}=\frac{K_{\mathrm{ss}}}{\mathrm{S}+K_{\mathrm{ss}}} .
$$

(iii) A relative expression of Eq. 8.6 is obtained by subtracting the nominators from the denominators (regula detri rule $1 \mathrm{~b}$ in Box 1.1) or dividing Eq. 8.6 with Eq. 8.7, thus:

$$
\frac{a r}{T R-a r}=\frac{\mathrm{S}}{\mathrm{S}+K_{\mathrm{ss}}-\mathrm{S}} \rightarrow \frac{a r}{r r}=\frac{\mathrm{S}}{K_{\mathrm{ss}}},
$$

(iv) taking the $\log$ on both sides of this equation, we obtain a so-called 'logit' of the ar function in Eq. 8.6:

$$
\log \frac{a r}{T R-a r}=\log \frac{a r}{r r}=\log \mathrm{S}-\log K_{\mathrm{ss}} .
$$

The linear and semi-log plots of Eqs. 8.6 and 8.9 are illustrated in Fig. 8.7A+B and 8.7C, respectively.
For the non-competitive interventor, the comparable expressions are:

(i) the air, a Langmuir-type equation:

$$
\frac{a i r}{T I R}=\frac{\mathrm{I}}{\mathrm{I}+K_{\mathrm{ii}}}
$$

(ii) its rir, a hyperbolic decay equation:

$$
1-\frac{\text { air }}{T I R}=1-\frac{\mathrm{I}}{\mathrm{I}+K_{\mathrm{ii}}} \rightarrow \frac{r i r}{T I R}=\frac{K_{i}}{\mathrm{I}+K_{\mathrm{ii}}} .
$$

Reciprocal transformations of this equation to linearity are no longer modern, but in passing, for historical reasons, I may mention that two of its linearized forms are the so-called Dixon and Cornish-Bowden plots (see Table 8.3).

(iii) Using r.d. rule $1 \mathrm{~b}$ in Box 1.1, the relative expression for non-competitive inhibition in Eq. 8.10 becomes:

$$
\frac{\text { air }}{T I R-\text { air }}=\frac{\mathrm{I}}{\mathrm{I}+K_{\mathrm{ii}}-\mathrm{I}} \rightarrow \frac{\text { air }}{\text { rir }}=\frac{\mathrm{I}}{K_{\mathrm{ii}}},
$$

which may also be obtained by dividing Eq. 8.10 with Eq. 8.11.

Again (iv) taking the $\log$ on both sides of this expression yields the logit of air in Eq. 8.10:

$$
\log \frac{\text { air }}{\text { TIR }- \text { air }}=\log \frac{\text { air }}{\text { rir }}=\log \mathrm{I}-\log K_{\mathrm{ii}} .
$$

The linear and semi-log plots of Eqs. 8.10 and 8.13 are depicted in Figs. 8.7D $+\mathrm{E}$ and 8.7F, respectively.

In case the results from our experiments do not indicate that the agonist and inhibitor compete exclusively for the same sites (simple competitive type I antagonism), and further as assumed above, neither the agonist nor the inhibitor exhibit auto-intervention or cooperativity (no deviation from the $82-\%$ rule), then the above cited equations and related plots may be used for an analysis of our experimental data. Thus, if we have stimulated a system partially with an agonist, applying increasing concentrations of an inhibitor should yield plots such as Figs. 2.5 and 8.7. Further, we may use either Eqs. 8.10 or 8.11 in order to extract fitted parameter values for $T I R$ and $K_{\mathrm{ii}}$. Chapter 9 provides details on how to plot and analyze such expressions.

\subsubsection{Overlaid Intervention Involving Hill or Reverse Hill Equations. The Hill Plot}

In Chapter 10: 'Hill in Hell', the reaction scheme described by Hill's equation is most unlikely, since the implications of this formula is a simultaneous or complete occupancy at all the reaction sites before any response can take place, and such a scheme is probably not relevant in most equilibrium systems. On the other hand, Hill's principle may be used as a semi-quantitative 

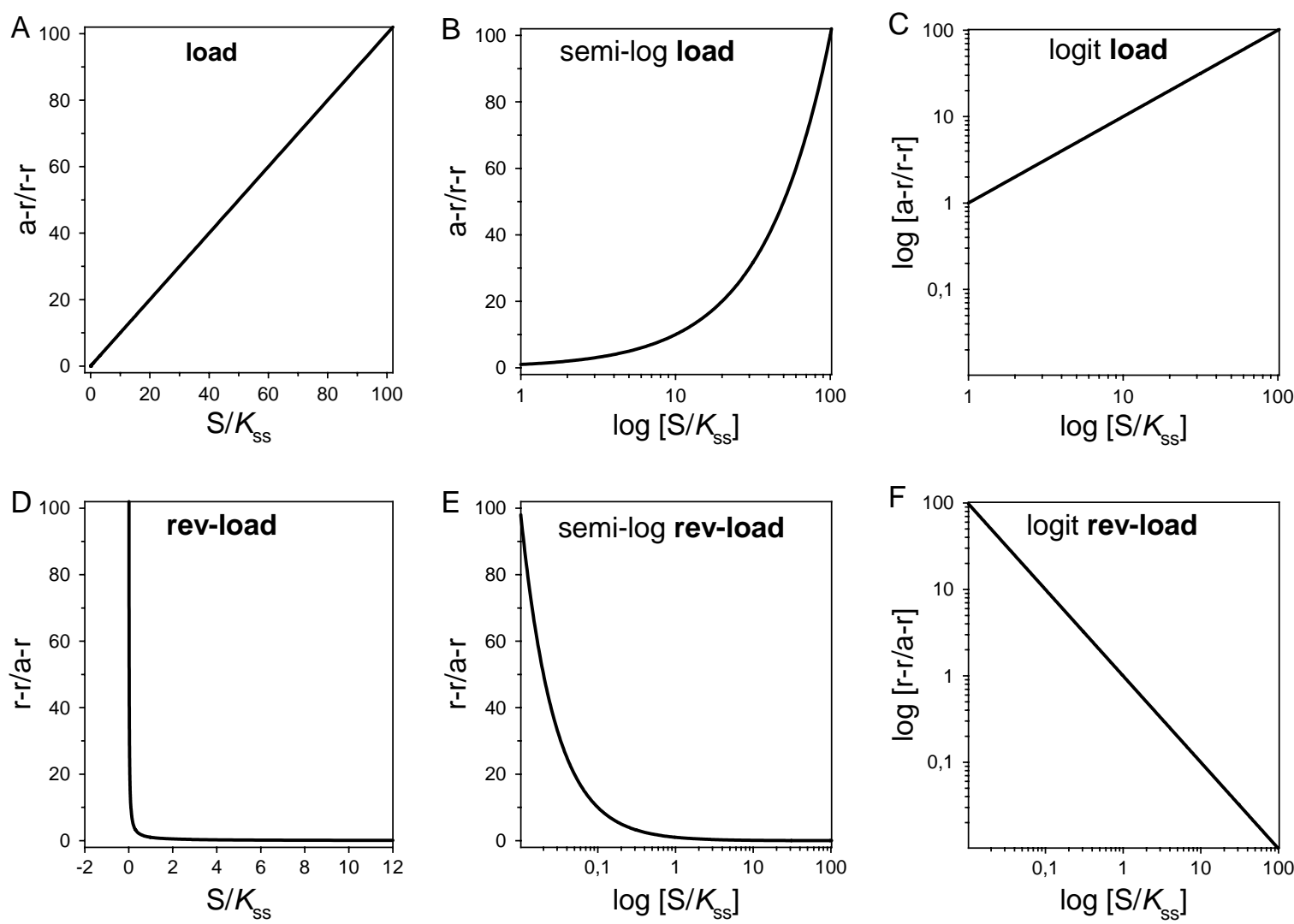

Figure 8.7. Three plot types are displayed. Linear, semi-log, and logit plots of relative load and relative hyperbolic decay. An example for each of the three plot types is shown for relative load: linear (A), semi-log (B), and logit (C). Additionally, an example for each of the three plot types for relative hyperbolic decay is shown: linear (D), semi-log (E), and logit (F).

measure of the underlying unknown synagic scheme and its possible interventory or modulatory behavior, as long as we recognize the limitations of the determined Hill exponents, $n_{\mathrm{H}}, n_{\mathrm{s}}$, or $n_{\mathrm{i}}$, and the extracted dissociation constants.

Therefore, let me continue by using the Hill expression, with the Hill exponent termed $n_{\mathrm{s}}$ for interactions of agonists at stimulatory sites and a reverse Hill expression with its Hill exponent dubbed $n_{\mathrm{i}}$ for interactions by non-competitive ant-agonists at inhibitory sites different from the stimulatory sites.

First, our theory is agonism with multi-site effects (the factor-squared rule is violated) and to analyze its doseresponse we have a Hillian equation:

(i) for the $a r$ :

$$
\frac{a r}{T R}=\frac{\mathrm{S}^{n_{\mathrm{s}}}}{\mathrm{S}^{n_{\mathrm{s}}}+K_{\mathrm{ss}}^{n_{\mathrm{s}}}}=\frac{1}{1+\left(K_{\mathrm{ss}} / \mathrm{S}\right)^{n_{\mathrm{s}}}},
$$

(ii) and for its $r$.

$$
1-\frac{a r}{T R}=1-\frac{\mathrm{S}^{n_{\mathrm{s}}}}{\mathrm{S}^{n_{\mathrm{s}}}+K_{\mathrm{ss}}^{n_{\mathrm{s}}}} \rightarrow \frac{r r}{T R}=\frac{1}{1+\left(\mathrm{S} / K_{\mathrm{ss}}\right)^{n_{\mathrm{s}}}} .
$$

(iii) The relative Hill equation of Eq. 8.14 is obtained by using r.d. rule $1 \mathrm{~b}$ in Box 1.1, or dividing Eq. 8.14 by Eq. 8.15:

$$
\frac{a r}{T R-a r}=\frac{\mathrm{S}^{n_{\mathrm{s}}}}{\mathrm{S}^{n_{\mathrm{s}}}+K_{\mathrm{ss}}^{n_{\mathrm{s}}}-\mathrm{S}^{n_{\mathrm{s}}}} \rightarrow \frac{a r}{r r}=\left(\frac{\mathrm{S}}{K_{\mathrm{ss}}}\right)^{n_{\mathrm{s}}} .
$$

The linear and semi-log plots of Eq. 8.14 are plotted in Fig. 8.8A + B.

(iv) Taking the log on both sides gives us the logit-Hill

$$
\log \frac{a r}{T R-a r}=\log \frac{a r}{r r}=n_{\mathrm{s}} * \log \mathrm{S}-n_{\mathrm{s}} * \log K_{\mathrm{ss}},
$$

which is a straight line with a slope of $n_{\mathrm{s}}$ (Fig. 8.8C). The logit-Hill (Cornish-Bowden \& Koshland 1975; De Lean et al. 1978) is also known as the 'Hill plot' (Table 8.3) (Taylor \& Insel 1990, p. 55).

Since non-linear fitting to data of the Hillian equation by modern software is as easy as analyzing its logit, we no longer have to make the logit transformation, and can ignore Eq. 8.17 and Fig. 8.8C. However, for statistical evaluation of synagics, logit as well as semi-log transformations may be advantageous (Finney 1971, Chapters 2-4; Motulsky \& Christopoulos 2004, pp. 263-264).

For the ant-agonists, we obtain similar Hillish expressions to be applied on experimental data for which we assume 'site interaction' due to multi-site effects with inhibitory sites 'affecting' each other or at stimulatory sites where the agonist operates and may display 

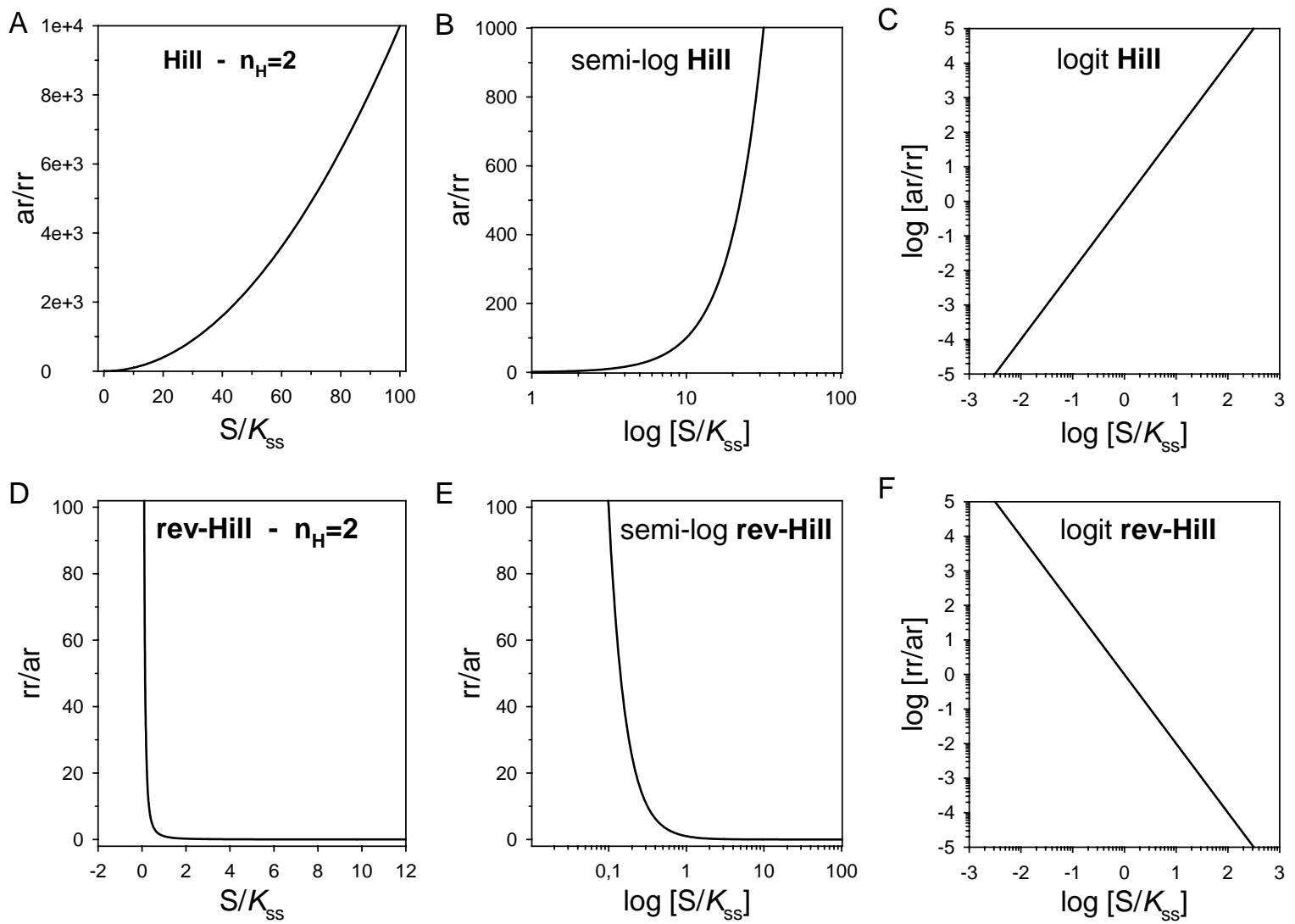

Figure 8.8. Three plot types are displayed. Linear, semi-log, and logit plots of relative Hill and relative reversed Hill. An example for each of the three plot types is shown for relative Hill: linear (A), semi-log (B), and logit (C). Addditionally, an example of each of the three plot types for relative reverse Hill is shown: linear $(D)$, semi-log $(E)$, and logit $(F)$. (F) is also a pseudo-Hill plot.

auto-multi-site effects. These two reaction schemes and their formulation can be used as tools when response data from increasing concentrations of an ant-agonist or the agonist itself seem to violate the factor-squared rule.

Next, to analyze such behavior of a non-competitive inhibitor, but viewed as an increasing action and operating at a multi-sited protein, we have:

(i) a Hill-inspired equation:

$$
\frac{a i r}{T I R}=\frac{\mathrm{I}^{n_{\mathrm{i}}}}{\mathrm{I}^{n_{\mathrm{i}}}+K_{\mathrm{ii}}^{n_{\mathrm{i}}}}=\frac{1}{1+\left(K_{\mathrm{ii}} / \mathrm{I}\right)^{n_{\mathrm{i}}}} .
$$

(ii) Its reverse Hillian type equation for the remaining inhibitory response is:

$$
1-\frac{\text { air }}{T I R}=1-\frac{\mathrm{I}^{n_{\mathrm{i}}}}{\mathrm{I}^{n_{\mathrm{i}}}+K_{\mathrm{ii}}^{n_{\mathrm{i}}}} \rightarrow \frac{r i r}{T I R}=\frac{1}{1+\left(\mathrm{I} / K_{\mathrm{ii}}\right)^{n_{\mathrm{i}}}} .
$$

(iii) With r.d. $1 \mathrm{~b}$ in Box 1.1, the relative expression for Eq. 8.18 is:

$$
\frac{\text { air }}{T I R-\text { air }}=\frac{\mathrm{I}^{n_{\mathrm{i}}}}{\mathrm{I}^{n_{\mathrm{i}}}+K_{\mathrm{ii}}^{n_{\mathrm{i}}}-\mathrm{I}^{n_{\mathrm{i}}}} \rightarrow \frac{\text { air }}{\text { rir }}=\left(\frac{\mathrm{I}}{K_{\mathrm{ii}}}\right)^{n_{\mathrm{i}}} .
$$

Eq. 2.20 may also be obtained by dividing Eq. 8.18 with Eq. 8.19.
As before, (iv) the logit of air in Eq. 8.18 is:

$$
\log \frac{\text { air }}{T I R-\text { air }}=\log \frac{\text { air }}{\text { rir }}=n_{\mathrm{i}} * \log \mathrm{I}-n_{\mathrm{i}} * \log K_{\mathrm{ii}}
$$

simply obtained by taking the $\log$ on both sides of Eq. 8.20. Fig. 8.8D $+\mathrm{E}$ are linear and semi-log plots of Eq. 8.18, and Fig. 8.8F is a plot of Eq. 8.21, a logit or a pseudo-Hill plot (Table 8.3) (Taylor \& Insel 1990, pp. 56-59). Again, the logit transformation is not necessary with modern computer software at hand, and we can skip Eq. 8.21 and Fig. 8.8F, although not in statistical evaluation, cf. with the arguments above about statistical analysis for ordinary Hill plots.

In summary, thus far, for synagic agonism deviating from load, i.e., breaking the factor-squared rule, a useful analytical tool is the Hill-type equation:

$$
\frac{a r}{T R}=\frac{1}{1+\left(K_{\mathrm{ss}} / \mathrm{S}\right)^{n_{\mathrm{s}}}}
$$

depicted in Figs. 8.5C and 8.8A $+\mathrm{B}$.

For a different compound with the effects of interventory inhibition and with its inhibitory response viewed as a rising response, one simple expression for its 
analysis is the reverse Hill dose-response equation for a non-competitive inhibitor:

$$
\frac{\text { air }}{T I R}=\frac{1}{1+\left(K_{\mathrm{ii}} / \mathrm{I}\right)^{n_{\mathrm{i}}}} .
$$

as shown in Figs. 8.5D and 8.8.C+ D.

The coefficients $n_{\mathrm{i}}$ and $n_{\mathrm{s}}$ are equal to 1 in case there is no intervention/modulation for either an interventor or a modulator or no auto-intervention/co-operativity for an agonist or a substrate. Chapter 9 contains guidelines for plotting and non-linear fitting that easily resolves best parameters in these two expressions, Eqs. 8.14 and 8.18 .

Of note, in the full intervention schemes of Subchapters 3.3 and 2.5, the constants $c^{\prime}$ and $c$ are in effect reinterpretations and replacements of the Hill coefficients $n_{\mathrm{s}}$ and $n_{\mathrm{i}}$, although the meaning of the two sets of parameters is totally different. Intervention parameters are for site-interaction, while the Hill parameters are for multi-sitedness.

\subsubsection{When Inhibition is Mutually Exclusive or that of Allostery}

Finally, what happens when the former analyses have to be evaluated under the expectation or suspicion of competitive inhibition type II or between neighboring receptive units with genuine heterotropic allostery, a reaction scheme allowing simultaneous binding of both agonist and modulator? Let us for simplicity assume a system with competitive interaction of the secondary type, i.e., a reaction scheme where individual receptive units affect each other by binding of ligands excluding each other and with no co-lateral binding (see Section 2.4.2 and Sub-chapter 2.6).

In this situation the hyperbolic decay equation must also respect possible different effects for agonist and inhibitor activity at different receptive unit, each possessing binding sites for either agonists or inhibitors; we do not anticipate reciprocal haptic affection between individual receptive units, where each unit has one or more binding sites, but rather sheer multi-site effects.

In the hyperbolic decay equation for non-competitive inhibition, the $K_{\mathrm{ii}}$ will be equal to an apparent dissociation constant for the inhibitor (Chapter 4). For competitive inhibition type II, the apparent dissociation constant $\operatorname{appK}_{\mathrm{ii}}\left(\mathrm{IC}_{50}\right)$ will differ from its $K_{\mathrm{ii}}$ when analyzing for parameter values $T I R, n_{\mathrm{i}}$, and $K_{\mathrm{ii}}$ based on experimental data. In order to acknowledge the influence of the 'interaction' between sites bound with agonist and inhibitor as elicited between two different receptive units, it is a tempting approach to include a correction term for the inhibitor dissociation constant by following a Hill-modified Cheng-Prusoff equation (Chapter 4): $\mathrm{IC}_{50}=\left(K_{\mathrm{ii}}\right)^{n_{\mathrm{i}}} \cdot\left(1+\left(\mathrm{S} / K_{\mathrm{ss}}\right)^{n_{\mathrm{s}}}\right)$. Inserting this expression in Eq. 8.18 yields Eq. $8.5 a$ suggested above, which is repeated here:

$$
y=\frac{y_{\max }}{1+\left(\mathrm{I}^{n_{\mathrm{i}}} / \mathrm{IC}_{50}\right)}=\frac{y_{\max }}{1+\frac{[\mathrm{I}]^{n_{\mathrm{i}}}}{K_{\mathrm{ii}}^{n_{\mathrm{i}}} \cdot\left(1+\left(\mathrm{S} / K_{\mathrm{ss}}\right)^{n_{\mathrm{s}}}\right)}} .
$$

A similar equation was derived but with a Schild slope factor included for the $n_{\mathrm{i}}$ parameter (Lazareno \& Birdsall 1993a) and used to analyze experimental data (Lazareno \& Birdsall 1993b).

We may also just take the ordinary competitive inhibition type II formula and raise its concentration terms with an exponent, as in Section 8.2.4. The derived formula is repeated here:

$$
y=\frac{y_{\max }}{1+\frac{1+\left([\mathrm{I}] / K_{\mathrm{ii}}\right)^{n_{\mathrm{i}}}}{\left([\mathrm{S}] / K_{\mathrm{ss}}\right)^{n_{\mathrm{s}}}}}
$$

Notice that the true $K_{\mathrm{ii}}$ is exponentiated with an inhibitor exponent $n_{\mathrm{i}}$, and the influence of an agonist is also exponentiated with agonist exponent $n_{\mathrm{s}}$.

Eqs. $8.5 a, b$ can easily be handled by modern computer software to extract fitted parameter values (see Chapter 9 for its execution). Examples of such analyses are shown in Figs. 8.9A-D and 8.9E-H.

With knowledge of $[\mathrm{S}]$ and the constants $n_{\mathrm{s}}$ and $K_{\mathrm{ss}}$, these parameter values should be inserted in the equation for hyperbolic decay in type II competitive inhibition (Eq. 8.5). This will yield a better estimate of $K_{\mathrm{ii}}$ although it is only correct when 'Hill interactions' are dominating, i.e., simultaneity in occupancy of all sites (Chapter 10: Hill-in-Hell), or concerted conformational change of all subunits before a response is obtained as in classical MWC-allostery (Chapter 15).

As stated previously, there is no argument for a transformation of Eq. 8.5 to either the logit-Hill or the logit-reverse Hill types of expression. In the examples above, these transformations were only carried out for pedagogic reasons and in order to expose their relevance for statistical evaluation as of today.

\subsubsection{Is it Worth it?}

Yes, as an excercise it is. Meanwhile, unfortunately, the entire exercise in Section 8.2.7 is somewhat futile. It gives no meaning to the attempt to obtain a Hillian type equation for competitive inhibition type II. The problem with Section 8.2.7 is as follows. Prerequisites for synagics of competitive ant-agonism or inhibition do not include possible site interaction in the accepted sense. Competitive inhibition simply competes by playing on multiple identical binding sites. The competitive inhibition schemes do not rely on site interaction. Therefore, it is a contradiction to speak about 'competitive intervention' 

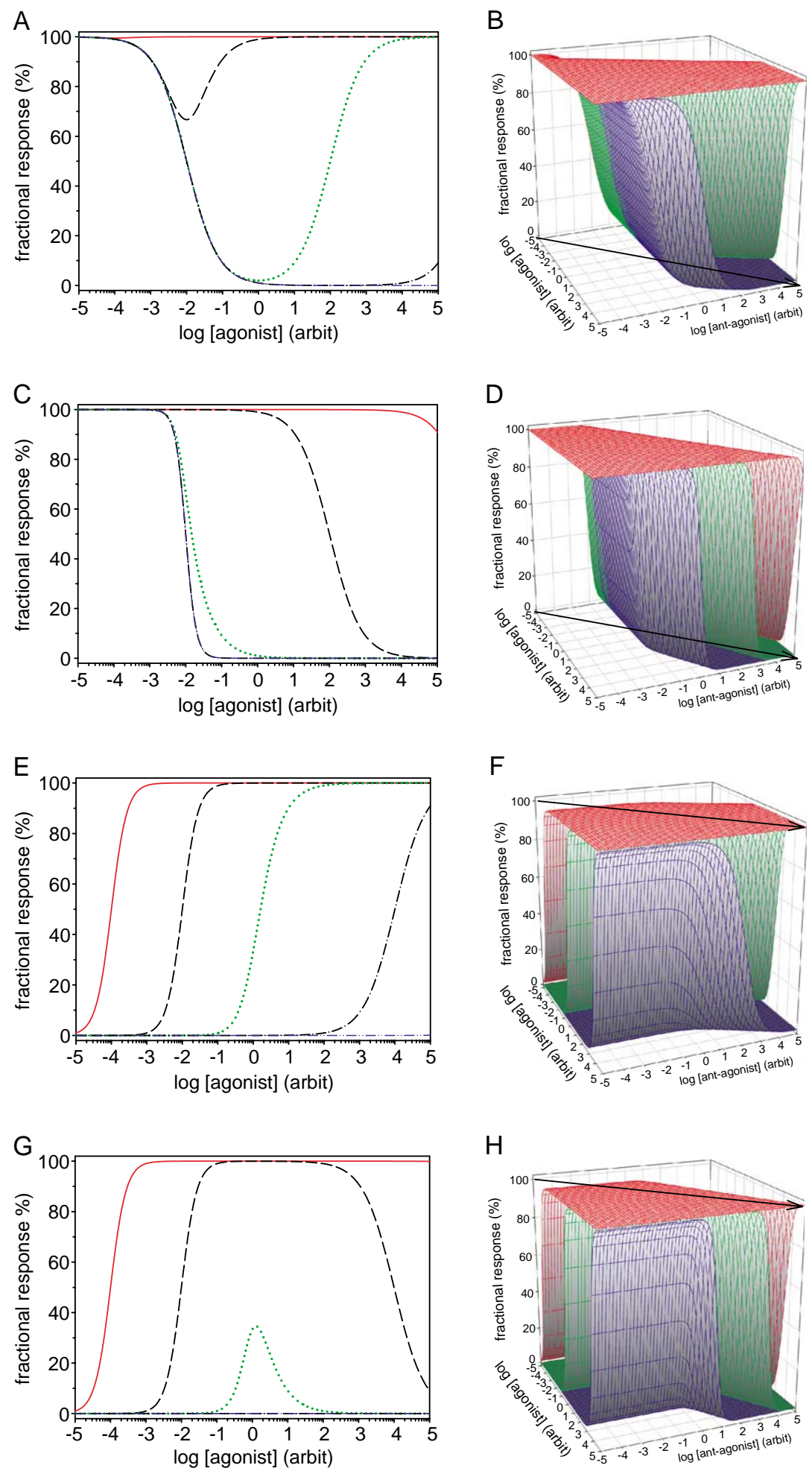

Figure 8.9. Examples of theoretical dose-response curves based on the vent-auto-vent-II equation (Eq. 8.5). Panels A-D for Eq. 8.5a and panels E-H for Eq. 8.5b. In 2-D graphs (panel A, C, E, and G), each plot in either graph is the fractional response as a function of simultaneously increasing both agonist and interventor ('ant-agonist') concentrations, i.e., the ratio [agonist]/[antagonist] is fixed for each plot. This corresponds to following the arrows shown in the concentration plane in the neighboring 3-D graphs (panels $B, D, F$ and $H$ ). The fixed concentration ratio is varied between five plots in 2-D graphs $A, C, E$, and $G$ and between three plots in 3-D graphs $\mathrm{B}, \mathrm{D}, \mathrm{F}$, and $\mathrm{H}$, cf. color code. In 2-D graphs the association constant $A_{\mathrm{s}}\left(=1 / K_{\mathrm{d}}\right.$ or $\left.=1 / K_{\mathrm{ss}}\right)$ is increased by a factor $10^{2}$ in five steps from $10^{-4}(-)$ to $10^{4}\left(-_{-} \cdot{ }^{-\cdot}-{ }_{-}\right)$, and in 3-D graphs by a factor $10^{4}$ in three steps from $10^{-4}$ to $10^{4}$ (red, green, blue) while equilibrium association constant $A_{\mathrm{i}}\left(=1 / K_{\mathrm{ii}}\right)$ is kept equal to 100 in $\mathrm{A}-\mathrm{D}$ and to 1 in $\mathrm{E}-\mathrm{H}$. Panels $\mathrm{A}+\mathrm{B}$ and $\mathrm{E}+\mathrm{F}$ are for $n_{\mathrm{s}}=2$ and $n_{\mathrm{i}}=1$. Panels $\mathrm{C}+\mathrm{D}$ and $\mathrm{G}+\mathrm{H}$ are for $n_{\mathrm{s}}=2$ and $n_{\mathrm{i}}=3$. The general effects of parameter changes are shown in Table 8.2 for the regime in Eq. $8.5 b$ (panels $\mathrm{E}-\mathrm{H}$ ). 
as well as to try to formulate it! Remember that parameters $c$ and $c^{\prime}$ are equal to zero in competitive inhibition (Chapters 2 and 3).

Competitive inhibition is also a contradiction to the intervention-Hillian schemes as the latter presuppose mutual inclusive binding, whereas the competitive inhibition/ant-agonism assumes mutually exclusive binding.

Competitive inhibition type II and its analysis in Subchapter 8.2 could be valid if we postulated a new receptor-substrate action, where for instance an earlier binding to a secondary receptive unit, no longer liganded, could still elicit a conformational change of some extended duration in the primary receptive unit, thereby preventing the agonist from binding here. However, in reality, that is abandoning the concept of competitive inhibition. Rather, to solve this dilemma, we may accept a move into issues on time-dependent (1) hysteresis (Paton 1961; Gosselin 1969; Frieden 1970; Storer \& Cornish-Bowden 1977; Kaiser 1980; Topham 1990; Topham \& Brocklehurst 1992); and (2) nonequilibrium effects (Cornish-Bowden \& Storer 1986; Lazareno \& Birdsall 1995; Lazareno et al. 2000; Lew et al. 2000; Avalani et al. 2004; May 2007). Certainly, its discussion will uproot us from synagics to kinetics, a topic beyond this text.

Thus, we learn that simple competitive inhibition with two binding sites cannot be transformed into an interventory or modulatory scheme, not to mention a possible Hillian transformation with several binding sites for the same ligand. Simple competitive inhibition does not match with intervention or, so to speak, with 'Hill-intervention'.

Another question arises in this connection. Since Schild analysis is based on simple competitive inhibition with $c=0$, can the Schild analysis be implemented for an intervention scheme, for instance an 'allosteric' ternary-complex model with two different binding sites and $c>0$ ?

Well, independent of the reader's answer, such an analysis has been carried out by Ehlert (1988).

\subsection{Scatchard's Plot and Non-reciprocal Tools with Non-linear Adjustments}

\subsubsection{The Scatchard-(Hofstee) Analysis}

A brief account of the derivation of the Scatchard(Hofstee) plot and its use in analysis can be found in several texts (e.g., Winzor \& Sawyer 1995, Chapter 7) and in greater detail in, for instance, Segel $(1975,1993)$.

To trigger the reader's curiosity and welcomed opposition, I classify the Scatchard plot as an obsolete plot (see Table 8.3).

\subsubsection{Historic Development of Reverse Plots}

Barnett Woolf is said to have originally suggested the three classical reciprocal forms of the HMM relation for response versus concentration ( $R$-vs-S) (Haldane \& Stern 1932; Haldane 1957), where HMM stands for the steadystate s-load equation (see note 1 in Chapter 10). Historically, succeeding Woolf, a $v /[\mathrm{S}]-\mathrm{vs}-[\mathrm{S}]$ form of the s-load relation was first suggested by Hanes (1932) (Fig. 8.10B), followed closely by Lineweaver-Burk (1934), who used the double reciprocal $1 / v$-vs- $1 /[\mathrm{S}]$ plot to analyze data (Fig. 8.10C). The $v$-vs- $v /[\mathrm{S}]$ Woolfplot was re-derived by Eadie (1942), then by Augustinsson (1948), and later by Hofstee (1952) (Fig. 8.10D). Scatchard (1949) suggested a reverse plot of the Eadie $v$-vs- $v /[\mathrm{S}]$ plot, viz. $v /[\mathrm{S}]-\mathrm{vs}-v$. Scatchard's plot is obtained by switching axes in Fig. 8.10D. Some ascribe this latter plot type to Eadie as well, and therefore it is designated 'an Eadie-Scatchard plot' (e.g., Segel 1975, 1993). However, as Eadie meant a $v$-vs- $v /[\mathrm{S}]$ and not a $v /[\mathrm{S}]-\mathrm{vs}-$ $v$ plot (see also Eadie et al. 1949), we ought to speak about an Eadie plot and a Scatchard plot. As the Eadie and Scatchard plots are mere mirror images of each other, in a sober-minded wor(1)d we may nevertheless talk about an Eadie-Scatchard plot. The plots are listed in Table 8.3.

In cotransport literature, as an example, the Eadie plot $(v-v s-v /[\mathrm{S}])$ is most often referred to as a Hofstee plot. However, the correct designation of an Eadie/ Hofstee plot is a Woolf-Eadie-Augustinsson-Hofstee plot (a WEAH plot).

\subsubsection{Comparing Semi-log Plots with Hanes, L\&B and Eadie-Scatchard Plots}

Figure 8.10 shows the four most general plots of presenting and evaluating concentration-binding or dose-response data according to load at equilibrium or steady-state - simple synagics. These plots are (1) the semi-log plot (Fig. 8.10A) which may be combined with non-reciprocal tools and non-linear parameter fitting; and the reciprocal plots of (2) Hanes (Fig. 8.10B), of (3) Lineweaver-Burk (L\&B) (Fig. 8.10C), and of (4) EadieScatchard (Fig. 8.10D). The three reciprocal plots may be combined with linear regression analysis. All three reciprocal plot types have had their proponents (Hofstee [Dixon \& Webb] 1959; Cressie \& Keightley 1981).

At this point, though stated several times before, I am tempted to reiterate some issues. Judging and comparing the above four analyses, the non-reciprocal transformed plots, semi-log and logit in Table III.1, combined with non-linear parameter fitting is the most reliable and useful method even for the simplest adsorption scheme (Burgisser 1984). 

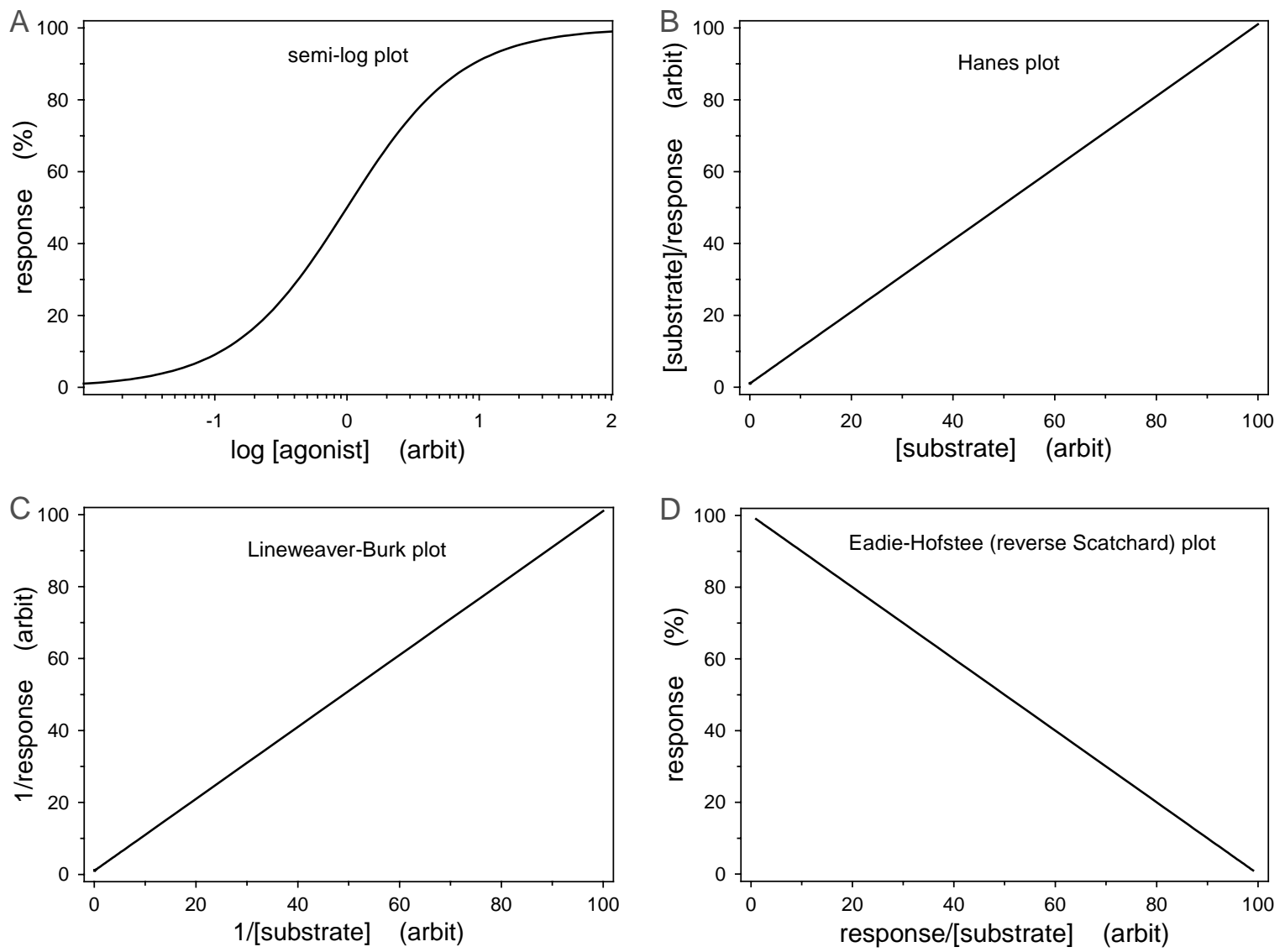

Figure 8.10. Four plot types of simple load dose-response. Semi-log (A), Hanes (B), double reciprocal or Lineweaver-Burk (C), and Eadie Scatchard (D). Details about plots B, C and D can be found in, for instance, Segel (1975, 1993).

Fitting parameters may be carried out on either a linear-linear plot or its semi-log form by software such as SigmaPlot, as demonstrated and effectuated in Chapter 9. Of the three remaining types of analyses, in general the Hanes plot is the most reliable with the L\&B plot the least reliable (Dowds \& Riggs 1965; Keightley \& Cressie 1980). Although the two L\&B coordinates are less meaningful than the co-ordinate axes of the Hanes and Eadie-Scatchard plots (Hofstee 1959; Eisenthal \& Cornish-Bowden 1974; Cornish-Bowden \& Eisenthal 1974; Cornish-Bowden 1995), analysis of data using the L\&B plot have been preferred by many researchers because its two co-ordinates, $1 /$ response vs $1 /$ concentration, are statistically independent, which they are not for either the Hanes or the EadieScatchard plots (Hofstee 1959, p. 1298). In addition, independent co-ordinates are required for a sound statistical analysis (Atkins \& Nimmo 1975, 1980). See Section 8.1.7 for more literature on tools for non-linear fitting of theory to experimental data and its statistical evaluation.

The arguments about the benefits of reciprocal plots do not hold for a comparison with a theory evaluated by non-linear parameter fitting to non-transformed experimental data in an ordinary linear-linear or a semi-log representation. Thus, of all four plots, the non-linear parameter fitting of theory is the most reliable in a semilog or logit transform of either load or Hill equations to experimental data.

Therefore, the conclusion is: Always use semi-log plotting of $R-v s-\mathrm{S}$ for an immediate evaluation and non-linear regression to further analyze your experimental concentration-binding or dose-response data.

\subsubsection{When Assumptions do not Hold}

Characteristics of how intervention, modulation or a distribution of receptors with non-homogeneous (heterogeneous) binding or response affect the four plots are shown in Figs. 8.11 and 8.12.

Clearly, hints about deviant behavior from simple load are revealed in all four types of plots (compare panels A$\mathrm{D}$ in Fig. 8.10 with identical panels in Figs. 8.11 and 8.12). At face value, reciprocal transforms of deviant data result in more spectacular theory-divergent plots than the semi-log plot. Notwithstanding, with experience and use of the factor-squared rule, the semi-log plot will reveal aberrant behavior just as well Fig. 9.28D.

However, researchers are often tempted to accept linear models and linear regression even when it is not 

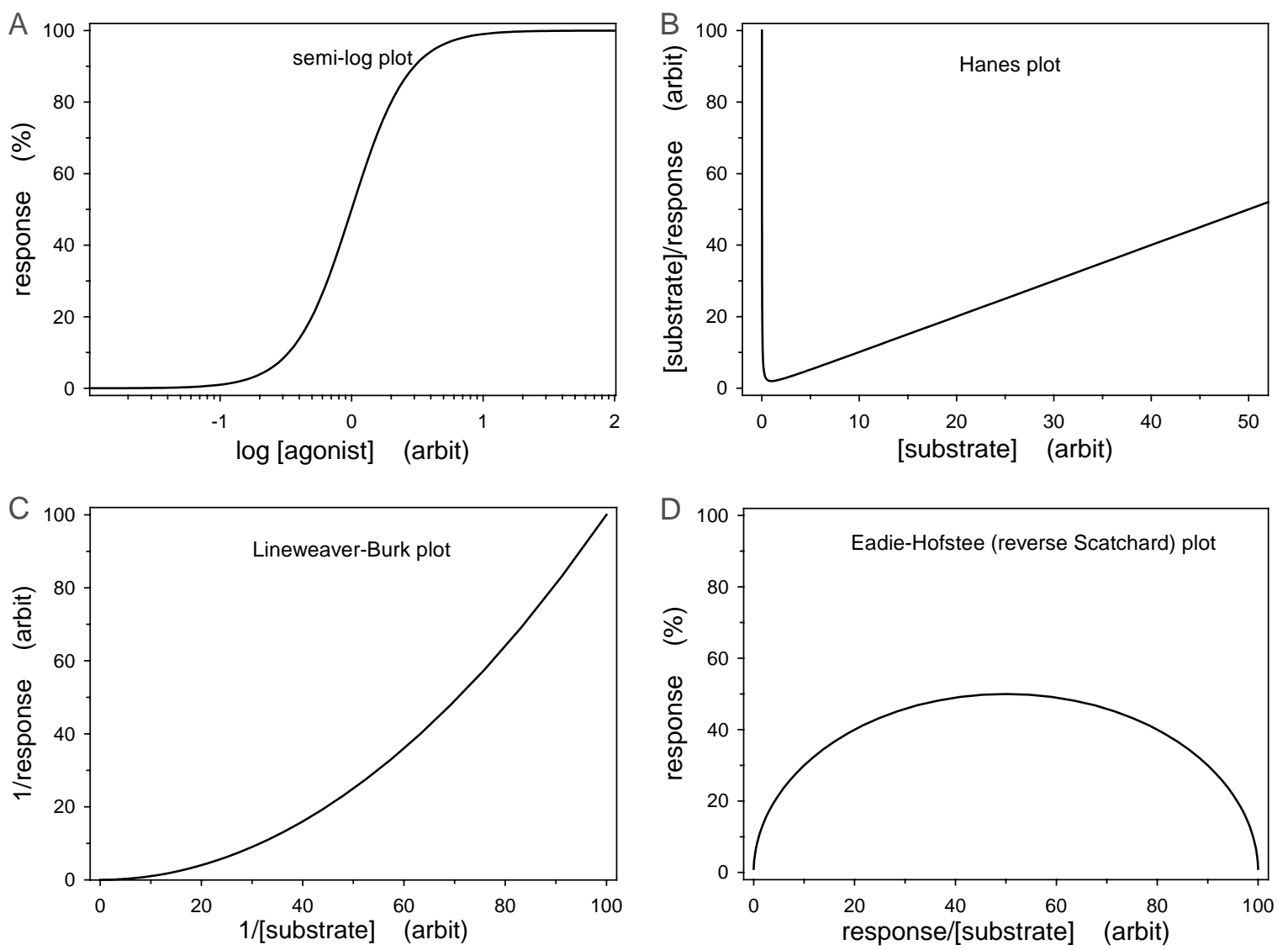

Figure 8.11. Four plot types of the Hill formulation. Semi-log (A), Hanes (B), double reciprocal or Lineweaver-Burk (C), and Eadie-Scatchard (D). The Hill coefficient is 2.

warranted. Furthermore, published results show that researchers employing Eadie-Scatchard plotting and evaluation by linear regression analysis are duped and overlook or ignore deviant behavior of equilibrium data (Klotz 1982). Examples of this are legio.

Deviation from linearity in the Scatchard plot due to experimental error has been summarized by Kermode (1989), while such error will of course also have to be dealt with in all other types of plots and their analyses. Kermode divided errors into types yielding either upward concave or downward concave Scatchard plots (Kermode 1989, Table 1).

\subsubsection{Analysis of Compounded Reaction Schemes}

Segel has derived the linear transforms for a host of more elaborate reaction schemes (Segel 1975, 1993, especially Chapter 9). The statistical confidence for the determined parameters from these linearly-transformed reciprocal models of compounded reaction schemes are even more uncertain than for the simple models, and will only further contribute to their inherent statistical problems. Therefore, when more complicated models or expressions are needed to analyze experimental data, i.e., data deviating from the simplest synagic schemes, then the only possible conclusion is: for statistical evaluation of your experimental concentration-binding or dose-response data always use linear, semi-log, or logit plotting and non-linear regression analysis.

\subsubsection{Eadie-Scatchard Wins Over Non-reciprocal}

Sometimes, with careful analysis of limited data, the Eadie-Scatchard plot, also referred to as the 'independent plot', may actually predict numbers of binding sites, where the semi-log plot of $R$-vs-S plus non-linear fitting to the load-theory are unable to resolve the number of binding sites. For an estimation of the number of binding sites to be extracted by a non-linear fitting procedure to a non-reciprocal load relationship, concentration-binding or dose-response data have to be collected for doses above $K_{\mathrm{d}}$ of the process, that is, above the inflection point of the $S$-shaped curve in a semi-log plot (Figs. 8.1B and 8.10A). A non-linear fitting of parameters to data in the semi-log plot (or an ordinary linear-linear plot as in Fig. 8.1A) is only possible with concentrations above the semi-log inflection point, that is, including co-ordinates above $\mathrm{IC}_{50}$ and $50 \%$ effect. 

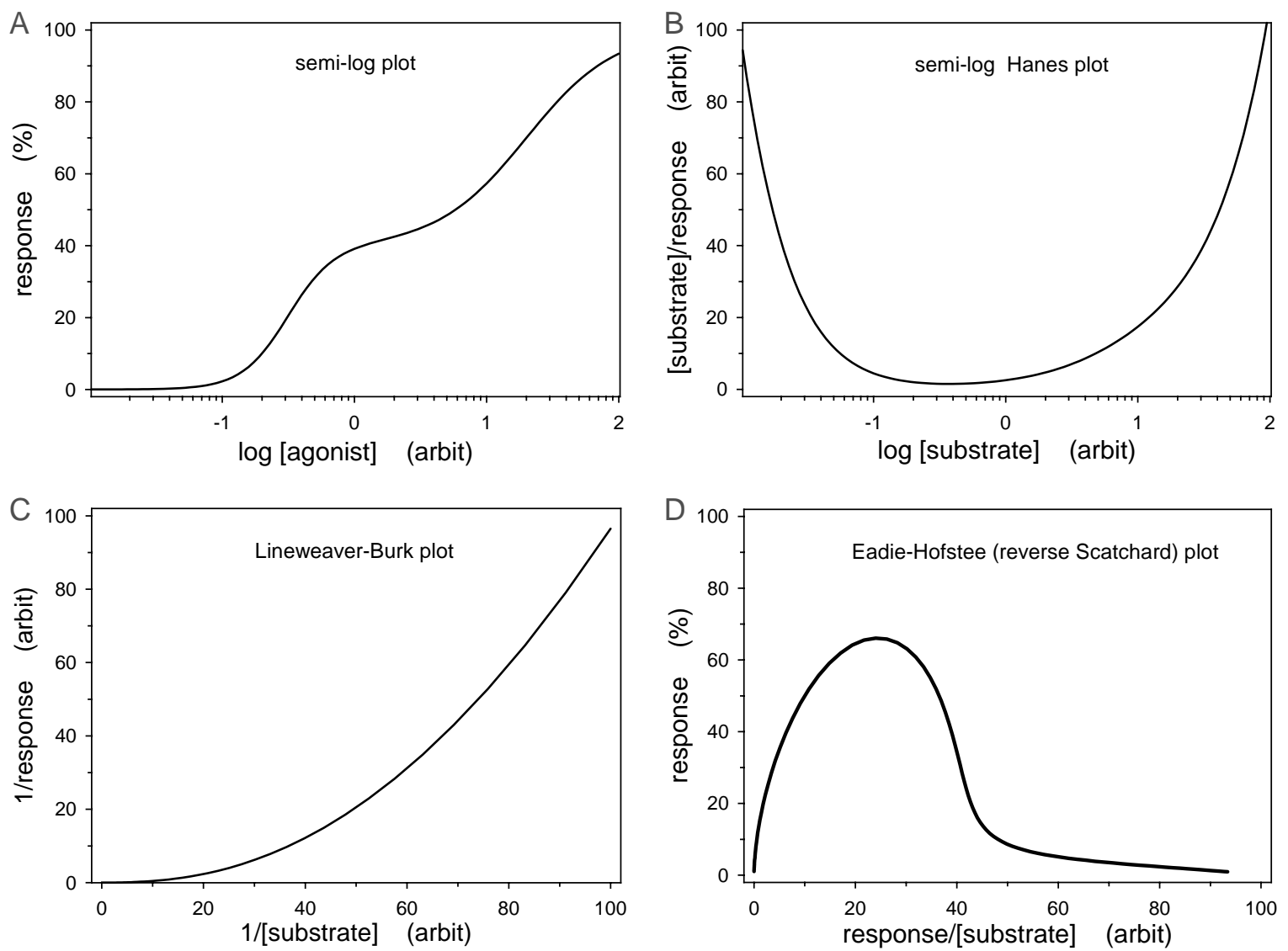

Figure 8.12. Four plot types of a dose-response relation with two receptive units and both with Hill type co-operativity. Semi-log (A), Hanes (B), double reciprocal or Lineweaver-Burk (C), and Eadie Scatchard (D). Parameters for the plots: $Y_{\max 1}=40, K_{\mathrm{d} 1}=$ 3.13, $n_{\mathrm{H} 1}=2.5, Y_{\max 2}=60, K_{\mathrm{d} 2}=20$, and $n_{\mathrm{H} 2}=1.3$.

This is not required for the Scatchard plot as explicitly demonstrated with the Feldman-Klotz discussion in 1982 and 1983 (Klotz 1982; Munson \& Rodbard 1983; Feldman 1983; Klotz \& Hunston 1984; Klotz 1985), although the debate involves a clear warning against sketchy Scatchard plots.

\subsubsection{A Perspective on Type I and Type II Competitive Inhibition}

Competitive ant-agonism/inhibition is based on either one or two binding sites (Fig. 2.4). This fact give a type of seamless transformation to the full intervention scheme discussed in Chapter 2, and is maybe the reason why competitive inhibition, non-competitive inhibition, and intervention schemes in their analysis are often mixed together when they should not be. Two examples of this may suffice. (1) It is problematic to combine a Hill type approach with competitive inhibition - the first requires simultaneous binding (Sub-chapter 8.2), the other requires mutually exclusive binding (see Chapter 2 ); and (2) it is inconsequent to combine a Schild analysis with an intervention model - the former requires competitive ant-agonism/inhibition where parameters $c$ or $c^{\prime}$ are equal to zero, the latter requires that these two parameters are different from zero (see last paragraphs in section 8.2.8 and Chapter 11).

\subsubsection{Conclusion on Reciprocal Plots}

As stated already, collection of concentration-response data from above an inflection point with for instance a load theory in mind, is not as necessary for an EadieScatchard analysis as it is for fitting to non-reciprocal data analysis, but the non-linear fitting is, in general, preferable to the Scatchard procedure, since parameter estimates based on non-reciprocal data will be more accurate (also demonstrated for instance by Bürgisser 1984). Observe also, that for this reason Marangoni completely skips reciprocal transformations for analysis of enzyme synagics (Marangoni 2003). Furthermore, as indicated several times, all kinds of synagic models and not just the simple agonism in the Eadie-Scatchard form may be tested and evaluated by non-linear parameter fitting to non-reciprocal data. 
Table 8.3. Table of plots plus current but obsolete plots ${ }^{\mathrm{a}}$. Examples of historically derived equations, their Linearization and further transformation including null methods

\begin{tabular}{|c|c|c|c|c|c|}
\hline \multicolumn{6}{|l|}{ Reciprocal and non-reciprocal plots } \\
\hline Historical name & Year 19 ‘x & Colloquial name & \multicolumn{3}{|c|}{ Equation: dependent and independent variables } \\
\hline \multicolumn{6}{|l|}{ Agonist plots } \\
\hline $\begin{array}{l}\text { (Hitchcock)-Woolf*-Hanes } \\
\text { Woolf*-Lineweaver-Burk }^{\star} \text { Woolf*-Eadie-Augustinsson-Hofstee }^{*} \\
\text { Scatchard } \\
\text { Logit-Hill }^{\star \star}\end{array}$ & $\begin{array}{l}\text { ('31)-'32-'32 } \\
\text { '32-'34 } \\
\text { '32-'42-'48-'52 } \\
\text { '49 } \\
\text { '13 }\end{array}$ & $\begin{array}{l}\text { Hanes } \\
\text { Reciprocal } \\
\text { Eadie-Hofstee } \\
\text { Scatchard } \\
\text { Hill plot }\end{array}$ & $\begin{array}{l}\mathrm{S} / v \text { versus } \mathrm{S} \\
1 / v \text { versus } 1 / \mathrm{S} \\
v \text { versus } v / \mathrm{S} \\
v / \mathrm{S} \text { versus } v \\
\text { See Eq. } 8.17 \text { in } \\
\text { section } 8.2 .6\end{array}$ & $\begin{array}{l}\text { or } \\
\text { or } \\
\text { or } \\
\text { or }\end{array}$ & $\begin{array}{l}F / B \text { versus } F \\
1 / B \text { versus } 1 / F \\
B \text { versus } B / F \\
B / F \text { versus } B\end{array}$ \\
\hline \multicolumn{6}{|l|}{ Ant-agonist/inhibitor plots } \\
\hline $\begin{array}{l}\text { Dixon }{ }^{\star \star \star} \\
\text { Cornish-Bowden } \\
\text { Gaddum } \\
\text { Schild } \\
\text { Logit of reverse-Hill }^{\star \star}\end{array}$ & $\begin{array}{l}' 53 \\
\prime 74 \\
\prime 37 \\
\text { '47 } \\
\text { See Chapter } 8\end{array}$ & $\begin{array}{l}\text { Dixon } \\
\text { Cornish-Bowden } \\
\text { Gaddum } \\
\text { Schild } \\
\text { Pseudo-Hill plot }\end{array}$ & $\begin{array}{l}1 / v \text { versus I } \\
\text { S } / v \text { versus I } \\
\text { See Chapter } 4 \\
\text { See Chapter } 11 \\
\text { See Eq. } 8.21, \mathrm{~S}\end{array}$ & 8.2 .5 & \\
\hline \multicolumn{6}{|c|}{$\begin{array}{l}\text { Specific agonists plots for binding response } \\
\text { assays }\end{array}$} \\
\hline $\begin{array}{l}\text { Klotz } \\
\text { Scatchard }\end{array}$ & $\begin{array}{l}\prime 46 \\
\prime 49\end{array}$ & $\begin{array}{l}\text { Klotz } \\
\text { Scatchard }\end{array}$ & $\begin{array}{l}1 / v \text { versus } 1 / / \\
\text { Bound }^{\$} \text { substrat } \\
\text { versus bound }^{\text {vers }}\end{array}$ & $\begin{array}{l}\text { s sub } \\
\text { trate }\end{array}$ & \\
\hline
\end{tabular}

$\mathrm{S}$ is the concentration of substrate or agonist and I is concentration of an inhibitor, interventor or modulator. $\mathrm{E}$, $\mathrm{T}$, and $\mathrm{R}$ designate enzyme, transporter and receptor while ' $V$ ' is the steady-state rate of enzymatic catalysis, transport flux, or time-dependent receptor response; ES $\cdot k_{\mathrm{p}}, \mathrm{TS} / t_{1 / 2}$, or RS. $k_{2}$.

As mentioned earlier, one of the purposes of this book, if not the purpose, is to convince the reader to use non-reciprocal/non-linear analysis and forget all about the classical linearized formulations (cf. Marangoni 2003).

${ }^{a}$ Most textbooks on the subject of analysis of equilibrium data from synagics rely heavily on reciprocal manipulation (see f.i. Segel 1975, 1993). This is obsolete with the advent of personal computers and their software, rendering easy access to generalized non-linear regression analysis.

*Woolf plots according to Haldane $(1932,1957)$.

** On 'logit' see for instance Finney (1971), Cornish-Bowden and Koshland (1975) and Cramer (2003).

***Dixon (1953).

${ }^{\star \star \star \star}$ Cornish-Bowden (1974). Applicability of the plot (Thompson \& Bachelard 1976).

‘'Klotz-plot' (Klotz 1946; Klotz et al. 1946), for multi-sited acceptors see Bobrovnik (2002).

On reciprocal and non-reciprocal plots, also see Boeyneams and Dumont (1975, pp. 123-42, equations p. 127, and plots p. 128)

\$'Bound' is a liganded enzyme, liganded transporter, or liganded receptor: ES, TS, or RS.

$\S$ In case the concentration of a ligand drops below the concentration of the binding sites, 'free' may refer to the non-liganded receptive unit $E$, $T$ or $R$.

For inhibitors, a more detailed discussion about the Schild method itself and about extracting inhibitor (antagonist) dissociation constants by this method is postponed to Chapter 11.

\section{REFERENCES}

Adair GS. The hemoglobin system. VI. The oxygen dissociation curve of hemoglobin. J Biol Chem 63: 529-545, 1925.

Atkins GL \& Nimmo IA. A comparison of seven methods for fitting the Michaelis-Menten equation. Biochem J 149: 775-777, 1975.

Atkins GL \& Nimmo IA. Current trends in the estimation of MichaelisMenten parameters. Anal Biochem 104: 1-9, 1980.

Augustinsson K-B. Cholinesterases. A study in comparative enzymology. Acta Physiol Scand 15 Suppl 52 (Dissertation): 1-182, 1948.

Avlani V, May LT, Sexton PM \& Christopoulos A. Application of a kinetic model to the apparently complex behavior of negative and positive allosteric modulators of muscarinic acetylcholine receptors. J Pharmacol Exp Ther 308: 1062-1072, 2004.

Bak P. How Nature Works. The Science of Self-organized Criticality. Oxford: Oxford University Press, 1997.

Berkson J. Application of the logistic function to bio-assay. J Am Statist Assoc 39: 357-365, 1944.
Bevington PR \& Robinson DK. Data Reduction and Error Analysis for the Physical Sciences. New York: McGraw-Hill, 1992.

Bjerrum J. Metal Ammine Formation in Aqueous Solution. Theory of the Reversible Step Reactions (Dissertation; pp. 1-298, Copenhagen University). Copenhagen: Haase \& Son, 1941.

Bobrovnik SA. Ligand-receptor interaction. Klotz-Hunston problem for two classes of binding sites and its solution. J Biochem Biophys Methods 52: 135-143, 2002.

Boeynaems JM \& Dumont JE. Quantitative analysis of the binding of ligands to their receptors. J Cyclic Nucleotide Res 1: 123-142, 1975.

Burgisser E. Radioligand-receptor binding studies: what's wrong with the Scatchard analysis? Trend Pharmacol Sci 5: 142-144, 1984

Cornish-Bowden A. A simple graphical method for determining the inhibition constants of mixed, uncompetitive and non-competitive inhibitors. Biochem J 137: 143-144, 1974.

Cornish-Bowden A. Fundamentals of Enzyme Kinetics. London: Portland Press, 1995.

Cornish-Bowden A. Fundamentals of Enzyme Kinetics. Colchester: Portland Press Ltd, 2004.

Cornish-Bowden A \& Eisenthal R. Statistical considerations in the estimation of enzyme kinetic parameters by the direct linear plot and other methods. Biochem J 139: 721-730, 1974.

Cornish-Bowden A \& Koshland DE Jr. Diagnostic uses of the Hill (Logit and Nernst) plots. J Mol Biol 95: 201-212, 1975. 
Cornish-Bowden A \& Storer AC. Mechanistic origin of the sigmoidal rate behaviour of rat liver hexokinase D ('glucokinase'). Biochem J 240: 293-296, 1986.

Cramer JS. Logit Models. From Economics and Other Fields. Cambridge: Cambridge University Press, 2003.

Cressie NAC \& Keightley DD. Analysing data from hormone-receptor assays. Biometrics 37: 235-249, 1981.

De Lean A, Munson PJ \& Rodbard D. Simultaneous analysis of families of sigmoidal curves: application to bioassay, radioligand assay, and physiological dose-response curves. Am J Physiol 235: E97E102, 1978.

Dixon M. The determination of enzyme inhibitor constants. Biochem J 55: 170-171, 1953.

Dixon M \& Webb EC. Non-inverted versus inverted plots in enzyme kinetics. Nature 184: 1296-1298, 1959.

Dowd JE \& Riggs DS. A comparison of estimates of Michaelis-Menten kinetic constants from various linear transformations. J Biol Chem 240: 863-869, 1965 .

Eadie GS. The inhibition of cholinesterase by phosphostigmine and prostigmine. J Biol Chem 146: 85-93, 1942.

Eadie GS, Bernheim F \& Bernheim MLC. The partial purification and properties of animal and plant hydantoinases. J Biol Chem 181: 449-458, 1949.

Ehlert FJ. Estimation of the affinities of allosteric ligands using radioligand binding and pharmacological null methods. Mol Pharmacol 33: 187-194, 1988.

Eisenthal R \& Cornish-Bowden A. The direct linear plot. Biochem J 139: $715-720,1974$.

Feldman HA. Statistical limits in Scatchard analysis. I Biol Chem 258: 12865-12867, 1983.

Finney DJ. Probit Analysis. Cambridge: Cambridge University Press, 1971.

Ford ED. Scientific Method for Ecological Research. Cambridge: Cambridge University Press, 2000

Fox J. Applied Regression Analysis and Generalized Linear Methods. Thousand Oaks: Sage Publications, 2008.

Frieden C. Kinetic aspects of regulation of metabolic processes. The hysteretic enzyme concept. J Biol Chem 245: 5788-5799, 1970.

Gaddum JH. The quantitative effects of antagonistic drugs. J Physiol 89: 7P-9P, 1937.

Gosselin RE. Drug-receptor interaction: a new kinetic model. In: Kinetics of Drug Action, edited by van Rossum JM. Berlin: Springer Verlag, 1977, pp. 325-356.

Grafen A \& Hails R. Modern Statistics for the Life Sciences. Oxford: Oxford University Press, 2002

Haldane JBS. Graphical methods in enzyme chemistry. Nature 179: 832, 1957.

Haldane JBS \& Stern K. Allgemeine Chemie der Enzyme. Leipzig: Steinkopf, 1932.

Hanes CS. Studies on plant amylases. I. The effect of starch concentration upon the velocity of hydrolysis by the amylase of germinated barley. Biochem J 26: 1406-1421, 1932.

Healey JF. The early alphabet. In: Reading of the Past. Ancient Writing from Cuneiform to the Alphabet, edited by The Trustees of the British Museum. London: British Museum Press, 1990, pp. 197-257.

Hill AV. The possible effects of aggregation of the molecules of haemoglobin on its dissociation curve. I Physiol 40: iv-vii, 1910.

Hofstee BHJ. On the evaluation of the constants $\mathrm{Vm}$ and $\mathrm{Km}$ in enzyme reactions. Science 116: 329-331, 1952.

Hofstee BHJ. Non-inverted versus inverted plots in enzyme kinetics. Nature 184: 1296-1298, 1959.

Hucka M, Finney A, Sauro H \& Bolouri H. Introduction to the systems biology workbench. 1. 2001. http://www.cds.caltech.edu/erato.

Jacquez JA. Compartmental Analysis in Biology and Medicine. Kinetics of Distribution of Tracer-labeled Materials. Amsterdam: Elsevier, 1972.
Jacquez JA. Compartmental Analysis in Biology and Medicine. Ann Arbor: The University of Michigan Press, 1985.

Johnson ML \& Frasier SG. Nonlinear least-squares analysis. Methods Enzymol 117: 301-342, 1985.

Kaiser PM. Substrate inhibition as a problem of non-linear steady state kinetics with monomeric enzymes. J Mol Catal 8: 431-442, 1980.

Keightley DD \& Cressie NA. The Woolf plot is more reliable than the Scatchard plot in analysing data from hormone receptor assays. J Steriod Biochem 13: 1317-1323, 1980.

Keller EF. Making Sense of Life. Explaining Biological Development with Models, Metaphors, and Machines. Harvard: Harvard University Press, 2002.

Kenakin T. A Pharmacology Primer: Theory, Application, and Methods. Amsterdam: Elsevier Academic Press, 2004.

Kermode JC. The curvilinear Scatchard plot. Experimental artifact or receptor heterogeneity? Biochem Pharmacol 38: 2053-2060, 1989.

Klotz IM. The application of the law of mass action to binding in proteins. Arch Biochem 9: 109-117, 1946.

Klotz IM. Numbers of receptor sites from Scatchard graphs: facts and fantasies. Science 217: 1247-1249, 1982.

Klotz IM. Ligand-receptor interactions: facts and fantasies. $Q$ Rev Biophys 18: 227-259, 1985

Klotz IM \& Hunston DL. Mathematical models for ligand-receptor binding. Real sites, ghost sites. J Biol Chem 259: 10060-10062, 1984.

Klotz IM, Walker FM \& Pivan RB. The binding of organic ions by proteins. J Am Chem Soc 68: 1486-1490, 1946.

Kuhn R. Über spezifität der enzyme. I. Zur theorie der zeitwertquotienten. Z Physiol Chem 125: 1-27, 1923.

Kuhn R. Über spezifität der enzyme. II. Saccharase- und raffinasewirkung des invertins. Z Physiol Chem 125: 28-92, 1923.

Kurganov BI. Allosteric Enzymes. Kinetic Behaviour. Chichester: Wiley \& Sons, 1982

Lawson CL \& Hanson RJ. Solving Least Squares Problems. SIAM (Society for Industrial \& Applied Mathematics), 1995.

Lazareno S \& Birdsall NJ. Estimation of antagonist $\mathrm{Kb}$ from inhibition curves in functional experiments: alternatives to the ChengPrusoff equation. Trends Pharmacol Sci 14: 237-239, 1993.

Lazareno S \& Birdsall NJ. Estimation of competitive antagonist affinity from functional inhibition curves using the Gaddum, Schild and Cheng-Prusoff equations. Br J Pharmacol 109: 1110-1119, 1993.

Lazareno S \& Birdsall NJ. Detection, quantitation, and verification of allosteric interactions of agents with labeled and unlabeled ligands at G protein-coupled receptors: interactions of strychnine and acetylcholine at muscarinic receptors. Mol Pharmacol 48: 362 378, 1995.

Lazareno S, Popham A \& Birdsall NJ. Allosteric interactions of staurosporine and other indolocarbazoles with $N$-[methyl- $\left.{ }^{3} \mathrm{H}\right] \mathrm{sco}-$ polamine and acetylcholine at muscarinic receptor subtypes: identification of a second allosteric site. Mol Pharmacol 58: 194 207, 2000.

Lew MJ \& Angus JA. An improved method for analysis of competitive agonist/antagonist interactions by non-linear regression. Ann NY Acad Sci 812: 179-181, 1997.

Lew MJ, Ziogas J \& Christopoulos A. Dynamic mechanisms of non-classical antagonism by competitive AT(1) receptor antagonists. Trends Pharmacol Sci 21: 376-381, 2000.

Lineweaver H \& Burk D. The determination of enzyme dissociation constants. J Am Chem Soc 56: 658-666, 1934.

Marangoni AG. Enzyme Kinetics: A Modern Approach. New York: WileyInterscience, 2003

May LT, Avlani VA, Langmead CJ, Herdon HJ, Wood MJ, Sexton PM \& Christopoulos A. Structure-function studies of allosteric agonism at M2 muscarinic acetylcholine receptors. Mol Pharmacol 72: 463$476,2007$. 
May RM. Simple mathematical models with very complicated dynamics. Nature 261: 459-467, 1976.

May RM. Uses and abuses of mathematics in biology. Science 303: 790793, 2004.

Michaelis L \& Davidsohn H. Die wirkung der wasserstoffionen auf das invertin. Biochem Zeitschrift 35: 386-412, 1911.

Motulsky H \& Christopoulos A. Fitting Models to Biological Data Using Linear and Nonlinear Regression: A Practical Guide to Curve Fitting. Oxford: Oxford University Press, 2004.

Munson PJ \& Rodbard D. Number of receptor sites from Scatchard and Klotz graphs: a constructive critique. Science 220: 979-981, 1983.

Neubig RR, Spedding M, Kenakin T \& Christopoulos A. International Union of Pharmacology Committee on Receptor Nomenclature and Drug Classification. XXXVIII. Update on terms and symbols in quantitative pharmacology. Pharmacol Rev 55: 597-606, 2003.

Paton WDM. A theory of drug action based on the rate of drug/ receptor combination. Proc R Soc Lond B 154: 21-69, 1961.

Peitgen H-O, Jürgens H \& Saupe D. Classical fractals and self-similarity. In: Chaos and Fractals: New Frontiers of Science, Berlin: SpringerVerlag, 1992

Ratkowsky DA. Handbook of Nonlinear Regression Models. New York: Marcel Dekker, Inc, 1990.

Reed LJ \& Berkson J. The application of the logistic function to experimental data. J Phys Chem 33: 760-779, 1929.

Riggs DS. Control Theory and Physiological Feedback Mechanisms. Baltimore: The Williams and Wilkins Co., 1970.

Samuels ML \& Witmer JA. Statistics for the Life Sciences. Upper Saddle River, NJ: Prentice Hall, 2002.

Scatchard G. The attractions of proteins for small molecules and ions. Ann NY Acad Sci 51: 660-672, 1949.

Schild HO. pA, a new scale for the measurement of drug antagonism. Br J Pharmacol 2: 189-206, 1947.

Schild HO. pAx and competitive drug antagonism. Br J Pharmacol 4: 277-280, 1949.

Segel IH. Enzyme Kinetics. Behavior and Analysis of Rapid Equilibrium and Steady-state Enzyme Systems. New York: Wiley \& Sons (reissued 1993), 1975

Severini TA. Likelihood Methods in Statistics (Oxford Statistical Science S.). Oxford: Oxford University Press, 2000.

Siok WT, Perfetti CA, Jin Z \& Tan LH. Biological abnormality of impaired reading is constrained by culture. Nature 432: 71-76, 2004 .
Storer AC \& Cornish-Bowden A. Kinetic evidence for a 'mnemonical' mechanism for rat liver glucokinase. Biochem J 165: 61-69, 1977.

Sørensen SPL. Études enzymatiques; II. Sur la mesure et l'importance de la concentration des ions hydrogène dans les réactions enzymatiques. Comp Rend Travaux Laboratoire Carlsberg 8: 1-168, 1909.

Taketa K \& Pogell BM. Allosteric inhibition of rat liver fructose 1,6diphosphatase by adenosine 5'-monophosphate. J Biol Chem 240: 651-662, 1965.

Taylor P \& Insel PA. Molecular basis of pharmacological selectivity. In: Principles of Drug Action: The Basis of Pharmacology, edited by Pratt WB \& Taylor P. Philadelphia: Churchill Livingstone, 1990, pp. 1102 .

Thompson MF \& Bachelard HS. Determination of true Ki values in enzyme dead-end inhibition kinetic studies. Anal Biochem 71: 507518, 1976.

Topham CM. A generalized theoretical treatment of the kinetics of an enzyme-catalysed reaction in the presence of an unstable irreversible modifier. J Theor Biol 145: 547-572, 1990.

Topham CM \& Brocklehurst K. In defence of the general validity of the Cha method of deriving rate equations. The importance of explicit recognition of the thermodynamic box in enzyme kinetics. Biochem J 282: 261-265, 1992.

Waud DR. Analysis of dose-response curves. In: Methods in Pharmacology: Smooth Muscle, Volume 3, edited by Daniel \& Paton. New York: Plenum Press, 1975.

Waud DR. Analysis of dose-response relationships. In: Advances in General and Cellular Pharmacology, edited by Narahashi $\mathrm{T}$ and Bianchi CP. New York: Plenum Press, 1976, pp. 145-178.

Waud DR, Son SL \& Waud BE. Kinetic and empirical analysis of doseresponse curves illustrated with a cardiac example. Life Sci 22: $1275-1285,1978$

Wells JW. Analysis and interpretation of binding at equilibrium. In: Receptor-ligand Interactions. A Practical Approach, edited by Hulme EC. Oxford: IRL Press at Oxford University Press, 1992, pp. 289 395.

Winzor DJ \& Sawyer H. Quantitive Characterization of Ligand Binding: New York: Wiley-Liss, 1995.

Witte RS \& Witte JS. Statistics. Hoboken, NJ: John Wiley \& Sons, Inc, 2004 
Part III: Test of Tool for Data Analysis 


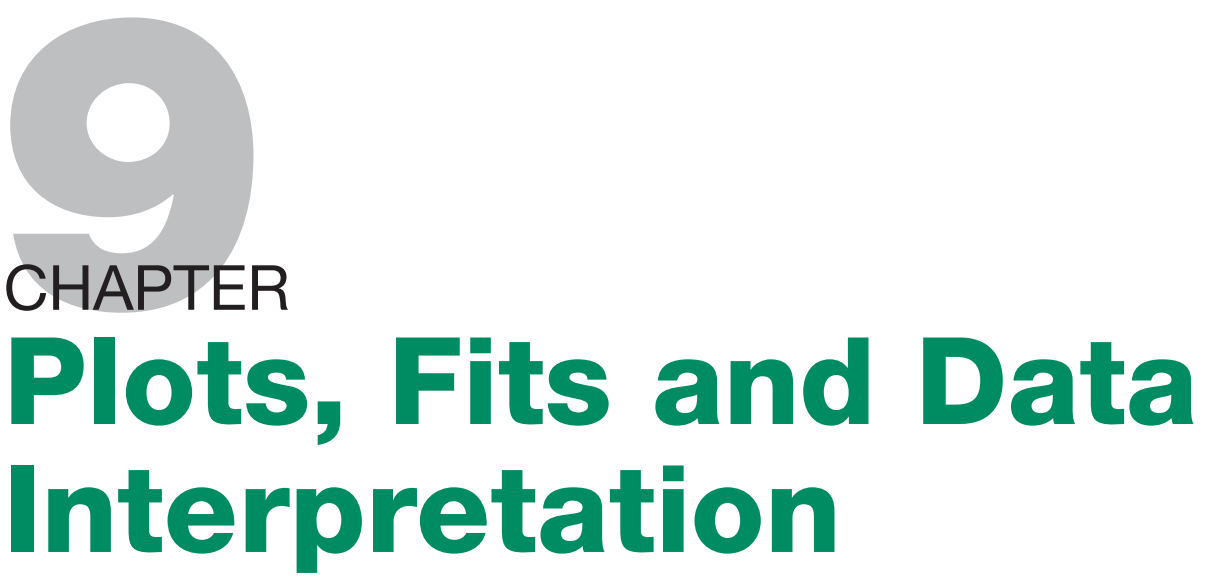

Several software packages may be used for the analysis of dose-response relations at equilibrium, also referred to as synagics. As an example of this, the SigmaPlot software version 9 (SP9) has been selected to present data plotting, parameter fitting, and curve generation. The SP9 is dedicated to these tasks and, at the same time, it allows for analysis of user-defined theories, presentation of 3-dimensional (3-D) mesh-plots of theories with two independent variables, and even plots of multiple 3-D plots in the same graph. For our purpose, we will need all these capabilities. A SigmaPlot version 10 is now available.

Subchapters 9.1-9.3 are brief courses on how to use the software as an analytical tool for dose-response relationships; the courses include making plots of experimental data, fitting theories to curves, and generating 2- and 3-D plots of theoretical reaction schemes for comparison with experimental curves.

In conclusion, Subchapter 9.4 explains the use of the factor-squared rule, described in Chapter 8, for an estimate on theory deviation from reality. Furthermore, the testing of a theory on another theory is demonstrated.

Several of the ideas in the four subchapters are general and should not be disturbed by adherence to a specific software package. However, to gain maximum benefit from the description in this chapter, a copy of the SigmaPlot software should be running on an accessible computer.

\subsection{Sigmaplot Software to Plot, Fit and Generate Curves}

The objective of this subchapter is to make a plot of a data set you supply, and then make a best-fit of a theory to the supplied data. The fit calculates parameters such that the theory most closely follows the data points.

At the end of the subchapter, the obtained best-fit parameters are used to generate a curve on top of the supplied experimental data.

\subsubsection{Using the Sigmaplot Software to Generate a Plot}

We assume that you have a data set of concentration and corresponding fractional response (in \%) from either a binding or a functional experiment.

Insert the data set of concentration $(\mathrm{S})$ and effect $(R)$ in the worksheet of SP9 (Column 1 and 2, Fig. 9.1), ${ }^{1}$ and select the Graph command (Fig. 9.1, arrow 1). This opens a menu for graphs (Fig. 9.1). Select Create Graph (Fig. 9.1, arrow 2). A new menu appears (Fig. 9.2A). Select the Scatter Plot (arrow 1), press Next (arrow 2), and arrive at a new window (Fig. 9.2B). Select the Simple Scatter (Fig. 9.2B, arrow 3), press Next (arrow 4), and find the opening menu of a Create Graph-Data Format window (Fig. 9.3A, arrow 1). Select XY Pair (Fig. 9.3A, arrow 2), and press Next (Fig. 9.3A, arrow 3). A new window for Create Graph-Select Data appears (Fig. 9.3B, arrow 4). Data selection (as required in Fig. 9.3B, arrow 5) is carried out by putting the cursor at the top of column 1 (concentration data) in the worksheet and left clicking (Fig. 9.4A, arrow 1), followed by positioning the cursor at the top of column 2 (response data) and left clicking (Fig. 9.4B, arrow 2). Press Finish (Fig. 9.4B, arrow 3), and a plot of $S$-values against $R$-values is displayed (Fig. 9.5).

The result in Fig. 9.5 is a linear-linear plot of the data, with concentration data (co-ordinates) on the $x$-axis and activity or binding data (co-ordinates) on the $y$-axis. At this point, the dose-response data seem to follow a simple hyperbolic relationship.

\footnotetext{
${ }^{1}$ The SP9 software is 'European', therefore fractions are comma rather than period delimited.
} 


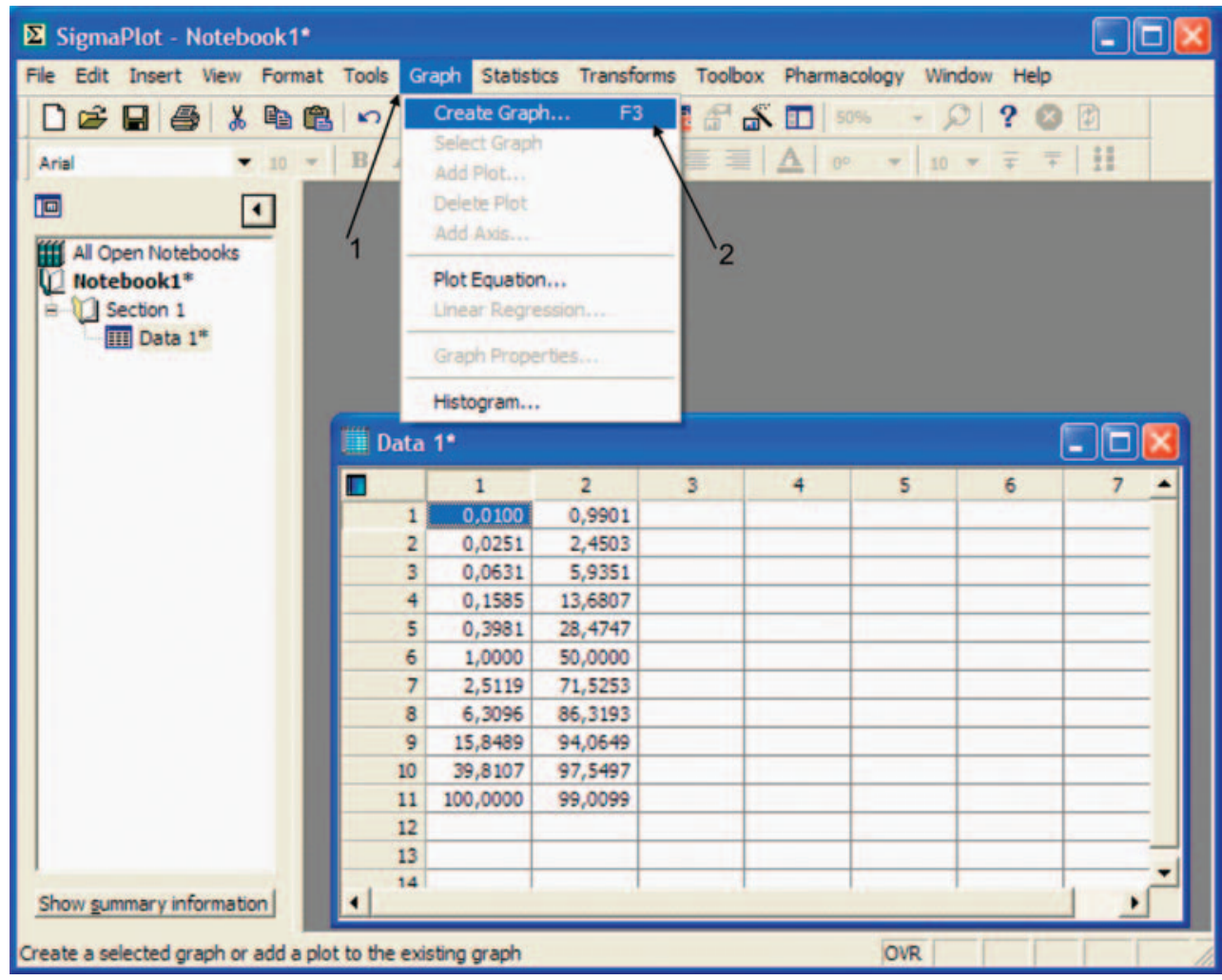

Figure 9.1. SigmaPlot data-sheet with inserted data in two columns.

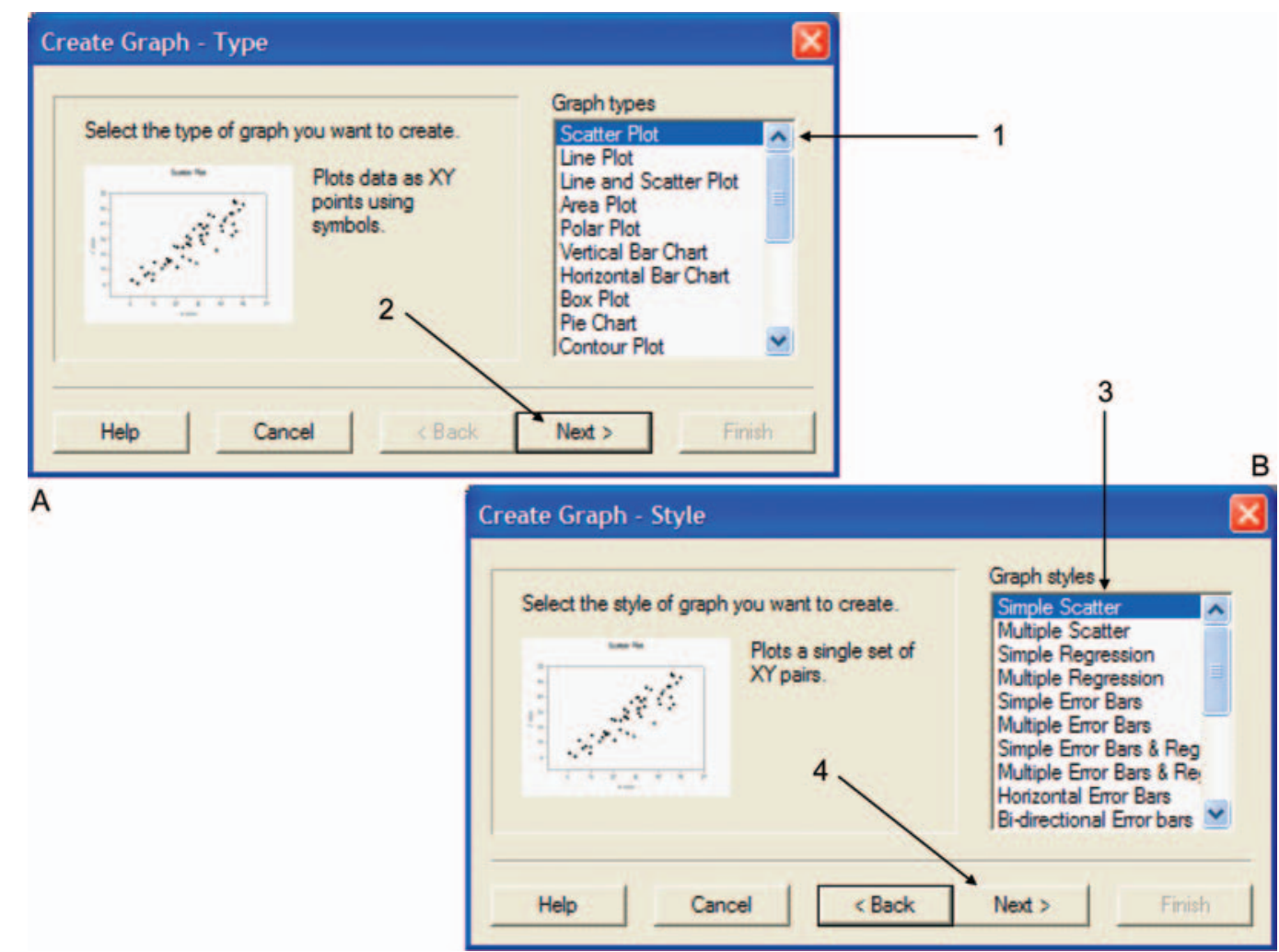

Figure 9.2. Menu to create graph in SigmaPlot software. 


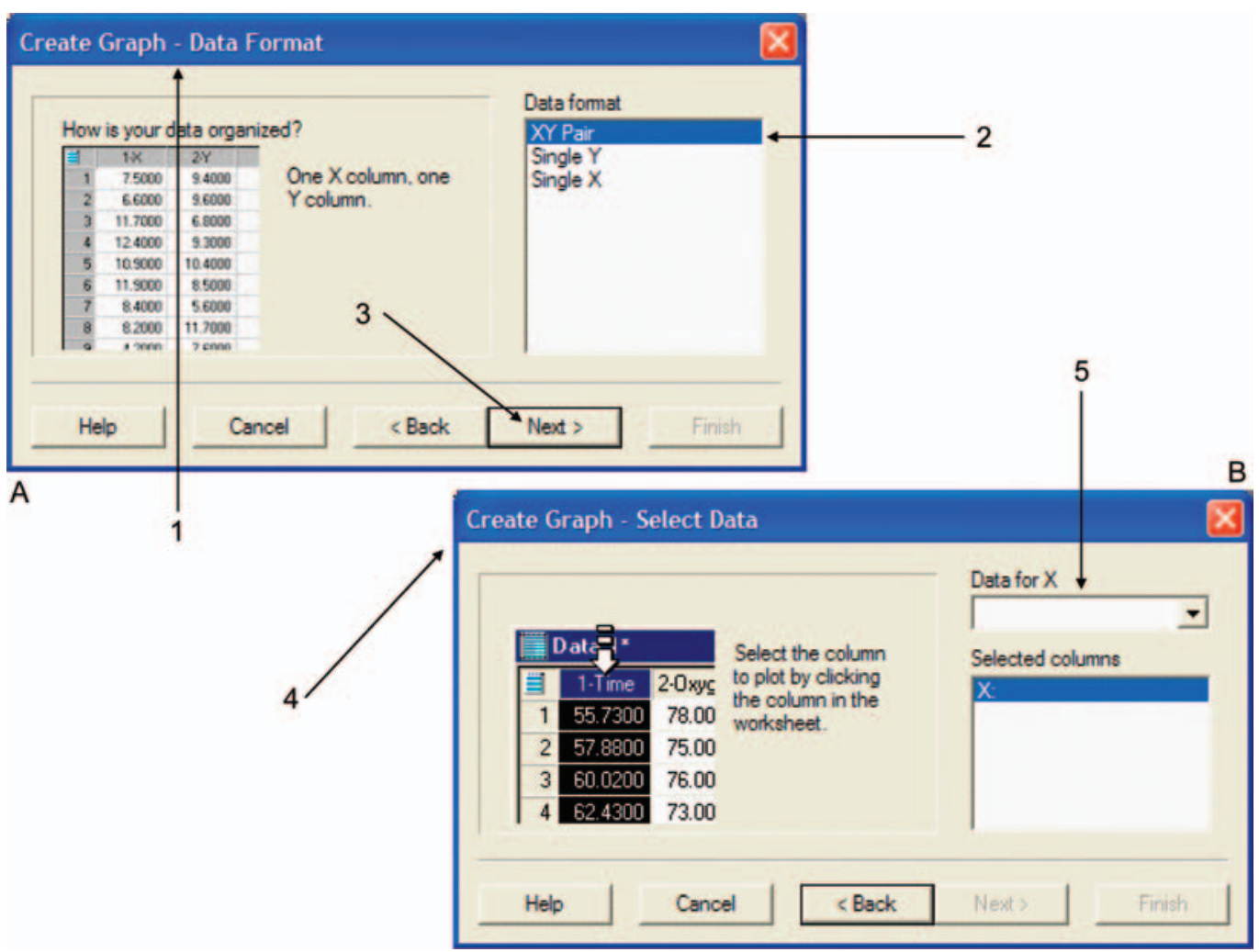

Figure 9.3. Format of data for graph in SigmaPlot software.

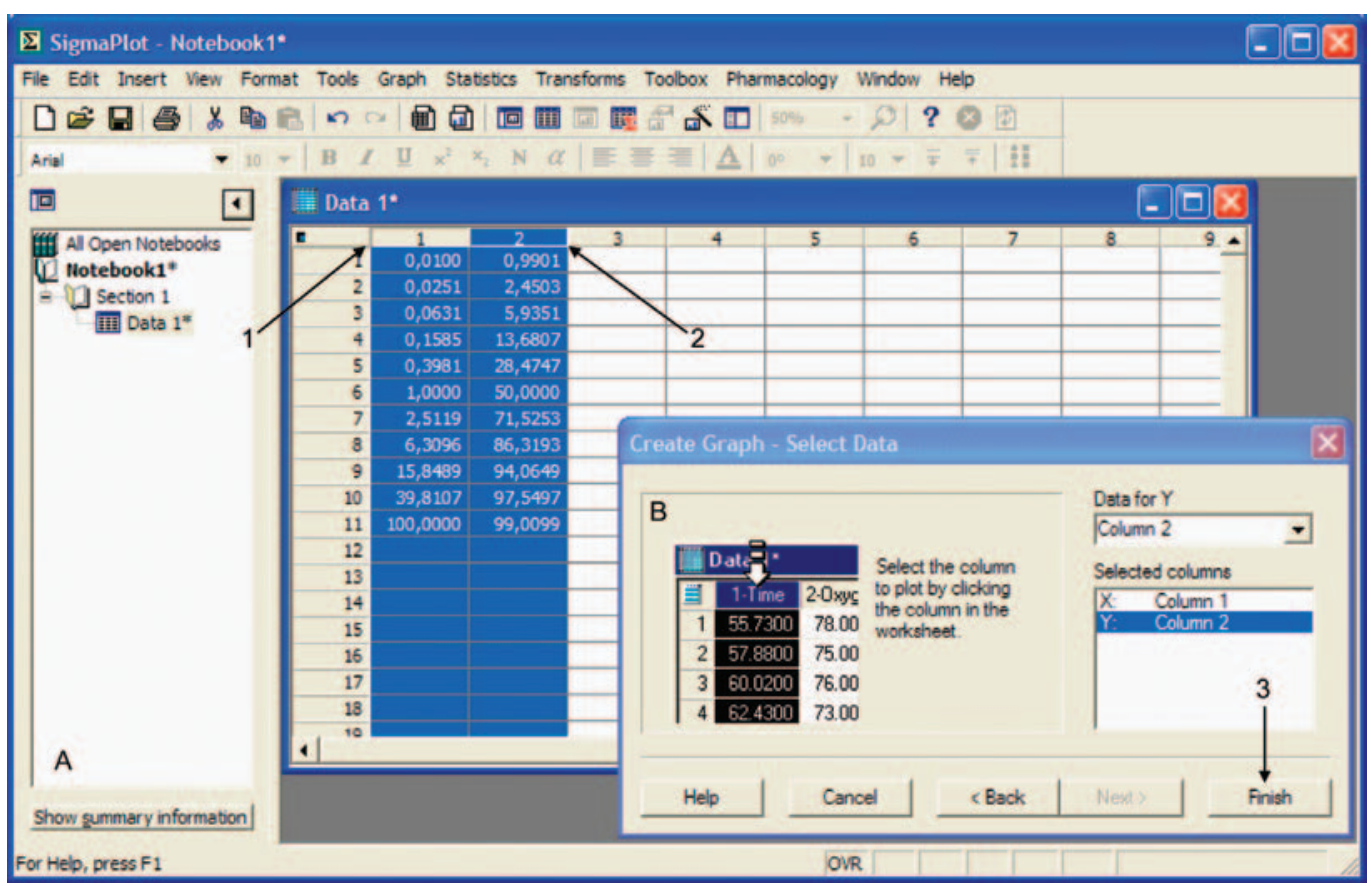

Figure 9.4. Selection of data columns for plot in SigmaPlot graph. 


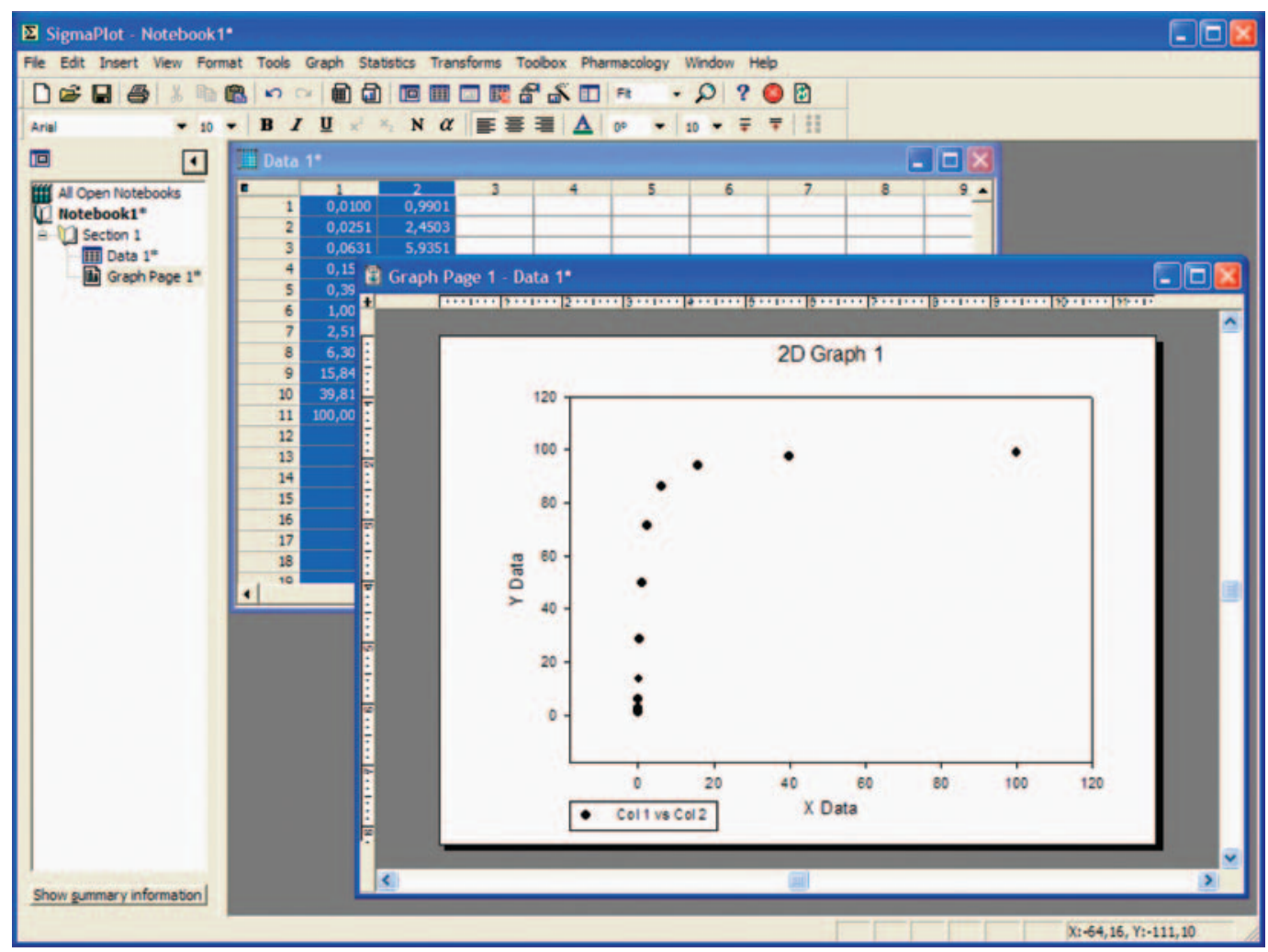

Figure 9.5. Display of 'Scatter' plot.

\subsubsection{Using the Sigmaplot Software to Generate a Semi-log Plot}

To test that data in Fig. 9.5 represents a rectangular hyperbola, we will convert the plot into a semi-log plot where the $x$-axis is logarithmic. Left click twice on the $x$ axis (arrow in Fig. 9.6). A menu appears (Fig. 9.7A). In selecting Scaling (Fig. 9.7A, arrow 1), a Scale type curtain appears (Fig. 9.7B, arrow 2). Open the Scale type menu, select the Log (common) option (Fig. 9.7B, arrow 3), and continue by clicking on OK (Fig. 9.7B, arrow 4). The plot, a semi-log plot of your data, will be obtained (Fig. 9.8).

Note that the $x$-axis in Fig. 9.8 is logarithmic.

We can efficiently test if the dose-response curve in this plot fulfils the $82 \%$ or factor-squared rule, as described in Sections 8.1.2 and 8.1.4. To test for deviations from the factor-squared rule, see Section 9.4.1.

\subsubsection{Using the Sigmaplot Software to Curve-fit to Data}

Based on the 'scatter' plot in Section 9.1.2 (Fig. 9.8), we will allow the SP9 software fit a curve to our data points. The curve will be based on the theory that data points come from a load function, as described in Chapter 1. As the SP9 software already has such a theory in its toolbox, the task is simple. Start by selecting the scatter plot (curve) in Fig. 9.8. This is done by setting the cursor above one of the data points in the plot (linear-linear or semi-log plot) and left-clicking once with the mouse (Fig. 9.9, arrow 1). Next, select Statistics on the command line (Fig. 9.9, arrow 2), and in the Statistics menu select Regression Wizard (Fig. 9.9, arrow 3). A Regression Wizard window appears with an Equation Category (Fig. 9.10, arrow 1), in which we select 'Hyperbola' (Fig. 9.10, arrow 2), and in the appearing Equation Name curtain, select 'Single Rectangular, 2 Parameters' (Fig. 9.10, arrow 3). Note, that the selected theory is shown to the left in the Regression Wizard window, labeled by the present author with 'Your theory:'. Next, press Next (Fig. 9.10, arrow 4). The program suggests columns 1 and 2 for the variables to be used (Fig. 9.11A, arrow 1), and since that is also where we have our variables, press Next (Fig. 9.11A, arrow 2). The program calculates the theory parameters a and $b$ equal to the maximum of the fractional activity or binding (a) and the dissociation constant (b), and displays the results in a new Regression Wizard window (Fig. 9.11B).

Fig. 9.11B shows the estimated values for maximal response (a) arrow 3, and for the dissociation constant (b) arrow 4. Press Next (Fig. 9.11B, arrow 5), and the Regression Wizard window appears (Fig. 9.12); here it is possible to save the estimated parameters and the statistical information to the spreadsheet by ticking 


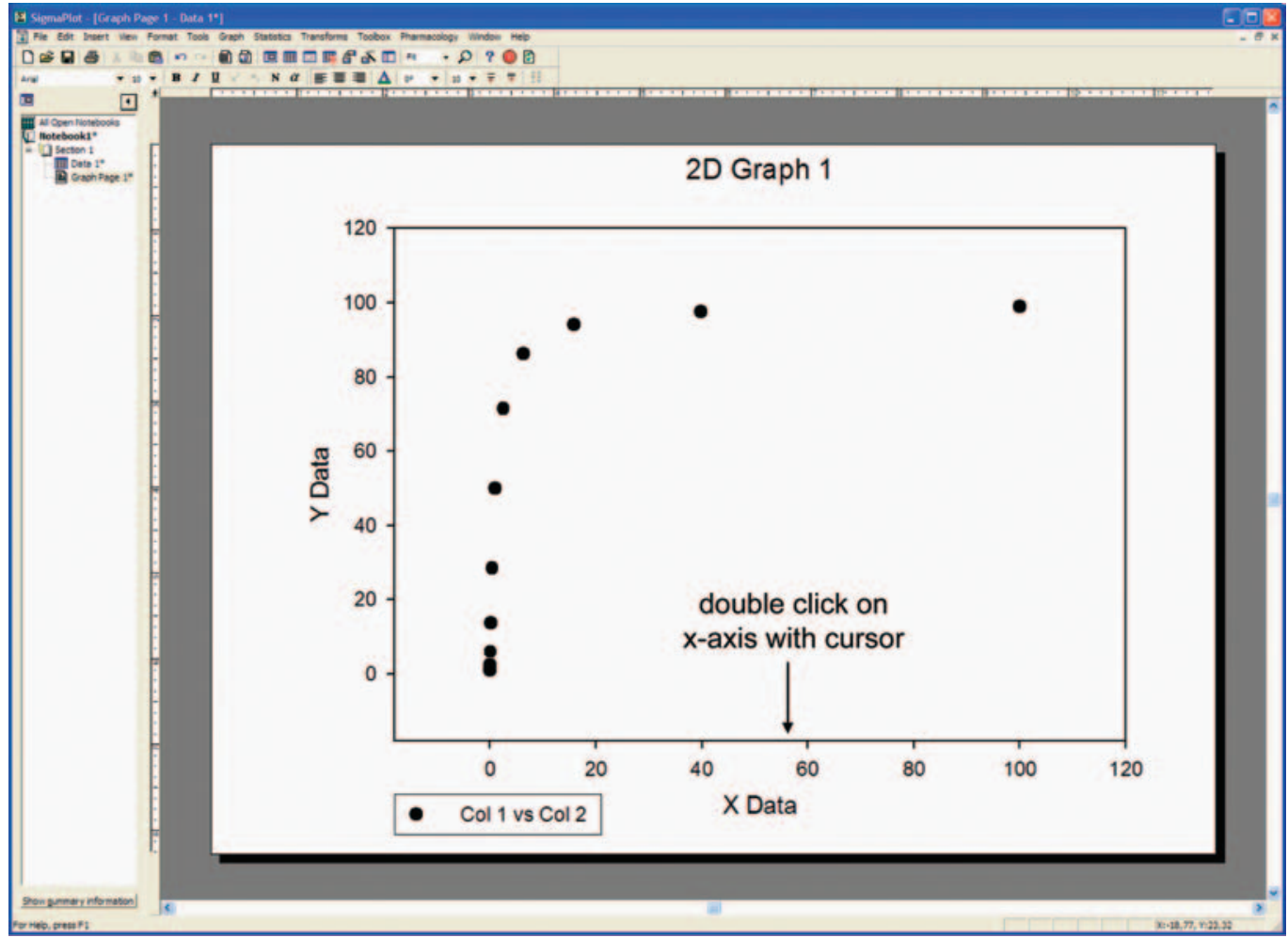

Figure 9.6. Selecting the $x$-axis for re-scaling.

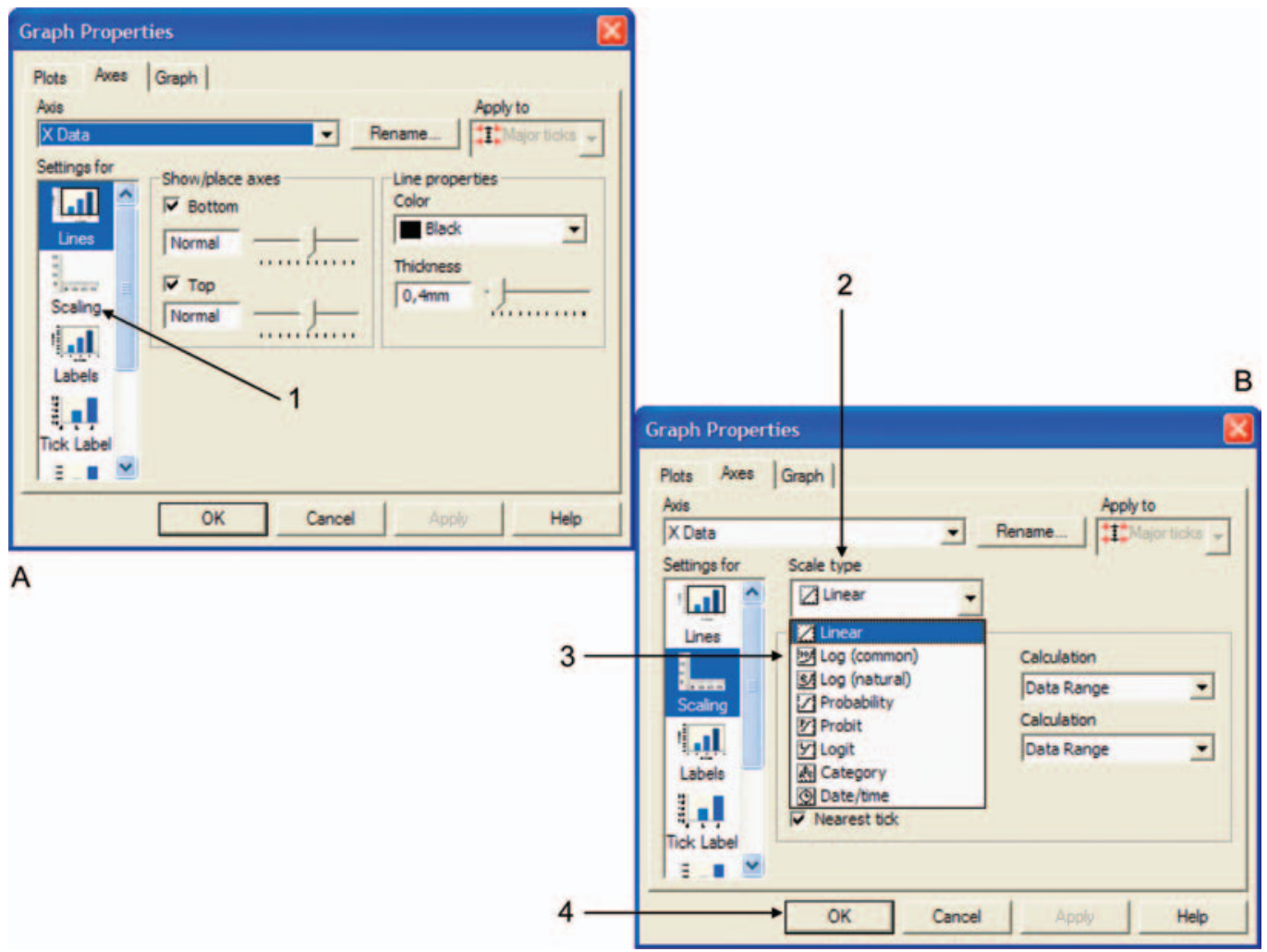

Figure 9.7. Logarithmic re-scaling of the $x$-axis. 


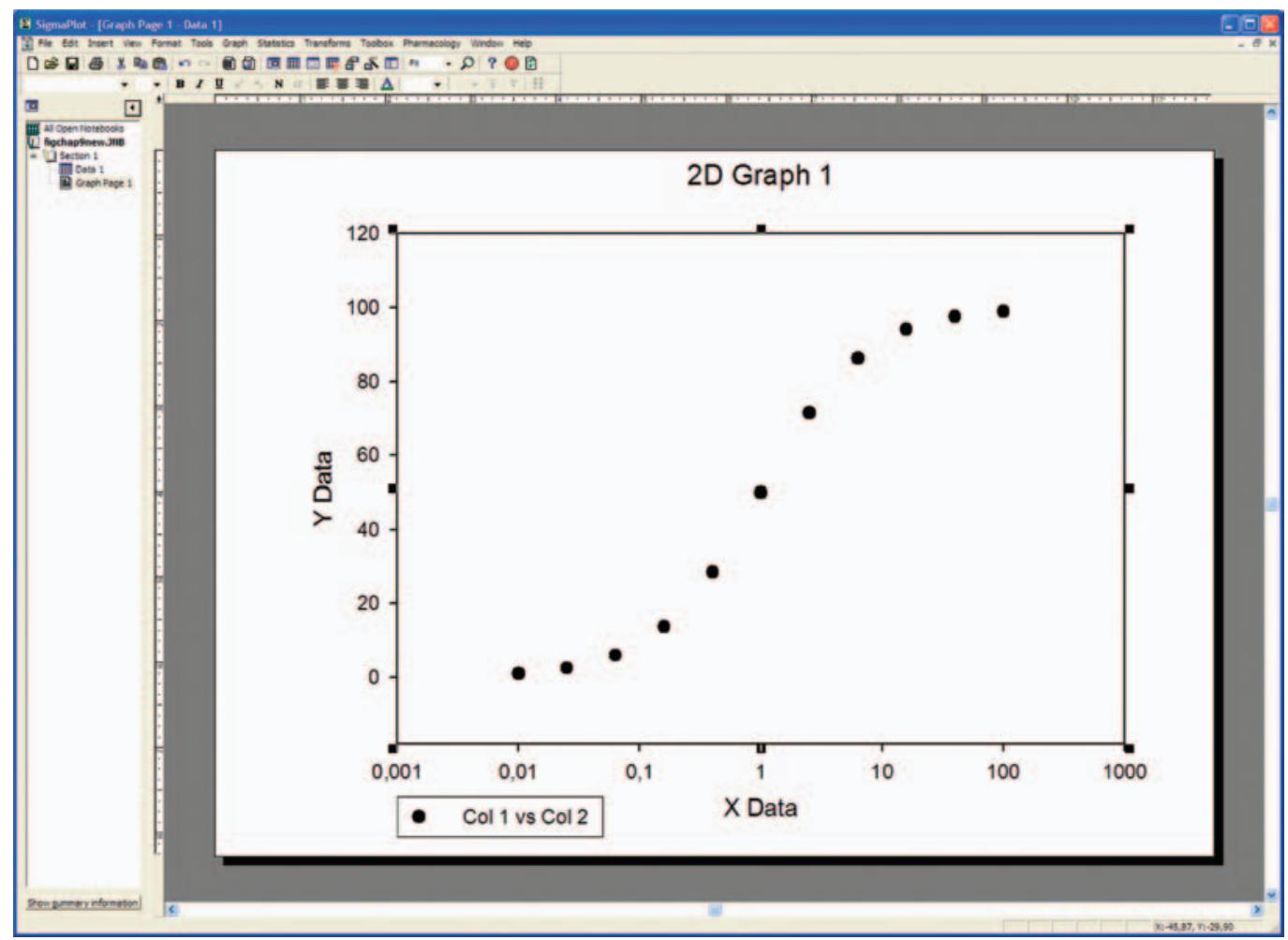

Figure 9.8. Display of semi-log re-scaled plot.

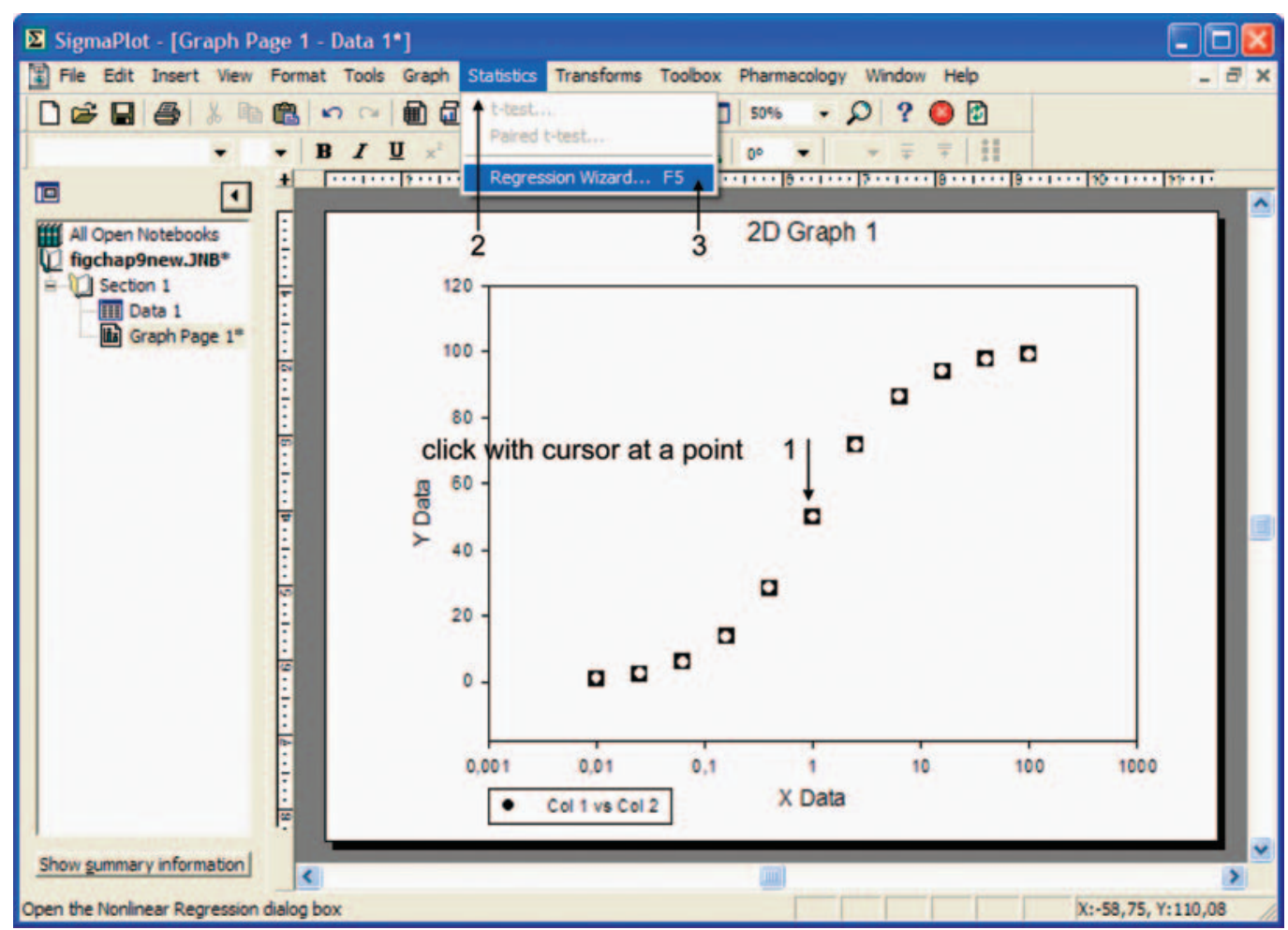

Figure 9.9. Selection of a plot for analysis in theory. 


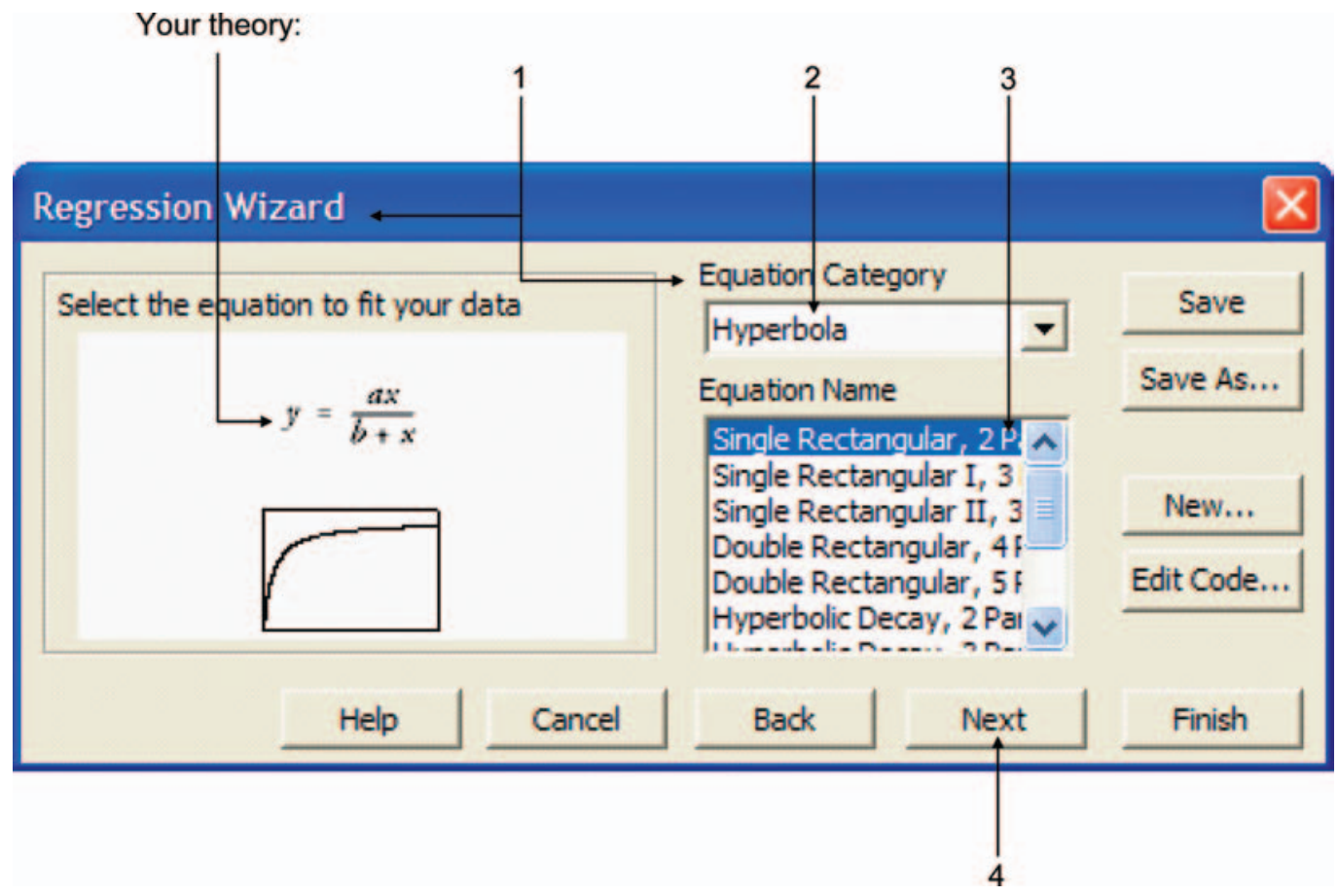

Figure 9.10. Selecting a theory to fit to plot.
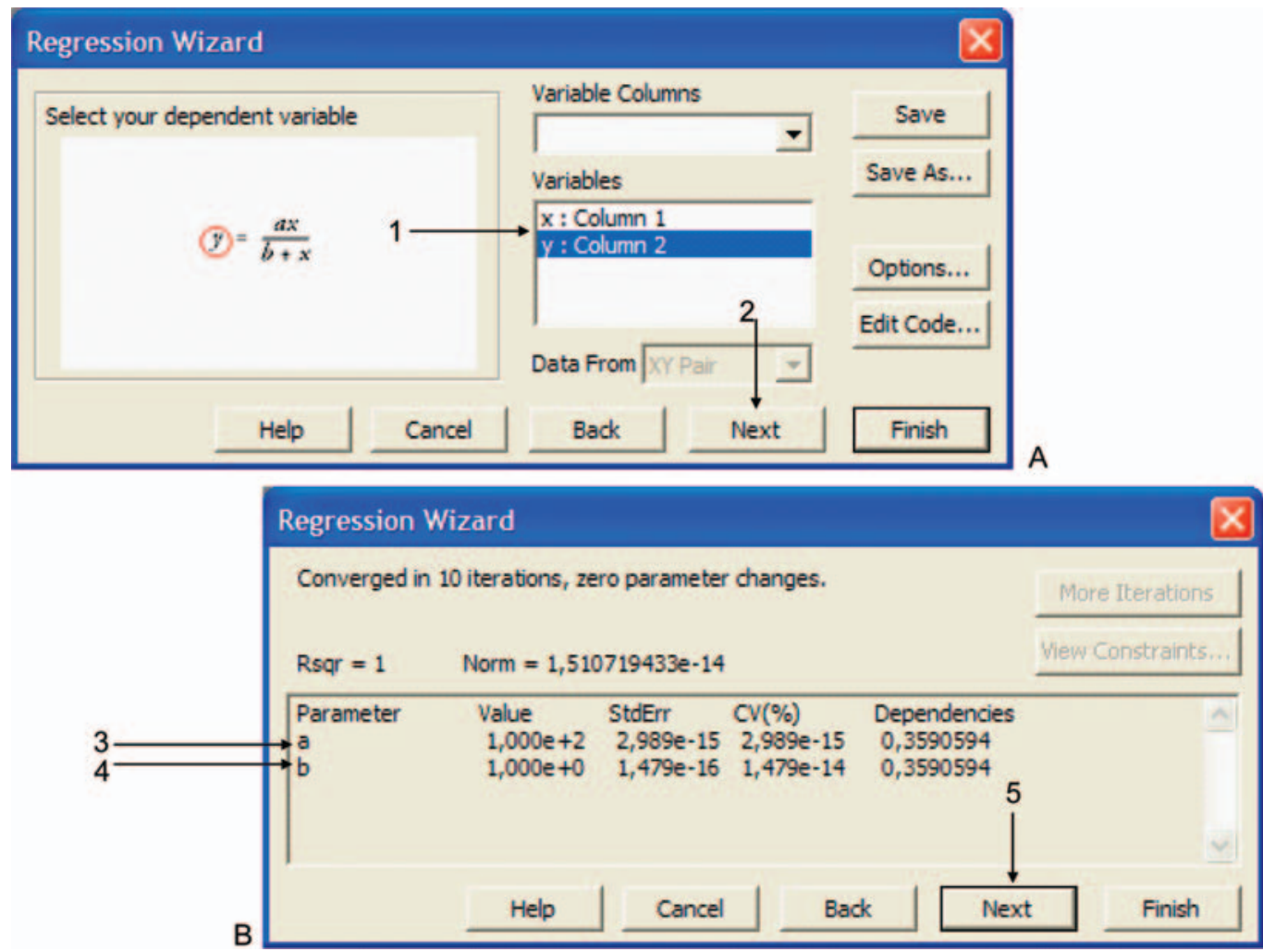

Figure 9.11. Run a fit routine by selecting the appropriate variable columns and obtain the fitted parameters of the theory. 


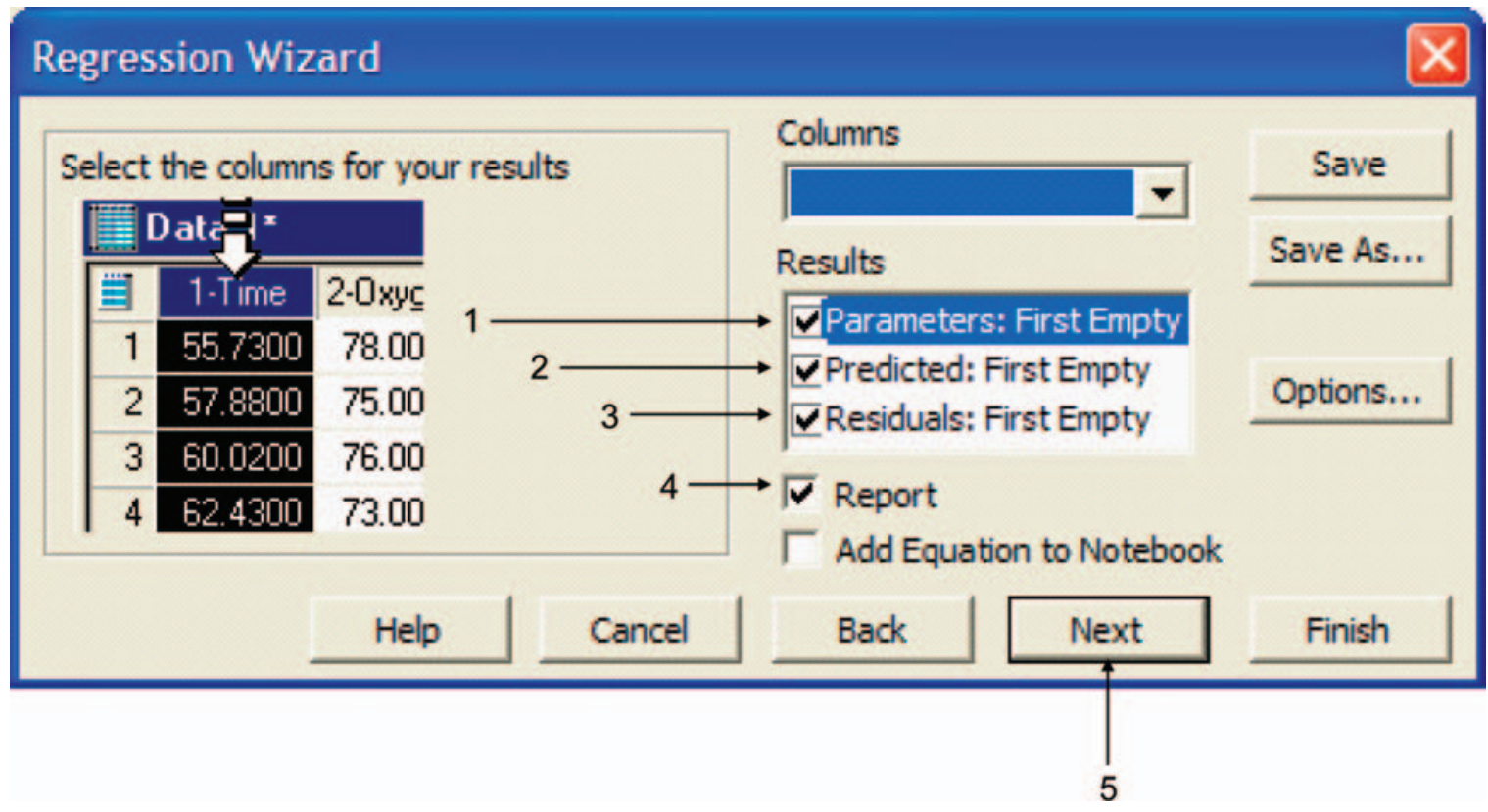

Figure 9.12. Display of fitted parameters and their placement in the data-sheet.

(shown by arrows 1-3 in Fig. 9.12), as well as creating a report on the fitted parameters and the statistics for the non-linear fitting procedure (Fig. 9.12, arrow 4).

\subsubsection{Using the Sigmaplot Software to Generate the Best-fit Curve}

Continue with Next (Fig. 9.12, arrow 5). The display in Fig. 9.13A appears and you can tick for generation of a best-fit-theory curve for your data (Fig. 9.13A, arrow 1). Click Next (Fig. 13A, arrow 2), and reach the Regression Wizard window in Fig. 9.13B. The program suggests to place the data it will generate for the best-fit plot in the 'first empty' columns of the spreadsheet (Fig. 9.13B, arrows 3 and 4). This is fine, and we accept by clicking on Finish (as indicated by arrow 5 in Fig. 9.13B). The result is given in Fig. 9.14, where a best-fit curve is placed on top of your experimental data points. As can be observed, there is a good match between theory and experiment (Fig. 9.14).

The best-fit curve is generated internally by the SP9 software which places $257 X-Y$ data points in the two 'first empty' columns. These theoretical data points are calculated automatically in SP9 by inserting the estimated parameter values into the theory. To view these data points in the spread-sheet, click at the spread-sheet icon at arrow 1 in Fig 9.14. The 257 sets of curve data are displayed in columns 6 and 7 of the SP9 datasheet (Fig. 9.15). Estimated parameter values are in column 3, predicted values for the independent variable are in column 4 , and the squared residuals are in column 5 .

Of course, the columns where such data appear may vary. Finally, a report of the fitting is presented in a
Report scheme (Fig. 9.16). You can switch between the datasheet, the graph-page and the Report-sheet, as pointed to by arrows $1-3$ in Fig. 9.16.

The employed theory is listed in the Report (Fig. 9.16, arrow 4), and the statistical information is pointed to by arrow 5 in Fig. 9.16.

The statistical evaluation is not dealt with here. For statistical analysis, see for instance the SYSTAT 11 software (www.systat.com) or the Statistica 7 software (www.statsoft.com). The SP9 Regression Wizard is based on the Marquardt-Levenberg algorithm (Marquardt 1963) and for greater accuracy can include weighted least squares. References to texts on statistics in general are listed in Section 8.1.7.

Our objective of plotting experimental data points, fitting a theory, thereby obtaining best-fit parameters, and generating a best-fit curve for the data points based on the theory with inserted best-fit parameters is completed.

\subsubsection{Non-linear Fitting}

There are several methods to fit in a non-linear fashion. As mentioned, to generate the curve described in Section 9.1.3, Sigma-Plot uses the Marquardt-Levenberg least squares fitting procedure, and generates plots based on 257 data points. See Nash (1990) for more on non-linear fitting of theory parameters to adjust to experimental data points, including modifications to the Marquardt method of fitting. Overall, the rules for using non-linear fitting can be found in Marangoni (2003, pp. 33-36). 

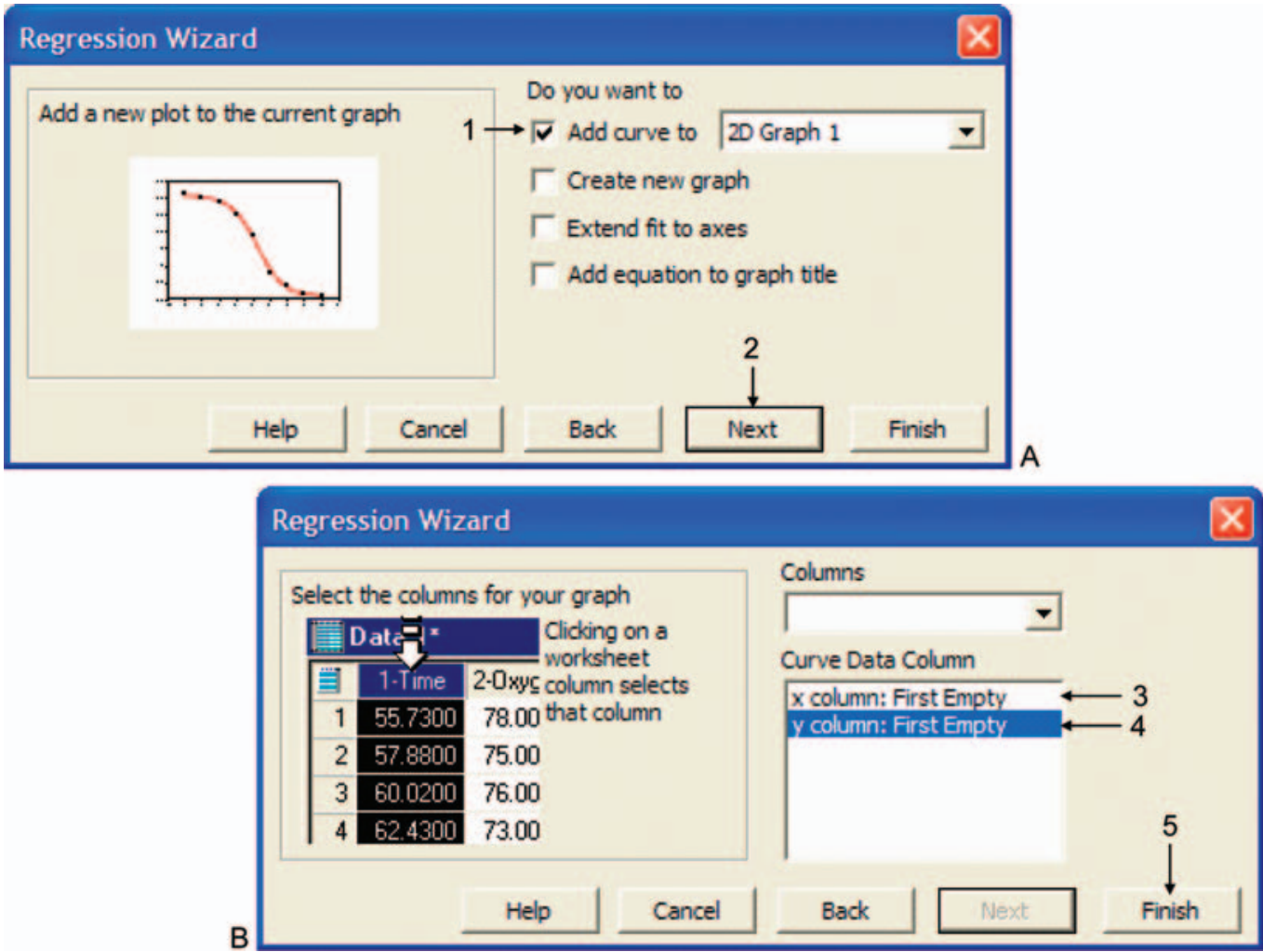

Figure 9.13. Generating data for best theoretical curve to experimental points.

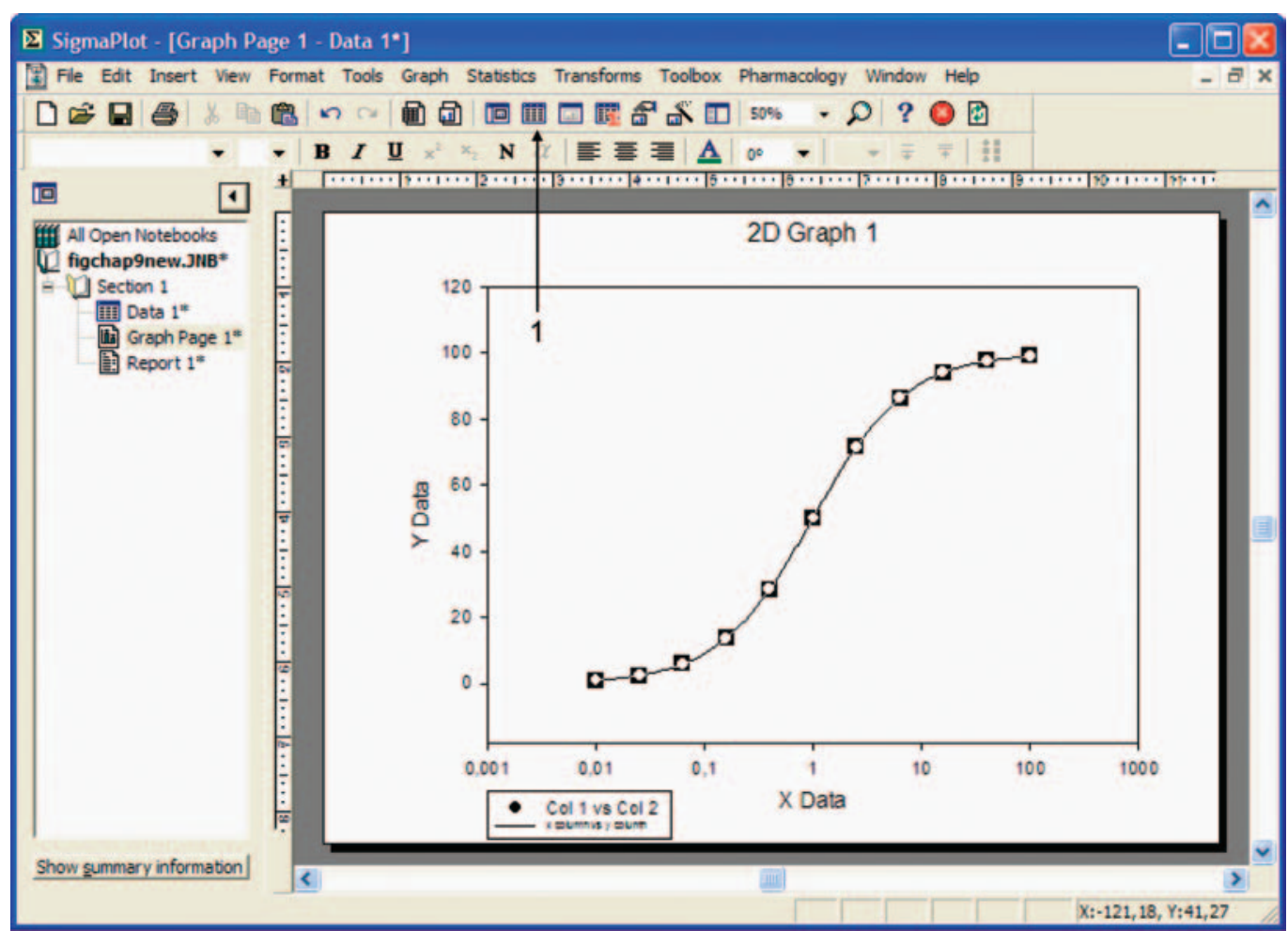

Figure 9.14. Display of best fitted theoretical plot to experimental data points. 


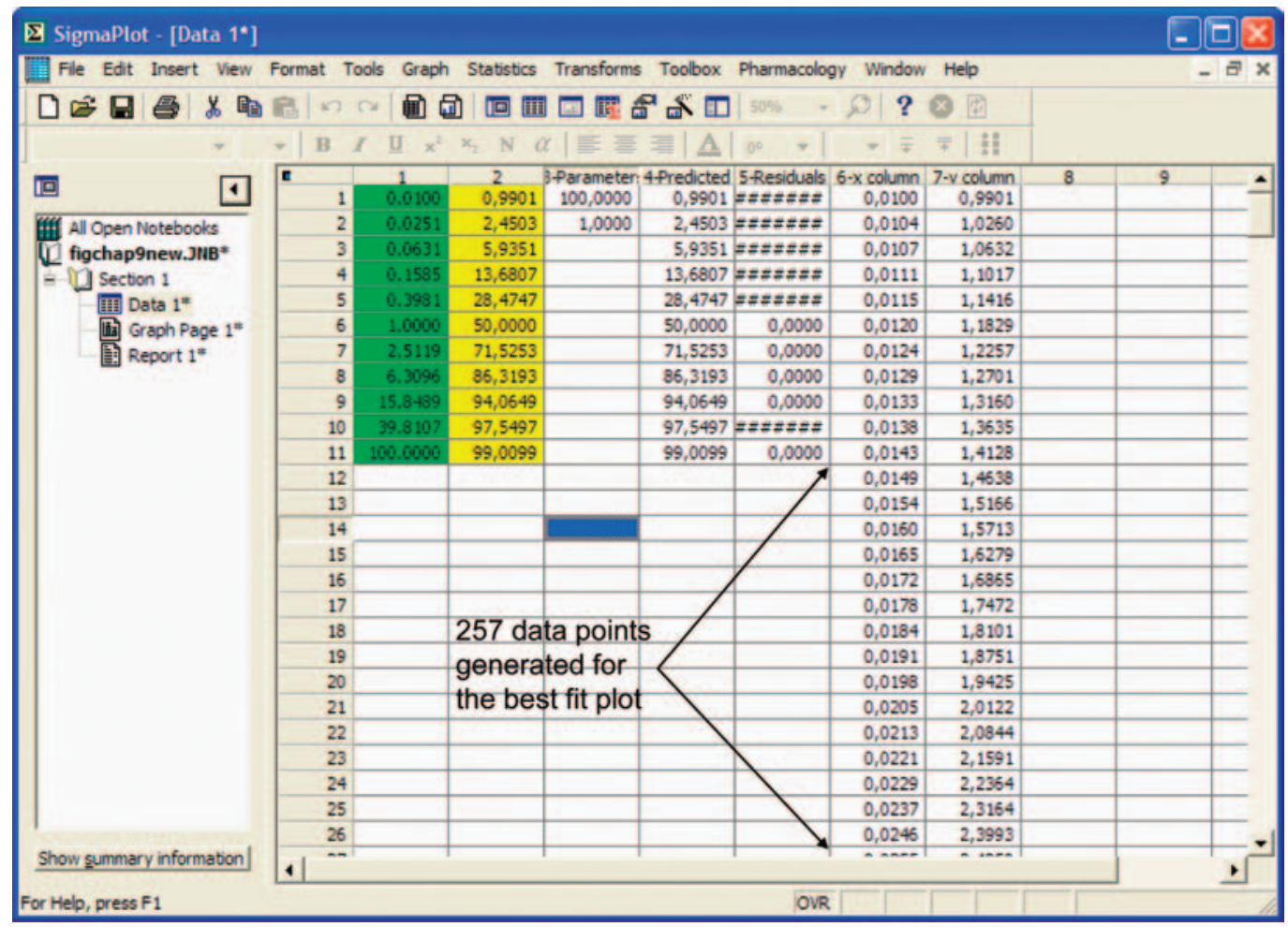

Figure 9.15. Parameters, predicted data point values, residuals, and data for theoretical curve.

\subsection{A User-defined Theory Fitted to Data Points}

\subsubsection{The Theory}

The procedures in Subchapter 9.1 were easy to follow. The simplicity was because the SP9 already covered the theory we assumed to be the right one. Now, instead, assume we have dose-response data that we believe follow another theory than for instance the load at equilibrium or the HMM steady-state situation described in Chapter 1. A theory that is not part of our software package.

One simple equation to represent such a situation is the intervention theory for binding or activity, where intervention parameter $c$ or $c^{\prime}$ is $>1$ (Chapters 2 and 3). This theory is generally not represented in ordinary software. Another possible theory could be the HMM combined with a diffusion process. To solve such a situation with the SP9 package, we can write our own program, i.e., a program of a novel theory and let the SP9 adjust the program's parameter values for a best-fit to experimental data. However, now we must use our arithmetic skills.

Let us imagine that we assume that our experimental data are for the transport of molecules and come from a system governed by a simple steady-state HMM reaction plus a parallel process of simple diffusion. This may be formulated as:

$$
f l u x=\frac{\text { Flux }_{\max } \cdot \mathrm{S}}{\mathrm{S}+K_{s}}+P \cdot \mathrm{S},
$$

where $P$ is a permeability. Both the flux and the $P \cdot S$ product have the dimension: mass $\cdot \mathrm{cm}^{-2} \cdot \mathrm{s}^{-1}$.

The parameters to be determined for our theory in Eq. 9.1 are: the maximal flux $\left(\right.$ Flux $\left._{\max }\right)$, a dissociation constant $\left(K_{\mathrm{s}}\right)$, and the permeability $(P)$, and we will incorporate this theory into our Sigma-Plot program. Another example of parallel fluxes is given by RoqueMalherbe (2007, Chapter 5).

\subsubsection{Create a User-defined Fit-routine}

As shown in Fig. 9.17A, we have read 11 data sets from an imagined experiment into our SP9 datasheet (arrows 1 and 2), and we used the GRAPH command to plot a curve of the data. ${ }^{2}$ To connect the data points with a line, we open the Graph Properties command (Fig. 9.17A, arrow 4) and arrive at Fig. 9.17B in which we select Lines (arrow 1) as Medium dash (arrow 2) and continue to Fig. 9.17C by pressing OK (arrow 3). The data points are now connected with a medium dashed line (Fig. 9.17C, arrow 4).

From a supposed knowledge about our experiment, let us assume that due to the context-dependent situation from where the data are obtained and by inspecting the plot closely, we imagine that the data are

\footnotetext{
${ }^{2}$ On how to make a 'Line and Scatter' plot in Sigma-Plot, see Section 9.1.1 and Fig. 9.2.
} 


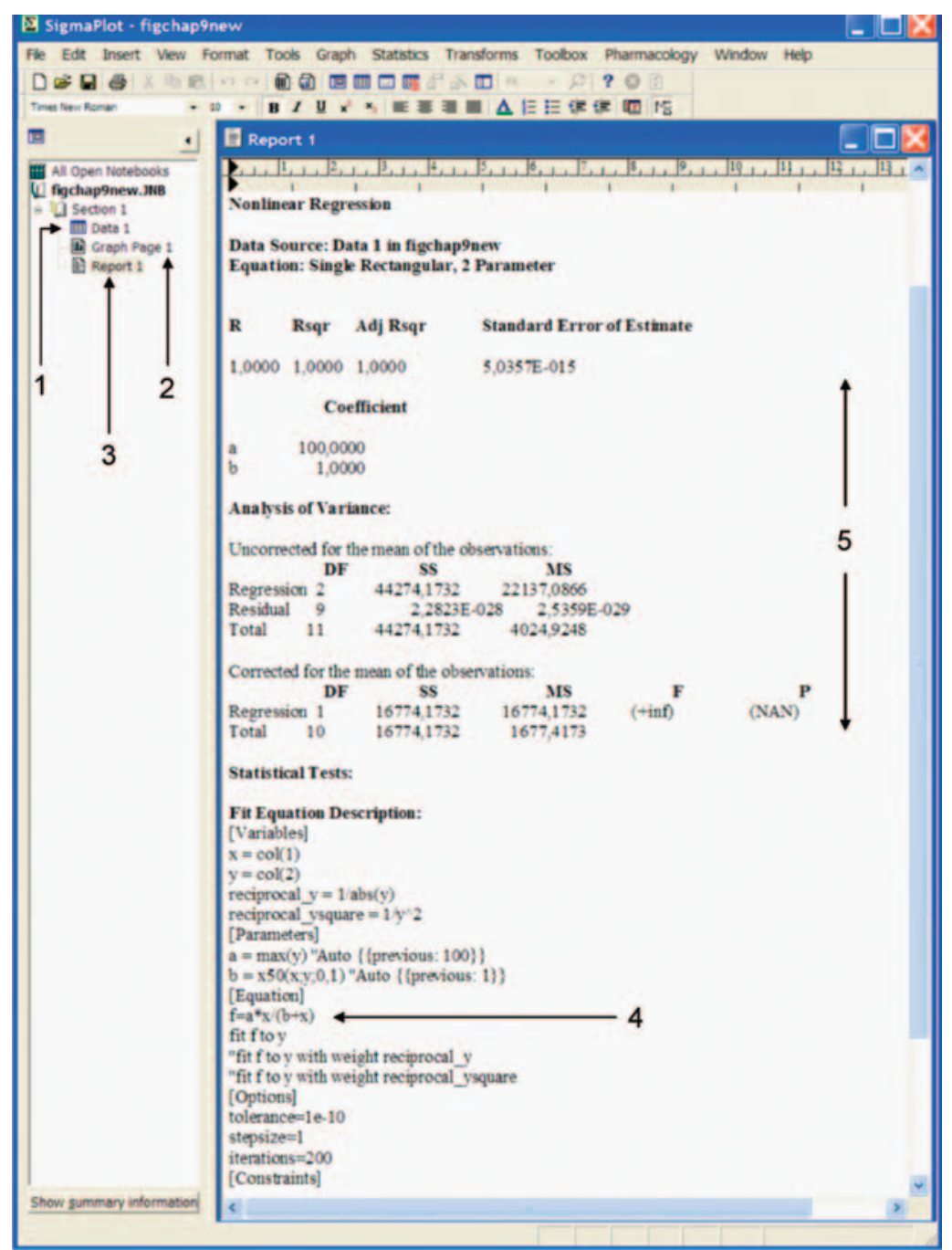

Figure 9.16. Report sheet for the fitting, including the selected theory.

best described as the sum of an HMM-type process in parallel with a diffusion process (Eq. 9.1). This type of response is seen in many applications, e.g., transport across epithelia (Menon \& Barr 2003; Fernandez-Teruel et al. 2005), and specific plus non-specific binding of ligands (Doo et al. 1998; Wigton \& Kilduff 2004).

To fit our theory in Eq. 9.1 to the data in Fig. 9.17A, we must first point and click at a data point in our Scatter plot (Fig. 9.17C, arrow 5) and then select the STATISTICS command (Fig. 9.9, arrow 1), and its submenu Regression Wizard (Fig. 9.9, arrow 2). This opens the Regression Wizard submenu (Fig. 9.18A). On finding the Equation category 'Hyperbola' (arrow 1), and Single Rectangular, 2 Parameter function (arrow 2), click on the Edit code (Fig. 9.18A, arrow 3) and Fig. 9.18B will appear. At this point, we want to adjust the Equation (arrow 4), the Initial Parameters (arrow 5), and the Constraints (arrow 6). However, in order not to disturb the SP9 supplied function, in reality we will edit a copy of the selected function. This is done by clicking on 'Add as' (Fig. 9.18B, arrow 7), and an 'Add as' menu appears (Fig. 9.19A). In this menu, reformulate the equation name to one which suits you, for instance load-diff 3 param (Fig. 9.19A, arrow 1 ), and notice on pressing $\mathbf{O K}$ (arrow 2), that a window with the name load-diff $\mathbf{3}$ param (arrow 3 ) is opened for the editing of our function (Fig. 9.19B, arrow 4). To first save our new function, press Add As..., (Fig. 9.19B, arrow 5 ), and jump to the editing in Fig. 9.20A. Here we change the function by adding: $+P * x$ (Fig. 9.20A, arrow 1 ), we define and initialize the new parameter $P$ by writing $P=1$ (Fig. 9.20A, arrow 2), and we include some constraints (Fig. 9.20A, arrow 3). Press the OK button, arrow 4 after the adjustments at arrows $1-3$ in Fig. 9.20A, and a Regression Wizard window appears (Fig. 9.20B).

Observe that a function now appears in the Equation category 'User-defined' with the name load-diff 3 param (Fig. 9.20B, arrow 5).

\subsubsection{Fit with Your Own Theory}

To fit your own theory to data, the new function load-diff 3 param is selected by pressing the Next key (Fig. 9.20B, 

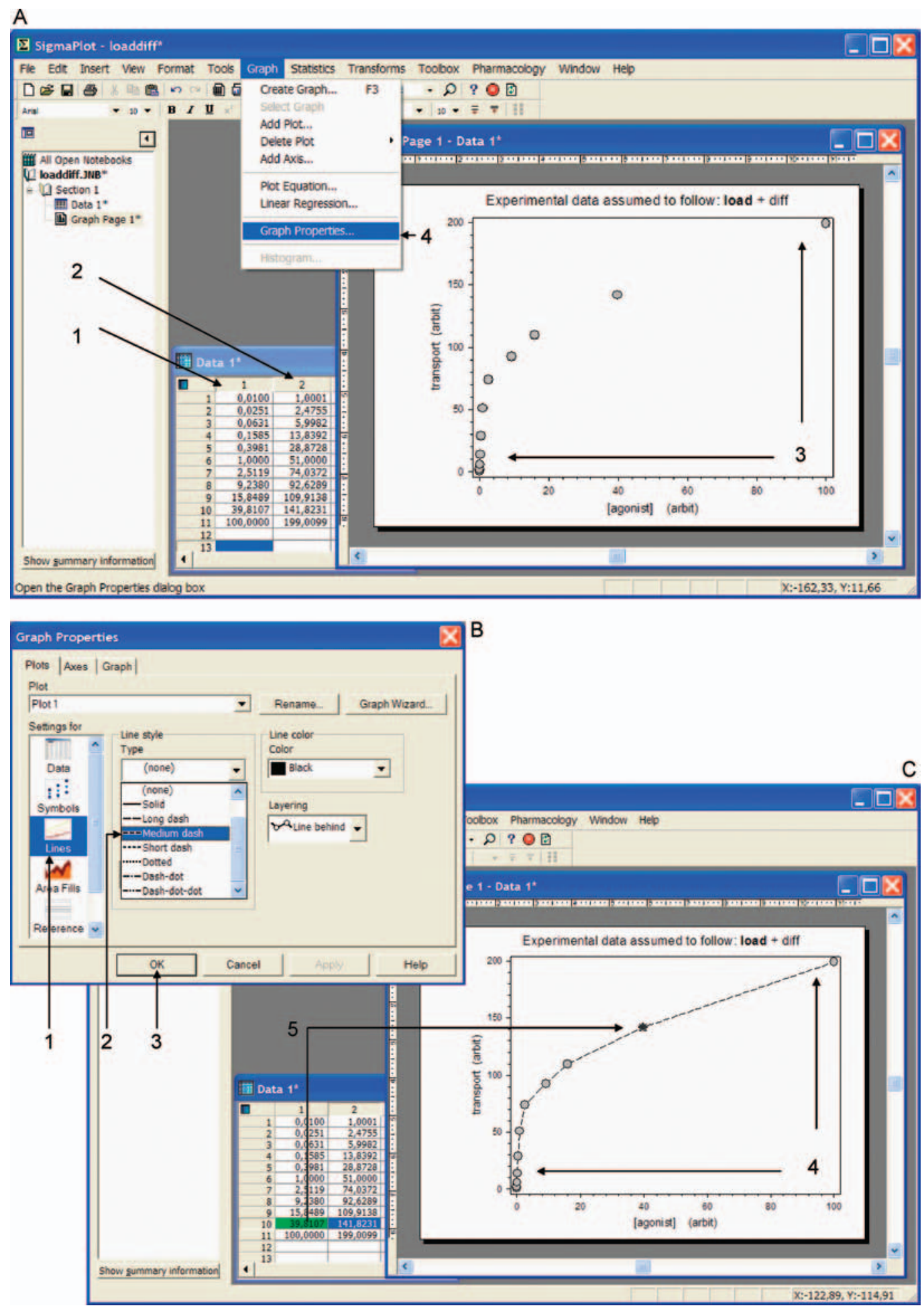

Figure 9.17. A 'Line and Scatter' plot of data based on a load plus diffusion reaction scheme. The menu for Graph types including Line and Scatter in SigmaPlot software is shown in Fig. 9.2A.

arrow 6). Control that the selected data for the fit are columns 1 and 2 where we placed our plot data in the SP9 worksheet (Fig. 9.20C, arrow 7), press Next (Fig. 9.20C, arrow 8) and obtain the best-fit parameter values (as shown in Fig. 9.20D). Press Next at arrow 9 in Fig. 9.20D and continue in the same manner as in Section
9.1.3 by repeating the menu functions shown in Figs. 9.12B-9.16. The final result is shown in Fig. 9.21.

Fig. 9.21A shows the data point connected with a dashed line, the 'Line and Scatter' plot, and on top of this is the plot of a non-linear regression best-fit theory, the red full line curve. 


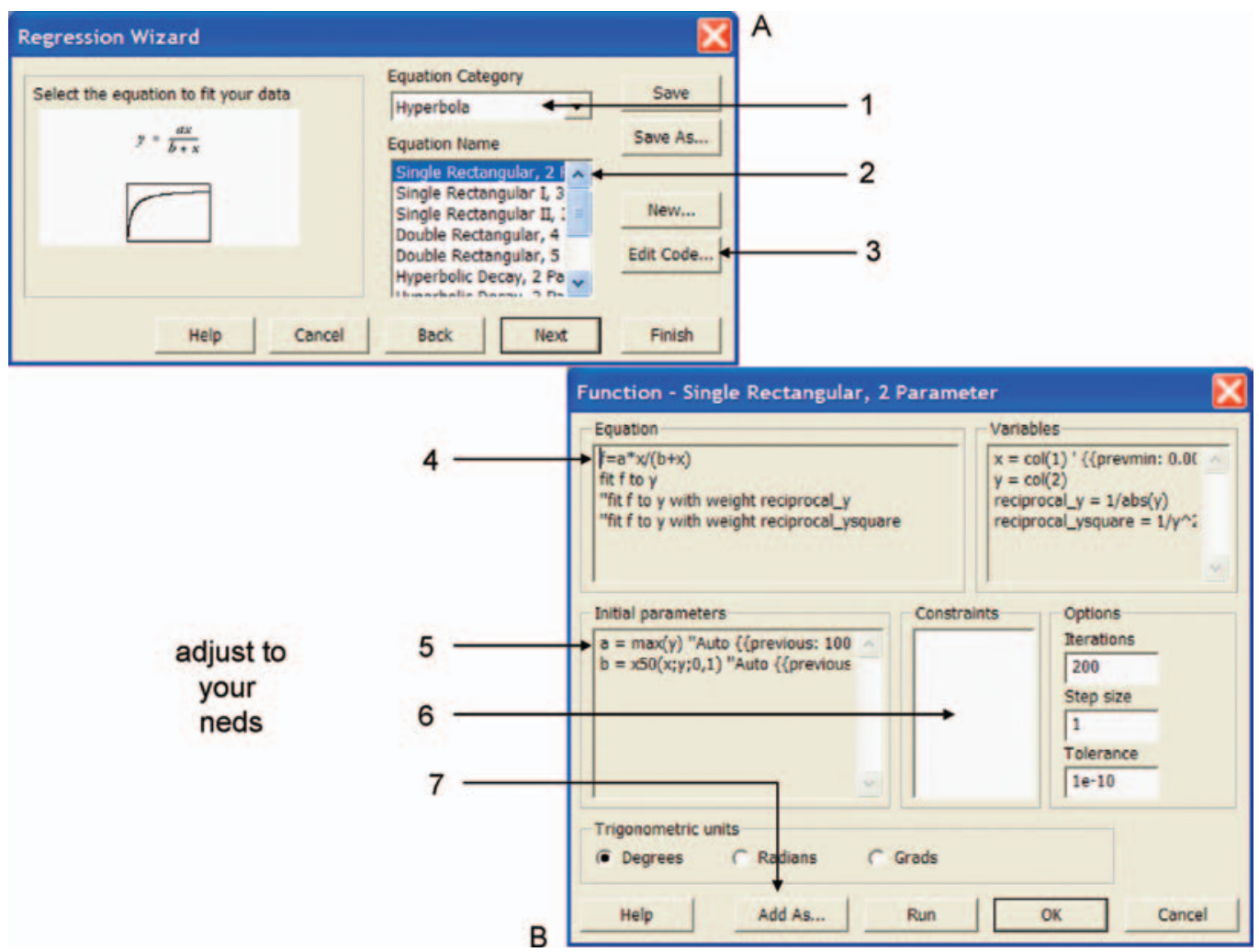

Figure 9.18. Selecting a suitable equation to be adjusted for load+diffusion theory.

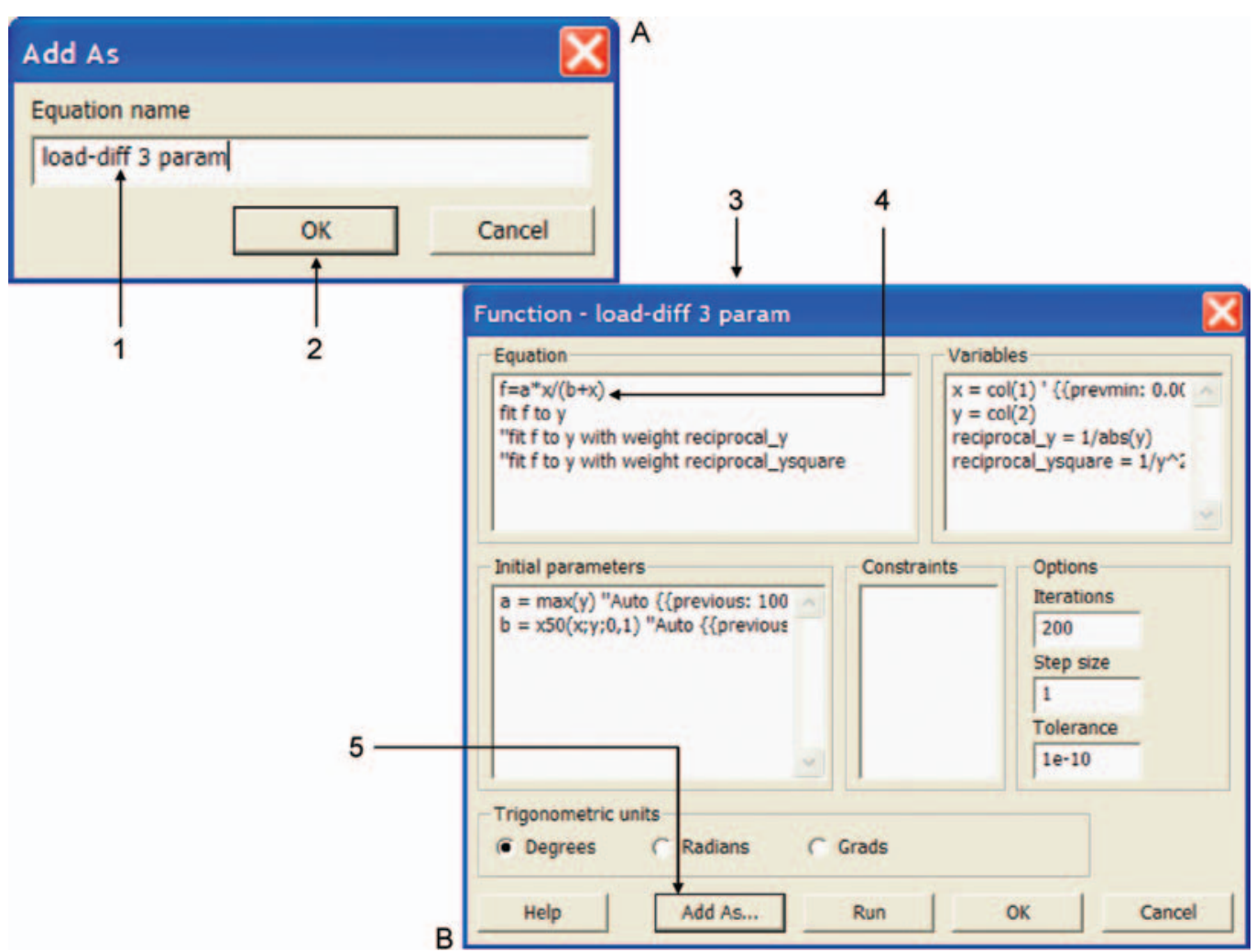

Figure 9.19. Baptizing new user-defined Function - named load-diff 3param. 


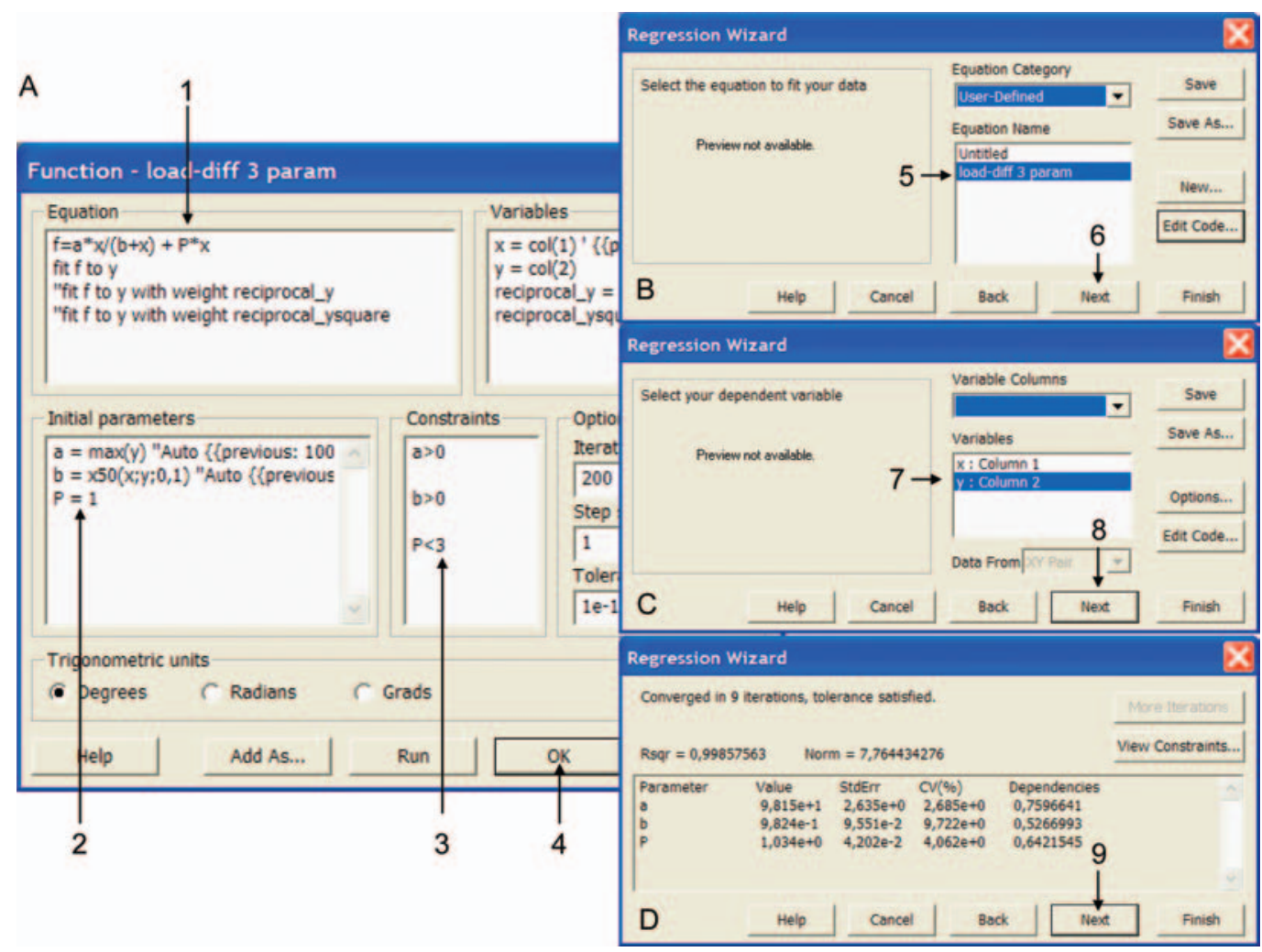

Figure 9.20. Creating a new user-defined Function, named load-diff 3param, and run this routine to obtain fits for parameters in the load+diffusion theory, Flux $x_{\max }, K_{\mathrm{s}}$, and $P$.

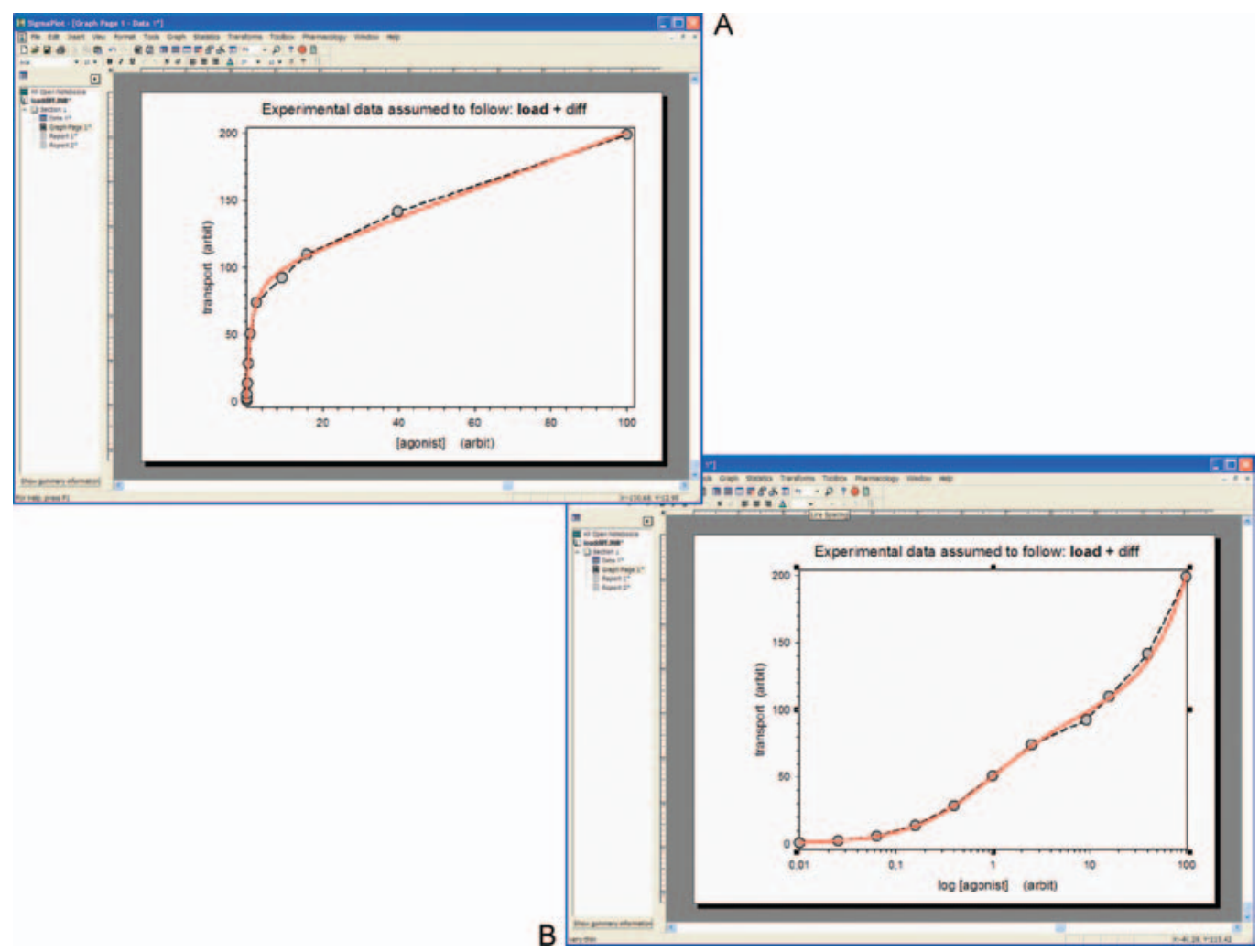

Figure 9.21. Plot of the best fit (red curve) on top of the experimental data for the load+diffusion theory. Presentation of the fit both as $(A)$ a linear-linear and (B) a semi-log plot. 
Fig. 9.21B shows the same as in Fig. 9.21A, except in this case we have selected a logarithmic $x$-axis for our concentration values, i.e., a semi-log plot of the data connected with a broken line and the non-linear regression plot presented as an unbroken red line. Notice the logarithmic scaled $x$-axis in Fig. 9.21B, and the linear scaled $x$-axis in Fig. 9.21A.

\subsubsection{Control and Complete Capability}

Since in principle our load-diff $\mathbf{3}$ param is the same as SP9s Single Rectangular II, 3 Parameter function under Equation Category 'Hyperbola', the result should be the same as it would be if the official SP9 Single Rectangular II, 3 Parameter function had been selected. Thus, in this situation we have an internal control on what we have carried out, which is comforting as long as we are pupils.

This concludes the second course in using SP9-tools for theoretical analysis of synagics. Of course, implementing new functions can be more complicated, but for now this will suffice as a demonstration of what we need for our analysis of new theories.

For complete capability covering all aspects of analyzing data, we still need to look at how we can generate data for any theory and be able to make plots of the theory in both 2-D and 3-D. This will give us the freedom to test our theories and impart new ones based on their realism and applicability to experimental data. Thus, experiment and theory should go hand-inhand.

How to generate theoretical data for 2-D and 3-D plots is demonstrated in the next course in subchapter 9.3.

\subsection{Test the Realism of User-defined Theories by Their 2-D and 3-D Plots}

\subsubsection{Introduction of the Theory}

It is important and essential to have easy access to analytical tools that can immediately visualize how theoretical dose-response relationships behave when the system constants are changing. This section describes how to generate your own dose-response curves from theoretical reaction schemes supplied by the user (you), after your theory has been formulated into a distribution equation (a function). An SP9 program 'datagen.xfm' is presented in Box 9.1 for generating data with which one can plot a curve of any theory with a single independent variable, including theories for synagic relationships. See later for an abbreviated version of the program in Fig. 9.23C+Box 9.3. An additional subroutine for the datagen. $\mathrm{xfm}$ program is listed in Box 9.2, which allows the program to operate with two independent variables and to generate data that can be used for plotting theories with two independent variables in 3-D surface plots. For instance, taking synagics as an example, it is advantageous to be able to visualize the behavior of simultaneously varying the concentration of an agonist ligand and of a modulator molecule. A compressed form of this subroutine is listed in Fig. 9.26A and Box 9.3.

The theory to be implemented in the program is the simple load equation: $y=Y_{\max } \cdot \mathrm{S} /\left(\mathrm{S}+K_{\mathrm{d}}\right)$.

The program in Box 9.1 consists of five subroutines, and each subroutine has a single program-line (compare with Fig. 9.23A). Thus, the program datagen.xfm consists of five executable program lines, and includes lines of commentaries. Only a few simple alterations are necessary for the program to work on any dose-response theory for immediate plotting, while varying parameter values. Switching to any theory is by rewriting subroutines 4 and 5 .

The range of the independent and dependent variables, i.e., concentration and response for synagics, and the number of data sets that plots can be based on are easily changed as well (Box 9.1 and Box 9.2).

\subsubsection{Writing an SP9 Program}

Programs written in SP9 language containing functions are referred to as 'Transforms'. Opening an SP9 datasheet (Fig. 9.14, arrow 1) we are able to activate the Transform command (Fig. 9.22A, arrow 1) and from its menu select User-Defined... transform (Fig. 9.22A, arrow 2). Observe that the Transform command is not activatable when viewing a Graph page. In the UserDefined Transform page (Fig. 9.22B), we can write two program line such as $x=\operatorname{DATA}(2 ; 5 ; 1)$ (Fig. 9.22B, arrow 3), and $\operatorname{col}(1)=x$ (Fig. 9.22B, arrow 4). The first number in the argument for $\operatorname{DATA}(\ldots)$ is 2 , the next is 5 , and the third is 1 (Fig. 9.22B, arrows 5-7). The meaning of this is: generate the numbers from 2 to 5 in steps of 1 . When we RUN this transform (Fig. 9.22B, arrow 8), the datasheet reappears with the values $2,3,4,5$ in column 1 (Fig. 9.22C). If we write a user-defined transform, such as $x=\operatorname{DATA}(0 ; 20 ; 2)$ and $\operatorname{col}(3)=x$, and execute the program, we obtain 11 data values from 0 to 20 in column 3, in jumps of 2 (Fig. 9.22D).

\subsubsection{Subroutine for Equidistant Concentration Values on a Log-scale}

The situation now becomes more difficult. First, we must generate suitable values for an independent variable ( $\mathrm{S}=$ concentration). On how to generate numbers for equidistant log-doses, i.e., values increasing with a constant factor, you should consult subroutines Nos. 1-3 in the datagen. xfm program listed in Box 9.1, and 


\section{BOX 9.1}

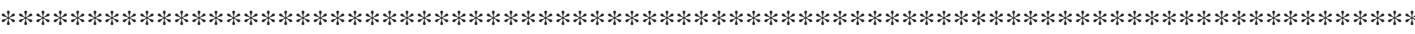

SP-program to generate 2D plots from equations of your theory C t'f 2007

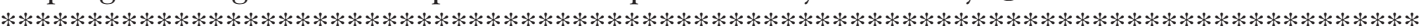

'Subroutine \#1 generates $N+1$ integers to cover a range of $10^{n}$. Integers from 0 to $N$ are 'placed in program variable pv1. The power $n$ is assigned to later generate the range $10^{n}$ :

$N=10 \quad \operatorname{pv} 1=\operatorname{DATA}(0 ; N ; 1) \quad n=6$

'Subroutine \#2 generates $N+1$ numbers that are spaced from 1 to $10^{n}$ by an equal factor, 'useful for log-scales, and placed in program variable pv2:

pv2 $=10^{\wedge}(n *(\mathrm{pv} 1 / N)) \quad$ 'The symbol $\wedge$ means raise to a power.

'Subroutine \#3 spreads $N$ values equally around independent variable $\mathrm{S}=1$; and places these

'values for $\mathrm{S}$ in the spreadsheet column 1:

$\mathrm{S}=\mathrm{pv} 2 * 10^{\wedge}(-n / 2) \quad \operatorname{col}(1)=\mathrm{S}$

'Rescaling S to a semi-log plot is obtained by log capabilities in SigmaPlot's 'Graph function'.

'Next, generate values of dependent variable $y$ from $S$, for example based on a load equation

'for binding: $y=Y_{\max } * \mathrm{~S} /\left(\mathrm{S}+K_{\mathrm{d}}\right)$. But first, give values to parameters:

'Subroutine \#4 assigns parameter values. For instance:

$Y \max =100 \quad K_{\mathrm{s}}=1$

'Subroutine \#5 generates $N+1$ values of $y$ from THE-ORY. Insert your theoretical equation here.

$y=Y_{\max } * \mathrm{~S} /\left(\mathrm{S}+K_{\mathrm{s}}\right) \quad \operatorname{col}(2)=y$

'Alternative subroutine \#5

'Additionally, we may vary one of the parameters in $x$ steps and place related $y$ values in columns $1+x$;

'to make $x$ curves of THE THE-ORY. We replace parameter $K_{\mathrm{s}}$ with $c=f(x)$ and can write:

'for $x=1$ to 5 do $c=10^{\wedge}(x-3) * K_{\mathrm{S}} \quad y=Y_{\max } * \mathrm{~S} /(\mathrm{S}+c) \quad \operatorname{col}(1+x)=y$ end for

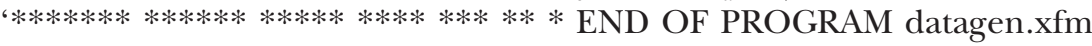

Comments to the datagen.xfm program:

1. Values $N$ and $n$ may be changed to operate with other numbers of data and other ranges of the independent variable $=\mathrm{S}$. To obtain smooth curves increase $N$ from 10 to 1000 , and to expand the range for the independent variable $\mathrm{S}$ increase from $n=6$ to $n=10$.

2. By placing an apostrophe in front of the executable-line in subroutine 5 and removing it from its last line, we can open for construction of five plots of the THE-ORY where $K_{\mathrm{s}}$ varies between $10^{-2}$ and $10^{2}$. The responses are placed in columns $x+1(=2,3,4,5$ and 6$)$ (see Fig. 9.23A).

3. Subroutines \#2 and \#3 may be combined, thus $\mathrm{S}=10^{\wedge}(n *((\mathrm{pv} 1 / N)-(1 / 2)))$.

written in a compressed form in Fig. 9.23A. ${ }^{3}$ The three executable lines of the program are indicated by arrow 1 in Fig. 9.23A. The program variable ' $n$ ' in the first line (arrow 2), will set the range of values for our data for the independent theory-variable, which is concentration ' $\mathrm{S}$ ' in line 3 . Here as an example, the range ' $n$ ' is chosen as 6 (arrow 2). The program command 'DATA' in line 1 will generate numbers of data from 0 to $N$ (Fig. 9.23A, arrow 3), equal to $N+1$ (cf. Section 9.3.1). Since $N$ is sat to 10 , we should expect 11 data. In case $N$ is changed to 1000 , we will get 1001 data.

When you RUN this part of the datagen.xfm program (Fig. 9.23A, arrow 4), you obtain the values shown in Fig. 9.23B.

\footnotetext{
3 'Interpretive delirium begins only when man, ill-prepared, is taken by a sudden fear in the forest of symbols'. André Breton, L'Amour fou (1937).
}

The values of our independent variable ' $S$ ' are spread over a range of 6 orders of magnitude $(n=6)$ with 11 data points $(N=0$ to 10$)$, and with the same factor between data points. The median data is unity (row 6 , Fig. 9.23B). These types of concentration values are suitable for logarithmic scales. Note that logarithmic axes are in principle also dimensionless, i.e., normalized or relative.

Lines 2 and 3 in the program (Fig. 9.23A) may be combined to a single statement (see also the third comment in Box 9.1 and later 2-D routine line 2 in Box 9.3).

\subsubsection{Subroutine for System Constants, the So-called Parameters}

Next, we must formulate an equation for our reaction scheme or theory. However, in the program, we first 


\section{BOX 9.2}

Subroutine \#6 for THREE DIMENSIONAL PLOTTING

To obtain smooth curves increase $N$ from 10 to 1000 , and to expand the range for the independent variable $\mathrm{S}$ increase from $n=6$ to $n=10$ in subroutine \#1 in BOX 9.1.

'Subroutine \#6. Generates data to produce 3-D mesh plots (C) t'f 2007

oo $=30 \quad$ 'The value of 'oo' determines the number of gridlines

for $m=0$ to oo do for $k=0$ to oo do

$\mathrm{s} 3=10^{\wedge}(-n / 2) *\left(10^{\wedge}(n / \mathrm{oo})\right)^{\wedge} k$

$\mathrm{s} 4=10^{\wedge}(-n / 2) *\left(10^{\wedge}(n / \mathrm{oo})\right)^{\wedge} m$

put s3 into $\operatorname{col}(x+2 ; 1+(o o+1) * m+k)$

put s4 into $\operatorname{col}(x+3 ; 1+($ oo +1$) * m+k)$

'We will assume a non-competitive reaction scheme with two independent variables,

'see Eq. 2.3, and further that the interventor constant $K_{\mathrm{ii}}$ can vary in five steps.

'Since the $K_{\mathrm{ii}}$ constant is not defined in subroutine \#4 we define it here:

$K_{\mathrm{ii}}=1$

'FUNC-TION

for $j=1$ to 5 do $g=\left(10^{\wedge}(j-3)\right) * K_{\text {ii }}$

$Z=Y_{\max } *\left(\mathrm{~s} 3 /\left(\mathrm{s} 3+K_{\mathrm{s}}\right)\right) *(g /(\mathrm{s} 4+g)) \quad \operatorname{col}(j+x+3 ; 1+(\mathrm{oo}+1) * m+k)=Z$

end for

end for end for

،

Comments to subroutine \#6:

1. To implement subroutine \#6, just insert the open lines into the program datagen. $\mathrm{xfm}$ in BOX 9.1 after subroutine \#5. The datagen. xfm program will now generate extra columns for the two variables $\mathrm{S}(=\mathrm{s} 3)$ in column $x+2(=7)$ and interventor $\mathrm{I}(=\mathrm{s} 4)$ in column $x+3(=8)$ plus the related response $(=Z)$ in columns $j+x+3(=9,10,1112$, and 13) for plotting several 3 -D plots of the FUNC-TION in a graph (see Fig. 9.26C).

2. A compressed form of the program datagen. $x$ fm generating data for 2-D plots including 3-D plots is given in Box 9.3 and shown in Fig. 9.26A. Two of the generated 3-D dose-responses are shown in Fig. 9.27.

3. The number of gridlines in the MESH-plots is here sat to 30 , but can be varied simply by changing the value of program-variable 'oo', line 6 in Fig. 9.26A. Thus, for instance, a value 40 for 'oo' instead creates a grid of $40 \times 40$ meshes for the 3 -D plots.

need a routine that will define the system constants of our theory. Defining and initializing parameters $Y_{\max }$ and $K_{\mathrm{d}}$ for our theory in Section 9.3.1 is carried out in subroutine \#4 in the datagen.xfm program (see Box 9.1 and line 4 in Fig. 9.23C, arrow 5).

\subsubsection{Subroutine for the Theory}

Now a subroutine \#5 is introduced that includes the theory we believe covers our experimental reaction scheme and formulated as an equation, equal to our function. Our theory is the aforementioned simple load function in Section 9.3.1. Subroutine \#5 will calculate the values of the dependent variable, $y=$ response, and place them in column 2 of the spreadsheet. (See subroutine \#5 in Box 9.1 or line 5 in Fig. 9.23C, arrow 6).
Eventually, we will allow the system constants to vary in a few steps in order to test their influence on the function. The values of dependent variable ' $y$ ' will vary both with the independent variable ' $\mathrm{S}$ ' as well as with the variations introduced for the system constants. The statement for this is presented in the program-line of the Alternative subroutine \#5 (Box 9.1), and in the executable program-line number 5 shown in the compressed program in Fig. 9.23C (arrow 7). ${ }^{4}$

By inserting generated values from subroutines \#1-3 of our independent variable into our formulation of the theory, the function, and also varying a single parameter in steps, as done in the 'for-end for' program loop in Alternative subroutine \#5 (Box 9.1) or in Fig. 23C

\footnotetext{
$\overline{4}$ Program-lines in SP9 are made executable by removing a preceding apostrophe.
} 

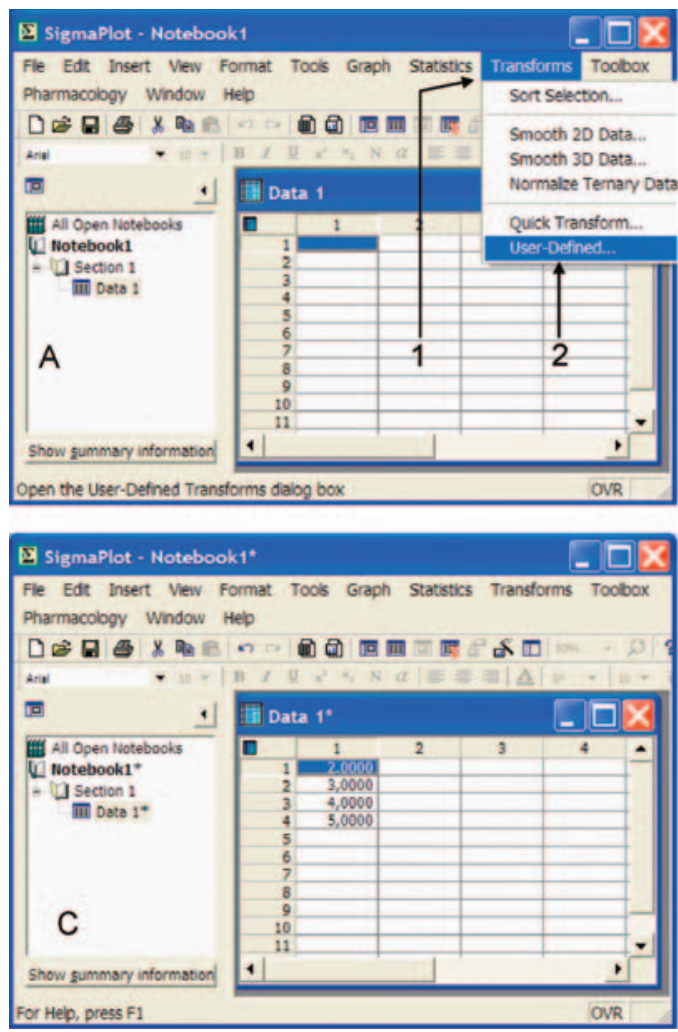
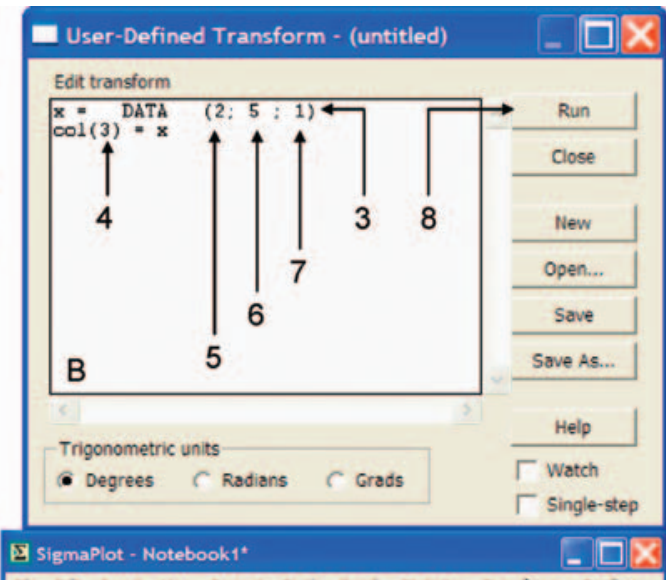

Fle Ede Insert Vew Format Tools Graph Statstics Transforms Toobox Pharmacology window Hel

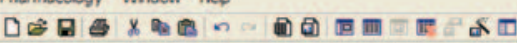
핑 All Open Notebooks W Motebook1

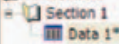

\section{D}

Show summary information For Help, press F1

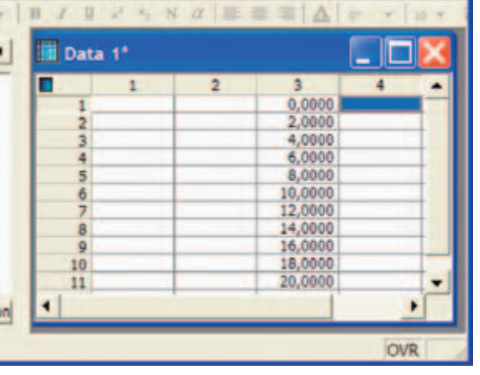

Figure 9.22. Working with user-defined Transforms. Data in $D$ from $x=\operatorname{DATA}(0 ; 20 ; 2)$ and $\operatorname{col} 3=x$.
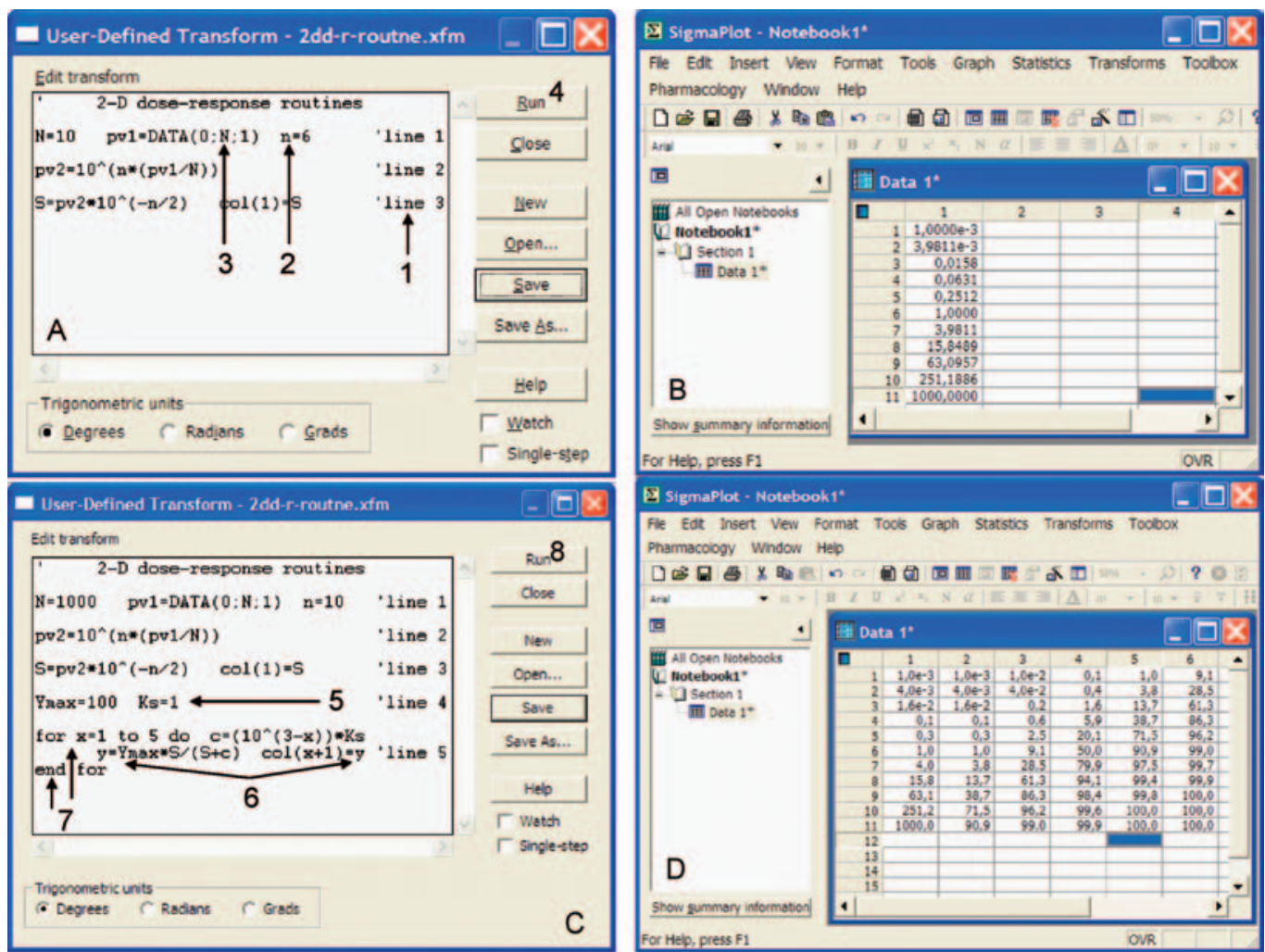

Figure 9.23. Equidistant data points. Generation of logarithmically equidistant data points (panels A and B), for use as an independent variable in load function included in a user-defined Transform (panel C). Data points for this load function with variation of its dissociation constant ' $K_{\mathrm{s}}$ ' in five steps are listed in panel $D$. 


\section{BOX 9.3}

Compressed version of SP-program datagen. xfm to generate data for plotting of any theory in 2-D line-plot and 3-D mesh- or contour-plot

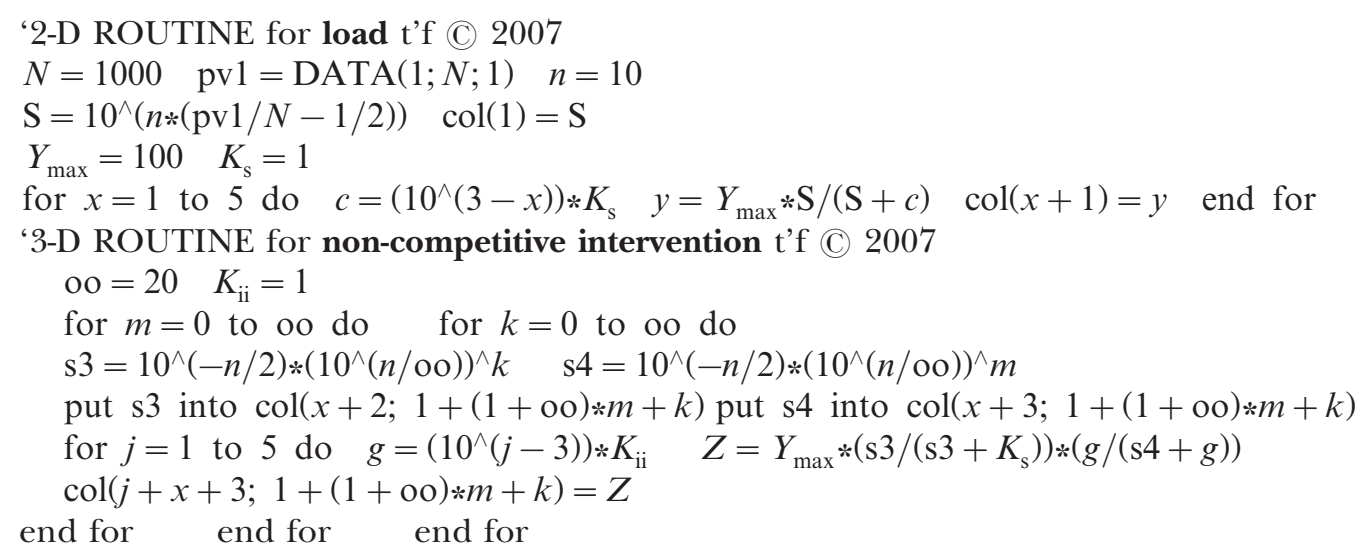

Comment: the number of grid-lines in the MESH-plots is here sat to 20, but can be varied simply by changing the value of program-variable 'oo' in the 5 th executable line above or in line 6 in Fig. 9.26A. Thus, for instance, a value 60 for 'oo' instead creates a grid of $60 \times 60$ meshes for the 3-D plots.

(arrow 7), we can simultaneously generate data for the dependent variable $(y=$ response $)$ changing with the system independent variable $(\mathrm{S}=$ concentration) and the parameter $K_{\mathrm{s}}$ (Fig. 9.23C, arrow 5). The generated $\mathrm{S}-y$ data sets are written into two columns, column 1 for $\mathrm{S}$ and column 2 for $y$ (Fig. 9.23D). In case we also alter the value of a parameter in steps, the dependent variable ' $y$ ' may appear in as many columns as there are steps, here for the parameter $K_{\mathrm{s}}=c$ is changing in five steps (Box 9.1 or arrow 7 in Fig. 9.23C), and response-values are placed in columns 2-6 in the datasheet in Fig. 9.23D.

\subsubsection{Simultaneous Presentation of Several Plots for the User-defined Theory}

Based on the generated data sets in Fig. 9.23D, we can plot a dose-response curve for each step in a given parameter, or all the curves (plots) for each parameter step may be presented in a single graph. The first plot we make is based on the data we have in columns 1 and 2 in Fig. 9.23D. These data are for $\mathrm{S}$ versus $y$ for $K_{\mathrm{s}}=10^{-2}$. The plot we want is a Line Plot instead of a Scatter Plot (Fig. 9.24A, arrow 1) in the Create Graph menu. After selecting the right columns for $x$-axis and $y$-axis, the plot is shown in Fig. 24B (arrow 2). As demonstrated earlier, this Line Plot in Fig. 24B with linear scaled axes is changed to a semi-log plot by double clicking at the $x$-axis, and in the Scaling menu selecting $\mathbf{L o g}$ (common) (see arrows 1-4 in Figs. 9.6 and 9.7). The semi-log plot of the curve in Fig. 24B is shown in Fig. 24C (arrow 3).

A Graph with several plots (curves) is obtained by the SP-command Graph and its Add Plot submenu (Fig. 9.24D, arrow 4). Again, the columns are selected for each new plot, as we did for the first plot. A 'Graph' in SP terminology is a single co-ordinate system, while a 'Plot' is a single curve in such a co-ordinate system. Several curves (plots) can be displayed in a Graph and several Graphs may be visualized on an SP9 Graph-Page. A presentation of the data for a change in $K_{\mathrm{s}}$ is now displayed in Fig. 9.24E (arrow 5), which makes it easy to judge the behavior of a parameter as it changes in a theory or a function. In Fig. 9.24E (arrow 5 ), $K_{\mathrm{s}}$ changes by a factor 10 in five equal steps from $10^{-2}$ to $10^{2}$.

\subsubsection{Shaping the Graph}

The procedures in Section 9.3.6 generate a template for presenting several plots at a time. Plots from the template are based on data placed in column 1 for the independent variable, and in columns 2-6 for the dependent variable. Plot templates may be refined in order to optimally present the $d-r$ curves, which can be effectuated by adjusting the scaling of the axes, implementing new ticks at the axes, rewriting the labels of the axes, and other details which I will omit here and just demonstrate the adjustment in a figure after supplementing an increased number of data sets for the two variables. To increase the number of generated data sets 

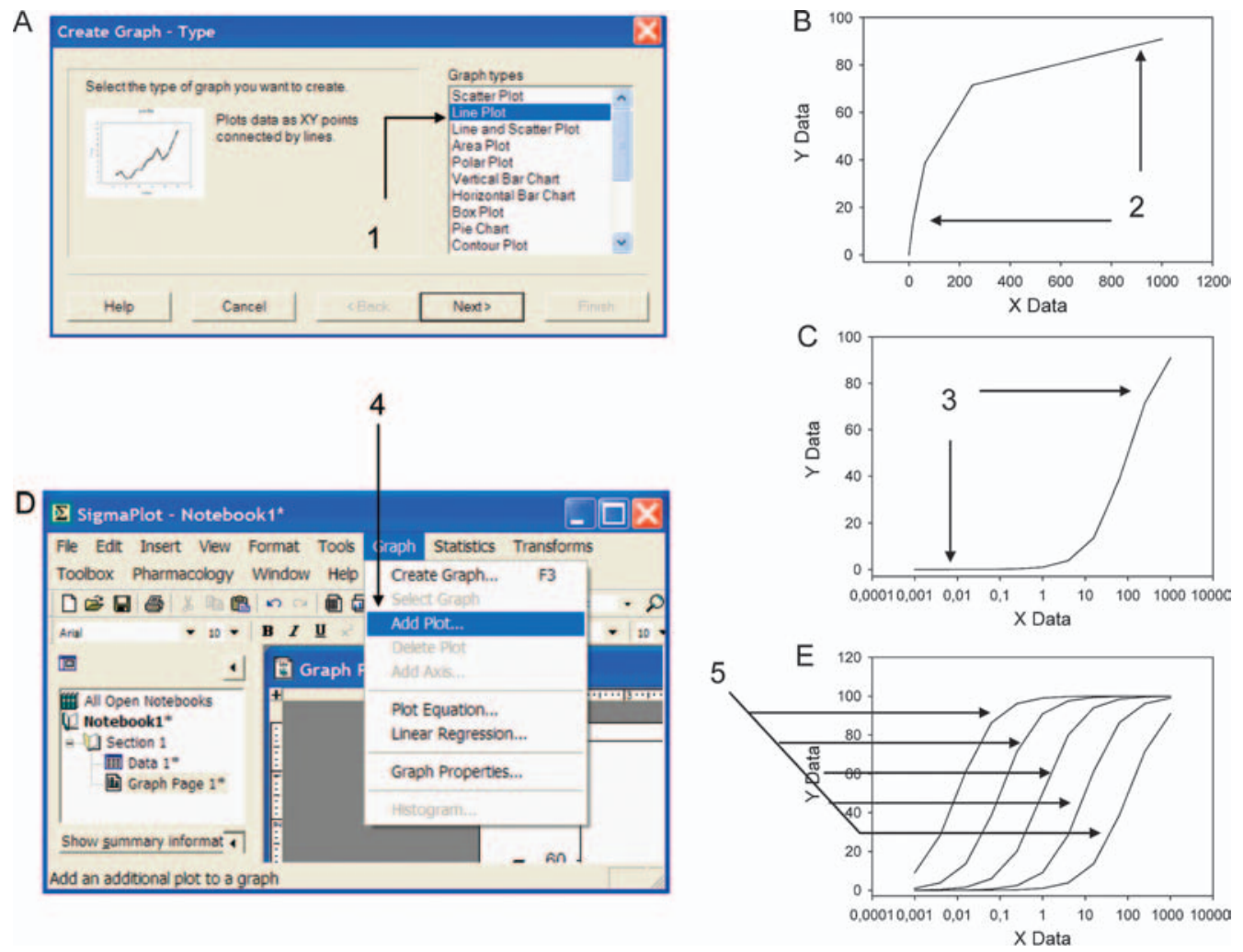

Figure 9.24. Several plot curves in a Graph. Plots of five data-sets in Fig. 9.23 are generated through procedures from panel A to $\mathrm{E}$.

for each of the five already displayed plots, just increase the value of ' $N$ ' from the original 10 to, for instance, 1000 and further increase the data range $n=6$ given by the original to perhaps $n=10$ (see Boxes 9.1 and 9.2, and arrows 1 and 2, Fig. 9.25A). Then rerun the program.

So, without specifying how I have adjusted the plot template of Fig. 9.24E, except for the adjustment of the $x$-axis to cover a data range from $10^{-5}$ to $10^{5}$ (Fig. 9.25B, arrow 3) using this new template on an expanded data sets $(N=1000, n=10)$ we obtain the plots as shown in Fig. 9.25C.

In Fig. 9.25C the labels of the axes are updated (arrows 4 and 5) and the variation in $K_{\mathrm{s}}$ that give us the five plots is explained by labeling the curves as shown at arrows 6 and 7. In addition, the Graph figure in 9.25C has been Exported from SP9 as a TIFF-file before reaching this page.

When you have tried the above-described procedures twice, generating your own 2-D plots becomes very simple. On the other hand, to understand the following section on generating 3-D presentations may cause some problems, but it is worth testing out.

\subsubsection{Three Dimensional Plots and Contour Plots}

It is visually convenient to be able to present doseresponse relationships when our theory involve two independent variables, for instance the concentrations of an agonist [S] and a modulator molecule [M], both affecting the resultant response R. With the Sigma-Plot9 program, a 3-D representation may be effectuated by its Mesh Plot command after data generation from the background theory. Unfortunately, the 3-D data-generator command in SP9 only allows symmetrical 3-D Mesh Plot solutions. Therefore, I have included the simple subroutine, listed in Box 9.2 and in an alternate form in Fig. 9.26A, to generate data of our 'twoindependent-variable' theory for a 3-D Mesh Plot. The subroutine in Box 9.2 may be added to existing programs, similar to that in Box 9.1, thereby employing the same function parameters of the program in Box 9.1; defined and initialized in its subroutine \#4.

For an implementation of 3-D Mesh Plots or Contour plots based on data generated by the 3-D subroutine in Box 9.2, consult the procedures of Fig. 9.26B (arrow 2).

\subsubsection{A User-defined 3-D Subroutine}

A subroutine that works together with the datagen. $x \mathrm{fm}$ program in Box 9.1 is presented in Box 9.2. The two programs in Boxes 9.1 and 9.2 are assembled in a compressed form in Fig. 9.26A, where the 3-D subroutine of Box 9.2 (Fig. 9.26A, arrow 1) is included in the 2-D program of Box 9.1 which is also presented in a compressed form in Fig. 9.25A. 


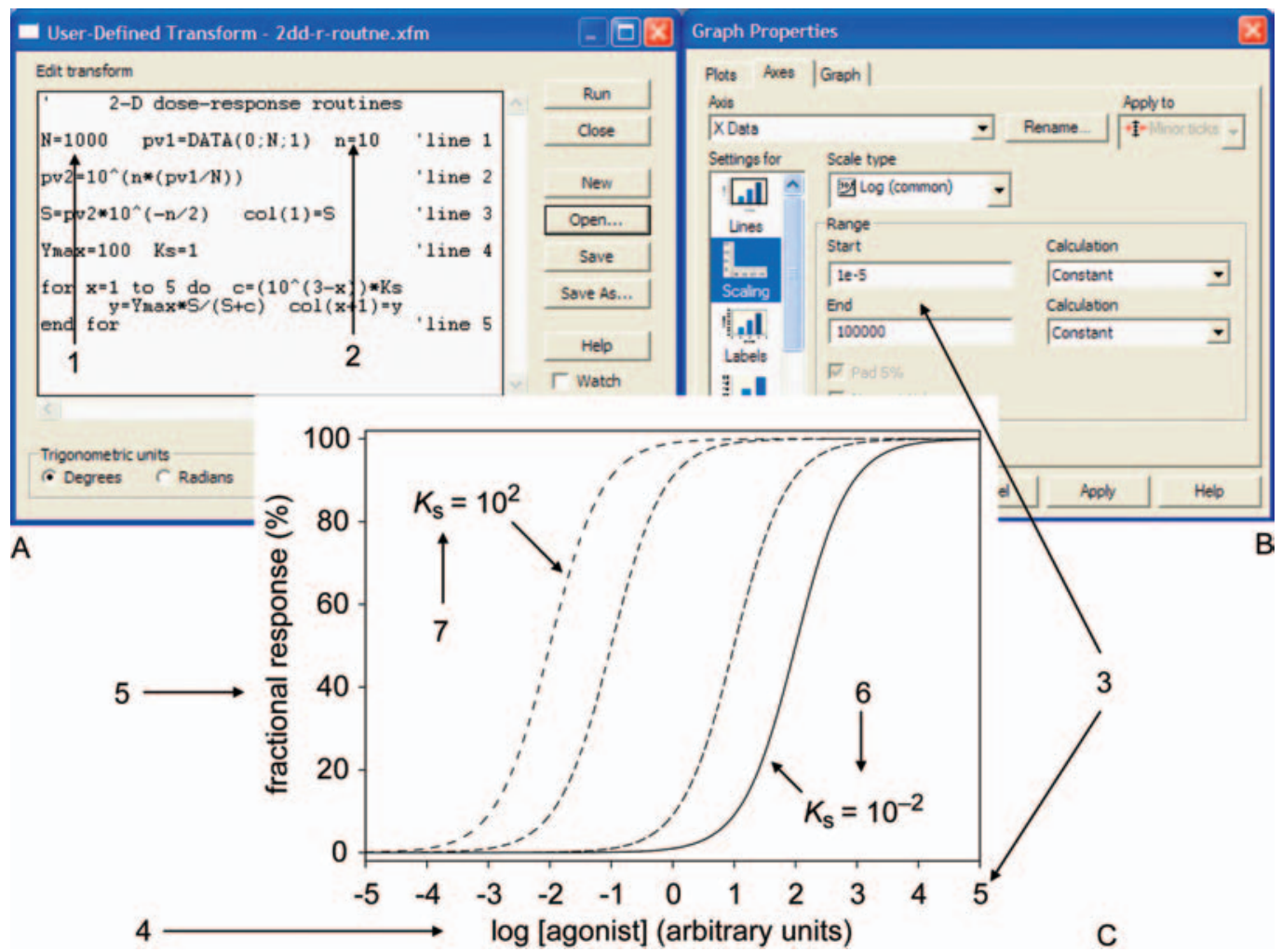

Figure 9.25. Improved data presentation. Increasing the number of generated data for plots in Fig. 9.24 (panel A), and adjusting the axes for better presentation (panel B), result in the data-plots in panel C.

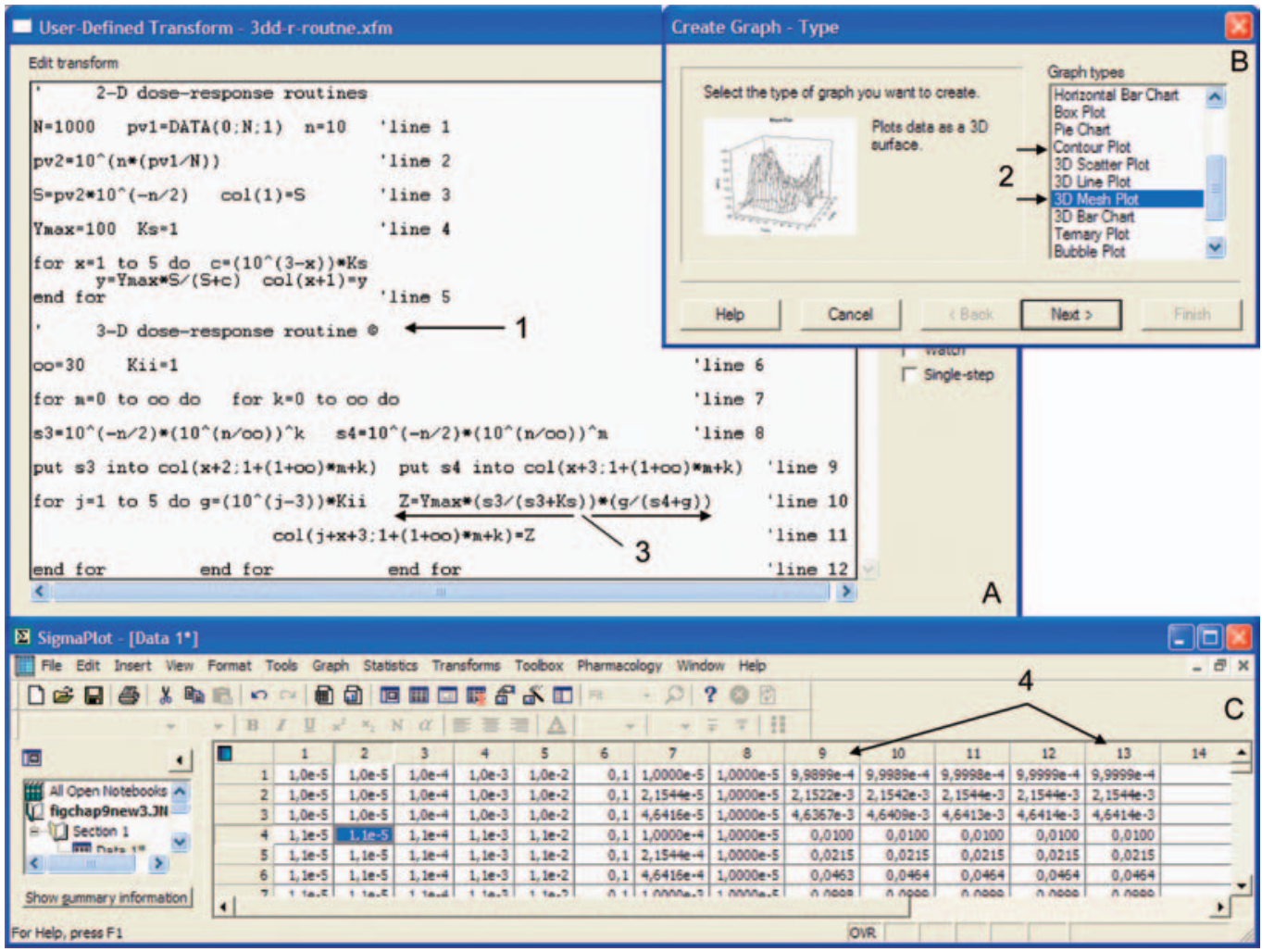

Figure 9.26. User-defined Transform and its generated data for producing 3-D plots of a theory. 
For the 3-D subroutine in Fig. 9.26A (arrow 1), I have selected the non-competitive reaction scheme (see Eq. 2.3, Chapter 2). The non-competitive intervention scheme operates with two independent variables, both affecting the response of the system. Therefore, the simple non-competitive scheme is suitable for a demonstration of 3-D plot presentations.

The 3-D subroutine in Fig. 9.26A is somewhat complex as it operates with three imbedded 'for-end for' loops. Meanwhile, observe our non-competitive function in line 10 of the 3-D subroutine (Fig. 9.26A, arrow 4$), Z=Y_{\max } *\left(\mathrm{~s} 3 /\left(\mathrm{s} 3+K_{s}\right)\right) *(g /(\mathrm{s} 4+g))$. Here $Z$ is the actual response, $Y_{\max }$ is the maximal response, s3 is the agonist concentration as one independent variable, $K_{s}$ is the agonist dissociation constant, and $g$ is the interventor dissociation constant $K_{\mathrm{ii}}$ defined as a value-varying parameter which jumps in 5 steps from $10^{-2}$ to $10^{2}$ by a factor of 10 . The interventor concentration s4 is the second independent variable. Line 11 of the subroutine ensures that the five generated data sets for 3-D Mesh Plots have their responses, $Z$, inserted in empty columns of the spreadsheet. In the present example, these columns are 9-13 (Fig. 9.26C, arrow 4), while the agonist concentrations for the 3-D Mesh Plots are in column 7, and the interventor concentrations are in column 8 (Fig. 9.26C). Our theory for the non-competitive intervention is displayed by two plots in a 3-D Mesh Graph (Fig. 9.27). The 'front- shell' in Fig. 9.27A is generated based on substrate concentration in column 7 , interventor concentration in column 8, and fractional response in column 13 $\left(K_{\mathrm{ii}}=10^{2}\right)$.

The 'rear-shell' in Fig. 9.27A is based on a fractional response for the inventor as an activator by rewriting line 10 of the 3-D subroutine in Fig. 9.26A to: $Z=$ $Y_{\max } *(\mathrm{~s} 4 /(\mathrm{s} 4+g)) *\left(K_{s} /\left(\mathrm{s} 3+K_{s}\right)\right)$ and at the same time save generated response data into columns $15-19$ by changing ' 3 ' in line 11 of the 3-D subroutine, Fig. 9.26A, to '9' (data not shown). The fractional response in the 'rear-shell' is based on such generated data in column $15\left(K_{\mathrm{ii}}=10^{-2}\right)$.

The 3-D Mesh Graph can be rotated, tilted, and afforded perspective, but I do not include instructions for such refinements. The SP9 manual (SigmaPlot-9 2004) will explain these possibilities.

Contour Plots (Fig. 26B, arrow 2) may be generated based on the same data as for 3-D Mesh Plots. Examples of this will appear in Chapter 12 on Synergy.

\subsubsection{Conclusion}

This concludes our three courses on (1) plotting experimental data, (2) fitting user-defined theory parameters to the obtained data, and (3) evaluating experimental synagics by simulations in 2-D and 3-D of possible

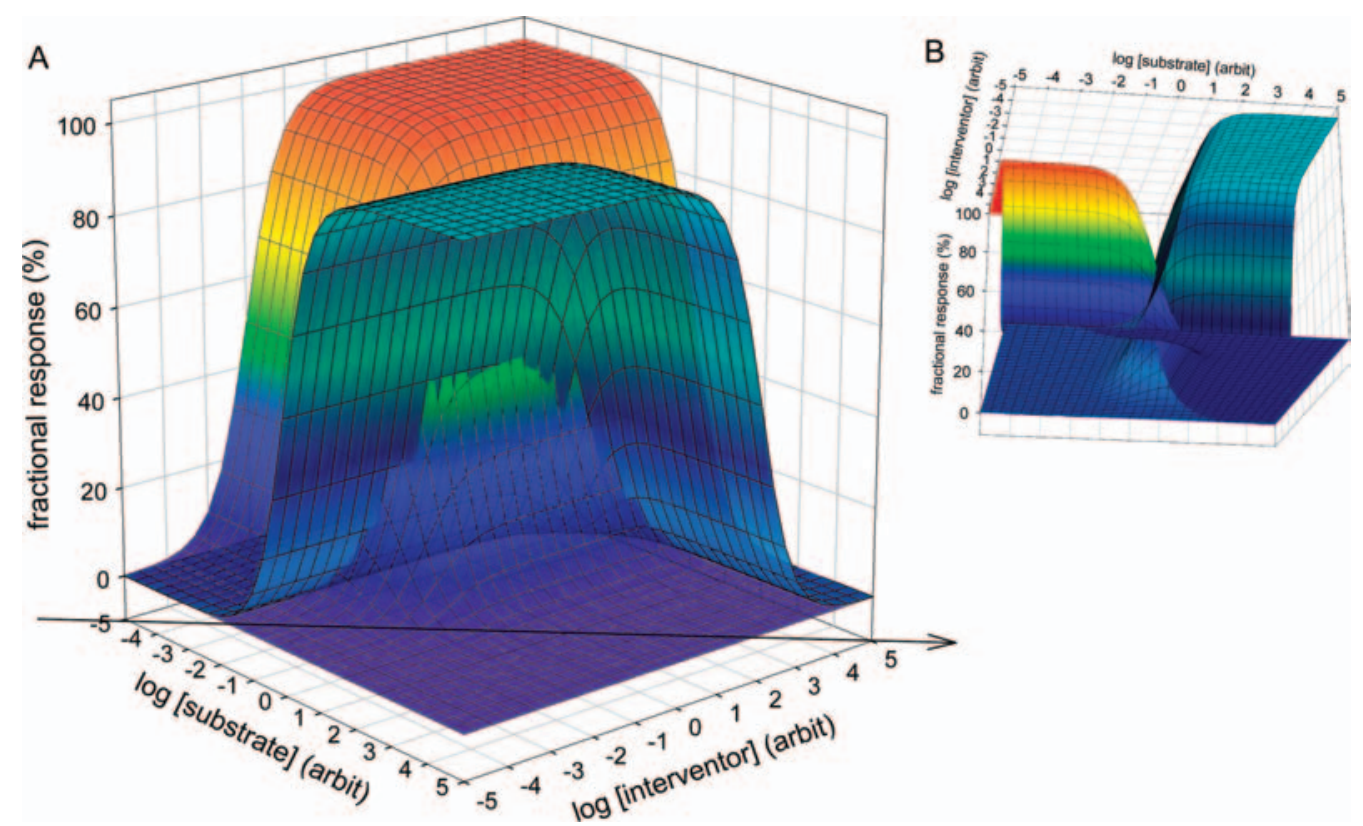

Figure 9.27. Two 3-D Mesh plots in a graph. The two surface plots in panel A are based on non-competitive inhibition theory. The front mesh-plot (ocean colors) is for substrate-activation with intervention by an interventor. The rear mesh-plot is for interventor-activation affected by a substrate (spectral colors). For details, see section 9.3.9. Arrow through the concentration plane indicate path for fixed ratio of concentrations for substrate and interventor. Panel $B$ is another aspect of the double meshplot in panel A seen from below. 
theories through user-defined data generation in SP9 Transform programs.

\subsection{Test for Load-deviant Behavior and Test of Theory on Theory}

\subsubsection{Tests by the Factor-squared Rule}

The factor-squared rule is described in Section 8.1.4. With the SP9 TOOL command and its option DRAW line (Fig. 9.28A, arrow 1), we can draw two horizontal lines (arrow 2), and two vertical lines (arrow 3) as shown in Fig. 9.28B. The two vertical lines representing concentration can be spaced by two orders of magnitude using the log-scaled $x$-axis (Fig. 9.28B). For responses, one of the horizontal lines is placed at $9.1 \%$ effect and the other horizontal line at $91 \%$ effect (Fig. 9.28B, arrow 2 ). Now select the two vertical lines by clicking at the lines while holding the shift-key down. Then activate the FORMAT-command and its GROUP option (Fig. 9.28C, arrow 4), which locks the two lines together for joint movement. The square formed by the four lines may now be overlain dose-response plots in the graph by moving the two vertical lines either left or right. The dose-response does not follow a simple load function, unless the intersections at the $9.1 \%$ and $91 \%$ with the two vertical lines can be brought to match with the intersection between horizontal lines and data curve (Fig. 9.28D). If the intersection at arrows 5 and 6 do not match with the dose-response curve, then the data deviates from load theory. An example of a loaddeviant dose-response is shown in Fig. 9.28D (arrows 5 and 6$)$.

In the same manner, a test for a Hill-type reaction scheme as describe in Section 8.1.4 may be implemented by the general form of the factor-squared rule. Centered around the $K_{\mathrm{d}}$, a factor for concentrations above and below $K_{\mathrm{d}}$, read of the $x$-axis in Fig. 9.28D, will be equal to $10^{2 / n_{\mathrm{H}}}$.

\subsubsection{Fit the Hill-theory to Your Theory}

As a final point of interest, it is worth keeping in mind when evaluating data generated by user-defined reaction schemes or program-supplied theories, i.e., functions, that software packages, such as the SP9, allow you to characterize your theory by another 'standard' theory in the Statistics/Regression Wizard menu. As an example, the Hill equation is a theory used as a 'standard' to
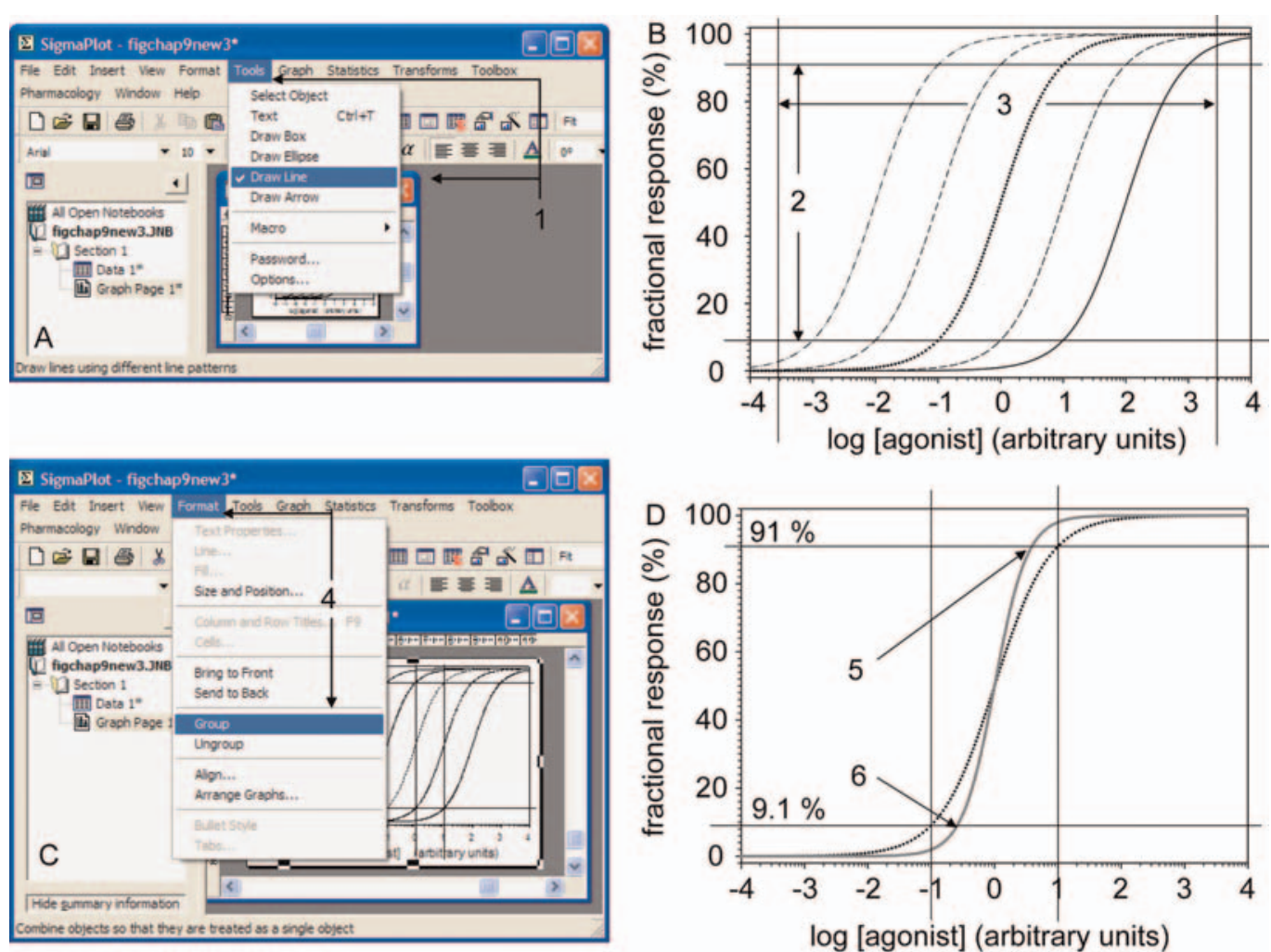

Figure 9.28. Test for load with factor-squared rule. Test for deviation from the factor-squared rule or $82 \%$ rule of the load theory by drawing two horizontal lines at 9.1 and $91 \%$ of absolute effect, and two vertical lines separated by two orders of magnitude on the concentration axis (see Sections 8.1.2 and 8.1.4 for details). Fixing the two vertical lines as a group with a factor 100 between them, they may be moved to either side for a test of curves matching the load (full line curve). 
evaluate the magnitude of co-operativity of experimental data; but, in addition, it may characterize the properties of theoretically generated data that are supplied based on your own theory.

\subsection{Conclusion}

On completion of subchapters 9.1-9.4, you will be equipped with theoretical pliers in your box of analytical tools to probe into the heart of regulatory means in biology as described in Chapters 2, 3, 7, and 15 .

In case you do not have access to a SigmaPlot program, implement the overall ideas and concepts in this chapter with other graphing software packages.

With the program in Box 9.3 and the Graph templates described in Sections 9.3.6-9.3.9, you can within minutes study the effects of varying a parameter from any theory involving either one or two independent variables. Without limits, the theories may emerge from synagics or any other field of science.

\section{REFERENCES}

Do DD. Adsorption Analysis: Equilibria and Kinetics. London, Imperial College Press, 1998.

Fernandez-Teruel C, Gonzalez-Alvarez I, Casabo VG, Ruiz-Garcia A \& Bermejo M. Kinetic modelling of the intestinal transport of sarafloxacin. Studies in situ in rat and in vitro in Caco-2 cells. $J$ Drug Target 13: 199-212, 2005.

Marangoni AG. Enzyme Kinetics: A Modern Approach. New York: WileyInterscience, 2003

Marquardt DW. An algorithm for least squares estimation of parameters. J Soc Indust Appl Math 11: 431-441, 1963.

Menon RM \& Barr WH. Comparison of ceftibuten transport across Caco-2 cells and rat jejunum mounted on modified Ussing chambers. Biopharm Drug Dispos 24: 299-308, 2003.

Nash JC. Compact Numerical Methods for Computers: Linear Algebra and Function Minimisation. Bristol: Adam Hilger, 1990.

Roque-Malherbe RMA. Adsorption and Diffusion in Nanoporous Materials. Boca Raton, CRC Press, 2007.

SigmaPlot 8. Transforms $\mathcal{E}^{2}$ Regressions: Reference Manual. Chicago, IL: SPSS Inc, 2000.

Wigton A \& Kilduff JE. Modeling trichloroethylene adsorption by activated carbon preloaded with natural dissolved organic matter using a modified IAST approach. Environ Sci Technol 38: 58255833, 2004. 


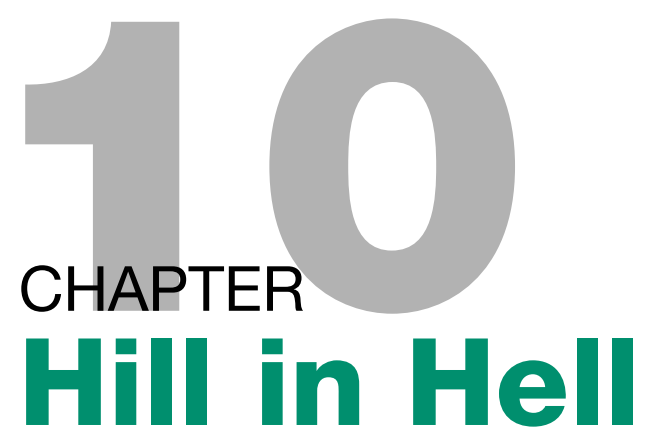

Chapter 10 begins with an account of how and why the Hill equation was introduced. The purpose of 'the Hill' was if not to explain, then at least to describe the aberrant behavior of dose-response relations and ease calculations. In Sub-chapter 10.2, I illustrate and discuss a frequently observed failure of linearity for experimental data in the so-called Hill plot Section 10.2.6. In Sub-chapter 10.3, there is an analysis and critique of what has been termed the 'logistic' Hill equation. The 'logistic' Hill is rendered perspective against the original logistic equation. An explanation is given of how the 'logistic' Hill equation is a mere semilog presentation-possibility of data when it is inserted into the Hill scheme. The chapter concludes with a list of facts about the use of the Hill formulation (Subchapter 10.4). Finally, the reader is taken on a brief tour through the history and use of the term logistic in data analyses, summarized in a Sub-chapter, Appendix 10.A, including statistical terms such as 'logistic regression' and 'polytomous logistic regression'.

Detailed descriptions of statistical theories, methods, and transformations can be found in various texts (see Sub-chapter 10.A). However, my purpose here is to illustrate in a superior way the terms logistic and 'logistic', and thus maybe even resolve some of the obfuscation about their use.

This chapter presents non-mechanistic models. Mechanistic models are detailed in Chapter 7.

\subsection{Deviations from the Law-of- adsorption-and-desorption}

Dose-response relations that follow the simple law-ofadsorption-desorption (load) give a plot that is a fraction of a rectangular hyperbolic curve (Fig. 1.3). In a semi-log plot, this fraction of the hyperbolic curve is an S-shaped and symmetric curve. An example is the dotted line curves in Fig. 10.1A+B (see also Fig. 8.1A+B).

\subsubsection{Start of De-ri-va-tion of Load}

From around 1850 on, somewhat different descriptions of various dose-response relations were formulated by chemists/physicists (Wilhelmy 1850; Biot 1860; van't Hoff 1877; Guldberg \& Waage 1967), physiologists working on for instance hemoglobin-oxygen binding (Hufner 1890; Bohr 1904a), and biochemists working with enzymes (Brown 1902; Brown \& Glendinning 1902; Henri 1903).

Biot, a French physicist (1776-1862), first realized that chemical reactions in solution follow a load-type rectangular hyperbolic function (Fig. 10.2) (Biot 1860, pp. 237-243 and Plate II). He probably reached his preHill/pre-Langmuir load-equation on reading Wilhelmy's quantitative insight on chemical reactions (1850). As early as 1884, Hüfner also described binding of carbonmonoxide to hemoglobin by a load-type equation (Hufner 1884) (Fig. 10.3).

After 1900, the dose-response relation in the form of the load started to reappear in works by Ostwald (1902), Henri (1903), Hill (1909), and Micahelis-Menten (1913). Meanwhile, at equilibrium, the hyperbolic load reaction theory is usually referred to as the 'isotherms of Langmuir'. This is due to Langmuir's explicit theoretical formulation of the load and his confirmation of the load by its experimental corroboration (Langmuir 1918).

Here I shall refer to the 'hyperbolic' interaction both at equilibrium and at steady-state as the law of adsorption-desorption (load). ${ }^{1}$ The law is based on the fact that binding sites are limited (see Sections 1.1.2 and 1.1.8).

\footnotetext{
1 Since Henri (1903) and Micahelis-Menten (1913) formulated steadystate expressions of the load, while Biot (1860), Hüfner (1884, 1890), Hill (1909), and Langmuir (1918) formulated equilibrium equations for the load, we may divide the law-of-adsorption-desorption into two types: an s-load (HMM-theory) and an e-load (BHHL-theory).
} 
A

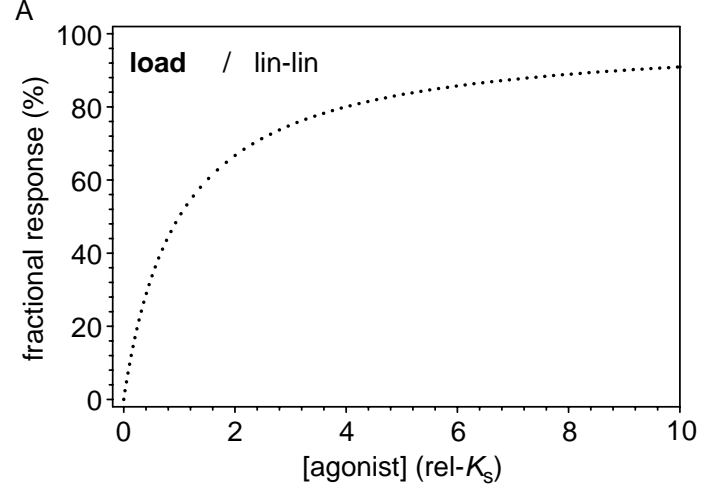

C

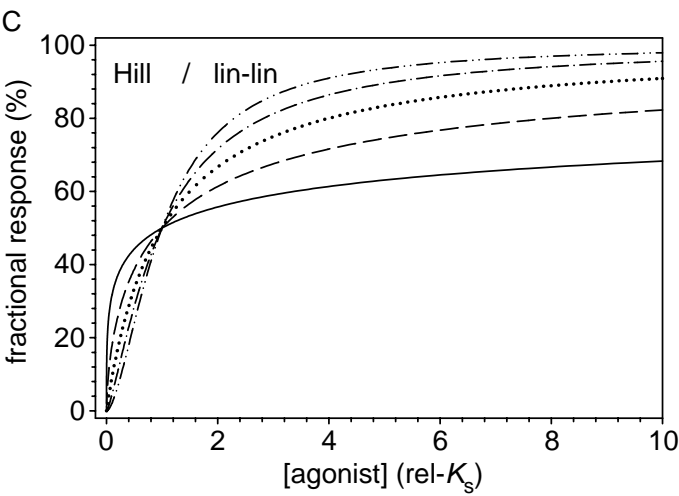

B

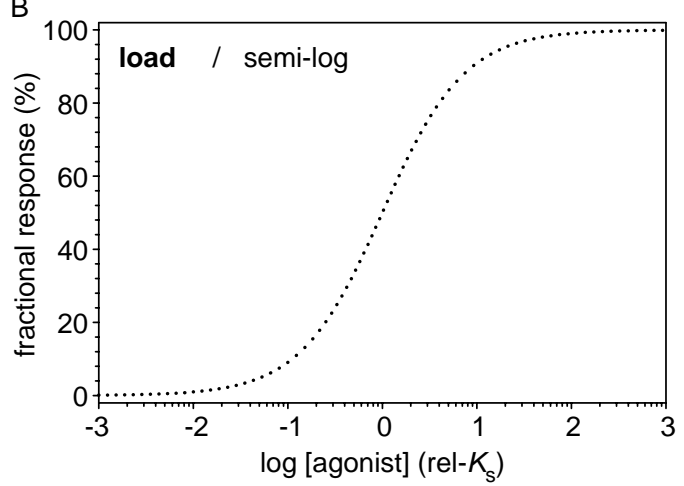

$\mathrm{D}$

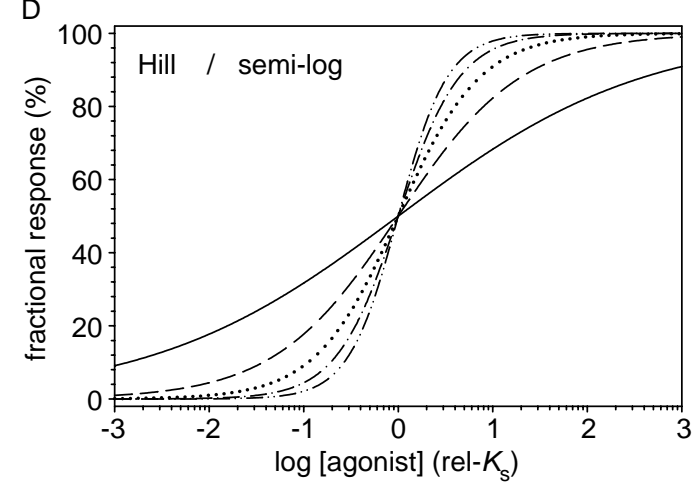

Figure 10.1. The load and modified Hill equations in linear and semi-log plots. (A) Linear-linear plot of load. (B) Semi-log ('logistic') plot of load. (C) Linear-linear plot of Hill. (D) Semi-log ('logistic') plot of Hill. The five Hill plots in (C) and (D) are generated by the modified Hill equation with $Y_{\max }=100, K_{\mathrm{s}}=1$ and $n_{\mathrm{H}}$ changing in five steps with the values $0.3333(-), 0.6667$, $1.0,1.3333$, and $1.6667(-\cdot-)$. Hill coefficient $=1.0$ generates the plots in panel $A$ and $B$.

\subsubsection{Start of De-vi-a-tions from Load. Formulation of Hill's Equation}

At the turn of the last century, it had already been observed that an experimental dose-response relation deviated from the simple hyperbolic rectangular doseresponse relation. Synagics for the hemoglobin- $\mathrm{O}_{2}$ binding as a function of $\mathrm{O}_{2}$ tension did not follow the simple load (Fig. 10.4) (Bohr 1904a,b; Bohr et al. 1904). Instead, the $\mathrm{Hb}-\mathrm{O}_{2}$ saturation against $\mathrm{O}_{2}$ tension gave a sigmoidal dose-response relation, equal to positive cooperativity. A theory for this behavior that is now accepted was formulated in a preliminary equation by Adair (1925). ${ }^{2}$ In 1910, Hill had also formulated an Adair-type equation (see Eq. 10.6), but due to the tedious work of calculating all the constants, Hill advocated for a simplified version, namely Eq. 10.1 given here and henceforth referred to as the Hill equation (Hill 1910). His equation reads:

\footnotetext{
${ }^{2}$ Edsall gives a fine account of this story (Edsall 1980), see also Klotz (2004).
}

$$
y=\frac{K \cdot \mathrm{x}^{n}}{1+K \cdot \mathrm{x}^{n}},
$$

in which $y$ is the observed saturation (fully-oxygensaturated hemoglobin aggregates) as a fraction of all hemoglobin conformations, bound or unbound, ' $x$ ' is the free ligand concentration (oxygen tension), and $K$ in this equation has no physical meaning, but relates to its origin, an equilibrium association constant. The power $n$ is known as Hill's coefficient. When Hill's coefficient is 1, Hill's mathematical expression is identical to the Langmuirian mechanistic expression for adsorption of gases, the load, Eq. 1.11. Thus, Hill just elevated the concentration (oxygen tension) to an arbitrary power $n$ (or $n_{\mathrm{H}}$ for 'the power of Hill').

In the linear scaled Cartesian coordinate system, dose-responses that follow the Hill equation with a Hill slope $\neq 1$ (Fig. 10.1C) give plots that deviate from a hyperbolic curve (dotted curves in Figs. $10.1 \mathrm{~A}+\mathrm{C}$ ), while these plots in a semi-log diagram are symmetric and S-shaped curves just as the simple load (Figs. 10.1B + D, also see Figs. 8.1 and 8.8).

As mentioned, employing the Hill equation was only meant as a quick way to estimate the co-operativity in experiments without any mechanistic interpretation, as 


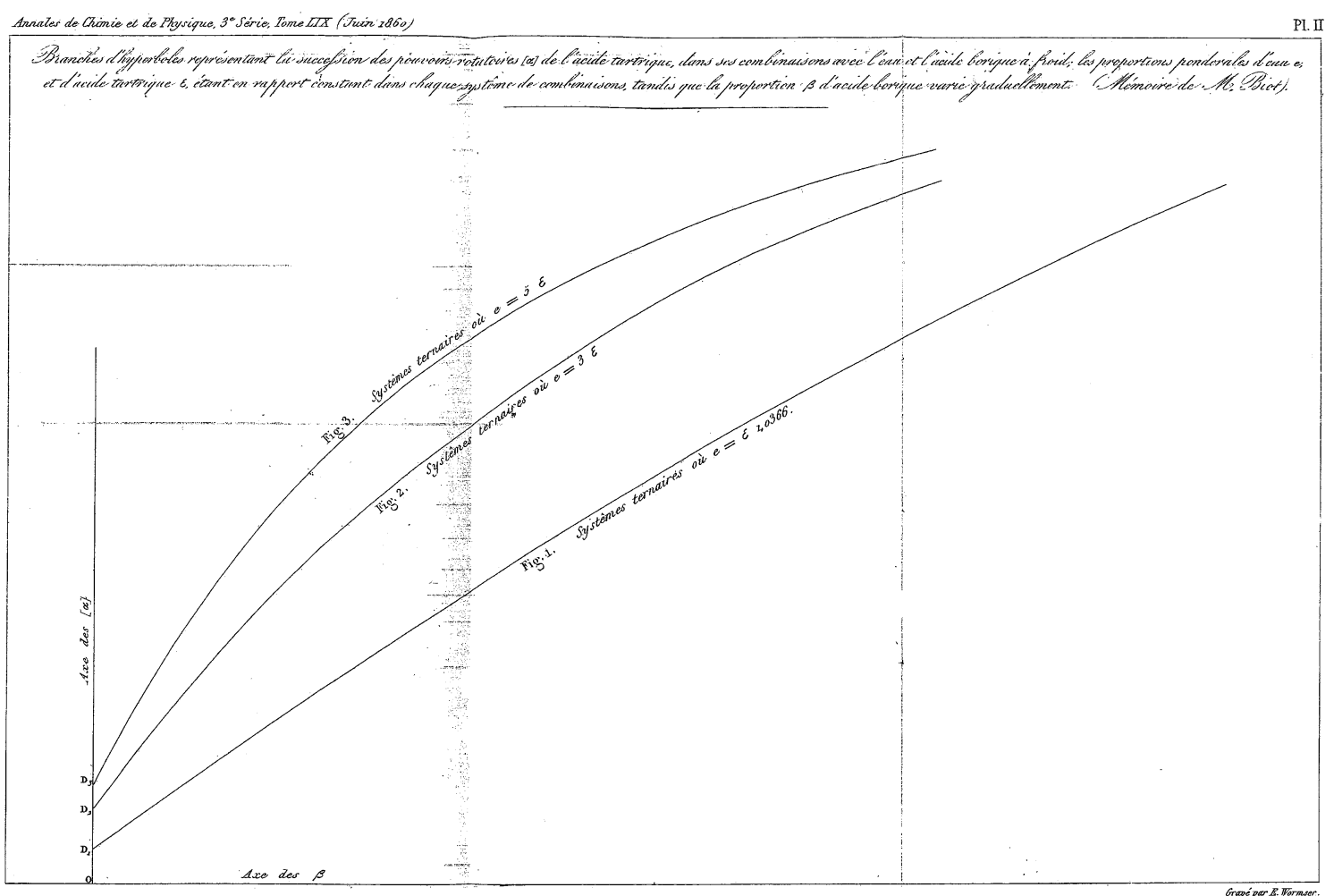

Figure 10.2. The origin of load curves. From Biot (1860, Plance II).

its inventor also attested to (Hill 1910). He writes 'My object was rather to see whether an equation of this type can satisfy all the observations, than to base any direct physical meaning on $n$ and $K^{\prime}$.

Notice in this context, that there are three Hillconcepts: (1) the Hill equation (Eq. 10.1), (2) the Hill coefficient, -factor, -power, or -slope, $n_{\mathrm{H}}$, and (3) the Hill plot (see Section 8.2.5 and Fig. 8.8C + F). The Hill plot is a plot of the logit formulation (see Section 10.2.2).

\subsubsection{Hill's Aggregation Theory for Hemoglobin Complexation Crumbles}

For complexation of a multi-unit hemoglobin, Hill postulated a so-called 'aggregation theory' (Hill 1910).

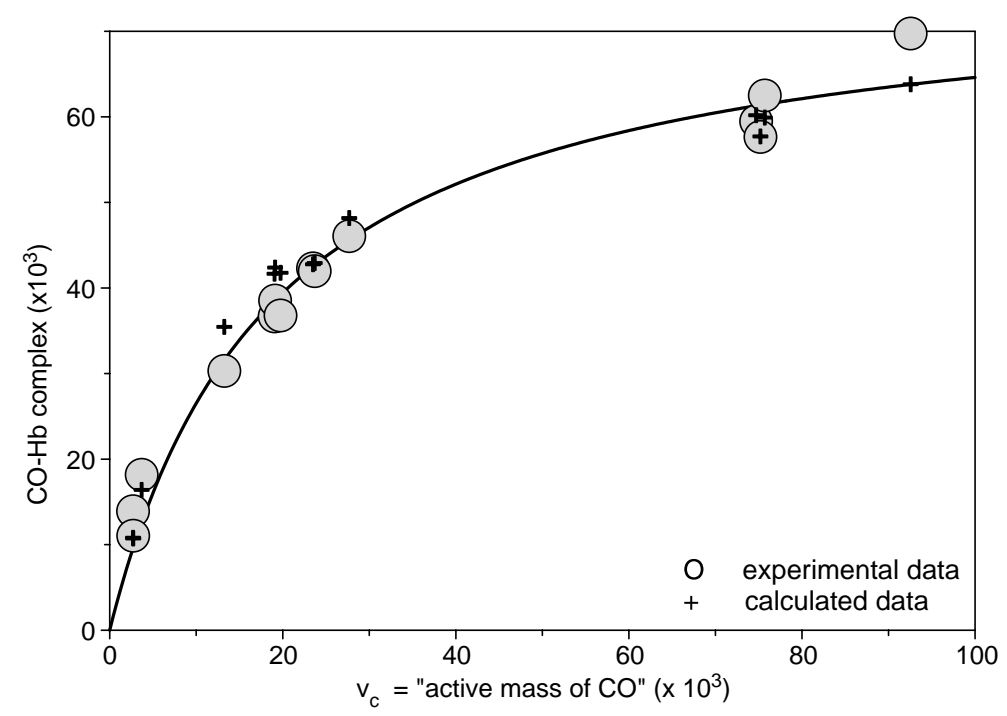

Figure 10.3. The origin of experimental data plots combined with the load theory. Carbon-monoxide binding to hemoglobin. Maximal binding $=76.9 \pm 3.4 \times 10^{-3}, \mathrm{EC}_{50}=19 \pm 1 \times 10^{-3}$. After Hüfner (1884). 


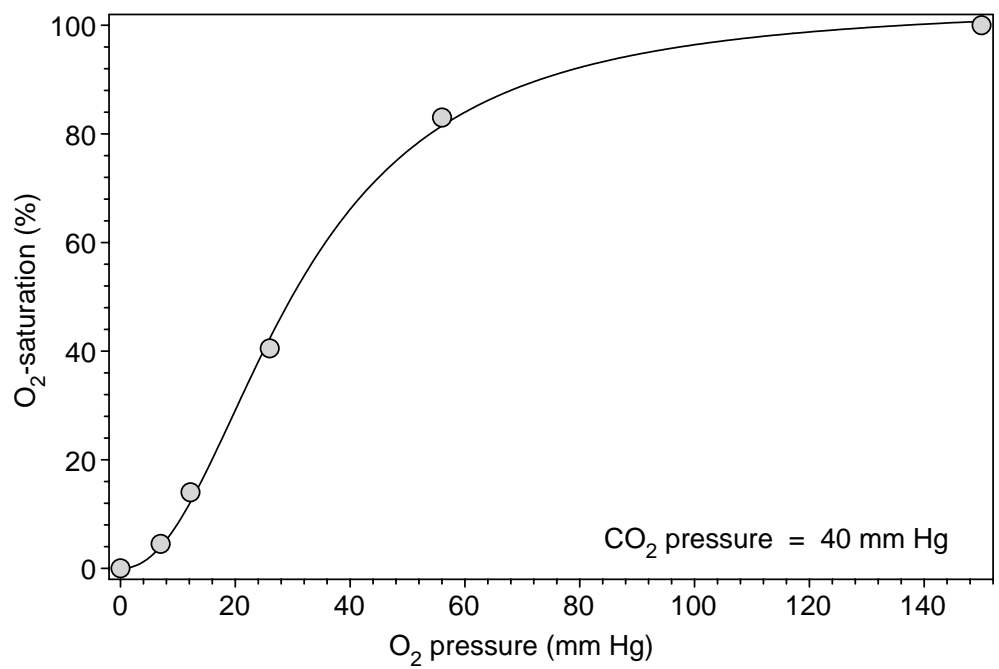

Figure 10.4. The origin of load-deviant behavior. Demonstration of a genuine sigmoidal dose-response relationship. Oxygen binding to hemoglobin in linear-linear dose-response plot. The relationship is determined at $40 \mathrm{mmHg} \mathrm{CO}_{2}$ with a maximal $\mathrm{O}_{2}$-saturation at $104 \pm 2 \%, \mathrm{EC}_{50}=31 \pm 1 \mathrm{mmHg} \mathrm{O}_{2}$ and a Hill coefficient $n_{\mathrm{H}}$ equal to $2.17 \pm 0.14$. Redrawn from Bohr et al. (1904).

In this theory, hemoglobin units, each with one atom of iron, can aggregate when $\mathrm{O}_{2}$ is bound. Thus, for all hemoglobin units, the actual binding site in a single and free hemoglobin unit associates or dissociates one molecule of $\mathrm{O}_{2}$ with identical association or dissociation constants regardless of the status of neighboring binding sites in hemoglobin aggregates. In the physical model of Hill's aggregation there is an average number of $\mathrm{O}_{2}$-complexed hemoglobin molecules equal to $n_{\mathrm{H}}$, which is therefore also the average number of bound $\mathrm{O}_{2}$ molecules per hemoglobin aggregate. Hill's equation thus describes a coincidence - or simultaneity model, based on the aggregation of only liganded units. Hill indeed realized that his simple formula was a mathematical expression without any physical reality, a short-cut to analyze his aggregation theory with oxygen bound to hemoglobin complexes (Hill 1910).

In the mid-1920s, based on experiments, Adair (1925) and Svedberg and Fåhreus (1926) argued that hemoglobin as such consisted of four subunits with four separate binding sites for oxygen, a fact that, as indicated, was generally accepted and soon proven correct. Therefore, Hill's aggregation theory crumbled. Together, the models by Adair (1925) and Pauling (1935), the X-ray work by Perutz and colleagues (1942, 1960; Muirhead \& Perutz 1963), and the determination of four association constants for oxygen binding to hemoglobin by Roughton et al. (1955), finally convinced Hill to give up his aggregation theory (Hill 1965, p. 105). For hemoglobin, four units are bound together allowing interaction between their binding sites.

In 1965, 55 years after suggesting the quick test in the form of Hill's equation, Hill catalogued his life as a scientist in a book entitled 'Trails and Trials in
Physiology'. Herein Hill commented: 'There can, however, be little doubt now that my equation $y=K x^{n} /(1+$ $K \mathrm{x}^{n}$ ), based on the aggregation theory, is wrong, or at least a serious over-simplification'. He continues: 'The theory that the earth is a sphere is also wrong, but for practical purposes it is often convenient'.

The story continues. Hill noted with surprise that his equation was still in use and the Hill coefficient maintained as a quick-quantifier of load-deviation. He writes 'The equation originally deduced in 1910 from the aggregation theory had been laid decently to rest in the 1920s; its body lay mouldering in the grave, but apparently its soul goes marching on' (Hill 1965, pp. 105-106). Currently, it still marches on.

We may conclude that the aggregation theory for hemoglobin is falsified and the physical meaning of Hill's equation is inappropriate for almost any adsorptiondesorption system due to the coincidence requirement and its lack of site interaction. Meanwhile, applying Hill's equation and using its coefficient as a quantifier for deviation from simple concentration-binding or dosefunction schemes is convenient.

\subsubsection{All-or-none is Often Forgotten}

We may suppose that the aggregation theory for hemoglobin is more likely than the coincidence resonance-theory to bring four hydrogen atoms together at once to form helium and followed by three helium atoms to combine into carbon in stardust (Burbridge et al. 1957; Davies 2006, pp. 151-158). Nonetheless, even if the coincidence formulated by Hill's equation is a remote possibility found in nature, his theory still seems to require continued luck. Or does it? Consider 
this. Di-merization and multi-merization for functionality of only liganded receptors are ceaseless processes for the tyrosine kinase receptor superfamily, with the insulin receptors, IRs, as a prototype example (de Myets 2004; Stoker 2005; Blanquart et al. 2006; Bublil \& Yarden 2007), and could certainly be described by a Hillesque aggregation theory in which coincidence is inherent. The resonance required for carbon-formation in stars is here replaced by stability of the insulin-IR complex. On the other hand, subunits of $\mathrm{G}$ proteincoupled receptors (GPCRs) seem to be aggregated before ligand binding (Ma et al. 2007).

In the meantime, the use of Hill's formulation may be justified for a fast and easy documentation of loaddeviation as long as: (1) it is realized that the synagic model behind Hill's equation is irrelevant for a host of binding and functional dose-response systems with intermediate interaction; and (2) the conclusions about the Hill equation at the end of this chapter are taken into account.

Numerous researchers have exposed their data to the 'Hill'-analysis and used the obtained Hill coefficient as a simple handle to express their data's deviation from the load. Many scientists have probably more or less recognized the limitations of Hill's equation, but most likely again, have quickly suppressed and forgotten all about its tacit assumptions.

\subsubsection{The Fickle Use of Hill's Equation}

What are the conclusions about the use of Hill's equation today? Hill's argument for introducing the Hill equation, as justified due to the cumbersome work in 1910 of calculating dose-responses for systems with many constants, is of course no longer valid (see Subchapter 8.3) (Weiss 1997). The argument for continuing to use the Hill equation as a tool to analyze one's experimental data is based on the fact that very often there is too little information on studied systems to justify a detailed description of the system constants. Here Hill's coefficient is a convenient measure. The schism between convenience and realism has been the story for Hill's equation ever since its emergence. Therefore, the real problem with a choice of the Hill equation is once it works as a silencer of possible explorative investigations and hence prevents a penetrating analysis.

\subsection{Deviations from the Linear Hill Plot}

\subsubsection{The Modified Hill Equation}

The following equation is nearly identical to Eq. 10.1, with slightly different symbols:

$$
y=\frac{K^{n_{\mathrm{H}}} \cdot[\mathrm{S}]^{n_{\mathrm{H}}}}{1+K^{n_{\mathrm{H}}} \cdot[\mathrm{S}]^{n_{\mathrm{H}}}}=\frac{1}{1+\frac{1}{(K \cdot[\mathrm{S}])^{n_{\mathrm{H}}}}} .
$$

In this expression, $y$ is the fractional response or fractional binding, $K$ is now an equilibrium association constant, itself raised to the power of a Hill coefficient, $n_{\mathrm{H}}-$ assuming all single-step association constants to be identical, and [S] is the free ligand concentration. Eq. 10.2 is a slightly modified form of the original Hill equation (see Hill 1910, 1913). I will refer to Eq. 10.2 as the modified Hill equation.

\subsubsection{The Logit or the Hill Plot}

If you subtract the nominator from the denominator on both sides of the equal sign in Eq. 10.2, i.e., invoking regula detri rule $1 \mathrm{~b}$ (Section 1.1.7 and Box 1.1) you have:

$$
\frac{y}{1-y}=[\mathrm{S}]^{n_{\mathrm{H}}} \cdot K^{n_{\mathrm{H}}} .
$$

As no intermediate steps of occupation are allowed in Eq. 10.3, the fraction on the left of the equation explicitly expresses that the fraction of fully bound receptive sites, $y$, over fraction of free receptive sites, $1-y$, is equal to the product of ligand concentration and association constant between ligand and receptor, as equated on the right side in Eq. 10.3; and both raised to the power of $n_{\mathrm{H}}$. Thus, the ratio of bound over free receptive sites is proportional to the normalized concentration $[\mathrm{S}] \cdot K$ raised to a power. ${ }^{3}$

On a $\log -\log$ form, Eq. 10.3 is a linear function:

$$
\log \left[\frac{y}{1-y}\right]=n_{\mathrm{H}} \log [\mathrm{S}]+n_{\mathrm{H}} \log K
$$

with a slope of $n_{\mathrm{H}}$ and a 'cut-off' at the ordinate of $n_{\mathrm{H}}$. $\log K$

The expression ' $\log [y /(1-y)]$ ' is a well-known term, called the 'logit'. Compare this with the logit derived in Section 8.2.4 (Eqs. 8.9 and 8.13) and Section 8.2.5 (Eqs. 8.17 and 8.21). Plots of Eq. 10.4 are referred to as 'logit plots' or in synagics also as 'Hill plots' (Fig. 8.8) (Hill 1913; Brown \& Hill 1923; Adair 1925). See Section 8.2.5 and Appendix 10.A for the naming and benefits of the logit formulation.

As pointed out by Cornish-Bowden and Koshland (1975), the 'logit' plot in pharmacology is a plot identical to the Hill plot, and formally equivalent to Nernst's plot of redox electrochemistry (Reed \& Berkson 1929, their example 5; Malmstrom 1974). The logit plot is also equivalent to the gating transitions of voltage

\footnotetext{
${ }^{3}[\mathrm{~S}] \cdot K=[\mathrm{S}] / K_{\mathrm{s}}$ is a normalized concentration, where $K_{\mathrm{s}}$ is a dissociation constant.
} 
dependent ion channels (Yifrach 2004). See Section 10.3.9 for more on the relationship between the Nernst and the Hill equations.

In former times, linear transformation of theoretical expressions was the simplest mean of analyzing loaddeviant dose-response data. Levitzki (1978) has given a detailed account of the development and usefulness of Hill's analysis, arguing for the continued use of the Hill plot. For more on the use of the Hill slope as a measure of co-operativity, see Hill (1985, pp. 64-66) and Giraldo and co-workers (Giraldo et al. 2002; Giraldo 2003).

In the world of formulae, the term 'logit' is sometimes mixed up with the term 'logistic'. However, the 'logit' is not to be confounded with the concepts 'logistic', 'logistic equation', or 'logistic function' (Tables 10.3 and 10.4 and Appendix 10.A) (Berkson 1944; Prentice 1976; Collins et al. 1992). Evidence that 'logit' is easily mixed with 'logistic' can be seen in an article on 'Generalized linear models' in Wikipedia (updated 2 March 2007), in which a link function as the logit or 'logit model' for logistic regression is mistakenly quoted as a logistic function.

Factually, as formulated in Eq. 10.13, the original logistic equation is the one for growth as a function of time and reproduced here from Section 10.3.2:

$$
y=\frac{1}{1+\exp \left(-k \cdot\left(\mathrm{t}-\mathrm{t}_{0}\right)\right)} .
$$

Here 'exp' means exponentiation of the parenthesis with the base for the natural logarithm, $\mathrm{e}=2.71828$. The logistic equation will be discussed further in Subchapter 10.3 and Appendix 10.A, as well as the term 'logistic' which is sometimes just a cover-up for a simple semi-log re-scaling.

\subsubsection{Non-linear Hill Plots}

One assumption about linearity of the logit (Hill) plot is that all sites have the same affinity for the tested ligands, i.e., association constants $K_{1}=K_{2}=K_{3} \cdots=K_{\mathrm{n}}$. Systems with different association constants between binding sites, which is the most likely, will have curvilinear logit (Hill) plots when experimental dose-response data are drawn according to the logit equation (Eq. 10.4) (see Figs. $8.7 \mathrm{C}+\mathrm{F}$ and $8.8 \mathrm{C}+\mathrm{F}$ for linear examples). At the extreme ends of such a non-linear experimental curve based on the logit, you may estimate the association constant for the site with the highest affinity, $=K_{\mathrm{n}}$, for small values of $[\mathrm{S}]$, while for very high values of $[\mathrm{S}]$, the affinity may be estimated for the binding site with lowest affinity, $K_{1}$ (Cornish-Bowden \& Koshland 1975).

Therefore, when the logit plot of an observed doseresponse is not a straight line, the actual reaction schemes behind the relation do not follow a simple theory excluding interactions between binding sites. In order to analyze such a system seriously for its equilibrium reactions, you may consider formulas derived and described for random or ordered sequential binding and function in Chapter 6 (Table 6.1 and 6.2) (Tuk \& van Ostenbruggen 1996; Weiss 1997), and for two-state models presented in Chapter 7.

\subsubsection{Hill's Formulation for Multi-sited Hemoglobin}

Hill (1910) and Adair (1925) derived a general formula for multi-sited receptive units; both different from the Hill equation and with possible site-interaction at binding. Their type of formula was later expanded according to Pascal's triangle as summarized in Table 6.1, and described for the random reaction scheme as the 'reformulated occupancy model' (Tuk \& vanOostenbruggen 1996), and by Weiss (1997) for both random (independent) and ordered (sequential) reaction schemes (see Chapter 6, Figs. 6.13-6.15, for details).

Hill (1910) gave the following general formulation:

$$
y=\sum_{\mathrm{r}}^{\mathrm{n}} a_{\mathrm{r}} \cdot \frac{K_{\mathrm{r}} \cdot \mathrm{x}^{\mathrm{r}}}{1+K_{\mathrm{r}} \cdot \mathrm{x}^{\mathrm{r}}},
$$

where $y$ is the total fractional occupancy, factor $a_{\mathrm{r}}$ is the maximal fraction of receptive units with a number of ' $r$ ' ligands bound, and $K_{\mathrm{r}}$ is the association constant for ligand binding with $r-1$ ligands already bound. The implications of equating $K$ with subscript ' $r$ ' allow for co-operativity, as the association constants may vary independently. That is, alternating site-interaction upon binding.

It is interesting to note that even with ten binding sites in the random binding regime with full occupancy but without site interaction, the fit-determined Hill coefficient never rises above 2.1 (Fig. 6.11) (Weiss 1997).

\subsubsection{Site-interaction in One and Two-state Models}

Numerous researchers have equated explicit expressions for such multi-sited receptive units with site-interaction in order to describe observed co-operativity, some in one-state models (Hill 1910; Adair 1925; Pauling 1935; Allen et al. 1950; Cornish-Bowden \& Koshland 1975; Segel 1975/93; Levitzki 1978; Dixon \& Webb 1979, pp 79-138; Wyman \& Gill 1990; Wells 1992; Tuk \& vanOostenbruggen 1996; Weiss 1997) and others in twostate models (Koshland et al. 1966; Eisenthal 1975), while at the same time a whole tradition was built around a two-state reaction scheme without site-interaction upon binding (Monod et al. 1965; Karlin 1967; Janin 1970; Perutz 1970; Thron 1973; Colquhoun 1973; Perutz et al. 1998). 
For two-states models, Perutz and co-workers paved the path taken by others (Monod et al. 1963, 1965; Harber \& Koshland 1967; Perutz 1990; Perutz et al. 1998) by presenting the quaternary structural changes of oxy/deoxy-hemoglobin consisting of four hemoglobin subunits (Perutz 1942, 1960; Muirhead \& Perutz 1963, Perutz et al. 1964).

\subsubsection{Adair's Equation Predicts Non-linear Hill Plots}

Both Hill's multi-sited Eq. 10.6 and Adair's equation with individual association constants predict non-linear Hill plots. Historically, the Hill plot was used by Adair (1925) to demonstrate that Hill's aggregation theory with no site interaction was too simple. The aggregation theory should show straight Hill plots, but Adair's experimental data gave a non-linear Hill (logit) plot (Fig. 10.5) (Adair 1925, Fig. 2).

Based on the most recent theories for multi-sited receptive units, it is interesting that each association constant, as binding increases or decreases, has different values and therefore must be considered separately in an expression for the magnitude of a response as indicated by $K_{\mathrm{r}} \mathrm{s}$ in Eq. 10.6. When $K_{\mathrm{r}} \mathrm{s}$ are not identical, the Hill plot (the logit plot) can never be a straight line, except for a Hill coefficient $=1$. In addition, as Cornish-Bowden and Koshland point out 'It is therefore rather puzzling that straight Hill plots have so often been published in which the Hill coefficients are appreciably different from unity' (Cornish-Bowden \& Koshland 1975). Of course, an escape explanation is that a narrow range of concentrations may yield a linear logit plot and an assumption of intermediate steps in the process could explain the observed non-linearity (Levitzki 1978). However, according to a linearized Hill plot, the random or ordered sequential schemes with intermediate binding steps involved before full occupancy of function (Table 6.2), does not define straight lines even if all the association (or dissociation) constants are equal (see Fig. 6.15B). In addition, as already stated, to find identical association constants as the binding process progresses is expected to be a seldom event. Therefore, the multitude of published linear Hill plots remains puzzling.

\subsection{The Original Logistic Equation and the Original Hill Equation}

How are equations born? For all applications of data analyses it is an art to find the formulation that best describes and most faithfully interprets the results of an experiment. However, due to lack of sufficient

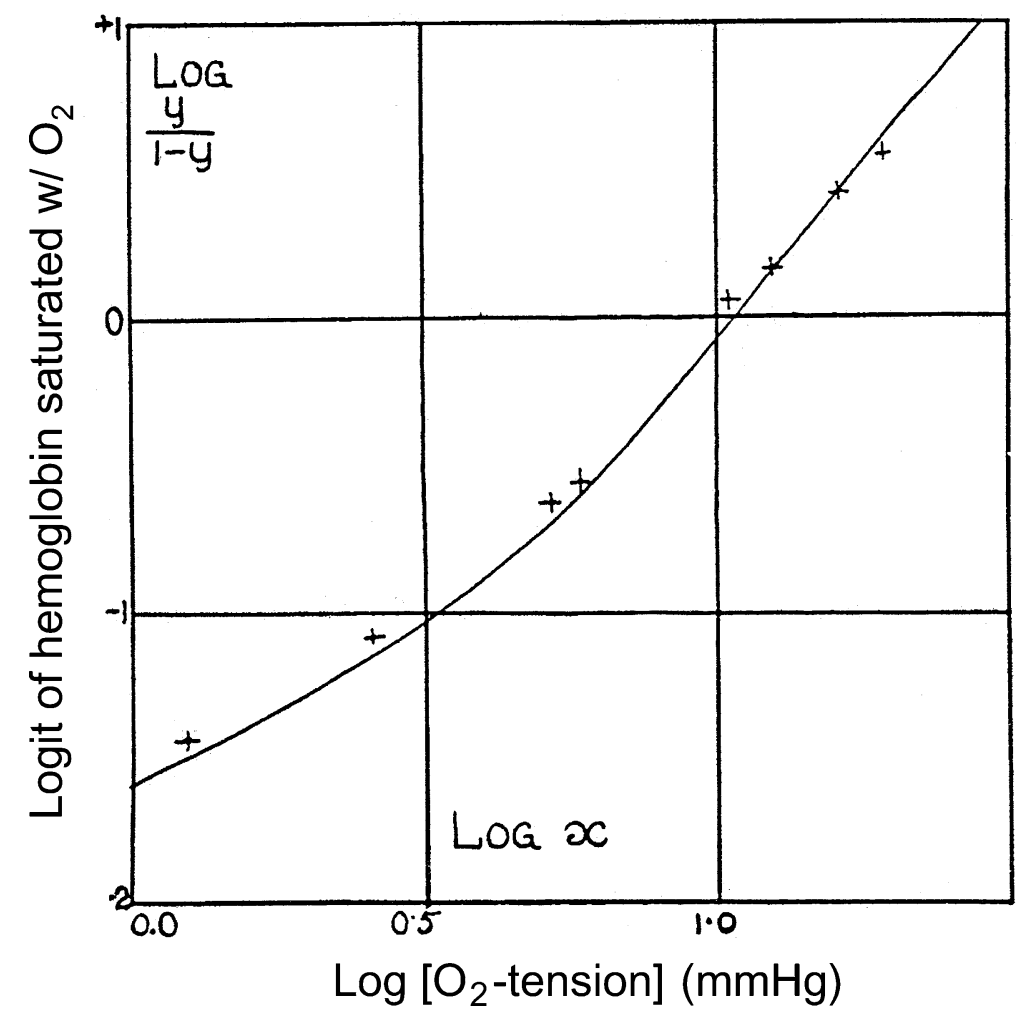

Figure 10.5. Deviation by hemoglobin saturation with molecular oxygen from a linear logit, Hill, plot. The curve fitted to the data points is a plot of Adair's equation with adjusted association constants. Taken from Adair (1925, Fig. 2). 
information, a possible mechanistic interpretation obtained by implementing a realistic equation is often ignored in favor of a simple formulation that gives a quick pseudo-qualitative description of one's data. In the biological sciences, a prototype example of this is the Hill equation from 1910 and its more recent transformation into a so-called 'logistic' equation (Jenkinson et al. 1995; Jenkinson 2003; Neubig et al. 2003).

In order to evaluate theories for dose-responses that are applied to experimental data - theories expressed as equations - there may be an advantage in transforming either the independent or the dependent variable, i.e., the $x$-axis or the $y$-axis, or both axes simultaneously. This is also called meta-metric re-scaling. Examples of such transformations are (1) a normal distribution re-scaling of the response axis as suggested by Gaddum (1933; 1953) equal to a probit scaling; that is, a Gaussian cumulative distribution of the dependent variable (see Appendix 10.A) (Finney 1971). (2) A semi-logarithmic re-scaling of the dose axis as suggested by Berkson (1944) and Klotz (1982) equal to the semi-log plots in Fig. 10.1B + D and Fig. 8.1B. (3) A logarithmic re-scaling of both axes in a logit transformation as described early on by several authors (Adair 1925; Berkson 1944; Finney
1971; DeLean et al. 1978) and depicted in Figs 8.7C+F and $8.8 \mathrm{C}+\mathrm{F}$ (see also Sub-chapter 10.A).

\subsubsection{A Logistic Equation, What is That?}

The classical logistic equation (Verhulst 1845; Pearl \& Reed 1920) is a formulation dealing with the description of population growth and auto-catalytic chemical processes as functions of time (Verhulst 1845; Ostwald 1883; Robertson 1908; Pearl \& Reed 1920; Reed \& Berkson 1929; Smith 1968). Development of this formulation is described in Section 10.3.2, and its history is recounted by Cramer 2003b ((http://www.cambridge.org/resources/ 0521815886/1208_default.pdf).

Meanwhile, Berkson who was familiar with the logistic growth curve transformed the classical dose-response relation (load) for drug-dose versus death-rate data into an equation which he termed 'logistic function' due to the resemblance of his semi-log transformed load equation with the logistic growth curve (Berkson 1944). In the Berksonian transformation of the load, the independent variable concentration $\mathrm{S}$ is expressed as the logarithm of normalized [S]. Therefore, the Berkson 'logistic function' means semi-log plotting of load (Fig. 10.6).
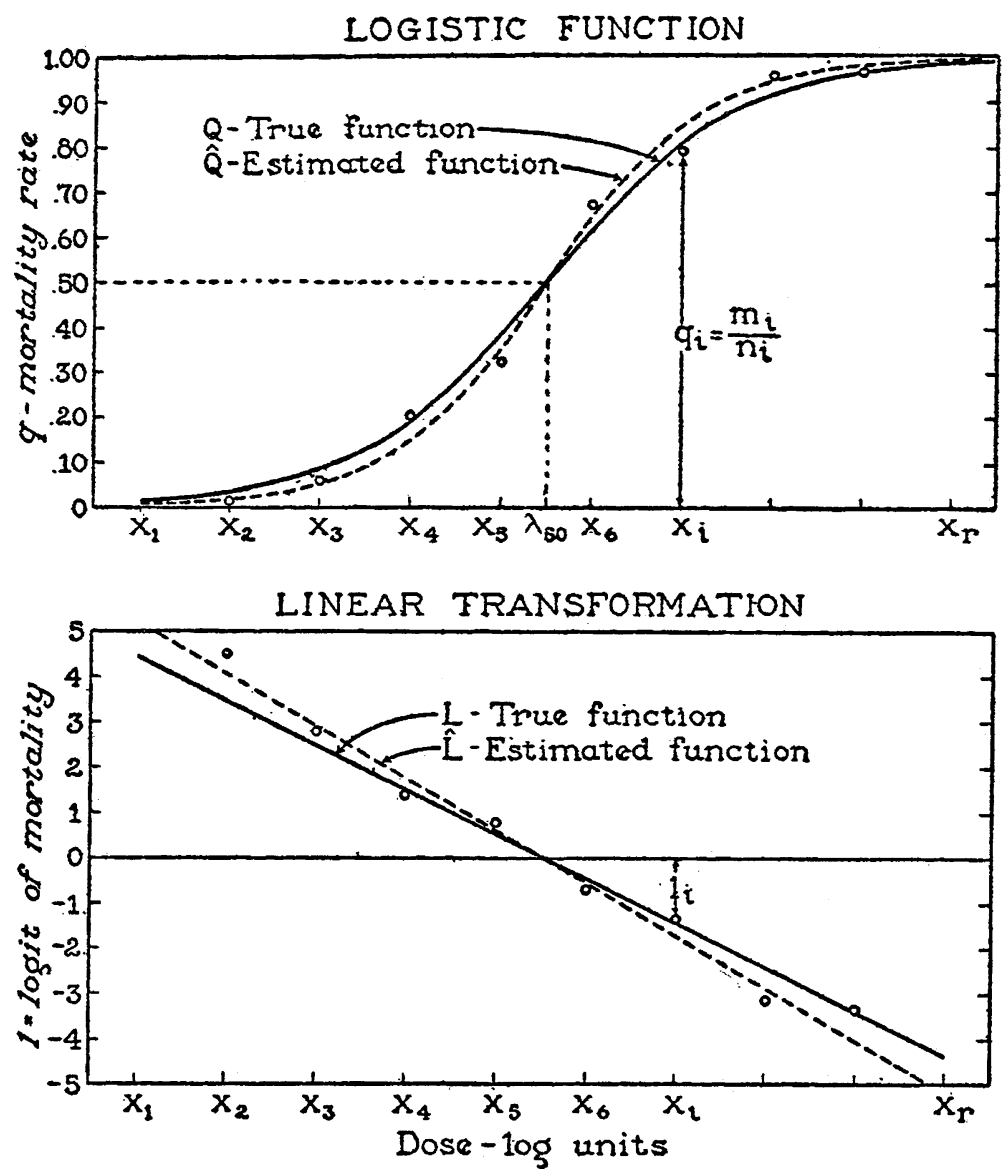

Figure 10.6. The logistic equation as analyzed by Berkson for dose-death relations and termed a 'logistic function'. Taken from Berkson (1944, Chart 1) with permission from the Journal of the American Statistical Association. 


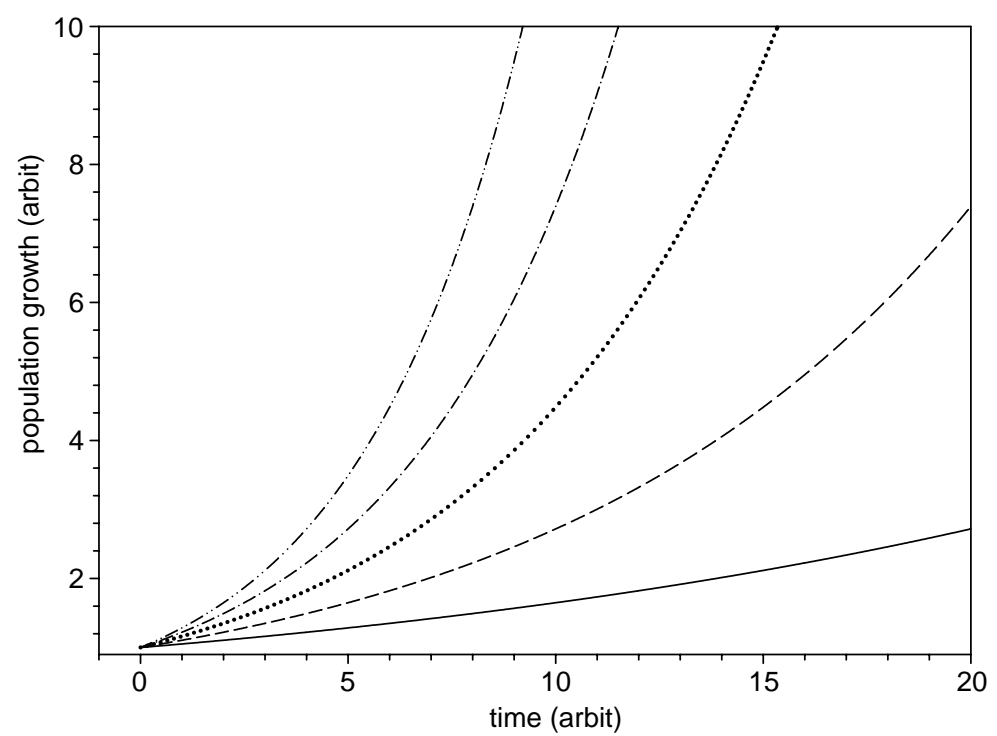

Figure 10.7. Malthusian growth curve. Malthus' geometrical (exponential) relationship according to the equation in Table 10.2 . By some termed Gompertz curve. The rate parameter $r$, equal to the Malthusian parameter, is varied in five steps from 1.0 .05 $(-)$ to $5.0 .05(-\cdot-)$, increasing by 0.05 between steps. See Malthus (1798).

In fact, Berkson's intention with the transformation was to show that the 'logistic function' of the load and its $\operatorname{logit}^{4}$ formulation yielded better statistics for the parameters than the probit analysis suggested by Gaddum (1933).

Since Berkson's semi-log transformation, the 'logistic' re-scaling of load has also been adopted for Hill-type formulations (van Rossum 1966) and characterized as a 'logistic equation' (De Lean et al. 1978; Jenkinson et al. 1995; Neubig et al. 2003; Motulsky \& Christopoulos 2004) (see also Sub-chapter 10.A).

Today, the purpose is to plot and analyze the hyperbola of the load or sigmoidality of dose-responses by the Hill equations in semi-log displays (Figs. 10.1B and $8.1 \mathrm{D}$ ), for better statistical evaluation of the function parameters (Finney 1971; Motulsky \& Christopou$\operatorname{los} 2004)$.

The next sections will reveal that when we perform a so-called logarithmic transformation of the modified Hill equation (Eq. 10.2), to a 'logistic-Hill' equation, it is a mere focus on logarithmic re-scaling of the independent variable for semi-log plotting and parameter evaluation, rather than the formulation of a new equation for synagics. Furthermore, it is not in any way congruent with the original logistic equation dealt with in analysis of growth. To illustrate, I will begin with the logistic equation for growth.

Note: in case there is no interest in logistic growth curves, you should go directly to Section 10.3.3 dealing with the 'logistic' Hill equation.

\footnotetext{
${ }^{4}$ The term 'logit' was coined by Berkson (1944) to differentiate it from the 'probit'.
}

\subsubsection{When Math meets Nature's Self-limiting Systems}

Setting up a mathematical formulation to describe processes in Nature and human society is an everdeveloping issue (Nowak 2006; Weisstein 2007 http:// scienceworld.wolfram.com). As an example, we can take different types of formulations for growth. As such, formulations for population growth are often referred to as logistic equations. Nearly 200 years ago, Malthus described population growth by a simple exponential function (Fig. 10.7) (Malthus 1798, revised in 1803, introduction by MP Fogarty). Since the Malthusian approach resulted in an unlikely unlimited growth, Gompertz (1825) invented and presented a population-mortality (growth) theory formulated as:

$$
N=N_{\max } \cdot \exp \left(-\exp \left(-\left(\mathrm{t}-\mathrm{t}_{0}\right) / b\right)\right)
$$

Here $N$ is the 'response' equal to the actual number of deaths and $N_{\max }$ is the total possible deaths, $\mathrm{t}_{0}$ the start of time, and $b$ a time constant. The Gompertz equation may also be used to describe population growth (Fig. 10.8). This growth is normalized to vary between zero and one; one representing the maximum possible population. Gompertz's theory has a rather steep increase in population at the beginning (Fig. 10.8), a phenomenon not characteristic of real population growth. Therefore, as we shall see, Verhulst introduced yet another growth theory.

Populations have maxima that depend on several conditions - one being available volume or surface. As real populations reach a maximum and do not expand rapidly with time at the beginning, which is the case for 


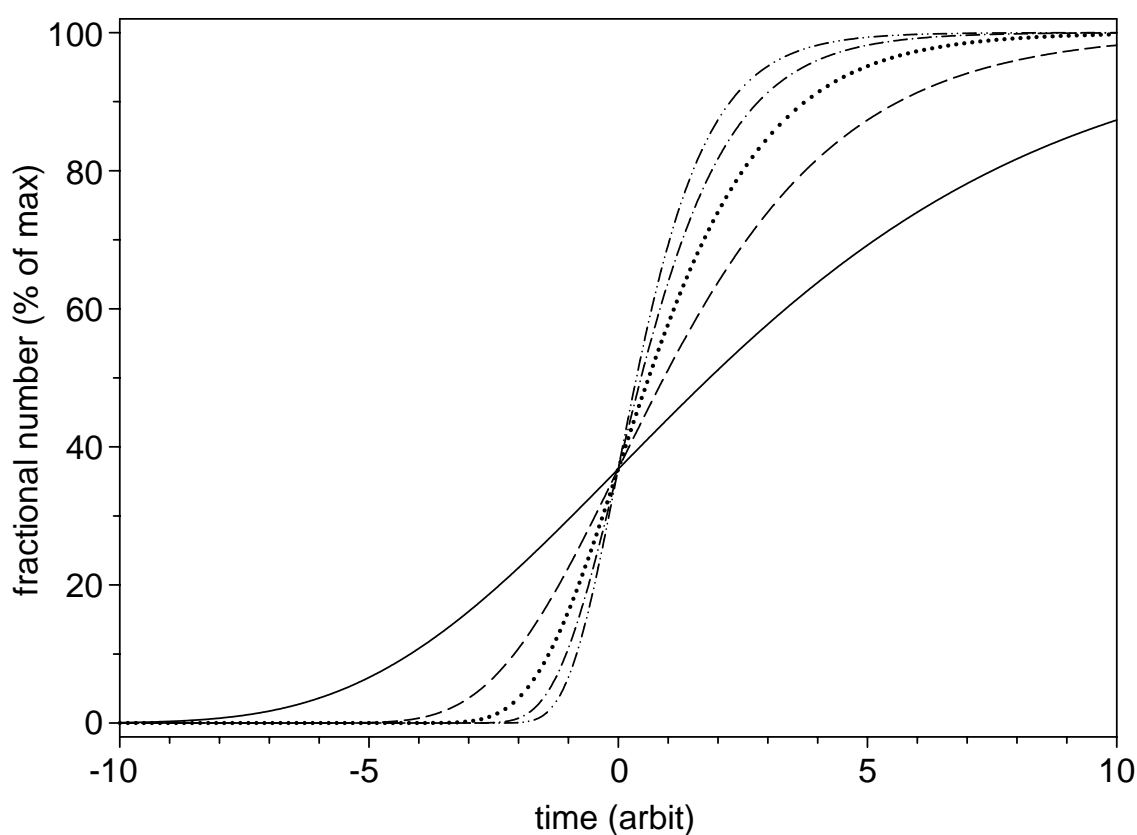

Figure 10.8. Gompertzian growth curve. Plots of the growth law also known as Gompertz function (Table 10.2). Here the Malthusian parameter $r$ is varied in five steps from 1.0.2 (-) to $5.0 .2(-\cdot \cdot)$, increasing by 0.2 between steps. After Gompertz (1825).

the Gompertz equation, the model by Gompertz was soon revised by Verhulst employing a differential equation (Verhulst 1838, 1845, 1847) and here expressed in homology with a first order difference equation:

$$
N_{\mathrm{t}+1}=C \cdot N_{\mathrm{t}} \cdot\left(1-N_{\mathrm{t}}\right),
$$

where $N_{\mathrm{t}}$ is the running number and $C$ is a system constant (Table 10.2) (Peitigen et al. 1992, pp. 63-134; Kingsland 1995; Camazine et al. 2001, pp. 42-44; Turchin 2003). Verhulst coined Eq. 10.8 a 'logistic' equation (Verhulst 1845). The logistic equation was independently rediscovered in 1920 by Pearl and Reed (1920). Almost simultaneously, Pearl recognized Verhulst' earlier discovery of the difference equation (Pearl 1922). In an exchange of ideas with Pearl, Yule reintroduced the term logistic for the Verhulst/Pearl \& Reed equation (Yule 1925; Kingsland 1995). Since then, formulations on growth have been known as logistic equations.

Accordingly, the transformed Verhulst formula (Eq. 10.8 ) is a new logistic equation, generally referred to as the logistic equation (Weisstein 2007; http://math world.wolfram.com). Eq. 10.8 may be compared with a first order differential equation used by Ostwald (1883) to describe auto-catalytic progression with time. Thus, an auto-catalytic ${ }^{5}$ and mono-molecular reaction is formulated by:

\footnotetext{
5 There is modern use of autocatalytic processes in networks, see, e.g., King (1981), Lee et al. (1997), and Goldstein (2006).
}

$$
\frac{\delta(a r)}{\delta \mathrm{t}}=R_{\max } \cdot a r \cdot(1-a r)
$$

(Verhulst 1938; Wilhelmy 1850; Ostwald 1883, 1902; Robertson 1908a) and limited by a maximal value of events, $R_{\max }$. Here ar is the actual reaction, homologous to the response in the form of $N$ in Eq. 10.7 and $N_{\mathrm{t}+1}$ in Eq. 10.8. Eq. 10.9 is a differential version of Eq. 10.8. We have now moved from integer math to thermodynamic ensemble math.

Inserting $T R$ for $R_{\max }$ in Eq. 10.9 and integrating yields:

$$
\ln \left(\frac{a r}{T R-a r}\right)=k \cdot\left(\mathrm{t}-\mathrm{t}_{0}\right)
$$

in which $k$ is a rate constant, see for instance Robertson (1908a,b), who used this modified logistic equation to describe growth in individuals with time.

Note that the logistic equation in Eq. 10.10 has a 'logit-like' expression to the left of the equal sign, but a non-logarithmic expression to the right of the equal sign for its independent variable time. Compare Eq. 10.10 with Eq. 10.4 for the Hill (logit) plot in Section 10.2.2. The Hill plot equation with its logit expression is logarithmic on both sides. On the other hand, as we shall see, the 'logistic Hill' equation (Eq. 10.15) has only a logarithmic re-scaled independent variable. Thus, Eq. 10.15 merely has a semi-log adjusted concentration. 
Before proceeding, we must first reformulate Eq. 10.10 to its non-logit distribution form with a term as ar/TR. The anti-log of Eq. 10.10 gives us:

$$
\frac{a r}{T R-a r}=\exp \left(k \cdot\left(\mathrm{t}-\mathrm{t}_{0}\right)\right) \text {. }
$$

Then by using regula detri rule 1a from Chapter 1 (Box 1.1), we get:

$$
\frac{a r}{T R}=\frac{\exp \left(k \cdot\left(\mathrm{t}-\mathrm{t}_{0}\right)\right)}{1+\exp \left(k \cdot\left(\mathrm{t}-\mathrm{t}_{0}\right)\right)},
$$

which, by dividing terms at the right with $\exp (k$. $\left.\left(\mathrm{t}-\mathrm{t}_{0}\right)\right)$, we can rewrite to:

$$
\frac{a r}{T R}=\frac{1}{1+\exp \left(-k \cdot\left(\mathrm{t}-\mathrm{t}_{0}\right)\right)} .
$$

Eq. 10.13 is also generally considered as the logistic equation (Fig. 10.9). Here time $\mathrm{t}$ is the independent variable. The level of growth varies as time goes by between zero growth and the maximum growth expressed as unity or $100 \%$. If time is only defined in the range $0 \leq$ time $<\infty$, then the rate constant $k$ is related to the initial level of growth, where constant $t_{0}$ is the time for maximal growth rate at the inflection of the growth curve (Fig. 10.9).

I have to admit that to equalize the plots in Fig. 10.9 with the plot in Fig. 10.1D is immensely tempting as they seem identical. Nevertheless, recognize that the meaning behind the two graphs is quite different.

Eq. 10.13 was the form of equation used for the growth of the USA population with time (Pearl \& Reed 1920). Nearly 200 million individuals, equal to $T R$ or 'the maximal carrying capacity', was the estimated maximal population found by data obtained for the USA until 1910 (Fig. 10.10). Location at the time axis of the inflection point for the fitted growth curve turned out to be the year 1914; four years ahead of the latest obtained population record (Fig. 10.10).

The general form of Eq. 10.13 is given in Eq. 10.14:

$$
y=\frac{1}{1+\mathrm{e}^{a-b \cdot \mathrm{x}}},
$$

in which $y$ is the level of sigmoidal increase as a function of any independent variable $\mathrm{x}$, while $a$ and $b$ are system constants. When the independent variable $\mathrm{x}$ is time, $b$ is a rate constant. Using Fig. 10.10 as an example, growth of the American population before 1920 had time constant $1 / b$ equal to 31.9 and its 'half-time' was 31.9 . $0.693=22.1$ years. The 'half-time' in the logistic equation is not the time it takes to double a population, therefore it is better to use a term as 'standard interval' for $1 / b$ (Yule 1925). Parameter $b$ is also known as the Malthusian parameter. The US population followed the logistic equation nicely in the beginning of the 20th century (Yule 1925, Fig. 4) and until the 1940s, still with an inflection point around year 1914 (Snedecor \& Cochran 1967). However, after 1940, luckily or unluckily, the rate constant $b$ for growth of the US population did not stay constant; it increased. Furthermore, the US population did not saturate at around 200 million individuals, as suggested in Fig. 10.10, but is now over 300 million and still growing. In addition, the logistic equation is no longer a good descriptor for the growth of inhabitants in the USA. The two major reasons are a post-war baby boom in the 1940s and an improved health care system (Snedecor \& Cochran 1967) not

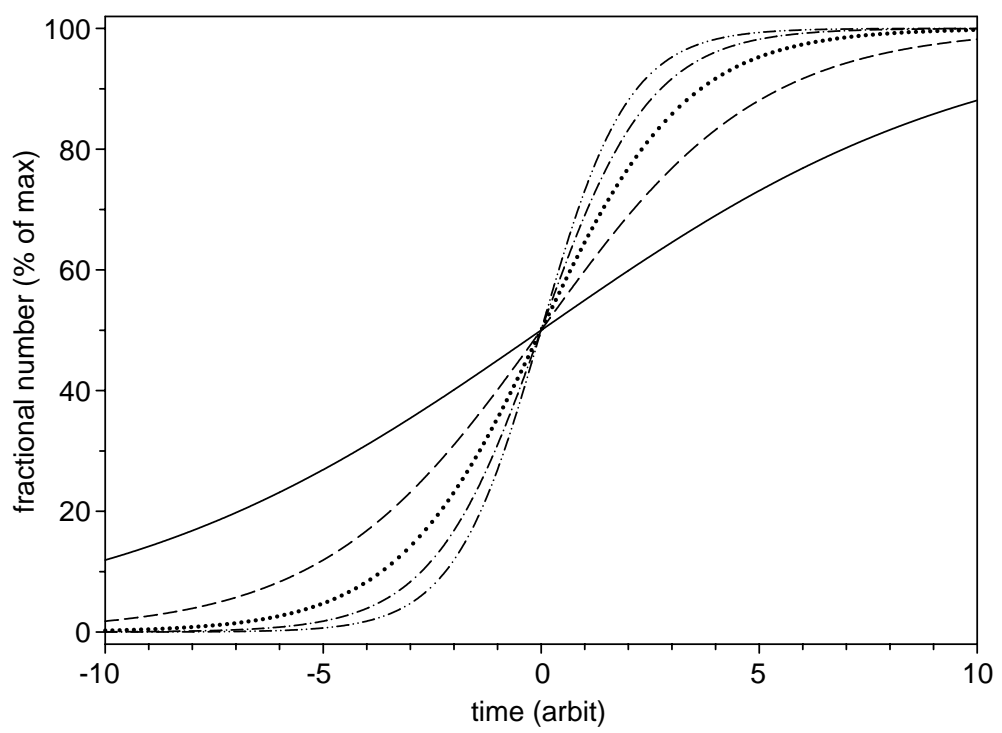

Figure 10.9. Verhulstian growth curve. Logistic equation for simulation of population growth. Plots of the logistic equation according to Verhulst (see Table 10.2). Plots are spaced by changing parameter in five steps from $1.0 .2(-)$ to $5.0 .2(-. \cdot-)$, increasing by 0.2 between steps. After Verhulst $(1838,1845)$. 


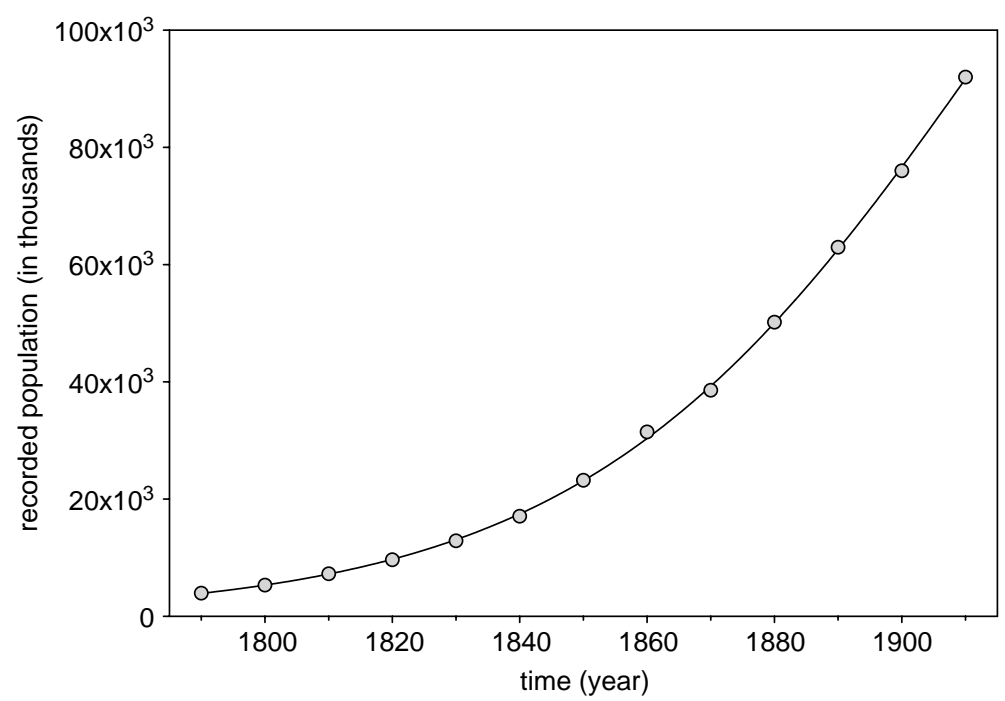

Figure 10.10. Population growth in the USA from 1790 to 1910. Analysis using the logistic equation. Maximal US population $=$ $196 \pm 10 \cdot 10^{6}$ individuals, 'time constant' $=32 \pm 1$ years, year of inflection point $=1914 \pm 3$. Based on Pearl and Reed (1920). None of the logistic functions, including the sigmoid function for population growth match the actual development of population growth in the 20th century (Snedecor \& Cochran 1967; Kingsland 1995; Turchin 2003).

incorporated in the formulation in Eq. 10.14. In fact, the logistic equation is not a good descriptor for population growth anywhere (Kingsland 1995; Turchin 2003), but may still be used for predicting an increase in industry products such as cellular phones (Cramer 2003a).

\subsubsection{Transforming Logistic Equations to Hill's Equations and Vice Versa}

Using mathematical manipulations, we can transform the above logistic equation with time as independent variable (Eq. 10.14), into an expression that is identical to the modified Hill equation (Eq. 10.2), where $K$ is an association constant equal to $1 / K_{\mathrm{s}}$, while the ligand concentration is the independent variable. Thus, assuming $a$ in Eq. 10.14 to be a new constant equal to $n_{\mathrm{H}}$. $\ln \left[K_{\mathrm{s}}\right]$, and $b$ yet another novel constant equal to $n_{\mathrm{H}}$, and, in addition, the independent variable $\mathrm{x}$ converted from time $\mathrm{t}$ as a variable to a different variable, viz. the natural $\log$ of the ligand concentration, $\ln [\mathrm{S}]$, we can rewrite the original logistic Eq. 10.14 as:

$$
y=\frac{1}{1+\mathrm{e}^{\left(n_{\mathrm{H}} \cdot \ln \left[K_{\mathrm{S}}\right]-n_{\mathrm{H}} \cdot \ln [\mathrm{S}]\right)}}=\frac{1}{1+\left(K_{\mathrm{S}} / \mathrm{S}\right)^{n_{\mathrm{H}}}},
$$

in which $y$ is the fraction of bound receptive units or the fraction of active effectors as a function of the ligand concentration $\mathrm{S}, K_{\mathrm{s}}$ is an equilibrium dissociation constant, and $n_{\mathrm{H}}$ is the Hill coefficient for a modified Hill equation, equal to our earlier parameter $n_{\mathrm{s}}$ in Chapter 8 .

The right hand-side of Eq. 10.15 is identifiable with the modified Hill equation (Eq. 10.2), and hence, the left hand side of this expression is a 'logistic' version of Hill's modified equation, i.e., the logistic equation conjured into the 'logistic' version of the modified Hill equation.

Fig. 10.1D is a graph of Eq. 10.15 with $\ln [\mathrm{S}]$ as the independent variable (in the figure it is $\log [\mathrm{S}]$ ) and $n_{\mathrm{H}}$ varying in five steps (see figure legend). In this, the graph in Fig. 10.1D is also just a simple semi-log presentation of the modified Hill equation.

If we assign a sign in reverse, i.e., a minus sign, for the exponent $\left(n_{\mathrm{H}} \cdot \ln \left[K_{\mathrm{S}}\right]-n_{\mathrm{H}} \cdot \ln [\mathrm{S}]\right)$ in Eq. 10.15 , then the transformation of the 'logistic' equation yields the wellknown reverse modified Hill equation in Eq. 8.4:

$$
y=\frac{1}{1+\mathrm{e}^{-\left(n_{\mathrm{H}} \cdot \ln \left[K_{\mathrm{i}}\right]-n_{\mathrm{H}} \cdot \ln [\mathrm{S}]\right)}}=\frac{1}{1+\left(\mathrm{S} / K_{\mathrm{i}}\right)^{n_{\mathrm{H}}}},
$$

with a Hill slope factor $n_{\mathrm{H}}$ equal to $n_{\mathrm{i}}$ described in Chapter 8, a dissociation constant for an inhibitor $K_{\mathrm{i}}$ instead of one for an agonist, and examples of its curves depicted in Fig. 8.8D-F.

The initiation of this 'logistic' transformation of the Hill equation in Eqs. 10.15 and 10.16 can be traced back to a comparison between a general form of logistic equations and Hill's equation (van Rossum 1966; De Lean et al. 1978). However, one can argue that the logistic-Hill-calamity if-you-will started with Joseph Berkson switching use of the logistic equation for time-dependent autocatalytic processes to processes dependent on a concentration-like variable for data of mortality rate (Reed \& Berkson 1929; Berkson 1944).

The above example on how the original logistic equation (Eq. 10.13), where time is the natural independent variable, can be transformed into its dose-response counterpart (Eq. 10.15), is described in Jenkinson et al. (1995) and Neubig et al. (2003), see also for instance Giraldo et al. (2002) and Giraldo (2003). 
Observe though, that the significance of parameters $K_{\mathrm{s}}$ and $n_{\mathrm{H}}$ and of independent variable $\mathrm{S}$ in the Hill and reverse-Hill 'logistic' equations is totally different from the physical meaning of parameters $a$ and $b$ and of independent variable $\mathrm{x}$ in the original logistic equation (compare Fig. 10.1D and Fig. 10.9). The new parameter $\ln \left[K_{\mathrm{s}}\right]$ and the variable $\ln [\mathrm{S}]$ have no physical meaning.

In addition, see comments by Jenkinson (2003, pp. 16-17) on the difference between the 'logistic'-Hill equation and the logistic equation for growth.

\subsubsection{Practical Use of a 'Logistic' Hill Equation}

The similarity between the original logistic equation and the 'logistic' transformed Hill/reverse-Hill equation has led users of the Hill equations into quoting the modified Hill equation in its 'logistic' form (Accamazzo et al. 2002; Hornigold et al. 2003; Motulsky \& Christopoulos 2004; May et al. 2007) or just describing the Hill equation as 'logistic' (De Lean et al. 1978; Barlow \& Blake 1989; Lazareno \& Birdsall 1993; Kenakin 2004, pp. 94 and 144; Ehlert 2005).

The modified Hill Eq. 10.2 in its 'logistic' form is formulated as: ${ }^{6}$

$$
y=\frac{1}{1+10^{n_{\mathrm{H}} \cdot\left(\log \left[K_{\mathrm{S}}\right]-\log [\mathrm{S}]\right)}},
$$

in which $\log \left[K_{\mathrm{s}}\right]$ might also be $\log \left(\mathrm{EC}_{50}\right)$.

In my opinion, Eq. 10.17 does not make things any easier to grasp. Rather, it will confound new users into a belief of some special significance. This is not the case. It appears merely as vestiges of 'The emperor's new clothes'. We are dealing with a simple pleonasm, since the $\log [\mathrm{S}]$ is equated as a power for a base of 10 , meaning that $\mathrm{S}$ still operates as straight forward $\mathrm{S}$ in Eq. 10.17.

Of course, one can argue that there is logic in the 'logistic' form of Hill's equation when plotting data in a semi-log plot, where it may be said that by converting the independent variable now expressed by $\log [\mathrm{S}]$ is the same as formulating Eq. 10.17, which is true (Giraldo et al. 2002; Giraldo 2003).

Meanwhile, we have obtained nothing by just quoting the 'logistic' transformation of the Hill equation compared with the ordinary modified Hill equation, except for a focus on the semi-log re-scaling for improved presentation and statistical evaluation. If this is the purpose, why not just say as much in a Methods section? And, further, remember that for hyperbolic

\footnotetext{
${ }^{6}$ A section in the appendix at the end of the chapter gives references to concentration dependent 'logistic' equations in Sigma-Plot software (Section 10.A.13 and Table 10.3).
}

dose-response relations the 'logit' actually is often a better method than the semi-log ('logistic') transformation for statistical evaluation of $\log \left(\mathrm{EC}_{50}\right)$. Therefore, why not quote and use the 'logit' instead of the 'logistic' semi-log re-scaling? See section 10.A.11. ${ }^{7}$

Notice that $\mathrm{S}=0$ is not defined in the 'logistic'-Hill or in the semi-log plot of Hill (Table 10.1). Thus, the 'logistic'-Hill has a disadvantage compared with a plot of Hill's equation in the linear-linear form. For all the listed equations in Table 10.1, the dependent variable ' $y$ ' varies between zero and unity. The range of independent variable $\mathrm{x}$, on the other hand, alternates between types of logistic equations (Table 10.1 and 10.2). For instance, there is a difference between the range of the independent variable $\mathrm{x}$ for non-transformed and transformed forms of Hill equations (Table 10.1). The independent variable, concentration, is not defined for $\mathrm{x}=0$ in the 'logistic' transformed versions, while in reality the physical process is defined for $\mathrm{x}=0$; equal to the control response and at zero ligand concentration.

Therefore, when fitting to a semi-log plot of synagic data, it is recommended to insert an extra data point at a low ligand concentration value and with zero response. That is, include a fictive data point at zero response two or three orders of magnitude below the concentration of the first data point with an actual ligand concentration eliciting a small response and instead of the data point at zero ligand concentration (see also Motulsky \& Christopoulos 2004, p. 264).

\subsection{5. 'Logistic-Hill' is a Technical Term}

It is true that a semi-log plot of data from synagic experiments analyzed by non-linear fitting of the Hill theory yields better statistics than in its linear-linear form (Motulsky \& Christopoulos 2004, pp. 256-265). This is correct in principle for synagic data if carried out according to the probability laws (Finney 1971, Chapters 2 and 3), but not necessarily for other types of data. For instance, population growth data analyzed by the logistic equation is best handled by a linear-linear scaling of data (see Fig. 10.9).

Taken as a whole, it is the semi-log, the logit, or the probit technique used on synagic data that yields the better statistics, rather than just quoting exotic expressions for the Hill equation. It may be said that it all boils down to a matter of taste. My preference is to give the Hill equation as the modified Hill equation (Eq. 10.2),

\footnotetext{
${ }^{7}$ For $n_{\mathrm{H}}>1$, Hill and 'logistic'-Hill are both sigmoidal curves in linear-linear plots, but symmetrically S-shaped when represented as curves in semi-log plots (Fig. 10.1). Also, see Chapter 8 on S-shape versus sigmoidality. The original logistic growth curve in Eq. 10.13 is a sigmoidal and symmetric curve in the relative linear-linear plot but an S-shaped and non-symmetric curve in the semi-log plot (see Section 10.3.6, Fig. 10.9 and Table 10.1).
} 
Table 10.1. Various types of 'logistic' equations (for comments see Section 10.3.5)

\begin{tabular}{|c|c|c|c|c|c|}
\hline & $\begin{array}{l}\text { Dependent } \\
\text { Variables }\end{array}$ & $\begin{array}{l}\text { Independent } \\
\text { Variables }\end{array}$ & Plot type & Plots in figures & Range of $x$ \\
\hline \multicolumn{6}{|l|}{ Logistic equations } \\
\hline \multirow[t]{2}{*}{ The original } & $\frac{1}{1+\mathrm{e}^{(a-b \cdot \mathrm{x})}}$ & $x$ & Symmetric sigmoid & 10.9 & $-\infty<x<\infty$ \\
\hline & & $\log x$ & Skewed & & $0<x<\infty$ \\
\hline \multirow[t]{2}{*}{ Modified Hill } & $\frac{1}{1+\left(K_{\mathrm{s}} / \mathrm{x}\right)^{n_{\mathrm{H}}}}$ & $x$ & Skewed sigmoid & $10.1 \mathrm{~A} ; 8.8 \mathrm{~A}$ & $-\infty<\mathrm{x}<\infty^{*}$ \\
\hline & & $\log x$ & Symmetric S-shaped & $8.8 \mathrm{~B}$ & $0<x<\infty$ \\
\hline \multirow[t]{2}{*}{ Semi-log transformed Hill } & $\frac{1}{1+10^{n_{\mathrm{H}}} \cdot\left(\log K_{\mathrm{s}}-\log \mathrm{x}\right)}$ & $x$ & Skewed sigmoid & $10.1 \mathrm{~A} ; 8.8 \mathrm{~A}$ & $0<x<\infty$ \\
\hline & & $\log x$ & Symmetric S-shaped & $8.8 \mathrm{~B}$ & $0<x<\infty$ \\
\hline \multirow[t]{2}{*}{ Semi-log transformed reverse Hill } & $\frac{1}{1+10^{-n_{\mathrm{H}} \cdot\left(\log K_{i}-\log \mathrm{x}\right)}}$ & $x$ & Skewed sigmoid & $8.8 \mathrm{D}$ & $0<x<\infty$ \\
\hline & & $\log x$ & Symmetric S-shaped & $8.8 \mathrm{E}$ & $0<x<\infty$ \\
\hline \multirow[t]{2}{*}{ Reverse Hill } & $\frac{1}{1+\left(x / K_{\mathrm{i}}\right)^{n_{\mathrm{H}}}}$ & $x$ & Skewed sigmoid & $8.8 \mathrm{D}$ & $-\infty<\mathrm{x}<\infty^{*}$ \\
\hline & & $\log x$ & Symmetric S-shaped & $8.8 \mathrm{E}$ & $0<x<\infty$ \\
\hline
\end{tabular}

Constants $a$ and $b$ exist for all values. ${ }^{*}$ Only range $0 \leq \mathrm{x}<\infty$ is relevant. $K_{\mathrm{s}}$ only for $0<K_{\mathrm{s}}<\infty$.

and plot data in so-called semi-log plots with appropriate statistical evaluation of $\log \left(\mathrm{EC}_{50}\right)$ in either 'logistic' or logit transforms. The transforms may not be needed with use of modern statistical software.

The term 'logistic' in connection with Hill's equation is a technical term referring to semi-logging, while for

Table 10.2. Terminology for logistic growth - examples of formulae for population growth equal to logistic equations

\begin{tabular}{|c|c|}
\hline Name of equation & Equation \\
\hline $\begin{array}{l}\text { Malthus' equation with Malthusian } \\
\text { parameter } r^{\dagger}\end{array}$ & See Gompertz curve \\
\hline Gompertz curve & $y=a \cdot b^{q^{x}} \vee y=a \cdot e^{b \cdot e^{c \cdot t}}$ \\
\hline $\begin{array}{l}\text { Gompertz standard model also known } \\
\text { as the law of growth }\end{array}$ & $N(t)=N_{0} \cdot \mathrm{e}^{r \cdot \mathrm{t}}$ \\
\hline Gompertz modified model & $N(\mathrm{t})=C+N_{0} \cdot \mathrm{e}^{r \cdot \mathrm{t}}$ \\
\hline $\begin{array}{l}\text { Logistic curve or when normalized } \\
\text { called the logistic distribution }\end{array}$ & $y=\frac{a}{1+b \cdot q^{\mathrm{t}}}$ \\
\hline $\begin{array}{l}\text { Logistic equation also called sigmoid } \\
\text { function or Verhulst' model }\end{array}$ & $y=\frac{1}{1+\left(1 / y_{0}-1\right) \cdot \mathrm{e}^{-r t}}$ \\
\hline $\begin{array}{l}\text { Sigmoid function also called the sigmoid } \\
\text { curve, logistic function or sigmoid } \\
\text { equation }\end{array}$ & $y=\frac{1}{1+\mathrm{e}^{-\mathrm{x}}}$ \\
\hline Verhulst' équation logistique & $\frac{d N}{d t}=\frac{r N \cdot(K-N)}{K}$ \\
\hline $\begin{array}{l}\text { Differential version of the logistic model } \\
\text { and its discrete quadratic version also } \\
\text { known as the logistic map; which leads } \\
\text { into deterministic chaos }\end{array}$ & $X_{n+1}=r \cdot N_{\mathrm{n}} \cdot\left(1-X_{\mathrm{n}}\right)$ \\
\hline
\end{tabular}

'Parameter $r$ is the maximum population growth rate, equal to parameter $c$ in Gompertz' curve equation, where parameters $b$ and $c$ are negative numbers. $\mathrm{e}=$ base of the natural logarithm, $\mathrm{t}=$ time, and $\mathrm{x}=$ any independent variable.

\#'Sigmoid equation' in SigmaPlot manual (2004). instance the term 'distribution equation' is a functional descriptor with more meaning, as it also discriminates the Hill equation from the original logistic equation. In line with this, Jenkinson also has a warning against mixing the logistic equation and the Hill 'logistic' equation (Jenkinson 2003, Eq. 1.2.44 and Appendix 1.2D, pp. 16-17).

In summary, quoting a 'logistic' Hill equation simply implies semi-log re-scaling for better presentation and statistics.

\subsubsection{The Richards Equation}

Giraldo has specifically analyzed equations for asymmetry, lacking in Hill's function (Giraldo et al. 2002; Giraldo 2003). One such equation is the Richards equation (Richards 1959), ${ }^{8}$ where the whole denominator is raised to a power (Van der Graaf \& Schoemaker 1999). Thus, for instance:

$$
y=\frac{1}{\left[1+\left(K_{\mathrm{s}} / \mathrm{x}\right)^{n_{\mathrm{H}}}\right]^{n_{\mathrm{R}}}},
$$

\footnotetext{
8 Reference to a Richards equation in this section is due to Giraldo et al. (2002). I must admit that I do not see the correspondence between Richards' formulations (Richards 1959) and those by Giraldo et al. (2002). Meanwhile, in this text I shall stick with the Giraldonomenclature. Note, the conventional 'Richards equation' is used in a different field; namely hydrology (Scienceworld.wolfram.com).
} 


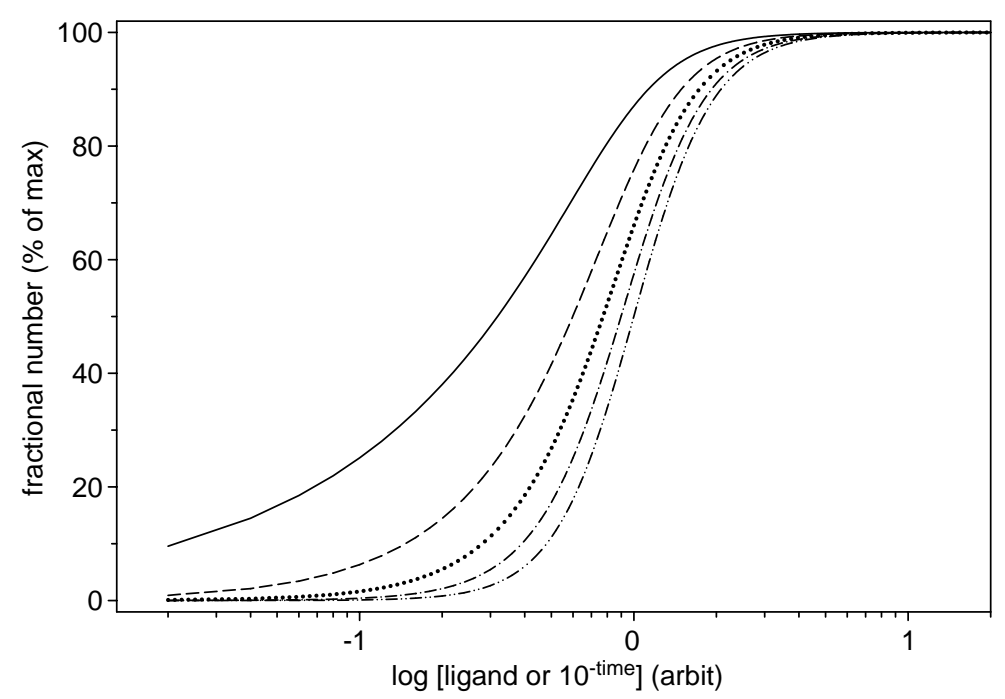

Figure 10.11. A Richards' function also called the generalized Hill equation. Here the Richards coefficient $n_{\mathrm{R}}$ is varied in five steps from $1.0 .2(-)$ to $5.0 .2(-\cdot \cdot)$, increasing by 0.2 between steps, while keeping the Hill's coefficient $=3$. Terminology after Giraldo et al. (2002).

where $y$ is a response as a function of independent variable $\mathrm{x}$. System constant $n_{\mathrm{R}}$ signifies a Richards coefficient. Both Hill's symmetric equation and Gompertz's asymmetric equation (http://mathworld. wolfram.com) are included in the Richards equation (Giraldo et al. 2002). The effect of varying the Richards coefficient, $n_{\mathrm{R}}$, on sigmoidality is shown in Fig. 10.11, depicting the skewed sigmoidality of Richards' equation for changing values of Richards' coefficient in semilog plotting. For $n_{\mathrm{H}}=1$, this type of equation is also called a 'generalized logistic curve' (Table 10.3). As an example, the Richards equation with $n_{\mathrm{H}}=1$ has been used frequently to analyze ion-pump data (Garay \& Garrahan 1973; Eisener \& Richards 1981). Meanwhile, since the Richards equation is purely an empiri-

Table 10.3. A comparison of expressions for various equations used with those in the SigmaPlot software, some incorporating the term 'logistic'

\begin{tabular}{|c|c|}
\hline Present terminology & SigmaPlot $^{\circledR}$ terminology ${ }^{\star}$ \\
\hline Hill equation (modified) & Hill equation \\
\hline Reverse Hill equation & $\begin{array}{l}\text { Logistic equation or four parameter } \\
\text { logistic curve }\end{array}$ \\
\hline Logistic equation" & Sigmoid equation \\
\hline Gompertz curve & Gompertz growth model \\
\hline $\begin{array}{l}\text { Generalized logistic curve }{ }^{\S} \text { or } \\
\text { Richard's equation }\end{array}$ & Modified hyperbola III \\
\hline $\begin{array}{l}\text { Load plus diffusion as used in } \\
\text { Chapter } 9\end{array}$ & $\begin{array}{l}\text { Rectangular hyperbola II or one site } \\
\text { saturation + non-specific }\end{array}$ \\
\hline
\end{tabular}

cal formulation (Richards 1959), its relevance as a mechanistic tool should be questioned.

Eq. 10.18 can be applied to a sequential process of $n_{\mathrm{R}}$ steps, in which only the final step renders activity or a conformational switch with change in ligand binding, $\mathrm{RS}_{\mathrm{n}} \rightleftarrows \mathrm{R} * \mathrm{~S}_{\mathrm{n}}$, governed by an isomerization constant equal to $L^{\text {ns }}$, where $L^{\text {ns }}$ is assumed small compared to the other forward parameters as association constants denoted $K_{\mathrm{ax}}=1 / K_{\mathrm{dx}}$. Besides assuming $L^{\mathrm{ns}}<<K_{\mathrm{ax}}$, we can further simplify the system by assuming that all the forward equilibrium association constants are identical. Compare with the Weiss model described in Chapter 6.

Giraldo and coworkers (2002) show that the exponent under these assumptions is equal to the number of steps. ${ }^{9}$ The slope at the point of inflection for four synagic models has been derived as a general expression, equal to $4 \cdot\left\{(\delta \mathrm{R} / \delta \log [\mathrm{S}])_{50}\right\} /\left\{\ln 10 \cdot R_{\max }\right\}$ and includes the Hill and Richards equations (Berkson 1944; Hill 1985, pp. 64-66; Giraldo 2003). However, both the Hill equation and the Richard type of equations as stated are empirical equations that may be used for semiquantitative estimates of deviation from the simple load formulation, but as mechanistic models they are only relevant for systems with full co-operativity or complete simultaneity.

See Giraldo et al. (2002) for a more detailed account of the Hill, the modified Hill, the Gompertz, and Richards equations as well as their asymptotic location, midpoint, and inflection points.

\footnotetext{
${ }^{9}$ Compare this with the meaning of $n_{\mathrm{H}}$ in Hill's aggregation theory described in Section 10.1.2.
} 


\subsubsection{Use of Double Hill or Seriatim Hill Equations}

Hill first suggested use of summed Hill equations (Eq. 10.6) (Hill 1910). Using the sum of two or more Hill equations to describe dose-response data with bellshaped or reverse bell-shaped appearance is even further from the mechanism of a functional system, although this type of analysis is rather popular (Pliska 1994; Taleb \& Betz 1994; Rovati \& Nicosia 1994; Vogel et al. 1995; Bronnikow et al. 1999; Accomozzo et al. 2002; Tucek et al. 2002; Hornigold et al. 2003; May et al. 2007), requiring determination of between six and seven system constants when two Hill equations are combined. There are other examples for the use of seriatim Hill equations. They appear as products or sums of Hill equations in analysis of genetic regulatory networks (Meir et al. 2002) and in pharmaco-dynamic and pharmaco-kinetic analyses of drug-drug interaction in signal transduction, in ligand binding studies (Wells 1992), and in clinical drug treatments (Scaramellini et al. 1997; Short et al. 2002; Jonker et al. 2003), as well as for synergy described in Chapter 12 (Chou \& Talalay 1981, 1984; Berenbaum 1989; Greco et al. 1995; Chou 2006).

Recently, Michel (2007) derived equations for a combined Hill scheme and Adair approach with varying association constants due to binding interactions.

Bell-shaped synagics may also be analyzed by a series of simple load functions (Szabadi 1977; Ehlert 1988; Jarv 1994; Williams et al. 2000; Cladman \& Chidiac 2002; Griffin et al. 2003), requiring determination of between four and five parameters when operating with the sum of two loads.

Ultra-sensitivity and switch-like function, for instance in signaling cascades, may be obtained by several levels of enzyme-induction and described by embedded Hill equations (Huang \& Ferrell 1996; Ferrell 1997).

Combining the Hill equation as well as other models with a term for feed-forward and feedback was introduced by Hofmeyr and Cornish-Bowden (1997; CornishBowden 2004, Sub-chapter 12.10; Hofmeyr et al. 2006). However, these models are non-mechanistic.

For a description of models for bell-shaped responses, but with a mechanistic implication, it is recommended to try other models such as the allosteric two-state model (ATSM) and the homotropic two-state model (HOTSM) presented in Chapter 7, or the random and ordered reaction schemes including their expanded versions in Chapter 6 (Table 6.2).

\subsubsection{Use of Hill-Richards Type Formulations}

Just as we use Hill-type equations in analysis when agonism deviates from the load and seemingly involves an auto-intervention or co-operative process, likewise we use reversed Hill-type equations when the action of an interventor also indicates an intervention process or a modulator a modulatory process that deviates from the simple load (Lazareno \& Birdsall 1993, 1995; Trankle et al. 2005).

For the effects of combining agonists and interventors or modulator molecules there are suggestions in the literature for the use of embedded Hill-Richards formulations in order to characterize parameters (Lazareno \& Birdsall 1995; Trankle et al. 2005). Meanwhile, if probing for a physical interpretation of synagic data, once again my preference would be to try the intervention model or the ATSM by Hall, and possible extensions thereof with different binding constants due to site interactions (Chapters 2 and 7).

\subsubsection{On the Similarity Between the Hill Equation and the Modified Boltzmann Equation}

Gibbs free energy (Fig. 1.5) may be represented by electrical potentials, concentration gradients, temperature gradients, pressure and other forms of physical or mechanical forces.

Boltzmann's equation for probability distribution at equilibrium ${ }^{10}$ recast into a chemical form is the Nernst equation, which we can write as:

$$
\mathrm{E}_{\mathrm{eq}}=\mathrm{E}_{1}-\mathrm{E}_{2}=\frac{R T}{\mathrm{z} F} \cdot \log \frac{[\mathrm{S}]_{1}}{[\mathrm{~S}]_{2}},
$$

in which $\mathrm{E}_{\mathrm{eq}}$ is equal to the equilibrium potential measured for example in milivolt given as the difference between the potential at side 1 and $2, \mathrm{E}_{1}-\mathrm{E}_{2} . R T / \mathrm{z} F$ is a constant equal to $61.5 \mathrm{mV}$ at $37^{\circ} \mathrm{C}$ and $[\mathrm{S}]_{1}$ and $[\mathrm{S}]_{2}$ are the passively distributed concentrations of $\mathrm{S}$ at side 1 and side 2 (Nernst 1889; Hille 2001, p. 13).

For voltage operated ion channels (VOCs), a modified form of the Boltzmann equation dictates a ratio of open $(\mathrm{O})$, to the sum of both open and closed $(\mathrm{C})$ channels (Fig. 5.2B), that is:

$$
\frac{\mathrm{O}}{\mathrm{O}+\mathrm{C}}=\frac{1}{1+\exp \left[(\mathrm{z} F / R T) \cdot\left(\mathrm{V}_{1 / 2}-\mathrm{E}\right)\right]},
$$

where 'exp' is the natural base e and z $F / R T$ a constant as before. When the potential is equal to $\mathrm{V}_{1 / 2}$ then $50 \%$ of the channels are open. $\mathrm{V}_{1 / 2}$ is similar to the so-called Boltzmann constant.

The relation in Eq. 10.20 is often plotted in a loglinear form (Hille 2001, p. 55).

\footnotetext{
${ }^{10}$ In fact, developing this distribution equation was done nearly simultaneously by Maxwell (for rate of molecules) and by Boltzmann (for energy of adsorption). Thus, this distribution equation is also known as das Maxwell-Boltzmannsches verteilungsgesetz.
} 
Eq. 10.20 clearly resembles the Verhulst growth expression, the logistic Eq. 10.13, and since from the Nernst relation in Eq. 10.19 we have E proportional to $\ln \left([\mathrm{S}] / K_{\mathrm{d}}\right)$, it is tempting to replace $\mathrm{E}$ in Eq. 10.20 with $\ln \left([\mathrm{S}] / K_{\mathrm{d}}\right)$ and rewrite Eq. 10.20 such that the expression for the number of charges, $=\mathrm{z}$, moved in the voltage gating is replaced by the number of ions binding and equated with Hill's coefficient $n_{\mathrm{H}}$. Therefore, we can write:

$$
\frac{\mathrm{O}}{\mathrm{O}+\mathrm{C}}=\frac{a r}{T R}=\frac{1}{1+\left(K_{\mathrm{d}} /[\mathrm{S}]^{n_{\mathrm{H}}}\right)},
$$

where $\ln \left(K_{\mathrm{d}}\right)$ replaces the $\mathrm{V}_{1 / 2}$. A transformation of the modified Boltzmann equation to the Hill equation.

In fact, this transformation has its own life in analysis of VOCs (Spivak 1995; Yifrach 2004; Kubokawa et al. 2005; Chapman et al. 2006; Zhang et al. 2007), and will not be discussed further here, except that the transformation does not bring the logistic equation with time as an independent variable any closer to the Hill equation for dose-response analysis in synagics.

\subsection{Conclusions}

\subsubsection{Details on the Use of Hill's Equation}

We can summarize the advantages and problems of using Hill's equation and its derived versions in the following seven paragraphs.

(1) As a physical model, the modified Hill equation (Eq. 10.2) describes a coincidence system. The coincidence may come about by a simultaneous and saturating binding of ligands to a multisited preexisting complex or in the instant formation of a multi-subunit complex where each subunit is already liganded. The latter possibility may be a scenario for di-merization of growth factor receptors (GFRs) (Bublil \& Yarden 2007), though unlikely for GPCRs (Ma et al. 2007). Thus, for some GFRs, liganding of single unit receptors might increase the likelihood of di-merization or multi-merization. Hill's aggregation theory revitalized. The Hill equation may also be used for a multi-sited system with a final dominating step for binding or function. Thus, the Hill coefficient can be equal to the number of binding sites, when reaction models behave as an all-or-non association/dissociation reaction scheme, e.g., when the final binding step has a much higher affinity than the preceding binding steps, as in hemoglobin- $\mathrm{O}_{2}$ binding (Table 5.3) (Roughton et al. 1955; Monod et al. 1965; Weiss, 1997).
(2) The Hill analysis is a practical and convenient measure of co-operativity when information about a system is limited.

(3) Obtaining a non-linear Hill plot, i.e., a nonlinear logit plot, is one way to uncover deviations of experimental data away from the modified Hill equation. In case the Hill (logit) plot of data with $n \neq 1$ deviate significantly from linearity, try the Pascal triangle expansion for multi-sited receptor complexes where individual association constants are allowed to vary (Adair 1925; Ricard \& Cornish-Bowden 1987) (see also Tables 6.1 and 6.2, or try allosteric models such as the HOTSM for single ligand applications, Chapter 7).

(4) The Hill equation is a special distribution equation for synagics rather than a logistic equation. The 'logistic' formulation of Hill's equation is a technical term for semi-log plotting and for statistical evaluation of data. It is a mere reference to semi-log analysis of synagic data, on the same line as a logit or a probit analysis. Semi-log presentation of dose-response results, synagic data, is recommended.

(5) Analysis may be performed for instance in semi$\log$ plots of synagic data, thus evaluating the $\log \left(\mathrm{EC}_{50}\right)$. Semi-log plot of experimental data analyzed by non-linear fitting to synagic data yields better statistical evaluation for the system constant $\mathrm{EC}_{50}$, i.e., $\log \mathrm{EC}_{50}$, compared to linear-linear analysis of data (Motulsky \& Christopoulos 2004, pp. 256-265). This is true in principle if carried out according to the statistical laws, even when the Hill coefficient is 1. On the estimation of the median effective dose, $\mathrm{ED}_{50}$, and its logarithmic transformation, $\log \mathrm{ED}_{50}$, see for instance Finney $(1971,1978$, Chapters 3 and 4; Berkson 1944). The probit and especially the logit methods for parameter evaluation should also be considered. In theory, the logit method is even better than the semi$\log$ ('logistic') method in using the modified Hill equation as a base for analysis of dose response data (Berkson 1944). Modern software may overcome such difficulties.

(6) In publishing results based on an analysis using Hill's modified equation and combined with either semi-log, logit, or probit transformation, write the following: modified Hill's equation was used as theory, and the statistical evaluation was performed by semi-log (logistic) transformation, by logit (double log of relative bound over free receptors) transformation, or by probit (normal equivalent deviate of dependent 
variable versus $\log$ of independent variable) transformation.

7. The modified Boltzmann equation (Eq. 10.20) resembles the logistic equation (Eq. 10.13), but the two are used in different applications with different meanings. The electrical potential as a force appears similar to time as a 'force' and may mathematically be transformed to concentration as a force.

In summary: (1) quoting 'logistic Hill' ought to cover the same as a semi-log analysis of data and based on the modified Hill equation, and (2) the real problem with a choice of the modified Hill equation for theory is when it works as a silencer of explorative investigations, thereby preventing possible penetrating analyses.

\subsubsection{Is Hill in Hell?}

A century celebration of Hill's equation - now, then, and in 2010, alia equibus. ${ }^{11}$

When co-operative logistics has to come 'true', ill shivers us as a hellish flu.

For whether you don't or you do have a clue, what do we do with a Hill of two?

We use him here, we use him there, for Bells the scientist uses 'im everywhere.

Is Hill in Heaven, is Hill in Hell - as he's hill-hidden no one can tell.

(to be cont'd....)

\section{A. Appendix A: A Short Course on the Term 'Logistic' and its Uses in Statistics}

Here the term 'logistic' is put in a slightly different perspective compared to the term 'logistic' discussed in the preceding Sub-chapters; although several of the related concepts are the same and also repeated here.

\section{A.1. Statistical Analysis}

Statistics is the science of analyzing, interpreting, and understanding data. There are various statistical methods for analyzing data. Which method to choose will depend on the distribution of data and the theory selected for their analysis. Before an analysis, the selected theory may further be transformed such that a plot of data according to the transformation is more equally distributed, 'linearized' and skewness of residuals thus reduced. Examples of this are given below.

\footnotetext{
11 alia equibus = everything else equal.
}

From a narrower perspective, statistics may be said to be an analysis of the validity of data sets, testing probabilities, and comparing models. Examples of hypotheses and methods for data evaluation are chisquare-, $t$ - or $F$-tests, least square and/or maximum likelihood fitting, and data transformations (Quinn \& Keough 2002; Samuels \& Witmer 2003; Sokal \& Rohlf $1995,2004)$, and frequentists or Bayesians interpretation of probability (Cox 2001; Quinn \& Keough 2002; Winkler 2003; Gelman et al. 2003; Lee 2004; Sivia \& Skilling 2006). As an example, Bayesian interpretations relying on inference and filtering is now also used for optimal exclusion of spam E-mails (Zorkadis et al. 2005). When the variance alters for data in the data set with time or another independent variable, this is an entirely separate subject. Its analysis in economics won Robert Engle the Nobel prize in 2003, solving the autoregressive conditional heteroskedasticity (http:// nobelprize.org/nobel_prizes/economics/laureates/2003/ engle-lecture.html). On more general aspects of data analysis see Ford (2000).

\section{A.2. Statistical Methods}

Names for some of the statistical methods are listed here: linear regression, curvilinear regression, non-linear regression, and logistic regression (Snedecor \& Cochran 1967; Pampel 2000; Hosmer \& Lemeshow 2000; Cramer 2003a; O’Connel 2005; Vittinghoff et al. 2005). Logistic regression is for discontinuous and often binary responses as 'dead' or 'alive' dependent on an independent predictor variable as for instance 'level of toxic drug' (Hosmer \& Lemeshow 2000; Vittinghoff et al. 2005). Other theories or techniques are for instance Multinominal Logistic Regression, multi-way frequency analysis (MFA), Multivariate analysis, and Repeated Measures Models (Hosmer \& Lemeshow 2000; Vittinghoff et al. 2005; Kleinbaum et al. 2007). Viewing many of the statistical methods under the same framework led researchers to the generalized linear modeling (GLM) ${ }^{12}$ (Dobson 2001; Quinn \& Keough 2005), not to be detailed here.

The more recent Bayesian method is yet another approach using inference and filtering in decision (Quinn \& Keough 2002; Winkler 2003; Marin \& Robert 2007; Bernado \& Smith 2007; Lage et al. 2007).

\section{A.3. Statistical Theory - the Logistic Equation}

For analysis of data sets, the selected theory is an equation. An example is the logistic equation (Eq. 10.13). This equation with its curvilinear regression is used for analysis of population growth, although now recognized as insufficient (Kingsland 1995; Turchin

\footnotetext{
${ }^{12}$ GLM is different from the general liner models in the SAS package.
} 
2003). Parameters in the logistic equation are the 'maximum carrying capacity of the population that is approached as times runs, equal $100 \%$, the standard interval $1 / r$ or reciprocal Malthusian parameter (Table 10.2), and the location of the inflection point on the time axis. The description obtained by the logistic equation is mathematical not physical. Oppositely, the adsorption-desorption process equated by the load formulation is a physical description.

\section{A.4. 'Logistic' and Logistic Equations}

Presently there are expanding uses of the word 'logistic'. Pertinent to our discussion, you can find formulations listed as 'logistic' functions and 'logistic' or logistic equations when the independent variable is raised to a power or the independent variable itself is included in an exponent. Other examples are terms as 'logistic maps', 'logistic distribution', 'logistic regression', and 'polytomous logistic regression' (Table 10.5). An example of the 'logistic' equation is the modified Hill equation (Eqs. 10.15-10.17), also referred to as a 'logistic function' when $n_{\mathrm{H}}=1$ (Berkson 1944). We also have the Verhulst/Pearl-Reed logistic equation for population growth (Eqs. 10.13 and 10.14).

Berkson used the logistic equation to analyze doseresponse data and called the equation 'the logistic function', in which his coefficient $\alpha / \beta$ for the independent variable he claimed to be to equal to L.D. $50\left(\mathrm{EC}_{50}\right)$ although it is equal to $\log$ L.D. $50\left(\log \mathrm{EC}_{50}\right)$ (Fig. 10.6) (Berkson 1944).

Furthermore, when equations have their independent and/or dependent variables re-scaled to a logarithmic dimension, such functions are also referred to as 'logistic' equations or functions. For the history of the development of the original logistic equation, see Kingsland (1995), Turchin (2003), and Cramer (2003b at http:// www.cambridge.org/resources/0521815886/1208_ default.pdf).

For statistical analysis, the logistic equation (Eq. 10.13) appears relevant for many applications with a limited number for response, which on a relative scale will vary between 0 and 1 . The term 'logistic regression', on the other hand, is a statistical analysis based mainly on discontinuous binary variables (Hosmer \& Lemeshow 2000; Quinn \& Keough 2002; Samuels \& Witmer 2003, pp. 582-585; Cramer 2003a; Vittinghoff et al. 2005).

Logistic regression is also referred to as 'logit analysis' covered by logit models (Table 10.4) (Cramer 2003a).

\section{A.5. 'Logistic' Hill's Equation}

As mentioned in Section 10.3.4, there is no need for an explicit use of the expression 'logistic equation' when
Table 10.4. Concept examples from fields of statistics, some including the term logistic

\begin{tabular}{ll}
\hline Equation terms & Parallel terms \\
\hline $\begin{array}{l}\text { Logistic equation } \\
\text { Hill/Reverse Hill }\end{array}$ & Growth curves \\
$\begin{array}{l}\text { Transformations } \\
\text { Logarithmic re-scaling including } \\
\text { semi-log }\end{array}$ & 'Logistic dose-response curve \\
$\begin{array}{l}\text { Logit } \\
\text { Probit }\end{array}$ & (Mistakenly called logistic) \\
$\begin{array}{l}\text { Power, including reciprocal } \\
\text { Analysis }\end{array}$ & Hanes, Lineweaver-Burk, Eadie- \\
$\begin{array}{l}\text { Logistic regression } \\
\text { Logistic map }\end{array}$ & Scatchard \\
Logistic distribution & 'Logit analysis' \\
\hline
\end{tabular}

operating with the Hill modified equation. The situation is similar to the analysis of density spectra by the Lorentzian probability function: $y=1 /\left(1+\left(\mathrm{x} / \mathrm{x}_{\mathrm{c}}-\mathrm{x}_{0}\right)^{2}\right)$ with the independent variable $\mathrm{x}$ as a frequency, $\mathrm{x}_{\mathrm{c}}$ as a 'corner frequency', and $\mathrm{x}_{0}$ a location parameter (Verveen \& DeFilice 1974). With the Lorentzian equation, it is natural to look at a semi-log distribution of data rather than a linear-linear distribution (Fig. 10.12). Meanwhile, no devil would dare to dream or to talk about the Lorentzian equation as a 'logistic' equation. Note further that the load equation with $n_{\mathrm{H}}$ equal 1 , which was termed 'logistic' by Berkson is seldomly referred to as a 'logistic' equation (Ehlert 2008). In fact, it is a hyperbolic function, see Fig. 1.3.

In conclusion, for a dose-response formulation, the term 'logistic function' as introduced by Berkson (1944) and transferred to the Hill equation is a misnomer, a nuisance, and a bad habit.

Remarkably, the modified Boltzmann distribution function (Eq. 10.20), which is a true logistic equation, does not carry this designator.

\section{A.6. Logistic Regression}

'Logistic regression' is also frequently used as synonymous with the logit or probit analysis, although most use the term 'logistic regression' specifically for the analysis of single or multiple predictor(s) with discontinuous outcome in contrast to single or multi-way analyses for continuous variables.

Dependent on the type of outcome, 'logistic regression' appears in various connections (see Table 10.5).

\section{A.7. Transformations}

The theory for data in the form of an equation may be transformed by altering the expression of the independent (predictor) and/or the dependent (outcome) 


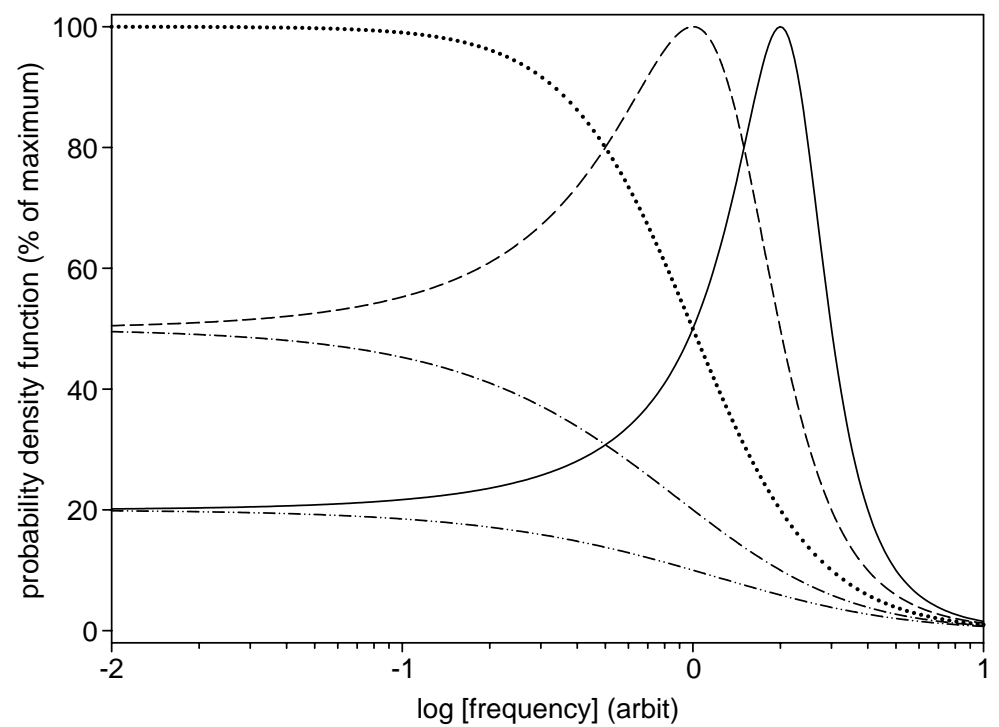

Figure 10.12. Plots of the Lorentzian relation given by $y=Y_{\max } /\left(1+(x-C)^{2}\right)$. Constant $C$ is varied in five steps: from $-2(-)$ to 2 $(-\cdot-)$, increasing by 1 between steps. For values of $C>0$ the Lorentzian has a peak value. See also Christensen and Bindslev (1982).

variable (Sokal \& Rohlf 1994, 2004; Quinn \& Keough 2002). Examples are:

Linear-linear (no transformation).

Semi-log ('logistic' function, logarithmic transformation of the axis for the independent variable).

Log-linear (logarithmic transformation of the axis for the dependent variable as a response).

Log-log (logarithmic scale of both axes).

Logit (equal to use of regula detri rule $1 \mathrm{~b}$ in Box 1.1 and a logarithmic transformation of both axes).

Probit (normal distribution of the dependent variable and logarithmic transformation of both axes).

Power (including the power -1 for reciprocal transformations as the Hanes, Lineweaver-Burk, EadieScatchard, or Schild plot in Fig. 11.7).

Table 10.5. Regression models divided according to the experimental outcome*

\begin{tabular}{lll}
\hline $\begin{array}{l}\text { Response } \\
\text { classification }\end{array}$ & Response type & Regression model \\
\hline Numerical & $\begin{array}{l}\text { Continuous* } \\
\text { Count } \\
\text { Time-to-event }\end{array}$ & $\begin{array}{l}\text { Linear, curvi- and non-linear } \\
\text { Poisson } \\
\text { Proportional hazards }\end{array}$ \\
& Binary & Logistic \\
& Ordinal & Proportional odds \\
& Nominal & Polytomous logistic \\
\hline
\end{tabular}

*Modeled after Vittinghoff et al. (2005, pp. 318-319).

\section{A.8. Logarithmic Re-scaling}

In statistical analysis, a logarithmic transformation of either the dependent or the independent variable, or both at a time, often have advantages over an analysis based on a linear-linear representation, especially when variables are log-normal, i.e., when the relationship between dependent and independent variables is relative. As already mentioned, semi-log, log-linear, and logit analysis are examples of such 'logistic' transformations (Hosmer \& Lemeshow 2001).

\section{A.9. Semi-log Re-scaling}

The load equation was designated as a 'logistic function' when its independent variable, concentration, was rescaled by logarithmic transformation (Berkson 1944).

Transformation of the modified Hill equation (Eq. 10.2) to a so-called 'logistic' function (van Rossum 1966; De Lean et al. 1978; Giraldo et al. 2002; Giraldo 2003) is the same as transforming the independent variable dose to a log-scale, and therefore equal to a semi-log transformation. Semi-log plotting is for better visualization and/or improved statistical analysis. Semi-log plots of the modified Hill's equation and its use in analysis is the same as 'logistic-Hill analysis'.

\section{A.10. Log-linear Re-scaling}

Log-linear transformation is a $\log$ (-istic) transformation of the dependent variable often used in statistical evaluation of numerical (Vittinghoff et al. 2005)/categorical (Quinn \& Keough 2002) variables with Poisson 
distribution, typical for economic and social sciences, but certainly also relevant in biological sciences (Finney 1971, 1978; Hille 2001, p. 55; Quinn \& Keough 2002; Vittinghoff et al. 2005). Log-linear models are something different (see for instance Quinn \& Keough 2002, Sub-chapter 14.3).

Log(-istic)-linear transformation is commonly used for dependent variables that are continuous, such as the response in the washout or loading of drugs in compartmental analysis often following mono- or multi-exponential decay or rise (Riggs 1970; Jacquez 1972 or 1996 - 3rd edition of his book). In addition, dependent responses in time-dependent pharmacokinetic studies are suitable for log-transformation as well (Chou et al. 2006).

\section{A.11. Logit Re-scaling}

The term 'logit' does not always refer to the logit rescaling. Logit transformation is taking the ratio of positive responses over negative responses for dependent categorical variables, also designated 'odds ratio', and where both sides of the formulations are logarithmically re-scaled. Or, the ratio of actual response or probability over remaining potential response or probability for dependent quantitative variables is formulated in an equation and thereafter both sides of the equation are re-scaled logarithmically, a 'logit' transformation. In effect, it is a logarithmic re-scaling of a transformed dependent variable (Figs. 8.7C $+\mathrm{F}$ and $8.8 \mathrm{C}+\mathrm{F}$ ) (Liao 1994; Chou 2006). Meanwhile, observe that in a fine text on 'logit models' in econometrics, a 'logit analysis' is the same as logistic regression (Cramer 2003a). Beware, a logit analysis can involve a logit transformation as a link function in logistic regression! See Table 10.4.

\section{A.12. Probit Re-scaling and Integration}

In probit analysis the dependent variable, response, is re-scaled according to the inverse standard normal cumulative distribution function, also designated 'the normal equivalent deviate', and the independent variable can be re-scaled percentage-wise or logarithmically depending on the analyzed theory (Finney 1971). This is an alternative way of logarithmic transformation compared to the examples above and is often used in individual effective dose (IED) experiments (Newman \& McCloskey 2000; Pena-Llopis et al. 2003).

Methods involving integration of the area 'under' the dose-response curve are used for better statistics as in the probit analysis (Gaddum 1933). Similar integration approaches have been employed for better statistics in dose-response relations in noise analysis of the Lorentzian type (Christensen \& Bindslev 1982; Lindemann 1984).

\section{A.13. SigmaPlot logistic equations}

A catalog of equations for the SigmaPlot software product refers to an equation as a 'non-linear logistic dose-response' equation. This SigmaPlot Logistic equation, listed under Regression Wizard-Equation Catalogue, Sigmoid Equations, is in fact the reverse Hill equation (see Section 8.2.2 and Table 10.3). Thus, some of the terminology for equations in the SigmaPlot User's guide is at variance with their use in this book.

\section{REFERENCES}

Accomazzo MR, Cattaneo S, Nicosia S \& Rovati GE. Bell-shaped curves for prostaglandin-induced modulation of adenylate cyclase: two mutually opposing effects. Eur J Pharmacol 454: 107-114, 2002.

Adair GS. The hemoglobin system. VI. The oxygen dissociation curve of hemoglobin. J Biol Chem 63: 529-545, 1925.

Allen DW, Guthe KF \& Wyman J Jr. Further studies on the oxygen equilibrium of hemoglobin. J Biol Chem 187: 393-410, 1950.

Ariëns EJ. Molecular Pharmacology. The Mode of Action of Biologically Active Compounds, Vol. I+ II, 1st ed. New York: Academic Press, 1964.

Barlow R \& Blake JF. Hill coefficients and the logistic equation. Trends Pharmacol Sci 11: 440-441, 1989.

Berenbaum MC. What is synergy? Pharmacol Rev 41: 93-141, 1989.

Berkson J. Application of the logistic function to bio-assay. J Am Statist Assoc 39: 357-365, 1944.

Bernado J \& Smith AFM. Bayesian Theory, 2nd ed., Chichester, Wiley \& Sons, 2007.

Biot JB. Introduction aux recherches de méanique chemique, dans lesquelles la lumière polarisée est employée auxiliairement comme réactif. Ann Chim Phys (3) 59: 206-345, 1860.

Blanquart C, Gonzalez-Yanes C \& Issad T. Monitoring the activation state of insulin/insulin-like growth factor-1 hybrid receptors using bioluminescence resonance energy transfer. Mol Pharmacol 70: 1802-1811, 2006.

Bohr C. Theoretische behandlung der quantitativen verhältnisse bei der sauerstoff aufnahme des hämoglobins. Zentralblatt Physiol 17: 682-688, 1904a.

Bohr C. Die sauerstoff aufnahme des genuinen blutfarbstoffes und des aus dem blute dargestellten hämoglobins. Zentralblatt Physiol 17: 688-691, 1904b.

Bohr C, Hasselbalch K \& Krogh A. Ueber einen in biologischer beziehung wichtigen einfluss, den die kohlensäuerspannung des blutes auf dessen sauerstoffbindung übt. Scand Arch Physiol 16: 402-412, 1904.

Bronnikov GE, Zhang SJ, Cannon B \& Nedergaard J. A dual component analysis explains the distinctive kinetics of cAMP accumulation in brown adipocytes. J Biol Chem 274: 37770-37780, 1999.

Brown AJ. Enzyme action. J Chem Soc 81: 373-388, 1902.

Brown HT \& Glendinning TA. The velocity of hydrolysis of starch by diastase, with some remarks on enzyme action.J Chem Soc 81: 388400, 1902.

Brown WEL \& Hill AV. The oxygen/dissociation curve of blood, and its thermodynamical basis. Proc Roy Soc B 94: 297-334, 1923.

Bublil EM \& Yarden Y. The EGF receptor family: spearheading a merger of signaling and therapeutics. Curr Opin Cell Biol 19: 124 134, 2007.

Burbridge EM, Burbridge GR, Fowler WA \& Hoyle F. Synthesis of the elements in stars. Revs Modern Phys 29: 547-650, 1957.

Camazine S, Deneubourg J-L, Franks NR, Sneyd J, Theraulaz G \& Bonabeau E. Self-organization in Biological Systems, 1st ed. Princeton, NJ: Princeton University Press, 2001. 
Changeux JP \& Edelstein SJ. Allosteric mechanisms of signal transduction. Science 308: 1424-1428, 2005.

Chapman H, Piggot C, Andrews PW \& Wann KT. Characterisation of large-conductance calcium-activated potassium channels $(\mathrm{BK}(\mathrm{Ca}))$ in human NT2-N cells. Brain Res 1129: 15-25, 2007.

Chou TC. Theoretical basis, experimental design, and computerized simulation of synergism and antagonism in drug combination studies. Pharmacol Rev 58: 621-681, 2006.

Chou TC \& Talalay P. A simple generalized equation for the analysis of multiple inhibitions of Michaelis-Menten kinetic systems. J Biol Chem 252: 6438-6442, 1977.

Chou TC \& Talalay P. Generalized equations for the analysis of inhibitions of Michaelis-Menten and higher-order kinetic systems with two or more mutually exclusive and nonexclusive inhibitors. Eur J Biochem 115: 207-216, 1981.

Christensen O \& Bindslev N. Fluctuation analysis of short-circuit current in a warm-blooded sodium-retaining epithelium: site current, density, and interaction with triamterene. J Membr Biol 65: $19-30,1982$.

Cladman W \& Chidiac P. Characterization and comparison of RGS2 and RGS4 as GTPase-activating proteins for $\mathrm{m} 2$ muscarinic receptor-stimulated G(i). Mol Pharmacol 62: 654-659, 2002.

Collins J, Mancilulli M, Hohlfeld R, Finch D, Sandri G \& Shtatland E. A random number generator based on the logit transform of the logistic variable. Comput Phys 6: 630-632, 1992.

Colquhoun D. Lectures on Biostatistics, 1st ed. Oxford: Oxford University Press, 1971.

Colquhoun D. The relation between classical and cooperative models for drug action. In: Drug Receptors. A Symposium, 1st ed., edited by Rang HP. London: MacMillan Press, 1973.

Cornish-Bowden A \& Koshland DE Jr. Diagnostic uses of the Hill (Logit and Nernst) plots. J Mol Biol 95: 201-212, 1975.

Cox RT. Algebra of Probable Inference. Baltimore, MA: Johns Hopkins University Press, 2001.

Cramer JS. Logit Models. From Economics and Other Fields, 1st ed. Cambridge: Cambridge University Press, 2003a.

Cramer JS. The Origins and Development of the Logit Model. Cambridge: Cambridge University Press, 2003b.

Davies P. The Goldilocks Enigma. Why is the Universe Just Right for Life? London: Penguin/Allen Lane, 2006.

De Lean A, Munson PJ \& Rodbard D. Simultaneous analysis of families of sigmoidal curves: application to bioassay, radioligand assay, and physiological dose-response curves. Am J Physiol 235: E97E102, 1978.

De Meyts P. Insulin and its receptor: structure, function and evolution. Bioessays 26: 1351-1362, 2004.

Dixon M and Webb EC. Enzymes. London: Longman Group Ltd, 1979.

Dobson AJ. Introduction to Generalized Linear Models, 2nd ed. London: Chapman and Hall/CRC, 2001

Edsall JT. Hemoglobin and the origins of the concept of allosterism. Fed Proc 39: 226-235, 1980 .

Ehlert FJ. Analysis of allosterism in functional assays. J Pharmacol Exp Ther 3: 740-754, 2005.

Ehlert FJ. On the analysis of ligand-directed signaling at G proteincoupled receptors. Naunyn Schmiedebergs Arch Pharmacol Feb 6; [Epub ahead of print]: 2008.

Eisner DA \& Richards DE. The interaction of potassium ions and ATP on the sodium pump of resealed red cell ghosts. J Physiol 319: 403-418, 1981.

Ferrell JE. How responses get more switch-like as you move down a protein cascade. Trend Biochem Sci 22: 288-289, 1997.

Finney DJ. Probit Analysis, 3rd ed. Cambridge: Cambridge University Press, 1971.

Ford ED. Scientific Method for Ecological Research (paperback ed.). Cambridge: Cambridge University Press, 2004.
Gaddum JH. Reports on biological standards III. Methods of biological assay depending on a quantal response. Med Res Council Spec Report No 183: 5-46, 1933.

Gaddum JH. Bioassays and mathematics. Pharmacol Rev 5: 87-134, 1953.

Garay RP \& Garrahan PJ. The interaction of sodium and potassium with the sodium pump in red cells. J Physiol 231: 297-325, 1973.

Gelman A, Carlin JB, Stern HS \& Rubin DB. Bayesian Data Analysis. Boca Raton, Chapman Hall/CRC, 2003.

Giraldo J. Empirical models and Hill coefficients. Trend Pharmacol Sci 24: 63-65, 2003.

Giraldo J, Vivas NM, Vila E \& Badia A. Assessing the (a)symmetry of concentration-effect curves: empirical versus mechanistic models. Pharmacol Ther 95: 21-45, 2002.

Goldstein RA. Emergent robustness in competition between autocatalytic chemical networks. Orig Life Evol Biosph 36: 381-389, 2006.

Gompertz B. On the nature of the function expressive of the law of human mortality, and on a new mode of determining the value of life contingencies. Philos Trans Roy Soc (Lond) 36: 513-585, 1825.

Grafen A \& Hails R. Modern Statistics for the Life Sciences, 1st ed. Oxford: Oxford University Press, 2002.

Greco WR, Bravo G \& Parsons JC. The search for synergy: a critical review from a response surface perspective. Pharmacol Rev 47: 331-385, 1995

Griffin MT, Hsu JC, Shehnaz D \& Ehlert FJ. Comparison of the pharmacological antagonism of M2 and M3 muscarinic receptors expressed in isolation and in combination. Biochem Pharmacol 65: 1227-1241, 2003.

Guldberg CM \& Waage P. Études sur les Affinités Chimiques. Programme de l'Université pour le 1er Semestre 1867. Christiania: Brøgger \& Christie, 1867.

Hatzimanikatis V, Emmerling M, Sauer U \& Bailey JE. Application of mathematical tools for metabolic design of microbial ethanol production. Biotechnol Bioeng 58: 154-161, 1998.

Heijnen JJ. Approximative kinetic formats used in metabolic network modeling. Biotechnol Bioeng 91: 534-545, 2005.

Heinrich R \& Rapoport SM. The utility of mathematical models for the understanding of metabolic systems. Biochem Soc Trans 11: 31-35, 1983.

Henri V. Lois Générales de l'Action des Diastases, 1st ed. Paris: A. Hermann, 1903.

Hill AV. The mode of action of nicotine and curari, determined by the form of the concentration curve and the method of temperature coefficients. J Physiol (Lond.) 39: 361-373, 1909.

Hill AV. The possible effects of aggregation of the molecules of haemoglobin on its dissociation curve. J Physiol 40: iv-vii, 1910.

Hill AV. The combinations of haemoglobin with oxygen and carbon monoxide. Biochem J 7: 471-480, 1913.

Hill AV. Trails and Trials in Physiology, 1st ed. London: Edward Arnold Ltd., 1965.

Hill TL. Cooperativity Theory in Biochemistry. Steady-state and Equilibrium Systems. Berlin: Springer-Verlag, 1985.

Hille B. Ionic Channels of Excitable Membranes, 3rd ed. Sunderland: Sinauer Associates, 2001.

Hofmeyr JH \& Cornish-Bowden A. The reversible Hill equation: how to incorporate cooperative enzymes into metabolic models. Comput Appl Biosci 13: 377-385, 1997.

Hofmeyr JH, Rohwer JM \& Snoep JL. Conditions for effective allosteric feedforward and feedback in metabolic pathways. Syst Biol (Stevenage) 153: 327-331, 2006.

Hornigold DC, Mistry R, Raymond PD, Blank JL \& Challiss RA. Evidence for cross-talk between M2 and M3 muscarinic acetylcholine receptors in the regulation of second messenger and extracellular signal-regulated kinase signalling pathways in Chinese hamster ovary cells. Br J Pharmacol 138: 1340-1350, 2003. 
Hosmer DW \& Lemeshow S. Applied Logistic Regression. Hoboken, NJ: John Wiley \& Sons, 2000.

Huang CY \& Ferrell JE Jr. Ultrasensitivity in the mitogen-activated protein kinase cascade. Proc Natl Acad Sci USA 93: 10078-10083, 1996

Hufner G. Ueber die verteilung des blutfarbstoffs zwischen kohlenoxyd und sauerstoff; ein beitrag zur lehre von der chemischen massenwirkung. J Pract Chem 30: 68-84, 1884.

Hufner G. Ueber das gesetz der dissociation des oxyhaemoglobins und über einige daran sich knüpfende wichtige fragen aus der biologie. Arch Anat Physiol Physiol Abt 1-27, 1890.

Jacquez JA. Compartmental Analysis in Biology and Medicine. Kinetics of Distribution of Tracer-labeled Materials, 1st ed. Amsterdam: Elsevier, 1972.

Janin J. The study of allosteric proteins. Prog Biophys Mol Biol 27: 77120, 1973.

Jardel Scientific. Table Curve 3D. Automated Surface Fitting Software, 1st ed. San Rafael, CA: Jardel Scientific, 1993.

Jarv J, Toomela T \& Karelson E. Dual effect of carbachol on the muscarinic receptor. Biochem Mol Biol Int 30: 649-654, 1993.

Jenkinson DH. Classical approaches to the study of drug-receptor interactions. In: Textbook of Receptor Pharmacology, 2nd ed., edited by Foreman JC \& Johansen T. Boca Raton, FL: CRC Press, 2003.

Jenkinson DH, Barnard EA, Hoyer D, Humphrey PP, Leff P \& Shankley NP. International Union of Pharmacology Committee on Receptor Nomenclature and Drug Classification. IX. Recommendations on terms and symbols in quantitative pharmacology. Pharmacol Rev 47: 255-266, 1995.

Jonker DM, Vermeij DA, Edelbroek PM, Voskuyl RA, Piotrovsky VK \& Danhof M. Pharmacodynamic analysis of the interaction between tiagabine and midazolam with an allosteric model that incorporates signal transduction. Epilepsia 44: 329-338, 2003.

Kacser H \& Burns JA. The control of flux. Biochem Soc Trans 23: 341366, 1995.

Karlin A. On the application of 'a plausible model' of allosteric proteins to the receptor for acetylcholine. J Theor Biol 16: 306320, 1967.

King GA. Growth of a hypercycle and comparison with conventional autocatalysis. Biosystems 13: 225-234, 1981.

Kingsland SE. Modeling Nature. Episodes in the History of Population Ecology, 2nd ed. Chicago, IL: The University of Chicago Press, 1995.

Kleinbaum DG \& Klein M. Logistic Regression, 2nd ed. New York: Springer, 2005.

Kleinbaum DG, Kupper LL, Nizam A \& Muller KE. Applied Regression Analysis and Multivariable Methods, 4th ed. Pacific Grove, CA: Duxbury Press, 2007.

Klotz IM. Numbers of receptor sites from Scatchard graphs: facts and fantasies. Science 217: 1247-1249, 1982.

Klotz IM. Ligand-receptor complexes: origin and development of the concept. J Biol Chem 279: 1-12, 2004.

Kubokawa M, Sohma Y, Hirano J, Nakamura K \& Kubota T. Intracellular Mg2 + influences both open and closed times of a native $\mathrm{Ca}(2+)$-activated $\mathrm{BK}$ channel in cultured human renal proximal tubule cells. J Membr Biol 207: 69-89, 2005.

Lage K, Karlberg EO, Storling ZM, Olason PI, Pedersen AG, Rigina O, Hinsby AM, Tumer Z, Pociot F, Tommerup N, Moreau Y and Brunak S. A human phenome-interactome network of protein complexes implicated in genetic disorders. Nat Biotechnol 25: 309-316, 2007.

Langmuir I. The adsorption of gases on plane surfaces of glass, mica and platinum. J Am Chem Soc 40: 1361-1403, 1918.

Lazareno S \& Birdsall NJ. Estimation of competitive antagonist affinity from functional inhibition curves using the Gaddum, Schild and Cheng-Prusoff equations. Br J Pharmacol 109: 1110-1119, 1993.
Lazareno S \& Birdsall NJ. Detection, quantitation, and verification of allosteric interactions of agents with labeled and unlabeled ligands at $\mathrm{G}$ protein-coupled receptors: interactions of strychnine and acetylcholine at muscarinic receptors. Mol Pharmacol 48: 362 378, 1995.

Lee DH, Severin K \& Ghadiri MR. Autocatalytic networks: the transition from molecular self-replication to molecular ecosystems. Curr Opin Chem Biol 1: 491-496, 1997.

Lee PM. Bayesian Statistics: An Introduction. London, Hodder Arnold Publications, 2004.

Levitzki A. Cooperativity in Multisubunit Proteins - The Basic Concepts, 1st ed. Berlin: Springer-Verlag, 1978.

Lindemann B. Analysis of additively contaminated Lorentzians by integration. Biophys J 46: 409-411, 1984.

Ma AW, Redka DS, Pisterzi LF, Angers S \& Wells JW. Recovery of Oligomers and Cooperativity When monomers of the $\mathrm{M}(2)$ muscarinic cholinergic receptor are reconstituted into phospholipid vesicles. Biochemistry 46: 7907-7927, 2007.

Malmstrøm BG. Cytochrome c oxidase: some current biochemical and biophysical problems. Q Rev Biophys 6: 389-431, 1974.

Malthus TR. An Essay on the Principle of Population, 2nd ed. London: St Paul's Church Yard, 1798, 1803. Reprinted by Dent \& Sons, London, 1967.

Marin JM \& Robert CP. Bayesian Core: A Practical Approach to Computational Bayesian Statistics. New York: Springer, 2007.

May LT, Avlani VA, Langmead CJ, Herdon HJ, Wood MJ, Sexton PM, et al. Structure-function studies of allosteric agonism at M2 muscarinic acetylcholine receptors. Mol Pharmacol 72: 463-476, 2007.

May RM. Simple mathematical models with very complicated dynamics. Nature 261: 459-467, 1976.

McFadden DL. http://nobelprize.org/economics/laureates/2000/ mcfadden-lecture.pdf, 2003.

McFadden DL. Economic choices. In: From Nobel Lectures, Economics 1996-2000, Editor, edited by Persson T. Singapore: World Scientific Publishing Co., 2003.

Meir E, Munro EM, Odell GM \& Von Dassow G. Ingeneue: a versatile tool for reconstituting genetic networks, with examples from the segment polarity network. J Exp Zool 294: 216-251, 2002.

Michaelis L \& Menten ML. Die kinetik der invertinwirkung. Biochem Zeitsch 49: 333-369, 1913.

Michel D. Cooperative equilibrium curves generated by ordered ligand binding to multi-site molecules. Biophys Chem 129: 284-288, 2007.

Monod J, Changeux JP \& Jacob F. Allosteric proteins and cellular control systems. J Mol Biol 6: 306-329, 1963.

Monod J, Wyman J \& Changeux J-P. On the nature of allosteric transitions: a plausible model. J Mol Biol 12: 88-118, 1965.

Motulsky H \& Christopoulos A. Fitting Models to Biological Data Using Linear and Nonlinear Regression: A Practical Guide to Curve Fitting, 1st ed. Oxford: Oxford University Press, 2004.

Muirhead H \& Perutz M. Structure of haemoglobin. A three-dimensional Fourier synthesis of reduced human haemoglobin at 55 a resolution. Nature 199: 633-639, 1963.

Nernst W. Die elektromotorische wirksamkeit der ionen. Z Phys Chem 4: 129-181, 1889

Neubig RR, Spedding M, Kenakin T \& Christopoulos A. International Union of Pharmacology Committee on Receptor Nomenclature and Drug Classification. XXXVIII. Update on terms and symbols in quantitative pharmacology. Pharmacol Rev 55: 597-606, 2003.

Newman MC \& McCloskey JT. The individual tolerance concept is not the sole explanation for the probit dose-effect model. Environ Toxicol Chem 19: 520-526, 2000.

Nowak MA. Evolutionary Dynamics. Exploring the Equations of Life, 1st ed. Cambridge, MA: The Belknap Press of Harvard University Press, 2006 
O’Connel AA. Logistic Regression Models for Ordinal Response Variables. Thousand Oaks, CA: Sage Publications Ltd., 2006.

Ostwald W. Studien zur chemischen dynamik. Zweite Abhandlung. Die einwirkung der säuren auf methylacetat. J Pract Chem 28 (136): 449-495, 1883.

Ostwald W. Lehrbuch der Allgemeinen Chemie. Zweiten Bandes Zweiter Teil: Verwandtschaftslehere - Erster Teil, Vol. 2. Leipzig: Verlag von W. Engelmann, 1902.

Pampel FC. Logistic Regression: A Primer. Sage Quantitative Applications in the Social Sciences, Vol. Series No. 132, 1st ed. Thousand Oaks, CA: Sage Publications, 2000.

Pauling L. The oxygen equilibrium of hemoglobin and its structural interpretation. Proc Nat Acad Sci USA 21: 186-191, 1935.

Pearl R \& Reed LJ. On the rate of growth of the population of the United States since 1790 and its mathematical representation. Proc Natl Acad Sci USA 6: 275-288, 1920.

Pearl R. The Biology of Death, 1st ed. Philadelphia, PA: Lippincott Co., 1922.

Peitgen H-O, Jürgens H \& Saupe D. Classical Fractals and Self-similarity, Chaos and Fractals: New Frontiers of Science, 1st ed. Berlin: SpringerVerlag, 1992.

Pena-Llopis S, Ferrando MD \& Pena JB. Fish tolerance to organophosphate-induced oxidative stress is dependent on the glutathione metabolism and enhanced by $\mathrm{N}$-acetylcysteine. Aquat Toxicol 65: 337-360, 2003.

Perutz M. X-ray analysis of hæmoglobin. Nature 149: 491-494, 1942.

Perutz M. Structure of hemoglobin. Brookhaven Symp Biol 13: 165-183, 1960.

Perutz M. Stereochemistry of cooperative effects in haemoglobin. Nature 228: 726-739, 1970.

Perutz M. Mechanisms of Cooperativity and Allosteric Regulation in Proteins, 1st ed. Cambridge: Cambridge University Press, 1990.

Perutz M, Bolton W, Diamond R, Muirhead H \& Watson HC. Structure of haemoglobin. An X-ray examination of reduced horse haemoglobin. Nature 203: 687-690, 1964.

Perutz M, Wilkinson AJ, Paoli M \& Dodson GG. The stereochemical mechanism of the cooperative effects in hemoglobin revisited. Annu Rev Biophys Biomol Struct 27: 1-34, 1998.

Pliska V. Models to explain dose-response relationships that exhibit a downturn phase. Trends Pharmacol Sci 15: 178-181, 1994.

Prentice RL. A generalization of the probit and logit methods for dose response curves. Biometrics 32: 761-768, 1976.

Quinn GP \& Keough MJ. Experimental Design and Data Analysis for Biologists, 1st ed. Cambridge: Cambridge University Press, 2002.

Ragozzino D, Woodward RM, Murata Y, Eusebi F, Overman LE \& Miledi R. Design and in vitro pharmacology of a selective gammaaminobutyric acidC receptor antagonist. Mol Pharmacol 50: 1024 1030, 1996.

Reed LJ \& Berkson J. The application of the logistic function to experimental data. J Phys Chem 33: 760-779, 1929.

Richards FJ. A flexible growth function for empirical use. J Exp Bot 10: 290-300, 1959.

Robertson TB. On the normal rate of growth of an individual and its biochemical significance. Arch Entwickmech Organ 25: 581-614, $1908 \mathrm{a}$.

Robertson TB. Further remarks on the normal rate of growth of an individual and its biochemical significance. Arch Entwickmech Organ 26: 108-118, 1908b.

Roughton FJW, Otis $\mathrm{AB} \&$ Lyster RLJ. The determination of the individual equilibrium constants of the four intermediate reactions between oxygen and sheep haemoglobin. Proc Roy Soc B 144: $29-54,1955$

Rovati GE \& Nicosia S. Lower efficacy: interaction with an inhibitory receptor or partial agonism? Trends Pharmacol Sci 15: 140-144, 1994.
Sak K, Barnard EA \& Jarv J. Dual effect of nucleotides on P2Y receptors. IUBMB Life 50: 99-103, 2000.

Samuels ML \& Witmer JA. Statistics for the Life Sciences, 3rd ed. Upper Saddle River, NJ: Prentice Hall, 2002.

Scaramellini C, Bennett G \& Leff P. Analysis of agonist-agonist interactions: the crucial influence of curve shape. J Pharmacol Toxicol Methods 37: 167-178, 1997.

Segel IH. Enzyme kinetics. Behavior and analysis of rapid equilibrium and steady-state enzyme systems. New York: Wiley \& Sons (reissued 1993), 1975.

Short TG, Ho TY, Minto CF, Schnider TW \& Shafer SL. Efficient trial design for eliciting a pharmacokinetic-pharmacodynamic model-based response surface describing the interaction between two intravenous anesthetic drugs. Anesthesiology 96: 400-408, 2002.

SigmaPlot 8. SigmaPlot ${ }^{\circledR} 9.0$ User's Manual, 1st ed. Point Richmond: Systat Software, Inc., 2004.

Sivia D \& Skilling J. Data Analysis: A Bayesian Tutorial. Oxford: Oxford University Press, 2006.

Smith JM. Mathematical Ideas in Biology, 1st ed. Cambridge: Cambridge University Press, 1968.

Snedecor GW \& Cochran WG. Statistical Methods, 6th ed. Ames, IA: The Iowa State University Press, 1967.

Sokal RR \& Rohlf FJ. Biometry. The Principles and Practice of Statistics in Biological Research, 3rd ed. New York: WH Freeman, 1994.

Sorribas A, Hernandez-Bermejo B, Vilaprinyo E \& Alves R. Cooperativity and saturation in biochemical networks: a saturable formalism using Taylor series approximations. Biotechnol Bioeng 97: 259-277, 2007

Spivak CE. Correlation among Hill parameters reflect models of activating ligand-gated ion channels. Trend Pharmacol Sci 16: 3942, 1995 .

Stoker AW. Protein tyrosine phosphatases and signalling. I Endocrinol 185: 19-33, 2005.

Svedberg T \& Fahraeus R. A new method for the determination of the molecular weights of the proteins. J Am Chem Soc 48: 430-438, 1926.

Szabadi E. A model of two functionally antagonistic receptor populations activated by the same agonist. J Theor Biol 69: 101-112, 1977.

Taleb O \& Betz H. Expression of the human glycine receptor alpha 1 subunit in Xenopus oocytes: apparent affinities of agonists increase at high receptor density. EMBO J 13: 1318-1324, 1994.

Thron CD. On the analysis of pharmacological experiments in terms of an allosteric receptor model. Mol Pharmacol 9: 1-9, 1973.

Trankle C, Dittmann A, Schulz U, Weyand O, Buller S, Johren K, Heller E, Birdsall NJ, Holzgrabe U, Ellis J, Holtje HD \& Mohr K. Atypical muscarinic allosteric modulation: cooperativity between modulators and their atypical binding topology in muscarinic M2 and M2/M5 chimeric receptors. Mol Pharmacol 68: 1597-1610, 2005.

Tucek S, Michal P \& Vlachova V. Modelling the consequences of receptor-G-protein promiscuity. Trends Pharmacol Sci 23: 171-176, 2002.

Tuk B \& van Oostenbruggen MF. Solving inconsistencies in the analysis of receptor-ligand interactions. Trend Pharmacol Sci 17: 403-409, 1996.

Turchin P. Complex Population Dynamics: A Theoretical/Empirical Synthesis. Princeton, NJ: Princeton University Press, 2003.

Van't Hoff JH. Die grenzebene, ein beitrag zur kenntniss der esterbildung. Berichte D Chem Gesellshaft 10: 669-678, 1887.

Van der Graaf PH \& Schoemaker RC. Analysis of asymmetry of agonist concentration-effect curves. J Pharmacol Toxicol Methods 41: 107115, 1999.

van Rossum JM. Receptor theory in enzymology. In: Molecular Pharmacology. The Mode of Action of Biologically Active Compounds, 1st ed., edited by Ariëns EJ. New York: Academic Press, 1964. 
van Rossum JM. Limitations of molecular pharmacology. Some implications of the basic assumptions underlying calculations on drug-receptor interactions and the significance of biological drug parameters. Adv Drug Res 3: 189-234, 1966.

Verhulst P-F. Notice sur la loi que la population suit dans son accroissement. Corrsp Math Phys 10: 113-116, 1838.

Verhulst P-F. Recherches mathématiques sur la loi d'accroissement de la population. Bruxelles, Acad Sci Mém 18: 1-41, 1845.

Verhulst P-F. Deuxième mémoire sur la loi d'accroissement de la population. Mém l'Academie Roy Sci, Lett $\mathcal{E}{ }^{\circ}$ Baux-Arts Belgique 20: 1-32, 1847.

Verveen AA \& DeFelice LJ. Membrane noise. In: Progress in Biophysics and Membrane Biology, Vol. 28, edited by Butler JAV \& Noble D. Oxford: Pergamon, 1974.

Visser D, Schmid JW, Mauch K, Reuss M \& Heijnen JJ. Optimal redesign of primary metabolism in Escherichia coli using linlog kinetics. Metab Eng 6: 378-390, 2004.

Vittinghoff E, Shiboski SC, Glidden DV \& McCulloch CE. Regression Methods in Biostatistics: Linear, Logistic, Survival, and Repeated Measures Models. New York: Springer Verlag, 2005.

Vogel WK, Mosser VA, Bulseco DA \& Schimerlik MI. Porcine m2 muscarinic acetylcholine receptor-effector coupling in Chinese hamster ovary cells. J Biol Chem 270: 15485-15493, 1995.

Voit EO. Computational Analysis of Biochemical Systems: A Practical Guide for Biochemists and Molecular Biologists, 1st ed. Cambridge: Cambridge University Press, 2000.

Weiss JN. The Hill equation revisited: uses and misuses. FASEB J 11: 835-841, 1997.
Wells JW. Analysis and interpretation of binding at equilibrium. In: Receptor-ligand Interactions. A Practical Approach, 1st ed., edited by Hulme EC. Oxford: IRL Press at Oxford University Press, 1992.

Williams BR, Barber R \& Clark RB. Kinetic analysis of agonist-induced down-regulation of the beta(2)-adrenergic receptor in BEAS-2B cells reveals high- and low-affinity components. Mol Pharmacol 58 : 421-430, 2000

Winkler RL. An Introduction to Bayesian Inference and Decision, 2nd ed. Sugar Land, TX: Probabilistic Publishing; 2003.

Witte RS \& Witte JS. Statistics, 7th ed. Hoboken, NJ: John Wiley \& Sons, Inc., 2004.

Wyman J \& Gill SJ. Binding and Linkage. Functional Chemistry of Biological Macromolecules, 1st ed. New York: Freeman, 1990.

Yifrach O. Hill coefficient for estimating the magnitude of cooperativity in gating transitions of voltage-dependent ion channels. Biophys J 87: 822-830, 2004.

Yule GU. The growth of population and the factors that control it. JRoy Statist Soc 88: 1-57, 1925.

Zhang Y, Niu X, Brelidze TI \& Magleby KL. Ring of negative charge in BK channels facilitate $\mathrm{s}$ block by intracellular $\mathrm{Mg}^{+}$and polyamines through electrostatics. J Gen Physiol 128: 185-202, 2006

Zorkadis V, Karras DA \& Panayotou M. Efficient information theoretic strategies for classifier combination, feature extraction and performance evaluation in improving false positives and false negatives for spam e-mail filtering. Neural Netw 18: 799 $807,2005$. 
Part III: Test of Tools for Data Analysis 


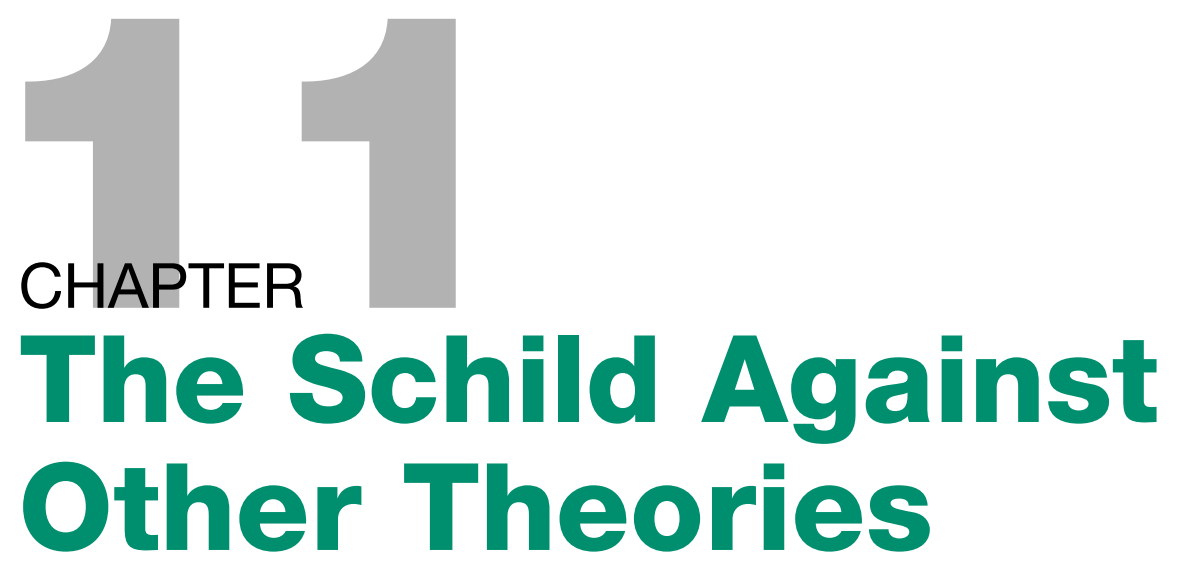

In this chapter, we shall review some of the features of null methods and, in particular, the null method of Schild that is popular in pharmacology. I argue for a reduced use of the Schild method for synagic analysis of ant-agonists in favor of other strategies.

\subsection{The Schild Analysis}

\subsubsection{General Introduction to the Schild Analysis}

Alone, due to regulatory demands imposed by evolution, biological reactions in real life are known and supposed to involve many interactions of high complexity. In functional studies of dose-responses it was realized early on that docking of metabolites on enzymes for hydrolysis and occupancy of receptors by ligands for response were followed by intricate steps controlling the effects of effector molecules. As one example, reflect on the transcription factors controlling binding sites in the DNA-string of gene promotors (Nelson \& Cox 2004) or the sophisticated interdependence in epigenetic networks (Allis et al. 2007, pp. 23-61).

In the formulation of systems with feed-forward and backward regulation, Arïens (1954) and Stephenson (1956) assembled unknown reactions together by concepts such as 'intrinsic activity' and 'efficacy'. Currently, the molecular basis for these terms are being searched and resolved with astonishing ingenuity and speed at a molecular-to-atomic scale by combining tools of electrophysiology and molecular biology (Figs. 11.1 and 11.2) (Taglialatela et al. 1992; Taglialatela \& Stefani 1993; Burzomato et al. 2004; Colquhoun \& Silvilotti 2004; Mitra et al. 2005; Paas et al. 2005; Campos et al. 2007), advanced visualization techniques such as color-overlay immunohistochemistry, fluorescence resonance energy transfer, and atomic force microscopy (Fig. 11.3) (Greenleaf et al.
2007; Zoffmann et al. 2007), structural Ångstrøm resolution by X-ray crystallography (Figs. 6.6 and $6.9 \mathrm{~A}+\mathrm{B}$ ) (MacKinnock 2004, 2005; Unwin 2005; Valiyaveetil 2006), combinatorial chemistry and recombinant DNA technology (Kellem 2006; Rowell 2006), and swift computational power in systems biology and bioinformatics employing results from genomics and proteomics (Fig. 11.4) (see, e.g., von Dassow \& Odell 2002; Alberts \& Odell 2004; Jensen et al. 2006; Olsen et al. 2006). This has led to insights into some of the basic interactions and regulatory steps even at levels of exceptionally entangled entities in the cell interior (Kodadek et al. 2005; Longabaugh et al. 2005; Schulte \& Levy 2007) as well as for clinical settings (Lage et al. 2007).

Based on a search for high affinity drugs and run partly by pecuniary addiction, a compulsive drive has haunted and is still steering many minds in the pharmaceutical industry. To determine affinity for developed drugs, reliable and accurate measures of agonist and ant-agonist equilibrium dissociation constants are essential. These constants allow for comparison and selection of lead compounds between drugs before they proceed onto preclinical and clinical trials, and eventually become medicaments for sale. In addition, these affinity constants are valuable for receptor subtype identification and classification.

Of late, the experimental methods on ant-agonists have changed into high throughput testing on functional rather than binding systems (Kenakin 2003), and into use of allosteric modulators in addition to classical ant-agonists (Birdsall \& Lazareno 2005; Kenakin 2005; May et al. 2007) also analyzing time-dependent events (Paton \& Rang 1965; Wreggett \& Wells 1995; Lew et al. 2000; Avlani et al. 2004; Kenakin et al. 2006; Mathiesen et al. 2006), while the theoretical evaluation of affinity constants for ant-agonists at equilibrium is still performed by the Schild method. The Schild method has 


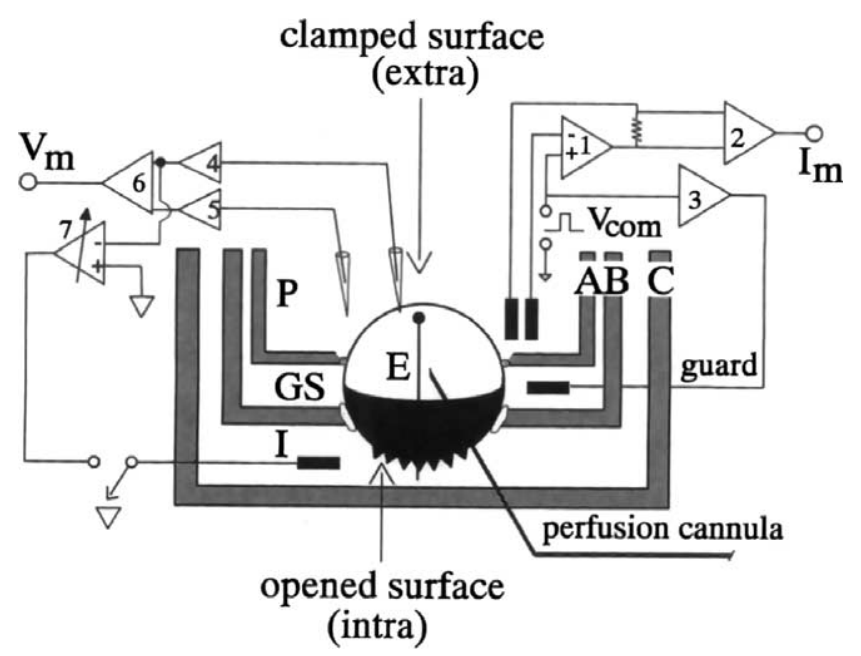

Figure 11.1. The cut-open expression system. Functionality of effectors involved in possible potential changes and expressed in the egg surface-membrane of Xenopus Laevis (African frog) may be studied dynamically at the molecular level by site-directed mutagenesis (Taglialatela et al. 1992, Fig. 1) with permission.

also mutated into the determination of parameters for non-competitive ant-agonists (Ehlert 2005), as well as for the allosteric modulators and combined with analysis in the genuine allosteric two-state model (ATSM) described in Chapter 7 (Hall 2000; Kenakin 2002; May et al. 2007).

In the drug hunt, when it comes to assessing and quoting equilibrium affinity constants for ant-agonistic drugs $\mathrm{pA}_{2} \mathrm{~s}$, Schild's method has achieved an aura of the ultimate truth (Kenakin 1997, p. 336; Wyllie \& Chen 2007) and is continuously recommended (Neubig et al. 2003; Colquhoun 2007).

\subsubsection{Null Methods - Comparison of Equi-active Responses in Schild Analysis}

In order to extract synagic parameters for potential drugs, one way to proceed is to equate identical activities, and without knowledge of all the intermediate processes, arrange that these processes cancel out in the formulation. Such manipulations are known as 'null methods' or ratio methods. The idea is to record an effect $\left(\mathrm{Eff}_{1}\right)$ which is a function of, let us say, concentrations of an agonist and an ant-agonist at one level, Eff $_{1}=f\left(\mathrm{~S}_{1}, K_{\mathrm{ss}}, \mathrm{I}_{1}, K_{\mathrm{si}}\right.$, [complex steps] $)$, and by taking the ratio with another effect $\left(\mathrm{Eff}_{2}\right)$ obtained at a different concentration level of agonist and ant-agonist, but reaching the same effect as $\mathrm{Eff}_{1}, \mathrm{Eff}_{2}=f\left(\mathrm{~S}_{2}, K_{\mathrm{ss}}, \mathrm{I}_{2}\right.$, $K_{\text {si }}$, [complex steps]), and let the function of intermediate steps, $f\left(\right.$ [complex steps]) for Eff $_{1}$ and Eff Cancel, as $_{2}$ they are matched to be identical in the two situation.

Based on this idea, we can formulate the following equation:

$$
\frac{\mathrm{Eff}_{1}}{\mathrm{Eff}_{2}}=\frac{f\left(\mathrm{~S}_{1}, K_{\mathrm{ss}}, \mathrm{I}_{1}, K_{\mathrm{si}},[\text { complex steps }]\right)}{f\left(\mathrm{~S}_{2}, K_{\mathrm{ss}}, \mathrm{I}_{2}, K_{\mathrm{si}},[\text { complex steps] })\right.}=1,
$$

in which $K_{\mathrm{ss}}$ and $K_{\mathrm{si}}$ are the two equilibrium dissociation constants for agonist $\mathrm{S}$ and inhibitor I at a common site of the receptor/enzyme for an initial or dominant process in the chain of reactions.

From a recording of $\mathrm{Eff}_{1}$ and $\mathrm{Eff}_{2}$ as identical, equiactive, and the knowledge of agonist and ant-agonist concentrations in situation 1 and 2, we may determine $K_{\mathrm{ss}}$ and $K_{\mathrm{si}}$, the system parameters we seek. It turns out that for simple competitive kinetics, $K_{\mathrm{ss}}$ cancels out by the ratio-null method and a determination of $K_{\mathrm{si}}$ may be reached, see the derivation below. This was our primary goal.

Gaddum and Clark worked with null methods in the 1920s and 1930s for receptor reactions in the presence of both agonists and ant-agonists (Gaddum 1926, 1937; Clark 1926, 1933, 1937). Ten years later, their ideas for determining an ant-agonist dissociation constant $K_{\mathrm{B}}$ were further developed and substantiated by Schild's affinity measure, $\mathrm{pA}_{x}$ (1947). Over the next decade, related conceptions were developed and presented, some in collaboration with Arunlakshana (Schild 1949; Arunlakshana \& Schild 1959). Today, this body of theory is referred to as 'The Schild method'. Schild's method is a null method, ${ }^{1}$ and by definition based on competitive ant-agonism (see Chapter 2).

Null methods are of course also invoked in today's means for disentangling complex interactions as, for instance, proteome - transcriptome correlations (e.g., Castrillo et al. 2007, and additional document 7), but that is another story.

\subsubsection{Schild's $p A_{\mathrm{x}}$ in Schild Analysis}

The Schild yields the so-called $\mathrm{pA}_{x}$ value, where ' $\mathrm{p}$ ' is equal to the negative $\log$ and taken of the ant-agonist concentration ' $A$ ', needed to displace an agonist-doseresponse curve obtained in the absence of the antagonist by a factor ' $x$ ' to the right on the agonist concentration axis (Figs. 11.5 and 11.6). Factor $x$ is equal to the subscript $x$ of $\mathrm{pA}_{x}{ }^{2}$ Note that the use of ' $\mathrm{A}$ ' as a symbol in Schild's analysis for an ant-agonist matches the use of 'I' for an ant-agonist throughout this book. Thus, $\mathrm{pA}_{x}$ is equal to $\mathrm{pI}_{x}$.

\footnotetext{
1 Another null method is the Ussing-flux ratio, well-known to transport physiologists, which was developed at the same time as the Schild method (Ussing 1949).

${ }^{2}$ According to Schild (1947) ' $\mathrm{pA}_{x}$ is defined as the negative logarithm to base 10 of the molar concentration of an antagonistic drug which will reduce the effect of a multitude dose $(x)$ of an active drug to that of a single dose'.
} 
A

$K_{\mathrm{R}}=520 \mu \mathrm{M} \quad K_{\mathrm{R}}=520 \mu \mathrm{M} \quad K_{\mathrm{R}}=520 \mu \mathrm{M}$

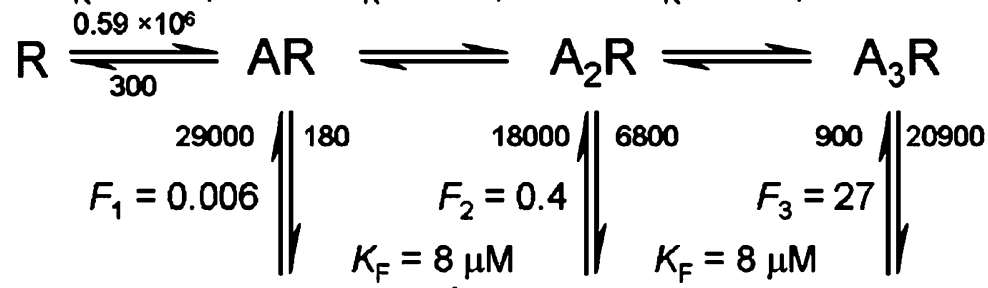

$\mathrm{AF} \stackrel{150 \times 10^{\circ}}{\stackrel{1200}{\rightleftharpoons}} A_{2} F \rightleftharpoons A_{3} F$

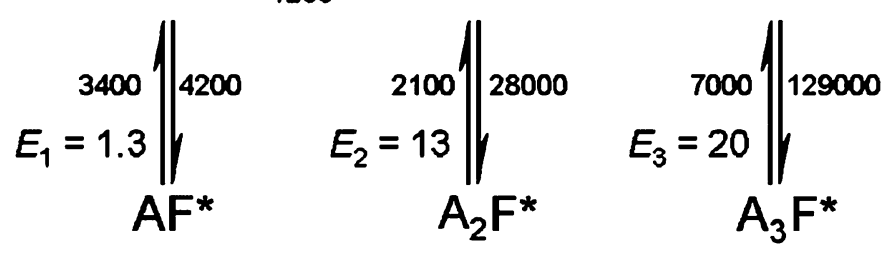

B

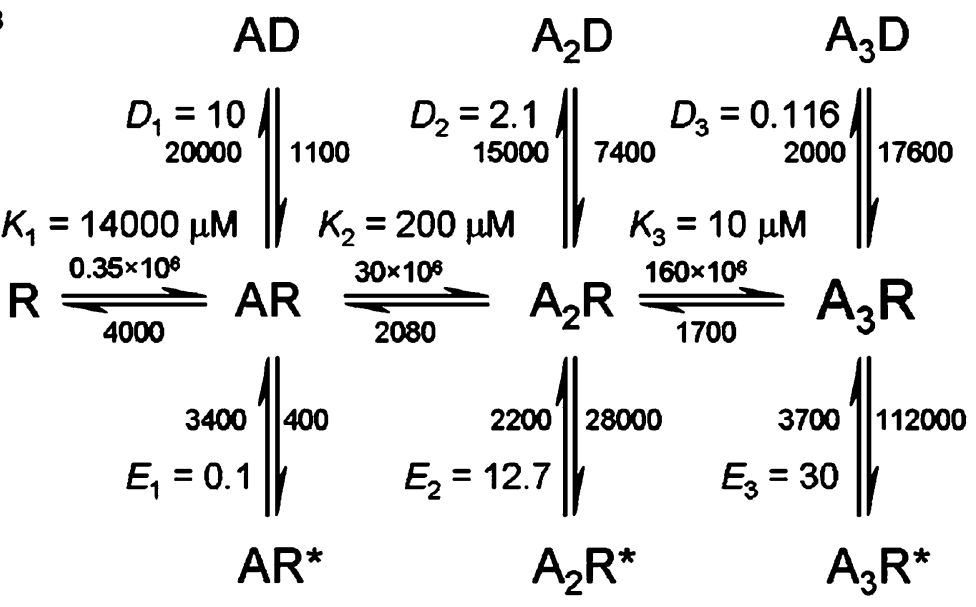

Figure 11.2. Electro-physiological determination of 18 model parameters. From Burzomato et al. (2004, Fig. 12) with permission.

It is important to differentiate between Schild's 'A' and ' $A$ ' for an association constants. When the agonist dose-response curve in the presence of an ant-agonist ' $A$ ' is displaced by a factor of 2 to the right on the agonist concentration axis $(x=2)$, compared to in the absence of an ant-agonist, then the term $\mathrm{pA}_{2}$ is identical to the $\log$ of an ant-agonist equilibrium association constant, the $\log A_{\mathrm{si}}$ (Fig. 11.7A). Why? Because the mathematical operator 'p' switches a negative logarithmic dissociation constant to a positive logarithmic association constant. As $\mathrm{pA}_{2}$ or $\mathrm{pI}_{2}$ is the same as $\mathrm{p} K_{\mathrm{si}}$, and often referred to as $\mathrm{p} K_{\mathrm{B}}$, it is also equal to the reciprocal logarithmic association constant, the $\log A_{\mathrm{si}}$, where ' $A$ ' is an association constant. Thus, $\mathrm{p} K_{\mathrm{B}}=\log A_{\mathrm{si}}$.

Let us scrutinize the Schild analysis and its aura.

\subsubsection{Aim of the Schild Analysis}

The objective of the Schild analysis is to obtain affinity constants (reciprocal equilibrium dissociation constants) for ant-agonists in functional studies. Studies of

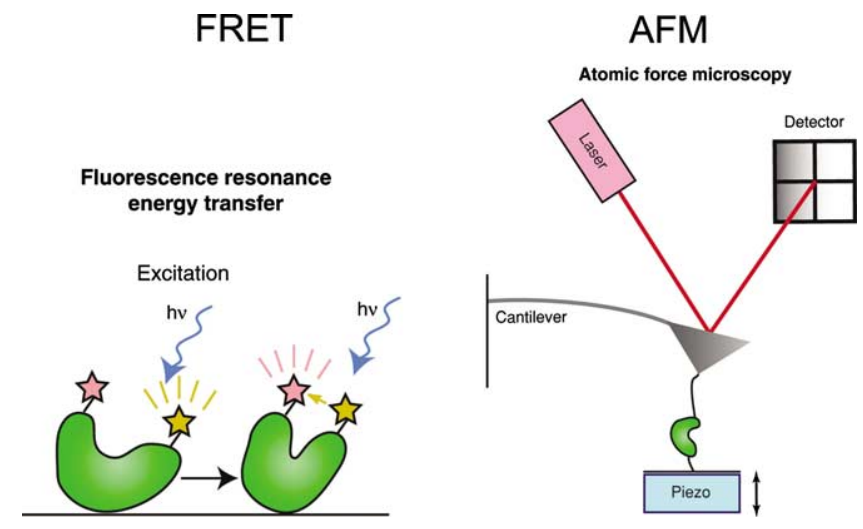

Figure 11.3. Principle diagrams for fluorescence resonance energy transfer (FRET) and atomic force microscopy (AFM). Taken from Greenleaf et al. (2007, Figs 2 and 7) with permission.

functionally active systems inherently have efficacies regulated through underlying added complexing factors compared to studies of plain association-dissociation interactions; plain association-dissociation interactions as often visualized primarily for binding experiments. 


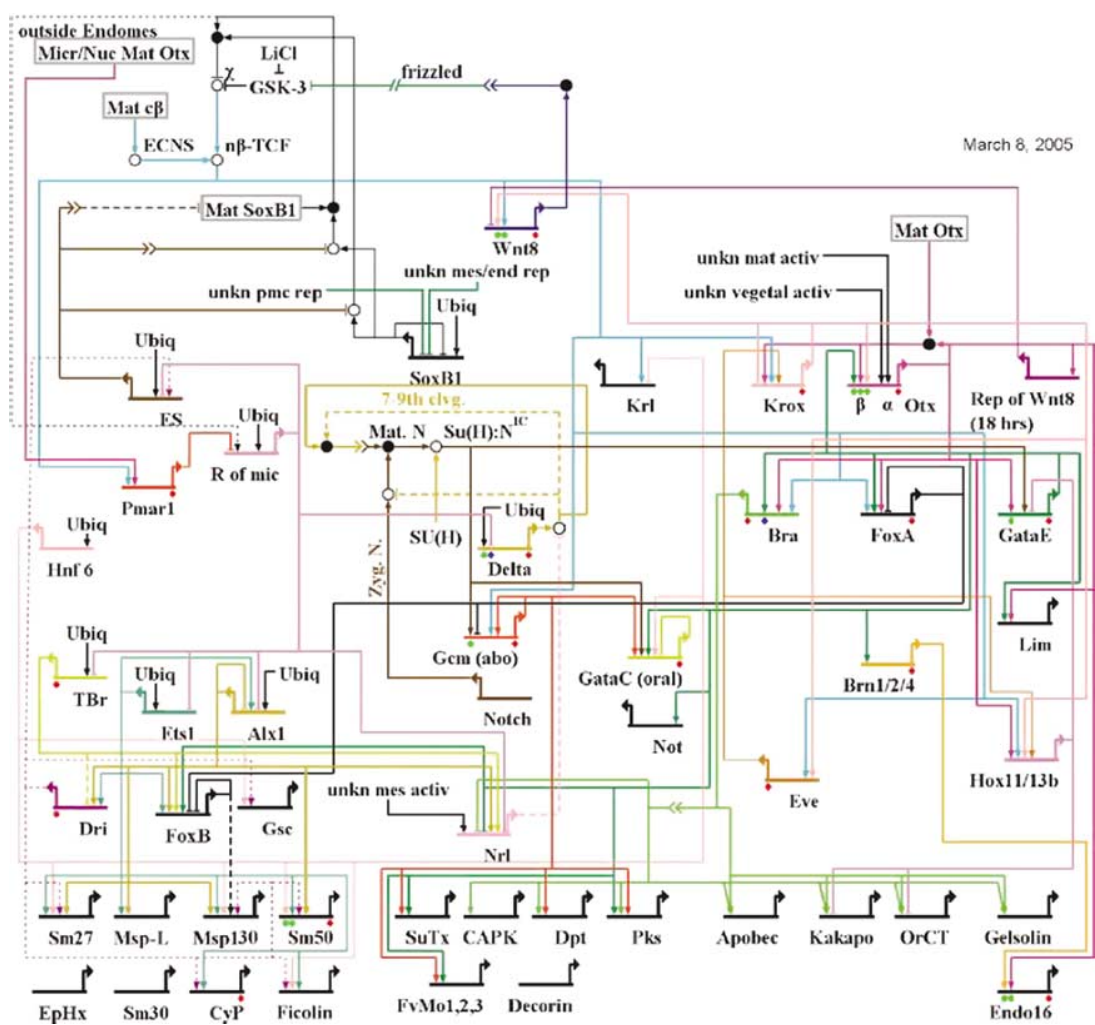

Figure 11.4. Network analysis and molecular biology combined. From Longabaugh et al. (2005, Fig. 1) with permission.

\subsubsection{Limits and Applicability of the Schild Analysis}

Schild analysis requires functional agonist/ant-agonist receptor interactions that follow simple competitive synagics (Section 2.3.1 and Fig. 2.4):

Competitive type I $\mathrm{RI} \rightleftharpoons \mathrm{I}+\mathrm{R}+\mathrm{S} \rightleftharpoons \mathrm{RS} \rightarrow$ signal Competitive type II $\mathrm{IR} \rightleftharpoons \mathrm{I}+\mathrm{R}+\mathrm{S} \rightleftharpoons \mathrm{RS} \rightarrow$ signal

However, does that mean that the 'Schild' may be implemented only in:

1. studies of simple competitive ant-agonism with no mutual binding, and only in

2. experimental setups of functional studies?

Ad 1: 'Simple competitive synagics' include the following: (i) no diffusion-limited access of ligands to the receptor, (ii) equilibrium for ligand/receptor association, this being a faster process than the ensuing activation of the receptor/effector complex, (iii) only one receptor type present, ${ }^{3}$ (iv) no receptor reserve, (v) ligands are mono-molecular agents, (vi) there is no reuptake or enzymatic elimination of the ligands, and (vii) no interventory or modulatory effects (negative or

\footnotetext{
${ }^{3}$ Colquhoun (2007) recently demonstrated that the Schild method is valid even if the single receptor has two or more identical binding sites.
}

positive) at or between receptor entities by the antagonists. The Schild will reveal a deviant behavior by a non-linearity of the Schild curve and a slope of the Schild curve that is not unity, should one of the seven assumptions above be violated. The type of skewedness by the Schild curve may provide hints on which aberrant conditions are involved (see Section 11.2.5), however the Schild analysis per se will not yield a purposeful suggestion of what to do next, even in the case of a simple non-competitive synagics.

Therefore, Schild is for simple-competitive synagics. The Schild analysis is derived from competitive synagics, and normally only reactions of this type are adoptable to the Schild. The Schild can only yield meaningful values for equilibrium affinity constants from reaction schemes of competitive ant-agonism type I: $1 / K_{\mathrm{s}}$, or type II: $1 / K_{\mathrm{ii}}$.

Ad 2: The Schild analysis is meant to be implemented in functional studies with complex efficacy steps in order to extract genuine equilibrium dissociation constants for ant-agonist binding. Meanwhile, nothing prevents Schild's method from being used in binding studies as well, as long as the ant-agonist does not change the conformation of involved receptive units. So much so, with the realization that there may be an 'intrinsic activity' in binding studies as well, where rather localized and delimited effects takes place directly related to the receptive molecule itself - selfinduced control mechanisms. Examples of this may be based on the existence of two or more spontaneous states 

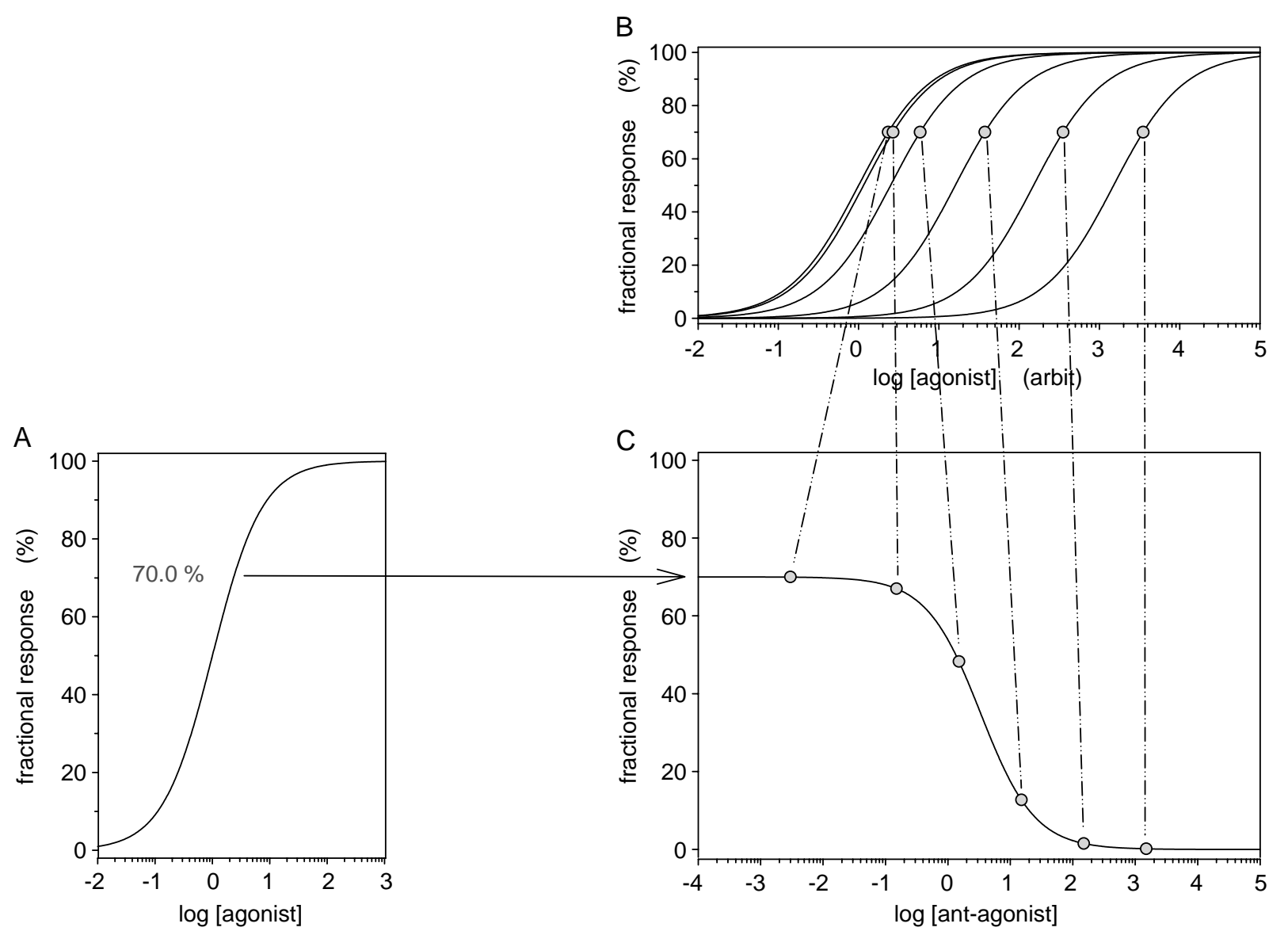

Figure 11.5. Simple Schild analysis with equi-active levels at $70 \%$ effect. (A) Simple agonism. (B) Schild analysis with five fixed doses of ant-agonist, from 0.15 to 1500 in steps of a factor 10 moving the simple agonism curves to the right. (C) The comparable inhibition curve for the five fixed doses of ant-agonist in panel B. Arbitrary concentration axes.

in a protein (Volkman et al. 2001). Another example is a $\mathrm{G}$ protein binding to or dissociating from its receptor, thereby changing the receptors affinity for the ligand (Baker \& Hill 2007). Another example is intrinsic desensitization, where binding of the primary ligand to its receptor elicits a molecular conformational change per $s e$ or due to a potential difference as the driving force affecting the receptor or an effector and is recorded as a gating current (Almers 1978; Galzi et al. 1998; Andreeva et al. 2006; Ben-Chaim et al. 2006; Campos et al. 2007; Moore et al. 2007; see also Chapter 3 on auto-intervention and Chapter 15 on auto-modulation). Some of these complicating conditions are meant to be eliminated by the Schild method when assessing the affinity for genuine competitive ant-agonists.

Note in passing, the term 'functional studies' involving ant-agonists is not to be confounded with the term 'functional ant-agonism', which is an interaction beyond the receptor at a point in the chain of events (Pratt \& Taylor 1990, p. 61; Kenakin 1993, pp. 238-239, 1997, pp. 277-279; Lutz \& Kenakin 1999, p. 165), and in the studies of whole organisms also termed 'physiological ant-agonism' (for a definition of the latter, see Rang et al. (2007, p. 19) and Bourne \& and von Zastrov 2007, p. 16). Another ant-agonism is 'chemical ant-agonism' which is seen when two drugs combine and become non-reactive (Rang et al. 2007, p. 19).

\subsubsection{Derivation of the Schild}

To simplify matters, I shall only work with the type I form of competitive ant-agonism with its dissociation constant $K_{\mathrm{si}}$ (see Section 11.1.5). In order to obtain the $K_{\mathrm{s}}$, the idea in Schild's formulation is to look for concentrations of an agonist $\mathrm{S}$, in the presence of an ant-agonist $\mathrm{I}$, that elicit a response $R$, identical to another response $R^{\prime}$, for an agonist concentration $\mathrm{S}^{\prime}$, in the absence of an ant-agonist (Figs. 11.5 and 11.6). In other words, we need to increase an agonist concentration from $S^{\prime}$ to a new level $S$ in order to obtain the response $R$, identical to the one we had before adding the ant-agonist with response level $R$. The new response $R$ should equal $R^{\prime}$. 


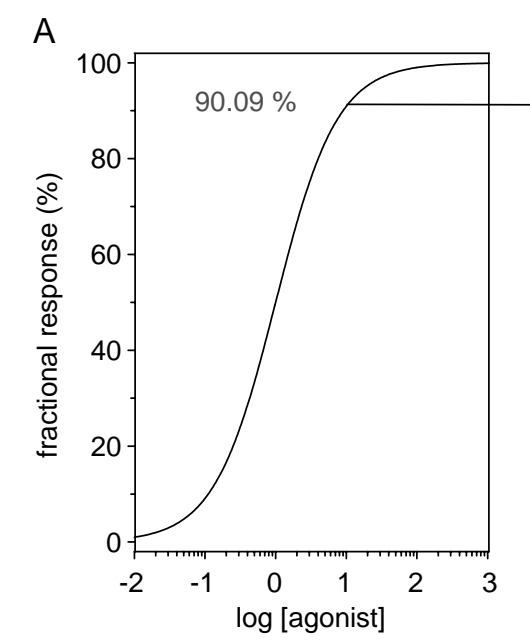

B
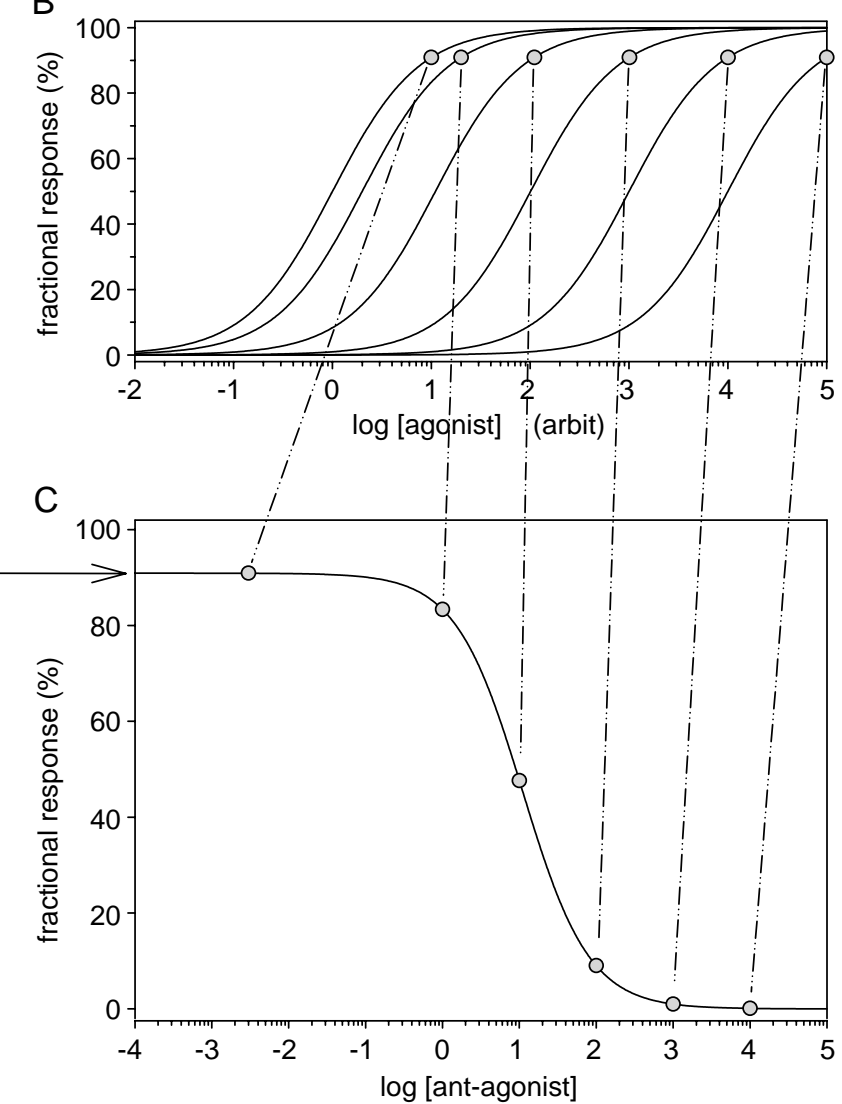

Figure 11.6. Simple Schild analysis with equi-active levels at $90.1 \%$ effect. (A) Simple agonism. (B) Schild analysis with five fixed doses of ant-agonist, from 1 to 10,000 in steps of a factor 10 moving the simple agonism curve to the right. (C) The comparable inhibition curve for the five fixed doses of ant-agonist in panel B. Arbitrary concentration axes.

In mathematical terms for simple competitive synagics we have:

$$
\begin{aligned}
& R^{\prime}=\frac{\mathrm{S}^{\prime} / K_{\mathrm{ss}}}{1+\mathrm{S}^{\prime} / K_{\mathrm{ss}}} \quad(\text { ant-agonist absent }) \\
& R=\frac{\mathrm{S} / K_{\mathrm{ss}}}{1+\mathrm{S} / K_{\mathrm{ss}}+\mathrm{I} / K_{\mathrm{si}}} \text { (ant-agonist present), }
\end{aligned}
$$

see Section 2.3.9 in which $\mathrm{S}^{\prime}$ and $\mathrm{S}$ are agonist concentrations, $K_{s \mathrm{~s}}$ is the agonist dissociation constant at the primary site, $R^{\prime}$ is the fractional effect due to an agonist in the absence of an ant-agonist, I is the antagonist concentration, $K_{\mathrm{si}}$ is the ant-agonist dissociation constant at the primary site, and $R$ is the fractional effect due to an agonist concentration $\mathrm{S}$ in the presence of an ant-agonist concentration I. In the right-hand expression of Eq. 11.2, the agonist concentration is allowed to vary freely, thus for a certain $\mathrm{S}$ we may obtain the response $R$ (Eq. 11.2).

Symbols with an apostrophe are terms in the absence of an ant-agonist, while symbols without an apostrophe are signifiers in the presence of an ant-agonist.
By setting $R^{\prime}=R$ and reversing the two fractions in Eq. 11.2 we obtain:

$$
\frac{1+\mathrm{S}^{\prime} / K_{\mathrm{ss}}}{\mathrm{S}^{\prime} / K_{\mathrm{ss}}}=\frac{1+\mathrm{S} / K_{\mathrm{ss}}+\mathrm{I} / K_{\mathrm{si}}}{\mathrm{S} / K_{\mathrm{ss}}} .
$$

Invoking the subtraction rule for fractions ${ }^{4}$ yields:

$$
\frac{1}{\mathrm{~S}^{\prime} / K_{\mathrm{ss}}}=\frac{1+\mathrm{I} / K_{\mathrm{si}}}{\mathrm{S} / K_{\mathrm{ss}}},
$$

which, after eliminating $K_{\mathrm{ss}}$ from both denominators, may be rewritten to:

$$
\frac{\mathrm{S}}{\mathrm{S}^{\prime}}-1=\frac{\mathrm{I}}{K_{\mathrm{si}}} .
$$

$\mathrm{S} / \mathrm{S}^{\prime}$ is also called the dose-ratio $(D R)$ (Gaddum et al. 1955; Gaddum 1957). Based on studies carried out by

${ }^{4}$ The reader is reminded of a simple subtraction rule for two equal fractions that many of us learned in high school. It states that you can subtract the denominator from its nominator if you do it on both sides of the equal sign - equal to an inverse regula detri $1 \mathrm{~b}$ in Box 1.1:

$$
\frac{1+a}{a}=\frac{Z+b}{b} \rightarrow \frac{1}{a}=\frac{Z}{b}
$$



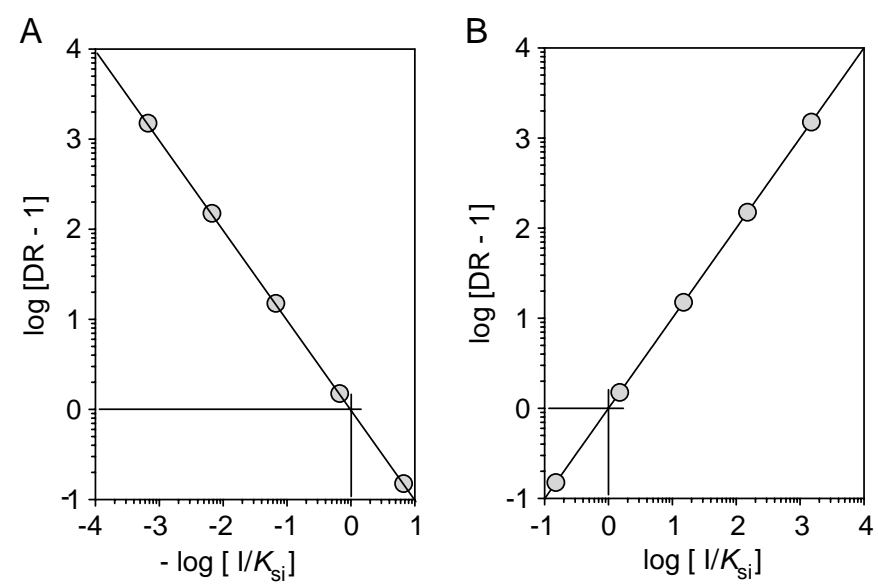

Figure 11.7. A Schild plot and a Schild regression. Based on data from Fig. 11.5. The graph in panel $A$ is a Schild plot. The graph in panel $B$ is a Schild regression. For a $\log (D R-1)=0$ the $K_{\mathrm{si}}$ may be read of the $x$-axis (negative logarithm in panel A) as indicated by horizontal and vertical lines. In panel $A$ this is a direct $\log$-measure of the ant-agonist's affinity, $\mathrm{p} K_{\mathrm{si}}=\mathrm{pA}_{2}=\log A_{\mathrm{si}}$.

Gaddum (1926) and Clark (1926), this ratio was first introduced by Gaddum (1937) and Clark and Raventos (1937) and may be derived from simple competitive synagics, as demonstrated above.

Taking the $\log$ on both sides of Eq. $11.5 a$ yields the Schild equation:

$$
\log (D R-1)=\log (\mathrm{I})-\log \left(K_{\mathrm{si}}\right) .
$$

See Fig. 11.7 for plots of Eq. 11.5b.

In case two molecules of the same drug bind to a single acceptor, the formulation will require a power of 2 for the ligand concentration (Hill 1910; Langmuir $1917,1918)$. In the analyses of Clark (1933) and Gaddum (1937, 1943), and later in Schild's analysis (Arunlakshana \& Schild 1959), the ant-agonist concentration term on the right-hand side in Eq. 11.5 was tentatively raised to a power, here designated $n_{\mathrm{i}}$. In raising the activating ligand concentration to a power $n_{\mathrm{s}}$, we may write:

$$
\left(\frac{\mathrm{S}}{\mathrm{S}^{\prime}}\right)^{n_{\mathrm{s}}}-1=\left(\frac{\mathrm{I}}{K_{\mathrm{si}}}\right)^{n_{\mathrm{i}}},
$$

which means that either auto-intervention and cooperativity as well as possible intervention or heterotropic allosteric effects are provisionally taken into account, but now for drugs operating at both primary and non-primary sites. Meanwhile, it is unclear exactly how this is to be conceived under the Schild-assumption of competitiveness at sites with mutual exclusive binding in both type I and type II competitive ant-agonism.

Inserting dose-ratio $D R$ for $\mathrm{S} / \mathrm{S}^{\prime}$ and taking the $\log$ on both sides of this particular Schild function in Eq. 11.6, see also Giraldo et al. (2007), yields a linearized form:

$$
\log \left(D R^{n_{\mathrm{s}}}-1\right)=n_{\mathrm{i}} \log (\mathrm{I})-n_{\mathrm{i}} \log \left(K_{\mathrm{si}}\right),
$$

where $n_{\mathrm{i}}$ is called the 'Schild slope' (Pratt \& Taylor 1990 , pp. 63-64; Kenakin 2004, pp. 95-104).

Eq. 11.7 is the base for a 'powered' Schild analysis, and a plot of the relation in Eq. 11.7 is shown in Fig. 11.8.

Since $\mathrm{S} / \mathrm{S}^{\prime}-1$ (Eq. 11.5) is the same as $\left(\mathrm{S}-\mathrm{S}^{\prime}\right) / \mathrm{S}^{\prime}$, it resembles a logit expression in reverse when both sides of Eq. 11.5 are logarithmic (see Section 10.2). However, $\log (D R-1)$ or $\log \left(\left(\mathrm{S}-\mathrm{S}^{\prime}\right) / \mathrm{S}^{\prime}\right)$ are not logit expressions.

\subsubsection{Performing the Schild Analysis}

Gaddum worked with dose proportions for the active drug adrenalin obtained from equi-active responses at increasing concentration of the ant-agonist ergotamine compared with no ant-agonist present. Gaddum drew a straight-line relationship between these proportions against the incrementing ant-agonist concentrations ' $D R$ ' $\propto[\mathrm{I}]$ (Gaddum 1926: Fig. 6), while this relationship was later changed to $D R-1 \propto[\mathrm{I}]$ (Gaddum 1937).

The Schild analysis is usually performed by producing first a dose-response curve for an agonist to be used during the analysis in the absence of an ant-agonists-tobe-characterized, and then in the presence of several fixed concentrations of the ant-agonist (Figs. 11.5 and 11.6). Besides the clean agonist dose-response curve, a proper Schild analysis requires a determination of at least three agonist dose-responses, each at a fixed antagonist concentration. The range of fixed ant-agonist concentrations should span at least an order of magnitude in order to judge the linearity and slope of the Schild curve (Fig. 11.7). The obtained agonist doseresponses must be parallel (Figs. 11.5 and 11.6). If this is not the case, the recommendation by Schild in 1949 was to employ as wide a concentration range for the antagonist as possible but only employing $50 \%$ activation by the agonist (Schild 1949). Ten years later, Arunlakshana and Schild (1959) had a different solution to the analysis of non-parallel dose-response curves. Their solution is not recommended.

\subsubsection{A Schild Analysis}

Figures 11.5 and 11.6 demonstrate a Schild analysis where several fixed concentrations of one ant-agonist have been employed. For practical reasons, the axis for agonist concentrations was chosen relative to the agonist dissociation constant $K_{\mathrm{ss}}{ }^{5}$ In the examples, an equiactivation effect of about 70 or $91 \%$ of the maximal

\footnotetext{
${ }^{5}$ If the Schild analysis is carried out properly, the $K_{\mathrm{ss}}$ for the agonist will be known, such that the assumption about pure competitiveness is ascertained for the particular agonist-ant-agonist pair to be characterized by the analysis.
} 

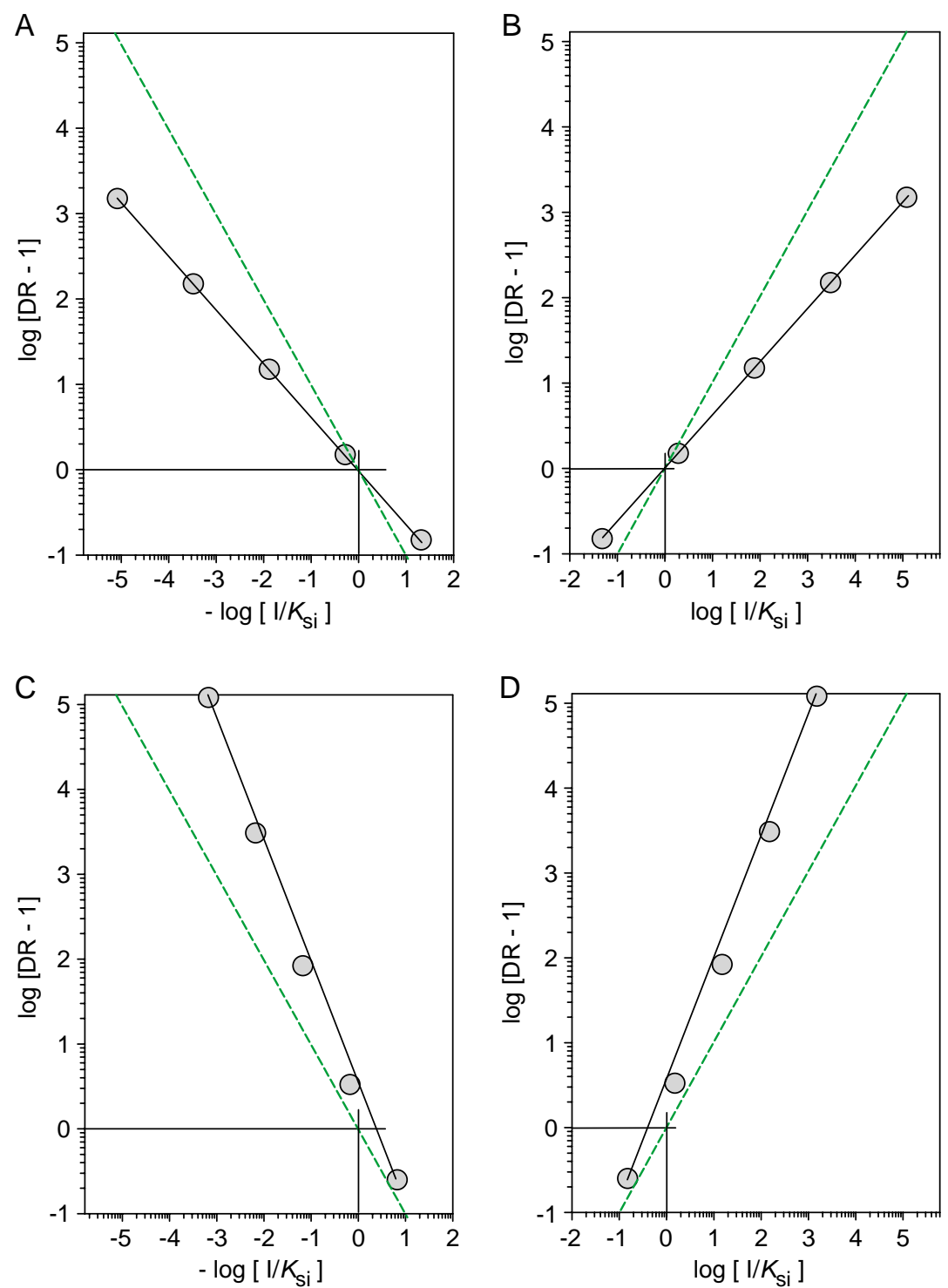

Figure 11.8. Examples of 'powered' Schild regression. Data points generated according to Eq. 11.7 based on data in Fig. 11.5, competitive antagonism. Panel A is a Schild plot and panel B is a Schild regression for the ant-agonist concentration [I], raised with a power 1.6 in both $\left(n_{\mathrm{i}}=1.6\right)$. The affinity measure is unperturbed while the slope deviates. Stippled lines are for [I] with power coefficient of unity. Panel $C$ is a Schild plot and panel $D$ is a Schild regression for the dose-ratio raised with a power 1.6 in both $\left(n_{\mathrm{s}}=1.6\right)$. Slope and affinity deviate form ideality. Stippled lines are for $D R$ with power coefficient of unity. Normalized concentration axes.

effect was arbitrarily selected as the level for comparison, and five ant-agonist concentrations were used, $\mathrm{I}_{1}, \mathrm{I}_{2}, \mathrm{I}_{3}$, $\mathrm{I}_{4}$, and $\mathrm{I}_{5}$. Each ant-agonist concentration was fixed during the determination of its pertaining agonist doseresponse relation, and each fixed concentration displaced the single agonist dose-response curve by a constant factor (Figs. 11.5 and 11.6). The new agonist concentrations, $\mathrm{S}_{1}, \mathrm{~S}_{2}, \mathrm{~S}_{3}, \mathrm{~S}_{4}$, and $\mathrm{S}_{5}$, corresponding to either $70 \%$ in Fig. 11.5 or $91 \%$ effect in Fig. 11.6 are read off the $x$-axis. Each of these increasing agonist concentrations, $\mathrm{S}_{70}$ or $\mathrm{S}_{91}$, relative to the concentration of agonist in the absence of any ant-agonist, $\mathrm{S}^{\prime}$, also eliciting either 70 or $91 \%$ effect, yields a $D R$. The obtained two sets of five DRs may be plotted against the five fixed ant-agonist concentrations employed for each agonist dose-response curve. For $70 \%$ this is shown in Fig. 11.7.

\subsubsection{Plots from the Schild Analysis}

The Schild function in Eq. $11.5 b$ may be plotted as illustrated in Fig. 11.7 based on values derived from the Schild experiment depicted in Fig. 11.6. In Figs. 11.5 and 11.6, the ant-agonist concentration axis has a scale relative to the dissociation constant for the ant-agonist used for constructing the figure. Since a determination of the parameter $K_{\mathrm{si}}$ is normally the aim of the whole 
analysis $-K_{\mathrm{si}}$ is the unknown - a relative concentration of the ant-agonist in Figs. 11.5 and 11.6 is not the smartest choice from a pedagogical point of view. On the other hand, by making this choice clear to the reader, such relative values should not disturb the understanding of how the 'Schild' works.

The ant-agonist concentration I, which forces a doubling of the agonist concentration (equal to a dose ratio of $2, D R=2$ ) in order to render the same effect as before the addition of any ant-agonist, is the dissociation constant of the ant-agonist, the $K_{\mathrm{si}}$ (Fig. 11.7), since for a dose-ratio $D R=\mathrm{S} / \mathrm{S}^{\prime}$ equal 2 , the $\log$ of $(D R-1)$ at the left side of Eq. 11.7 is $=0$. This means that $\log \left(K_{\mathrm{si}}\right)=$ $\log (\mathrm{I})$, and thus $\mathrm{I}=K_{\mathrm{si}}$.

In double-log plotting the Schild relation from Eq. 11.7, where the $\log ([$ concentration] $)$ on an absolute scale yields negative values, since realistic concentrations are in the sub-molar range, the scaling is prevented from being negative by invoking 'p', the negative log scale, just as for $\mathrm{pH}$ values. ${ }^{6}$ Such plots of Schild's analysis with pI for the ant-agonist concentration axis is called a 'Schild plot' (Fig. 11.7A), whereas plots of Schild with the ordinary log axis are 'Schild regressions' (Fig. 11.7B) (Pratt \& Taylor 1990, p. 63) with a direct estimate of affinity on a log-scale (Fig. 4.4).

For $\log (D R-1)=0$ in the two plots of Fig. 11.7 the related $\log ([$ concentration] $)$ of the ant-agonist is zero, which means that $\mathrm{I}$ is sat to 1 . As already mentioned, the reason is that the ant-agonist concentration axis is given relative to the ant-agonist dissociation constant $K_{\mathrm{si}}$. The chosen dimension of the $x$-axis is I/ $K_{\mathrm{si}}$. In real life, the $x$-axis in Fig. 11.7 will be in absolute concentration measures, since $K_{\mathrm{si}}$ is the unknown.

\subsection{To Schild or Not to Schild}

\subsubsection{Inhibition Curve Experiments Versus the Schild Analysis}

In the following two sub-sections, I shall argue that nonlinear fitting to experimental data from a single inhibition curve obtained by an ant-agonist or an interventor is more simple and, at a minimum, as informative as performing a Schild analysis. Also see the discussion of non-reciprocal versus reciprocal analysis in Chapter 8.3 and the

\footnotetext{
${ }^{6}$ To this author the pH scale introduced by Sørensen (1909) is an unfortunate and illogical choice, since $\mathrm{pH}$ falls as the concentration of protons increase. When it comes to handling sub-molar concentrations of ions other than $\mathrm{H}^{+}$, paradoxically we have no problem not involving a pscale. However, contrary to the $\mathrm{pH}$ concept, it is recommended to use the Schild plot and its p-scale instead of a Schild regression, since affinity measures are reciprocal to dissociation constants. Thus the $\mathrm{pA}_{x}$ is a logical measure of ant-agonist affinity (see also Kenakin 2004, pp. 14-15). Ultimately, make your own conscious choice. In synagics, I prefer inhibition curves combined with non-linear parameter fitting of semi-log or logit transformed data instead of a Schild.
}

discussion and experiments on the subject by Lazareno and Birdsall (1993a,b) and Calderone et al. (1999).

The conclusion is that, in general, the inhibition curve experiment is preferable to the Schild analysis.

\subsubsection{First: Inhibition Curves}

First inhibition curves of the combined agonist-antagonist dose response experiment is simple to perform (Figs. 11.5C and 11.6C). The exact same information as derived by a Schild analysis may be obtained by first determining the agonist dose-response curve in the absence of any ant-agonist as illustrated by the curve in panel A of Figs. 11.5 and 11.6, and identical to the start of Schild's analysis. The remainder of the analysis is simply carried out by continuing the experiment with increasing concentrations of an ant-agonist, as demonstrated by the curve in panel C of Figs. 11.5 and 11.6 from a 'full response' (see Section 2.1.3), here chosen at 70 and $91 \%$ down to a level near $100 \%$ inhibition, a so-called inhibition curve. In addition, if advantageous, the ant-agonist curve may be obtained from levels of agonist activation lower than the $70 \%$ 'full-response' used here. Meanwhile, increased accuracy comes with use of sizable 'full-response' plateaus.

\subsubsection{Second: Schild Analysis}

Schild analysis may require four near-complete agonist dose-response relations to demonstrate that the underlying synagic scheme is competitive, one in the absence and three in the presence of an ant-agonist. A possible analysis for competitive dose-response for an agonist is shown in Fig. 11.7, illustrated by the six dose-response curves in panel B of Fig. 11.5. First, a dose-response curve for the agonist in the absence of any ant-agonist. Next, it is necessary to displace this agonist doseresponse curve with at least three fixed and arbitrarily chosen concentrations of an ant-agonist, and then again determine the agonist dose-response curves up to a suitable level of response. In Figs. 11.5 and 11.6 displacement of agonist dose-response relations is obtained with five fixed concentrations of an ant-agonist, $\mathrm{I} / K_{\mathrm{si}}=1.5 \times 10^{-1}$ to $1.5 \times 10^{3}$ in steps of a factor 10 . From these results it is possible to construct five points in a Schild plot as shown in Fig. 11.7. As we have already seen, in this plot an extrapolation to zero on the relative $\log$ dose-ratio axis $\log (D R-1)=0$, is the same as $D R=2$, and the pertaining negative $\log$ concentration of the ant-agonist yields the parameter $\mathrm{pA}_{2}\left(\mathrm{pI}_{2}\right)$, which is the corresponding concentration on the ant-agonist concentration axis, equal to $-\log \left[K_{\mathrm{si}}\right]$ (Fig. 11.7A), and the same as the logarithm of the ant-agonist affinity constant $A_{\text {si }}$. The minus sign in front of the log-operator handles the reverse relation between $K_{\mathrm{si}}$ and $A_{\mathrm{si}}$.

A series of inhibition curves may be obtained from any level of varied agonist effect (Figs. 11.5 and 11.6). This 
second part of the experiment obtaining inhibition curves yields the exact same $\mathrm{pA}_{2}$ value as a Schild analysis, which requires more data points, and at higher concentrations of agonist. A deviation from competitive antagonism will show up in this analysis just as non-linearity is revealed in the Schild plot where the slope must be unity and the relationship between $(D R-1)$ and antagonist concentration must be a straight line. Deviations from linearity and slopes of non-unity are discussed later, see also Kenakin (1993: Chapter 9, 1997: Chapter 10, 2004: Chapter 6).

The complications of a possible receptor reserve will not necessarily show up in this form of analysis and neither will it necessarily reveal itself in the Schild analysis. No matter what method is employed, one must test separately for this possibility, just as other assumptions have to be substantiated or verified before accepting a certain reaction scheme.

\subsubsection{Other Concerns About the Schild Analysis}

It has been suggested that the Schild analysis does not require a determination of the agonist dissociation constant (Kromer 1991). This is partially true when screening a series of ant-agonists with the same agonist. Nevertheless, in principle, a Schild cannot be performed without a full characterization of the system's agonist dose-response curve in order to reveal, for instance, interventory-/modulatory behavior, receptor heterogeneity, or auto-intervention/co-operativity for each agonist in order to judge the validity of the analysis. Parallel time-controls are also necessary to judge possible timedependent changes (Lew \& Angus 1997).

Leff and Dougall (1993: Fig. 1) expressed concerns about a risk of misinterpreting experiments as based on a competitive agonist-ant-agonist scheme instead of a noncompetitive interventor relation. In order to avoid such a misinterpretation, they recommended use of a Schild protocol. However, the calamity is more easily avoided with an inhibition curve analysis by adapting the following procedure to systems with unknown reaction schemes:

1. Bring the agonist response to $80-90 \%$ of the maximal effect.

2. Determine the inhibition curve for the actual antagonist/interventor and obtain a pre- $K_{\mathrm{i}}$, as in Figs. 11.5 and 11.6.

3. In a new experiment, before introducing the agonist, add a concentration of the ant-agonist/ interventor equal to its pre- $K_{\mathrm{i}}$ in the media, and after equilibration, increase the concentration of the agonist from zero to its $K_{\mathrm{s}}$ or eventually to 10 times its $K_{\mathrm{s}}$.
The result of this procedure, as exemplified in Fig. 11.9, will clearly differentiate between competitive ant-agonism and non-competitive intervention.

Stone and Angus (1978) and Stone (1980) described a plot different from the Schild plot and based on a slightly modified plot of data from Clark (1926), which they termed a 'Clark plot'. They demonstrated that this Clark plot, $\log [\mathrm{S}]-\mathrm{vs}-\log \left[K_{\mathrm{si}}+\mathrm{I}\right]$, is statistically more valid than the Schild plot. Kenakin (1993, pp. 311-312, 1997, pp. 361-363) describes several advantages of the Clark plot over the Schild plot.

Furthermore, Lew and Angus (1995) demonstrated that analysis by a global regression method for the Clark plot would further improve accuracy compared to Schild's analysis. The inclusion of all data for better statistical analysis was suggested already by Trist and Leff (1985) and may be compared with an area method for density power spectra. ${ }^{7}$

\subsubsection{Inhibition Curve Strategy Against Schild Analysis}

The following are some advantages of the inhibition curve strategy compared with drawbacks of the Schild method including straightforward load analysis of appropriate synagics and employing non-linear parameter fitting.

1. The inhibition curve method is more easily carried out with one dose-response curve for the agonist and one inhibition curve for each ant-agonist as shown in Figs. 11.5 and 11.6, rather than the minimum of four agonist dose-response curves in the Schild method. For each potential competitive ant-agonist, control for deviations from the assumptions should of course be performed prior to the analysis and independent of the chosen method.

2. Another advantage of the inhibition curve method is that it may also be used for non-competitive synagics as well as for any other hypothesis, and its analysis is based on the presumed synagics. Schild analysis yields usable results in studies with simple competitive synagics, but may only hint at other schemes.

3. A non-linear fit to load activation and inhibition curves yields the most accurate determination of $K_{\text {si }}$, due to better statistics than in the Schild analysis (Lew \& Angus 1995; Kenakin 1997).

4. One more disadvantage of Schild in comparison with the inhibition curve method is that in order to perform the Schild to completion, the experimenter often has to go to ridiculously high agonist

\footnotetext{
7 Integrating all data in an analysis of a dose-response relation might be done as for power spectra with better resolution (Christensen \& Bindslev 1982) and even possible separation of two overlaid data sets (Lindemann 1984).
} 


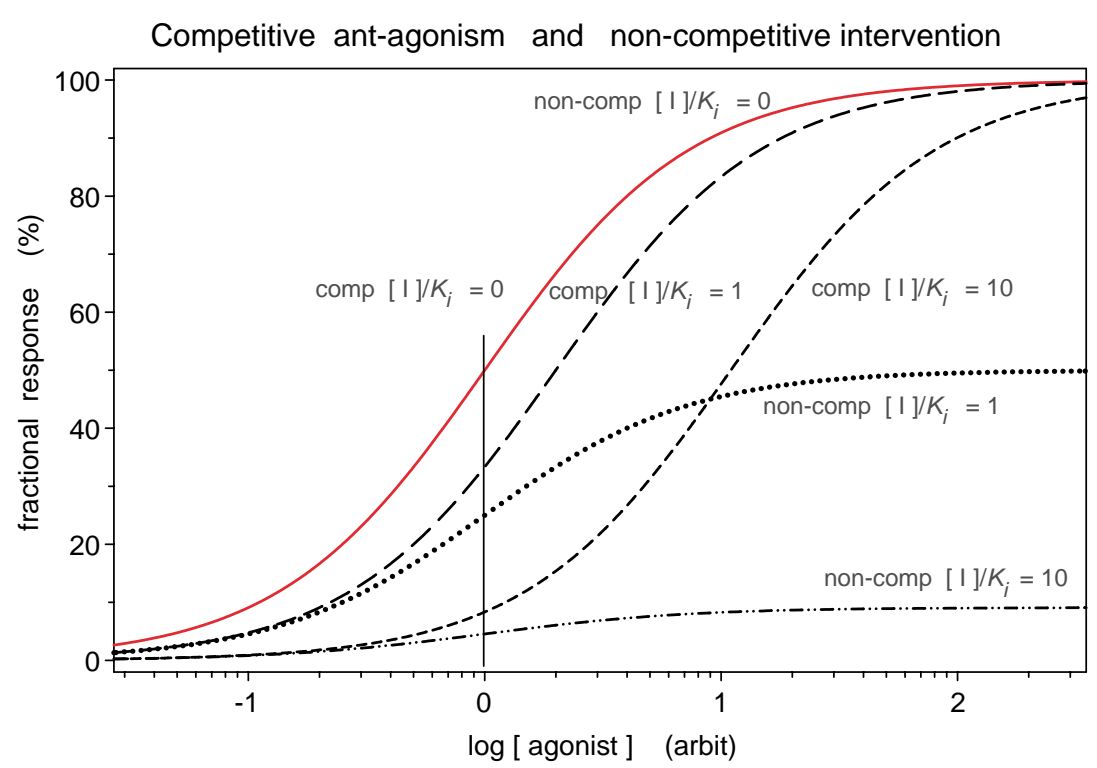

Figure 11.9. Differentiating between competitive ant-agonism and non-competitive intervention is easy. Without ant-agonist there is no difference (red curve). See also Kenakin (2004, 2007 'orthosteric ant-agonism' and 'allosteric modulation'). Arbitrary concentration axis.

concentrations. An example of meaningless use of high agonist concentrations in a Schild analysis is illustrated in Section 11.2.4. In addition, it is known that high concentrations of agonist or antagonist concentrations may very well elicit allosteric and other effects not relevant for the primary binding site effects (Melchiorre 1988), thus interfering with the determination of $-\log \left(K_{\mathrm{si}}\right)$.

5. When it is hard to washout test compound, the Schild analysis requires new preparations. To circumvent the problem introduced by several preps, the Schild analysis may be expanded with a re-sampling technique, bootstrapping (Lutz et al. 1995). This problem does not hamper the inhibition curve method.

6. Finally, Schild's method operates optimally for DRs of a factor 2 and above. In contrast, the Schild is poorly defined for values of the $D R$ below 2 , it yields negative values for the $\log (D R-1)$ (see hatched areas in Figs. 4.3 and 4.4). The domain below a factor 2 for $D R$ represents data point spread over the upper half of an inhibition curve (Figs. $11.5 \mathrm{~B}+\mathrm{C}$ and $11.6 \mathrm{~B}+\mathrm{C}$ ).

\subsubsection{An Experimental Evaluation of $p K_{B}$ by the Schild Analysis}

Figures 11.10 and 11.11 show an example of a Schild analysis for the neurokinin inhibitor CP 99,994 on the Sar'-substance P-induced secretion in pig jejunum (Thorboll et al. 1998). The determined Schild slope deviates significantly from unity, $n_{\mathrm{i}}=1.59$ (Fig. 11.11). Therefore, the authors had to conclude that they were unable to estimate the $\mathrm{p} K_{\mathrm{B}}\left(\mathrm{p} K_{\mathrm{i}}\right)$.
Note that in order to make the Schild analysis for CP 99,994 it was necessary to employ excessively high doses of the neurokinin agonist $\mathrm{Sar}^{9} \mathrm{SP}$, approximately 100 $\mu \mathrm{M}$.

Alternatively, an inhibition curve analysis originating from the left-most curve in Fig. 11.10 and simply increasing the CP 99,994 concentration to near complete inhibition (Fig. 11.10), would merely have required a maximal agonist concentration of around $1 \mu \mathrm{M}$.

Furthermore, employing data from the inhibition curve methodology and an analysis also including a noncompetitive inhibition scheme might easily have solved the author's calamity and yielded a meaningful affinity, $\mathrm{p} K_{\mathrm{i}}$, for CP 99,994.

\subsubsection{Deviation from Slopes of Unity and from Linearity in the Schild Analysis}

Consult Kenakin for a thorough and detailed evaluation of possible forms of Schild plots when handling systems that do not follow simple competitive dose-responses (Kenakin 1993: Chapter 9, 1997: Chapter 10, 2004: Chapter 6).

\subsubsection{To Schild or Not to Schild?}

All in all, the answer to the question 'to Schild or not to Schild' is given by a summary of the listed points in Section 11.2.3, which concludes: Do not Schild, instead use the inhibition curve protocol and a realistic reaction scheme. 


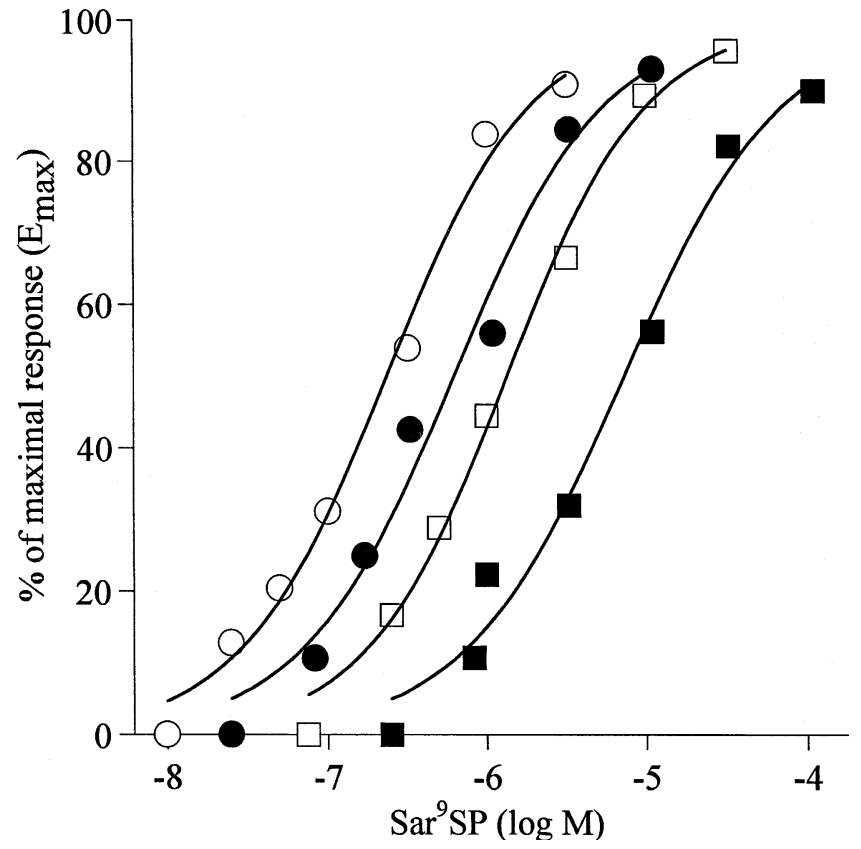

Figure 11.10. Schild analysis of $\mathrm{pA}_{2}$ for $\mathrm{CP} 99,994$ by use of Sar ${ }^{9} \mathrm{SP}$ as agonist. Taken from Thorbøll et al. (1998) with permission.

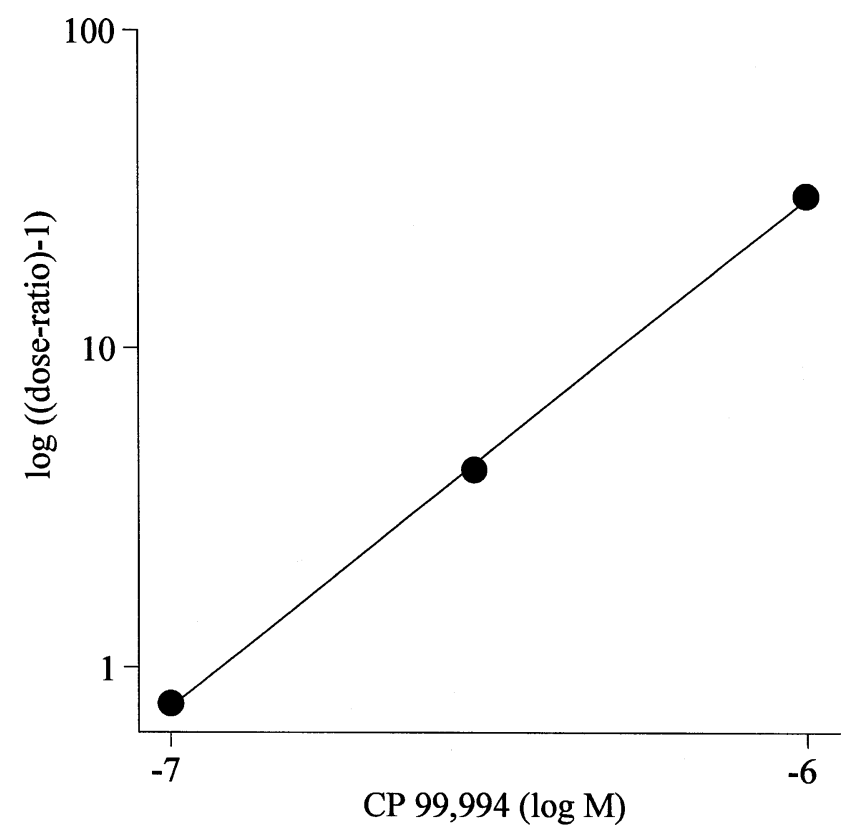

Figure 11.11. Schild regression for CP99,994 by use of Sar ${ }^{9} \mathrm{SP}$ as agonist. Taken from Thorbøll et al. (1998) with permission.

\subsection{Time to Abandon the Schild}

\subsubsection{How You Can Fool Yourself with a Schild Analysis}

As there is enormous pressure on research performance, one easily becomes biased. The demand is to come up with affinities for newly developed ant-agonists measured through use of the Schild analysis. It has become prestigious to be able to present $\mathrm{pA}_{2}$ values from a Schild analysis, since, in practice, a quoted $\mathrm{pA}_{2}$ value equal to the negative logarithm of the dissociation constant $-\log K_{\mathrm{si}}$ for an ant-agonist, is considered a pharmaceutical truism and a good sales argument for the drug. A high $\mathrm{pA}_{2}$ is equal to having a high affinity of the drug for the system under study.

Before employing an agonist/ant-agonist pair to be characterized in a Schild analysis, it is necessary to prove to yourself that the pair consists of a full agonist and an ant-agonist with strict competitive synagics. These requirements are often not fulfilled, however due to the above-mentioned pressure, a 'Schild' is forced on the experimental data anyway.

When carried out correctly and with the required assumptions fulfilled, we can have nothing against a Schild analysis, it is a powerful tool. The problem is that users of the analysis are in difficulties if their system does not comply with underlying assumptions. In such situations, use of a restricted ant-agonist concentration range will often allow you to obtain linear Schild plots with a slope of unity. However, if you are honest, you are in deep trouble, since there seems to be no way out of the dilemma.

As a rescue, several authors have suggested 'modified Schild plots' or 'enhanced Schild analysis' introducing power transformations (Cheng 2002, 2004; Cheng \& Lai 2003; Kenakin 2004, p. 106; see also Eq. 11.7 and Fig. 11.8). Again, these types of 'power departure' have the same twist as described for the Hill equation in Chapter 10. You get a better mathematical resolution by escaping the physical world.

The question is: when is a system 'Schildable', when does 'Schild' deviate from linearity and a slope of unity, in case the underlying mechanism is not a simple competitive system, which it frequently is not? I have illustrated this question in Section 11.2.4.

The solution to this Gordian knot is simple. Try another theory based for instance on data from an inhibition curve experiment, now also suggested by more and more authors (e.g., Calderone 1998; Calderone et al. 1999; Kenakin 2004).

\subsubsection{Other Theoretical Schemes and Their Schild Formulation}

The Schild null hypothesis has been extended to theoretical models that do not follow the simple competitive ant-agonist reaction scheme (Schild 1949; Arunlakshana \& Schild 1959; Ehlert 1988; Leff 1995; Kenakin 2004: Section 6.8.6).

The Schild principle can logically be applied to the simple two-state model where both ant-agonists and inverse agonists function as competitive inhibitors (Leff 1995). 
Contrary to this, implementing the Schild principle for the ternary-complex model - i.e., the intervention scheme described in Chapter 2 - is a bit more tricky, if not meaningless. For all that, Ehlert (1988) assumed equi-activity between two different functional terms as well as between two binding terms, that is, formulations for activation/occupancy equal to a function $f$ in the absence of inhibitor, $\mathrm{I}=0$, i.e., response $=f_{\mathrm{I}=0}\left(e_{0}, \mathrm{~S}_{0}\right)$, is the same as a formulation for activation/occupancy equal to another function $g$ in the presence of an interventor, i.e., response $=g_{\mathrm{I}>0}\left(e_{1}, \mathrm{~S}_{1}, \mathrm{I}\right)$. Here $e_{0}$ and $e_{1}$ are efficacy constants in the presence and absence of an interventor, $S_{0}$ and $S_{1}$ are agonist concentrations in the absence and presence of an interventor, and $\mathrm{I}$ is the concentration of an interventor. By further assuming the efficacy terms as simple factors (see discussions in Chapter 1.3 and Section 5.6.2) and thus the outcome of operators $f_{\mathrm{I}=0}$ and $g_{\mathrm{I}>0}$ to be identical, an expression for the equi-active agonist concentrations $S_{1}$ in the presence and $S_{0}$ in the absence of the interventor could be obtained (Ehlert's Eq. 18) and used for a Schild-type analysis of both binding and activity (Ehlert 1988). However, as indicated, the validity of calling it a Schild procedure is doubtful, since Schild is not for noncompetitive or intervention schemes as the TCM with so-called 'allosteric modulators' (Kanakin 2004; Ehlert 2005; May 2007).

Meanwhile, a better approach than Schild's analysis for the ternary-complex model and working both for function and binding could be to compare equi-activity in the Hall (2000) ATSM in Chapter 7 (see also May et al. 2007).

\subsubsection{It is Time to Reduce Use of Schilds}

In parallel with the above description of my idiosyncratic feel for the Schild analysis, simultaneously I get a parasympathetic sensation about Schild's paradigm for the following reason. In attending meetings, I have witnessed how I earlier swore to the 'Schild'. Self-satisfaction emanated from my body language as I got up to announce to the world the $\mathrm{pA}_{2}$ s for studied ant-agonists, and I proudly assured the audience that 'the slope of the Schild plot was not significantly different from unity'. This statement is a hallmark for Schild-aficionados indicating that the studied system with employed agonist and ant-agonists behaved competitively - justifying the analysis.

The trend setting focus on Schild analysis seemed to have blinded me to much more general and fecund approaches to ligand-receptor interactions. Approaches such as modeling based on reaction schemes illustrated in Chapter 7 (Fig. 7.1) that can cover many more systems and might give meaningful results far beyond the suffocating constraints of the requirements for the 'Schild'.

For me, interrogative modeling is now the Tao for synagic analyses, rather than quoting $\mathrm{pA}_{2}$ values from Schild's analysis with self-contented body language and added 'slope of unity'.

The popularity of the 'Schild' stems from a paradigm or picture that its use is a fairly simple way to obtain correct values for ant-agonist potencies, circumventing several obstacles such as required knowledge about the dissociation constant for the agonist(s) used in the analysis (Kromer 1991).

However, modern synagic theory tells us that affinity and efficacy parameters are reciprocally interfused (Colquhoun 1998). Hence, my suggestion is to implement the most likely formulation of a model for your system with non-linear fitting and not necessarily the 'Schild'; unless you have a simple competitive system at hand.

Sixty years ago, 'Schild' was developed as an elegant analysis based on severe restrictions and before the computing power we possess today. However, competitive ant-agonism is often too simple a reaction scheme for the description of studied systems.

The justification of a Schild analysis is its use of the ratio method to eliminate effects other than competitive ant-agonism with a formulation that is based on competitive ant-agonism. Do we have a contradiction here - a circular argument?

I would suggest reading Jenkinson (2003, pp. 43-53). His conjecture of the Schild story is somewhat different from that provided here, but he also emphasizes the difference between $\mathrm{p} K_{\mathrm{B}}, \mathrm{p} K_{\mathrm{i}}$, and $\mathrm{pA}_{2}$.

To conclude, synagic analysis of inhibition ought to be conducted by a combination of straightforward nonlinear fitting of parameters in the most likely reaction scheme to data from experimental inhibition curves, and possibly in a semi-log or logit transformation.

\section{REFERENCES}

Alberts JB \& Odell GM. In silico reconstitution of Listeria propulsion exhibits nano-saltation. PLoS Biol 2: e412, 2004.

Allis CD, Jenuwein T \& Reinberg D. Epigenetics, 1st ed. Cold Spring Harbor, NY: Cold Spring Harbor Laboratory Press 2007.

Almers W. Gating currents and charge movements in excitable membranes. Rev Physiol Biochem Pharmacol 82: 96-190, 1978.

Andreeva IE, Nirthanan S, Cohen JB \& Pedersen SE. Site specificity of agonist-induced opening and desensitization of the Torpedo californica nicotinic acetylcholine receptor. Biochemistry 45: 195204, 2006.

Ariëns EJ. Affinity and intrinsic activity in the theory of competitive inhibition. Part I. Problems and theory. Arch Int Pharmacodyn 99: 32-49, 1954.

Arunlakshana O \& Schild HO. Some quantitative uses of drug antagonism. Br J Pharmacol 14: 48-58, 1959.

Avlani V, May LT, Sexton PM \& Christopoulos A. Application of a kinetic model to the apparently complex behavior of negative and 
positive allosteric modulators of muscarinic acetylcholine receptors. J Pharmacol Exp Ther 308: 1062-1072, 2004.

Baker JG \& Hill SJ. A comparison of the antagonist affinities for the Giand Gs-coupled states of the human adenosine Al-receptor. J Pharmacol Exp Ther 320: 218-228, 2007.

Ben-Chaim Y, Chanda B, Dascal N, Bezanilla F, Parnas I \& Parnas H. Movement of 'gating charge' is coupled to ligand binding in a Gprotein-coupled receptor. Nature 444: 106-109, 2006.

Birdsall NJ \& Lazareno S. Allosterism at muscarinic receptors: ligands and mechanisms. Mini Rev Med Chem 5: 523-543, 2005.

Bourne HR \& von Zastrov M. Drug receptors and pharmacodynamics. In: Basic $\mathcal{E}$ Clinical Pharmacology, 10th ed, edited by Katzung BG. New York: McGraw-Hill, 2007, pp. 11-33.

Burzomato V, Beato M, Groot-Kormelink PJ, Colquhoun D \& Sivilotti LG. Single-channel behavior of heteromeric alphalbeta glycine receptors: an attempt to detect a conformational change before the channel opens. J Neurosci 24: 10924-10940, 2004.

Calderone V. An alternative method to evaluate the Nature of an antagonist and its potency: a theoretical approach. J Pharmacol Toxicol Methods 39: 129-135, 1998.

Calderone V, Baragatti B, Breschi MC \& Martinotti E. Experimental and theoretical comparisons between the classical Schild analysis and a new alternative method to evaluate the pA2 of competitive antagonists. Naunyn Schmiedebergs Arch Pharmacol 360: 477-487, 1999.

Campos FV, Chanda B, Roux B \& Bezanilla F. Two atomic constraints unambiguously position the $\mathrm{S} 4$ segment relative to $\mathrm{S} 1$ and $\mathrm{S} 2$ segments in the closed state of Shaker K channel. Proc Natl Acad Sci USA 140: 7904-7909, 2007.

Castrillo JI, Zeef LA, Hoyle DC, Zhang N, Hayes A, Gardner DC, Cornell MJ, Petty J, Hakes L, Wardleworth L, Rash B, Dunn WB, Broadhurst D, O'donoghue K, Hester SS, Dunkley TP, Hart SR, Swainston N, Li P, Gaskell SJ, Paton NW, Lilley KS, Kell DB \& Oliver SG. Growth control of the eukaryote cell: A systems biology study in yeast. J Biol 6 (1): 4, 2007.

Cheng HC. The power issue: determination of KB or Ki from IC50. A closer look at the Cheng-Prusoff equation, the Schild plot and related power equations. J Pharmacol Toxicol Methods 46: 61-71, 2001.

Cheng HC. The influence of cooperativity on the determination of dissociation constants: examination of the Cheng-Prusoff equation, the Scatchard analysis, the Schild analysis and related power equations. Pharmacol Res 50: 21-40, 2004.

Cheng HC \& Lai RW. Use of the proportionality equations for analyses of dose-response curves. Pharmacol Res 47: 163-173, 2003.

Christensen O \& Bindslev N. Fluctuation analysis of short-circuit current in a warm-blooded sodium-retaining epithelium: site current, density, and interaction with triamterene. J Membr Biol 65: 19-30, 1982.

Clark AJ. The antagonism of acetyl choline by atropine. J Physiol 61: 547-556, 1926.

Clark AJ. The Mode of Drugs on Cells. London: Arnold \& Co, 1933.

Clark AJ. General Pharmacology, Vol. 4, 1st ed. Berlin: Springer, 1937.

Clark AJ \& Raventos J. The antagonism of acetyl choline and of quartenary ammonium salts. O J Exp Physiol 26: 375-392, 1937.

Colquhoun D. Binding, gating, affinity and efficacy: the interpretation of structure-activity relationships for agonists and of the effects of mutating receptors. Br J Pharmacol 125: 924-947, 1998.

Colquhoun D. Why the Schild method is better than Schild realised. Trends Pharmacol Sci 28: 608-614, 2007.

Colquhoun D \& Sivilotti LG. Function and structure in glycine receptors and some of their relatives. Trends Neurosci 27: 337344,2004

Ehlert FJ. Estimation of the affinities of allosteric ligands using radioligand binding and pharmacological null methods. Mol Pharmacol 33: 187-194, 1988.
Ehlert FJ. Analysis of allosterism in functional assays. J Pharmacol Exp Ther 315: 740-754, 2005.

Gaddum JH. The action of adrenalin and ergotamine on the uterus of the rabbit. J Physiol 61: 141-150, 1926.

Gaddum JH. The quantitative effects of antagonistic drugs. J Physiol 89: 7P-9P, 1937.

Gaddum JH. Introductory address. Part I. Biological aspects: the antagonism of drugs. Trans Faraday Soc 39: 323-332, 1943.

Gaddum JH. Theories of drug antagonism. Pharmacol Rev 9: 211-218, 1957.

Gaddum JH, Hameed KA, Hathway DE \& Stephens FF. Quantitative studies of antagonists for 5-hydroxytryptamine. Q J Exp Physiol 40: 49-74, 1955.

Galzi JL, Edelstein SJ \& Changeux J. The multiple phenotypes of allosteric receptor mutants. Proc Natl Acad Sci USA 93: 1853-1858, 1996.

Giraldo J, Serra J, Roche D \& Rovira X. Assessing receptor affinity for inverse agonists: Schild and Cheng-Prusoff methods revisited. Curr Drug Targets 8: 197-202, 2007.

Greenleaf WJ, Woodside MT \& Block SM. High-resolution, singlemolecule measurements of biomolecular motion. Annu Rev Biophys Biomol Struct 36: 171-190, 2007.

Hill AV. The possible effects of aggregation of the molecules of haemoglobin on its dissociation curve. J Physiol 40: iv-vii, 1910.

Hall DA. Modeling the functional effects of allosteric modulators at pharmacological receptors: an extension of the two-state model of receptor activation. Mol Pharmacol 58: 1412-1423, 2000.

Jenkinson DH. Classical approaches to the study of drug-receptor interactions. In: Textbook of Receptor Pharmacology, 2nd ed, edited by Foreman JC \& Johansen T. Boca Raton, FL: CRC Press, 2003, pp. 3-62.

Jensen LJ, Jensen TS, de Lichtenberg U, Brunak S \& Bork P. Coevolution of transcriptional and post-translational cell-cycle regulation. Nature 443: 594-597, 2006.

Kellam B. Combinatorial chemistry: a tool for drug discovery. In: Smith and William's Introduction to the Principles of Drug Design and Action, 4th ed, edited by Smith HJ. Boca Raton, FL: CRC Press, 2006, pp. $355-376$

Kenakin T. Pharmacologic Analysis of Drug-receptor Interaction, 2nd ed. New York: Raven Press, 1993.

Kenakin T. Pharmacological Analysis of Drug-receptor Interaction, 3rd ed. Philadelphia, PA: Lippincott-Raven Publishers, 1997.

Kenakin T. Efficacy at G-protein-coupled receptors. Nat Rev Drug Discov 1: 103-110, 2002.

Kenakin T. Predicting therapeutic value in the lead optimization phase of drug discovery. Nat Rev Drug Discov 2: 429-438, 2003.

Kenakin T. A Pharmacology Primer: Theory, Application, and Methods, 1st ed. Amsterdam: Elsevier Academic Press, 2004.

Kenakin T. New concepts in drug discovery: collateral efficacy and permissive antagonism. Nat Rev Drug Discov 4: 919-927, 2005.

Kenakin T. Allosteric agonist modulators. J Recept Signal Transduct Res 27: 247-259, 2007.

Kenakin TP, Jenkinson S \& Watson C. Determining the potency and molecular mechanism of action of insurmountable antagonists. J Pharmacol Exp Ther 319: 710-723, 2006.

Kodadek T, Duroux-Richard I \& Bonnafous JC. Oxidative cross-linking as an emergent tool for the analysis of receptor-mediated signalling events. Trends Pharmacol Sci 26: 210-217, 2005.

Kromer W. Receptor classification using antagonists without determining pA2 values. Trends Pharmacol Sci 12: 11-13, 1991.

Lage K, Karlberg EO, Storling ZM, Olason PI, Pedersen AG, Rigina O, Hinsby AM, Tumer Z, Pociot F, Tommerup N, Moreau Y \& Brunak S. A human phenome-interactome network of protein complexes implicated in genetic disorders. Nat Biotechnol 25: 309-316, 2007.

Langmuir I. Abstract. J Am Chem Soc 39 Issue 11 Abstr: 2422, 1917. 
Langmuir I. The adsorption of gases on plane surfaces of glass, mica and platinum. J Am Chem Soc 40: 1361-1403, 1918.

Lazareno S \& Birdsall NJ. Estimation of antagonist Kb from inhibition curves in functional experiments: alternatives to the ChengPrusoff equation. Trends Pharmacol Sci 14: 237-239, 1993.

Lazareno S \& Birdsall NJ. Estimation of competitive antagonist affinity from functional inhibition curves using the Gaddum, Schild and Cheng-Prusoff equations. Br J Pharmacol 109: 1110-1119, 1993.

Leff P. The two state model of receptor activation. Trends Pharmacol Sci 16: 89-97, 1995 .

Leff P \& Dougall IG. Further concerns over Cheng-Prusoff analysis. Trends Pharmacol Sci 14: 110-112, 1993.

Lew MJ \& Angus JA. Analysis of competitive agonist-antagonist interactions by non-linear regression. Trends Pharmacol Sci 16: 328-337, 1995.

Lew MJ \& Angus JA. An improved method for analysis of competitive agonist/antagonist interactions by non-linear regression. Ann NY Acad Sci 812: 179-181, 1997.

Lew MJ, Ziogas J \& Christopoulos A. Dynamic mechanisms of nonclassical antagonism by competitive AT(1) receptor antagonists. Trends Pharmacol Sci 21: 376-381, 2000.

Lindemann B. Analysis of additively contaminated Lorentzians by integration. Biophys J 46: 409-411, 1984.

Longabaugh WJ, Davidson EH \& Bolouri H. Computational representation of developmental genetic regulatory networks. Dev Biol 283: $1-16,2005$.

Lutz M \& Kenakin T. Quantitative Molecular Pharmacology and Informatics in Drug Discovery, 1st ed. Chichester: Wiley \& Sons, 1999.

Lutz MW, Kenakin TP, Corsi M, Menius JA, Krishnamoorthy C, Rimele $\mathrm{T} \&$ Morgan $\mathrm{PH}$. Use of resampling techniques to estimate the variance of parameters in pharmacological assays when experimental protocols preclude independent replication: an example using Schild regressions. J Pharmacol Toxicol Methods 34: 37-46, 1995 .

MacKinnon R. Nobel Lecture. Potassium channels and the atomic basis of selective ion conduction. Biosci Rep 24: 75-100, 2004.

MacKinnon R. Structural biology. Membrane protein insertion and stability. Science 307: 1425-1426, 2005.

Mathiesen JM, Christopoulos A, Ulven T, Royer J, Campillo M, Heinemann A, Pardo L \& Kostenis E. On the mechanism of interaction of potent, surmountable and insurmountable antagonists with the prostaglandin D2 receptor CRTH2. Mol Pharmacol 69: 1441-1453, 2006.

May LT, Leach K, Sexton PM \& Christopoulos A. Allosteric modulation of g protein-coupled receptors. Annu Rev Pharmacol Toxicol 47: $1-51,2007$.

Melchiorre C. Polymethylene tetramines: a new generation of selective muscarinic antagonists. Trends Pharmacol Sci 9: 216-220, 1988

Mitra A, Cymes GD \& Auerbach A. Dynamics of the acetylcholine receptor pore at the gating transition state. Proc Natl Acad Sci USA 102: 15069-15074, 2005.

Moore RH, Millman EE, Godines V, Hanania NA, Tran TM, Peng H, Dickey BF, Knoll BJ \& Clark RB. Salmeterol stimulation dissociates beta2-adrenergic receptor phosphorylation and internalization. Am J Respir Cell Mol Biol 36: 254-261, 2007.

Nelson DL \& Cox MM. Lehninger Principles of Biochemistry, 4th ed. New York: WH Freeman, 2005

Neubig RR, Spedding M, Kenakin T \& Christopoulos A. International Union of Pharmacology Committee on Receptor Nomenclature and Drug Classification. XXXVIII. Update on terms and symbols in quantitative pharmacology. Pharmacol Rev 55: 597-606, 2003.

Olsen JV, Blagoev B, Gnad F, Macek B, Kumar C, Mortensen P \& Mann M. Global, in vivo, and site-specific phosphorylation dynamics in signaling networks. Cell 127: 635-648, 2006.

Paas Y, Gibor G, Grailhe R, Savatier-Duclert N, Dufresne V, Sunesen M, Prado de Carvalho L, Changeux JP \& Attali B. Pore conforma- tions and gating mechanism of a Cys-loop receptor. Proc Natl Acad Sci USA 102: 15877-15882, 2005.

Paton WD \& Rang HP. The uptake of atropine and related drugs by intestinal smooth muscle of the guinea-pig in relation to acetylcholine receptors. Proc R Soc Lond B Biol Sci 163: 1-44, 1965.

Pratt WB \& Taylor P. Principles of Drug Action: The Basis of Pharmacology, 3rd ed. Philadelphia, PA: Churchill Livingstone, 1990.

Rang HP, Dale MM, Ritter JM \& Flower R. Rang Eं Dale's Pharmacology, 6th ed. Edinburgh: Churchill-Livingstone, 2007.

Rowell FJ. Recombinant DNA technology: monoclonal antibodies. In: Smith and William's Introduction to the Principles of Drug Design and Action, 4th ed, edited by Smith HJ. Boca Raton, FL: CRC Press, 2006, pp. 377-393.

Schild HO. pA, a new scale for the measurement of drug antagonism. Br J Pharmacol 2: 189-206, 1947.

Schild HO. pAx and competitive drug antagonism. Br J Pharmacol 4: 277-280, 1949.

Schulte G \& Levy FO. Novel aspects of G-protein-coupled receptor signalling - different ways to achieve specificity. Acta Physiol (Oxf) 190: 33-38, 2007

Stephenson RP. A modification of receptor theory. Br J Pharmacol 11 : 379-393, 1956.

Stone M. The Clark plot: a semi-historical case study. J Pharm Pharmacol 32: 81-86, 1980.

Stone M \& Angus JA. Developments of computer-based estimation of pA2 values and associated analysis. J Pharmacol Exp Ther 207: 705$718,1978$.

Sørensen SPL. Études enzymatiques; II. Sur la mesure et l'importance de la concentration des ions hydrogène dans les réactions enzymatiques. Comp Rend Travaux Laboratoire Carlsberg 8: 1-168, 1909.

Taglialatela M \& Stefani E. Gating currents of the cloned delayedrectifier K+ channel DRK1. Proc Natl Acad Sci USA 90: 4758 4762, 1993.

Taglialatela M, Toro L \& Stefani E. Novel voltage clamp to record small, fast currents from ion channels expressed in Xenopus oocytes. Biophys J 61: 78-82, 1992.

Thorboll JE, Bindslev N, Hansen MB, Schmidt P \& Skadhauge E. Functional characterisation of tachykinin receptors mediating ion transport in porcine jejunum. Eur J Pharmacol 359: 271-279, 1998.

Trist DG \& Leff P. Quantification of H2-agonism by clonidine and dimaprit in an adenylate cyclase assay. Agents Actions 16: 222-226, 1985.

Unwin N. Refined structure of the nicotinic acetylcholine receptor at 4A resolution. J Mol Biol 346: 967-989, 2005.

Ussing HH. Distinction by means of tracers between active transport and diffusion. The transfer of iodide across the isolated frog skin. Acta Physiol Scand 19: 43-56, 1949.

Valiyaveetil FI, Leonetti M, Muir TW \& MacKinnon R. Ion selectivity in a semisynthetic $\mathrm{K}+$ channel locked in the conductive conformation. Science 314: 1004-1007, 2006.

Volkman BF, Lipson D, Wemmer DE \& Kern D. Two-state allosteric behavior in a single-domain signaling protein. Science 291: 2429 2433, 2001

Von Dassow G \& Odell GM. Design and constraints of the Drosophila segment polarity module: robust spatial patterning emerges from intertwined cell state switches. J Exp Zool 294: 179-215, 2002.

Wreggett KA \& Wells JW. Cooperativity manifest in the binding properties of purified cardiac muscarinic receptors. J Biol Chem 270: 22488-22499, 1995.

Wyllie DJ \& Chen PE. Taking the time to study competitive antagonism. Br J Pharmacol 150: 541-545, 2007.

Zoffmann S, Bertrand S, Do QT, Bertrand D, Rognan D, Hibert M \& Galzi JL. Topological analysis of the complex formed between neurokinin A and the NK2 tachykinin receptor. J Neurochem 101: 506-516, 2007. 
Part III: Test of Tools for Data Analysis 


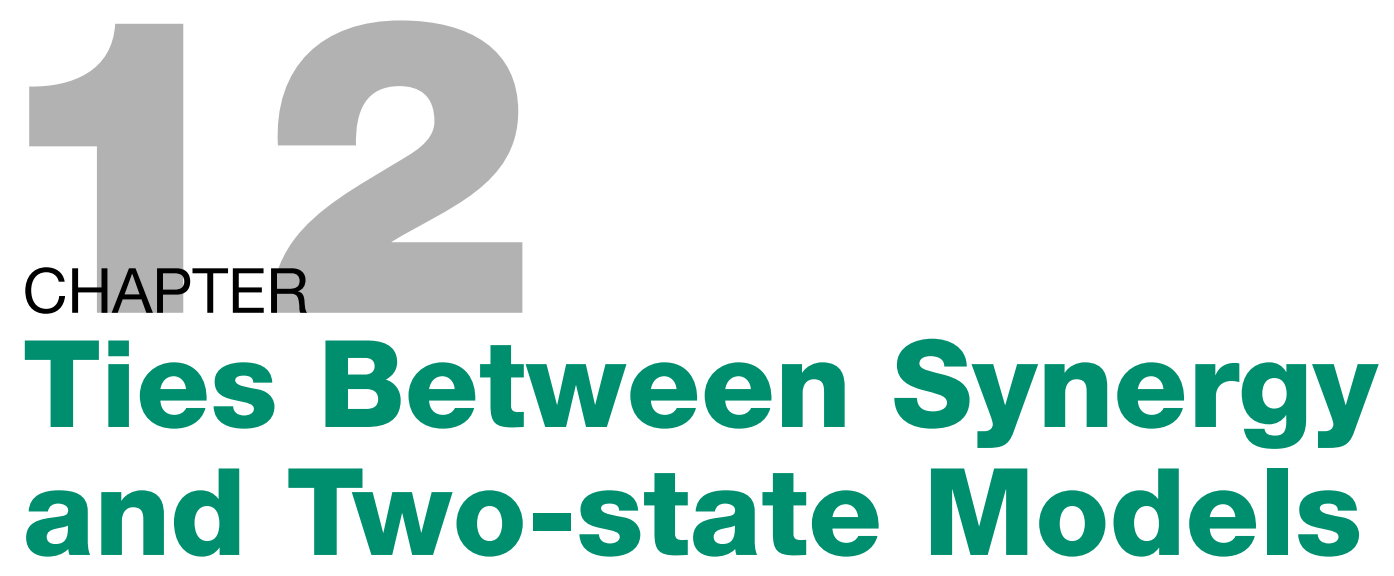

\subsection{General Synergy. A Non-mechanistic Approach}

\subsubsection{Outlook of Synergy}

'Synergy' covers the effects above those expected for a simple additive effect of drug combinations.

Describing and understanding the effects of combining two or more drugs, two or more substrates, and in general two or more ligands has challenged a broad segment of scientists in a number of research fields (Berenbaum 1989; Greco et al. 1995; Tallarida 2000, 2007; White et al. 2003; Chou 2006; Lee et al. 2007; Brun et al. 2007). Table 12.1 lists some examples of drug combinations from the human disease field that cover therapies for cancer, AIDS, and auto-immunological diseases, such as asthma, diabetes, inflammatory bowl disease, and rheumatoid arthritis, as well as many others. For multi-drug treatment or survival of other organisms there is, for instance, the use of fertilizers, pesticides and weed killers.

Although there are advances in, for instance, combinatorial cancer chemotherapy (Hellebrekers et al. 2007; Messeguer et al. 2007; Sanchez-Escribano Morcuende et al. 2007), models predicting the correct combination of drugs and their mutual concentration and timing are still in great demand.

\subsubsection{Three Levels: Organ, Cell, and Receptor}

The subject of how we are to understand an elicited effect mechanistically or empirically when two or more drugs are combined should be divided into sub-themes based on either physical and pure mathematical models or on sketchy diagrams, in order to bring some sort of sanity to a 'madhouse' (Table 12.2). It is necessary to divide and describe the subject of combinatorial therapy at a minimum of three different system levels: (1) whole organisms, (2) cells, and (3) receptors. One may argue that ultimately understanding what occurs at the receptor level will also explain the operations at the whole body level. Meanwhile, in integrated systems there are links and networkings whose operational predictions are not necessarily given by understanding the underlying parts. Furthermore, it turns out that the nomenclature and concepts at these three levels do not overlap, although some of the principles in mathematical formulation, including mechanistic terms, are the same. One area that seems to bring all three levels together is the theme of multi-drug-resistance (MDR) (Kellen 1993; Goldie \& Coldman 1998; Buolamwini \& Adjei 2003; Michor et al. 2006; McDevitt \& Callaghan 2007).

In two-drug applications at the integrated organ level we will find expressions for concepts such as isoboles, isobolograms, median-effect plots, response surface modeling, synergy, cross-tolerance, pharmaco-kinetics, and tachyphylaxia, while at the receptor level is more likely to see expressions such as allosteric-two-state model, ternary-complex model, concentration-occupancy curve, partial and mixedinhibition, and insurmountable ant-agonism. Many terms are the same but sometimes cover different meanings, e.g., dose-response relations, desensitization, co-operativity and pharmaco-dynamics.

The main focus of this book is on receptive units, and I shall concentrate on themes relevant to this level. Even so, for perspective, I will introduce the concepts of synergy and isobolograms that are more frequently used in studies on organs and whole organisms. Compared to mechanistic models at the receptor level, I foresee that a critical discussion of the synergy concept and its actual non-mechanistic models for analysis will open our minds further to the meaning and validity of both approaches. 
Table 12.1. 'Order in the SYNERGY - madhouse I' (Possible effects of combinatorial drug therapy in some specific fields of human disease and nutrition. Two drugs or food-stuffs ingested by whole organisms or applied to cells)

\begin{tabular}{|c|c|c|}
\hline Research field & Drug examples & References \\
\hline Acute lymphoblastic leukemia & Combination therapy & Reynolds et al. (2007) \\
\hline AIDS/HIV & $\begin{array}{l}\text { Anti-retroviral drugs, } \\
\text { integrase inhibitor }\end{array}$ & $\begin{array}{l}\text { Yeni et al. (2006); Yeung et al. (2007); } \\
\text { Shimura et al. (2007); Traynor (2007) }\end{array}$ \\
\hline Alzheimer & & van Dyck et al. (2006) \\
\hline Arrhythmia - long QT syndrome & $\mathrm{K}$ channel modulators & Thomsen (2007) \\
\hline Asthma & Steroids and beta-adrenergic drug & Hancox (2006) \\
\hline Atherosclerosis & Statins and ezitimibe & Genest (2006); lughetti et al. (2007) \\
\hline Cancer, colon and breast & $\begin{array}{l}\text { Ningalin, -estradiol, -norspermine, tamoxifen, } \\
\text { cetuximab, erbitux }{ }^{\circledR} \text {, herceptin }\end{array}$ & $\begin{array}{l}\text { Chou et al. (2005); Nair et al. (2007); } \\
\text { Hoffmann et al. (2007); Mahtani \& } \\
\text { Macdonald (2008) }\end{array}$ \\
\hline Cystic fibrosis & Poly-antibiotics & Thelin \& Boucher (2007) \\
\hline Diabetes type 1 , diabetes type 2 & $\begin{array}{l}\text { Insulin, GIP, GLP-1, glitazone DPP-IV-4 } \\
\text { inhibitors }\end{array}$ & Knudsen et al. (2007) \\
\hline Epilepsia & Gabapentin, oxcarbazepine & $\begin{array}{l}\text { Bouwman et al. (2004); } \\
\text { Jonker et al. (2007) }\end{array}$ \\
\hline Fever & NOS rofecoxib mefenamic & Gowen et al. (2006) \\
\hline Food satiety and obesity & CCK, Leptin, Ghrelin, rimonabant & Atkinson (2008) \\
\hline Hypertension, kidney diseases & $\begin{array}{l}\text { Thiazides, ACE inhibitors + angiotensin- } \\
\text { receptor blocker }\end{array}$ & Kjeldsen et al. $(2005 a, b)$ \\
\hline $\begin{array}{l}\text { Inflammatory bowl diseases (IBD) } \\
\text { Crohn's, ulcerative colitis }\end{array}$ & TNF- $\alpha$ antibody (Infliximab ${ }^{\circledR}$ ) other biologics & Hanauer (2007) \\
\hline $\begin{array}{l}\text { Inflammation and infection: bacterial, } \\
\text { helmetic, viral and fungal or due to } \\
\text { antigens, toxins }\end{array}$ & Antibiotics, antihelmetics, palivizumab & Lin et al. (2004); Khanna et al. (2008) \\
\hline Multi-drug-resistance & Ningalines + , paclitaxel, tariquidar + & $\begin{array}{l}\text { Chou et al. (2005); Michor et al. (2006); } \\
\text { McDevitt and Callaghan (2007); } \\
\text { Hubensack et al. (2007); Brun et al (2007); } \\
\text { Fox \& Bates (2007) }\end{array}$ \\
\hline Multiple myeloma & Bortezomib combination-therapy & \\
\hline Nutrition & Flavonoids, probiotics & Ward (2005); Ward and Fonseca (2007) \\
\hline Osteoporosis & Calcium, biphosphate, D-vitamin & Dudakovic et al. (2008); Morishita et al. (2008) \\
\hline Oncology: small cell carcinoma, ovarian & $\begin{array}{l}\text { Epigenetics: RNA interference and demethylation. } \\
\text { Cisplatin, paclitaxel, doxorubicin }\end{array}$ & Chou (2006); Hellebrekers et al. (2007) \\
\hline Pain general, pain neuropathic & Opiods, NSAIDs & Guindon et al. $(2006 a, b)$ \\
\hline Parkinson & Ergopeptide & Vendrell et al. (2007) \\
\hline Postoperative nausea vomiting (PONV) & Propofol, setrones, dexamethasone & Antonetti et al. (2007) \\
\hline Rheumatoid arthritis & $\begin{array}{l}\text { DMARDs, biologics, Infliximab, methotrexate, } \\
\text { sulfasalazine, hydroxy-chloroquine }\end{array}$ & Jenks et al. (2007) \\
\hline Schizophrenia & Clozapine + amisulpride & Horner et al. (2007) \\
\hline Ulcer & Pump inhibitor + antibiotics & de Leest et al. (2007) \\
\hline
\end{tabular}

Communications on combinatorial drug effects and treatment surpass 100,000 in the MedLine database, therefore this table is just an illustration of the complexity of the synergy subject.

If unfamiliar with synergy at any system level, it is highly recommended to consult the reviews by Berenbaum (1989), Greco et al. (1995) and Chou (2006, Erratum in Pharmacol Rev 59: 124, 2007). Synergy has also been treated by differential approximation (Salvador 2000a,b). Read especially the excellent review by Greco and coworkers. Furthermore, realize that due to the complexity of analyzing possible synergy in whole organisms, Berenbaum gave up and advocated for a mathematical but non-mechanistic analysis of synergy, thus leaving a physical interpretation to a distant future. The aim here is to bring that future to the present.

\subsubsection{Some History on the Synergy Concept}

The development of induction and treatment by the effects of combinatorial drug application has followed a tortuous path. This also applies to the analysis for possible synergy in combinatorial drug application as, for instance, in survival from toxic drugs, in treatment of cancer, AIDS, and atherosclerosis, and in induction of anesthesia, analgesia, and anti-angiogenesis for tumor control (Fraser 1871; Frei 1913; Loewe 1928, 1953, 1959; Berenbaum 1977, 1989; Tallarida 1992, 2000; Chou et al. 1994, 2003, 2005, 2006; Lopez-Munoz 1993; LopezMunoz et al. 1994; Greco et al. 1995; van der Graaf \& 
Table 12.2. 'Order in the SYNERGY - madhouse II' (Some models in use and other potential models for synergy in general)

\begin{tabular}{|c|c|c|}
\hline Subjects & Sub-themes and related models & Associated terms or models to be implemented \\
\hline $\begin{array}{l}\text { Basic physiology, pharmacology, and } \\
\text { pato-physioplogy, Vira, bacteria, } \\
\text { yeasts, plants, and animals }\end{array}$ & $\begin{array}{l}\text { Receptor level, cellular level, organ level, } \\
\text { whole organisms }\end{array}$ & $\begin{array}{l}\text { Pharmaco-dynamics, synagics; cross-tolerance; } \\
\text { pharmaco-kinetics, synergy, tolerance; nutrition } \\
\text { and psychology (Spinella 2002; Belitz et al. 2004; } \\
\text { McGee 2004; Ward 2005) }\end{array}$ \\
\hline $\begin{array}{l}\text { Historic development of models at the } \\
\text { receptor/cellular level for: one ligand } \\
\text { and one or two states }\end{array}$ & $\begin{array}{l}\text { HMM-Haldane; modified Hill equation; dC\&K, } \\
\text { B\&L, cTSM, MWC-KNF, HOTSM }\end{array}$ & $\begin{array}{l}\text { Auto-ant-agonism, auto-intervention (pos or neg); } \\
\text { co-operativity (pos or neg); auto-modulation (pos } \\
\text { or neg); bell-shaped and terraced dr-relation }\end{array}$ \\
\hline $\begin{array}{l}\text { Historic development of models at the } \\
\text { receptor/cellular level for: two li- } \\
\text { gands and one or two states }\end{array}$ & $\begin{array}{l}\text { TCM, FP-OSM; mixed-inhibition-mixed } \\
\text { activation*; ETCM-CTCM; ATSM- } \\
\text { FP-TSM }\end{array}$ & $\begin{array}{l}\text { Mixed-inhibition, model for GPCRs; ETCM, CTCM, } \\
\text { and CQCM; ant-agonism, intervention, modula- } \\
\text { tion, OFCOR }{ }^{\star \star}\end{array}$ \\
\hline $\begin{array}{l}\text { Historic development of models at the } \\
\text { receptor/cellular/whole organ level } \\
\text { for: synergy }\end{array}$ & $\begin{array}{l}\text { Loewe (1926, p. 59); Bliss (1939); Chou and } \\
\text { Talalay (1977, p. 84); Berenbaum (1989); } \\
\text { Tallarida (1992, 2007); Greco et al. (1995, } \\
\text { 2007); White et al. (2003); Brun et al. (2007) }\end{array}$ & $\begin{array}{l}\text { Isobologram, response surface modeling; probabil- } \\
\text { ity model; Hill exponentiation, median-effect plots } \\
\text { and Combination Index, competitive and non- } \\
\text { competitive TCM with fixed ratio doses, combi- } \\
\text { nation therapy. Interaction Index, comparison of } \\
\text { models for synergy }\end{array}$ \\
\hline $\begin{array}{l}\text { Mechanistic models to be tried for: } \\
\text { synergy }\end{array}$ & $\begin{array}{l}\text { Intervention model. FP-OSM (Fig. 2.4). ATSM } \\
\text { (Fig. 7.1A). FP-TSM (Fig. 7.2) }\end{array}$ & $\begin{array}{l}\text { Intervention (pos or neg) (Figs. 12. 4-12.19). } \\
\text { Simple OFCOR model (Figs. 12.20 and 21) }{ }^{\star \star} \text {. } \\
\text { Heterotropic allostery (Figs. 12.24-12.27) with } \\
\text { the potential of an OFCOR model }\end{array}$ \\
\hline
\end{tabular}

Abbreviations: HMM = Henri-Michaelis-Menten; dC\&K = delCastillo \& Katz; B\&L =Black \& Leff; cTSM = cyclic-two-state model; MWC =Monod-Wyman-Changeux $\mathrm{KNF}=$ Koshland-Nemethy-Filmer; TCM = tertiary-complex model; FP-OSM =four-pane one-state model; $\mathrm{ETCM}=$ extended tertiary-complex model; CTCM =cubic tertiary-complex model; CQCM = cubic quaternary-complex model; ATSM =allosteric two-state model; HOTSM =homotropic two-state model; FP-TSM, four-pane two-state model; OFCOR =optimal fixed concentration ratio.

*Equal to TCM, Ehlert's model (1988), or the intervention model in Chapter 2.

**Winding and Bindslev (1993) used the FP-OSM for an analysis of OFCOR, see also Wells (1992), Wood et al. (1995), Hoare et al. (2000), and Giraldo (2004).

Schoemaker 1999; Chan et al. 2000; Lorenzen et al. 2002; Deciga-Campos et al. 2003, 2004; Barrera et al. 2005; Gasparini et al. 2005; Gouwy et al. 2005; Jonker et al. 2005, 2007). Meanwhile, synergy's modern interpretation is largely based on the synagics as described by exclusive/non-exclusive dose-responses with additional mathematical principles as the Hill exponentiation for the modeling of ligand-receptor interactions (Chou \& Talalay 1977, 1981, 1983, 1984; Chou 2006).

Fraser (1871) was the first to describe the life-saving effect of combining physiostigmine and atropine in survival studies of their toxic effects (Fig. 12.1). In the first quarter of the 20th century, such combinatorial drug application became popular (Langley 1905; Schulmann et al. 1913; Frei 1913; Clark 1926, 1937). Frei (1913) discussed in clear terms the difference between 'iso-addition' and 'hetero-addition', thus laying the groundwork in the field of synergy for an ingenious summary of concepts and terms by Loewe $(1928,1953)$ (Figs. 12.2 and 12.3). Isobolograms and isoboles (see Section 12.1.4) were coined as concepts by Loewe and Muischnek (1926). Serious analysis of combinatorial drug effects, with synergy, additivism, and ant-agonism, began with Loewe's pioneering understanding of the issue (Loewe \& Muischnek 1926; Kaer \& Loewe 1926a,b; Loewe 1928, 1938). In Estonia's short interval of independence from 1919 to 1940 , Loewe managed to lay the groundwork for modern day analysis of synergy (Loewe 1928). Although Loewe realized diversions (Kaer \& Loewe 1926a,b; Loewe 1955a,b, 1957) from

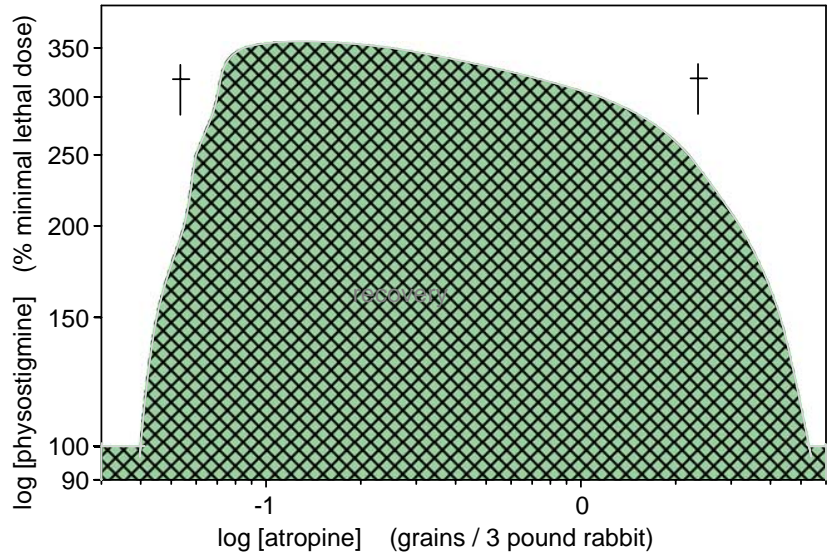

Figure 12.1. An area relation between survival and drug combinations of a toxic drug mixed with an antidote. Modified from Fraser (1871).

his own simple rules (Fig. 12.2), his definitions remain standard equipment for modern-day therapeutics in disease-fields as diverse as plant pests, diarrhea in domestic animals, human hypertension, astringent asthma, killing cancer, and infectious inflammation with related maladies as pain and edema (Tallarida 2000: Chapter 1; Guindon et al. 2006; Miranda et al. 2006). See Table 12.1 for further examples.

\subsubsection{Isobologram and Isoboles}

Fig. $12.4 \mathrm{~A}$ is a response surface plot in three dimensions of the additive response of two drugs applied 

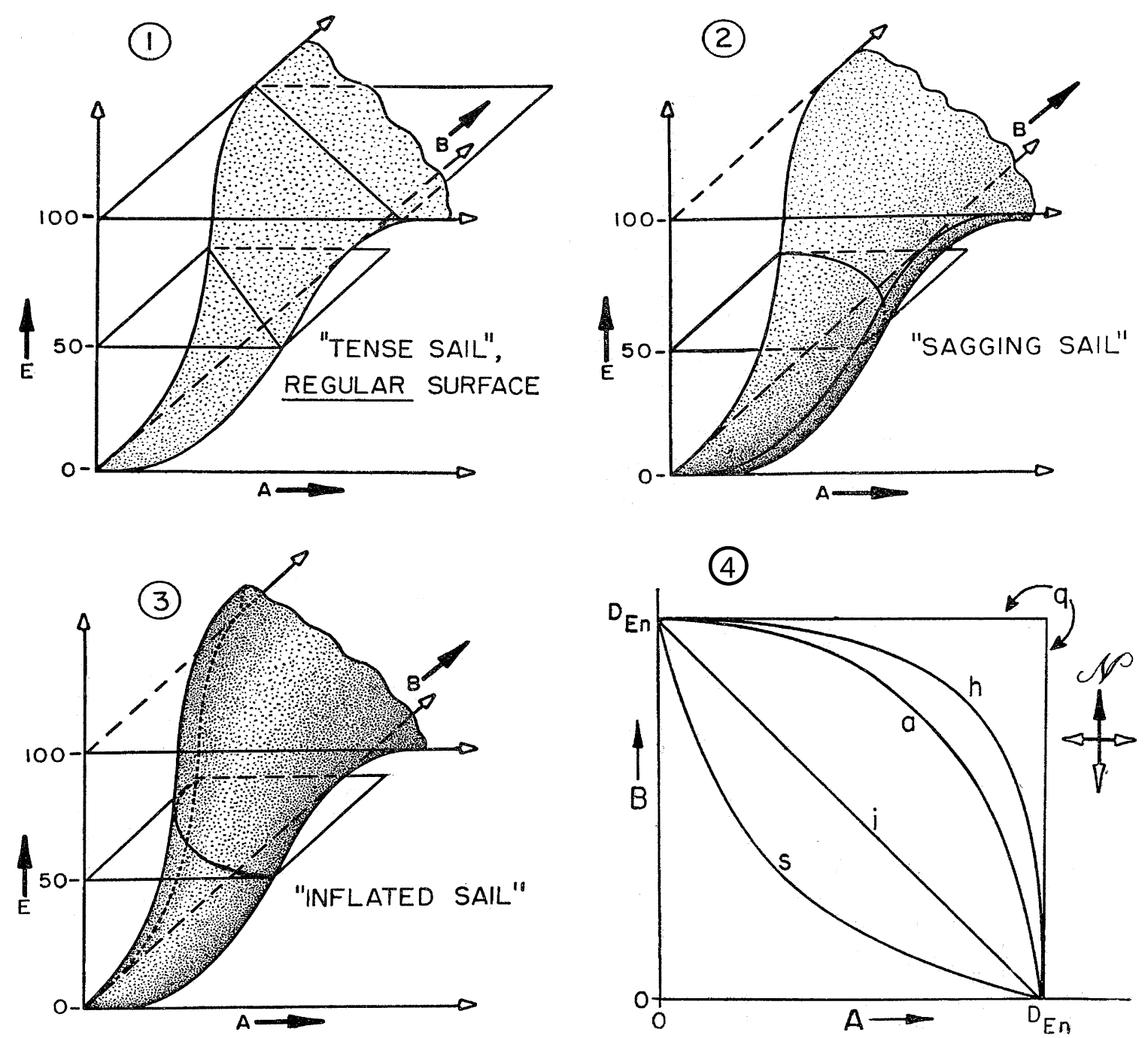

Figure 12.2. Space-bolograms or 'raumbologrammen' and their contour projections. Examples: (1) simple additivity (tense sail), (2) ant-agonism (sagging sail), and (3) synergism (inflated sail). Panel 4 is a two-dimensional projection of the horizontal 'sail'responses in 1,2, and 3. Straight cord 'i' represents additivity, line ' $s$ ' bending into the south-west represents an example of synergy, while the curves ' $a$ ' and ' $h$ ' bending into the north-east represent examples of ant-agonism. Based on Loewe and coworkers analysis of combinatorial drug effects. From Loewe (1953, Figs 1 and 2) with permission.

simultaneously and both assumed to elicit a load reaction, the simple hyperbolic reaction, when each drug is applied on its own, and furthermore reacting according to a simple competitive scheme when combined, as described in Chapter 2. Applying increasing doses of a mixture of two competitive drugs with a fixed concentration ratio $1: 1$ results in a competitive reaction scheme for its 'additive'-form (Fig. 12.4C). Finally, when the surface response plot is converted to a 2-D contour plot of the combined effects we get a plot as in Fig. 12.4B. Note that the concentrations of both drugs are on axes scaled in a linear dimension in all three graphs in Fig. 12.4. The lines in the contour plot are the socalled isoboles. When various combinations of concentration for the two drugs elicit iso-effects, we move along contour lines (see graph B in Fig. 12.4). Fig. 12.4B is a so-called isobologram and its contour lines are the isoboles (Loewe \& Muischnek 1926; Loewe 1928).

In accordance with certain standards in the synergy community, isoboles deviate from the line of additivity of a competitive reaction scheme (Fig. 12.2). Thus, there is synergism when isoboles bend into the southwestern area (Fig. 12.2-3), additivism when following the straight isobole in Fig. 12.2-1, and ant-agonism as the isoboles curve into the north-eastern corner of the graph in Fig. 12.2-2 (Loewe 1928, 1953, Tallarida 2000). Other terms for synergism are super-additivity and supra-additivity, and for ant-agonism, sub-additivity or infra-additivity.

However, in reality, these definitions depend on the chosen algebraic model (see Section 12.3.1). Except for the simple intervention model described in Chapter 2, 
there are several kinds of isobolograms not in line with the one depicted in Fig. 12.2 (Table 12.3). That this is true for experimental data, as well, was already pointed

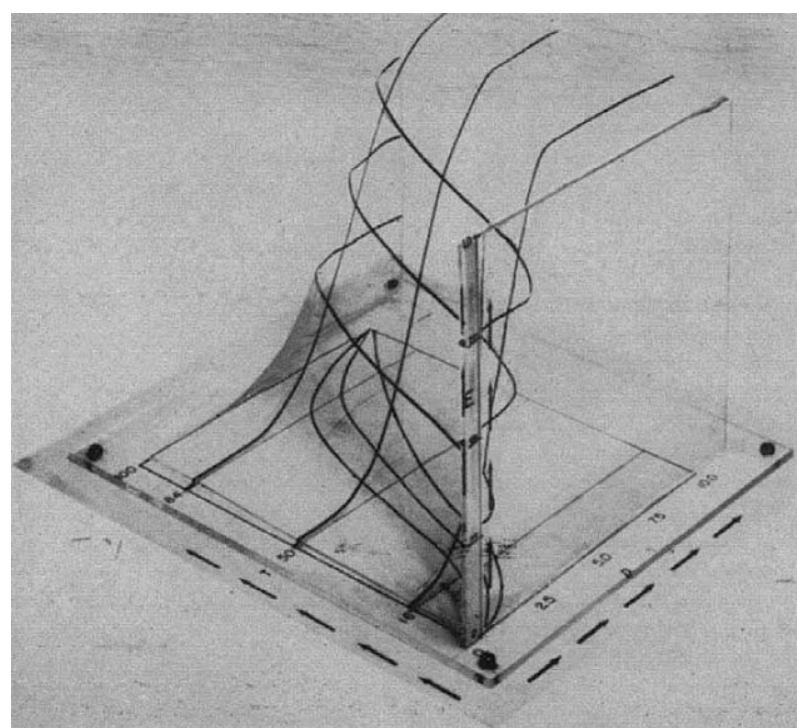

Figure 12.3. Model of Loewe's space-bolograms equal to response-surface plots. The three horizontal curves represent the dose-tolerance curves, isoboles. From Loewe (1959, Fig 2) with permission. out by Loewe (Kaer \& Loewe 1926a,b; Loewe 1928, 1955a,b, 1957).

Examples of simple models that follow Loewe's definitions in Fig. 12.2 are the competitive additivity model (Fig. 12.4), and the non-competitive synergy model (Fig. 12.5). However, models that deviate from the definitions in Fig. 12.2 are easily obtained. An example is the isoboles of a competitive ant-agonism model shown in Fig. 12.6 for two simultaneously applied drugs.

\subsubsection{Loewe's Relationship}

Assume that a drug D1 added alone in concentration [D1] elicits a response equal to the response of another drug D2 when this drug is added alone in a concentration [D2]; not necessarily equal to [D1]. Then, a potency ratio $\left(P_{\mathrm{R}}\right)$ between these two drugs can be formulated as $[\mathrm{D} 1] /[\mathrm{D} 2]$. For instance, if we assume that D1 is 100-fold more efficient than D2, then our potency ratio $P_{\mathrm{R}}=[\mathrm{D} 1] /[\mathrm{D} 2]=1 / 100$. For drug 2 , we need 100 times the concentration of drug 1 to obtain the same response by either drug applied alone. In case

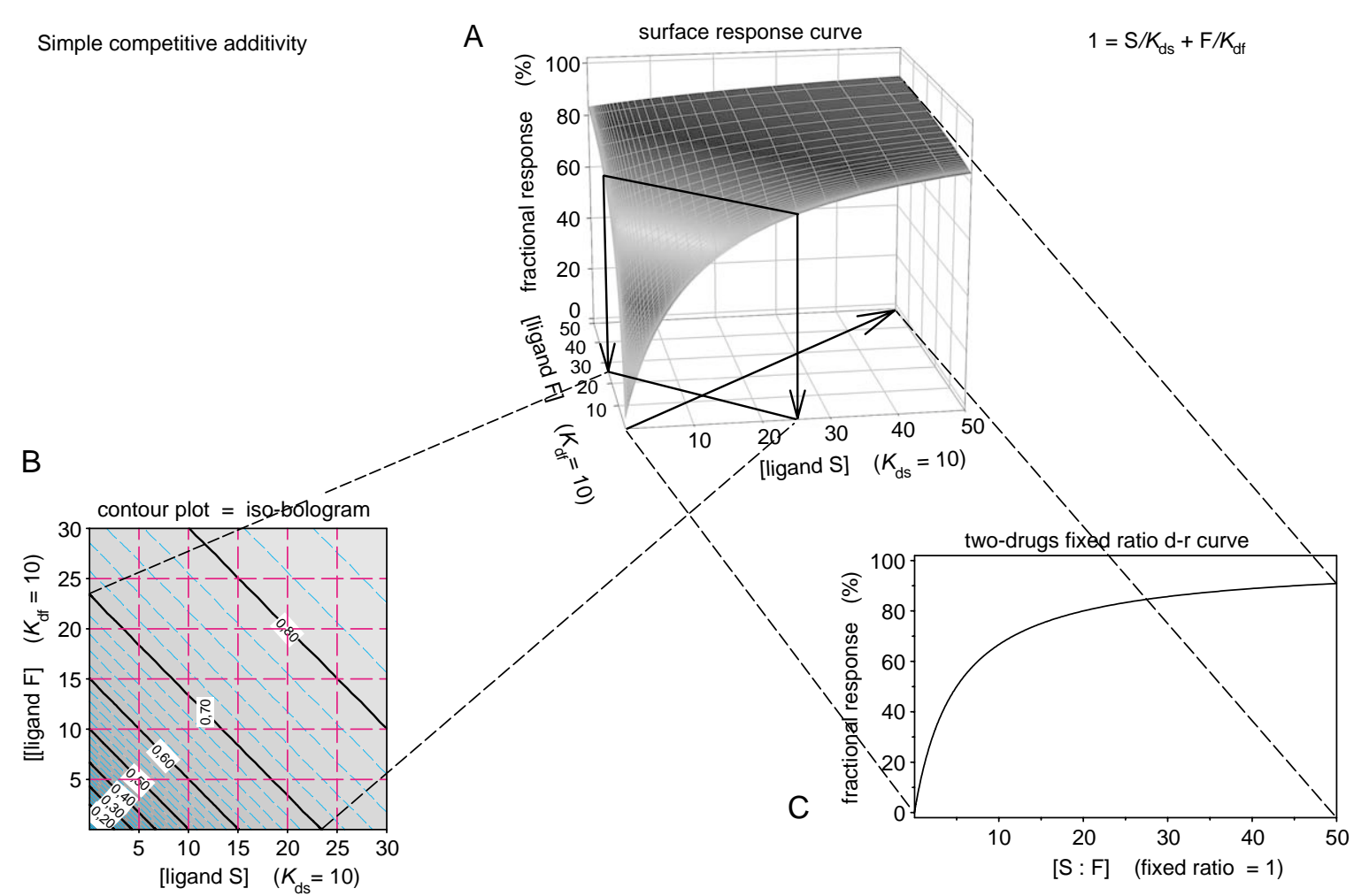

Figure 12.4. Simple competitive additivity for two ligands in three representations. The model is based on $(S+F) /(1+S+F)$, where ' $S$ ' stands for the receptive units with bound S-ligand and ' $F$ ' is the receptive units bound with $F$-ligand. Parameters are as indicated in Table 12.3. (A) A 3-D surface topography, (B) its related contour plot, and (C) its 2-D dose-response curve for fixed ratio between increasing concentrations of drugs. Two vertical arrows in panel $A$ indicate the projection of the $70 \%$ isobole and is represented in panel B. Raising a plane vertical through the arrow in panel A's concentration plane for two ligands, i.e., following a fixed ratio of drug concentrations, cuts a curve in A's surface response plot as reproduced in panel C. 
Table 12.3. Fourteen functional models for the simple intervention scheme - including binding abbreviated as + occ.

\begin{tabular}{|c|c|c|c|c|}
\hline Type of reaction scheme (see code below) & Value of const $c$ & Characteristics of the distribution equation & Fig. 12\# & Loewe's isoboles \\
\hline Competitive ant-agonism + occ & 0 & $S /(1+S+F)$ & 6 & No \\
\hline Competitive additivity & 0 & $(\mathrm{~S}+\mathrm{F}) /(1+\mathrm{S}+\mathrm{F})$ & $4+7$ & Yes \\
\hline Non-compet. ant-agonism & 1 & $\mathrm{~S} /(1+\mathrm{S}+\mathrm{F}+\mathrm{SF})$ & 8 & No \\
\hline Non-compet. Additivity & 1 & $(\mathrm{~S}+\mathrm{F}) /(1+\mathrm{S}+\mathrm{F}+\mathrm{SF})$ & 9 & No/yes* \\
\hline Non-compet. synergism I+occ & 1 & $(\mathrm{~S}+\mathrm{SF}) /(1+\mathrm{S}+\mathrm{F}+\mathrm{SF})$ & 10 & No \\
\hline Non-compet. synergism II & 1 & $(\mathrm{~S}+\mathrm{F}+\mathrm{SF}) /(1+\mathrm{S}+\mathrm{F}+\mathrm{SF})$ & $5+11$ & No/yes** \\
\hline Pos interv. ant-agonism & 10 & $S /(1+S+F+S F)$ & 12 & No \\
\hline Neg interv. ant-agonism & 0.1 & $\mathrm{~S} /(1+\mathrm{S}+\mathrm{F}+\mathrm{SF})$ & 13 & No \\
\hline Pos interv. additivity & 10 & $(\mathrm{~S}+\mathrm{F}) /(1+\mathrm{S}+\mathrm{F}+\mathrm{SF})$ & 14 & No/yes* \\
\hline Neg interv. additivity & 0.1 & $(\mathrm{~S}+\mathrm{F}) /(1+\mathrm{S}+\mathrm{F}+\mathrm{SF})$ & 15 & $\mathrm{No}^{\star \star \star *}$ \\
\hline Pos interv. synergism I+occ & 10 & $(\mathrm{~S}+\mathrm{SF}) /(1+\mathrm{S}+\mathrm{F}+\mathrm{SF})$ & 16 & No/yes** \\
\hline Neg interv. synergism I+occ & 0.1 & $(\mathrm{~S}+\mathrm{SF}) /(1+\mathrm{S}+\mathrm{F}+\mathrm{SF})$ & 17 & No \\
\hline Pos interv. synergism II & 10 & $(\mathrm{~S}+\mathrm{F}+\mathrm{SF}) /(1+\mathrm{S}+\mathrm{F}+\mathrm{SF})$ & 18 & No/yes** \\
\hline Neg interv. synergism II & 0.1 & $(\mathrm{~S}+\mathrm{F}+\mathrm{SF}) /(1+\mathrm{S}+\mathrm{F}+\mathrm{SF})$ & 19 & No/yes** \\
\hline
\end{tabular}

Parameter values: as indicated on the figure axes, the dissociation constant for ligand $\mathrm{S}, K_{\mathrm{ds}}$, and for ligand $\mathrm{F}, K_{\mathrm{df}}$, are fixed at 10 and the values for parameter $c$ are in column 2. Column 1+2: Nomenclature and designators for the intervention model: competitive: $c=0$; non-competitive: $c=1$; intervention: $c>0$ and $\neq 1$; positive intervention: $c>1$; negative intervention: $0<c<1$. Column 3: Distribution equations with indicated receptive species: $\mathrm{S}=$ primary ligand bound to $\mathrm{R}, \mathrm{F}=$ interventor (competitive/non-competitive) ligand bound to $\mathrm{R}, \mathrm{SF}=$ both $\mathrm{S}$ and $\mathrm{F}$ are bound to the receptive unit $\mathrm{R}$. In the nominator: $\mathrm{S}=$ 'ant-agonism' (for $c=0$ also 'occupancy'); $\mathrm{S}+\mathrm{F}=$ 'additivity'; $\mathrm{S}+\mathrm{SF}=$ either 'non-competitive synergism I', 'non-competitive occupancy', 'interventory synergism l', or 'intervention occupancy'; $\mathrm{S}+\mathrm{F}+\mathrm{SF}=$ either 'non-competitive synergism Il' or 'intervention synergism II'. In accord with the above codes, there is no 'competitive synergism' possible. The different types of models within the intervention scheme, including both $c=0$ and $c=1$, result in 14 different possibilities for the functional intervention scheme. Four of these schemes are equal to the intervention scheme in binding studies and are listed as '+occ' in the column 1. Column 4: Figure numbers showing the listed model. Column 5: Resemblance with Loewe's isoboles.

${ }^{*}$ At small values of $S=[D 1]$ and $F=[D 2]$, isoboles resemble ant-agonism in Fig. 12.2

${ }^{* *}$ Recognizing the model behind, curved contour lines are the 'additivity' isoboles.

${ }^{\star \star \star *}$ The isobologram appears as Loewe's ant-agonism (Fig. 12.2).

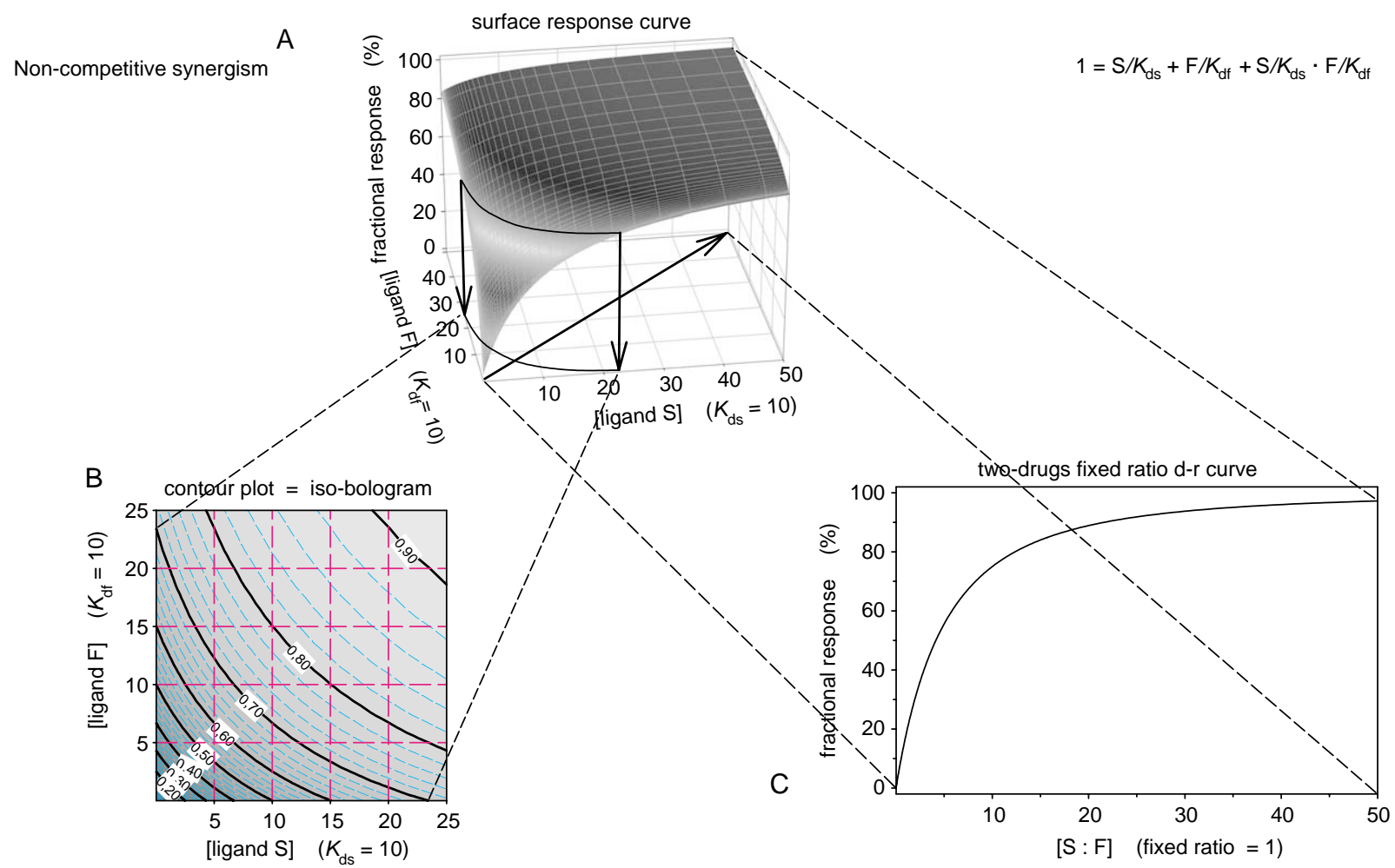

Figure 12.5. Non-competitive 'synergy' for two ligands in three representations. The model is based on $(S+F+S F) /(1+S+F+$ $\mathrm{SF}$ ), where ' $\mathrm{S}$ ' stands for the receptive units with bound S-ligand and ' $\mathrm{F}$ ' is the receptive units bound with $\mathrm{F}$-ligand. Parameters are as indicated in Table 12.3. (A) A 3-D surface topography, (B) its related contour plot, and (C) its 2-D dose-response curve for fixed ratio between increasing concentrations of drugs. Two vertical arrows in panel $A$ indicate the projection of the $70 \%$ isobole, and is represented in panel $\mathrm{B}$. This isobole is curved representing classical 'synergy'. Raising a plane vertical through the arrow in panel A's concentration plane for two ligands, i.e., following a fixed ratio of drug concentrations, cuts a curve in A's surface response plot as reproduced in panel $\mathrm{C}$. 
A

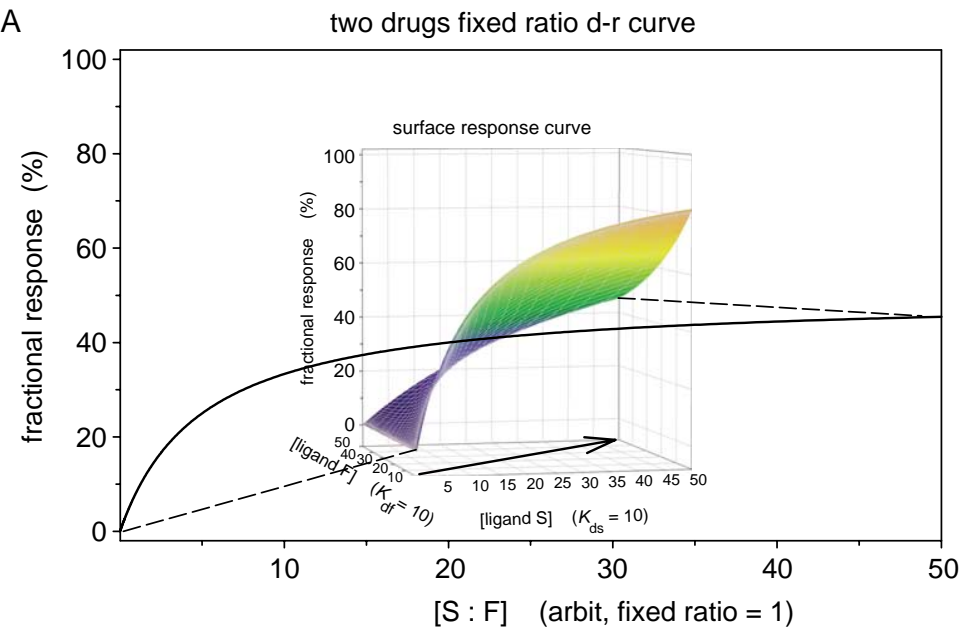

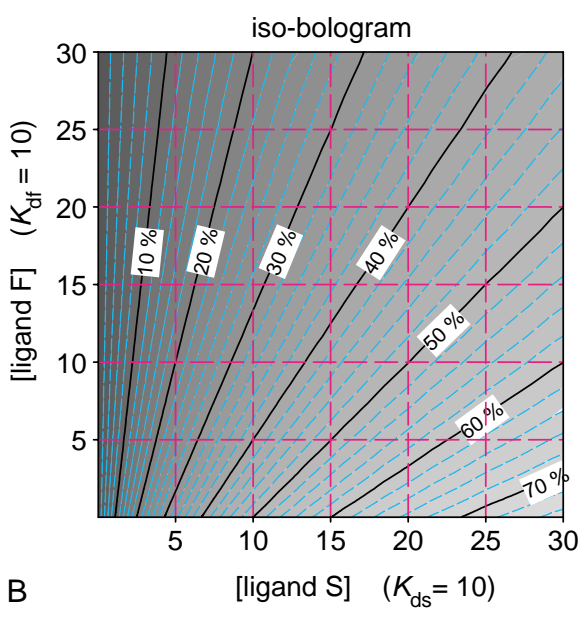

B

\section{Competitive ant-agonism}

Figure 12.6. Dose-response curve for competitive ant-agonism with its surface plot and related isobologram. The competitive ant-agonism is based on $S /(1+S+F)$, where ' $S$ ' stands for receptive units with bound $S$-ligand and ' $F$ ' is receptive units bound with F-ligand. Parameters are as indicated in Table 12.3. Panel A shows the dose-response for increasing concentrations of both drugs in a fixed ratio (1:1), with the related 3-D surface plane for varying concentrations of each drug as insert. Panel $B$ is the contour plot that drops under the 3-D surface. Intervention co-operativity coefficient $c$ is 0 . See Table 12.3.

the response is due to simple competition between the two drugs added simultaneously, then $P_{\mathrm{R}}$ is identical to the ratio of drug dissociation constants, i.e., $K_{\mathrm{d} 1} / K_{\mathrm{d} 2}$.

In mixtures of the two drugs, their concentrations are designated by [d1] for drug 1 and [d2] for drug 2. Therefore, in a mixture of drug 1 and drug 2, assuming simple additivity in effect for the drugs, we may replace an amount of drug 1 in the mixture by drug 2, which together generates the same response as elicited by either [D1] of D1 alone or [D2] of D2 alone. Similarly, in a mixture of the two drugs, we may replace an amount of [D2] by an amount of drug 1 to obtain the same response as for D2 alone or $\mathrm{D} 1$ alone. Thus, when we compare doses, in case of simple competitive additivity we get:

$$
\begin{aligned}
& {[\mathrm{D} 1]=[\mathrm{d} 1]+\left([\mathrm{d} 2] \cdot P_{\mathrm{R}}\right) \vee} \\
& {[\mathrm{D} 2]=[\mathrm{d} 2]+\left([\mathrm{d} 1] / P_{\mathrm{R}}\right),}
\end{aligned}
$$

which is the same as:
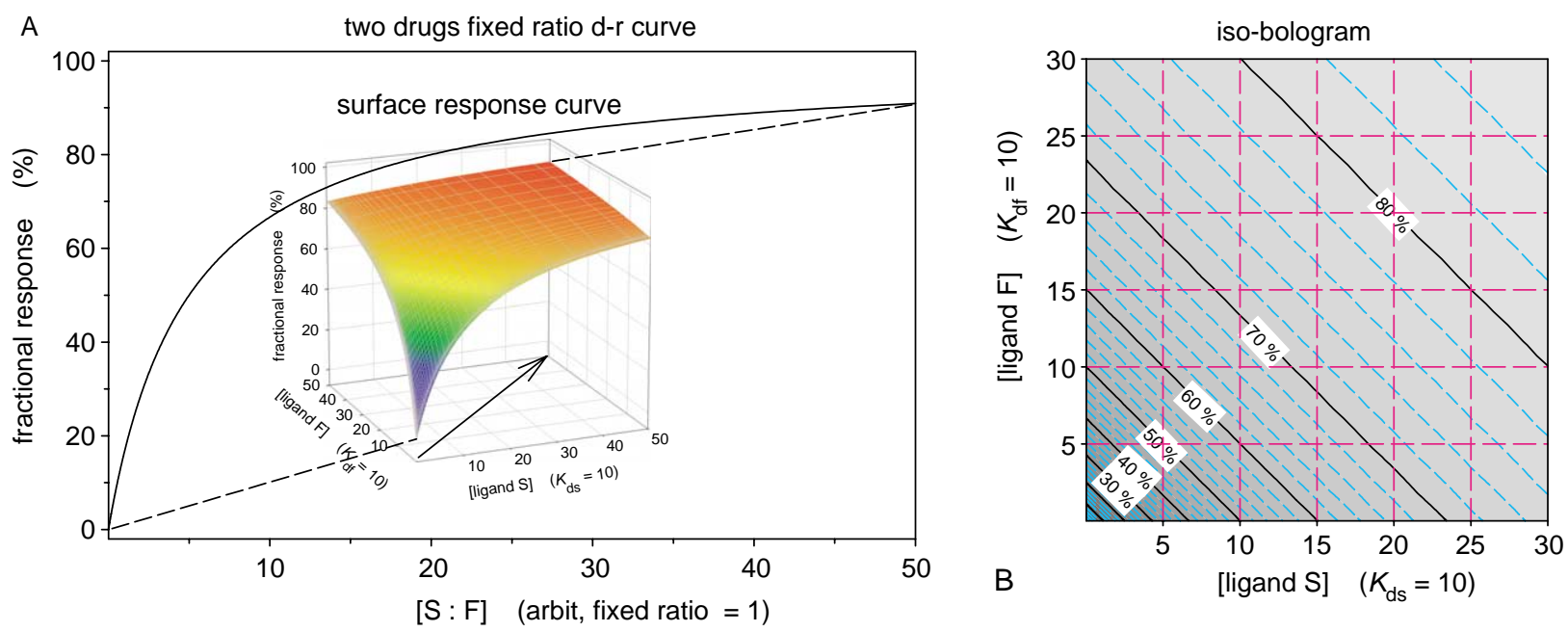

\section{Competitive "additivity"}

Figure 12.7. Dose-response curve for competitive additivity with its surface plot and related isobologram. The competitive additivity is based on $(S+F) /(1+S+F)$, where ' $S$ ' stands for the receptive units with bound S-ligand and ' $F$ ' is the receptive units bound with F-ligand. Parameters are as indicated in Table 12.3. Panel A shows the dose-response for increasing concentrations of both drugs in a fixed ratio (1:1), with the related 3-D surface plane for varying concentrations of each drug as insert. Panel $B$ is the contour plot that drops under the 3-D surface. Intervention co-operativity coefficient $c$ is 0 . See Table 12.3. 

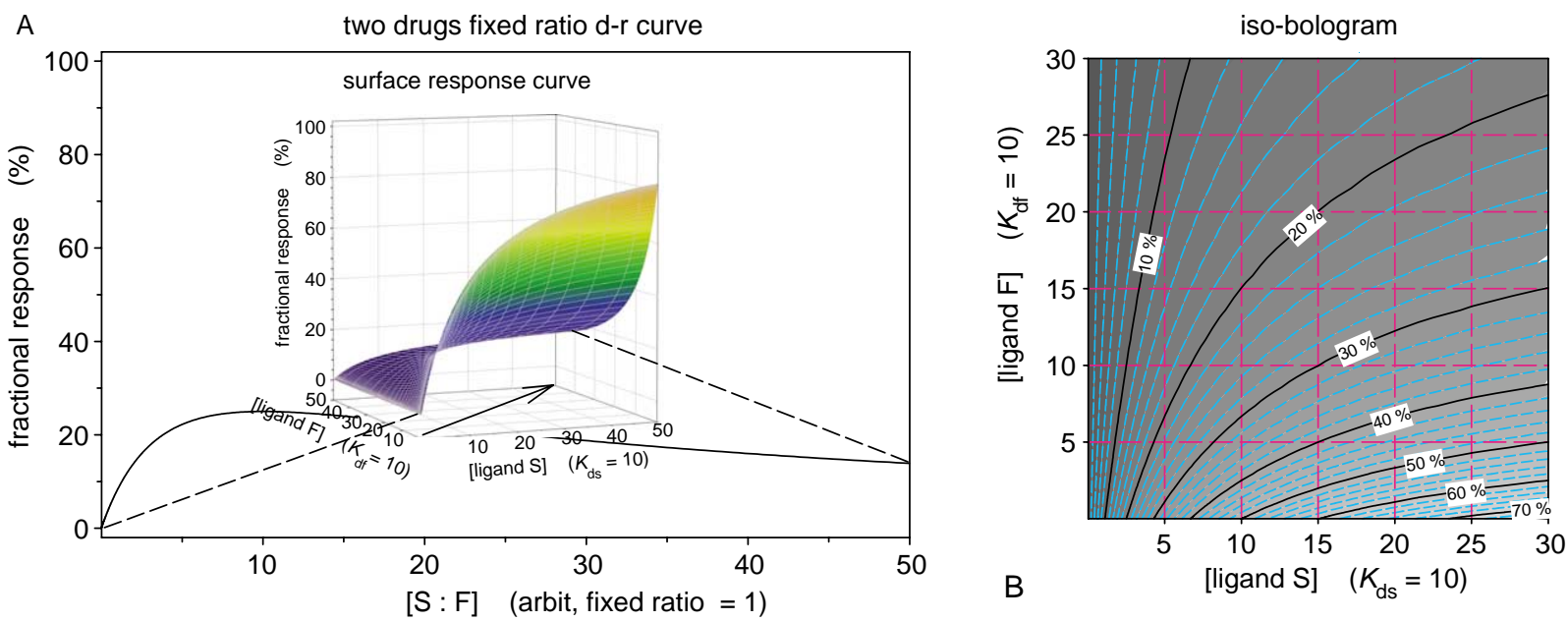

Non-competitive "ant-agonism"

Figure 12.8. Dose-response curve for non-competitive 'ant-agonism' with its surface plot and related isobologram. The noncompetitive ant-agonism is based on $S /(1+S+F+S F)$, where ' $S$ ' stands for the receptive units with bound $S$-ligand, ' $F$ ' is the receptive units bound with F-ligand, and 'SF' represent receptive units with both and $\mathrm{F}$ bound. Parameters are as indicated in Table 12.3. Panel A shows the dose-response for increasing concentrations of both drugs in a fixed ratio (1:1), with the related 3D surface plane for varying concentrations of each drug as insert. Panel B is the contour plot that drops under the 3-D surface. Intervention co-operativity coefficient $c$ is 1 . See Table 12.3.

$$
\begin{aligned}
& {[\mathrm{D} 1]=[\mathrm{d} 1]+[\mathrm{d} 2] \cdot([\mathrm{D} 1] /[\mathrm{D} 2]) \vee} \\
& {[\mathrm{D} 2]=[\mathrm{d} 2]+[\mathrm{d} 1] \cdot([\mathrm{D} 2] /[\mathrm{D} 1]),}
\end{aligned}
$$

where [d1] and [d2] are doses of drug 1 and 2 when combined in a mixture to elicit the same effect as that of a single drug's application. Both expressions in Eq. 12.1 may be rewritten to:

$$
1=\frac{[\mathrm{d} 1]}{[\mathrm{D} 1]}+\frac{[\mathrm{d} 2]}{[\mathrm{D} 2]} .
$$

A similar relationship to Eq. 12.2 was deduced from simple geometry by Loewe and Muischnek (1926) and Loewe (1928). The relation in Eq. 12.2 has been adopted by successor synergy modelers (Chou \& Talalay 1984; Berenbaum 1989, 1991; Tallarida 1992, 2000; Greco et al. 1995; Chou 2006).
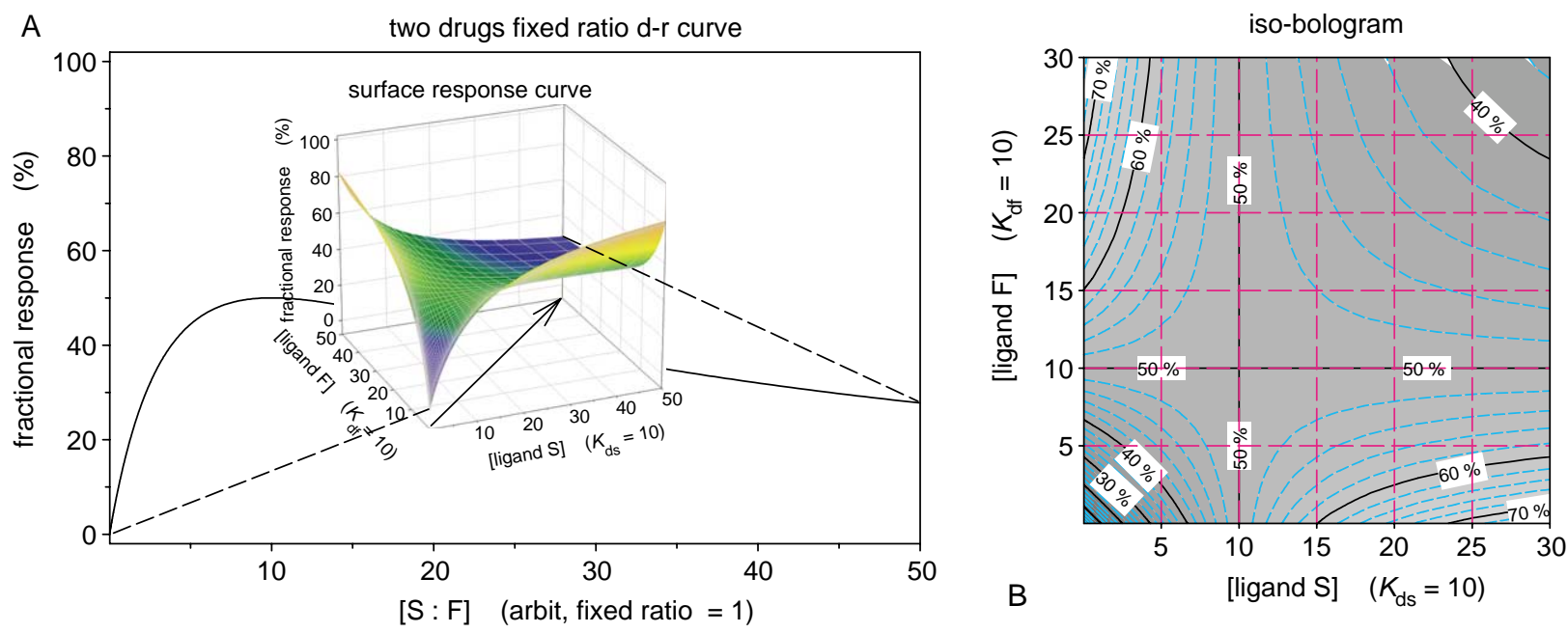

Non-competitive "additivity"

Figure 12.9. Dose-response curve for non-competitive 'additivity' with its surface plot and related isobologram. The noncompetitive additivity is based on $(S+F) /(1+S+F+S F)$, where ' $S$ ' stands for the receptive units with bound $S$-ligand, ' $F$ ' is receptive units bound with F-ligand, and 'SF' represent receptive units with both $\mathrm{S}$ and $\mathrm{F}$ bound. Parameters are as indicated in Table 12.3. Panel A shows the dose-response for increasing concentrations of both drugs in a fixed ratio (1:1), with the related 3D surface plane for varying concentrations of each drug as insert. Panel B is the contour plot that drops under the 3-D surface. Intervention co-operativity coefficient $c$ is 1 . See Table 12.3. 

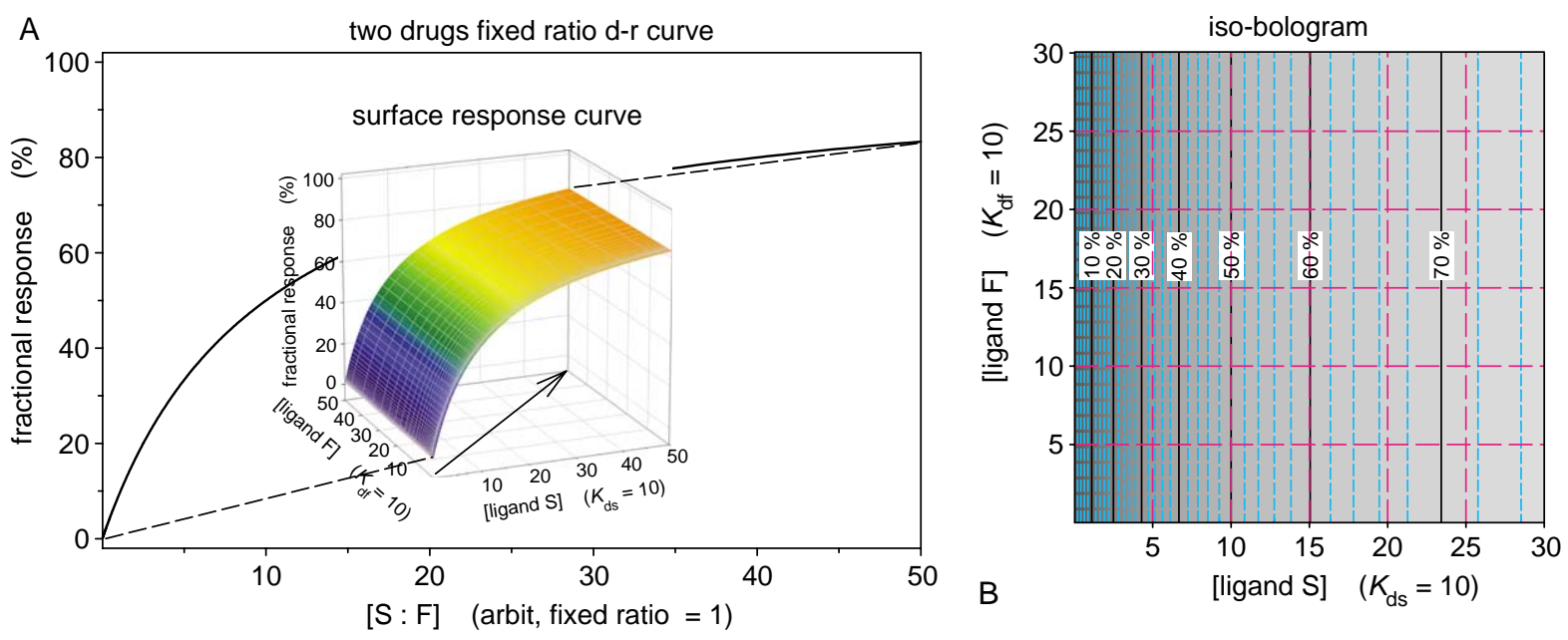

Non-competitive occupancy

Figure 12.10. Dose-response curve for non-competitive occupancy with its surface plot and related isobologram. The noncompetitive occupancy is based on $(S+S F) /(1+S+F+S F)$, where ' $S$ ' stands for the receptive units with bound $S$-ligand, ' $F$ ' is receptive units bound with F-ligand, and 'SF' represent receptive units with both $S$ and $F$ bound. Parameters are as indicated in Table 12.3. Panel A shows the dose-response for increasing concentrations of both drugs in a fixed ratio (1:1), with the related 3D surface plane for varying concentrations of each drug as insert. Panel B is the contour plot that drops under the 3-D surface. Intervention co-operativity coefficient $c$ is 1 . See Table 12.3.

At face value the relations in Eqs. 12.1 and 12.2 are always a matter of summation of concentrations of two or more drugs in which the single drug can elicit the same effect alone or in combination. In case the level of equi-effect is $50 \%$, i.e., the median effect (Trevan 1927), the combination of concentrations for two drugs for this effect may be equated as:

$$
1=\frac{[\mathrm{d} 1]}{\mathrm{ED}_{50,1}}+\frac{[\mathrm{d} 2]}{\mathrm{ED}_{50,2}}
$$

in which $\mathrm{ED}_{50,1}$ and $\mathrm{ED}_{50,2}$ are the effective doses of the two drugs eliciting the same response (50\% of maximal) due to either drug; while in combination it is [d1]+ [d2].

Equations 12.1-12.3 are based on an additivecompetition scheme as demonstrated in Fig. 12.4. In Fig. 12.4 (graph A) the $70 \%$ isobole was selected instead of a $50 \%$ effect, but this should not disturb the meaning.
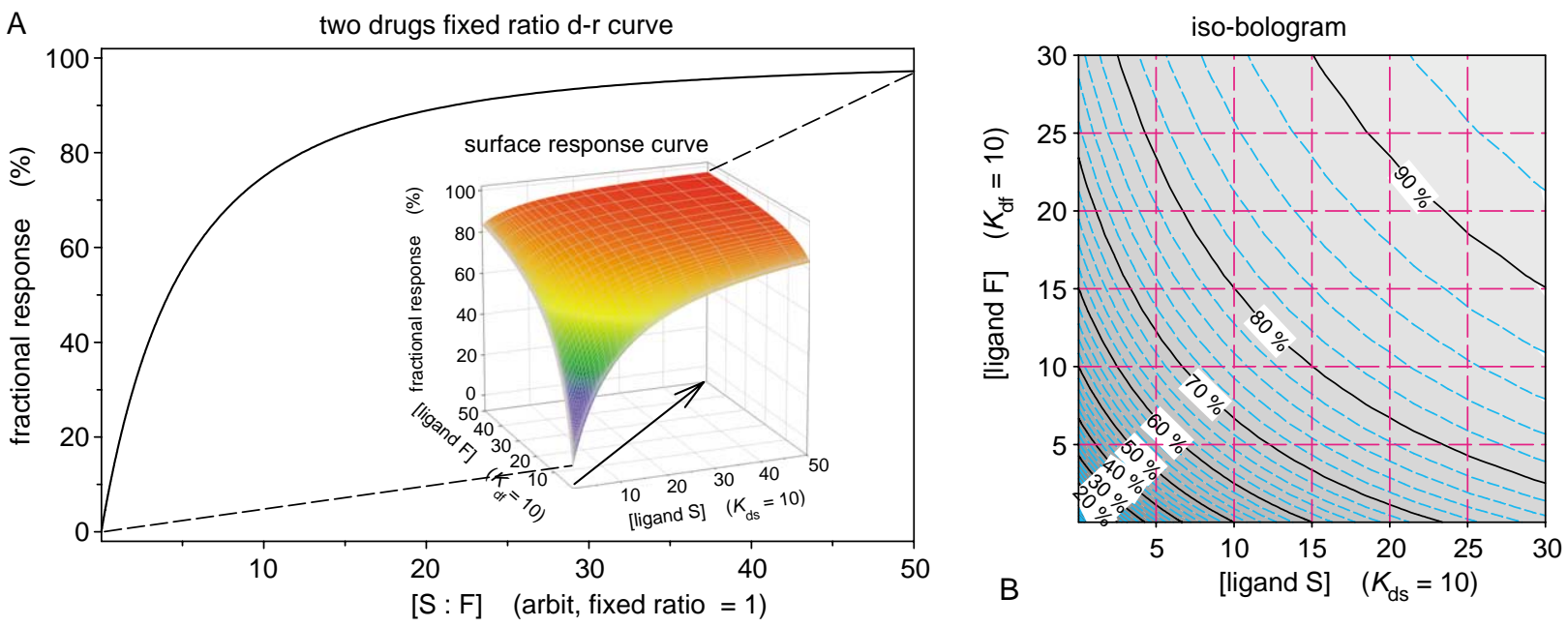

Non-competitive synergism

Figure 12.11. Dose-response curve for non-competitive 'synergism' with its surface plot and related isobologram. The noncompetitive synergism is based on $(S+F+S F) /(1+S+F+S F)$, where ' $S$ ' stands for receptive units with bound $S$-ligand, ' $F$ ' is receptive units bound with F-ligand, and 'SF' represent receptive units with both $S$ and $F$ bound. Parameters are as indicated in Table 12.3. Panel A shows the dose-response for increasing concentrations of both drugs in a fixed ratio (1:1), with the related 3D surface plane for varying concentrations of each drug as insert. Panel B is the contour plot that drops under the 3-D surface. Intervention co-operativity coefficient $c$ is 1 . See Table 12.3 . 
A

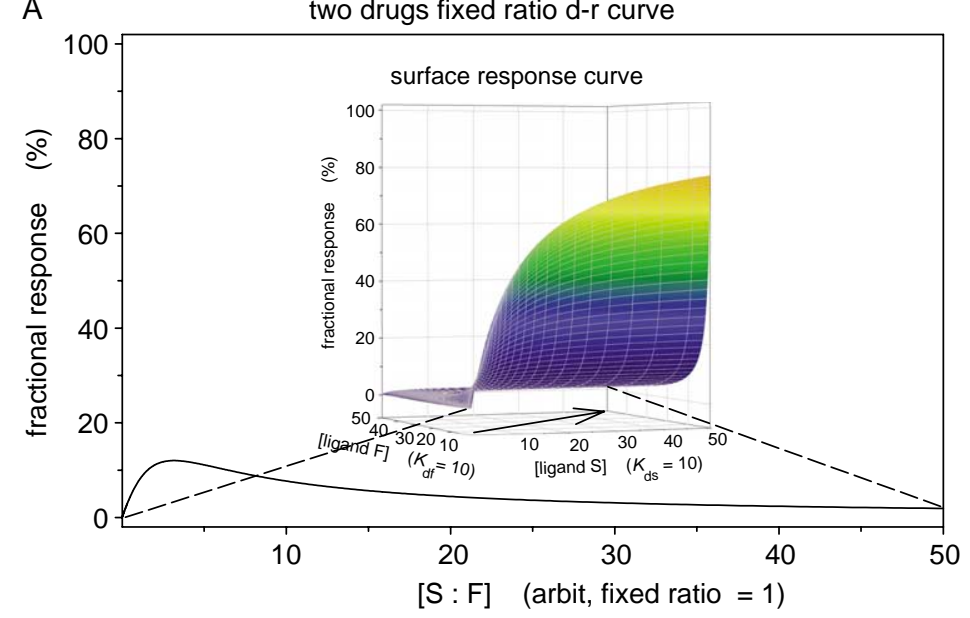

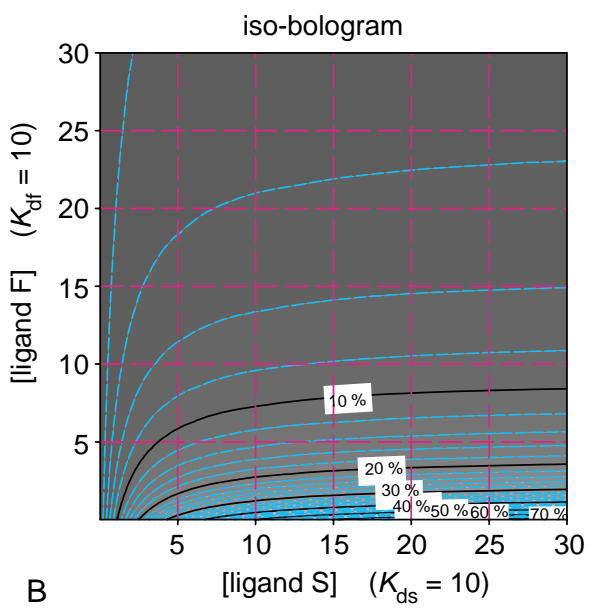

B

Positive interventory "ant-agonism"

Figure 12.12. Dose-response curve for positive interventory 'ant-agonism' with its surface plot and related isobologram. The positive interventory ant-agonism is based on $S /(1+S+F+S F)$, where ' $S$ ' stands for receptive units with bound $S$-ligand, ' $F$ ' is receptive units bound with F-ligand, and 'SF' represent receptive units with both $\mathrm{S}$ and $\mathrm{F}$ bound. Parameters are as indicated in Table 12.3. Panel A shows the dose-response for increasing concentrations of both drugs in a fixed ratio (1:1), with the related 3D surface plane for varying concentrations of each drug as insert. Panel B is the contour plot that drops under the 3-D surface. Intervention co-operativity coefficient $c$ is 10 . See Table 12.3.

\subsubsection{Interaction Index and Combination Index}

Ideas about synergy, additivity and ant-agonism are the basis for other expressions of drug interaction, e.g., the Interaction Index (Berenbaum 1977, 1985; Tallarida 2000: Chapter 10) and the Combination Index (CI) (Chou \& Talalay 1984).

The Interaction Index is a simple rewriting of the Loewe formulation. Thus $\left([\mathrm{d} 1]+[\mathrm{d} 2] P_{\mathrm{R}}\right) / a$, in which $a$ is an index of interaction and $P_{\mathrm{R}}$ is the relative potency and assumed constant. For $a=1$ (or is it $a=1 /[\mathrm{D} 1]$ ?) this formula is identical to the Loewe formulation, meaning simple competitive additivity. The value of $a$ is the Interaction Index. If $a<1$ there is synergy, and if $a>1$ there is ant-agonism. Tallarida writes that $a$ 'indicates the degree of dosage reduction in a combination in order to get the effect of [D1] alone or [D2] alone' (Tallarida 2000: Chapter 10). The CI by Chou and Talalay is a slightly expanded form of the Interaction Index based on the median-effect formulation (see Section 12.2.2). The CI
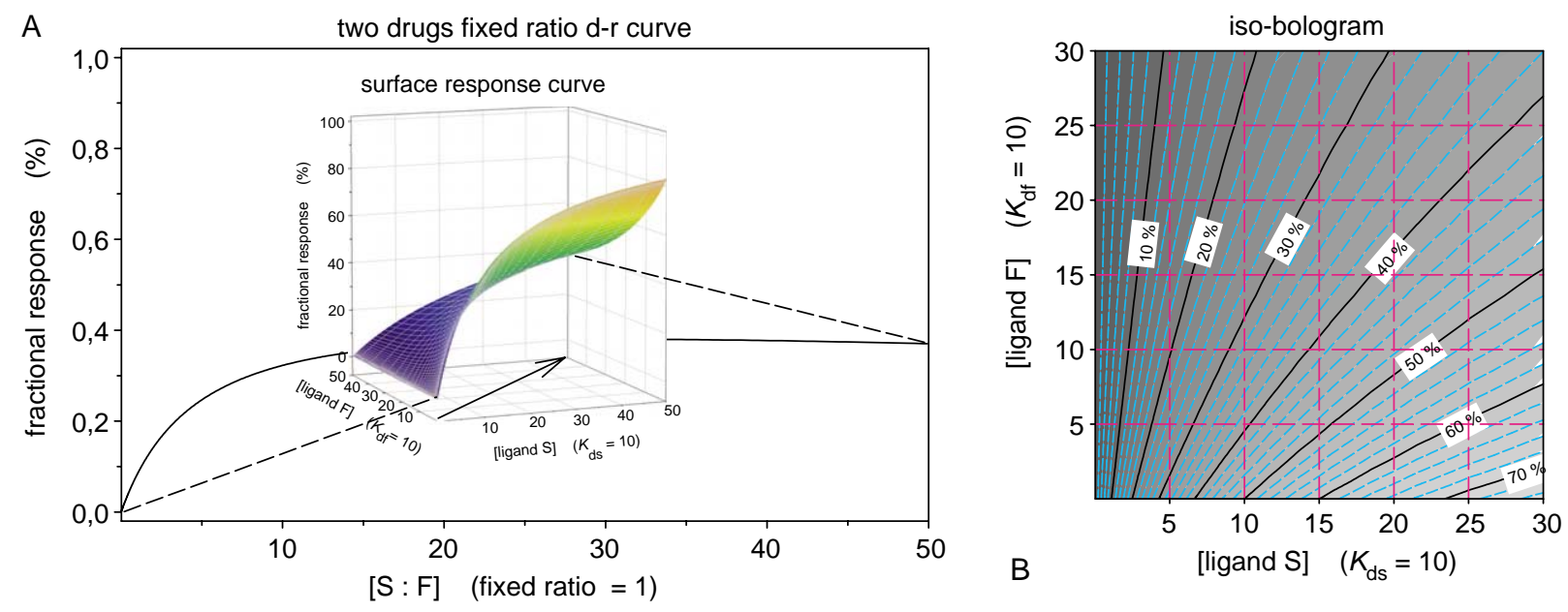

Negative interventory "ant-agonism"

Figure 12.13. Dose -response curve for negative interventory 'ant-agonism' with its surface plot and related isobologram. The positive interventory ant-agonism is based on $S /(1+S+F+S F)$, where ' $S$ ' stands for the receptive units with bound $S$-ligand, ' $F$ ' is receptive units bound with F-ligand, and 'SF' represent receptive units with both $\mathrm{S}$ and $\mathrm{F}$ bound. Parameters are as indicated in Table 12.3. Panel A shows the dose-response for increasing concentrations of both drugs in a fixed ratio (1:1), with the related 3D surface plane for varying concentrations of each drug as insert. Panel B is the contour plot that drops under the 3-D surface. Intervention co-operativity coefficient $c$ is 0.1 . See Table 12.3 . 
A

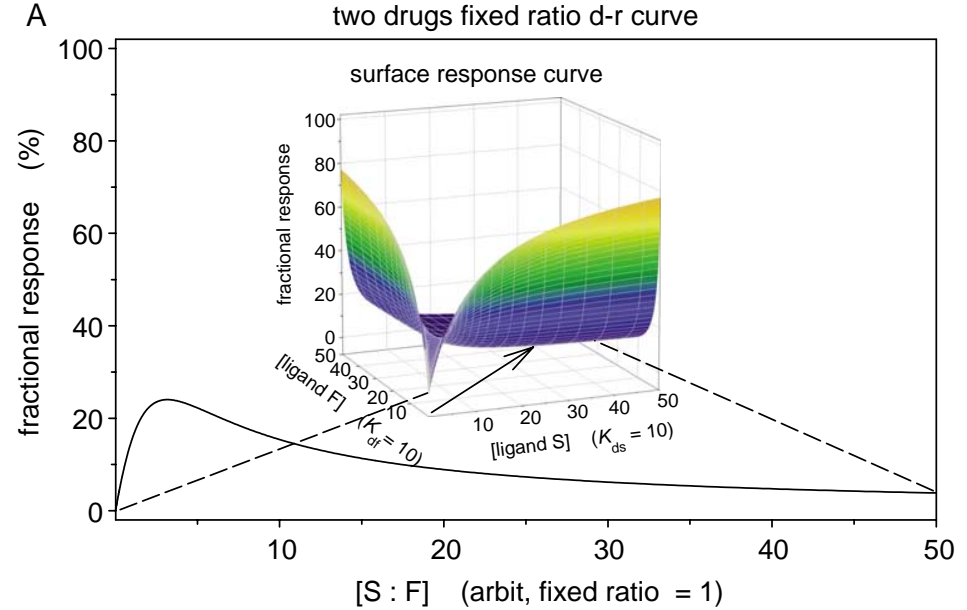

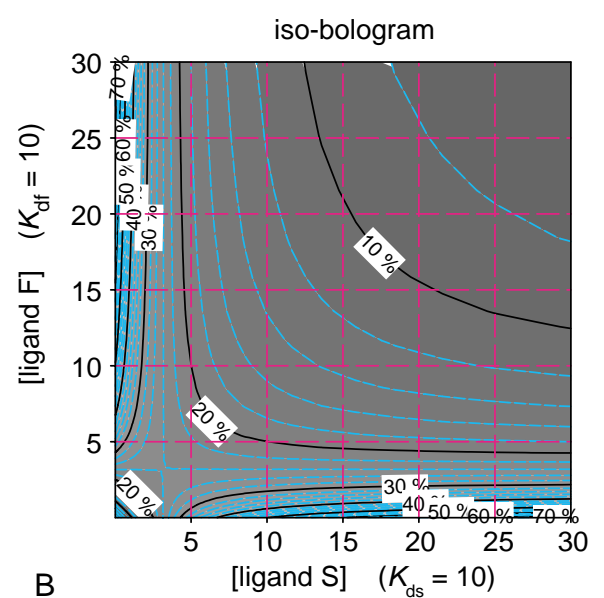

Positive interventory "additivity"

Figure 12.14. Dose-response curve for positive interventory 'additivity' with its surface plot and related isobologram. The positive interventory additivity is based on $(S+F) /(1+S+F+S F)$, where ' $S$ ' stands for the receptive units with bound $S$-ligand, ' $F$ ' is receptive units bound with $F$-ligand, and 'SF' represent receptive units with both $S$ and $F$ bound. Parameters are as indicated in Table 12.3. Panel A shows the dose-response for increasing concentrations of both drugs in a fixed ratio (1:1), with the related 3-D surface plane for varying concentrations of each drug as insert. Panel B is the contour plot that drops under the 3D surface. Intervention co-operativity coefficient $c$ is 10 . See Table 12.3 .

takes care of both competitive and non-competitive interactions and possible multi-sited receptive units (Chou \& Talalay 1984). For competitive interactions, the CI is identical to its Interaction Index.

The isobologram, the Interaction Index (Loewe's equation), and the CI (relative fractional-effect), all at the organ level, may be said to be the answer to Schild's null-method for competitive interactions at the receptor level (Chapter 11). Therefore, when it comes to the effects of drugs at integrated levels in the whole body, CI rather than Schild is the answer. Although the Schild

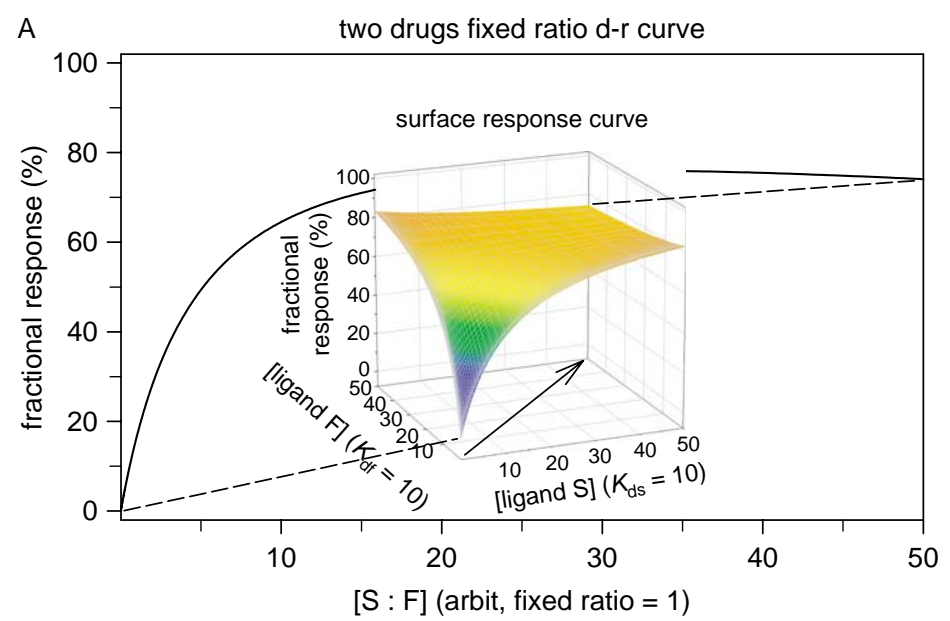

tool is valid for receptors with more than one binding site (Colquhoun 2007), at the level of receptor, cell, or whole organism, it is only workable for competitive synagics. In addition, competitive synagics is often not relevant in combinatorial drug therapy.

\subsubsection{Random Choice of Models for Integrated Systems}

Models of reaction schemes are needed when analyzing the affects of adding two or more drugs simultaneously

B

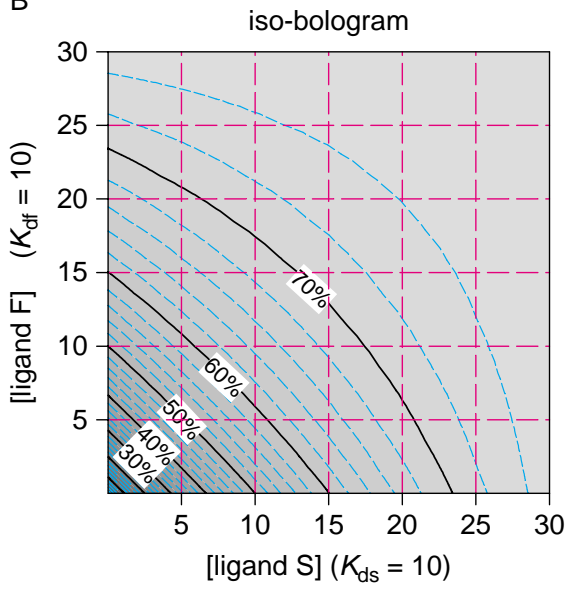

Negative interventory "additivity"

Figure 12.15. Dose response curve for negative interventory 'additivity' with its surface plot and related isobologram. The negative interventory additivity is based on $(S+F) /(1+S+F+S F)$, where ' $S$ ' stands for receptive units with bound $S$-ligand, ' $F$ ' is receptive units bound with F-ligand, and 'SF' represent receptive units with both $S$ and $F$ bound. Parameters are as indicated in Table 12.3. Panel A shows the dose-response for increasing concentrations of both drugs in a fixed ratio (1:1), with the related 3D surface plane for varying concentrations of each drug as insert. Panel $B$ is the contour plot that drops under the 3-D surface. Intervention co-operativity coefficient $c$ is 0.1 . See Table 12.3 . 
A

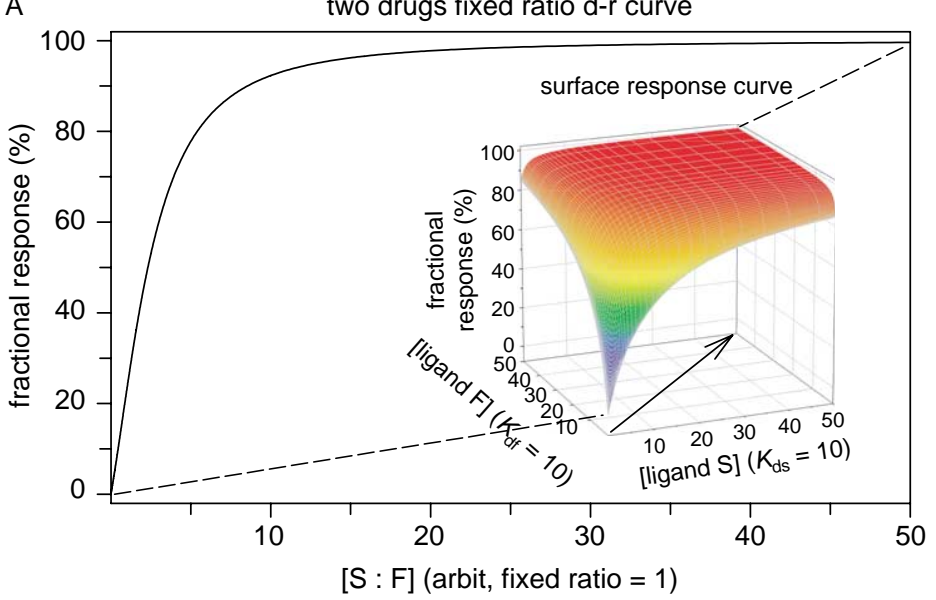

B

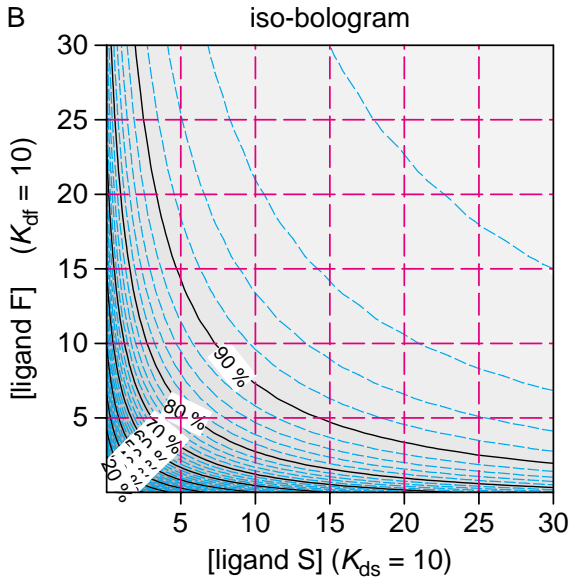

Positive interventory synergism

Figure 12.16. Dose-response curve for positive interventory 'occupancy' with its surface plot and related isobologram. The positive interventory occupancy is based on $(S+S F) /(1+S+F+S F)$, where ' $S$ ' stands for receptive units with bound $S$-ligand, ' $F$ ' is receptive units bound with F-ligand, and 'SF' represent receptive units with both $S$ and $F$ bound. Parameters are as indicated in Table 12.3. Panel A shows the dose-response for increasing concentrations of both drugs in a fixed ratio (1:1), with the related 3D surface plane for varying concentrations of each drug as insert. Panel B is the contour plot that drops under the 3-D surface. Intervention co-operativity coefficient $c$ is 10 . See Table 12.3.

to complex systems as whole organisms with their integrated regulatory networks. Greco et al. (1995) presented a splendid survey and comparison of sixteen different synergy models. Due to the complexity of the studied systems, their conclusion on the correct model to use seems to be anybody's guess. Therefore, the selection of a 'pertinent' model is generally empirical and random. It illustrates the impossibility of deducing and choosing mechanisms significantly behind observed actions of drug combinations. As an example, for chemotherapy in whole organisms a certain model is

A

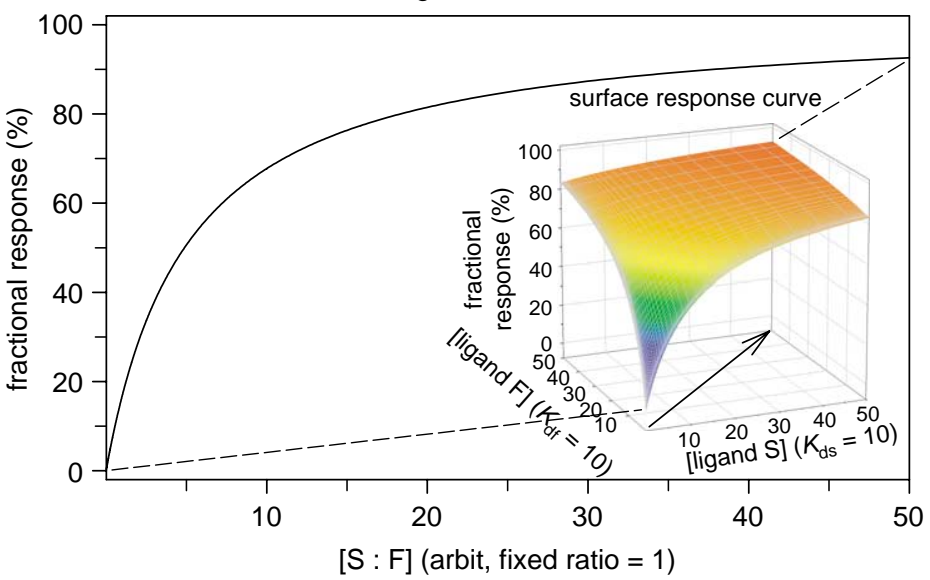

often selected unexpectedly (Teicher 2003). This applies to combinations of several drugs, for just two drugs, or even for the effects of single drug applications in, for instance, humans. Standard algorithms suggested for the complex integrated systems are often based on the exponential function, the Poisson distribution, the binominal distribution, or the very popular modified Hill equation (Chou \& Talalay 1981; Greco et al. 1995).

I shall not comment on these various approaches, however I would ask the interested reader to look in the three aforementioned recent reviews which contain an

Negative interventory synergism

Figure 12.17. Dose-response curve for negative interventory 'occupancy' with its surface plot and related isobologram. The negative interventory occupancy is based on $(S+S F) /(1+S+F+S F)$, where ' $S$ ' stands for receptive units with bound $S$-ligand, ' $F$ ' is receptive units bound with F-ligand, and 'SF' represent receptive units with both $S$ and $F$ bound. Parameters are as indicated in Table 12.3. Panel A shows the dose-response for increasing concentrations of both drugs in a fixed ratio (1:1), with the related 3-D surface plane for varying concentrations of each drug as insert. Panel B is the contour plot that drops under the 3$\mathrm{D}$ surface. Intervention co-operativity coefficient $c$ is 0.1 . See Table 12.3 . 
A

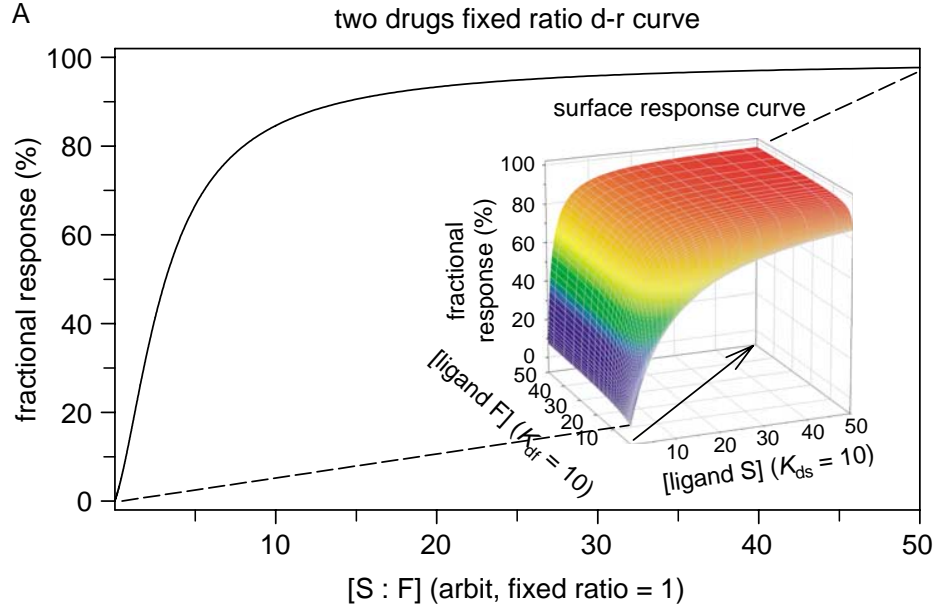

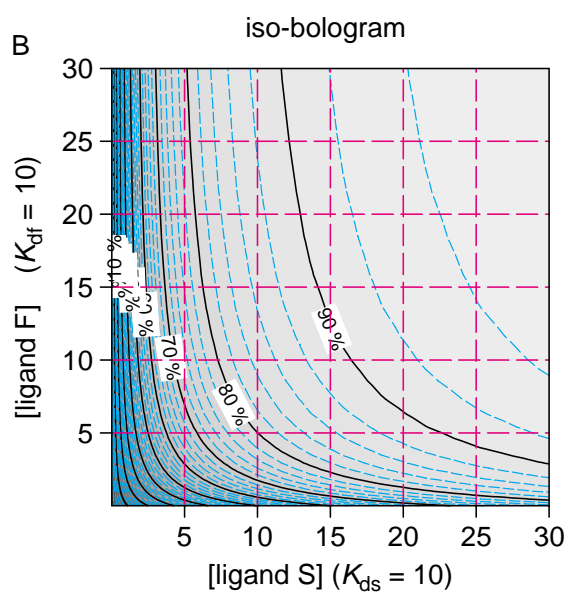

Positive interventory occupancy

Figure 12.18. Dose-response curve for positive interventory 'functional synergism' with its surface plot and related isobologram. The positive interventory synergism is based on $(S+F+S F) /(1+S+F+S F)$, where ' $S$ ' stands for receptive units with bound $S$ ligand, ' $F$ ' is receptive units bound with $F$-ligand, and 'SF' represent receptive units with both $S$ and $F$ bound. Parameters are as indicated in Table 12.3. Panel A shows the dose-response for increasing concentrations of both drugs in a fixed ratio (1:1), with the related 3-D surface plane for varying concentrations of each drug as insert. Panel B is the contour plot that drops under the 3D surface. Intervention co-operativity coefficient $c$ is 10 . See Table 12.3.

excellent comparison between different mathematical models and their mutual evaluation by methods as the response surface modeling, the simple isobologram, median effect/Combination Index, and other means (Berenbaum 1989; Greco et al. 1995: with a summary in Table 5; Chou 2006).

In reading these reviews, it should become obvious that the weak point in analysis is the haphazard selection of reaction schemes for the combined action of two drugs in most cases of synergy - the super-additivity to subadditivity world at the level of cells or the whole body.
The mathematical model with the best fit to experimental data is obviously the preferred one (Greco et al. 1995).

In addition, the difficulties mount to hardships in the real world when aiming at a description of concentrationeffect relations for mixtures of more than two drugs given to whole organisms in combinatorial drug therapy. Experimental chemotherapy often involves three compounds in combination (Snyder et al. 2000; Ciombor \& Lima 2005; Gillenwater et al. 2005; Rougier \& Lepere 2005), sometimes with synergy-like beneficial effects as found in the first two referred studies. Therefore,
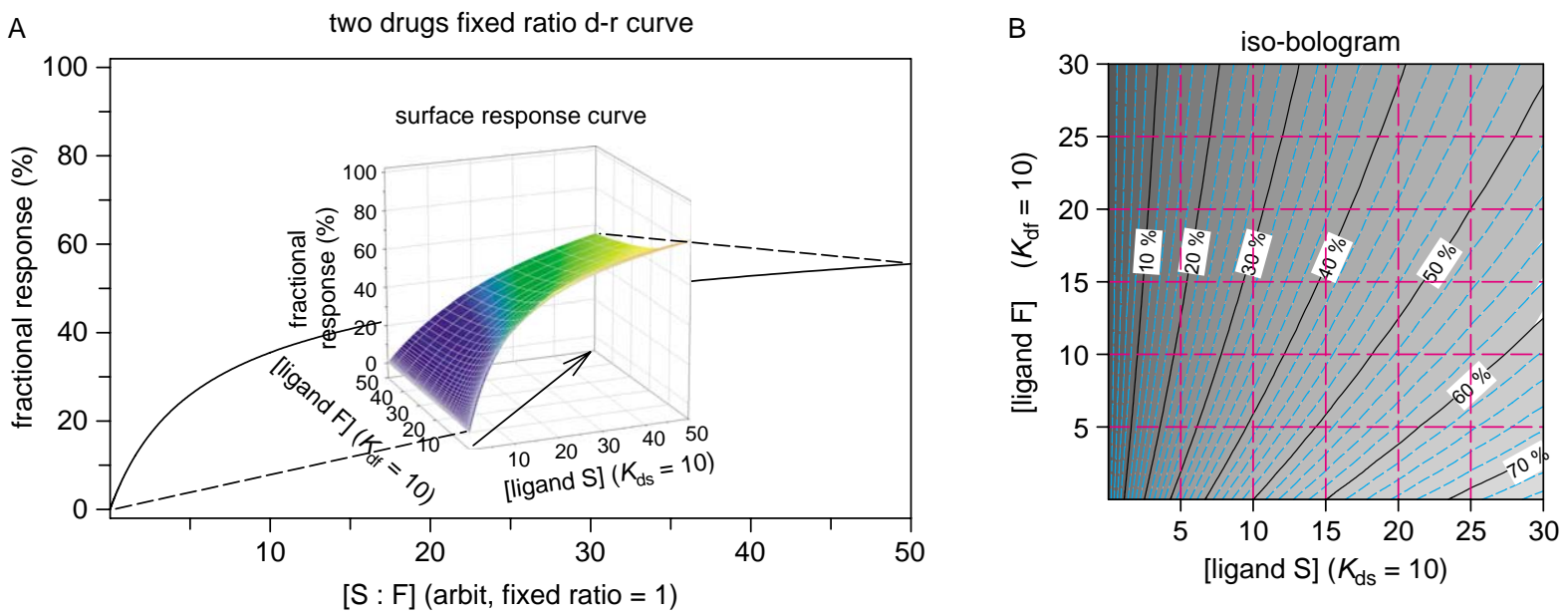

Positive interventory occupancy

Figure 12.19. Dose-response curve for negative interventory 'functional synergism' with its surface plot and related isobologram. The negative interventory synergism is based on $(S+F+S F) /(1+S+F+S F)$, where ' $S$ ' stands for receptive units with bound S-ligand, ' $F$ ' is receptive units bound with F-ligand, and 'SF' represent receptive units with both $S$ and $F$ bound. Parameters are as indicated in Table 12.3. Panel A shows the dose-response for increasing concentrations of both drugs in a fixed ratio (1:1), with the related 3-D surface plane for varying concentrations of each drug as insert. Panel $B$ is the contour plot that drops under the 3-D surface. Intervention co-operativity coefficient $c$ is 0.1 . See Table 12.3 . 


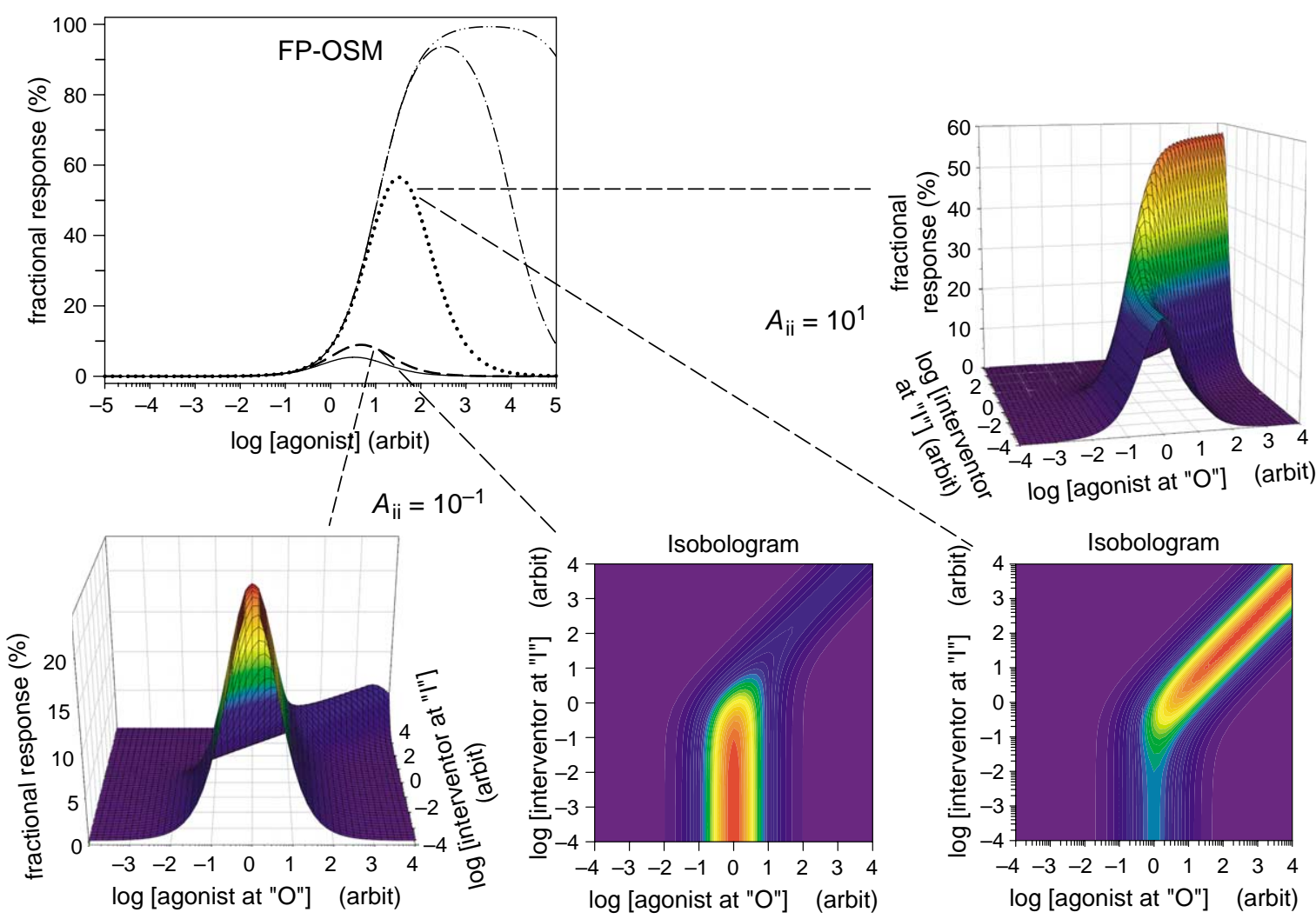

Figure 12.20. Dose-response plots of the four-pane one-state model (FP-OSM) with active conformations RS and IRS. To simplify, it is assumed that parameters $A_{\mathrm{ss}}, A_{\mathrm{ss}}^{\text {is }}$, and $A_{\mathrm{ss}}^{\text {is }}$ are equal, while $A_{\mathrm{si}}$ is also equal to $A_{\mathrm{si}}^{\mathrm{is}}$ and $A_{\mathrm{si} \text {. The parameters are thus }}^{\mathrm{i} \text {. The }}$ independent of what is bound to the opposite sites. Five 2-D plots are generated with an interventor concentration [I] equal 10 and parameters $A_{\mathrm{ss}}=1, A_{\mathrm{si}}=1, A_{\mathrm{is}}=1$, while $A_{\mathrm{ii}}$ is varied from $10^{-3}(-)$ to $10^{5}(-\cdots-)$ in five steps by a factor $10^{2}$. As indicated with dashed lines, the 2-D plot for $A_{\mathrm{ii}}=10^{-1}$ may be transformed to a related set of a 3-D surface plot and an isobologram plus a similar set for the 2-D plot at $A_{\mathrm{ii}}=10$. The 3-D surface plot at $A_{\mathrm{ii}}=10^{-1}$ is similar to the profile as generated by Winding and Bindslev (1993, Fig 10) also assuming active conformations RS and IRS in the FP-OSM, but with somewhat different parameters in analysis for an optimal-fixed-concentration-ratio (OFCOR) between [S] and [I].

difficulties in model selection are even more grotesque and insurmountable when it is realized that actual regimes require reaction schemes with more than two drugs applied simultaneously. The same is true when it is further recognized that these schemes should also consider possible significant and important displacement of responses for the different drugs with time (Barrera et al. 2005).

\subsubsection{My Strategy for Synergy}

The vast obstacles for a genuine understanding of the underlying mechanisms described in Section 12.1.7 are not the subject of this chapter, rather I will focus on a few simple and fairly well-described mechanistic reaction schemes involving just two drugs: the intervention model described in Chapter 2 and the allosteric twostate model (ATSM) described in Chapter 7. Let us learn from an examination of their kind of synergy at the receptor level, and afterwards judge if the conclusions are relevant for analysis of synergy when combining several drugs at the level of whole organisms.
Therefore, to put the issue in perspective, I shall start in Sub-chapter 12.2 with an ultra-brief survey of the analysis of the synagics for exclusive and non-exclusive reaction schemes - as it was developed for the classic synergy approach by Chou and Talalay (1977, 1981, 1984) and Chou (2006). 'Exclusive' is the same as 'competitive' or 'non-inclusive', while 'non-exclusive' is the same as 'non-competitive' or 'inclusive' (see Chapter 2 ). Variations in these standard models are mostly obtained by including Hill's exponentiation.

Then as an alternative, this Hill-adaptation of models is replaced by allowing an induced variation in binding constants. In Sub-chapter 12.3, I will demonstrate how one may obtain diverse isobolograms from a very simple ternary component model, where two drugs ( $\mathrm{S}$ and $\mathrm{F}$ ) can bind simultaneously to a third component, leading to isoboles that deviate somewhat from those adopted in Fig. 12.2. The model referred to is the intervention reaction scheme with its interaction constant $c$ (see Chapter 2), which opens for induced variations in binding constants. Contrary to the non-competitive model that also has simultaneous binding, in the 


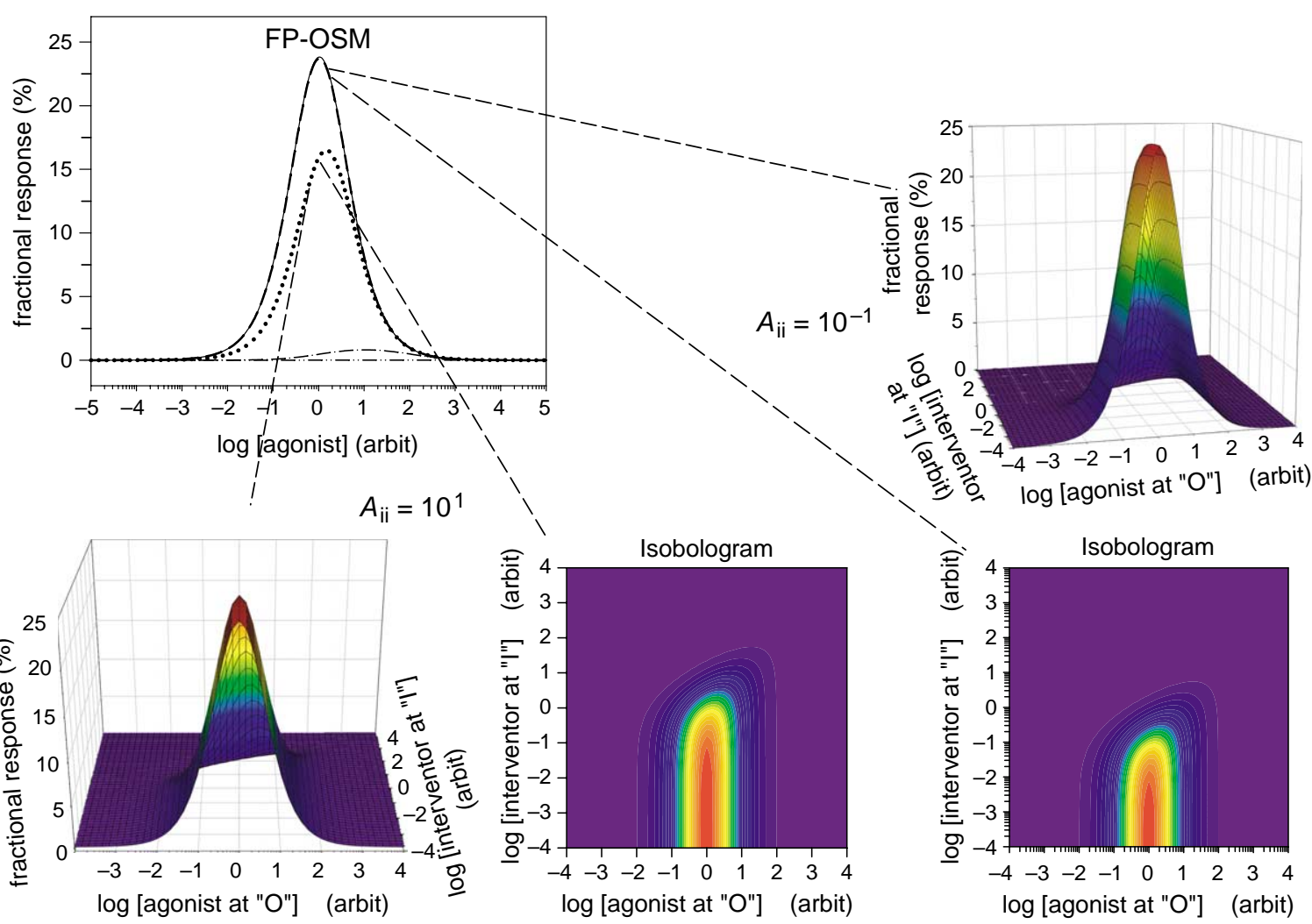

Figure 12.21. Dose-response plots of the four-pane one-state model (FP-OSM) with RS as the sole active conformation. To simplify, it is assumed that parameter $A_{\mathrm{ss}}$ is also equal to $A_{\mathrm{ss}}^{\mathrm{is}}$ and $A_{\mathrm{ss}}^{\mathrm{ii}}$ while $A_{\mathrm{si}}$ is also equal to $A_{\mathrm{si}}^{\mathrm{is}}$ and $A_{\mathrm{ss}}^{\mathrm{ii}}$, thus independent of what is bound to the opposite binding sites. Five 2-D plots are generated with an interventor concentration [I] equal $10^{-1}$ and parameters $A_{\mathrm{ss}}=1, A_{\mathrm{si}}=1, A_{\mathrm{is}}=1$ while $A_{\mathrm{ii}}$ is varied from $10^{-3}(-)$ to $10^{5}(-\cdot-)$ in five steps by a factor $10^{2}$. As indicated with dashed lines, the 2-D plot for $A_{\mathrm{ii}}=10^{-1}$ may be transformed to a related set of a 3-D surface plot and an isobologram plus a similar set for the 2-D plot at $A_{\mathrm{ii}}=10$.

intervention model it is the binding constants that vary upon binding at neighboring sites. Constant $c$ deviates from unity. The intervention model is too simple for what occurs in the whole body. On the other hand, it seems correct to have a sense of what the predictions are at the most simplistic level before we move on to more advanced systems, as an entire organism, and their analyses of drug combinations.

\subsection{Mechanistic Synergy at the Receptor Level. 1. Classic Models}

\subsubsection{A Modern-classic Synergy Approach}

To handle synergy, additivity, and ant-agonism, general equations were derived for modes of competitive, noncompetitive, and un-competitive inhibition, as well as for models of sequential and ping-pong type reaction schemes. These equations for different forms of drug ant-agonism and drug synergism at the single receptor level were then combined into one expression (Chou \& Talalay 1977):

$$
\frac{1}{a r_{1,2, \ldots n}}=\left[\sum \frac{1}{a r_{\mathrm{j}}}\right]-\frac{n-1}{a r_{0}}
$$

where $a r_{1,2, \ldots n}$ is the actual response induced by $n$ inhibitors, $a r_{\mathrm{j}}$ is the actual response of the $j$ th inhibitor, and $a r_{0}$ the actual response in the absence of any inhibitor. ${ }^{1}$

Equation 12.4 was subsequently reformulated into its relative relationships (Chou \& Talalay 1981). The relative relations by Chou and Talalay may be formulated as:

$$
\frac{a r_{\mathrm{j}}}{r r_{\mathrm{j}}}=\frac{a r_{\mathrm{j}}}{1-a r_{\mathrm{j}}},
$$

where $a r_{\mathrm{j}}$ is the actual response for compound $\mathrm{j}$, and $r r_{\mathrm{j}}$ is the remaining response expected when the concentration of $j$ is increased, as also formulated in Subchapter 8.3 . 


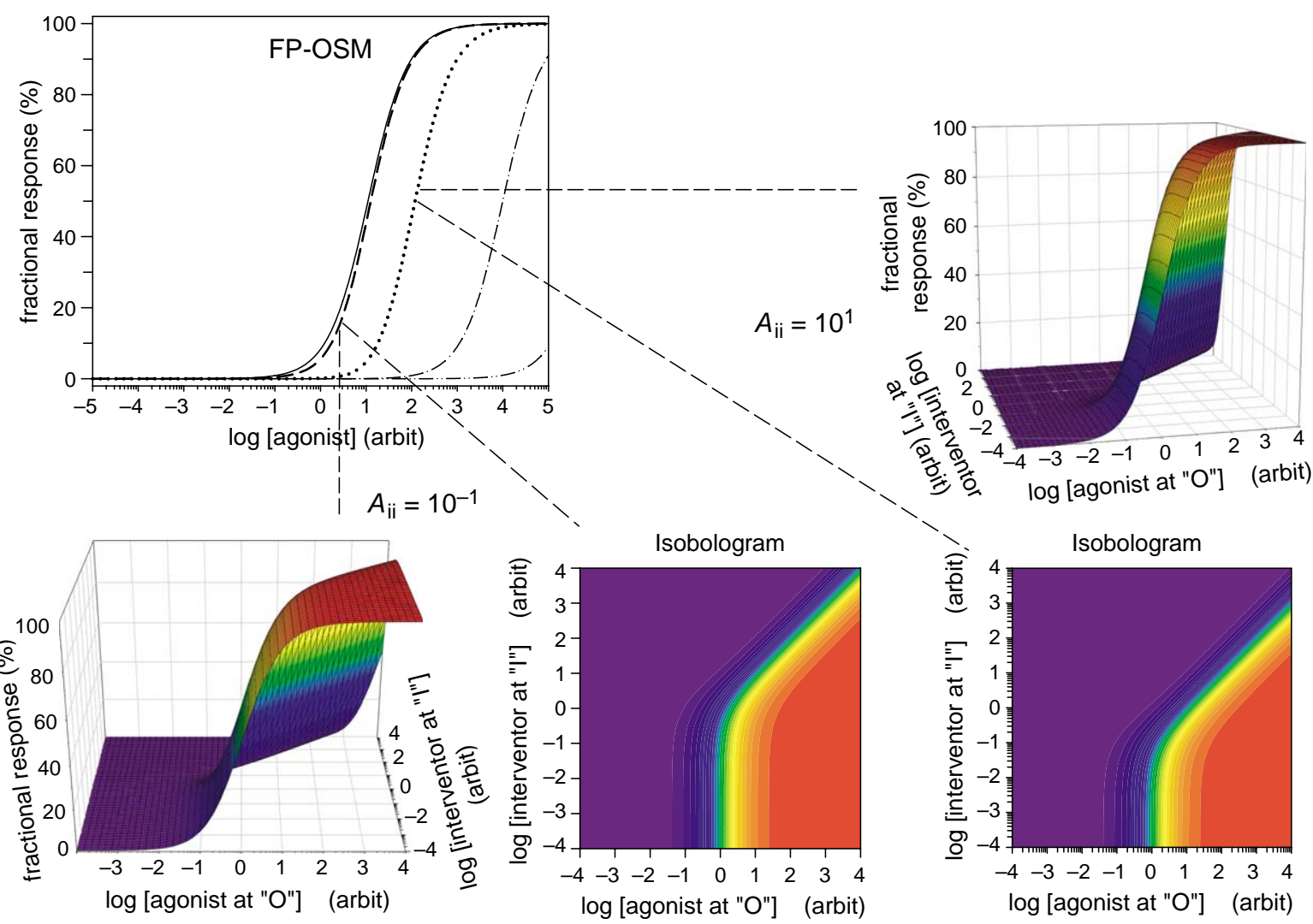

Figure 12.22. Plots of the four-pane one-state model (FP-OSM) with active conformations RS and SRS. To simplify, it is assumed that parameter $A_{\mathrm{ss}}$ is equal to $A_{\mathrm{ss}}^{\mathrm{is}}$ and $A_{\mathrm{ss}}^{\mathrm{ii}}$, while $A_{\mathrm{si}}$ is equal to $A_{\mathrm{si}}^{\mathrm{is}}$ and $A_{\mathrm{si}}^{\mathrm{ii}}$. Parameters are thus independent of what is bound to the opposite binding sites. Five 2-D plots are generated with an interventor concentration [I] equal 10 and parameters $A_{\mathrm{ss}}=1, A_{\mathrm{si}}=1, A_{\mathrm{is}}=1$, while $A_{\mathrm{ii}}$ is varied from $10^{-3}(-)$ to $10^{5}(-\cdot-)$ in five steps by a factor $10^{2}$. As indicated with dashed lines, the 2-D plot for $A_{\mathrm{ii}}=10^{-1}$ may be transformed to a related set of a 3-D surface plot and an isobologram plus a similar set for the 2-D plot at $A_{\mathrm{ii}}=10$. Compare the plots with plots in Fig. 12.23.

A reformulation of Eq. 12.5 for two competitive inhibitors as supplemented by Chou and Talalay (1981) and rewritten in our terminology is:

$$
\frac{a r_{12}}{r r_{12}}=\frac{a r_{1}}{r r_{1}}+\frac{a r_{2}}{r r_{2}}
$$

where $r r_{12}, r r_{1}$, and $r r_{2}$ are the remaining responses after the addition of inhibitors 1 and 2 simultaneously or one at a time as indicated in the subscripts, and the actual response $(a r)$ is also subscripted according to the presence of either ligands 1 or 2 , or both.

Based on the fact that the distribution equations for systems with a limited number of receptive units can always be formulated by listing the sum of all possible receptor conformations in the denominator of their distribution equation (Segel 1975, 1993; Chou \& Tallay 1981), we thus have the following relation for the noncompetitive (inclusive) reaction scheme (as depicted in Fig. 2.4-1 $\square$ ), where constant $c=1$ :

$$
\frac{a r}{T R}=\frac{\text { nominator }}{1+\mathrm{S} / K_{\mathrm{s}}+\mathrm{F} / K_{\mathrm{f}}+\left(\mathrm{S} / K_{\mathrm{s}}\right) \cdot \mathrm{F} / K_{\mathrm{f}}} .
$$

'Nominator' is determined by the type of receptor complexes participating in the measured response. $\mathrm{S}$ as usual represents one type of ligand and $\mathrm{F}$ another type. Subscript $\mathrm{f}$ refers to the secondary ligand $\mathrm{F}$, and $K_{\mathrm{f}}$ is the dissociation constant for $\mathrm{F}$. The term ' $\mathrm{ar} / \mathrm{TR}$ ' is the actual response over total response. Compare Eq. 12.7 with Eq. 2.3 in Chapter 2.

Thus, for the non-competitive (non-exclusive or inclusive) situation, with a Chou and Talalay (1981) formulation, where $\mathrm{S}$ is replaced by 1 and $\mathrm{F}$ by 2 , we get:

$$
\frac{a r_{12}}{r r_{12}}=\frac{a r_{1}}{r r_{1}}+\frac{a r_{2}}{r r_{2}}+\frac{a r_{1}}{r r_{1}} \cdot \frac{a r_{2}}{r r_{2}} .
$$

Formulations for adding more than two inhibitory ligands of both competitive, non-competitive, and uncompetitive action may be developed and these formulations may further be expanded in the paradigm of the Hill equation. Based on such relations, the general equations for multi-drug application and receptors with several binding sites, $m$, is given by Chou and Talalay (1981). 


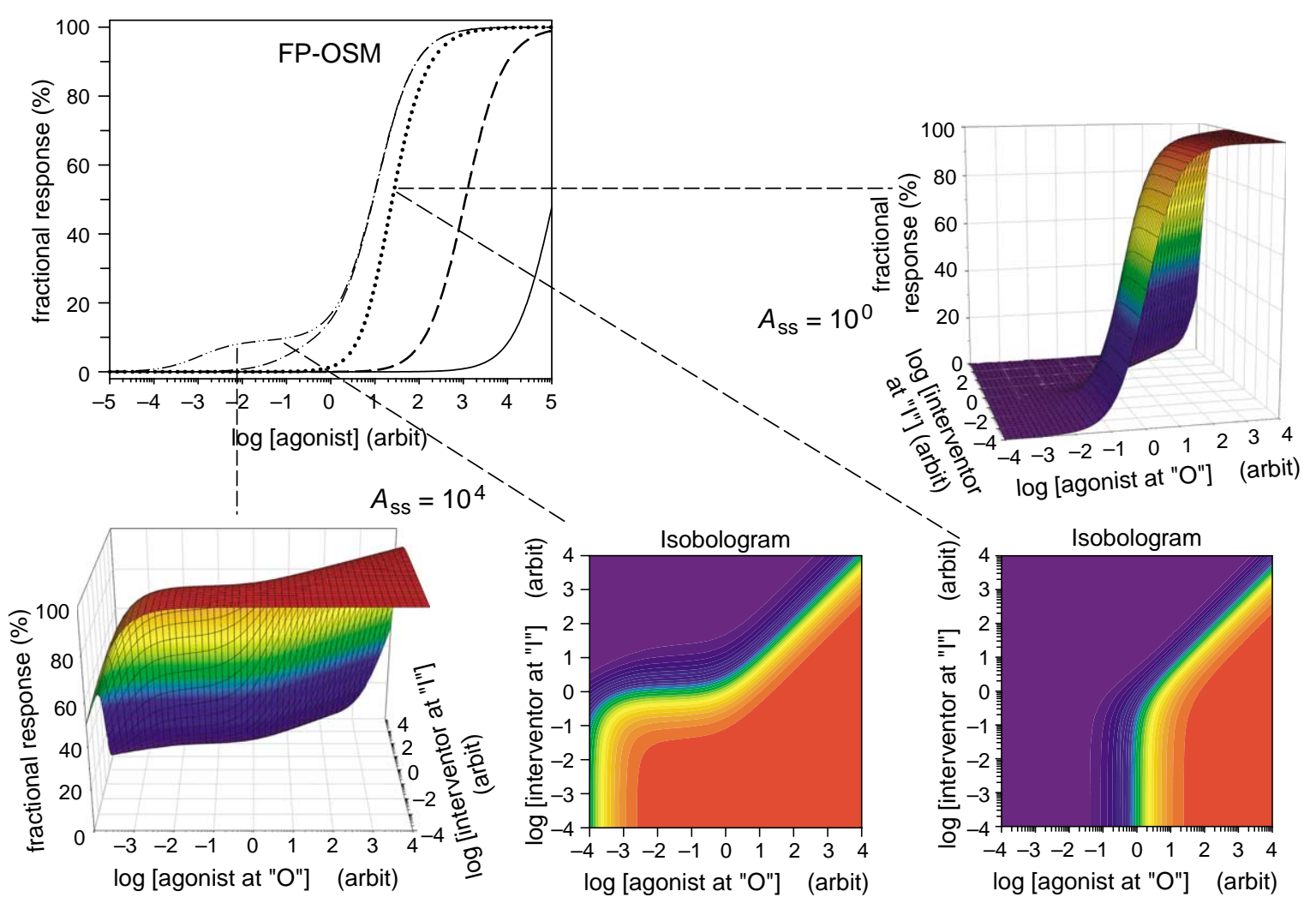

Figure 12.23. Plots of the four-pane one-state model (FP-OSM) with active conformations RS and SRS. To simplify, it is assumed that parameter $A_{\mathrm{ss}}$ is equal to $A_{\mathrm{ss}}^{\mathrm{is}}$ and $A_{\mathrm{ss}}^{\mathrm{ii}}$, while $A_{\mathrm{si}}$ is equal to $A_{\mathrm{si}}^{\mathrm{is}}$ and $A_{\mathrm{si}}^{\mathrm{ii}}$. Parameters are thus independent of what is bound to the opposite binding sites. Five 2-D plots are generated with an interventor concentration [I] equal 10 and parameters $A_{\mathrm{ii}}=1, A_{\mathrm{si}}=1, A_{\mathrm{is}}=1$, while $A_{\mathrm{ss}}$ is varied from $10^{-4}(-)$ to $10^{4}(-\cdot-)$ in five steps by a factor $10^{2}$. As indicated with dashed lines, the 2-D plot for $A_{\mathrm{ss}}=10^{4}$ may be transformed to a related set of a 3-D surface plot and an isobologram plus a similar set for the 2-D plot at $A_{\mathrm{ss}}=1$. For $A_{\mathrm{ss}}=10^{4}$, the dose-response curve has an intermediate plateau. Compare the plots with plots in Fig. 12.22 .

Simply exponentiate or raise-to-a-power each term in Eq. 12.8 or its expanded forms. If the receptor has $m$ identical binding sites allowing for more complex modeling, the exponent will be $1 / m$ for each term (Chou \& Talalay 1981).

For the non-competitive situation with two-inhibitors, the equation by a Hill-type expansion becomes:

$$
\left[\frac{a r_{12}}{r r_{12}}\right]^{1 / m}=\left[\frac{a r_{1}}{r r_{1}}\right]^{1 / m}+\left[\frac{a r_{2}}{r r_{2}}\right]^{1 / m}+\left[\frac{a r_{1}}{r r_{1}} \cdot \frac{a r_{2}}{r r_{2}}\right]^{1 / m}
$$

where $m$ is a Hill coefficient and under certain conditions equal to the number of binding sites; here equal 2. Consult Chou and Talalay (1981: Appendix II) for a detailed derivation of Eq. 12.9. Although, Chou and Talalay specifically noted that the formulation in Eq. 12.9 requires binding of inhibitors in one step, therefore Eq. 12.9 is not a real mechanistic formulation, relations based on similar ideas as expressed in Eq. 12.9 are in general use today (Chou et al. 1994; Greco et al. 1995; Scaramellini et al. 1997; Minto et al. 2000; Short et al. 2002; Jonker et al. 2003, 2005; Bouillon et al. 2004; Lin et al. 2004; Chou 2006; Adusumilli et al. 2006; Nair et al. 2007).

\subsubsection{The Median-effect Plot}

The median-effect at effective dose $\mathrm{ED}_{50}$ (Trevan 1927) introduces the relation between the relative terms in Eqs. 12.8 and 12.9 and the concentrations of ligands relative to their apparent dissociation constants, $\mathrm{ED}_{50,1}$ and $\mathrm{ED}_{50,2}$. Thus, the median-effect equation for noncompetitive interaction for two drugs at 'higher order conditions' is given by Chou and Talalay (1984):

$$
\begin{aligned}
{\left[\frac{a r_{12}}{r r_{12}}\right]^{1 / m}=} & \frac{[\mathrm{d} 1]}{\left[\mathrm{ED}_{50}\right]_{1}}+\frac{[\mathrm{d} 2]}{\left[\mathrm{ED}_{50}\right]_{2}} \\
& +\frac{[\mathrm{d} 1] \cdot[\mathrm{d} 2]}{\left[\mathrm{ED}_{50}\right]_{1} \cdot\left[\mathrm{ED}_{50}\right]_{2}},
\end{aligned}
$$

and also for inhibitors (Chou 2006). It must be emphasized that exponentiation is a mathematical tool, but not necessarily a mechanistic tool, as it presupposes simultaneity.

This kind of simultaneity is a 'Hillian ghost', as described in Chapter 10, constantly prevailing in the above models (Eqs. 12.9 and 12.10). 
Berenbaum (1989) and Greco et al. (1995) have interesting discussions and reservations about the accuracy and validity of the Chou and Talalay equation as repeated in Eq. 12.10 (Chou \& Talalay 1981, 1984), and its meaning in relation to synergy. Meanwhile, since the Chou and Talalay formulation refers to the ratio between actual response and remaining response it is derived correctly. Although briefly commented upon by Chou and Talalay (1981), a remaining concern by Greco et al. (1995: Appendix A) is about restrictions imposed by introducing a Hill coefficient, as also discussed by Berenbaum (1989, pp. 108-116).

However, the obtained indication or pseudo-quantification of a system's co-operative interaction using Eq. 12.10 (Chou 2006) remains useful as with the ordinary Hill analysis described in Chapters 8 and 10.

\subsubsection{Bliss's Formulation of Independence a la Greco}

According to a formulation by Bliss, the 'independent joint action' of ligand interaction of a combined signal for two drugs was formulated as a sum of the fractional effect, $f$, for each individual ligand minus their product. The equation writes:

$$
f_{12}=f_{1}+f_{2}-f_{1} \cdot f_{2},
$$

Bliss (1939: Eq. 2). It is based on Abbott's formula, which came from studies by Tattersfield and Morris as well as by Abbott (Finney 1971, pp. 125-126).

In terms of probabilistic 'non-occurrence' we have:

$$
f_{12}=f_{1} \cdot f_{2},
$$

where $f$ now stands for the fractional response of the subscripted ligand(s) (Greco et al. 1995). The 'product' formulation in Eq. 12.12 is not surprising considering its origin from a field on probabilities of survival. The conceptual leap from Eq. 12.11 to Eq. 12.12, i.e., from fractional responses to probabilities is shrewdly described by Berenbaum (1989: Eqs. 13 and 14).

In case the appropriate expression of response as a function of drug concentration for each individual drug is given by a Hill-type expression, then for the product in Eq. 12.12 we have:

$$
\begin{aligned}
f_{12} & =\frac{a r}{T R} \\
& =\frac{\left([\mathrm{d} 1] /\left[\mathrm{ED}_{50}\right]_{1}\right)^{m_{1}} \cdot\left([\mathrm{d} 2] /\left[\mathrm{ED}_{50}\right]_{2}\right)^{m_{2}}}{\left.\left(1+\left([\mathrm{d} 1] /\left[\mathrm{ED}_{50}\right]_{1}\right]\right)^{m_{1}}\right) \cdot\left(1+\left([\mathrm{d} 2] /\left[\mathrm{ED}_{50}\right]_{2}\right)^{m_{2}}\right)},
\end{aligned}
$$

which is equal to Eq. 12 in Greco et al. (1995), in which their term $\mathrm{E} / \mathrm{Econ}$ is equal to $a r / T R$.

\subsubsection{Flagship Formulation by El Greco}

Based on suggestions by Berenbaum (1985), Greco et al. derived an equation for non-exclusive independence similar to the formulation by Chou and Talalay (Eq. 12.10), but with two important modifications (Greco et al. 1995: Eq. 5). First, the number of binding sites, $m$, for either ligand was made independent of the binding sites for other ligands, thus for ligand $1=m_{1}$ and for ligand $2=m_{2}$, etc. Second, a proportionality factor, $\alpha$, was multiplied onto the cross-term of the formulation for a non-exclusive, i.e., non-competitive, reaction scheme. Thus, Eq. 5 of Greco et al. for non-competitive agonists in the present terminology may be written as:

$$
\begin{aligned}
1= & \frac{\mathrm{d}_{1}}{\mathrm{EC}_{50,1} \cdot\left[\frac{t r}{T R-t r}\right]^{1 / m_{1}}}+\frac{\mathrm{d}_{2}}{\mathrm{EC}_{50,2} \cdot\left[\frac{t r}{T R-t r}\right]^{1 / m_{2}}} \\
& +\frac{\alpha \cdot \mathrm{d}_{1} \cdot \mathrm{d}_{2}}{\mathrm{EC}_{50,1} \cdot \mathrm{EC}_{50,2} \cdot\left[\frac{t r}{T R-t r}\right]^{\left(1 / 2 m_{1}\right)+\left(1 / 2 m_{2}\right)}} \cdot
\end{aligned}
$$

Eq. 12.14 was quoted as a 'flagship equation' in the Greco et al. (1995) paper. It is a plain mathematical formulation, not a mechanistic one. Clearly the possibilities for differentiating between a number of possible binding sites for different ligands provides a more versatile approach, but increases the number of parameters to be determined or guessed. The El Greco flagship equation still has the inherent wandering 'Hillghost' (Chapter 10). Another concern of mine about the Greco flagship equation is the insertion of the proportionality factor $\alpha$ in the numerator of the right-most fraction in Eq. 12.14. Simply multiplying one of the terms with a new system constant give associations to an Ariënsfactor for 'algebraic balancing' (Ariens 1954); an approach left many years ago by mechanistic receptor analysts.

Meanwhile, our purpose is different. We will adhere to the simple intervention scheme covering both competitive, non-competitive, and positive or negative intervention synagics, ${ }^{2}$ in the presence of two different drugs and analyze their synergy by contour plots equal to isobolograms.

\subsection{Mechanistic Synergy at the Receptor Level. 2. The Intervention Model}

Thus far, the synergy models discussed in Sub-chapter 12.2 have implicated Hill coefficients when experimental data present relationships more complex than the 
competitive or non-competitive reaction schemes. Contrary to the Hill-type approach, in this Sub-chapter we will focus on a model that confines possible interaction upon binding of different ligands to operating with binding constants sensitive to preceding adsorption in the receptive unit of different ligands. The model is the heterointervention scheme described in Sub-chapter 2.5.

\subsubsection{Synergy and the Intervention Reaction Scheme}

By plotting in three dimensions the effects of applying two drugs simultaneously to a receptive system with two binding sites, we are merely depicting the combined effect of the two drugs in an intervention model and its various possibilities as developed in Chapter 2. The intervention model includes simultaneous binding, but excludes co-lateral binding (see Section 2.6.1). Equieffective responses in such a system with varying mixtures of ligand concentration, isoboles, are easily obtained with modern graphics software by a transformation of 3-D response surface plots in panel A in Figs. 12.4 and 12.5 , and converted to 2 -D contour plots, as also demonstrated in panel B of Figs. 12.4 and 12.5.

In order to obtain some systematization into modeling with rapidly expanding possibilities, I will divide the intervention scheme and its subdivisions of non-competitive and competitive action into different models. The intervention scheme can be subdivided based on a change in its parameter $c$. Parameter $c$ (see Chapter 2 ), can be (a) $=0$, i.e., equal to competitive synagics, (b) = unity, i.e., equal to non-competitive synagics, or (c) $>0$ and different from unity, i.e., equal to what may be termed synergism of intervention (Sub-chapter 2.5).

From this, fourteen models emerge by a change in parameter $c$ and further selecting different receptor conformations for the actual response, since the response varies with the receptor complexes involved. The fourteen possibilities are listed in Table 12.3 and the corresponding plots are in Figs. 12.6-12.19. Isobolograms are generated for each subdivision and results are commented upon in the legend to Table 12.3. Note that all isobolograms in Figs. 12.4-12.19 are linear-linear plots.

From Table 12.3 and its related figures it should be obvious that even with a model as simple as the chosen intervention scheme, it is not possible to maintain the original standards for synergy, additivity, and ant-agonism as stipulated by Loewe (1928, 1953, 1959), Chou and Talalay (1984), Bernenbaum (1989), Greco et al. (1995) and Tallarida (2000: Chapter 10). Rather, a reinterpretation is required, which will probably involve isobolograms, i.e., contour plots, defined separately for each model implicated in a multi-drug reaction scheme - be it at either of the three levels defined in Section 12.1.2, the body, organ or receptor level. Recognition of realistic isobolograms deviating from the simple form as in Fig. 12.2 was already evident to Loewe (1928, 1955a,b, 1957).

From the analysis of expected isobolograms for the simple intervention model, it seems more relevant to discuss whole body and multi-drug experiments based on the isobolograms pertaining to each individual synagic model implied instead of a generalized geometric construction as the Loewe formulation of additive competition (Eq. 12.2), shown in Figs. 12.4 and 12.7 (Tallarida 2000) or mathematical constructions as those by Chou and Talalay in Eq. 12.9 and Greco et al. in Eq. 12.14.

This will also be the conclusion in Sub-chapter 12.5 on isobolograms for the ATSM.

\subsubsection{Modeling of Two Drugs with Inclusive and Co-lateral Binding}

To cover all possibilities, modeling of two different drugs with inclusive and co-lateral binding requires a four-pane one-state model (FP-OSM) (Fig. 2.4). The model has eight independent variables and may further be evaluated according to which conformations are involved in the measured response. Results of a preliminary analysis of FP-OSM are transformed into contour plots (Figs. 12.20-12.23) in order to demonstrate the potential isobolograms of the model and allow for a comparison with classical isobolograms described in Section 12.1.4.

For certain parameter values in the FP-OSM with active conformations RS and SRS, there are intermediate plateaus for the dose-response curves (for an example see Fig. 12.23).

Table 12.4 lists some of the preliminary results for the isobolograms of FP-OSM. An analysis of this model has been partially covered in Chapter 2 and variations thereof discussed by Segel (1975, 1993), Wells (1992), Waelbroeck (1994), Hoare et al. (2000) among others.

\subsubsection{Auto-intervention and Synergy}

The auto-intervention model was developed in Subchapter 3.3. As in the preceding section, it is variations in three parameters, $A_{\mathrm{s}}, A_{\mathrm{m}}$, and $c^{\prime}$, which determines the behavior of the model. Two-dimensional plots are given in Figs. 3.3 and 3.4. Furthermore, the active conformations of the receptive unit may vary between the three types (cf Section 12.3.1).

Here we could supply the model with isobolographs in the form of contour plots based on 3-D plotting, but in reality this is only relevant on models for two drugs 
Table 12.4. Parameter-dependent behavior of three types of the four-pane one-state model (FP-OSM) with fixed-dose of interventor [l]

\begin{tabular}{|c|c|c|c|c|c|}
\hline \multirow{2}{*}{$\begin{array}{l}\text { Type of FP-OSM } \\
\text { RS + IRS are functional bell-shaped } \\
\text { (see Fig. } 12.20 \text { for explanation) }\end{array}$} & \multicolumn{2}{|c|}{$\begin{array}{l}\text { Parameter increase* } \\
\text { (Fig. 12)\# }\end{array}$} & \multirow{2}{*}{$\begin{array}{l}\text { Bell broadens }(\mathrm{B}) \text { or } \\
\text { narrows }(\mathrm{N})\end{array}$} & \multirow{2}{*}{$\begin{array}{l}\text { Dose-response curve } \\
\text { or 'peak' } \\
\text { Increase }\end{array}$} & \multirow{2}{*}{$\begin{array}{l}\text { Dose -response curve or } \\
\text { 'peak' move }\end{array}$} \\
\hline & $A_{\mathrm{ii}}$ & 20 & & & \\
\hline & $A_{\mathrm{ss}}$ & & $\mathrm{N} \rightarrow \mathrm{B}$ & Increase & Left \\
\hline & $A_{\text {is }}$ & & $\mathrm{N} \rightarrow \mathrm{B}$ & Decrease & Left \\
\hline & $A_{\mathrm{si}}$ & & $\mathrm{N} \rightarrow \mathrm{B}$ & Decrease & Right \\
\hline \multirow{5}{*}{$\begin{array}{l}\text { Only RS is functional bell-shaped } \\
\text { (see Fig. } 12.21 \text { for explanation) }\end{array}$} & $A_{\mathrm{ii}}$ & 21 & B & Decrease & Right \\
\hline & $A_{\mathrm{ss}}$ & & $\mathrm{N} \rightarrow \mathrm{B}$ & Increase & Left \\
\hline & $A_{\text {is }}$ & & $\mathrm{N} \rightarrow \mathrm{B}$ & Decrease & Left \\
\hline & $A_{\mathrm{si}}$ & & $\mathrm{B}$ & Decrease & Right \\
\hline & & & $R_{\max }$ & Hill coefficient & \\
\hline \multirow{4}{*}{$\begin{array}{l}\text { RS }+ \text { SRS are functional, reach } \\
\text { max } 100 \% \text { (see Fig. } 12.22 \text { for } \\
\text { explanation) }\end{array}$} & $A_{\mathrm{ii}}$ & 22 & $100 \%$ & $1.0 \rightarrow \approx 1.1 \rightarrow 1.0$ & Right \\
\hline & $A_{\mathrm{ss}}$ & 23 & $100 \%$ & $\begin{aligned} 1.0 & \rightarrow \approx 1.12 \rightarrow \\
& \approx 0.97\end{aligned}$ & Left \\
\hline & $A_{\text {is }}$ & & $100 \%$ & $\approx 0.95 \rightarrow 1.1 \rightarrow 1.0$ & Left \\
\hline & $A_{\mathrm{si}}$ & & $100 \%$ & $1.0 \rightarrow \approx 1.1 \rightarrow 1.0$ & Right \\
\hline
\end{tabular}

The value of [I] was fixed at 10 , while varying the value of ligand [S] from $10^{-5}$ to $10^{5}$. Parameters as in Fig. 2.4. To simplify, it is assumed that parameter $A_{\mathrm{SS}}, A_{\mathrm{SS}}^{\text {is }}$, and $A_{\mathrm{ss}}^{\mathrm{ii}}$ are equal, while $A_{\mathrm{si}}$ is also equal to $A_{\mathrm{si}}^{\mathrm{is}}$ and $A_{\mathrm{si}}^{\mathrm{ii}}$. Parameters are thus independent of what is bound to the opposite binding sites.

*The actual parameter was changed in five steps from $10^{-4}$ to $10^{4}$, by a factor $10^{2}$ per step, while the other parameters were kept at 1 and $A_{\mathrm{ii}}$ at 10 . Thus, for instance, when $A_{\mathrm{ii}}$ varied the values were $10^{-3}, 10^{-1}, 10^{1}, 10^{3}$, and $10^{5}$.

${ }^{*}$ When the value of $A_{\text {ii }}$ was fixed at 1 , there was an intermediate dose-response plateau for $A_{\mathrm{ss}}=10^{4}$ as shown in Fig. 12.23 , a plateau for $A_{\text {is }}$ at $10^{-4}$ (not shown), and no plateau for $A_{\text {si }}$ between $10^{-4}$ to $10^{4}$ (not shown)

Ignoring log-log graphing, none of the generated contour plots in Figs. 12.20-12.23 of the FP-OSM resemble Loewe's linear-linear isoboles in Fig. 12.2.

and a receptor that allows both inclusive and co-lateral interactions, as described in Section 12.3.2. Hence, since isobolograms are not relevant for auto-intervention, they are not developed further.

\subsection{Mechanistic Synergy at the Receptor Level. 3. The Operational Model}

\subsubsection{Black and Leff's Operational Model}

The formulation of a simple so-called operational model was described by Black and Leff (1983), known as simple Black \& Leff (sB\&L). The sB\&L model is identical to the formulation of the pre-allosteric model by Del Castillo and Katz (dC\&K) (Del Castillo \& Katz 1957) (see Sections 1.3.4 and 1.3.5).

While the dC\&K model is straightforward mechanistic, the $\mathrm{SB} \& \mathrm{~L}$ is based on the idea of embedded steps of the load function. Thus, a stimulus is based on loaddependent occupation and an ensuing response is based on this load-dependent stimulus. Nevertheless, the resultant formulation of the sB\&L model is identical to the dC\&K model, in which the isomerization constant $L^{\prime}$ is now the same as the transducer ratio $\tau=R_{\max } / K_{\mathrm{E}}$ of the sB\&L model (Table 1.2).
The operational model is also described in more detail in Chapter 5.10.

\subsubsection{Towards a Mechanistic-based Analysis}

In order to describe synergy in a mechanistic context, Jonker et al. (2005) returned to so-called operational models by Black \& Leff (1983: B\&L models) and combined their sB\&L model with the intervention model for two interacting drugs, thus including a 'cooperativity factor' $\gamma$; the same parameter as system constant $c$ described in Chapter 2.5 and Section 12.3.1.

Jonker et al. (2005) further implemented the classical non-mechanistic Hillian exponentiation onto their formulations as reintroduced by others (Chou \& Talalay 1981; Leff et al. 1990; van der Graaf \& Danhof 1997; van der Graaf \& Stam 1999), thus actually departing from a mechanistic-based analysis. Moreover, Jonker et al. (2005) expressed the stimulus terms for two drugs, $S_{A}$ and $S_{B}$, with a so-called pre-amplification term given as $\mathrm{S}_{\mathrm{A}, \mathrm{B}}=\mathrm{S}_{\mathrm{A}}\left(1+\delta \mathrm{S}_{\mathrm{B}}\right)$, where $\mathrm{S}_{\mathrm{A}, \mathrm{B}}$ is the stimulus of the two drugs combined, and $\delta$ is defined as the 'degree of response modulation'. Such linear additivity of stimuli resembles a model suggested by MacKay (1981). The tenets of this kind of thinking on the interaction involved in two-ligand applications, unfortunately, again 
brings us back to ideas of intrinsic activity in monoligand analysis by Ariëns (1954), which was left many years ago in favor of Stephenson's approach for stimuli (Sections 1.3.2 and 1.3.3). In addition, the Stephenson approach and its results are either the dC\&K model or the sB\&L operational model (see Sections 5.6.2 and 5.6.3), both characterized by explicitly including a new conformation in formulating distribution equations for the models. That is, an implementation of mechanics.

What is the solution? An obvious alternative to synergy modeling initiated by Chou and Talalay (1977, 1981, 1984), including the use of Hillian exponentiation, is the genuine ATSM by Hall (2000). Hall's model describes in translucent mechanistic terms the synergy of two interacting drugs. Therefore, I shall continue with an analysis of synergy based on the Hall model.

\subsection{Mechanistic Synergy at the Receptor Level. 4. The ATSM}

We now move to models with the un-liganded and liganded receptors both appearing in two conformations, two-states, participating in the response, and formulated in equations for the reaction schemes. The two-state models were developed in Chapters 5 and 7.

\subsubsection{Synergy with the ATSM}

The ATSM is the simplest model for two interacting drugs, including genuine allostery with two-states for unliganded receptor complexes, but excluding possible colateral binding. It involves a maximum of seven system constants. As in the intervention model, the "co-operative factor' $c$ in the ATSM deals with interaction between the two drugs-receptor conformations, and further a new interaction efficacy constant $d$ emerges. The ATSM was developed and analyzed in Chapter 7. Therefore, in the present chapter we merely have to look at contour plots of the ATSM for a comparison with the synergy modeling for the intervention model presented in Sub-chapter 12.3.

In comparing cases with due alteration to the details, van Rijn and Willems-van Bree (2004) have studied three interacting ligands in a one-state hyper-cube model (Fig. 7.5B).

\subsubsection{Contour Plots of the ATSM Without Co-lateral Binding}

Three-dimensional plots of occupancy in the ATSM have been developed and characterized in Chapter 7 for varying values of each single parameter, i.e. $a, b, c, d$, $A_{\mathrm{s}}, A_{\mathrm{m}}, L$ (see Figs. 7.7-7.8 and 7.15-7.20). Thus, it is a simple procedure to obtain isobolograms for the ATSM as its seven parameters vary. It only requires construction of contour plots as the value of these parameters varies
Table 12.5. Four parameters varied for the functional type allosteric two-state model (ATSM)

\begin{tabular}{cccc}
\hline $\begin{array}{c}\text { Type of } \\
\text { reaction } \\
\text { scheme }\end{array}$ & $\begin{array}{c}\text { Vary } \\
\text { con- } \\
\text { stant }\end{array}$ & $\begin{array}{c}\text { Presented as } \\
\text { B/D) 3-dimension } \\
\text { in Fig. 12\# }\end{array}$ & $\begin{array}{r}\text { Resembles Loewe's } \\
\text { isobologram when } \\
\text { in linear-linear con- } \\
\text { tour graphs }\end{array}$ \\
\hline & $b$ & 24 & $\mathrm{No}^{*}$ \\
& $c$ & 25 & $\mathrm{No}^{*}$ \\
& $d$ & 26 & $\mathrm{No}^{*}$ \\
& $L$ & 27 & $\mathrm{No}^{*}$ \\
\hline
\end{tabular}

${ }^{*}$ For restricted parameter values, yes.

Note that related contour plots in Figs. 12.24-12.27 are log-log graphs.

one by one. To obtain these contour plots, isobolograms, merely requires one touch on a button of the keyboard of a personal computer equipped with the right software for a 3-D plot. Three-dimensional plots in Chapter 7 only require a single stroke of a key to become a contour plot instead. With a 3-D program for ATSM ready, ${ }^{3}$ the preparation consists of simple adjustments for parameter values in the software program. The question is: what does the contour plots for such-and-such a response surface modeling (3-D plot) and such-and-such a parameter value look like? Some results of such a study are presented in Table 12.5 and Figs. 12.24 - 12.27.

As with the conclusions drawn for the intervention model described in Sub-chapter 12.2, it is clear that the original definition of what 'synergy' and 'ant-agonism' as presented in Section 12.1.6 should mean is, to put it mildly, obsolete when simple drug competition is excluded.

For the future in combinatorial drug therapy, it is recommended to analyze for the single most likely model involved and study what its contour plots have to say about synergy, additivism and ant-agonism.

What is more, the terms 'synergy' and 'ant-agonism' may have to be replaced by terms as positive and negative 'intervention' or positive and negative 'modulation'.

\subsubsection{Contour Plots of the ATSM with Co-lateral Binding}

Contour plots of the ATSM with co-lateral binding are covered by the FP-TSM model shown in Figs. 5.9 and 15.2. The FP-TSM seems to be the reaction scheme at the most basic level involving co-lateral binding and interactions of intervention - auto-intervention as well as allostery with modulation and/or co-operativity. Therefore, the future for mechanistic-based synergy analysis should start with the FP-TSM. This model has yet to be developed to completion. However, recognizing its immense importance, a full analysis of the FP-TSM,

\footnotetext{
${ }^{3}$ Subroutine \#6 at http://www.synagics.ku.dk contains such a program for SigmaPlot.
} 

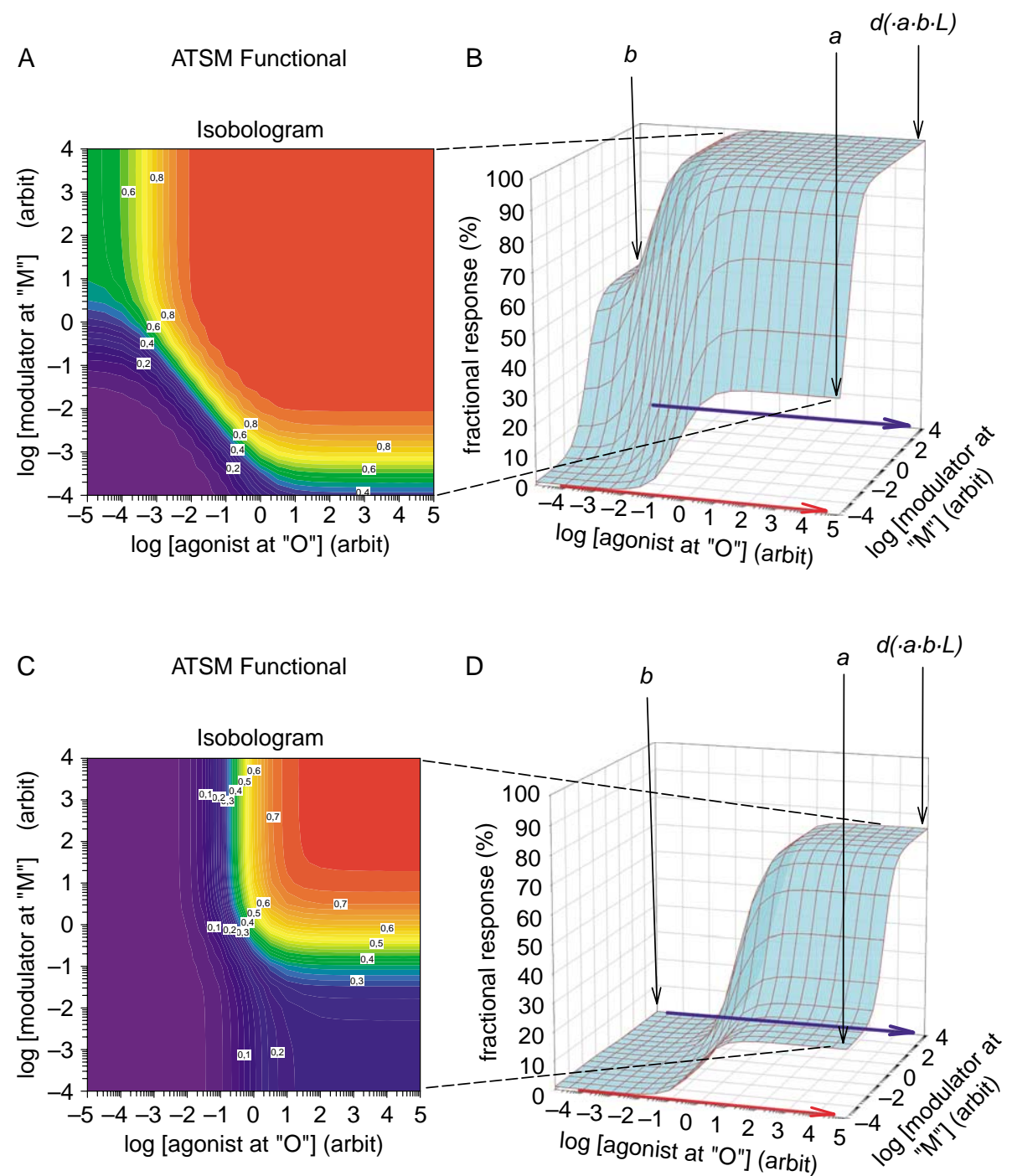

Figure 12.24. Examples of isobolograms for the functional form of the allosteric two-state model (ATSM) varying parameter $b$. In $\mathrm{A}$ and $\mathrm{B}, b=100$; in $\mathrm{C}$ and $\mathrm{D}, b=0.1$. Other parameters as in Fig. 7.10.

covering mechanistic synergy, is expected within less than a decade.

For the clinics, development of the FP-TSM will improve our understanding of medication by allosteric measures and in avoiding adverse effects. For individual therapy of patients, besides pharmaco-genetics, analysis with use of the OFCOR principle may also be helpful in avoiding adverse effects (Bindslev 2004). ${ }^{4}$

\subsubsection{Synergy, Allosteric Therapy, Fixed-dose, and the OFCOR Principle}

In combinatorial drug therapy, there is a connection between the phenomena covering synergy, allosteric drug modulation, a fixed-dose regime, and the OFCOR principle. However, thus far the terms have merely been used at separate levels of understanding. Therefore, what are their connections?

The OFCOR principle and ASTM are described in Chapter 7, and related to the application of two compounds interacting at the same or nearby receptors including co-lateral binding. With a mixture of two drugs at a fixed ratio of concentration between the two, it is possible to avoid adverse effects that would appear at a much lower concentration level of one of the drugs. This is part of the OFCOR principle. An explanation for this phenomenon is given in Chapter 7 , and is based on a combination of the ATSM, and the homotropic two-state model (HOTSM). In fixed-dose medication to patients, the idea is again to avoid adverse effects, although the explanation for the obtained results is less clear than for the OFCOR. Still, it is possible that the OFCOR principle and the advantages of a fixed-dose ratio medication play 

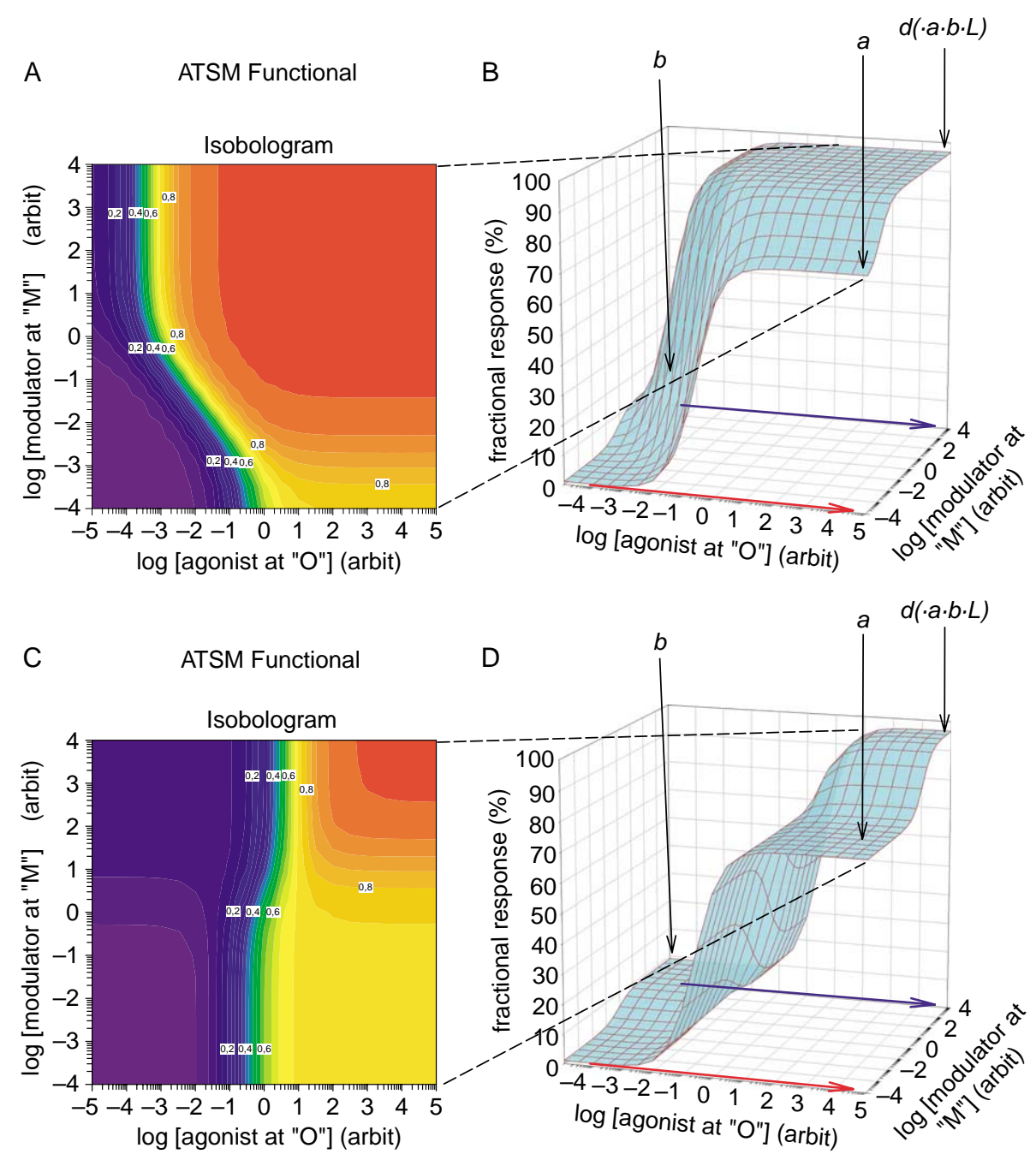

Figure 12.25. Examples of isobolograms for the functional form of the allosteric two-state model (ATSM) varying parameter $c$. In $\mathrm{A}$ and $\mathrm{B}, c=100$; in $\mathrm{C}$ and $\mathrm{D}, c=0.01$. Other parameters as in Fig. 7.11.

on the same mechanisms and possibly can be optimized for each individual profile (Winding \& Bindslev 1993; Bindslev 2004). A prescription we could call the 'dividualdrug-designer coctail'.

Overall, concepts of synergy, ATSM, and OFCOR in combinatorial medication with two substances and in a two-state model may be linked through the interactions in the FP-TSM at the receptor level.

An analysis of OFCOR in the FP-OSM was performed by Winding and Bindslev (1993) (Fig. 7.30) and may be compared with plots in Fig. 12.20.

A summary of two-ligand and one- or two-state models at the receptor level that should be implemented for synergy analysis is incorporated in Table 12.2.

\subsubsection{Contour Plots of the HOTSM}

Contour plots of the HOTSM are not applicable, since the contour plots, isobolograms, require involvement of two different and independent ligands.

\subsubsection{A Discussion of Linear-linear versus Log-log Contour Plots}

Graphing contour plots in $\log -\log$ format is just an expansion of Loewe's isobolograms, thus, covering a larger range of ligand concentrations, but the log-log contour plots can, in principle, be compared with linear-linear isoboles (Figs. 12.2, 12.4-12.19).

\subsection{Recent Developments for Isobolographic Analysis}

\subsubsection{Synergy Goes Non-mechanistic}

In the field of combinatorial drug therapy, there is a consensus to down-play mechanistic models in the analysis of possible synergy, and rather relate to Loewe's equation (Eq. 12.2), and other pure mathematical 

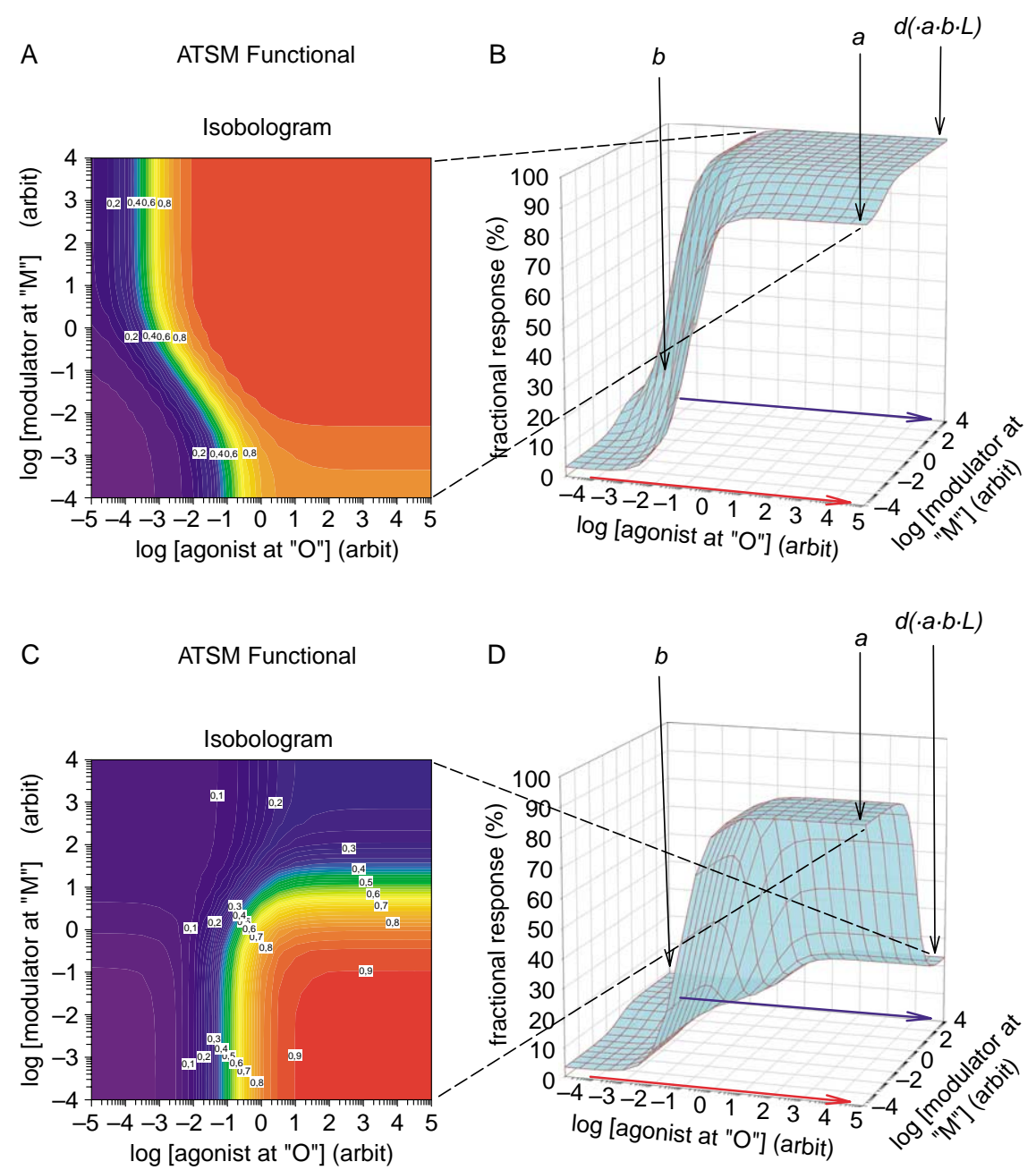

Figure 12.26. Examples of isobolograms for the functional form of the allosteric two-state model (ATSM) varying parameter $b$. In $A$ and $B, d=100$; in $C$ and $D, d=0.01$. Other parameters as in Fig. 7.12.

adaptations (Berenbaum 1989; Greco et al. 1995; Tallarida 2000). This is understandable in the light of the complexities of the studied systems. However, vigilance must be maintained in attempts to make mechanistic inferences about possible synergy, see Subchapter 12.7 .

\subsubsection{Synergy in 3-D}

Plotting the effect of two independent drugs in combinatorial therapy results in so-called 'additive response surface' representations. Evaluation of these response surfaces for synergy analysis has focused on the response of combining two drugs and its conversion from a representation in 3-D to a 2-D contour plot (Tallarida et al. 1999c, Tallarida 2000, Chapter 10); similar to the method described in Section 12.5.2, but now with the mathematical ideas from competitive additivity as reference. The analysis has a so-called Interaction Index as a measure of deviation from additivity (see Section
12.1.6). This type of analysis adds nothing to the analysis already described in Section 12.5.2, except for a comparison between theory in 3-D with isobolograms and actual experimental data in isobolographic plots (Tallarida et al. 1999c, Tallarida 2001, 2002).

\subsubsection{Actual Software for Synergy}

A Windows ${ }^{\circledR}$ program based on the multi-drug formulations by Chou and Talalay is commercially available under the name 'Calcusyn' ${ }^{\circledR}$ from Biosoft ${ }^{\circledR}$ (http://www.biosoft.com/biosoft (Chou \& Hayball 1996; Chou \& Martin 2005)). Its latest version can handle up to six interacting drugs, and the program provides a 'Combination Index' based on plots of median-effects. That is, an evaluation of a possible degree of interaction (Chou \& Talalay 1984). A Google ${ }^{\circledR}$ search of the web for 'calcusyn' resulted in over 7500 hits (December 2007; Medline December $2007=27$ hits). It should be realized that some of Calcusyn's algorithms are based on the Hillian paradigm. The CI 

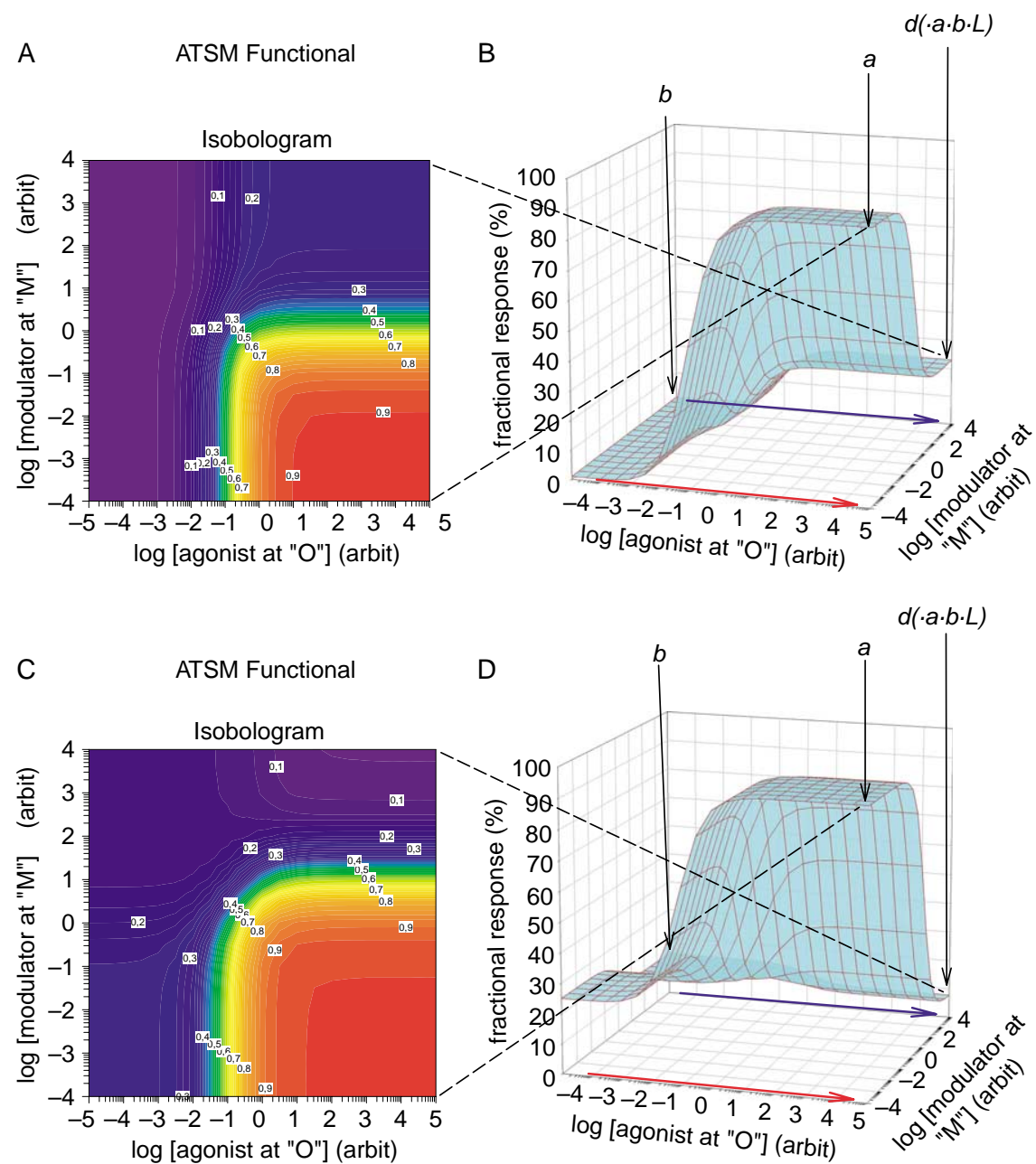

Figure 12.27. Examples of isobolograms for the functional form of the allosteric two-state model (ATSM) varying parameter $b$. In $A$ and $B, L=1 / 100$; in $C$ and $D, L=1 / 3$. Other parameters as in Fig. 7.13.

and Interaction Index remain popular measures of synergy, while new approaches are also requested (Lee et al. 2007; Reynolds et al. 2007).

Some authors prefer other means of characterizing drug interactions (Poch et al. 1990a,b,c, 1999; Snyder et al. 2000; Dawson et al. 2002). As an example, inspired by Greco et al. (1995), Snyder et al. (2000) employed a triple compound non-competitive interaction model based on earlier formulations by Chou and Talalay (1981) and used the ADAPT II ${ }^{\circledR}$ software for parameter resolution (http://bmsr.usc.edu/Software/Adapt/usergde. html). However, once again, an expanded ATSM is probably a better model for triple compound analysis.

\subsubsection{Synergy in Partial Agonism with Co-operativity}

Grabowsky and Tallarida recently introduced a formulation for isobolograms adjusted to comparing combinatorial effects of full and partial agonists (Grabowsky \& Tallarida 2004; Tallarida 2007). Since the used drugs by themselves further displayed deviations from the simple load function, the authors introduced a mathematical adjustment in the form of the Hill potentiation. Their isobolographic analysis was carried out on a hypothermic response elicited by a selective cannaboid agonist, WIN 55212-2, in combination with an NMDA antagonist, dextromethophan (Grabowsky \& Tallarida 2004). This novel type of isobolographic analysis with mathematical correction for partial agonism and cooperativity has now also been applied with successful fitting of theory to experimental isobolographic data for combinations of inhibitors of vascular smooth muscle cell proliferation (Parry et al. 2006) and hypertension (Rawls et al. 2006).

I am aware that for the moment no other general theory is obvious to judge synergy or infra-additivity in these complex experimental models, therefore, as a measure of synergy, the CI and the Interaction Index are as good as anything else. My sole objection is on theoretical grounds, since the employed measures follow in the non-mechanistic footsteps of Ariens's 
intrinsic activity and Hill's exponentiation, and this seems to be a trend in the synergy field (Jonker et al. 2005, 2007; Chou 2006; Lee et al. 2007; Tallarida 2007; Brun et al. 2007). My question is, is it not time for novel approaches?

\subsection{Conclusions on the Analysis of Synergy Analysis}

\subsubsection{Conclusions by Berenbaum}

Berenbaum's main recommendation for analysis of possible synergy in combinatorial drug therapy is to use non-mechanistic mathematical models, since mechanistic interpretations will be spurious anyway for complex systems as the entire body (Berenbaum 1989). His conclusion must be refuted. I suggest that you should try and try again to obtain a reasonable mechanistic model.

\subsubsection{Conclusions by Greco et al.}

Until new initiatives are taken, conclusions by Greco et al. (1995) might suffice. Thus, a roundup of their efforts of thoughtful and clever insight can be listed as:

a. only use the general combined-action mathematical models in assessment of complex systems if mechanistic models do not exist;

b. the degree of departure from 'no interaction' is a quantitative measure of the ignorance of the investigator;

c. for an initial step of analysis use Loewe's additivity, Bliss's independence, or their own derived response surface interaction models;

d. while a later step of useful interaction assessment may, through mechanistic arguments, involve application of Loewe's synergy, additivity, antagonism, or coalism (Loewe \& Muischnek 1926; Loewe 1938) and Bliss's synergy, independence, or ant-agonism.

Finally, since it is seldom that we will have a mechanistic understanding for responses in whole bodies and therefore a mechanistic reaction scheme of our system

e. the alternative use of a general combinatorialaction analysis may:

1. summarize a large amount of data with joint concentration-effect surface relying on relatively few parameters;

2. facilitate good predictions of joint effects in regions with no data;
3. possibly find and characterize agent combinations with intense synergy or ant-agonism; and

4. provide a lead to mechanistic explanations of joint action.

\subsubsection{Conclusions on New Ways to Go}

If, on completion of Chapter 12, the reader has the feeling that its content encourages a new kind of approach for analyses in synergy, an alternative from the present non-mechanistic type, the reader is not completely wrong. The chapter is an invitation to start synergy analysis right from the beginning with mechanistic models, such as the ATSM, and then continue by combining it with the HOTSM, into the FP-TSM. For instance, in comparison with all the models summarized above (Greco et al. 1995). The ATSM, HOTSM, and FPTSM as a novelty include spontaneous activity combined with non-static binding constants of which $c$ is the same as in the intervention model where its parameter $c$ can differ from unity. Implementing these two-state models will keep a mechanistic framework alive for the analysis of synergy in combinatorial drug therapy.

\section{REFERENCES}

Adli M, Baethge C, Heinz A, Langlitz N \& Bauer M. Is dose escalation of antidepressants a rational strategy after a medium-dose treatment has failed? A systematic review. Eur Arch Psychiatry Clin Neurosci 255: 387-400, 2005.

Adusumilli PS, Chan MK, Chun YS, Hezel M, Chou TC, Rusch VW \& Fong Y. Cisplatin-induced GADD34 upregulation potentiates oncolytic viral therapy in the treatment of malignant pleural mesothelioma. Cancer Biol Ther 5: 48-53, 2006.

Antonetti M, Kirton O, Bui P, Ademi A, Staff I, Hudson-Civetta JA \& Lilly $\mathrm{R}$. The effects of preoperative rofecoxib, metoclopramide, dexamethasone, and ondansetron on postoperative pain and nausea in patients undergoing elective laparoscopic cholecystectomy. Surg Endosc 21: 1855-1861, 2007.

Ariëns EJ. Affinity and intrinsic activity in the theory of competitive inhibition. Part I. Problems and theory. Arch Int Pharmacodyn 99: 32-49, 1954.

Atkinson TJ. Central and peripheral neuroendocrine peptides and signalling in appetite regulation: considerations for obesity pharmacotherapy. Obes Rev 9: 108-120, 2008.

Barrera NP, Morales B, Torres S \& Villalon M. Principles: mechanisms and modeling of synergism in cellular responses. Trends Pharmacol Sci 26: 526-532, 2005.

Bauer M, Heinz A \& Whybrow PC. Thyroid hormones, serotonin and mood: of synergy and significance in the adult brain. Mol Psychiatry 7: 140-156, 2002.

Belitz HD, Grosch W, Schleberle P \& Burghagen MM. Food Chemistry, 3rd ed. Berlin: Springer, 2004, pp. 1-1071.

Berenbaum MC. Synergy, additivism and antagonism in immunosuppression. A critical review. Clin Exp Immunol 28: 1-18, 1977.

Berenbaum MC. The expected effect of a combination of agents: the general solution. J Theor Biol 114: 413-431, 1985.

Berenbaum MC. What is synergy? Pharmacol Rev 41: 93-141, 1989.

Berenbaum MC. Concepts for describing the interaction of two agents. Radiat Res 126: 264-268, 1991. 
Bhat AS, Tandan SK, Kumar D, Krishna V \& Prakash VR. Interaction between inhibitors of inducible nitric oxide synthase and cyclooxygenase in Brewer's yeast induced pyrexia in mice: an isobolographic study. Eur J Pharmacol 511: 137-142, 2005.

Bindslev N. A homotropic two-state model and auto-antagonism. BMC Pharmacol 4: 11, 2004.

Black JW \& Leff P. Operational models of pharmacological agonism. Proc R Soc Lond B 220: 141-162, 1983.

Bliss CI. The toxicity of poisons applied jointly. Ann Appl Biol 26: 585$615,1939$.

Bouillon TW, Bruhn J, Radulescu L, Andresen C, Shafer TJ, Cohane C \& Shafer SL. Pharmacodynamic interaction between propofol and remifentanil regarding hypnosis, tolerance of laryngoscopy, bispectral index, and electroencephalographic approximate entropy. Anesthesiology 100: 1356-1372, 2004.

Bouwman BM, Heesen E \& van Rijn CM. The interaction between vigabatrin and diazepam on the electroencephalogram during active behaviour in rats: an isobolic analysis. Eur J Pharmacol 495: 119-128, 2004.

Brun YF, Dennis CG, Greco WR, Bernacki RJ, Pera PJ, Bushey JJ, Youn RC, White DB \& Segal BH. Modeling the combination of amphotericin B, micafungin, and nikkomycin $\mathrm{Z}$ against Aspergillus fumigatus in vitro using a novel response surface paradigm. Antimicrob Agents Chemother 51: 1804-1812, 2007.

Bruzzese F, Di Gennaro E, Avallone A, Pepe S, Arra C, Caraglia M, Tagliaferri P \& Budillon A. Synergistic antitumor activity of epidermal growth factor receptor tyrosine kinase inhibitor gefitinib and IFN-\{alpha\} in head and neck cancer cells in vitro and in vivo. Clin Cancer Res 12: 617-625, 2006.

Buolamwini JK \& Adjei AA. Novel Anticancer Drug Protocols, 1st ed. Totowa, NJ: Humana Press, 2003.

Chan MM, Mattiacci JA, Hwang HS, Shah A \& Fong D. Synergy between ethanol and grape polyphenols, quercetin, and resveratrol, in the inhibition of the inducible nitric oxide synthase pathway. Biochem Pharmacol 60: 1539-1548, 2000.

Chen X, McClatchy DB, Geller EB, Tallarida RJ \& Adler MW. The dynamic relationship between mu and kappa opioid receptors in body temperature regulation. Life Sci 78: 329-333, 2005.

Chou TC. Theoretical basis, experimental design, and computerized simulation of synergism and antagonism in drug combination studies. Pharmacol Rev 58: 621-681, 2006.

Chou TC \& Talalay P. A simple generalized equation for the analysis of multiple inhibitions of Michaelis-Menten kinetic systems. J Biol Chem 252: 6438-6442, 1977.

Chou TC \& Talalay P. Generalized equations for the analysis of inhibitions of Michaelis-Menten and higher-order kinetic systems with two or more mutually exclusive and nonexclusive inhibitors. Eur J Biochem 115: 207-216, 1981.

Chou TC \& Talalay P. Analysis of combined drug effects: a new look at a very old problem. Trend Pharmacol Sci 4: 450-454, 1983.

Chou TC \& Talalay P. Quantitative analysis of dose-effect relationships: the combined effects of multiple drugs or enzyme inhibitors. Adv Enzyme Regul 22: 27-55, 1984.

Chou TC \& Hayball MK. Calcusyn for Windows, Multiple-drug-effect Analyzer and Manual, 1st ed. Cambridge: Biosoft Cambridge Place, 1996.

Chou TC \& Martin N. Compusyn for Drug Combinations: PC Software and User's Guide: A Computer Program for Quantitation of Synergism and Antagonism in Drug Combinations, and the Determination of IC50 and EC50 and LD50 Values. NJ: Combosyn, Paramus, 2005.

Chou TC, Motzer RJ, Tong Y \& Bosl GJ. Computerized quantitation of synergism and antagonism of taxol, topotecan, and cisplatin against human teratocarcinoma cell growth: a rational approach to clinical protocol design. J Natl Cancer Inst 86: 1517-1524, 1994.

Chou TC, Guan Y, Soenen DR, Danishefsky SJ \& Boger DL. Potent reversal of multidrug resistance by ningalins and its use in drug combinations against human colon carcinoma xenograft in nude mice. Cancer Chemother Pharmacol 56: 379-390, 2005.

Ciombor KK \& Rocha Lima CM. Management of small cell lung cancer. Curr Treat Options Oncol 7: 59-68, 2006.

Clark AJ. The antagonism of acetylcholine by atropine. J Physiol 61: 547-556, 1926.

Clark AJ. General Pharmacology, Vol. 4, 1st ed. Berlin: Springer, 1937, pp. $1-228$.

Colquhoun D. Why the Schild method is better than Schild realised. Trends Pharmacol Sci 28: 608-614, 2007.

Danilenko M \& Studzinski GP. Enhancement by other compounds of the anti-cancer activity of vitamin $\mathrm{D}(3)$ and its analogs. Exp Cell Res 298: 339-358, 2004.

Dawson DA, Rinaldi AC \& Poch G. Biochemical and toxicological evaluation of agent-cofactor reactivity as a mechanism of action for osteolathyrism. Toxicology 177: 267-284, 2002.

de Leest HT, Steen KS, Lems WF, Bijlsma JW, van de Laar MA \& Huisman AM. Eradication of Helicobacter pylori does not reduce the incidence of gastroduodenal ulcers in patients on long-term NSAID treatment: double-blind, randomized, placebo-controlled trial. Helicobacter 12: 477-485, 2007.

Deciga-Campos M, Lopez UG, Reval MI \& Lopez-Munoz FJ. Enhancement of antinociception by co-administration of an opioid drug (morphine) and a preferential cyclooxygenase-2 inhibitor (rofecoxib) in rats. Eur J Pharmacol 460: 99-107, 2003.

Deciga-Campos M, Diaz-Reval MI, Ventura-Martinez R \& Lopez-Munoz FJ. Participation of the serotonin system in rofecoxib-induced antinociception. Proc West Pharmacol Soc 47: 100-102, 2004.

del Castillo J \& Katz B. Interaction at end-plate receptors between different choline derivatives. J Physiol 146: 369-381, 1957.

Delage N, Maaliki H, Beloeil H, Benhamou D \& Mazoit JX. Median effective dose (ED50) of Nefopam and Ketoprofen in postoperative patients: a study of interaction using sequential analysis and isobolographic analysis. Anesthesiology 102: 1211-1216, 2005.

Dudakovic A, Wiemer AJ, Lamb KM, Vonnahme LA, Dietz SE \& Hohl RJ. Inhibition of Geranylgeranyl Diphosphate Synthase Induces Apoptosis through Multiple Mechanisms and Displays Synergy with Inhibition of other Isoprenoid Biosynthetic Enzymes. $J$ Pharmacol Exp Ther JPET107132217V1: 2008. (no vol yet)

Figul M, Soling A, Dong HJ, Chou TC \& Rainov NG. Combined effects of temozolomide and the ribonucleotide reductase inhibitors didox and trimidox in malignant brain tumor cells. Cancer Chemother Pharmacol 52: 41-46, 2003.

Finney DJ. Probit Analysis, 3rd ed. Cambridge: Cambridge University Press, 1971, pp. 1-333.

Fox E \& Bates SE. Tariquidar (XR9576): a P-glycoprotein drug efflux pump inhibitor. Expert Rev Anticancer Ther 7: 447-459, 2007.

Fraser TR. An experimental research on the antagonism between the action of physostigma and atropia. Proc R Soc Edin 7: 506-511, 1871.

Frei W. Versuche uber kombination von desinfektionsmitteln. Z Hyg Infektionskrank 75: 433-496, 1913.

Gan TJ, Coop A \& Philip BK. A randomized, double-blind study of granisetron plus dexamethasone versus ondansetron plus dexamethasone to prevent postoperative nausea and vomiting in patients undergoing abdominal hysterectomy. Anesth Analg 101: 1323-1329, 2005.

Gasparini G, Longo R, Fanelli M \& Teicher BA. Combination of antiangiogenic therapy with other anticancer therapies: results, challenges, and open questions. J Clin Oncol 23: 1295-1311, 2005.

Genest J. Combination of statin and ezetimibe for the treatment of dyslipidemias and the prevention of coronary artery disease. Can J Cardiol 22: 863-868, 2006.

Gillenwater HH, McCune JS, Lindley C, Faucette S, Shord S, Donahue A, Socinski MA, Stewart CF, Zamboni WC, Kirstein MN \& Moore D. A phase I trial defining the maximum tolerated systemic 
exposure of topotecan in combination with Carboplatin and Etoposide in extensive stage small cell lung cancer. Cancer Invest 23: 511-519, 2005.

Goldie JH \& Coldman AJ. Drug Resistance in Cancer: Mechanisms and Models, 1st ed. Cambridge: Cambridge University Press, 1998, pp. $1-247$.

Gouwy M, Struyf S, Proost P \& Van Damme J. Synergy in cytokine and chemokine networks amplifies the inflammatory response. Cytokine Growth Factor Rev 16: 561-580, 2005.

Gowen BB, Smee DF, Wong MH, Pace AM, Jung KH, Bailey KW, Blatt LM \& Sidwell RW. Combinatorial ribavirin and interferon alfacon-1 therapy of acute arenaviral disease in hamsters. Antivir Chem Chemother 17: 175-183, 2006.

Grabovsky Y \& Tallarida RJ. Isobolographic analysis for combinations of a full and partial agonist: curved isoboles. J Pharmacol Exp Ther 310: 981-986, 2004.

Greco WR, Bravo G \& Parsons JC. The search for synergy: a critical review from a response surface perspective. Pharmacol Rev 47: 331-385, 1995.

Greco WR. New practices in computational modeling. Clin Cancer Res 13: 1074-1075, 2007.

Guindon J, De Lean A \& Beaulieu P. Local interactions between anandamide, an endocannabinoid, and ibuprofen, a nonsteroidal anti-inflammatory drug, in acute and inflammatory pain. Pain 121: 85-93, 2006.

Guindon J, LoVerme J, De Lean A, Piomelli D \& Beaulieu P. Synergistic antinociceptive effects of anandamide, an endocannabinoid, and nonsteroidal anti-inflammatory drugs in peripheral tissue: a role for endogenous fatty-acid ethanolamides? Eur J Pharmacol 550: 68-77, 2006.

Hall DA. Modeling the functional effects of allosteric modulators at pharmacological receptors: an extension of the two-state model of receptor activation. Mol Pharmacol 58: 1412-1423, 2000.

Hanauer SB. Risks and benefits of combining immunosuppressives and biological agents in inflammatory bowel disease: is the synergy worth the risk? Gut 56: 1181-1183, 2007.

Hancox RJ. Interactions between corticosteroids and beta2-agonists. Clin Rev Allergy Immunol 31: 231-246, 2006.

Head J \& Johnston SR. New targets for therapy in breast cancer: farnesyltransferase inhibitors. Breast Cancer Res 6: 262-268, 2004.

Hellebrekers DM, Griffioen AW \& van Engeland M. Dual targeting of epigenetic therapy in cancer. Biochim Biophys Acta 1775: 76-91, 2007.

Hoare SR, Coldwell MC, Armstrong D \& Strange PG. Regulation of human D(1), d(2(long)), d(2(short)), D(3) and D(4) dopamine receptors by amiloride and amiloride analogues. Br J Pharmacol 130: 1045-1059, 2000.

Hoffmann D, Bayer W \& Wildner O. Local and distant immunemediated control of colon cancer growth with fusogenic membrane glycoproteins in combination with viral oncolysis. Hum Gene Ther 18: 435-450, 2007.

Honer WG, Thornton AE, Sherwood M, MacEwan GW, Ehmann TS, Williams R, Kopala LC, Procyshyn R \& Barr AM. Conceptual and methodological issues in the design of clinical trials of antipsychotics for the treatment of schizophrenia. CNS Drugs 21: 699714, 2007.

Hubensack M, Müller C, Höcherl P, Fellner S, Spruss T, Bernhardt G \& Buschauer A. Effect of the ABCB1 modulators elacridar and tariquidar on the distribution of paclitaxel in nude mice. J Cancer Res Clin Oncol Oct 12: 2007. (no vol yet)

Iughetti L, Predieri B, Balli F \& Calandra S. Rational approach to the treatment for heterozygous familial hypercholesterolemia in childhood and adolescence: a review. J Endocrinol Invest 30, 700-719, 2007.

Jakubik J, Bacakova L, El-Fakahany EE \& Tucek S. Positive cooperativity of acetylcholine and other agonists with allosteric ligands on muscarinic acetylcholine receptors. Mol Pharmacol 52: 172-179, 1997.

Jenks KA, Stamp LK, O’Donnell JL, Savage RL \& Chapman PT. Leflunomide-associated infections in rheumatoid arthritis. $J$ Rheumatol 34: 2201-2203, 2007.

Jonker DM, Vermeij DA, Edelbroek PM, Voskuyl RA, Piotrovsky VK \& Danhof M. Pharmacodynamic analysis of the interaction between tiagabine and midazolam with an allosteric model that incorporates signal transduction. Epilepsia 44: 329-338, 2003.

Jonker DM, Voskuyl RA \& Danhof M. Pharmacodynamic analysis of the anticonvulsant effects of tiagabine and lamotrigine in combination in the rat. Epilepsia 45: 424-435, 2004.

Jonker DM, Visser SA, van der Graaf PH, Voskuyl RA \& Danhof M. Towards a mechanism-based analysis of pharmacodynamic drug?drug interactions in vivo. Pharmacol Ther 106: 1-18, 2005.

Jonker DM, Voskuyl RA \& Danhof M. Synergistic combinations of anticonvulsant agents: what is the evidence from animal experiments? Epilepsia 48: 412-434, 2007.

Kaer E \& Loewe S. Über kobinationswirkugen III. Die wirkungsvariationen in veronal-antipyrin-gemischen. Arch Exp Path Pharmak 114: 339-347, 1926.

Kaer E \& Loewe S. Über kobinationswirkugen II. Wirkungen von diathylbarbitursauer-pyramidon-gemischen. Arch Exp Path Pharmak 114: 237-338, 1926.

Kampf P, Agelink MW \& Naber D. Augmentation of clozapine with amisulpride: a promising therapeutic approach to refractory schizophrenic symptoms. Pharmacopsychiatry 38: 39-40, 2005.

Kellen JA. Reversal of Multidrug Resistance in Cancer, 1st ed. Boca Raton, FL: CRC, 1994, pp. 1-148.

Khanna N, Widmer AF, Decker M, Steffen I, Halter J, Heim D, Weisser M, Gratwohl A, Fluckiger U \& Hirsch HH. Respiratory syncytial virus infection in patients with hematological diseases: singlecenter study and review of the literature. Clin Infect Dis 46: 402412, 2008.

Kjeldsen SE, Os I, Hoieggen A, Beckey K, Gleim GW \& Oparil S. Fixeddose combinations in the management of hypertension: defining the place of angiotensin receptor antagonists and hydrochlorothiazide. Am J Cardiovasc Drugs 5: 17-22, 2005.

Kjeldsen SE, Westheim AS \& Os I. Prevention of cardiovascular events and diabetes with angiotensin-receptor blockers in hypertension: LIFE, SCOPE, and VALUE. Curr Hypertens Rep 7: 155-157, 2005.

Langley NJ. On the reaction of cells and of nerve-endings to certain poisons, chiefly as regards the reaction of striated muscle to nicotine and to curari. J Physiol 33: 374-413, 1905.

Lee JJ, Kong M, Ayers GD \& Lotan R. Interaction index and different methods for determining drug interaction in combination therapy. J Biopharm Stat 17: 461-480, 2007.

Leff P, Prentice DJ, Giles H, Martin GR \& Wood J. Estimation of agonist affinity and efficacy by direct, operational model-fitting. J Pharmacol Methods 23: 225-237, 1990.

Lin K, Kwong AD \& Lin C. Combination of a hepatitis C virus NS3NS4A protease inhibitor and alpha interferon synergistically inhibits viral RNA replication and facilitates viral RNA clearance in replicon cells. Antimicrob Agents Chemother 48: 4784-4792, 2004.

Loboz KK \& Shenfield GM. Drug combinations and impaired renal function - the 'triple whammy'. Br J Clin Pharmacol 59: 239-243, 2005 .

Loewe S. Die quantitativen probleme der pharmakologie. Erg Physiol 27: 47-187, 1928.

Loewe S. Coalitive effects of drug combinations. J Pharmacol 63: 24, 1938.

Loewe S. The problem of synergism and antagonism of combined drugs. Arzneimittelforschung 3: 285-290, 1953.

Loewe S. Isobols of dose-effect relations in the combination of pentylenetetrazole and phenobarbital. J Pharmacol Exp Ther 114: 185-191, 1955 . 
Loewe S. Isobols of dose-effect relations in the combination of nikethamide (coramine) and phenobarbital. J Pharmacol Exp Ther 115: 6-15, 1955.

Loewe S. Antagonisms and antagonists. Pharmacol Rev 9: 237-242, 1957.

Loewe S. Relationship between stimulus and response. Science 130: 692-695, 1959.

Loewe S \& Muischnek H. Über kobinationswirkugen I. Hilfsmittel der fragestellung. Arch Exp Path Pharmak 114: 313-336, 1926.

Lopez-Munoz FJ. A new model to assess analgesic activity: pain/ induced functional impairment in the rat (PIFIR). Drug Dev Res 28: 169-175, 1993.

Lopez-Munoz FJ. Surface of synergistic interaction between dipyrone and morphine in the PIFIR model. Drug Dev Res 33: 26-32, 1994.

Lorenzen A, Beukers MW, van der Graaf PH, Lang H, van MuijlwijkKoezen J, de Groote M, Menge W, Schwabe U \& IJzerman AP. Modulation of agonist responses at the $\mathrm{A}(1)$ adenosine receptor by an irreversible antagonist, receptor-G protein uncoupling and by the G protein activation state. Biochem Pharmacol 64: 12511265, 2002.

Luszczki JJ, Andres MM \& Czuczwar SJ. Synergistic interaction of gabapentin and oxcarbazepine in the mouse maximal electroshock seizure model - an isobolographic analysis. EurJ Pharmacol 515: 54-61, 2005.

MacKay D. An analysis of functional antagonism and synergism. $\mathrm{Br} J$ Pharmacol 73: 127-134, 1981.

Mahtani RL \& Macdonald JS. Synergy between cetuximab and chemotherapy in tumors of the gastrointestinal tract. Oncologist 13: 39-50, 2008.

McDevitt CA \& Callaghan R. How can we best use structural information on P-glycoprotein to design inhibitors? Pharmacol Ther 113: 429-441, 2007.

McGee H. Food and Cooking. The Science and Lore of the Kitchen, 2nd ed. New York: Scribner, 2004, pp. 1-884.

Messeguer A \& Cortes N. Combinatorial chemistry in cancer research. Clin Transl Oncol 9: 83-92, 2007.

Michor F, Nowak MA \& Iwasa Y. Evolution of resistance to cancer therapy. Curr Pharm Des 12: 261-271, 2006.

Minto CF, Schnider TW, Short TG, Gregg KM, Gentilini A \& Shafer SL. Response surface model for anesthetic drug interactions. Anesthesiology 92: 1603-1616, 2000.

Miranda HF, Puig MM, Prieto JC \& Pinardi G. Synergism between paracetamol and nonsteroidal anti-inflammatory drugs in experimental acute pain. Pain 121: 22-28, 2006.

Morishita M, Nagashima M, Wauke K, Takahashi H \& Takenouchi K. Osteoclast inhibitory effects of vitamin $\mathrm{K} 2$ alone or in combination with etidronate or risedronate in patients with rheumatoid arthritis: 2-year results. J Rheumatol Feb 1: 2008. (no vol yet)

Nair SK, Verma A, Thomas TJ, Chou TC, Gallo MA, Shirahata A \& Thomas T. Synergistic apoptosis of MCF-7 breast cancer cells by 2methoxyestradiol and bis(ethyl)norspermine. Cancer Lett 250: 311-322, 2007.

Parry TJ, Thyagarajan R, Argentieri D, Falotico R, Siekierka J \& Tallarida RJ. Effects of drug combinations on smooth muscle cell proliferation: An isobolographic analysis. Eur J Pharmacol 532: 3843, 2006.

Poch G, Reiffenstein RJ \& Unkelbach HD. Application of the isobologram technique for the analysis of combined effects with respect to additivity as well as independence. Can J Physiol Pharmacol 68: 682-688, 1990.

Poch G, Dittrich P, Reiffenstein RJ, Lenk W \& Schuster A. Evaluation of experimental combined toxicity by use of dose-frequency curves: comparison with theoretical additivity as well as independence. Can J Physiol Pharmacol 68: 1338-1345, 1990.
Poch G, Dittrich P \& Holzmann S. Evaluation of combined effects in dose-response studies by statistical comparison with additive and independent interactions. J Pharmacol Methods 24: 311-325, 1990.

Poch G, Vychodil-Kahr S \& Petru E. Sigmoid model versus medianeffect analysis for obtaining dose-response curves for in vitro chemosensitivity testing. Int J Clin Pharmacol Ther 37: 189-192, 1999.

Pui CH \& Evans WE. Treatment of acute lymphoblastic leukemia. $N$ Engl J Med 354: 166-178, 2006.

Qadir M, O'Loughlin KL, Fricke SM, Williamson NA, Greco WR, Minderman H \& Bear MR. Cyclosporin A is a broad-spectrum multidrug resistance modulator. Clin Cancer Res 11: 2320-2326, 2005.

Raffa RB, Clark-Vetri R, Tallarida RJ \& Wertheimer AI. Combination strategies for pain management. Expert Opin Pharmacother 4: 16971708, 2003.

Rawls SM, Jacobs K \& Tallarida RJ. Baclofen and NOS inhibitors interact to evoke synergistic hypothermia in rats. Life Sci 78: 669672, 2006.

Reynolds CP, Kang MH, Keshelava N \& Maurer BJ. Assessing combinations of cytotoxic agents using leukemia cell lines. Curr Drug Targets 8: 765-771, 2007.

Rougier P \& Lepere C. Metastatic colorectal cancer: first- and secondline treatment in 2005. Semin Oncol 32: 15-20, 2005.

Sacchi A, Gasparri A, Gallo-Stampino C, Toma S, Curnis F \& Corti A. Synergistic antitumor activity of cisplatin, paclitaxel, and gemcitabine with tumor vasculature-targeted tumor necrosis factoralpha. Clin Cancer Res 12: 175-182, 2006.

Salvador A. Synergism analysis of biochemical systems. I. Conceptual framework. Math Biosci 163: 105-129, 2000.

Salvador A. Synergism analysis of biochemical systems. II. Tensor formulation and treatment of stoichiometric constraints. Math Biosci 163: 131-158, 2000.

Sanchez-Escribano Morcuende R, Ales-Martinez JE \& Aramburo Gonzalez PM. Low dose Gemcitabine plus cisplatin in a weeklybased regimen as salvage therapy for relapsed breast cancer after taxane-anthracycline-containing regimens. Clin Transl Oncol 9: 459-464, 2007.

Scaramellini C, Bennett G \& Leff P. Analysis of agonist-agonist interactions: the crucial influence of curve shape. $J$ Pharmacol Toxicol Methods 37: 167-178, 1997.

Segel IH. Enzyme kinetics. Behavior and Analysis of Rapid Equilibrium and Steady-state Enzyme Systems. New York: Wiley \& Sons (reissued 1993), 1975.

Shapiro GI. Preclinical and clinical development of the cyclindependent kinase inhibitor flavopiridol. Clin Cancer Res 10: $4270 \mathrm{~s}-4275 \mathrm{~s}, 2004$

Shenfield GM. Fixed combination drug therapy. Drugs 23: 462-480, 1982.

Shimura K, Kodama E, Sakagami Y, Matsuzaki Y, Watanabe W, Yamataka K, Watanabe Y, Ohata Y, Doi S, Sato M, Ikeda S \& Matsuoka M. Broad anti-retroviral activity and resistance profile of a novel human immunodeficiency virus integrase inhibitor, elvitegravir (JTK-303/GS-9137). J Virol 82:764-774, 2007.

Short TG, Ho TY, Minto CF, Schnider TW \& Shafer SL. Efficient trial design for eliciting a pharmacokinetic-pharmacodynamic modelbased response surface describing the interaction between two intravenous anesthetic drugs. Anesthesiology 96: 400-408, 2002.

Smalley KS \& Herlyn M. Targeting intracellular signaling pathways as a novel strategy in melanoma therapeutics. Ann N Y Acad Sci 1059: $16-25,2005$.

Snyder S, D'Argenio DZ, Weislow O, Bilello JA \& Drusano GL. The triple combination indinavir-zidovudine-lamivudine is highly synergistic. Antimicrob Agents Chemother 44: 1051-1058, 2000.

Spinella M. The importance of pharmacological synergy in psychoactive herbal medicines. Altern Med Rev 7: 130-137, 2002. 
Staffileno BA. Treating hypertension with cardioprotective therapies: the role of ACE inhibitors, ARBs, and beta-blockers. I Cardiovasc Nurs 20: 354-364, 2005.

Tallarida RJ. Statistical analysis of drug combinations for synergism. Pain 49: 93-97, 1992.

Tallarida RJ. Drug Synergism and Dose-effect Data Analysis, 1st ed. Boca Raton, FL: Chapman \& Hall/CRC, 2000, pp. 1-247.

Tallarida RJ. Drug synergism: its detection and applications. J Pharmacol Exp Ther 298: 865-872, 2001.

Tallarida RJ. The interaction index: a measure of drug synergism. Pain 98: 163-168, 2002.

Tallarida RJ. Interactions between drugs and occupied receptors. Pharmacol Ther 113: 197-209, 2007.

Tallarida RJ \& Raffa RB. Receptor regulation, competitive antagonism and pA2. Life Sci 51: PL61-PL65, 1992.

Tallarida RJ, Stone DJ Jr \& Raffa RB. Efficient designs for studying synergistic drug combinations. Life Sci 61: PL417-PL425, 1997.

Tallarida RJ, Stone DJ Jr, McCary JD \& Raffa RB. Response surface analysis of synergism between morphine and clonidine. J Pharmacol Exp Ther 289: 8-13, 1999.

Teicher BA. Assays for in vitro and in vivo synergy. In: Novel Anticancer Drug Protocols, 1st ed., edited by Buolamwini JK \& Adjei AA. Totowa, NJ: Humana Press, 2003, pp. 297-321.

Tham SM, Angus JA, Tudor EM \& Wright CE. Synergistic and additive interactions of the cannabinoid agonist CP55,940 with mu opioid receptor and alpha2-adrenoceptor agonists in acute pain models in mice. Br J Pharmacol 144: 875-884, 2005.

Thelin WR \& Boucher RC. The epithelium as a target for therapy in cystic fibrosis. Curr Opin Pharmacol 7: 290-295, 2007.

Thomsen MB. Double pharmacological challenge on repolarization opens new avenues for drug safety research. Br J Pharmacol 151: 909-911, 2007.

Traynor K. Integrase inhibitor gains FDA approval. Am J Health Syst Pharm 64: 2310, 2007.

Tremblay CL \& Hirsch MS. The basic principles for combination therapy. In: Combination Therapy of AIDS, 1st ed., edited by De Clercq EDA \& Vandamme AMI. Basel: Birkhauser Verlag, 2004, pp. $41-51$.

Trevan JW. The error of determination of toxicity. Proc Roy Soc B 101: 483-514, 1927.

Turchin P. Complex Population Dynamics: A Theoretical/empirical Synthesis. Boston, MA: Princeton University Press, 2003. van der Graaf PH \& Danhof M. On the reliability of affinity and efficacy estimates obtained by direct operational model fitting of agonist concentration-effect curves following irreversible receptor inactivation. J Pharmacol Toxicol Methods 38: 81-85, 1997.

van der Graaf PH \& Stam WB. Analysis of receptor inactivation experiments with the operational model of agonism yields correlated estimates of agonist affinity and efficacy. J Pharmacol Toxicol Methods 41: 117-125, 1999.

van Dyck CH, Schmitt FA \& Olin JT; Memantine MEM-MD-02 Study Group. A responder analysis of memantine treatment in patients with Alzheimer disease maintained on donepezil. Am J Geriatr Psychiatry 14: 428-437, 2006.

van Rijn CM \& Willems-van Bree E. A four-ligand hypercube model to quantify allosteric interactions within the GABAA receptor complex. Eur J Pharmacol 485: 43-51, 2004.

Vendrell M, Angulo E, Casadó V, Lluis C, Franco R, Albericio F \& Royo M. Novel ergopeptides as dual ligands for adenosine and dopamine receptors. J Med Chem 50: 3062-3069, 2007.

Ward WE (editor). Food-drug Synergy and Safety, 1st ed. Boca Raton, FL: CRC Press, 2007, pp. 1-500.

Ward WE \& Fonseca D. Soy isoflavones and fatty acids: effects on bone tissue postovariectomy in mice. Mol Nutr Food Res 51: 824-831, 2007.

Wells JW. Analysis and interpretation of binding at equilibrium. In: Receptor?ligand Interactions. A Practical Approach, 1st ed., edited by Hulme EC. Oxford: IRL Press at Oxford University Press, 1992, pp. $289-395$.

White DB, Slocum HK, Brun Y, Wrzosek C \& Greco WR. A new nonlinear mixture response surface paradigm for the study of synergism: a three drug example. Curr Drug Metab 4: 399-409, 2003.

Winding B \& Bindslev N. Desensitization and reactivation of AChregulated exocrine secretion in hen tracheal epithelium. Am J Physiol 264: C342-C351, 1993.

Wood SC, Tonner PH, de Armendi AJ, Bugge B \& Miller KW. Channel inhibition by alkanols occurs at a binding site on the nicotinic acetylcholine receptor. Mol Pharmacol 47: 121-130, 1995.

Yeni P. Update on HAART in HIV. J Hepatol 44 suppl 1: S100-S103, 2006.

Yeung ML, Bennasser Y, Le SY \& Jeang KT. RNA interference and HIV1. Adv Pharmacol 55: 427-438, 2007. 


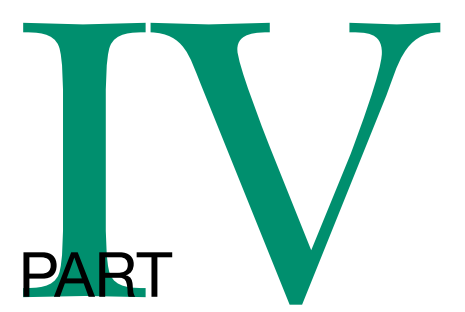

\section{BIOLOGICAL REGULATION AND ALLOSTERY}

As a start-up, for the last three chapters with a discussion of biological regulation and allostery in more detail, here I present molecular and dynamic forces operating at equilibrium and at steady-state in a nano-world. At a nano-scale, things are not static. This is also illustrated by observing the physics of the cell wall.

\section{IV.1. Flicks and Forces at a Nano-scale}

Before descriptions of general regulatory means, as exemplified in Chapter 13, allow me to remind you of the flicks and forces operating for molecules at or below a nano-dimension. Recognize that movement of molecules, also when operating at equilibrium, are not static from an angstrom-nano scale perspective, that is a scale of $10^{-9}-10^{-10} \mathrm{~m}$. Even at equilibrium, molecules flicker forth and back at high speed typically governed by noncovalent forces such as dipole moment (van der Waal force), ionic interaction (Coulombic force), hydrogen bonding (Pauling force), hydrophobic effects, and 'exchange repulsion' (Davies 2006b). The physics of the nano-world differs from our mechanistic reality. Thus, the tools in nano-technology are specialized equipment using techniques such as tunnel-scanning microscopy (TSM) and atomic-force microscopy (AFM) (Di Ventra et al. 2004; Dupres et al. 2007). Similarly, resolution by X-ray crystallography operates at this level. Nevertheless, observe that all three techniques yield static pictures of a flickering world, whereas techniques such as dual polarization interferometry, fluorescence resonance energy transfer (FRET) (Granier et al. 2007), flash-flow laser-beam exposure and recording, together with high-field electron paramagnetic resonance spectroscopy studies (Budamagunta et al. 2007) can give us genuine dynamic nano-images.

\section{IV.2. The Cell Wall}

Structures like the cell membrane are held together by the above-mentioned non-covalent forces. They render the resting membrane extremely elastic and fluidly flexible. We can illustrate this by comparing the thickness of the cell membrane of an epithelial columnar cell with the walls of the Leaning Tower of Pisa (Fig. IV.1). Moreover, at an angstrom-nano-scale with dynamics in and around the cell membrane, equilibrium still permits molecules to jump on and off the membrane at a flickering rate faster than $10^{10} \mathrm{~Hz}$. Therefore, at ordinary membrane potentials, ion channels inserted in a cell membrane can move up to $10^{8}$ molecules per second across the wall, and that in a selective fashion!

\section{IV.3. Equilibrium is Not 'Static'. Synagics Versus Statics}

A note on terminology. According to Lotka (1956, p. 53) equilibrium is 'Statics'. However, since movement at a molecular scale, even at equilibrium, is a very fast and dynamic process brought about by Brownian movements, I prefer the term 'synagics' for descriptions of dose-responses at either equilibrium or steady-state (see also sub-chapter I.3 of Part I for the term 'synagics').

\section{IV.4. Last Three Chapters}

Chapter 13 is on aspects of bio-regulation. Themes are on networks introduced in sub-chapter 13.2, on timedependent signaling by morphogens outlined in sub-chapter 13.3, and on evolution-development. In 


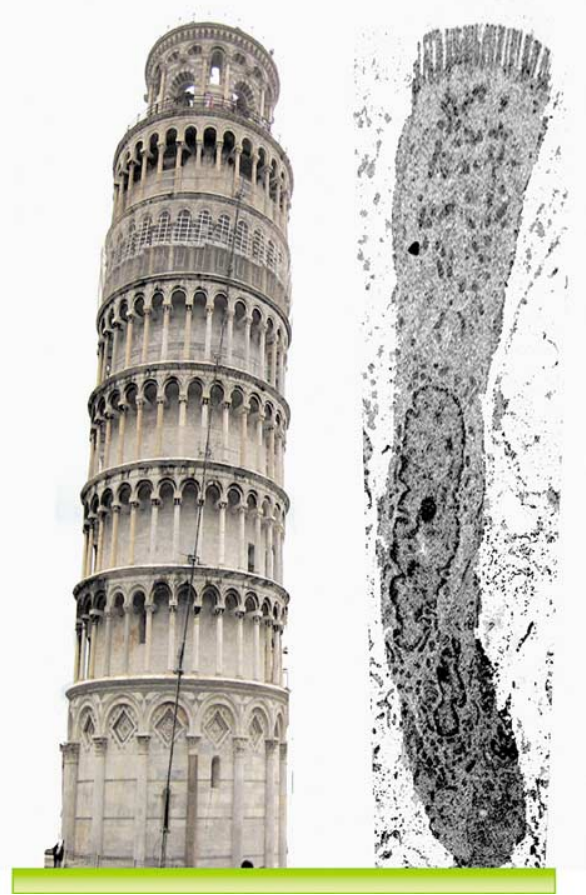

Figure IV.1. Cell wall thickness. (A) The leaning tower of Pisa is $56 \mathrm{~m}$ high and its wall thickness is between 2.5 and $4 \mathrm{~m}$. (B) A columnar epithelial cell from the small intestine is some $28 \mathrm{~mm}$ tall and its cell wall thickness is $4 \mathrm{~nm}$. When we multiply the cell values with $2 \times 10^{6}$, the epithelial cell becomes the height of Pisa' leaning tower, whilst the wall thickness for this giant cell remains at $<1 \mathrm{~cm},=0.8 \mathrm{~cm}$. Clearly it is another combination of forces than in ordinary mortar which keeps bricks together in the cell wall and makes it flexible and elastic. Figures and composition kindly provided by Dr. Steen Seier Poulsen, BMI, University of Copenhagen.

evo-devo, 'evolvability' is a regulatory term for the general capacity to adapt. I shall return to this and other aspects of evolution in sub-chapter 13.4. One brief survey on the present state of control theory with allosteric modulation and covalent modification is presented in sub-chapter 13.5.

In the next two chapters on synagics, chapters 14 and 15, we will concentrate on the so-called 'allosteric model' for biological co-operativity and modulation.

Chapter 14 focuses on definitions of the doseresponse concepts 'allostery' and 'co-operativity'. In sub-chapter 14.1, we commence with some historic accounts of the two terms. Then in sub-chapter 14.2, I impart strict definitions of the concepts 'allostery' and 'co-operativity' as they relate to modeling in synagic relations. The strict definitions of allostery and cooperativity are again contrasted with the looser use of the terms in studies where the model description is phenomenological rather than physical (sub-chapter 14.3). In sub-chapter 14.4 , the meaning of the concept 'allostery' is further illuminated by relating it to the following subjects: (1) multi-meric effectors, (2) monomeric effectors, (3) down-stream measures in signaling pathways, (4) multi-ligand cotransporters, (5) monoligand studies with reverse bell-shaped synagics, (6) 'functional selectivity' or so-called ligand-dependent differentiated functionality (Berg et al. 2008) and finally (7) to an intrasteric functional control. It is indicative and of note that in two modern and excellent texts on biochemistry, covering enzyme action, it is hard to find a useful survey on meanings and models of allostery except for a repetitious repeat of the Monod-WymanChangeux equations for occupancy and function from 1965 (Monod et al. 1965; Berg et al. 2002, 2006; Nelson \& Cox 2005).

Chapter 15 is rather difficult to understand. However, if you are able to extract the essentials with a clear sense of 'what should be done for model-building in the future', you are well off.

Chapter 15 begins with a brief account of the origin of the allosteric model and its development by MonodWyman-Changeux, the MWC model. In sub-chapter 15.3, I go through the classic description of the MWC model in a homotropic setting with a single type of ligand. In Sections 15.3.4-15.3.6, as a novelty, I demonstrate how the functional aspect of the MWC model has been diverted in a spurious direction by the original MWC paper, and instead I offer an operative formulation for the functional aspect of the MWC model; a formulation which should be followed in order to handle catalytic activity in enzymes and molecular movement by co-transporters in the spirit of a genuine allosteric model. Hopefully, Sections 15.3.4-15.3.6 will perplex the reader - followed by enlightenment. At the outset, heterotropy is briefly introduced by Eq. 15c in Section 15.3.4. The MWC model in a heterotropic setting with at least two different types of ligands is dealt with in sub-chapter 15.4. This is followed in subchapter 15.5 by a short account on the term 'desensitization' as used by two schools. Sub-chapter 15.6 presents a short discussion of other allosteric models such as the Koshland et al. reaction schemes, KNF models, in line with the Hill-Adair-Pauling tradition of varying interactions between subunits interactions. Although these schemes are less restrictive and more comprehensive than the MWC model, working with them is more difficult. In sub-chapter 15.8, amongst other model expansions, I present a Pascal extension of the modified Hill equation, offer a comparison between the MWC model and the Katz and Thesleff two-state model, and further specify what differentiates the MWC model from other models. I conclude with a section on how to model in the future. Sub-chapter 15.9 is a brief summary on which theoretical tools to choose in order to evaluate experimental equilibrium effects. 


\subsection{Allostery in Perspective}

\subsubsection{Networks, Morphogens, and Evolution}

In advance of discussing issues and models on regulation at the receptor level, focusing on allostery, I will briefly present three aspects related to process control, ${ }^{1}$ in order to put allosteric regulation in perspective. The three aspects are (i) network control, (ii) morphogen signaling with pattern formation, and (iii) evolution of bio-logical regulation. All three aspects operate within cells, at cells, between cells, and beyond - that is, at and above the receptor level.

\subsubsection{Process Control}

For process control, I shall adhere to the traditional physiological interpretation of information processing of, for instance, input-output-feedback without contingent codes and meaning.

Conversely, in the semiotic sign-theory, an interpretation is needed for the information processing with codes and a kind of meaning as 'survival of the fittest'. Presumably, sporadic and simple biosemiotics (Barbieri 2007; Hoffmeyer 2008 (in Danish 2005)) appeared early on in the organic realm followed by the codon of transfer-RNA and methylation of DNA with histone modifications for epigenetic regulation of cell differentiation. Later on, slowly transmuting into the outmost abstraction of reflection and self-reflection in and on human language/psyche and inter-subjectivity of souls (Lacan 1969; Deacon 1997; Jablonka \& Lamb 2005). ${ }^{2}$

\footnotetext{
${ }^{1}$ See David Fell (1997, Chapter 1) for a definition of the terms 'control' and 'regulation' in biological and metabolic control theory.

2 Consiousness in humans is best explained by the limits of our language - a Gödelian loop.
}

\subsubsection{The Rate-limiting Step}

In reality, for metabolic control and signal transduction in networks it is not enough to gaze at a few or a single step, 'the rate-limiting step'. As advocated by Fell (1997, Chapter 5), in regulation and control of metabolism, the whole network of involved elements and events must be taken into consideration (Cornish-Bowden et al. 2007; Henry et al. 2007); even though many triggered responses including steps in the bio-synthetic process (Cornish-Bowden 2004a,b) may still be viewed through a looking-glass, narrowing in at a rate-limiting process with a the 'committed step' (Straub \& Sharp 2004; Johnson et al. 2005; Levin 2006; Harris 2006a, p. 404).

On the level of organic auto-regulation or self-reflection, consider the proof-reading, maintenance, and repair of DNA by proteins encoded by DNA.

\subsection{Principles of Strategy. Networks and Systems Biology}

\subsubsection{Network Motifs}

Interdependence takes place at many levels and has many forms and patterns in the living world. The allosteric twostate model (ATSM), a synagic scheme presented in Chapter 7, is just one such connective network on a small scale. The ATSM may be taken for more general descriptions of regulatory and complex networks, built on simple nodes and edges (Milo et al. 2002, 2004; Kashtan et al. 2004; Kasthan \& Alon 2005; Zhang et al. 2005), with for instance a total of six quads fully connected (see Table 13.1 and Fig. 13.1). In such networks, one can find motifs. Identified motifs are (a) three-node motifs such as chains, feed-forward, feedback, feedback with mutual dyads, uplinked mutual dyads, including fully connected triads, and (b) four-node motifs as the 
Table 13.1. Examples of network motifs and node subgraphs*

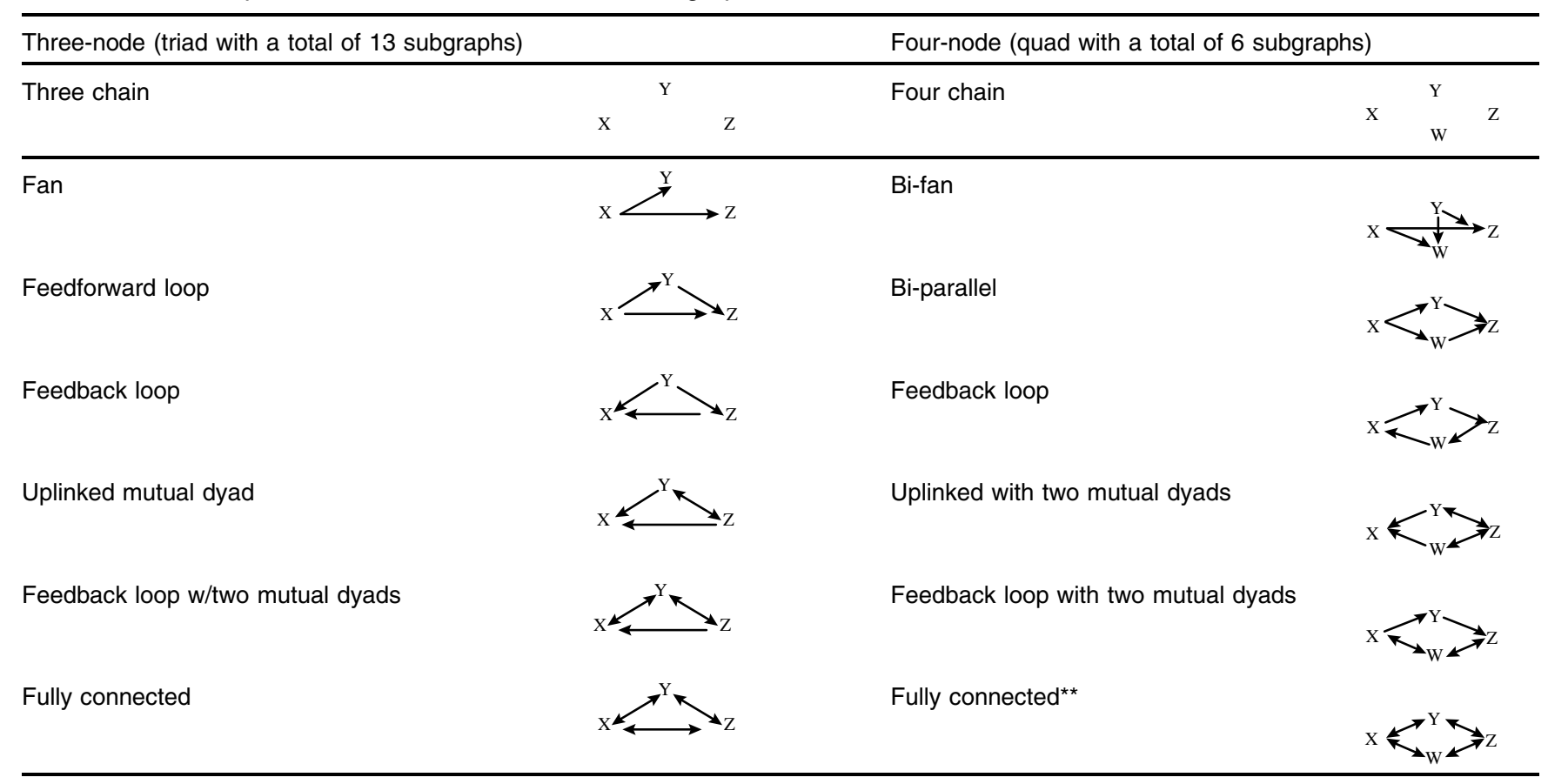

${ }^{\star}$ Freely after Milo et al. (2002)

**The ternary-complex model in chapter 2 and the cyclic two-state model in chapter 5 are examples of a fully connected triad and a dyad.

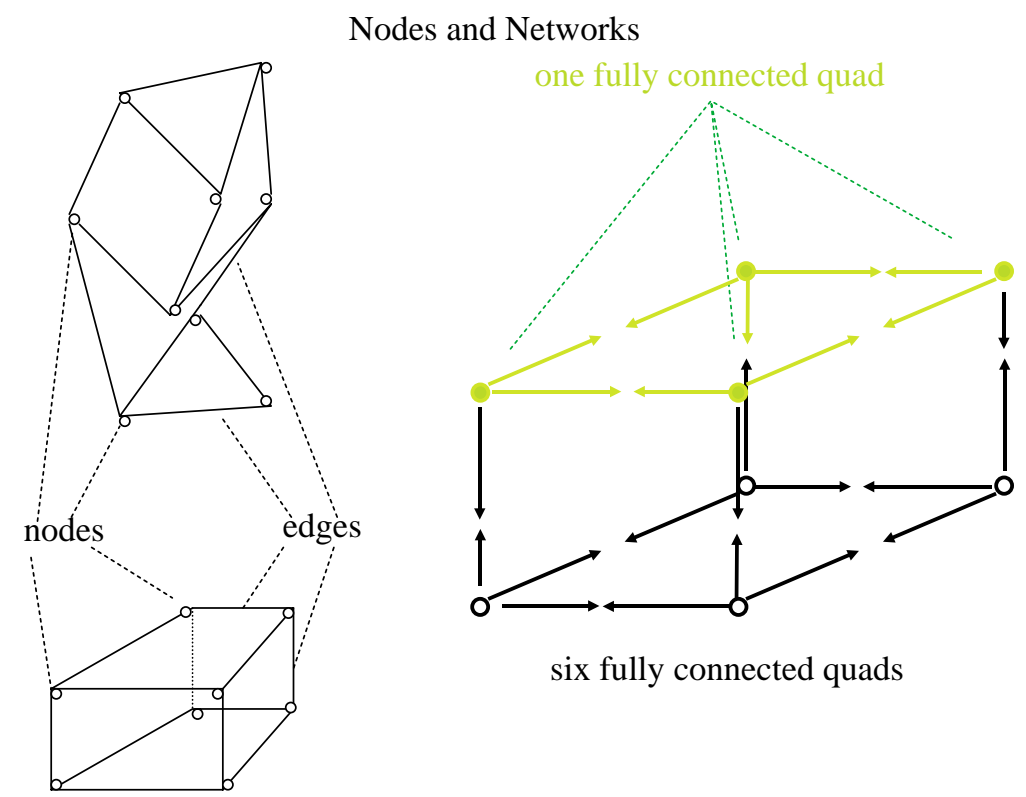

Figure 13.1. Examples of simple network modules with nodes and edges. On the right, the six fully connected quads comprise a cubic reaction scheme resembling the allosteric two-state model (ATSM), the homotropic two-state model (HOTSM), or the cubic ternary complex model (CTCM), described in Chapter 7.

bi-fan, bi-parallel, and feedback loops (Table 13.1) (Milo et al. 2002). Network motifs are recurring significant patterns of interconnections and may be found in fields as disparate as regulated gene networks, transcriptional expression, social nets, economic evaluation, ecological analysis, neuronal interconnectivity, electronic circuits, world wide web linking, food supply webs (Dobrin et al. 2004; Milo et al. 2002, 2004) and also in simple networks of chemical processes described by enzymologists with their generalized grids 
for regulated catalysis. Descriptions with nodes and edges for enzyme catalysis appeared early. Compare motifs in Table 13.1 with the King and Altman (1956), Cleland (1956) and Cha (1968) schematic description of enzymatic reaction patterns (Volkenstein \& Goldstein 1966). In other fields, models for the behavior of simple networks started with the description by Kauffman (1969) of genetic stability, and the simultaneously developed 'Game of Life' by John Horton Conway (Gardner 1970).

The general applicability of the motifs approach has been questioned (Artzy-Randrup et al. 2004), however, to mention a few examples from cellular signal transduction and stem cell self-renewal and commitment, the network approach is now exploding for explications of among others the activity of mitogen activating protein kinases (MAPKs) (Kholendenko et al. 2006; Santos et al. 2007), new network levels of regulation (Ciesla 2006; Meshi et al. 2007), and the transcriptional regulation of embryonic stem cells (Walker et al. 2007).

\subsubsection{Network Quantification}

Formulated quantization in space and time of the different pathways in a soup of mutual dominancesubordinance for the overall process is still sparse and trailing behind popular flow diagrams and high throughput screens on micro-array chips for identifying new gene products and their functional synergistic interactions - the products structure-activity relationship (PSAR). Gene expressions may be analyzed by consensus clustering of microarray data (Swift et al. 2004), and furthermore, gene function and their PSAR are examined by the Gene Ontology Toolbox (Martin et al. 2004).

Suggestive arrow-schemes of networks without quantization are ubiquitous (e.g., see Figs. 13.2 and 13.3). Of course, arrow diagrams are useful (Meir et al. 2002; Hornberg et al. 2006; Kohn et al. 2006) and when realized even useful with misleading interfaces (von Dassow \& Odell 2002).

However, it is the quantization of the depicted arrows and their interaction that is falling behind and desperately needed; the answer is Systems Biology.

\subsubsection{Examples of Systems Biology}

Indeed, initiatives to describe network control quantitatively are increasing, notably with an example as the study of the whole cell metabolism by Tomita and coworkers (Tomita et al. 1997). Keranen (2004), in another example of quantization, has demonstrated that stable patterns depend on the distribution of regulatory interactions as well as on negative interactions. Early on, Tyson and Mackey (2001) introduced a whole issue of the journal Chaos with non-linear modeling as a tool for quantifying complex reaction schemes for newcomers. In addition to the already quoted references, other examples may be found in papers by Reid and Bourke (2003a,b), Ciliberto et al. (2003), Salvador and Savageau (2003), Zhigulin (2004), Roberts and Stone (2004), Yu and Bernadino (2004), and in recent reviews by Tan et al. (2008) and Komili and Silver (2008).

This entire subject is now collected under the novel field term 'Systems Biology'. ${ }^{3}$ Part of this field consists of studies in genome-networks controlling biochemical reactions that take place inside cells. Thus, Systems Biology is assigning numbers on the arrows of network diagrams for biological processes, integrating them and evaluating the outcome of the integration. Systems Biology is integrative biology (physiology) with a new kick-start due to the functional genomics of the 1990s and additional advances in high-throughput studies and repositories of bioinformatics (Alon 2006; Palsson 2006; Konopka 2007). Nevertheless, modeling processes of integrative biology and its quantitation have been around for much longer (e.g., Kauffman 1969; Kacser \& Burns 1973).

Nonetheless, Systems Biology seems to have replaced the hype about molecular biology in the 1980s and 1990s. Hits on 'Systems Biology' in PubMed were 1203 in early January 2007 of which 1200 appeared by the year 2001 or later, and over 2000 in early January 2008. Table 13.2 lists examples of fields covered by Systems Biology.

\subsubsection{Brief Statements on Bioinformatics, Networks, and Systems Biology}

Bioinformatics is the acquiring and organizing of information about genes, proteins, and their regulatory components such as transcription factors by highthroughput screens of genomes and proteomes, and placing the results in data-repositories for easy access.

Biological networks are generated based on information obtained from experimentation including micro-array screens of functionality links in genome, transcriptome, proteome, metabolome and other interactomes, such as signal transduction in regulatory systems (e.g., Clemens et al. 2000; Friedman \& Perrimon 2006, 2007); the latter we may call the 'signalome' by a generalizing term (see also arrowomics, nodomics, and wiromics in Table 13.2).

\footnotetext{
${ }^{3}$ Definition from Wikipedia: 'the defining feature of Systems Biology is the ability to obtain, integrate and analyze complex data from multiple experimental sources using interdisciplinary tools'. In addition, I would like to add: 'and with quantification of arrows in network diagrams'.
} 


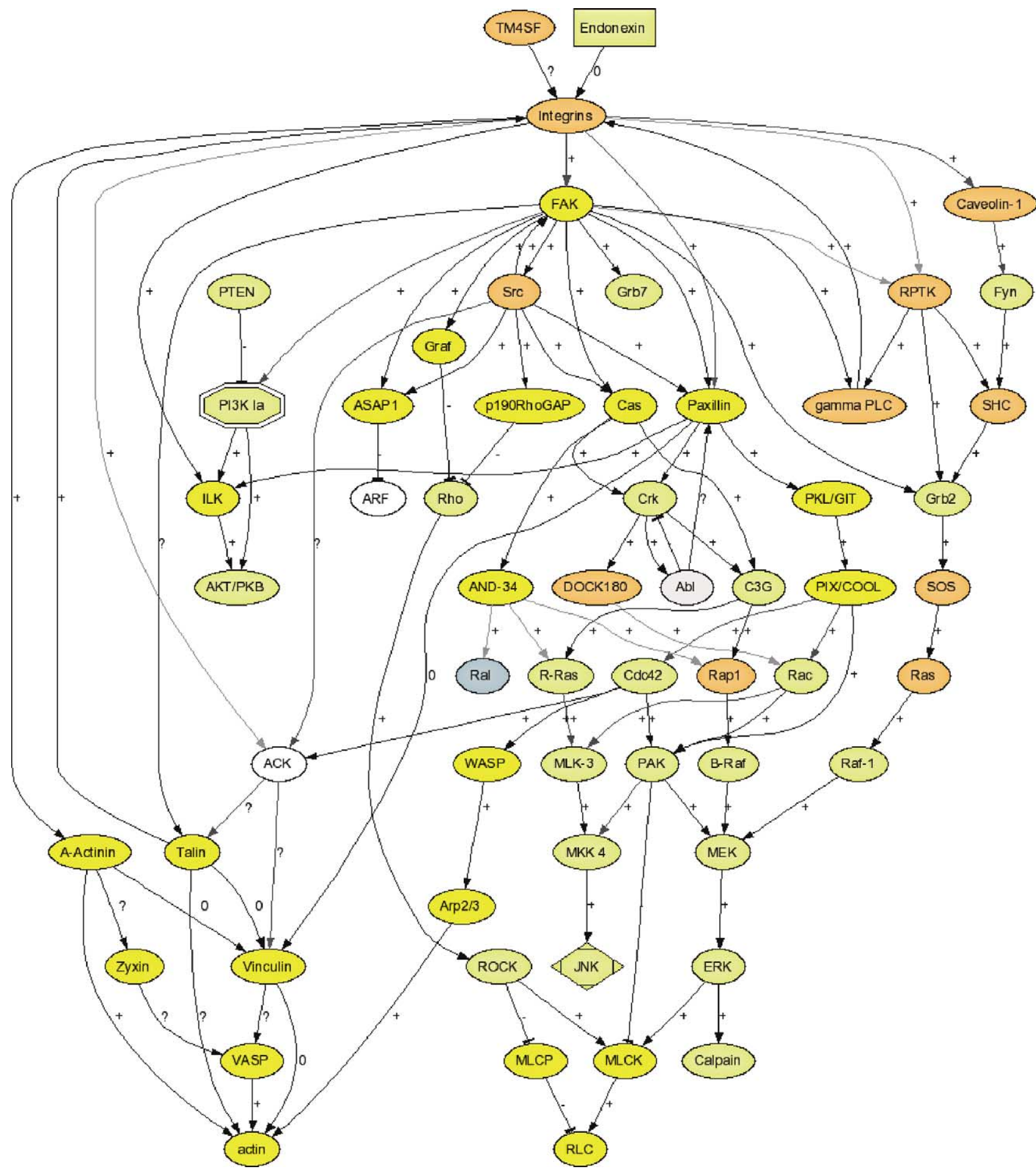

Figure 13.2. Arrow diagram of the integrin signaling pathway from K.H. Martin, J.K. Slack, S.A. Boerner, C.C. Martin, J.T. Parsons. Integrin Signaling Pathway Sci. Signal. (Connections Map in the Database of Cell Signaling as seen 29 February 2008). http://stke.sciencemag.org/cgi/cm/stlecm/CMP_6880, with permission.

Additionally, 'connectomics' is a coming term covering wiring in the nerve system (http://biblion.epfl.ch/EPFL/ theses/2005/3230/EPFL_TH3230.pdf).

Systems Biology uses bioinformatics from established and anticipated network diagrams. Systems Biology is just a modeling of these networks and attempts to quantify the connecting arrows of the diagrams by applying pertinent and optimized network rules (Kholodenko 2007). The links or arrows of the network diagrams are also their parameters. Systems Biology is thus a general term for approaches to estimate parameters of interaction in biology. 


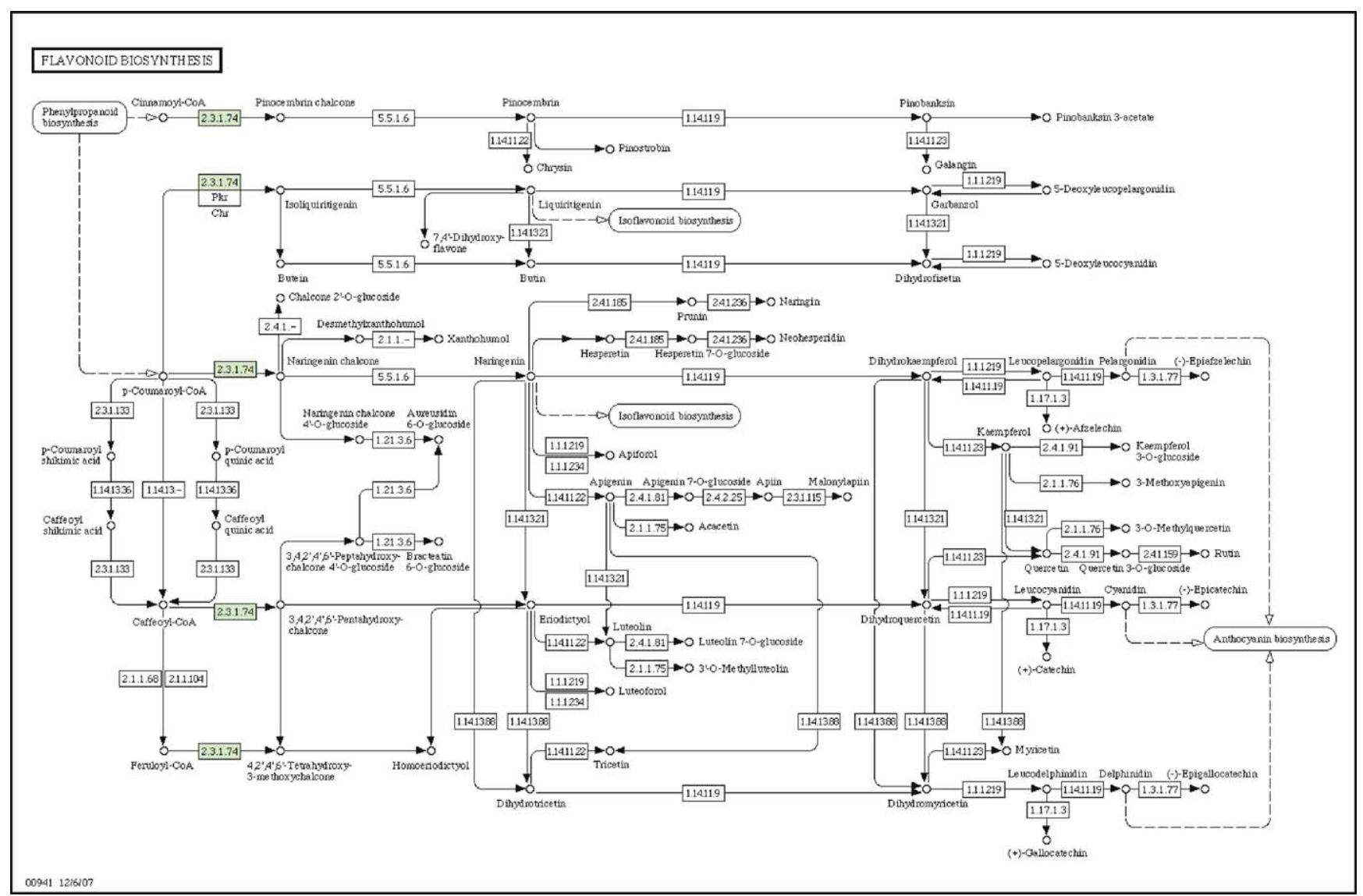

Figure 13.3. Node and edge diagram of flavonoid biosynthesis in Arthrobacter aurescens. Map 00941 from the KEGG database (reproduced from http://www.genome.jp/kegg/xml/map/index2.html) with permission.

Table 13.2. '-Omics'. Bio-fields covered by Systems Biology 9

\begin{tabular}{|c|c|c|c|}
\hline The '-omics' & Field of interest & Society & Reference examples \\
\hline Arrowomics* ${ }^{*}$ & Networks in general & - & Mak et al. (2007) \\
\hline Babelomics & Suit of web tools & & Al-Shahrour et al. (2006) \\
\hline Biomics & Many omics & & Romero et al. (2006) \\
\hline Blobomics & Omics in general & & Colquhoun (2005) \\
\hline Cytomics & Cytometry & & Herrera et al. (2007) \\
\hline Genomics & DNA sequences & & Jurka et al. (2007) \\
\hline Glycomics & Glucose metabolism & & Pilobello \& Mahal (2007) \\
\hline Histocytomics & Histo-cyto-chemistry & & Coulton (2004) \\
\hline Interactomics & $\begin{array}{l}\text { Interaction studies in general, e.g., networks of } \\
\text { protein or signaling }\end{array}$ & & Cesareni et al. (2005); Yip et al. (2006) \\
\hline Kinomomics & Kinome & STKE/Science & Ray \& Gough (2002) \\
\hline Metabolomics & Metabolism & Boston 2006 & Goodacre (2005) \\
\hline Metabonomics & Small molecules drug discovery & & Robertson et al. (2007) \\
\hline Nodomics* & Networks in general & - & - \\
\hline Pharmaco-genomics & Drug development for individuals & $\begin{array}{l}\text { Pharmacogenomics } \\
\text { Focus and Network }\end{array}$ & Giacomini et al. (2007) \\
\hline Pharmacomics & 'Analytical pharmacology’ & & Urban et al. (2006) \\
\hline Phosphoproteomics & Protein sequences and more & & Paradela \& Albar (2008) \\
\hline $\begin{array}{l}\text { Proteomics } \\
\text { STYK-omics }{ }^{* *}\end{array}$ & $\begin{array}{l}\text { Protein sequences and more } \\
\text { Signal transduction knowledge environment }\end{array}$ & & $\begin{array}{l}\text { Ahn et al. (2007) } \\
\text { Ray \& Gough (2002); Adler et al. (2007) }\end{array}$ \\
\hline $\begin{array}{l}\text { STYK-omics } \\
\text { Traitomics }\end{array}$ & $\begin{array}{l}\text { Signal transduction knowledge environment } \\
\text { Ancestry-info markers (in human development) }\end{array}$ & STKE/science STYKs & $\begin{array}{l}\text { Ray \& Gough (2002); Adler et al. (2007) } \\
\text { Mao et al. (2007) }\end{array}$ \\
\hline Transcriptomics & RNA expression levels & & $\begin{array}{l}\text { Carter (2006); Tuggle et al. (2007); Heidecker \& } \\
\quad \text { Hare (2007) }\end{array}$ \\
\hline Wiromics* & Networks in general & - & - \\
\hline
\end{tabular}

See also Petsko (2007).

${ }^{*}$ These network -omics are what Systems Biology is all about (Sections 13.2.3-13.2.4).

** The interactome of signaling is often referred to as the 'signalome' or for regulated phosphorylation by kinases at serine, threonine, or tyrosine it is the 'STYKome'. 


\subsection{Short-term Strategy. Pattern Formation}

\subsubsection{Morphogen Signaling and Pattern Formation}

The second issue is morphogen signaling with pattern formation. Morphogens are signaling molecules released during differentiation and development. Morphogens control the shape of organs and whole organisms in embryogenesis and ontogenesis. An example is the construction of the colon epithelium (van de Wetering et al. 2002; Battle et al. 2002; Laprise et al. 2004; van den Brink \& Offerhaus 2007). The morphogen molecules are produced in and released from restricted regions of tissue and move away to form a long-range concentration gradient. Differentiation of cells, induced by morphogens, operates by timing, tuning, and turning on-'n-off the nodes in transcriptional networks; networks dependent on the spatial arrangement of cells in the extracellular morphogen concentration gradient (Teleman et al. 2001; Rogulja \& Irvine 2005).

\subsubsection{Production, Release, and Movement of Morphogens}

The production, release, and travel of morphogens are not only due to simple processes such as constitutive secretion and diffusion. The synthesis and release of these signaling molecules are well-orchestrated, and their concentration is controlled both in time and space. For instance, the release of membrane bound morphogens is dependent on specialized exocytotic mechanisms (Burke et al. 1999; Lewis et al. 2001; Wang et al. 2007). In addition to diffusion, the movement of morphogen molecules takes place by several other mechanisms such as transcytosis (Entchev et al. 2000; Strigini \& Cohen 2000; Entchev \& GonzalezGaitan 2002; Jude et al. 2004), intracellular transport assisted by members of the kinesin superfamily (Hirokawa 2000; Hirokawa \& Takemura 2004; Weaver \& Kimelman 2004), transport in cytoplasmic extensions, the cytonemes (Ramirez-Weber \& Kornberg 1999; Williams et al. 2004; Galkina et al. 2006) (Fig. 13.4). Intracellular vesicular transport in endovesicles, called argosomes (Greco et al. 2001; Christian 2002), by trancytotic movement (Fig. 13.4B; Tabata \& Takei 2004) and possibly also by the wandering of morphogen-containing cells (Tashiro et al. 2006); all end up by releasing morphogens for signaling in the paracrine pathways of particular pattern-plays. The regulated presentation of morphogens in the extra cellular matrix (ECM) will also depend on the binding of morphogens to cell surface receptors and ECM components along the long-range gradients (The et al. 1999; Eldar et al. 2003;

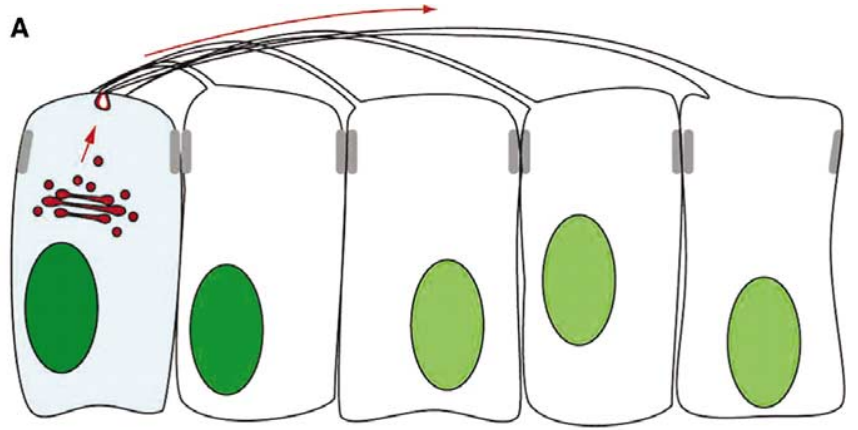

B

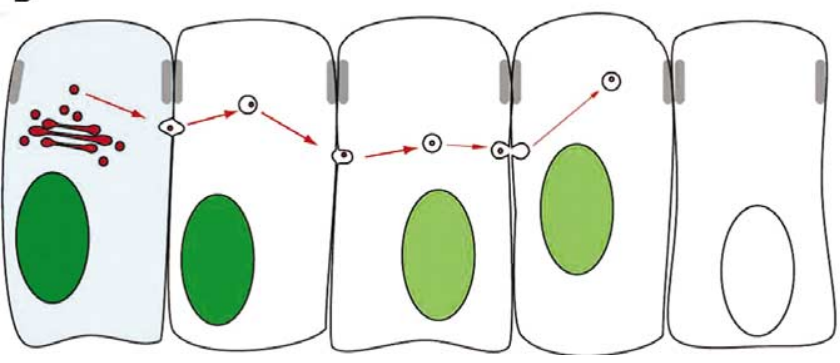

Figure 13.4. Cartoons of cytonemes (A) and agrosomes (B) in morphogenesis (from Tabata and Takei (2004, Fig. 9) Reproduced with Permission of the Company of Biologists). See also Sasaki et al. (2007).

Seiki \& Yana 2003; Tabata \& Takei 2004; Hinsby et al. 2004; Wang et al. 2007).

\subsubsection{Structural Genes or Homeo-box Genes and Their Morphogens}

Examples of differentiation ligands in the fruit fly Drosophilia, so-called morphogens, are hedgehog, wingless, and decapentaplegic. For more examples on fly-morphogens consult http://sdb.bio.pudue.edu/fly/aimorph/ imagdisc.htm, where you will also find examples on the involved transcription factors and their genes.

These structural genes are homeo-box genes also designated HOX-genes and are controlled by their own networks involving gap and notch genes (Hombria \& Lovegrove 2003; Duboule \& Deschamps 2004).

\subsubsection{Regulated Release of Morphogens and Extracellular Binding or Removal}

As an illustration of the complexity of intracellular trafficking, membrane transfer, and extracellular binding of morphogens, shortly I will present some of the current knowledge on the secretion and binding of hedgehog $(\mathrm{Hh})$ and Notch ligands in the respective signaling pathway of hedgehog and Notch.

Hedgehogs are morphogens important for embryonic development and their misbehavior results in, for instance, congenital defects and cancers (McMahon et al. 2003). 
The hedgehog morphogens are synthesized as precursor proteins, cleaved auto-catalytically, double lipidated (palmitoyl/cholestrol), and released as a 19-kDa complex from cavaolin-coated lipid rafts, maybe, or possibly via an apical sorting receptor mechanism (Rodriguez-Boulan et al. 2005; Reynwar et al. 2007), involving a 12TM RND-type $\mathrm{H}^{+}$-exchanger Disp1 (Burke 1999; Ma et al. 2002; Nakano et al. 2004; Higgins 2007) (Fig. 6.16) and/or the V0 sector of a vacuolar type $\mathrm{H}^{+}$ATPase (Liegeois et al. 2006). The secreted Hh lipoproteins are bound in a larger oligomeric lipoprotein complex (Fig. 13.5). This complex (dl-Hh-C) may either be removed from the ECM by internalization, effectuated through megalin receptors for lysosomal degradation, or bound to its receptor at nearby or distant cells. The dl-Hh-C receptor can be the transmembrane Ihog/ Boi proteins in invertebrates or the Boc/Cdo proteins in vertebrates. The complex of dl-Hh-C and its receptor then derepress a neighboring 7TM Smo receptor via a co-expressed and inhibitory 12TM Ptch1 protein, presumably involving the $\mathrm{G}$ protein-coupled receptor kinase 2 (GRK2) (Melino et al. 2006), thus activating family Ci/ Gli of transcriptional factors (Fig. 13.5) (Svard et al. 2006; Wilson \& Chuang 2006; Wang et al. 2007).
In the Notch signaling pathway, the secretory mechanism of polarized exocytosis differs from the hedgehog secretion. A significant difference is that Notch ligands stay in the cell membrane of the cell in which they were synthesized, and thus only interact with neighboring cells expressing the Notch receptor (Levine et al. 2007). Notch proteins are maternal morphogens involved in several aspects of cell movement and cancers (Androutsellis-Theotokis et al. 2006; Bray 2006; Roy et al. 2006; Sasaki et al. 2007; Bolos et al. 2007). Notch and gap genes control pair-rule segmentation and HOX-genes including hedgehog genes (Tour \& McGinnis 2006).

The superfamily of Semaphorins is just another group of morphogens with both free-floating and membranebound ligand-receptor characteristics (Yazdani \& Terman 2006).

\subsection{Long-term Strategy. Evolution}

\subsubsection{Evolution of Bio-logical Regulation}

The third theme is biological regulation of evolution or vice versa.

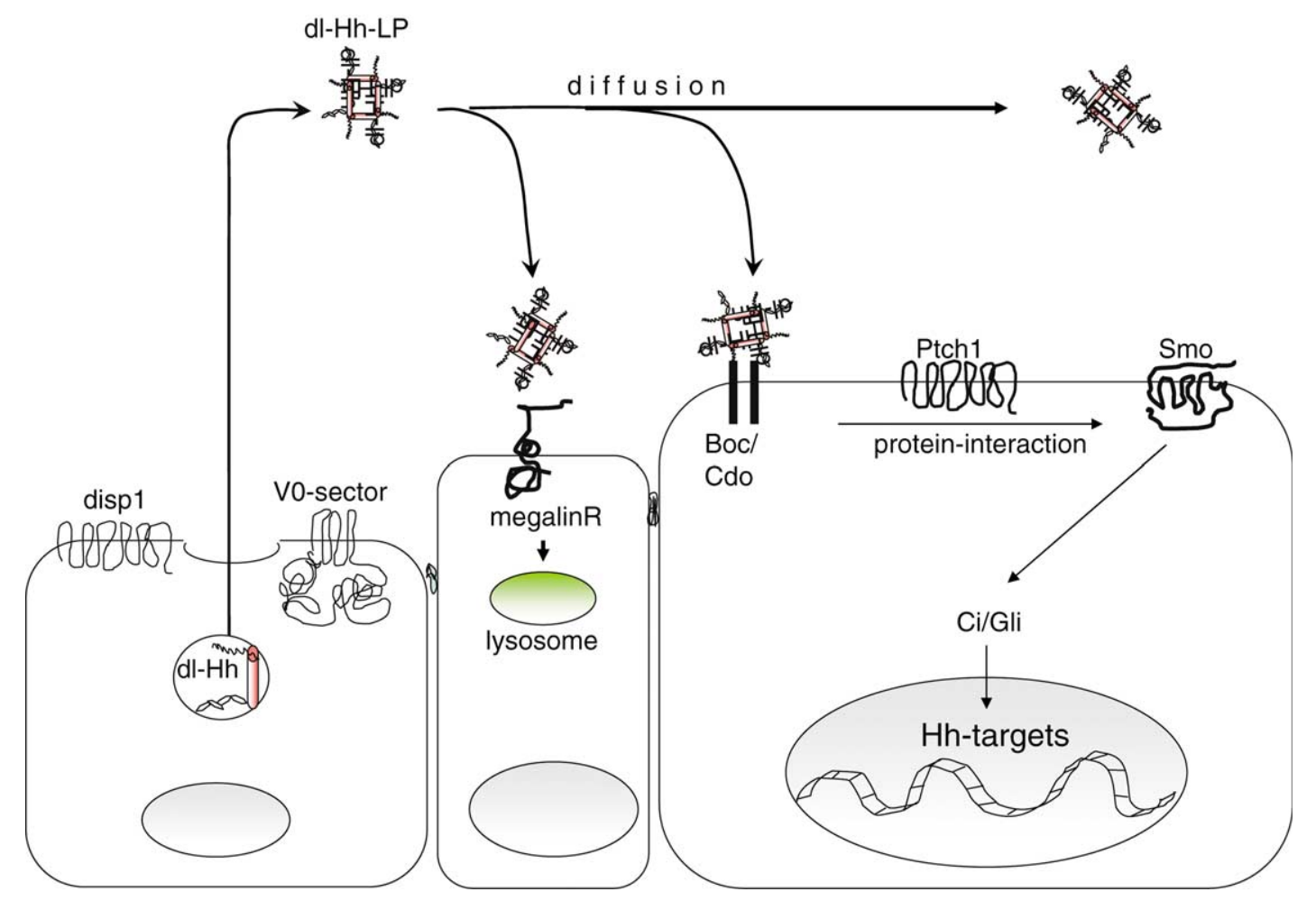

Figure 13.5. Schematic drawing of the release and binding of hedgehog $(\mathrm{Hh})$ morphogens inducing transcriptional modulations. $\mathrm{dl}-\mathrm{Hh}=$ dilipidated hedgehog, Disp1=dispatched 1 protein (a RND-type $\mathrm{H}^{+}$-exchanger), V0-sector=the $\mathrm{V} 0$ entity from a vacuolar type $\mathrm{H}^{+}$-ATPase, dl-Hh-LP=dilipidated hedgehog lipoprotein complex, Boi/Cdo=the vertebrate dl-Hh-LP receptor, Ptch1 $=12 \mathrm{TM}$ patched protein, Smo=7TM Smoothened receptor protein, $\mathrm{Ci} / \mathrm{Gli}=$ the $\mathrm{Ci} / \mathrm{Gli}$ family of transcriptional factors (the figure is created based on Butler et al. 1999; Nakano et al. 2004; Rodriguez-Boulan et al. 2005; Wilson \& Chuang 2006; Liegeois et al. 2006; Guerrero \& Chiang 2007; Wang et al. 2007). 
At the effector level, principles for glycogen phosphorylation carried out by phosphorylase enzymes ${ }^{4}$ have developed from bacteria to man as a good example of the evolutionary progress of bio-regulation from simple or no control to more complex strategies (Buschbinder et al. 2001). One difference between man and bacteria is the internal control of the glycogen phosphorylation process, which is a first step in recruiting energy, converting D-glucose subunits from the non-reducible end of glycogen to free D-glucose-1-phosphate. The bacterial consumption of glycogen is purely regulated by the exogenous supply situation and simple product (glucose-6-P) feedback, whereas in multi-cellular organisms from yeast to vertebrates there are complex internal regulatory mechanisms 'built' around the catalytic activity in order to comply optimally with energy demands at all times and with energy supply provided from internal stores. Covalent phosphorylation of the phosphorylase itself, inducing conformational changes, is part of the regulation of human glycogen phosphorylase (Nelson \& Cox 2005, pp. 583-591); as this kind of phosphorylation is for thousands of other controlled systems as for instance phosphorylation of 1,6-phophofructokinase (Fig. 13.6), by 2,6-phosphofructokinase in the ensuing steps of D-glucose breakdown (Fig. 13.7). Thus, Fig. 13.7 shows in more detail the regulation of subsequent steps in glycolysis in glucose breakdown. The regulatory mechanism of the committed step in glycogenolysis by phosphorylation of the human glycogen phosphorylase enzyme is missing in Fig. 13.7 and in the bacterial counterpart. That is not to say that intrinsic and extrinsic allostery is absent in bacteria. The whole concept of allostery was developed based on the behavior of bacterial enzymes (Cori et al. 1943; Umbarger 1956; Yates \& Pardee 1956) and one of the most complex allosteric enzymes is glutamine synthetase (GS), found amongst others in the bacterium E. coli (Berg et al. 2002; Nelson \& Cox 2005). Both the GS allosteric mechanisms and the E. coli are found in higher organisms as well. GS is the key-enzyme in nitrogen metabolism (Fig. 4.5). GS has at least eight different modulator sites, and is further covalently adenylated for regulatory purposes by another allosterically regulated enzyme, adenylyl-transferase with its allosteric ligand protein PII. PII is activated allosterically by uridinylyl transferase regulated allosterically by ... and on, and on and on ... (Nelson \& Cox 2005, pp. 838-340).

So far, metabolic modulation of bacterial GS is one of the most complex regulatory mechanisms. In addition, the possible interactions of all these regulatory means

\footnotetext{
${ }^{4}$ Recapitulate that phosphorylases add inorganic $\mathrm{P}$ to molecules (Berg et al. 2002, pp. 582-583), while kinases transfer the $\gamma$ P of ATP to amino acid residues (serine, threonine, and tyrosine) in proteins.
}

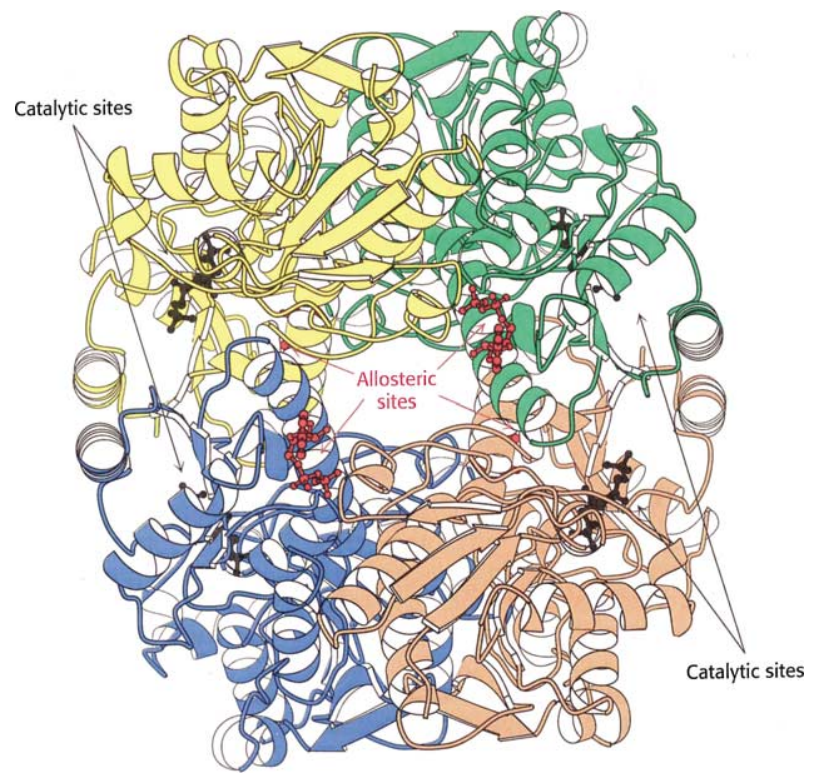

Figure 13.6. Allosteric modulation of 6-phosphofructo-1-kinase (PF-1-K (a)) by ATP/AMP ratio. ATP in red, fructose-6phosphate in black. From Berg et al. (2002, Fig. 16.16) with permission.

are staggering (Savageau 1976; Ni \& Savageau 1996; Salvador \& Savageau 2003).

Another spectacular example of evolutionary adjustment, now at the organ level, is the manner in which organism rid themselves of waste products from the nitrogen metabolism. When the end product of nitrogen metabolism is urea with a high solubility, it is upconcentrated in the kidneys. Contrary to this, for uric acid with a low solubility, creatures use a cloaca with a large diameter to concentrate their urine. Examples of animals in this category are dinosaurs and birds. In the kidney where water is absorbed, uric acid would be concentrated and become a thick white paste without a chance to pass through the tiny dimensions of the kidney tubules. Therefore, to solve this mechanical problem, uricoteles pass a low-concentrated urine into the lower intestine, cloaca, with a large dimension and up-concentrate the urine here (Bindslev \& Skadhauge 1971; Laverty \& Skadhauge 2008). The white paste on bird droppings is uric acid.

The development of different strategies in Nature for biological regulation and control is an integral part of evolution itself, and many questions need to be answered in this connection. Three such questions follow.

\subsubsection{Regulatory Intensions. HOX or Hoax}

First question: is it so that during evolution of the biofield, mechanisms for various kinds of regulatory feedback at different levels, including self-organization or autopoiesis (Maturana \& Varela 1980; Camazine et al. 


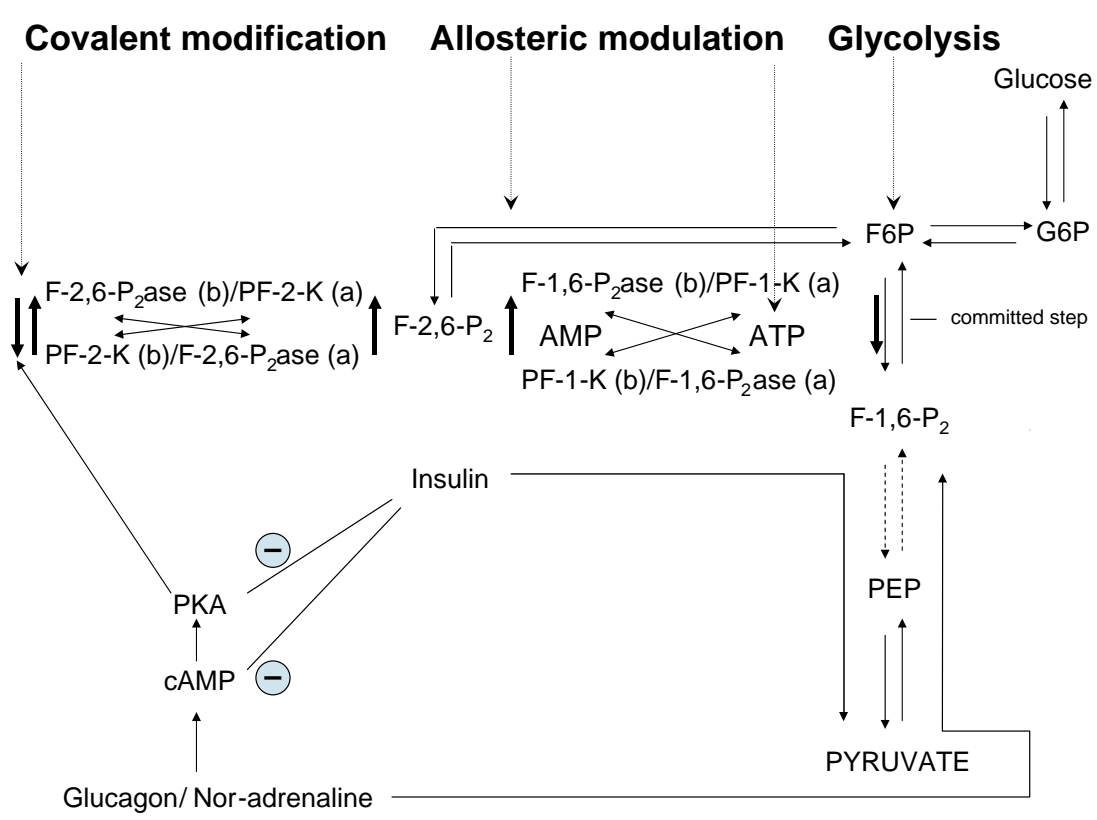

Figure 13.7. Covalent and allosteric modulation through two bifunctional enzymes in glycolysis. The enzyme fructo-1,6bisphosphatase/6-phosphofructo-1-kinase (F-1,6- $\mathrm{P}_{2}$ ase/PF-1-K) in the active kinase form (F-1,6- $\mathrm{P}_{2}$ ase (b)/PF-1-K (a)) phosphorylates fructose-6-phosphate (F6P) to fructose-1,6-bisphosphate ( $\left.\mathrm{F}-1,6-\mathrm{P}_{2}\right)$ - see the indicated 'committed step' in glycolysis (Harris 2006a). This enzyme is positively regulated in an allosteric fashion by the side-way reaction compound fructo-2,6-bisphosphate $\left(\mathrm{F}-2,6-\mathrm{P}_{2}\right)$, which is a product of another bifunctional enzyme fructo-2,6-bisphosphatase/6phosphofructo-2-kinase (F-2,6- $\mathrm{P}_{2}$ ase/PF-2-K), that in its active kinase form (F-2,6- $\mathrm{P}_{2}$ ase (b)/PF-2-K (a)) produces the allosteric modulator F-2,6- $\mathrm{P}_{2}$ from F6P. This second enzyme is covalently regulated towards increased phosphatase activity (PF-2-K (b)/F-2,6- $\mathrm{P}_{2}$ ase (a)) through phosphorylation by stimulated protein kinase $A(P K A)$ activated by cAMP. For evolution of the cAMP binding site, see Kannan et al. (2007). In addition, cAMP is generated through signaling via glucagon or noradrenaline or reduced due to insulin inhibition of PKA or activation of cAMP-phosphodiesterase (not shown). Thus, glucagon and nor-adrenaline covalent-allosterically reduce glycolysis while insulin augments glycolysis.

2001; Feltz et al. 2006) were invented with a purpose ${ }^{5}$ by an 'intelligent designer' or were these particular regulatory mechanisms blindly selected by Nature (Monod 1971; Dawkins 1987, 2003), leading to the marvel of the function of an eye as displayed in front of our eyes? That is a big question that arouses strong feelings.

I am in line with the criticism of 'intelligent design' (ID) as a hoax due to its lack of experimentation, as meticulously detailed by Coyne $(2005,2006)$ and at length by Forrest and Gross (2003). Thus, I favor the homeo-box (Hox) and modified Darwinian theory (Dawkins 1987, 1995; Gould 2002; Cornish-Bowden 2004b; Jablonska \& Lamb 2005; White 2006) as provisional mechanisms for our understanding of evolution. One may ask 'since the intelligent designer is not God, who is he?' Here Paul Davies (2006a, Chapter 10) takes the middle ground with a summary of different universes including multi-verse theory, Dennett (2006,

\footnotetext{
5 'Purpose' is to be taken as meaning 'final cause' from Peircian terminology as discussed in biosemiotics. See Peircean biosemiotics quoted in Hoffmeyer (1996, 2005, 2008) and Kantian 'purposiveness in Nature' (Walsh 2006) as opposed to the postulates of 'intelligent design'; even claiming experimentations into the matter (Dembski 2004). Intelligent design, unfortunately, has to be refuted as a science based on its lack of a tradition for experimental falsification.
}

2007) has an explication, while Harris (2006b), Dawkins (2006), and Hitchens (2007) are merciless on the God delusion, with Dawkins tentatively opposed by McGrath (2007).

A thorough presentation and discussion of multi-verse theory and the Neo-Darwinism-versus-ID debate is beyond the scope and commentaries of this book. Therefore, luckily, I do not need supportive reference to the Holy Book with a gospel prophecy from the Prolog of St John (Chapter 1, verses 1-3 and 18). However, when it comes to moral and ethical values, evolution in the scientific sense is of course insufficient. These values must be recognized and discussed in other forums as well. Normative regulation of political power must be exercised through social guidance that is governed by human rights and plights, not religion (Ratzinger 2005).

\subsubsection{Regulatory Evolution - Regu-lution}

Second question: do the different principles of bioregulation have a chrono-logical stem-tree that has been a logical prerequisite for our evolution; for instance for mono-cellularity to multi-cellularity (Fig. 13.8) (King 2004) or for the case of regu-lution (sic) of the above- 
A simplified history of life on Earth

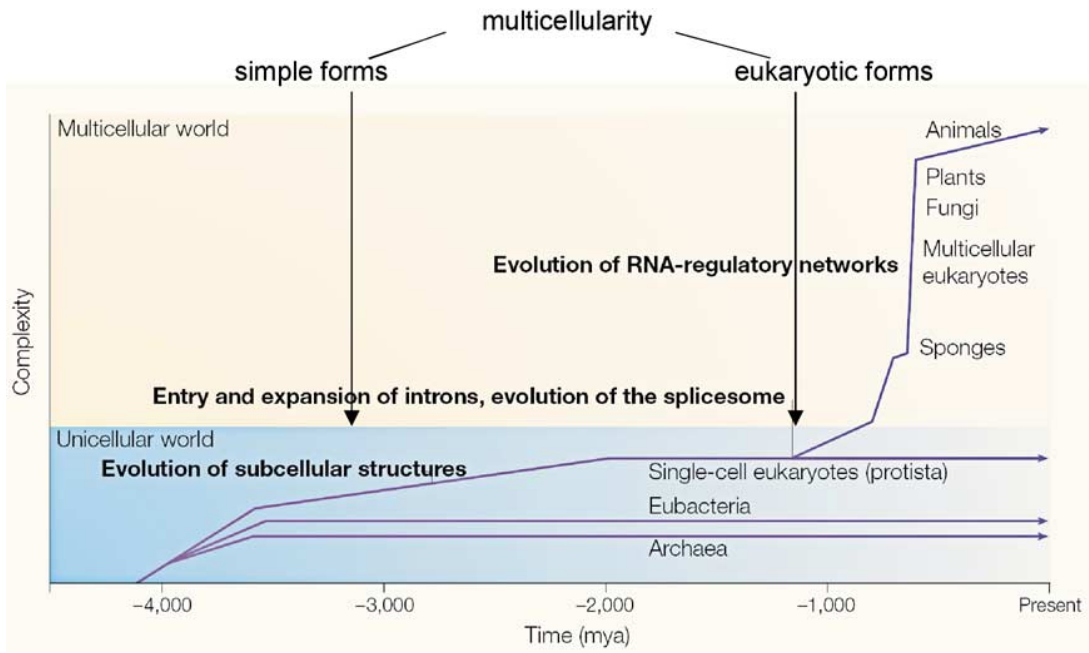

Figure 13.8. From unicellular to multicellular organisms during evolution of the biosphere. Simple forms of multi-cellularity started forming more than 3 billion years ago, while eukaryotic multi-cellularity started around 700 million years ago. Modified from Mattick (2004, Fig. 3) with permission. On the transition from uni- to multi-cellularity, see also Kirk (1998), King (2004) and Duncan et al. (2006).

mentioned glycogen phosphorylase and the flow of $\mathrm{N}_{2}$ through the works of the glutamine synthetase (GS) complex (Miflin \& Habas 2002)? That is, does the timing of the appearance of regulatory proteins and mechanisms matter in evolution? How important in development was the timed emergence of this metabolic mechanism (Battistuzzi et al. 2004; Vitkup et al. 2006), this hormonal induction, or that neuronal regulatory control? Was the timing of this operon and that HOXgene $^{6}$ a sine qua non? In addition, at the epigenetic network level, how are we to understand the timing in $98.5 \%$ of our genome as junk-DNA (Brosius 2003a), the timing of some exons representing bad genes (Brosius 2003b), and the timing of microRNAs involved in regulatory networks (Mattick 2004; Martin \& Caplen 2007; Bushati \& Cohn 2007)?

Furthermore, new questions arise as we now realize that over $20 \%$ of the junk-DNA is coding for regulatory RNA (Royo \& Cavaillé 2008; Umlauf \& Fraser 2008).

\subsubsection{Regulatory Death}

Third question: how does regulated cell death, apoptosis with caspase-mediated proteolysis (Nicotera \& Melino 2004; Riedl \& Salvesen 2007; Taylor et al. 2008) and species extinction (Gould 2002, pp. 738-741) come in

\footnotetext{
${ }^{6}$ Operon and HOX-genes. The concerted level of expression of a set of enzymes that contribute to an adaptive function due to an environmental change is based on a coordinated gene expression. An operon is a unit of such coordinated gene expression. Genes of operons especially related to development and differentiation are HOX-genes (Monod \& Jacob 1961; Berg et al. 2002, 2006).
}

as logical principles of regu-lution in the actual view of bio-evolution? Was and is regulated death of cells and species an immanent part of our ability to adapt - of our evolvability?

\subsubsection{Mapping Evolution. Human Races?}

A combination of maps and markers of single nucleotide polymorphism and short tandem repeats from the string of our genetic code combined with highly sophisticated computing techniques enables us to link human traits, often related to the so-called human race, to specific genetic composition (Fig. 13.9), which allows us to speak of human diversity [races] based on 'sick' genes among others (Smith et al. 2004). Analysis in this field of human traits and genetics with mapping-byadmixture-linkage-disequilibrium (Patterson et al. 2004) may soon be ripe for the '-omic post-fix', imparting the term 'traitomics' (see Section 13.4.6 and Table 13.2).

Let us hope that it will not be possible to link human psychological traits, such as angst, hysteria, obsessive neurosis, as well as inclinations for suicide and terrorism with a specific genetic composition. Possibilities for linking such traits will tempt smart-alecs into a scrupleless play with human knockins and knockouts; replacing sound and speculative Lacanian psych-analysis as well as relevant forms of cognitive psycho-therapy. The fear we may have of a genome-wide approach to and resolution of these issues is dampened (drugged) by the contents of the next section on epigenetics. 


\subsubsection{Epigenetic Inheritance Systems (EISs)}

The background for our humanness consists of regulatory networks upon regulatory networks (Feinberg 2007). Our composite phenotype is dependent on rather complex interactions between linear and nonlinear genetics such as crossing-over and recombination modification (Poyatos \& Hurst 2006; Wilson et al. 2007) - as well as epigenetic-, behavioral-, and symbolic inheritance systems. Epigenetic inheritance systems, EISs, include (1) self-sustaining information loops,
(2) structural inheritance as self-templating cellular entities and prions, (3) organizing chromatin markers, and (4) in particular RNA interference (Jablonka \& Lamb 2005, see especially Chapter 4). Thus, EISs alone bring uncanny designators such as 'Neo-Lamarckism' into the discussion of evolution. On 'epi-genetics', a current buzz-word, see also the papers in the 2007 May issue of Nature (pp 395-440), and its definition (Feinberg 2007). A general survey is in Allis et al. (2007). The epigenetic scheme is illustrated in Fig. 13.10.
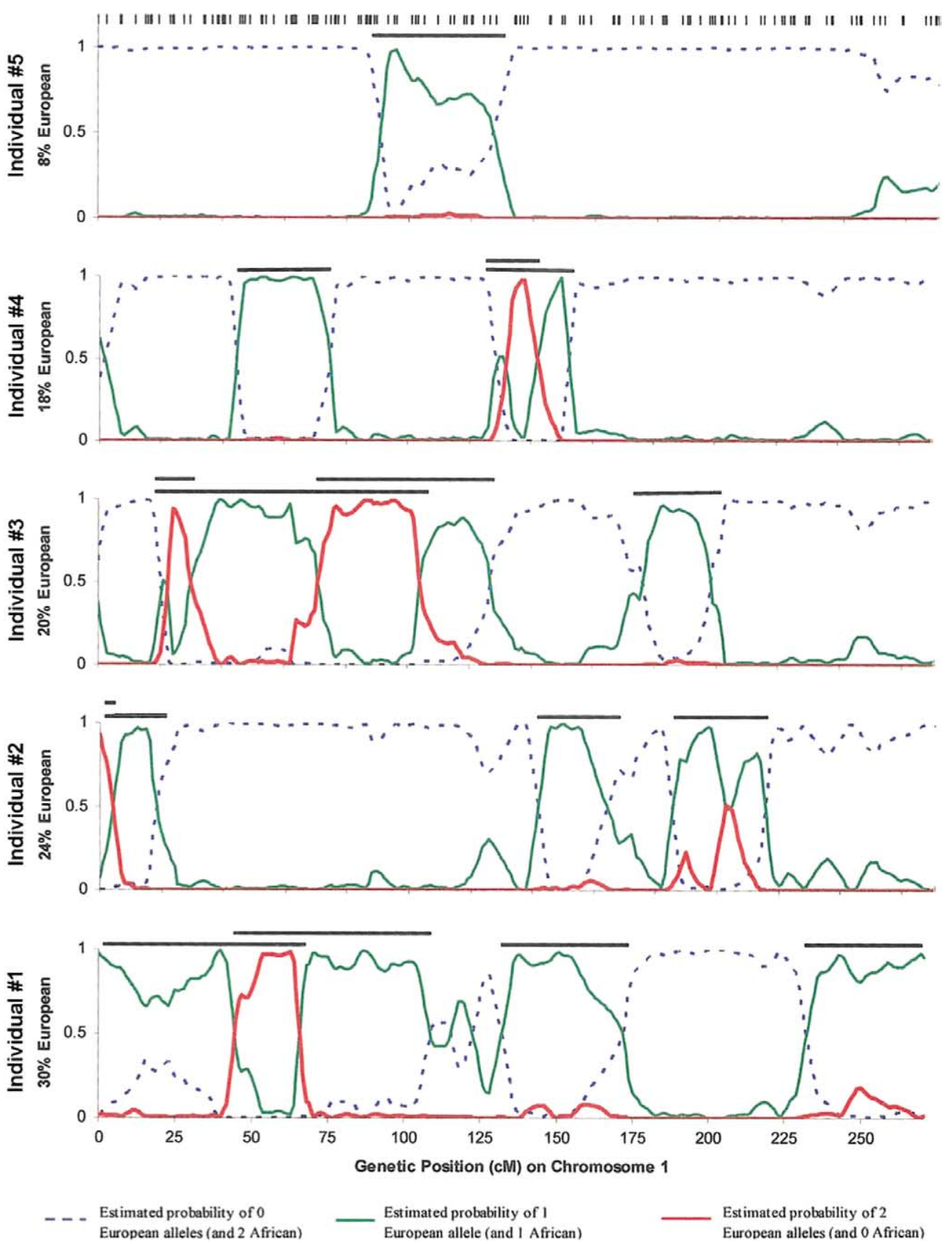

Figure 13.9. Distribution of human traits or race characteristics in various populations (after Smith et al. (2004) with permission). 

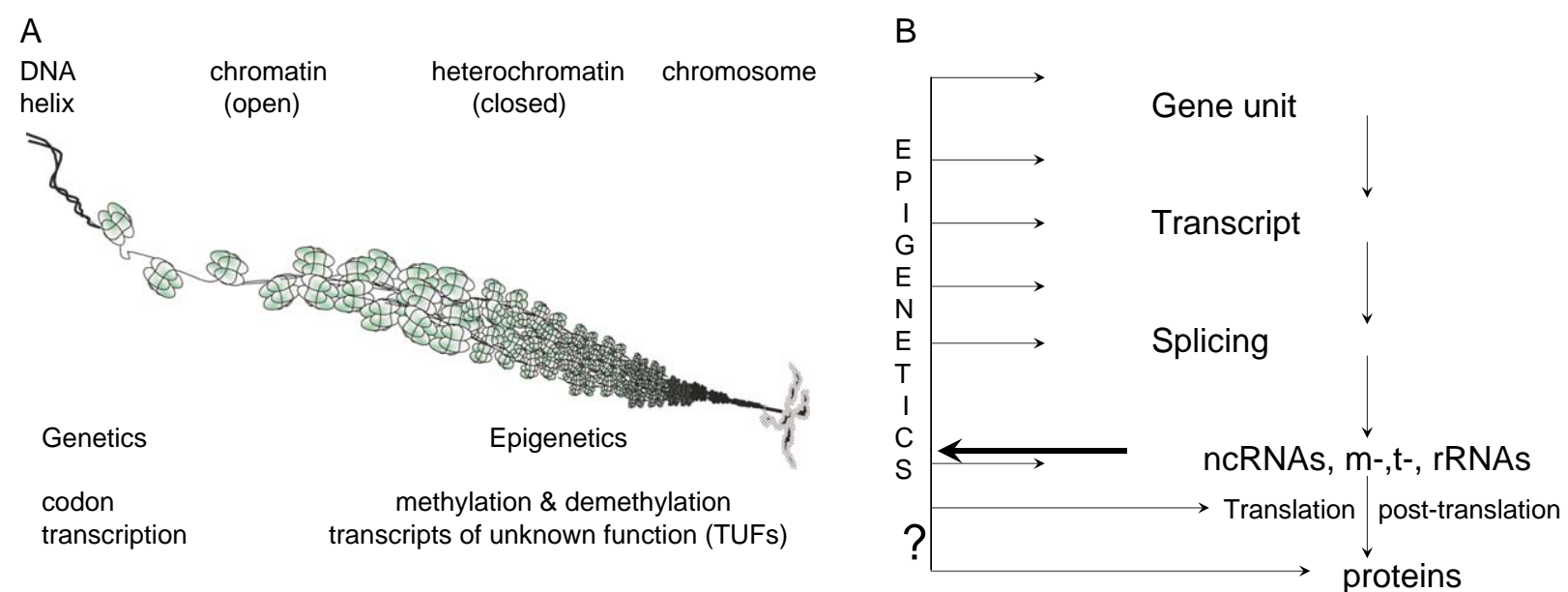

Figure 13.10. Genetic and epi-genetic regulation. (A) Cartoon of DNA and its packing into chromosomes. (B) Tentative noncoding RNAs (ncRNAs) regulatory network. One form of epi-genetic regulation of genetic expression is through ncRNA interfering with all levels of expressing proteins. Some other regulatory means are the genetic regulation through promoters (not shown) and the epi-genetic regulation by methylation-imprinting (not shown).

In light of predictions of close to 100,000 (Prasanth \& Spector 2007) or more non-coding RNA (ncRNA) transcripts for regulation (Gingeras 2007), we still know very little about the epigenetic possibilities.

I highly recommend the Jablonka and Lamb (2005) book, 'Evolution in Four Dimensions. Genetic, Epigenetic, Behavioral, and Symbolic Variation in the History of Life'. Although its text is a bit lengthy, the book is a sovereign survey, thought-provoking and enlightening for those of us who supposed that pharmaco-genetics would soon solve all our individual somatic and mental calamities (Hall \& Pirmohamed 2006; George 2008). Genetics will indeed have the answer to a few special cases of human traits, but understanding most human traits, not to mention treating their wrong-doing, is fortunately still a long way off.

\subsubsection{Omics}

It has become modern to characterize scientific fields with the suffix '-omics'. In semantic fields of free association, we now operate with genomics, proteomics, and even arrowomics (network analysis of arrow diagrams); 'omics' blossoming without boundaries it seems - see for instance Wikipaedia on 'omics' and Table 13.2. Our evolvability as scientists will be judged by our genome-wide, proteome-wide, or metabolome-wide approaches to solutions (Conradie et al. 2006; Stegmann et al. 2006; Gutteridge et al. 2007; Carlson 2007; Llaneras \& Pico 2007; Petsko 2007).

For an issue to be '-omic', a technique must take a global and holistic view of its field.

For instance in biology, 'biomics' should address biological complexity by synthesizing multi-parameters into predictive network models. Biomics is thus the integrated application of science into a coherent strategy for resolving biological complexity (Coulton 2005). Can you define the difference between biomics and Systems Biology or earlier 'integrated physiology'?

Table 13.2 lists some of the new and future '-omics', some with founded societies, some more speculative.

\subsection{The Present State of Regulation by Allostery and Other Affairs}

\subsubsection{To be or Not to be Regulated}

Meantime, while waiting for answers and solutions to the above questions, we may simply divide the formulation of kinetic processes into two: (A) reaction kinetics without built-in regulation; and $(\mathbf{B})$ reaction kinetics with built-in regulation as feedback (rüch-coupling).

Examples of the formulation of kinetic processes belonging to (A) are: (1) Henri-Michaelis-Menten kinetics (HMM) (Section 1.1.9), (2) TIME-dependent loading or washout processes, and (3) COMPARTMENTAL analyses described in pharmaco-kinetic texts and elsewhere (Jacquez 1985; Taylor 2006), and examples belonging to (B) are: (1) spontaneous INACTIVATION following activation and intrinsic/extrinsic DESENSITIZATION, (2) intrasteric regulation such as PSEUDO-SUBSTRATE SWAPPING and PROTEASE ACTIVATION (zymogen regulation) (Table 13.3 and Chapter 14), and (3) regulation by additional ligands in schemes such as mixed-inhibition equal to INTERVENTION (Chapter 2) and ALLOSTERY including concentration-dependent AUTO-REGULATORY CONTRIVANCES such auto-intervention and 
Table 13.3. Some examples of regulatory strategies in biology with increasing complexity

(1) Heterotropic intervention and heterotropic allostery

Modification by ligands other than substrates and primary ligands

Ex: Modification by products and released ions, $\mathrm{H}^{+}, \mathrm{Ca}^{2+}$, auxilliary proteins including calmodulin, $\mathrm{CaM},{ }^{*} \mathrm{G}_{\alpha}, \mathrm{G}_{\beta \gamma}$, and many so-called $\beta$ subunits. Or, by cAMP, cGMP and cyclic-di-GMP/and phospho-diesterases in prokaryotes including their two-component signaling system with aspartic acid and histidine kinases (Stock et al. 2000; Jernal \& Malone 2006; Bekker et al. 2006; Mascher et al. 2006)

(2) Homotropic intervention and homotropic allostery

Auto-modulation by substrates or primary ligands (auto-ant-agonism, feed-forward, auto-inhibition) at secondary sites

Specific Ex: $\mathrm{O}_{2}$ on $\mathrm{Hgb}$, substrate-inhibition, neuro-transmitters auto-modulation

(3) Non-competitive interaction

Steric interactions at primary sites

Steric interactions at secondary (allosteric) sites

(4) Pseudosubstrates

Intramolecular steric interactions at primary sites

Intramolecular steric interactions at secondary (allosteric) sites

Ex: substrate similarities of sequences in PKA and PKC

(5) Intrinsic desensitization - activation enhancement

Time-dependent alterations in receptor transduction, enzyme catalysis, channel transport

Ex: inactivation of VOCs through h-gates and ball-and-chain channel terminals

(6) Phosphorylation/dephosphorylation by kinases and phophatases

Alterations at primary sites

Alterations at secondary (allosteric) sites

General examples: two-component signaling through aspatic acid and histidine kinases in prokaryotes (Galperin 2007) or through STY-kinases in all kinds of living organisms, including prokaryotes (Gangreasse et al. 2007)

Specific examples: glycogen becomes active, glycogen synthetase and phospho-fructokinase, glutamine synthetase reactive on phosphorylation

(7) Phosphorylation-independent attenuation of GCR-signaling (Ferguson et al. 2007)

(8) Extrinsic desensitization or inactivation

Ex. G protein signaling kinases and arrestins (DeWire et al. 2007)

(9) Beyond the level of effectors such as receptors, enzymes, or transporters

Ex: Genetics transcription involving regulatory pathways with transcriptional factors as SMADs (Xu 2006), translation, post-translational modification, translocation, and trafficking of substrates, ligands, modificators and effectors and supply of nutrients and exogenously supplied chemicals. Many of these are regulated by neurotransmitters, hormones, and paracrine signaling molecules as chemokines (Zlotnik et al. 2006)

Fig. 13.6 is an example from glycolysis regulated by noradrenalin, insulin (Litwack \& Schmidt 2006, Chapter 23 and Figs. 23.27-29), and glucagon. Auxilliary proteins in the membrane and in the internal and external cell milieu such as RAMPs, (Parameswaran \& Spielman 2006) anchoring proteins, scaffolding elements, other cytoskeletal components, extracellular matrix molecules as collagen (Volloch \& Kaplan 2002) and cell-substrate adhesion molecules such as fibronectin, laminin, and NCAM (Danen \& Yamada 2001; Yamada et al. 2003), integrin and integrin receptors (Arnaout 2002; for an integrin map see Martin et al. 2002) also involved in adhesion signaling (Hinsby et al. 2004; Evans \& Calderwood 2007)

On networks of further complexity as networks of cross-talk, see e.g., Natarajan et al. (2006); Gavi et al. (2006), and Agnati et al. (2007a,b), and Figs. 13.2 and 13.3.

At higher levels of regulation, we have for instance epigenetics (Thomas \& D'Ari 1990; Jablonka \& Lamb 2005; Allis et al. 2007; Feinberg 2007).

*See calmodulin like activity in delta subunit of Gly phosphorylase (Harris 2006a, pp. 619-620).

auto-modulation (see Chapters 3, 7 and 15), and regulatory kinetics as DOWN-REGULATION, PHOSPHORYLATION and DEPHOSPHORYLATION, processes of turnoff-'n-on; notated schematically elsewhere (Table 13.5).

A more comprehensive, although certainly not complete, list of bio-regulatory means at some of the mentioned levels is given in Tables 13.3-13.5. The tables are only meant as an illustration of the bioregulatory subject's complexity. Table 13.3 is a general listing, while Table 13.4 is on non-covalent and Table 13.5 on covalent regulatory principles. Noncovalent allostery, pseudo-substrate locking, and protein-GTP binding as well as covalent activation by proteases (e.g., Burns et al. 2007), phosphorylation and dephosphorylation (Tables 13.4 and 13.5), are some of the regulatory principles in biology which will be detailed in subsequent sub-chapters and sections of Chapters 14 and 15. Moreover, see for instance CornishBowden (2004b) for regulated biosynthetic supply and product demand contrary to non-modulated waste 'demand'.

\subsubsection{Control Theory and the Rate-limiting Step Concept}

The main part of this book deals with a single or a few steps of reaction where the focus is on the unique modulatory mechanism related to concepts such as the 'rate-limiting step' or 'committed step', where both noncovalent and covalent modifications may take place. As mentioned at the beginning of the chapter, a different approach such as Biological Control or Metabolic 
Table 13.4. Examples of NON-COVALENT, reversible, regulatory principles, at the level of receptors, enzymes and transporters

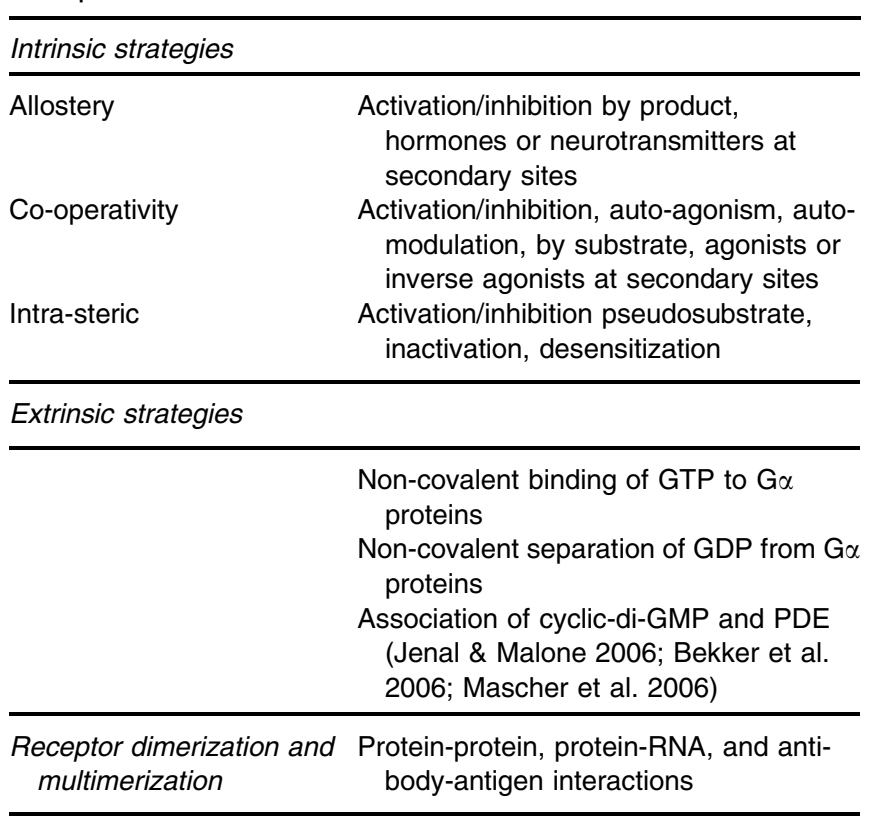

Control Theory (Metabolic Control Analysis) is presented in Fell (1997, Chapters 1 and 5; Cornish-Bowden 1995, Chapter 10 or 2004a, Chapter 12), where the focus is on the overall control process (also see Harris 2006a, Chapter 15); likewise characterized in models of selforganization (Camazine et al. 2001; Feltz et al. 2006), epigenetic regulation (Thomas \& D'Ari 1990; Allis et al. 2007) and network analysis by Systems Biology (see sections 13.2.3-13.2.4).

In Metabolic and Biological Control Theory operating with complex networks, Fell and those who share his views certainly have a point when promoting their nonrate-limiting step theories. On the other hand, in many fast receptor reactions such as signaling to effectors with trigger-mechanisms involving for instance action potentials or the opening of $\mathrm{Ca}^{2+}$-channels, the controlled feedback is at a more isolated level of complexity and the entire process leading to a resultant reaction may be described as a 'committed step' or a rate-limiting step. Therefore, in discussing biological control and regulation it is necessary to find a balance between a network approach with concomitant up- and down-grading of enzyme activity levels through operon-steering (Fell 1997) and the more classical 'rate-limiting step' and 'commitment step' approaches (Staub \& Sharp 2004; Johnson et al. 2005; Brandmann et al. 2005; Levin 2006; Harris 2006a).

\subsubsection{The Present State of Analysis for Allostery}

As a research theme, the analysis of single molecule allostery in enzymology has been pushed aside by other
Table 13.5. Examples of COVALENT regulatory means in biology - including activation by synthetase condensation, hydrolysis or phosphorylation

\begin{tabular}{|c|c|}
\hline \multicolumn{2}{|l|}{ Covalent regulation } \\
\hline Covalent binding & Covalent separation or comment \\
\hline $\begin{array}{l}\text { Phosphorylation (ATP } \gamma \mathrm{P} \text { by } \\
\text { kinases) }^{\dagger}\end{array}$ & $\begin{array}{l}\text { De-phosphorylation (by phospha- } \\
\text { tases) }\end{array}$ \\
\hline Adenylation & Deadenylation \\
\hline ADP-ribosylation & Blood clotting enzymes activation \\
\hline $\begin{array}{l}\text { Amidation (stabilization of } \\
\text { peptides) }\end{array}$ & Restriction enzymes DNA-cutting \\
\hline Farnesylation/myristoylation & $\mathrm{S}-\mathrm{S} \rightarrow \mathrm{HS}-\mathrm{SH}$ \\
\hline Methylation & $\begin{array}{l}\text { DNA-repair, protection, and gene- } \\
\text { regulated expression }\end{array}$ \\
\hline $\begin{array}{l}\text { Protease activation; protease- } \\
\text { activating receptors (PARs); } \\
\text { zymogen activation of enzymes } \\
\text { by hydrolysis, prohormone } \\
\text { activation }\end{array}$ & $\begin{array}{l}\text { Removal of ENaC self-inhibition } \\
\text { by furin and prostasin (Burns } \\
\text { et al. 2007) }\end{array}$ \\
\hline Polymerase sequence elongation & $\begin{array}{l}\text { Restriction enzyme sequence } \\
\text { cutting }\end{array}$ \\
\hline S-nitrosylation* & Denitrosylation $^{\star *}$ \\
\hline Sulphatation & $\begin{array}{l}\text { Structural protein activation } \\
\text { (procollagen-ase) }\end{array}$ \\
\hline SUMOylation & DeSUMOylation $\$$ \\
\hline Transposonation ${ }^{\&}$ & Jumping genes \\
\hline Ubiquitination & $\begin{array}{l}\text { Juxtacrine activation (proteinase, } \\
\text { GF, protein shedding, striptase) }\end{array}$ \\
\hline
\end{tabular}

TOf the covalent types of activation in regulation, phosphorylation by kinases is the most ubiquitous, (Paradela \& Albar 2008), carried out by well over 500 kinase enzymes (Kinome map at STKE, see Manning et al. (2002) and Niedner et al. (2006)).

Other types of enzyme regulation are covalent regulation by swapping, clipping or phosphorylation and cis-trans-retinal, flip-flop, including proenzyme-enzymedegradation and pre-pro-hormone and pro-hormone-degradation. *Whalen et al. (2007).

${ }^{* *}$ Foster et al. (2006), Sun et al. (2006) and Yoshida et al. (2006).

\$See Martin et al. (2007).

\&Lin et al. (2007).

regulatory studies that have become possible through newly available techniques in modern molecular biology and computation. Much effort is being put into the transcription-translation of effector genes and its network regulation, leaving functional regulation within effector proteins as such to selective niches of research. As an example, major reviews on allostery for the aforementioned glutamine synthetase (GS) enzyme goes back to the late 1980s (Rhee et al. 1989), while recent publications are available on the regulated gene expression of GS (Miflin \& Habash 2002; Gebhardt et al. 2007). In addition, the literature on morphogens is another example of how molecular biology and Systems Biology now dominate over simple allosteric control studies, see for example sub-chapter 13.3 on morphogens where allosteric analysis by models is lost in favor of arrow-diagrams and complex computation. Notwithstanding, allostery is being studied intensively for enzymes, transporters and receptive units (Weinstein 2006), however it lacks novel innovative modeling of 
simple reaction schemes. Compared to sprouting network modeling (Table 13.2), there seems to be an artificial gap to the sporadic research in the synagic models of intervention and allostery, as described in Chapters 2 and 7. Instead of developments in the field of synagic modeling, inventions here are mere repeats.

\subsubsection{Will We be Able to Combine Complexity, Chaos, and Criticality with Allostery?}

New techniques such as DNA microarrays and tissue microarrays reveal cell components simultaneously present at any given time. Combined with other techniques such as yeast-two-hybrid screens, protein chips, and advanced cellular visualization, including laser scanning cytometry and layered expression screening, microarrays give detailed information about the quantitative interactions between individual components (Coulton 2004; Davierwala et al. 2005).

The development in detecting possible interactions between linear cascades in cell signaling now reflects the observed divergence and crosstalk between pathways, and the complexity of integrated cell signaling.

Therefore, in wet-laboratory bench work, integrative physiology at the cellular level is a reality (Thiery \& Sleeman 2006; Taniguchi et al. 2006) requiring theoretical complex network modeling, which fortunately is also undergoing rapid progression (Dorogovtsev \& Mendes 2003; Almaas et al. 2005), even into descriptions of 'networks-of-networks' responsible for the performance of cells and organisms (Barabasi \& Oltvai 2004; Gunsalus et al. 2005; Myers et al. 2006).

Furthermore, since natural evolution is a highly accomplished designer of diversity, and the designer of biological building blocks, studying for instance the plasticity of promiscuous enzymes and receptors gives advice on how to construct new enzymes and receptors with insertional mutagenesis (Yoshikuni et al. 2006; Zhang \& Bishop 2007; Cherezov et al. 2007) or in drug discovery of new ligands with better specificity and differential activity (Urban et al. 2007; May et al. 2007; Xia et al. 2007).

\subsubsection{More Means of Bio-regulation}

The demand on modern day researchers seems to be a necessary upgrade of our earlier reductionistic view based on discrete biological function attributed to individual molecules and simple nodes or linear cascades. In fact, building networks of nodes and networksof-networks or staying at the single node is a mirror of mutual enrichment, or a clash between holistic and reductionist approaches. Therefore, in our quest to understand cell signaling and function, new network models of more or less integrated complexities await to be implemented in what looks to be a bright and exciting future (Barabasi \& Oltvai 2004; Goodacre 2007; Mak et al. 2007).

\subsubsection{Synthetic Biology}

Constructing optimized enzymes (Yoshikuni et al. 2006; Zhang \& Bishop 2007) and other biological effectors (Fig. 13.11) (Cherezov et al. 2007) of naturally occurring entities, is an entire field of its own, squeezed between personalized drug development and stem cell repair.

Although clinical results based on the above efforts of molecular manipulations seem to be in the distant future, it looks promising when combined with the feasible treatment of multi-factorial diseases by RNA-interference techniques based on the cellular immune-system with
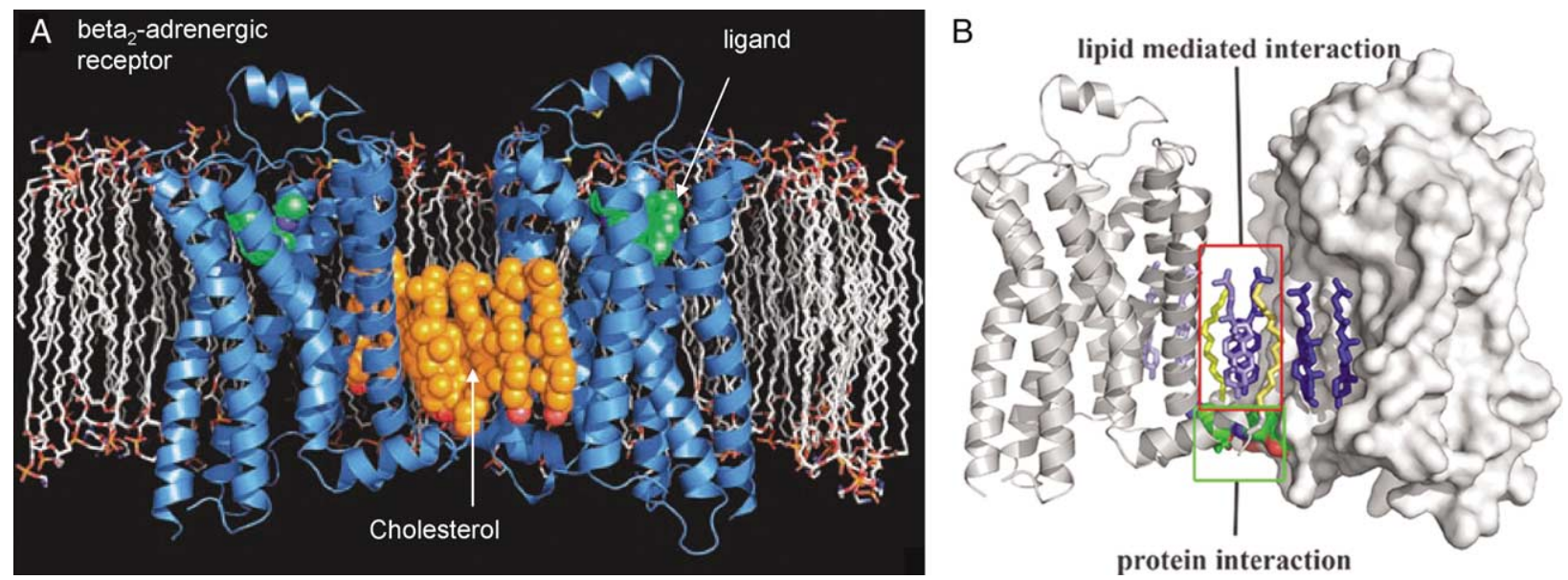

Figure 13.11. Structural resolution at $2.4 \AA$ of human $\beta_{2}$-adrenergic receptor. Engineered $G$ protein-coupled receptor for synthetic biology, structural resolution, and membrane-lipid-interaction. Composed from Cherezov et al. (2007, author's summary figure (A) and Fig. $2(B))$ with permission. 
microRNAs (miRNAs) consisting of small developmental RNAs and small interference RNAs (Bushati \& Cohn 2007; Martin \& Caplen 2007; Perrimon \& Mathey-Prevot 2007), and possible new approaches to obtain stem cells from somatic cells (Maherali et al. 2007; Okita et al. 2007; Werning et al. 2007) that might repair the loss of differentiated cells.

A recent example of biological bottom-up synthetic technology is the microbial production of an antimalarial drug, artemisinin, by engineered E. Coli (Keasling 2008). A flashy example of synthetic biology, is a topdown complete chemical synthesis, assembly, and coding of the whole DNA-string for the Mycoplasma genome, consisting of 582,970 base pairs (Gibson et al. 2008).

\subsubsection{Insecurity about Allostery}

There are always scales of complexity and criticality to every problem. Answering questions on stock market analyses, on the function of brains, on the birth of galaxies, on the operation of genomes, on the eruption of earthquakes and volcanoes, on climate and weather forecasts, as well as on cell signaling and epigenetics all require dynamic and complex theories whereas answering questions on synagics, it appears, must start with limited allosteric modeling.

In Chapters 14 and 15, I shall return to models with a simplistic view of only a few interacting nodes, viz. the binding of two ligands and a conformational change induced or selected for enzymes, receptors, transducers, transporters, and other effectors in what I have termed a 'genuine allosteric model'. The reason for revisiting, in spite of fancy network models, is not nostalgia, but a sensation of permanent confusion or insecurity in the interpretation of allosteric models (Jaffe 2005; Atkins et al. 2006).

In Chapters 14 and 15, you will note my opposition to jump solely into justified demands to switch from synagic models to kinetic models in order to understand the complex ligand-receptor interactions in 'functional selectivity' or ligand-differential responses (Lew et al. 2000; Urban et al. 2006).

\subsubsection{Allostery and Synagics}

It is my intention in Chapters 14 and 15 to reintroduce allostery, its definitions, its meanings, its modes and models, its related reaction schemes, its history, and several other pertinent subjects. The treatment is at the 'statics' level of equilibrium or with examples taken from steady-state conditions. Thus, we will stay at the level of synagics, simple though complex.

\section{REFERENCES}

Adler EM, Gough NR \& Ray LB. 2006: signaling breakthroughs of the year. Sci STKE 367: eg1, 2007.

Agnati LF, Guidolin D, Leo G \& Fuxe K. A Boolean network modelling of receptor mosaics relevance of topology and cooperativity. $J$ Neural Transm 114: 77-92, 2007a.

Agnati LF, Guidolin D \& Fuxe K. The brain as a system of nested but partially overlapping networks. Heuristic relevance of the model for brain physiology and pathology. J Neural Transm 114: 3-19, 2007b.

Ahn NG, Shabb JB, Old WM \& Resing KA. Achieving in-depth proteomics profiling by mass spectrometry. ACS Chem Biol 2: 39-52, 2007.

Al-Shahrour F, Minguez P, Tarraga J, Montaner D, Alloza E, Vaquerizas JM, Conde L, Blaschke C, Vera J \& Dapazo J. BABELOMICS: a systems biology perspective in the functional annotation of genome-scale experiments. Nucleic Acids Res 34: W472-W476, 2006.

Allis CD, Jenuwein T \& Reinberg D. Epigenetics. Cold Spring Habor: Cold Spring Harbor Laboratory Press, 2007.

Almaas E, Oltvai ZN \& Barabasi AL. The activity reaction core and plasticity of metabolic networks. PLoS Comput Biol 1: e68, 2005.

Alon U. An Introduction to Systems Biology. Design Principles of Biological Circuits. Boca Raton, Chapman \& Hall/CRC, 2006.

Androutsellis-Theotokis A, Leker RR, Soldner F, Hoeppner DJ, Ravin R, Poser SW, Rueger MA, Bae SK, Kittappa R \& McKay RD. Notch signalling regulates stem cell numbers in vitro and in vivo. Nature 442: 823-826, 2006.

Arnaout MA. Integrin structure: new twists and turns in dynamic cell adhesion. Immunol Rev 186: 125-140, 2002.

Artzy-Randrup Y, Fleishman SJ, Ben-Tal N \& Stone L. Comment on 'Network motifs: simple building blocks of complex networks' and 'Superfamilies of evolved and designed networks'. Science 305: $1107 \mathrm{c}, 2004$.

Atkins WM. Current views on the fundamental mechanisms of cytochrome P450 allosterism. Expert Opin Drug Metab Toxicol 2: 573-579, 2006.

Barabasi AL \& Oltvai ZN. Network biology: understanding the cell's functional organization. Nat Rev Genet 5: 101-113, 2004.

Barbieri M. Biosemiotics: Information, Codes and Signs in Living Systems. New York: Nova Science Publishers, 2007.

Batlle E, Henderson JT, Beghtel H, van den Born MM, Sancho E, Huls G, Meeldijk J, Robertson J, van de WM, Pawson T \& Clevers H. Beta-catenin and TCF mediate cell positioning in the intestinal epithelium by controlling the expression of EphB/ephrinB. Cell 111: 251-263, 2002.

Battistuzzi FU, Feijao A \& Hedges SB. A genomic timescale of prokaryote evolution: insights into the origin of methanogenesis, phototrophy, and the colonization of land. BMC Evol Biol 4: 44.144.25, 2004.

Bekker M, Teixeira de Mattos MJ \& Hellingwerf KJ. The role of twocomponent regulation systems in the physiology of the bacterial cell. Sci Prog 89: 213-242, 2006.

Berg JM, Tymoczko JL \& Stryer L. Biochemistry. New York: WH Freeman, 2002.

Berg JM, Tymoczko JL \& Stryer L. Biochemistry. New York: WH Freeman and Company, 2006.

Berg KA, Dunlop J, Sanchez T, Silva M and Clarke WP. A conservative, single-amino acid substitution in the second cytoplasmic domain of the human Serotonin2C receptor alters both ligand-dependent and -independent receptor signaling. J Pharmacol Exp Ther 324: $1084-1089,2008$.

Bindslev N \& Skadhauge E. Sodium chloride absorption and solutelinked water flow across the epithelium of the coprodeum and 
large intestine in the normal and dehydrated fowl (Gallus domesticus). In vivo perfusion studies. $J$ Physiol 216: 753-768, 1971.

Bolos V, Grego-Bessa J \& de la Pompa JL. Notch signaling in development and cancer. Endocr Rev 28: 339-363, 2007.

Brandman O, Ferrell JE Jr, Li R \& Meyer T. Interlinked fast and slow positive feedback loops drive reliable cell decisions. Science 310: 496-498, 2005.

Bray SJ. Notch signalling: a simple pathway becomes complex. Nat Rev Mol Cell Biol 7: 678-689, 2006.

Brosius J. How significant is $98.5 \%$ 'junk' in mammalian genomes? Bioinformatics 19 Suppl 2: ii35, 2003a.

Brosius J. From Eden to a hell of uniformity? Directed evolution in humans. Bioessays 25: 615-821, 2003b.

Bruns JB, Carattino MD, Sheng S, Maarouf AB, Weisz OA, Pilewski JM, Hughey RP \& Kleyman TR. Epithelial $\mathrm{Na}+$ channels are fully activated by furin- and prostasin-dependent release of an inhibitory peptide from the gamma-subunit. I Biol Chem 282: 61536160, 2007.

Buchbinder JL, Rath VL \& Fletterick RJ. Structural relationships among regulated and unregulated phosphorylases. Annu Rev Biophys Biomol Struct 30: 191-209, 2001

Budamagunta M, Hess J, Fitzgerald P \& Voss J. Describing the structure and assembly of protein filaments by EPR spectroscopy of spinlabeled side chains. Cell Biochem Biophys 48: 45-53, 2007.

Burke R, Nellen D, Bellotto M, Hafen E, Senti KA, Dickson BJ \& Basler K. Dispatched, a novel sterol-sensing domain protein dedicated to the release of cholesterol-modified hedgehog from signaling cells. Cell 99: 803-815, 1999.

Bushati N \& Cohen SM. microRNA functions. Annu Rev Cell Dev Biol 23: 175-205, 2007.

Camazine S, Deneubourg J-L, Franks NR, Sneyd J, Theraulaz G \& Bonabeau E. Self-organization in Biological Systems. Princeton, NJ: Princeton University Press, 2001.

Carlson RP. Metabolic systems cost-benefit analysis for interpreting network structure and regulation. Bioinformatics 23: 1258-1264, 2007.

Carter D. Cellular transcriptomics - the next phase of endocrine expression profiling. Trends Endocrinol Metab 17: 192-198, 2006.

Cesareni G, Ceol A, Gavrila C, Palazzi LM, Persico M \& Schneider MV. Comparative interactomics. FEBS Lett 579: 1828-1833, 2005.

Cha S. A simple method for derivation of rate equations for enzymecatalyzed reactions under rapid equilibrium assumptions or combined assumptions of equilibrium and steady state. $J$ Biol Chem 243: 820-825, 1968.

Cherezov V, Rosenbaum DM, Hanson MA, Rasmussen SG, Thian FS, Kobilka TS, Choi HJ, Kuhn P, Weis WI, Kobilka BK \& Stevens RC. High-resolution crystal structure of an engineered human beta2adrenergic G protein-coupled receptor. Science 318: 1258-1265, 2007.

Christian JL. Argosomes: intracellular transport vehicles for intercellular signals? Sci STKE 19: PE13, 2002.

Ciesla J. Metabolic enzymes that bind RNA: yet another level of cellular regulatory network? Acta Biochim Pol 53: 11-32, 2006.

Ciliberto A, Novak B \& Tyson IJ. Mathematical model of the morphogenesis checkpoint in budding yeast. J Cell Biol 163: 1243-1254, 2003.

Cleland WW. The kinetics of enzyme-catalyzed reactions with two or more substrates or products. Biochim Biophys Acta 67: 104-137, 1963.

Clemens JC, Worby CA, Simonson-Leff N, Muda M \& Maehama T. Use of double-stranded RNA interference in Drosophilia cell lines to dissect signal transduction pathways. Proc Nat Acad Sci USA 97: 6499-6503, 2000.

Colquhoun D. From shut to open: what can we learn from linear free energy relationships? Biophys J 89: 3673-3675, 2005.
Conradie R, Westerhoff HV, Rohwer JM, Hofmeyr JH \& Snoep JL. Summation theorems for flux and concentration control coefficients of dynamic systems. Syst Biol (Stevenage) 153: 314-317, 2006.

Cori CF, Cori GT \& Green AA. Crystalline muscle phosphorylase. III. Kinetics. J Biol Chem 199: 39-55, 1943.

Cornish-Bowden A. Fundamentals of Enzyme Kinetics. London: Portland Press, 1995.

Cornish-Bowden A. Fundamentals of Enzyme Kinetics. Colchester: Portland Press, 2004a.

Cornish-Bowden A. The Pursuit of Perfection. Aspects of Biochemical Evolution. Oxford: Oxford University Press, 2004b.

Cornish-Bowden A, Cardenas ML, Letelier JC \& Soto-Andrade J. Beyond reductionism: metabolic circularity as a guiding vision for a real biology of systems. Proteomics 7: 839-845, 2007.

Coulton G. Are histochemistry and cytochemistry 'Omics'? J Mol Histol 35: 603-613, 2004.

Coyne JA. The faith that dare not speak its name. The case against intelligent design. The New Republic (August 22-29): 21-33, 2005.

Coyne JA. Intelligent design: the faith that dare not speak its name. In: Intelligent Thought. Science Versus the Intelligent Design Movement, edited by Brockman J. London: Vintage Books, 2006.

Danen EH \& Yamada KM. Fibronectin, integrins, and growth control. $J$ Cell Physiol 189: 1-13, 2001.

Davierwala AP, Haynes J, Li Z, Brost RL, Robinson MD, Yu L, Mnaimneh S, Ding H, Zhu H, Chen Y, Cheng X, Brown GW, Boone C, Andrews BJ \& Hughes TR. The synthetic genetic interaction spectrum of essential genes. Nat Genet 37: 11471152, 2005.

Davies P. The Goldilocks Enigma. Why is the Universe Just Right for Life? London: Penguin/Allen Lane, 2006a.

Davies RH. Intermolecular forces and molecular modeling. In: Smith $\mathcal{E}$ Williams' Introduction to the Principles of Drug Design and Action, edited by Smith HJ. Boca Raton, FL: CRC Press, 2006b.

Dawkins R. The Blind Watchmaker: Why the Evidence of Evolution Reveals a Universe Without Design. London: WW Norton \& Company, 1987.

Dawkins R. River Out of Eden. A Darwinian View of Life. London: Phoenix/Orion, 1995.

Dawkins R. A Devil's Chaplain: Selected Essays. London: Orion Books Ltd., 2003.

Dawkins R. The God Delusion. London: Bantam Press, 2006.

Deacon TW. The Symbolic Species. The Co-evolution of Language and the Brain. New York: WW Norton, 1997.

Dembski WA. The Design Revolution. Answering the Toughest Questions about Intelligent Design. Downers Grove, IL: InterVarsity Press, 2004.

Dennett DC. The hoax of intelligent design and how it was perpetrated. In: Intelligent Thought. Science Versus the Intelligent Design Movement, edited by Brockman J. London: Vintage Books, 2006.

Dennett DC. Breaking the Spell. Religion as a Natural Phenomenon. New York: Penguin, 2007.

DeWire SM, Ahn S, Lefkowitz RJ \& Shenoy SK. Beta-arrestins and cell signaling. Annu Rev Physiol 69: 483-510, 2007.

Di Ventra M, Evoy S \& Heflin JR Jr. Introduction to Nanoscale Science and Technology. NewYork, Springer Verlag, 2004.

Dobrin R, Beg QK, Barabasi AL \& Oltvai ZN. Aggregation of topological motifs in the Escherichia coli transcriptional regulatory network. BMC Bioinformatics 5,10: 1-8, 2004.

Dorogovtsev SN \& Mendes JFF. Evolution and Networks: From Biological Nets to the Internet and WWW. Oxford: Oxford University Press, 2003.

Duboule D \& Deschamps J. Colinearity loops out. Dev Cell 6: 738-740, 2004

Duncan L, Nishii I, Howard A, Kirk D \& Miller SM. Orthologs and paralogs of regA, a master cell-type regulatory gene in Volvox carteri. Curr Genet 50: 61-72, 2006. 
Dupres V, Verbelen C \& Dufrêne YF. Probing molecular recognition sites on biosurfaces using AFM. Biomaterials 28: 2393-2402, 2007.

Eldar A, Rosin D, Shilo BZ \& Barkai N. Self-enhanced ligand degradation underlies robustness of morphogen gradients. Dev Cell 5: 635-646, 2003.

Entchev EV \& Gonzalez-Gaitan MA. Morphogen gradient formation and vesicular trafficking. Traffic 3: 98-109, 2002.

Entchev EV, Schwabedissen A \& Gonzalez-Gaitan M. Gradient formation of the TGF-beta homolog Dpp. Cell 103: 981-991, 2000.

Evans EA \& Calderwood DA. Forces and bond dynamics in cell adhesion. Science 316: 1148-1153, 2007.

Feinberg AP. Phenotypic plasticity and the epigenetics of human disease. Nature 447: 433-440, 2007.

Fell D. Understanding the Control of Metabolism. London: Portland Press, 1997.

Feltz B, Crommelinck M \& Goujon P. Self-organization and Emergence in Life Sciences. Dordrecht: Springer, 2006.

Ferguson SS. Phosphorylation-independent attenuation of GPCR signalling. Trends Pharmacol Sci 28: 173-179, 2007.

Forrest BC \& Gross PR. Creationism's Trojan Horse: The Wedge of Intelligent Design. Oxford: Oxford University Press, 2003.

Foster MW, Hess DT \& Stamler JS. S-nitrosylation TRiPs a calcium switch. Nat Chem Biol 2: 570-571, 2006.

Friedman A \& Perrimon N. A functional RNAi screen for regulators of receptor tyrosine kinase and ERK signalling. Nature 444: 230-234, 2006.

Friedman A \& Perrimon N. Genetic screening for signal transduction in the era of network biology. Cell 128: 225-231, 2007.

Galkina SI, Sud'ina GF \& Klein T. Metabolic regulation of neutrophil spreading, membrane tubulovesicular extensions (cytonemes) formation and intracellular $\mathrm{pH}$ upon adhesion to fibronectin. Exp Cell Res 312: 2568-2579, 2006.

Galperin MY. A census of membrane-bound and intracellular signal transduction proteins in bacteria: bacterial IQ, extroverts and introverts. BMC Microbiol 5: 35.1-35.53, 2007.

Gardner M. The fantastic combinations of John Conway's new solitaire game 'Life'. Sci Am 223: 120-123, 1970.

Gavi S, Shumay E, Wang HY \& Malbon CC. G-protein-coupled receptors and tyrosine kinases: crossroads in cell signaling and regulation. Trends Endocrinol Metab 17: 48-54, 2006.

Gebhardt R, Baldysiak-Figiel A, Krugel V, Ueberham E \& Gaunitz F. Hepatocellular expression of glutamine synthetase: an indicator of morphogen actions as master regulators of zonation in adult liver. Prog Histochem Cytochem 41: 201-266, 2007.

George A, Roden D \& Wood A. Clinical Pharmacogenetics. New York: McGraw-Hill Professional, 2008.

Giacomini KM, Brett CM, Altman RB, Benowitz NL, Dolan ME, Flockhart DA, Johnson JA, Hayes DF, Klein T, Krauss RM, Kroetz DL, McLeod HL, Nguyen AT, Ratain MJ, Relling MV, Reus V, Roden DM, Schaefer CA, Shuldiner AR, Skaar T, Tantisira K, Tyndale RF, Wang L, Weinshilboum RM, Weiss ST \& Zineh I. The pharmacogenetics research network: from SNP discovery to clinical drug response. Clin Pharmacol Ther 81: 328-345, 2007.

Gibson DG, Benders GA, Andrews-Pfannkoch C, Denisova EA, BadenTillson H, Zaveri J, Stockwell TB, Brownley A, Thomas DW, Algire MA, Merryman C, Young L, Noskov VN, Glass JI, Venter JC, Hutchison CA 3rd \& Smith HO. Complete Chemical Synthesis, Assembly, and Cloning of a Mycoplasma genitalium Genome. Science 319: 1215-1220, 2008.

Gingeras TR. Origin of phenotypes: genes and transcripts. Genome Res 17: 682-690, 2007.

Goodacre R. Metabolomics of a superorganism. J Nutr 137: 259S-266S, 2007.

Gould SJ. The Structure of Evolutionary Theory. Cambridge, MA: Harvard University Press, 2002.
Grangeasse C, Cozzone AJ, Deutscher J \& Mijakovic I. Tyrosine phosphorylation: an emerging regulatory device of bacterial physiology. Trends Biochem Sci 32: 86-94, 2007.

Granier S, Kim S, Shafer AM, Ratnala VR, Fung JJ, Zare RN \& Kobilka B. Structure and conformational changes in the C-terminal domain of the beta2-adrenoceptor: insights from fluorescence resonance energy transfer studies. J Biol Chem 282: 13895-13905, 2007.

Greco V, Hannus M \& Eaton S. Argosomes: a potential vehicle for the spread of morphogens through epithelia. Cell 106: 633-645, 2001.

Guerrero I \& Chiang C. A conserved mechanism of Hedgehog gradient formation by lipid modifications. Trends Cell Biol 17: 1$5,2007$.

Gunsalus KC, Ge H, Schetter AJ, Goldberg DS, Han JD, Hao T, Berriz GF, Bertin N, Huang J, Chuang LS, Li N, Mani R, Hyman AA, Sonnichsen B, Echeverri CJ, Roth FP, Vidal M \& Piano F. Predictive models of molecular machines involved in Caenorhabditis elegans early embryogenesis. Nature 436: 861-865, 2005.

Gutteridge A, Kanehisa M \& Goto S. Regulation of metabolic networks by small molecule metabolites. BMC Bioinformatics 8: 88.1, 2007.

Hall IP \& Pirmohamed M. Phamacogenetics. New York: Informa Healthcare. Francis \& Taylor Group, 2006a.

Harris RA. Carbohydrate metabolism I: Major metabolic pathways and their control. In: Textbook of Biochemistry with Clinical Corrolations, edited by Devlin TM. New York: Wiley-Liss, 2006b.

Harris S. The End of Faith: Religion, Terror, and the Future of Reason. London, Simon \& Schuster, Inc., 2006.

Heidecker B \& Hare JM. The use of transcriptomic biomarkers for personalized medicine. Heart Fail Rev 12: 1-11, 2007.

Henry CS, Broadbelt LJ \& Hatzimanikatis V. Thermodynamics-based metabolic flux analysis. Biophys J 92: 1792-1805, 2007.

Herrera G, Diaz L, Martinez-Romero A, Gomes A, Villamon E, Callaghan RC \& O'Connor JE. Cytomics: a multiparametric, dynamic approach to cell research. Toxicol In Vitro 21: 176-182, 2007.

Higgins CF. Multiple molecular mechanisms for multidrug resistance transporters. Nature 446: 749-757, 2007.

Hinsby AM, Lundfald L, Ditlevsen DK, Korshunova I, Juhl L, Meakin SO, Berezin V \& Bock E. ShcA regulates neurite outgrowth stimulated by neural cell adhesion molecule but not by fibroblast growth factor 2: evidence for a distinct fibroblast growth factor receptor response to neural cell adhesion molecule activation. J Neurochem 91: 694-703, 2004.

Hirokawa N. Stirring up development with the heterotrimeric kinesin KIF3. Traffic 1: 29-34, 2000.

Hirokawa N \& Takemura R. Kinesin superfamily proteins and their various functions and dynamics. Exp Cell Res 301: 50-59, 2004

Hitchens C. God is not Great. How Religion Poisons Everything. NewYork, Twelve Books, 2007.

Hoffmeyer J. Signs of Meaning in the Universe. Bloomington Indiana University Press, 1996.

Hoffmeyer J. Biosemiotik. En Afhandling om Livets Tegn og Tegnenes Liv. Copenhagen: Ries Forlag, 2005.

Hoffmeyer J. Biosemiotics: An Examination into the Signs of Life and the Life of Signs. Scranton, University of Scranton Press, 2008.

Hombria JC-G \& Lovegrove B. Beyond homeosis - HOX function in morphogenesis and organogenesis. Differentiation 71: 461-476, 2003.

Hornberg JJ, Bruggeman FJ, Westerhoff HV \& Lankelma J. Cancer: a systems biology disease. Biosystems 83: 81-90, 2006.

Jablonka E \& Lamb MJ. Evolution in Four Dimensions. Genetic, Epigenetic, Behavioral, and Symbolic Variation in the History of Life. Cambridge, MA: MIT Press, 2005.

Jacquez JA. Compartmental Analysis in Biology and Medicine. Ann Arbor, MI: The University of Michigan Press, 1985. 
Jaffe EK. Morpheeins - a new structural paradigm for allosteric regulation. Trends Biochem Sci 30: 490-497, 2005.

Jenal U \& Malone J. Mechanisms of cyclic-di-GMP signaling in bacteria. Annu Rev Genet 40: 385-407, 2006.

Johnson CM, Roderick SL \& Cook PF. The serine acetyltransferase reaction: acetyl transfer from an acylpantothenyl donor to an alcohol. Arch Biochem Biophys 433: 85-95, 2005.

Jurka J, Kapitonov VV, Kohany O \& Jurka MV. Repetitive sequences in complex genomes: structure and evolution. Annu Rev Genomics Hum Genet 8: 241-259, 2007.

Kacser H \& Burns JA. The control of flux. Symp Soc Exp Biol 27: 62-104, 1973.

Kannan N, Wu J, Anand GS, Yooseph S, Neuwald AF, Venter JC \& Taylor SS. Evolution of allostery in the cyclic nucleotide binding module. Genome Biol 8: R264, 2007.

Kashtan N \& Alon U. Spontaneous evolution of modularity and network motifs. Proc Natl Acad Sci USA 102: 13773-13778, 2005.

Kashtan N, Itzkovitz S, Milo R \& Alon U. Topological generalizations of network motifs. Phys Rev E Stat Nonlin Soft Matter Phys 70: 031909, 2004.

Kauffman SA. Metabolic stability and epigenesis in randomly constructed genetic networks. J Theor Biol 22: 437-467, 1969.

Keasling JD. Synthetic biology for synthetic chemistry. ACS Chem Biol 3: 64-76, 2008

Keranen SV. Simulation study on effects of signaling network structure on the developmental increase in complexity. J Theor Biol 231: 321,2004

Kholodenko BN. Cell signalling dynamics in time and space. Nat Rev Mol Cell Biol 7: 165-176, 2006

Kholodenko BN. Untangling the signalling wires. Nat Cell Biol 9: 247249, 2007.

King EL \& Altman C. A scematic method of deriving the rate laws for enzyme-catalyzed reactions. J Am Chem Soc 60: 1377-1381, 1956.

King N. The unicellular ancestry of animal development. Dev Cell 7: 313-325, 2004.

Kirk D. Volvox. Molecular-genetic Origins of Multicellularity and Cellular Differentiation. Cambridge: Cambridge University Press, 1998.

Kohn KW, Aladjem MI, Kim S, Weinstein JN \& Pommier Y. Depicting combinatorial complexity with the molecular interaction map notation. Mol Syst Biol 2: 51.1-51.12, 2006.

Komili S \& Silver PA. Coupling and coordination in gene expression processes: a systems biology view. Nat Rev Genet 9: 38-48, 2008.

Konopka AK. Systems Biology: Principles, Methods, and Concepts. Boca Raton, FL: CRC Press/Taylor \& Francis, 2007.

Lacan J. The Seminar of Jacques Lacan. Book XVII. Psychoanalysis Upside Down/The Reverse Side of Psychoanalysis. Sem 1-15. (Private edition, translated by Gallagher C 2001). 1969/1970.

Laprise P, Viel A \& Rivard N. Human homolog of disc-large is required for adherens junction assembly and differentiation of human intestinal epithelial cells. J Biol Chem 279: 10157-10166, 2004.

Laverty G \& Skadhauge E. Adaptive strategies for post-renal handling of urine in birds. Comp Biochem Physiol A Mol Integr Physiol 149: 246-254, 2008.

Levin BE. Metabolic sensing neurons and the control of energy homeostasis. Physiol Behav 30: 86-89, 2006.

Levine B, Jean-Francois M, Bernardi F, Gargiulo G \& Dobens L. Notch signaling links interactions between the $\mathrm{C} / \mathrm{EBP}$ homolog slow border cells and the GILZ homolog bunched during cell migration. Dev Biol 305: 217-231, 2007.

Lew MJ, Ziogas J \& Christopoulos A. Dynamic mechanisms of nonclassical antagonism by competitive AT(1) receptor antagonists. Trends Pharmacol Sci 21: 376-381, 2000.

Lewis PM, Dunn MP, McMahon JA, Logan M, Martin JF, St Jacques B \& McMahon AP. Cholesterol modification of sonic hedgehog is required for long-range signaling activity and effective modulation of signaling by Ptc1. Cell 105: 599-612, 2001.

Liegeois S, Benedetto A, Garnier JM, Schwab Y \& Labouesse M. The V0-ATPase mediates apical secretion of exosomes containing Hedgehog-related proteins in Caenorhabditis elegans. J Cell Biol 173: 949-961, 2006.

Lin R, Ding L, Casola C, Ripoll DR, Feschotte C \& Wang H. Transposase-derived transcription factors regulate light signaling in Arabidopsis. Science 318: 1302-1305; Erratum in: Science 318: 1866, 2007.

Litwack G. \& Schmidt TJ. Biochemistry of hormones I: Poypeptide hormones. In: Textbook of Biochemistry with Clinical Corrolations, edited by Devlin TM. New York: Wiley-Liss, 2006.

Llaneras F \& Pico J. An interval approach for dealing with flux distributions and elementary modes activity patterns. J Theor Biol 246: 290-308, 2007.

Lotka AJ. Elements of Mathematical Biology. Reprint of 'Elements of Physical Biology' From 1924. New York: Dover Press, 1956.

Ma Y, Erkner A, Gong R, Yao S, Taipale J, Basler K \& Beachy PA. Hedgehog-mediated patterning of the mammalian embryo requires transporter-like function of dispatched. Cell 111: 63-75, 2002.

Maherali N, Sridharan R, Xie W, Utikal J, Eminli S, Arnold K, Stadtfeld M, Yachechko R, Tchieu J, Jaenisch R, Plath K \& Hochedlinger K. Directly reprogrammed fibroblasts show global epigenetic remodeling and widespread tissue contribution. Cell Stem Cell 1: 55-70, 2007.

Mak HC, Daly M, Gruebel B \& Ideker T. CellCircuits: a database of protein network models. Nucleic Acids Res 35: D538-D545, 2007.

Manning G, Whyte DB, Martinez R, Hunter T \& Sudarsanam S. The protein kinase complement of the human genome. Science 298 : 1912-1934, 2002.

Mao X, Bigham AW, Mei R, Gutierrez G, Weiss KM, Brutsaert TD, Leon-Velarde F, Moore LG, Vargas E, McKeigue PM, Shriver MD \& Parra EJ. A genomewide admixture mapping panel for hispanic/latino populations. Am J Hum Genet 80: 1171-1178, 2007.

Martin D, Brun C, Remy E, Mouren P, Thieffry D \& Jacq B GOToolBox: functional analysis of gene datasets based on gene ontology. Genome Biol 5: R101, 2004.

Martin KH, Slack JK, Boerner SA, Martin CC \& Parsons JT. Integrin connections map: to infinity and beyond. Science 296: 1652-1653, 2002.

Martin S, Nishimune A, Mellor JR \& Henley JM. SUMOylation regulates kainate-receptor-mediated synaptic transmission. Nature 447: 321-325, 2007.

Martin SE \& Caplen NJ. Applications of RNA interference in mammalian systems. Annu Rev Genomics Hum Genet 8: 81-108, 2007.

Mascher T, Helmann JD \& Unden G. Stimulus perception in bacterial signal-transducing histidine kinases. Microbiol Mol Biol Rev 70 910-938, 2006.

Mattick JS. RNA regulation: a new genetics? Nat Rev Genet 5: 316-323, 2004

Maturana HR \& Varela FJ. Autopoiesis and Cognition: The Realization of the Living. Dordrecht, Holland: Reidel Publishing Co., 1980.

May LT, Leach K, Sexton PM \& Christopoulos A. Allosteric modulation of g protein-coupled receptors. Annu Rev Pharmacol Toxicol 47: 151, 2007.

McGrath A. The Dawkins Delusion. London: SPCK Publishing, 2007.

McMahon AP, Ingham PW \& Tabin CJ. Developmental roles and clinical significance of hedgehog signaling. Curr Top Dev Biol 53 : 1-114, 2003. 
Meir E, Munro EM, Odell GM \& Von Dassow G. Ingeneue: a versatile tool for reconstituting genetic networks, with examples from the segment polarity network. J Exp Zool 294: 216-251, 2002.

Meloni AR, Fralish GB, Kelly P, Salahpour A, Chen JK, Wechsler-Reya RJ, Lefkowitz RJ \& Caron MG. Smoothened signal transduction is promoted by G protein-coupled receptor kinase 2. Mol Cell Biol 26: 7550-7560, 2006.

Meshi O, Shlomi T \& Ruppin E. Evolutionary conservation and overrepresentation of functionally enriched network patterns in the yeast regulatory network. BMC Systems Biology 1: 1, 2007.

Miflin BJ \& Habash DZ. The role of glutamine synthetase and glutamate dehydrogenase in nitrogen assimilation and possibilities for improvement in the nitrogen utilization of crops. J Exp Bot 53: 979-987, 2002.

Milo R, Shen-Orr S, Itzkovitz S, Kashtan N, Chklovskii D \& Alon U. Network motifs: simple building blocks of complex networks. Science 298: 824-827, 2002.

Milo R, Itzkovitz S, Kashtan N, Levitt R, Shen-Orr S, Ayzenshtat I, Sheffer M \& Alon U. Superfamilies of evolved and designed networks. Science 303: 1538-1542, 2004.

Monod J. Chance and Necessity: An Essay on the Natural Philosophy of Modern Biology. New York: Knopf, 1971.

Monod J \& Jacob F. Teleonomic mechanisms in cellular metabolism, growth, and differentiation. Cold Spring Harb Symp Quant Biol 26: 389-401, 1961.

Monod J, Wyman J \& Changeux J-P. On the nature of allosteric transitions: a plausible model. J Mol Biol 12: 88-118, 1965.

Myers CL, Barrett DR, Hibbs MA, Huttenhower C \& Troyanskaya OG. Finding function: evaluation methods for functional genomic data. BMC Genomics 7: 187, 2006.

Nakano Y, Nystedt S, Shivdasani AA, Strutt H, Thomas C \& Ingham PW. Functional domains and sub-cellular distribution of the Hedgehog transducing protein smoothened in Drosophila. Mech Dev 121: 507-518, 2004.

Natarajan M, Lin KM, Hsueh RC, Sternweis PC \& Ranganathan R. A global analysis of cross-talk in a mammalian cellular signalling network. Nat Cell Biol 8: 571-580, 2006.

Nelson DL \& Cox MM. Lehninger Principles of Biochemistry. New York: WH Freeman, 2005

Ni TC \& Savageau MA. Application of biochemical systems theory to metabolism in human red blood cells. Signal propagation and accuracy of representation. J Biol Chem 271: 7927-7941, 1996.

Nicotera P \& Melino G. Regulation of the apoptosis-necrosis switch. Oncogene 23: 2757-2765, 2004.

Niedner RH, Buzko OV, Haste NM, Taylor A, Gribskov M \& Taylor SS. Protein kinase resource: an integrated environment for phosphorylation research. Proteins 63: 78-86, 2006.

Okita K, Ichisaka T \& Yamanaka S. Generation of germline-competent pluripotent stem cells. Nature 448: 313-317, 2007.

Palsson BØ. Systems Biology. Properties of Reconstructed Networks. Cambridge: Cambridge University Press, 2006.

Paradela A \& Albar JP. Advances in the Analysis of Protein Phosphorylation. J Proteome Res [Epub ahead of print]: 2008.

Parameswaran N \& Spielman WS. RAMPs: the past, present and future. Trends Biochem Sci 31: 631-638, 2006.

Patterson N, Hattangadi N, Lane B, Lohmueller KE, Hafler DA, Oksenberg JR, Hauser SL, Smith MW, O'Brien SJ, Altshuler D, Daly MJ \& Reich D. Methods for high-density admixture mapping of disease genes. Am J Hum Genet 74: 979-1000, 2004.

Perrimon N \& Mathey-Prevot B. Application of high-throughput RNA interference screens to problems in cell and developmental biology. Genetics 175: 7-16, 2007.

Petsko GA. Inconvenient truths. Genome Biol 8: 106-107, 2007.

Pilobello KT \& Mahal LK. Deciphering the glycocode: the complexity and analytical challenge of glycomics. Curr Opin Chem Biol 11: 300-305, 2007.
Poyatos JF \& Hurst LD. Is optimal gene order impossible? Trends Genet 22: 420-423, 2006.

Prasanth KV \& Spector DL. Eukaryotic regulatory RNAs: an answer to the 'genome complexity' conundrum. Genes Dev 21: 11-42, 2007.

Ramirez-Weber FA \& Kornberg TB. Cytonemes: cellular processes that project to the principal signaling center in Drosophila imaginal discs. Cell 97: 599-607, 1999.

Ratzinger J. Vorpolitische moralische grundlage eines freiheitlichen staaten. In: Dialektik der Säkularisierung, edited by Schuller F. Freiburg: Herder, 2005.

Ray LB \& Gough NR. Orienteering strategies for a signaling maze. Science 296: 1632-1633, 2002.

Reid BL \& Bourke CA. Control of metabolic energy by space systems. Part 1: theoretical reports of space flow structure and function. Med Hypotheses 61: 351-365, 2003a.

Reid BL \& Bourke CB. Control of metabolic energy by space systems. Part 2: possible distribution at metabolic cycle chemicals. Med Hypotheses 61: 366-377, 2003b.

Reynwar BJ, Illya G, Harmandaris VA, Muller MM, Kremer K \& Deserno M. Aggregation and vesiculation of membrane proteins by curvature-mediated interactions. Nature 447: 461-464, 2007.

Rhee SG, Chock PB \& Stadtman ER. Regulation of Escherichia coli glutamine synthetase. Adv Enzymol Relat Areas Mol Biol 62: 37-92, 1989.

Riedl SJ \& Salvesen GS. The apoptosome: signalling platform of cell death. Nat Rev Mol Cell Biol 8: 405-413, 2007.

Roberts A \& Stone L. Advantageous indirect interactions in systems of competition. J Theor Biol 228: 367-375, 2004.

Robertson DG, Reily MD \& Baker JD. Metabonomics in pharmaceutical discovery and development. J Proteome Res 6: 526-539, 2007.

Rodriguez-Boulan E, Kreitzer G \& Musch A. Organization of vesicular trafficking in epithelia. Nat Rev Mol Cell Biol 6: 233-247, 2005.

Rogulja D \& Irvine KD. Regulation of cell proliferation by a morphogen gradient. Cell 123: 449-461, 2005.

Romero R, Espinoza J, Gotsch F, Kusanovic JP, Friel LA, Erez O, Mazaki-Tovi S, Than NG, Hassan S \& Tromp G. The use of highdimensional biology (genomics, transcriptomics, proteomics, and metabolomics) to understand the preterm parturition syndrome. BJOG 133 Suppl 3: 118-135, 2006.

Roy M, Pear WS \& Aster JC. The multifaceted role of Notch in cancer. Curr Opin Genet Dev 17: 1-8, 2006.

Royo H \& Cavaillé J. Non-coding RNAs in imprinted gene clusters. Biol Cell 100: 149-166, 2008.

Salvador A \& Savageau MA. Quantitative evolutionary design of glucose 6-phosphate dehydrogenase expression in human erythrocytes. Proc Natl Acad Sci USA 100: 14463-14468, 2003.

Santos SDM, Verveer PJ \& Bastiaens PIH. Growth factor-induced MAPK network topology shapes Erk response determining PC-12 cell fate. Nat Cell Biol 9: 324-330, 2007.

Sasaki N, Sasamura T, Ishikawa HO, Kanai M, Ueda R, Saigo K \& Matsuno K. Polarized exocytosis and transcytosis of Notch during its apical localization in Drosophila epithelial cells. Genes Cells 12: 89-103, 2007.

Savageau MA. Biochemical Systems Analysis: a Study of Function and Design in Molecular Biology. Reading, MA: Addison-Wesley, 1976.

Seiki M \& Yana I. Roles of pericellular proteolysis by membrane type-1 matrix metalloproteinase in cancer invasion and angiogenesis. Cancer Sci 94: 569-574, 2004.

Smith MW, Patterson N, Lautenberger JA, Truelove AL, McDonald GJ Waliszewska A, Kessing BD, Malasky MJ, Scafe C, Le E, De Jager PL, Mignault AA, Yi Z, De The G, Essex M, Sankale JL, Moore JH, Poku K, Phair JP, Goedert JJ, Vlahov D, Williams SM, Tishkoff SA, Winkler CA, De La Vega FM, Woodage T, Sninsky IJ, Hafler DA, Altshuler D, Gilbert DA, O'Brien SJ \& Reich DA. A high-density admixture map for disease gene discovery in African Americans. Am J Hum Genet 74: 1001-1013, 2004. 
Stegmann A, Hansen M, Wang Y, Larsen JB, Lund LR, Ritie L, Nicholson JK, Quistorff B, Simon-Assmann P, Troelsen JT \& Olsen J. Metabolome, transcriptome, and bioinformatic ciselement analyses point to HNF-4 as a central regulator of gene expression during enterocyte differentiation. Physiol Genomics 27: 141-155, 2006.

Stock AM, Robinson VL \& Goudreau PN. Two-component signal transduction. Annu Rev Biochem 69: 183-215, 2000.

Straub SG \& Sharp GW. Hypothesis: one rate-limiting step controls the magnitude of both phases of glucose-stimulated insulin secretion. Am J Physiol Cell Physiol 287: C565-C571, 2004.

Strigini M \& Cohen SM. Wingless gradient formation in the Drosophila wing. Curr Biol 10: 293-300, 2000.

Sun J, Steenbergen C \& Murphy E. S-nitrosylation: NO-related redox signaling to protect against oxidative stress. Antioxid Redox Signal 8: 1693-1705, 2006.

Svard J, Heby-Henricson K, Persson-Lek M, Rozell B, Lauth M, Bergstrom A, Ericson J, Toftgard R \& Teglund S. Genetic elimination of suppressor of fused reveals an essential repressor function in the mammalian Hedgehog signaling pathway. Dev Cell 10: 187-197, 2006.

Swift S, Tucker A, Vinciotti V, Martin N, Orengo C, Liu X \& Kellam P. Consensus clustering and functional interpretation of geneexpression data. Genome Biol 5: R94, 2004.

Tabata T \& Takei Y. Morphogens, their identification and regulation. Development 131: 703-712, 2004.

Tan K, Tegner J \& Ravasi T. Integrated approaches to uncovering transcription regulatory networks in mammalian cells. Genomics 91: 219-231, 2008.

Taniguchi CM, Emanuelli B \& Kahn CR. Critical nodes in signalling pathways: insights into insulin action. Nat Rev Mol Cell Biol 7: 8596, 2006.

Tashiro S, Sedohara A, Asashima M, Izutsu Y \& Maeno M. Characterization of myeloid cells derived from the anterior ventral mesoderm in the Xenopus laevis embryo. Dev Growth Differ 48: 499-512, 2006.

Taylor G. Fundamental pharmacokinetics. In: Smith \& Williams' Introduction to the Principles of Drug Design and Action, edited by Smith HJ. Boca Raton: CRC Press, 2006.

Taylor RC, Cullen SP \& Martin SJ. Apoptosis: controlled demolition at the cellular level. Nat Rev Mol Cell Biol 9: 231-241, 2008.

Teleman AA, Strigini M \& Cohen SM. Shaping morphogen gradients. Cell 105: 559-562, 2001.

The I, Bellaiche Y \& Perrimon N. Hedgehog movement is regulated through tout velu-dependent synthesis of a heparan sulfate proteoglycan. Mol Cell 4: 633-639, 1999.

Thiery JP \& Sleeman JP. Complex networks orchestrate epithelialmesenchymal transitions. Nat Rev Mol Cell Biol 7: 131-142, 2006.

Thomas R \& D'Ari R. Biological Feedback. Boca Raton, FL: CRC, 1990.

Tomita M, Hashimoto K, Takahashi K, Shimitzu T, Matsuzaki Y, Miyoshi F, Saito K, Tanida S, Yugi K, Venter JC \& Hutchison CA. E-CELL: software environment for whole cell simulation. Genome Inform Ser Workshop Genome Inform 8: 147-155, 1997.

Tour E \& McGinnis W. Gap peptides: a new way to control embryonic patterning? Cell 126: 448-449, 2006.

Tuggle CK, Wang Y \& Couture O. Advances in swine transcriptomics. Int J Biol Sci 3: 132-152, 2007.

Tyson JJ \& Mackey MC. Molecular, metabolic, and genetic control: an introduction. Chaos 11: 81-83, 2001.

Umbarger HE. Evidence for a negative-feedback mechanism in the biosynthesis of isoleucine. Science 123: 848, 1956.

Umlauf D \& Fraser P. The role of long non-coding RNAs in chromatin structure and gene regulation: variations on a theme. Biol Chem [Epub ahead of print]: 2008.
Urban JD, Clarke WP, von Zastrow M, Nichols DE, Kobilka B, Weinstein H, Javitch JA, Roth BL, Christopoulos A, Sexton PM, Miller KJ \& Mailman RB. Functional selectivity and classical concepts of quantitative pharmacology. J Pharmacol Exp Ther 320: $1-13,2007$.

van de Wetering M., Sancho E, Verweij C, de Lau W, Oving I, Hurlstone A, van der HK, Batlle E, Coudreuse D, Haramis AP, Tjon-Pon-Fong M, Moerer P, van den BM, Soete G, Pals S, Eilers M, Medema R \& Clevers H. The beta-catenin/TCF-4 complex imposes a crypt progenitor phenotype on colorectal cancer cells. Cell 111: 241-250, 2002.

van den Brink GR \& Offerhaus GJ. The morphogenetic code and colon cancer development. Cancer Cell 11: 109-117, 2007.

Vitkup D, Kharchenko P \& Wagner A. Influence of metabolic network structure and function on enzyme evolution. Genome Biol 7: R39.1-R39.9, 2006.

Volkenstein MV \& Goldstein BN. Allosteric enzyme models and their analysis by the theory of graphs. Biochim Biophys Acta 115: 478$485,1966$.

Volloch V \& Kaplan D. Matrix-mediated cellular rejuvenation. Matrix Biol 21: 533-543, 2002.

Von Dassow G \& Odell GM. Design and constraints of the Drosophila segment polarity module: robust spatial patterning emerges from intertwined cell state switches. J Exp Zool 294: 179-215, 2002.

Walker E, Ohishi M, Davey R, Zhang W, Cassar PA, Tanaka TS, Der SD, Morris Q, Hughes TR, Zandstra PW \& Stanford WL. Prediction and testing of novel transcriptional networks regulating embryonic stem cell self-renewal and commitment. Cell Stem Cell 1: 71-86, 2007.

Walsh DM. Organisms as natural purposes: the contemporary evolutionary perspective. Stud Hist Philos Biol Biomed Sci 37: 771-791, 2006.

Wang Y, McMahon AP \& Allen BL. Shifting paradigms in Hedgehog signaling. Curr Opin Cell Biol 19: 159-165, 2007.

Weaver C \& Kimelman D. Move it or lose it: axis specification in Xenopus. Development 131: 3491-3499, 2004.

Wernig M, Meissner A, Foreman R, Brambrink T, Ku M, Hochedlinger $\mathrm{K}$, Bernstein $\mathrm{BE}$ \& Jaenisch R. In vitro reprogramming of fibroblasts into a pluripotent ES-cell-like state. Nature 448: 318 324, 2007.

Whalen EJ, Foster MW, Matsomoto A, Ozawa K, Violin JD, Que LG, Nelson CD, Benhar M, Keys JR, Rockman HA, Koch WJ, Daaka Y, Lefkowitz RJ \& Stamler JS. Regulation of $\beta$-adrenergic receptor signaling by S-nitrosylation of G-protein-coupled receptor kinase 2. Cell 129: 511-522, 2007.

White TD. Human evolution: the evidence. In: Intelligent Thought. Science Versus the Intelligent Design Movement, edited by Brockman J. London: Vintage Books, 2006.

Williams PH, Hagemann A, Gonzalez-Gaitan M \& Smith JC. Visualizing long-range movement of the morphogen xnr2 in the Xenopus embryo. Curr Biol 14: 1916-1923, 2004.

Wilson CJ, Zhan H, Swint-Kruse L \& Matthews KS. The lactose repressor system: paradigms for regulation, allosteric behavior and protein folding. Cell Mol Life Sci 64: 3-16, 2007.

Wilson CW \& Chuang PT. New 'hogs' in Hedgehog transport and signal reception. Cell 125: 435-438, 2006.

Xia CQ Milton MN \& Gan LS. Evaluation of drug-transporter interactions using in vitro and in vivo models. Curr Drug Metab 8: 341-363, 2007.

Xu L. Regulation of Smad activities. Biochim Biophys Acta 1759: 503513, 2006.

Yamada S, Takayama Y, Seki T, Okada M \& Nagai K. Changes in L1 and NCAM expression in the rat suprachiasmatic nucleus during growth and after orbital enucleation. Brain Res Dev Brain Res 143: 189-198, 2003. 
Yates RA \& Pardee AB. Control of pyrimidine biosynthesis in Escherichia Coli by a feed-back mechanism. J Biol Chem 221: 757-770, 1956.

Yazdani U \& Terman JR. The semaphorins. Genome Biol 7: 211, 2006.

Yip KY, Yu H, Kim PM, Schultz M \& Gerstein M. The tYNA platform for comparative interactomics: a web tool for managing, comparing and mining multiple networks. Bioinformatics 22: 2968-2970, 2006.

Yoshida T, Inoue R, Morii T, Takahashi N, Yamamoto S, Hara Y, Tominaga M, Shimizu S, Sato Y \& Mori Y. Nitric oxide activates TRP channels by cysteine S-nitrosylation. Nat Chem Biol 2: 596$607,2006$.

Yoshikuni Y, Ferrin TE \& Keasling JD. Designed divergent evolution of enzyme function. Nature 440: 1078-1082, 2006.

Yu J \& Bernardo R. Metabolic control analysis as a mechanism that conserves genetic variance during advanced cycle breeding. Theor Appl Genet 108: 1614-1619, 2004
Zhang LV, King OD, Wong SL, Goldberg DS, Tong AH, Lesage G, Andrews B, Bussey H, Boone C \& Roth FP. Motifs, themes and thematic maps of an integrated Saccharomyces cerevisiae interaction network. J Biol 4: 6.1-6.13, 2005.

Zhang X-Y \& Bishop AC. Site-specific incorporation of allostericinhibition sites in a protein tyrosine phosphatase. J Am Chem Soc 129: 3812-3813, 2007.

Zhigulin VP. Dynamical motifs: building blocks of complex dynamics in sparsely connected random networks. Phys Rev Lett 92: 238701, 2004.

Zlotnik A, Yoshie O \& Nomiyama H. The chemokine and chemokine receptor superfamilies and their molecular evolution. Genome Biol 7: 243, 2006. 


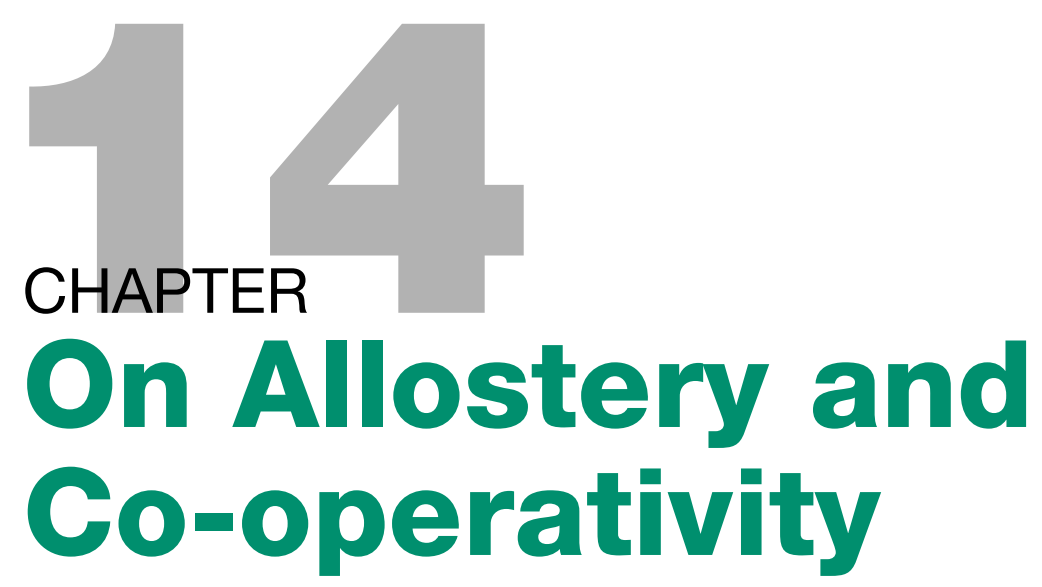

\subsubsection{Allostery and Co-operativity for the Modeler}

In defining the two terms allostery and co-operativity for synagics, I have chosen not to advance directly to the heart of the matter, but to take a tortuous path, thereby delaying an answer to the question which immediately springs to mind: What is the difference between the two terms 'co-operativity' and 'allostery'; are they not the same?

First some history on terminology.

Hopefully, Chapter 14 will prepare you, the synagic modeler, for what allosteric analysis might be after the year 2007 as detailed further in Chapter 15.

\subsection{Historic Accounts on the Use of 'Allosteric' and 'Co-operative'}

\subsubsection{The 'Effector' Concept}

When comparing mechanisms in different fields such as enzymology, receptology, and molecular transportology, ideally the same term should be used for comparable components - just as an identical expression should cover equal concepts in order not to separate our different but overlapping fields. Nowadays, there are differences to be noted.

In enzymology, it is customary to use the term 'effector' about regulatory ligands in general, and about positive 'allosteric ligands' in particular; an unfavorable tradition that goes back to Jacques Monod and collaborators (Monod et al. 1963). Since this use of the term 'effector' is not customary in other fields of biology, I will make one point clear. Contrary to enzymology, in my text the term 'effector' is used as a common designator for proteins in the final step of function; proteins such as enzymes, molecular motors, and molecular transporters including ion channels, pumps, and cotransporters. Receptive entities, such as the $G$ protein coupled receptors (GPCRs) and the tyrosine kinase receptors (TKRs), are also considered effectors. Conversely, in general I refer to regulatory ligands as 'modifiers'. Modifiers are ligands that bind to a secondary binding site, thereby altering effector function. This alteration may be in the presence or absence of primary ligands.

Modifiers for allostery are 'modulators' and modifier molecules in intervention schemes (Chapters 2 and 3) are designated 'interventors'. The modifiers, including interventors, may be either stimulative or inhibitory. Figs. 14.1, 14.2 and Table 14.1 specify the use of nomenclature, differentiating primary ligands from modulators and interventors as well as from 'effectors'. The separate use of 'modulator' and 'interventor' for modifier ligands in models is further detailed in Section 14.3.1.

\subsubsection{The 'Allosteric' Concept}

In discussing models for the regulated control of enzyme function and accelerated binding in carriers, a concept for conformational switching of effectors in an un-liganded state was designated by the adjective 'allosteric' in the early 1960s (Monod \& Jacob 1961; Monod et al. 1963, 1965). This understanding of allostery was exquisitely summarized for enzymes and hemoglobin by Mahler and Cordes (1966, Chapters 6 and 7) more than 40 years ago, and later by many others (e.g., Wong 1975, Chapters 7 and 8; Segel 1975, 1993; Levitzki 1978; Dixon \& Webb 1979; Perutz 1990; Copeland 2000, Chapter 12; Changeux \& Edelstein 2001, 2005), and readdressed in a mature form by Cornish-Bowden (1995, 


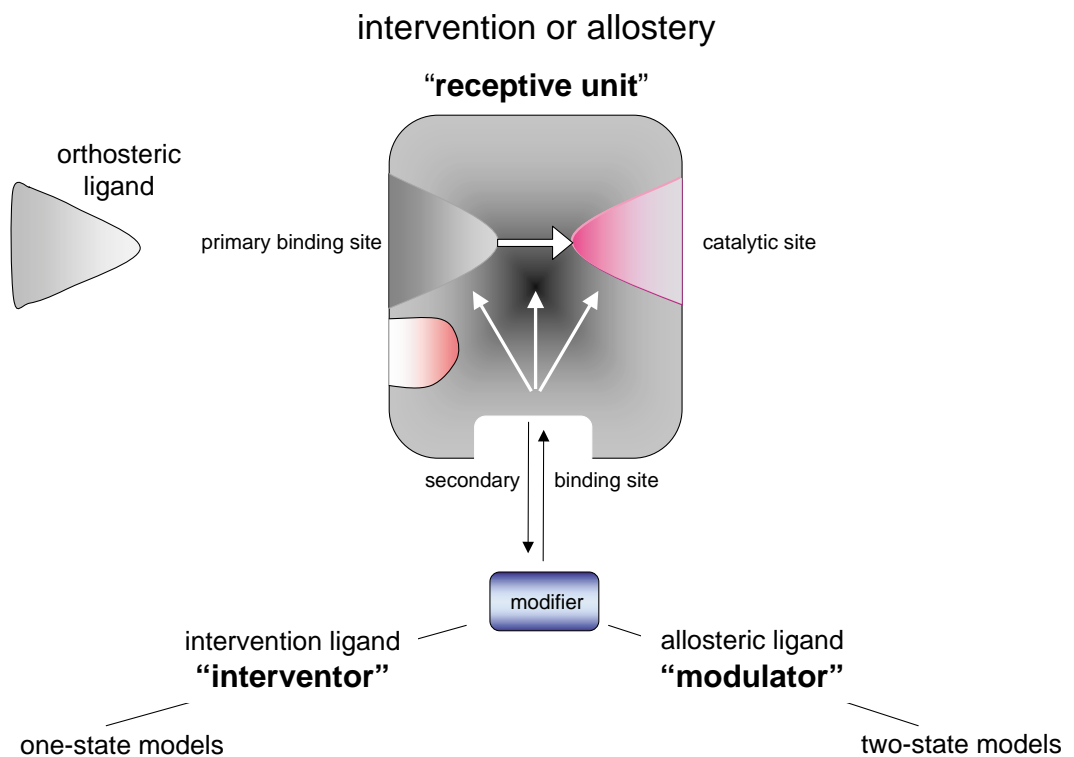

Figure 14.1. Drawing of modifiers and their receptive unit with a catalytic site. Based on an assumed reaction scheme of either a one-state model (intervention) or a two-state model (allostery), modifier ligands (blue) are designated as either interventors or modulators. They bind in their receptive unit to a secondary site sterically separate from the primary (orthosteric) binding site.

Chapter 9, 2004, Chapter 11). Since then, some additional insights have been gained, especially in the fields of transmembrane receptors and transport molecules as channels, which justify a comparison and an update.

In attempts to define allosteric ligands in relation to effectors such as enzymes, it is often emphasized that the allosteric modulators of enzymes, resulting in either increased or decreased activity, are not themselves changed as a result of enzymatic activity, in contrast to the substrate, the 'primary ligand', which is altered.

However, this is different for effectors, such as transporters and receptors, since both the allosteric ligand and the primary ligand are unchanged after moving through the cycle of binding, transfer, and release. Thus, ions or nutrients moving through a transporter are unaltered after passage, just as the receptor ligands, both primary and secondary, are unaltered after binding-to, signal-transduction-through, and release-from receptors. Therefore, the fact that a ligand is unchanged after release from its binding site does not qualify it as an allosteric ligand.

\subsubsection{The Adjective 'Allosteric' and its Noun 'Allostery' or 'Allosterism'}

In 1961, the term 'allosteric' was introduced at the famous meeting entitled 'Cold Spring Harbor Symposia on Quantitative Biology' essentially to cover the newly discovered product-inhibition in a host of enzyme studies (Monod \& Jacob 1961).

Table 14.1. Nomenclature for one-state and genuine two-state models

\begin{tabular}{|c|c|c|}
\hline & One-state models & Genuine two-state models \\
\hline \multicolumn{3}{|l|}{ Modifier molecules } \\
\hline Primary ligand & Auto-interventor & Auto-modulator \\
\hline Secondary ligand & Interventor & Modulator \\
\hline \multicolumn{3}{|l|}{ Receptive unit/effector } \\
\hline $\begin{array}{l}\text { Homo/hetero - mono-mer di-mer; } \\
\text { homo/hetero - multi-mer }\end{array}$ & All types of complexes are possible & $\begin{array}{l}\text { All types of complexes are } \\
\text { possible }\end{array}$ \\
\hline \multirow[t]{2}{*}{ Mechanisms } & Auto-intervention (pos + neg), one ligand & $\begin{array}{l}\text { Co-operativity (homotropic } \\
\text { allostery) }\end{array}$ \\
\hline & Intervention (pos + neg), two ligands & Allostery (heterotropic allostery) \\
\hline Affected molecule & Effector & Effector \\
\hline Self-stimulative response & Positive auto-intervention (mixed auto-activation) & Positive co-operativity \\
\hline Self-inhibitory response & Negative auto-intervention (mixed auto-inhibition)* & Negative co-operativity \\
\hline Stimulative response & Positive intervention (mixed activation) & Positive allostery \\
\hline Inhibitory response & Negative intervention (mixed inhibition) ${ }^{\star \star}$ & Negative allostery \\
\hline
\end{tabular}

${ }^{*, * *}$ Mixed inhibition also covers competitive auto-ant-agonism, auto-non-competitive inhibition, competitive ant-agonism, and non-competitive inhibition. For mixedinhibition or -activation, see, e.g., Cornish-Bowden $(1999,2004)$. 
Changeux (1993) gave an informative clue as to why the adjective 'allosteric' frequently appears, while the nouns 'allostery' and 'allosterism' are only sporadically used in scientific texts. The nouns do not exist in the French language. This fact has influenced its use in English writings as well (Galzi et al. 1996) where the nouns are seldom used, 110 hits for 'allosterism' and around 320 hits for 'allostery' compared to $>13,300$ for 'allosteric' (PubMed Jan 2008). Meanwhile, I use the noun 'allostery' quite often in this text. In enzymology, both nouns are employed (Pardee \& Reddy 2003; Atkins 2005), while in pharmacology 'allosterism' seems to be the preferred noun (Christopoulos 2002; Christopoulos \& Kenakin 2002; Kenakin 2004a,b; Ehlert 2005; Birdsall \& Lazareno 2005).

Here, I shall use the term 'allostery' as a noun, identical to the word 'allosterism'.

\subsubsection{Transformations in the Use of 'Allosteric'}

Due to developments after 1965, use of the term 'allosteric' has undergone a transformation and is now often employed in a loose sense, just referring to supposed ligand-induced conformational change in a receptive unit or a ligand-selection of a preferred conformation of the receptive unit when ligands supposedly bind to a secondary site.

The loose application of the adjective 'allosteric' is due to a lack of detailed insight into the results from a plethora of experiments with ligand occupancy, and a lack of understanding of the mechanisms of numerous functional systems. Therefore, the theoretical claim of two conformations in un-liganded enzymes or carriers (Monod et al. 1965) was not obligatory withheld in discussing 'allosteric' mechanisms, thus a logic linguistic degeneration process has evolved for the term 'allosteric'. In addition, the allosteric effects restricted to multi-meric forms of receptive units in the original definition of allostery by Monod and co-workers lost precision, since mono-meric forms of enzymes and other receptive units probably also display synagics and kinetics that do deviate from simple load relationships (Kaiser 1980; Aleshin et al. 2000; Turk et al. 2000; Sintchak et al. 2002; Kamata et al. 2004; Sack \& Aldrich 2006; Mitriphanov et al. 2007).

However, for conceptual accuracy and stringency, when 'allostery' is used in connection with formalized analysis of dose-response data, it is recommended that the models are genuine two-state reaction schemes for functional as well as occupancy studies. The effector is thus assumed to attain a minimum of two conformational states for the un-liganded species (see Sections 14.2.1-14.2.2).
On the other hand, the original strict definition involving multi-meric effector proteins has been replaced by an understanding that 'allostery' means control of activity in effectors by binding of a modulator molecule to sites in the effector protein that are distinct from and with no overlap of the primary binding site, the catalytic site, or the active site - although the two sites are not necessarily on different subunits (protomers). Thus, multi-sited monomers can be allosteric effectors (Fig. 14.2).

By now, a revision of the meaning of the word 'allosteric' has become mandatory in relation to formalistic analyses of synagic models (see Section 14.2.1).

\subsubsection{The Term 'Co-operativity'}

At face value, the term co-operativity is associated with selfstimulatory processes and a coordinated and augmented synergistic interaction between two or several different ligands. Historically, the concept 'co-operative' has often been connected with the positive non-Michaelian binding of $\mathrm{O}_{2}$ to hemoglobin. In the present text, co-operativity is used to specifically characterize auto-modulatory reactions. Therefore, self-stimulatory processes are equal to positive co-operativity, and self-inhibitory processes are equal to negative co-operativity. On 'co-operativity' as a term for homotropic allostery, consult for instance Cornish-Bowden (1995, Chapter 9, 2004, Chapter 11).

In addition, regarding allostery, a tightening of the meaning of the word 'co-operativity' has become mandatory in relation to synagic formulation and analysis of dose-responses, and its revision is presented in Section 14.2.2.

\subsubsection{A Comment on Conformational Selection Versus Conformational Induction}

Between the concepts of conformational selection (Monod et al. 1965) and conformational induction (Koshland et al. 1966), conformational selection was recently favored for the GPCRs - although imprecise wording in relation to non-formalistic modeling was used, such as 'promote a conformational distribution' and 'promotes significant conformational changes', 'mediate' or 'stabilize' conformational changes (e.g., Kobilka et al. 1999; Gether 2000; Loland et al. 2004); just to be replaced again by the 'induction' and 'sequential' vocabulary (Swaminath et al. 2004; Kobilka 2006; Granier et al. 2007). Thus, under natural and experimental conditions the conception of allosteric control is still exerted either through selection and stabilization of certain states of the effector molecule or it is induced by ligand binding in sequentially ordered steps. 
intervention or allostery

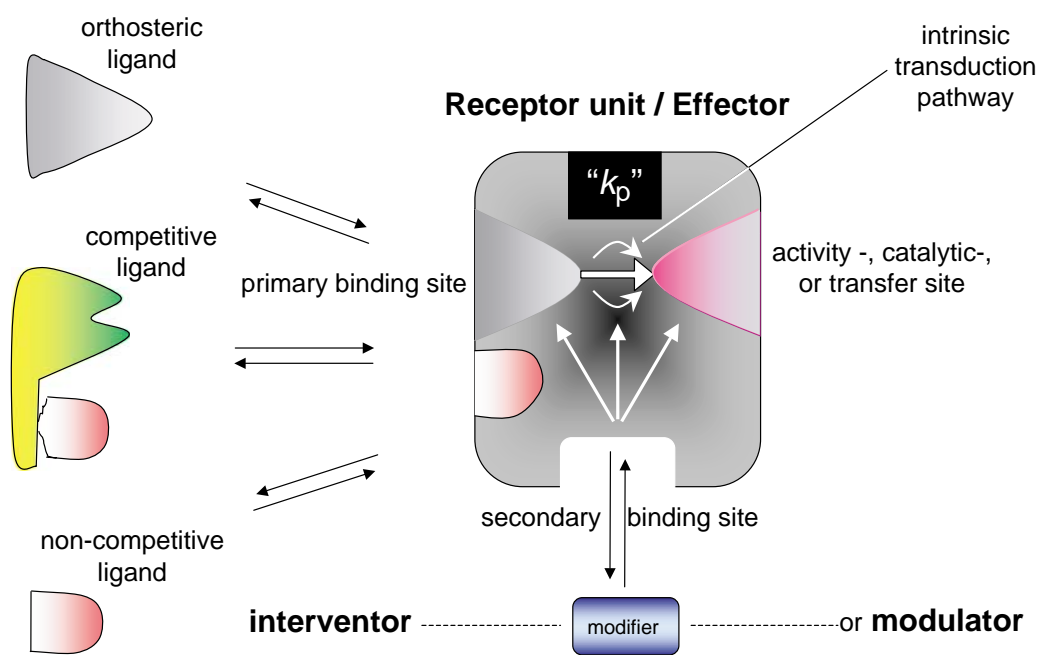

Figure 14.2. Cartoon of a combined receptive unit and effector (reactive-active entity). Ligands designated 'competitive', 'noncompetitive' and 'inteventor' all relate to one-state formulations (see Chapter 2 and 3 ), and the ligand designated 'modulator' relates to two-state formulations (see Chapters 5, 7 and 15). First, when the model for analysis is selected, the secondary ligand (modifier) becomes either an interventor or a modulator. Modification by a secondary ligand, interventor or modulator, may either affect (1) the binding at the primary site, (2) the intrinsic transduction of signal information, or (3) the catalysis and transfer per se, $k_{\mathrm{p}}$, in the active site. In case the primary ligand is also a modifier molecule, there is auto-intervention or auto-modulation.

This affirms the dichotomy and general bewilderment about conformational selection versus induction as rounded up in sub-chapter 5.11 and further substantiates that both points of view are equally valid. Therefore, to simplify matters, I shall often use the paradigm of 'induction' in descriptions of mechanisms without discrimination between conformational selection and conformational induction.

\subsubsection{The Classical Terms 'Allostery' and 'Co-operativity'}

Allostery implies an alteration in ligand-binding or in ligand-elicited function, and furthermore that this modulation takes place through binding of a second ligand to a site at a distance from the primary binding site (Ricard \& Cornish-Bowden 1987). Thus, allostery implies interactions from a secondary binding site through a conformational change in the primary binding site or in the signal-transduction pathway from the primary binding site to the site of activation (Fig. 14.2).

The allosteric (modulatory) ligand, which binds to a site remote from the primary binding site, can be the primary ligand itself, i.e., substrate, agonist, or transportee, or it can be an entirely different molecule. In the first case, with primary ligands as modulators (automodulators), allostery is identical to homotropic allostery and covers co-operative phenomena (co-operativity) as described in Section 14.1.5. Conversely, in the second case with modulators other than primary ligands, allostery is known as heterotropic allostery or just allostery and the ligands are heterotropic modulators or just modulators.

\subsubsection{Genuine Two-state Models}

Thus, for most users of the term 'allosteric', allostery is observed when a non-primary binding site is occupied and this binding induces a change in the primary binding site, in the site of activation, or in the transmission of information from a primary binding site to a site of activation, thus affecting catalysis, transduction, transfer, or binding itself (Fig. 14.2).

However, as originally formulated by Monod et al. (1965), besides binding to a secondary site, the keyconcept for allostery is that it requires an explicitly expressed conformational switch between two un-liganded states of the receptive unit - this is a genuine two-state mechanism (Chapter 5 - Fig. 5.4). In a strict sense, formulation of allostery must be based on genuine twostate reaction schemes, discussed further in Chapter 15 (see for instance Fig. 15.3). As a bonus, genuine twostate models segregate allosteric models from intervention models (compare Sections 14.2.1 and 2.5.1).

Unfortunately, according to the classical understanding of allostery, two-state reaction schemes of allostery were not requested to be cyclic (Fig. 15.3) (Monod et al. 1965), as in the Koshland models (Katz \& Thesleff 1957; Botts \& Drain 1958; Koshland et al. 1966; Harber \& Koshland 1967). Several authors maintained the noncyclic scheme (Karlin 1967; Colquhoun 1973; Thron 1973; Kurganov 1982), while others have mistakenly 
presented the MWC-model as cyclic (Cornish-Bowden 1995, Fig. 9.2 or 2004, Fig. 11.6, Neet 1995). For the simplest cyclic two-state model (cTSM) confer with subchapters 5.7 and 5.8 and Figs. 5.4 and 14.3B.

We can summarize this section by recognizing that when constructing models, as a minimum, the concept of allostery requires (1) an explicit formulation of two un-liganded conformation of its receptive unit, and (2) the presence of at least two separate binding sites in the receptive unit.

\subsection{Definitions of Allostery and Co-operativity}

\subsubsection{The Definition of Allostery Related to Modeling}

Of necessity, a definition of allostery in a strict sense is: allostery is occupancy of a secondary binding site that (1) alters orthosteric binding to or (2) alters elicited functional behavior of an effector in a genuine two-state reaction scheme.

On 'genuine two-state' see Section 14.1.8.

Observe in this connection, especially for agonists binding to receptors such as $\mathrm{G}$ protein-coupled receptors and to transporters such as ligand-gated channels, that primary ligands on binding to their primary sites may induce or select an active conformation of the receptor or channel molecule, but this conformational induction or selection is generally not considered an allosteric mechanism, even though this primary binding site is remote from the sites of activity or transfer and the reaction scheme involves a genuine two-state mechanism. For instance, consider channels and receptors for GABA, $\mathrm{ACh}$, glutamate, and serotonin. Thus, for GABA activating either the GABA-A channel or the GABA-B receptor and for ACh activating either the mAChR receptor or the nAChR channel, these ligand-operated activations are generally not described as allosteric, but may certainly be formulated by the cTSM, which is a pro-allosteric model with only one binding site (see cTSM in Figs. 5.4 and 14.3). It is when several identical or near-identical primary binding sites start to interact, that we glide into the realm of co-operativity and therefore allostery. In relation to a description of binding domains for primary ligand attachment, at least three different types of sites will appear: (1) primary ligand binding sites for metabolites, endogenous signaling molecules, or drugs, (2) activation sites for catalysis, transport, or transduction, and (3) allosteric binding sites for heterotropic ligands or for auto-modulators (Fig. 14.2) (e.g., Galzi et al. 1996). Accordingly, often there is no strict distinction between the use of these terms for binding sites.

For a different definition of allostery see Laidler and Bunting (1973, p 370).

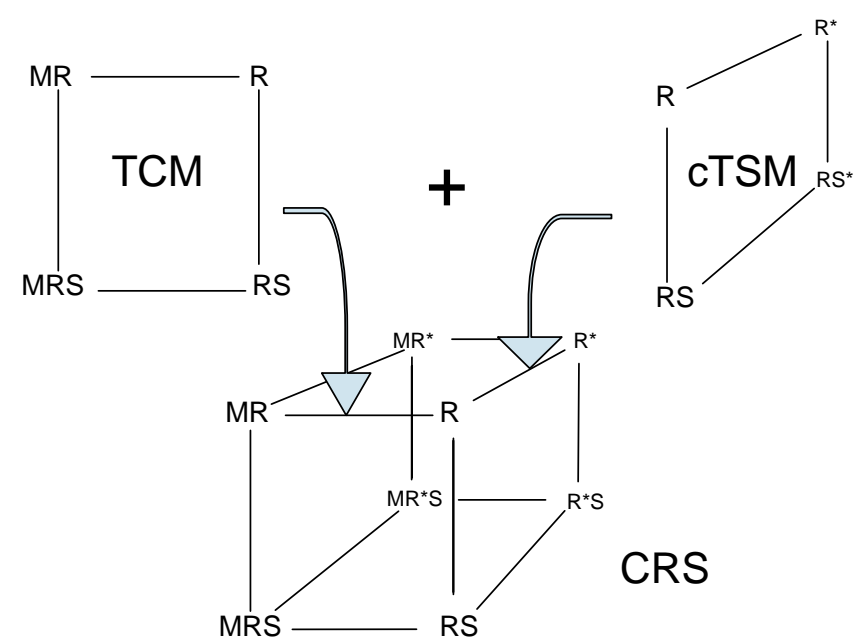

Figure 14.3. The cubic reaction scheme (CRS) for allostery. CRS is a basic model for allostery. The reaction scheme is composed of a complete thermodynamic combination of the cyclic two-state model (cTSM), and the ternary-complex model (TCM). An asterix at the receptor symbol $\left(\mathrm{R}^{*}\right)$ indicates an active conformation, while receptor symbols without an asterix (Rs) indicate re-active conformations. CRS is a prototype model for the allosteric two-state model (ATSM), for the homotropic two-state model (HOTSM) where $\mathrm{M}=\mathrm{S}$, and for the cubic ternary-complex model (CTCM) (Weiss et al. 1996; May et al. 2007a). In modeling the activity of the $G$ protein coupled receptors for the CTCM only the $\mathrm{MR}^{*}\left(\mathrm{GR}^{\star}\right)$ and $M R^{\star} S\left(G R^{\star} S\right)$ conformations are actively involved.

Evidently, in the use of 'allostery' we must cope with a fact: semantic shutters are never tight.

\subsubsection{The Definition of Co-operativity Related to Modeling}

The definition of co-operativity also requires a genuine two-state mechanism when 'co-operativity' is included under allostery in the strict sense. Furthermore, cooperativity is suggested to only cover homotropic allostery.

Therefore, again of necessity, a definition of cooperativity in a strict sense is: co-operativity is occupancy of a secondary binding site by a primary ligand that (1) alters its own orthosteric binding to or (2) alters its own elicited functional behavior of an effector in a genuine two-state reaction scheme.

On 'genuine two-state' see Section 14.1.8.

It is important to realize that the two strict definitions for allostery and co-operativity are needed when we want to formulate the concepts of allostery and co-operativity in explicit expressions (Table 14.2).

Table 14.3 lists algebraic formulations for strict allostery and strict co-operativity.

Co-operativity may be an effect of substrates at binding-catalysis in enzymes, of agonists at bindingsignaling in receptors, of transportees at binding-transfer in transporters, and of ligands at binding-binding in 
Table 14.2. Listing of terms for allosteric dose-responses in a strict sense

\begin{tabular}{lll}
\hline & \multicolumn{2}{c}{ Allosteric response including co-operativity } \\
\hline $\begin{array}{c}\text { Secondary ligand }= \\
\text { non-primary ligand } \\
\text { Heterotropic allostery }\end{array}$ & $\begin{array}{c}\text { Hill coefficient for } \\
\text { synagics }\end{array}$ & $\begin{array}{c}\text { Secondary ligand }= \\
\text { primary ligand } \\
\text { Homotropic }- \text { co-operative }\end{array}$ \\
\hline Positive & $>1$ & Positive \\
Negative & $<1$ & Negative \\
& - & Bell-shaped \\
& - & Reverse bell-shaped \\
\hline
\end{tabular}

carriers. In addition, co-operativity can be an enhancement or an attenuation of the response as the concentration of the primary ligand increases.

Allostery requires multi-sited systems, so that at least two ligands - in the case of co-operativity at least two identical substrates, agonists, or transportees - can bind simultaneously to the functional unit and mutually modulate binding or function. Thus, the regulatory phenomenon of co-operativity is just one aspect of the allosteric concept - viz. homotropic allostery. ${ }^{1}$

The models that best describe these definitions of allostery and co-operativity are the allosteric two-state model (ATSM) and the homotropic two-state model (HOTSM) in Chapter 7.

\subsection{Additional Aspects of Allostery and Co-operativity}

\subsubsection{To Select Allosteric Schemes Instead of Intervention Schemes}

The loss of distinction in the meaning of allostery at a theoretical level, as described in Section 14.1.4, further comes from the confounding of two basic models instead of combining them. The two models are the genuine two-state model described in Chapter 5 and the intervention model described in Chapters 2 and 3 . The models are shown and combined in a cubic reaction scheme in Fig. 14.3. The simple intervention model, also denoted the ternary complex model (TCM)

\footnotetext{
1 Here, definitions of allostery and co-operativity are somewhat at variance with those of other authors (e.g., Gutfreund 1995, p. 93; Copeland 2000, pp. 367-368). As described in Section 14.3.4, the cooperative effect, homotropic allostery, both in binding and in functional studies is affected by heterotropic allosteric ligands - for chorismate mutase (CM) by tryptophan and tyrosine (Helmstaedt et al. 2004), for aspartate transcarbamoylase (ATCase) by ATP and CTP (Alam et al. 2004), and for hemoglobin by $\mathrm{H}^{+}, \mathrm{CO}_{2}$, and 2,3-DPG (Berg et al. 2002, 2006). That is, three relatively complex systems. Fortunately, for now, my definitions of allostery and co-operativity seem adequate to tackle the synagic interactions for these and other systems.
}

or mixed-load reaction scheme (Fig. 14.3), is often referred to as an 'allosteric' scheme (Reinhart 1983; Kwong et al. 1996; Pham \& Reinhart 2001; Verhamme et al. 2002; Fenton et al. 2004; Ehlert 1988, 2001, 2005; Kenakin 2002, 2005, 2007; Birdsall \& Lazareno 2005; Price et al. 2005; May et al. 2007), although the model is not allosteric in our strict sense. It is the combination of TCM and cTSM that results in a true allosteric reaction scheme with two binding sites and two conformations of the un-liganded receptive unit.

For a description of allostery by equations, the two basic requirements are (1) the binding of 'two' ligands as in the TCM (Figs. 2.1A, 3.1B + C and 14.3), and (2) an explicitly formulated conformational switch of an unliganded receptive unit as in the cTSM (Figs. 5.4 and 14.3). Thus, TCM and cTSM may be characterized respectively as a pre-allosteric model and a pro-allosteric model. Only when an explicitly expressed conformational switch and a ternary complexing are combined, as in models by Monod's laboratory (Fig. 15.3) (MWC Monod et al. 1965) and Koshland's laboratory (Fig. 15.13) (KNF - Koshland et al. 1966; Harber \& Koshland 1967), can we speak about an allosteric model (see the cubic model in Fig. 14.3). ${ }^{2}$

For allostery, as indicated above, the two principles from TCM and cTSM are best formulated in models by simply combining these two reaction schemes, as in the ATSM by Hall (2000) for heterotropic ligands and in the HOTSM by Bindslev (2004) for a single type of ligand (Chapter 7).

Once again, as described in Chapters 2 and 3 for onestate mixed-load models, where receptive units are exposed to two ligands, with either one different from or both identical to a primary ligand, the modifier effects of a ligand binding to a secondary site are characterized as 'intervention' or 'auto-intervention', whilst terms such as 'allosteric' and 'co-operative' are saved for genuine two-state models in modeling. Likewise, the secondary ligand in one-state models is designated an 'interventor', while in genuine two-state models the secondary ligand is a 'modulator' (Table 14.1).

It is a good idea to make this nomenclature your choice too. The reason is that it opens up possibilities for exact model descriptions as illustrated in Table 14.3 where the difference in expression between one-state intervention models (pre-allosteric TCMs) and cyclic two-state models (pro-allosteric cTSMs) are contrasted for both binding and function, and for 'homo-' and 'hetero-' as well.

\footnotetext{
${ }^{2}$ In allosteric models as in the MWC and KNF models of ternary complexes (or higher), site 'interactions' become co-operative as they involve a single type of ligand and two un-liganded states.
} 
Table 14.3. Comparison of parameters $B_{\max }, R_{\max }$ and appK $\mathrm{ss}_{\mathrm{ss}}$ between one- and two-state models

\begin{tabular}{|c|c|c|c|}
\hline Type of experiment & Model name & Fractional $B_{\max }$ or $R_{\max }$ & $\mathrm{appK}_{\mathrm{ss}}$ \\
\hline \multicolumn{4}{|l|}{ Binding of ONE ligand } \\
\hline Binding/two-states & Cyclic TSM; pro-allosteric model & 1 & $\frac{1+L}{A_{\mathrm{S}} \cdot(1+a \cdot L)}$ \\
\hline Function/two-states & Cyclic TSM; pro-allosteric model & $\frac{a \cdot L}{1+a \cdot L}$ & $\frac{1+L}{A_{\mathrm{s}} \cdot(1+a \cdot L)}$ \\
\hline \multicolumn{4}{|c|}{ Binding of TWO different or identical ligands } \\
\hline Binding/one-state different ligands & $\begin{array}{l}\text { Intervention/ modulation }{ }^{\#} \text { or classic } \\
\mathrm{TCM}^{\# \# \#} \text { pre-allosteric }\end{array}$ & 1 & $\frac{1+A_{\mathrm{M}} \cdot \mathrm{M}}{A_{\mathrm{s}} \cdot\left(1+c \cdot A_{\mathrm{M}} \cdot \mathrm{M}\right)}$ \\
\hline Binding/one-state identical ligands & $\begin{array}{l}\text { Intervention/modulation\# or classic } \\
\mathrm{TCM}^{\# \# \# ~ p r e-a l l o s t e r i c ~}\end{array}$ & & \\
\hline $\begin{array}{l}\text { Different ligand; binding/two-states } \\
\text { Identical ligand; binding/two-states }\end{array}$ & $\begin{array}{l}\text { ATSM } \\
\text { HOTSM }\end{array}$ & $\begin{array}{l}\text { See Chapter } 7 \\
\text { See Chapter } 7\end{array}$ & \\
\hline Function/one-state different ligands & $\begin{array}{l}\text { Intervention/modulation" or classic } \\
\mathrm{TCM}^{\# \# \#} \text { pre-allosteric }\end{array}$ & $\frac{1}{1+A_{\mathrm{M}} \cdot \mathrm{M}}$ & $\frac{1+A_{\mathrm{M}} \cdot \mathrm{M}}{A_{\mathrm{s}} \cdot\left(1+c \cdot A_{\mathrm{M}} \cdot \mathrm{M}\right)}$ \\
\hline Function/one-state identical ligands & $\begin{array}{l}\text { Intervention/modulation }{ }^{\#} \text { or classic } \\
\mathrm{TCM}^{\# \# \#} \text { pre-allosteric }\end{array}$ & & \\
\hline Function/two-states different ligands & ATSM $^{\# \# \#}$ & Cf. Chapter 7 & \\
\hline Function/two-states identical ligands & HOTSM & Cf. Chapter 7 & \\
\hline
\end{tabular}

Nomenclature is as defined for the mixed-competitive OSM in Chapter 2 and the CTSM in Chapter 5

\#'Intervention' by modifier molecules has replaced nomenclature such as 'mixed inhibition', 'mixed competition', and 'mixed activation' as we move into the world of augmenting as well as attenuating behavior intervened by interventory ligands.

\#\#Ehlert (1988) in his ternary complex model (TCM) for ligand and G protein interactions has a constant $\alpha$ equal to reciprocal constants $\gamma$ in Hall (2000) and $c$ in Bindslev (2004). These are all intervention constants, whilst the $c$ constant here is a heterotropic allosteric constant. Ehlert uses the term 'allosteric' to indicate binding to a secondary site and his $\alpha$ constant is a measure for his heterotropic 'co-operativity'. Both lines of thought are not to be attacked! Meanwhile, for the TCM, I prefer 'intervention' instead of heterotropic 'allostery' and 'positive intervention' instead of 'positive co-operativity'.

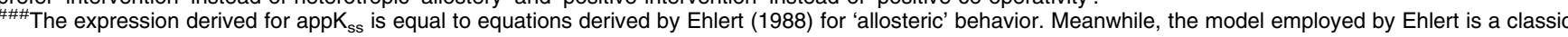
mixed competitive reaction scheme (Laidler 1958; Segal 1959; Frieden 1964; Cornish-Bowden 1995, 2004) and not a genuine two-state model. Therefore, Ehlert's derived parameters belong to the two-ligand intervention reaction scheme as detailed in Chapter 2 and not for heterotropic allostery as defined here in Chapter 14. Heterotropic allostery is better described by the allosteric two-state model (ATSM) (Hall 2000) (Chapter 7).

The ternary-complex model (TCM) in the classic sense (Cornish-Bowden 1995) should not be confused with the ternary-complex model involving G proteins as establish around the 1980s (De Lean et al. 1980). Classic-TCM consists of a tripartite complexation between a receptor and two different ligands, an agonist S and an intervention molecule I. In the latter model, a G protein replaces the interventor I, and formulation of the two models are not identical (Weiss et al. 1996). In comparison with the classic-TCM for catalysis, the G protein-TCM signaling has the constraint that only complexes with G proteins can be part of the active receptor.

\subsubsection{To Choose a Loose Use of Terms}

As a relief to the reader, the use of both adjective terms 'co-operative' and 'allosteric' is frequently adapted in a colloquial fashion without strict insight and for no particular model application (e.g., Bindslev \& Wright 1984). The main reason is that the understanding of regulatory mechanisms for most bio-molecular systems is far from complete (Soudijn et al. 2004; Schwartz \& Holst 2006; Urban et al. 2007).

While 'co-operative' is often used in a loose sense for single ligand responses, 'allostery' is mostly taken to mean heterotropic allostery if nothing else is specified. In addition, as long as the degree of looseness in the use of these terms is recognized, there should be no problem. Thus, deviation from simple load-synagics is designated co-operativity or homotropic allostery in binding and functional assays with a single ligand present, while when it is a ligand different from the primary ligand that renders the deviation from simple load-synagics, we talk about heterotropic allostery or just allostery.
For other approaches to and interpretations of the use of the term 'allosteric', see 'A note on the term allosteric' by Jenkinson (2003, Appendix 1.6A, pp. 64-66).

The co-operative effect may display a shallow doseresponse relation compared with the hyperbolic curve of a simple load dose-response - equal to negative co-operativity, while positive co-operativity (Table 14.2) enhances dose-response relations which are steeper compared to simple load-relationships (Fig. 14.4). Both are referred to as 'non-Michaelian'.

Nevertheless, in the loose sense, an example of positive co-operativity for transporters is the transport of sodium in the sodium pump (Fig. 14.5) (Cornelius \& Skou 1988; Cornelius et al. 1998; Balshaw et al. 2000; Morth et al. 2007), while an example of negative cooperativity, as well as in the relaxed understanding, is the self-inhibition by sodium of its own transport through the epithelial sodium channel ENaC (Fig. 14.6) (Fuchs et al. 1977; Sheng et al. 2004a,b; Quinton 2007). The underlying mechanism of both these reaction may of course be a genuine allosteric 


\section{Examples of dose-response relations}

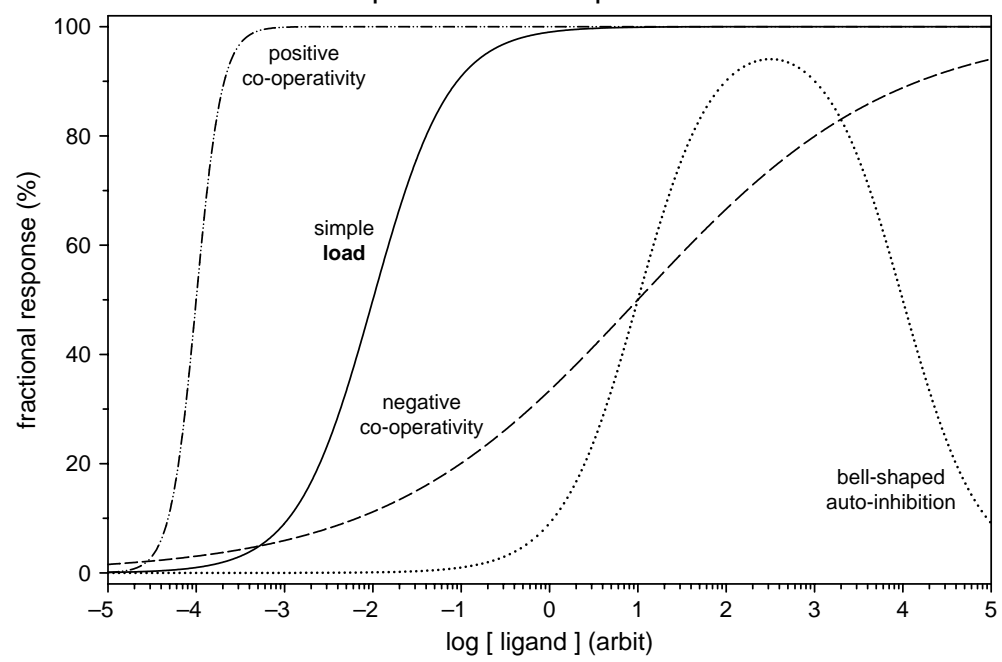

Figure 14.4. Principal diagram for various dose-response (d-r) curves. The $d$-r curve of positive co-operativity is steeper and the $d$-r curve of negative co-operativity is shallower than the $d-r$ curve for simple load. The co-operative effects are assumed due to auto-modulation by the primary ligand itself. Thus, co-operative effects only involve a single type of ligand. The bell-shaped d-r curve of auto-inhibition, as seen for instance in substrate inhibition, is either an auto-intervention (Chapter 3) or an automodulation (Chapter 7) effect, depending on the model selected for analysis. The term 'negative co-operativity' does not usually cover bell-shaped auto-inhibition.

scheme. Therefore, as presented in Sections 14.2.1 and 14.2.2, when we want to analyze data of dose-responses deviating from simple load in a physical scheme by mathematical expressions of either homotropic or heterotropic allostery, the synagic model ought to - or rather must - be a genuine two- or multi-state model.

Consult Chapters 3 and 7 for the difference between negative co-operativity and either a complete substrateinhibition or an auto-inhibition with a bell-shaped doseresponse curve, as in Figs. 14.4 and 14.6E.

\subsubsection{To Lean Towards Either V-or K-systems}

In comparing one-state load models involving two different ligands (described in Part I, Chapter 2) with ATSMs (described in Part 2, Chapters 5 and 7), an immediate similarity emerges between some mixed-load models and allosteric V-systems, where there are variations only in $V_{\max }$ and not in the dissociation constant $K_{\mathrm{d}}$. For other mixed-load one-state models, they cover the same ground as allosteric K-systems, where only the $K_{\mathrm{d}}$ varies while the $V_{\max }$ is constant. A more detailed analysis of the differences between one-and two-state models when it comes to formulation, as demonstrated in Table 14.3, may relate to allosteric V- and K-systems for enzymes, or K-, gamma, or L-systems for receptors (Galzi et al. 1996). However, the relevance of those V-and K-systems (Monod et al. 1965; Nelson \& Cox 2005; Weiner 2006, p. 401) has been questioned (Cornish-Bowden 1995, 2004).

\subsubsection{To Cause Allostery-upon-allostery}

It should be realized that co-operativity, equal to homotropic allostery, might be affected by heterotropic ligands. For example, in Baker's yeast, Sarccomyces cerviciae chorismate mutase (ScCM) is an enzyme where its substrate chorismic acid, chorismate, display positive co-operativity, while two heterotropic ligands, tyrosine and tryptophan, can affect the co-operative dose-response curve for chorismate (Fig. 14.7). Thus, tryptophan has a stimulatory effect on the balance in ScCM between a reactive T-conformation and a catalytic active R-conformation - an assumed T-R transition in the enzyme - while tyrosine has an inhibitory effect on the T-R transition induced from a common allosteric binding site (Helmsteadt et al. 2001). Furthermore, in ScCM, tryptophan has an inhibitory effect on the positive co-operative effect induced by the substrate, whereas tyrosine has an enhancing effect on the positive co-operative effect of chorismate (Fig. 14.7). The modulation by tyrosine and tryptophan seems to travel through different pathways of molecular switches (Schnappauf et al. 1998).

Both tryptophan and tyrosine can be products of ScCM activity. Therefore, there exist complex interactions between homotropic and heterotropic ligands in the ScCM system, as well as in many other functional systems with product allostery (e.g., see Berg et al. 2002, 2006 or Nelson \& Cox 2005). 

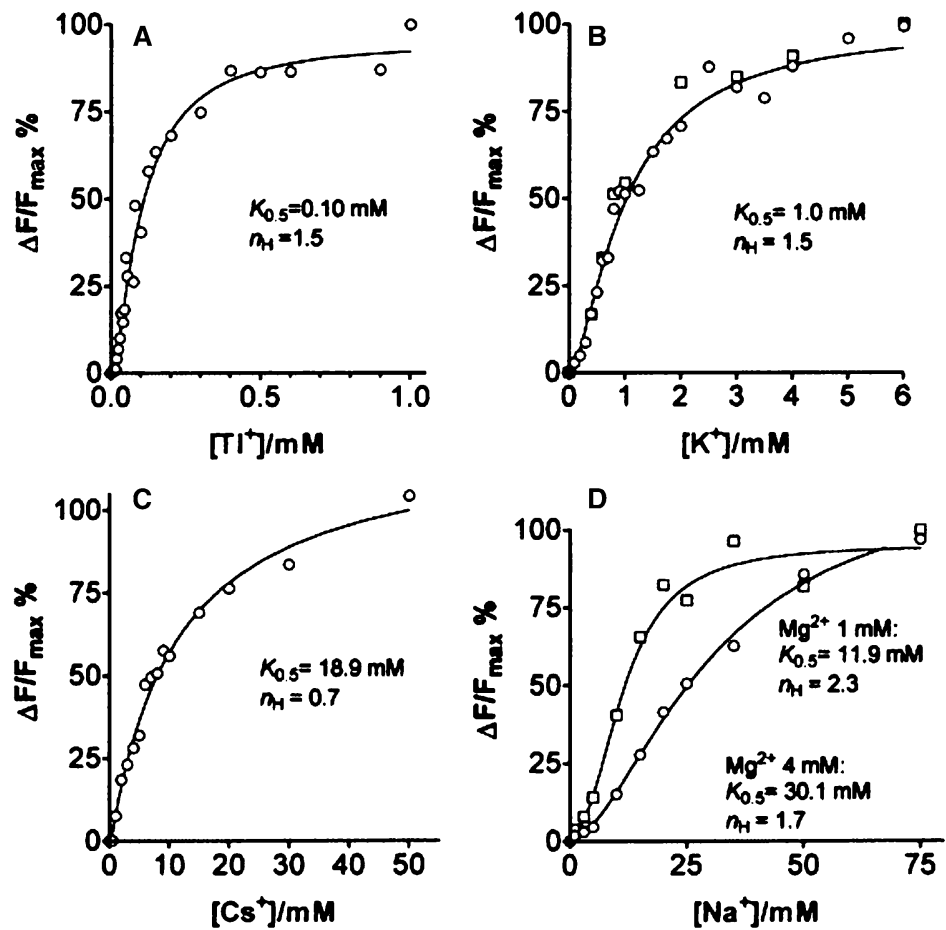

Figure 14.5. Co-operativity in the sodium pump ( $\mathrm{Na}, \mathrm{K}-\mathrm{ATPase}$ ). The shark rectal gland $\mathrm{Na}, \mathrm{K}-\mathrm{ATP} a s e$ displays positive cooperativity for both potassium (panel $\mathrm{B}, n_{\mathrm{H}}=1.5$ ) and sodium (panel $\mathrm{D}, n_{\mathrm{H}}=1.7-2.3$ ). Taken with permission from Cornelius et al. (1998, Fig. 10).

A

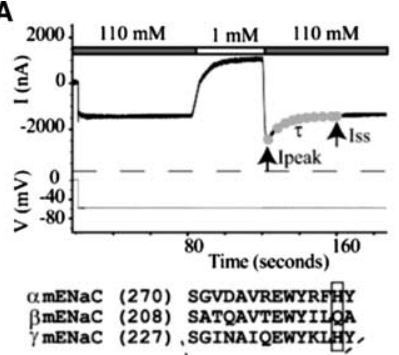

B
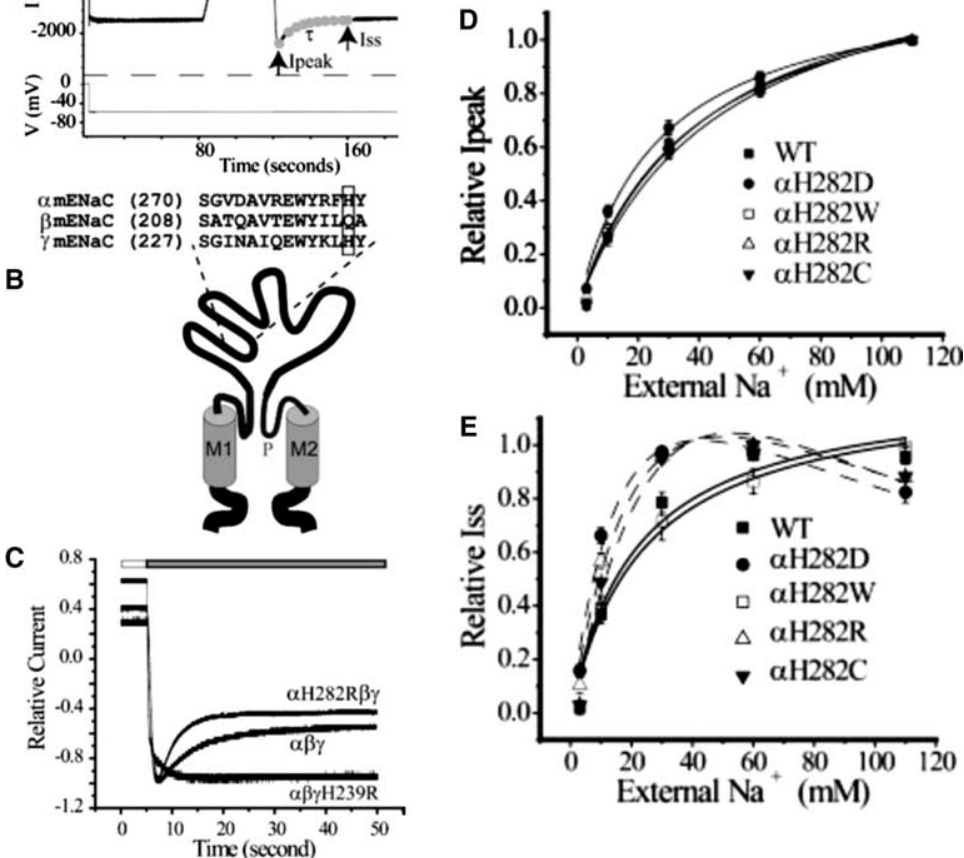

Figure 14.6. Typical recordings of self-inhibition by sodium in the epithelial sodium channel (ENaC). The hetero-tetrameric ENaC composed of $2 \alpha \beta \gamma$ subunits (panel B) display desensitization in entities with a histidyl residue at position 282 replaced by arginine in $\alpha$-subunits while a similar replacement in the $\gamma$-subunit at position 239 prevents the desensitization process (panel C). With single point mutations in position 282 of the $\alpha$-subunits replacing a histidyl residue with arginine, cysteine or glutamic acid, $\mathrm{ENaC}$ also displays bell-shaped auto-inhibition of the steady-state current, $\mathrm{I}_{\mathrm{ss}}$, with increasing sodium concentrations (panel $\mathrm{E}$ ). Taken with permission from Sheng et al. (2004b, Figs 1+3B, C). 


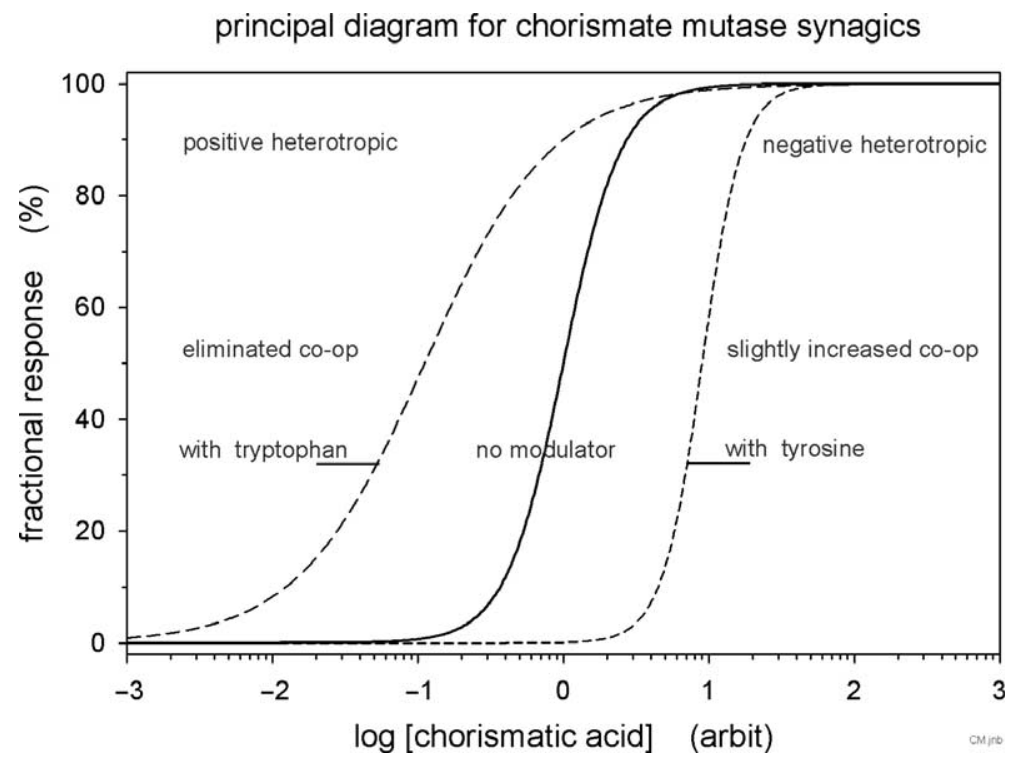

Figure 14.7. Heterotropic modified activity of chorismate mutase. Tryptophan reduces the co-operative effect of the substrate chorismatic acid, but at the same time increase the affinity for the substrate. Tyrosine has the opposite effect as it increases the chorismatic acid co-operative effect, while simultaneously reducing its overall affinity.

\subsubsection{New Epithets for Allostery}

Of late, epithets are in the making for modulators with observed activation and inhibition in the absence and/or presence of primary ligands, since it is now en vogue to discuss the spontaneous activity for receptors and ligandgated transporters as part of allosteric models. Suggestions for some of the modulators binding to an allosteric entity have been 'allosteric agonists' (Soudijn et al. 2004) and 'inverse allosteric agonists' (Bindslev 2004). Even simple terms, such as positive and negative modulators (Pin et al. 2004), may be better when ligands acting at a secondary site can activate or attenuate the binding or function of effectors in the presence or absence of primary ligands. Furthermore, we may operate with neutral (positive or negative) modulators when having an effect from a secondary site, but only in the presence of primary ligands as for co-agonism (Johnson \& Ascher 1987; Corsi et al. 1996; Trist \& Corsi 2000) (see Table 14.4).

Ligands can have more than one function, and Schwartz and Holst (2006) have suggested the name 'ago-allosteric modulators' for compounds that are both agonist and modulators (see also May et al. 2007b). The therapeutic benefits of drugs with such complex behavior await confirmatory proof, and furthermore a theoretical basis is lacking for the supposed advantages of this type of drugs. The conclusion to the story on agoallosteric modulators must await further experimentation and research.

\subsection{More on the Meaning of Allostery}

\subsubsection{Allostery with Multi-subunit Complexes or Multi-sited Units}

On a casual level, to a substantial number of scientists, the definition of allostery has changed completely, since its introduction in the mid-1960s. Thus, 'allosteric' essentially just indicates the same as either positive co-operativity or negative (inhibitory) non-competitive interactions, where accelerator or decelerator modifiers bind to secondary sites on the effector molecule, and not necessarily to secondary sites in a subunit different

Table 14.4. Terminology for ligands with effects at primary (orthosteric) and secondary (modulator, allosteric) binding sites

\begin{tabular}{lccc}
\hline At the primary site & \multicolumn{2}{c}{ At a secondary site } \\
\hline $\begin{array}{l}\text { In presence or absence of other } \\
\text { ligands }\end{array}$ & $\begin{array}{c}\text { Only in presence of other } \\
\text { ligands }\end{array}$ & $\begin{array}{c}\text { In presence or absence of other } \\
\text { ligands }\end{array}$ & Only in presence of other ligands \\
AGONIST & NEUTRAL ANT-AGONIST & POSITIVE MODULATOR & NODULATOR \\
INVERSE AGONIST & NEGATIVE MODULATOR & NEUTRAL MODULATOR & NEUTRAL NEGATIVE MODULATOR \\
\hline
\end{tabular}

Ligands that work at both primary and secondary binding sites may be positive and named enhancers or they may be negative and named inhibitors or as recently suggested 'ago-allosteric modulators' (Schwartz \& Holst 2006; 2007) or 'allosteric agonists' (May et al. 2007b) with both positive and negative effects between 'ago' and 'allo'. For 'co-agonist' see Trist and Corsi (2000). 
from the subunit possessing the primary site. The fact that allosteric phenomena also takes place in monomeric effectors, contrary to fulfilling the strict definition of allostery in the MWC model, has no doubt played a role in loosening the definition of 'allostery' in receptology, where until recently most receptors were thought of as monomeric entities. Next, I illustrate three examples of this dilemma of meaning.

(1) First example of allostery for a ligand at GPCRs or channels. Acetylcholine is the endogenous ligand at mucarinic G protein-coupled receptors (mAChRs) and acetylcholine-dependent nicotinic effector channels (nAChRs). The muscarinic receptor subtype M2 is an example of a single-subunit receptor molecule that demonstrates accelerated allostery as well as heterotropic inhibition (Vogtländer et al. 2003). This must require at least two binding sites. Where these sites are in the M1 and M2 receptor molecules is still an enigma (Johren \& Holtje 2002; Krejci et al. 2004; Huang \& Ellis 2007), although copious searches for binding sites are closing in (Fig. 14.8) (Trankle et al. 2005; Han et al. 2005; Wess 2005; Peng et al. 2006). The allosteric binding site in mAChRs even seems able to adapt two different heterotropic modulators simultaneously (Fig. 14.8) (Trankle et al. 2005).

Meanwhile, di-merization or multi-merization of muscarinic receptor units (Zeng \& Wess 1999; Park \& Wells 2004) as the cause of muscarinic allostery is still a possibility for consideration (Novi et al. 2004), although it seems evident that there are several binding sites in the single mAChR subunits (Trankle et al. 2003, 2005; Avalani et al. 2004). Even so, for functionality, Tränkle et al. (2003) have also suggested M2 dimers or oligomers as possibilities.

In a recent paper on a phospholipid assembly system, co-operativity is first established when mono-meric M2 receptor units are tetra-merized (Ma et al. 2007).

Due to the crystallization capabilities of the nicotinic receptor, where major parts of the $\mathrm{nAChR}$ have been resolved at a 4-Å level (Fig. 14.9A + B) (Miyazawa et al. 1999, 2003; Unwin 2005), its edifice, function, desensitization, and location of primary binding sites in relation to gating (Mitra et al. 2005; Lee \& Sine 2005; Cymes et al. 2005; Mukhtasimova et al. 2005; Elenes et al. 2006;
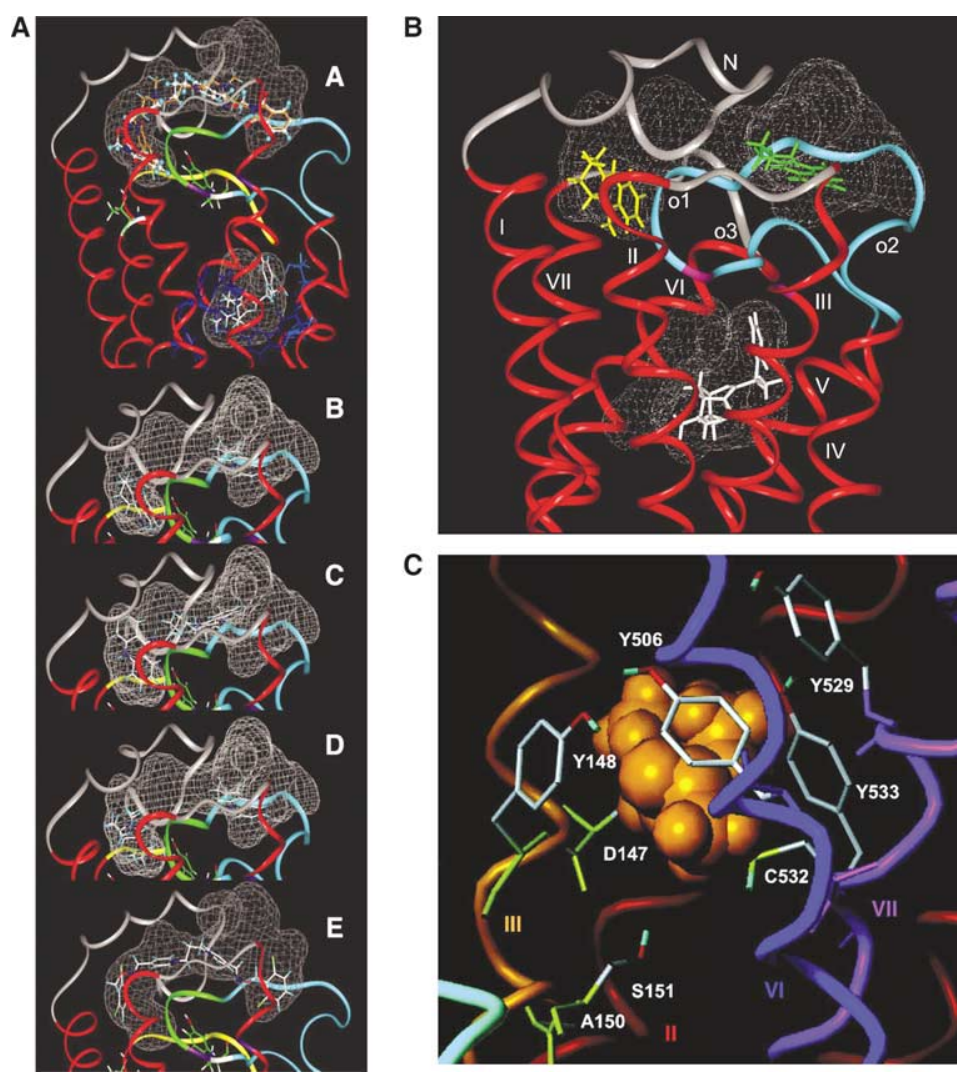

Figure 14.8. Docking simulation of modulators and agonists in muscarinic receptors. Panel A shows docking at the allosteric site for various combinations of two modulators simultaneously (sub-panel A: NMS at the lower orthosteric site with WDuo3 and W84 in the upper allosteric site) in human M2 receptor subtype (from Trankle et al 2005, Fig. 8). Panel B shows two tacrine modulator molecules (yellow and green) in the allosteric site of the M2 receptor subtype (similar to sub-panel B in Panel A) and NMS (white) in the orthosteric site (from Wess 2005, Fig. 1). Panel C is a simulation of acetylcholine (golden orange) bound in the orthosteric site of an M3 receptor subtype (from Han et al 2005, Fig. 8). All figures reproduced with permission. 
Mukhtasimova \& Sine 2007) are ahead of a similar location of binding sites and mechanism of action for the mAChRs.

(2) Second example of allostery for a ligand at GPCRs or channels. The dilemma of choosing between models of multi-meric entities and multi-sited mono-meric unit is related to the orthosteric binding sites for GABA as primary ligand and the secondary binding sites for allosteric ligands, such as benzodiazepines and anesthetics, in the GABA-A channel molecule. The functional GABA-A (and GABA-C) effector consists of five subunits, just as the next of kin in the family of cys-loop ligand-gated $\mathrm{nAChR}$, glycine- $\mathrm{R}$, and $5-\mathrm{HT}_{3} \mathrm{R}$ channels (Thomson \& Lummis 2006). With inferences and parallel information from the structural resolution and location of binding sites in the nAChR channel receptor (Fig. 14.9) (Miyazawa et al. 1999, 2003; Unwin 2003, 2005; Lee \& Sine 2005), the primary binding sites of GABA-A are being tracked down, although the exact structure and location of both primary binding sites and allosteric binding sites in GABA-A/C remain uncertain (Fig. 14.10A + B) (Olsen et al. 2004; Jensen et al. 2005; Lummis et al. 2005; Chen \& Olsen 2007).

Reverting the path from channel to receptor for GABA, the primary binding site in the dimeric GABA$B$ receptor is on one subunit $(\mathrm{B} 1)$, while allostery is elicited by the second subunit (B2), Fig. 14.11A (Pin et al. 2004; Binet 2004) and Fig. 14.11B (Kubo \&
Tateyama 2005). Furthermore, again challenging our imagination is the naming and modeling of another channel-receptor pair for glutamate, iGluR and mGluR, see for instance Dingleline et al. (1999), Planells-Cases et al. (2006), and Pin et al. (2005; Chen et al. 2007; Frauli et al. 2007). Both the GABA-B receptor and the mGluRs are from class C of GPCRs.

(3) Third example of allostery for enzymes and their substrates. Although multi-subunits are the dominating scenario for allosteric functionality (Goodsell \& Olson 2000), allosteric behavior has been claimed for two mono-meric enzymes: ribonucleoside diphosphate reductase, RNR, and pyruvate-UDP-N-acetyl-glucosamine transferase (York 2002), and for others such as members of the hexokinase family (Kamata et al. 2004; Nishimasu et al. 2007), as well as for over 50 enzymes supposed to be mono-meric (Kaiser 1980); although the claims by Kaiser probably no longer hold for all the enzymes.

In passing, one of the above mentioned mono-meric enzymes, RNR, only functions as a hetero-dimer (Cory 2002, 2006, p. 807). Therefore, the quest comes to the fore: is the functional form of RNR a mono-meric or a dimeric complex? Section 14.4.2 provides a solution to this controversy.

Note: glutamine 5-phosphoribosyl-1-pyrophosphate amidotransferase is an example of an enzyme more active in its mono-meric than in its dimeric form! (Cory 2002, p. 833 or 2006, p. 792).

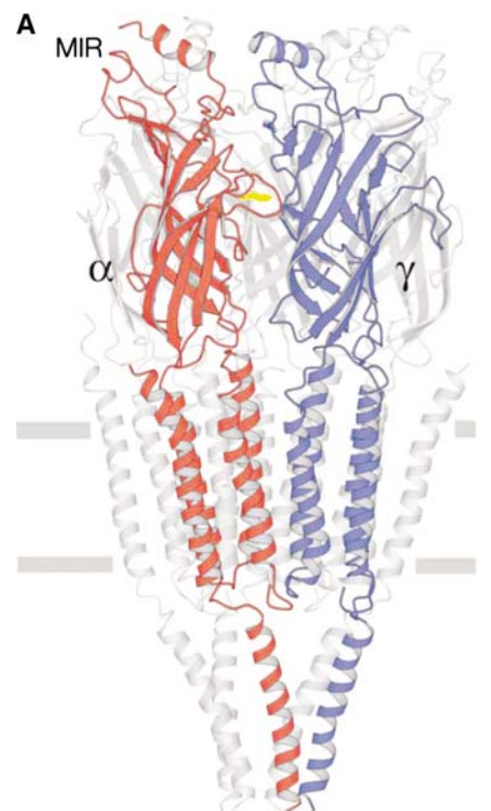

B

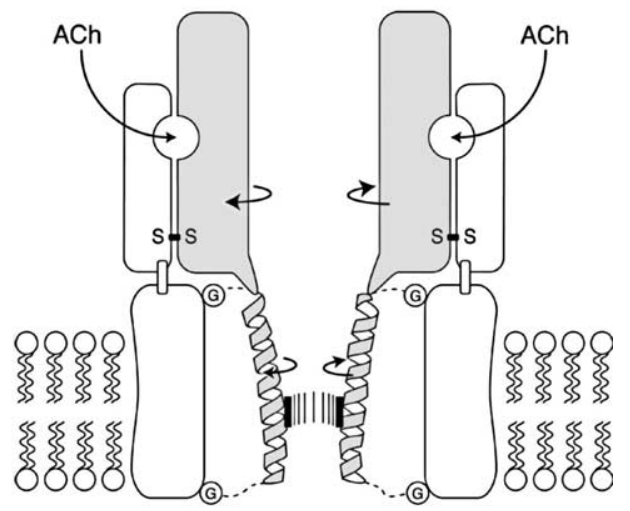

Figure 14.9. Structure of the nicotinic acetylcholine receptor channel, nAChR. Models are built based on X-ray diffraction studies and cryo-fractional studies at a structural resolution of about $4 \AA$. Detailed information about the coupling between binding-gating in this channel structure is now available (Lee \& Sine 2005; Cymes et al 2005). Panel A is a ribbon diagram of the whole nAChR with a view parallel to the cell membrane (from Unwin 2005, Fig. 3). Panel B illustrates the acetylcholine-(ACh)induced rotation transmitted to the gate - a hydrophobic segment in the middle of the membrane passage encased by five M2 helices of which only two are shown (from Miyazawa et al 2003, Fig. 6). Both figures with permission. 

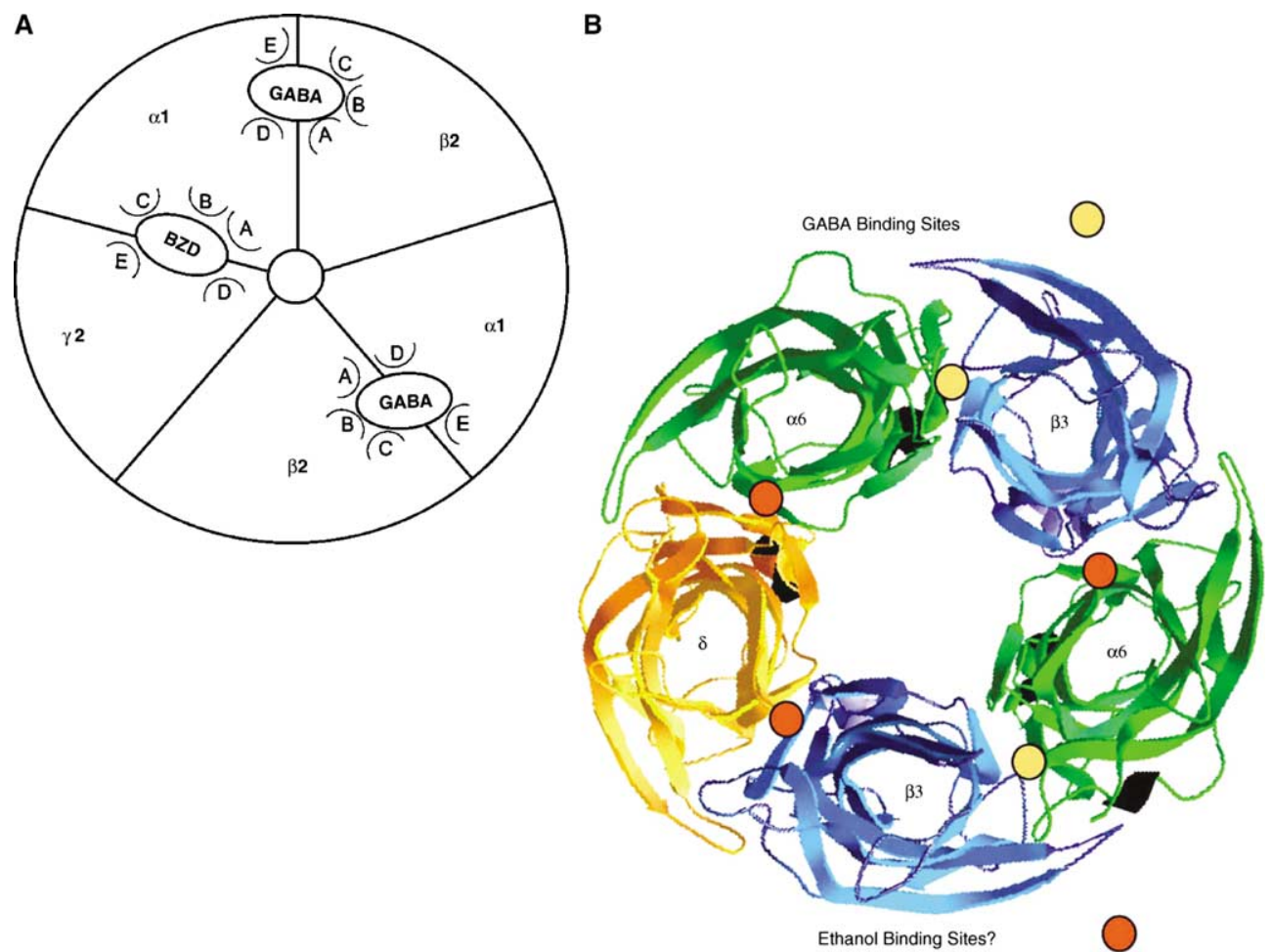

Figure 14.10. Structure of the GABA-A receptor channel. The likely positions of binding sites for GABA and benzodiazepines (B2D) are illustrated on a donut model (panel A) and potential ethanol binding sites (red dots) on a 3-D graph (panel B). Both panels are taken from Olsen et al. (2004, Figs. 3 and 5) with permission.

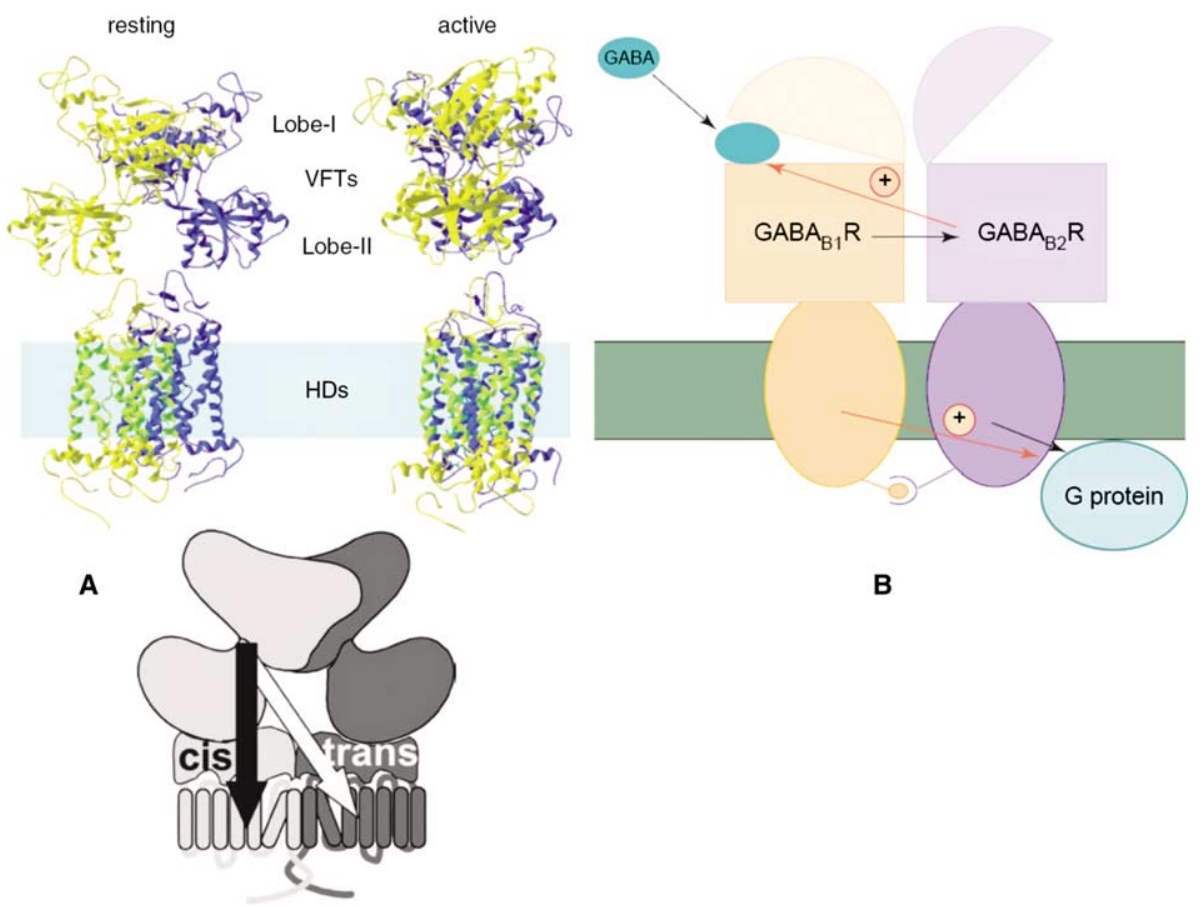

Figure 14.11. Ribbon model and cartoons of allosteric transmission in a GABA-B receptor. The primary ligand GABA binds extracellularly in Venus fly traps (VFTs) while allosteric modulators bind in the membrane-passing helical domains (HDs) of subunit B1. The Venus fly traps switch closer to each other in the homo-dimer upon binding of an allosteric ligand. Cartoon A illustrates activity on ligand binding of the HD in the same subunit (cis) as well as in the other subunit (trans) for homo-dimers. In hetero-dimers only trans-activation occurs. In cartoon B, B1 facilitates the coupling of B2 to G protein whilst B2 increases the affinity of B1 for GABA. Panel A taken with permission from Pin et al (2005, Fig. 2) and Panel B from Kubo and Tateyama (2005, Fig. 1). 


\subsubsection{Allostery with Mono-mers}

The key-question is: are there mono-meric effector molecules where the localization of both a primary site and an allosteric site has unquestionably been demonstrated on the same subunit? The answer seems to be a resounding 'Yes'. For example, the enzyme ribonucleoside triphosphate reductase (RTPR, EC 1.17.4.2) belonging to the class II ribonucleotide reductases (RNR), responsible for conversion of ribonucleotides to the deoxy-form, operates as a monomer. Instead of an allosteric site established at the interface between two subunits, as for class I and III RNRs, class II RNR has an allosteric site conserved in its monomeric sequence. The 3-D structure of its modifier site for binding of ATP or deoxy-nucleoside triphosphate modulators is resolved at the 1.7-A level (Sintchak et al. 2002). The modulator binding site involves a stretch of 130 residues plus shorter flanking segments, residues 147-158 and 298313 , of the monomeric sequence for class II RTPR (Fig. 14.12). Thus, the functional monomer RNR mentioned by York (2002) is not the ribonucleoside diphosphate reductase, but the ribonucleoside triphosphate reductase.

Another classic example of allostery in a monomeric enzyme is hexokinase, allosterically regulated and even with an 'induce fit' upon ligand binding (Nelson \& Cox 2005, p. 218; Weiner 2006, p. 371).

With the surge in our acceptance of di-merization, trimerization and multi-merization as requisites for regulated functionality of receptors (Brady \& Limbird 2002; Franco et al. 2007; Milligan \& Smith 2007; Niv \& Filizola 2007), enzymes and carriers (Berg et al. 2002, 2006; Nelson \& Cox 2005), channel transporters and pumps (Unwin 2005; McKinnon 2003, 2004; Long et al. 2005; Gouaux \& McKinnon 2005; Chen 2005) and maybe even non-channel transporters (Zelcher et al. 2003; Simard et al. 2004; Brunet et al. 2005), it has become more convincing that allostery as a regulatory mean is a consequence connected to a complex of multiple subunits in effector proteins rather than in mono-meric effectors.

Nonetheless, in spite of the current tendency to assume in general that several subunits must come together for modulatory effects in receptors, enzymes, and transporters, it still lingers that some single-subunit receptive molecules may also display genuine allosteric effects (e.g., Fig. 14.8) (Trankle et al. 2005; Wess 2005; Grossmuller et al. 2006)

Whether di-merization or multi-merization of receptive systems in general is an absolute prerequisite for allosteric behavior, except for a few odd cases of monomeric enzymes as the RTPR of class II RNRs, small soluble enzymes like the D or IV hexokinase I (Aleshin et al.
2000; Lunin et al. 2004; Kamata et al. 2004), and possibly the mAChRs (Trankle et al. 2005), only time will tell.

\subsubsection{Allostery with Two Different Primary Ligands}

Allostery may of course be a matter of definitions. For example, there is a concerted translocation of sodium and D-glucose in the sodium/D-glucose cotransporter SGLT1, both types of transportees affecting the transfer of each other. Based on studies with the $\mathrm{Na}^{+} / \mathrm{D}$-glucose cotransporter from bacterium Vibrio para-haemolyticus (vSGLT), the SGLT1 is supposed to be a functional monomer (Turk et al. 2000). In fact, a more detailed understanding of this and other cotransporters may be imminent after a recent and dramatically high resolution at $1.65 \AA$ of the binding sites for a family member of cotransporters from the bacterium Aquifex aeolicus (Fig. 14.13) (Yamashita et al. 2005). Will the day come, where we describe the interactions between sodium and D-glucose in the SGLT cotransporter (Wright et al. 2004; Eskandari et al. 2005) as allosteric? For this to happen, we will probably need a demonstrable constitutive activity in SGLTs (Fig. 14.14), and an acceptance of 'allostery' assigned to the transfer of primary ligands. The most recent understanding for $\mathrm{Na}$ and $\mathrm{D}$-glucose transfer in SGLT is actually a four-state allosteric model (Figs. 14.14 and $6.9 \mathrm{E})$.

\subsubsection{Allostery with Reverse Bell-shaped PTX-sensitive Synagics}

Reverse bell-shaped behavior is an initial lowering of spontaneous activity at low ligand concentration followed by a surge in activity at higher concentrations. Let us look in more detail at the reverse bell-shaped relation in relation to the allostery concept. Question: is reverse bell-shaped synagics due to multi-meric allostery or is it due to bifurcation elicited down-stream in the signaling path, where bifurcation is 'signal trafficking' in Kenakin's terminology or some other mechanism as the G protein-independent signaling (Sun et al. 2007)? The following possibilities discussed to explain mono-ligand induced reverse bell-shaped behavior are listed in Table 14.5.

Reverse bell-shaped dose-responses have been found for $\mathrm{m} 2, \mathrm{~m} 3$, and $\mathrm{m} 4$ receptor subtypes each expressed as the sole receptor in CHO-cells and other cell types, producing cAMP when stimulated with carbachol alone (Migeon \& Nathanson 1994; Hornigold et al. 2003; Griffin et al. 2003). When only a single receptor subtype is expressed, the reverse bell-shaped behavior may be due to a promiscuous interaction by the expressed receptor subtype interacting with both $G_{i / o}$ and $G_{s}$ 

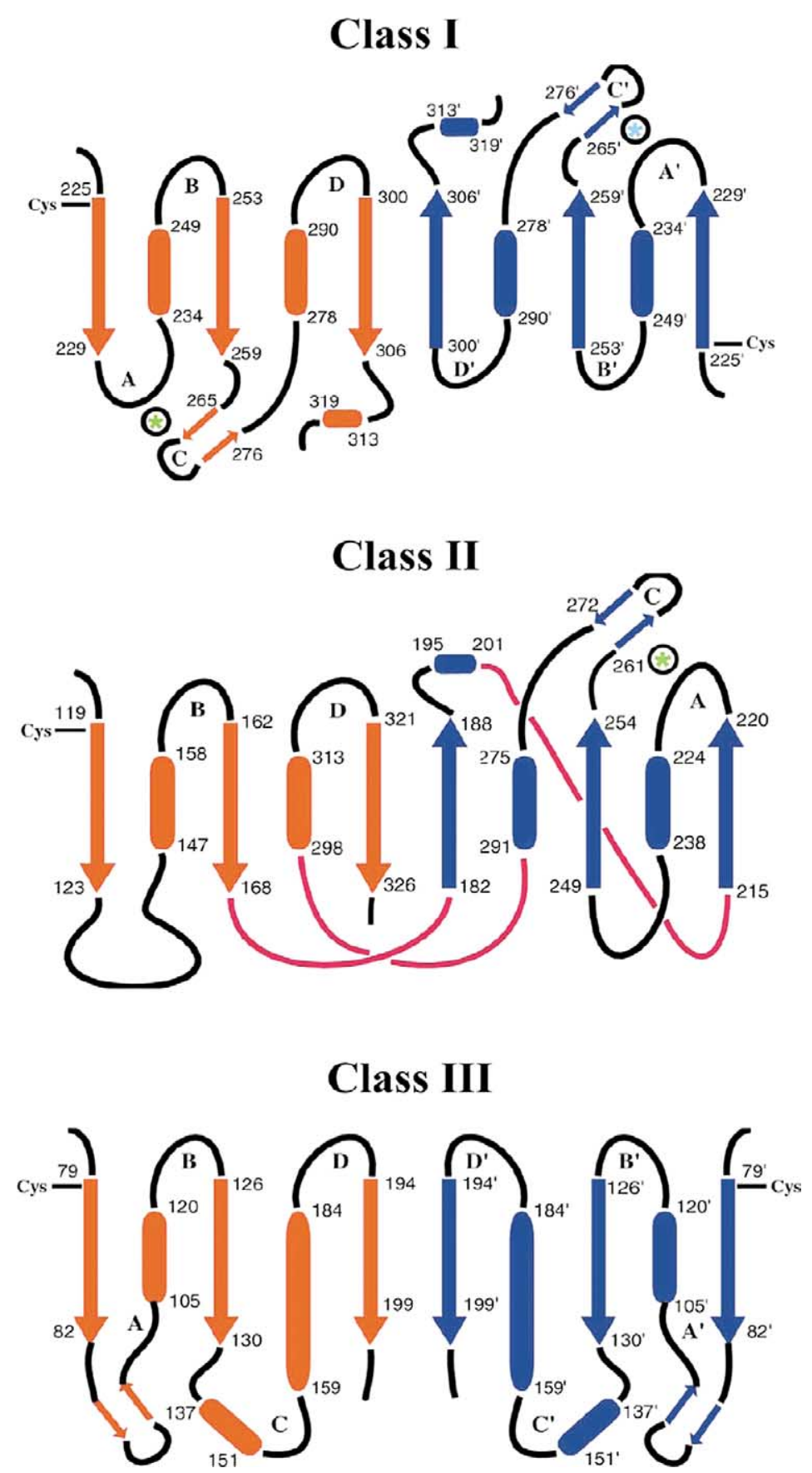

Figure 14.12. Allosteric backbone segment in a monomer. Snake-diagrams of the three classes of ribonucleotide reductases (RNRs) are aligned. In class II there is an intrinsic back-bone segment in the monomer to mediate allosteric behavior of the enzymes. The corresponding segment in class I and III RNRs exists as a separate $\beta$-subunit. Taken with permission from Sintchak et al. (2002, Fig. 6).

proteins. As nicely shown, very often, reversed bellshaped dose-responses may be eliminated by inhibiting $\mathrm{G}_{\mathrm{i} / \mathrm{o}-\alpha}$ GTPase activity with pertussis toxin (PTX), thus indicating bifurcation between $G$ proteins in the transduction as an explanation for the bell-shaped behavior of response (Fig. 14.15) (Michal et al. 2001; Accomazzo et al. 2002; Griffin et al. 2003). In certain studies this is likely the case, since PTX poisoning eliminates the inhibitory leg of the reverse bell-shape dose-response curves in its low concentration range in several studies (e.g., Jones et al. 1991; Migeon \& Nathanson 1994; Schindler et al. 1998; Carruthers et al. 1999; Michal et al. 2001; Accomazzo et al. 2002; Griffin et al. 2003; Cordeaux et al. 2004). Another interpretation is that PTX interferes with certain allosteric binding sites on the muscarinic receptor subtype, especially for the m3 


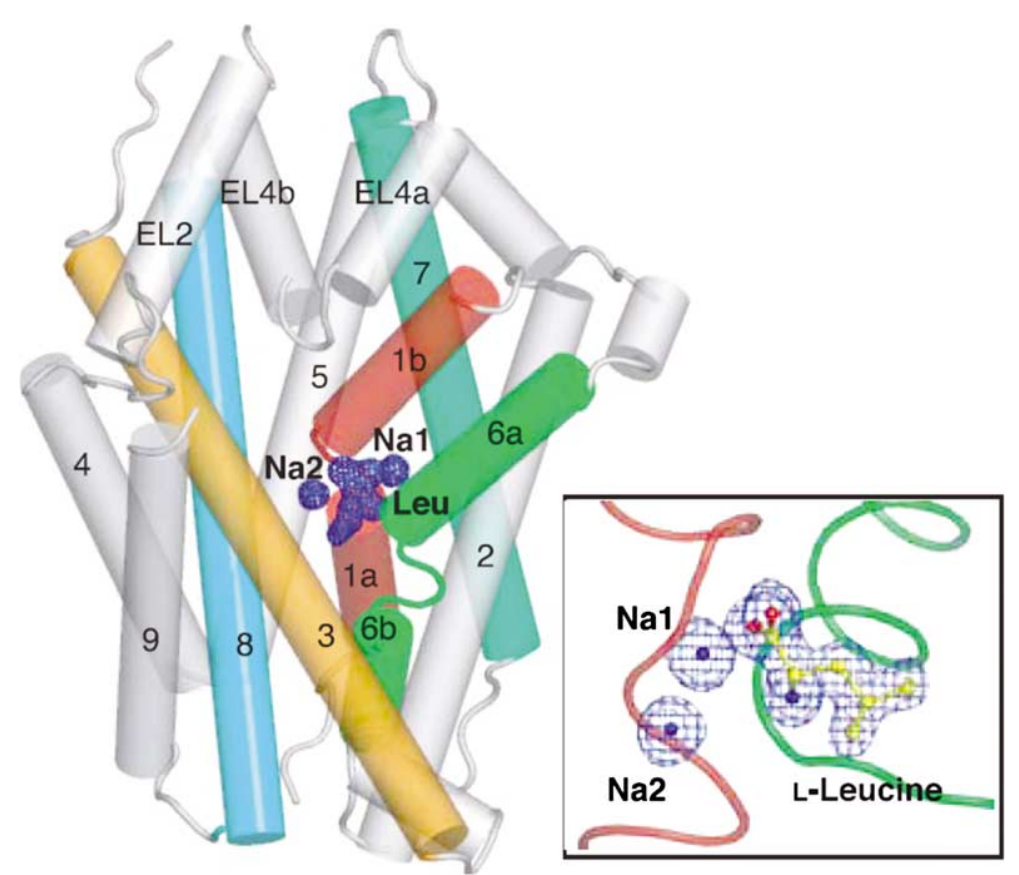

Figure 14.13. Leucine-sodium-binding site of a $\mathrm{Na} / \mathrm{Cl}$ cotransporter. Structure simulation by X-ray diffractions from a crystal of the sodium/chloride/lysine cotransporter in bacterium Aquipex aeolicus. The binding site for two sodium ions and the lysine molecule is resolved at a crystal-clear 1.65 Ångstrøm. This exquisite study should certainly spark and spur structural models for other cotransporters in the near future (Hirayama et al. 2007; Indarte et al. 2007). Figure from Yamashita et al. (2005, Fig. 3a) with permission.

A

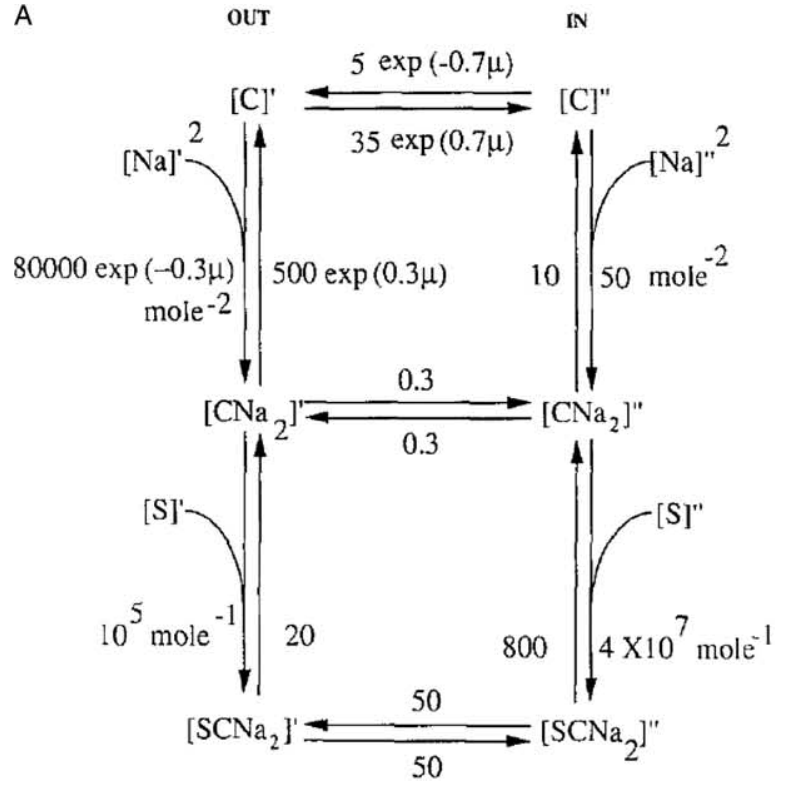

B

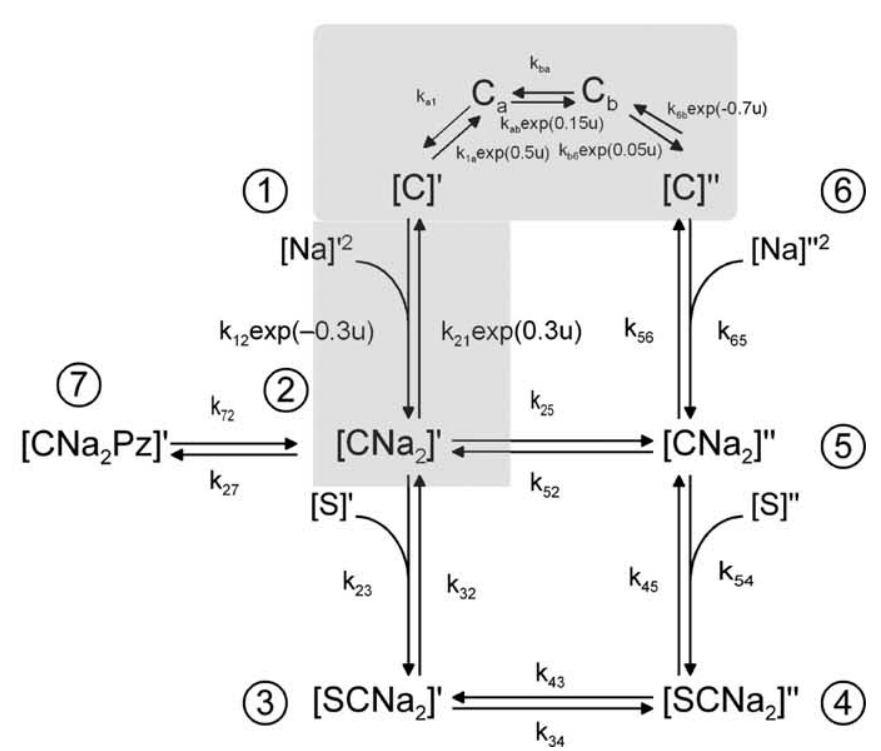

Figure 14.14. Four-state allosteric model for the SGLT cotransporter. Panel A is a two-state model for SGLT from Parent et al. (1992, Fig 2). Panel B is a novel four-state reaction scheme to explain movements of two $\mathrm{Na}^{+}$ions and D-glucose in SGLT. From Loo et al. (2006, Fig 9). Both panels reproduced with permission.

subtype which until recently was known not to interfere with the $G_{i / o}$ protein, although, now that is a possibility (Akam et al. 2001).

Nevertheless, another possible bifurcation that may display reverse bell-shaped dose-responses is signaling induced by both the $\mathrm{G}$ protein alfa subunit and the beta- gamma complex released at activation (Clapham \& Neer 1997; Mirshahi et al. 2003; Du et al. 2004). This other possibility is an inhibitory action of the released $G_{i / 0-\beta \gamma}$ (Migeon \& Nathanson 1994). Meanwhile, this pathway will also be eliminated by the action of PTX (Table 14.5). 
Table 14.5. Possible mechanisms for reverse bell-shaped synagics

\begin{tabular}{ll}
\hline Mechanism & $\begin{array}{l}\text { Pertussis } \\
\text { toxin-sensitive }\end{array}$ \\
\hline Involvement of several subtype receptors & Yes and No \\
Subtype receptor coupling to several G proteins & Yes and No \\
$\mathrm{Gs}_{\alpha}$ and $\mathrm{Gs}_{\beta \gamma} / \mathrm{G}_{\mathrm{q} / 11^{-} \alpha}$ and $\mathrm{G}_{\mathrm{q} / 11^{-} \beta \gamma}$ & No \\
$\mathrm{G}_{0 / \mathrm{i}^{-} \alpha}$ and $\mathrm{G}_{\mathrm{O} / \mathrm{i}^{-} \beta \gamma}$ & Yes \\
Bifurcation downstream of $\mathrm{G}$ proteins & No \\
$\begin{array}{l}\text { Negative leg of the reverse bell removed } \\
\text { allosterically by PTX }\end{array}$ & Yes \\
$\begin{array}{l}\mathrm{G} \text { protein-dependent and } \mathrm{G} \text { protein-independent } \\
\text { pathways }\end{array}$ & No \\
Allostery in the single subtype receptor according \\
to HOTSM
\end{tabular}
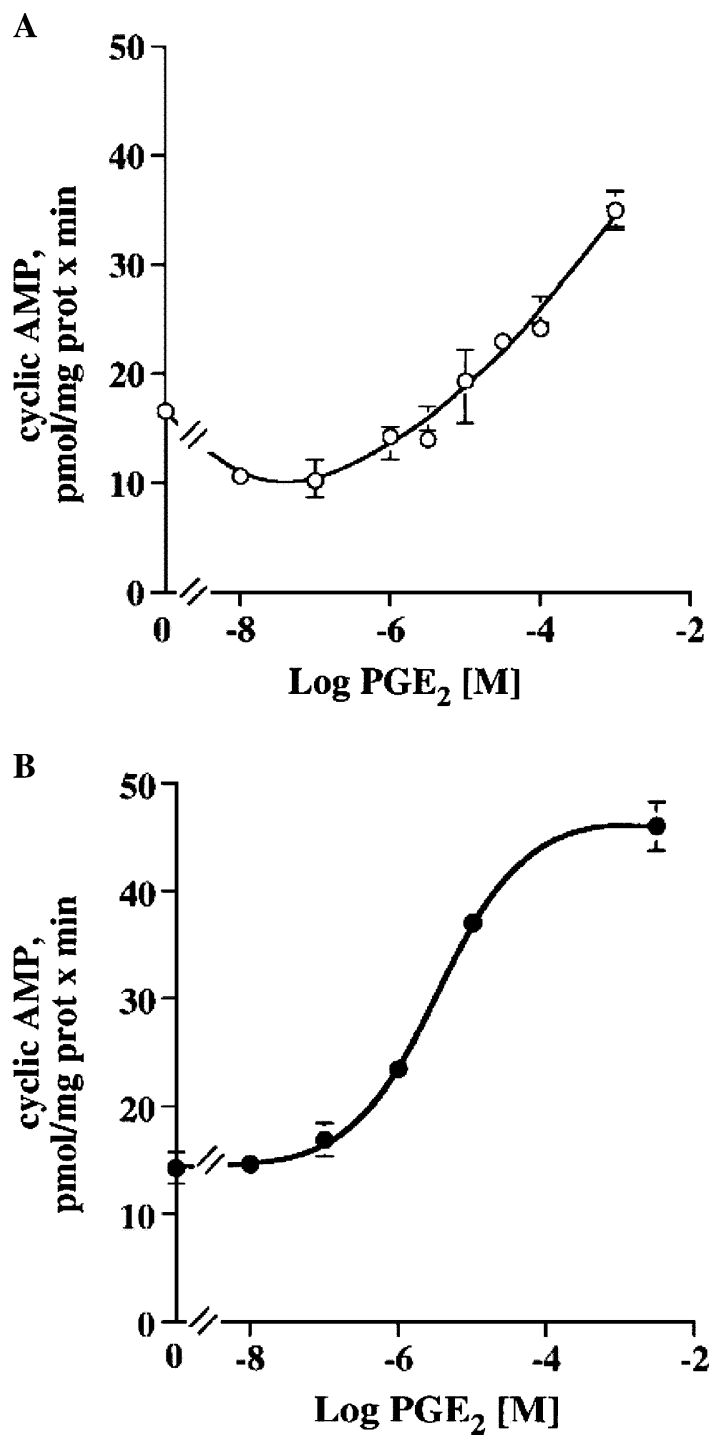

Figure 14.15. Reverse bell-shaped dose-response relation for $\mathrm{PGE}_{2}$ stimulation. Pertussis toxin (PTX) in panel $B$ eliminates the negative leg of the control dose-response in panel A. Modified from Accomazzo et al. (2002, Fig. 4) with permission.
A novel possibility for reverse bell-shaped synagics, independent of PTX poisoning in single receptor subtype expression systems, is the $G$ protein-independent GPCR signaling as detected by several research groups (Zhai et al. 2005; Rajagopal et al. 2005; Sun et al. 2007).

\subsubsection{Allostery with PTX-resistant Reverse Bell-shaped Synagics}

Experiments that will challenge our imagination are studies with expression of a sole subtype receptor where PTX poisoning does not eliminate the inhibitor leg of reverse bell-shaped dose-response. Such studies are, for instance, a recent study on the muscarinic receptor (mAChR) subtype 3 showing reverse bell-shaped synagics resistant to PTX poisoning (Fig. 14.16). Therefore, a challenge to modeling is the observed reverse bellshaped dose-response curves that are independent of PTX poisoning. An example of experiments with monoligand and mono-subtype receptor induced reverse bellshaped dose-responses are depicted in Fig. 14.16A. Thus, even with PTX poisoning, mono-ligand induced reverse bell-shaped dose-responses have been observed for stimulation of the $\mathrm{m} 4$ subtype receptor (Migeon \& Nathanson 1994) and more explicit for the $m 3$ receptor subtype (Fig. 14.16B) (Hornigold et al. 2003). These studies temporarily exclude bifurcation via $G_{q / 11}$ and $\mathrm{G}_{\mathrm{i} / \mathrm{o}}, \mathrm{G}_{\mathrm{i} / \mathrm{o}-\alpha}$ and $\mathrm{G}_{\mathrm{i} / 0-\beta \gamma}$ or the allosteric effect of PTX per $s e$ as explanations for the phenomenon of reverse bellshaped relationships with a single ligand.

The explanation I favor for the mono-ligand and PTX-independent reverse bell-shaped synagics in Fig. 14.16B (Hornigold et al. 2003) is a genuine HOTSM scheme, as an intrinsic mechanism for the reverse bell of mAChRs function. The HOTSM can easily describe the observed reverse bell-shaped behavior without the help of PTX poisoning (Fig 7.27) (Bindslev 2004).

\subsubsection{Allostery with Covalent and Intra-steric Modifications}

Examples of non-covalent modification are listed in Table 13.4 and examples of covalent modification are listed in Table 13.5. Activation of AMP-kinase (AMPK) with both covalent and non-covalent regulatory modifications is one such example (Adams et al. 2004). The covalent phosphorylation of AMPK regulates the enzyme activity, while ATP as a ligand by cross-binding to both AMPK's $\alpha$ - and $\gamma$-subunits interacts with the allosteric effects of another ligand, AMP, at AMPK. ATP is thus both an allosteric, non-covalent ligand and used for covalent regulation as phosphorylation.

Another non-covalent regulation, the intra-steric regulation, is a widespread phenomenon in several protein 

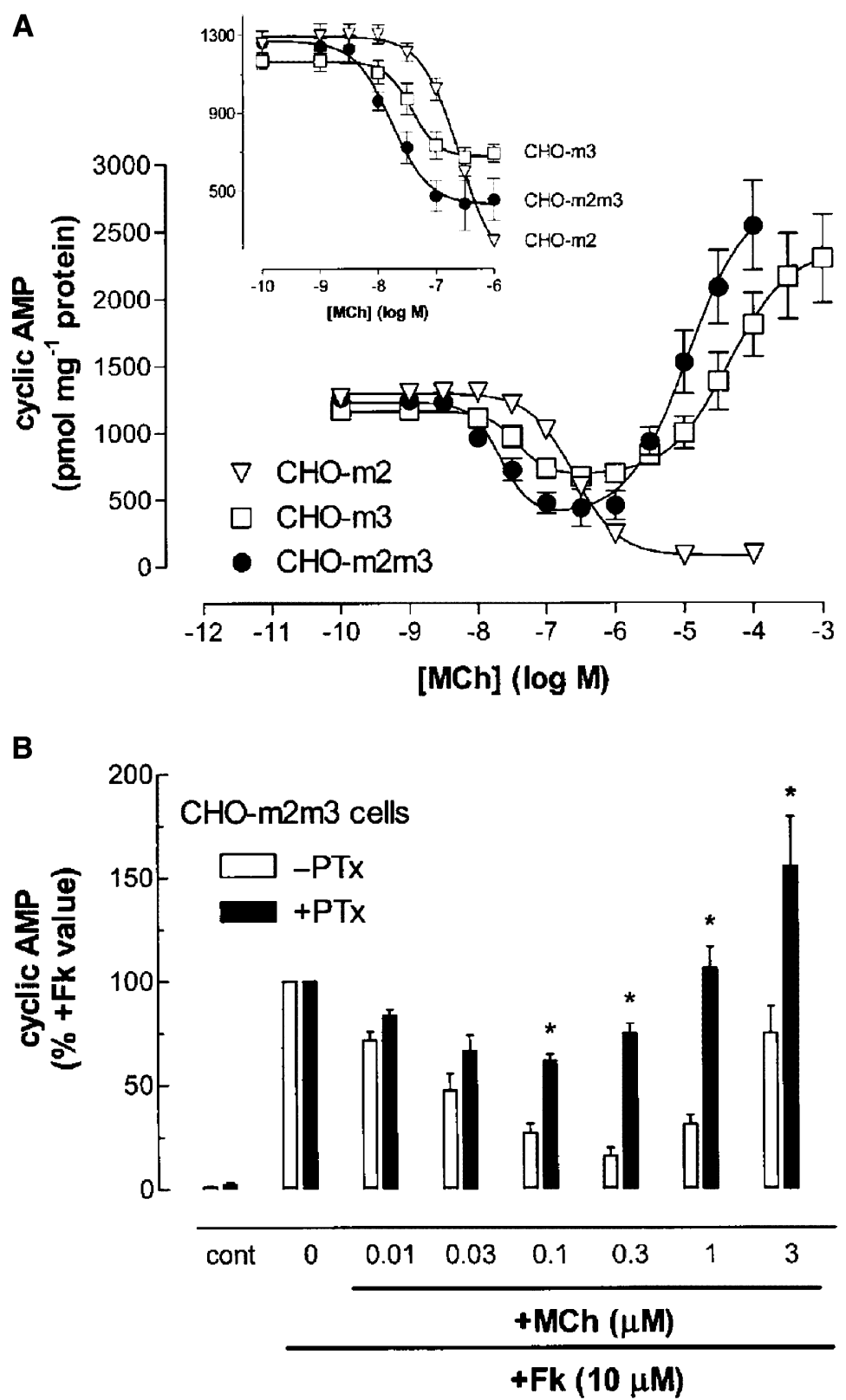

Figure 14.16. Reverse bell-shaped synagics independent of pertussis toxin poisoning. The depicted study is an example of a sole receptor (either muscarinic receptor subtype M2, M3, or both) rendering reverse bell-shaped dose-responses of cAMP production induced by methacholine (MCh) (panel A). Modified from Hornigold et al. (2003, Fig. 4). The reverse bell-shaped synagics by the expressed M3 receptor subtype alone is not eliminated by pretreatment with pertussis toxin (PTX) (panel B). + FK indicate forskolin background activation. From Hornigold et al. (2003, Fig. 5a) with permission.

effector families, with the kinase superfamily and its family members (Niedner et al. 2006) as major contributors.

\subsubsection{Allostery with Unknown Mechanisms}

In the loose sense of allostery, there are still many unexplained observations of aberrant behavior that need a closer look. Some are listed as 'functional selectivity' (Urban et al. 2007), while others have their ligands characterized as 'ago-allosteric modulators' or 'allosteric agonists' (Schwartz \& Holst 2006, 2007; May et al. 2007b).

\subsection{Summing Up Some Views on Allostery and Co-operativity}

\subsubsection{Soft Use of Terms}

In conclusion, due to historical developments, groups of scientists from different fields will use the adjective 'allosteric' and its noun 'allostery' or 'allosterism' with 
variable meanings. Some will relate to microscopically defined intermolecular conformational changes between subunits (Monod et al. 1965) or mono-meric antibodies brought together (Metzger 2002), some to non-specified intra-molecular conformational changes within cotransporters (Bindslev \& Wright 1984) some modeled (Eskandari et al. 2005) and others to specified intra-molecular conformational changes in monomers (Aleshin et al. 2000; Turk et al. 2000; Yamashita et al. 2005). Some find overlaid intra-steric and allosteric control (Adams et al. 2004), others even allostery without a conformational change but with entropy effects (Harris et al. 2001; Vistoli et al. 2007), while others will use the terms referring to dose-responses that deviate from a simple hyperbolic relationship without specifying molecular mechanisms for an explanation. Additionally, others will even use the term allostery in connection with co-agonism and concave/convex bellshaped synagics (Corsi et al. 1996; Bindslev 2004) (Chapter 7). The so-called intervention behavior in the TCM (Chapters 2 and 3) is often equated with allostery in pharmacology (Kwong et al. 1996; Pham \& Reinhart 2001; Ehlert 1988, 2005; Kenakin 2005, 2007; Birdsall \& Lazareno 2005; Price et al. 2005; May et al. 2007a).

\subsubsection{Homo- and Heterotropic Allostery}

Allosteric entities have different mechanisms for cooperative (homotropic) effects and for allosteric (heterotropic) behavior. The precise mechanism for a host of allosteric interactions have more or less been established, e.g., CM, ATCase, and Hb (Berg et al. 2002; Nelson \& Cox 2005), although an unambiguous opinion about many of these mechanisms cannot be reached. For most systems, homotropic and heterotropic mechanisms of allostery remain in the dark.

'Co-operativity' is mostly used as a phenomenologically characterizing term when a single ligand does more than induce simple hyperbolic dose-responses. Thus, numerous researchers use 'co-operativity' to signify when heterotropic modulators induce odd behavior not following a simple load dose-response relation, also characterized as non-Michaelian.

As stated already, the allosteric ligands are molecules that bind to remote sites, physically detached from the primary binding site, and by binding induce conformational changes or select a preestablished conformational state in primary binding or active sites - even of the unliganded effector (Figs. 14.3 and 15.3). Thus, the designation of allosteric isomerization is a concept about a mechanism linking topographically distinct sites. In certain effectors, even though their primary binding sites are also at a distance from the site of activation, as in ligand-gated ion channels, these 'modulator' sites at primary binding sites are not considered allosteric.

\subsubsection{From a Modeler's Point of View}

The terms 'allostery' and 'co-operativity' in a strict sense are defined in Sections 14.2.1 and 14.2.2. It is recommended when formulating allosteric models with the purpose of deriving equations for data analysis, that allostery, both hetero- and homo-tropic, should include a genuine two-state mechanism explicitly expressing two conformations of the un-liganded receptive unit. Contrary to this, we may use terms as 'non-competitive', 'mixed inhibition/activation', 'ternary complex model', or 'intervention schemes' when formulating one-state models.

\subsubsection{Strict Allosteric Models}

The simplest allosteric model, which is also thermodynamically complete, is the ATSM by Hall (2000). In homotropic allostery, equal to co-operativity, this model is converted to the HOTSM as described by Bindslev (2004).

\subsubsection{Allo- Upon - Allo-}

Regulator mechanisms via allosteric contrivances can be extremely intricate. Examples are the chorismate mutase enzyme (Fig. 14.7 and Section 14.3.4), and the glutamine synthetase complex (Section 13.4.1, Fig 4.5). Furthermore, ligands may have pleiotropic functions and become ago-allosteric modulators (Schwartz \& Holst 2006) or multi-functional ligands in ligand-dependent functional diversity, with so-called 'functional selectivity' (Urban et al. 2007).

\subsubsection{6. $K$-, V-, and L-systems}

There is a tendency to further evolve the concepts of Kand V-systems originally based on the ideas of systems following specified synagic models (Galzi et al. 1996). Meanwhile, these classifications are seldom represented in nature (Cornish-Bowden 1995) and can probably be abandoned in the future.

\subsubsection{Reverse Bell-shaped Synagics and PTX Poisoning}

Reverse bell-shaped dose-responses may have several explanations. Bifurcation via $G_{q / 11}$ and $G_{i / o}, G_{i / o-\alpha}$ and $\mathrm{G}_{\mathrm{i} / 0-\beta \gamma}$, or due to simple homotropy as described in the HOTSM (Fig. 7.1B). An alfa/beta-gamma bifurcation was rejected in an $\mathrm{m} 4$ expression system as explanation for reverse bell-shaped synagics (Migeon \& Nathanson 1994). The 'alfa/beta-gamma-bifurcation' explanation may also be rejected for the m3-methacholine reverse bell-shaped dose-response study of Hornigold et al. (2003), since the PTX-poisoning did not eliminate the negative leg of dose-response curves (Fig. 14.16). Reverse bell-shaped synagics persisting after PTX poisoning can be analyzed by the HOTSM (Bindslev 2004). For the heterotrimeric $\mathrm{G}$ proteins, the beta-gamma dimers 
other than that from the $\mathrm{G}_{\mathrm{i} / \mathrm{o}}$ hetero-trimer may be players. Furthermore, part of the GPCR signalling may circumvent G proteins (Zhai et al. 2005; Sun et al. 2007).

\section{REFERENCES}

Accomazzo MR, Cattaneo S, Nicosia S \& Rovati GE. Bell-shaped curves for prostaglandin-induced modulation of adenylate cyclase: two mutually opposing effects. Eur J Pharmacol 454: 107-114, 2002.

Adams J, Chen ZP, Van Denderen BJ, Morton CJ, Parker MW, Witters LA, Stapleton D \& Kemp BE. Intrasteric control of AMPK via the gammal subunit AMP allosteric regulatory site. Protein Sci 13: 155-165, 2004.

Akam EC, Challiss RA \& Nahorski SR. G(q/11) and G(i/o) activation profiles in CHO cells expressing human muscarinic acetylcholine receptors: dependence on agonist as well as receptor-subtype. $\mathrm{Br}$ Pharmacol 132: 950-958, 2001.

Alam N, Stieglitz KA, Caban MD, Gourinath S, Tsuruta H \& Kantrowitz ER. 240s loop interactions stabilize the T state of Escherichia coli aspartate transcarbamoylase. J Biol Chem 279: 23302-23310, 2004.

Aleshin AE, Kirby C, Liu X, Bourenkov GP, Bartunik HD, Fromm HJ \& Honzatko RB. Crystal structures of mutant monomeric hexokinase I reveal multiple ADP binding sites and conformational changes relevant to allosteric regulation. J Mol Biol 296: 1001$1015,2000$.

Atkins WM. Non-Michaelis-Menten Kinetics in cytochrome P450catalyzed reactions. Annu Rev Pharmacol Toxicol 45: 291-310, 2005.

Avlani V, May LT, Sexton PM \& Christopoulos A. Application of a kinetic model to the apparently complex behavior of negative and positive allosteric modulators of muscarinic acetylcholine receptors. J Pharmacol Exp Ther 308: 1062-1072, 2004.

Balshaw DM, Millette LA, Tepperman K \& Wallick ET. Combined allosteric and competitive interaction between extracellular $\mathrm{Na}(+)$ and $\mathrm{K}(+)$ during ion transport by the alpha(1), alpha(2), and alpha(3) isoforms of the Na, K-ATPase. Biophys $J$ 79: 853-862, 2000.

Berg JM, Tymoczko JL \& Stryer L. Biochemistry, 5th ed. New York: WH Freeman, 2002.

Berg JM, Tymoczko JL \& Stryer L. Biochemistry, 6th ed. New York: WH Freeman and Company, 2006.

Bindslev N. A homotropic two-state model and auto-antagonism. BMC Pharmacology 4: 11, 2004.

Bindslev N \& Wright EM. Histidyl residues at the active site of the $\mathrm{Na}$ / succinate co-transporter in rabbit renal brush borders. J Membr Biol 81: 159-170, 1984.

Binet V, Goudet C, Brajon C, Le Corre L, Acher F, Pin JP \& Prezeau L. Molecular mechanisms of $\mathrm{GABA}(\mathrm{B})$ receptor activation: new insights from the mechanism of action of CGP7930, a positive allosteric modulator. Biochem Soc Trans 32: 871-872, 2004.

Birdsall NJ \& Lazareno S. Allosterism at muscarinic receptors: ligands and mechanisms. Mini Rev Med Chem 5: 523-543, 2005.

Botts J \& Drain GF. An illustration of a kinetic analysis: the myosin BATP-EDTA system. The Committee of Muscle Chemistry of Japan. 33-41. 1958. Tokyo, Igaku Shoin: Proceedings from Conference on the Chemistry of Muscular Contraction (1957).

Brady AE \& Limbird LE. G protein-coupled receptor interacting proteins: emerging roles in localization and signal transduction. Cell Signal 14: 297-309, 2002.

Brunet GM, Gagnon E, Simard CF, Daigle ND, Caron L, Noel M, Lefoll $\mathrm{MH}$, Bergeron $\mathrm{MJ}$ \& Isenring $\mathrm{P}$. Novel insights regarding the operational characteristics and teleological purpose of the renal $\mathrm{Na}+-\mathrm{K}+-\mathrm{Cl} 2$ cotransporter (NKCC2s) splice variants. J Gen Physiol 126: 325-337, 2005.

Carruthers AM, Warner AJ, Michel AD, Feniuk W \& Humphrey PP. Activation of adenylate cyclase by human recombinant sst5 receptors expressed in CHO-K1 cells and involvement of Galphas proteins. Br J Pharmacol 126: 1221-1229, 1999.

Changeux J \& Edelstein SJ. Allosteric mechanisms in normal and pathological nicotinic acetylcholine receptors. Curr Opin Neurobiol 11: 369-377, 2001

Changeux JP. Allosteric proteins: from regulatory enzymes to receptors - personal recollections. Bioessays 15: 625-634, 1993.

Changeux J-P \& Edelstein SJ. Nicotinic Acetylcholine Receptors: From Molecular Biology to Cognition. Baltimore: Johns Hopkins University Press, 2005.

Chen TY. Structure and function of clc channels. Annu Rev Physiol 67: 809-839, 2005

Chen Y, Nong Y, Goudet C, Hemstapat K, de Paulis T, Pin JP \& Conn PJ. Interaction of novel positive allosteric modulators of metabotropic glutamate receptor 5 with the negative allosteric antagonist site is required for potentiation of receptor responses. $\mathrm{Mol}$ Pharmacol 71: 1389-1398, 2007.

Chen ZW \& Olsen RW. GABAA receptor associated proteins: a key factor regulating GABAA receptor function. J Neurochem 100: 279294, 2007.

Christopoulos A. Allosteric binding sites on cell-surface receptors: novel targets for drug discovery. Nat Rev Drug Discov 1: 198-210, 2002.

Christopoulos A \& Kenakin T. G protein coupled receptor allosterism and complexing. Pharmacol Rev 54: 323-374, 2002.

Clapham DE \& Neer EJ. G protein beta gamma subunits. Annu Rev Pharmacol Toxicol 37: 167-203, 1997.

Colquhoun D. The relation between classical and cooperative models for drug action. In: Drug receptors. A symposium, edited by Rang HP. London: MacMillan Press, 1973.

Copeland RA. Enzymes. A Practical Introduction to Structure, Mechanisms and Data Analysis. New York: Wiley-VCH, 2000.

Cordeaux Y, Ijzerman AP \& Hill SJ. Coupling of the human Al adenosine receptor to different heterotrimeric $\mathrm{G}$ proteins: evidence for agonist-specific G protein activation. Br J Pharmacol 143: 705-714, 2004

Cornelius F \& Skou JC. The sided action of $\mathrm{Na}+$ on reconstituted shark $\mathrm{Na}+/ \mathrm{K}+$-ATPase engaged in $\mathrm{Na}+-\mathrm{Na}+$ exchange accompanied by ATP hydrolysis. II. Transmembrane allosteric effects on $\mathrm{Na}+$ affinity. Biochim Biophys Acta 944: 223-232, 1988.

Cornelius F, Fedosova NU \& Klodos I. E2P phosphoforms of $\mathrm{Na}$, K-ATPase. II. Interaction of substrate and cation-binding sites in Pi phosphorylation of Na,K-ATPase. Biochemistry 37: 16686-16696, 1998.

Cornish-Bowden A. Fundamentals of Enzyme Kinetics. London: Portland Press, 1995.

Cornish-Bowden A. Fundamentals of Enzyme Kinetics. Colchester: Portland Press Ltd., 2004.

Corsi M, Fina P \& Trist DG. Co-agonism in drug-receptor interaction: illustrated by the NMDA receptors. Trends Pharmacol Sci 17: 220222, 1996.

Cory JG. Purine and pyrimidine nucloetide metabolism. In: Textbook of Biochemistry with Clinical Correlations, edited by Devlin TM. New York: Wiley-Liss, 2002.

Cory JG. Purine and pyrimidine nucloetide metabolism. In: Textbook of Biochemistry with Clinical Correlations, edited by Devlin TM. New York: Wiley-Liss, 2006.

Cymes GD, Ni Y \& Grosman C. Probing ion-channel pores one proton at a time. Nature 438: 975-980, 2005.

De Lean A, Stadel JM \& Lefkowitz RJ. A ternary complex model explains the agonist specific binding properties of the adenylate cyclase coupled beta-adrenergic receptor. J Biol Chem 255: 71087117, 1980.

Dingledine R, Borges K, Bowie D \& Traynelis SF. The glutamate receptor ion channels. Pharmacol Rev 51: 7-61, 1999

Dixon M \& Webb EC. Enzymes. London, Longman, 1979. 
Du X, Zhang H, Lopes C, Mirshahi T, Rohacs T \& Logothetis DE. Characteristic interactions with phosphatidylinositol 4,5-bisphosphate determine regulation of kir channels by diverse modulators. J Biol Chem 279: 37271-37281, 2004.

Ehlert FJ. Estimation of the affinities of allosteric ligands using radioligand binding and pharmacological null methods. Mol Pharmacol 33: 187-194, 1988.

Ehlert FJ. The ternary complex model. In: Biomedical Application of Computer Modeling, edited by Christopoulos A. Boca Raton, FL: CRC Press, 2001.

Ehlert FJ. Analysis of allosterism in functional assays. J Pharmacol Exp Ther 315: 740-754, 2005.

Elenes S, Ni Y, Cymes GD \& Grosman C. Desensitization contributes to the synaptic response of gain-of-function mutants of the muscle nicotinic receptor. J Gen Physiol 128: 615-627, 2006.

Eskandari S, Wright EM \& Loo DD. Kinetics of the reverse mode of the $\mathrm{Na}+$ /glucose cotransporter. J Membr Biol 204: 23-32, 2005.

Fenton AW, Paricharttanakul NM \& Reinhart GD. Disentangling the web of allosteric communication in a homotetramer: heterotropic activation in phosphofructokinase from Escherichia coli. Biochemistry 43: 14104-14110, 2004

Franco R, Casadó V, Cortés A, Ferrada C, Mallol J, Woods A, Lluis C, Canela EI \& Ferré S. Basic concepts in G-protein-coupled receptor homo- and heterodimerization. Sci World J 7: 48-57, 2007.

Frauli M, Hubert N, Schann S, Triballeau N, Bertrand HO, Acher F, Neuville P, Pin JP \& Prezeau L. Amino-pyrrolidine tricarboxylic acids give new insight into group III metabotropic glutamate receptor activation mechanism. Mol Pharmacol 71: 704-712, 2007.

Frieden C. Treatment of enzyme kinetic data. I. The effect of modifiers on the kinetic parameters of single substrate enzymes. J Biol Chem 239: 3533-3543, 1964

Fuchs W, Larsen EH \& Lindemann B. Current-voltage curve of sodium channels and concentration dependence of sodium permeability in frog skin. I Physiol 267: 137-166, 1977.

Galzi JL, Edelstein SJ \& Changeux J. The multiple phenotypes of allosteric receptor mutants. Proc Natl Acad Sci USA 93: 1853-1858, 1996.

Gether U. Uncovering molecular mechanisms involved in activation of G protein-coupled receptors. Endocr Rev 21: 90-113, 2000.

Goodsell DS \& Olson AJ. Structural symmetry and protein function. Annu Rev Biophys Biomol Struct 29: 105-153, 2000.

Gouaux E \& MacKinnon R. Principles of selective ion transport in channels and pumps. Science 310: 1461-1465, 2005.

Granier S, Kim S, Shafer AM, Ratnala VR, Fung IJ, Zare RN \& Kobilka B. Structure and conformational changes in the C-terminal domain of the beta2-adrenoceptor: insights from fluorescence resonance energy transfer studies. J Biol Chem 282: 13895-13905, 2007.

Griffin MT, Hsu JC, Shehnaz D \& Ehlert FJ. Comparison of the pharmacological antagonism of M2 and M3 muscarinic receptors expressed in isolation and in combination. Biochem Pharmacol 65: 1227-1241, 2003.

Grossmuller M, Antony J, Trankle C, Holzgrabe U \& Mohr K. Allosteric site in M2 acetylcholine receptors: evidence for a major conformational change upon binding of an orthosteric agonist instead of an antagonist. Naunyn Schmiedebergs Arch Pharmacol 372: 267-276, 2006.

Gutfreund H. Kinetics for the Life Sciences. Receptors, Transmitters and Catalysts. Cambridge: Cambridge University Press, 1995.

Haber JE \& Koshland DE Jr. Relation of protein subunit interactions to the molecular species observed during cooperative binding of ligands. Proc Natl Acad Sci USA 58: 2087-2093, 1967.

Hall DA. Modeling the functional effects of allosteric modulators at pharmacological receptors: an extension of the two-state model of receptor activation. Mol Pharmacol 58: 1412-1423, 2000.
Han SJ, Hamdan FF, Kim SK, Jacobson KA, Bloodworth LM, Li B \& Wess J. Identification of an agonist-induced conformational change occurring adjacent to the ligand-binding pocket of the M(3) muscarinic acetylcholine receptor. J Biol Chem 280: 34849 34858, 2005.

Harris SA, Gavathiotis E, Searle MS, Orozco M \& Laughton CA. Cooperativity in drug-DNA recognition: a molecular dynamics study. J Am Chem Soc 123: 12658-12663, 2001.

Helmstaedt K, Krappmann S \& Braus GH. Allosteric regulation of catalytic activity: Escherichia coli aspartate transcarbamoylase versus yeast chorismate mutase. Microbiol Mol Biol Rev 65: 404-421, 2001.

Helmstaedt K, Heinrich G, Merkl R \& Braus GH. Chorismate mutase of Thermus thermophilus is a monofunctional AroH class enzyme inhibited by tyrosine. Arch Microbiol 181: 195-203, 2004.

Hirayama BA, Loo DD, Díez-Sampedro A, Leung DW, Meinild AK, Lai-Bing M, Turk E \& Wright EM. Sodium-dependent reorganization of the sugar-binding site of SGLT1. Biochemistry 46: 1339113406, 2007.

Hornigold DC, Mistry R, Raymond PD, Blank JL \& Challiss RA. Evidence for cross-talk between M2 and M3 muscarinic acetylcholine receptors in the regulation of second messenger and extracellular signal-regulated kinase signalling pathways in Chinese hamster ovary cells. Br J Pharmacol 138: 1340-1350, 2003.

Huang XP \& Ellis J. Mutational disruption of a conserved disulfide bond in muscarinic acetylcholine receptors attenuates positive homotropic cooperativity between multiple allosteric sites and has subtype-dependent effects on the affinities of muscarinic allosteric ligands. Mol Pharmacol 71: 759-768, 2007.

Indarte M, Madura JD \& Surratt CK. Dopamine transporter comparative molecular modeling and binding site prediction using the LeuT(Aa) leucine transporter as a template. Proteins 70: 10331046, 2007.

Jenkinson DH. Classical approaches to the study of drug-receptor interactions. In: Textbook of Receptor Pharmacology, edited by Foreman JC \& Johansen T. Boca Raton, FL: CRC Press, 2003.

Jensen ML, Pedersen LN, Timmermann DB, Schousboe A \& Ahring PK. Mutational studies using a cation-conducting GABAA receptor reveal the selectivity determinants of the Cys-loop family of ligand-gated ion channels. J Neurochem 92: 962-972, 2005.

Johnson JW \& Ascher P. Glycine potentiates the NMDA response in cultured mouse brain neurons. Nature 325: 529-531, 1987.

Johren K \& Holtje HD. A model of the human M2 muscarinic acetylcholine receptor. J Comput Aided Mol Des 16: 795-801, 2002.

Jones SV, Heilman CJ \& Brann MR. Functional responses of cloned muscarinic receptors expressed in CHO-K1 cells. Mol Pharmacol 40: 242-247, 1991.

Kaiser PM. Substrate inhibition as a problem of non-linear steady state kinetics with monomeric enzymes. J Mol Catal 8: 431-442, 1980.

Kamata K, Mitsuya M, Nishimura T, Eiki J \& Nagata Y. Structural basis for allosteric regulation of the monomeric allosteric enzyme human glucokinase. Structure (Camb) 12: 429-438, 2004.

Karlin A. On the application of 'a plausible model' of allosteric proteins to the receptor for acetylcholine. I Theor Biol 16: 306320, 1967.

Katz B \& Thesleff S. A study of the desensitization produced by acetylcholine at the motor end-plate. J Physiol 138: 63-80, 1957.

Kenakin T. Efficacy at G-protein-coupled receptors. Nat Rev Drug Discov 1: 103-110, 2002.

Kenakin T. Allosteric modulators: the new generation of receptor antagonist. Mol Interv 4: 222-229, 2004a.

Kenakin T. G-protein coupled receptors as allosteric machines. Receptors Channels 10: 51-60, 2004b.

Kenakin T. New concepts in drug discovery: collateral efficacy and permissive antagonism. Nat Rev Drug Discov 4: 919-927, 2005.

Kenakin T. Allosteric agonist modulators. I Recept Signal Transduct Res 27: 247-259, 2007. 
Kobilka B, Gether U, Seifert R, Lin S \& Ghanouni P. Characterization of ligand-induced conformational states in the beta 2 adrenergic receptor. J Recept Signal Transduct Res 19: 293-300, 1999.

Kobilka BK. G protein coupled receptor structure and activation. Biochim Biophys Acta 1768: 794-807, 2007.

Koshland DE Jr, Nemethy G \& Filmer D. Comparison of experimental binding data and theoretical models in proteins containing subunits. Biochemistry 5: 365-385, 1966.

Krejci A, Michal P, Jakubik J, Ricny J \& Dolezal V. Regulation of signal transduction at M2 muscarinic receptor. Physiol Res 53 Suppl 1: S131-S140, 2004.

Kubo Y \& Tateyama M. Towards a view of functioning dimeric metabotropic receptors. Curr Opin Neurobiol 15: 289-295, 2005.

Kurganov BI. Allosteric enzymes. Kinetic behaviour. Chichester: Wiley \& Sons, 1982.

Kwong DW, Leung WN, Xu M, Zhu SQ \& Cheng CH. Modulatory effects of peroxovanadates on insulin receptor binding. J Inorg Biochem 64: 163-180, 1996.

Laidler KJ. The Chemical Kinetics of Enzyme Action. London: Oxford University Press, 1958.

Laidler KJ and Bunting PS. The Chemical Kinetics of Enzyme Action. Oxford: Oxford University Press, 1973.

Lee WY \& Sine SM. Principal pathway coupling agonist binding to channel gating in nicotinic receptors. Nature 438: 243-247, 2005.

Levitzki A. Quantitative Aspects of Allosteric Mechanisms. Berlin: SpringerVerlag, 1978.

Loland CJ, Granas C, Javitch JA \& Gether U. Identification of intracellular residues in the dopamine transporter critical for regulation of transporter conformation and cocaine binding. $J$ Biol Chem 279: 3228-3238, 2004.

Long SB, Campbell EB \& MacKinnon R. Crystal structure of a mammalian voltage-dependent Shaker family $\mathrm{K}+$ channel. Science 309: 897-903, 2005.

Loo DD, Hirayama BA, Karakossian MH, Meinild AK \& Wright EM. Conformational dynamics of hSGLT1 during $\mathrm{Na}+$ /glucose cotransport. J Gen Physiol 128: 701-720, 2006.

Loo DDF, Hirayama BA, Cha A, Bezanilla F \& Wright EM. Pertubation analysis of the voltage-sensitive conformational changes of the $\mathrm{Na}^{+}$/glucose cotransporter. J Gen Physiol 125: 13-36, 2005.

Lummis SC, L Beene D, Harrison NJ, Lester HA \& Dougherty DA. A cation-pi binding interaction with a tyrosine in the binding site of the GABAC receptor. Chem Biol 12: 993-997, 2005.

Lunin VV, Li Y, Schrag JD, Iannuzzi P, Cygler M \& Matte A. Crystal structures of Escherichia coli ATP-dependent glucokinase and its complex with glucose. J Bacteriol 186: 6915-6927, 2004.

Ma AW, Redka DS, Pisterzi LF, Angers S \& Wells JW. Recovery of oligomers and cooperativity when monomers of the $\mathrm{M}(2)$ muscarinic cholinergic receptor are reconstituted into phospholipid vesicles. Biochemistry 46: 7907-7927, 2007.

MacKinnon R. Potassium channels. FEBS Lett 555: 62-65, 2003.

MacKinnon R. Nobel Lecture. Potassium channels and the atomic basis of selective ion conduction. Biosci Rep 24: 75-100, 2004.

Mahler HR \& Cordes EH. Biological Chemistry. London: Harper \& Row Ltd., 1966.

May LT, Leach K, Sexton PM \& Christopoulos A. Allosteric modulation of G protein-coupled receptors. Annu Rev Pharmacol Toxicol 47: 151, 2007a.

May LT, Avlani VA, Langmead CJ, Herdon HJ, Wood MJ, Sexton PM \& Christopoulos A. Structure-function studies of allosteric agonism at M2 muscarinic acetylcholine receptors. Mol Pharmacol 72: 463476, $2007 \mathrm{~b}$.

Metzger H. Molecular versatility of antibodies. Immunol Rev 185: 186205, 2002.

Michal P, Lysikova M \& Tucek S. Dual effects of muscarinic (M2) acetylcholine receptors on the synthesis of cyclic AMP in CHO cells: dependence on time, receptor density and receptor agonists. Br J Pharmacol 132: 1217-1228, 2001.

Migeon JC \& Nathanson NM. Differential regulation of cAMPmediated gene transcription by $\mathrm{m} 1$ and $\mathrm{m} 4$ muscarinic acetylcholine receptors. Preferential coupling of $\mathrm{m} 4$ receptors to $\mathrm{Gi}$ alpha-2. J Biol Chem 269: 9767-9773, 1994.

Milligan G \& Smith NJ. Allosteric modulation of heterodimeric Gprotein-coupled receptors. Trends Pharmacol Sci 28: 615-620, 2007.

Mirshahi T, Jin T \& Logothetis DE. G beta gamma and KACh: old story, new insights. Sci STKE 2003: PE32, 2003.

Mitra A, Cymes GD \& Auerbach A. Dynamics of the acetylcholine receptor pore at the gating transition state. Proc Natl Acad Sci USA 102: 15069-15074, 2005.

Mitrophanov AY, Churchward G \& Borodovsky M. Control of Streptococcus pyogenes virulence: modeling of the CovR/S signal transduction system. I Theor Biol 246: 113-128, 2007.

Miyazawa A, Fujiyoshi Y, Stowell M \& Unwin N. Nicotinic acetylcholine receptor at $4.6 \mathrm{~A}$ resolution: transverse tunnels in the channel wall. J Mol Biol 288: 765-768, 1999.

Miyazawa A, Fujiyoshi Y \& Unwin N. Structure and gating mechanism of the acetylcholine receptor pore. Nature 423: 949-955, 2003.

Monod J \& Jacob F. Teleonomic mechanisms in cellular metabolism, growth, and differentiation. Cold Spring Harb Symp Quant Biol 26: 389-401, 1961 .

Monod J, Changeux JP \& Jacob F. Allosteric proteins and cellular control systems. J Mol Biol 6: 306-329, 1963.

Monod J, Wyman J \& Changeux J-P. On the nature of allosteric transitions: a plausible model. J Mol Biol 12: 88-118, 1965.

Morth JP, Pedersen BP, Toustrup-Jensen MS, Sørensen TL, Petersen J, Andersen JP, Vilsen B \& Nissen P. Crystal structure of the sodiumpotassium pump. Nature 450: 1043-1049, 2007.

Mukhtasimova N \& Sine SM. An intersubunit trigger of channel gating in the muscle nicotinic receptor. J Neurosci 27: 4110-4119, 2007.

Mukhtasimova N, Free C \& Sine SM. Initial coupling of binding to gating mediated by conserved residues in the muscle nicotinic receptor. J Gen Physiol 126: 23-39, 2005.

Neet KE. Cooperativity in enzyme function: equilibrium and kinetic aspects. Series editor Purich DL. Enzyme Kinetics and mechansims. Editors. Abelson JN and Simon MI. Methods Enzymol 249: 519-567. New York, Acad Press, 1995.

Nelson DL \& Cox MM. Lehninger Principles of Biochemistry. New York: WH Freeman, 2005.

Niedner RH, Buzko OV, Haste NM, Taylor A, Gribskov M \& Taylor SS Protein kinase resource: an integrated environment for phosphorylation research. Proteins 63: 78-86, 2006.

Nishimasu H, Fushinobu S, Shoun H \& Wakagi T. Crystal structures of an ATP-dependent hexokinase with broad substrate specificity from the hyperthermophilic archaeon Sulfolobus tokodaii. I Biol Chem 282: 9923-9931, 2007.

Novi F, Scarselli M, Corsini GU \& Maggio R. The paired activation of the two components of the muscarinic M3 receptor dimer is required for induction of ERK1/2 phosphorylation. I Biol Chem 279: 7476-7486, 2004.

Olsen RW, Chang CS, Li G, Hanchar HJ \& Wallner M. Fishing for allosteric sites on GABA(A) receptors. Biochem Pharmacol 68: 1675-1684, 2004.

Pardee AB \& Reddy GP. Beginnings of feedback inhibition, allostery, and multi-protein complexes. Gene 321: 17-23, 2003.

Parent L, Supplisson S, Loo DDF \& Wright EM. Electrogenic properties of the cloned $\mathrm{Na}+$ /glucose cotransporter. II. A transport model under nonrapid equilibrium conditions. J Membr Biol 125: 63-79, 1992.

Park PS \& Wells JW. Oligomeric potential of the M2 muscarinic cholinergic receptor. J Neurochem 90: 537-548, 2004. 
Peng JY, Vaidehi N, Hall SE \& Goddard WA 3rd. The predicted 3D structures of the human M1 muscarinic acetylcholine receptor with agonist or antagonist bound. Chem Med Chem 1: 878-890, 2006.

Perutz M. Mechanisms of Cooperativity and Allosteric Regulation in Proteins. New York: Cambridge University Press, 1990.

Pham AS \& Reinhart GD. Pre-steady state quantification of the allosteric influence of Escherichia coli phosphofructokinase. J Biol Chem 276: 34388-34395, 2001.

Pin JP, Kniazeff J, Binet V, Liu J, Maurel D, Galvez T, Duthey B, Havlickova M, Blahos J, Prezeau L \& Rondard P. Activation mechanism of the heterodimeric GABA(B) receptor. Biochem Pharmacol 68: 1565-1572, 2004.

Pin JP, Kniazeff J, Liu J, Binet V, Goudet C, Rondard P \& Prezeau L. Allosteric functioning of dimeric class C G-protein-coupled receptors. FEBS J 272: 2947-2955, 2005.

Planells-Cases R, Lerma J \& Ferrer-Montiel A. Pharmacological intervention at ionotropic glutamate receptor complexes. Curr Pharm Des 12: 3583-3596, 2006.

Price MR, Baillie GL, Thomas A, Stevenson LA, Easson M, Goodwin R, McLean A, McIntosh L, Goodwin G, Walker G, Westwood P, Marrs J, Thomson F, Cowley P, Christopoulos A, Pertwee RG \& Ross RA. Allosteric modulation of the cannabinoid CB1 receptor. Mol Pharmacol 68: 1484-1495, 2005.

Quinton PM. Cystic fibrosis: lessons from the sweat gland. Physiology (Bethesda) 22: 212-225, 2007.

Rajagopal K, Lefkowitz RJ \& Rockman HA. When 7 transmembrane receptors are not G protein-coupled receptors. J Clin Invest 115: 2971-2974, 2005.

Reinhart GD. The determination of thermodynamic allosteric parameters of an enzyme undergoing steady-state turnover. Arch Biochem Biophys 224: 389-401, 1983.

Ricard J \& Cornish-Bowden A. Co-operative and allosteric enzymes: 20 years on. Eur J Biochem 166: 256-272, 1987.

Sack JT \& Aldrich RW. Binding of a gating modifier toxin induces intersubunit cooperativity early in the Shaker K channel's activation pathway. J Gen Physiol 128: 119-132, 2006.

Schindler M, Kidd EJ, Carruthers AM, Wyatt MA, Jarvie EM, Sellers LA, Feniuk W \& Humphrey PP. Molecular cloning and functional characterization of a rat somatostatin sst2(b) receptor splice variant. Br J Pharmacol 125: 209-217, 1998.

Schnappauf G, Lipscomb WN \& Braus GH. Separation of inhibition and activation of the allosteric yeast chorismate mutase. Proc Natl Acad Sci USA 95: 2668-2673, 1998.

Schwartz TW \& Holst B. Ago-allosteric modulation and other types of allostery in dimeric 7TM receptors. J Recept Signal Transduct Res 26: 107-128, 2006.

Schwartz TW \& Holst B. Allosteric enhancers, allosteric agonists and ago-allosteric modulators: where do they bind and how do they act? Trends Pharmacol Sci 28: 366-373, 2007.

Segal HL. The development of enzyme kinetics. In: The Enzymes, edited by Boyer PD, Lardy H \& Myrbäck K. New York: Academic Press, 1959.

Segel IH. Enzyme Kinetics. Behavior and Analysis of Rapid Equilibrium and Steady-state Enzyme Systems. New York: Wiley \& Sons (reissued 1993), 1975.

Sheng S, Perry CJ \& Kleyman TR. Extracellular Zn2+ activates epithelial $\mathrm{Na}+$ channels by eliminating $\mathrm{Na}+$ self-inhibition. $J$ Biol Chem 279: 31687-31696, 2004a.

Sheng S, Bruns JB \& Kleyman TR. Extracellular histidine residues crucial for $\mathrm{Na}+$ self-inhibition of epithelial $\mathrm{Na}+$ channels. J Biol Chem 279: 9743-9749, 2004b.

Simard CF, Brunet GM, Daigle ND, Montminy V, Caron L \& Isenring P. Self-interacting domains in the $\mathrm{C}$ terminus of a cation-Clcotransporter described for the first time. I Biol Chem 279: 40769-40777, 2004.
Sintchak MD, Arjara G, Kellogg BA, Stubbe J \& Drennan CL. The crystal structure of class II ribonucleotide reductase reveals how an allosterically regulated monomer mimics a dimer. Nat Struct Biol 9: 293-300, 2002.

Soudijn W, Van Wijngaarden I \& IJzerman AP. Allosteric modulation of $\mathrm{G}$ protein-coupled receptors: perspectives and recent developments. Drug Discov Today 9: 752-758, 2004.

Sun Y, Huang J, Xiang Y, Bastepe M, Juppner H, Kobilka BK, Zhang JJ \& Huang XY. Dosage-dependent switch from G proteincoupled to $\mathrm{G}$ protein-independent signaling by a GPCR. EMBO J26: 53-64, 2007.

Swaminath G, Xiang Y, Lee TW, Steenhuis J, Parnot C \& Kobilka BK. Sequential binding of agonists to the beta2 adrenoceptor. Kinetic evidence for intermediate conformational states. J Biol Chem 279: 686-691, 2004.

Thompson AJ \& Lummis SC. 5-HT3 receptors. Curr Pharm Des 12 $3615-3630,2006$

Thron CD. On the analysis of pharmacological experiments in terms of an allosteric receptor model. Mol Pharmacol 9: 1-9, 1973.

Trankle C, Weyand O, Voigtlander U, Mynett A, Lazareno S, Birdsall NJ \& Mohr K. Interactions of orthosteric and allosteric ligands with $[3 \mathrm{H}]$ dimethyl-W84 at the common allosteric site of muscarinic M2 receptors. Mol Pharmacol 64: 180-190, 2003.

Trankle C, Dittmann A, Schulz U, Weyand O, Buller S, Johren K, Heller E, Birdsall NJ, Holzgrabe U, Ellis J, Holtje HD \& Mohr K. Atypical muscarinic allosteric modulation: cooperativity between modulators and their atypical binding topology in muscarinic M2 and M2/M5 chimeric receptors. Mol Pharmacol 68: 1597-1610, 2005.

Trist DG \& Corsi M. Mechanisms of non-competitive antagonism and co-agonism. In: The Pharmacology of Functional, Biochemical, and Recombinant Receptor Systems, edited by Kenakin T \& Angus JA. Berlin: Springer-Verlag, 2000.

Turk E, Kim O, le Coutre J, Whitelegge JP, Eskandari S, Lam JT, Kreman M, Zampighi G, Faull KF \& Wright EM. Molecular characterization of Vibrio parahaemolyticus vSGLT: a model for sodium-coupled sugar cotransporters. J Biol Chem 275: 2571125716,2000

Unwin N. Structure and action of the nicotinic acetylcholine receptor explored by electron microscopy. FEBS Lett 555: 91-95, 2003.

Unwin N. Refined structure of the nicotinic acetylcholine receptor at 4A resolution. J Mol Biol 346: 967-989, 2005.

Urban JD, Clarke WP, von Zastrow M, Nichols DE, Kobilka B, Weinstein H, Javitch JA, Roth BL, Christopoulos A, Sexton PM, Miller KJ \& Mailman RB. Functional selectivity and classical concepts of quantitative pharmacology. J Pharmacol Exp Ther 320: $1-13,2007$.

Verhamme IM, Olson ST, Tollefsen DM \& Bock PE. Binding of exosite ligands to human thrombin. Re-evaluation of allosteric linkage between thrombin exosites I and II. J Biol Chem 277: 6788-6798, 2002.

Vistoli G, Pedretti A, Testa B \& Matucci R. The conformational and property space of acetylcholine bound to muscarinic receptors: an entropy component accounts for the subtype selectivity of acetylcholine. Arch Biochem Biophys 464: 112-121, 2007.

Voigtlander U, Johren K, Mohr M, Raasch A, Trankle C, Buller S, Ellis J. Holtje HD \& Mohr K. Allosteric site on muscarinic acetylcholine receptors: identification of two amino acids in the muscarinic M2 receptor that account entirely for the M2/M5 subtype selectivities of some structurally diverse allosteric ligands in N-methylscopolamine-occupied receptors. Mol Pharmacol 64: 21-31, 2003.

Weiner H. Enzymes: classification, kinetics, and control. In: Textbook of Biochemistry, edited by Devlin TM. Hoboken, NJ: Wiley-Liss, 2006.

Weiss JM, Morgan PH, Lutz MW \& Kenakin TP. The Cubic Ternary Complex Receptor-Occupancy Model I. model description. J Theoret Biol 178: 151-167, 1996. 
Wess J. Allosteric binding sites on muscarinic acetylcholine receptors. Mol Pharmacol 68: 1506-1509, 2005.

Wong JT-F. Kinetics of Enzyme Mechanisms. London: Academic Press, 1975.

Wright EM, Loo DD, Hirayama BA \& Turk E. Surprising versatility of $\mathrm{Na}+$-glucose cotransporters: SLC5. Physiology (Bethesda) 19: 370376, 2004.

Yamashita A, Singh SK, Kawate T, Jin Y \& Gouaux E. Crystal structure of a bacterial homologue of $\mathrm{Na}+/ \mathrm{Cl}-$-dependent neurotransmitter transporters. Nature 437: 215-223, 2005.

York JL. Enzymes: classification, kinetics, and control. In: Textbook of Biochemistry with Clinical Correlations, edited by Devlin TM. New York: Wiley-Liss, 2002.
Zelcer N, Huisman MT, Reid G, Wielinga P, Breedveld P, Kuil A, Knipscheer P, Schellens JH, Schinkel AH \& Borst P. Evidence for two interacting ligand binding sites in human multidrug resistance protein 2 (ATP binding cassette C2). I Biol Chem 278: 23538-23544, 2003.

Zeng FY \& Wess J. Identification and molecular characterization of m3 muscarinic receptor dimers. J Biol Chem 274: 19487-19497, 1999.

Zhai P, Yamamoto M, Galeotti J, Liu J, Masurekar M, Thaisz J, Irie K, Holle E, Yu X, Kupershmidt S, Roden DM, Wagner T, Yatani A, Vatner DE, Vatner SF \& Sadoshima J. Cardiac-specific overexpression of AT1 receptor mutant lacking Galphaq/Galphai coupling causes hypertrophy and bradycardia in transgenic mice. J Clin Invest 115: 3045-3056, 2005. 


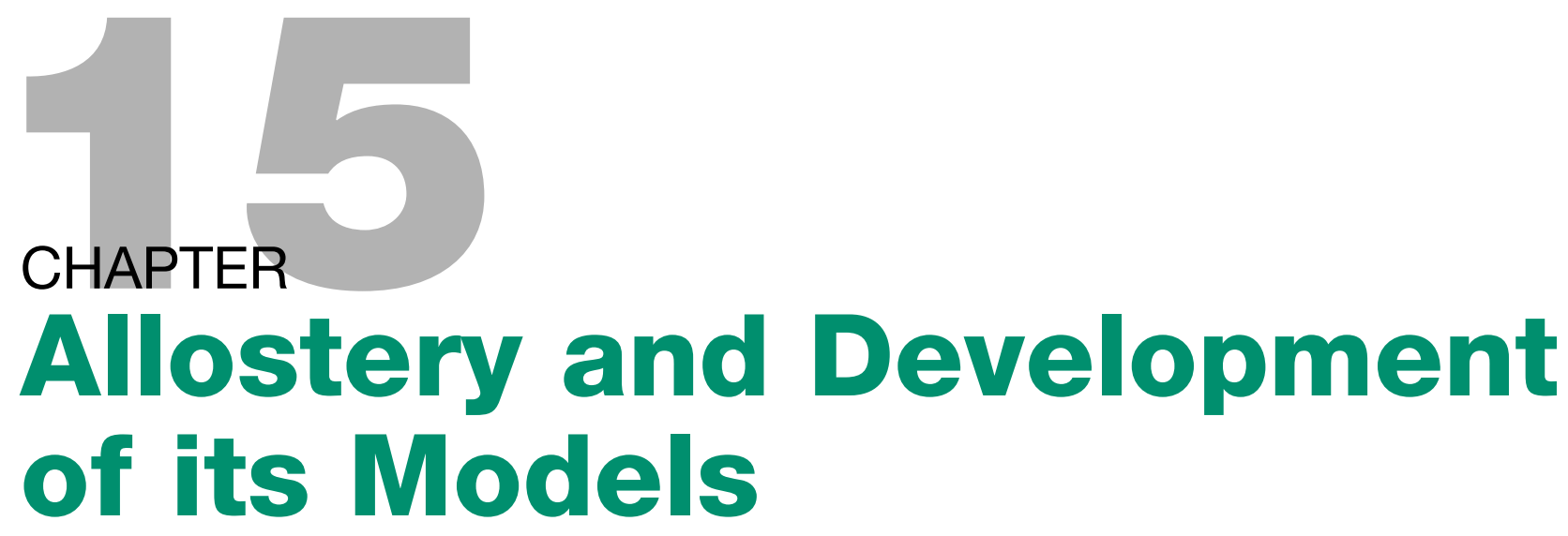

\subsection{Use, Non-use, and Misuse of the Function of State R}

\subsubsection{Hill-Adair-Pauling and Monod-Koshland Models}

It is over 100 years since Christian Bohr and collaborators published papers on the sigmoidality ${ }^{1}$ of concentrationresponse relations at equilibrium, synagics, for $\mathrm{O}_{2}$-binding to hemoglobin (Bohr 1904a,b; Bohr et al. 1904). A re-plot of their data is given in Fig. 15.1. The experimental results in Fig. 15.1 display a clear deviation from load synagics. This deviation is not the so-called 'Bohr-effect'. The Bohr-effect is the rightward shift of the whole hemoglobin- $\mathrm{O}_{2}$ binding curve due to respiratory acidosis as demonstrated in Fig. 15.1 by increasing $\mathrm{CO}_{2}$-tension.

Since Bohr's discoveries, possible explanations for the sigmoidality of interaction between $\mathrm{O}_{2}$ and hemoglobin as well as for non-hyperbolic behavior of enzymes and other receptive systems have engaged a host of researchers, who have developed allosteric models for the loadaberrant behavior (see Box 15.1).

The analytical challenge of the non-Michaelian synagics in Fig. 15.1 has provoked numerous theoretical models. These models have a long and colorful history (Hill 1910; Adair 1925; Pauling 1936; Wyman \& Allen 1951; Monod et al. 1965; Koshland et al. 1966; Wyman \& Gill 1990; Perutz 1990, 1998; Changeux 1993; Bettati et al. 1998). The challenge still prompts the ongoing debate on mechanisms of allostery and co-operativity for enzymes and carriers (Cornish-Bowden 1995, 2004; Papa et al. 2004; Tsuruta et al. 2005; Fetler 2007), for receptors (Christopoulos 2002; Christopoulos et al. 2004; Kenakin 2004; Urban et al. 2006; May et al.

\footnotetext{
${ }^{1}$ See Section 8.1.1 and Fig. 8.2 about sigmoidality.
}

2007), for ligand-gated transporters (DiFrancesco 1999, 2006; Altomare et al. 2001; Changeux \& Edelstein 2001, 2003, 2005; Burzomato et al. 2004), as well as for other types of transporters (Paucek \& Jaburek 2004; Eskandari et al. 2005; Yusef et al. 2006; Ye et al. 2006; Miller 2006; Meyer et al. 2007; Alexander et al. 2007).

Theoretical reaction schemes in formulated expressions such as the Hill-Adair-Pauling (HAP) models and the Monod-Koshland (MK or 'Mon-land') models have often been the base for analyses of dose-response data, and several extensions of these models have flourished.

To mention a few illustrative examples, see for instance Kurganov (1982), Hill (1985), Tuk and van Ostenbruggen (1996), Weiss (1997), Cornish-Bowden (2004), and Michel (2007).

Synagic and kinetic theories have been one-, two-, and multi-state models for binding and for function (Grosman \& Auerbach 2000; Altomare et al. 2001; Scaramellini \& Leff 2002; Giraldo 2004; Avlani et al. 2004; Zhou et al. 2005; Colquhoun 2006; Loo et al. 2006; Jager et al. 2006).

Recently, new powerful models to explain allosteric phenomena at equilibrium have been suggested. They include a cubic allosteric two-state model (ATSM) in relation to heterotropic allostery (Hall 2000), a cubic homotropic two-state model (HOTSM) related to homotropic allostery, equal to co-operativity (Bindslev 2004), and a cubic ternary- and a cubic quaternary-complex model (CTCM) and (CQCM), relevant for G proteincoupled receptors (Weiss et al. 1996a,b,c; Christopoulos et al. 1998; Kenakin et al. 2000, pp. 147-169; Christopoulos \& Kenakin 2002). These new models are treated in more detail in Chapter 7.

The mentioned cubic two-state models may be characterized as analog schemes in comparison with the HAP- and MK-models, which could be said to be based on digital reaction schemes. While the HAP schemes are one-state models, the 'Mon-land' and cubic reactions 


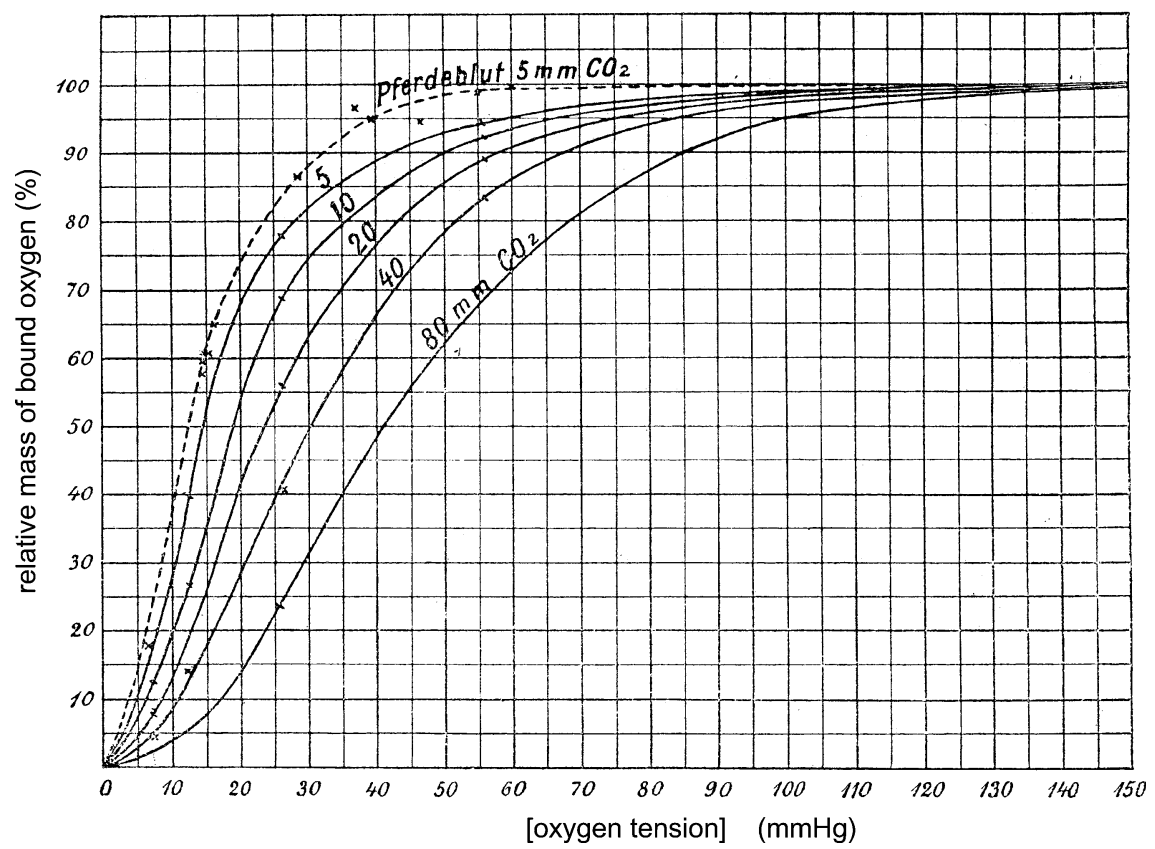

Figure 15.1. Molecular oxygen binding to horse hemoglobin with increasing $\mathrm{CO}_{2}$ tension. Modified from Bohr and coworkers (1904).

schemes are mostly two-state models with a different terminology (Table 15.1).

\subsubsection{New Allostery Models FP-TSM and CQCM, and Their Hybrid Model}

The HOTSM and the ATSM can be combined into a socalled four-pane two-state model (FP-TSM) (Fig. 15.2), where a receptive unit, $\mathrm{R}$, has an orthosteric and an allosteric (modulator) binding site presented to two different ligands (see also Figs. 5.9, 7.2, and 7.3). In the FP-TSM, ligands can (a) bind to either site, the same as 'co-lateral' binding, and (b) bind simultaneously, equal to mutually 'inclusive' binding. Binding in this scheme yields nine conformations (front of Fig. 15.2). Furthermore, (c) all nine reactive conformations can switch to an active state and back, therefore the FP-TSM consists of 18 conformations (Fig. 15.2).

Indeed, in the context of future analysis, one objective of this book is to spark research in an area that combines the HOTSM and the ATSM into the FP-TSM as in Fig. 15.2. The FP-TSM has an alarming 17 independent system constants (Fig. 7.3). Although the 17 parameters may be reduced to about 12 constants by making some simplifying assumptions, analysis of the FP-TSM is still terrifying. Or is it? Maybe not, when we think of the people, the efforts, and the funding that has gone into and continues to go into solving 3-D structural conformations of protein molecules for the purpose of understanding their function, the so-called structureactivity-relationships (SARs) in the drug discovery busi- ness with over 26 million compounds synthesized and tested so far (Lameijer et al. 2006).

A more distant aim of this book is to provoke an appetite for an attack on solutions to the CQCM, on top of a FP-TSM. The goal is that these two models are worked out separately, and, additionally, scrutinized when combined. Note in this connection that just as $\mathrm{G}$ proteins are allosteric modulators (Sprang et al. 2007) G protein-coupled receptor molecules may themselves be thought of as allosteric modulators, i.e., modulators for the heterotrimeric $\mathrm{G}$ protein complex (Bourne et al. 1990; Kenakin 2004). This view is at least a theoretical reality and therefore will affect models such as the CQCM as well as hybrid models of CQCM and FP-TSM.

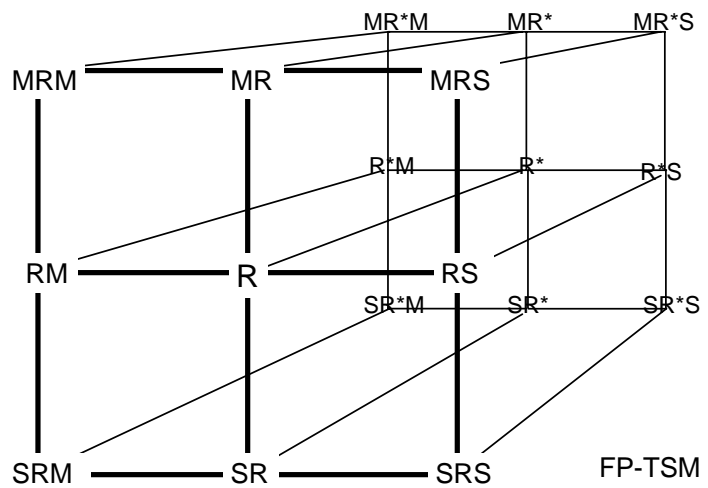

Figure 15.2. The four-pane two-state model (FP-TSM). The reaction scheme is a combination of the allosteric two-state model (ATSM) and the homotropic two-state model (HOTSM) in a thermodynamic regime with complete reciprocity. 
Table 15.1. The intervention scheme (simple TCM) versus ATSM/MWC

\begin{tabular}{|c|c|c|c|c|}
\hline \multirow[b]{2}{*}{ Type of experiment } & Type of ligand & One-state & Type of ligand & Two-state \\
\hline & & Positive-negative & & Positive-negative \\
\hline Function & Same & Auto-intervention partially competitive* & Homotropic & Allosteric (co-operative) \\
\hline Binding & Different & Intervention & Heterotropic & Allosteric \\
\hline Function & Different & Intervention & Heterotropic & Allosteric \\
\hline
\end{tabular}

*See Tipton (1996).

However, these objectives are for the future. In the meantime, here is a short account of some models that have dominated the analytical scene for non-Michaelian synagics over the last forty years, and a few consequences of these models.

\subsection{The MWC Model}

\subsubsection{The Origin of MWC}

In a concluding section of the transactions from a meeting in Cold Spring Harbour in 1961, Monod and Jacob introduced the term 'allosteric' for co-operativity (Monod \& Jacob 1961) on referring to discoveries by J-P Changeux and work by other groups on enzymatic regulation (Yates \& Pardree 1956; Umbarger 1956; Umbarger \& Brown 1957; Changeux 1961; Gerhart \& Pardee 1962). In the following years, the concept of allostery and its model were modified to a more strict definition referring to multi-subunit proteins (Monod et al. 1963). In 1965, Monod, Wyman and Changeux introduced their famous 'concerted model' and further tightened the definition of allostery (Monod et al. 1965). ${ }^{2}$

\subsubsection{Details of Monod-Wyman-Changeux's Model, the MWC Model}

In the renowned article 'On the nature of allosteric transition: A plausible model' the allosteric effect was defined and a model for its mechanism proposed (Monod et al. 1965). Detailed presentations and analyses of this model can be found in Mahler and Cordes (1966, Chapter 6), Weber (1972), Boeynaems and Dumont (1980), Kurganov (1982), Cornish-Bowden (1995, 2004) and Fersht (1999), as well as in numerous other accounts (e.g., Eigen 1968; Rubinow 1975; Segel 1975; Wong 1975; Fromm 1975; Levitzki 1978; Cantor \& Schimmel 1980; Hill 1985; Perutz 1990; Wyman \& Gill 1990; Perutz et al. 1998; Ben-Naim 2001; Berg et al. 2006, Chapter 7). In order to demonstrate the popularity and repetitious use of MWC, Box 15.1 elaborates slightly more on references, though not an exhaustive listing.

\footnotetext{
2 'Concerted', a term coined by Koshland et al. (1966).
}

The genesis of 'A plausible model' is recounted in a beautiful essay by Changeux (1993).

Here, I present a semi-brief overview of the 1965 MWC paper.

First, the paper has a general statement about allosteric effects, that is: an indirect interaction between distinct specific binding sites is responsible for the regulatory function.

Second, two terms are defined:

I. Homotropic (allosteric) effects are due to identical ligands.

II. Heterotropic (allosteric) effects are due to ligands that are not identical.

Regulatory enzymes are called homotropic when substrate and modulator are identical. When the modulator is a molecule other than the substrate, the effect of the enzyme is said to be heterotropic. Some enzymes have two or more modulators, a fact that is now also true for many receptors and transporters (Balshaw et al. 2000; Weber et al. 2001; John et al. 2003; Burzomato et al. 2004; Hamborck et al. 2004; Kew 2004; Jensen et al. 2005; May 2005; van Aubel et al. 2005; Birdsall \& Lazareno 2005; Holzgrabe et al. 2006; to mention a few).

Third, the paper lists five properties for allosteric systems (Box 15.2).

Fourth, in modeling the allosteric mechanism, six axioms were put forward (Box 15.3).

The MWC model yields two key-equations for homotropic allostery, the 'saturation-function' $\mathbf{Y}$ for occupancy and the 'function of state' $\mathbf{R}$ for functional activity (Eqs. 15.1 and 15.2).

\subsubsection{Allostery and Spontaneous Activity}

At the time of introducing the allosteric model it was mostly the binding of ligands to enzymes and the binding of $\mathrm{O}_{2}$ to hemoglobin that were in focus. Accordingly, although the MWC model allows for spontaneous activity in its function of state $\mathbf{R}$ (R-function) (see Eq. 15.2), spontaneous activity was rarely considered when the R-function attracted attention. Of course, exceptions can be found (Rubin \& Changeux 1966; Changeux et al. 1967; Changeux \& Rubin 1968; Eisenstein et al. 1995; 


\section{Box 15.1. Some papers on HAP- and Mon-land models for allostery and co-operativity}

Hill-Adair-Pauling (HAP) and Monod-Koshland (Mon-land) models as analytical tools were developed for enzymes and circulating carriers, beginning in the first decade of the 20th century (e.g., Hill 1910, 1913; Douglas et al. 1912; Brown \& Hill 1923; Adair 1925, Pauling 1935, Roughton et al. 1955; Botts \& Drain 1958; Monod et al. 1963; Frieden 1964; 1970; Monod et al. 1965; Koshland et al. 1966; Haber \& Koshland 1967; Kirtley \& Koshland 1967; Eigen 1967, 1968; Gerhardt 1970; Weber 1972, 1975; Janin 1973; Edelstein 1975; Fromm 1975; Segel 1975/93; Wong 1975; Wyman 1975; Kurganov 1977, 1982; Levitzki 1978; Hill 1985, Chapters 24 and 25; Ricard \& Cornish-Bowden 1987; Ricard 1989; Perutz 1990; Wyman \& Gill 1990; CornishBowden 1995; Neet 1995; Perutz et al. 1998; Jin et al. 1999; Kurganov 2000; Helmsteadt et al. 2001 ; Macol et al. 2001; Stieglitz et al. 2004; Changeux \& Edelstein 2005; Tsuruta et al. 2005; Wang et al. 2005, 2007 ; Fetler et al. 2007). Hill's book on co-operativity is a general introduction to equilibrium and steady-state synagic models. Hill has nicely divided the subject into reaction schemes without and with interactive co-operativity (Hill 1985).

Somewhat later, similar models were developed for transporters of the ligand-gated channel type and for receptors (e.g., DelCastillo \& Katz 1956; Katz \& Thesleff 1957; Karlin 1967; Changeux \& Podleski 1968; Colquhoun 1973, 1998; Thron 1973; Boeynaems \& Dumont 1977, 1980; Birdsall et al. 1978; Birnbaum et al. 1980; DeLean et al. 1980; Stockton et al. 1983; Ehlert 1988; Lefkowitz et al. 1993; Tucek \& Proska 1995; Lazareno \& Birdsall 1995; Christopoulos et al. 1998, 1999; 2004; Changeux \& Edelstein 1998, 2001, 2005; Scaramelli \& Leff 2002; Tucek et al. 2002; Lazareno et al. 2002; Parmentier et al. 2002; Hatton et al. 2003; Beato et al. 2004; Kenakin 2004; Kew 2004; Suzuki et al. 2004; Hlavackova et al. 2005; Leach et al. 2007 ; May et al. 2007; Springael et al. 2007) to mention a few, though not all of the presented models are genuine two-state models as required from a rigorous point of view (see Sections 14.2.1 and 14.2.2).

Examples may likewise be found for non-ligand-gated transporters, i.e., pumps (Balshaw et al. 2000; Wang et al. 2004; van Aubel et al. 2005; Pinkett et al. 2007; Jones \& George 2007; Morth et al. 2007; Olesen et al. 2007), coand counter-transporters including the RND-type, the SMR-type, and the MFS-type $\mathrm{H}^{+}$-driven systems (Ferrell et al. 2000, Weber et al. 2001; Berteloot 2003; Loo 2006; Higgins 2007), and uniporters (Zottola et al. 1995; Poirier et al. 2007).

\section{Box 15.2. Features of an allosteric system according to MWC}

(1) Allosteric systems are often an oligomer involving several identical subunits.

Comment: For many receptor systems with allostery, statement (1) is no longer entirely true, as subunits are not identical. On the other hand, the statement above may have a renaissance and turn out to frequently embody some truism, as more and more receptors seem to be either homo-dimers or homo-oligomers in their functional state. Meanwhile, a significant number of functional systems are also hetero-dimers and heteromultimers (Min et al. 2002; Pin et al. 2004; Jensen et al. 2005; Milligan \& Smith 2007; Springael et al. 2007 ). Monomers with both primary and secondary binding sites also exist (Aleshin et al. 2000; Sintchak et al. 2002; Kamata et al. 2004; Trankle et al. 2005; Lazareno \& Bidsall 2005).

(2) Subunits have allosteric interactions, which are frequently due to alterations in the bonding between subunits, i.e., in the quaternary structure. See comment in point 6 in Box 15.3.

(3) Heterotropic allostery (i.e., allosteric effects due to different ligands) may be either accelerative or decelerative, while homotropic allostery (i.e., allosteric effects due to identical ligands) always appears to be positively co-operative.

Comment: This third statement was based on observations for $\mathrm{O}_{2}$-binding to hemoglobin but completely overlooked the so-called 'substrate inhibition', auto-ant-agonism or auto-inhibition (also see Table 15.2, column 3).

(4) Nearly all the allosteric systems that exhibit heterotropic effects also possess homotropic effects. Comment: Separations of homotropic and heterotropic effects from co-operativity effects are encountered (Fig. 15.6) (Helmstaedt et al. 2001).

(5) Conditions altering the heterotropic effects also affect the homotropic effects.

Comment: This is still true, even though there are examples to negate this statement (Chan \& Enns 1979; Stebbins et al. 1990; Peterson et al. 1992; Schnappauf et al. 1998; Helmstaedt et al. 2001). 


\section{Box 15.3. A priories for allosteric systems according to $\mathrm{MWC}^{9}$}

(1) Allosteric proteins are oligomers containing identical subunits and all these allosteric subunits occupy equivalent positions, axial and rotational symmetry.

Comments: This axiom has an esthetism often not fulfilled in real life. Separation of primary binding sites and modulator binding sites extends to being placed on different subunits - for enzymes often in catalytic and regulatory subunits and at interfaces between subunits as well (Changeux \& Edelstein 2005).

(2) Only one stereospecific ligand binding site on each allosteric subunit. An allosteric entity with a single binding site was also termed a protomer (see first comment in Box 15.2 and the comment to point 1 in this Box).

(3) The conformation of each of these protomers is constrained by its association with the other protomers.

Comment: Inter-subunit or inter-protomer bonds may relate both to the quaternary and tertiary structure of proteins. Thus regulatory mechanisms as the allosteric effects lie in the quaternary and tertiary structure related to inter- 'allosteric subunit' -bonds or conformational restraints, comparable to intrasteric constraints of the regulatory subunits in, for instance, PKA-, PKG kinases, and others (Cheetham 2004; Peterson \& Golemis 2004).

(4) At least two states are reversibly accessible to the whole system and dependent on intersubunit bonds.

Comment: In the formulation of the original model the reversible transition between states was only allowed for the un-bound (non-liganded) states. The allosteric MWC 1965 model is a genuine two-state and concerted model (see point 5 below).

Furthermore, tacitly 'selection' instead of 'induction' by ligands was assumed. As Changeux (JP-C) writes in his memoire paper from 1993, 'in Monod's writing all the way back from 1949, I find the idea of selection of protein states by ligand for that of enzymatic adaptation' (Changeux 1993). JP-C further attests that the idea about conformational isomerization for enzymes prior to ligand binding, i.e., selection as claimed for the MWC model, was confirmed in 1967 by Gerhardt and Changeux (1968a see abstract in Oslo Conference; Changeux et al. 1967; Changeux \& Rubin 1968). Meanwhile, see a perspectivated discussion of this subject in sub-chapter 5.11.

Pertaining to this axiom 4, with due respect I may add that a crucial missing point, viz. the omission of a cyclic reaction, was not explicitly stated although it appears in reaction schemes and formulations of the 1965 Monod et al. paper. The missing point, a reversal flip, or even just a flip, between liganded states was not allowed, in spite of the finding of Roughton et al. of a deviating concerted step for the tri- $\mathrm{O}_{2}$-liganded hemoglobin (Roughton et al. 1955). Accordingly the model by MWC ought to be designated the 'sole un-liganded step flipflop model'. This model was also maintained in models by Karlin (1967), Thron (1973), and Colquhoun (1973), while incorrectly presented as fully thermodynamically reversible in many other publications (e.g., Cornish-Bowden 2004, Fig. 11.6; Changeux \& Edelstein 2005, Fig. A.1C).

The 'sole un-liganded step flip-flop' constraint listed for the MWC model is a major advantage in that it reduces the complex of the concerted system to include only four parameters for its description, viz. $L^{\mathrm{MWC}}, K_{\mathrm{T}}, K_{\mathrm{R}}$, and $n$ (Monod et al. 1965).

(5) Concerted-ness. Transition between states alters the conformation and the affinity for binding sites at all of the allosteric protomers concomitantly.

(6) During the transition between states, the symmetry of conformational constraints imposed on each allosteric protomer is conserved. Thus, regulatory mechanisms of certain allosteric effects lie in the quaternary structure related to inter- 'allosteric subunit' -bonds or conformational restraints. See a- and b-glycogen phosphorylases, which are both in R and T states! (Kruganov 1982; Berg et al. 2002, p. 584; Cornish-Bowden 1995, 2004).

Comment: Statements 5 and 6 are taken to mean that when the transition between states alters the affinity constant of one binding site, the affinity constant for the rest alters equivalently and simultaneously and it is due to quaternary redistribution of subunits affecting the binding constants. However, tertiary relaying is certainly also involved (Perutz 1990; Iwata et al. 1994; Changeux \& Edelstein 1998, 2005; Steiglitz et al. 2003).

Functionally, the general MWC includes intrinsic (microscopically) efficacy constants (Kurganov 1982, p. 150).

${ }^{9}$ Listed as four points in Monod's Nobel lecture (Monod 1972).

Changeux \& Edelstein 2005). However, in general, constitutive activity inherent in the MWC-model is almost completely ignored by modelers. A likely explanation for this disregard is that enzymes in an experiment do not overtly display spontaneous catalytic activity in the absence of a substrate. This is probably also one reason why models on spontaneous activity are only sporadically treated in composite texts on enzyme synagics and kinetics (e.g., Segel 1975, 1993; Cornish-Bowden 2004; Berg et al. 2006). Furthermore, constitutive activity in receptors was first reinvented in 1989 (Costa \& Herz) and here the cyclic two-state and the ternary-complex models 
took the lead (DeLean 1980; Ehlert 1988; Leff 1995) rather than the MWC model. Spontaneous activity immanent in the R-function is debated further in Sections 15.3.4, 15.3.5, 15.4.3, and 15.4.4.

\subsection{Homotropic Allostery of the MWC Model}

\subsubsection{Consequences of the MWC Model for Homotropic Allostery $=$ Co-operativity}

The MWC model with its $\mathrm{R}$ - and $\mathrm{T}$ states is shown in Fig. 15.3, where the normalized concentration $\alpha$ and parameters $L^{\mathrm{MWC}}, c^{\mathrm{MWC}}$, and $n$ are defined.

Based on the simplifying assumptions for the MWC model, the authors derived two equations, one for the total number of binding sites occupied by a single type of ligand relative to all possible conformations, the 'saturation-function' $\mathbf{Y}$ :

$\boldsymbol{Y}=\frac{L^{\mathrm{MWC}} \cdot c^{\mathrm{MWC}} \cdot \alpha \cdot\left(1+c^{\mathrm{MWC}} \cdot \alpha\right)^{n-1}+\alpha \cdot(1+\alpha)^{n-1}}{L^{\mathrm{MWC}} \cdot\left(1+c^{\mathrm{MWC}} \cdot \alpha\right)^{n}+(1+\alpha)^{n}}$,

and one for the total number of active sites or high affinity conformations in the $\mathrm{R}$ state, including the unliganded $\mathrm{R}_{0}$ state, relative to all the possible receptor conformations, the 'function of state' $\mathbf{R}$ (R-function) when a single type of ligand is present:

$$
\boldsymbol{R}=\frac{(1+\alpha)^{n}}{L^{\mathrm{MWC}} \cdot\left(1+c^{M W C} \cdot \alpha\right)^{n}+(1+\alpha)^{n}}
$$

(Monod et al. 1965). $L^{\mathrm{MWC}}$ is the MWC allosteric constant equal to the ratio between non-ligated tense (T) forms, $T_{0}$, and non-ligated relaxed $(R)$ forms, $R_{0}$, i.e., $L^{\mathrm{MWC}}=\mathrm{T}_{0} / \mathrm{R}_{0}$ (see footnote 3 in Chapter 5 ). For large values of $L^{\mathrm{MWC}}, \mathrm{T}$ conformations are the dominating form. $K_{\mathrm{R}}$ and $K_{\mathrm{T}}$ are 'microscopic' dissociation constants for ligand binding to states $\mathrm{R}$ and $\mathrm{T}$, and parameter $c^{\mathrm{MWC}}$ is the ratio between the two microscopic dissociation constants, $K_{\mathrm{R}} / K_{\mathrm{T}}$. The term 'microscopic' indicates that a parameter cannot be separately determined in experiments. The symbol $\alpha$ just stands for the normalized agonist concentration, $=[\mathrm{S}] / K_{\mathrm{R}}$, and finally parameter $n$ is the number of binding sites in a single receptive entity (Fig. 15.3).

I have omitted a derivation of these two equations (Eqs. 15.1 and 15.2). The equations can be found quoted and derived in many places (e.g., Eigen 1968, Rubinow 1975, pp. 46-103; Segel 1975, Chapter 7; Cantor \& Schimmel 1980, Chapter 15; Kurganov 1982, Chapter 3; Wyman \& Gill 1990; Cornish-Bowden 1995; Ben-Naim 2001; Berg et al. 2006, Chapter 7), and of course can be found in the original paper (Monod et al. 1965).

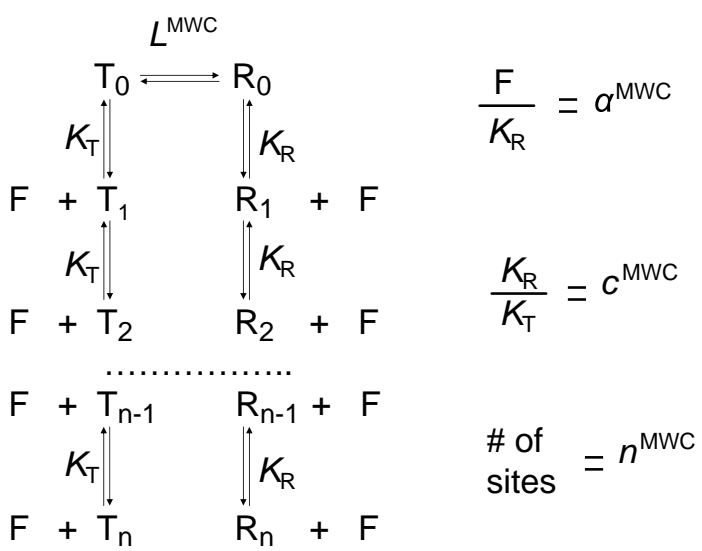

Figure 15.3. The MWC model. A receptive unit, an enzyme, exists in two conformations, a $\mathrm{T}$ (tense) and an $\mathrm{R}$ (relaxed) state. Only the unbound forms of the enzyme, $T_{0}$ and $R_{0}$, can make the switch to the other conformation. The ratio of $T_{0} / R_{0}$ is given by the allosteric constant $L^{\mathrm{MWC}}$. ' $\mathrm{F}$ ' is the substrate or its concentration $K_{\mathrm{R}}$ the dissociation constant for $\mathrm{F}$ binding to the $R$ states and $K_{\mathrm{T}}$ the dissociation constant for $\mathrm{F}$ binding to $\mathrm{T}$ states. Constant $n^{\mathrm{MWC}}$, or just $n$, indicates the number of subunits (protomers) equal to the total number of binding sites in the enzyme complex. Drawn according to Monod et al. (1965).

\subsubsection{Homotropic Binding Studies}

The saturation-function $\mathbf{Y}$ (Eq. 15.1) covers equilibrium binding experiments towards complete saturation as the agonist concentration increases. ${ }^{3}$ Examples of behavior of this function are depicted in Fig. 15.4. It is valid for receptors, enzymes, transporters, and carriers such as hemoglobin, when only one type of ligand is present, and very importantly, all the sites in a given state have the same dissociation constant for ligand binding, i.e., either $K_{\mathrm{T}}$ or $K_{\mathrm{R}}$.

The Y-function is generously quoted as a tool for analysis of binding, although with justified opposition at the perimeter (Koshland et al. 1966; Eisenstein et al. 1995). The opposition is due to strangulating assumptions about constants $K_{\mathrm{R}} \mathrm{s}$ and $K_{\mathrm{T}} \mathrm{s}$ for consecutive binding processes, namely that the constants are invariant from binding of the first until the last bound ligand.

\footnotetext{
${ }^{3}$ In a modern biochemistry textbook the Y-function for homotropic synagics is described as 'a function for fraction of active sites bound to substrate (fractional saturation, $\mathbf{Y}_{\mathrm{S}}$ )' (Berg et al. 2002, p. 268). Regrettably, in this brilliant textbook, the above statement is incorrect. The Y-function, equal to fractional saturation, is for the fraction of all bound effectors some of which are not active (viz. all the liganded $\mathrm{T}$ forms). A better function covering the fraction of active sites bound to ligand is actually the cotr-enz function in Eq. 15.4. Except for this conceptual slip there is also an unfortunate printing error. The Y-function in Berg et al. (2002) appears erroneously with a minus sign, although correctly quoted in earlier editions (Stryer 1995, p. 167) and a later edition (Berg et al. 2006, pp. 200-201).
} 

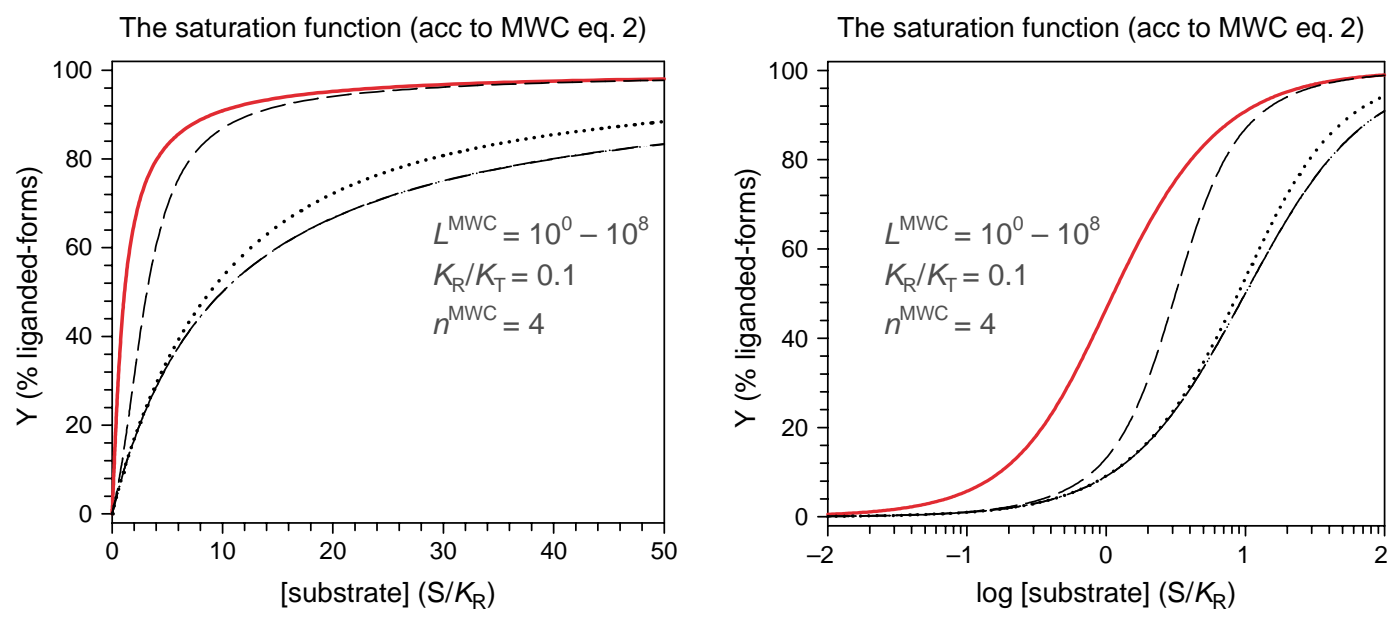

The saturation function (acc to MWC eq. 2)

The saturation function (acc to MWC eq. 2)
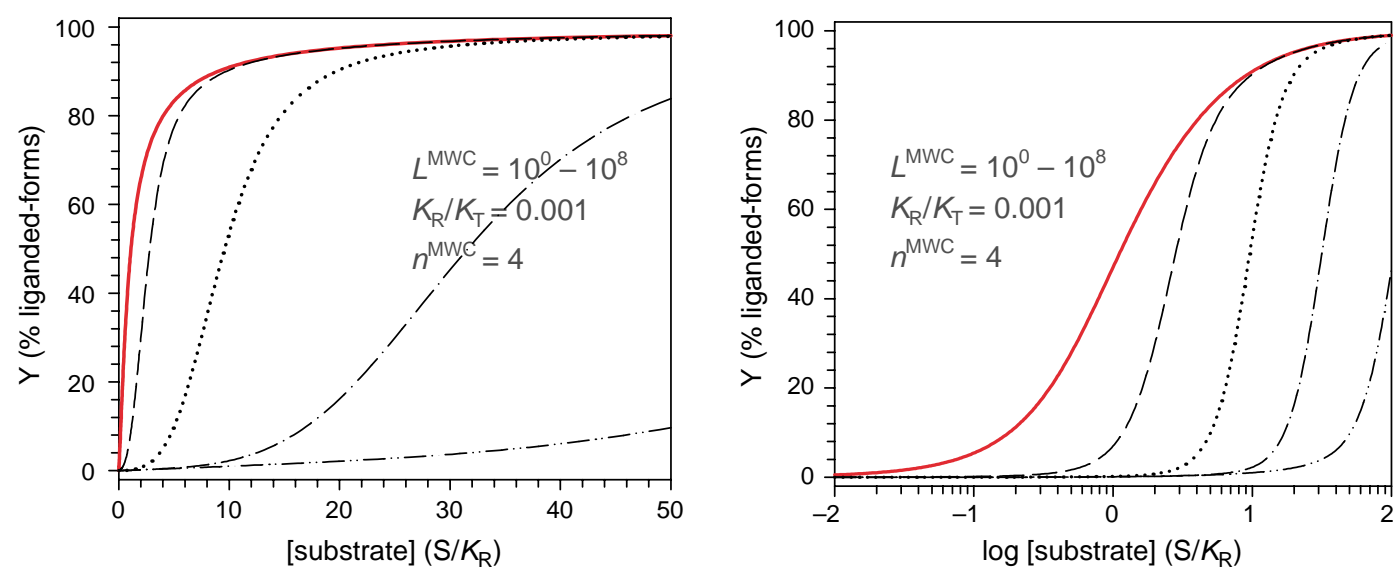

The saturation function (acc to MWC eq. 2)

The saturation function (acc to MWC eq. 2)
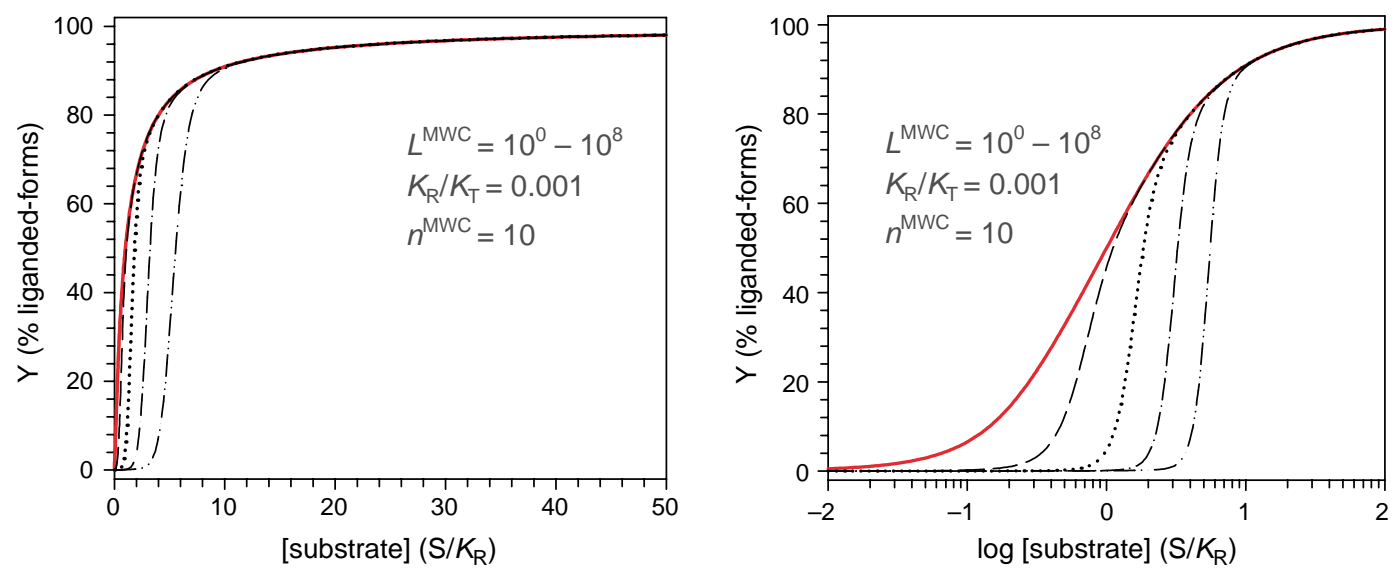

Figure 15.4. Examples of dose-response curves for the 'saturation function' $\mathrm{Y}$. Values for the three parameters $L^{\mathrm{MWC}}, K_{\mathrm{R}} / K_{\mathrm{T}}=$ $c^{M W C}$, and $n$ are indicated in each panel and with the normalized substrate concentration $\mathrm{S} / K_{\mathrm{R}}=\alpha$ as the independent variable. Parameter $L^{\text {MWC }}$ varies in five steps between $10^{\circ}(-)$ to $10^{8}(-\cdot \cdot-)$ by a factor $10^{2}$. Left panels show the concentration axis with a linear scale, while the right panels have logarithmically scaled concentration axes. 


\subsubsection{Validity of the Homotropic $\boldsymbol{Y}$-model}

Even for $n>2$, pronounced sigmoidality for binding in the MWC model, i.e., using the Y-function equal to Eq. 15.1, can only be obtained by assuming fairly skewed values for parameters $L^{\mathrm{MWC}}$ and $c^{\mathrm{MWC}}$. For instance, observe the graph in Fig. 15.4 where plots are for four binding sites, $n=4$. Here, parameter $c^{\mathrm{MWC}}$ has to be $<1 / 100$ and $L^{\mathrm{MWC}}>100$ in order for there to be recognizable sigmoidality, in spite all liganded receptor molecules are included in the Y-formulation. The question is whether these parameter values are reasonable for natural systems. The answer, thus far, is blowing in the wind.

\subsubsection{Functional Studies Including Some Heterotropy}

We shall now discuss some confounding concepts developed in MWC'65 for the modeling and formulation of effector activity. Since the presentation of the MWC model, there has been a silent acceptance of the approach chosen by the authors in 1965 for their model of activity in enzymes. To clarify, on the functional aspect of the MWC model there has been and still is a repetitive tendency to use a constrained saturation function for activity instead of a straightforward formulated activity.

For enzymes and hemoglobin there is a problem with the 'function of state', R, used for activity. The MWC'65 'function of state' equal to $\mathbf{R}$ displays spontaneous activity (Fig. 15.5) (also see Rubin \& Changeux 1966). However, in many studies, enzymes and carriers do not overtly exhibit spontaneous activity. This is the dilemma.

Following in the footsteps of MWC'65 in order to solve this problem, the spontaneous activity appearing in Fig. 15.5 and inherent in Eq. 15.2 may vanish by operating with (1) very large values for the MWC allosteric constant, $L^{\mathrm{MWC}}$, and/or (2) a very low affinity for ligands at the non-active sites, T sites. Since $c^{\mathrm{MWC}}$ is equal to the ligand dissociation constant for active sites over the dissociation constant for non-active sites, $=K_{\mathrm{R}} / K_{\mathrm{T}}$, small values of $c^{\mathrm{MWC}}$ mean low affinity for $\mathrm{T}$ states. Therefore, in analysis, working with large values for $L^{\mathrm{MWC}}$ and small values for $c^{\mathrm{MWC}}$, you can suppress basal activity, which is convenient in classic biochemistry.

Assuming that $c^{\text {MWC }}$ is nearly equal to zero, the Yfunction reduces to the following expression, which was quoted in a footnote of the MWC paper:

$$
\boldsymbol{Y}_{\boldsymbol{F}}=\frac{\alpha \cdot(1+\alpha)^{n-1}}{L^{\mathrm{MWC}}+(1+\alpha)^{n}} .
$$

As stated in the MWC paper, this formulation may be used for homotropic (co-operative) effects in functional studies. Examples of the behavior for this formulation of homotropic activity are shown in Fig. 15.6.
For heterotropic activity, again assuming that $c^{\mathrm{MWC}}$ is negligible, the saturation function $\mathbf{Y}$ (see Eq. 15.1), can be simplified to a new saturation function, $\mathbf{Y}_{\mathrm{S}}$ :

$$
\boldsymbol{Y}_{S}=\frac{\alpha \cdot(1+\alpha)^{n-1}}{L^{\prime \mathrm{MWC}}+(1+\alpha)^{n}},
$$

where $L^{\mathrm{MWC}}$ is an apparent allosteric constant (Monod et al. 1965, their Eq. 3). This apparent allosteric constant $L^{\prime \text { MWC }}$ is suggested to be equal to the allosteric $L^{\mathrm{MWC}}$ constant multiplied by a factor $(1+\beta)^{n} /(1+\gamma)^{n}$, in which $\beta$ is equal to $\mathrm{I} / K_{\mathrm{I}}$ and $\gamma$ is equal to $\mathrm{A} / K_{\mathrm{A}}$. Here, ' $\mathrm{I}$ ' is an inhibitor and 'A' an activator. $K_{\mathrm{I}}$ and $K_{\mathrm{A}}$ are the respective dissociation constants. By inserting the apparent allosteric constant $L^{\mathrm{MWC}}$ as expressed by $L^{\mathrm{MWC}}$, we and MWC obtain:

$$
\boldsymbol{Y}_{\boldsymbol{S}}=\frac{\alpha \cdot(1+\alpha)^{n-1}}{L^{\mathrm{MWC}} \cdot \frac{(1+\beta)^{n}}{(1+\gamma)^{n}}+(1+\alpha)^{n}} .
$$

Eq. $15.1 c$ is identical to the MWC Eq. 4. Now this equation is used by MWC to model heterotropic modulation of enzyme functionality (activity), see MWC'65's experimental Figs. 4a,b compared with their theoretical Figs. 4c,d, as well as MWC'65's experimental Fig. $7 \mathrm{~b}$ compared with their theoretical Fig. 7a.

By choosing $L^{\mathrm{MWC}} \gg 1$ and $c^{\mathrm{MWC}} \ll 1$, all non-liganded and non-active conformations disappear from the numerator in Eq. 15.1. This leaves exclusively the active sites of interest in the numerator of Eqs. 15.1 $a-c$, that is, unliganded active conformations disappear and with them spontaneous activity.

The behavior of the model in Eq. $15.1 c$ is given by examples in Fig. 15.7.

The general acceptance or negligence of the MWC approach described in Eqs. 15.1 $a-c$ for both homotropic and heterotropic activity (Mahler \& Cordes 1966, Chapter 6; Rubin \& Changeux 1966; Eigen 1967; Karlin 1967; Colquhoun 1973; Rubinow 1975; Segel 1975, 1993; Fromm 1975; Wong 1975; Cantor \& Schimmel 1980; Kurganov 1982; Hill 1985; Cornish-Bowden 1995, 2004; Neet 1995; Ben-Naim 2001) is extremely unfortunate as it is very confounding in relation to the use of the original MWC model for functionality or effector ${ }^{4}$ activity.

A more versatile expression for functionality is derived in Section 15.3.6 for enzymes and non-ligand-gated porters that allow $L^{\mathrm{MWC}}$ and $c^{\mathrm{MWC}}$ to vary freely, while spontaneous activity is excluded. However, first we take a look at the MWC 'function of state' $\mathbf{R}$ with its restrictions (Eq. 15.2), which is actually relevant for receptive units such as G protein-coupled receptors and ligand-gated porters displaying spontaneous activity.

\footnotetext{
${ }^{4}$ Recap that 'effector' does not mean modulator in my text.
} 

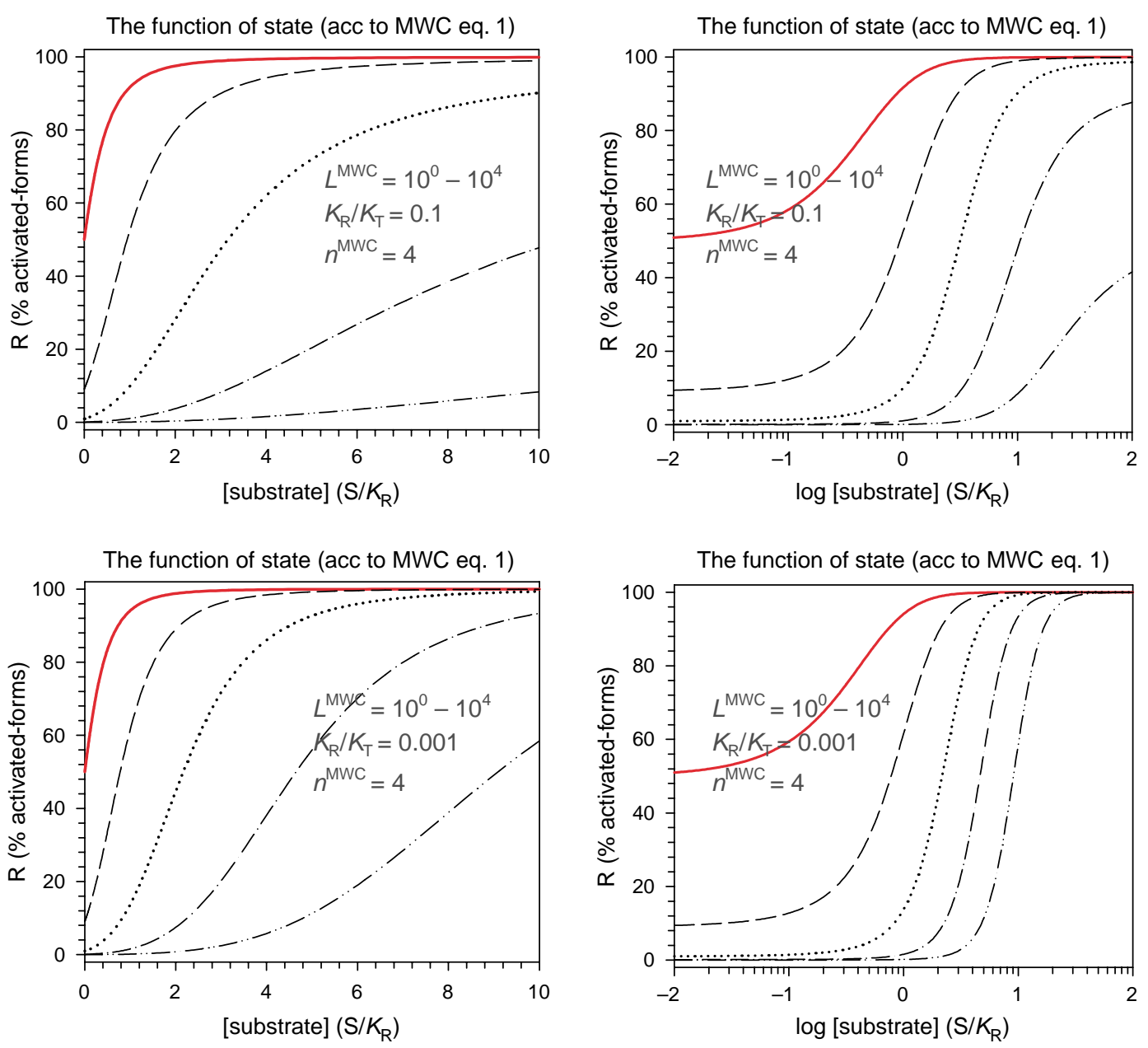

The function of state (acc to MWC eq. 1)

The function of state (acc to MWC eq. 1)
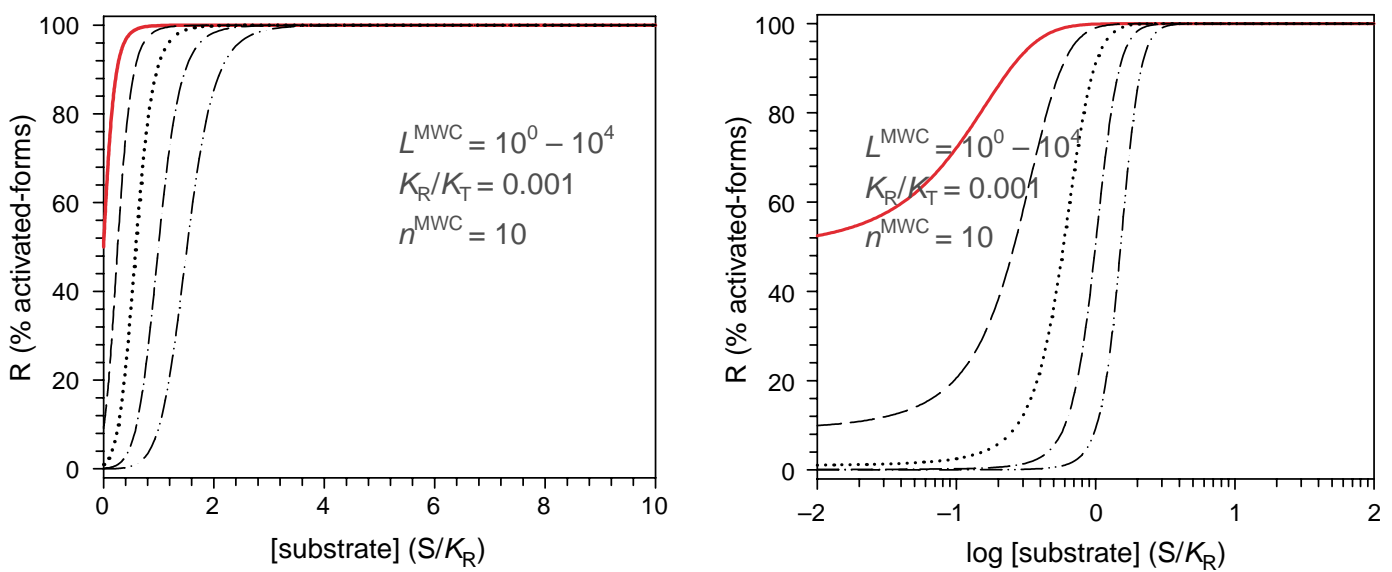

Figure 15.5. Examples of dose-response curves for the 'state of function' $\mathbf{R}$. Values for the three parameters $L^{\mathrm{MWc}}, K_{\mathrm{R}} / K_{\mathrm{T}}=$ $c^{\mathrm{MWC}}$, and $n$ are indicated in each panel and with the normalized substrate concentration $\mathrm{S} / K_{\mathrm{R}}=\alpha$ as the independent variable. Parameter $L^{\mathrm{MWC}}$ varies in five steps between $10^{\circ}(-)$ to $10^{4}(-\cdot \cdot)$ by a factor 10 . Left panels show the concentration axis with a linear scale, while the right panels have logarithmically scaled concentration axes. At low values of $L^{\mathrm{MWC}}$ there is spontaneous activity. 


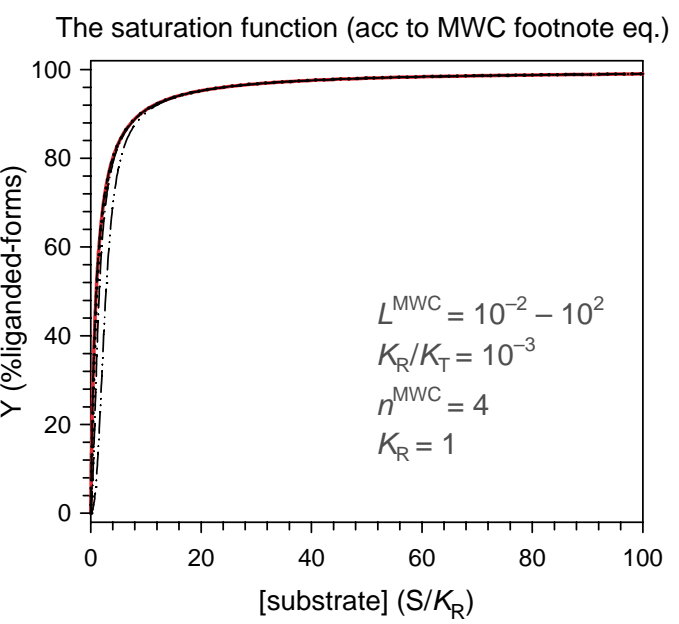

The saturation function (acc to MWC footnote eq.)

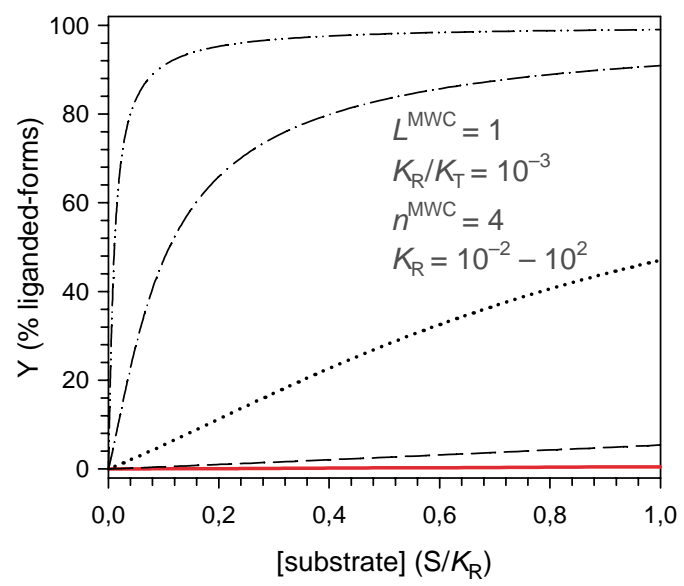

The saturation function (acc to MWC footnote eq.)

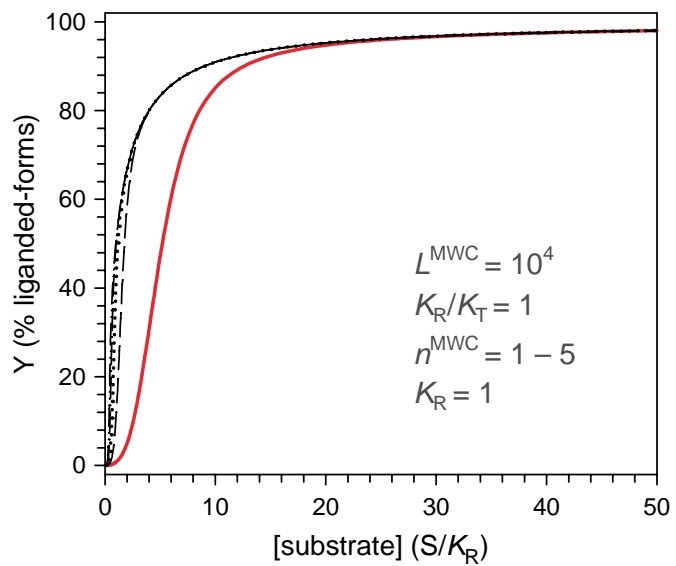

The saturation function (acc to MWC footnote eq.)

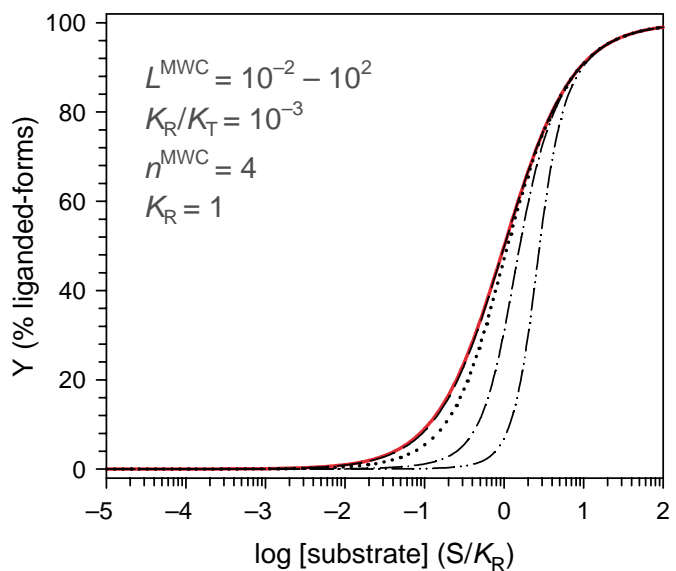

The saturation function (acc to MWC footnote eq.)

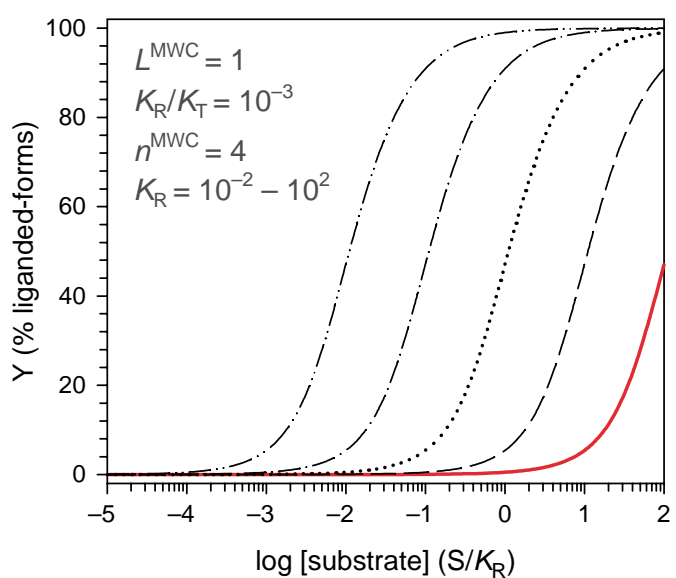

The saturation function (acc to MWC footnote eq.)

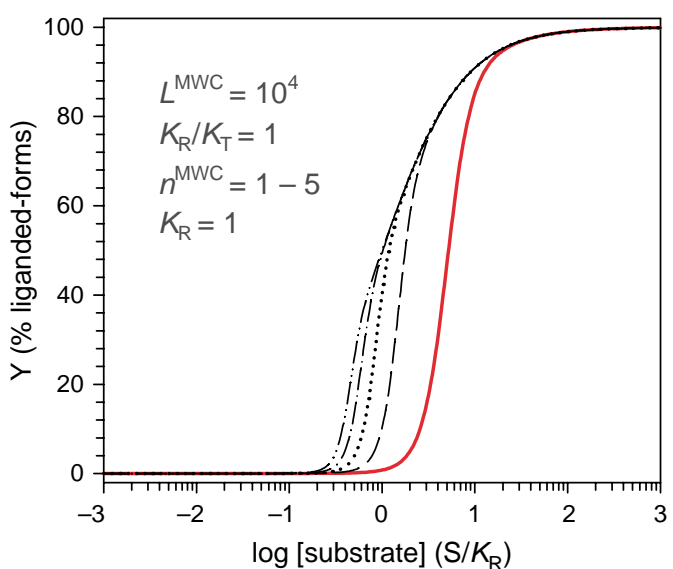

Figure 15.6. Examples of dose-response curves for the saturation function $\mathbf{Y}_{\mathbf{F}}$ (Footnote Eq. 15.1a). Values for the three parameters $L^{\mathrm{MWC}}, K_{\mathrm{R}} / K_{\mathrm{T}}=c^{\mathrm{MWC}}$, and $n$ are indicated in each panel and with the normalized substrate concentration $\mathrm{S} / K_{\mathrm{R}}=\alpha$ as the independent variable. In the two upper panels, parameter $L^{\mathrm{MWC}}$ varies in five steps from $10^{-2}(-)$ to $10^{2}(-\cdot \cdot-)$ by a factor 10. In the two middle panels $K_{\mathrm{R}}$ varies in five steps from $10^{-2}(-)$ to $10^{2}(-\cdot-)$ by a factor 10 . In the two lower panels, $n$ varies in five steps from $1(-)$ to $5(-\cdot-)$ increasing by 1 between steps. Left panels show the concentration axis with a linear scale, while the right panels have logarithmically scaled concentration axes. 

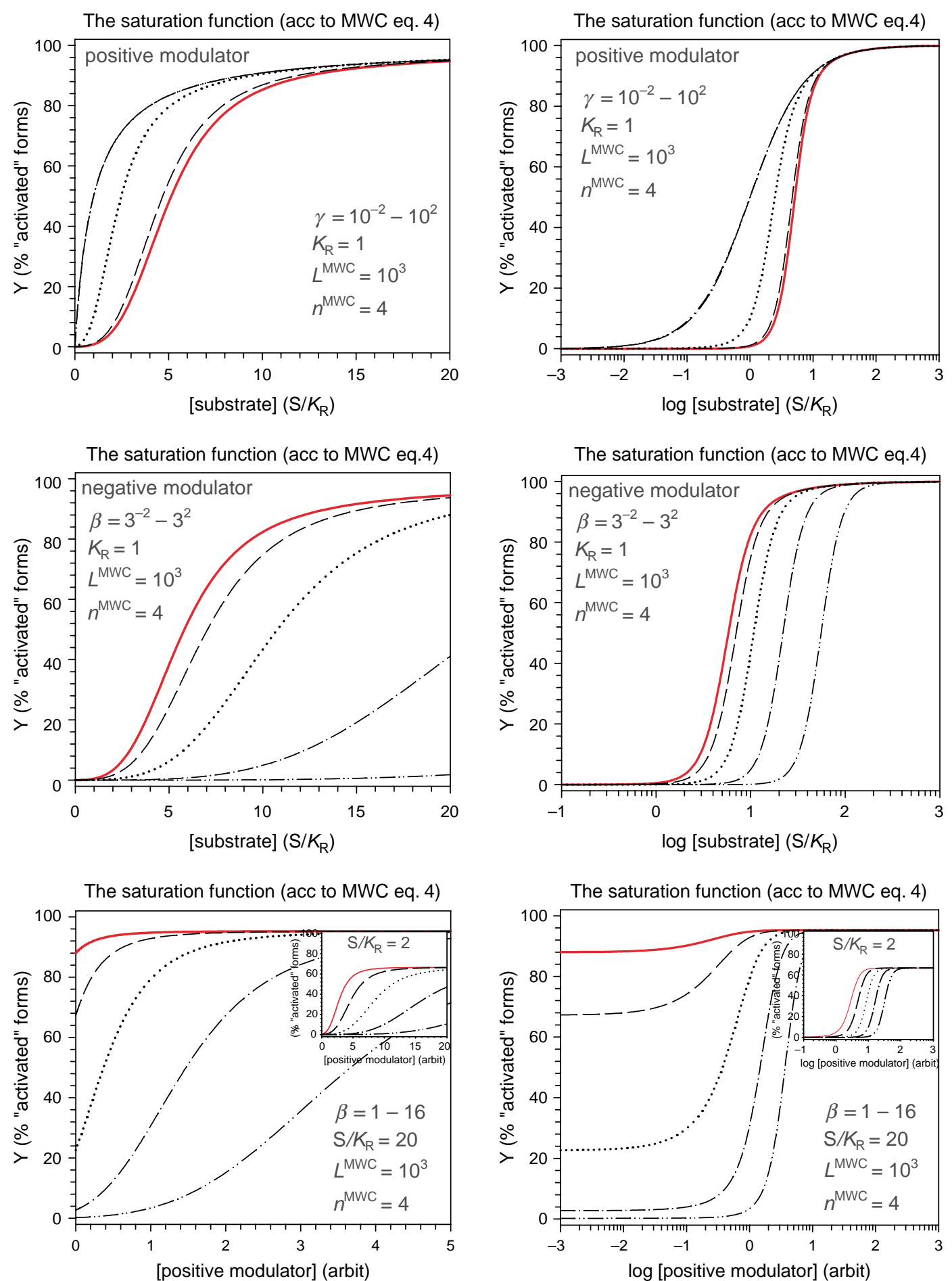

Figure 15.7. Examples of dose-response curves for the saturation function $\mathbf{Y}_{\mathbf{S}}$ (MWC-footnote Eq. 15.1c). Values for the five parameters $L^{\mathrm{MWC}}, K_{\mathrm{R}} / K_{\mathrm{T}}=c^{\mathrm{MWC}}, n, \gamma$, and $\beta$ are indicated in each panel. In the two upper panels parameter $\gamma$ for positive modulators varies in five steps from $10^{-2}(-)$ to $10^{2}(-\cdot \cdot)$ by a factor 10 . In the two middle panels, parameter $\beta$ for negative modulators varies in five steps from $1 / 9(-)$ to $9(-\cdot-)$ by a factor 3 . In these four panels the normalized substrate concentration $\mathrm{S} / K_{\mathrm{R}}=\alpha$ is the independent variable. In the two lower panels, the positive modulator concentration is the independent variable, while the negative modulator parameter $\beta$ varies in five steps from $1(-)$ to $16(-\cdot \cdot)$ increasing by a factor 2 between steps. For a normalized concentration of substrate fixed at 20, there is an increase in already 'active' forms at the low concentration of positive modulator towards higher concentrations. 'Active' forms at the low concentration of a positive modulator may be suppressed close to zero for a concentration of the normalized substrate 10 fold lower, $S / K_{R}=\alpha=2$, see inserts. Left panels show the concentration axis with a linear scale, while the right panels have logarithmically scaled concentration axes. 


\subsubsection{Homotropic Functional Studies. A. Receptor Studies}

For functional studies we need equations to describe the situation other than those derived for binding. In receptor studies on function, the total number of active receptors is given by the 'function of state $\mathbf{R}$ ' (Rfunction) in the MWC paper (MWC Eq. 1), a function that includes non-ligated spontaneously active receptors, $\mathrm{R}_{0}$ (Monod et al. 1965). The R-function (Eq. 15.2) describes the activity level as the concentration rises (Fig. 15.5). Thrillingly, the MWC R-function for functional activity is acceptable for receptor synagics including LGC- and GPCR systems, ${ }^{5}$ as it covers all active receptor conformations, bound $R_{x} s$ plus unbound $R_{0} s$. Thus, $R_{0}$ is actual for receptor and ligand-gated transporter studies, as $R_{0}$ is the unbound and active form of the system yielding spontaneous activity that modulator ligands can play on, even in the absence of primary ligands. Examples of the behavior of MWC-Eq. 1 (Eq. 15.2), with spontaneous activity are shown in Fig. 15.8. See also Changeux and Edelstein (2005, Appendix Figs. A3 and A4) where $L^{\mathrm{MWC}}$ is allowed to decrease from 800,000 to 20 , which equals an increase of $L$ from 1.25 . $10^{-6}$ to $5 \cdot 10^{-2}$, but not further, thereby missing marked spontaneous activity.

When values of $L^{\text {MWC }}$ decrease below 100, spontaneous activity starts to appear for the function-of-state $\mathbf{R}$, and for values of $c^{\mathrm{MWC}}=K_{\mathrm{R}} / K_{\mathrm{T}}$ increasing above unity, the dose-response curve for the MWC R-function will show inverse agonism as the ligand concentration increases. Spontaneous activity and inverse agonism should be expected (Fig. 15.8), since both phenomena are integrated in two-state synagic schemes as in the cTSM (Chapter 5, K\&T model 5), of which the MWC model is a subtype, viz. for $n=1$.

As depicted in Fig. 15.8, the R-function allows for simulation and analysis of inverse agonism. In particular, I found no examples of such an analysis in the literature. Surprisingly, even Changeux and co-workers do not analyze this possibility. Henceforth, I will keep looking.

In the meantime, GABA-A channels as well as GABA-B receptors, mGlu1R, $\mathrm{mAChR}$ and many other receptors present spontaneous activity (Pin et al. 2004; Hlavackova et al. 2005; Sergeeva et al. 2005; Costa \& Cotecchia 2005; Holst et al. 2006; Bond \& Ijzerman 2006; Cotecchia 2007; Smit et al. 2007), and their functional synagics may be analyzed by the 1965-R-function.

In the literature, for obvious reasons mentioned above, there are only a few examples of the use of MWC's R-function for functionality, i.e., enzymatic

\footnotetext{
${ }^{5}$ LGC $=$ ligand-gated channel and GPCR $=\mathrm{G}$ protein-coupled receptor.
}

catalytic activity. In spite of this, Kurganov (1982) can write 'The model of Monod et al. occupies a special position amongst models of allosteric enzymes. It possesses a clear physical significance, is relatively simple, and enables predictions to be made concerning the nature of both homotropic and heterotropic interactions between the ligand-binding sites in the enzyme molecule. For these reasons, the model of Monod et al. is widely used for quantitative description of the kinetic behavior of allosteric enzymes'. The last sentence should be perceived with caution, insight, and reservation. The MWC Y-function has been employed repeatedly for binding synagics, but its derived function-of-state $\mathbf{R}$ for 'kinetics' is nearly useless for systems without spontaneous activity, and therefore rarely employed in enzymology. In fact, maybe the MWC model is too simple or too advanced when it comes to functional activity.

Uncertainty about the R-function in enzymology did not prevent Changeux and Rubin (1968) from using the spontaneous activity aspect of the R-function as an argument against sequential models developed by Koshland et al. (1966) (see also Changeux \& Edelstein 2005). This point is further discussed in Section 15.4.3.

\subsubsection{Homotropic Functional Studies. B. Enzyme and Non-gated Porter Studies}

Functional studies for enzymes and non-ligand-gated transporters normally require the presence of a substrate or a transportee to assess the catalytic activity in enzymes or the transfer of molecules by transporters like pumps, cotransporters, uniporters, and membranebound artificial carriers. In the absence of primary ligands, neither of these effectors is spontaneously active in a manner that such activity can be measured directly. Indeed, for enzymes the $R_{0}$ is active but it does not necessarily count as functional! From an experimental dose-response and non-structural point of view, $\mathrm{R}_{0}$ conformations are not functional in the sense of the word 'active' for catalysis or transport.

Therefore, for these effectors we need a model and an equation that contains only bound forms of the active $\mathrm{R}$ conformation in the numerator, excluding non-ligated forms even though these conformations exist and are in an active state, $\mathrm{R}_{0}$, based on structural analyses (see Section 15.4.4).

Expressions that satisfy these requirements may be obtained by a simple modification of the MWC Yfunction. This modification, which follows, is different from the MWC approach in Section 15.3.4 where parameter values were chosen to avoid 'the problem' of constitutive activity.

The Y-function covers all bound conformations, both non-active and active, and explicitly excludes un-liganded $\mathrm{T}_{0}$ and $\mathrm{R}_{0}$ forms in the numerator. Thus, to satisfy a 

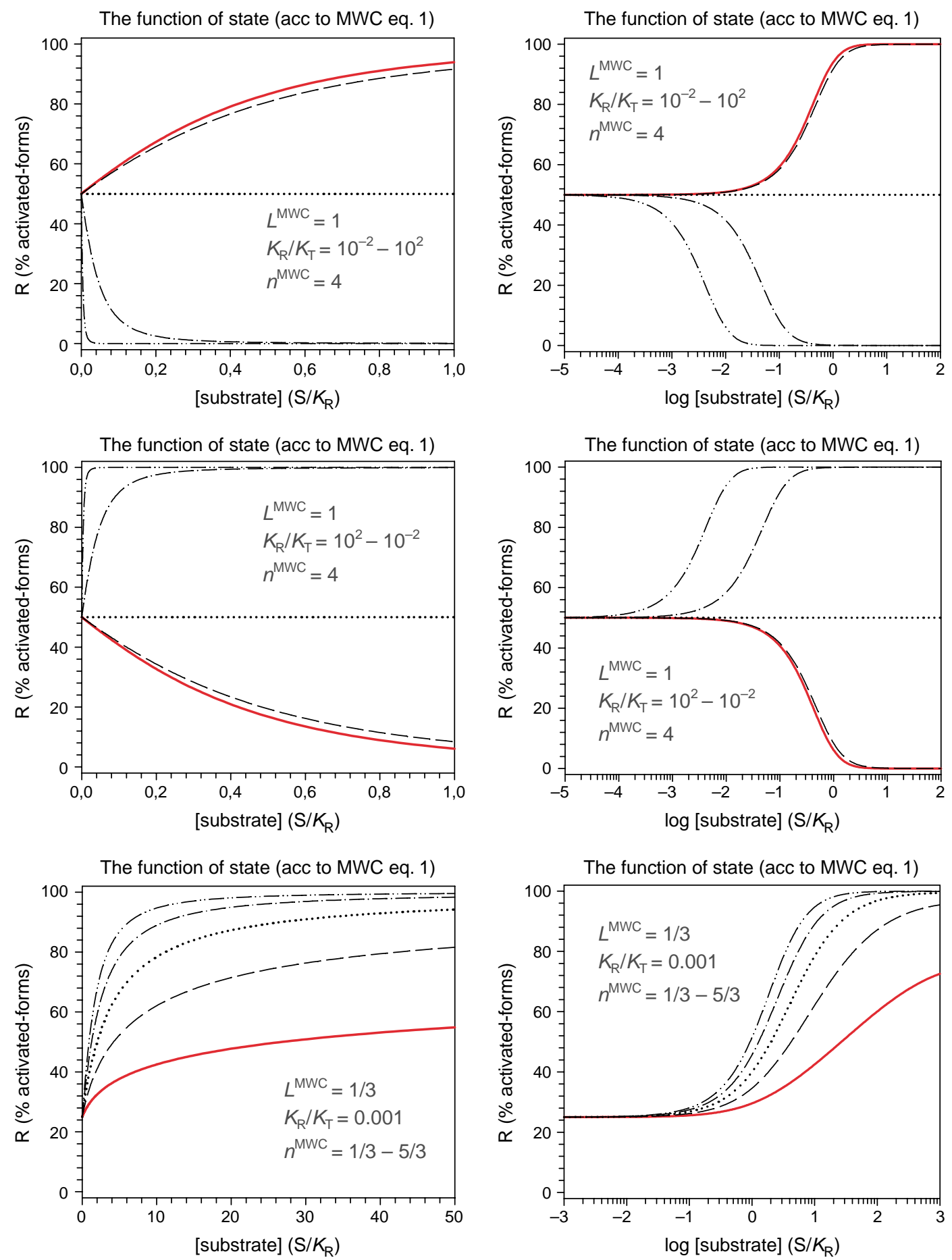

Figure 15.8. Examples of dose-response curves for the 'state of function' $\mathbf{R}$ demonstrating inverse agonism. Values for the three parameters $L^{\mathrm{MWC}}, K_{\mathrm{R}} / K_{\mathrm{T}}=c^{\mathrm{MWC}}$, and $n$ are indicated in each panel and with the normalized substrate concentration $\mathrm{S} /$ $K_{\mathrm{R}}=\alpha$ as the independent variable. In the two upper panels, parameter $K_{\mathrm{R}} / K_{\mathrm{T}}$ varies in five steps from $10^{-2}(-)$ to $10^{2}(-. \cdot-)$ by a factor 10 . In the two middle panels $K_{\mathrm{R}} / K_{\mathrm{T}}$ varies in five steps from $10^{2}(-)$ to $10^{-2}(-\cdot-)$ by a factor $10^{-1}$. In the two lower panels, $n$ is varied in five steps from $1 / 3(-)$ to $5 / 3(-. \cdot)$ increasing by $1 / 3$ between steps. Left panels show the concentration axis with a linear scale, while the right panels have logarithmically scaled concentration axes. Note that for values of $L$ close to or equal unity in all the panels, spontaneous activity is hard to conceal at low values of substrate concentration. Compare with Fig. 15.5. 
formulation for enzyme and certain transport activities, we just have to eliminate all liganded non-active $\mathrm{T}$ forms in the numerator of the Y-function.

All the bound T-forms are equal to a sum of Ts:

$$
\sum_{1}^{n} \mathrm{~T}=\mathrm{T} \cdot \frac{\mathrm{S}}{K_{\mathrm{T}}} \cdot\left(1+\frac{\mathrm{S}}{K_{\mathrm{T}}}\right)^{n-1}
$$

Here, $\mathrm{S} / K_{\mathrm{T}}$ is the normalized ligand concentration in relation to T forms. In case we replace $L^{\mathrm{MWC}}$ and $c^{\mathrm{MWC}}$ with $\mathrm{T} / \mathrm{R}$ and $K_{\mathrm{R}} / K_{\mathrm{T}}$ in the Y-formulation (Eq. 15.1), then the first product term in the numerator of the Y-function is equal to Eq. 15.3. Therefore, eliminating the term shown in Eq. 15.3 from the numerator of Eq. 15.1, we obtain the actual and fractional catalytic capacity in enzymes, c-capacity, or the actual and fractional transfer capacity in certain transporters, $t-$ capacity, but without spontaneous activity. For homotropy, we can formulate these capacities as:

$$
\begin{aligned}
\text { c-capacity } & \vee t \text {-capacity } \\
& =\frac{\alpha \cdot(1+\alpha)^{n-1}}{L^{\mathrm{MWC}} \cdot\left(1+c^{\mathrm{MWC}} \cdot \alpha\right)^{n}+(1+\alpha)^{n}} .
\end{aligned}
$$

Compare this functional expression, Eq. 15.4, with Eqs. 15.1 and 15.1 $a$.

Eq. 15.4, which is drawn as curves in Fig. 15.9, we may call the 'cotr-enz' MWC relation for fractional transport capacity in pumps, cotransporters, uniporters, and artificial membrane-carriers or for the normalized fractional catalytic capacity in enzymes.

Eq. 15.4 is for liganded activity. Un-liganded activity can be studied with specialized experimentation for enzymes (Jager et al. 2006; Fetler et al. 2007) and for non-ligand-gated transporters (e.g., Wright et al. 1994; Larrajoz et al. 2006). As indicated, there is more in Section 15.4.3 about spontaneous activity in such effectors.

Based on the sum of two ordinary Michaelis-Menten kinetics combined with a generalized MWC, Kurganov (1982) has developed a whole set of possible equations for functional co-operativity for enzymes. His generalized MWC models for function are simply a multiplication of rate constants, $k_{\mathrm{T}}$ and $k_{\mathrm{R}}$, onto expressions for the $\mathrm{T}$ and $\mathrm{R}$ forms of the enzyme. Meanwhile, Kurganov's functional models are analyzed with rather high values of $L^{\mathrm{MWC}}$ (Kurganov 1982, his Figs. 3.4b, 3.7b and $3.8 \mathrm{~b}$ ), thus suppressing the inherent spontaneous activity not ordinarily observed for enzyme activity. Obviously, with this kind of approach we are back to square one.

\subsubsection{Functional Bell-shaped Synagics of the Cotr-enz Formulation}

Fig. 15.10 depicts other examples of the cotr-enz relation for homotropic dose-responses. It can be seen that the cotr-enz function has a biphasic behavior in a narrow window of parameter values - it can display upright bell-shaped synagics (Fig. $15.10 \mathrm{~B}+\mathrm{C}$ ) ${ }^{6}{ }^{6}$ For this to occur, one requirement is that the $c^{\mathrm{MWC}}=K_{\mathrm{R}} / K_{\mathrm{T}}>1$ (see Fig. 15.8), as for the inverse agonism with the MWC model for function mentioned above. When lacking or not measuring spontaneous activity, Eq. 15.4 is a correct functional MWC-equation for both enzymes and for nonligand-gated transporters, but has, to my knowledge, never been invoked on functional data for enzymes or transporters. Also of note is that the constrained form of the MWC saturation-model (Eq. 15.1a) for homotropic activity, avoiding spontaneous activity by parameter manipulations, was not and has not been employed in analyses of homotropic enzyme regimes starting from its inception in the mid-1960s. The lack of use of MWC 'footnote-equation' $15.1 \mathrm{a}$ is already in Monod et al. (1965) and Rubin and Changeux (1966).

\subsubsection{Other Methods of Suppressing Spontaneous Activity in the R-function}

Soon after the heterotropic model for function had been stated (Eq. 15.1c) (Monod et al. 1965), Rubin and Changeux (1966) expanded it with inclusive binding of different ligands. Inspired by the Rubin-Changeux reformulation for an activity-model (Rubin \& Changeux 1966; Changeux \& Rubin 1968), as an example, Eisenstein et al. (1995) derived a new functional form for the R-function for homotropic co-operativity in a different attempt to 'correct' for spontaneous activity in the original R-function. Moreover, in the Eisenstein et al. equation, the regulator sites in an enzyme are equipped with new dissociation constants for binding homotropic ligands to modulator sites both for the $\mathrm{R}$ and T states; in their terminology $-K_{\mathrm{Ract}, \mathrm{X}}$ and $K_{\mathrm{tact}, \mathrm{X}}$. These two dissociation constants were assumed invariant, i.e., independent of liganded or non-liganded forms of $\mathrm{R}$ and $\mathrm{T}$ states, similar to $K_{\mathrm{R}}$ and $K_{\mathrm{T}}$.

In the terminology of this book, the Eisenstein et al. equation for non-exclusive function with new dissociation constants for a homotropic ligand at a modulator site $\mathrm{M}$ may be written as:

\footnotetext{
${ }^{6}$ Eigen has suggested a bell-shaped figure for auto-inhibitory doseresponses of enzyme synagics with an outline of an elephant (Eigen 1968). This form, Eigen claims, will be possible with four parameters, and I guess a MWC paradigm. However, he did not specify what he meant. Meanwhile, I show that bell-shaped synagic relationships, even with broad plateaus, are possible with only three parameters (see the intervention reaction scheme, sub-chapter 2.5 ).
} 

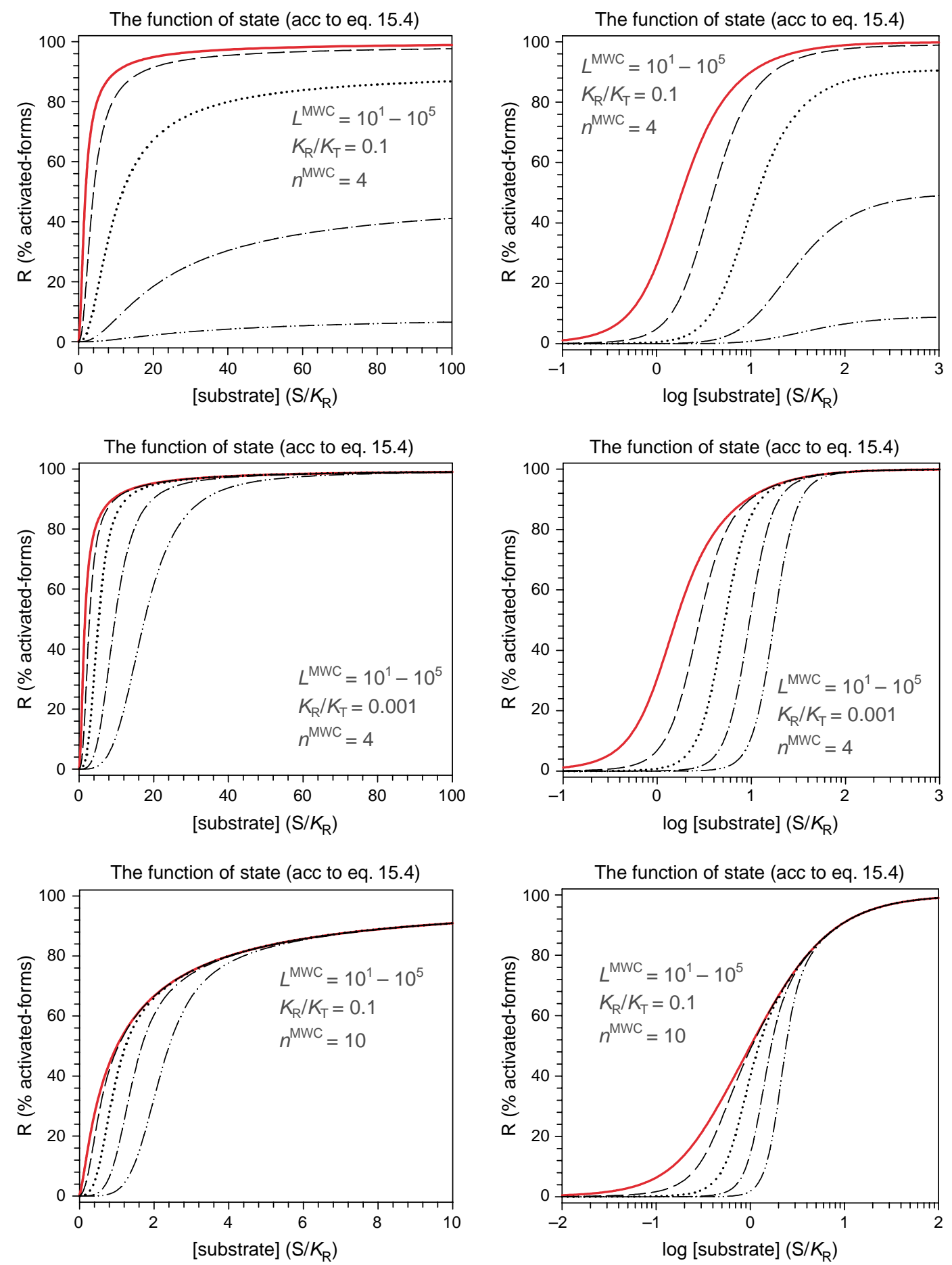

Figure 15.9. Examples of dose-response curves for the 'cotr-enz' function. Values for the three parameter $L^{\mathrm{MWC}}, K_{\mathrm{R}} / K_{\mathrm{T}}=c^{\mathrm{MWC}}$ and $n$ are indicated in each panel and with the normalized substrate concentration $\mathrm{S} / K_{\mathrm{R}}=\alpha$ as the independent variable. Parameters $L^{\mathrm{MWC}}$ varies in five steps between $10^{1}(-)$ to $10^{5}(-\cdot-)$ by a factor 10 . Left panels show the concentration axis with a linear scale, while the right panels have logarithmically scaled concentration axes. 

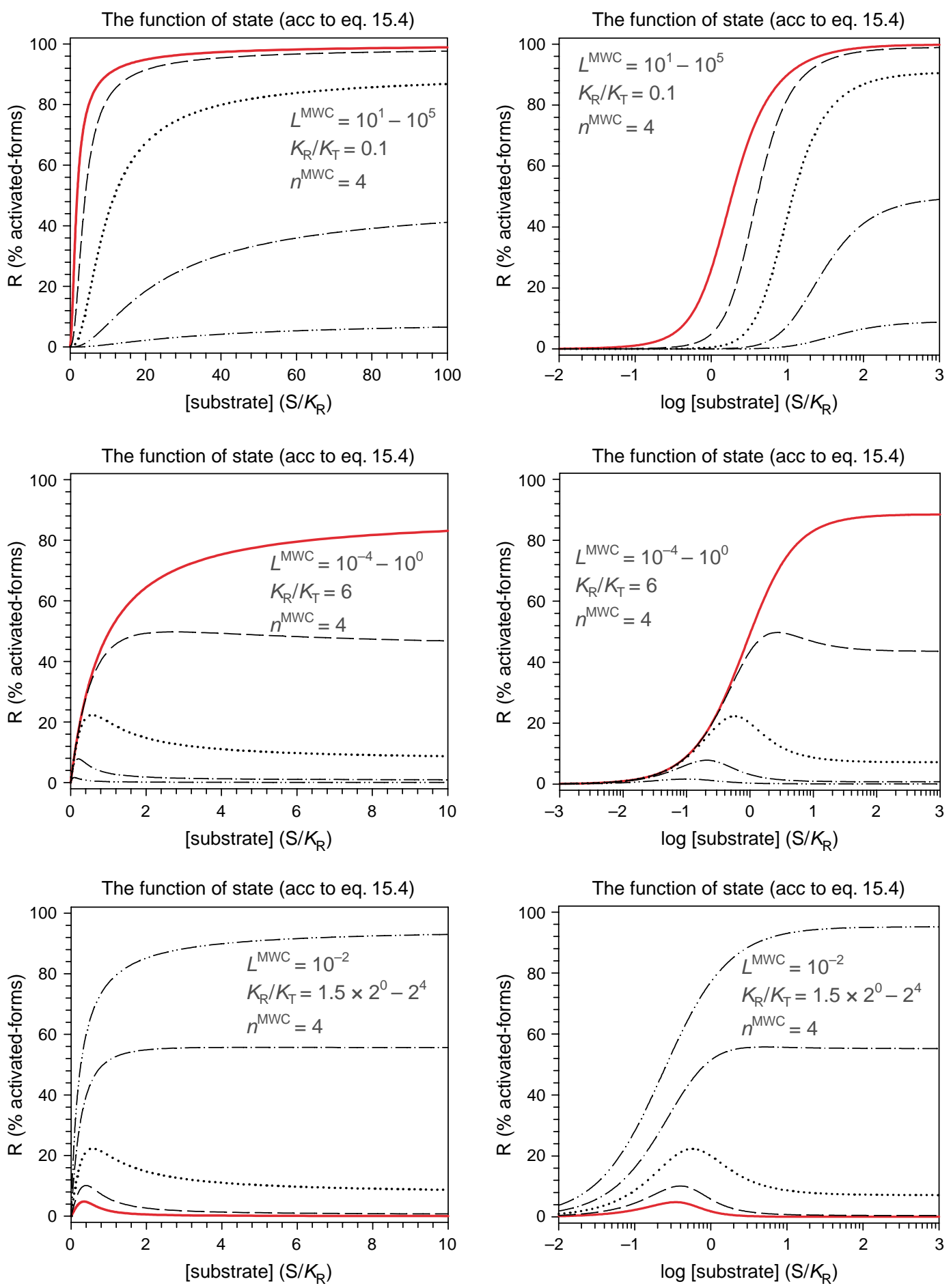

Figure 15.10. Examples of dose-response curves for the 'cotr-enz' function demonstrating bell-shaped synagics. Values for the three parameters $L^{\mathrm{MWC}}, K_{\mathrm{R}} / K_{\mathrm{T}} c^{\mathrm{MWC}}$, and $n$ are indicated in each panel and with the normalized substrate concentration $\mathrm{S} / K_{\mathrm{R}}=\alpha$ as the independent variable. The two upper panels are identical to the comparable panels in Fig. 15.9. In the middle panels, parameter $L^{\mathrm{MWC}}$ varies in five steps between $10^{-4}(-)$ to $10^{0}(-\cdot-)$ by a factor 10 . In the lower panel $K_{\mathrm{R}} / K_{\mathrm{T}}=c^{\mathrm{MWC}}$ is increased in five steps from 1.5.2 (- to $1.5 \cdot 2^{4}(-\cdot-)$ by a factor 2 . Left panels show the concentration axis with a linear scale, while the right panels have logarithmically scaled concentration axes. Active forms, $R$ states, first increase then decrease with an increase in substrate concentration. The cotr-enz function can analyze negative co-operativity and bell-shaped dose-response relationships. 


$$
\begin{aligned}
& \text { c-capacity } \vee \text { t-capacity } \\
& \quad=\frac{a A_{\mathrm{S}} \mathrm{S} \cdot\left(1+a A_{\mathrm{S}} \mathrm{S}\right)^{n-1}}{\left(1+a A_{\mathrm{S}} \mathrm{S}\right)^{n}+(1 / L) \cdot\left[\left(1+A_{\mathrm{M}} \mathrm{S}\right) /\left(1+b \cdot A_{\mathrm{M}} \mathrm{S}\right)\right]^{n}}
\end{aligned}
$$

For $n=4$, Eq. 15.5 was applied to kinetic data (Fig. 6 in Eisenstein et al. 1995) assuming $c^{\mathrm{MWC}}=1 / a<<1$ (Einsenstein 1994) and $L^{\mathrm{MWC}}=1 / L>>1$; maybe in order to keep spontaneous activity insignificant. The characteristics of Eq. 15.5 are shown in Fig. 15.11.

The Eisenstein extension of the classical MWC is a slight opening to more variation for the static dissociation constants as defined for the MWC model; in line with the HAP models. Meanwhile, a better choice of reaction scheme to start with is the ATSM in Chapter 7 or Koshland and coworker's sequential models described in sub-chapter 15.6.

Other examples aimed at eliminating the basal activity in the function of state $\mathbf{R}$ can be found (e.g., Mooser 1980). Since the basal activity (spontaneous activity, amount of $\mathrm{R}_{0}$ conformations) at low concentrations of substrate, $S \rightarrow 0$, in the MWC regime approaches $1 /(1+$ $\left.L^{\mathrm{MWC}}\right)$, Mooser suggested to simply subtract this fraction from the function of state $\mathbf{R}$. This may be done, but it is an incorrect correction as demonstrated in Fig. 15.12. For instance, for values of $c^{\mathrm{MWC}}=K_{\mathrm{R}} / K_{\mathrm{T}}=1$, the $\mathbf{R}$ function is zero for all values of $L^{\mathrm{MWC}}$ over the entire range of substrate concentrations (not shown). For values of $c^{\mathrm{MWC}}>1$, the $\mathbf{R}$-function becomes negative (see the two lower panels in Fig. 15.12).

In summary: in homotropy the $\mathbf{R}$-function (Eq. 15.1) is for systems with observable constitutive activity, while the cotr-enz function (Eq. 15.4) is for systems with nonobservable spontaneous activity.

\subsection{Heterotropic Allostery of the MWC Model}

\subsubsection{Heterotropic Allostery for Binding in the MWC Model}

Expressions already in the original development of the allosteric model, were formulated for the fractional saturation, i.e., occupancy studies, under heterotropic conditions with a primary ligand, an inhibitor, and an enhancer present for exclusive binding of the different ligands. An example is Eq. 15.1c (see the $\mathbf{Y}_{\mathrm{S}}$ function in Eq. 4 in Monod et al. 1965). Boeynaems and Dumont (1980) derived a general form of this expression for exclusive binding for attenuating (I) and augmenting (A) modulators (their Eq. 8.37).

By replacing factor $(1+\beta)^{\mathrm{n}} /(1+\gamma)^{\mathrm{n}}$ (Section 15.3.4), Rubin and Changeux (1966) modified and expanded the MWC'65 Eq. 4 by including both exclusive and non-exclusive binding of heterotropic modulators and obtained their Eq. 3 for the apparent allosteric constant $L^{\text {MWC: }}$

$$
\begin{aligned}
L^{\prime \mathrm{MWC}}= & L^{\mathrm{MWC}} \\
& \cdot\left(\frac{\left(1+d \cdot \mathrm{I} / K_{\mathrm{IR}}\right)}{\left(1+\mathrm{I} / K_{\mathrm{IR}}\right)} \cdot \frac{\left(1+e \cdot \mathrm{A} / K_{\mathrm{AR}}\right)}{\left(1+\mathrm{A} / K_{\mathrm{AR}}\right)}\right)^{n},
\end{aligned}
$$

(Changeux \& Rubin 1968). In Eq. 15.6 ' $\mathrm{A}$ ' is the concentration of an augmenting modulator, ' $\mathrm{I}$ ' is the concentration of an attenuating modulator, $d=K_{\mathrm{IR}} /$ $K_{\mathrm{IT}}>1$ and $e=K_{\mathrm{AR}} / K_{\mathrm{AT}}<1$. The basis for this derivation of $L^{\prime \text { MWC }}$ is the assumption that heterotropic influence in the MWC is only possible through a pertubation of the isomerization constant $L^{\mathrm{MWC}}$ (Rubin \& Changeux 1966). For me, the usefulness of this and similar formulations is dubious, since binding and function as well as homotropy and heterotropy are mixed together. Compare Eq. 15.6 with $L^{\prime \mathrm{MWC}}$ in Eq. 4 in Monod et al. (1965) and in Eq. 15.1b, as well as with $L^{\mathrm{MWC}}$ in Eq. 15.1 $a$. They are all based on the same seductive assumption that 'for simplicity... the substrate (S) has significant affinity only for the sites in one of the two states (for example R)', cited from Monod et al. (1965). With this, $c^{\mathrm{MWC}}$ is assumed zero in all analyses of functional studies. The statement in parenthesis '(for example R)' sounds innocent, but in reality - it functions to the effect of eliminating spontaneous activity. At least in my judgement it does. However, I suggest a careful reading of the MWC'65 paper before making a ruling. It is an elegant milestone paper of a powerful model presented with flaws.

\subsubsection{Heterotropic Allostery for Function in the MWC Model}

Monod et al. (1965) also derived the functional correlate for a heterotropic regime, again based on the constrained $\mathbf{Y}_{\mathrm{s}}$-function (Eq. 15.1b), although not for non-exclusive binding, as done by Rubin and Changeux (1966). In Monod et al. (1965) the apparent $L^{\text {MWC }}$ allosteric constant (already stated in Section 15.3.4) is $=L^{\mathrm{MWC}} \cdot\left[\left(1+\mathrm{I} / K_{\mathrm{I}}\right) /\left(1+\mathrm{A} / K_{\mathrm{A}}\right)\right]^{n}$, and used in functional analyses displayed in Monod et al.'s Figs. 4b, 6c, and $7 \mathrm{a}$ (1965).

Following Monod and co-workers (Monod et al. 1965; Rubin \& Changeux 1966), Kurganov (1982) has derived the heterotropic functional correlate to the binding/ function for both exclusive and non-exclusive heterotropic allostery and arrives at an expression for the apparent allosteric constant equivalent to Eq. 15.6. I remain skeptical about the relevance of these approaches. 

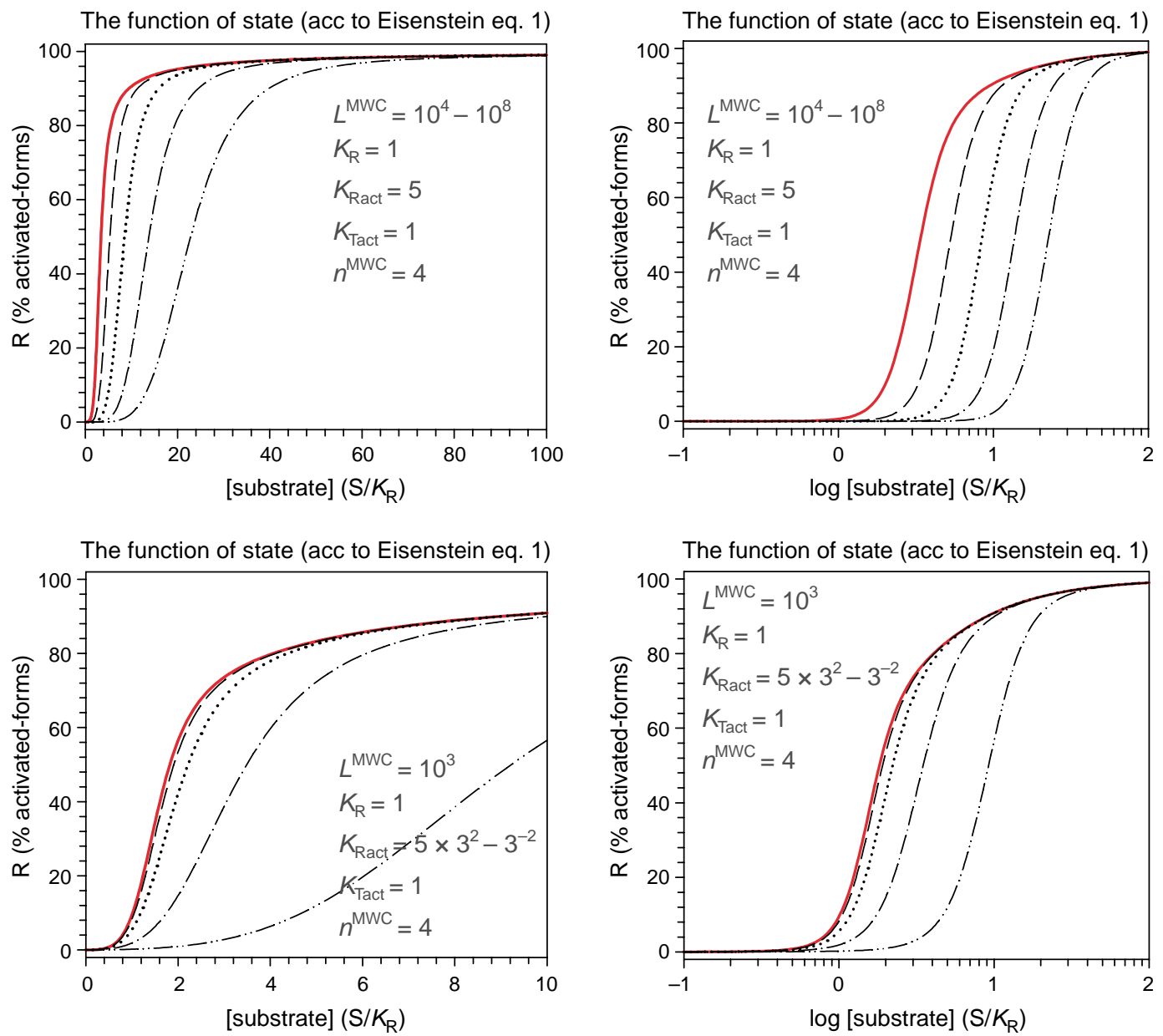

The function of state (acc to Eisenstein eq. 1)
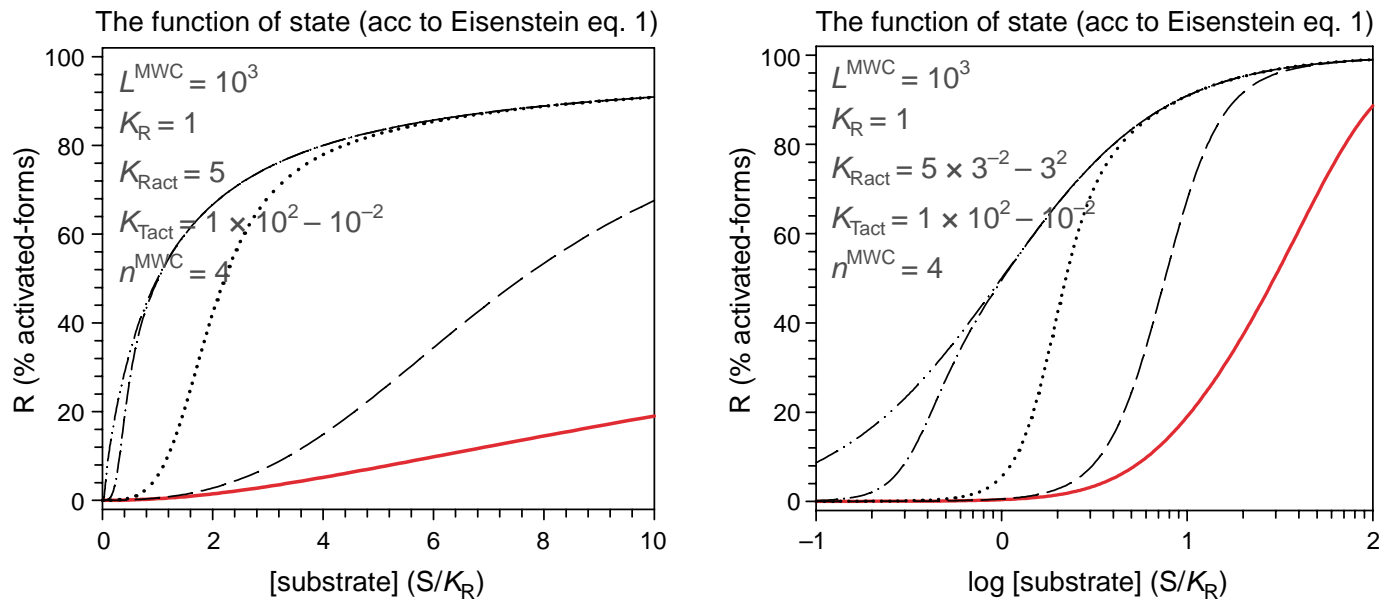

Figure 15.11. Examples of dose-response curves in the Eisenstein regime. This regime introduces two new parameters compared to the MWC model, $K_{\text {Ract }}$ and $K_{\text {Tact }}$. Values for the five parameters $L^{\mathrm{MWC}}, K_{\mathrm{R}} / K_{\mathrm{T}}=c^{\mathrm{MWC}}, n, K_{\text {Ract }}$ and $K_{\mathrm{T} a c t}$ are indicated in each panel and with the normalized substrate concentration $\mathrm{S} / K_{\mathrm{R}}=\alpha$ as the independent variable. In the two upper panels, $L^{\text {MWC }}$ varies in five steps from $10^{4}(-)$ to $10^{8}(-\cdot-)$ by a factor 10 . In the two middle panels, $K_{\text {Ract }}$ varies in five steps from $5 \cdot 3^{2}(-)$ to $5 \cdot 3^{-2}(-\cdot-)$ by a factor $1 / 3$. In the two lower panels, $K_{\text {Tact }}$ varies in five steps from $10^{2}(-)$ to $10^{-2}(-\cdot \cdot)$ decreasing by a factor 10 between steps. Left panels show the concentration axis with a linear scale, while the right panels have logarithmically scaled concentration axes. By the right selection of parameter values, $L^{\mathrm{MWC}}>>1$, spontaneous activity is gone. 

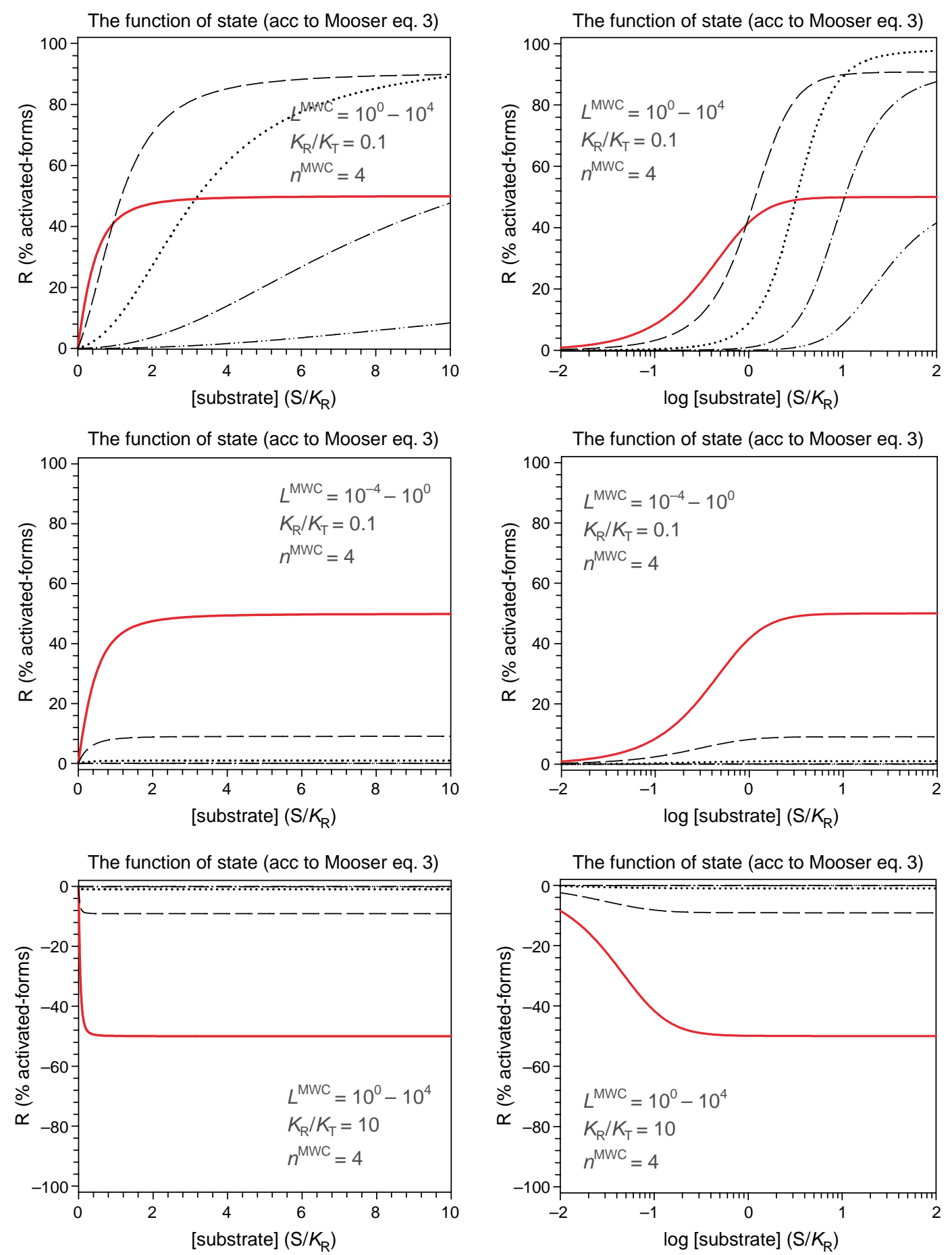

Figure 15.12. Examples of dose-response curves for Mooser's Eq. 3 (Mooser 1980). In this formulation $\mathrm{R}_{0}$ conformations are simply (and incorrectly) subtracted from the R function. Values for the three parameters $L^{\mathrm{MWC}}, K_{\mathrm{R}} / K_{\mathrm{T}}=c^{\mathrm{MWC}}$, and $n$ are indicated in each panel and with the normalized substrate concentration $S / K_{R}=\alpha$ as the independent variable. In the two upper and two lower panels, $L^{\mathrm{MWC}}$ varies in five steps from $10^{\circ}(-)$ to $10^{4}(-\cdot-)$ by a factor 10 . In the two middle panels, $L^{\mathrm{MWC}}$ varies in five steps from $10^{\circ}(-)$ to $10^{-4}(-\cdot-)$ by a factor $1 / 10$. In the upper four panels, $c^{\mathrm{MWC}}$ is below unity, $=0.1$, while in the two lower panels, the parameter $c^{\mathrm{MWC}}$ is above unity, $=10$. Left panels show the concentration axis with a linear scale, while the right panels have logarithmically scaled concentration axes. For $c^{\mathrm{MWC}}$ above unity, the R-function becomes negative. 


\subsubsection{Use of the R-function for Enzymes Displaying 'Spontaneous Activity'}

The spontaneous activity 'hidden' in the conformational function of state $\mathbf{R}$ was exposed by Rubin and Changeux (1966) and used as an argument for the MWC reaction scheme being a better model (Changeux \& Rubin 1968) than the original sequential reaction schemes suggested by Koshland and co-workers, KNF models (Koshland et al. 1966). The KNF models from 1966 did not really deal with or include the unliganded active states of receptive units, $\mathrm{R}_{0}$, in its formulations, and thus could not handle spontaneous activity.

For enzymes, Gerhart and Schachman (1968) obtained indirect evidence for a quantitative distribution between two states, $\mathrm{T}$ and $\mathrm{R}$, in the absence of substrates. These observations were based on measurements of the rate of sulfhydryl agents binding to Aspartate Transcarbamylase (Changeux et al. 1968; Gerhart \& Schachman 1968) and on sedimentation rates for the enzyme measured in the presence and absence of the substrate succinate (Gerhart \& Schachman 1968). Thus, the results of these studies could be interpreted as the enzyme being in two conformational states even in the absence of ligands, and thus could be presented by the function-of-state $\mathbf{R}$, demonstrating a distribution between $\mathrm{T}$ and $\mathrm{R}$ conformations in a ratio $4: 1$, i.e., $L^{\mathrm{MWC}}=4$. Therefore, a possible spontaneous active conformation could be assumed to represent around $20 \%$ of all the conformations (Changeux \& Rubin 1968, see their Figs. 5 and 7). In Fig. A.3C of Changeux and Edelstein (2005) it is about 5\%. This possible spontaneous activity for enzymes matched by the R-function is seldom exposed nowadays, but consistently brought to light by one of its inventors as an argument for the benefits of the MWC over the KNF (Changeux 2004, Chapter 8). Don't misunderstand me, the MWC model is a must in modeling.

\subsubsection{Two-states and Spontaneous Activity in Enzymes and Non-ligand-gated Transporters}

Un-liganded activity as demonstrated in receptors and ligand-gated receptor channels (Jackson 1984, 1986; Costa \& Herz 1989; Samama et al. 1993; DiFrancesco 1999; de Ligt et al. 2000; Grosman \& Auerbach 2000; Sablin et al. 2003; Bond \& Ijzerman 2005; Taly et al. 2006; Ye et al. 2006; Cotecchia 2007; Smit et al. 2007) is also found in enzymes, although not as obvious (Rypniewski \& Evans 1989; Perutz 1990; Iwata et al. 1994; Stieglitz et al. 2004; Fetler et al. 2007), carriers as hemoglobin (Perutz et al. 1998; Yonetani \& Tsuneshige 2003), and predicted in non-ligand-gated transporters as the cotransporters (Wright et al. 1994, 2007; Loo et al. 2006; Hirayama et al. 2007); pumps (Cornelius et al. 1998;
Einholm et al. 2005; Pinkett et al. 2007), and uniporters (Salas-Burgos et al. 2004).

Attention: the statement that: 'the $\mathbf{R}$-function is for receptors and ligand-gated ion channels, while the cotrenz-function is for enzymes and transporters such as pumps, co-transporters and uniporters' is an enunciation for homotropic but not for heterotropic studies.

\subsection{Desensitization' as Used by Two Different Schools}

\subsubsection{Desensitization l' as Used in Enzymology}

When treating enzymes with either high temperature or chemicals, the term 'desensitization' was used to describe the loss of allosteric regulatory potential to both homotropic and heterotropic ligands while the enzyme preserved the catalytic activity (Fig. 15.13) (Changeux 1961; Gerhardt \& Pardee 1962; Gerhardt 1970; Changeux 1993).

\subsubsection{Desensitization II' as Used in Receptology and for Some Ligand Gated Channels}

In GPCR and LGC literature, 'desensitization' is used as a general term for a spontaneous and time-dependent phenomenon, where during a maintained constant stimulus the response decays with time (Fig. 15.13). Desensitization with this meaning was coined by Katz and Thesleff (1957) for the ligand-gated nicotinicacetytcholine receptor channel (nAChR). In drug-gated channels, as for example the epithelial sodium channel $(\mathrm{ENaC})$, both time-dependent desensitization and concentration dependent auto-modulation takes place simultaneously, as demonstrated in Fig. 14.6D. For the reduced conductance in the epithelial sodium channel $(\mathrm{ENaC})$ by activated CFTR, ${ }^{7}$ it is also customary to use the term 'deactivation' (Table 15.2) (Reddy \& Quinton 1996, Quinton 2007). 'Deactivation' as illustrated in Fig. 15.13 is something else.

A molecular explanation was recently porvided for intrinsic desensitization in an ionotropic glutamate receptor (iGluR). Desensitization is excerted by agonist-rupture of the extensive domain 1-interface in iGluRs (Armstrong et al. 2006).

A time table for the various steps in GPCR activation, transduction, and desensitization is surveyed in a recent review (Lohse et al. 2008).

Historically and as convention, for voltage-operated channels (VOCs), an equivalent phenomenon to the LGC 'desensitization' behavior is 'inactivation' (Hille 2001).

\footnotetext{
${ }^{7}$ CFTR $=$ cystic fibrosis transmembrane conductance regulator .
} 

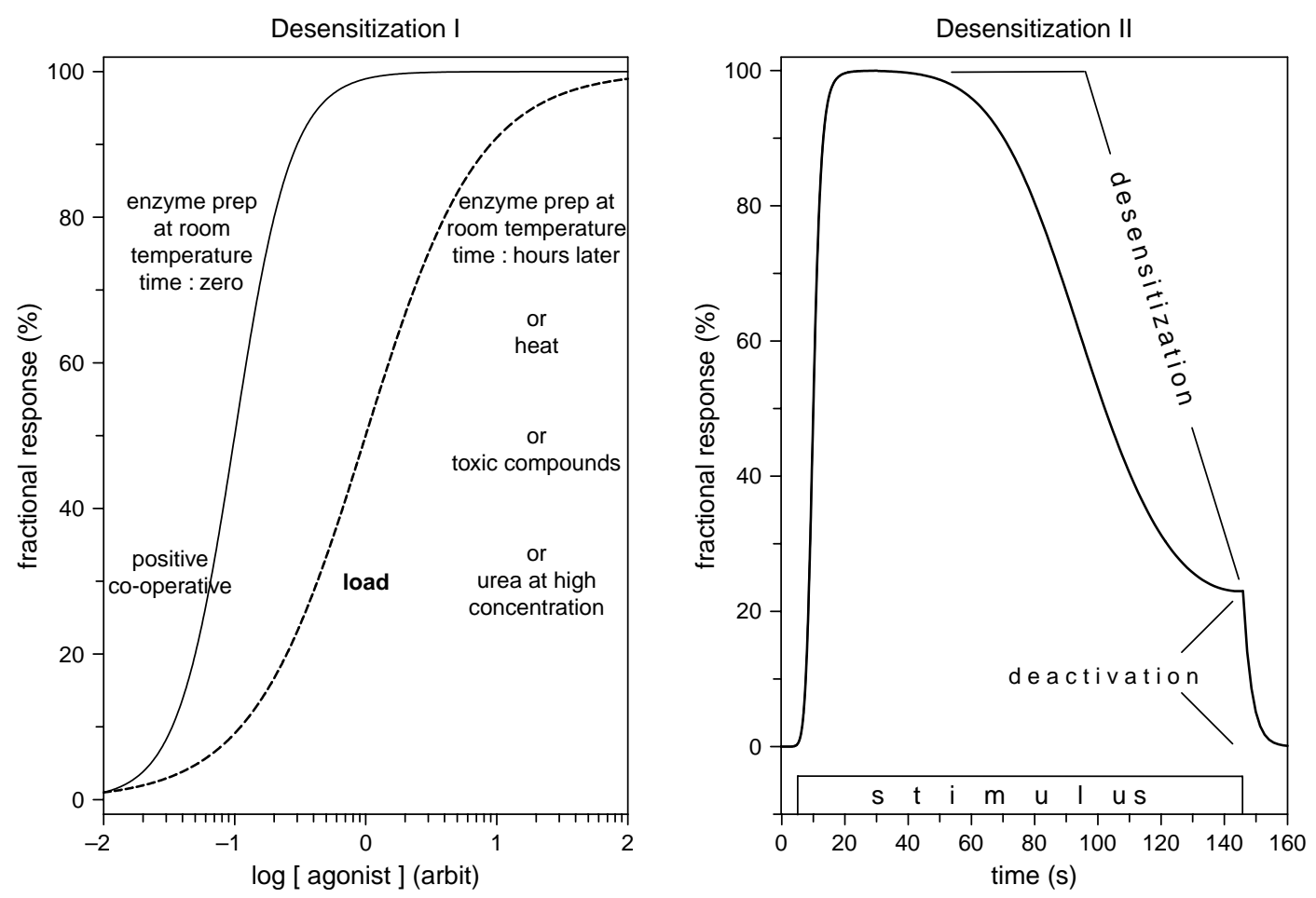

Figure 15.13. Principal graphs for desensitization I and II. Desensitization I (DI) has substrate concentration as the independent variable (Changeux 1961, 1993), whilst desensitization II (DII) has time as the independent variable (Katz \& Thesleff 1957; Lefkowitz 1986). Desensitization II is an attenuation of a drug-induced response with time, here with a maintained stimulus. Several recent reviews detail the mechanisms for DII (e.g., Gainetdinov et al. 2004; Hirsh et al. 2005; Giniatullin et al. 2005; Marie et al. 2006; Dhami \& Ferguson 2006; Schreiber \& Avissar 2007).

\subsection{3. 'Desensitization’}

The desensitization concept is still very much in use in receptology in the sense of desensitization II. Conversely, the concept desensitization I is only used sporadically in enzymology. Since desensitization I, like desensitization II, also include time as an independent variable, it is important to clearly realize that there is not a seamless transition between desensitization I and desensitization II, neither in reality nor in meaning. The two terms cover completely different conditions and concepts. Desensitization I is based on alterations in allosteric dose-responses with ligand concentration as the independent variable, while desensitization II is strictly dependent on time as the independent variable. A survey of the terms is given in Table 15.2.

\subsection{The KNF Models}

\subsubsection{Homotropic Binding Studies in the KNF Model}

Remarkably, one year after the MWC model was presented, Koshland and his collaborators presented competing theories - submitted August 1965, the Koshland-Nemeth-Filmer model, the 'sequential effect model', or the 'KNF model' (Koshland et al. 1965),

Table 15.2. Two schools for the term 'desensitization' and its related terms for concentration-dependent negative co-operativity

Receptive system

Enzymes

Voltage-operated channels

Receptive units as $\mathrm{G}$ protein-coupled receptors and ligand-gated channels
Epithelial sodium channel (ENaC)

$\begin{aligned} & \text { Kinetic designators for 'desensiti- } \\ & \text { zation' }\end{aligned}$
$\begin{aligned} & \text { Synagic designators for auto-inhibition or negative } \\ & \text { co-operativity }\end{aligned}$
$\begin{aligned} & \text { regulation } \\ & \text { Inactivation }\end{aligned}$
$\begin{aligned} & \text { Desensitization II loss of } \\ & \text { response with time }\end{aligned}$
$\begin{aligned} & \text { Segative auto-ant-agonism; negative auto-intervention; } \\ & \text { Sodium self-inhibition desensiti- } \\ & \text { zation II }\end{aligned}$

The epithelial sodium channel $(\mathrm{ENaC})$ is included as an example of an aberrant nomenclature.

${ }^{\#}$ CFTR = cystic fibrosis transmembrane conductance regulator (Reddy \& Quinton 1996; Quinton 2007). For a different 'deactivation' see Fig. 15.13. 
predicted in 1925 by Adair, modified by Pauling (1935), and reconceived in the late 1950s by Koshland (Koshland 1958, 2004). The KNF model (shown in Fig. 15.14) could also explain negative co-operativity (Conway \& Koshland 1968) although not bell-shaped synagics. Thus sequential induction of conformational changes may be said to go back to Adair's model for binding of $\mathrm{O}_{2}$ to hemoglobin (Adair 1925); or if you prefer, even to Hill in 1910 (see Chapter 10). Negative co-operativity in the form of shallow dose-response curves is not a possibility in the MWC 'concerted model' (Wyman 1967; Wong 1975).

One major difference between the simple MWC model and the more elaborate KNF model is the introduction of expressions for interaction between subunits of different binding arrangements affecting the occupancy and function of the individual subunit in the KNF reaction scheme. The term interaction constant (or coefficient) acknowledges this type of varied interaction between subunits. Thus for instance, one interaction between a reactive subunit $\mathrm{A}$ and an active subunit $\mathrm{B}$ in a quaternary subunit complex is given by the interaction coefficient $K_{\mathrm{AB}}=(\mathrm{AB}) \cdot(\mathrm{A}) /[(\mathrm{AA}) \cdot(\mathrm{B})]$, where $(\mathrm{AB})$ and $(\mathrm{AA})$ refer to interacting subunits, while (A) and (B) refer to non-interacting subunits (Koshland et al. 1966; Segel 1975, 1993; Neet 1995).

(1)

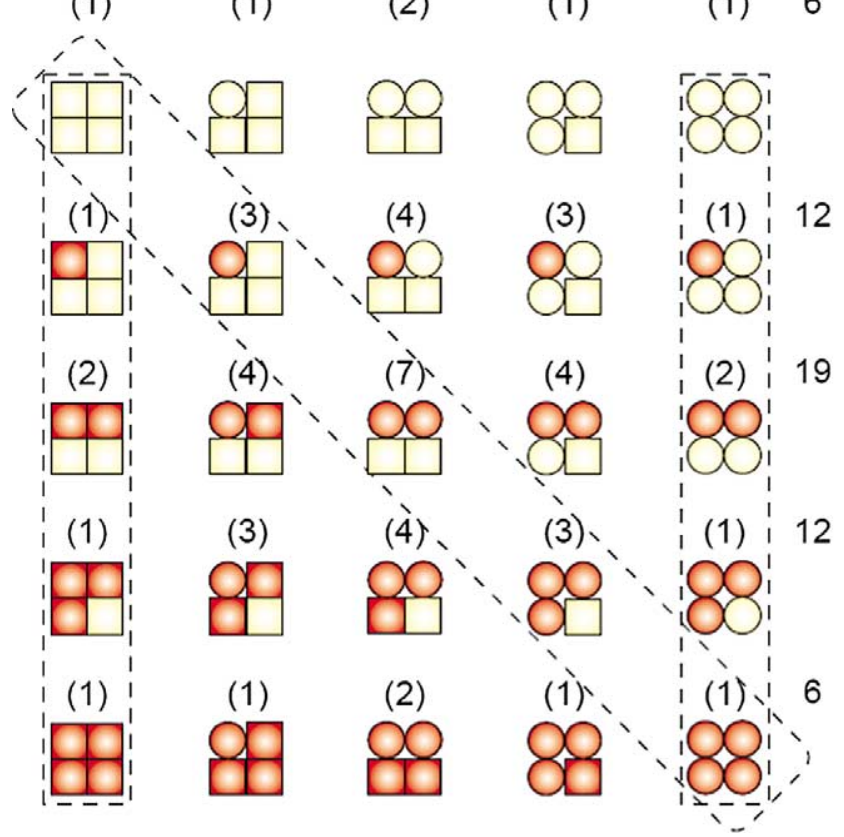

Figure 15.14. The general KNF model. This complete sequential model of the KNF scheme has a total of 44 independent dissociation and isomerization constants. The framed conformations indicate the original KNF model. Drawn according to Eigen (1968). Taken from Karpen and Ruiz (2002, Fig. 1) with permission.
In 1965, Atkinson and co-workers showed that even in the simplest possible model with four subunits present, allowing the effects of a single constant-of-interaction with values from 1 to 20 , at a value of 20 for maximum interaction, the model could predict an earlier observed Hill coefficient of 3.8 for catalytic activity in yeast DNFisocitrate dehydrogenase (Atkinson et al. 1965).

In this way, introducing constants-of-interaction is one key difference between the Monod and the Koshland approaches. These constants-of-interaction are similar to the complex affinity constant introduced by Hall in his ATSM, constant $\gamma$ (Hall 2000) that can vary on a graded and continuous scale.

Even though the KNF model is not an explicit 'genuine two-state model' as the MWC model, the KNF paper (Koshland et al. 1966) is still an intriguing score of intelligent software. The paper is on homotropic binding with the complexity of a four-site and sequentially alternating system as numbers of bound ligands increase. Koshland and co-worker showed that many models can actually explain the observed sigmoidality of oxygen binding to hemoglobin. Alone the conformations of a four-subunit molecule have several possibilities for subunit interactions: tetrahedral, square, linear, and even concerted (Koshland et al. 1966). The full model has 44 different conformations (Fig. 15.14). A square form of the quaternary KNF model is framed in Fig. 15.14 and shown in Fig. 15.15. This model was explicitly analyzed by Kirtley and Koshland (1967).

Further in the discussion of the KNF paper, there is a representation of a genuine two-state model. Meanwhile, it is used for a discussion of induction versus selection (see sub-chapter 5.11) or as it was called 'catalyzed transformation versus tautomerism' (Koshland et al. 1966).

In the following years, Koshland and co-workers also explicitly discussed genuine two-state models (Haber \& Koshland 1967).

It has been argued that the KNF model is ruled out if an effector molecule spontaneously presents two conformational states (Bloom et al. 1997). Notwithstanding, spontaneous flipping of site conformation is included in the general KNF model (Haber \& Koshland 1967; Segel 1975, 1993).

For practical implementation of the KNF models, it is the homotropic-binding-form that is most often used for synagic data analysis. To mention just a single example, see Wangensteen et al. (2001).

\subsubsection{Homotropic Functional Studies in the KNF Model}

Functionality was obtained for the homotropic KNF model by invoking an HMM-like formulation on the 


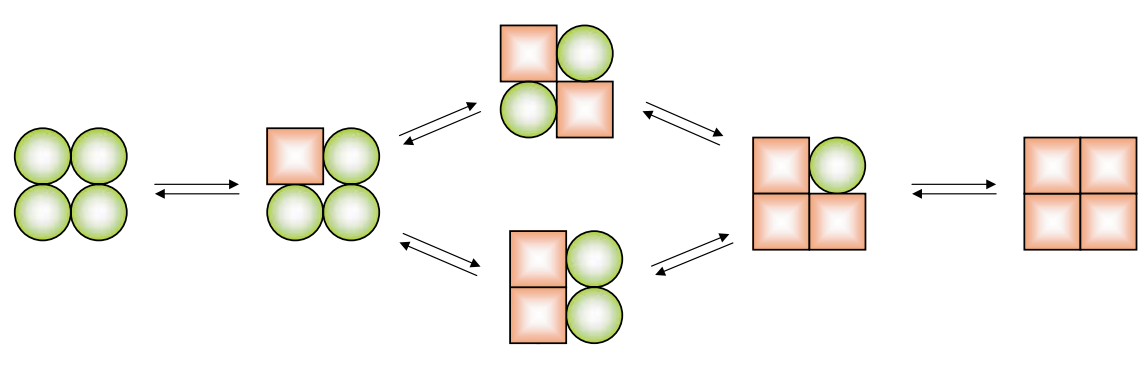

Koshland-Némethy-Filmer's Square model

Figure 15.15. The 'square' KNF model.

expressions obtained for binding (Teipel \& Koshland 1969). A similar approach for the homotropic functionality was taken by Kurganov (1982). Since the purpose of Teipel and Koshland's paper was to explain heterotropic effects of different ligands, their article is more relevant for heterotropic functionality, which will be discussed below.

Dose-response relationships with levels, or terraced synagics, were found for enzymes and characterized as 'negative co-operativity'. The negative co-operativity was easily explained by the KNF model (Conway \& Koshland 1968; Teipel \& Koshland 1969; Levitzki \& Koshland 1969), whilst as often cited, the MWC model could not explain such negative homotropic phenomena.

Although versions of the homotropic KNF model for single ligand interactions may be found scattered throughout the biological literature, it is moderately implemented for experimental activity (Shou et al. 1999; Houston \& Galetin 2005).

\subsubsection{Heterotropic Binding Studies in the KNF Model}

This theme is explicitly addressed in Kirtley and Koshland (1967) based on the square model (Fig. 15.15); introduced as one of the possible models in Koshland et al. (1966). Later examples implicating this type of analysis for theoretical analysis and experimental data are frequent and expanding (e.g., Wells 1992; Wreggert \& Wells 1995; Avalani et al. 2004; Cornish-Bowden 1995, Chapter 9, 2004, Chapter 11; Linder 2006; Choi \& Zocchi 2007; Lee et al. 2007).

\subsubsection{Heterotropic Functional Studies in the KNF Model}

Functionality was obtained as for the homotropic KNF model by invoking a HMM-like formulation (Teipel \& Koshland 1969). The aim was in fact to explain synagics with 'levels' ('negative co-operativity', leveling or plateau regions) and 'bumpy curves' (Levitzki \& Koshland 1969). Nowadays, synagic curves with intercalated plateaus are also referred to as 'terraced' relationships (Munson 1983).

Again, versions of the KNF model for heterotropic functionality may be found scattered throughout the biological literature, but as with the homotropic type of $\mathrm{KNF}$ it is also rarely used for analysis of experimental data on activity (Shou et al. 2001; Shou 2002; Galetin et al. 2005).

\subsubsection{Disappointments with Omitted Details for the KNF Model}

The zealous reader might have expected and wished for more details on the KNF models. However, further analyses and detailing of the KNF models should be developed in relation to specific projects, since a general description with no particular experimental objective it is a matter of ever expanding formulations based on the unconstrained variability and amplitude of interactions between dissociation constants in KNF models (Figs. 15.14 and 15.15).

In reality, matters are a bit more complicated. Consider as an example the molecular models used to describe allostery in the fructose-1,6-bisphosphatase enzyme shown in Fig. 15.16.

Therefore, in modeling, it is left to personal judgment to assign realistic possibilities and values for the implemented interactions of an actual experiment. Consider for example an analysis of forces and interactions in the 'cube' and the 'star' models of soap bubbles (Fig. 15.17).

\subsection{Some Notes on Concerted Versus Sequential}

\subsubsection{Evidence for Either Concerted or Sequential Synagics}

We have a concerted model, when (1) all subunits flip from one conformation to another at the same time, (2) all their binding constants change simultaneously and are invariant for the single state, and (3) spontaneous conformation flipping from one to another state only 
A

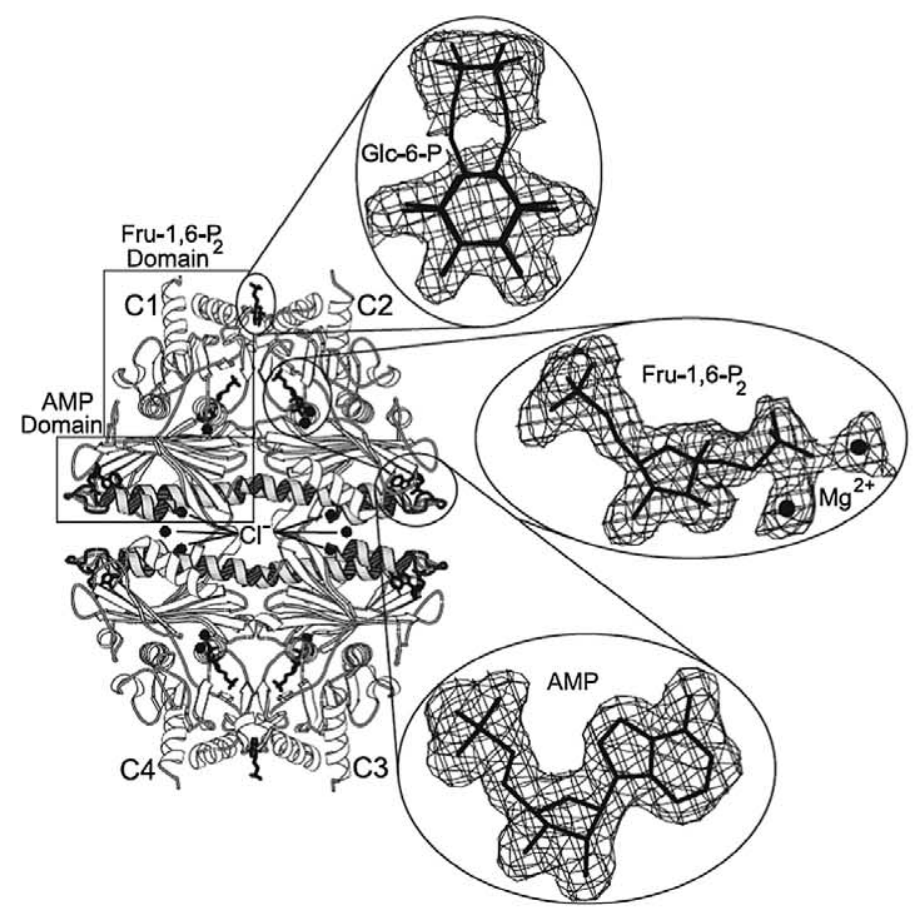

B
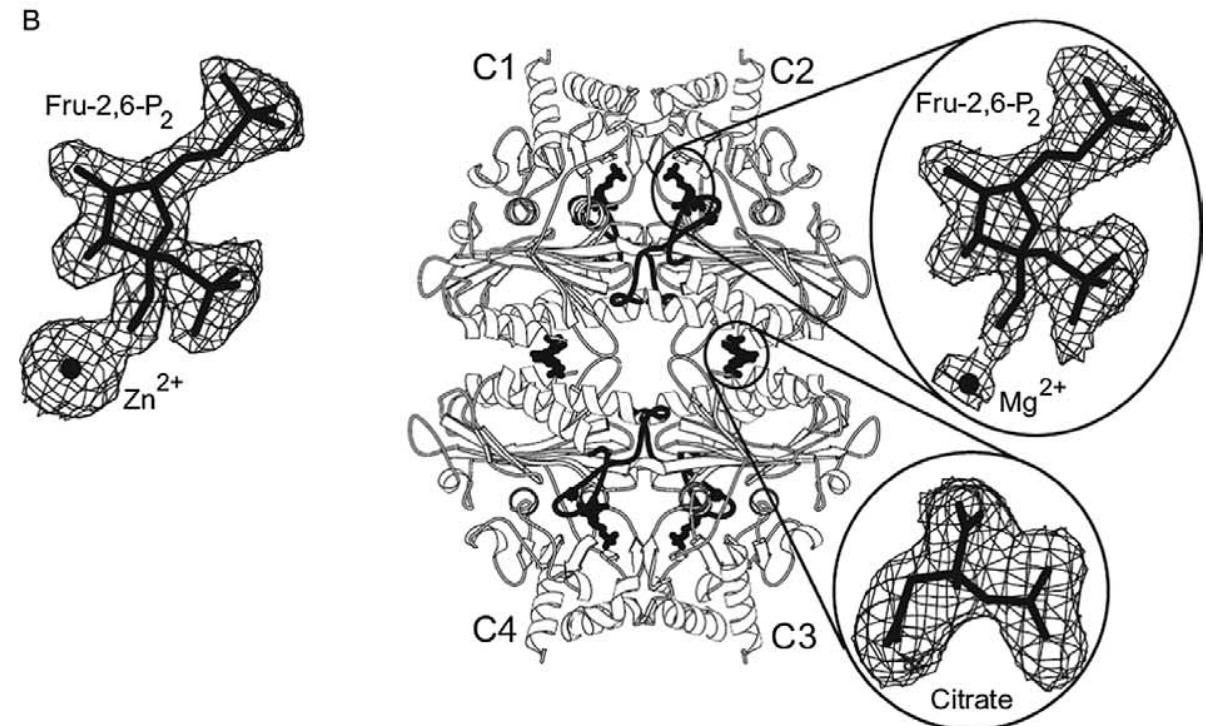

Figure 15.16. Allostery in complexes of the porcine and $E$. coli fructose-1,6-biphosphatases by modulators. Cut-off radius is at $2 \AA$. From Hines et al. (2007a,b, Fig. 2 (Panel A), Fig. 2B+C (Panel B)) with permission.

takes place in non-liganded receptive units (Fig. 15.3). Alternatively, we have a sequential model when (1) subunits flip conformationally one site at a time, (2) the interaction between different subunits vary as binding proceeds and affect the dissociation constants, and (3) the conformational isomerization is also induced by the presence of bound ligands (Fig. 15.14).

Concepts and their designators are easily confounded. Keep the term-pair 'concerted/vs/sequential' separate from the pair 'conformational induction/vs/conformational selection' or just 'induction-selection'. These two term-pairs of complementary action cover different conceptions for the function of effectors, but both pairs may be relevant for the same allosteric mechanism. The pair 'concerted-sequential' is treated next, while 'selection-induction' is treated in sub-chapter 5.11.

The concerted or symmetrical model proposed by MWC in 1965 and the sequential model or 'induced fit' 

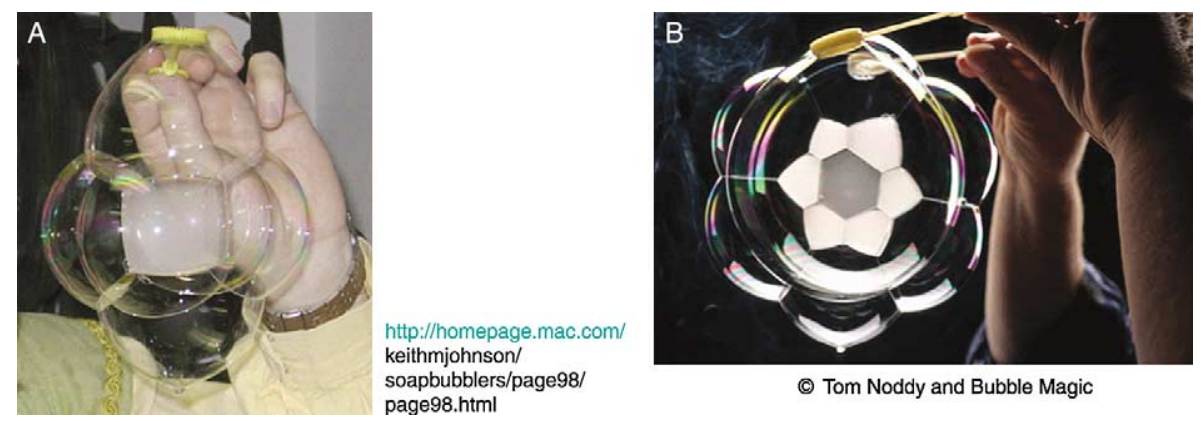

Figure 15.17. 'Cube' and 'Star' models in soap bubbles. An aspect of 'detailed balance in thermodynamics' is observed. Although soap films are restricted by external forces to sheets and spheres, constructions can be made such that cubes, stars and other forms appear. Soap models: are they homologous for synagic reaction schemes or just analogies? (A) From http:// homepage.mac.com/keithmjohnson and (B) from http://www.tomnoddy.com.

model proposed by KNF in 1966 were and still are the two dominating allosteric models for concerted-vssequential reaction schemes and for ideas on selectionvs-induction. As mentioned, concertedness was already established in the 1950s when four association constants for $\mathrm{O}_{2}$ binding to hemoglobin were determined (Roughton et al. 1955) and confirmed structurally by Perutz (1970). However, the conformational flipping was on binding of the 4th $\mathrm{O}_{2}$ molecule (Roughton et al. 1955), not before binding of the first $\mathrm{O}_{2}$ as assumed in the MWC model (see Table 5.3 in Chapter 5).

The debate between followers of these two models, concerted versus sequential, is still actual and very lively (Perutz et al. 1998; Bettati et al. 1998; Edelstein \& Changeux 1998; Macol et al. 2001; Helmstaedt et al. 2001; Fetler \& Vachette 2002; Koshland \& Hamadani 2002; Changeux 2004; Tsuruta et al. 2005; Fetler et al. 2007). On concerted-ness in protein folding, see Zhou and Bai (2007).

Most researchers on the aspartate transcarbamylase enzyme (ATCase) consider it to follow concerted behavior as assumed in the MWC model, where within a unspecified time frame around the binding of a single or the first substrate molecule it flips the whole ATCase enzyme complex, consisting of 12 subunits with two catalytic sites built by six subunits and three regulatory domains built by six other subunits, into its active $\mathrm{R}$ conformation (Jin et al. 1999; Macol et al. 2001; Stieglitz 2004; Tsuruta et al. 2005; Wang et al. 2005, 2007). Observe that concerted behavior in ATCase is not an argument against sequential stepping. Both concerted and sequential models may be valid for different effector complexes-and even expressed in the same protein complex, as discussed by Perutz et al. (1998).

\subsubsection{The 'Mon-land' Model}

Thorough and detailed analyses of the KNF and MWC models and a comparison between the two have been recapitulated again and again (Segel 1975, 1993; Kurga- nov 1982; Neet 1995; Koshland \& Hamadani 2002; Changeux 2004, Chapters 7 and 8). Perutz et al. (1998) have even unified the two models and designated a hybrid as the 'Mon-land' model.

Therefore, here, I will only sum up the situation by stating that the models of KNF has the advantage over the MWC model by including negative co-operativity (negative homotropic allostery) and negative heterotropic allostery. KNF models can handle interim effector states and the Koshland and coworker papers describe several of the possible subunit interactions, including intermediary complex states of differently arranged geometry for subunits. The general KNF model also switches between states in the liganded and non-liganded forms (Segel 1975, 1993), i.e., the KNF models really include both induction and selection, as in the simpler K\&T model discussed in Chapter 5. Furthermore, both sequential and concerted behavior is inherent in KNF models.

Although spontaneous activity was not explicitly dealt with in the 1966-KNF models, spontaneous activity was never excluded from the KNF model (Haber \& Koshland 1967). Evidently, some protein complexes obey the MWC concertedness when undergoing a conformational isomerization (Tsuruta et al. 2005; Wang et al. 2005, 2007). However, this may also be, as mentioned, formulated by versions of the general KNF model.

\subsubsection{Where to Go}

Both concept-pairs of 'concerted-sequential' and 'selection-induction' are included in thermo-dynamically sound schemes of the ATSM (Chapter 7). The ATSM as presented is limited by merely involving two binding sites. All in all, reaction schemes of ATSM and elaborations of this model, as in the FP-TSM, seem more relevant as starting material for further analysis of synagic studies than a base in the MWC model.

Strangely, the idea of 'selection' - where different conformational states of receptors are stabilized by the ligand binding - rather than ligand 'induction' has 
been favoured in recent years by receptologists, but kept in balance by others (Lutz \& Kenakin 1999, pp. 66-68). More details on this are given in sub-chapter 5.11.

I conclude that maybe some multi-sited enzymes operate in a concerted fashion, but as they do not follow the functional synagics derived in the MWC paper (Eq. 15.2), other models have to be invoked (see for instance Section 15.3.6).

\subsection{Pascal Triangle, Two-state, and Cubic Two-state Schemes}

I shall end Chapter 15 with a brief look at the differences between one-state Hill-Adair-Pauling-type models (HAP-models) and genuine two-state models as the cyclic two-state model and the Monod-WymanChangeux model. To conclude this sub-chapter, there is a summary on what differentiates the Monod-WymanChangeux model from other models (Section 15.8.3), and a suggestion for the future use of two-state cubic models (Section 15.8.4).

\subsubsection{Combinatorial Models and the Pascal-triangle Extension}

Invoking Hill's modified equation (Eq. 10.2) and general formulation for its use in data analysis is presented in Chapter 10.

The Hill type equations are in principle simpler than the MWC regime but may be expanded as in the Pascal triangle (Tuk \& vanOostenbruggen 1996; Weiss 1997). Compare this with Adair (1925) and Pauling (1935) derivations. The original KNF models are a prolongation, concatenation, and extension of the HAP-models, especially when the KNF models do not deal explicitly with two-state modeling, as was somewhat the case in the KNF paper (Koshland et al. 1966).

Tuk and van Oostenbruggen (1996) and Weiss (1997), in the same manner as KNF, investigate the model possibilities when focusing on interaction between binding sites without genuine two-state mechanisms. In these two papers, sequential effects are invoked by employing equilibrium dissociation constants $K_{\mathrm{d} 1}$ and $K_{\mathrm{d} 2}$ ( 1 and 2 indicate the first and second binding) that are not equal. This covers the idea that binding constants change as ligands bind. The general statement about binding site interactions is $K_{\mathrm{dx}} \neq K_{\mathrm{dx}+1}$. This is ruled out in the MWC model, where $K_{\mathrm{d} 1}, K_{\mathrm{d} 2}, \ldots K_{\mathrm{dn}-1}$ must all be identical with $K_{\mathrm{dn}}$.

For both (1) fully occupied and (2) all kinds of occupied receptors, fully and partially, Tuk and van Oostenbruggen (1996) in the foot-steps of Adair-Pauling-KNF-Wells (Wells 1992) derive the general expres- sions for fractional binding with any number of binding sites, $n$, using the Pascal-triangle. This type of equation is given in Chapter 6 (Table 6.2) for both occupancy and function.

Weiss (1997) also used the Pascal-triangle extension for the fraction of fully occupied receptors in ordered (sequential) and random (independent) reaction schemes. These two schemes are discussed in subchapters 6.2 and 6.3. Weiss's expression for the random model is identical to the Tuk and van Oostenbruggen expression. Weiss's two formulae are presented in Chapter 6 as equations in Table 6.2.

The isomerization constant for conformational change, $L$, in un-liganded receptive systems, i.e., genuine allosteric effects, is not considered in the Tuk-van Oostenbruggen and Weiss papers, and thus is different from the treatment by for instance Janin (1973), Thron (1973), and Colquhoun (1973). Meanwhile, Weiss (1997) analyze a model with a conformational step after $\mathrm{n}-1$ ligands have bound. This is comparable to $\mathrm{O}_{2}$ binding to hemoglobin as found by Roughton et al. (1955).

Derivations that are different from the Tuk and van Oostenbruggen and Weiss expansion for multi-sited binding at equilibrium can be found in several other publications (e.g., Wells 1992; Wreggett \& Wells 1995; Colozo et al. 2007).

The conclusion of Weiss (1997) on the use of Hill's equation is worth noting. Weiss writes 'Despite its appealing simplicity, the Hill equation is not a physically realistic reaction scheme, raising the question of whether it should be abandoned in favor of realistic reaction schemes; at the very least, its limitations should be more widely recognized in its frequent application to ligand-receptor interactions. The Hill coefficient is best thought of as an interaction coefficient among multiple binding sites'. I totally agree.

In continuation of the expanding interest in interaction coefficients, several authors have proposed new models within the realm of MWC. As an example, an empirical model with expanded interaction coefficients was suggested by Kurganov (2000), and as mentioned in Section 15.3.8, Eisenstein et al. (1995) in manipulating the MWC/Changeux and Rubin equation for heterotropic functionality also introduced new dissociation constants for co-operativity (homotropy).

\subsubsection{Two-states - the MWC Model Versus the $K \& T$ Model}

In any event, it is enlightening to compare the Katz and Thesleff model of cyclic two-states from 1957 (see K\&T

\footnotetext{
8 There is a misprinting in the Weiss formula for the ordered case, where the second term in the denominator should read $[L] / K_{\mathrm{d} 1}$, instead of $[L]$. Anyway, it is correctly quoted in Table 6.2.
} 
in Chapter 5), with the two-state model for allostery from 1965 by MWC, which, in contrast, is a non-cyclic although reversible two-state system. In the K\&T model, the two-state system was treated for one type ligand in a fully reversible cycle including both 'induction' and 'selection' of receptor states by ligand binding. In the MWC, which is a non-cyclic scheme in its original form, the two-state system was (also) first evaluated with one ligand, while reversibility was only between unbound states, tacitly assuming only 'selection'. Thus, the term 'selection' might be included in designating the MWC model as a two-state selection model. Next, a lemma was introduced for the MWC with simultaneous switching for all subunit binding constants in a concerted fashion. Accordingly, the MWC model has even been designated the two-state selection and concerted model (Koshland et al. 1966). This is in contrast to the $K \& T$ model that specifically operates with an interaction constant as in the KNF models due to the principle of thermodynamic cycles (Fersht 1999, his Chapter 3 section L). To avoid a contentious association to the term 'co-operativity', a term such as 'symmetry model' is also used for the MWC model instead of an 'allosteric model' (Cornish-Bowden 2004, p. 260).

For the MWC model, on leaving the K\&T model behind, there followed a treatment with two heterotropic ligands, one being an allosteric activator and the other an allosteric inhibitor (Monod et al. 1965; Rubin \& Changeux 1966). That was an advancement beyond the K\&T.

\subsubsection{What Differentiates the MWC Model from Other Models?}

It has been upheld and posited many times that the MWC model is characterized (1) by its description of constitutive activity in an un-liganded state, i.e., spontaneous activity (Changeux \& Edelstein 2005; Fetler et al. 2007), (2) by its protomer assemblies required for allosteric behavior, and (3) by its sole change of conformation before binding of ligands in a concerted fashion involving all binding sites.

1. Notwithstanding, spontaneous activity in the un-liganded state is not specific for the MWC. This was anticipated long before (Haurowitz 1938; Perutz 1942; Wyman \& Allen 1951), formulated earlier (Katz \& Thesleff 1957; Botts \& Drain 1958), and proven immediately before the MWC model appeared (Muirhead \& Perutz 1963). As an example, the KNF models also explicitly include un-liganded 'two-state' spontaneous activity (Haber \& Koshland 1967).

2. Furthermore, while arguments about axial and rotational symmetries were introduced with the
MWC model, the receptive entities with several identical subunits as in the original MWC model also does not differentiate this model from other models. Besides, binding sites at the interface between subunits were missed by the MWC model (Changeux \& Edelstein 2005).

3. The possible conformational switch before ligand binding is a whole paradigm of its own related to the selection-vs-induction debate as discussed in sub-chapter 5.11. In short, the conformational switch 'before' binding is a reality (Fetler et al. 2007), but in relation to the MWC model, it is the sole possibility. Regarding this constraint for the MWC, see below.

So, what differentiates the MWC model from other models? First, it is the ferreting out and weeding of variability in system binding constants that differentiates this model from other models. The MWC model only allows two dissociation constants, $K_{\mathrm{R}}$ and $K_{\mathrm{T}}$, thus dramatically simplifying synagic formulations for multisited systems. Second, it is the limitation of an isomerization of the receptive units exclusively for unbound conformations, as mentioned above; a particular form for concert.

These two assumed requirements in the MWC model render special distribution equations for its description (Monod et al. 1965), and these two hallmarks are the strength and the weakness of the model. The combined effect of the two hallmarks for MWC is also stated in point 6 of Box 15.3.

Regrettably though, as demonstrated in Section 15.3.6, the elegant formulations for occupancy and function in the MWC model were mixed and misguided for the function of state $\mathbf{R}$.

In subsequent redrawings of the MWC reaction scheme, its essential precondition of a sole $\mathrm{T}_{0} \rightleftharpoons \mathrm{R}_{0}$ flip-flop is not always carried and maintained (see, e.g., Cornish-Bowden 1995, Fig. 9.2, or 2004, Fig. 11.6; Changeux \& Edelstein 2005, Fig. A.1C; and Section 1.4.2).

\subsubsection{Graded FP-TSM and the Future}

The FP-TSM in Fig. 15.2, with graded system constants (Fig. 7.2B), is a novel type of reaction scheme, since it allows for co-lateral binding and varied interactions between different ligand-receptor complexes, similar to the KNF model, but in a full thermo-dynamically complete reaction scheme with an explicitly formulated two-state mechanism. Some types of both the KNF model and the MWC model are included in the FPTSM, although with severe constraints on the FP-TSM to accommodate in particular the MWC models. 
Opposite to this, so far, the bi-sited FP-TSM in Fig. 15.2 fails to match the multi-sites of both KNF and MWC models. Nevertheless, in the future, when there is detailed information about binding and/or function in dose-response relations for ligand-receptor interactions, the HOTSM, ATSM, and FP-TSM should be implemented as analytical tools. In case there are more than two sites for binding, an expansion of the HOTSM, ATSM, and FP-TSM will of course be necessary.

\subsection{Conclusions}

A. It is extremely important to keep analytical tools for binding studies apart from tools for functional studies.

B. If using the MWC model for analysis of homotropic functional studies for enzymes or nonligand-gated transporters, the newly derived Eq. 15.4 should be employed instead of twisted versions of the $15.1 a$ and $15.1 c$ formulation or the Eisenstein et al. formulation (Eq. 15.5). For analysis of similar but heterotropic studies it is recommended to drop the Changeux-Rubin and Kurganov formulations (sub-chapter 15.4) and instead employ expansions of the ATSM.

C. The MWC model is without cyclic two-states. When you compare the MWC model with the ATSM and HOTSM described in Chapter 7, it is my conviction that the ATSM and HOTSM are much more versatile models for analysis, although so far only developed for two-site reaction schemes. Expansions of the ATSM are begining to appear (Ehlert \& Griffin 2008).

D. In nature, many switch mechanisms exist, and on these lines, even covalently adapting multikinase systems requires several steps in order to become on-off switch-like with signal amplification (Bhalla et al. 2002; Li \& Qian 2003; Angeli et al. 2004; Bhalla 2004; Cornish-Bowden 2004, pp. 102-104; Cheng et al. 2005; Dinarina et al. 2005). Thus, in order to obtain molecular switches, it may be that the modified Hill and MWC-concerted multi-sited system has the immediate solution. Algebraic expressions for switch behavior can also be obtained by extensions of the HOTSM and ATSM reaction schemes; that is, an expansion from their present two binding sites to more binding sites.

E. Therefore, modeling in the future should avoid the Hill and MWC models and instead implement the new models for allostery, ATSM and the HOTSM, their combined scheme the FPTSM, and further develop these models into models with more than two binding sites.

\section{REFERENCES}

Adair GS. The hemoglobin system. VI. The oxygen dissociation curve of hemoglobin. J Biol Chem 63: 529-545, 1925.

Alexander RT, Malevanets A, Durkan AM, Kocinsky HS, Aronson PS, Orlowski J \& Grinstein S. Membrane curvature alters the activation kinetics of the epithelial $\mathrm{Na}+/ \mathrm{H}+$ exchanger, NHE3. J Biol Chem 282: 7376-7384, 2007.

Altomare C, Bucchi A, Camatini E, Baruscotti M, Viscomi C, Moroni A \& DiFrancesco D. Integrated allosteric model of voltage gating of HCN channels. J Gen Physiol 117: 519-532, 2001.

Angeli D, Ferrell JE Jr \& Sontag ED. Detection of multistability, bifurcations, and hysteresis in a large class of biological positivefeedback systems. Proc Natl Acad Sci USA 101: 1822-1827, 2004.

Armstrong N, Jasti J, Beich-Frandsen M \& Gouaux E. Measurement of conformational changes accompanying desensitization in an ionotropic glutamate receptor. Cell 127: 85-97, 2006.

Atkins WM. Non-Michaelis-Menten kinetics in cytochrome P450catalyzed reactions. Annu Rev Pharmacol Toxicol 45: 291-310, 2005.

Atkinson DE, Hathaway JA \& Smith EC. Kinetics of regulatory enzymes. Kinetic order of the yeast diphosphopyridine nucleotide isocitrate dehydrogenase reaction and a model for the reaction. $J$ Biol Chem 240: 2682-2690, 1965.

Avlani V, May LT, Sexton PM \& Christopoulos A. Application of a kinetic model to the apparently complex behavior of negative and positive allosteric modulators of muscarinic acetylcholine receptors. J Pharmacol Exp Ther 308: 1062-1072, 2004.

Balshaw DM, Millette LA, Tepperman K \& Wallick ET. Combined allosteric and competitive interaction between extracellular $\mathrm{Na}(+)$ and $\mathrm{K}(+)$ during ion transport by the alpha(1), alpha(2), and alpha(3) isoforms of the Na, K-ATPase. Biophys $J$ 79: 853-862, 2000.

Beato M, Groot-Kormelink PJ, Colquhoun D \& Sivilotti LG. The activation mechanism of alphal homomeric glycine receptors. J Neurosci 24: 895-906, 2004.

Ben-Amin A. Cooperativity and Regulation in Biochemical Processes. New York: Kluwer Academic/Plenum Publishers, 2001.

Berg JM, Tymoczko JL \& Stryer L. Biochemistry, 5th ed. New York: WH Freeman, 2002.

Berg JM, Tymoczko JL \& Stryer L. Biochemistry. 6th ed. New York: WH Freeman \& Company, 2007.

Berteloot A. Kinetic mechanism of $\mathrm{Na}+$-glucose cotransport through the rabbit intestinal SGLT1 protein. J Membr Biol 192: 89-100, 2003.

Bettati S, Mozzarelli A \& Perutz MF. Allosteric mechanism of haemoglobin: rupture of salt-bridges raises the oxygen affinity of the T-structure. J Mol Biol 281: 581-585, 1998.

Bhalla US. Models of cell signaling pathways. Curr Opin Genet Dev 14: 375-381, 2004

Bhalla US, Ram PT \& Iyengar R. MAP kinase phosphatase as a locus of flexibility in a mitogen-activated protein kinase signaling network. Science 297: 1018-1023, 2002.

Bindslev N. A homotropic two-state model and auto-antagonism. BMC Pharmacology 4: 11, 2004.

Birdsall NJ, Burgen AS \& Hulme EC. The binding of agonists to brain muscarinic receptors. Mol Pharmacol 14: 723-736, 1978.

Birnbaumer L, Swartz TL, Abramowitz J, Mintz PW \& Iyengar R. Transient and steady state kinetics of the interaction of guanyl nucleotides with the adenylyl cyclase system from rat liver plasma membranes. Interpretation in terms of a simple two-state model. J Biol Chem 255: 3542-3551, 1980.

Bloom CR, Kaarsholm NC, Ha J \& Dunn MF. Half-site reactivity, negative cooperativity, and positive cooperativity: quantitative considerations of a plausible model. Biochemistry 36: 12759 $12765,1997$. 
Boeynaems JM \& Dumont JE. The two-step model of ligand-receptor interaction. Mol Cell Endocrinol 7: 33-47, 1977.

Boeynaems JM \& Dumont JE. Outlines of Receptor Theory. Amsterdam: Elsevier, 1980

Bohr C. Die sauerstoff aufnahme des genuinen blutfarbstoffes und des aus dem blute dargestellten hämoglobins. Zentralbl Physiol 17: 688-691, 1904a.

Bohr C. Theoretische behandlung der quantitativen verhältnisse bei der sauerstoff aufnahme des hämoglobins. Zentralbl Physiol 17: 682-688, 1904b.

Bohr C, Hasselbalch K \& Krogh A. Ueber einen in biologischer beziehung wichtigen einfluss, den die kohlensäuerspannung des blutes auf dessen sauerstoffbindung übt. Skand Arch Physiol 16: 402-412, 1904.

Bond RA \& Ijzerman AP. Recent developments in constitutive receptor activity and inverse agonism, and their potential for GPCR drug discovery. Trends Pharmacol Sci 27: 92-96, 2006.

Botts J \& Drain GF. An illustration of a kinetic analysis: the myosin B-ATP-EDTA system. The committee of muscle chemistry of Japan. 33-41. 1958. Tokyo, Igaku Shoin. Proceedings from Conference on the chemistry of muscular contraction 1957.

Bourne HR, Sanders DA \& McCormick F. The GTPase superfamily: a conserved switch for diverse cell functions. Nature 348: 125-132, 1990.

Brown WEL \& Hill AV. The oxygen/dissociation curve of blood, and its thermodynamical basis. Proc Roy Soc B 94: 297-334, 1923.

Burzomato V, Beato M, Groot-Kormelink PJ, Colquhoun D \& Sivilotti LG. Single-channel behavior of heteromeric alphalbeta glycine receptors: an attempt to detect a conformational change before the channel opens. J Neurosci 24: 10924-10940, 2004.

Cantor CR \& Schimmel PR. Biophysical Chemistry. III The Behavior of Biological Macromolecules. New York: WH Freeman \& Co., 1980.

Chan WW \& Enns CA. Aspartate transcarbamoylase: loss of homotropic but not heterotropic interactions upon modification of the catalytic subunit with a bifunctional reagent. Can J Biochem 57: $798-805,1979$.

Changeux J-P. The Physiology of Truth: Neuroscience and Human Knowledge. Cambridge: Belkanp Press of Harvard University Press, 2004.

Changeux JP. The feedback control mechanisms of biosynthetic L-threonine deaminase by L-isoleucine. Cold Spring Harb Symp Quant Biol 26: 313-318, 1961.

Changeux JP. Allosteric proteins: from regulatory enzymes to receptors - personal recollections. Bioessays 15: 625-634, 1993.

Changeux JP \& Thiery J. On the mode of action of colicins: a model of regulation at the membrane level. J Theor Biol 17: 315-318, 1967.

Changeux JP \& Podleski TR. On the excitability and cooperativity of the electroplax membrane. Proc Natl Acad Sci USA 59: 944-950, $1968 \mathrm{a}$.

Changeux JP \& Rubin MM. Allosteric interactions in aspartate transcarbamylase. 3. Interpretation of experimental data in terms of the model of Monod, Wyman, and Changeux. Biochemistry 7: 553-561, 1968b.

Changeux JP \& Edelstein SJ. Allosteric mechanisms in normal and pathological nicotinic acetylcholine receptors. Curr Opin Neurobiol 11: 369-377, 2001

Changeux JP \& Edelstein SJ. Allosteric mechanisms of signal transduction. Science 308: 1424-1428, 2005.

Changeux JP, Thiery J, Tung Y \& Kittel C. On the cooperativity of biological membranes. Proc Natl Acad Sci USA 57: 335-341, 1967.

Changeux JP, Gerhart JC \& Schachman HK. Allosteric interactions in aspartate transcarbamylase. I. Binding of specific ligands to the native enzyme and its isolated subunits. Biochemistry 7: 531-538, 1968.

Cheetham GM. Novel protein kinases and molecular mechanisms of autoinhibition. Curr Opin Struct Biol 14: 700-705, 2004.
Chen YH, Li MH, Zhang Y, He LL, Yamada Y, Fitzmaurice A, Shen Y, Zhang H, Tong L \& Yang J. Structural basis of the alphal-beta subunit interaction of voltage-gated $\mathrm{Ca} 2+$ channels. Nature 429: 675-680, 2004.

Cheng A, Xiong W, Ferrell JE Jr \& Solomon MJ. Identification and comparative analysis of multiple mammalian Speedy/Ringo proteins. Cell Cycle 4: 155-165, 2005.

Choi B \& Zocchi G. Guanylate kinase, induced fit, and the allosteric spring probe. Biophys J 92: 1651-1658, 2007.

Christopoulos A. Allosteric binding sites on cell-surface receptors: novel targets for drug discovery. Nat Rev Drug Discov 1: 198-210, 2002.

Christopoulos A \& Kenakin T. G protein coupled receptor allosterism and complexing. Pharmacol Rev 54: 323-374, 2002.

Christopoulos A, Lanzafame A \& Mitchelson F. Allosteric interactions at muscarinic cholinoceptors. Clin Exp Pharmacol Physiol 25: 185194, 1998.

Christopoulos A, Parsons AM, Lew MJ \& El-Fakahany EE. The assessment of antagonist potency under conditions of transient response kinetics. Eur J Pharmacol 382: 217-227, 1999.

Christopoulos A, May LT, Avlani VA \& Sexton PM. G-protein-coupled receptor allosterism: the promise and the problem(s). Biochem Soc Trans 32: 873-877, 2004.

Colozo AT, Park PS, Sum CS, Pisterzi LF \& Wells JW. Cholesterol as a determinant of cooperativity in the $\mathrm{M}(2)$ muscarinic cholinergic receptor. Biochem Pharmacol 74: 236-255, 2007.

Colquhoun D. The relation between classical and cooperative models for drug action. In: Drug Receptors. A Symposium, edited by Rang HP. London: MacMillan Press, 1973.

Colquhoun D. Binding, gating, affinity and efficacy: the interpretation of structure-activity relationships for agonists and of the effects of mutating receptors. Br J Pharmacol 125: 924-947, 1998.

Colquhoun D. Agonist-activated ion channels. Br J Pharmacol 147: S17S26, 2006.

Conway A \& Koshland DE Jr. Negative cooperativity in enzyme action. The binding of diphosphopyridine nucleotide to glyceraldehyde 3-phosphate dehydrogenase. Biochemistry 7: 4011-4023, 1968.

Cornelius F, Fedosova NU \& Klodos I. E2P phosphoforms of Na,KATPase. II. Interaction of substrate and cation-binding sites in Pi phosphorylation of Na,K-ATPase. Biochemistry 37: 16686-16696, 1998.

Cornish-Bowden A. Fundamentals of Enzyme Kinetics, 2nd ed. London: Portland Press, 1995.

Cornish-Bowden A. Fundamentals of Enzyme Kinetics, 3rd ed. Colchester: Portland Press Ltd., 2004a.

Cornish-Bowden A. The Pursuit of Perfection. Aspects of Biochemical Evolution. Oxford: Oxford University Press, 2004b.

Costa T \& Herz A. Antagonists with negative intrinsic activity at delta opioid receptors coupled to GTP binding proteins. Proc Natl Acad Sci USA 86: 7321-7325, 1989.

Costa $\mathrm{T}$ \& Cotecchia S. Historical review: negative efficacy and constitutive activity of G-protein-coupled receptors. Trend Pharmacol Sci 26: 618-624, 2005.

Cotecchia S. Constitutive activity and inverse agonism at the alpha(1) adrenoceptors. Biochem Pharmacol 73: 1076-1083, 2007.

De Lean A, Stadel JM \& Lefkowitz RJ. A ternary complex model explains the agonist specific binding properties of the adenylate cyclase coupled beta-adrenergic receptor. J Biol Chem 255: 7108$7117,1980$.

de Ligt RA, Kourounakis AP \& Ijzerman AP. Inverse agonism at G protein-coupled receptors: (patho) physiological relevance and implications for drug discovery. Br J Pharmacol 130: 1-12, 2000.

del Castillo J \& Katz B. Interaction at end-plate receptors between different choline derivatives. J Physiol 146: 369-381, 1957. 
Dhami GK \& Ferguson SS. Regulation of metabotropic glutamate receptor signaling, desensitization and endocytosis. Pharmacol Ther 111: 260-267, 2006.

DiFrancesco D. Dual allosteric modulation of pacemaker (f) channels by cAMP and voltage in rabbit SA node. J Physiol 515: 367-376, 1999.

Dinarina A, Perez LH, Davila A, Schwab M, Hunt T \& Nebreda AR. Characterization of a new family of cyclin-dependent kinase activators. Biochem J 386: 349-355, 2005.

Douglas CG, Haldane JS \& Haldane JBS. The laws of combination of haemoglobin with carbon monoxide and oxygen. $J$ Physiol 44: 275-304, 1912.

Edelstein SJ. Cooperative interactions of hemoglobin. Annu Rev Biochem 44: 209-232, 1975.

Edelstein SJ \& Changeux JP. Allosteric transitions of the acetylcholine receptor. Adv Protein Chem 51: 121-184, 1998.

Ehlert FJ. Estimation of the affinities of allosteric ligands using radioligand binding and pharmacological null methods. Mol Pharmacol 33: 187-194, 1988.

Ehlert FJ and Griffin MT. Two-state Models and the Analysis of the Allosteric Effect of Gallamine at the M2 Muscarinic Receptor. J Pharmacol Exp Ther Feb 27; [Epub ahead of print]: 2008.

Eigen M. Kinetics of reaction control and information transfer in enzymes and nucleic acids. In: Fast Reactions and Primary Processes in Chemical Kinetics. Nobel Symposium 5, edited by Claesson S. Stockholm: Almquist \& Wiksell, 1967.

Eigen M. New looks and outlooks on physical enzymology. Q Rev Biophys 1: 3-33, 1968.

Einholm AP, Toustrup-Jensen M, Andersen JP \& Vilsen B. Mutation of Gly-94 in transmembrane segment $\mathrm{M} 1$ of $\mathrm{Na}+, \mathrm{K}+-\mathrm{ATPa} e$ interferes with $\mathrm{Na}+$ and $\mathrm{K}+$ binding in E2P conformation. Proc Natl Acad Sci USA 102: 11254-11259, 2005.

Eisenstein E. Energetics of cooperative ligand binding to the active sites of biosynthetic threonine deaminase from Escherichia coli. J Biol Chem 269: 29416-29422, 1994.

Eisenstein E, Yu HD \& Schwarz FP. Cooperative binding of the feedback modifiers isoleucine and valine to biosynthetic threonine deaminase from Escherichia coli. J Biol Chem 269: 29429, 1994.

Eisenstein E, Yu HD, Fisher KE, Iacuzio DA, Ducote KR \& Schwarz FP. An expanded two-state model accounts for homotropic cooperativity in biosynthetic threonine deaminase from Escherichia coli. Biochemistry 34: 9403-9412, 1995.

Eskandari S, Wright EM \& Loo DD. Kinetics of the reverse mode of the $\mathrm{Na}+$ /glucose cotransporter. J Membr Biol 204: 23-32, 2005.

Ferrell CM, Lauf PK, Wilson BA \& Adragna NC. Lithium and protein kinase $\mathrm{C}$ modulators regulate swelling-activated $\mathrm{K}-\mathrm{Cl}$ cotransport and reveal a complete phosphatidylinositol cycle in low $\mathrm{K}$ sheep erythrocytes. J Membr Biol 177: 81-93, 2000.

Fersht A. Structure and Mechanism in Protein Science. A Guide to Enzyme Catalysis and Protein Folding. New York: WH Freeman \& Co., 1999.

Fetler L \& Vachette P. Revisiting the allosteric mechanism of aspartate transcarbamoylase. Nat Struct Biol 9: 87-89, 2002.

Fetler L, Kantrowitz ER \& Vachette P. Direct observation in solution of a preexisting structural equilibrium for a mutant of the allosteric aspartate transcarbamoylase. Proc Natl Acad Sci USA 104: 495-500, 2007.

Frieden C. Treatment of enzyme kinetic data. I. The effect of modifiers on the kinetic parameters of single substrate enzymes. J Biol Chem 239: 3533-3543, 1964.

Frieden C. Kinetic aspects of regulation of metabolic processes. The hysteretic enzyme concept. J Biol Chem 245: 5788-5799, 1970.

Fromm HJ. Initial Rate Enzyme Kinetics. Berlin: Springer-Verlag, 1975.

Gainetdinov RR, Premont RT, Bohn LM, Lefkowitz RJ \& Caron MG. Desensitization of $\mathrm{G}$ protein-coupled receptors and neuronal functions. Annu Rev Neurosci 27: 107-144, 2004.
Galetin A, Ito K, Hallifax D \& Houston JB. Cyp3A4 substrate selection and substitution in the prediction of potential drug/drug interaction.J Pharmacol Exp Ther 314: 180-190, 2005.

Gerhart JC. A discussion of the regulatory properties of aspartate transcarbamlase from Escherichia coli. In: Current Topics in Cellular Regulation, edited by Horecker BL \& Stadtman ER. New York: Academic Press, 1970.

Gerhart JC \& Pardee AB. The enzymology of control by feedback inhibition. J Biol Chem 237: 891-896, 1962.

Gerhart JC \& Schachman HK. Allosteric interactions in aspartate transcarbamylase. II. Evidence for different conformational states of the protein in the presence and absence of specific ligands. Biochemistry 7: 538-552, 1968.

Giniatullin R, Nistri A \& Yakel JL. Desensitization of nicotinic ACh receptors: shaping cholinergic signaling. Trends Neurosci 28: 371378, 2005.

Giraldo J. Agonist induction, conformational selection, and mutant receptors. FEBS Lett 256: 13-18, 2004.

Grosman C \& Auerbach A. Kinetic, mechanistic, and structural aspects of unliganded gating of acetylcholine receptor channels: a singlechannel study of second transmembrane segment 12' mutants. J Gen Physiol 115: 621-635, 2000.

Haber JE \& Koshland DE Jr. Relation of protein subunit interactions to the molecular species observed during cooperative binding of ligands. Proc Natl Acad Sci USA 58: 2087-2093, 1967.

Hall DA. Modeling the functional effects of allosteric modulators at pharmacological receptors: an extension of the two-state model of receptor activation. Mol Pharmacol 58: 1412-1423, 2000.

Hambrock A, Kayar T, Stumpp D \& Osswald H. Effect of two amino acids in TM17 of Sulfonylurea receptor SUR1 on the binding of ATP-sensitive K+ channel modulators. Diabetes 53: S128-S134, 2004.

Hatton CJ, Shelley C, Brydson M, Beeson D \& Colquhoun D. Properties of the human muscle nicotinic receptor, and of the slow-channel myasthenic syndrome mutant epsilonL221F, inferred from maximum likelihood fits. J Physiol 547: 729-760, 2003.

Haurowitz F. Das gleichgewicht zwischen hämoglobin und sauerstoff. Hoppe-Seyler Z Physiol Chem 254: 266-274, 1938.

Helmstaedt K, Krappmann S \& Braus GH. Allosteric regulation of catalytic activity: Escherichia coli aspartate transcarbamoylase versus yeast chorismate mutase. Microbiol Mol Biol Rev 65: 404-421, 2001.

Hill AV. The mode of action of nicotine and curari, determined by the form of the concentration curve and the method of temperature coefficients. J Physiol (Lond) 39: 361-373, 1910.

Hill AV. The combinations of haemoglobin with oxygen and carbon monoxide. Biochem J 7: 471-480, 1913.

Hill TL. Cooperativity Theory in Biochemistry. Steady-state and Equilibrium Systems. Berlin: Springer-Verlag, 1985.

Hille B. Ionic Channels of Excitable Membranes. Sunderland: Sinauer Associates, 2001.

Hines JK, Kruesel CE, Fromm HJ \& Honzatko RB. Structure of inhibited fructose-1,6-bisphosphatase from Escherichia coli: distinct allosteric inhibition sites for AMP and glucose 6-phosphate and the characterization of a gluconeogenic switch. J Biol Chem 282: 24697-24706, 2007a.

Hines JK, Chen X, Nix JC, Fromm HJ \& Honzatko RB. Structures of mammalian and bacterial fructose-1,6-bisphosphatase reveal the basis for synergism in AMP/fructose 2,6-bisphosphate inhibition. J Biol Chem 282: 36121-36131, 2007b.

Hirayama BA, Loo DD, Díez-Sampedro A, Leung DW, Meinild AK, LaiBing M, Turk E and Wright EM. Sodium-dependent reorganization of the sugar-binding site of SGLT1. Biochemistry 46: 1339113406, 2007.

Hirsh L, Ben-Ami I, Freimann S, Dantes A, Tajima K, Kotsuji F \& Amsterdam A. Desensitization to gonadotropic hormones: a model 
system for the regulation of a G-protein-coupled receptor with 7-transmembrane spanning regions. Biochem Biophys Res Commun 326: 1-6, 2005.

Hlavackova V, Goudet C, Kniazeff J, Zikova A, Maurel D, Vol C, Trojanova J, Prezeau L, Pin JP \& Blahos J. Evidence for a single heptahelical domain being turned on upon activation of a dimeric GPCR. EMBO J 24: 499-509, 2005.

Holst B, Lang M, Brandt E, Bach A, Howard A, Frimurer TM, BeckSickinger A \& Schwartz TW. Ghrelin receptor inverse agonists: identification of an active peptide core and its interaction epitopes on the receptor. Mol Pharmacol 70: 936-946, 2006.

Holzgrabe U, De Amici M \& Mohr K. Allosteric modulators and selective agonists of muscarinic receptors. J Mol Neurosci 30: 165168, 2006.

Houston JB \& Galetin A. Modelling atypical CYP3A4 kinetics: principles and pragmatism. Arch Biochem Biophys 433: 351-360, 2005.

Iwata S, Kamata K, Yoshida S, Minowa T \& Ohta T. T and R states in the crystals of bacterial L-lactate dehydrogenase reveal the mechanism for allosteric control. Nat Struct Biol 1: 176-185, 1994.

Jackson MB. Spontaneous openings of the acetylcholine receptor channel. Proc Natl Acad Sci USA 81: 3901-3904, 1984.

Jackson MB. Kinetics of unliganded acetylcholine receptor channel gating. Biophys J 49: 663-672, 1986.

Jager M, Zhang Y, Bieschke J, Nguyen H, Dendle M, Bowman ME, Noel JP, Gruebele M \& Kelly JW. Structure-function-folding relationship in a WW domain. Proc Natl Acad Sci USA 103: 10648-10653, 2006.

Janin J. The study of allosteric proteins. Prog Biophys Mol Biol 27: 77120, 1973.

Jensen ML, Schousboe A \& Ahring PK. Charge selectivity of the Cysloop family of ligand-gated ion channels. J Neurochem 92: 217-225, 2005.

Jin L, Stec B, Lipscomb WN \& Kantrowitz ER. Insights into the mechanisms of catalysis and heterotropic regulation of Escherichia coli aspartate transcarbamoylase based upon a structure of the enzyme complexed with the bisubstrate analogue N-phosphonacetyl-lL-aspartate at 2.1 A. Proteins 37: 729-734, 1999.

John SA, Weiss JN, Xie LH \& Ribalet B. Molecular mechanism for ATPdependent closure of the $\mathrm{K}+$ channel Kir6.2. I Physiol 552: 2334, 2003.

Jones PM \& George AM. Nucleotide-dependent allostery within the ABC transporter ATP-binding cassette: a computational study of the MJ0796 dimer. I Biol Chem 282: 22793-22803, 2007.

Kamata K, Mitsuya M, Nishimura T, Eiki J \& Nagata Y. Structural basis for allosteric regulation of the monomeric allosteric enzyme human glucokinase. Structure (Camb) 12: 429-438, 2004.

Karlin A. On the application of 'a plausible model' of allosteric proteins to the receptor for acetylcholine. J Theor Biol 16: 306320, 1967.

Karpen JW \& Ruiz M. Ion channels: does each subunit do something on its own? Trends Biochem Sci 27: 402-409, 2002.

Katz B \& Thesleff S. A study of the desensitization produced by acetylcholine at the motor end-plate. J Physiol 138: 63-80, 1957.

Kenakin T. G-protein coupled receptors as allosteric machines. Receptors Channels 10: 51-60, 2004a

Kenakin T. Allosteric modulators: the new generation of receptor antagonist. Mol Interv 4: 222-229, 2004b.

Kenakin T, Morgan P, Lutz M \& Weiss J. The evolution of drugreceptor models: the cubic ternary complex model for $\mathrm{G}$ proteincoupled receptors. In: The Pharmacology of Functional, Biochemical, and Recombinant Receptor Systems, edited by Kenakin T \& Angus JA. Berlin: Springer Verlag, 2000.

Kew JN. Positive and negative allosteric modulation of metabotropic glutamate receptors: emerging therapeutic potential. Pharmacol Ther 104: 233-244, 2004.
Kirtley ME \& Koshland DE Jr. Models for cooperative effects in proteins containing subunits. Effects of two interacting ligands. J Biol Chem 242: 4192-4205, 1967.

Koshland DE Jr. Application of a theory of enzyme specificity to protein synthesis. Proc Natl Acad Sci USA 44: 98-104, 1958.

Koshland DE Jr. Crazy, but correct. Nature 432: 447, 2004.

Koshland DE Jr \& Hamadani K. Proteomics and models for enzyme cooperativity. J Biol Chem 277: 46841-46844, 2002.

Koshland DE Jr, Nemethy G \& Filmer D. Comparison of experimental binding data and theoretical models in proteins containing subunits. Biochemistry 5: 365-385, 1966.

Kurganov BI. The theoretical analysis of kinetic behaviour of 'hysteretic' allosteric enzymes. IV. Kinetics of dissociation-association processes of allosteric enzymes. J Theor Biol 68: 521-543, 1977.

Kurganov BI. Allosteric Enzymes. Kinetic Behaviour. Chichester: Wiley \& Sons, 1982.

Kurganov BI. New approach to analysis of deviations from hyperbolic law in enzyme kinetics. Biochemistry (Mosc) 65: 898-909, 2000.

Lameijer E-W, Kok JN, Back T \& Ijzerman AP. The molecule evaluator. An interactive evolutionary algorithm for the design of drug-like molecules. J Chem Inf Model 46: 545-552, 2006.

Larrayoz IM, Fernandez-Nistal A, Garces A, Gorraitz E \& Lostao MP. Characterization of the rat $\mathrm{Na}+$ /nucleoside cotransporter 2 and transport of nucleoside-derived drugs using electrophysiological methods. Am J Physiol Cell Physiol 291: C1395-C1404, 2006.

Lazareno S \& Birdsall NJ. Detection, quantitation, and verification of allosteric interactions of agents with labeled and unlabeled ligands at $\mathrm{G}$ protein-coupled receptors: interactions of strychnine and acetylcholine at muscarinic receptors. Mol Pharmacol 48: 362378, 1995.

Lazareno S, Popham A \& Birdsall NJ. Progress toward a high-affinity allosteric enhancer at muscarinic M1 receptors. J Mol Neurosci 20 363-367, 2003.

Leach K, Sexton PM \& Christopoulos A. Allosteric GPCR modulators: taking advantage of permissive receptor pharmacology. Trends Pharmacol Sci 28: 382-389, 2007.

Lee TW, Cherney MM, Liu J, James KE, Powers JC, Eltis LD and James MN. Crystal structures reveal an induced-fit binding of a substratelike aza-peptide epoxide to SARS coronavirus main peptidase. J Mol Biol 366: 916-932, 2007.

Leff P. The two-state model of receptor activation. Trends Pharmacol Sci 16: 89-97, 1995.

Lefkowitz RJ, Cotecchia S, Samama P \& Costa T. Constitutive activity of receptors coupled to guanine nucleotide regulatory proteins. Trends Pharmacol Sci 14: 303-307, 1993.

Levitzki A. Quantitative Aspects of Allosteric Mechanisms. Berlin: SpringerVerlag, 1978.

Levitzki A \& Koshland DE Jr. Negative cooperativity in regulatory enzymes. Proc Natl Acad Sci USA 62: 1121-1128, 1969.

Li G \& Qian H. Sensitivity and specificity amplification in signal transduction. Cell Biochem Biophys 39: 45-59, 2003.

Linder JU. Class III adenylyl cyclases: molecular mechanisms of catalysis and regulation. Cell Mol Life Sci 63: 1736-1751, 2006.

Lohse MJ, Nikolaev VO, Hein P, Hoffmann C, Vilardaga JP \& Bünemann M. Optical techniques to analyze real-time activation and signaling of G-protein-coupled receptors. Trends Pharmacol Sci 29: $159-165,2008$

Loo DD, Hirayama BA, Karakossian MH, Meinild AK \& Wright EM. Conformational dynamics of hSGLT1 during $\mathrm{Na}+$ /glucose cotransport. J Gen Physiol 128: 701-720, 2006.

Lutz M \& Kenakin T. Quantitative Molecular Pharmacology and Informatics in Drug Discovery. Chichester: Wiley \& Sons, 1999.

Macol CP, Tsuruta H, Stec B \& Kantrowitz ER. Direct structural evidence for a concerted allosteric transition in Escherichia coli aspartate transcarbamoylase. Nat Struct Biol 8: 423-426, 2001. 
Mahler HR \& Cordes EH. Biological Chemistry. London: Harper \& Row Ltd., 1966.

Marie N, Aguila B \& Allouche S. Tracking the opioid receptors on the way of desensitization. Cell Signal 18: 1815-1833, 2006.

May LT, Lin Y, Sexton PM \& Christopoulos A. Regulation of M2 muscarinic acetylcholine receptor expression and signaling by prolonged exposure to allosteric modulators. J Pharmacol Exp Ther 312: 382-390, 2005.

May LT, Leach K, Sexton PM \& Christopoulos A. Allosteric modulation of G protein-coupled receptors. Annu Rev Pharmacol Toxicol 47: $1-51,2007$.

Meyer S, Savaresi S, Forster IC \& Dutzler R. Nucleotide recognition by the cytoplasmic domain of the human chloride transporter ClC-5. Nat Struct Mol Biol 14: 60-67, 2007.

Michel D. Cooperative equilibrium curves generated by ordered ligand binding to multi-site molecules. Biophys Chem 129: 284-288, 2007.

Miller C. ClC chloride channels viewed through a transporter lens. Nature 440: 484-489, 2006.

Milligan G \& Smith NJ. Allosteric modulation of heterodimeric G-protein-coupled receptors. Trends Pharmacol Sci 28: 615-620, 2007.

Min G, Kim H, Bae Y, Petz L \& Kemper JK. Inhibitory cross-talk between estrogen receptor (ER) and constitutively activated androstane receptor (CAR). CAR inhibits ER-mediated signaling pathway by squelching p160 coactivators. J Biol Chem 277: 3462634633, 2002.

Monod J. From enzymatic adaptation to allosteric transition. In: Nobel Lectures, Physiology or Medicine 1963-1970. Amsterdam: Elsevier Publishing Co., 1972.

Monod J \& Jacob F. Teleonomic mechanisms in cellular metabolism, growth, and differentiation. Cold Spring Harb Symp Quant Biol 26 : 389-401, 1961.

Monod J, Changeux JP \& Jacob F. Allosteric proteins and cellular control systems. J Mol Biol 6: 306-329, 1963.

Monod J, Wyman J \& Changeux J-P. On the nature of allosteric transitions: a plausible model. J Mol Biol 12: 88-118, 1965.

Morth JP, Pedersen BP, Toustrup-Jensen MS, Sørensen TL, Petersen J, Andersen JP, Vilsen B \& Nissen P. Crystal structure of the sodiumpotassium pump. Nature 450: 1043-1049, 2007.

Muirhead H \& Perutz M. Structure of haemoglobin. A threedimensional Fourier synthesis of reduced human haemoglobin at 55 a resolution. Nature 199: 633-639, 1963.

Munson PJ. Ligand: a computerized analysis of ligand binding data. Methods Enzymol 92: 543-576, 1983

Neet KE. Cooperativity in enzyme function: equilibrium and kinetic aspects. Series editor Purich DL. Enzyme Kinetics and mechansims. Editors. Abelson JN and Simon MI. Methods Enzymol 249: 519-567. New York, Acad Press, 1995.

Olesen C, Picard M, Winther AM, Gyrup C, Morth JP, Oxvig C, Møller JV \& Nissen P. The structural basis of calcium transport by the calcium pump. Nature 450: 1036-1042, 2007.

Papa S, Capitanio N, Capitanio G \& Palese LL. Protonmotive cooperativity in cytochrome c oxidase. Biochim Biophys Acta 1658: 95-105, 2004.

Parmentier ML, Prezeau L, Bockaert J \& Pin JP. A model for the functioning of family 3 GPCRs. Trends Pharmacol Sci 23: 268-274, 2002.

Pauling L. The oxygen equilibrium of hemoglobin and its structural interpretation. Proc Nat Acad Sci 21: 186-191, 1935.

Perutz M. Mechanisms of Cooperativity and Allosteric Regulation in Proteins. Cambridge: Cambridge University Press, 1990.

Perutz MF. Stereochemistry of cooperative effects in haemoglobin. Nature 228: 726-739, 1970.

Perutz M, Wilkinson AJ, Paoli M \& Dodson GG. The stereochemical mechanism of the cooperative effects in hemoglobin revisited. Annu Rev Biophys Biomol Struct 27: 1-34, 1998.
Peterson CB, Burman DL \& Schachman HK. Effects of replacement of active site residue glutamine 231 on activity and allosteric properties of aspartate transcarbamoylase. Biochemistry 31: 85088515, 1992.

Peterson JR \& Golemis EA. Autoinhibited proteins as promising drug targets. J Cell Biochem 93: 68-73, 2004.

Pin JP, Kniazeff J, Binet V, Liu J, Maurel D, Galvez T, Duthey B, Havlickova M, Blahos J, Prezeau L \& Rondard P. Activation mechanism of the heterodimeric GABA(B) receptor. Biochem Pharmacol 68: 1565-1572, 2004.

Pinkett HW, Lee AT, Lum P, Locher KP \& Rees DC. An inward-facing conformation of a putative metal-chelate-type ABC transporter. Science 315: 373-377, 2007.

Plenge P, Gether U \& Rasmussen SG. Allosteric effects of R- and Scitalopram on the human 5-HT transporter: evidence for distinct high- and low-affinity binding sites. Eur J Pharmacol 567: 1-9, 2007.

Poirier A, Funk C, Lave T \& Noe J. New strategies to address drugdrug interactions involving OATPs. Curr Opin Drug Discov Dev 10 74-83, 2007.

Quinton PM. Cystic fibrosis: lessons from the sweat gland. Physiology (Bethesda) 22: 212-225, 2007.

Reddy MM \& Quinton PM. Deactivation of CFTR-Cl conductance by endogenous phosphatases in the native sweat duct. Am J Physiol 270: C474-C480, 1996.

Ricard J. Concepts and models of enzyme cooperativity. In: Allosteric Enzymes, edited by Herve G. Boca Raton, FL: CRC Press, 1989.

Ricard J \& Cornish-Bowden A. Co-operative and allosteric enzymes: 20 years on. Eur J Biochem 166: 256-272, 1987.

Roughton FJW, Otis $\mathrm{AB} \&$ Lyster RLJ. The determination of the individual equilibrium constants of the four intermediate reactions between oxygen and sheep haemoglobin. Proc Roy Soc B 144: $29-54,1955$.

Rubin MM \& Changeux JP. On the nature of allosteric transitions: implications of non-exclusive ligand binding. J Mol Biol 21: 265274, 1966.

Rubinow SI. Introduction to Mathematical Biology. New York: Wiley \& Sons, 1975

Rypniewski WR \& Evans PR. Crystal structure of unliganded phosphofructokinase from Escherichia coli. J Mol Biol 207: 805-821, 1989.

Sablin EP, Krylova IN, Fletterick RJ \& Ingraham HA. Structural basis for ligand-independent activation of the orphan nuclear receptor LRH-1. Mol Cell 11: 1575-1585, 2003.

Salas-Burgos A, Iserovich P, Zuniga F, Vera JC \& Fischbarg J. Predicting the three-dimensional structure of the human facilitative glucose transporter glut1 by a novel evolutionary homology strategy: insights on the molecular mechanism of substrate migration, and binding sites for glucose and inhibitory molecules. Biophys $J$ 87: 2990-2999, 2004.

Scaramellini C \& Leff P. A three-state receptor model: predictions of multiple agonist pharmacology for the same receptor type. Ann $N$ Y Acad Sci 861: 97-103, 1998.

Scaramellini C \& Leff P. Theoretical implications of receptor coupling to multiple $\mathrm{G}$ proteins based on analysis of a three-state model. Methods Enzymol 343: 17-29, 2002.

Schnappauf G, Lipscomb WN \& Braus GH. Separation of inhibition and activation of the allosteric yeast chorismate mutase. Proc Natl Acad Sci USA 95: 2668-2673, 1998.

Schreiber G \& Avissar S. Regulators of G-protein-coupled receptorG-protein coupling: antidepressants mechanism of action. Expert Rev Neurother 7: 75-84, 2007.

Segel IH. Enzyme Kinetics. Behavior and Analysis of Rapid Equilibrium and Steady-state Enzyme Systems. New York: Wiley \& Sons (reissued 1993), 1975.

Sergeeva OA, Andreeva N, Garret M, Scherer A \& Haas HL. Pharmacological properties of GABAA receptors in rat hypothalamic neurons expressing the epsilon-subunit. JNeurosci 25: 88-95, 2005. 
Shou M. Kinetic analysis for multiple substrate interaction at the active site of cytochrome P450. Methods Enzymol 357: 261-276, 2002.

Shou M, Mei Q, Ettore MW Jr, Dai R, Baillie TA \& Rushmore TH. Sigmoidal kinetic model for two co-operative substrate-binding sites in a cytochrome P450 3A4 active site: an example of the metabolism of diazepam and its derivatives. Biochem J 340: 845853, 1999.

Shou M, Dai R, Cui D, Korzekwa KR, Baillie TA \& Rushmore TH. A kinetic model for the metabolic interaction of two substrates at the active site of cytochrome P450 3A4. J Biol Chem 276: 22562262, 2001.

Sintchak MD, Arjara G, Kellogg BA, Stubbe J \& Drennan CL. The crystal structure of class II ribonucleotide reductase reveals how an allosterically regulated monomer mimics a dimer. Nat Struct Biol 9: 293-300, 2002.

Smit MJ, Vischer HF, Bakker RA, Jongejan A, Timmerman H, Pardo L \& Leurs R. Pharmacogenomic and structural analysis of constitutive g protein-coupled receptor activity. Annu Rev Pharmacol Toxicol 47: 53-87, 2007.

Sprang SR, Chen Z \& Du X. Structural basis of effector regulation and signal termination in heterotrimeric Galpha proteins. Adv Protein Chem. 74: 1-65, 2007.

Springael JY, Urizar E, Costagliola S, Vassart G \& Parmentier M. Allosteric properties of $\mathrm{G}$ protein-coupled receptor oligomers. Pharmacol Ther 115: 410-418, 2007.

Stebbins JW, Zhang Y \& Kantrowitz ER. Importance of residues Arg-167 and Gln-231 in both the allosteric and catalytic mechanisms of Escherichia coli aspartate transcarbamoylase. Biochemistry 29: 38213827, 1990.

Stieglitz K, Stec B, Baker DP \& Kantrowitz ER. Monitoring the transition from the $\mathrm{T}$ to the $\mathrm{R}$ state in $E$. coli aspartate transcarbamoylase by X-ray crystallography: crystal structures of the E50A mutant enzyme in four distinct allosteric states. $J$ Mol Biol 341: 853-868, 2004.

Stockton JM, Birdsall NJ, Burgen AS \& Hulme EC. Modification of the binding properties of muscarinic receptors by gallamine. Mol Pharmacol 23: 551-557, 1983.

Stryer L. Biochemistry. New York: WH Freeman \& Co., 1995.

Suzuki Y, Moriyoshi E, Tsuchiya D \& Jingami H. Negative cooperativity of glutamate binding in the dimeric metabotropic glutamate receptor subtype 1. J Biol Chem 279: 35526-35534, 2004.

Taly A, Corringer PJ, Grutter T, Prado de Carvalho L, Karplus M \& Changeux JP. Implications of the quaternary twist allosteric model for the physiology and pathology of nicotinic acetylcholine receptors. Proc Natl Acad Sci USA 103: 16965-16970, 2006.

Teipel J \& Koshland DE Jr. The significance of intermediary plateau regions in enzyme saturation curves. Biochemistry 8: 4656-4663, 1969.

Thron CD. On the analysis of pharmacological experiments in terms of an allosteric receptor model. Mol Pharmacol 9: 1-9, 1973.

Tipton KF. Patterns of enzyme inhibition. In: Enzymology, edited by Engel PC. San Diego, CA: Bios Scientific Publishers Ltd., 1996.

Tsuruta H, Kihara H, Sano T, Amemiya Y \& Vachette P. Influence of nucleotide effectors on the kinetics of the quaternary structure transition of allosteric aspartate transcarbamylase. J Mol Biol 348: 195-204, 2005

Tucek S \& Proska J. Allosteric modulation of muscarinic acetylcholine receptors. Trends Pharmacol Sci 16: 205-212, 1995.

Tucek S, Michal P \& Vlachova V. Modelling the consequences of receptor-G-protein promiscuity. Trends Pharmacol Sci 23: 171-176, 2002.

Tuk B \& van Oostenbruggen MF. Solving inconsistencies in the analysis of receptor-ligand interactions. Trend Pharmacol Sci 17: 403-409, 1996.

Umbarger HE. Evidence for a negative-feedback mechanism in the biosynthesis of isoleucine. Science 123: 848, 1956.
Umbarger HE \& Brown B. Threonine deamination in Escherichia coli. II. Evidence for two lL-threonine deaminases. J Bacteriol 73: 105112, 1957.

Valiyaveetil FI, Leonetti M, Muir TW \& MacKinnon R. Ion selectivity in a semisynthetic $\mathrm{K}+$ channel locked in the conductive conformation. Science 314: 1007, 2006.

Van Aubel RA, Smeets PH, van den Heuvel JJ \& Russel FG. Human organic anion transporter MRP4 (ABCC4) is an efflux pump for the purine end metabolite urate with multiple allosteric substrate binding sites. Am J Physiol Renal Physiol 288: F327-F333, 2005.

Wang C, Karpowich N, Hunt JF, Rance M \& Palmer AG. Dynamics of ATP-binding cassette contribute to allosteric control, nucleotide binding and energy transduction in ABC transporters. J Mol Biol 342: 525-537, 2004.

Wang J, Stieglitz KA, Cardia JP \& Kantrowitz ER. Structural basis for ordered substrate binding and cooperativity in aspartate transcarbamoylase. Proc Natl Acad Sci USA 102: 8881-8886, 2005.

Wang J, Eldo J \& Kantrowitz ER. Structural model of the R state of Escherichia coli aspartate transcarbamoylase with substrates bound. J Mol Biol 371: 1261-1273, 2007.

Wangensteen OS, Chueca A, Hirasawa M, Sahrawy M, Knaff DB \& Lopez Gorge J. Binding features of chloroplast fructose-1, 6-bisphosphatase-thioredoxin interaction. Biochim Biophys Acta 1547: 156-166, 2001

Weber CR, Ginsburg KS, Philipson KD, Shannon TR \& Bers DM. Allosteric regulation of $\mathrm{Na} / \mathrm{Ca}$ exchange current by cytosolic $\mathrm{Ca}$ in intact cardiac myocytes. J Gen Physiol 117: 119-131, 2001.

Weber G. Ligand binding and internal equilibria in proteins. Biochemistry 11: 864-878, 1972.

Weiss JM, Morgan PH, Lutz MW \& Kenakin TP. The cubic ternary complex receptor-occupancy model I. Model description. J Theoret Biol 178: 151-167, 1996a.

Weiss JM, Morgan PH, Lutz MW \& Kenakin TP. The cubic ternary complex receptor-occupancy model II. Understanding apparent affinity. J Theor Biology 178: 169-182, 1996b.

Weiss JM, Morgan PH, Lutz MW \& Kenakin TP. The cubic ternary complex receptor occupancy model. III. resurrecting efficacy. J Theor Biol 181: 381-397, 1996c.

Weiss JN. The Hill equation revisited: uses and misuses. FASEB $J$ 11: 835-841, 1997.

Wells JW. Analysis and interpretation of binding at equilibrium. In: Receptor-ligand Interactions. A Practical Approach, edited by Hulme EC. Oxford: IRL Press at Oxford University Press, 1992.

Wong JT-F. Kinetics of enzyme mechanisms. London: Academic Press, 1975 .

Wreggett KA \& Wells JW. Cooperativity manifest in the binding properties of purified cardiac muscarinic receptors. J Biol Chem 270: 22488-22499, 1995.

Wright EM, Loo DD, Panayotova-Heiermann M, Lostao MP, Hirayama BH, Mackenzie B, Boorer K \& Zampighi G. 'Active' sugar transport in eukaryotes. J Exp Biol 196: 197-212, 1994.

Wright EM, Hirayama BA and Loo DF. Active sugar transport in health and disease. J Intern Med 261: 32-43, 2007.

Wyman J. Allosteric linkage. J Am Chem Soc 89: 2202-2218, 1967.

Wyman J. The turning wheel: a study in steady state. Proc Natl Acad Sci USA 72: 3983-3987, 1975.

Wyman J \& Gill SJ. Binding and Linkage. Functional Chemistry of Biological Macromolecules. New York: Freeman, 1990.

Wyman J \& Allen DW. The problem of the heme interactions in hemoglobin and the basis of the Bohr effect. J Polym Sci 7: 499518, 1951.

Yates RA \& Pardee AB. Control of pyrimidine biosynthesis in Escherichia coli by a feed-back mechanism. $J$ Biol Chem 221: 757-770, 1956.

Ye S, Li Y, Chen L \& Jiang Y. Crystal structures of a ligand-free MthK gating ring: insights into the ligand gating mechanism of $\mathrm{K}+$ channels. Cell 126: 1161-1173, 2006. 
Yonetani T \& Tsuneshige A. The global allostery model of hemoglobin: an allosteric mechanism involving homotropic and heterotropic interactions. C R Biol 326: 523-532, 2003.

Yusef YR, Zuniga L, Catalan M, Niemeyer MI, Cid LP \& Sepulveda FV. Removal of gating in voltage-dependent ClC-2 chloride channel by point mutations affecting the pore and C-terminus CBS-2 domain. J Physiol 572: 173-181, 2006.

Zhou Y, Pearson JE \& Auerbach A. Phi-value analysis of a linear, sequential reaction mechanism: theory and application to ion channel gating. Biophys J 89: 3680-3685, 2005.
Zhou Z \& Bai Y. Structural biology: analysis of protein-folding cooperativity. Nature 445: E16-E17, 2007.

Zottola RJ, Cloherty EK, Coderre PE, Hansen A, Hebert DN \& Carruthers A. Glucose transporter function is controlled by transporter oligomeric structure. A single, intramolecular disulfide promotes GLUT1 tetramerization. Biochemistry 34: 9734 9747, 1995. 


\section{The mirror image, yes}

is based on physical projected-light as well as tête-à-tête expectancy thus, mirror-you has left to right, whilst foot is foot and heads without aberrancy 